\title{
Assessment of Carbon Tetrachloride Groundwater Transport in Support of the Hanford Carbon Tetrachloride Innovative Technology Demonstration Program
}
M. J. Truex
C. J. Murray
C. R. Cole
R. J. Cameron
M. D. Johnson
R. S. Skeen
C. D. Johnson

July 2001

Prepared for the U.S. Department of Energy

Under Contract DE-AC06-76RL01830 


\title{
DISCLAIMER
}

This report was prepared as an account of work sponsored by an agency of the United States Government. Neither the United States Government nor any agency thereof, nor Battelle Memorial Institute, nor any of their employees, makes any warranty, express or implied, or assumes any legal liability or responsibility for the accuracy, completeness, or usefulness of any information, apparatus, product, or process disclosed, or represents that its use would not infringe privately owned rights. Reference herein to any specific commercial product, process, or service by trade name, trademark, manufacturer, or otherwise does not necessarily constitute or imply its endorsement, recommendation, or favoring by the United States Government or any agency thereof, or Battelle Memorial Institute. The views and opinions of authors expressed herein do not necessarily state or reflect those of the United States Government or any agency thereof.

\author{
PACIFIC NORTHWEST NATIONAL LABORATORY \\ operated by \\ BATTELLE \\ for the \\ UNITED STATES DEPARTMENT OF ENERGY \\ under Contract DE-AC06-76RL01830
}

This document was printed on recycled paper. 


\title{
Assessment of Carbon Tetrachloride Groundwater Transport in Support of the Hanford Carbon Tetrachloride Innovative Technology Demonstration Program
}

\author{
M. J. Truex \\ C. J. Murray \\ C. R. Cole \\ R. J. Cameron \\ M. D. Johnson \\ R. S. Skeen \\ C. D. Johnson
}

July 2001

Pacific Northwest National Laboratory

Richland, WA 99352 


\section{Executive Summary}

Groundwater modeling was performed in support of the Hanford Carbon Tetrachloride Innovative Treatment Remediation Demonstration (ITRD) Program. The ITRD program is facilitated by Sandia National Laboratories for the Department of Energy (DOE) Office of Science and Technology. This report was prepared to document the results of the modeling effort and facilitate discussion of characterization and remediation options for the carbon tetrachloride (CT) plume among the ITRD participants.

As a first step toward implementation of innovative technologies for remediation of the CT plume underlying the 200 West Area, modeling was performed to provide an indication of the potential impact of the CT source on the compliance boundary approximately $5000 \mathrm{~m}$ away. The primary objective of the modeling was to bracket the amount of CT source that will most likely result in compliance/non-compliance at the boundary and the relative influence of the various model input parameters.

The modeling was based on the assumption that about $750,000 \mathrm{~kg}$ of CT were discharged to the soil in the 200 West Area. Previous work has shown that, of this $750,000 \mathrm{~kg}$, about $65 \%$ cannot be accounted for (after totaling atmospheric losses [21\%]; unsaturated zone inventory in soil gas, soil moisture, and adsorbed phases [12\%]; and the dissolved phase in the aquifer [2\%]). Therefore, model simulations were performed using $65 \%, 30 \%, 10 \%$, and $1 \%$ of the $750,000 \mathrm{~kg}$ as possible source amounts of CT that could reach the groundwater (approximately 1 to $2 \%$ of the original CT inventory now exists in the distal plume based on averaged CT groundwater measurements). The modeling simulations conducted for this study examine the migration of CT from the source area to the compliance boundary. The simulations did not examine migration of the existing distal plume and did not attempt to match the historical disposal and migration of CT (i.e., did not attempt to reproduce the current CT plume distribution).

Other model input parameters including the groundwater Darcy velocity; inlet concentration (the concentration of CT leaving the source area); porosity; soil/water equilibrium partition coefficient $\left(\mathrm{K}_{\mathrm{d}}\right)$; abiotic degradation rate $\left(\mathrm{K}_{\mathrm{a}}\right)$; dispersivity; and stream tube cross-sectional area were also varied to assess sensitivity of the results to each of the parameters.

The CT transport simulation was conservatively modeled as a stream tube $1000 \mathrm{~m}$ wide by $5000 \mathrm{~m}$ long in one-dimensional flow (i.e., no vertical or transverse dispersivity or convection). Regional flow-modeling results using the Hanford Site-Wide Groundwater Model (SGM) flow grid provided groundwater velocity estimates for the simulation. The basic assumptions used in developing the model were as follows:

- The major source of contamination is within a $500 \mathrm{~m} \times 500 \mathrm{~m}$ box.

- The contaminant plume was in equilibrium with the source immediately before pumpand-treat efforts.

- Processes considered in the model are one-dimensional convective-dispersive transport of reactive solutes subject to adsorption and first-order abiotic degradation (hydrolysis).

- Volatilization and first-order natural biodegradation are negligible. 
The one-dimensional van Genuchten model simulated convective-dispersive transport of CT through a homogenous medium along the centerline of the contaminant plume from the 200 West source area to the compliance boundary, approximately $5000 \mathrm{~m}$ distant. One thousand Monte Carlo realizations were carried out. Fixed and deterministic parameters included the following:

- Stream tube length: $5000 \mathrm{~m}$

- Base inventory of contaminant: $750,000 \mathrm{~kg} \mathrm{CT}$

- Base porosity: $0.10 \mathrm{~m}^{3} / \mathrm{m}^{3}$

- Bulk density: $1950 \mathrm{~kg} / \mathrm{m}^{3}$

- Inlet concentration: $1500,2000,2500$ or $3000 \mu \mathrm{g} / \mathrm{L}$

- Amount of source remaining: $1 \%, 10 \%, 30 \%$, or $65 \%$

- Groundwater Darcy velocity: mean, $\pm \sigma, \pm 2 \sigma$.

Stochastic parameters varied by Monte Carlo methods included the following:

- Porosity

- Soil/water equilibrium partition coefficient $\mathrm{K}_{\mathrm{d}}$

- Abiotic degradation rate $\mathrm{K}_{\mathrm{a}}$

- Dispersivity

- Stream tube cross-sectional area

Parameter limits for $\mathrm{K}_{\mathrm{a}}$ and $\mathrm{K}_{\mathrm{d}}$ were determined by methods detailed in the report entitled Literature Review: Natural Attenuation Mechanisms and Rates for Chloromethane Subsurface Contamination at Hanford previously provided to the ITRD Technical Advisory Group and included as Appendix $\mathrm{C}$ to this report. Parameter limits for porosity and dispersivity were determined by geophysical methods and transport field studies.

Simulations including transverse and vertical dispersion/dilution were conducted to determine how the one-dimensional modeling results could be interpreted to estimate the effect of a three-dimensional flow field. In $80 \%$ of these simulations, the concentration at the compliance boundary was reduced by a factor of greater than 5 . In about $20 \%$ of these simulations, the concentration was reduced by a factor of greater than 10 with a maximum reduction factor of about 30. The results of these simulations were used to postulate that interpretations of onedimensional modeling results using a $50-\mu \mathrm{g} / \mathrm{L}$ boundary concentration limit provided a pseudothree-dimensional (ptd) estimate of CT transport at the higher end of the observed concentration reduction factor. This ptd estimate was used to provide a reasonable bound for the possible three-dimensional flow effects during transport of CT from the source area to the compliance boundary. CT concentration limits of $5 \mu \mathrm{g} / \mathrm{L}$ and $50 \mu \mathrm{g} / \mathrm{L}$ at the compliance boundary are used in the report. Note that the $50-\mu \mathrm{g} / \mathrm{L}$ compliance boundary concentration limit is solely a calculational construct to approximate a three-dimensional model, not a proposed change in the actual $5-\mu \mathrm{g} / \mathrm{L}$ compliance concentration requirement. Selection of the $50-\mu \mathrm{g} / \mathrm{L}$ compliance boundary 
concentration is based on using a factor of 10 correlation between one- and three-dimensional transport that was calculated based on the components of dispersion that are not accounted for in the one-dimensional model.

The modeling produced 1000 Monte Carlo simulations that were analyzed to provide an estimate of the portion of the source area needing remediation. The simulations also provided a method for gauging sensitivity of predicted contaminant transport to variations in individual model parameters.

The results of the Monte Carlo simulations were charted as histograms showing resultant distributions for 1000 modeling realizations using stochastic parameters. Simulation outputs include the following:

- Required source cleanup percentage needed to meet compliance regulations

- Source inlet rate

- Compliance boundary concentration

- Compliance boundary flux

- Compliance boundary arrival times

- Source duration

- Arrival time of peak concentration

- Positional concentration profile.

The results show frequency distributions of the required source cleanup percentages for the Monte Carlo simulations (variations of transport parameters $\mathrm{K}_{\mathrm{d}}, \mathrm{K}_{\mathrm{a}}$, porosity, dispersivity and cross-sectional area) at the mean Darcy velocity and $3000 \mu \mathrm{g} / \mathrm{L}$ inlet-source concentration. The required source cleanup percentage for each simulation was calculated from the ratio of the maximum concentration at the compliance boundary to the selected compliance concentration. This calculation assumes that reducing the source quantity by this percentage would decrease the source concentration by this same percentage; thus the concentration at the compliance boundary would decrease by this percentage as well. The basis of this assumption is that by removing source within a portion of the defined source area volume so that this "cleaned up" portion now contributes only clean water within the source, the source is diluted.

Figure ES-1 shows the results of the 1000 realizations at $10 \%$ of source inventory reaching the groundwater and indicates for over $60 \%$ of the simulations (i.e., different groupings of transport parameter values) the $50-\mu \mathrm{g} / \mathrm{L}$ compliance concentration will be exceeded at the compliance boundary unless some CT is removed from the source area. Figure ES-2 shows the results of the 1000 realizations for $1 \%$ of the source inventory reaching the groundwater and indicates that, for over $70 \%$ of the simulations, the $50-\mu \mathrm{g} / \mathrm{L}$ compliance concentration will not be exceed without any CT source removal.

The break point for cleanup percentages clearly lies between $1 \%$ and $10 \%$ of source inventory remaining. The input parameters in the simulation having the greatest effect on the results 


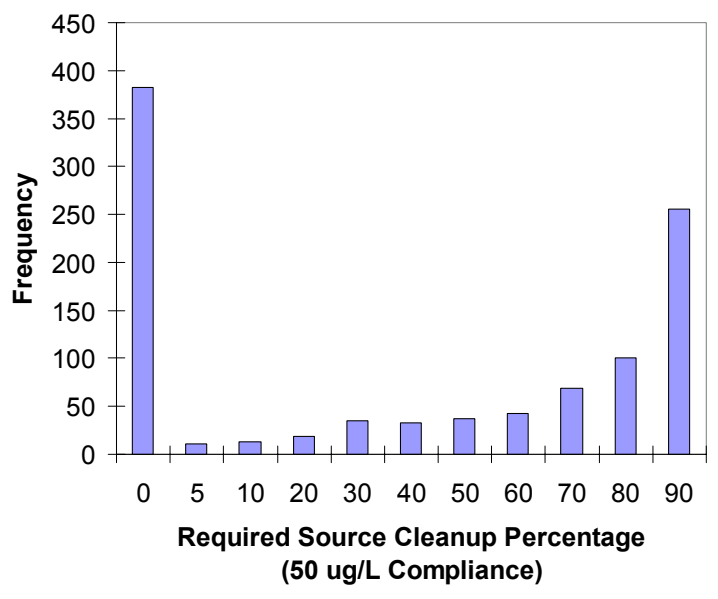

Figure ES-1. $10 \%$ Source Inventory Mean Velocity, $3000 \mu \mathrm{g} / \mathrm{L}$

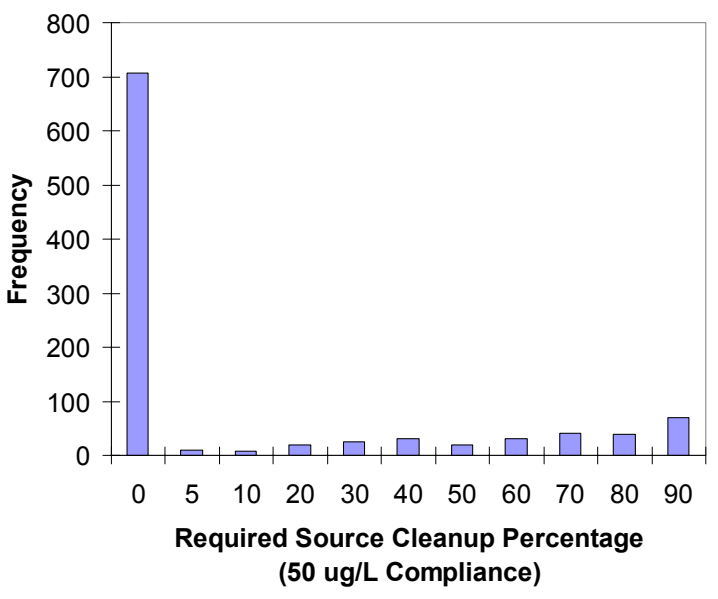

Figure ES-2. $1 \%$ Source Inventory Mean Velocity, $3000 \mu \mathrm{g} / \mathrm{L}$

appear to be porosity, $\mathrm{K}_{\mathrm{d}}$, and $\mathrm{K}_{\mathrm{a}}$; a multiple linear regression analysis of log maximum concentration at the boundary versus porosity, $\mathrm{K}_{\mathrm{d}}$, and $\mathrm{K}_{\mathrm{a}}$ gave standardized regression coefficients of $-0.145,-0.785$, and -0.504 , respectively, at $1 \%$ inventory remaining and -0.187 , -0.691 , and -0.601 , respectively, at $10 \%$ inventory. Thus, better definition of these parameters, primarily $\mathrm{K}_{\mathrm{d}}$, and $\mathrm{K}_{\mathrm{a}}$, would aid in refining the estimate of how much source requires treatment to avoid exceeding the compliance boundary concentration limit.

Several conclusions can be drawn from the modeling, as follows:

- If $1 \%$ of the discharged CT is all that ever reaches the groundwater, then it is likely the highest concentration of CT to arrive at the compliance boundary will not exceed the compliance concentration. However, it is possible the compliance concentration would be exceeded if the actual site parameters correspond to the lower porosity, lower $\mathrm{K}_{\mathrm{d}}$, and lower $\mathrm{K}_{\mathrm{a}}$ values used in this study.

- If $10 \%$ or more of the discharged CT reaches the groundwater, it is likely that the concentration of CT eventually arriving at the compliance boundary will exceed the compliance concentration (unless source removal efforts are used).

- There is a breakpoint between $1 \%$ and $10 \%$ of the discharged inventory that defines the amount of CT in the source at which source removal would be required to avoid exceeding the compliance concentration at the compliance boundary.

- Because source inventory remaining appears to be the quantity driving the amount of site cleanup required for compliance, source inventory characterization would be a milestone on the path forward to resolution of compliance issues.

- Laboratory experiments and site surveys can be used to better quantify values for the parameters controlling compliance boundary concentrations: $\mathrm{K}_{\mathrm{d}}, \mathrm{K}_{\mathrm{a}}$ and porosity; additional modeling, including use of a three-dimensional model, can then be performed using these improved values to give more accurate estimates of compliance boundary concentrations and source cleanup requirements. 


\section{Contents}

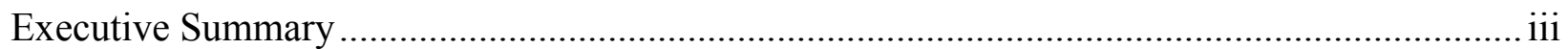

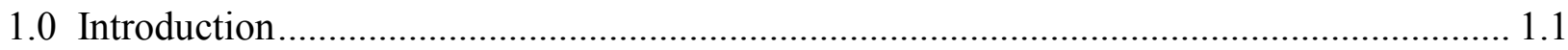

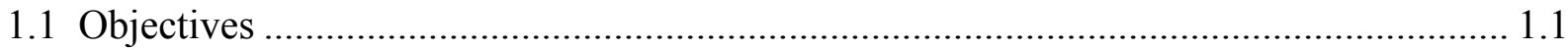

1.2 Hanford Carbon Tetrachloride Plume Summary ………................................................ 1.1

1.3 Hanford Carbon Tetrachloride ITRD Program Overview ……........................................ 1.2

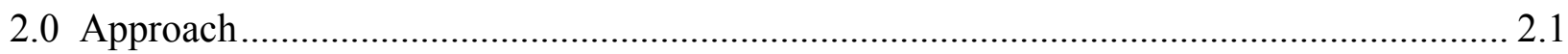

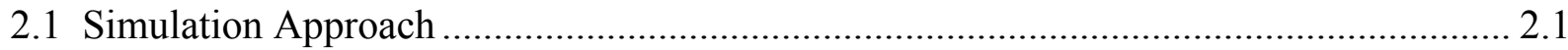

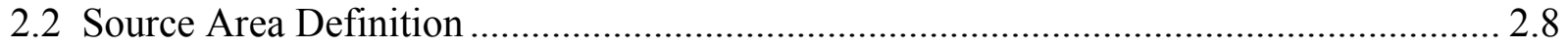

2.2.1 Equilibrium of Carbon Tetrachloride Source and Plume ........................................ 2.8

2.2.2 Three-Dimensional Simulation of Carbon Tetrachloride Distribution..................... 2.12

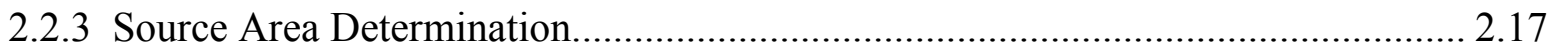

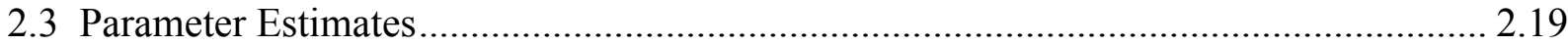

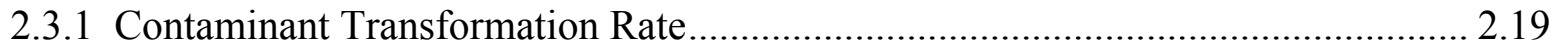

2.3.2 Solid/Liquid Partitioning ............................................................................... 2.21

2.4 Probability Distributions of Modeling Parameters ....................................................... 2.26

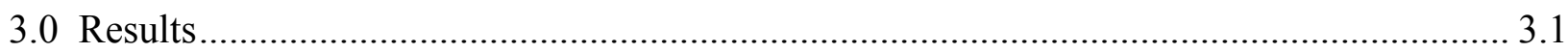

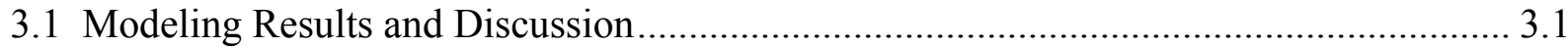

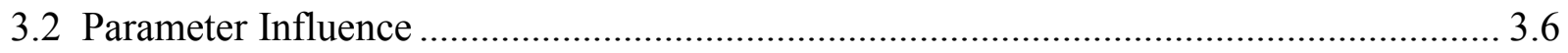

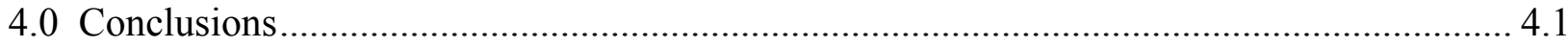

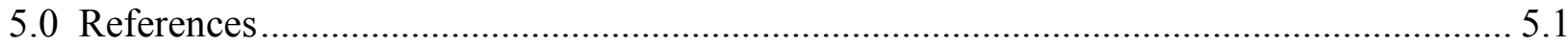

Appendix A: Groundwater Modeling Plan-Hanford Carbon Tetrachloride ITRD Project..... A.1

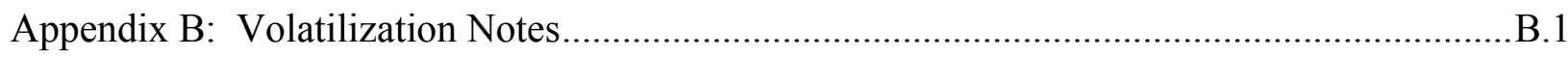

Appendix C: Literature Review .......................................................................................

Appendix D: Simulation Parameters and Results ................................................................. D.1 


\section{Figures}

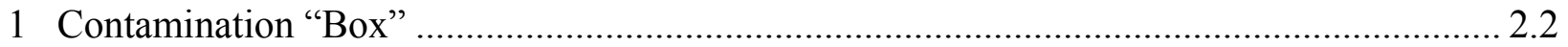

2 Carbon Tetrachloride Modeling Area............................................................................. 2.3

3 Concentration at the Compliance Boundary as a Function of Dispersivity ............................ 2.4

4 Concentration at the Compliance Boundary as a Function of Sorption .................................. 2.4

5 Concentration at the Compliance Boundary as a Function of Abiotic CT Degradation ........ 2.5

6 Concentration at the Compliance Boundary for Selected Simulation Scenarios..................... 2.6

7 Concentration at Compliance Boundary as Function of Contaminant Mass in Source Area. 2.6

8 Concentration at the Compliance Boundary as a Function of Source Volumes..................... 2.7

9 Concentration at Compliance Boundary as Function of Dirichlet Inlet Concentration.......... 2.7

10 Location Map of CT Samples at 200 West Area from 1988 to 1993 .................................... 2.9

11 Number of years CT Concentrations Exceeded $500 \mu \mathrm{g} / \mathrm{L}$ from 1988 to 1993 .................... 2.10

12 Number of Years CT Concentrations Exceeded $1000 \mu \mathrm{g} / \mathrm{L}$ from 1988 to 1993 at Each Grid Node in the Mapped Area......................................................................... 2.11

13 Mean CT Concentration Within the Areas of Exceedence.................................................. 2.12

14 Location of CT Concentration Data Used in the Geostatistical Analysis........................... 2.13

15 Exponential Decay Function for 3-D CT Data with a Decay Distance of 15m.................. 2.14

16 Experimental Horizontal Semivariogram of Normal Scores of Original 3-D CT Data

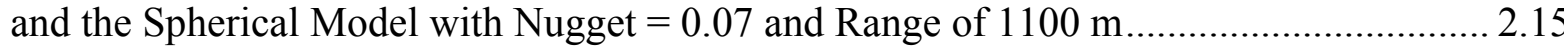

17 Block Diagrams Showing Median of First 100 Geostatistical Simulations of

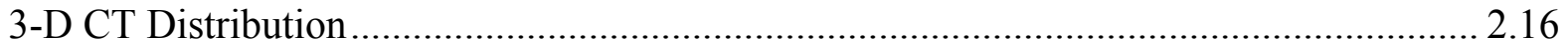

18 Vertical Summation of Simulated Median CT Concentration and the Proportion of Concentration Within Three Source Blocks to Total Summed Concentration.................... 2.17

19 Proportions of Total CT Inventory Within Area of Different Sizes for Three Blocks ........ 2.18

20 Volume above $3000 \mu \mathrm{g} / \mathrm{L}$ for 1000 Simulations of 3-D Distribution of CT ...................... 2.19

21 Neutral Homogeneous Hydrolysis of Carbon Tetrachloride in Distilled Water .................. 2.20

22 Histograms of 1000 Simulated Values of Four Input Parameters Used in

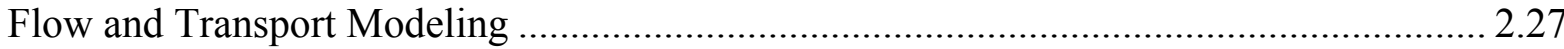

23 Estimated Additional Dilution due to Transverse and Vertical Spreading ........................... 3.2

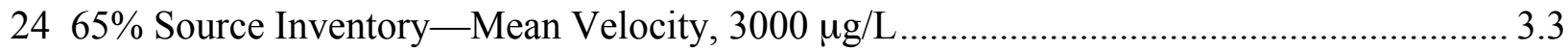

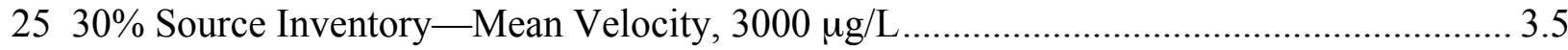

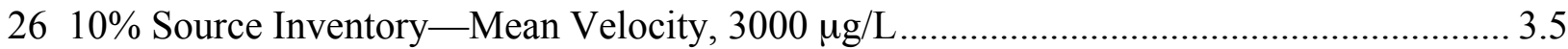

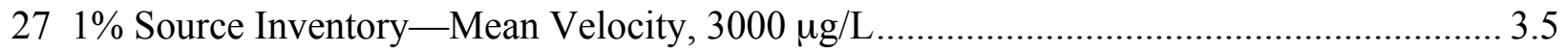

28 Notched Box Plots Showing Values of Transport Parameters for Cases that Did and Did Not Require Source Cleanup for the 1\% Inventory Case 3.7 


\section{Tables}

1 Estimated $\mathrm{K}_{\mathrm{oc}}$ Values for Chloromethanes .............................................................. 2.22

2 Published Values of Koc for Chloromethanes.................................................................. 2.23

3 Maximum Estimates for Solid/Liquid Equilibrium Partition Coefficient Based on

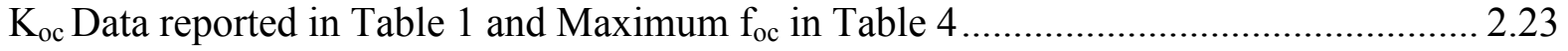

4 Organic Carbon Fraction Data for the Hanford Aquifer (Ringold Formation) ................... 2.24

5 Maximum Estimates for Solid/Liquid Equilibrium Partition Coefficient Based on $\mathrm{K}_{\mathrm{oc}}$ Data Reported in Table 2 and Maximum $\mathrm{f}_{\mathrm{oc}}$ in Table 4............................ 2.24

6 Minimum Estimates for Solid/Liquid Equilibrium Partition Coefficient Based on $\mathrm{K}_{\mathrm{oc}}$ Data Reported in Table 1 and an $\mathrm{f}_{\mathrm{oc}}$ of 0.00027 ........................................ 2.25

7 Minimum Estimates for Solid/Liquid Equilibrium Partition Coefficient Based

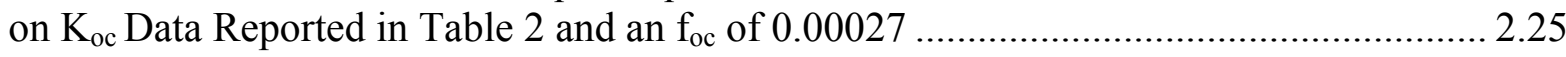

8 Cutoff Levels and Volume, Mass, and Proportions ...................................................... 2.26

9 Modeling Parameter Probability Distributions ............................................................... 2.27

10 Linear correlation coefficients between logarithm of maximum concentration at compliance boundary and input parameters for flow and transport simulations ................. 3.6 


\subsection{Introduction}

Groundwater modeling was performed in support of the Hanford Carbon Tetrachloride Innovative Treatment Remediation Demonstration (ITRD) Program. This report documents the results of the modeling effort and facilitates discussion of characterization and remediation options for the carbon tetrachloride (CT) plume among the ITRD participants.

Section 1.1 states the objectives of the study. Section 1.2 provides an overview of the CT plume, and Section 1.3 describes the Hanford CT ITRD program.

\subsection{Objectives}

The objectives of this modeling study were to predict CT concentrations at the selected compliance boundary, use these predictions to estimate the amount of source remediation needed to meet selected cleanup requirements, and identify the transport parameters having the greatest effect on these results.

\subsection{Hanford Carbon Tetrachloride Plume Summary}

In the 200 West Area at Hanford, CT was disposed to the soil at several sites adjacent to the Z Plant (Plutonium Finishing Plant) during operations from 1955 to 1973. The CT had been used in mixtures with other organics to recover plutonium from aqueous streams at Z Plant. The resultant organic liquid waste stream discharged to the disposal sites consisted primarily of CT in mixtures with tributyl phosphate, dibutyl butyl phosphate, and lard oil.

The areal extent of the dissolved CT plume is approximately $10 \mathrm{~km}^{2}$. Concentrations of dissolved CT detected in the groundwater have been estimated to account for approximately $2 \%$ of the original CT inventory.

Previous work (Swanson et al, 1999) considered an order-of-magnitude estimate of the 1990 inventory of CT remaining in the subsurface using available groundwater concentration data, soil-gas concentration data, and well venting data. For the rough-order-of-magnitude estimates, it was assumed that $750,000 \mathrm{~kg}$ of CT were discharged to the soil during 1955 to 1973 . Total atmospheric losses were estimated to be $21 \%$; the unsaturated zone inventory (in soil gas, soil moisture, and adsorbed phases) accounted for $12 \%$; and the dissolved phase in the aquifer was estimated at $2 \%$, leaving $65 \%$ of the original CT volume unaccounted for. However, the estimates did not consider nonaqueous-phase liquid organic residual saturation in the unsaturated zone, perched organic liquid on low-permeability lenses, or separate organic liquid present within the unconfined aquifer. Any or all of these forms of concentrated CT may be present within the subsurface, although none has been observed. 


\subsection{Hanford Carbon Tetrachloride ITRD Program Overview}

Beginning in January 1999, the Technical Advisory Group (TAG) of the Hanford CT ITRD Program began a series of discussions regarding the potential application of remediation technologies at the CT site in the 200 West Area. The remediation technologies discussed included those for both the saturated zone and the unsaturated zone. However, during the discussions it became evident the selection of remediation technologies needs to be based on the type of remediation (e.g., source removal from the saturated zone) and the extent to which the remediation needs to occur (i.e., the level to which the CT concentration must be reduced). To provide such information, the ITRD TAG determined that groundwater modeling (and site characterization) need to be performed.

This report provides the approach (Section 2) and results (Section 3) of that modeling effort. The modeling was performed to provide an indication of the potential impact of the CT source on the compliance boundary approximately $5000 \mathrm{~m}$ distant. The primary results of the modeling bracket the amount of CT source that will most likely result in compliance/non-compliance at the boundary and the relative influence of the various modeling parameters, thus providing a basis for additional characterization and remediation needs to be addressed by the ITRD TAG.

Section 4 expresses the conclusions reached as a result of the research. Cited references may be found in Section 5. Supporting documentation is provided in the Appendixes. 


\subsection{Approach}

The following overall approach was used to examine the transport of CT from the source area to the compliance boundary as a function of variation in CT source concentration and transport parameter values. A one-dimensional model was configured to estimate CT transport. Ranges for the value of transport parameters within the model (e.g., porosity, $\mathrm{K}_{\mathrm{d}}$ ) were determined from the literature and Hanford site data. The uncertainty in the concentration of CT in the source area was estimated based on geostatistical analysis of existing CT concentration data at the Hanford site. The parameter value ranges and source area $\mathrm{CT}$ concentration variability were used within a Monte Carlo approach where 1000 combinations of parameter values and CT concentration were simulated for selected cases of remaining source area inventory. Each transport simulation provided an estimate of the CT concentration at the compliance boundary over time. These estimated values were compared to the concentration limit selected by regulators for the compliance boundary to determine source cleanup requirements for each simulation. The entire set of simulations was used to determine the model parameters that had the greatest influence on the source cleanup requirements.

\subsection{Simulation Approach}

The CT transport simulation was conservatively modeled as a stream tube $1000 \mathrm{~m}$ wide by $5000 \mathrm{~m}$ long in one-dimensional flow (no vertical or transverse dispersivity or convection). Regional flow-modeling results using the Hanford Site-Wide Groundwater Model (SGM) flow grid provided groundwater velocity estimates for the simulation (see Appendix A). The basic assumptions used in developing the model are the following:

- The major source of contamination is within a $500 \times 500-\mathrm{m}$ box (see Figure 1).

- The plume was in equilibrium with the source just prior to pump-and-treat efforts.

- Processes considered in the model are one-dimensional convective-dispersive transport of reactive solutes subject to adsorption and first-order abiotic degradation (hydrolysis).

- Volatilization (Appendix B) and first-order biodegradation (Appendix C) are negligible.

A one-dimensional van Genuchten (1974) model simulated convective-dispersive transport of CT through a homogenous medium along the centerline of the contaminant plume from the 200 West source area to the compliance boundary approximately $5 \mathrm{~km}$ away (see Figure 2). One thousand Monte Carlo realizations were carried out. Fixed and deterministic parameters include:

- Stream tube length: $5.0 \mathrm{~km}$

- Base inventory of contaminant: $750,000 \mathrm{~kg} \mathrm{CT}$

- Base porosity: $0.10 \mathrm{~m}^{3} / \mathrm{m}^{3}$ (a)

- Bulk density: $1950 \mathrm{~kg} / \mathrm{m}^{3}$

- Inlet concentration: $1500,2000,2500$ or $3000 \mu \mathrm{g} / \mathrm{L}$.

(a) The formation is bimodal with the large cobble-size fraction fully packed and the smaller-size fraction packed in the void space between the cobbles. The porosity is about 0.3 for the cobbles, and about 0.3 for the remaining void. Thus the total porosity is about $0.3 \times 0.3=0.1$. 
- Amount of source remaining: $1 \%, 10 \%, 30 \%$, or $65 \%$

- Groundwater Darcy velocity: mean, $\pm \sigma, \pm 2 \sigma$.

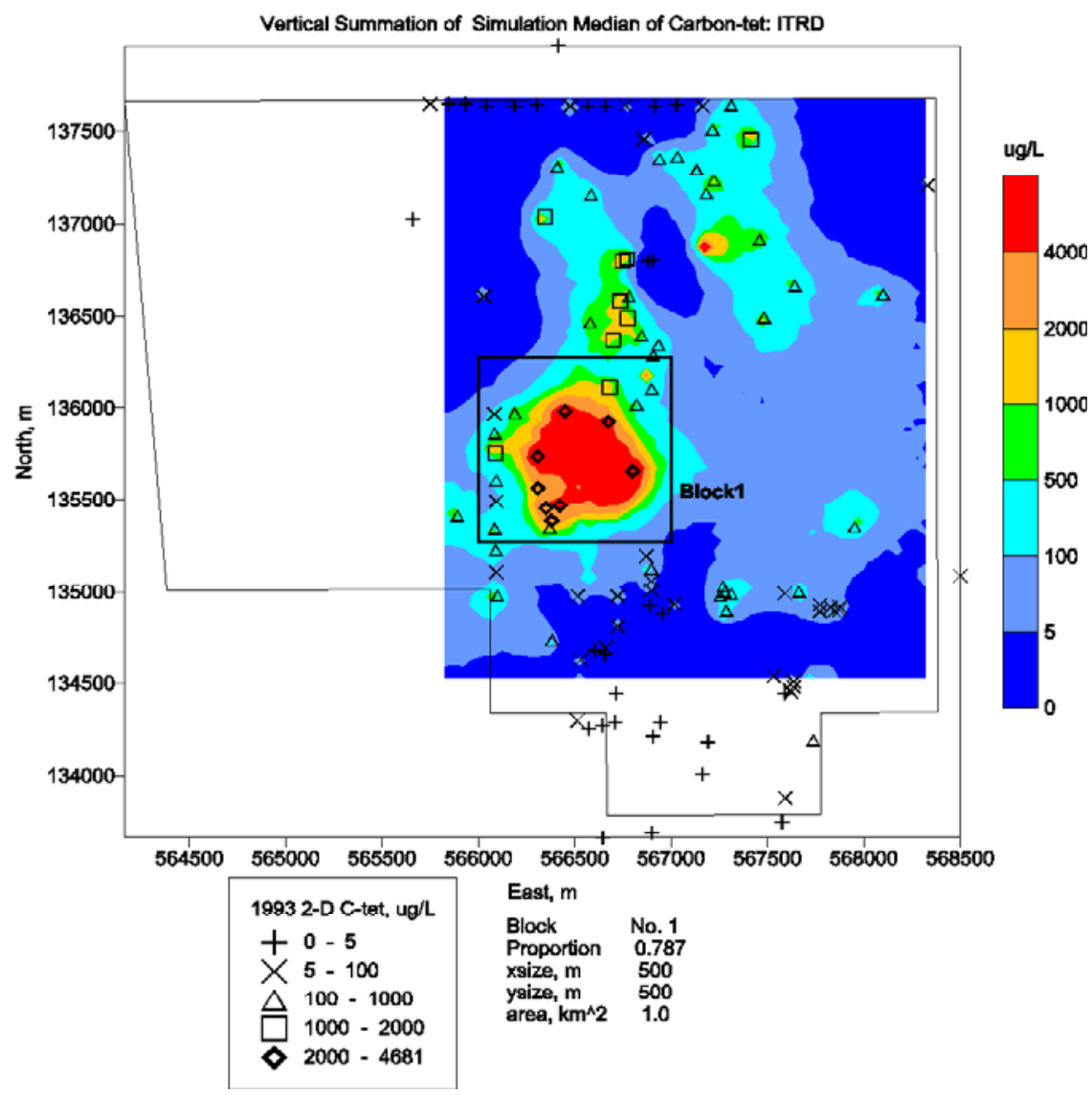

Figure 1. Contamination "Box"

Stochastic parameters varied by Monte Carlo methods include

- porosity

- soil/water equilibrium partition coefficient $\mathrm{K}_{\mathrm{d}}$

- abiotic degradation rate $\mathrm{K}_{\mathrm{a}}$

- dispersivity

- stream tube cross-sectional area.

Parameter limits for $\mathrm{K}_{\mathrm{a}}$ and $\mathrm{K}_{\mathrm{d}}$ were determined by methods detailed in Appendix C. Parameter limits for porosity and dispersivity were determined respectively by geophysical methods and transport field studies. (Gelhar et al. 1985)

An additional simulation of transverse and vertical dispersion/dilution was modeled using a transverse dispersivity ratio of 0.20 and a vertical dispersivity ratio of 0.02 ; the results of this simulation were used to postulate a $50-\mu \mathrm{g} / \mathrm{L}$ compliance boundary concentration limit to perform 
pseudo-three-dimensional (ptd) simulations using the van Genuchten model. The $50 \mu \mathrm{g} / \mathrm{L}$ compliance boundary concentration limit is solely a calculational construct to give a realistic approximation of a three-dimensional model, not a proposed change in actual compliance concentration requirements.

\section{Carbon Tetrachloride Modeling Area}

(current transport grid)

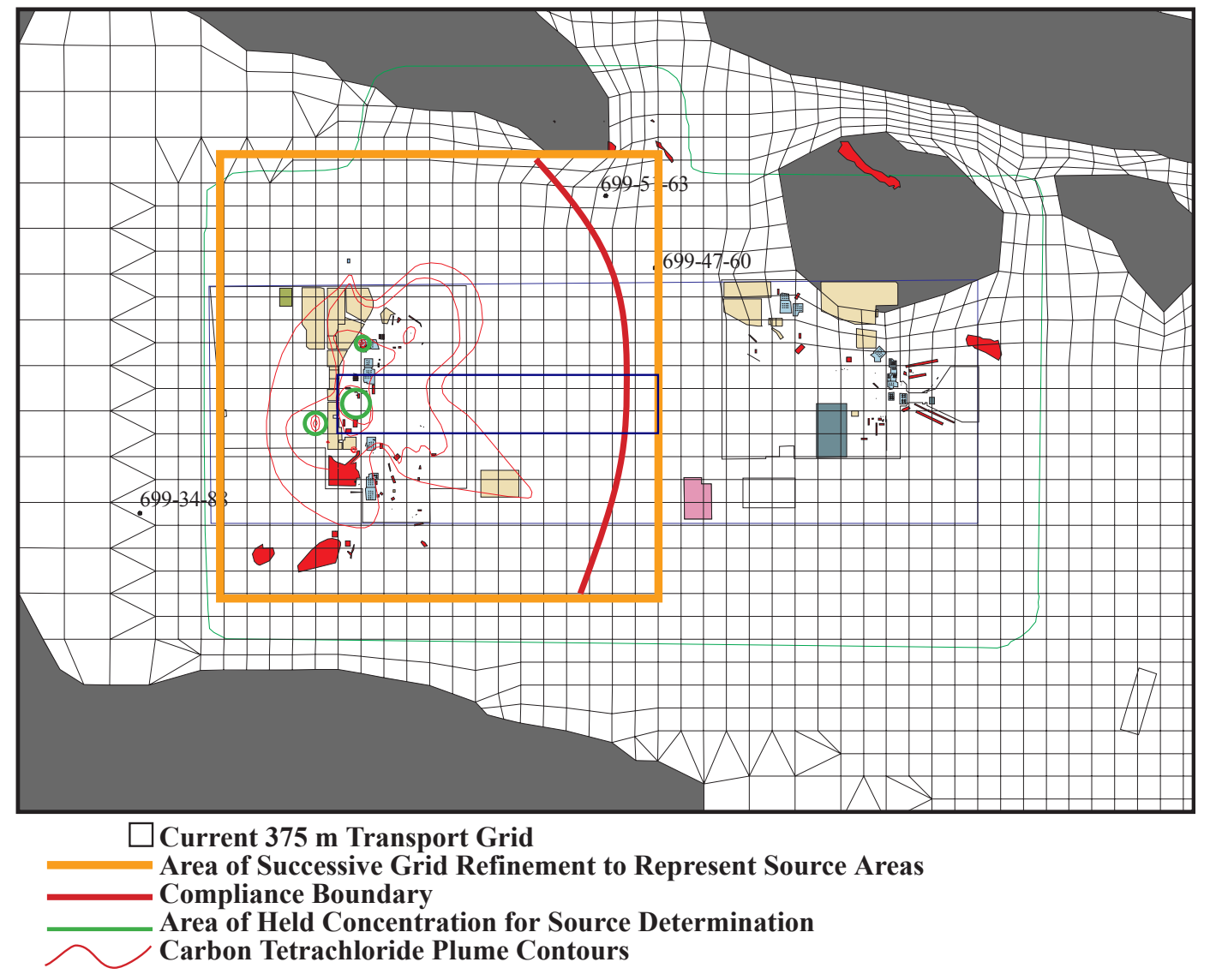

Figure 2. Carbon Tetrachloride Modeling Area

A summary of deterministic results serves to illustrate model performance, and the following tables and figures give details of simulation outputs. For these simulations, selected parameter values varied from low to high where the range of values was determined as described in Sections 2.3.1 and 2.3.2. The low, middle, and high parameter values in the simulations correspond to the minimum, most likely, and maximum values reported in these sections. The concentration of CT over time at the compliance boundary is presented in the figures to compare the effects of parameter variation on model output.

A simulation employing no abiotic decay and no sorption gives a visualization of transport solely through groundwater flow and dispersion. In this simulation, only dispersivity was varied (Figure 3). As dispersivity is increased $(1 \mathrm{~b}<1 \mathrm{a}<1 \mathrm{c})$, the concurrent change in both initial 
concentration increase at the boundary and time to reach peak concentration reflects the faster transport times and smearing of concentration pulses with higher dispersivity.

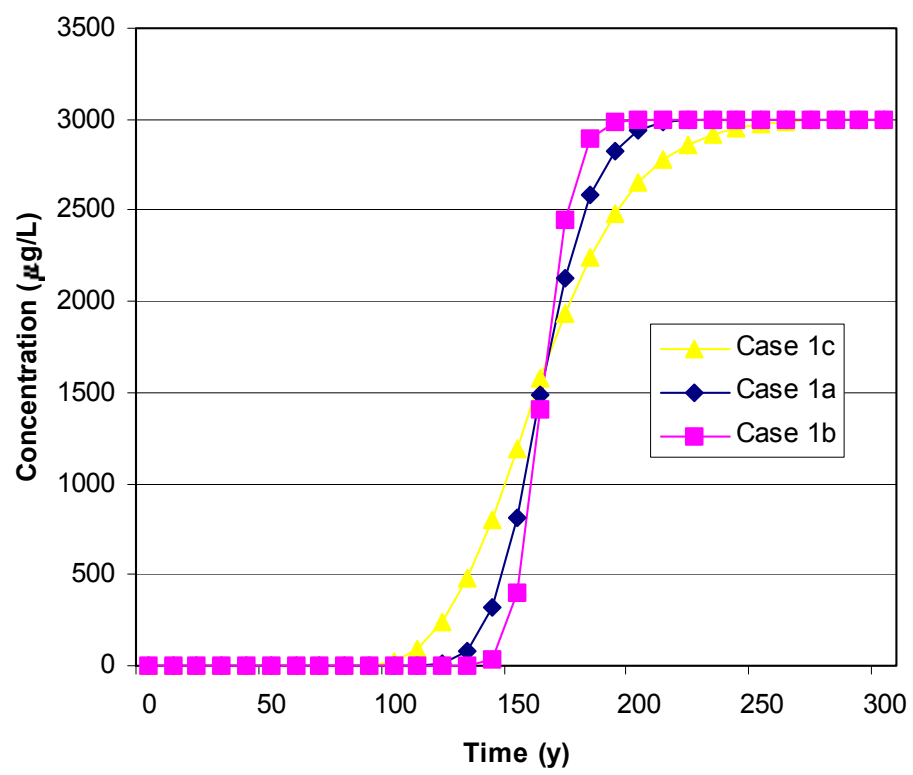

Figure 3. Concentration at the Compliance Boundary as a Function of Dispersivity

A simulation with a mid-range dispersivity, no abiotic decay, and varying sorption $\left(\mathrm{K}_{\mathrm{d}}\right)$ illustrates the delay in arrival times of the concentration peak with increasing sorption $\left(\mathrm{K}_{\mathrm{d}} \forall 2 \mathrm{a}\right.$ $<2 \mathrm{~b}<2 \mathrm{c}$; see Figure 4). As arrival times are delayed, dispersion-related spreading also increases. The mid-range transport-only case (1a) is included here and with other graphs for comparison.

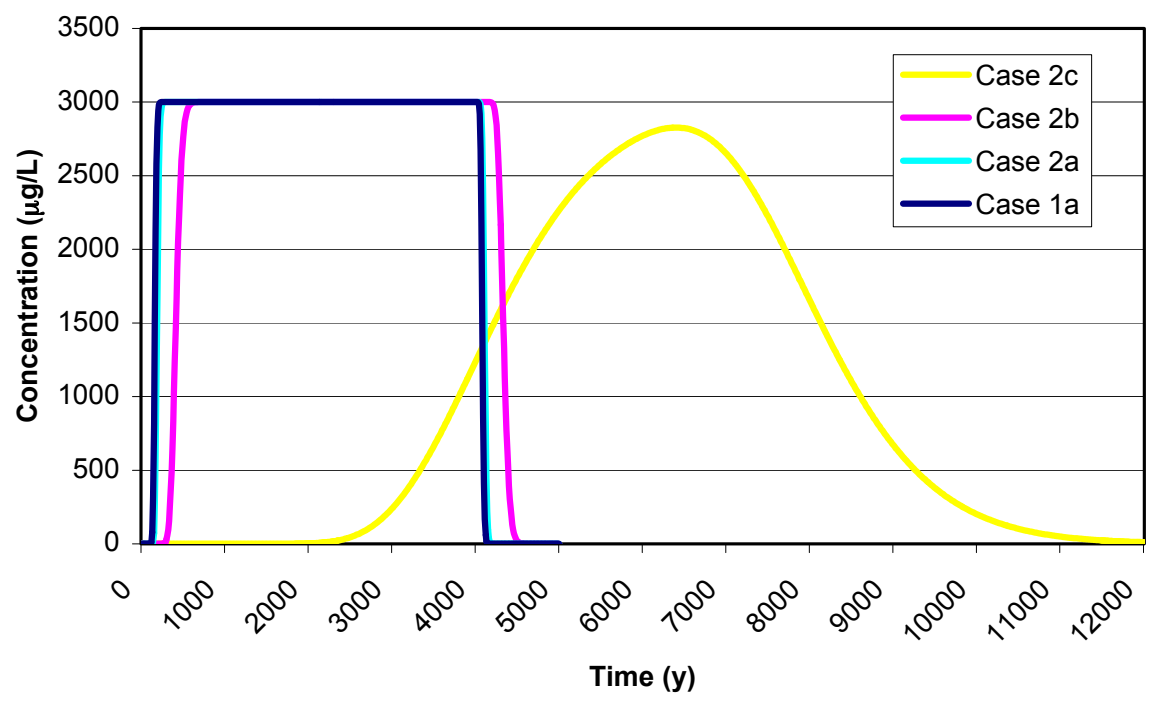

Figure 4. Concentration at the Compliance Boundary as a Function of Sorption 
A simulation with no sorption, mid-range dispersivity, and varying abiotic decay shows the effect on peak concentration for increasing $\mathrm{K}_{\mathrm{a}}\left(\mathrm{K}_{\mathrm{a}} \forall 3 \mathrm{a}<3 \mathrm{~b}<3 \mathrm{c}\right)$ (see Figure 5); the effect on the time of initial concentration change and time to reach peak concentration is minimal.

A series of simulations showing results for varying porosity, Darcy velocity, and cutoff volume using base-case values for sorption, decay and dispersion is given in Figures 6-9. Curve 4a shows base-case values for all fixed and deterministic parameters and is included on other figures for comparison; curve $4 \mathrm{~b}$ shows base-case values with zero dispersion as a check of travel-time effect alone on reactivity (note slight reduction of peak concentration and squared-off shoulders at peak inflection points). Curves $4 \mathrm{c} \& 4 \mathrm{~d}$ show the effect of base-case parameters with low and high porosity, respectively, with a near-600 $\mu \mathrm{g} / \mathrm{L}$ difference in concentration as well as noticeable lag in rise time for the low-porosity simulation. Curves $4 \mathrm{e} \& 4 \mathrm{f}$ simulate basecase parameters with high and low Darcy velocities, with the expected results in peak concentration and event duration.

Remaining contaminant-inventory percentages are the varied parameters in curves $4 \mathrm{~g}-\mathrm{i}$ ( $\mathrm{g}: 1 \%, \mathrm{~h}: 10 \%, \mathrm{i}: 30 \%$ ), with a clear break-point in peak concentration between $1 \%$ and $10 \%$ of inventory; the $4 \mathrm{a}$ base-case inventory of $65 \%$ is included here also. Note that for inventories of $10 \%, 30 \%$ and $65 \%$, the peak concentration remains constant at just over $500 \mu \mathrm{g} / \mathrm{L}$, with only the event duration changing. This presages the results of the Monte Carlo simulations discussed in Section 3 .

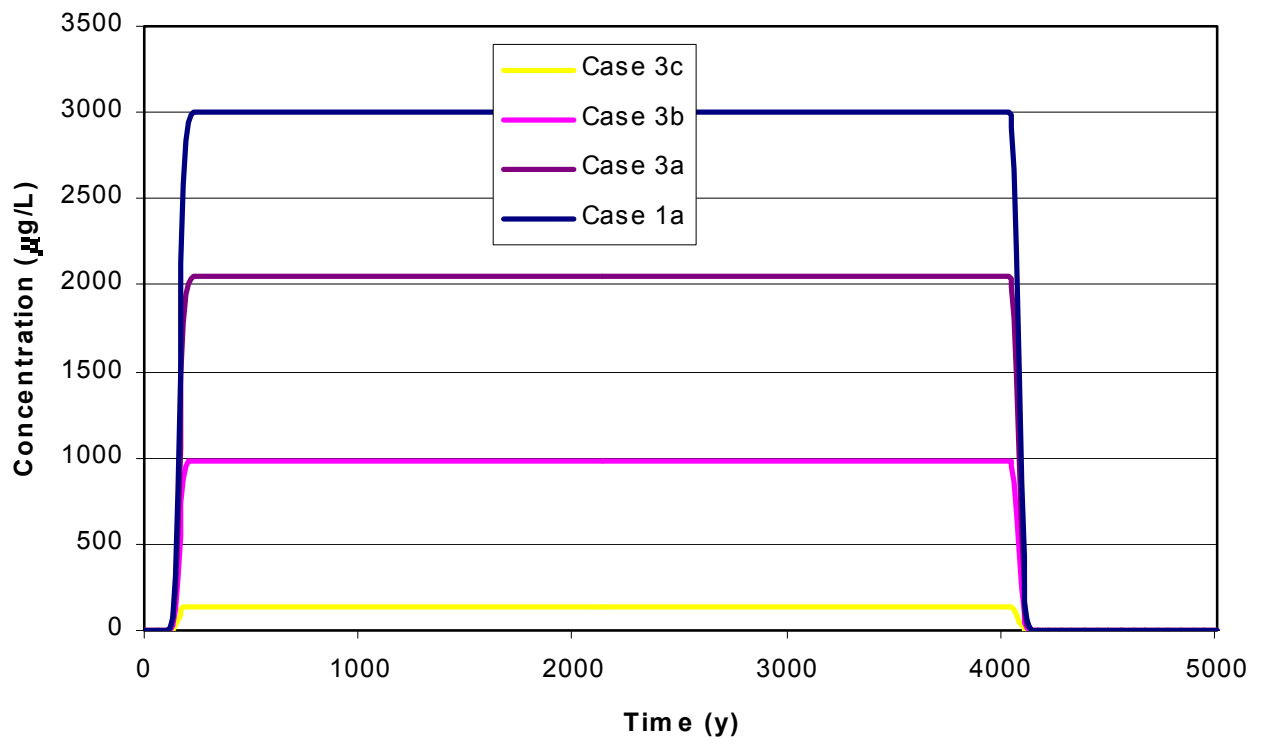

Figure 5. Concentration at the Compliance Boundary as a Function of Abiotic CT Degradation 


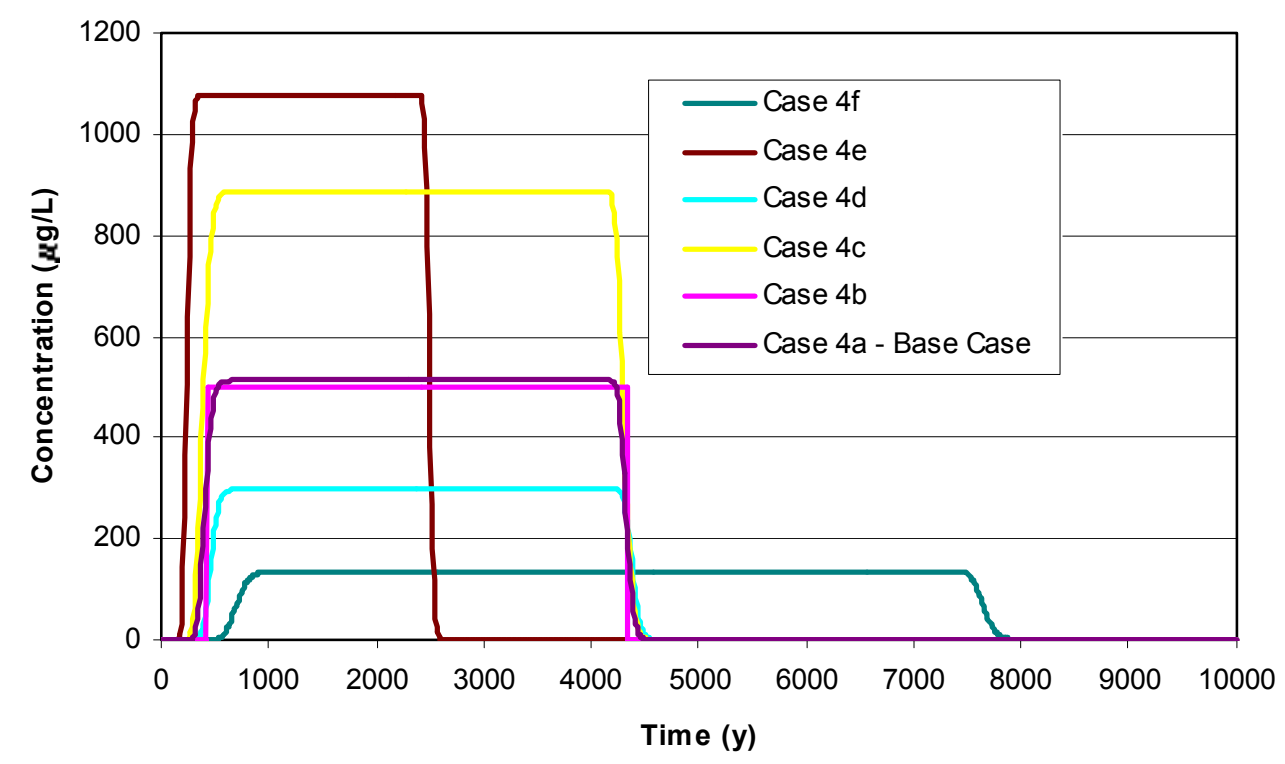

Figure 6. Concentration at the Compliance Boundary for Selected Simulation Scenarios

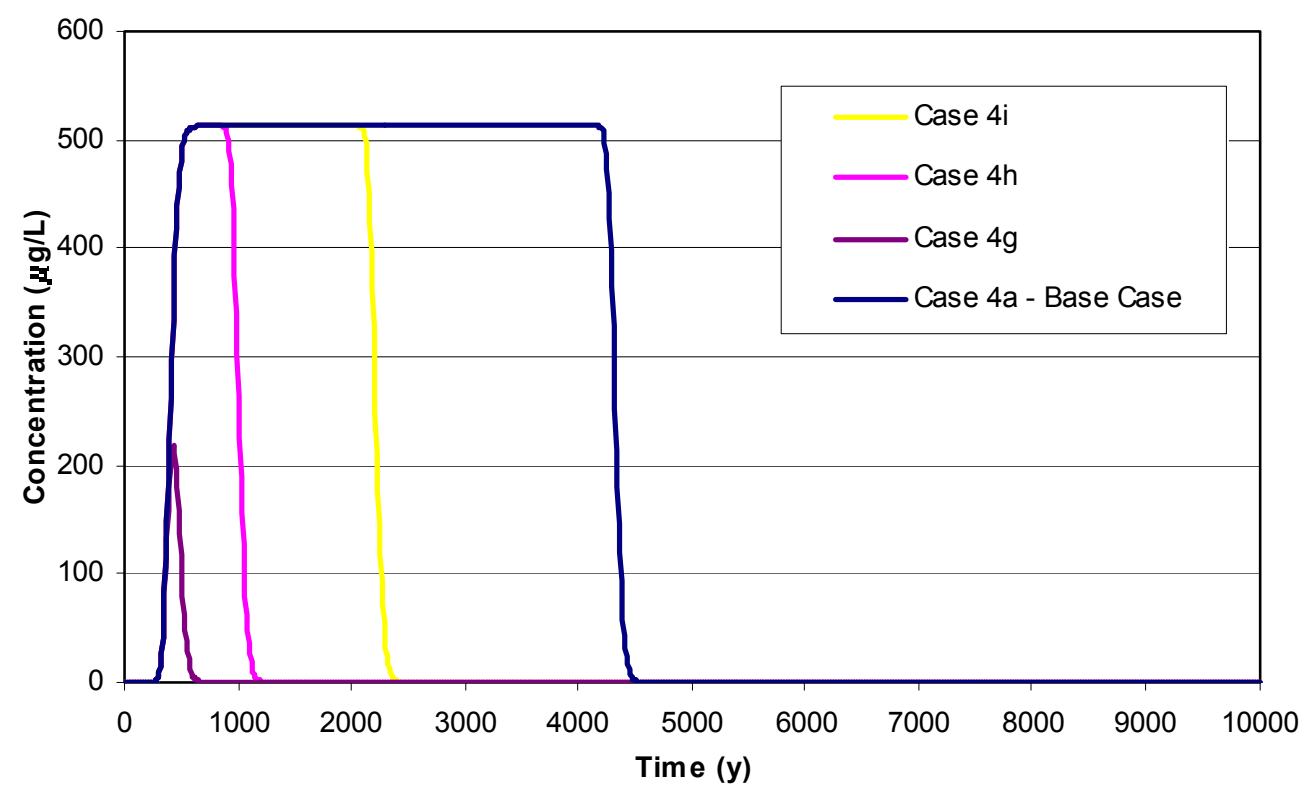

Figure 7. Concentration at the Compliance Boundary as a Function of Contaminant Mass in the Source Area

Curves $4 \mathrm{j}$ and $\mathrm{k}$ show simulations using base-case parameters and minimum and maximum cutoff volumes for a $3000 \mu \mathrm{g} / \mathrm{L}$ inlet Dirichlet concentration; peak concentrations remain constant while event durations change (cutoff volume affects influent mass to the model). 


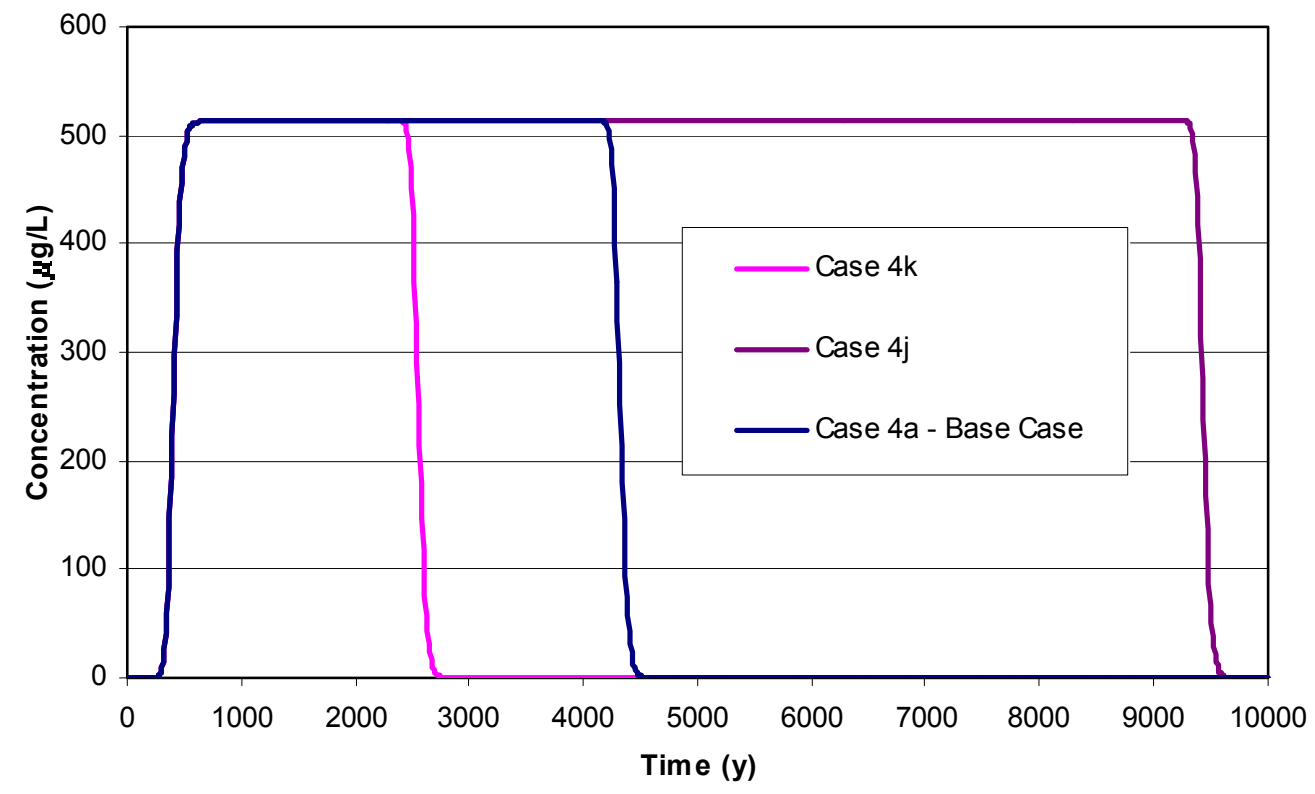

Figure 8. Concentration at the Compliance Boundary as a Function of Source Volumes

Curves 41 through o hold transport parameters constant while changing Dirichlet inlet concentrations $(1: 1000 \mu \mathrm{g} / \mathrm{L}, \mathrm{m}: 1500 \mu \mathrm{g} / \mathrm{L}, \mathrm{n}: 2000 \mu \mathrm{g} / \mathrm{L}, \mathrm{o}: 2500 \mu \mathrm{g} / \mathrm{L})$; event duration decreases with increasing peak concentrations as the Dirichlet inlet concentrations increase.

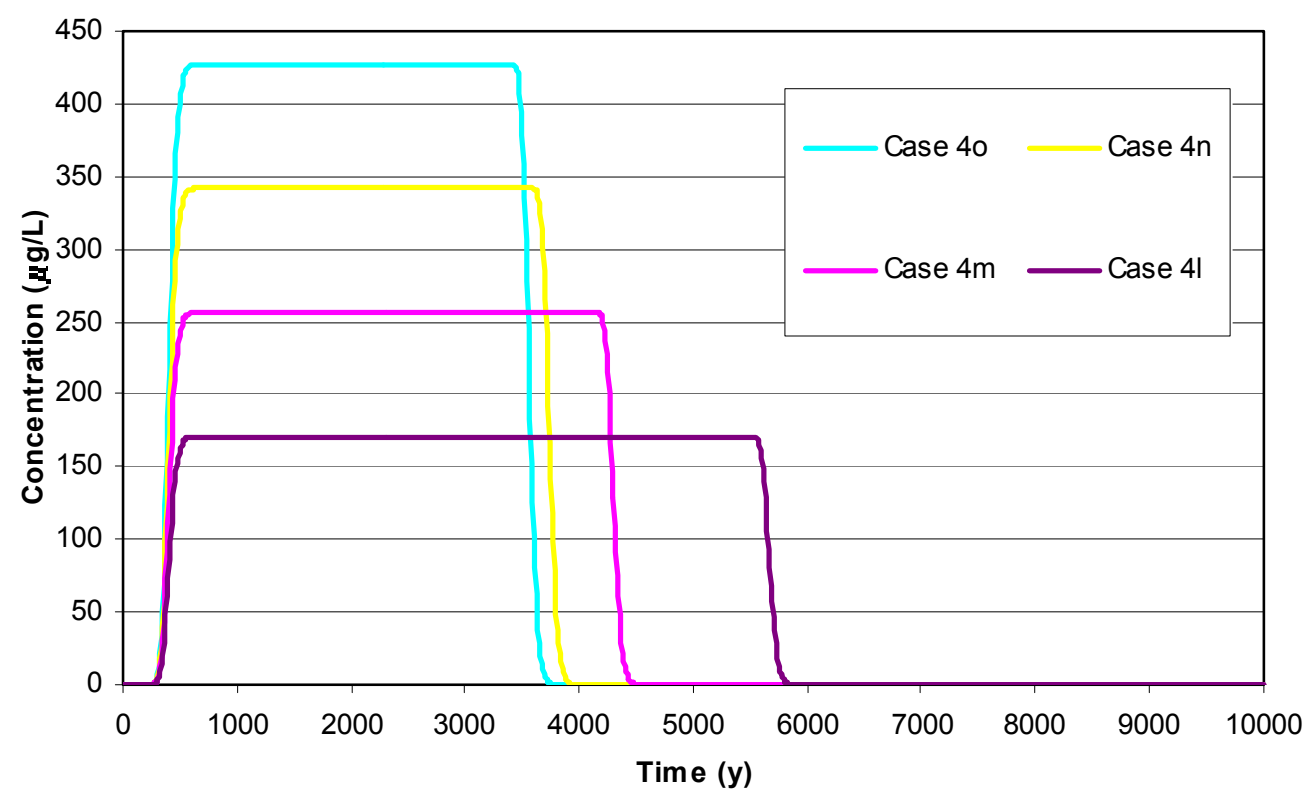

Figure 9. Concentration at the Compliance Boundary as a Function of Dirichlet Inlet Concentration 
The simulation results from varying deterministic parameters show that the model outputs change as expected:

- Increasing dispersivity decreases the arrival time of the initial concentration increase and produces smearing effects at high dispersivity.

- Increasing sorption $\left(\mathrm{K}_{\mathrm{d}}\right)$ delays the arrival time of the concentration peak.

- Increasing abiotic decay $\left(\mathrm{K}_{\mathrm{a}}\right)$ reduces peak concentrations with little effect on arrival times.

- Increasing porosity reduces peak concentrations with a slight increase in arrival times.

- Decreasing Darcy velocity reduces peak concentrations; arrival times and event duration increase.

- Increasing contaminant inventory produces a break point in peak concentration between $1 \%$ and $10 \%$ of inventory, with increasing event duration at constant peak concentration above the break point. This result suggests determining the amount of CT in the source area is important for establishing remediation requirements.

- Increasing cutoff volumes decreases event duration with no change in arrival times or peak concentrations.

- Increasing Dirichlet inlet concentrations decreases event duration and increases peak concentration.

These outputs verify model performance and integrity as well as the independence of parameter inputs. Results of input parameter analysis are detailed in Section 3.

\subsection{Source Area Definition}

\subsubsection{Equilibrium of Carbon Tetrachloride Source and Plume}

The behavior of the CT plume over time was examined to determine whether it appeared to be in equilibrium with a constant source in 1993, prior to the start of pump-and-treat remediation of the plume. This examination was performed because it is assumed that the plume was in equilibrium with the source in the flow and transport modeling. To evaluate that assumption, we mapped the spatial distribution of CT from 1988 to 1993 to determine whether the plume was migrating, expanding, or contracting.

The locations of CT samples from 1988 to 1993 are shown in Figure 10. The sample number ranges from 25 (1989) to 118 (1993). We then used inverse distance squared gridding to map the distribution of CT for each of the six years. Our examination of the maps (not shown) suggested that the center and extent of the CT plume remained constant. To visualize that, we examined each grid node in the map and counted the number of years for which the concentrations exceeded two concentration levels, 500 and $1000 \mu \mathrm{g} / \mathrm{L}$, which are mapped in Figures 11 and 12. Despite the uneven sampling coverage in the early years, the areas in which the concentrations exceeded each of the two cutoff levels remain constant throughout most of the six years. Only the portions of the study area with a good distribution of conditioning data are mapped. The bold 

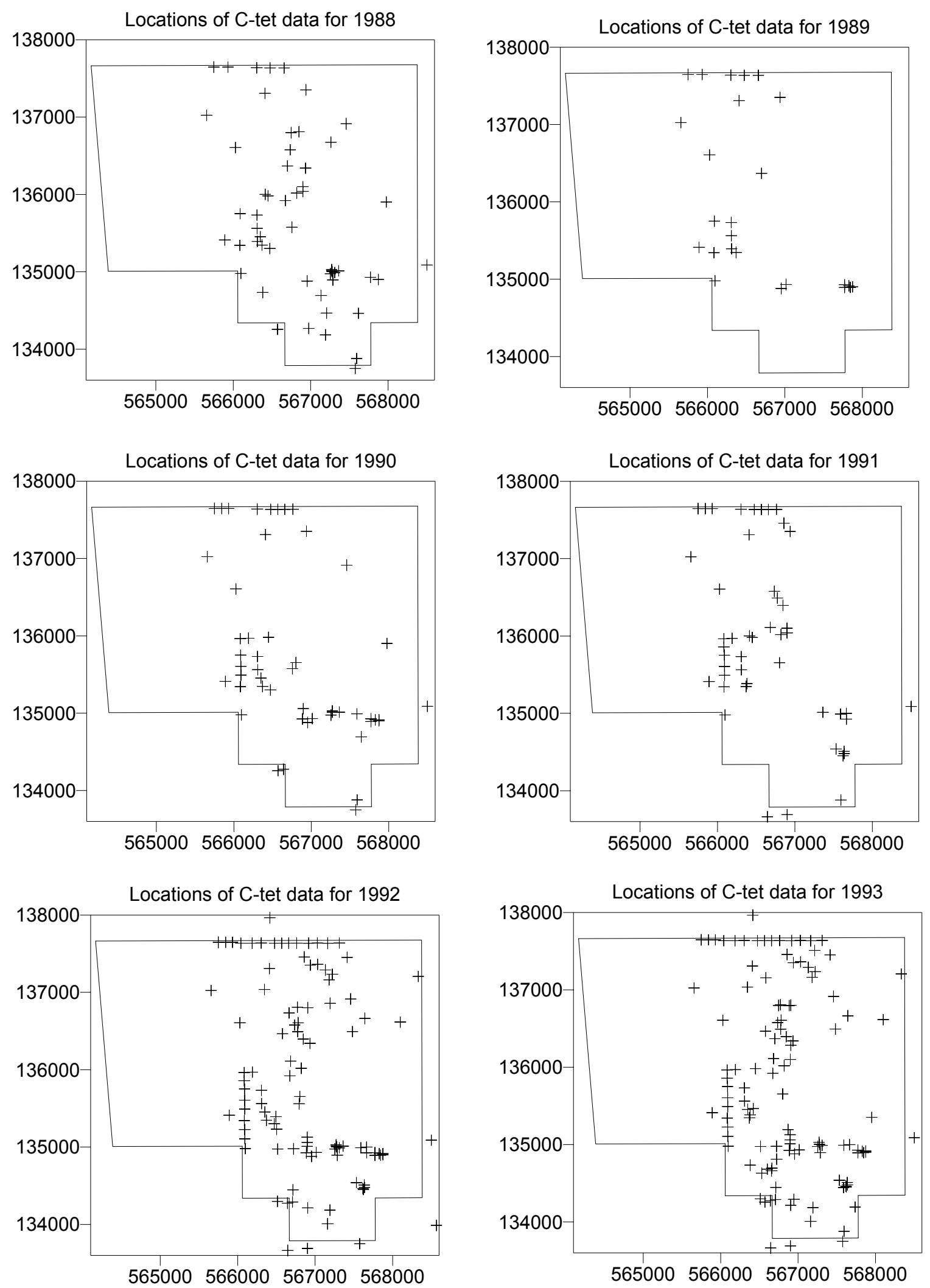

Figure 10. Location Map of CT Samples at 200 West Area from 1988 to 1993 


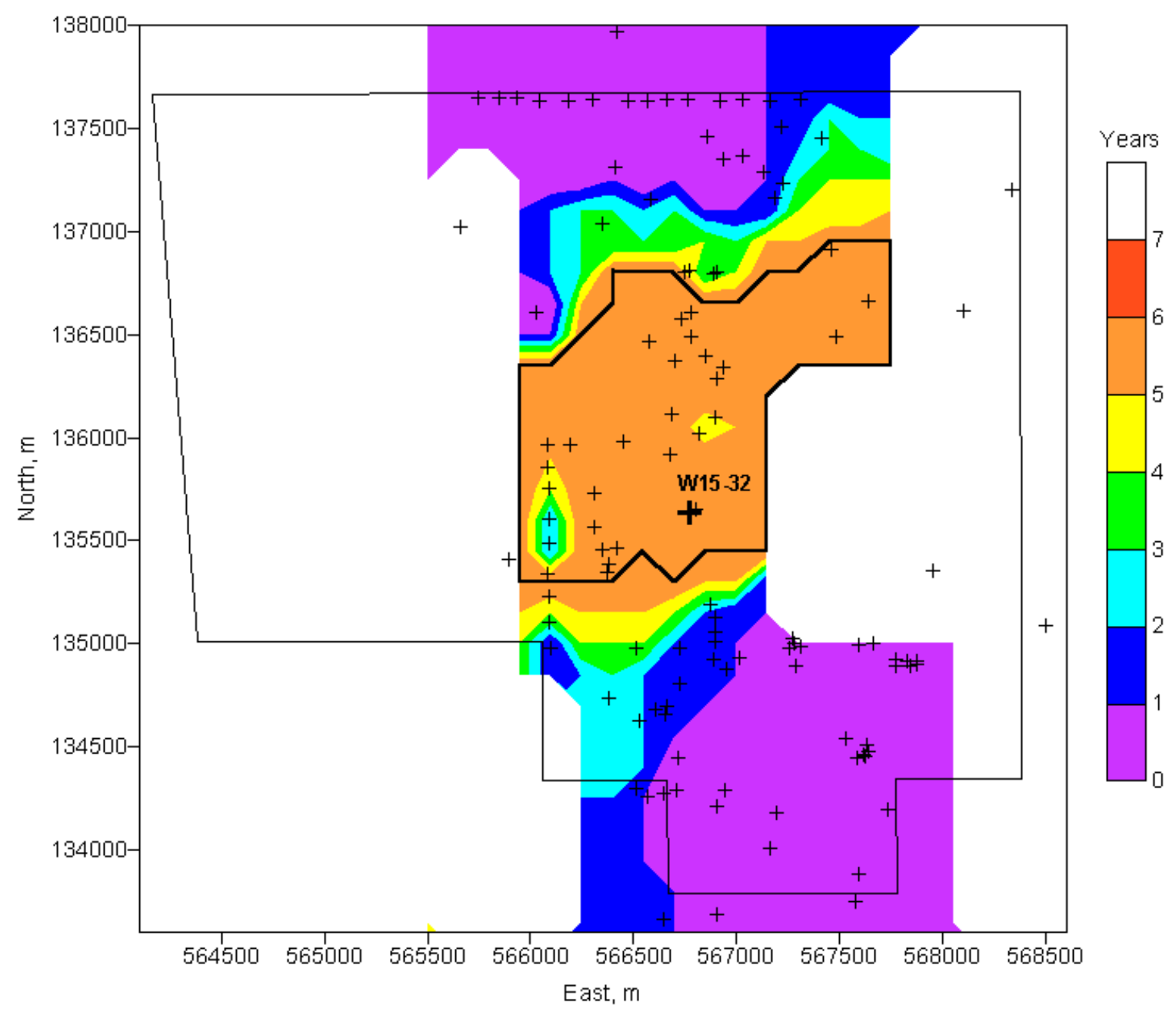

Figure 11. Number of Years CT Concentrations Exceeded $500 \mu \mathrm{g} / \mathrm{L}$ from 1988 to 1993 at Each Grid Node in Mapped Area. The 200 West Area fence is shown for reference. The cross indicates monitoring well W15-32 at the northeast corner of the Z-9 trench.

solid lines in Figures 11 and 12 outline the areas where the CT concentrations exceeded $500 \mu \mathrm{g} / \mathrm{L}$ for six years and $1000 \mu \mathrm{g} / \mathrm{L}$ for three or more years, respectively. The mean CT concentrations from 1988 to 1993 within the areas shown in bold solid lines in Figures 11 and 12 are shown in Figure 13. The mean CT concentration decreased from 1988 to 1991, then remained constant from 1991 to 1993. Figure 13 together with Figures 11 and 12 indicate that the center of the plume was not migrating with time; that the edges of the plume did not appear to be expanding or contracting; and that the mean concentration in the main portions of the plume was constant from 1991 to 1993 . These results suggest that assuming the plume was in equilibrium with the source prior to the start of remediation does not disagree with the available data. 


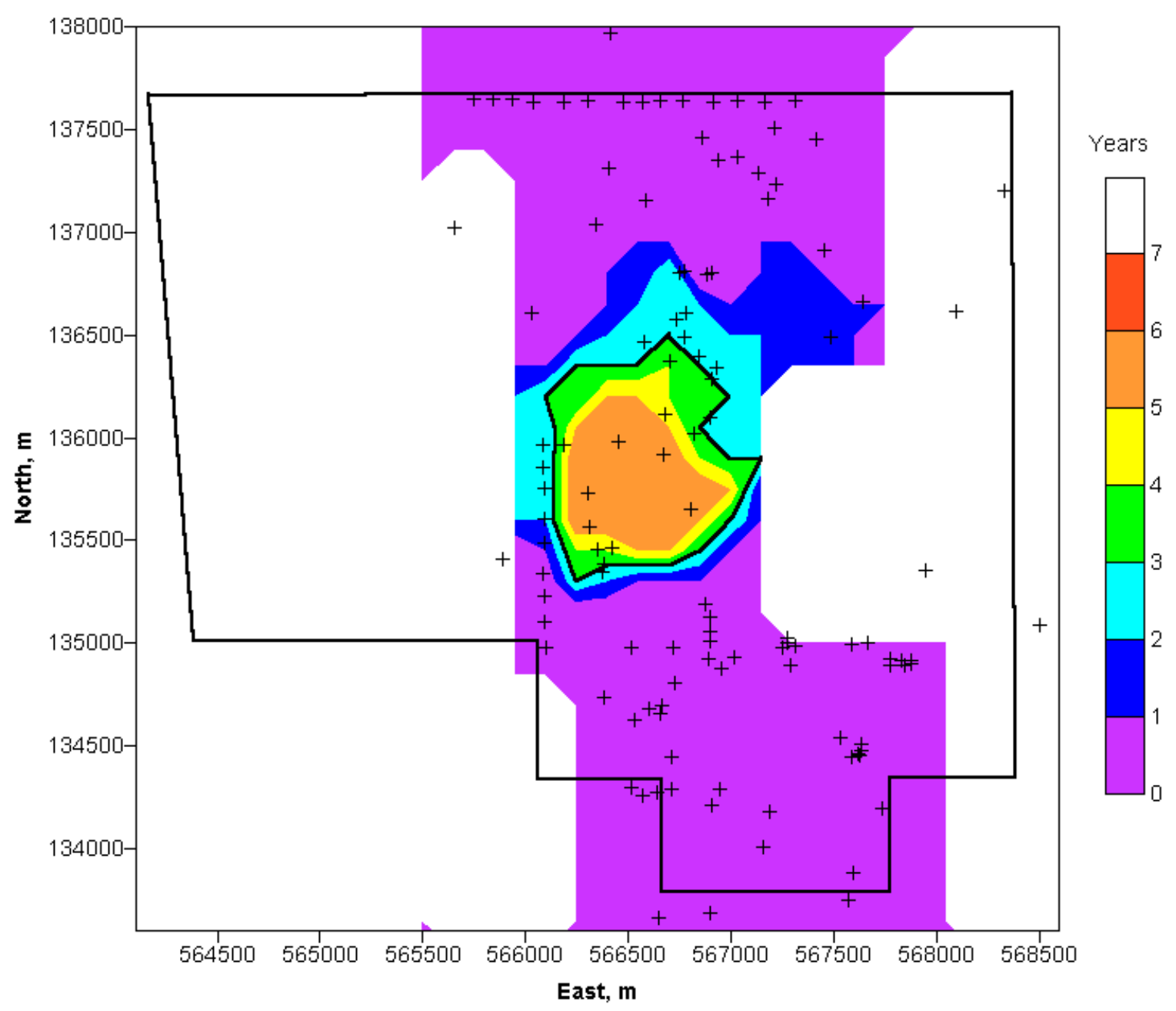

Figure 12. Number of Years that CT Concentrations Exceeded $1000 \mu \mathrm{g} / \mathrm{L}$ from 1988 to 1993 at Each Grid Node in the Mapped Area 

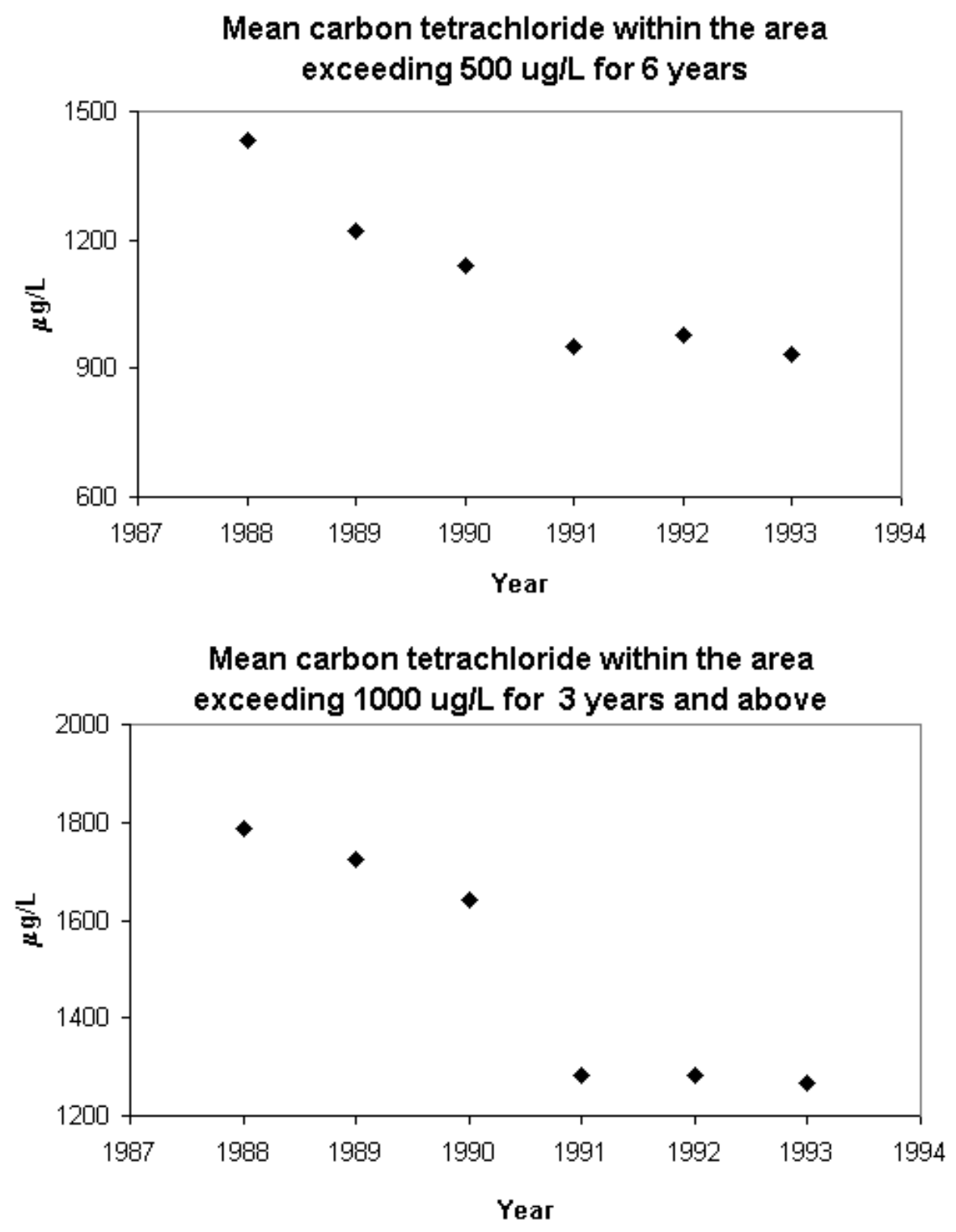

Figure 13. Mean CT Concentration Within the Areas of Exceedence

\subsubsection{Three-Dimensional Simulation of Carbon Tetrachloride Distribution}

We used geostatistical methods to provide a set of 1000 stochastic simulations of the CT concentration in the study area. The three-dimensional distribution of CT in 1993 was needed to provide initial conditions for the flow and transport modeling. Figure 14 is a map of the well locations used in the study. The wells are labeled as central wells if the concentration at any depth at the location exceeds $1000 \mu \mathrm{g} / \mathrm{L}$. For most of the wells in the area, information on distribution of CT with depth was not available, and only one measurement was available near 


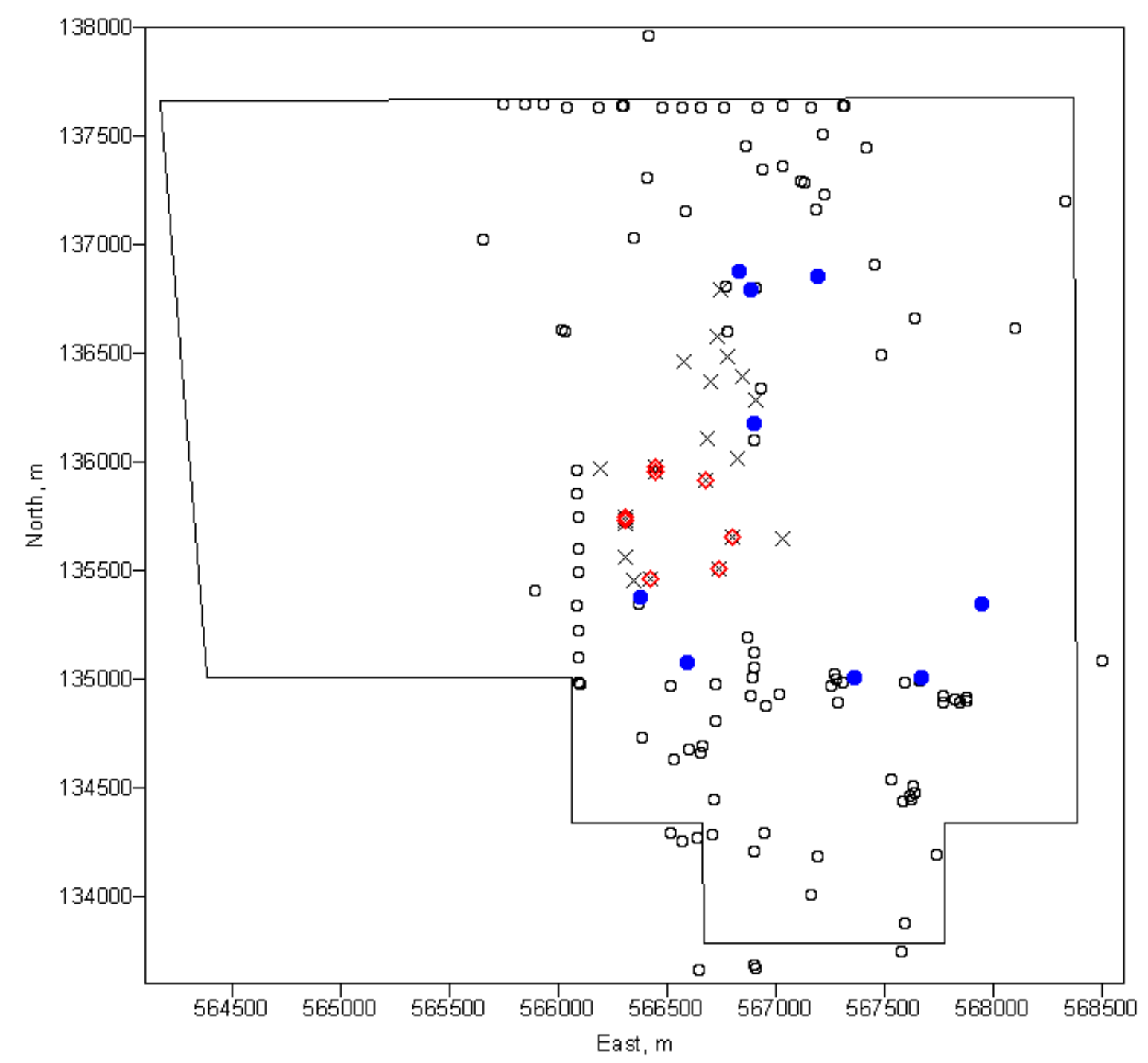

Figure 14. Location Map of CT Concentration Data Used in the Geostatistical Analysis. The 2-D outer and central wells are labeled as blank circles and crosses; the 3-D outer and central wells are labeled as blue solid circles and red diamonds. Central wells have CT concentrations exceeding $1000 \mu \mathrm{g} / \mathrm{L}$; 3-D wells have CT concentration data at depths greater than $5 \mathrm{~m}$.

the water table. CT concentration data for those 121 wells were taken from the database maintained by the Hanford Groundwater Monitoring Project and were restricted to concentrations measured in 1993. Data for the distribution of CT with depth were taken from Appendix A of Swanson et al. (1999). Only eight central wells and nine outer wells have available three-dimensional CT data in that dataset. Because of the scarcity of deep CT data where the location of the measurement is known with any confidence, it was not possible to restrict the data to 1993 for deep measurements, and the average of all measurements at a given three-dimensional location (i.e., for a given well and depth) were used. Even so, only 58 data points are available where the depth of the measurement was more than $5 \mathrm{~m}$ below the water table. The 58 data points are distributed among the 17 locations with deep CT measurements. 
Because of the scarcity of deep CT data, there were not enough data to model the vertical spatial distribution using normal geostatistical methods or three-dimensional trend analysis. However, analysis of the available three-dimensional CT data suggests that the concentration profile is not constant with depth but decreases rapidly with depth below the water table (Figure 15). A relationship between measured concentrations near the water table and concentrations at selected depths was developed using well locations where data were available at multiple depths. This relationship was used to estimate concentrations for other depths in the aquifer where only the concentration near the water table was known. An exponential decay function was used for this correlation and fit with a decay distance of $15 \mathrm{~m}$, as plotted in Figure 15 and shown in Eq. (1):

$$
C(h)=C_{0} * \operatorname{Exp}\left(\frac{-3 h}{a}\right)
$$

where $C(h)$ is the concentration at depth $h, C_{0}$ is the concentration at the water table (measured), and $a$ is the range of the exponential decay function. The parameters of the decay function were determined using available three-dimensional CT data from the central area plotted with depth for a large number of possible ranges of the decay function, and the root-mean-square error (RMSE) between the predicted and actual values was calculated for each possible range. The minimum RMSE was found for a15-m range and used to extrapolate the measured 1993 nearwater-table concentrations to depth within the aquifer for the 121 points where no deep CT data were available. The extrapolation was performed from the water table down to a maximum of 100 meters below the water table at 5-m intervals. No extrapolation was applied to the 17 locations that had three-dimensional CT data. The original three-dimensional CT data and the extrapolated water table concentration data were combined as the conditioning data set for threedimensional simulations of CT spatial distribution.

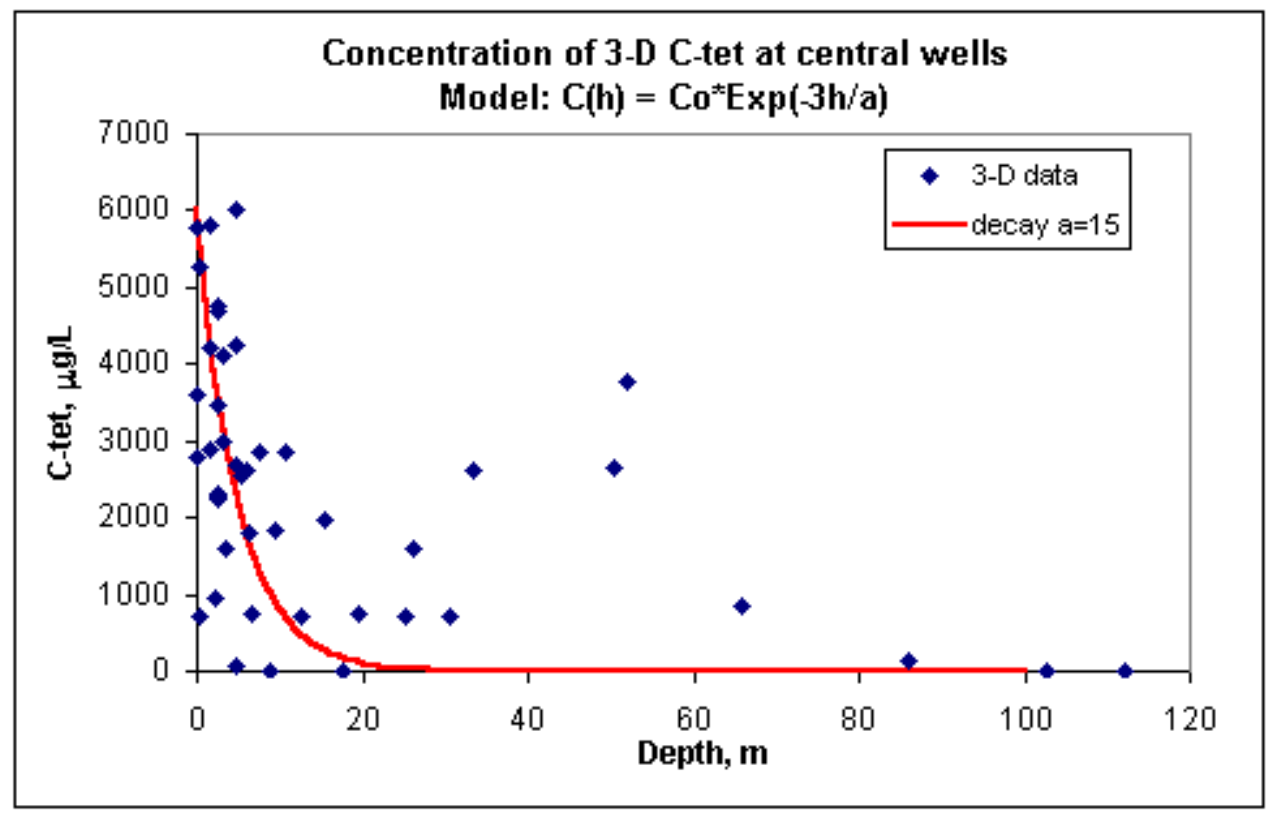

Figure 15. Exponential Decay Function for 3-D CT Data with a Decay Distance of $15 \mathrm{~m}$ 
We used variogram analysis to derive a mathematical model of the spatial continuity of the CT distributions (see Isaaks and Srivastava [1989] for a discussion of variogram analysis and modeling). Before performing the variogram analysis, a normal score transform was applied to the CT data to transform the data to a standard Gaussian distribution, which is required for the sequential Gaussian simulation technique used in the study (Deutsch and Journel 1998). Horizontal variograms were calculated using the GAMV program from the GSLIB software package (Deutsch and Journel 1998). No significant spatial anisotropy was found in the directional semivariograms (not shown). The omnidirectional horizontal semivariogram of the normal scores of the three-dimensional CT is plotted in Figure 16. This semivariogram was based only on the original data and does not include the extrapolated values for the locations that do not have CT measurements below the upper portion of the aquifer. A spherical model (Isaaks and Srivastava 1989) was fit to the horizontal semivariogram with a nugget of 0.07 and a range of $1100 \mathrm{~m}$. There were not sufficient data with depth to calculate a vertical variogram, so the range of spatial continuity in the vertical direction was set to be $1 / 10$ of the horizontal range, or $110 \mathrm{~m}$. The ratio of the horizontal range to the vertical range (10:1) is based on a previous study by Connelly et al. (1992) that found a 10:1 anisotropy ratio between the horizontal and vertical hydraulic conductivity. The sequential Gaussian simulation program, SGSIM, from GSLIB (Deutsch and Journel 1998) was applied to simulate the 3-D spatial distribution of CT concentration. The simulations were conditioned to the combined data and the variogram model parameters. We generated 100 realizations of the 3-D spatial distribution of the CT concentration at a grid node spacing of $50 \mathrm{~m}$ by $50 \mathrm{~m}$ horizontally and $5 \mathrm{~m}$ vertically. A total of 65,280 ( 51 by 64 by 20) grid nodes were simulated in each realization. Horizontal and vertical displays of the median CT from the first 100 realizations are shown in Figure 17.

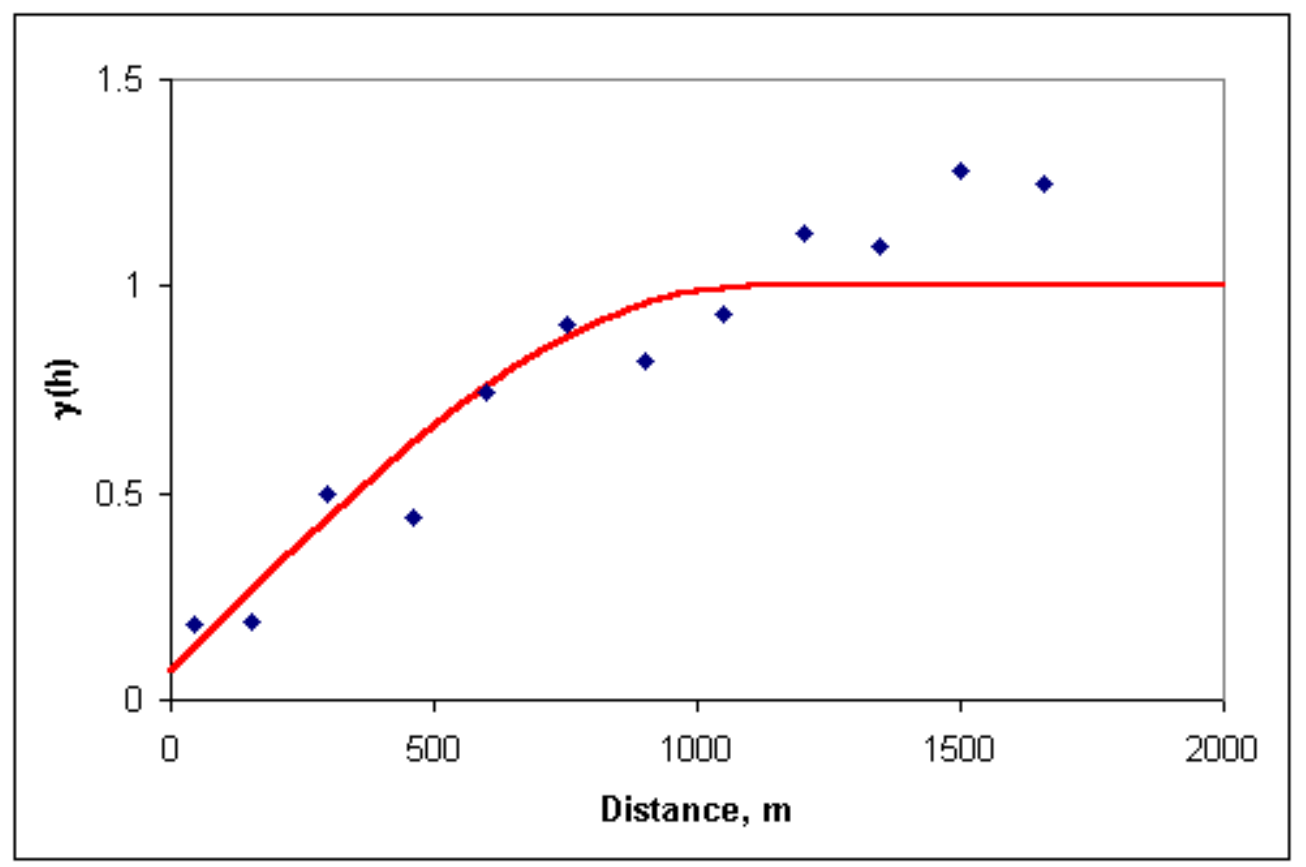

Figure 16. Experimental Horizontal Semivariogram of Normal Scores of Original 3-D CT Data and the Spherical Model with Nugget $=0.07$ and Range of $1100 \mathrm{~m}$ 

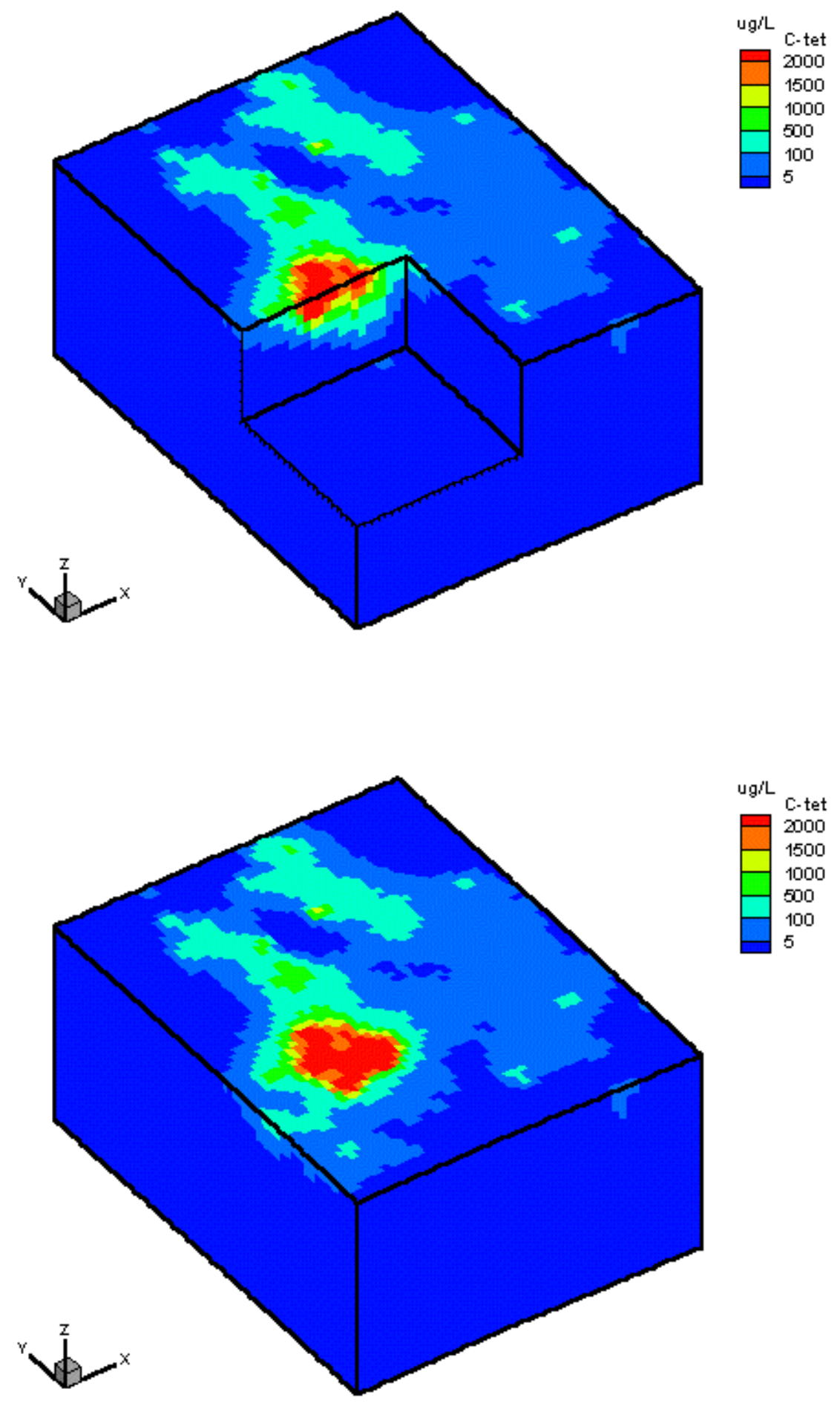

Figure 17. Block Diagrams Showing the Median of the First 100 Geostatistical Simulations of the 3-D CT Distribution 


\subsubsection{Source Area Determination}

To define the major source area of CT mass for the flow and transport modeling, the simulated three-dimensional median values were summed vertically at each $2-\mathrm{D}$ grid node. The summation of the simulated CT concentration was used as a proxy for the inventory of CT mass below each location. Any grid nodes with simulated CT concentrations that were equal to the detection limit of $1.0 \mu \mathrm{g} / \mathrm{L}$ were set to zero before the summation. Based on the 2-D distribution of the CT inventory, the outline of the source area can be defined. We calculated the inventory within several areas of different sizes and determined the proportion of that inventory to the total inventory. From the plot of the proportions of the inventory within different boundaries against the area, the size of the source area was determined such that the proportion of inventory remains nearly constant as the area increases.

The 2-D CT inventory from vertical summation of simulated three-dimensional median CT is shown in Figure 18. Three possible source areas were identified labeled as Blocks 1, 2, and 3. By varying the size of the blocks, the proportions of inventory within different block sizes were plotted against the area of the blocks (Figure 19). As a result, the proportion of CT inventory in

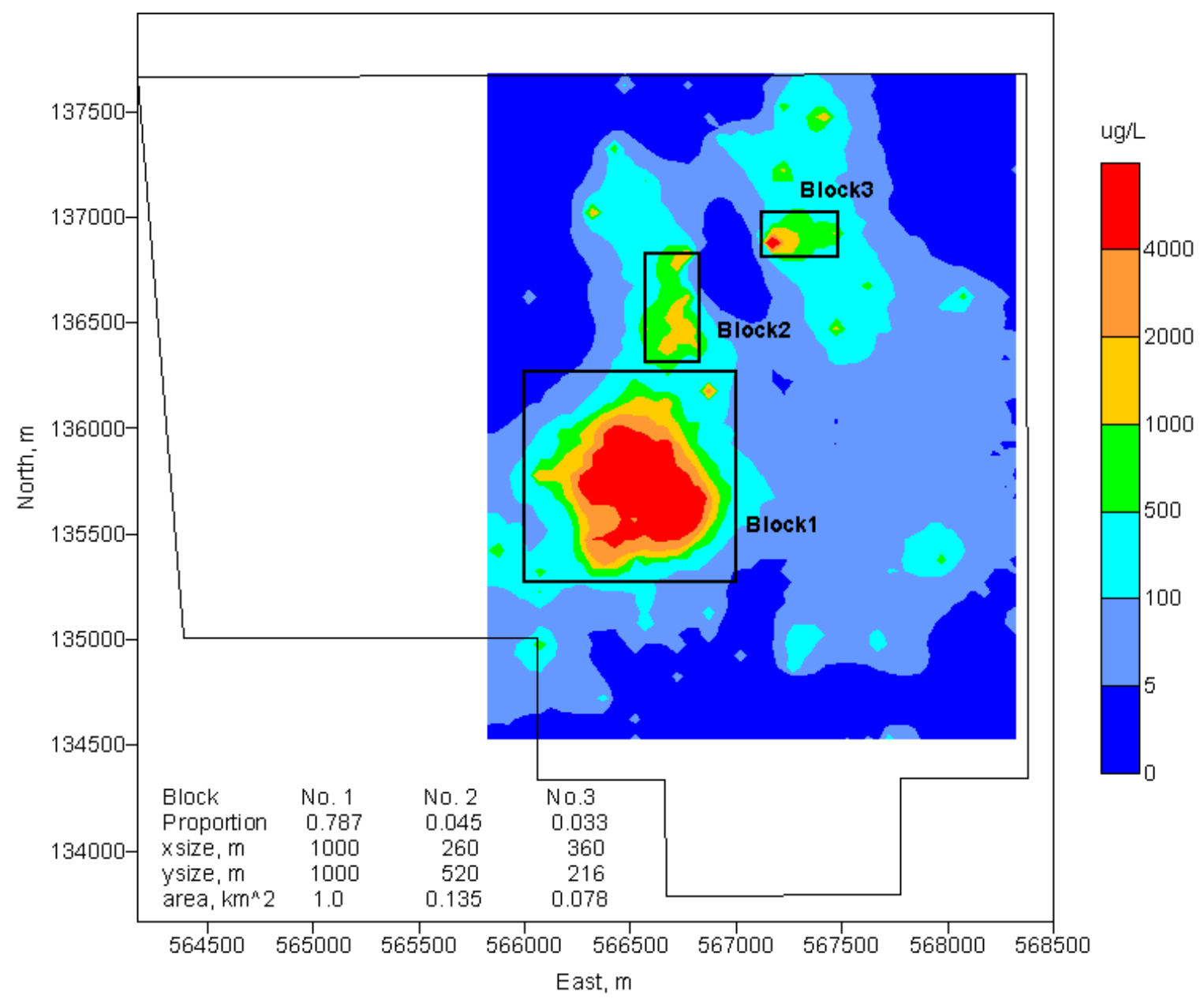

Figure 18. Vertical Summation of Simulated Median CT Concentration and the Proportion of the Concentration Within Three Source Blocks to the Total Summed Concentration 


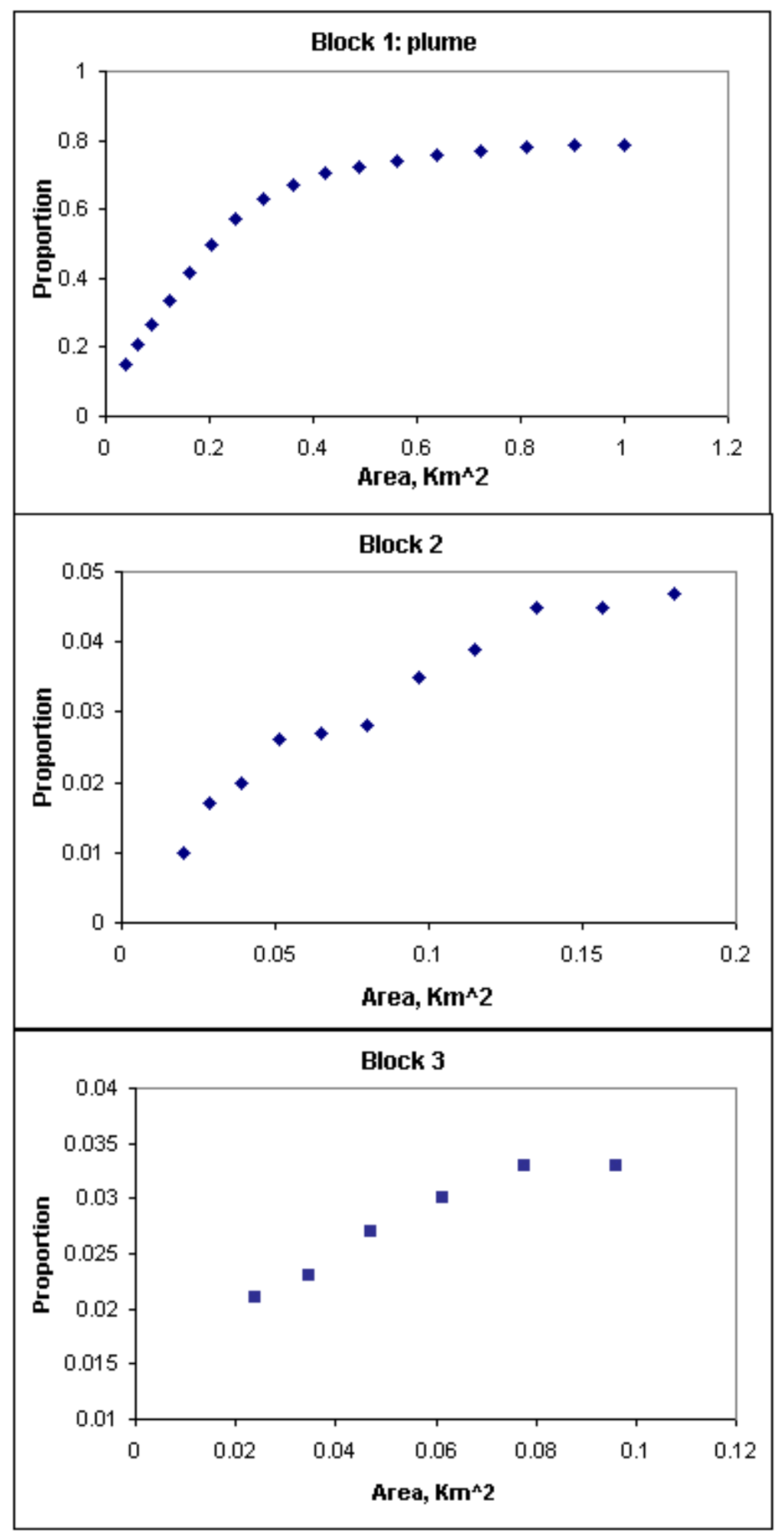

Figure 19. Proportions of Total CT Inventory Within Area of Different Sizes for the Three Blocks 
Block 1 remains nearly constant around 0.8 for areas approaching $1.0 \mathrm{~km}^{2}$. The proportion of CT inventory in Blocks 2 and 3 together remains below 0.1 of the total inventory. Therefore, a 1 by $1-\mathrm{km}$ square box covering Block 1 was defined as the major source area for the flow and transport modeling (see Figure 18).

A set of 1000 realizations of three-dimensional CT concentration was generated using the same parameters that were used in the three-dimensional simulation described above and summarized in Figure 17. The volume and mass of CT that exceeded various thresholds ranging from 250 to $3000 \mu \mathrm{g} / \mathrm{L}$ within the defined source area (i.e., Block 1 in Figure 18) were calculated and are detailed in Section 2.4. Figure 20 is a scatterplot of the volume above the $3000 \mu \mathrm{g} / \mathrm{L}$ threshold for the 1000 realizations.

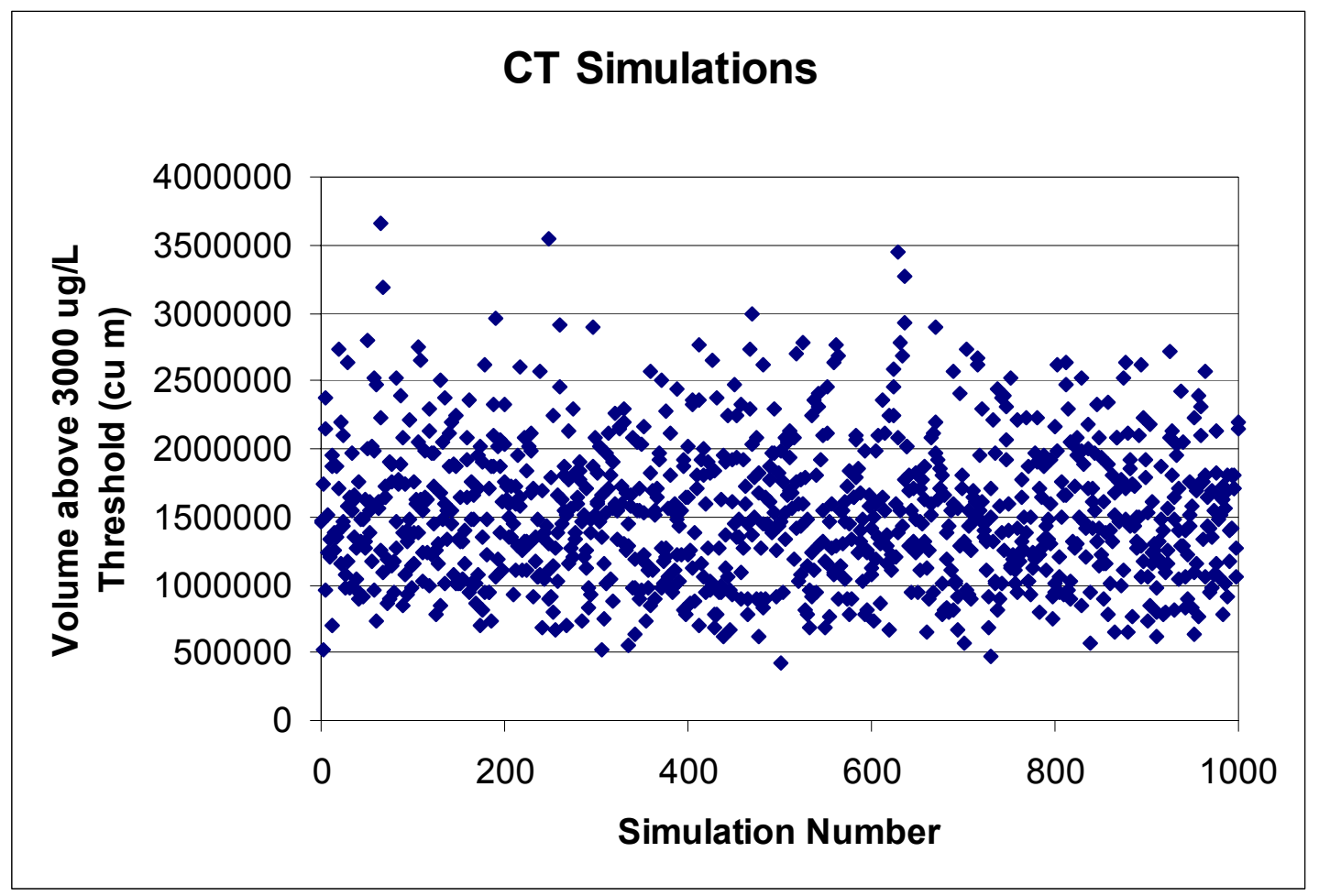

Figure 20. Volume Above a Threshold of $3000 \mu \mathrm{g} / \mathrm{L}$ for 1000 Simulations of the 3-D Distribution of CT

\subsection{Parameter Estimates}

\subsubsection{Contaminant Transformation Rate}

A summary of the natural mechanisms that may contribute to CT loss in the Hanford aquifer is provided in this section. Natural attenuation mechanisms were quantified for use as reaction parameters in the reactive transport simulations to estimate the fate of CT in the Hanford aquifer. Additional details about how the natural attenuation mechanisms were quantified are described in the reports include as part of Appendix C. 
Three specific natural attenuation mechanisms for $\mathrm{CT}$ were determined to have the potential for affecting the fate of $\mathrm{CT}$ in the Hanford aquifer: aqueous hydrolysis, biodegradation, and volatilization. Aqueous hydrolysis and biodegradation are discussed in the following subsections; volatilization is discussed in Appendix B.

\subsubsection{Aqueous Hydrolysis of Carbon Tetrachloride}

Aqueous hydrolysis rates for $\mathrm{CT}$ in deionized water have been measured previously. The most relevant results for determining the loss rate of $\mathrm{CT}$ due to aqueous hydrolysis are those by Jeffers $(1989,1996)$. Based on the literature and personal communication sources listed above, it is likely that aqueous hydrolysis of $\mathrm{CT}$ leads to formation of $\mathrm{CO}_{2}$ and $\mathrm{HCl}$ but no hazardous compounds. However, this assertion may need to be confirmed with site-specific testing.

The predicted half-life for CT using the Arrhenius parameters presented in Jeffers et al. $(1989,1996)$, which were shown to agree with data presented by Fells and Moelwyn-Hughes (1959) (as cited in the compilation by Mabey and Mill [1978]) over a temperature range of $10^{\circ}$ to $25^{\circ} \mathrm{C}$, is plotted in Figure 21. Included on this plot are the upper- and lower-bound predictions based on the uncertainty in the Arrhenius parameters listed in Jeffers (1996). This plot suggests that the half-life of CT in the aquifer at Hanford is likely between 36 and 290 years for a temperature of about $19^{\circ} \mathrm{C}$ (groundwater temperature measured by Newcomer et al. 1995). The best estimate for the half-life would be about 100 years. We have found no data for CT hydrolysis that were collected at ambient temperatures.

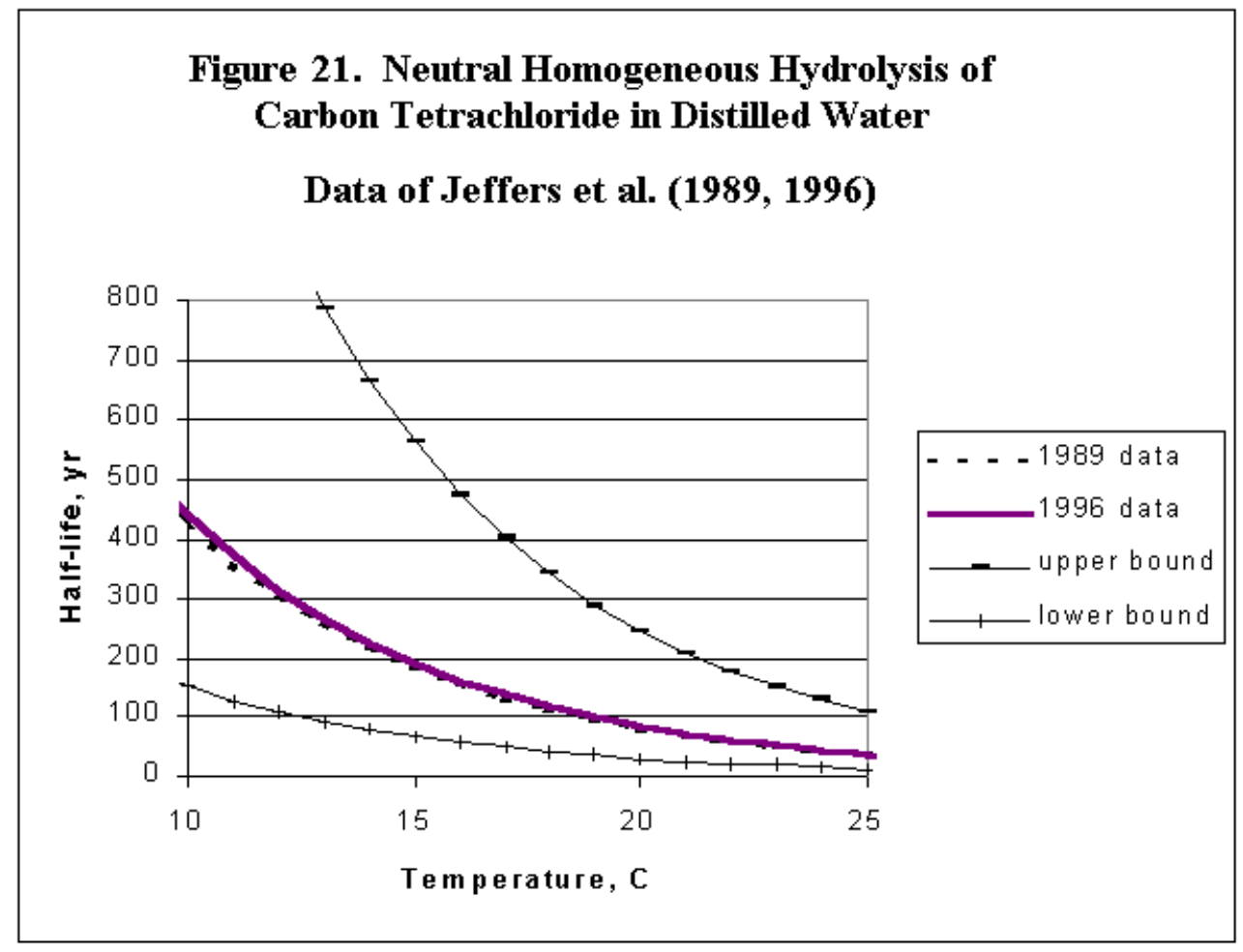

Figure 21. Neutral Homogeneous Hydrolysis of Carbon Tetrachloride in Distilled Water (Jeffers et al. 1989, 1996) 
Jeffers et al. (1994) also attempted to measure any possible effect of mineral surfaces on hydrolysis rate and found the rate unchanged even in the presence of sulfide minerals. Other literature on related compounds (Haag and Mill 1988; Deeley et al. 1991) corroborates this result and suggests that the hydrolysis rate determined in distilled ionized water should be a reasonable prediction of the rate of hydrolysis in the subsurface.

\subsubsection{Biodegradation of Carbon Tetrachloride}

The conditions that favor biodegradation of $\mathrm{CT}$ are predominantly anaerobic and require the presence of biodegradable organic carbon (Bouwer and McCarty 1983a; Hooker et al. 1998; Cobb and Bouwer 1991; Criddle et al. 1990a). In fact, there have been no published reports of aerobic transformation of CT. For the Hanford CT groundwater plume, which is both aerobic and in contact with sediments that have organic carbon levels below $0.5 \mathrm{wt} \%$ (Newcomer et al., 1995), these results suggest that no biological activity is currently contributing to the natural attenuation of $C T$.

\subsubsection{Solid/Liquid Partitioning}

Solid/liquid partitioning is simulated in the model used for this project using a linear equilibrium partitioning coefficient, $\mathrm{K}_{\mathrm{d}}$. This section discusses how estimates of this parameter were determined for use in the model.

Solid/liquid partitioning is primarily a sorption-desorption process governed by the solubility of the particular chemical in water and its affinity to the solid phase. In high-carbon soils, it has been demonstrated that the amount of nonionic organic chemicals sorbed varies from soil to soil and that such variations are primarily caused by the organic content of the soil (Jeng et al. 1992; Lyman et al. 1990; Bishop et al. 1989). However, mineral-driven sorption becomes important as organic carbon content diminishes to below $0.1 \%$ (Kile et al. 1995). At levels of organic carbon above $0.1 \%$, the normalized sorption coefficient $\left(\mathrm{K}_{\mathrm{oc}}\right)$ represents an important parameter that can be used to estimate the soil/water equilibrium partition coefficient $\left(\mathrm{K}_{\mathrm{d}}\right)$ with this equation:

$$
K_{d}=f_{o c} \times K_{o c}
$$

where $f_{o c}$ is the mass fraction of organic carbon (mass-oc/mass-soil) in the soil and $\mathrm{K}_{\mathrm{d}}$ is the soil/water equilibrium partition coefficient $[(\mathrm{mg} / \mathrm{kg}$-soil $) /(\mathrm{mg} / \mathrm{L})]$. It is important to note that at levels of organic carbon below approximately $0.1 \%$, this relationship will likely underestimate $\mathrm{K}_{\mathrm{d}}$ because it neglects mineral-driven sorption (Stephanatos et al. 1991). In these cases, the real quantity of attached material will be higher than those predicted by Equation (2).

Many researchers have developed methods for estimating $\mathrm{K}_{\mathrm{oc}}$ based on measurable properties such as the octanol/water partition coefficient $\left(\mathrm{K}_{\mathrm{ow}}\right)$. The two most applicable of these common correlations are given in the following equations (Lyman et al. 1990; Bishop et al. 1989):

$$
\log \left(K_{o c}\right)=3.64-0.55 \times \log (S)
$$




$$
\log \left(K_{o c}\right)=4.277-0.557 \times \log \left(S_{m}\right)
$$

where $\mathrm{S}$ is the water solubility of the organic compound $(\mathrm{mg} / \mathrm{L})$, and $\mathrm{S}_{\mathrm{m}}$ is the molar water solubility of the organic compound $(\mu \mathrm{mol} / \mathrm{L})$. Table 1 lists the values of $\mathrm{K}_{\mathrm{oc}}$ estimated by Equations (3) and (4) for chlorinated methane. The estimate of $K_{o c}$ can then be used to estimate the $K_{d}$ for an aquifer if the $f_{o c}$ is estimated or measured for the specific aquifer.

Table 1. Estimated $\mathrm{K}_{\mathrm{oc}}$ Values for Chloromethanes

\begin{tabular}{|l|c|c|}
\hline \multirow{2}{*}{ Compound } & \multicolumn{2}{c|}{ Koc $(\boldsymbol{\mu g} / \mathbf{g}-\mathbf{o c}) /(\boldsymbol{\mu g} / \mathbf{m L})$} \\
\hline CT & 110.48 & 161.11 \\
\hline CF & 30.72 & 38.26 \\
\hline DCM & 23.84 & 24.49 \\
\hline CM & 35.33 & 27.29 \\
\hline
\end{tabular}

An estimate for the range and most probable value for $\mathrm{K}_{\mathrm{d}}$ of each chloromethane at Hanford was derived from literature data. Equation (1) was used to calculate $\mathrm{K}_{\mathrm{d}}$ based on reported or calculated data for $\mathrm{f}_{\mathrm{oc}}$ and $\mathrm{K}_{\mathrm{oc}}$. However, the predicted $K_{d}$ from Equation (1) for Hanford may be an underestimate because the organic carbon fraction of the Hanford aquifer sediments are very close to the lower limit of applicability for this correlation. This potential to underpredict sorption is demonstrated by the fact that Zhao et al. (1999) report a measured $\mathrm{K}_{\mathrm{d}}$ value of 0.39 for $\mathrm{CT}$ in a soil with an organic carbon content below their detection limit of $0.03 \%$. In contrast, the predicted $\mathrm{K}_{\mathrm{d}}$ for $\mathrm{CT}$ in a soil with an organic carbon content of $0.03 \%$ ranges from 0.015 to $0.081(\mathrm{~L} / \mathrm{kg})$ based on the values of $\mathrm{K}_{\mathrm{oc}}$ reported in Tables 1 and 2 .

The estimated range of $\mathrm{K}_{\mathrm{d}}$ values for CT was determined by examining the range of calculated and reported $K_{d}$ values from the literature. Table 3 reports the maximum values of $K_{d}$ that are predicted from Equation (1) using the $\mathrm{K}_{\mathrm{oc}}$ values reported in Table 1 and the highest organic carbon fraction reported in Table 4 (0.0052). Maximum values for $\mathrm{K}_{\mathrm{d}}$ determined in field studies are listed in Table 5. These values are based on the highest organic carbon fraction reported in Table 4 (0.0052) and $\mathrm{K}_{\mathrm{oc}}$ (Table 2) values from the individual studies. For CT, $\mathrm{K}_{\mathrm{d}}$ values calculated using the $f_{o c}$ and $K_{o c}$ values measured in each study are shown in parentheses next to the maximum value. The maximum value of $K_{d}$ selected from the information in Table 3 is $0.83 \mathrm{~L} / \mathrm{kg}$. There is one $\mathrm{K}_{\mathrm{d}}$ value reported in Table 5 that is higher than the selected maximum, but the study conditions and results suggest that a $\mathrm{K}_{\mathrm{d}}$ calculated using the measured $\mathrm{K}_{\mathrm{oc}}$ and an $\mathrm{f}_{\mathrm{oc}}$ value from another source would not be reasonable.

Tables 6 and 7 provide minimum $K_{d}$ estimates using an $f_{o c}$ of 0.00027 , the lowest average $f_{o c}$ value determined from the data of Newcomer et al. (1995). Values in Table 6 are calculated using the $\mathrm{K}_{\mathrm{oc}}$ values reported in Table 1; values in Table 7 are calculated from field data using the $\mathrm{K}_{\mathrm{oc}}$ values from Table 2. Another estimate for the minimum $\mathrm{K}_{\mathrm{d}}$ was obtained by applying Equation (2) with the lowest average $f_{o c}$ value determined from the data reported by Newcomer et al. (1995) and a $\mathrm{K}_{\mathrm{oc}}$ value of 60 . As shown in Table 4, the low average of measured $\mathrm{f}_{\mathrm{oc}}$ corresponds to an organic fraction of $0.00027(0.027 \%)$. The $\mathrm{K}_{\mathrm{oc}}$ value $(60 \mu \mathrm{g} / \mathrm{g}-\mathrm{oc} / \mu \mathrm{g} / \mathrm{mL})$ was selected from an extensive survey of soils conducted by Kile et al. (1995) showing relatively 
Table 2. Published Values of Koc for Chloromethanes

\begin{tabular}{|c|c|c|c|c|c|}
\hline \multirow[b]{2}{*}{ Reference } & \multicolumn{4}{|c|}{ Koc $(\mu \mathrm{g} / \mathrm{g}-\mathrm{oc}) /(\mu \mathrm{g} / \mathrm{mL})$} & \multirow[b]{2}{*}{ Comment } \\
\hline & CT & CF & DCM & CM & \\
\hline Jeng et al. 1992 & 122 & 76.8 & 47.4 & $\mathrm{ND}^{(\mathrm{a})}$ & $\begin{array}{l}\text { Data are a compilation of } \\
\text { information measured by } \\
\text { others }\end{array}$ \\
\hline Walton et al. 1992 & $143.6 \pm 32.1^{(\mathrm{b})}$ & $37.4 \pm 8.6^{(\mathrm{b})}$ & ND & ND & $\begin{array}{l}\text { Silty loam with an } \\
\text { organic carbon content } \\
\text { of } 1.5 \%\end{array}$ \\
\hline Walton et al. 1992 & $48.9 \pm 16.2^{(b)}$ & $30.0 \pm 2.6^{(b)}$ & ND & ND & $\begin{array}{l}\text { Sandy loam with an } \\
\text { organic carbon content } \\
\text { of } 0.7 \%\end{array}$ \\
\hline Duffy et al. 1997 & $55.0 \pm 1.0^{(\mathrm{b})}$ & ND & ND & ND & $\begin{array}{l}\text { Soil with an organic } \\
\text { carbon content of } 1.5 \%\end{array}$ \\
\hline Duffy et al. 1997 & $77.6 \pm 1.3^{(\mathrm{b})}$ & ND & ND & ND & $\begin{array}{l}\text { Soil with an organic } \\
\text { carbon content of } 0.53 \%\end{array}$ \\
\hline Duffy et al. 1997 & $269 \pm 2^{(b)}$ & ND & ND & ND & $\begin{array}{l}\text { Soil with an organic } \\
\text { carbon content of } 0.14 \%\end{array}$ \\
\hline Peng and Dural 1998 & 121.9 & 153.5 & ND & ND & $\begin{array}{l}\text { Measured with Missouri } \\
\text { soil. }\end{array}$ \\
\hline Peng and Dural 1998 & 150.4 & 196.9 & ND & ND & $\begin{array}{l}\text { Measured with } \\
\text { California soil }\end{array}$ \\
\hline Peng and Dural 1998 & 121.0 & 190.0 & ND & ND & $\begin{array}{l}\text { Measured with Florida } \\
\text { Soil }\end{array}$ \\
\hline Kile et al. 1999 & $59.1 \pm 7.5^{(b)}$ & ND & ND & ND & $\begin{array}{l}\text { Average of } 17 \text { near } \\
\text { surface soils with } \\
\text { organic carbon ranging } \\
\text { from } 1.1 \% \text { to } 5.6 \%\end{array}$ \\
\hline Kile et al. 1999 & $106.7 \pm 13.44^{(\mathrm{b})}$ & ND & ND & ND & $\begin{array}{l}\text { Average of nine river } \\
\text { bottom sediments with } \\
\text { organic carbon ranging } \\
\text { from } 1.4 \% \text { to } 5.6 \%\end{array}$ \\
\hline Kile et al. 1995 & $60 \pm 7^{(\mathrm{b})}$ & ND & ND & ND & $\begin{array}{l}\text { Average of } 32 \text { near } \\
\text { surface soils with } \\
\text { organic carbon ranging } \\
\text { from } 0.16 \% \text { to } 6.1 \%\end{array}$ \\
\hline Kile et al. 1995 & $102 \pm 11^{(\mathrm{b})}$ & ND & ND & ND & $\begin{array}{l}\text { Average of } 36 \text { bed } \\
\text { sediments with organic } \\
\text { carbon ranging from } \\
0.11 \% \text { to } 4.7 \%\end{array}$ \\
\hline
\end{tabular}

Table 3. Maximum Estimates for the Solid/Liquid Equilibrium Partition Coefficient Based on $\mathrm{K}_{\mathrm{oc}}$ Data reported in Table 1 and the Maximum $\mathrm{f}_{\mathrm{oc}}$ in Table 4

\begin{tabular}{|l|c|c|}
\hline \multicolumn{3}{|c|}{$\begin{array}{c}\mathbf{K}_{\mathbf{d}}(\boldsymbol{\mu g} / \mathbf{g}-\mathbf{s o i l}) /(\boldsymbol{\mu g} / \mathbf{m L}) \\
\text { Compound }\end{array}$} \\
\hline CT & 0.57 & 0.83 \\
\hline CF & 0.16 & 0.20 \\
\hline DCM & 0.12 & 0.13 \\
\hline CM & 0.18 & 0.14 \\
\hline
\end{tabular}


Table 4. Organic Carbon Fraction Data for the Hanford Aquifer (Ringold Formation)

\begin{tabular}{|c|c|c|c|c|c|}
\hline Location & $\begin{array}{c}\text { Depth BGS } \\
\text { (m) }\end{array}$ & $\operatorname{Max} \mathbf{f}_{\mathrm{oc}}$ & $\operatorname{Min} \mathbf{f}_{\mathbf{~ o c}}$ & $\operatorname{Avg}_{ \pm}$Stdev & Reference \\
\hline $\begin{array}{l}299-w 11-30 \\
\text { (four samples) }\end{array}$ & 67 to 85 & $5.15 \times 10^{-3}$ & 0 & $1.5 \times 10^{-3} \pm 2.4 \times 10^{-3}$ & $\begin{array}{l}\text { Newcomer et al. } \\
1995\end{array}$ \\
\hline $\begin{array}{l}\text { 299-w11-32 } \\
\text { (five samples) }\end{array}$ & 74 to 77 & $7.6 \times 10^{-4}$ & 0 & $2.7 \times 10^{-4} \pm 2.9 \times 10^{-4}$ & $\begin{array}{l}\text { Newcomer et al. } \\
1995\end{array}$ \\
\hline $\begin{array}{l}\text { Quoted value } \\
\text { for Hanford, no } \\
\text { supporting data }\end{array}$ & $\mathrm{NA}^{(\mathrm{a})}$ & $1.00 \times 10^{-3}$ & $\mathrm{ND}^{(\mathrm{b})}$ & NA & WHC 1990 \\
\hline 299-w15-31 & 40 to 43 & $1.10 \times 10^{-3}$ & ND & NA & Ford 1996 \\
\hline
\end{tabular}

Table 5. Maximum Estimates for the Solid/Liquid Equilibrium Partition Coefficient Based on $\mathrm{K}_{\mathrm{oc}}$ Data Reported in Table 2 and the Maximum $\mathrm{f}_{\mathrm{oc}}$ in Table 4

\begin{tabular}{|c|c|c|c|c|c|}
\hline \multirow{2}{*}{ Reference } & \multicolumn{4}{|c|}{$K_{d}(\mu \mathrm{g} / g-s o i l) /(\mu \mathrm{g} / \mathrm{mL})$} & \multirow{2}{*}{ Comment } \\
\hline & CT & $\mathbf{C F}$ & DCM & CM & \\
\hline Jeng et al. 1992 & 0.63 & 0.40 & 0.24 & $\mathrm{ND}^{(\mathrm{a})}$ & $\begin{array}{l}\text { Data are a compilation of } \\
\text { information measured by } \\
\text { others }\end{array}$ \\
\hline Walton et al. 1992 & $0.74 \pm 0.17(2.1)$ & $0.19 \pm 0.04$ & ND & ND & $\begin{array}{l}\text { Silty loam with an organic } \\
\text { carbon content of } 1.5 \%\end{array}$ \\
\hline Walton et al. 1992 & $0.25 \pm 0.08(0.34)$ & $0.15 \pm 0.01$ & ND & ND & $\begin{array}{l}\text { Sandy loam with an } \\
\text { organic carbon content of } \\
0.7 \%\end{array}$ \\
\hline Duffy et al. 1997 & $0.28 \pm 0.01(0.83)$ & ND & ND & ND & $\begin{array}{l}\text { Soil with an organic } \\
\text { carbon content of } 1.5 \%\end{array}$ \\
\hline Duffy et al. 1997 & $0.40 \pm 0.01(0.41)$ & ND & ND & ND & $\begin{array}{l}\text { Soil with an organic } \\
\text { carbon content of } 0.53 \%\end{array}$ \\
\hline Duffy et al. 1997 & $1.4 \pm 0.01(0.38)$ & ND & ND & ND & $\begin{array}{l}\text { Soil with an organic } \\
\text { carbon content of } 0.14 \%\end{array}$ \\
\hline Peng and Dural 1998 & 0.63 & 0.79 & ND & ND & $\begin{array}{l}\text { Measured with Missouri } \\
\text { soil }\end{array}$ \\
\hline Peng and Dural 1998 & 0.77 & 1.0 & ND & ND & $\begin{array}{l}\text { Measured with California } \\
\text { soil }\end{array}$ \\
\hline Peng and Dural 1998 & 0.62 & 0.98 & ND & ND & Measured with Florida soil \\
\hline Kile et al. 1999 & $0.30 \pm 0.04^{(\mathrm{b})}$ & ND & ND & ND & $\begin{array}{l}\text { Average of } 17 \text { near surface } \\
\text { soils. }\end{array}$ \\
\hline Kile et al. 1999 & $0.55 \pm 0.07^{(\mathrm{b})}$ & ND & ND & ND & $\begin{array}{l}\text { Average of nine river } \\
\text { bottom sediments. }\end{array}$ \\
\hline Kile et al. 1995 & $0.31 \pm 0.04^{(\mathrm{b})}$ & ND & ND & ND & $\begin{array}{l}\text { Average of } 32 \text { near surface } \\
\text { soils with organic carbon } \\
\text { ranging from } 0.16 \% \text { to } \\
6.1 \%\end{array}$ \\
\hline Kile et al. 1995 & $0.53 \pm 0.06^{(\mathrm{b})}$ & ND & ND & ND & $\begin{array}{l}\text { Average of } 36 \text { bed } \\
\text { sediments with organic } \\
\text { carbon ranging from } \\
0.11 \% \text { to } 4.7 \% \text {. }\end{array}$ \\
\hline
\end{tabular}


Table 6. Minimum Estimates for the Solid/Liquid Equilibrium Partition Coefficient Based on $\mathrm{K}_{\mathrm{oc}}$ Data Reported in Table 1 and an $\mathrm{f}_{\mathrm{oc}}$ of 0.00027

\begin{tabular}{|c|c|c|}
\hline \multirow[b]{2}{*}{ Compound } & \multicolumn{2}{|c|}{$K_{d}(\mu g / g-s o i l) /(\mu g / m L)$} \\
\hline & Equation 2 & Equation 5 \\
\hline $\mathrm{CT}$ & $2.99 \times 10^{-2}$ & $4.35 \times 10^{-2}$ \\
\hline $\mathrm{CF}$ & $8.39 \times 10^{-3}$ & $1.05 \times 10^{-2}$ \\
\hline $\mathrm{DCM}$ & $6.29 \times 10^{-3}$ & $6.82 \times 10^{-3}$ \\
\hline $\mathrm{CM}$ & $9.44 \times 10^{-3}$ & $7.34 \times 10^{-3}$ \\
\hline
\end{tabular}

consistent $\mathrm{K}_{\mathrm{oc}}$ values over a wide range of soil types and organic carbon content. The minimum $\mathrm{K}_{\mathrm{d}}$ value calculated using this technique is $0.016 \mathrm{~L} / \mathrm{kg}$. This value is consistent with many of the lower $K_{d}$ values listed in Table 7 and was selected as the minimum $K_{d}$ value for use at Hanford.

Table 7. Minimum Estimates for the Solid/Liquid Equilibrium Partition Coefficient Based on $\mathrm{K}_{\mathrm{oc}}$ Data Reported in Table 2 and an $\mathrm{f}_{\mathrm{oc}}$ of 0.00027

\begin{tabular}{|c|c|c|c|c|c|}
\hline \multirow{2}{*}{ Reference } & \multicolumn{4}{|c|}{$K_{d}(\mu \mathrm{g} / \mathrm{g}-\mathrm{soil}) /(\mu \mathrm{g} / \mathrm{mL})$} & \multirow{2}{*}{ Comment } \\
\hline & $\mathrm{CT}^{(\mathbf{a})}$ & $C F^{(a)}$ & DCM & CM & \\
\hline Jeng et al. 1992 & 0.033 & $0.021 \pm 0.002$ & 0.013 & $\mathrm{ND}^{(\mathrm{b})}$ & $\begin{array}{l}\text { Data are a compilation of } \\
\text { information measured by } \\
\text { others }\end{array}$ \\
\hline Walton et al. 1992 & $0.039 \pm 0.009$ & $0.010 \pm 0.001$ & ND & ND & $\begin{array}{l}\text { Silty loam with an organic } \\
\text { carbon content of } 1.5 \%\end{array}$ \\
\hline Walton et al. 1992 & $0.013 \pm 0.004$ & 0.0079 & ND & ND & $\begin{array}{l}\text { Sandy loam with an organic } \\
\text { carbon content of } 0.7 \%\end{array}$ \\
\hline Duffy et al. 1997 & $0.015 \pm 0.003$ & ND & ND & ND & $\begin{array}{l}\text { Soil with an organic carbon } \\
\text { content of } 1.5 \%\end{array}$ \\
\hline Duffy et al. 1997 & $0.021 \pm 0.003$ & ND & ND & ND & $\begin{array}{l}\text { Soil with an organic carbon } \\
\text { content of } 0.53 \%\end{array}$ \\
\hline Duffy et al. 1997 & $0.073 \pm 0.005$ & ND & ND & ND & $\begin{array}{l}\text { Soil with an organic carbon } \\
\text { content of } 0.14 \%\end{array}$ \\
\hline Peng and Dural 1998 & 0.033 & 0.041 & ND & ND & Measured with Missouri soil \\
\hline Peng and Dural 1998 & 0.040 & 0.052 & ND & ND & Measured with California soil \\
\hline Peng and Dural 1998 & 0.033 & 0.051 & ND & ND & Measured with Florida soil \\
\hline Kile et al. 1999 & $0.016 \pm 0.002$ & ND & ND & ND & $\begin{array}{l}\text { Average of } 17 \text { near surface } \\
\text { soils }\end{array}$ \\
\hline Kile et al. 1999 & $0.029 \pm 0.004$ & ND & ND & ND & $\begin{array}{l}\text { Average of } 9 \text { river bottom } \\
\text { sediments }\end{array}$ \\
\hline Kile et al. 1995 & $0.016 \pm 0.002$ & ND & ND & ND & $\begin{array}{l}\text { Average of } 32 \text { near surface } \\
\text { soils with organic carbon } \\
\text { ranging from } 0.16 \% \text { to } 6.1 \%\end{array}$ \\
\hline Kile et al. 1995 & $0.028 \pm 0.003$ & ND & ND & ND & $\begin{array}{l}\text { Average of } 36 \text { bed sediments } \\
\text { with organic carbon ranging } \\
\text { from } 0.11 \% \text { to } 4.7 \%\end{array}$ \\
\hline
\end{tabular}


The selected range of values of $\mathrm{K}_{\mathrm{d}}$ for $\mathrm{CT}$ is 0.016 to $0.83 \mathrm{~L} / \mathrm{kg}$. The most probable value within this range was selected as $0.12 \mathrm{~L} / \mathrm{kg}$. This value corresponds a $\mathrm{K}_{\mathrm{d}}$ calculated using a $\mathrm{K}_{\mathrm{oc}}$ value measured from an extensive survey of soils conducted by Kile et al. (1995) showing relatively consistent $K_{o c}$ values over a wide range of soil types and organic carbon content. The $f_{o c}$ value used in the calculation of most probable $K_{d}$ was 0.002 . This value for $f_{o c}$ is the average of the maximum $\mathrm{f}_{\mathrm{oc}}$ values reported in Table 4 and is considered to be a reasonable compromise of $\mathrm{f}_{\mathrm{oc}}$ information for the Hanford Site.

\subsection{Probability Distributions of Modeling Parameters}

In addition to the geostatistical simulations of the $\mathrm{CT}$ source volumes that we just described, realizations of several other modeling parameters were needed for the Monte Carlo flow and transport study. Two of those parameters, the $\mathrm{K}_{\mathrm{d}}$ for $\mathrm{CT}$ and the abiotic degradation rate of $\mathrm{CT}$, were included in the parameter estimation portion of this study (see Section 2.3). As part of parameter estimation, the minimum, maximum, and most likely values of each parameter were identified and used to characterize the probability distributions of those two variables (Table 8). In each case, a triangular probability distribution was assumed to fit the distribution. Estimated characteristics of the probability distribution for the porosity of the aquifer in the 200 West Area were identified by F. Spane (PNNL), based on the assumption that the aquifer is dominated by Ringold gravels. Based on that input, the distribution of porosity in those sediments was assumed to be uniform with a minimum of $5 \%$ and a maximum of $15 \%$. The remaining parameter required for the modeling was the longitudinal dispersivity. The characteristics of the probability distribution for that parameter were supplied by C. Cole (PNNL), and were assumed to be a triangular distribution with a minimum of $10 \mathrm{~m}$, maximum of $100 \mathrm{~m}$, with a most probable value of $30 \mathrm{~m}$. Table 9 summarizes the assumed characteristics of the probability distributions of the 4 modeling parameters. These probability distributions were then sampled using the program STATGEN, developed by Paul Eslinger (PNNL). A suite of 1000 realizations of each parameter were generated by the program, assuming that the four parameters are independent of one another. Figure 21 displays histograms of the simulated values for each of the four parameters.

Table 8 shows the volume and mass exceeding cutoff levels within the source area. The table also shows the proportions of mass exceeding the cutoff levels within the source area to the total mass within the identified source area and the proportions of mass exceeding the cutoff levels within the source area to the entire simulated map area. Results shown are for the first three of 1000 simulations generated.

Table 8. Cutoff Levels and Volume, Mass, and Proportions

\begin{tabular}{|c|c|c|c|c|c|c|c|c|c|c|c|c|}
\hline \multirow{2}{*}{$\begin{array}{c}\text { Simulation } \\
\text { No. }\end{array}$} & \multicolumn{4}{|c|}{ Cutoff Level $=500 \Phi \mathrm{g} / \mathrm{L}$} & \multicolumn{4}{|c|}{ Cutoff Level $=1000 \Phi \mathrm{g} / \mathrm{L}$} & \multicolumn{4}{|c|}{ Cutoff Level $=2000 \Phi \mathrm{g} / \mathrm{L}$} \\
\hline & $\mathrm{Vol}(>\mathrm{CT})$ & Mass $(>\mathrm{CT})$ & Prop(box) & Prop(all) & $\operatorname{Vol}(>\mathrm{CT})$ & $\operatorname{Mass}(>\mathrm{CT})$ & Prop(box) & Prop(all) & $\operatorname{Vol}(>\mathrm{CT})$ & $\operatorname{Mass}(>\mathrm{CT})$ & Prop(box) & Prop(all) \\
\hline 1 & 6887500 & 14447 & 0.92 & 0.44 & 4500000 & 12756 & 0.81 & 0.39 & 3312500 & 11013 & 0.70 & 0.34 \\
\hline 2 & 4762500 & 8258 & 0.88 & 0.29 & 2975000 & 7031 & 0.75 & 0.24 & 1750000 & 5238 & 0.56 & 0.18 \\
\hline 3 & 8437500 & 17836 & 0.94 & 0.49 & 5775000 & 15962 & 0.84 & 0.43 & 4125000 & 13567 & 0.71 & 0.37 \\
\hline
\end{tabular}


Table 9. Modeling Parameter Probability Distributions

\begin{tabular}{|c|c|c|c|c|}
\hline Parameter & Distribution Type & Minimum & Maximum & Most Probable \\
\hline $\mathrm{CT} \mathrm{K}_{\mathrm{d}}(\mathrm{L} / \mathrm{kg})$ & Triangular & 0.016 & 0.83 & 0.12 \\
\hline $\mathrm{CT} \mathrm{K}_{\mathrm{a}}$ (per day) & Triangular & $6.50 \mathrm{E}-06$ & $5.30 \mathrm{E}-05$ & $1.90 \mathrm{E}-05$ \\
\hline Porosity & Uniform & $5 \%$ & $15 \%$ & $\mathrm{NA}^{\text {(a) }}$ \\
\hline Dispersity (m) & Triangular & 10 & 100 & 30 \\
\hline (a) NA equals not applicable.
\end{tabular}
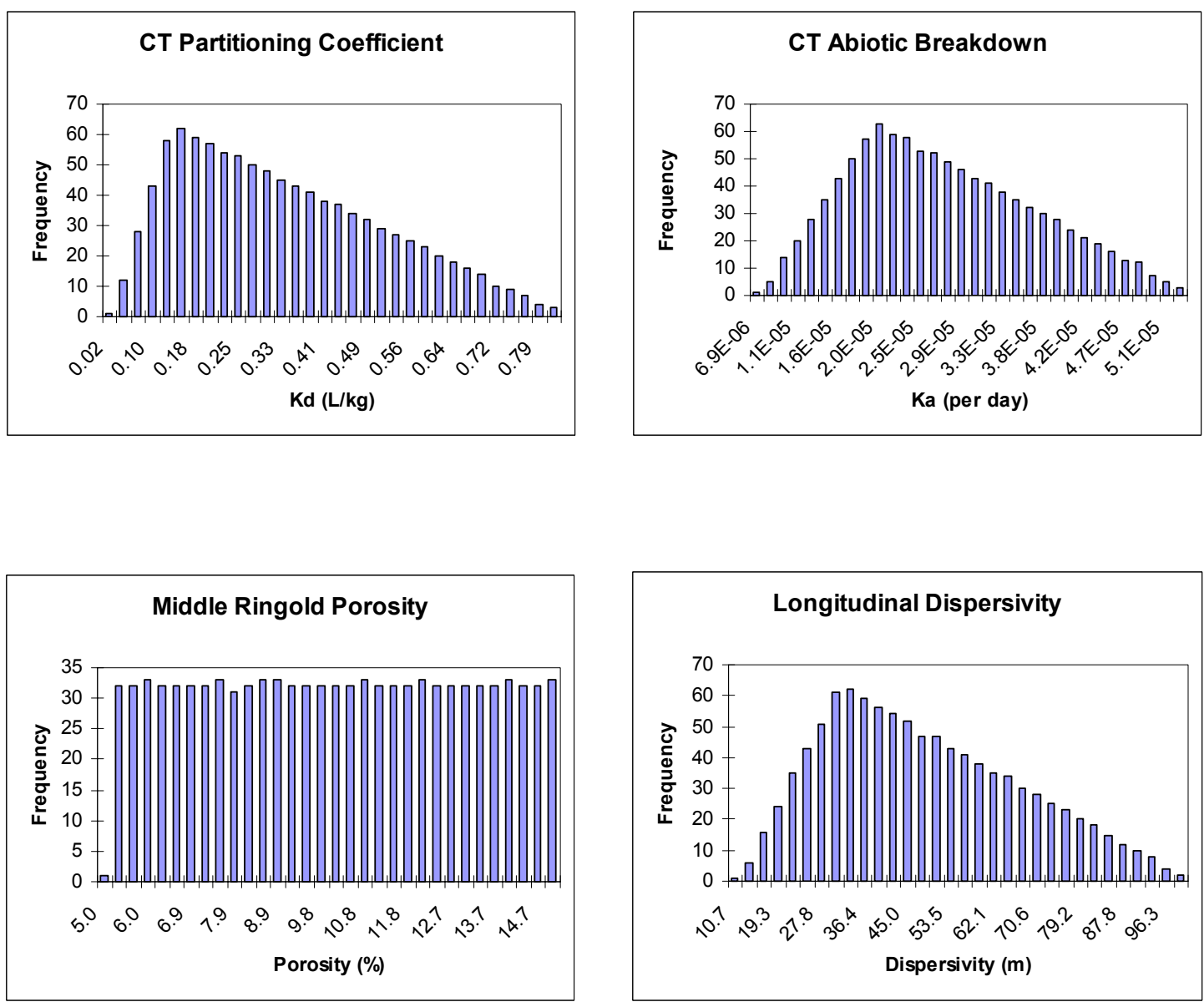

Figure 22. Histograms of 1000 Simulated Values of Each of the Four Input Parameters Used in the Flow and Transport Modeling 


\subsection{Results}

The results presented in this section show frequency distributions of the required source cleanup percentages for the Monte Carlo simulations (i.e., variations of transport parameters $\mathrm{K}_{\mathrm{d}}$,

$\mathrm{K}_{\mathrm{a}}$, porosity, dispersivity, and cross-sectional area) at the mean Darcy velocity and a $3000 \mu \mathrm{g} / \mathrm{L}$ inlet-source concentration. The required source cleanup percentage for each simulation was calculated from the ratio of the maximum concentration at the compliance boundary to the selected compliance concentration. This calculation assumes that reducing the source quantity by this percentage would decrease the source concentration by this same percentage; thus, the concentration at the compliance boundary would decrease by this percentage as well. The basis of this assumption is that by removing source within a portion of the defined source area volume so that this "cleaned up" portion now contributes only clean water within the source, the source is diluted.

Two boundary-concentration levels were used in the simulations: the $5-\mu \mathrm{g} / \mathrm{L}$ regulatory limit, and a higher $50-\mu \mathrm{g} / \mathrm{L}$ concentration used to account for three-dimensional dispersion. The probability distributions of source cleanup required as a function of source inventory were used to identify the likely limits of remediation; stochastic parameters were subjected to regression analysis to determine their relative influence on simulation outputs and identify variables needing further characterization to improve modeling accuracy.

\subsection{Modeling Results and Discussion}

The results of the 1000 Monte Carlo simulations using the van Genuchten model provide a basis for determining the portion of the source area needing remediation; the simulations also provide a method of gauging sensitivity of predicted contaminant transport to variations in individual parameters. (Simulation results are provided in Appendix D.) The break point between peak contaminant-concentration levels at $1 \%$ and $10 \%$ remaining source inventory seen in the determinant-parameter variation simulations is repeated in the Monte Carlo simulations. With more accurate parameter estimates and additional site characterization, the path forward to necessary site remediation can be mapped using improved modeling techniques and sourceinventory quantification technologies.

The one-dimensional model used for this study simulates the transport of CT from the source area to the compliance boundary assuming a steady-state, one-dimensional flow field. Thus the model is conservative in that CT travels in a direct path from the source area toward the compliance boundary. Based on the current and projected groundwater flow conditions in the 200 West Area, an assumption of uniform steady-state flow in one direction is conservative but reasonable. However, the one-dimensional van Genuchten model did not take into account transverse or vertical dispersivity and the additional reduction in contaminant-concentration that would occur with this additional plume spreading (i.e., increased vertical and lateral dispersion would result in the CT plume contacting more volume of the aquifer and therefore more sorption sites, delaying migration and allowing more time for hydrolysis to occur). 
To estimate three-dimensional dispersion effects on simulation outputs, transverse and vertical dispersivities were modeled as $20 \%$ and $2 \%$ (respectively) of longitudinal dispersion. Simulations including transverse and vertical dispersion/dilution were conducted to determine how the one-dimension modeling results could be interpreted for estimating the effect of a threedimensional flow field (see Figure 23). In $80 \%$ of these simulations, the concentration at the compliance boundary was reduced by a factor of greater than 5 . In about $20 \%$ of these simulations, the concentration was reduced by a factor of greater than 10 with a maximum reduction of a factor of about 30 . The results of these simulations were used to postulate that interpretations of one-dimensional modeling results using a $50-\mu \mathrm{g} / \mathrm{L}$ boundary concentration limit provided a ptd estimate of CT transport at the higher end of the observed concentration reduction factor. This ptd estimate was used to provide a reasonable bound for the possible three-dimensional flow effects during transport of CT from the source area to the compliance boundary. CT concentration limits of $5 \mu \mathrm{g} / \mathrm{L}$ and $50 \mu \mathrm{g} / \mathrm{L}$ at the compliance boundary are used in the report so that interpretations of the results under both conservative one-dimensional flow conditions and reasonable estimate of three-dimensional flow conditions (factor of 10 change due to three-dimensional flow) can be considered. Note that the $50-\mu \mathrm{g} / \mathrm{L}$ compliance boundary concentration limit is solely a calculational construct to give an approximation of a threedimensional model, not a proposed change in the actual 5- $\mu \mathrm{g} / \mathrm{L}$ compliance concentration requirement. Selection of the $50-\mu \mathrm{g} / \mathrm{L}$ compliance boundary concentration is based on using a factor of 10 correlation between one- and three-dimensional transport that was calculated based on the components of dispersion that are not accounted for in the one-dimensional model.

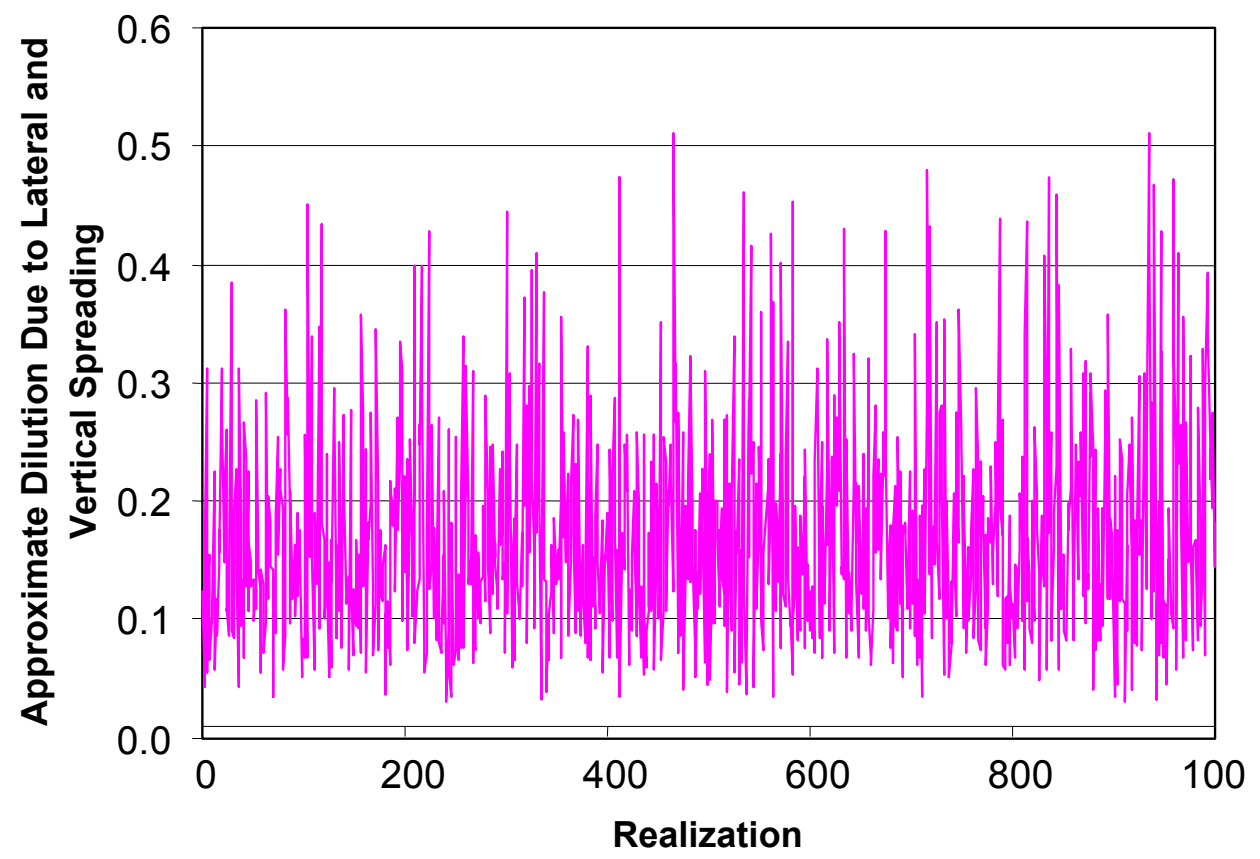

Fractional Dilution by Lateral and Vertical Spreading (due to increase in cross-sectional area of streamtube)

Figure 23. Estimated Additional Dilution due to Transverse and Vertical Spreading (3-D effect) 
The simulations conducted for this study examine the migration of CT from the source area to the compliance boundary; the simulations did not examine migration of the existing dispersed plume and did not attempt to match the historical disposal and migration of CT (i.e., did not attempt to reproduce the current $\mathrm{CT}$ plume distribution).

The results of the Monte Carlo simulations were charted as histograms showing frequency distributions for 1000 modeling realizations using stochastic parameters. Simulation outputs include

- required source cleanup percentage needed to meet compliance regulations

- source inlet rate

- compliance boundary concentration

- compliance boundary flux

- compliance boundary arrival times

- source duration

- arrival time of peak concentration

- concentration as a function of distance from the source.

The results considered here are the source cleanup percentages presented as realizations of the base-case deterministic parameters (mean Darcy velocity, $3000 \mu \mathrm{g} / \mathrm{L}$ inlet source concentration) with the variable deterministic parameter being the fraction of base inventory remaining.

Results for the required source cleanup frequency distribution for $65 \%$ of the source inventory remaining shows that more than 470 simulations out of 1000 require more than $70 \%$ source cleanup for the ptd simulation using the $50-\mu \mathrm{g} / \mathrm{L}$ ptd compliance boundary concentration. The conservative one-dimensional result ( $5-\mu \mathrm{g} / \mathrm{L}$ ptd compliance boundary concentration) shows that about 850 simulations out of 1000 require more than $70 \%$ source cleanup (see Figure 24 ). The
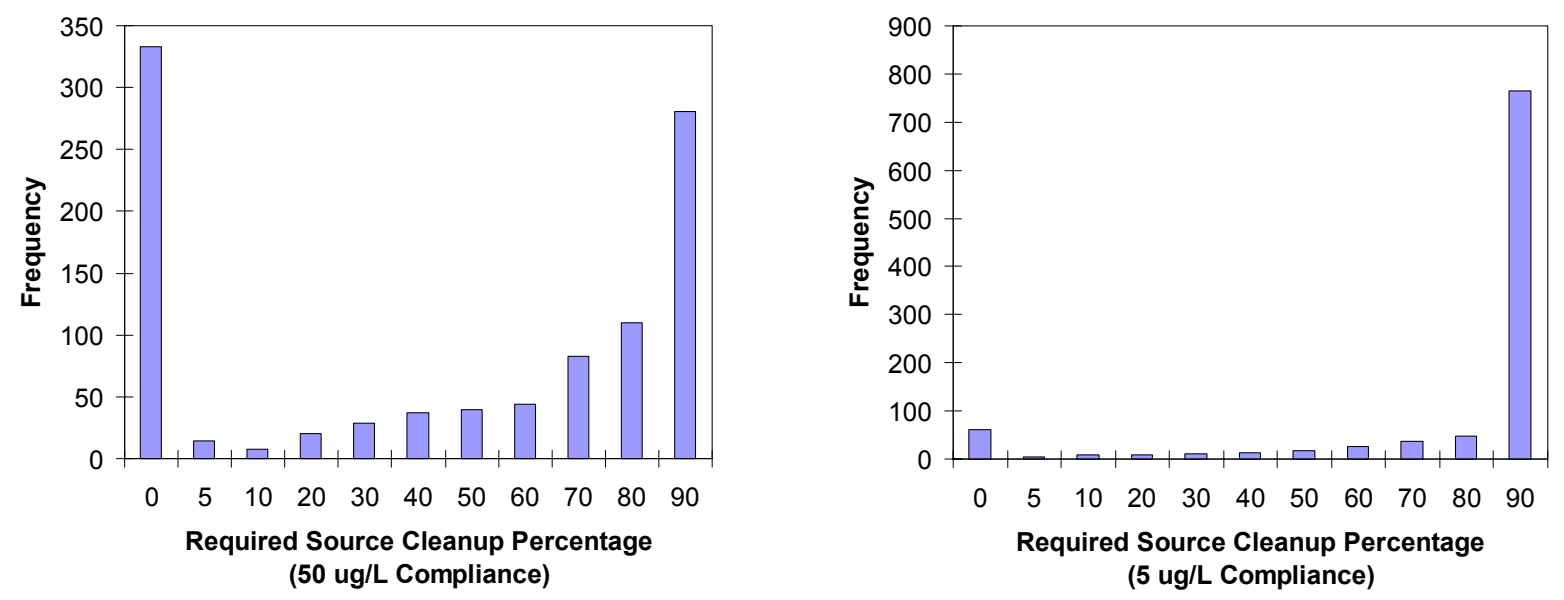

Figure 24. 65\% Source Inventory—Mean Velocity, $3000 \mu \mathrm{g} / \mathrm{L}$ 
results also show that more than 300 and more than 50 of the simulations required less than 5\% source cleanup for the $50-\mu \mathrm{g} / \mathrm{L}$ and $5-\mu \mathrm{g} / \mathrm{L}$ compliance concentrations, respectively.

The required source cleanup percentage for $30 \%$ of the source inventory remaining shows approximately the same results as the $65 \%$ case. The required source cleanup frequency distribution for $30 \%$ of source inventory remaining shows more than 470 simulations out of 1000 require greater than $70 \%$ source cleanup for the ptd simulation using the $50-\mu \mathrm{g} / \mathrm{L} \mathrm{ptd} \mathrm{compliance}$ boundary concentration; the conservative one-dimensional result $(5-\mu \mathrm{g} / \mathrm{L}$ ptd compliance boundary concentration) shows about 850 simulations out of 1000 require greater than $70 \%$ source cleanup (Figure 25). The results also show that more than 300 and more than 50 of the simulations required less than $5 \%$ source cleanup for the $50-\mu \mathrm{g} / \mathrm{L}$ and $5-\mu \mathrm{g} / \mathrm{L}$ compliance concentrations, respectively.

There is also little change in required source cleanup percentage from the former cases to the $10 \%$ source inventory remaining. The required source cleanup frequency distribution for $10 \%$ of source inventory remaining shows more than 420 simulations out of 1000 require greater than $70 \%$ source cleanup for the ptd simulation using the $50-\mu \mathrm{g} / \mathrm{L}$ ptd compliance boundary concentration; the conservative one-dimensional result $(5-\mu \mathrm{g} / \mathrm{L}$ ptd compliance boundary concentration) shows more than 800 simulations out of 1000 require greater than $70 \%$ source cleanup (see Figure 26). The results also show that more than 375 and about 100 of the simulations required less than 5\% source cleanup for the 50- $\mu \mathrm{g} / \mathrm{L}$ and $5-\mu \mathrm{g} / \mathrm{L}$ compliance concentrations, respectively.

At $1 \%$ source inventory remaining, a dramatic shift in the distributions is observed. The required source cleanup frequency distribution for $1 \%$ of source inventory remaining shows about 150 simulations out of 1000 require greater than $70 \%$ source cleanup for the ptd simulation using the $50-\mu \mathrm{g} / \mathrm{L}$ ptd compliance boundary concentration; the conservative one-dimensional result $(5-\mu \mathrm{g} / \mathrm{L}$ ptd compliance boundary concentration) shows about 550 simulations out of 1000 require greater than $70 \%$ source cleanup (Figure 27 ). The results also show that more than 700 and more than 300 of the simulations required less than $5 \%$ source cleanup for the $50-\mu \mathrm{g} / \mathrm{L}$ and $5-\mu g$ /L compliance concentrations, respectively.

The breakpoint for dramatic change in the cleanup percentages clearly lies between $1 \%$ and $10 \%$ of source inventory remaining. The input parameters in the simulation that had the greatest effect on the results appear to be porosity, $\mathrm{K}_{\mathrm{d}}$ and $\mathrm{K}_{\mathrm{a}}$; a multiple linear regression analysis of $\log$ maximum concentration at the boundary versus porosity, $\mathrm{K}_{\mathrm{d}}$ and $\mathrm{K}_{\mathrm{a}}$ gave standardized regression coefficients of $-0.145,-0.785$, and -0.504 , respectively, at $1 \%$ inventory remaining and -0.187 , -0.691 , and -0.601 , respectively, at $10 \%$ inventory remaining (see Table 10 ). 

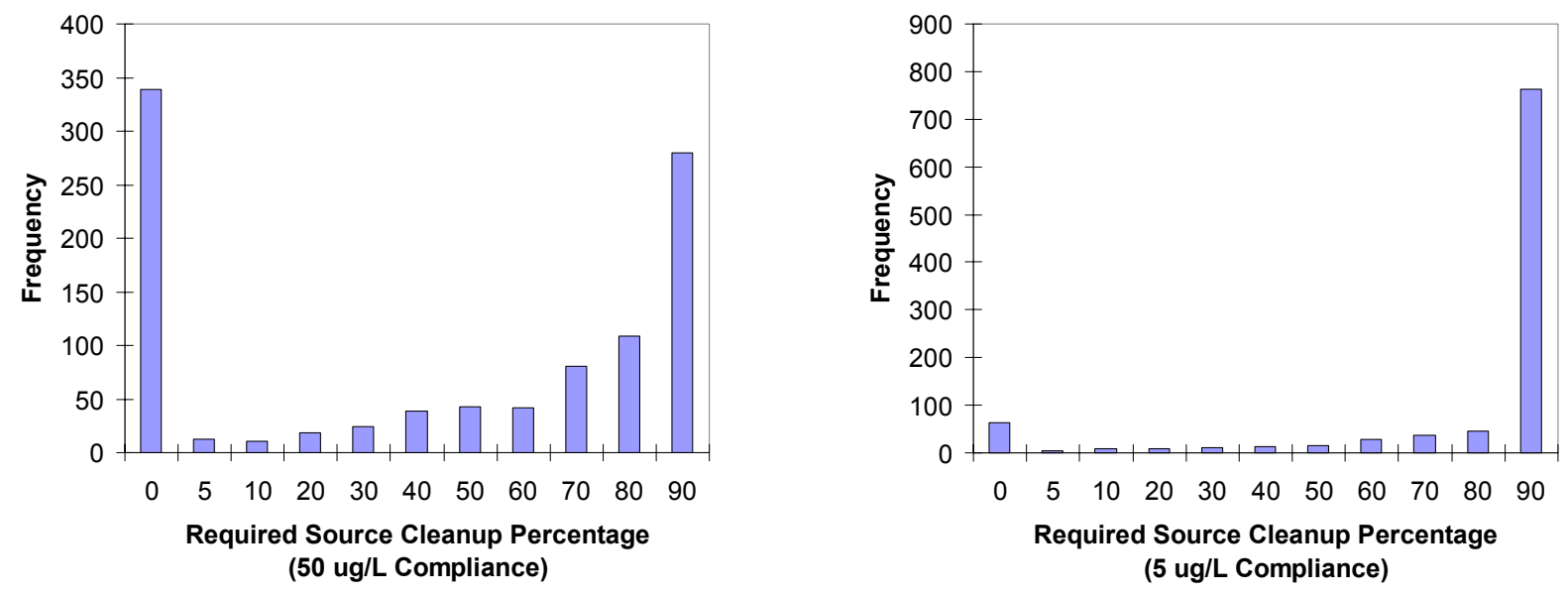

Figure 25. 30\% Source Inventory—Mean Velocity, $3000 \mu \mathrm{g} / \mathrm{L}$
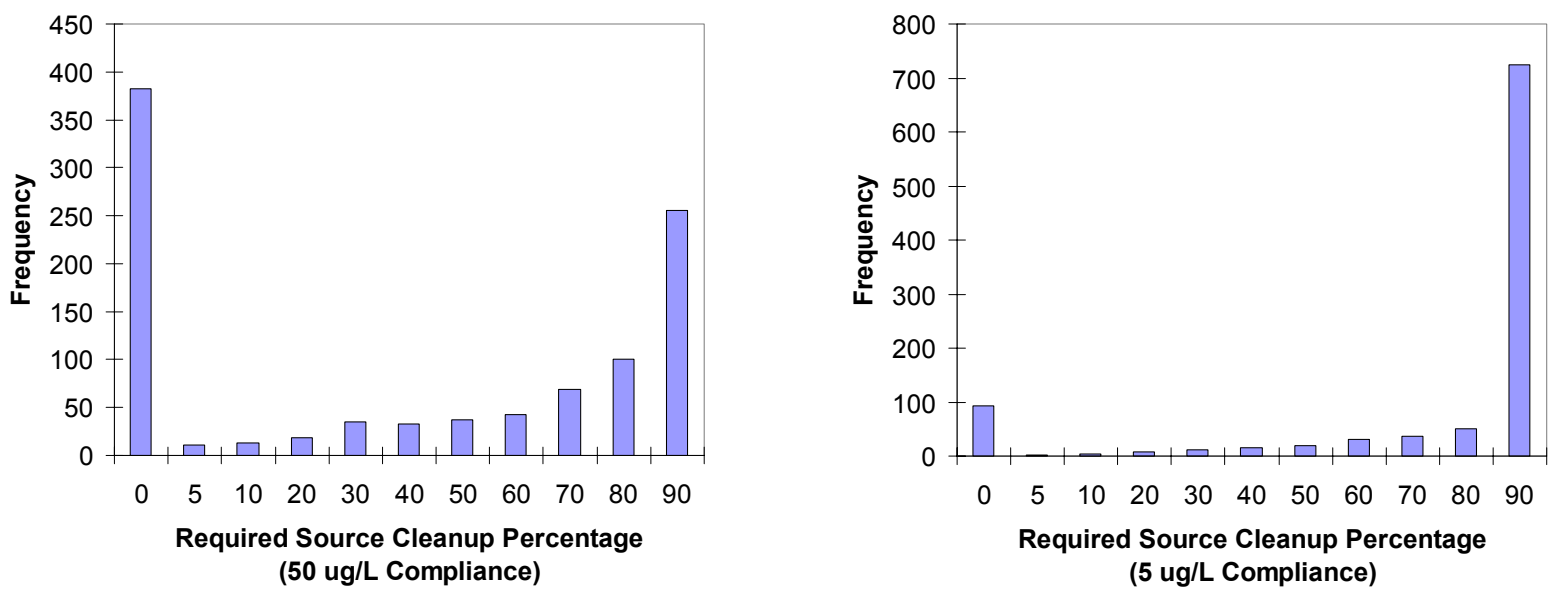

Figure 26. $10 \%$ Source Inventory—Mean Velocity, $3000 \mu \mathrm{g} / \mathrm{L}$
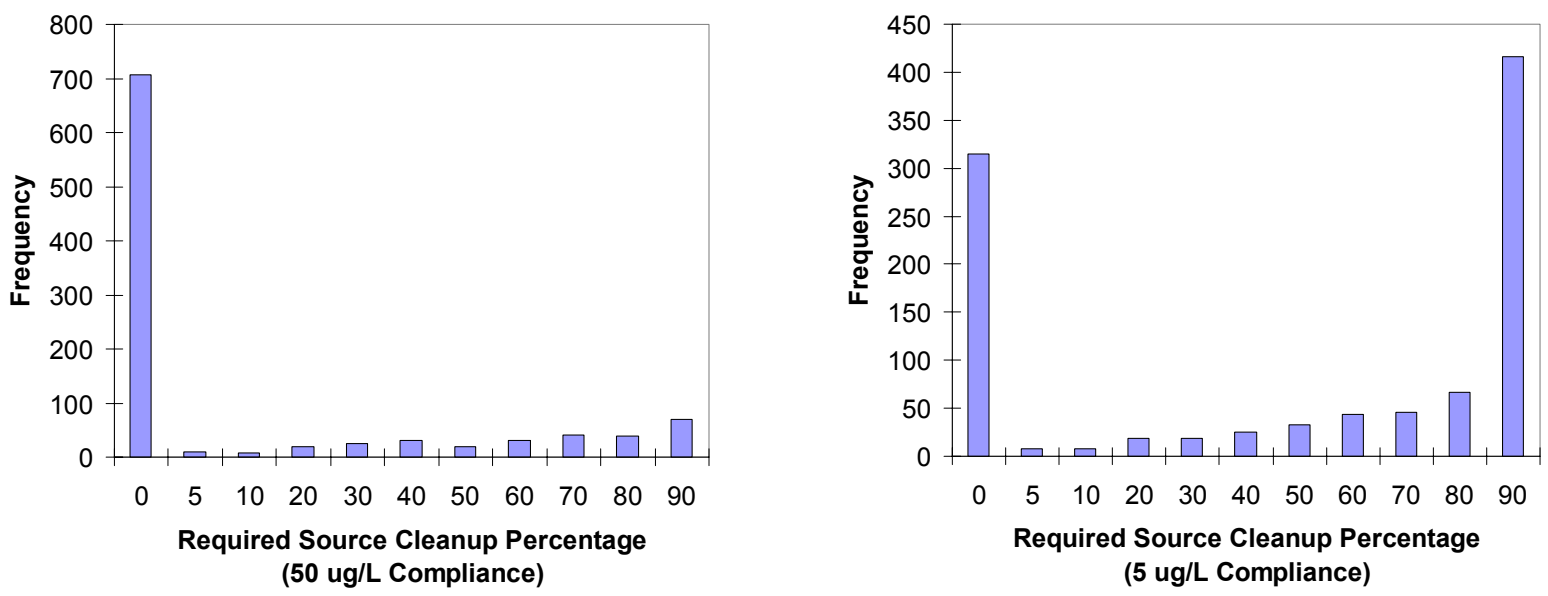

Figure 27. 1\% Source Inventory-Mean Velocity, $3000 \mu \mathrm{g} / \mathrm{L}$ 
Table 10. Linear Correlation Coefficients Between the Logarithm of Maximum Concentration at the Compliance Boundary and Input Parameters for the Set of 1000 1-D Flow and Transport Simulations of CT ( $2 \%$ remaining inventory case)

\begin{tabular}{|l|l|l|l|l|l|}
\hline & $\begin{array}{c}\text { Vol. over 3000 } \\
\boldsymbol{\mu g} / \mathbf{L}\end{array}$ & Porosity & \multicolumn{1}{|c|}{$\mathbf{K}_{\mathbf{d}}$} & $\boldsymbol{\alpha}_{\text {Long. }}$ & Abiotic t $_{\mathbf{1 / 2}}$ \\
\hline Volume above cutoff & 1.000 & & & & \\
\hline Porosity & 0.057 & 1.000 & & & \\
\hline $\mathrm{K}_{\mathrm{d}}$ & -0.070 & 0.053 & 1.000 & & \\
\hline$\alpha_{\text {Long. }}$ & -0.032 & $3.205 \mathrm{E}-4$ & 0.004 & 1.000 & \\
\hline Abiotic $\mathrm{t}_{1 / 2}$ & -0.016 & 0.004 & 0.012 & 0.026 & 1.000 \\
\hline $\log$ max. concentration & -0.054 & -0.184 & -0.787 & 0.025 & 0.493 \\
\hline
\end{tabular}

\subsection{Parameter Influence}

The simulation results suggest that $\mathrm{K}_{\mathrm{d}}$ has the greatest influence and porosity the least influence on boundary concentration levels as source inventory remaining decreases. Figure 28 displays notched box plots of the five input parameters for realizations that would require cleanup at the $50-\mu \mathrm{g} / \mathrm{L}$ threshold vs. those that would not, for the $1 \%$ inventory case. The plots indicate that the porosity and $\mathrm{Kd}$ are significantly lower and the abiotic half-life significantly higher for cases that require cleanup. The correlation coefficients for the logarithm of maximum concentration at the compliance boundary and the five input parameters for the $1 \%$ case (see Table 10) also suggest that the volume above the cutoff and the longitudinal dispersivity do not influence the maximum concentration at the compliance boundary, but that the porosity, $\mathrm{Kd}$, and abiotic half-life all do. The relative influence of the three variables that appear to control the maximum concentration was confirmed by multiple linear regression of the logarithm of the maximum concentration against the porosity, $\mathrm{Kd}$, and abiotic half-life. The standardized regression coefficients for the three input parameters are $-0.145,-0.785$, and 0.504 , suggesting that $\mathrm{Kd}$ has the greatest influence of the three input parameters on the maximum concentrations and porosity has the least influence after accounting for differences in the scale of the three input parameters. Because the input parameters are independent of one another (by construction), the standardized regression coefficients should provide a reasonable measure of the relative importance of the input parameters, which would not necessarily be the case if there were significant correlation between them. The total correlation coefficient for that multiple linear regression model is 0.945 , suggesting that almost $90 \%$ of the variation in the logarithm of the maximum concentration at the compliance boundary is explained by the linear regression using the porosity, $\mathrm{Kd}$, and abiotic half-life.

However, a significant shift occurs in standardized regression coefficients for increasing remaining inventory levels. In all cases up to the $65 \%$ inventory level, the porosity has the least influence on the maximum concentration, but the magnitude of its standardized correlation coefficient does increase from -0.145 to -0.235 . The relative importance of $\mathrm{Kd}$ and the abiotic half-life also shift, so the relative influence of the two parameters is more nearly equal for the $65 \%$ remaining inventory case (standardized regression coefficients are -0.598 and 0.656 , respectively). 

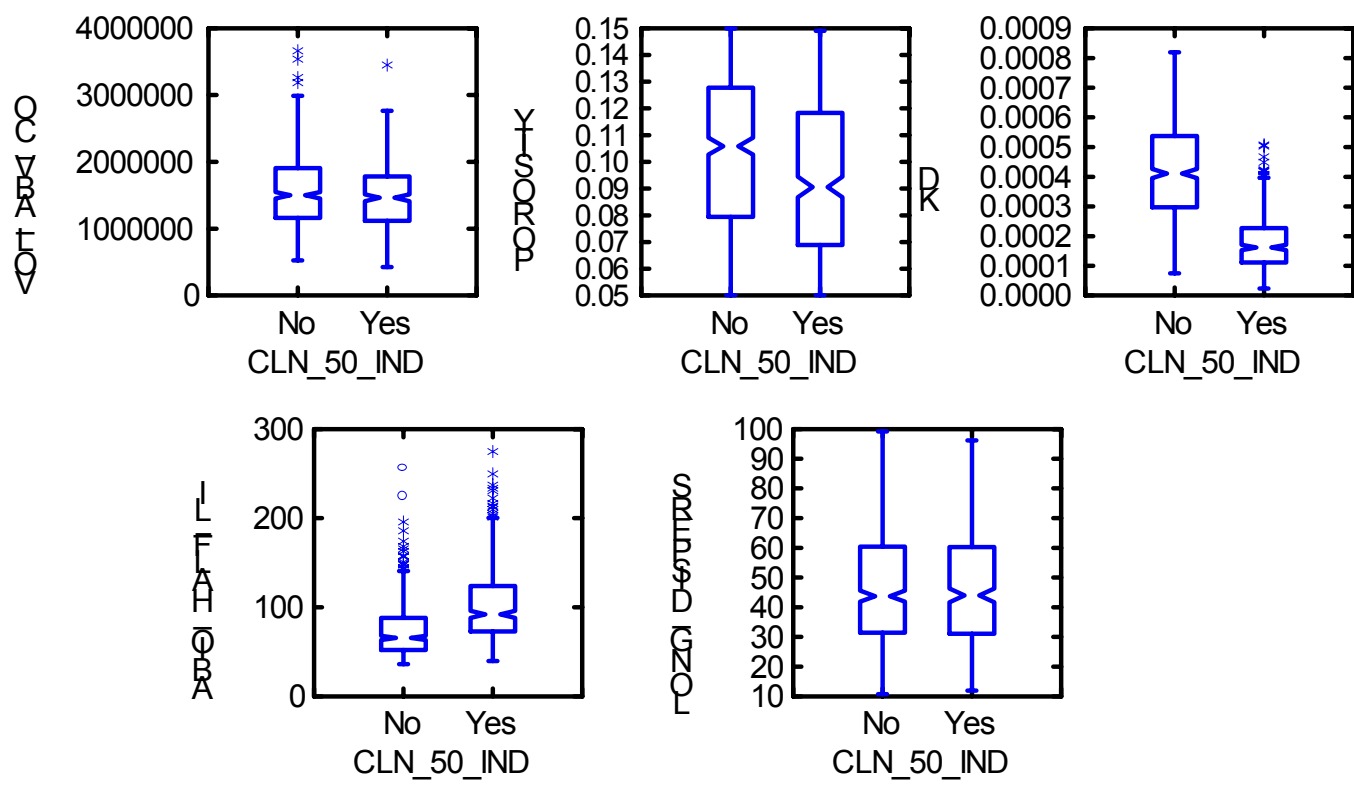

Figure 28. Notched Box Plots Showing Values of Transport Parameters for Cases that Did and Did Not Require Source Cleanup for the 1\% Inventory Case. Note that transport parameters with a significant effect on the need for cleanup are where the notches of the two boxes do not overlap. Notched box plots compare the values of input parameters, for those cases requiring cleanup and those that do not, for the $1 \%$ remaining inventory case. The upper edge of the box in each case marks the $75^{\text {th }}$ percentile; the lower edge of the box marks the $25^{\text {th }}$ percentile; and the middle of the notch denotes the median of the distribution. The upper and lower edges of the notches represent an approximate $95 \%$ confidence interval for the median of the distribution. If the notches overlap, as they do for the volume above the cutoff of $3000 \mu \mathrm{g} / \mathrm{L}$ and for the longitudinal dispersivity, the difference between the medians of the cases requiring cleanup and those not requiring cleanup is not significant. Significant differences exist for the porosity, abiotic half-life, and $\mathrm{K}_{\mathrm{d}}$, with the maximum separation occurring for the $\mathrm{K}_{\mathrm{d}}$.

These results suggest that $\mathrm{K}_{d}$ is the most important input parameter when there is only a small remaining inventory and the duration of the source term is relatively small, but that $\mathrm{K}_{\mathrm{d}}$ and the abiotic half-life both exert about equal influence on the maximum concentration at the compliance boundary for larger remaining inventory levels when the duration of the source term is much longer. 


\subsection{Conclusions}

The modeling was based on the assumption that about $750,000 \mathrm{~kg}$ of CT was discharged to the soil in the Z-crib area. Previous work has shown that of this $750,000 \mathrm{~kg}$, about $65 \%$ cannot be accounted for. Therefore, modeling was performed using $65 \%, 30 \%, 10 \%$, and $1 \%$ of the $750,000 \mathrm{~kg}$ as possible amounts of CT that could reach the groundwater. It is of value to note that approximately 1 to $2 \%$ of the CT inventory currently exists in the distal plume based on averaged CT groundwater measurements.

Several conclusions drawn from the modeling are discussed below:

- If $1 \%$ of the discharged CT is all that ever reaches the groundwater, it is likely that the highest concentration of CT to arrive at the compliance boundary will not exceed the compliance concentration. However, it is possible the compliance concentration would be exceeded if the actual site parameters correspond to the lower porosity, lower $\mathrm{K}_{\mathrm{d}}$, and lower $\mathrm{K}_{\mathrm{a}}$ values used in this study.

- If $10 \%$ or more of the discharged CT reaches the groundwater, it is likely that the concentration of CT eventually arriving at the compliance boundary will exceed the compliance concentration (unless source removal efforts are used).

- There is a breakpoint between $1 \%$ and $10 \%$ of the discharged inventory that defines the amount of CT in the source at which source removal would be required to avoid exceeding the compliance concentration at the compliance boundary.

- Because source inventory remaining appears to be the quantity driving the amount of site cleanup required for compliance, source inventory characterization using partitioning interwell tracer tests (or other source-quantity characterization technologies) would be a milestone on the path toward resolution of compliance issues.

- Laboratory experiments and site surveys can be used to better quantify values for the parameters controlling compliance boundary concentrations: $\mathrm{K}_{\mathrm{d}}, \mathrm{K}_{\mathrm{a}}$, and porosity; additional modeling, including use of a three-dimensional model, can then be performed using these improved values to give more accurate estimates of compliance boundary concentrations and source cleanup requirements. 


\subsection{References}

Bishop DJ, JP Knezovitch, and DW Rice Jr. 1989. Sorption Studies of VOCs Related to Soil/Ground Water Contamination at LLNL. Environmental Protection Agency Environmental Restoration Series, UCID-21651:1-12. Lawrence Livermore National Laboratory, Livermore, California.

Bouwer EJ and PL McCarty. 1983. Transformations of 1- and 2-Carbon Halogenated Aliphatic Organic Compounds Under Methanogenic Conditions. Applied and Environ. Microbiology, 45(4):1286-1294.

Cobb GD and EJ Bouwer. 1991. Effects of Electron Acceptors on Halogenated Organic Compound Biotransformations in a Biofilm Column. Environ. Sci. Technol. 25:1068-1074.

Connelly MP, BH Ford, and JV Borghese. 1992. Hydrogeologic Model for 200 West Groundwater Aggregate Area. WHC-SD-EN-TI-014, Westinghouse Hanford Company, Richland, Washington.

Criddle CS, JT DeWitt, and PL McCarty. 1990. Reductive Dehalogenation of Carbon Tetrachloride by Escherichia coli K-12. Applied and Environ. Microbiology, 56(11):3247-3254.

Deeley GM, M Reinhard, and SM Stearns. 1991. Transformation and sorption of 1,2-dibromo3-chloropropane in subsurface samples collected at Fresno, California. J. Environ. Qual. 20:547-556.

Deutsch CV and AG Journel. 1998. GSLIB: Geostatistical Software Library and User's Guide, $2^{\text {nd }}$ ed. Oxford University Press, New York.

Duffy CC, DL McCallister, and RR Renken. 1997. Carbon tetrachloride retention by modern and buried soil A horizons. J. Environ. Qual. 26:1123-1127.

Fells I and EA Moelwyn-Hughes. 1959. The kinetics of hydrolysis of the chlorinated methanes. J. Chem. Soc. 1959:398-409.

Ford HL. 1996. Impact of a low velocity field on soil vapor extraction of carbon tetrachloride. Master of Science Thesis, Washington State University, Pullman.

Gelhar LW, A Mantoglou, C Welty, and KR Rehfeldt. 1985. Report EA-4190, Elec. Power Res. Inst., Palo Alto, California.

Haag WR and T Mill. 1988. Some reactions of naturally occurring nucleophiles with haloalkanes in water. Environ. Toxicol. Chem. 7:917-924.

Hooker BS, RS Skeen, MJ Truex, CD Johnson, BM Peyton, and DB Anderson. 1998. In Situ Bioremediation of Carbon Tetrachloride: Field Test Results. Bioremed. Journal 1(3):181-182. 
Isaaks EH and RM Srivastava. 1989. An Introduction to Applied Geostatistics. Oxford University Press, New York, p. 561.

Jeffers PM, LM Ward, LM Woytowitch, and NL Wolf. 1989. Homogenous Hydrolysis Rate Constants for Selected Chlorinated Methanes, Ethanes, Ethenes, and Propanes. Env. Sci. Technol. 23(8):965-969.

Jeffers PM, P Coty, S Luczak, and NL Wolf. 1994. Halocarbon hydrolysis rates-a search for ionic strength and heterogeneous effects. J. Environ. Sci. Health. A29(4):821-831.

Jeffers PM, C Brenner, and NL Wolf. 1996. Hydrolysis of Carbon Tetrachloride. Environ. Toxicol. Chem. 15(7):1064-1065.

Jeng CY, DH Chen, and CL Yaws. 1992. Data Compilation for Soil Sorption Coefficient. Pollution Engineering, pp. 54-60.

Kile DE, RL Wershaw, and CT Chiou. 1999. Correlations of soil and sediment organic matter polarity to aqueous sorption of nonionic compounds. Environ. Sci. Technol. 33:2053-2056.

Kile DE, CT Chiou, H Zhou, H Li, and O Xu. 1995. Partition of nonpolar organic pollutants from water to soil and sediment organic matters. Environ. Sci. Technol. 29:1401-1406.

Lyman WJ, WF Reehl, and DH Rosenblatt, eds. 1990. Octanol/water partition coefficient. Handbook of Chemical Property Estimation Methods. American Chemical Society, Washington, D.C.

Mabey W and T Mill. 1978. Critical Review of Hydrolysis of Organic Compounds in Water Under Environmental Conditions. J. Phys. Chem. Ref. Data. 7(2):383-415.

Newcomer DR, LA Doreums, SH Hall, MJ Truex, VR Vermeul, and RE Engleman. 1995. Geology, Hydrology, Chemistry, and Microbiology of the In Situ Bioremediation Demonstration Site. PNL-10422, Pacific Northwest Laboratory, Richland, Washington.

Peng D and NH Dural. 1998. Multicomponent adsorption of chloroform, carbon tetrachloride, and 1,1,1-trichloroethane on soils. J. Chem. Eng. Data, 43:283-288.

Stephanatos BN, K Walters, A Funk, and A MacGregor. 1991. Pitfalls associated with the assumption of a constant partition coefficient in modeling sorbing solute transport through the subsurface. Proceedings of Internal Ground Water Synposium, Nashville, Tennesee, pp. 13-20.

Swanson LC, VJ Rohay, and JM Faurote. 1999. Hydrogeologic Conceptual Model for the Carbon Tetrachloride and Uranium/Technetium Plumes in the 200 West Area: 1994 Through 1999 Update. BHI-01311, Bechtel Hanford, Inc., Richland, Washington.

van Genuchten MT. 1974. "Mass transfer studies in sorbing porous media." Ph.D thesis, New Mexico State University, Las Cruces. 
Walton BT, MS Hendricks, TA Anderson, WH Griest, R Merriweather, JJ Beauchamp, and CW Francis. 1992. Soil sorption of volatile and semivolatile organic compounds in a mixture. J. Envrion. Qual. 21:552-558.

WHC. 1990. Liquid Effluent Study Final Project Report. WHC-EP-0367, Westinghouse Hanford Company, Richland, Washington.

Zhao XM, JSzafanski, MA Maraqa, and TC Voice. 1999. Sorption of Carbon Tetrachloride in a Low Organic Content Sandy Soil. Environ. Tox. and Chem. 18:1755-1762. 


\section{Appendix A \\ Groundwater Modeling Plan \\ Hanford Carbon Tetrachloride ITRD Project}




\section{Appendix A \\ Groundwater Modeling Plan \\ Hanford Carbon Tetrachloride ITRD Project}

\section{A.1 Approach}

The Hanford Carbon Tetrachloride ITRD project will use the Monte Carlo saturated groundwater modeling approach described in this modeling plan. The purpose of this effort is to quantify the uncertainties in the strength of the remaining source term feeding the carbon tetrachloride plume originating in the 200 West Area of the Hanford Site (Figure 1). For each Monte Carlo groundwater flow and transport realization created, the modeling team will identify the source term strength, and maximum concentration arriving at the compliance boundary through time (i.e., 2000 through 2200). The maximum concentration arriving at the compliance boundary will then be used to determine the fraction of source area requiring remediation. Results from the

\section{Carbon Tetrachloride Modeling Area}

(current transport grid)

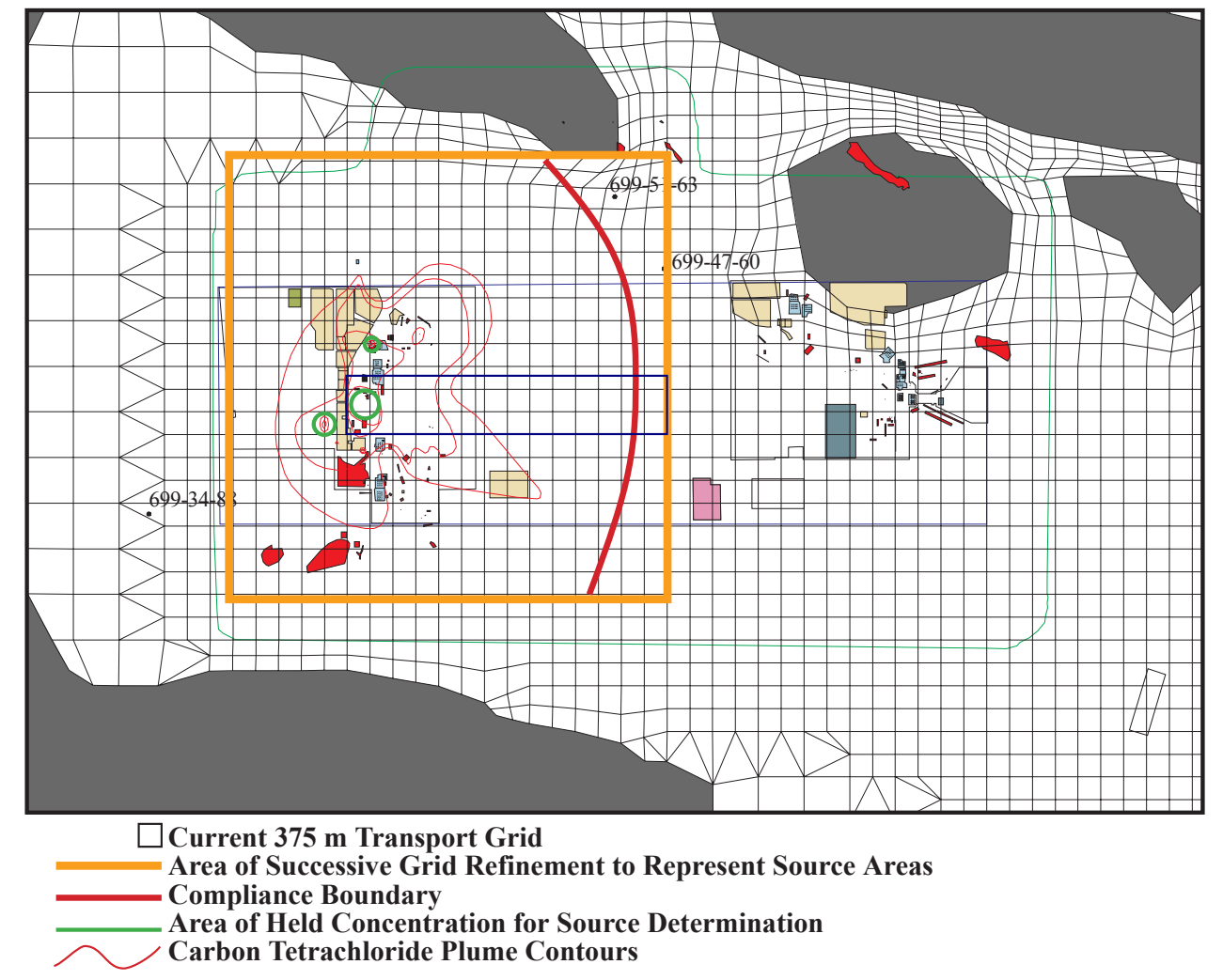

Figure A.1. Portion of the Current Hanford SGM Grid near the 200 East and 200 West Areas with Various Overlays that Illustrate: 1) the areal extent of the carbon tetrachloride plume (thin red lines), 2) the proposed area of grid refinement for this study (orange line), 3) the proposed location of the compliance boundary (thick red line) for this study, and 4) a blue box showing the proposed initial onedimensional modeling, which will examine parameter sampling strategies to develop a way to minimize the number of three-dimensional model runs. 
multiple realizations will provide uncertainty estimates for source term strength related to the uncertainty in $\mathrm{K}_{\mathrm{d}}$, porosity, dispersivity, and first-order abiotic reaction rates. Estimates of the uncertainty in the remaining source term and the fraction requiring treatment will provide information useful to the ITRD for estimating uncertainty in the costs associated with each of the proposed source term remediation strategies.

Mass balance derived from observations of existing plumes, amounts removed with already implemented initial remediations, and other considerations only account for $\sim 35 \%$ of the mass that was imported for use at Hanford. Estimates are that this remaining source could be $\sim 780,000 \mathrm{~kg}$ and that the remaining source(s) of contamination could be described as follows:

- Located only in the vadose zone with the source resulting from any combination of dissolved, vapor, and/or DNAPL phases

- Located only in the groundwater as a DNAPL source only

- Combinations of various vadose sources and groundwater DNAPL sources.

\section{A.1.1 Basic Assumption Used in Approach}

The pivotal assumption in the approach outlined in this modeling plan is that the plume immediately prior to the start of interim pump and treat was in equilibrium with the various possible types of potential sources.

The assumption has been investigated through a geostatistical evaluation of the data gathered before beginning the interim pump and treat. Chris Murray presented the geostatistical evaluation results at the March 2000 Hanford Carbon Tetrachloride ITRD project meeting. These results indicate that it is reasonable to assume that the groundwater plume was in equilibrium with the remaining source before the interim pump and treat began because the temporal analysis of the observations indicated the concentrations near the source area had stabilized. The spatial concentration distributions from the geostatistical evaluation will provide initial conditions for the Monte Carlo realizations and the location and values of specified concentrations that will represent the source term and its uncertainty as can be interpreted from existing data.

\section{A.1.2 Uncertainties Addressed}

This groundwater modeling effort, in conjunction with the geostatistical analysis of concentration presented at the March 2000 workshop by Chris Murray and the analysis of uncertainty in $\mathrm{Kd}$, biotic and abiotic reaction rates, and other natural attenuation factors presented at this same workshop by Rod Skeen will allow us to address source term uncertainty arising from uncertainty in the following parameters:

- plume definition (concentration distribution horizontally and vertically including assumptions about porosity)

- $\mathrm{K}_{\mathrm{d}}$

- first-order natural abiotic dechlorination rate

- dispersivity. 
Volatilization from saturated zone to unsaturated zone and first-order natural biodegradation were investigated by the team and determined to be negligible. As a result, the uncertainty related to these processes will not be included. Details are presented in Appendix C. As was discussed in the initial Hanford Carbon Tetrachloride ITRD project modeling workshops, uncertainties in the groundwater model will not be addressed because the current model is deterministic. Uncertainty related to the groundwater flow model will be reduced by limiting transport model calculations to movement through the less transmissive Ringold formation (Unit E and the lower units) from 200 West Area source locations to a compliance boundary at or near the contact with the highly transmissive saturated Hanford formation (Figures 1 and 2).

\section{A.1.3 Modeling Scales}

Two scales of modeling will be used in this assessment.

1. An analytical model for one-dimensional, convective-dispersive transport of reactive solutes, subject to adsorption, first-order degradation, and in a homogeneous medium will be used. The model is based on the analytical solution developed by van Genuchten (1974). It will be used to represent transport in a single flow tube along the centerline of the plume from the 200 West source area(s) (green circles in Figure 1) to the compliance boundary, a distance of $\sim 5 \mathrm{~km}$ (blue rectangle in Figure 1). This one-dimensional model will be used to develop an initial understanding of parameter interactions/sensitivity and to examine a large number of realizations of the uncertain parameters (source term volume/concentration, $\mathrm{K}_{\mathrm{d}}$, porosity, degradation half time, dispersivity) to develop an efficient sampling strategy to use with the threedimensional model.

2. A three-dimensional local scale model to simulate transport of the approximate scale shown in Figure 1 (orange box). This model will be used to represent transport from the source area(s) (thick green lines in Figure 1) to the compliance boundary (thick red line in Figure 1). Multiple realizations for the transport parameters and initial and source term boundary conditions will be implemented based on the sampling strategy developed with the one-dimensional streamtube model to address the uncertainty in source term strength.

\section{A.2 Regional Model}

The groundwater model used in this assessment is the three-dimensional deterministic model of the Hanford Site developed and maintained as part of the Hanford Site Groundwater Monitoring Program. The Sitewide Groundwater Model (SGM) is documented in Cole et al. (1997) and Wurstner et al. (1995). Figure 3 illustrates the SGM flow model grid ( $\sim 750 \mathrm{~m}$ regular grid) and the various lateral boundary conditions (green are specified heads based on river elevations, red are no flow, and blue are specified flux). Regional flow model results from the plume analysis discussed in Cole et al. (1997) will be used to provide information for the two scales of modeling for this ITRD effort. The regional SGM will provide velocity estimates for the one-dimensional streamtube analysis and boundary conditions for the local scale transport model (approximately the scale shown in Figure 1). 


\section{Geologic Units Present at the Water Table, June 1998.}

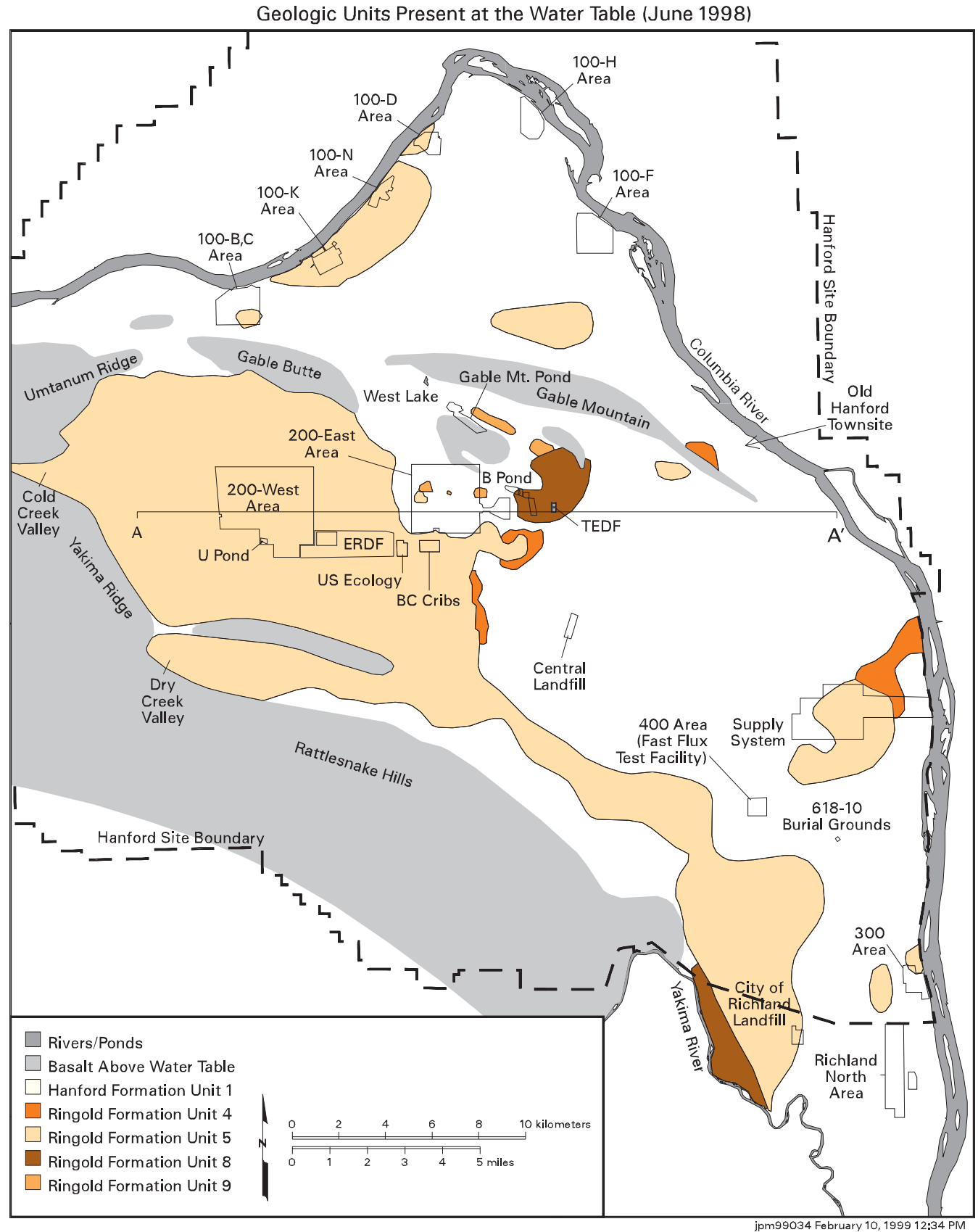

Figure A.2. Illustration of the Areal Extent of the Geologic Units Present at the Water Table (June 1998). Note that Ringold Unit 5 (i.e., middle Ringold conglomerate or Ringold E) is the hydrologic unit that contains the water table from 200 West to the western boundary of 200 East. 


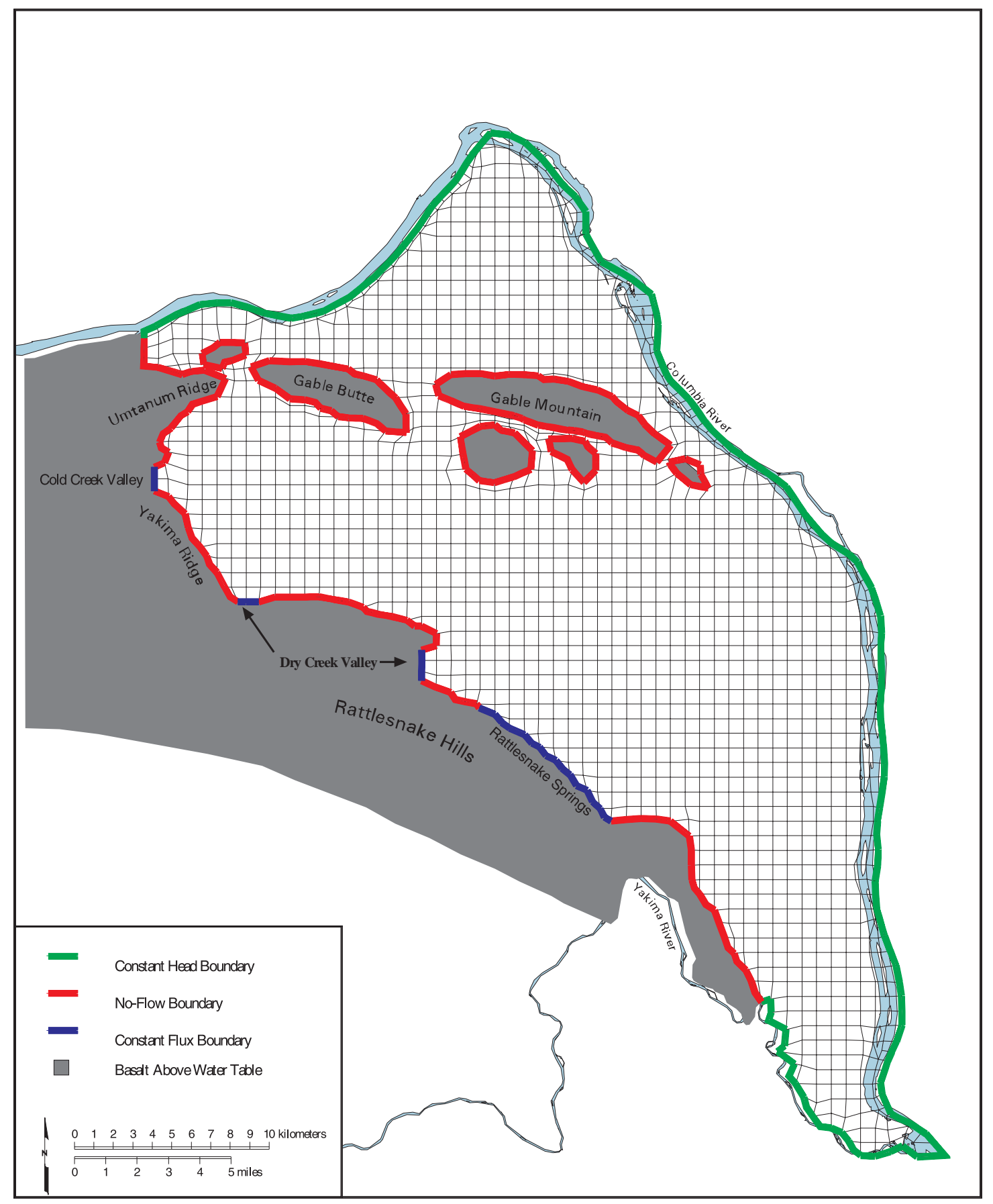

97skw017.eps November 26, 1997

Figure 3. SGM flow model grid ( $\sim 750 \mathrm{~m}$ regular grid) with the various lateral boundary conditions annotated. Green boundaries are specified heads based on river elevations, red boundaries are no-flow, and blue boundaries are specified flux. 


\section{A.3 Source Area Assumption}

Figure 4 illustrates the vertically summed median concentration contours (from 100 realizations) for the 1993 carbon tetrachloride plume(s) in the 200 West area. This result (from the geostatistical analysis presented at the March 2000 workshop by Chris Murray) indicates that the majority of the mass of carbon tetrachloride is within the $500 \mathrm{~m}$ by $500 \mathrm{~m}$ zone outlined by the small black box that surrounds the high concentration portion of the plume (Figure 4). As a result we will assume that the majority of the remaining source is within this area and only this single source area will be investigated in this modeling analysis. However, the entire interpretation of carbon tetrachloride concentration (i.e., the colored area) for each plume realization will be used to set the initial conditions for the three-dimensional modeling.

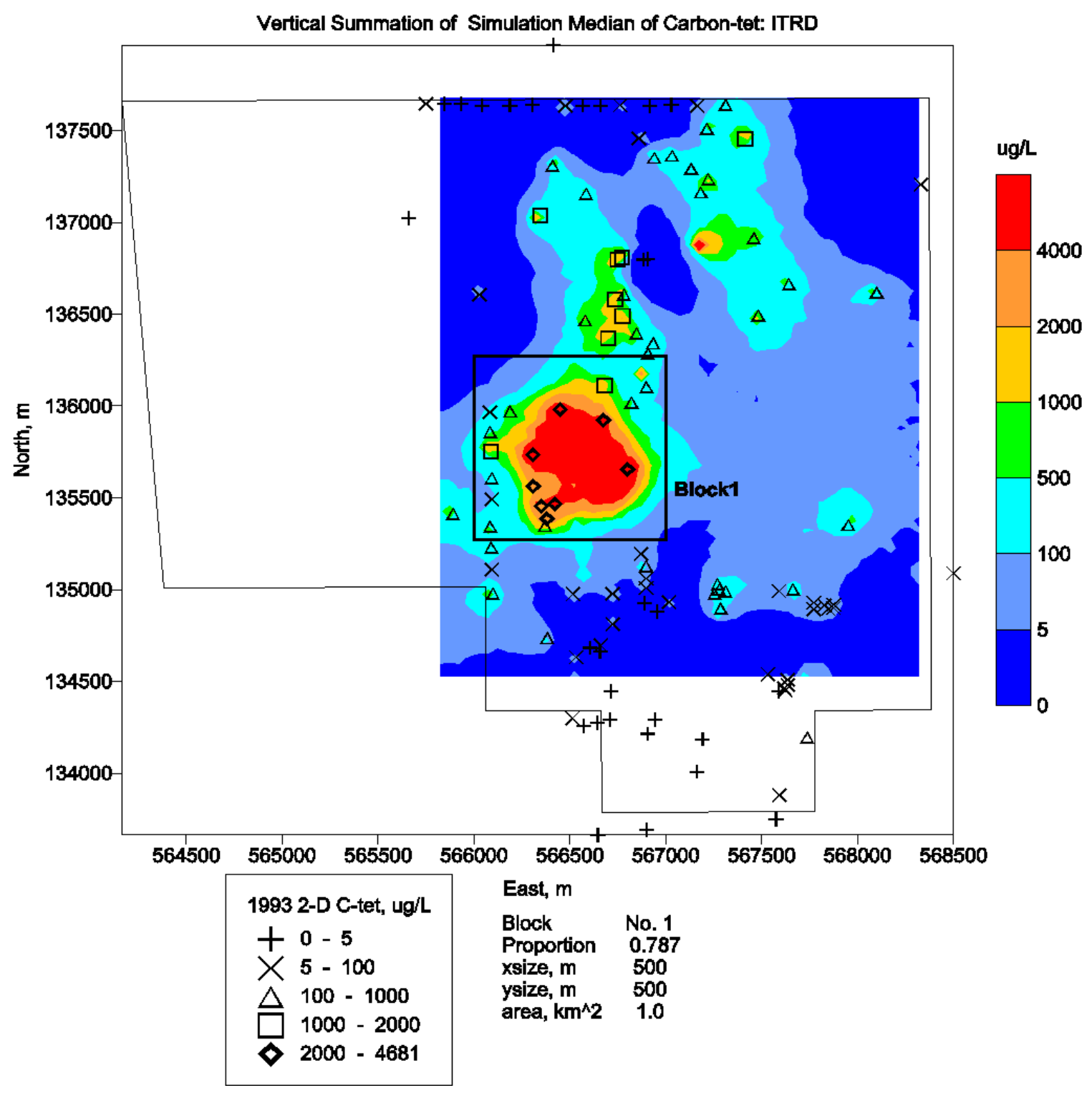

Figure 4. Vertical Summation of the Simulated Median of the Carbon Tetrachloride Concentration in the 200 West Area in $1993(\mu \mathrm{g} / \mathrm{L})$ 


\section{A.4 Realizations}

The 100 realizations of concentration distributions will be used in the one-dimensional modeling to develop a sampling strategy. This strategy will allow a smaller number of threedimensional model runs to be made while developing the uncertainties in the source term and the maximum concentration arriving at the compliance boundary over the next 200 years (i.e., 20002200). For each of the 100 concentration distribution realizations the probability distributions representing the uncertainty in the transport parameters $\left(\mathrm{K}_{\mathrm{d}}\right.$, porosity, first-order reaction rate, and dispersivity) will be used to prepare a parameter tablelike Table 1

Table 1. Example of a Realization Table

\begin{tabular}{|c|l|c|c|c|c|}
\hline $\begin{array}{c}\text { Realization } \\
\text { Number }\end{array}$ & $\begin{array}{c}\text { Name of Concentration } \\
\text { Distribution File }\end{array}$ & $\begin{array}{c}\text { Kd } \\
(\mathbf{m l} / \mathbf{g})\end{array}$ & Porosity & $\begin{array}{c}\text { 1st order reaction } \\
\text { half time (y) }\end{array}$ & Dispersivity (m) \\
\hline 1 & realization_1.fil & $\mathrm{xx}$ & $\mathrm{sxy}$ & $\mathrm{xyx}$ & $\mathrm{xxz}$ \\
\hline 2 & realization_2.fil & $\mathrm{yx}$ & $\mathrm{fxz}$ & $\mathrm{yyy}$ & $\mathrm{zxx}$ \\
\hline$\cdot$ & & & & & \\
\hline$\cdot$ & & & & & \\
\hline$\cdot$ & & & & & $\mathrm{zzz}$ \\
\hline 100 & realization_100.fil & $\mathrm{Zx}$ & $\mathrm{dxz}$ & $\mathrm{xxx}$ & \\
\hline
\end{tabular}

\section{A.5 One-Dimensional Streamtube Model}

The streamtube model will consider convective-dispersive transport of reactive solutes, subject to adsorption and first-order degradation in a homogeneous medium, and is based on the analytical solution developed by van Genuchten (1974). The model will be used in a Monte Carlo mode to address the effect of uncertainty in $\mathrm{K}_{\mathrm{d}}$, dispersivity, first-order abiotic reaction rate, and concentration distribution on the concentration of carbon tetrachloride reaching the compliance boundary during the 200 year model period. Uncertainty in travel time from the source area to the assumed compliance boundary will be addressed using a range of travel times along different streamlines from the source area to the compliance boundary. Uncertainty about what contour levels (at some distance from the source area) might be in equilibrium with the source will also be addressed by performing the set of Monte Carlo analysis runs for a range of concentration cutoff levels (e.g., 500, 750, 1000, 1250, and $1500 \mu \mathrm{g} / \mathrm{L}$ in the plume within the small black box shown in Figure 4).

\section{A.5.1 Domain and Inlet Boundary Concentration}

The streamtube model will consist of a $1000 \mathrm{~m}$ wide (see small black box in Figure 4) by $\sim 5000 \mathrm{~m}$ long area (see dark blue rectangle in Figure 1 going from source area to the assumed compliance boundary). The height of the streamtube will depend both on the concentration distribution developed for each realization and the concentration level selected for the upstream source area boundary as the specified concentration at this inlet boundary. If one assumes that the three-dimensional plume that develops around the source area were a cube, the downstream exit face area that would be at the concentration cutoff level (i.e., specified inlet concentration) would be equal to the $2 / 3$ root of the volume of the plume above this cutoff level, which is what we will assume. Depending on the actual shape of the three-dimensional plume, the downstream exit face will be proportional to the $2 / 3$ root of the volume of the plume at or above this cutoff 
concentration level time constant. Since our streamtube width is fixed at $1000 \mathrm{~m}$, the height will depend on the volume of the plume at or above the cutoff concentration level that will be used as the specified inlet concentration. As a result, the height of the streamtube for each realization will be equal to $1 / 1000^{\text {th }}$ of the $2 / 3$ root of the volume of the plume above the cutoff level.

Figure A.5 illustrates the variation of the mean and median volume above the cutoff level for the 100 concentration realizations as a function of the specified cutoff level. This figure also shows the variation in the projected source term rate considering only advective flow for an assumed square plume and a groundwater velocity of $0.04 \mathrm{~m} / \mathrm{d}$ which is close to the model predicted velocity near the source area. Figure A.6 illustrates the variability in volume above a cutoff level of $1000 \mu \mathrm{g} / \mathrm{L}$ for the various realizations as well as the mean and the mean plus and minus one standard deviation.

\section{A.5.2 Velocity}

Velocity for the streamtube will be estimated from the 1995 SGM predictions shown in Figure A.7. Table A.2 illustrates the average and range of values that will be considered. The line of velocity magnitudes down the actual streamtube (dark blue box in Figure A.7 and streamtube 2 in Table A.2) and lines of velocity magnitudes below and above the actual streamtube (dark blue box in Figure A.7 and streamtubes 1 and 3, respectively, in Table A.2) will be used to calculate the average, maximum, and minimum streamtube velocities for this study.

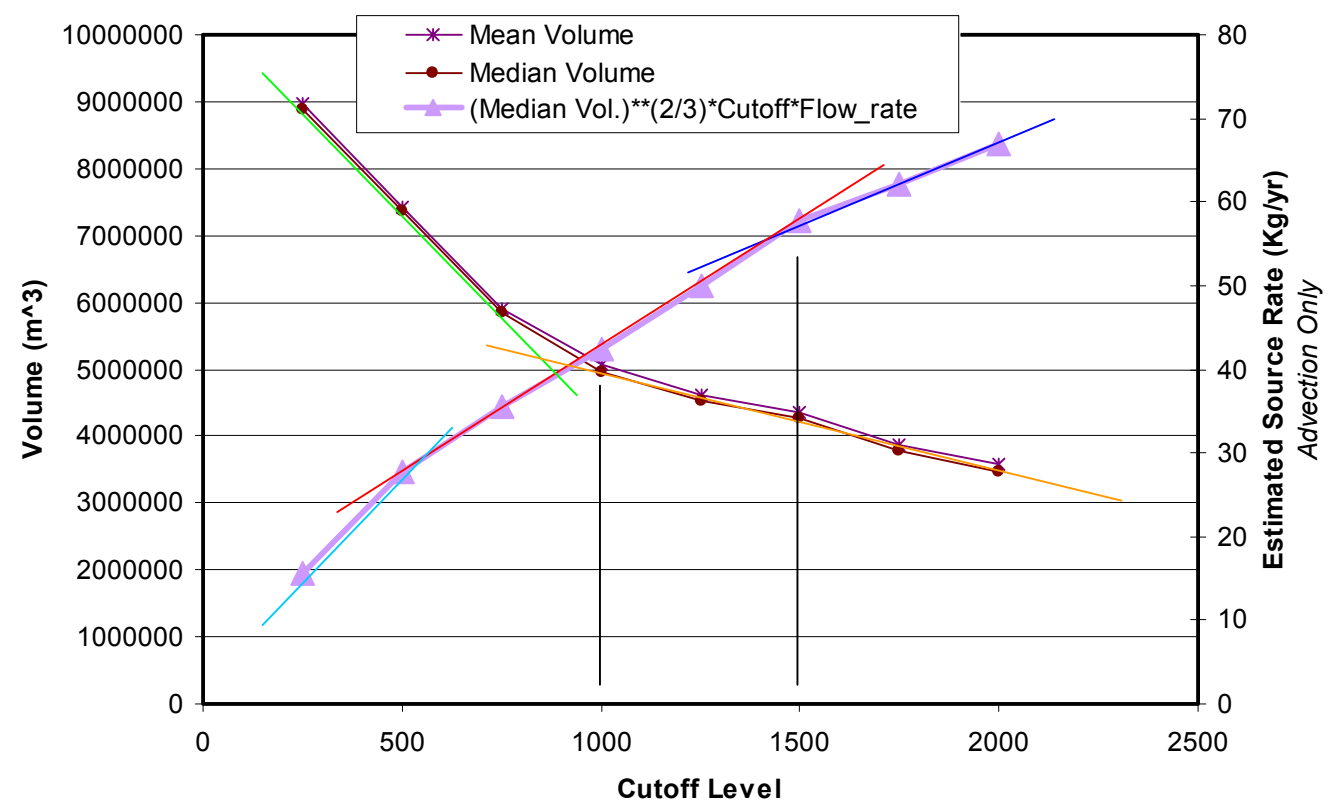

Figure A.5. Mean and Median Volume above a Specified Cutoff Level for the 100 Concentration Realizations and Projected Source Term Rate Considering only Advective Flow as a Function of the Specified Cutoff Level 


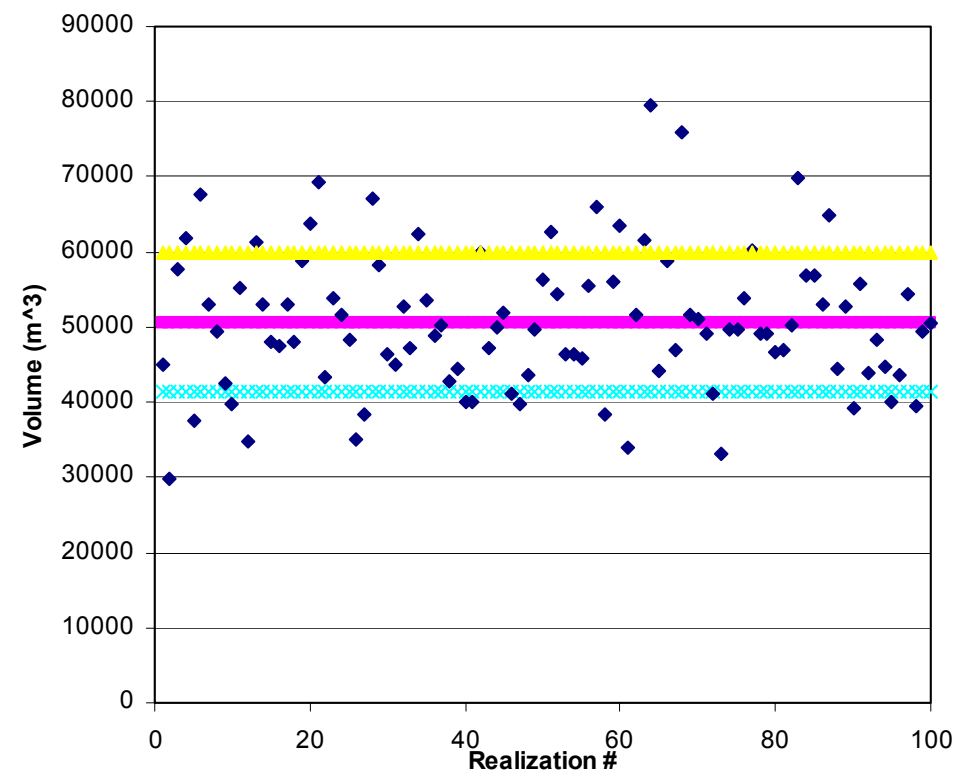

Figure A.6. Illustration of the Volume above a Cutoff Level of $1000 \mathrm{mg} / \mathrm{L}$ for the Various Realizations as well as the Mean (magenta) and the Mean plus (yellow) and minus (cyan) one Standard Deviation

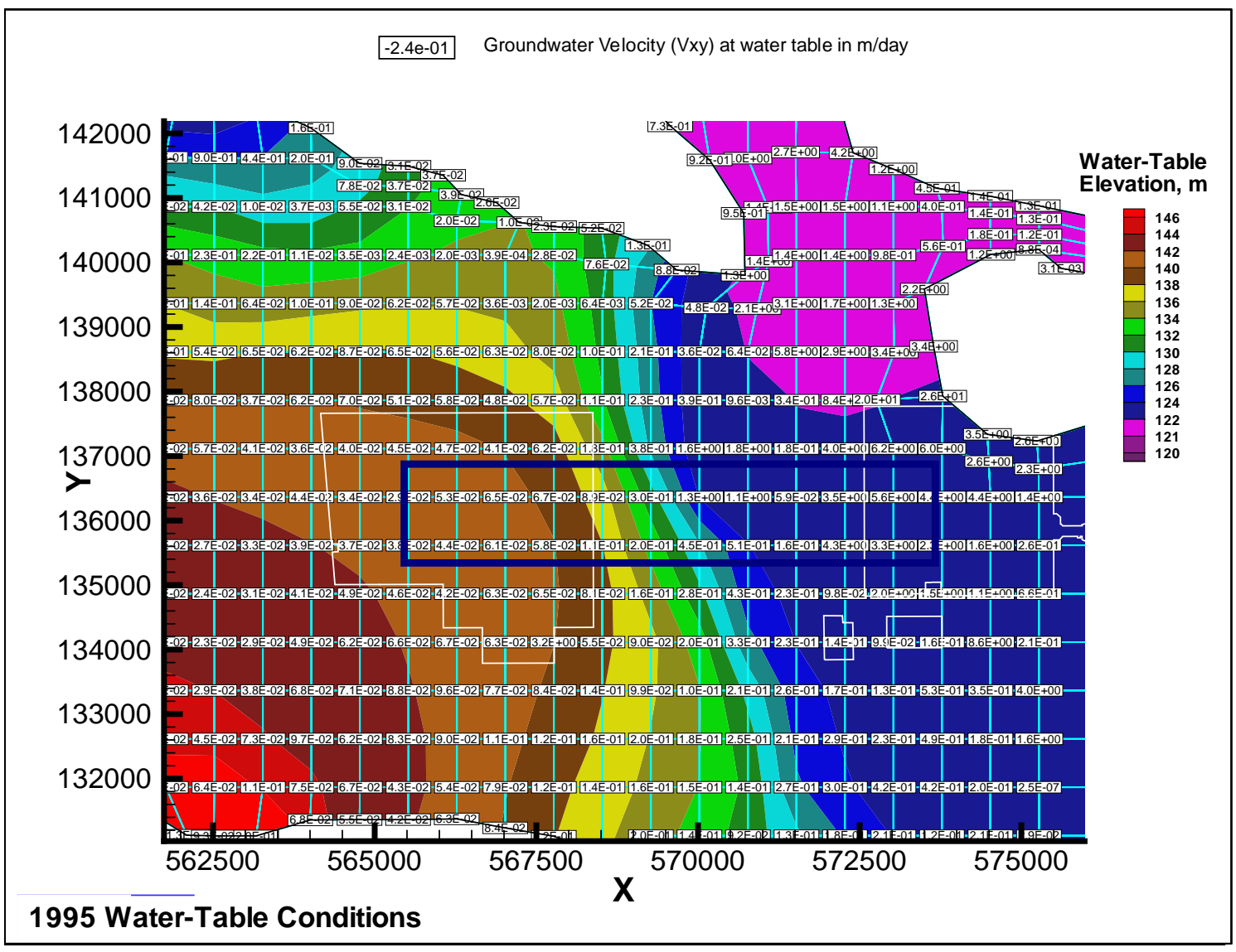

Figure A.7. SGM Predicted Velocities (posted) and Color-Coded Head (red-blue) 
Table A.2. Streamtube Velocity Table

\begin{tabular}{|c|c|c|c|c|c|c|}
\hline \multirow[t]{2}{*}{ Average } & \multirow{2}{*}{\multicolumn{4}{|c|}{\begin{tabular}{c|c}
$\boldsymbol{v}=\mathbf{0 . 0 9 6} \mathbf{m} /$ day $t=150 y$ \\
Streamtube 1
\end{tabular}}} & \multicolumn{2}{|c|}{$y$} \\
\hline & & & & & Streamtu & be 3 \\
\hline Grid Block & $\mid v(\mathrm{~m} / \mathrm{d})$ & $t(\mathrm{y})$ & $v(\mathrm{~m} / \mathrm{d})$ & $t(\mathrm{y})$ & $v(\mathrm{~m} / \mathrm{d})$ & $t(\mathrm{y})$ \\
\hline 1 & 0.042 & 48.89 & 0.044 & 46.67 & 0.053 & 38.74 \\
\hline 2 & 0.063 & 32.59 & 0.061 & 33.66 & 0.065 & 31.59 \\
\hline 3 & 0.065 & 31.59 & 0.058 & 35.40 & 0.067 & 30.65 \\
\hline 4 & 0.081 & 25.35 & 0.110 & 18.67 & 0.089 & 23.07 \\
\hline 5 & 0.160 & 12.83 & 0.200 & 10.27 & 0.300 & 6.84 \\
\hline 6 & 0.280 & 7.33 & 0.450 & 4.56 & 1.300 & 1.58 \\
\hline 7 & 0.430 & 4.78 & 0.510 & 4.03 & 1.100 & 1.87 \\
\hline Average & 0.088 & 163.37 & $\overline{0.094}$ & 153.26 & 0.107 & 134.34 \\
\hline
\end{tabular}

\section{A.5.2 Output}

Output will be frequency plots of source term strength and fraction of source requiring remediation, an understanding of parameter importance, and a strategy for realization sampling to reduce the number of three-dimensional model runs.

\section{A.5.3 Realizations and Time Domain}

Realizations will be the 100 realizations drawn from the various distributions as already discussed (see Table A.1). The model will be run from 2000-2300 to understand the longer-term nature of compliance boundary arrival curves, but the analysis will be for the 2200 time period.

\section{A.6 Three-Dimensional Local Scale Model}

The CFEST code (Cole et al. 1988) will be used to model a three-dimensional local scale model to simulate transport at the approximate scale shown in Figure A.1 (orange box). This model will use the SGM methodology to refine the grid and develop a refined (both laterally and vertically) local scale model as discussed in Barnett et al. (1997). Processes considered are the same as for the streamtube model and include convective-dispersive transport of reactive solutes subject to adsorption and first-order degradation. Uncertainty in the concentration contours that are in equilibrium with the source will be addressed in the same way as was proposed for the one-dimensional modeling. Uncertainty as to what contour levels (at some distance from the source area) might be in equilibrium with the source will also be addressed by examining a range of concentration cutoff levels used to represent the source term. The parameter distributions and other aspects of the three-dimensional flow model are not discussed because they are the same as used in the SGM. 


\section{A.6.1 Spatial Domain and Boundary Conditions}

An subregion of the SGM as approximately shown in Figure A.1 will be selected and a refined plan view grid prepared that corresponds exactly to the $50 \mathrm{~m}$ regular grid used to do the statistical interpretations around the source area (i.e., the 20 by 20 regular $50 \mathrm{~m}$ grid within the black box around the source in Figure A.4). The vertical model resolution will also be altered to correspond exactly to the $5 \mathrm{~m}$ vertical grid used in the geostatistical interpretation shown in Figure A.4. This will allow the concentration values from the geostatistical interpretation to be directly used to specify the concentration distribution in this high concentration source area and to provide a simple means to identify the nodes that will be specified concentration boundary conditions representing the source. The horizontal resolution of the subregion model will be decreased to $100 \mathrm{~m}$, then $200 \mathrm{~m}$, and it will finally correspond to the SGM transport model grid spacing of $375 \mathrm{~m}$ just beyond the compliance boundary. Similarly the vertical spatial resolution will be decreased to correspond to the transport model grid as we move away from the source area. Note that the grid in Figure A.1 is the SGM transport model grid. Boundary conditions for this subregion model will be supplied by the full=scale regional transport model.

As with the streamtube model, the representation of the source area depends on the realization. Within the source area (see small black box in Figure A.4), and for a selected cutoff level as discussed earlier all the nodes that are at or above the specified cutoff level will become specified concentration boundary condition nodes (value $=$ the cutoff value). As a result, the shape, location and distribution of the source will vary with each realization.

\section{A.6.2 Realizations and Time Domain}

The realization strategy developed from the one-dimensional modeling will be used to define the required subsampling of the 100 realizations examined in the one-dimensional modeling. The model time domain for the three-dimensional model will be just 200 years (from 2000 to 2200).

\section{A.6.3 Desired Output}

1. Determine the source term release for each realization that is consistent with the uncertainty in parameters drawn for that realization.

2. Produce a distribution of source term release rates conditioned on uncertainty in unknown parameters.

3. For each realization, determine concentrations at the compliance boundary at 200 years to determine the portion of the source that needs to be removed to achieve compliance.

\section{A.7 References}

Barnett DB, MD Freshley, MP Bergeron, SK Wurstner, and CR Cole. 1997. Tritium Monitoring in Groundwater and Evaluation of Model Predictions for the Hanford Site 200 Area Effluent Treatment Facility. PNNL-11665, Pacific National Northwest Laboratory, Richland, Washington. 
Cole CR, SB Yabusaki, and CT Kincaid. 1988. CFEST-SC, Coupled Fluid, Energy, and Solute Transport Code, SuperComputer Version, Documentation and User's Manual. Battelle, Pacific Northwest Laboratories, Richland, Washington.

Cole CR, SK Wurstner, MP Bergeron, MD Williams, and PD Thorne. 1997. Three-Dimensional Analysis of Future Groundwater Flow Conditions and Contaminant Plume Transport in the Hanford Site Unconfined Aquifer System: FY 1996 and 1997 Status Report. PNNL-11801, Pacific Northwest National Laboratory, Richland, Washington.

van Genuchten MT. 1974. "Mass transfer studies in sorbing porous media." Ph.D thesis, New Mexico State University, Las Cruces.

Wurstner SK, PD Thorne, MA Chamness, MD Freshley, and MD Williams. 1995. Development of a Three-Dimensional Groundwater Model of the Hanford Site Unconfined Aquifer System: FY 1995 Status Report. PNL-10886, Pacific Northwest Laboratory, Richland, Washington. 
Appendix B

\section{Volatilization Notes}




\section{Appendix B}

\section{Volatilization Notes}

A potential mechanism for decreasing carbon tetrachloride (CT) concentration in the groundwater is through volatilization of CT into the vadose zone. This mechanism was investigated for the dispersed portion of the plume. The investigation provides an estimate of the mass transfer rate of CT out of the groundwater and into the vadose zone. Thus, the investigation does not apply to areas of the vadose zone where CT sources in the vadose zone can maintain high CT vapor concentrations.

There are three mass transfer processes that must be quantified to estimate the overall transfer to CT from the groundwater to the gas phase. Figure B.1 illustrates the concept applied for mass transfer at the liquid-gas interface. At the liquid-gas interface, there is a mass transfer resistance associated with $\mathrm{CT}$ molecules crossing this interface. On either side of this interface, there are diffusion layers (gas film and liquid film) that effect the rate at which CT can reach the liquid-gas interface from the bulk groundwater and the rate at which CT can move away from the interface into the bulk vadose zone gas phase. The estimate of overall mass transfer rate is a function of the parameters that control these three mass transfer processes.

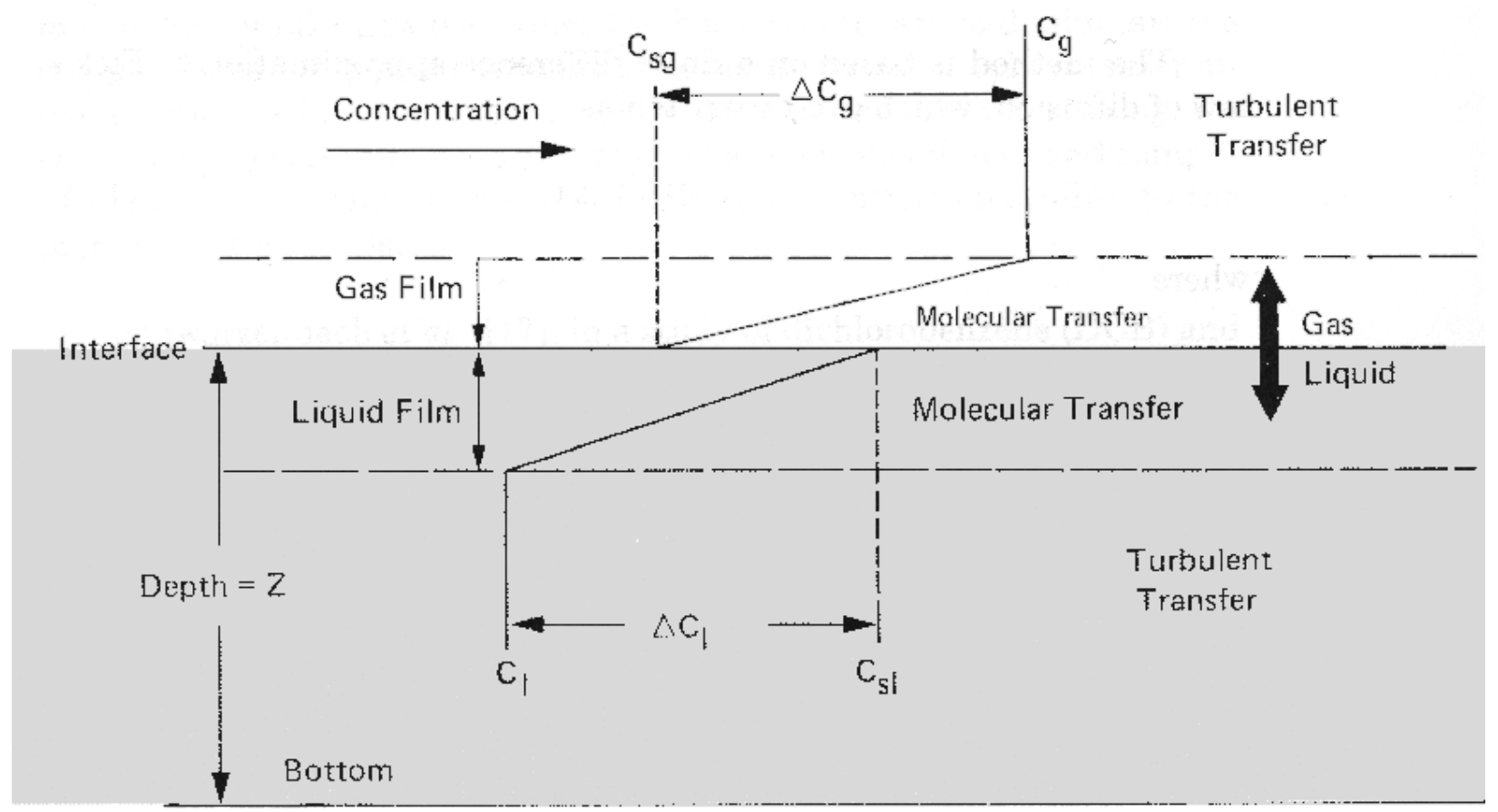

Figure B.1. Mass Transfer Concept at the Liquid-Gas Interface 
The parameter controlling mass transfer at the liquid-gas interface is the Henry's Law equilibrium partitioning coefficient. The Henry's Law coefficient is expressed as the concentration of a species in the vapor phase divided by the concentration of the species in the aqueous phase when these two phases are in equilibrium with respect to the species of interest. CT has a relatively high Henry's Law coefficient; consequently, CT readily partitions into the vapor phase across the liquid-gas interface.

The diffusion processes to and away from the liquid-gas interface are controlled by the diffusion coefficient in the medium (water or air) and the thickness of the diffusion layer. The diffusion coefficients for carbon tetrachloride are $7.9 \times 10^{-6} \mathrm{~cm}^{2} / \mathrm{s}$ and $7.3 \times 10^{-2} \mathrm{~cm}^{2} / \mathrm{s}$ in water and air, respectively (Lyman et al. 1990). The thickness of the diffusion layer is dependent on the hydraulic regime and physical nature of the liquid-gas system. In the Hanford vadose zone, it was assumed that the soil gas is mixed well enough that the concentration of CT was maintained at near zero within $1 \mathrm{~m}$ above the groundwater. Thus, the diffusion layer thickness in the gas phase was estimated as $1 \mathrm{~m}$. Because of the very slow groundwater flow rates and the physical nature of groundwater flow, the groundwater was considered to have minimal mixing over a scale of between $10 \mathrm{~cm}$ and $1 \mathrm{~m}$. Thus the diffusion layer thickness in the aqueous phase was estimated as being between $10 \mathrm{~cm}$ and $1 \mathrm{~m}$. The mass transfer rate is significantly influenced by the diffusion layer thickness in the groundwater and minimally influenced by the diffusion layer thickness in the gas phase.

The calculated mass transfer flux rates ranged from $9 \times 10^{-6} \mathrm{~g} / \mathrm{m}^{2} \cdot \mathrm{d}$ to $9 \times 10^{-7} \mathrm{~g} / \mathrm{m}^{2} \cdot \mathrm{d}$ based on an aqueous-phase diffusion layer thickness of $10 \mathrm{~cm}$ and $1 \mathrm{~m}$, respectively. These rates were converted to psuedo first-order reaction rates to compare the magnitude of rate of volatilization to the magnitude of the rate of abiotic hydrolysis. As described in the main report, the likely range of abiotic hydrolysis rates estimated for this modeling correspond to a CT half-life of between 36 and 290 years. The range of estimated volatilization rates correspond to a CT halflife of between 600 and 6000 years.

It was concluded that rate of CT volatilization was not significant compared with the other mechanisms of CT loss in the groundwater flow field.

\section{Reference}

Lyman WJ, WF Reehl, and DH Rosenblatt, eds. 1990. Handbook of Chemical Property Estimation Methods. American Chemical Society, Washington, D.C. 


\section{Appendix C}

\section{Literature Review:}

Natural Attenuation Mechanisms and Rates for Chloromethane Subsurface Contamination at Hanford 
Literature Review:

Natural Attenuation Mechanisms and Rates for Chloromethane Subsurface

Contamination at Hanford

$6 / 30 / 2000$

Prepared for:

Sandia National Laboratory

Innovative Treatment Remediation Demonstration Program, Hanford Carbon Tetrachloride Project

Point of Contact:

Michael J. Truex

Pacific Northwest National Laboratory

P.O. Box 999, MSIN K2-10

Richland, WA 99352

Phone: (509) 375-2636

Fax: (509) 372-4660 


\section{Table of Contents for Appendix C}

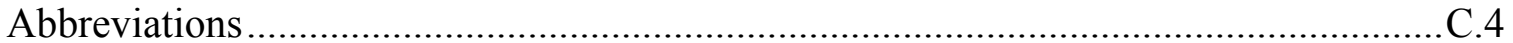

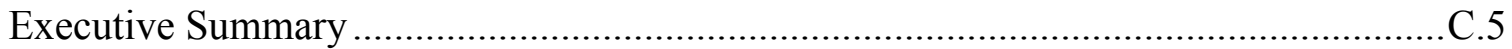

Review of Chloromethane Transformation ….......................................................... 5

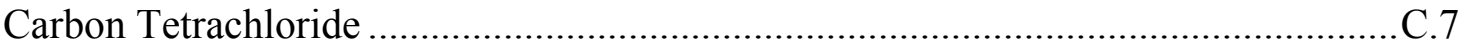

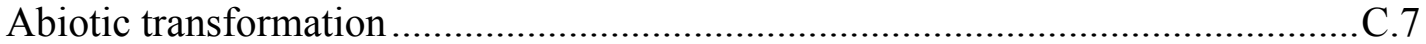

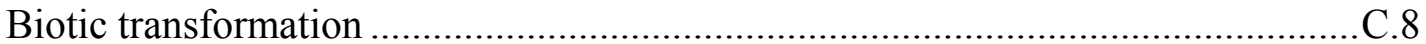

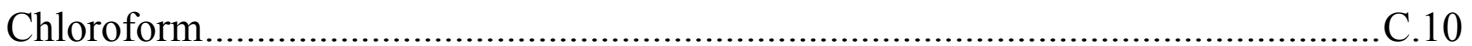

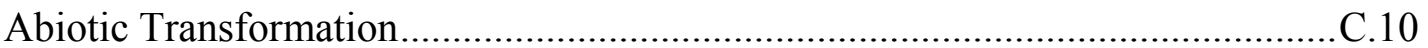

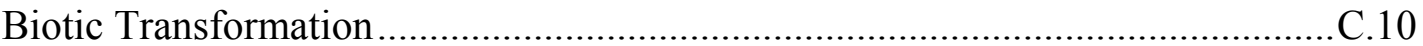

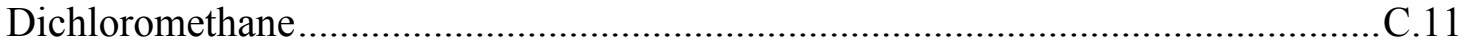

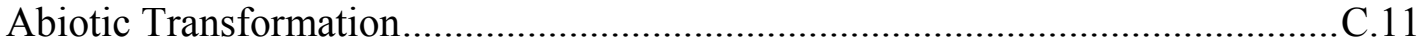

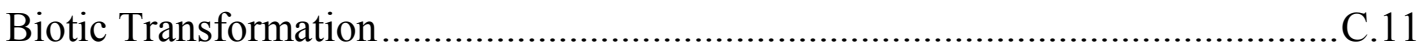

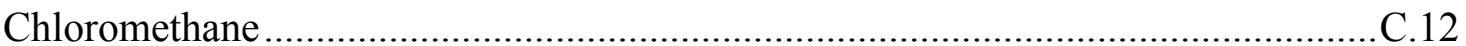

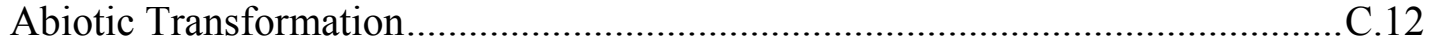

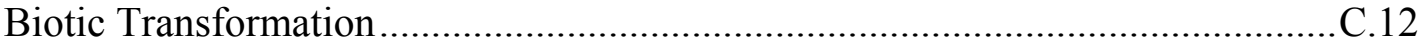

Chloromethane Transformation in Groundwater Systems ............................................ 13

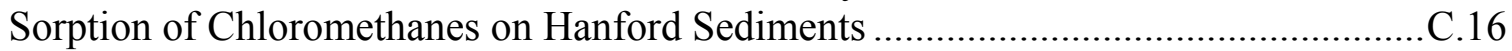

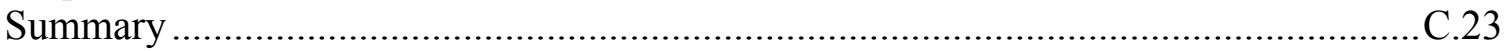

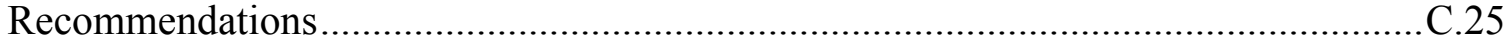

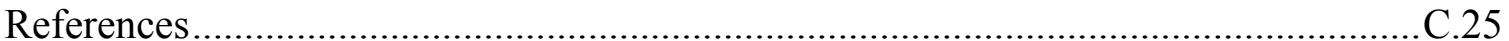




\section{List of Tables and Figures in Appendix C}

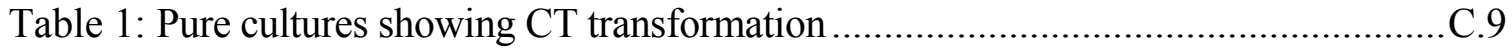

Table 2: Metabolisms that co-metabolically degrade CF under aerobic conditions......C. 10

Table 3: Cultures showing anaerobic CF biotransformation ...................................... 11

Table 4: List of organisms showing the ability to co-metabolically degrade DCM under

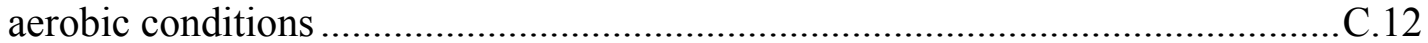

Table 5: List of organisms showing ability to degrade CM under aerobic conditions ..C.13

Table 6: First order dehalogenation rates measured for CT. ........................................ 14

Table 7: First order dehalogenation rates measured for CF........................................ 15

Table 8: First order dehalogenation rates measured for DCM. ................................. 15

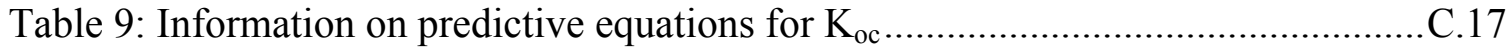

Table 10: Physical properties used to estimate organic carbon sorption coefficient .....C.17

Table 11: Estimated $\mathrm{K}_{\mathrm{oc}}$ values for chloromethanes................................................... 17

Table 12: Published values of Koc for chloromethanes ................................................18

Table 13: Organic carbon fraction data for the Hanford aquifer. ...................................19

Table 14: Maximum estimates for the solid/liquid equilibrium partition coefficient based on $\mathrm{K}_{\mathrm{oc}}$ data reported in Table 12 and the maximum $\mathrm{f}_{\mathrm{oc}}$ in Table $14 \ldots \ldots \ldots \ldots \ldots \ldots . . . . . .20$

Table 15: Maximum estimates for the solid/liquid equilibrium partition coefficient based on $\mathrm{K}_{\mathrm{oc}}$ data reported in Table 13 and the maximum $\mathrm{f}_{\mathrm{oc}}$ in Table 14 ......................2.20

Table 16: Minimum estimates for the solid/liquid equilibrium partition coefficient based on $\mathrm{K}_{\mathrm{oc}}$ data reported in Table 12 and and an $\mathrm{f}_{\mathrm{oc}}$ of 0.00027 .................................2.22

Table 17: Minimum estimates for the solid/liquid equilibrium partition coefficient based

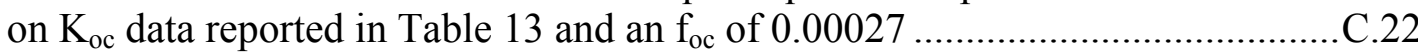

Table 18: Suggested range of parameters for modeling the fate of chloromethanes in the Hanford Aquifer

Figure 1: Transformation pathways for chlorinated methanes...................... 6 Figure 2: Neutral homogeneous hydrolysis of carbon tetrachloride in distilled water...C.8

Figure 3: Transformation pathways that can be expected to occur at Hanford C.25 


\section{Abbreviations}

\begin{tabular}{|l|l|}
\hline CT & Carbon Tetrachloride \\
\hline CF & Chloroform \\
\hline DCM & Dichloromethane \\
\hline CM & Chloromethane \\
\hline TeCA & $1,1,2,2$-Tetrachloroethane \\
\hline TCA & $1,1,1$-Trichloroethane \\
\hline DCA & 1,2 -Dichloroethane \\
\hline PCE & Tetrachloroethene \\
\hline DI & Deionized water \\
\hline $\mathrm{t}_{1 / 2}$ & Half-life \\
\hline
\end{tabular}




\section{Executive Summary}

This report provides a review of the current published literature on potential natural attenuation processes that could affect the concentration of chloromethane contaminants in the groundwater of the Hanford Site. The processes discussed in this report include biotic and abiotic reactions and solid/liquid equilibrium partitioning. Contaminants that are included in the analysis are CT and its potential reaction products $\mathrm{CF}, \mathrm{DCM}$, and $\mathrm{CM}$.

It was found that the natural processes that are likely to affect chloromethanes in the groundwater at Hanford include abiotic hydrolysis of CT and CF; biotic and abiotic transformation of DCM; and sorption of all these compounds to the soil matrix. Substantial levels of natural biotic transformation of CT are unlikely at Hanford because this is an anaerobic process that is inhibited by the dissolved oxygen present in the Hanford aquifer. Furthermore, the Hanford aquifer has very low levels of the organic carbon needed to support biotic transformation of CT. Similarly, biotic conversion of CF will be minimal in the Hanford aquifer because although the reaction can occur in the presence of oxygen, it also requires an additional electron donor. In contrast, biotic conversion of DCM may occur at Hanford because it has been reported to be used as an aerobic growth substrate for a variety of microorganisms. In general, the products from abiotic conversion of the chloromethanes are predominately nonchlorinated species. Products from the aerobic biotransformation of DCM are also nontoxic, nonchlorinated species.

A substantial amount of published information was found for the equilibrium sorption of chloromethanes on soils. These data include several studies with soil organic carbon levels similar to Hanford (below $0.5 \%$ ). Experimentally measured values were found to correlate well with the traditional predictive equations that were developed using chlorinated $\mathrm{C}_{1}$ and $\mathrm{C}_{2}$ compounds in the regression data sets. The most probable values for $\mathrm{K}_{\mathrm{d}}$ for the Hanford aquifer were estimated to be $0.12,0.06$, and $0.1 \mathrm{~L} / \mathrm{kg}$ for CT, CF, and DCM, respectively.

\section{Review of Chloromethane Transformation}

Figure 1 outlines the published biotic and abiotic reactions that have been observed for chlorinated methanes. The following sections discuss the published data that supports the reaction pathways detailed in this figure. 


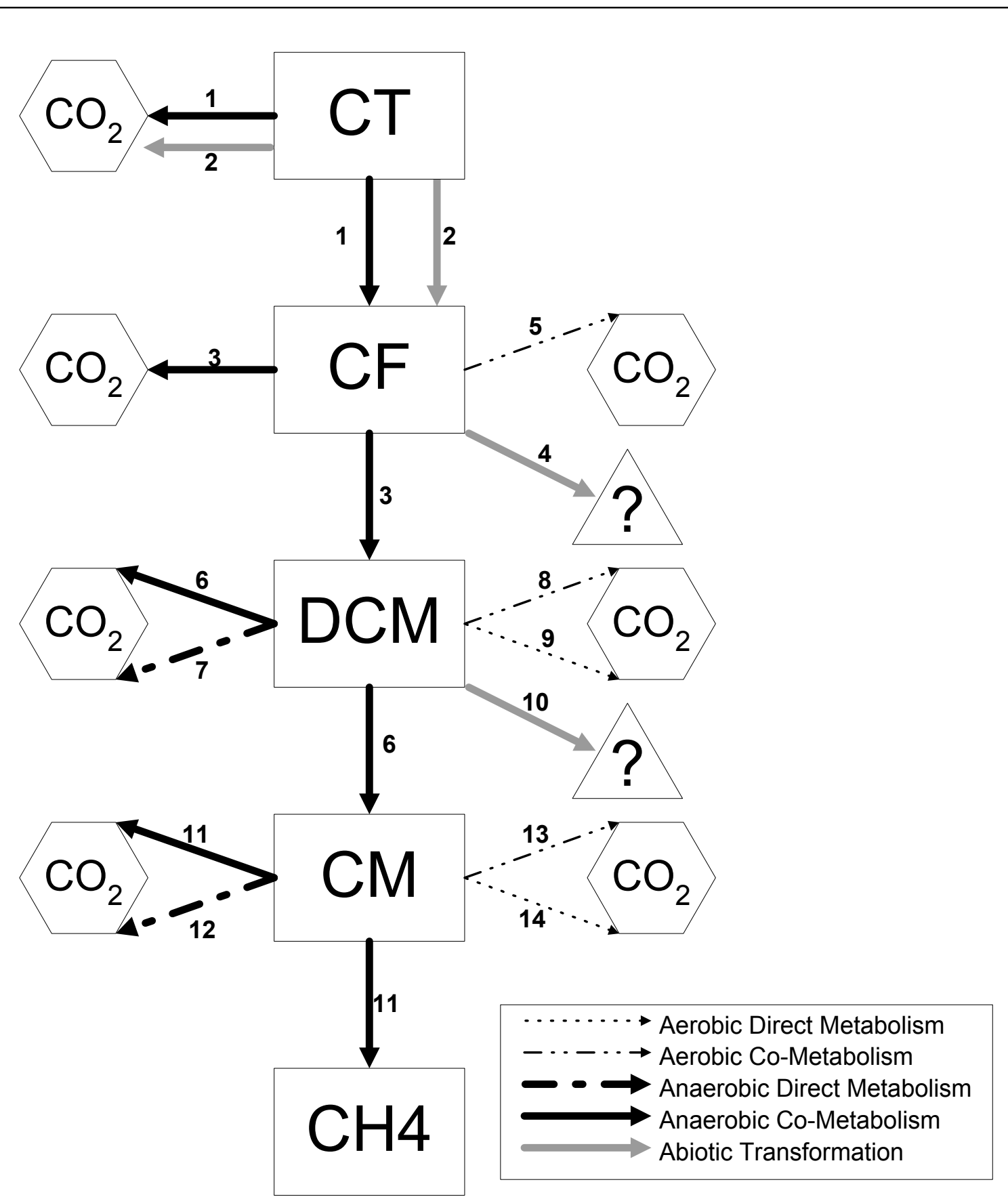

Figure 1: Transformation pathways for chlorinated methanes. Numbers next to each reaction refer to reaction numbers that are referenced in the text. 


\section{Carbon Tetrachloride}

\section{Abiotic transformation}

There is some conflicting information in the literature for on the hydrolysis rate of CT at 25C. For instance, Fells and Moelwyn-Hughes (1959), as cited in the compilation by Mabey and Mill (1978), reported a CT half-life of 7000 years at 25C; whereas, Jeffers et al., (1989) reported a CT half-life of 40 years at $25 \mathrm{C}$. It should be noted that these data were collected at temperatures of $70-180 \mathrm{C}$ and extrapolated to $25 \mathrm{C}$ using the Arrhenius equation.

A later article by Jeffers et al. (1996) addresses the discrepancy between the previous two sources. Essentially, through further testing of reaction order, Jeffers et al. (1996) show that the hydrolysis reaction for CT is first order, as proposed in the 1989 paper and in contrast to the second-order interpretation that generated the 7000-year half-life in Fells and Moelwyn-Hughes (1959). Both the new Jeffers et al. (1996) data and a reanalysis of original data that were the basis for the Fells and Moelwyn-Hughes (1959) half-life calculation agree with the half-life reported in Jeffers et al. (1989). That is, Jeffers et al. (1996) assert and provide evidence to support that the extrapolated half-life of CT at $25 \mathrm{C}$ is approximately 40 years.

Both Peter Jeffers and Ted Mill, the primary researchers in this field, were personally contacted. They agree that the estimate of 40 years for the half-life of CT in water seemed reasonable and that the hydrolysis reaction is appropriately modeled as a first-order reaction. A further paper by Jeffers et al. (1994) attempted to measure any possible effect of mineral surfaces on hydrolysis rate and found the rate unchanged even in the presence of sulfide minerals. Other literature on related compounds (Haag and Mill 1988; Deeley et al. 1991) corroborates this result and suggests that the hydrolysis rate determined in DI water should be a reasonable prediction of the rate of hydrolysis in the subsurface. However, both of these individuals thought that further laboratory work to verify this general assumption with Hanford sediments under ambient conditions would be a good idea before making a remediation decision.

The predicted half-life for CT using the Arrhenius parameters presented in Jeffers et al. (1989) and Jeffers et al. (1996) over a temperature range of 10 to $25 \mathrm{C}$ is plotted in Figure 2. Included on this plot are the upper and lower bound predictions based on the uncertainty in the Arrhenius parameters listed in Jeffers (1996). This plot suggests that the half-life of CT in the aquifer at Hanford is likely between 36 and 290 years for a temperature of about $19 C$. The best estimate for the half-life would be about 100 years. It should be noted, however, that we have found no data for CT hydrolysis that were collected at ambient temperatures. As this seems to be an issue with the other chlorinated methanes (see below), it cannot be ignored for CT.

Based on the literature and personal communication sources listed above, it is likely that neutral hydrolysis of CT leads to formation of $\mathrm{CO}_{2}$ and $\mathrm{HCl}$, not any hazardous compounds. However, reductive processes leading to the formation of other chlorinated intermediates such as $\mathrm{CF}$, and the possible presence and persistence of any chlorinated intermediates formed during hydrolysis of CT under conditions in the Hanford aquifer (including groundwater and sediment interactions) should be confirmed. 


\section{Figure 2. Neutral Homogeneous Hydrolysis of Carbon Tetrachloride in Distilled Water}

Data of Jeffers et al. $(1989,1996)$
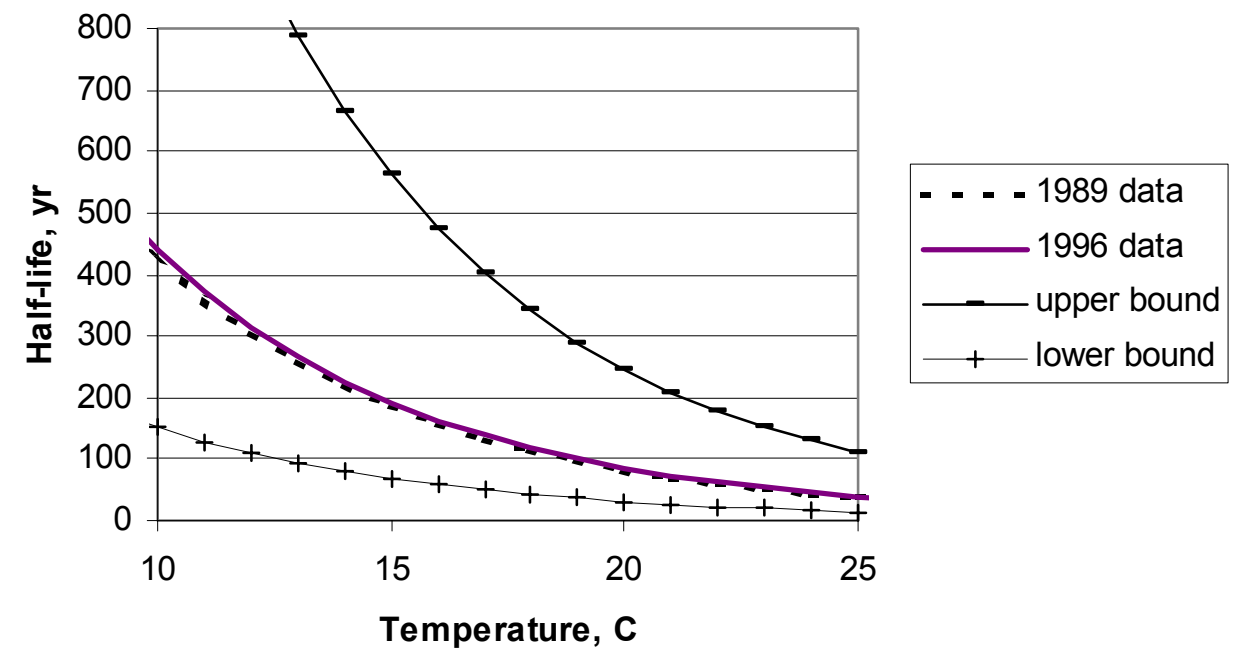

Biotic transformation

Carbon tetrachloride biotransformation has been demonstrated with a number of different bacteria in both the laboratory and the field. The conditions that favor biodegradation of CT are predominantly anaerobic. In fact, there have been no published reports of aerobic transformation of CT. In contrast, anaerobic transformation of CT has been demonstrated in a wide range of microbes. Table 1 provides details on pure cultures that have demonstrated CT transformation. As is evident from this table, CT destruction has been observed using denitrifying, sulfate-reducing, acetogenic, fermentative, and methanogenic organisms. Unlike chloroethenes, CT biotransformation does not strictly follow a reductive dechlorination pattern with sequential removal of single chlorines to produce CF, DCM, CM, and methane. Rather, the presence of chloromethanes, carbon dioxide, and other non-chlorinated organics suggest both reductive and substitutional reactions.

Various authors studying mixed cultures of anaerobic organisms have also reported a similar distribution of products. For example, Bouwer and McCarty reported that cultures of sewage treatment bacteria biodegraded CT to carbon dioxide and other non-chlorinated metabolites under methanogenic conditions using acetate as the electron donor (Bouwer and McCarty 1983a). These authors also demonstrated CT transformation to CF and carbon dioxide by an ethanol-fed, denitrifying consortium (Bouwer and McCarty 1983b). Similar products were reported by researchers studying CT biotransformation under denitrifying conditions at the Hanford Site (Hooker et al. 1998; Sherwood et al. 1996). A sulfate-reducing consortium was also reported to transform CT (Cobb and Bouwer 1991). In addition, Semprini speculated that sulfate-reducing bacteria were responsible for the CT degradation they observed during a field test of in situ bioremediation (Semprini et al. 1991). In both cases CF production only accounted for approximately $50 \%$ to $60 \%$ of the reduction in CT concentration. Other products were not identified. 
It is important to note that in each of the above systems, CT was transformed by anaerobic cultures that were actively metabolizing another organic compound as an energy and carbon source. To date, it has never been demonstrated that CT can be used as a growth substrate. For example, Semprini et al. observed only minimal reductions in CT concentration during the initial phase of their field test of CT in situ bioremediation before primary substrates were added to the aquifer (Semprini et al. 1992). In contrast once substrate addition commenced there was rapid reduction in $\mathrm{CT}$ concentration with a simultaneous production of $\mathrm{CF}$.

Conversion of CT to less chlorinated methanes has also been observed in the field in anaerobic aquifers where $\mathrm{CT}$ is present as a co-contaminant with other organic compounds that can serve as an electron donor. Liang and Grbic-Galic (1993) observed reductions in CT concentration in a laboratory microcosm study using sediments from two CT and fuel hydrocarbon contaminated sites. These researchers also saw similar responses from an uncontaminated, but actively methanogenic, aquifer sediment. Chloroform formation could only account for between 15 and $50 \%$ of the reduction in CT. No DCM or chloromethane were detected.

Table 1: Pure cultures showing CT transformation

\begin{tabular}{|c|c|c|c|c|}
\hline Organism & Condition & Reported Products & $\begin{array}{l}\text { CM Fraction of } \\
\text { Products }^{\mathrm{a}}\end{array}$ & Reference \\
\hline $\begin{array}{l}\text { Desulfobacterium } \\
\text { autotrophicum }\end{array}$ & $\begin{array}{c}\text { Sulfate } \\
\text { Reducing }\end{array}$ & CF, DCM & $60 \%$ & Egli et al. 1988 \\
\hline $\begin{array}{l}\text { Acetobacterium } \\
\text { woodii }\end{array}$ & Acetogenic & $\mathrm{CF}, \mathrm{DCM}, \mathrm{CO}_{2}$ & $8 \%$ & Egli et al. 1988 \\
\hline $\begin{array}{l}\text { Methanobacterium } \\
\text { thermoautotrophicum }\end{array}$ & Methanogenic & $\begin{array}{c}\mathrm{CF}, \underset{\mathrm{DCM}, \mathrm{CO}_{2},}{\mathrm{WSC}^{\mathrm{b}}}\end{array}$ & $34 \%$ & Egli et al. 1990 \\
\hline $\begin{array}{l}\text { Methanosarcina } \\
\text { thermophila }\end{array}$ & Methanogenic & $\mathrm{CF}, \mathrm{CO}_{2}$ & $13 \%$ & $\begin{array}{l}\text { Andrews and } \\
\text { Novak } 1999\end{array}$ \\
\hline Clostridium sp. & Fermentative & CF, DCM & $8 \%$ & $\begin{array}{l}\text { Galli and } \\
\text { McCarty } 1989\end{array}$ \\
\hline $\begin{array}{l}\text { Pseudomonas strain } \\
\mathrm{KC}\end{array}$ & Denitrifying & $\mathrm{CO}_{2}$ & $0 \%$ & $\begin{array}{l}\text { Criddle et al. } \\
\text { 1990a }\end{array}$ \\
\hline Escherichia coli $\mathrm{K}-12$ & Fermentative & $\mathrm{CF}, \mathrm{CO}_{2}, \mathrm{CBF}^{\mathrm{c}}$ & $38 \%$ & $\begin{array}{l}\text { Criddle et al. } \\
1990 \mathrm{~b}\end{array}$ \\
\hline
\end{tabular}




\section{Chloroform}

\section{Abiotic Transformation}

The literature on hydrolysis rates of CF also contains conflicting data. For example, a roomtemperature sealed-ampule study of CF hydrolysis (Dilling et al. 1975) yielded a half-live of 1.25 years. In contrast, the extrapolated high-temperature (100-150C) data of Fells and MoelwynHughes (1959) for CF show a half-live of 3500 years at 25C. Jeffers et al., (1989) reported a half-life for CF of 1850 years based on data collected at 50-170 C.

Dilling et al. (1975) stated that the faster rates obtained at $25 \mathrm{C}$ were possibly due to different mechanisms operating at low temperature than at the elevated temperature. This possibility is supported by the failure of the Fells and Moelwyn-Hughes (1959) data to be adequately described by the standard two-constant Arrhenius equation. Other differences in the high- and low-temperature data sets are 1) that a large headspace (49-72\% of the total ampule) was used in the low-temperature work, whereas Jeffers et al. (1989) restricted headspace to the minimum needed to prevent ampule breakage (about 5\%), and 2) initial concentrations were 1 ppm in the low-temperature work, and 1125 ppm for the Fells and Moelwyn-Hughes (1959) hightemperature data. It is unclear what the initial CF concentration was in the Jeffers et al. (1989) work at high temperature, although it was probably between $1 \%$ and $10 \%$ of aqueous saturation (i.e., $82-820 \mathrm{ppm}$ ).

\section{Biotic Transformation}

Unlike CT, chloroform can be biologically transformed under both aerobic and anaerobic conditions. Aerobic destruction of CF is a co-metabolic process that is mediated by a variety of organisms. Table 2 provides a list of some of the most common types of co-substrates that have demonstrated the ability to support aerobic CF transformation. As is evident from Table 2, aerobic co-metabolism of $\mathrm{CF}$ results in complete mineralization of the toxin.

Table 2: Metabolisms that co-metabolically degrade CF under aerobic conditions

\begin{tabular}{|c|c|c|c|}
\hline Organism & Co-Substrate & Reported Products & Reference \\
\hline Methylosinus trichosporium $\mathrm{OB} 3 \mathrm{~b}$ & Methane & $\mathrm{CO}_{2}$ & Speitel et al. 1993 \\
\hline $\begin{array}{l}\text { Rhodococcus rhodochrous ATCC } \\
21197\end{array}$ & Propane & $\mathrm{CO}_{2}{ }^{\mathrm{a}}$ & Malachowsky et al. 1994 \\
\hline Pseudomonas sp. strain ENVPC1 & Toluene & $\mathrm{CO} 2,{ }^{b}, \mathrm{CBF}^{\mathrm{c}}$ & McClay et al. 1996 \\
\hline Nitrosomonas europaea & Ammonia & Not Reported & Ely et al. 1997 \\
\hline
\end{tabular}

Anaerobic transformation of CF is similar to that for CT with the exception that it is not transformed by denitrifying bacteria (Bouwer and McCarty 1983; Criddle et al. 1990). CF biotransformation as been demonstrated by sulfate reducing (Gupta et al. 1996a; Egli et al. 1987), acetogenic (Egli et al. 1990), fermentative (Gali and McCarty 1989), and methanogenic cultures (Gupta et al. 1996; Bagley and Gossett 1995; Mikesell and Boyd 1990; Bouwer and 
McCarty 1983). In each case, CF was not acting as a growth substrate and its destruction requires the presence of a co-substrate. The last entry in this table represents a methanogenic river sediment that was not amended with an anthropogenic electron donor. Hence, the energy source for $\mathrm{CF}$ transformation is natural organic carbon. Products from biotransformation of CF in well-controlled laboratory studies are shown in Table 3. As is evident from this table, both reductive dechlorination and oxidation products are produced by the various anaerobic organisms.

Table 3: Cultures showing anaerobic CF biotransformation

\begin{tabular}{|c|c|c|c|c|}
\hline Organism & Condition & Reported Products & $\begin{array}{l}\text { CM Fraction of } \\
\text { Products }^{\text {a }}\end{array}$ & Reference \\
\hline Mixed culture & Sulfate reducing & DCM & $\begin{array}{c}5 \%(\mathrm{CM} \text { not } \\
\text { measured so could } \\
\text { be higher })\end{array}$ & Gupta et al. 1996b \\
\hline $\begin{array}{l}\text { Acetobacterium } \\
\text { woodii }\end{array}$ & Acetogenic & $\mathrm{DCM}, \mathrm{CO}_{2}$ & $28 \%$ & Egli et al. 1988 \\
\hline Clostridium sp. & Fermentative & DCM & $20 \%$ & $\begin{array}{l}\text { Galli and McCarty } \\
1989\end{array}$ \\
\hline $\begin{array}{l}\text { Methanosarcina } \\
\text { barkeri } 227\end{array}$ & Methanogenic & $\mathrm{DCM}, \mathrm{CO}_{2}$ & $20-60 \%$ & $\begin{array}{l}\text { Bagley and Gossett } \\
1995\end{array}$ \\
\hline $\begin{array}{l}\text { Methanosarcina } \\
\text { strain DCM }\end{array}$ & Methanogenic & $\mathrm{DCM}, \mathrm{CM}, \mathrm{CO}_{2}$ & $65 \%$ & $\begin{array}{l}\text { Mikesell and Boyd } \\
1990\end{array}$ \\
\hline $\begin{array}{l}\text { Methanosarcina } \\
\text { mazei S6 }\end{array}$ & Methanogenic & $\mathrm{DCM}, \mathrm{CM}, \mathrm{CO}_{2}$ & $78 \%$ & $\begin{array}{l}\text { Mikesell and Boyd } \\
1990\end{array}$ \\
\hline Mixed culture & Methanogenic & $\mathrm{CO}_{2}$ & $\sim 80 \%$ & $\begin{array}{l}\text { VanBeelen and } \\
\text { VanKeulen } 1990\end{array}$ \\
\hline
\end{tabular}

\section{Dichloromethane}

\section{Abiotic Transformation}

Little published data is available on abiotic transformation of DCM. No data was found for reaction in a mixed mineral and aqueous system. One report was found for aqueous hydrolysis of DCM in DI water and a half-life of 690 years was observed at 25 C (Maybey and Mills 1978). No products were reported for this work. In addition, it is not known whether the experiments were conducted with, or without oxygen.

\section{Biotic Transformation}

Microorganisms transform dichloromethane under both aerobic and anaerobic conditions. Transformation under both conditions has been observed both cometabolically and with DCM acting as a growth substrate (Leisinger and Braus-Stromeyer 1995; Freedman and Gossett 1991; Yanghao 1990). Table 4 provides list of the reported laboratory microbial systems that transform DCM. It is important to note that, with the exception of the acetogen, Acetobacterium woodii, the predominant products are nonchlorinated compounds. This finding is consistent with the lack of chloromethane in groundwater where DCM is degrading (Mehran and Wolf 1999; Mechaber et al. 1998). This observation implies that it may be reasonable to assume that CM 
can be neglected as a degradation product while modeling the fate of $\mathrm{CT}$ in the Hanford aquifer. Such an assumption would eliminate reactions 6, 11, 12, 13, and 14 from the reaction scheme displayed in Figure 1.

Table 4: List of organisms showing the ability to co-metabolically degrade DCM under aerobic conditions

\begin{tabular}{|c|c|c|c|c|}
\hline Organism & Condition & Co-Substrate & Reported Products & Reference \\
\hline $\begin{array}{l}\text { Rhodococcus } \\
\text { rhodochrous ATCC } \\
21197\end{array}$ & $\begin{array}{l}\text { Aerobic; } \\
\text { cometabolic }\end{array}$ & Propane & $\mathrm{CO}_{2}^{\mathrm{a}}$ & $\begin{array}{l}\text { Malachowsky et al. } \\
1994\end{array}$ \\
\hline Soil microcosm & $\begin{array}{l}\text { Aerobic; } \\
\text { cometabolic? }\end{array}$ & $\begin{array}{l}\text { None added, } \\
\text { but } \mathrm{SOM}^{\mathrm{a}} \\
\text { was present }\end{array}$ & $\mathrm{CO}_{2}(15-75 \%)$ & Davis and Madsen 1991 \\
\hline Soil microcosm & $\begin{array}{l}\text { Aerobic; } \\
\text { cometabolic? }\end{array}$ & $\begin{array}{l}\text { None added, } \\
\text { but } \mathrm{SOM}^{\mathrm{a}} \\
\text { was present }\end{array}$ & $\mathrm{CO}_{2}(15-75 \%)$ & Davis and Madsen 1991 \\
\hline Mixed culture & $\begin{array}{l}\text { Aerobic; } \\
\text { cometabolic }\end{array}$ & Glucose & $\mathrm{CO}_{2}(\sim 100 \%)$ & Klecka 1982 \\
\hline $\begin{array}{l}\text { Pseudomonas strain } \\
\text { DM1 }\end{array}$ & $\begin{array}{l}\text { Aerobic; growth } \\
\text { substrate }\end{array}$ & None & $\begin{array}{l}\text { Complete release of } \\
\qquad \mathrm{Cl}^{-}\end{array}$ & Yanghao 1990 \\
\hline Mixed culture & $\begin{array}{l}\text { Methanogenic; } \\
\text { growth substrate }\end{array}$ & None & $\mathrm{CO}_{2}, \mathrm{CH}_{4}$, Acetate & $\begin{array}{l}\text { Freedman and Gossett } \\
1991\end{array}$ \\
\hline $\begin{array}{l}\text { Acetobacterium } \\
\text { woodii }\end{array}$ & $\begin{array}{l}\text { Acetogenic; co- } \\
\text { metabolic }\end{array}$ & Fructose & $\mathrm{CM}$ & Egli et al. 1988 \\
\hline Mixed culture & $\begin{array}{l}\text { Methanogenic; } \\
\text { cometabolic }\end{array}$ & Acetate & Not reported & Gupta et al. 1996a \\
\hline Mixed culture & $\begin{array}{l}\text { Sulfate } \\
\text { reducing; co- } \\
\text { metabolic }\end{array}$ & Acetate & Not reported & Gupta et al. 1996b \\
\hline Strain DMC & $\begin{array}{c}\text { Acetogenic; } \\
\text { growth substrate }\end{array}$ & None & Acetate & Magli et al. 1995 \\
\hline Mixed culture & $\begin{array}{l}\text { Acetogenic; } \\
\text { growth substrate }\end{array}$ & None & $\begin{array}{c}\text { Acetate }(23 \%), \mathrm{CO}_{2} \\
(58 \%), \text { Formate } \\
(11 \%)\end{array}$ & $\begin{array}{l}\text { Braus-Stromeyer et al. } \\
1993\end{array}$ \\
\hline
\end{tabular}

\section{Chloromethane}

\section{Abiotic Transformation}

Little published data is available on abiotic transformation of CM. No data could be found for reaction in a mixed mineral and aqueous system. One report was found for aqueous hydrolysis of $\mathrm{CM}$ at $25^{\circ} \mathrm{C}$, and a half-life of 0.93 years was observed (Maybey and Mills 1978). No products were reported for these experiments nor whether the experiments were conducted in the presence of oxygen..

\section{Biotic Transformation}

Chloromethane can be transformed by a number of biological processes. This compound is cometabolized both oxidatively (Rasche et al. 1990; Stirling and Dalton 1979) and hydrolytically 
(Keuning et al. 1985). In addition, several bacteria have been characterized which are able to use chloromethane as a growth substrate. These organisms include the strictly anaerobic homoacetogenic bacterium strain MC (Messmer et al. 1993) and several aerobic methylotrophs (Connell-Hancock et al. 1998; Doronia and Trotsenko 1997; Doronia et al. 1996; Hartmans et al. 1986). Table 5 outlines the pertinent properties of these reactions including the reported products from CM biotransformation.

Table 5: List of organisms showing the ability to degrade CM under aerobic conditions

\begin{tabular}{|l|l|l|l|l|}
\hline Organism & Condition & Co-Substrate & Reported Products & Reference \\
\hline $\begin{array}{l}\text { Methylobacterium } \\
\text { sp. Strain CM4 }\end{array}$ & Aerobic & None & Complete $\mathrm{Cl}^{-}$release to media & $\begin{array}{l}\text { Vannelli et al. } \\
1998\end{array}$ \\
\hline Strain IMB-1 & Aerobic & None & $\begin{array}{l}\text { Not reported, but } \mathrm{MeBr}^{\mathrm{a}} \\
\text { transformed to CO2, and } \\
\text { biomass }\end{array}$ & $\begin{array}{l}\text { Connell-Hancock } \\
\text { et al. 1998 }\end{array}$ \\
\hline Hyphomicrobium & Aerobic & None & Complete $\mathrm{Cl}^{-}$release to media & $\begin{array}{l}\text { Hartmans et al. } \\
1986\end{array}$ \\
\hline $\begin{array}{l}\text { Nitrosomonas } \\
\text { europaea }\end{array}$ & Aerobic & Ammonia & Formate & Rasche et al. 1990 \\
\hline $\begin{array}{l}\text { Methylococcus } \\
\text { capsulatus }\end{array}$ & Aerobic & Methane & ND $^{\mathrm{b}}$ & $\begin{array}{l}\text { Stirling and } \\
\text { Dalton 1979 }\end{array}$ \\
\hline $\begin{array}{l}{ }^{\mathrm{a}} \text { MeBr denotes methyl bromide. } \\
\text { b ND denotes no data was reported. }\end{array}$ & & \\
\hline
\end{tabular}

\section{Chloromethane Transformation in Groundwater Systems}

Tables 6, 7, and 8 summarize the published first order rate data (reported as a half-life) for biotransformation of CT, CF, and DCM in actual, or simulated, aquifer systems. No published data could be found for CM degradation rates in an aquifer system. For CT, the reported halflife ranges from 2.8 days to 4300 days. The range of values for both CF and DCM is much smaller at 12 to 63 days and 2.3 to 70 days, respectively.

The reaction products and conditions listed in Tables 6 through 8 are consistent with those discussed in the preceding sections for highly enriched and pure cultures. Namely, a co-substrate was present in all systems showing CT and CF transformation. In contrast, DCM was transformed in systems without a co-substrate, suggesting it was used as a growth substrate. Furthermore, anaerobic conditions were present in all systems showing $\mathrm{CT}$ and $\mathrm{CF}$ degradation while DCM was degraded in both aerobic and anaerobic systems. Finally, products of transformation for each compound consisted of a mixture of both less chlorinated methanes and (presumably in many cases) non-chlorinated compounds.

For the Hanford CT groundwater plume, which is both aerobic and in contact with sediments that have organic carbon levels below $0.5 \mathrm{wt} \%$ (Newcomer et al. 1995), these results suggest that no biological activity is currently contributing to the natural attenuation of either CT or CF. Hence only the abiotic reactions indicated in Figure 1 (Numbers 2, 4, and 10) and aerobic DCM transformation (Number 9) should be considered when evaluating the impact of natural attenuation on the Hanford CT plume. 
Table 6: First-order dehalogenation rates measured for CT

\begin{tabular}{|c|c|c|c|c|c|c|c|c|}
\hline Location & $\begin{array}{l}\text { Temp } \\
\left({ }^{\circ} \mathrm{C}\right)\end{array}$ & Condition & $\begin{array}{l}\text { Lab or Field } \\
\text { Data? }\end{array}$ & $\begin{array}{c}\text { Initial } \\
\text { Concentration } \\
(\mu \mathrm{M})\end{array}$ & Products & $\begin{array}{l}\text { Half Life } \\
\text { (day) }\end{array}$ & Co-Substrate & Reference \\
\hline Brazil Manufacturing Site & $\mathrm{ND}^{\mathrm{b}}$ & Anaerobic & Field & 65 to 650 & CF, DCM & 90 to 130 & $\mathrm{DCP}^{\mathrm{a}}$ & Hardy et al. 1999 \\
\hline Tyndal AFB & 35 & Anaerobic & Lab & 10 & $\mathrm{CF}$ & 4.6 & Jet fuel & $\begin{array}{l}\text { Liang and Grbic-Galic } \\
1993\end{array}$ \\
\hline Traverse City MI & 35 & Anaerobic & Lab & 8 & $\begin{array}{c}\mathrm{CF}(50 \%)^{\mathrm{c}}, \\
\text { other? }\end{array}$ & 22 & Gasoline & $\begin{array}{l}\text { Liang and Grbic-Galic } \\
1993\end{array}$ \\
\hline Pensacola FL & 35 & Anaerobic & Lab & 10 & $\begin{array}{l}\mathrm{CF}(14 \%)^{\mathrm{c}}, \\
\text { other? }\end{array}$ & 14 & High carbon soil & $\begin{array}{l}\text { Liang and Grbic-Galic } \\
1993\end{array}$ \\
\hline $\begin{array}{l}\text { Grinsted Landfill, } \\
\text { Denmark }\end{array}$ & $\mathrm{ND}^{\mathrm{b}}$ & Anaerobic & Field & 0.5 to 2.3 & $\mathrm{ND}^{\mathrm{b}}$ & 2.8 & $\mathrm{ND}^{\mathrm{b}}$ & Rugge et al. 1995 \\
\hline Vejen Landfill, Denmark & $\mathrm{ND}^{\mathrm{b}}$ & Anaerobic & Field & 0.8 & $\mathrm{ND}^{\mathrm{b}}$ & 3.5 & $\mathrm{DOC}^{\mathrm{e}}$ & Nielsen et al. 1992 \\
\hline Tucumcari NM & $\mathrm{ND}^{\mathrm{b}}$ & Anaerobic & Field & 6.5 & $\begin{array}{l}\mathrm{CF}, \mathrm{DCM}, \\
\mathrm{CO}_{2} ?^{\mathrm{d}}\end{array}$ & 4300 & Gasoline & McQuillian et al. 1998 \\
\hline Necco Park, NY & 10 & Anaerobic & Field & 65 to 650 & CF, DCM & 340 & Landfill leachate & Lee et al. 1995 \\
\hline \multicolumn{9}{|c|}{$\begin{array}{l}\text { a DCP denotes dichloropropane. } \\
{ }^{\mathrm{b}} \text { ND denotes no data was reported. } \\
\mathrm{c} \text { Values in brackets indicate the quantity of the transformed parent compound present as a less chlorinated species. } \\
{ }^{\mathrm{d}} \text { Carbon dioxide implicated by lack of mass balance on CF and DCM, not verified with direct measurement. } \\
{ }^{\mathrm{e}} \text { DOC denotes dissolved organic carbon. }\end{array}$} \\
\hline
\end{tabular}


Table 7: First-order dehalogenation rates measured for CF

\begin{tabular}{|l|c|c|c|c|c|c|l|l|}
\multicolumn{1}{|c|}{ Location } & $\begin{array}{c}\text { Temp } \\
\left({ }^{\circ} \mathbf{C}\right)\end{array}$ & Condition & $\begin{array}{c}\text { Lab or } \\
\text { Field } \\
\text { Data? }\end{array}$ & $\begin{array}{c}\text { Initial } \\
\text { Concentration } \\
(\mu \mathrm{M})\end{array}$ & \multicolumn{1}{|c|}{ Products } & $\begin{array}{c}\text { Half } \\
\text { Life } \\
(\mathbf{d a y})\end{array}$ & \multicolumn{1}{c|}{ Co-Substrate } \\
\hline $\begin{array}{l}\text { Merwede River, } \\
\text { Netherlands }\end{array}$ & 10 & Anaerobic & Lab & 34 & $\mathrm{CO}_{2}(60 \%)^{\mathrm{a}}$ & 12 & High carbon soil & $\begin{array}{l}\text { VanVeelen and } \\
\text { Keulem 1990 }\end{array}$ \\
\hline CA chemical plant & 20 & Anaerobic & Field & 268 & DCM, $\mathrm{CO}_{2}{ }^{\mathrm{b}}$ & 63 & $\begin{array}{l}\text { Yes, other GW } \\
\text { contaminants }\end{array}$ & Cox et al. 1998 \\
\hline
\end{tabular}

Table 8: First-order dehalogenation rates measured for DCM

\begin{tabular}{|c|c|c|c|c|c|c|c|c|}
\hline Location & $\begin{array}{l}\text { Temp } \\
\left({ }^{\circ} \mathrm{C}\right)\end{array}$ & Condition & $\begin{array}{c}\text { Lab or } \\
\text { Field } \\
\text { Data? }\end{array}$ & $\begin{array}{c}\text { Initial } \\
\text { Concentration } \\
(\mu \mathrm{M})\end{array}$ & Products & $\begin{array}{l}\text { Half } \\
\text { Life } \\
\text { (day) }\end{array}$ & Co-Substrate & Reference \\
\hline Not stated & $\mathrm{ND}^{\mathrm{a}}$ & Anaerobic & Lab & 1200 & $\mathrm{CO}_{2}, \mathrm{Cl}^{-}$ & 2.3 & None & Biehle et al. 1999 \\
\hline Los Angeles, CA & $\mathrm{ND}^{\mathrm{a}}$ & $\begin{array}{c}\text { Sequential } \\
\text { Anaerobic/ } \\
\text { Aerobic }\end{array}$ & Field & 12,000 & $\mathrm{NCh}^{\mathrm{b}}$ & 4 & None & $\begin{array}{l}\text { Mehran and Wolf } \\
1999\end{array}$ \\
\hline $\begin{array}{l}\text { Near surface soil, location not } \\
\text { stated }\end{array}$ & 25 & Aerobic & Lab & 6 & $\mathrm{CO}_{2}$, Others? & 7 to 70 & None & $\begin{array}{l}\text { Davis and Masen } \\
1991\end{array}$ \\
\hline $\begin{array}{l}\text { Near surface soil, location not } \\
\text { stated }\end{array}$ & 25 & Anaerobic & Lab & $\mathrm{ND}^{\mathrm{a}}$ & $\mathrm{ND}^{\mathrm{a}}$ & 22 & None & $\begin{array}{l}\text { Davis and Masen } \\
1991\end{array}$ \\
\hline Not stated & $\mathrm{ND}^{\mathrm{a}}$ & Anaerobic & Field & $<15$ & $\mathrm{ND}^{\mathrm{a}}$ & 63 & $\begin{array}{l}\text { Unspecified co- } \\
\text { contaminants }\end{array}$ & $\begin{array}{l}\text { Mechaber et al. } \\
1998\end{array}$ \\
\hline
\end{tabular}




\section{Sorption of Chloromethanes on Hanford Sediments}

Movement of chemicals across the water/sediment interface is primarily a sorption-desorption process governed by the solubility of the particular chemical in water and its affinity to the solid phase. In high carbon soils, it has been demonstrated that the amount of nonionic organic chemicals sorbed varies from soil to soil and that such variations are primarily caused by the organic content of the soil (Chang et al. 1992; Lyman et al. 1990; Bishop et al. 1989). However, mineral driven sorption also becomes important as organic carbon content diminishes to below $0.1 \%$ (Kile et al. 1995). At levels of organic carbon above $0.1 \%$, the normalized sorption coefficient $\left(\mathrm{K}_{\mathrm{oc}}\right)$ represents an important parameter that can be used to estimate the soil/water equilibrium partition coefficient $\left(\mathrm{K}_{\mathrm{d}}\right)$ by the equation:

$$
K_{d}=f_{o c} \times K_{o c}
$$

Where $f_{o c}$ is the mass fraction of organic carbon (mass-oc/mass-soil) in the soil and $K_{d}$ is the soil/water equilibrium partition coefficient $[(\mathrm{mg} / \mathrm{kg}$-soil $) /(\mathrm{mg} / \mathrm{L})]$. It is important to note that at levels of organic carbon below approximately $0.1 \%$, this relationship will likely under estimate $\mathrm{K}_{\mathrm{d}}$ since it neglects mineral driven sorption (Stephanatos et al. 1991). In these cases the real quantity of attached material will be higher than those predicted by Equation (1).

Many researchers have developed methods for estimating $\mathrm{K}_{\mathrm{oc}}$ based on measurable properties such as the octanol/water partition coefficient $\left(\mathrm{K}_{\mathrm{ow}}\right)$. Four of the most common correlations are given in the following equations (Lyman et al. 1990; Bishop et al. 1989).

$$
\begin{aligned}
& \log \left(K_{o c}\right)=3.64-0.55 \times \log (S) \\
& K_{o c}=0.63 \times K_{o w} \\
& \log \left(K_{o c}\right)=5.3-0.54 \times \log \left(W \times 10^{9}\right) \\
& \log \left(K_{o c}\right)=4.277-0.557 \times \log \left(S_{m}\right)
\end{aligned}
$$

Where $\mathrm{S}$ is the water solubility of the organic compound $(\mathrm{mg} / \mathrm{L}), \mathrm{S}_{\mathrm{m}}$ is the molar water solubility of the organic compound ( $\mu \mathrm{mol} / \mathrm{L})$, and $\mathrm{W}$ the water solubility in mole fraction (molecompound/mole-water). Table 9 provides information on the data used to develop each of these predictive equations. Table 10 details the value of the required input parameters for the chlorinated methanes while Table 11 lists the values of $\mathrm{K}_{\mathrm{oc}}$ estimated by equations 2 and 5 for chlorinated methane. Equations 2 and 5 are likely to provide the most reliable predictions of $\mathrm{K}_{\mathrm{oc}}$ for chloromethanes because the value of the input parameters is within the range used to develop these equations. In addition, halogenated $\mathrm{C}_{1}$ and $\mathrm{C}_{2}$ compounds were included in the regression data used to develop Equations 2 and 5, but not Equations 3 and 4. Experimentally measured values of $\mathrm{K}_{\mathrm{oc}}$ for chloromethanes from a variety of studies are given in Table 12 for comparison. 
Table 9: Information on predictive equations for $\mathrm{K}_{\mathrm{oc}}$

\begin{tabular}{|c|c|c|c|}
\hline $\begin{array}{l}\text { Equation } \\
\text { Number }\end{array}$ & $\begin{array}{l}\text { Range of } \\
\text { Independent } \\
\text { Parameter }^{\mathrm{a}}\end{array}$ & Range of $K_{o c}{ }^{a}$ & Comment \\
\hline 2 & $5 \times 10^{-4} \leq \mathrm{S} \leq 1 \times 10^{6}$ & $1 \leq \mathrm{K}_{\mathrm{oc}} \leq 1 \times 10^{6}$ & $\begin{array}{l}\text { Wide variety of compounds, dibromomethane } \\
\text { was only halogenated } C_{1} \text { or } C_{2}\end{array}$ \\
\hline 3 & $1 \times 10^{2} \leq \mathrm{K}_{\mathrm{ow}} \leq 4 \times 10^{6}$ & $100 \leq \mathrm{K}_{\mathrm{oc}} \leq 1 \times 10^{6}$ & $\begin{array}{l}\text { Mostly aromatic compounds, no halogenated } \\
C_{1} \text { or } C_{2}\end{array}$ \\
\hline 4 & $3 \times 10^{-11} \leq \mathrm{W} \leq 4 \times 10^{-5}$ & $80 \leq K_{o c} \leq 1 \times 10^{6}$ & $\begin{array}{l}\text { Mostly aromatic compounds, no halogenated } \\
C_{1} \text { or } C_{2}\end{array}$ \\
\hline 5 & $2 \times 10^{-3} \leq S_{m} \leq 1 \times 10^{5}$ & $30 \leq K_{o c} \leq 1 \times 10^{6}$ & $\begin{array}{l}\text { Chlorinated hydrocarbons including TeCA, } \\
\text { TCA, DCA, PCE, dibromomethane }\end{array}$ \\
\hline
\end{tabular}

Table 10: Physical properties used to estimate organic carbon sorption coefficient

\begin{tabular}{|l|c|c|c|c|c|}
\hline Compound & Molecular Weight & $\begin{array}{c}\mathbf{S} \\
(\mathrm{mg} / \mathrm{L})\end{array}$ & $\begin{array}{c}\mathbf{S}_{\mathrm{m}} \\
(\mu \mathrm{mol} / \mathrm{L})\end{array}$ & $\begin{array}{c}\mathbf{W} \\
(\mathrm{mol} / \mathrm{mol})\end{array}$ & $\mathbf{K}_{\text {ow }}$ \\
\hline CT & 153.8 & 800 & 5200 & $9.4 \times 10^{-5}$ & 537 \\
\hline CF & 119.4 & 8200 & $6.9 \times 10^{4}$ & $1.2 \times 10^{-3}$ & 85.1 \\
\hline DCM & 84.9 & 13000 & $1.5 \times 10^{5}$ & $2.8 \times 10^{-3}$ & 14.1 \\
\hline CM & 50.5 & 6400 & $1.3 \times 10^{5}$ & $2.3 \times 10^{-3}$ & 26.9 \\
\hline
\end{tabular}

(a) Koc values were taken from those compiled by Chang et al. 1992.

Table 11: Estimated $\mathrm{K}_{\mathrm{oc}}$ values for chloromethanes

\begin{tabular}{|l|c|c|}
\hline \multirow{2}{*}{ Compound } & \multicolumn{2}{|c|}{ Koc $(\mu \mathrm{g} / \mathrm{g}-\mathbf{0 c}) /(\mu \mathrm{g} / \mathrm{mL})$} \\
\hline Eq. 2 & $\mathbf{E q} \mathbf{5}$ \\
\hline CF & 110.48 & 161.11 \\
\hline DCM & 30.72 & 38.26 \\
\hline CM & 23.84 & 24.49 \\
\hline
\end{tabular}


Table 12: Published values of Koc for chloromethanes

\begin{tabular}{|c|c|c|c|c|c|}
\hline \multirow{2}{*}{ Reference } & \multicolumn{4}{|c|}{ Koc $(\mu \mathrm{g} / \mathrm{g}-\mathbf{0 c}) /(\mu \mathrm{g} / \mathrm{mL})$} & \multirow{2}{*}{ Comment } \\
\hline & CT & CF & nCM & $\mathbf{C M}$ & \\
\hline Jeng et al. 1992 & 122 & 76.8 & 47.4 & $\mathrm{ND}^{\mathrm{a}}$ & $\begin{array}{l}\text { Data is a compilation of } \\
\text { information measured by } \\
\text { others. }\end{array}$ \\
\hline Walton et al. 1992 & $143.6 \pm 32.1^{b}$ & $37.4 \pm 8.6^{b}$ & $\mathrm{ND}^{\mathrm{a}}$ & $\mathrm{ND}^{\mathrm{a}}$ & $\begin{array}{l}\text { Silty loam with an organic } \\
\text { carbon content of } 1.5 \% \text {. }\end{array}$ \\
\hline Walton et al. 1992 & $48.9 \pm 16.2^{b}$ & $30.0 \pm 2.6^{b}$ & $\mathrm{ND}^{\mathrm{a}}$ & $\mathrm{ND}^{\mathrm{a}}$ & $\begin{array}{l}\text { Sandy loam with an organic } \\
\text { carbon content of } 0.7 \% \text {. }\end{array}$ \\
\hline Duffy et al. 1997 & $55.0 \pm 1.0^{b}$ & $\mathrm{ND}^{\mathrm{a}}$ & $\mathrm{ND}^{\mathrm{a}}$ & $\mathrm{ND}^{\mathrm{a}}$ & $\begin{array}{l}\text { Soil with an organic carbon } \\
\text { content of } 1.5 \% \text {. }\end{array}$ \\
\hline Duffy et al. 1997 & $77.6 \pm 1.3^{b}$ & $\mathrm{ND}^{\mathrm{a}}$ & $\mathrm{ND}^{\mathrm{a}}$ & $\mathrm{ND}^{\mathrm{a}}$ & $\begin{array}{l}\text { Soil with an organic carbon } \\
\text { content of } 0.53 \% \text {. }\end{array}$ \\
\hline Duffy et al. 1997 & $269 \pm 2^{b}$ & $\mathrm{ND}^{\mathrm{a}}$ & $\mathrm{ND}^{\mathrm{a}}$ & $\mathrm{ND}^{\mathrm{a}}$ & $\begin{array}{l}\text { Soil with an organic carbon } \\
\text { content of } 0.14 \% \text {. }\end{array}$ \\
\hline Peng and Dural 1998 & 121.9 & 153.5 & $\mathrm{ND}^{\mathrm{a}}$ & $\mathrm{ND}^{\mathrm{a}}$ & Measured with Missouri Soil. \\
\hline Peng and Dural 1998 & 150.4 & 196.9 & $\mathrm{ND}^{\mathrm{a}}$ & $\mathrm{ND}^{\mathrm{a}}$ & $\begin{array}{l}\text { Measured with California } \\
\text { Soil. }\end{array}$ \\
\hline Peng and Dural 1998 & 121.0 & 190.0 & $\mathrm{ND}^{\mathrm{a}}$ & $\mathrm{ND}^{\mathrm{a}}$ & Measured with Florida Soil. \\
\hline Kile et al. 1999 & $59.1 \pm 7.5^{b}$ & $\mathrm{ND}^{\mathrm{a}}$ & $\mathrm{ND}^{\mathrm{a}}$ & $\mathrm{ND}^{\mathrm{a}}$ & $\begin{array}{l}\text { Average of } 17 \text { near surface } \\
\text { soils with organic carbon } \\
\text { ranging from } 1.1 \% \text { to } 5.6 \% \text {. }\end{array}$ \\
\hline Kile et al. 1999 & $106.7 \pm 13.44^{b}$ & $\mathrm{ND}^{\mathrm{a}}$ & $\mathrm{ND}^{\mathrm{a}}$ & $\mathrm{ND}^{\mathrm{a}}$ & $\begin{array}{l}\text { Average of } 9 \text { river bottom } \\
\text { sediments with organic carbon } \\
\text { ranging from } 1.4 \% \text { to } 5.6 \% \text {. }\end{array}$ \\
\hline Kile et al. 1995 & $60 \pm 7^{b}$ & $\mathrm{ND}^{\mathrm{a}}$ & $\mathrm{ND}^{\mathrm{a}}$ & $\mathrm{ND}^{\mathrm{a}}$ & $\begin{array}{l}\text { Average of } 32 \text { near surface } \\
\text { soils with organic carbon } \\
\text { ranging from } 0.16 \% \text { to } 6.1 \% \text {. }\end{array}$ \\
\hline Kile et al. 1995 & $102 \pm 11^{b}$ & $\mathrm{ND}^{\mathrm{a}}$ & $\mathrm{ND}^{\mathrm{a}}$ & $\mathrm{ND}^{\mathrm{a}}$ & $\begin{array}{l}\text { Average of } 36 \text { bed sediments } \\
\text { with organic carbon ranging } \\
\text { from } 0.11 \% \text { to } 4.7 \% \text {. }\end{array}$ \\
\hline
\end{tabular}

Estimating the soil/water equilibrium partition coefficient for Hanford requires a knowledge of the organic carbon of aquifer sediments. Values for $\mathrm{f}_{\mathrm{oc}}$ for the Hanford aquifer are listed in Table 13. The first two entries in this table correspond to values determined at the in situ bioremediation test site approximately $1.5 \mathrm{~km}$ northeast of the Plutonium Finishing Plant. The third entry represents the value used in the 1990 assessment of liquid effluents at the Hanford Site (WHC 1990). No reference was given in this document to the source of the value. The final value corresponds to that measured for a Hanford silty sand used by Washington State University will study the effects of soil vapor extraction on CT. 
Table 13: Organic carbon fraction data for the Hanford aquifer

\begin{tabular}{|c|c|c|c|c|c|}
\hline Location & Depth BGS (m) & $\operatorname{Max} \mathbf{f}_{o c}$ & $\operatorname{Min} f_{o c}$ & Av \pm St dev & Reference \\
\hline $\begin{array}{l}\text { 299-w11-30 } \\
\text { (4 samples) }\end{array}$ & 67 to 85 & $5.15 \times 10^{-3}$ & 0 & $1.5 \times 10^{-3} \pm 2.4 \times 10^{-3}$ & $\begin{array}{l}\text { Newcomer et al. } \\
1995\end{array}$ \\
\hline $\begin{array}{l}\text { 299-w11-32 } \\
\text { (5 samples) }\end{array}$ & 74 to 77 & $7.6 \times 10^{-4}$ & 0 & $2.7 \times 10^{-4} \pm 2.9 \times 10^{-4}$ & $\begin{array}{l}\text { Newcomer et al. } \\
1995\end{array}$ \\
\hline $\begin{array}{l}\text { Quoted value } \\
\text { for Hanford, no } \\
\text { supporting data }\end{array}$ & NA & $1.00 \times 10^{-3}$ & $\mathrm{ND}^{\mathrm{a}}$ & $\mathrm{NA}^{\mathrm{b}}$ & WHC 1990 \\
\hline 299-w15-31 & 40 to 43 & $1.10 \times 10^{-3}$ & $\mathrm{ND}^{\mathrm{a}}$ & $\mathrm{NA}^{\mathrm{b}}$ & Ford 1996 \\
\hline
\end{tabular}

An estimate for the range and most probable value for $\mathrm{K}_{\mathrm{d}}$ of each chloromethane at Hanford was derived from the data and information presented in this report. Equation 1 was used to calculate $\mathrm{K}_{\mathrm{d}}$ based on reported or calculated data for $\mathrm{f}_{\mathrm{oc}}$ and $\mathrm{K}_{\mathrm{oc}}$. However, it is important to note that the predicted $K_{d}$ from Equation 1 for Hanford may be an under estimate since the organic carbon fraction of the Hanford aquifer sediments are very close to the lower limit of applicability for this correlation. This potential to under predict sorption is demonstrated by the fact that Zhao et al. (1999) report a measured $\mathrm{K}_{\mathrm{d}}$ value of 0.39 for CT in a soil with an organic carbon content below their detection limit of $0.03 \%$. In contrast, the predicted $\mathrm{K}_{\mathrm{d}}$ for CT in a soil with an organic carbon content of $0.03 \%$ ranges from 0.013 to $0.12(\mathrm{~L} / \mathrm{kg})$ based on the values of $\mathrm{K}_{\mathrm{oc}}$ reported in Table 11 and Table 12.

The estimated range of $\mathrm{K}_{\mathrm{d}}$ values for $\mathrm{CT}$ was determined by examining the range of calculated and reported $\mathrm{K}_{\mathrm{d}}$ values from the literature. Table 14 reports the maximum values of $\mathrm{K}_{\mathrm{d}}$ that are predicted from Equation 1 using the $\mathrm{K}_{\mathrm{oc}}$ values reported in Table 11, along with the highest organic carbon fraction reported in Table $13(0.0052)$. Maximum values for $\mathrm{K}_{\mathrm{d}}$ determined in field studies are listed in Table 15. These values are based on the highest organic carbon fraction reported in Table $13(0.0052)$ and $\mathrm{K}_{\mathrm{oc}}$ (Table 12) values from each individual study. For $\mathrm{CT}, \mathrm{Kd}$ values calculated using the $\mathrm{f}_{\mathrm{oc}}$ and $\mathrm{K}_{\mathrm{oc}}$ values measured in each study are shown in parentheses next to the maximum value. The maximum value of $\mathrm{K}_{\mathrm{d}}$ selected from this information is 0.83 $\mathrm{L} / \mathrm{kg}$. There is one $\mathrm{K}_{\mathrm{d}}$ value reported in Table 15 that is higher than the selected maximum, but the study conditions and results suggest that a $\mathrm{K}_{\mathrm{d}}$ calculated using the measured $\mathrm{K}_{\mathrm{oc}}$ and an $\mathrm{f}_{\mathrm{oc}}$ value from another source would not be reasonable.

Table 16and Table 17 provide minimum $\mathrm{K}_{\mathrm{d}}$ estimates using an $\mathrm{f}_{\mathrm{oc}}$ of 0.0027 , the lowest average $\mathrm{f}_{\mathrm{oc}}$ value determined from the data reported by Newcomer et al. (1995). Values in Table 16 are calculated using the $\mathrm{K}_{\mathrm{oc}}$ values reported in Table 11. Values in Table 17 are calculated from field data using the $\mathrm{K}_{\mathrm{oc}}$ values reported in Table 12. Another estimate for the minimum $\mathrm{K}_{\mathrm{d}}$ was obtained by applying Equation 1 with the lowest average $f_{o c}$ value determined from the data reported by Newcomer et al. (1995) and a $\mathrm{K}_{\mathrm{oc}}$ value of 60. As shown in Table 13, the low average of measured $\mathrm{f}_{\mathrm{oc}}$ corresponds to an organic fraction of $0.00027(0.027 \%)$. The $\mathrm{K}_{\mathrm{oc}}$ value $(60(\mathrm{ug} / \mathrm{g}-\mathrm{oc}) /(\mathrm{ug} / \mathrm{mL}))$ was selected from an extensive survey of soils conducted by Kile et al. (1995) showing relatively consistent $\mathrm{K}_{\mathrm{oc}}$ values over a wide range of soil types and organic carbon content.. The minimum $\mathrm{K}_{\mathrm{d}}$ value calculated using this technique is $0.016 \mathrm{~L} / \mathrm{kg}$. This 
value is consistent with many of the lower $\mathrm{K}_{\mathrm{d}}$ values listed in Table 17 and was selected as the minimum $\mathrm{K}_{\mathrm{d}}$ value for use at Hanford.

The selected range of values of $\mathrm{K}_{\mathrm{d}}$ for $\mathrm{CT}$ is 0.016 to $0.83 \mathrm{~L} / \mathrm{kg}$. The most probable value within this range was selected as $0.12 \mathrm{~L} / \mathrm{kg}$. This value corresponds a $\mathrm{K}_{\mathrm{d}}$ calculated using a $\mathrm{K}_{\mathrm{oc}}$ value measured from an extensive survey of soils conducted by Kile et al. (1995) showing relatively consistent $\mathrm{K}_{\mathrm{oc}}$ values over a wide range of soil types and organic carbon content. The $\mathrm{f}_{\mathrm{oc}}$ value used in the calculation of most probable $K_{d}$ was 0.002 . This value for $f_{o c}$ is the average of the maximum $f_{o c}$ values reported in Table 13 and is considered to be a reasonable compromise of $f_{o c}$ information for the Hanford Site.

Similarly, Tables 14 through 17 show range of $\mathrm{K}_{d}$ values for CF and DCM. For CF, the range of $\mathrm{K}_{\mathrm{d}}$ values selected from this information is 0.008 to $0.39 \mathrm{~L} / \mathrm{kg}$. For the maximum $\mathrm{K}_{\mathrm{d}}$ value, the data from Peng and Dural (1998)was not considered due to the study conditions. The most probable $K_{d}$ value of $0.06 \mathrm{~L} / \mathrm{kg}$ was selected using the same $f_{o c}$ as used for the CT estimate and a $\mathrm{K}_{\mathrm{oc}}$ value of $30(\mu \mathrm{g} / \mathrm{g}-\mathrm{oc}) /(\mu \mathrm{g} / \mathrm{mL})$ (experimental value of Walton et al. 1992). Only limited data are available for DCM. The selected range of $\mathrm{K}_{d}$ values is 0.013 to $0.24 \mathrm{~L} / \mathrm{kg}$ based on the available $\mathrm{K}_{\mathrm{oc}}$ value $(47(\mu \mathrm{g} / \mathrm{g}-\mathrm{oc}) /(\mu \mathrm{g} / \mathrm{mL}))$ and the minimum and maximum $\mathrm{f}_{\mathrm{oc}}$ values used in the CT calculations. The most probable $\mathrm{K}_{\mathrm{d}}$ value of $0.13 \mathrm{~L} / \mathrm{kg}$ is a simple average of the minimum and maximum values.

For comparison, the values for $\mathrm{K}_{\mathrm{d}}$ evaluated in the previous Hanford CT modeling report by Chiaramonte et al. (1996) were 0 and $0.114(\mathrm{~L} / \mathrm{kg})$ for CT and 0 and $0.016(\mathrm{~L} / \mathrm{kg})$ for CF. Clearly, these are somewhat lower that those predicted from the analysis described in this report.

Table 14: Maximum estimates for the solid/liquid equilibrium partition coefficient based on $\mathrm{K}_{\mathrm{oc}}$ data reported in Table 12 and the maximum $\mathrm{f}_{\mathrm{oc}}$ in Table 14

\begin{tabular}{|l|c|c|}
\hline \multicolumn{1}{|c|}{ Compound } & \multicolumn{2}{c|}{$\mathbf{K}_{\mathrm{d}}(\mu \mathrm{g} / \mathrm{g}$-soil $) /(\mu \mathrm{g} / \mathrm{mL})$} \\
CT & 0.57 & 0.83 \\
\hline CF & 0.16 & 0.20 \\
\hline DCM & 0.12 & 0.13 \\
\hline CM & 0.18 & 0.14 \\
\hline (a) ND = no data was reported for this compound. \\
\hline
\end{tabular}


Table 15: Maximum estimates for the solid/liquid equilibrium partition coefficient based on $\mathrm{K}_{\mathrm{oc}}$ data reported in Table 13 and the maximum $\mathrm{f}_{\text {oc }}$ in Table 14

\begin{tabular}{|c|c|c|c|c|c|}
\hline \multirow[b]{2}{*}{ Reference } & \multicolumn{4}{|c|}{$K_{d}$ (ug/g-soil)/(ug/mL) } & \multirow[b]{2}{*}{ Comment } \\
\hline & CT & CF & DCM & CM & \\
\hline Jeng et al. 1992 & 0.63 & 0.40 & 0.24 & $\mathrm{ND}^{\mathrm{a}}$ & $\begin{array}{l}\text { Data is a compilation of } \\
\text { information measured by } \\
\text { others. }\end{array}$ \\
\hline Walton et al. 1992 & $\begin{array}{l}0.74 \pm 0.17 \\
\quad(2.1)\end{array}$ & $0.19 \pm 0.04$ & $\mathrm{ND}^{\mathrm{a}}$ & $\mathrm{ND}^{\mathrm{a}}$ & $\begin{array}{l}\text { Silty loam with an } \\
\text { organic carbon content } \\
\text { of } 1.5 \%\end{array}$ \\
\hline Walton et al. 1992 & $\begin{array}{l}0.25 \pm 0.08 \\
\quad(0.34)\end{array}$ & $0.15 \pm 0.01$ & $\mathrm{ND}^{\mathrm{a}}$ & $\mathrm{ND}^{\mathrm{a}}$ & $\begin{array}{l}\text { Sandy loam with an } \\
\text { organic carbon content } \\
\text { of } 0.7 \%\end{array}$ \\
\hline Duffy et al. 1997 & $\begin{array}{l}0.28 \pm 0.01 \\
\quad(0.83)\end{array}$ & $\mathrm{ND}^{\mathrm{a}}$ & $\mathrm{ND}^{\mathrm{a}}$ & $\mathrm{ND}^{\mathrm{a}}$ & $\begin{array}{l}\text { Soil with an organic } \\
\text { carbon content of } 1.5 \%\end{array}$ \\
\hline Duffy et al. 1997 & $\begin{array}{l}0.40 \pm 0.01 \\
\quad(0.41)\end{array}$ & $\mathrm{ND}^{\mathrm{a}}$ & $\mathrm{ND}^{\mathrm{a}}$ & $\mathrm{ND}^{\mathrm{a}}$ & $\begin{array}{l}\text { Soil with an organic } \\
\text { carbon content of } 0.53 \%\end{array}$ \\
\hline Duffy et al. 1997 & $\begin{array}{c}1.4 \pm 0.01 \\
(0.38)\end{array}$ & $\mathrm{ND}^{\mathrm{a}}$ & $\mathrm{ND}^{\mathrm{a}}$ & $\mathrm{ND}^{\mathrm{a}}$ & $\begin{array}{l}\text { Soil with an organic } \\
\text { carbon content of } 0.14 \%\end{array}$ \\
\hline Peng and Dural 1998 & 0.63 & 0.79 & $\mathrm{ND}^{\mathrm{a}}$ & $\mathrm{ND}^{\mathrm{a}}$ & $\begin{array}{l}\text { Measured with Missouri } \\
\text { Soil }\end{array}$ \\
\hline Peng and Dural 1998 & 0.77 & 1.0 & $\mathrm{ND}^{\mathrm{a}}$ & $\mathrm{ND}^{\mathrm{a}}$ & $\begin{array}{l}\text { Measured with } \\
\text { California Soil }\end{array}$ \\
\hline Peng and Dural 1998 & 0.62 & 0.98 & $\mathrm{ND}^{\mathrm{a}}$ & $\mathrm{ND}^{\mathrm{a}}$ & $\begin{array}{l}\text { Measured with Florida } \\
\text { Soil }\end{array}$ \\
\hline Kile et al. 1999 & $0.30 \pm 0.04^{b}$ & $\mathrm{ND}^{\mathrm{a}}$ & $\mathrm{ND}^{\mathrm{a}}$ & $\mathrm{ND}^{\mathrm{a}}$ & $\begin{array}{l}\text { Average of } 17 \text { near } \\
\text { surface soils. }\end{array}$ \\
\hline Kile et al. 1999 & $0.55 \pm 0.07^{b}$ & $\mathrm{ND}^{\mathrm{a}}$ & $\mathrm{ND}^{\mathrm{a}}$ & $\mathrm{ND}^{\mathrm{a}}$ & $\begin{array}{l}\text { Average of } 9 \text { river } \\
\text { bottom sediments. }\end{array}$ \\
\hline Kile et al. 1995 & $0.31 \pm 0.04^{b}$ & $\mathrm{ND}^{\mathrm{a}}$ & $\mathrm{ND}^{\mathrm{a}}$ & $\mathrm{ND}^{\mathrm{a}}$ & $\begin{array}{l}\text { Average of } 32 \text { near } \\
\text { surface soils with } \\
\text { organic carbon ranging } \\
\text { from } 0.16 \% \text { to } 6.1 \% \text {. }\end{array}$ \\
\hline Kile et al. 1995 & $0.53 \pm 0.06^{b}$ & $\mathrm{ND}^{\mathrm{a}}$ & $\mathrm{ND}^{\mathrm{a}}$ & $\mathrm{ND}^{\mathrm{a}}$ & $\begin{array}{l}\text { Average of } 36 \text { bed } \\
\text { sediments with organic } \\
\text { carbon ranging from } \\
0.11 \% \text { to } 4.7 \% \text {. }\end{array}$ \\
\hline
\end{tabular}


Table 16: Minimum estimates for the solid/liquid equilibrium partition coefficient based on $\mathrm{K}_{\mathrm{oc}}$ data reported in Table 12 and an $f_{o c}$ of 0.00027

\begin{tabular}{|l|c|c|}
\multicolumn{1}{c}{ Compound } & \multicolumn{2}{c|}{$\mathbf{K}_{\mathrm{d}}$ (ug/g-soil)/(ug/mL) } \\
\hline CT & $2.99 \times 10^{-2}$ & $4.35 \times 10^{-2}$ \\
\hline CF & $8.39 \times 10^{-3}$ & $1.05 \times 10^{-2}$ \\
\hline DCM & $6.29 \times 10^{-3}$ & $6.82 \times 10^{-3}$ \\
\hline CM & $9.44 \times 10^{-3}$ & $7.34 \times 10^{-3}$ \\
\hline (a) ND $=$.
\end{tabular}

(a) $\mathrm{ND}=$ no data was reported for this compound

Table 17: Minimum estimates for the solid/liquid equilibrium partition coefficient based on $\mathrm{K}_{\mathrm{oc}}$ data reported in Table 13 and an $f_{\text {oc }}$ of 0.00027

\begin{tabular}{|c|c|c|c|c|c|}
\hline \multirow[b]{2}{*}{ Reference } & \multicolumn{4}{|c|}{$\mathbf{K}_{\mathrm{d}}$ (ug/g-soil)/(ug/mL) } & \multirow[b]{2}{*}{ Comment } \\
\hline & $\mathrm{CT}^{\mathrm{b}}$ & $\mathrm{CF}^{\mathrm{b}}$ & DCM & $\mathbf{C M}$ & \\
\hline Jeng et al. 1992 & 0.033 & $0.021 \pm 0.002$ & 0.013 & $\mathrm{ND}^{\mathrm{a}}$ & $\begin{array}{l}\text { Data is a compilation of } \\
\text { information measured by } \\
\text { others. }\end{array}$ \\
\hline Walton et al. 1992 & $0.039 \pm 0.009$ & $0.010 \pm 0.001$ & $\mathrm{ND}^{\mathrm{a}}$ & $\mathrm{ND}^{\mathrm{a}}$ & $\begin{array}{l}\text { Silty loam with an } \\
\text { organic carbon content } \\
\text { of } 1.5 \%\end{array}$ \\
\hline Walton et al. 1992 & $0.013 \pm 0.004$ & 0.0079 & $\mathrm{ND}^{\mathrm{a}}$ & $\mathrm{ND}^{\mathrm{a}}$ & $\begin{array}{l}\text { Sandy loam with an } \\
\text { organic carbon content } \\
\text { of } 0.7 \%\end{array}$ \\
\hline Duffy et al. 1997 & $0.015 \pm 0.003$ & $\mathrm{ND}^{\mathrm{a}}$ & $\mathrm{ND}^{\mathrm{a}}$ & $\mathrm{ND}^{\mathrm{a}}$ & $\begin{array}{l}\text { Soil with an organic } \\
\text { carbon content of } 1.5 \%\end{array}$ \\
\hline Duffy et al. 1997 & $0.021 \pm 0.003$ & $\mathrm{ND}^{\mathrm{a}}$ & $\mathrm{ND}^{\mathrm{a}}$ & $\mathrm{ND}^{\mathrm{a}}$ & $\begin{array}{l}\text { Soil with an organic } \\
\text { carbon content of } 0.53 \%\end{array}$ \\
\hline Duffy et al. 1997 & $0.073 \pm 0.005$ & $\mathrm{ND}^{\mathrm{a}}$ & $\mathrm{ND}^{\mathrm{a}}$ & $\mathrm{ND}^{\mathrm{a}}$ & $\begin{array}{l}\text { Soil with an organic } \\
\text { carbon content of } 0.14 \%\end{array}$ \\
\hline Peng and Dural 1998 & 0.033 & 0.041 & $\mathrm{ND}^{\mathrm{a}}$ & $\mathrm{ND}^{\mathrm{a}}$ & $\begin{array}{l}\text { Measured with Missouri } \\
\text { Soil }\end{array}$ \\
\hline Peng and Dural 1998 & 0.040 & 0.052 & $\mathrm{ND}^{\mathrm{a}}$ & $\mathrm{ND}^{\mathrm{a}}$ & $\begin{array}{l}\text { Measured with } \\
\text { California Soil }\end{array}$ \\
\hline Peng and Dural 1998 & 0.033 & 0.051 & $\mathrm{ND}^{\mathrm{a}}$ & $\mathrm{ND}^{\mathrm{a}}$ & $\begin{array}{l}\text { Measured with Florida } \\
\text { Soil }\end{array}$ \\
\hline Kile et al. 1999 & $0.016 \pm 0.002$ & $\mathrm{ND}^{\mathrm{a}}$ & $\mathrm{ND}^{\mathrm{a}}$ & $\mathrm{ND}^{\mathrm{a}}$ & $\begin{array}{l}\text { Average of } 17 \text { near } \\
\text { surface soils. }\end{array}$ \\
\hline Kile et al. 1999 & $0.029 \pm 0.004$ & $\mathrm{ND}^{\mathrm{a}}$ & $\mathrm{ND}^{\mathrm{a}}$ & $\mathrm{ND}^{\mathrm{a}}$ & $\begin{array}{l}\text { Average of } 9 \text { river } \\
\text { bottom sediments. }\end{array}$ \\
\hline Kile et al. 1995 & $0.016 \pm 0.002$ & $\mathrm{ND}^{\mathrm{a}}$ & $\mathrm{ND}^{\mathrm{a}}$ & $\mathrm{ND}^{\mathrm{a}}$ & $\begin{array}{l}\text { Average of } 32 \text { near } \\
\text { surface soils with } \\
\text { organic carbon ranging } \\
\text { from } 0.16 \% \text { to } 6.1 \% \text {. }\end{array}$ \\
\hline Kile et al. 1995 & $0.028 \pm 0.003$ & $\mathrm{ND}^{\mathrm{a}}$ & $\mathrm{ND}^{\mathrm{a}}$ & $\mathrm{ND}^{\mathrm{a}}$ & $\begin{array}{l}\text { Average of } 36 \text { bed } \\
\text { sediments with organic } \\
\text { carbon ranging from } \\
0.11 \% \text { to } 4.7 \% \text {. }\end{array}$ \\
\hline
\end{tabular}




\section{Summary}

Natural processes that are likely to affect chloromethane contamination in the groundwater at Hanford include abiotic hydrolysis of CT and CF; biotic and abiotic transformation of DCM, and sorption of these compounds to the soil matrix. The reaction processes are summarized in Figure 3.

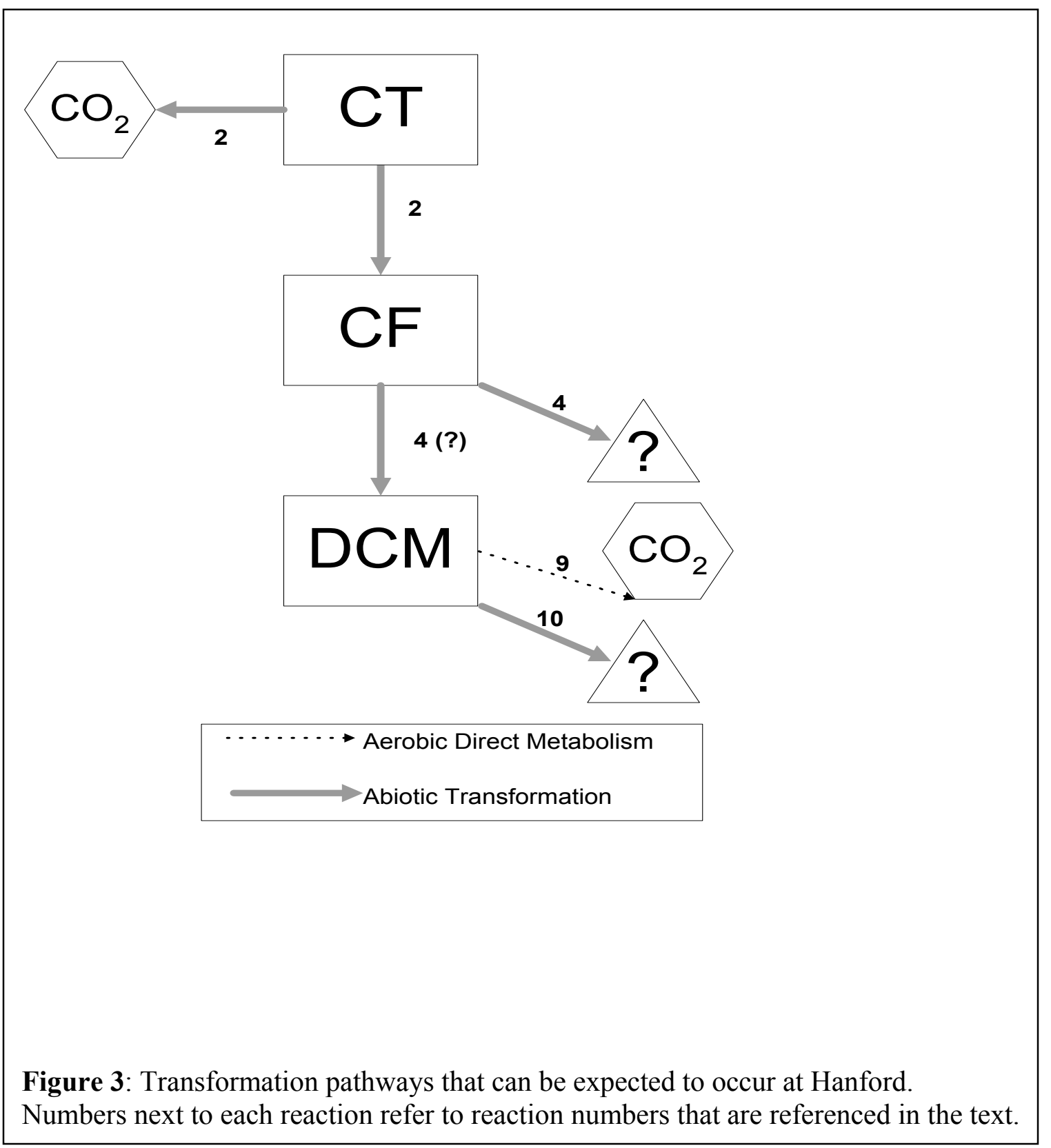


Assuming first order reactions, the following equations describe the potential effects of abiotic and biotic transformation on $\mathrm{CT}, \mathrm{CF}$, and DCM in the Hanford groundwater.

$$
\begin{aligned}
& \left.\frac{\partial[\boldsymbol{C T}]}{\partial \boldsymbol{t}}\right|_{\text {reaction }}=\boldsymbol{k}_{\boldsymbol{a}}^{\boldsymbol{C T}} \cdot[\boldsymbol{C T}] \\
& \left.\frac{\partial[\boldsymbol{C F}]}{\partial \boldsymbol{t}}\right|_{\text {reaction }}=\boldsymbol{k}_{\boldsymbol{a}}^{\boldsymbol{C T}} \cdot[\boldsymbol{C T}] \cdot \boldsymbol{Y}_{(\boldsymbol{C F} / \boldsymbol{C T})}-\boldsymbol{k}_{\boldsymbol{a}}^{\boldsymbol{C F}} \cdot[\boldsymbol{C F}] \\
& \left.\left.\frac{\partial[D C M]}{\partial t}\right|_{\text {reaction }}=k_{a}^{C F} \cdot[C F] \cdot Y_{(D C M} / C F\right)-\left(k_{a}^{D C M}+k_{b}^{D C M}\right) \cdot[D C M]
\end{aligned}
$$

where $\mathrm{k}_{\mathrm{a}}{ }^{\mathrm{i}}$ is the first order abiotic transformation rate for the $\mathrm{i}^{\text {th }}$ compound, $\mathrm{k}_{\mathrm{b}}{ }^{\mathrm{i}}$ is the first order biotic transformation rate for the $\mathrm{i}^{\text {th }}$ compound, $\mathrm{Y}_{(\mathrm{CF} / \mathrm{CT})}$ is the yield of $\mathrm{CF}$ from abiotic transformation of CT, and $\mathrm{Y}_{(\mathrm{DCM} / \mathrm{CF})}$ is the yield of DCM from abiotic transformation of $\mathrm{CF}$.

Ranges for each parameter listed in Equations 6 through 8 are given in Table 18. These values are based on the results reviewed in the previous sections. Table 18 also provides the expected range of values for the soil/water equilibrium partition coefficient for $\mathrm{CT}, \mathrm{CF}$, and DCM. As described in the preceding sections, $\mathrm{CM}$ is ignored since the products from both biotic and abiotic transformation of DCM are predominately nonchlorinated (Braus-Stromeyer et al. 1993; Freedman and Gossett 1991; Yanghao 1990). In addition, CM is readily degraded as a growth substrate by many aerobic organisms, and, even if formed, it will be rapidly consumed in the Hanford aquifer.

Table 18: Range of parameters for modeling fate of chloromethanes in Hanford Aquifer

\begin{tabular}{|l|c|c|c|l|}
\hline Parameter & $\begin{array}{c}\text { Maximum } \\
\text { Value }\end{array}$ & $\begin{array}{c}\text { Minimum } \\
\text { Value }\end{array}$ & $\begin{array}{c}\text { Most } \\
\text { Probable } \\
\text { Value }\end{array}$ & Comment \\
\hline $\mathrm{k}_{\mathrm{a}}{ }^{\mathrm{CT}}\left(\mathrm{day}^{-1}\right)$ & $4.6 \times 10^{-5}$ & $2.7 \times 10^{-7}$ & $4.6 \times 10^{-5}$ & $\begin{array}{l}\text { Work by Jeffers et al. (1989) } \\
\text { appears to be more reliable. }\end{array}$ \\
\hline $\mathrm{k}_{\mathrm{a}}{ }^{\mathrm{CF}}\left(\mathrm{day}^{-1}\right)$ & $1.1 \times 10^{-6}$ & $5.9 \times 10^{-7}$ & $1.1 \times 10^{-6}$ & $\begin{array}{l}\text { Work by Jeffers et al. (1989) } \\
\text { appears to be more reliable. }\end{array}$ \\
\hline $\mathrm{k}_{\mathrm{a}}{ }^{\mathrm{DCM}}\left(\mathrm{day}^{-1}\right)$ & $2.8 \times 10^{-6}$ & $2.8 \times 10^{-6}$ & $2.8 \times 10^{-6}$ & $\begin{array}{l}\text { Only one measured value } \\
\text { available for this parameter. }\end{array}$ \\
\hline $\mathrm{k}_{\mathrm{b}}{ }^{\mathrm{DCM}}\left(\mathrm{day}^{-1}\right)$ & $3.0 \times 10^{-1}$ & $9.9 \times 10^{-3}$ & $1.7 \times 10^{-1}$ & $\begin{array}{l}\text { Most probable value is based field } \\
\text { test by Mehran and Wolf (1999) } \\
\text { in an aerobic aquifer. }\end{array}$ \\
\hline $\mathrm{Y}_{(\mathrm{CF} / \mathrm{CT})}(\mathrm{mole} / \mathrm{mole})$ & 0.2 & 0.1 & 0.1 & $\begin{array}{l}\text { Most studies showed CF } \\
\text { production to be closer to the } \\
\text { lower value. }\end{array}$ \\
\hline $\mathrm{Y}_{\text {(DCM/CF) }}(\mathrm{mole} / \mathrm{mole})$ & 0.2 & 0.1 & 0.1 & $\begin{array}{l}\text { No data available for this, so } \\
\text { based on CT to CF reaction. }\end{array}$ \\
\hline $\mathrm{K}_{\mathrm{d}}^{\mathrm{CT}}(\mathrm{L} / \mathrm{kg})$ & 0.83 & 0.016 & 0.12 & See text. \\
\hline $\mathrm{K}_{\mathrm{d}}{ }^{\mathrm{CF}}(\mathrm{L} / \mathrm{kg})$ & 0.39 & 0.008 & 0.06 & See text. \\
\hline $\mathrm{K}_{\mathrm{d}}{ }^{\text {DCM }}(\mathrm{L} / \mathrm{kg})$ & 0.24 & 0.013 & 0.10 & See text. \\
\hline
\end{tabular}




\section{Recommendations}

Based on a review of the available site data and published literature, it is recommended that sitespecific values for both the soil/water equilibrium partition coefficient and the abiotic hydrolysis half-life be determined for CT, CF, and DCM. Neither parameter has been measured for the Hanford system.

\section{References}

Andrews EJ and PJ Novak. 1999. Carbon Tetrachloride Product Shift in a Methanogenic, IronContaining System. Engineered Approaches for In Situ Bioremediation of Chlorinated Solvent Contamination. Fifth International In Situ and Onsite Bioremediation Symposium, pp. 35-40.

Bagley DM and JM Gossett. 1995. Chloroform Degradation in Methanogenic Methanol Enrichment Cultures and by Methanosarcina Barkeri 227. Applied and Environmental Microbiology 61(9):3195-3201.

Biehle AA, JM Odom, WB Hall, BE Caldwell, DE Ellis, and GE Gregory. 1999. Monitored Natural Attenuation of a Methylene Chloride Plume. Natural Attenuation of Chlorinated Solvents, Petroleum Hydrocarbons, and Other Organic Compounds. Proceedings of the Fifth International In Situ and Onsite Bioremediation Symposium, Battelle Press, pp. 35-40.

Bishop DJ, JP Knezovich, and DW Rice Jr. 1989. Sorption Studies of VOCs Related to Soil/Groundwater Contamination at LLNL. UCID-21651:1-12, Environmental Protection Agency Environmental Restoration Series, Washington, D.C. .

Bjerg PL, K Rugge, J Cortsen, PH Nielsen, and TH Christensen. 1999. Degradation of Aromatic and Chlorinated Aliphatic Hydrocarbons in the Anaerobic Part of the Grindsted Landfill Leachate Plume: In Situ Microcosm and Laboratory Batch Experiments. Ground Water. 37(1):113-121.

Bouwer EJ and JP Wright. 1988. Transformations of Trace Halogenated Aliphatics in Anoxic Biofilm Columns. Journal of Contaminant Hydrology 2:155-169.

Bouwer EJ and PL McCarty. 1985. Utilization Rates of Trace Halogenated Organic Compounds in Acetate-Grown Biofilms. Biotechnology and Bioengineering 82:1564-1571.

Bouwer EJ and PL McCarty. 1983. Transformations of 1- and 2-Carbon Halogenated Aliphatic Organic Compounds under Methanogenic Conditions. Applied and Environmental Microbiology 45(4):1286-1294.

Bouwer EJ and PL McCarty. 1983. Transformations of Halogenated Organic Compounds under Denitrification Conditions. Applied and Environmental Microbiology 45(4):1295-1299.

Bouwer EJ, BE Rittmann, and PL McCarty. 1981. Anaerobic Degradation of Halogenated 1and 2-Carbon Organic Compounds. Environmental Science \& Technology. 15(5):596-599. 
Braus-Stromeyer SA, R Hermann, AM Cook, and T Leisinger. 1993. Deichloromethane as the Sole Carbon Source for an Acetogenic Mixed Culture and Isolation of a Fermentative, Dichloromethane-Degrading Bacterium. Applied and Environmental Microbiology 59(11)3790-3797.

Chappelle FH and PM Bradley. 1998. Selecting remediation goals by assessing the natural attenuation capacity of groundwater systems. Bioremediation Journal, 2(3\&4):227-238.

Chiaramonte GR, CW Denslow, AJ Knepp, RD Landon, and S Panday. 1996. Hanford site-wide groundwater remediation strategy - groundwater contaminant predictions. BHI-00469, Bechtel Hanford, Inc., Richland, Washington.

Christensen TH, P Kjeldsen, HJ Albrechtsen, G Heron, PH Nielsen, PL Bjerg, and PE Homl. 1994. Attenuation of Landfill Leachate Pollutants in Aquifers. Critical Reviews in Environmental Science and Technology 24(2):119-202.

Clement TP, CD Johnson, Y Sun, GM Klecka, and C Bartlett. 1999. Simulation of natural attenuation at a chlorinated solvent contaminated site. Submitted to Journal of Contaminant Transport Hydrology.

Cobb GD and EJ Bouwer. 1991. Effects of Electron Acceptors on Halogenated Organic Compound Biotransformations in a Biofilm Column. Environ. Sci. Technol. 25:1068-1074.

Connell Hancock TL, AM Costello, ME Lidstrom, and RS Oremland. 1998. Strain IMB-1, a Novel Bacterium for the Removal of Methyl Bromide in Fumigated Agricultural Soils. Applied and Environmental Microbiology 64(8)2899-2905.

Cox EE, M McMaster, and DW Major. 1998. Natural Attenuation of 1,2-Dichloroethane and Chloroform in Groundwater at a Superfund Site. Natural Attenuation: Chlorinated and Recalcitrant Compounds. First International Conference on Remediation of Chlorinated and Recalcitrant Compounds, pp. 309-314.

Cox E, E Edwards, L Lehmicke, and D Major. 1995. Intrinsic biodegradation of trichloroethylene and trichloroethane in a sequential anaerobic-aerobic aquifer, RE Hinchee, JT Wilson, and DC Downey, eds. Intrinsic Bioremediation, pp. 223-231. Battelle Press, Columbus, Ohio.

Criddle CS, JT DeWitt, and PL McCarty. 1990. Reductive Dehalogenation of Carbon Tetrachloride by Escherichia coli K-12. Applied and Environmental Microbiology 56(11):3247-3254.

Criddle CS, JT DeWitt, D Grbic-Galic, and PL McCarty. 1990. Transformation of Carbon Tetrachloride by Pseudomonas sp. Strain KC under Denitrification Conditions. Applied and Environmental Microbiology 56(11): 3240-3246.

Davis JW and SS Madsen. 1991. The Biodegradation of Methylene Chloride In Soils. Environmental Toxicology and Chemistry 10:463-474. 
Deeley GM, M Reinhard, and SM Stearns. 1991. Transformation and sorption of 1,2-dibromo3-chloropropane in subsurface samples collected at Fresno, California. J. Environ. Qual. 20:547-556.

Devlin JF and D Muller. 1999. Field and Laboratory Studies of Carbon Tetrachloride Transformation in a Sandy Aquifer under Sulfate Reducing Conditions. Environ. Sci. Technol. 33:1021-1027.

Dilling WL, NB Tefertiller, and GJ Kallos. 1975. Evaporation rates and reactivities of methylene chloride, chloroform, 1,1,1-trichloroethane, trichloroethylene, tetrachloroethylene, and other chlorinated compounds in dilute aqueous solutions. Environ. Sci. Technol. 9:833-838.

Doronia NV and YA Trotsenko. 1997. Isolation and characterization of aerobic degraders of methyl chloride. Microbiology, 66:57-64.

Doronia NV, AP Sokolov, and YA Trotsenko. 1996. Isolation and initial characterization of aerobic chloromethane-utilizing bacteria. FEMS Microbiol. Lett. 142:179-183.

Duffy CC, DL McCallister, and RR Renken. 1997. Carbon tetrachloride retention by modern and buried soil A horizons. J. Environ. Qual. 26:1123-1127.

Egli C, S Stromeyer, AM Cook, and T Leisinger. 1990. Transformation of tetra- and trichloromethane to $\mathrm{CO}_{2}$ by anaerobic bacteria is a non-enzymatic process. FEMS Microbiology Letters 68:207-212.

Egli C, R Scholtz, AM Cook, and T Leisinger. 1987. Anaerobic Dechlorination of tetrachloromethane and 1,2-dichloroethane to degradable products by pure cultures of Desulfobacterium sp. and Methanobacterium sp. FEMS Microbiology Letter 43:257-261.

Egli C, T Tschan, R Scholtz, AM Cook, and T Leisinger. 1988. Transformation of tetrachloromethane to dichloromethane and carbon dioxide by Acetobacterium woodii. Appl. Environ. Microbiol. 54:2819-2824.

Ely RL, KJ Williamson, MR Hyman, and DJ Arp. 1997. Cometabolism of Chlorinated Solvents by Nitrifying Bacteria: Kinetics, Substrate Interactions, Toxicity Effects, and Bacterial Response. Biotechnology and Bioengineering 54(6):520-534.

EPA. 1995. Background Document for EPACMTP: Finite methodology for degrading chemicals and transformation products, U.S. Environmental Protection Agency, Office of Solid Waste, Washington, D.C.

Fathepure BZ, GA Youngers, DL Richter, and CE Downs. 1995. In Situ Bioremediation of Chlorinated Hydrocarbons under Field Aerobic-Anaerobic Environments. Bioremediation of Chlorinated Solvents, pp. 169-186. 
Fells I and EA Moelwyn-Hughes. 1959. The kinetics of hydrolysis of the chlorinated methanes. J. Chem. Soc. 1959:398-409.

Ford HL. 1996. Impact of a low velocity field on soil vapor extraction of carbon tetrachloride. Master of Science Thesis, Washington State University, Pullman.

Freedman DL and JM Gossett. 1991. Biodegradation of Dichloromethane and Its Utilization as a Growth Substrate under Methanogenic Conditions. Applied and Environmental Microbiology 57(10):2847-2857.

Freedman DL, M Lasecki, S Hashsham, and R Scholze. 1995. Accelerated Biotransformation of Carbon Tetrachloride and Chloroform by Sulfate-Reducing Enrichment Cultures. Bioremediation of Chlorinated Solvents, pp. 123-138.

Galli R and PL McCarty. 1989. Biotransformation of 1,1,1-Trichloroethane, Trichloromethane, and Tetrachloromethane by a Clostridium sp. Applied and Environmental Microbiology pp. 837844.

Garbarini DR and LW Lion. 1986. Influence of the nature of soil organics on the sorption of toluene and trichloroethylene. Environ. Sci. Technol. 20:1263-1269.

Grathwohl P. 1990. Influence of organic matter from soils and sediments from various origins on the sorption of some chlorinated aliphatic hydrocarbons: implications of $\mathrm{K}_{\mathrm{oc}}$ correlations. Environ. Sci. Technol. 24:1687-1693.

Gupta M, A Gupta, MT Suidan, and GD Sayles. 1996. Biotransformation Rates of Chloroform under Anaerobic Conditions-II. Sulfate Reduction. Wat. Res. 30(6):1387-1394.

Gupta M, D Sharma, MT Suidan, and GD Sayles. 1996. Biotransformation Rates of Chloroform under Anaerobic Conditions-I. Methanogenesis. Wat. Res. 30(6):1377-1385.

Haag WR and T Mill. 1988. Some reactions of naturally occurring nucleophiles with haolalkanes in water. Environ. Toxicol. Chem. 7:917-924.

Hardy L, E Moeri, and MC Salvador. 1999. Rapid Intrinsic Degradation of Chlorinated Solvents at a Manufacturing Site in Brazil. Natural Attenuation of Chlorinated Solvents, Petroleum Hydrocarbons, and Other Organic Compounds. Fifth International In Situ and Onsite Bioremediation Symposium, pp. 19-28.

Harkov R, SJ Gianti Jr, JW Bozzelli, and JE LaRegina. 1985. Monitoring Volatile Organic Compounds at Hazardous and Sanitary Landfills in New Jersey. J. Environ. Sci. Health. A20(5):491-501.

Hartmans S, A Schmuckle, AM Cook, and T Leisinger. 1986. Methyl chloride: natural occurring toxicant and C-1 growth substrate. J. Gen. Microbiol. 132:1139-1142. 
Hooker BS, RS Skeen, and JN Petersen. 1994. Biological destruction of $\mathrm{CCl}_{4}$ part II: kinetic modeling. Biotechnol. Bioeng. 44:211-218.

Hooker BS, RS Skeen, MJ Truex, CD Johnson, BM Peyton, and DB Anderson. 1998. In Situ Bioremediation of Carbon Tetrachloride: Field Test Results. Bioremediation Journal. 1(3):181182.

Hughes JB and GF Parkin. 1996. Concentration Effects on Chlorinated Aliphatic Transformation Kinetics. Journal of Environmental Engineering, pp. 92-98.

Jeffers PM, LM Ward, LM Woytowitch, and NL Wolfe. 1989. Homogeneous Hydrolysis Rate Constants for Selected Chlorinated Methanes, Ethanes, Ethenes, and Propanes. Environ. Sci. Technol. 23(8):965-969.

Jeffers PM, P Coty, S Luczak, and NL Wolfe. 1994. Halocarbon hydrolysis rates - a search for ionic strength and heterogeneous effects. J. Environ. Sci. Health. A29(4):821-831.

Jeffers PM, C Brenner, and NL Wolfe. 1996. Hydrolysis of carbon tetrachloride. Environ. Toxicol. Chem. 15(7):1064-1065.

Jeng CY, DH Chen, and CL Yaws. 1992. Data Compilation for Soil Sorption Coefficient. Pollution Engineering, pp. 54-60.

Kile DE, RL Wershaw, and CT Chiou. 1999. Correlations of soil and sediment organic matter polarity to aqueous sorption of nonionic compounds. Environ. Sci. Technol. 33:2053-2056.

Kile DE, CT Chiou, H Zhou, H Li, and O Xu. 1995. Partition of nonpolar organic pollutants from water to soil and sediment organic matters. Environ. Sci. Technol. 29:1401-1406.

Klecka GM. 1982. Fate and Effects of Methylene Chloride in Activated Sludge. Applied and Environmental Microbiology. 44(3)701-707.

Kriegman-King MR and M Reinhard. 1994. Transformation of Carbon Tetrachloride by Pyrite in Aqueous Solution. Environ. Sci. Technol. 28(4)692-700.

Kriegman-King MR and M Reinhard. 1992. Transformation of Carbon Tetrachloride in the Presence of Sulfide, Biotite, and Vermiculite. Environ. Sci. Technol. 26:2198-2206.

Kriegman-King MR and M Reinhard. 1991. Reduction of Hexachloroethane and Carbon Tetrachloride at Surfaces of Biotite, Vermiculite, Pyrite, and Marcasite. Organic Substances and Sediments in Water, Volume 2: Processes and Analytical. 16:349-364.

Leahy MC and GJ Skladany. 1998. Assessment of Intrinsic Biodegradation of Multiple Chlorinated Hydrocarbons. Natural Attenuation; Chlorinated and Recalcitrant Compounds, the First International Conference on Remediation of Chlorinated and Recalcitrant Compounds, pp. $165-170$. 
Lee MD, PF Mazierski, RJ Buchanan Jr, DE Ellis, and LS Sehayek. 1995. Intrinsic In Situ Anaerobic Biodegradation of Chlorinated Solvents at an Industrial Landfill. Intrinsic Bioremediation, pp. 205-222.

Leisinger T and SA Braus-Stromeyer. 1995. Bacterial growth on chlorinated methanes. Environ. Health Perspect. 103:33-36.

Liang LN and D Grbic-Galic. 1993. Biotransformation of Chlorinated Aliphatic Solvents in the Presence of Aromatic Compounds under Methanogenic Conditions. Environmental Toxicology and Chemistry. 12:1377-1393.

Lyman WJ, WF Reehl, and DH Rosenblatt, eds. 1990. Octanol/water partition coefficient. Handbook of Chemical Property Estimation Methods. American Chemical Society, Washington, D.C.

Mabey W and T Mill. 1978. Critical Review of Hydrolysis of Organic Compounds in Water under Environmental Conditions. J. Phys. Chem. Ref. Data. 7(2):383-415.

Magli A, FA Rainey, and T Leisinger. 1995. Acetogenesis from Dichloromethane by a TwoComponent Mixed Culture Comprising a Novel Bacterium. Applied and Environmental Microbiology, pp. 2943-2949.

Malachowy KJ, TJ Phelps, AB Teboli, DE Minnikin, and DC White. 1994. Aerobic Mineralization of Trichloroethylene, Vinyl Chloride, and Aromatic Compounds by Rhodococcus Species. Applied and Environmental Microbiology. 60(2):542-548.

McClay K, BG Fox, and RJ Steffan. 1996. Chloroform Mineralization by Toluene-Oxidizing Bacteria. Applied and Environmental Microbiology. 62(8):2716-2722.

McQuillan DM, BH Faris, and BH Swanson. 1998. Intrinsic Cometabolism of Carbon Tetrachloride with Gasoline: Regulatory Site-Closure. Natural Attenuation: Chlorinated and Recalcitrant Compounds, the First International Conference on Remediation of Chlorinated and Recalcitrant Compounds, pp. 263-268.

Mechaber RA, BY Su, and E Cox. 1998. Intrinsic Bioremediation of Chlorinated and NonChlorinated VOCS at a RCRA Landfill. Natural Attenuation: Chlorinated and Recalcitrant Compounds, the First International Conference on Remediation of Chlorinated and Recalcitrant Compounds, pp. 275-280.

Mehran M and JL Wolf Jr. 1999. Natural Attenuation of Methylene Chloride in Ground Water. Natural Attenuation of Chlorinated Solvents, Petroleum Hydrocarbons, and Other Organic Compounds. Fifth International In Situ and Onsite Bioremediation Symposium, pp. 13-18.

Messmer M, G Wohlfarth, and G Diekert. 1993. Methyl chloride metabolism of the strictly anaerobic methyl chloride-utilizing homoacetogen strain MC. Arch Microbiol. 160:383-387. 
Mikesell MD and SA Boyd. 1990. Dechlorination of Chloroform by Methanosarcina Strains. Applied and Environmental Microbiology. 566(4):1198-1201.

Mikessel MD and SA Boyd. 1990. Dechlorination of Chloroform by Methanosarcina Strains. Applied and Environmental Microbiology, pp. 1198-1201.

Newcomer DR, LA Doreums, SH Hall, MJ Truex, VR Vermuel, and RE Engleman. 1995. Geology, Hydrology, Chemistry, and Microbiology of the In Situ Bioremediation Demonstration Site. PNL-10422, Pacific Northwest Laboratory, Richland, Washington.

Nielsen PH, PE Holm, and TH Christensen. 1992. A Field Method for Determination of Groundwater and Groundwater-Sediment Associated Potentials for Degradation of Xenobiotic Organic Compounds. Chemosphere. 25(4):449-462.

Peng D and NH Dural. 1998. Multicomponent adsorption of chloroform, carbon tetrachloride, and 1,1,1-trichloroethane on soils. J. Chem. Eng. Data, 43:283-288.

Rasche ME, RE Hicks, MR Hyman, and DJ Arp. 1990. Oxidation of monohalogenated ethanes and n-chlorinated alkanes by whole cells of Nitrosomonas europaea. J. Bacteriol. 172:53685373 .

Rugge K, PL Bjerg, H Mosbaek, and TH Christensen. Natural Attenuation of Xenobiotic Compounds: Anaerobic Field Injection Experiment. Intrinsic Bioremediation, pp. 127-133.

Rugge K, PL Bjerg, JK Pedersen, H Mosbaek, and TH Christensen. 1999. An anaerobic field injection experiment in a landfill leachate plume, Grindsted, Denmark. 1. Experimental setup, tracer movement, and fate of aromatic and chlorinated compounds. Water Resources Research. 35(4):1231-1246.

Semprini L, GD Hopkins, DB Jansen, M Lang, PV Roberts, and PL McCarty. 1991. In-Situ biotransformation of carbon tetrachloride under anoxic conditions. EPA Report No. EPA/290/060, U.S. EPA, Ada, Oklahoma.

Semprini L, GD Hopkins, PL McCarty, and PV Roberts. 1992. In-Situ Transformation of Carbon Tetrachloride and Other Halogenated Compounds Resulting from Biostimulation under Anoxic Conditions. Environ. Sci. Technol. 26(12):2454-2461.

Sherwood JL, JN Petersen, RS Skeen, and NB Valentine. 1996. Effects of Nitrate and Acetate Availability on Chloroform Production During Carbon Tetrachloride Destruction. Biotechnology and Bioengineering. 51:551-557.

Speitzel GE Jr, RC Thompson, and D Weissman. 1993. Biodegradation Kinetics of Methylosinus Trichosporium OB3b at Low Concentrations of Chloroform in the Presence and Absence of Enzyme Competition by Methane. Wat. Res. 27(1):15-24. 
Stephanatos BN, K Walters, A Funk, and A MacGregor. 1991. Pitfalls associated with the assumption of a constant partition coefficient in modeling sorbing solute transport through the subsurface. Proceedings of the Internal Ground Water Symposium, Nashville, pp. 13-20.

Stirling DI, and H Dalton. 1979. The fortuitous oxidation and cometabolism of various carbon compounds by whole cell suspensions of Methylococcus capsulatus (Bath). FEMS Microbiol. Lett. 5:315-318.

Van Beelen P and F Van Keulen. 1990. The Kinetics of the Degradation of Chloroform and Benzene in Anaerobic Sediment from the River Rhine. Hydrobiol. Bull. 24(1):13-21.

Van Eekert MHA, MC Viega, JA Field, AJM Stams, and G Schraa. 1995. Removal of Tetrachloromethane by Granular Methanogenic Sludge. Bioremediation of Chlorinated Solvents, pp. 139-145.

Vannelli T, M Messmer, A Studer, S Vuilleumier, and T Leisinger. 1999. A corrinoiddependent catabolic pathway for growth of a Methylobacterium strain with chloromethane. Proc. Natl. Acad. Sci. 96:4615-4620.

Vannelli T, A Studer, M Kertesz, and T Leisinger. 1998. Chloromethane Metabolism by Methylobacterium sp. Strain CM4. Applied and Environmental Microbiology. 64(5)1933-1936.

Walton BT, MS Hendricks, TA Anderson, WH Griest, R Merriweather, JJ Beauchamp, and CW Francis. 1992. Soil sorption of volatile and semivolatile organic compounds in a mixture. J. Environ. Qual. 21:552-558.

Weathers LJ and GF Parkin. 1995. Metallic Iron-Enhanced Biotransformation of Carbon Tetrachloride and Chloroform under Methanogenic Conditions. Bioremediation of Chlorinated Solvents, pp. 117-123.

WHC. 1990. Liquid Effluent Study Final Project Report. WHC-EP-0367, Westinghouse Hanford Company, Richland, Washington.

Yanghao G. 1990. Microbial kinetics of Pseudomonas SP. Straub DM1 during dichloromethane biodegradation. Chinese Journal of Biotechnology. 6:75-85.

Zhao X, MJ Szafranski, MA Maraqa, and TC Voice. 1999. Sorption of carbon tetrachloride in a low organic content sandy soil. Environ. Tox. and Chem. 18:1755-1762. 
Appendix D

Simulation Parameters and Results 


\begin{tabular}{|c|c|c|c|c|c|c|c|c|c|c|c|c|c|c|c|c|c|c|c|c|c|c|}
\hline & $\begin{array}{c}\text { Velocity } \\
(\mathrm{m} / \mathrm{y})\end{array}$ & $\begin{array}{l}\text { Fixed Source } \\
\text { Concentration } \\
(\mathrm{mg} / \mathrm{L})\end{array}$ & $\begin{array}{c}\text { Time of } \\
\text { Maximum } \\
\text { at } \\
\text { Compliance } \\
\text { Boundary } \\
\text { (y) }\end{array}$ & $\mid \begin{array}{c}\text { Maximum } \\
\text { Concentration } \\
\text { Compliance } \\
\text { Boundary } \\
(\mu \mathrm{g} / \mathrm{L})\end{array}$ & $\begin{array}{c}\text { Maximum } \\
\text { Release Rate } \\
\text { at } \\
\text { Compliance } \\
\text { Boundary } \\
(\mathrm{Kg} / \mathrm{y})\end{array}$ & $\begin{array}{l} \\
\text { Sourc } \\
\text { e Rate } \\
\text { (Kg/y) }\end{array}$ & \begin{tabular}{|c|} 
Source \\
Duration \\
(y)
\end{tabular} & $\begin{array}{c}\text { 1-D } \\
\text { Streamtube } \\
\text { Length } \\
(\mathrm{m})\end{array}$ & \begin{tabular}{|} 
Base \\
Porosity \\
$\left(\mathrm{m}^{3} / \mathrm{m}^{3}\right)$
\end{tabular} & $\begin{array}{c}\text { Bulk } \\
\text { Densty } \\
\left(\mathrm{Kg} / \mathrm{m}^{3}\right)\end{array}$ & \begin{tabular}{|c|} 
Remaining \\
Carbon \\
Tetrachloride \\
Source \\
$(\mathrm{Kg})$
\end{tabular} & $\begin{array}{c}\text { Volume at or } \\
\text { above Fixed } \\
\text { Source } \\
\begin{array}{c}\text { Concentratio } \\
n(m 3)\end{array}\end{array}$ & $\begin{array}{l}\text { Porosity } \\
\left(\mathrm{m}^{3} / \mathrm{m}^{3}\right)\end{array}$ & $\left(\begin{array}{c}\mathrm{Kd} \\
\left(\mathrm{m}^{3} / \mathrm{kg}\right)\end{array}\right.$ & Retardation & $\begin{array}{c}\text { First } \\
\text { Order } \\
\text { Rate } \\
\text { Constant } \\
(1 / y)\end{array}$ & $\begin{array}{l}\text { Longitudinal } \\
\text { Dispersivity } \\
\text { (m) }\end{array}$ & $\begin{array}{c}\text { Required } \\
\text { Source } \\
\text { Cleanup } \\
\text { Percentage } \\
(5 \mu \mathrm{g} / \mathrm{L} \\
\text { Compliance })\end{array}$ & $\begin{array}{c}\text { Required } \\
\text { Source } \\
\text { Cleanup } \\
\text { Percentage } \\
(50 \mu \mathrm{g} / \mathrm{L} \\
\text { Compliance })\end{array}$ & \begin{tabular}{|c} 
Required \\
Source Cleanup \\
Percentage \\
(500 $\mu \mathrm{g} / \mathrm{L}$ \\
Compliance)
\end{tabular} & \begin{tabular}{|c|} 
Abiotic \\
Reaction \\
Half Time \\
(y)
\end{tabular} & $\begin{array}{l}\text { Carbon } \\
\text { Tetrachloride } \\
\text { Travel Time } \\
\text { (y) }\end{array}$ \\
\hline & & & & \begin{tabular}{|l|}
4.428796 \\
\end{tabular} & \begin{tabular}{|l|}
0.3348 \\
\end{tabular} & 226.8 & \begin{tabular}{|l|}
33.07 \\
\end{tabular} & 5125 & & 1950 & & 3662500 & & \#\#\#\#+ & 1.673 & $1.23 \mathrm{E}-02$ & $\begin{array}{r}52.78 \\
\end{array}$ & & & & & \\
\hline 65 & 24.66 & 3000 & 1130 & 14.566434 & 0.5377 & 110.7 & 67.72 & 5125 & 0.1 & 1950 & 7500 & 1250000 & 0.129 & 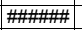 & 2.562 & $6.42 \mathrm{E}-03$ & 52.21 & 65.67 & 0 & & 108 & 532.4 \\
\hline 66 & 46.17 & 3000 & 210 & 126.276218 & 6.8467 & 162.7 & 46.11 & 5125 & 0.1 & 1950 & 7500 & 2225000 & 0.0689 & \#\#\#\#+ & \begin{tabular}{l|l|}
1.405 \\
\end{tabular} & $1.62 \mathrm{E}-02$ & 72.97 & 96.04 & 60.4 & & 42.8 & 156 \\
\hline 67 & 49.79 & 3000 & 1080 & 39.542806 & 1.3303 & 100.9 & 74.31 & 5125 & 0.1 & 1950 & 7500 & 1087500 & 0.0639 & \#\#\#\#+1 & 3.368 & $\begin{array}{c}7.65 \mathrm{E}-03 \\
\end{array}$ & 21.89 & 87.36 & & & 90.6 & 346.7 \\
\hline 68 & 26.34 & 3000 & 1990 & \begin{tabular}{|c|} 
\\
\end{tabular} & 0.0364 & 206.7 & 36.28 & 5125 & 0.1 & 1950 & 7500 & 3187500 & 0.1208 & 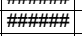 & 3.918 & $8.11 \mathrm{E}-03$ & 50.27 & 0 & & & 85.5 & 762.5 \\
\hline 69 & 41 & 3000 & 460 & 23.962752 & 1.0965 & $\begin{array}{l}237.3 \\
137.1\end{array}$ & 54.64 & 5125 & 0.1 & 1950 & 7500 & 1725000 & 0.0776 & \#\#\#\#+1 & 2.075 & $1.42 \mathrm{E}-02$ & 64.47 & 79.13 & & & 48.9 & 259.5 \\
\hline 70 & 56.81 & 3000 & 1730 & \begin{tabular}{|l|}
0.197101 \\
\end{tabular} & 0.0087 & $\begin{array}{l}132.6 \\
\end{array}$ & 56.56 & 5125 & 0.1 & 1950 & 7500 & 1637500 & 0.056 & 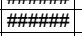 & 7.404 & \begin{tabular}{|c|}
$1.71 \mathrm{E}-02$ \\
\end{tabular} & 82.08 & & & & 40.6 & 667.9 \\
\hline 71 & 25.31 & 3000 & 1200 & 0.074735 & 0.0033 & 133.3 & 56.28 & 5125 & 0.1 & 1950 & 7500 & 1650000 & 0.1257 & \#\#\#\# & 2.961 & $1.68 \mathrm{E}-02$ & 45.79 & & & & 41.2 & 599.6 \\
\hline 72 & 27.21 & 3000 & 2040 & \begin{tabular}{|c|}
0.867229 \\
728022
\end{tabular} & 0.0309 & 107 & 70.08 & 5125 & 0.1 & 1950 & 7500 & 1187500 & 0.1169 & \#\#\#\#年曲 & 3.975 & $8.47 E-03$ & 44.95 & & 0 & & 81.8 & 748.6 \\
\hline 73 & 29.32 & 3000 & 1150 & $\begin{array}{l}7.380022 \\
\end{array}$ & 0.2127 & $\begin{array}{l}86.48 \\
\end{array}$ & 86.73 & 5125 & 0.1 & 1950 & 7500 & 862500 & 0.1085 & \#\#\#\#+ & 2.682 & $9.99 \mathrm{E}-03$ & 22.54 & 32.25 & 0 & & 69.4 & 468.5 \\
\hline 74 & 46.04 & 3000 & 450 & 112.761638 & 5.5031 & 146.4 & 51.23 & 5125 & 0.1 & 1950 & 7500 & 1900000 & 0.0691 & \#\#\#\#+ & 2.054 & $9.13 \mathrm{E}-03$ & 34.26 & 95.57 & & & 75.9 & 228.6 \\
\hline 75 & 33.49 & 3000 & 280 & 375.591504 & 11.1383 & 88.97 & 84.3 & 5125 & 0.1 & 1950 & 7500 & 900000 & 0.095 & \#\#\#\#曲 & 1.282 & $9.81 \mathrm{E}-03$ & 32.53 & \begin{tabular}{ll|}
98.67 \\
\end{tabular} & 86.69 & & 70.6 & 196.1 \\
\hline 76 & 23.85 & 3000 & 1250 & & 0.4394 & $\begin{array}{l}139.3 \\
\end{array}$ & 53.86 & 5125 & 0.1 & 1950 & 7500 & 1762500 & 0.1334 & \#\#\#\#+ & 2.644 & $6.26 \mathrm{E}-03$ & 46.73 & 47.18 & 0 & & 110.8 & 568.2 \\
\hline & 43.94 & 3000 & 670 & 52.15927 & 2.5343 & $\begin{array}{l}145.8 \\
\end{array}$ & 51.45 & 5125 & 0.1 & 1950 & 7500 & 1887500 & & \#\#\#\#+ & 2.493 & $8.38 \mathrm{E}-03$ & 32.24 & 90.41 & & & 82.7 & 290.7 \\
\hline 78 & 22.28 & 3000 & 790 & \begin{tabular}{|l|l|}
57.690186 \\
\end{tabular} & 1.9999 & 104 & 72.12 & 5125 & 0.1 & 1950 & 7500 & 1137500 & & \#\#\#\#+ & 1.899 & $6.00 \mathrm{E}-03$ & 33.34 & \begin{tabular}{|c|}
91.33 \\
\end{tabular} & 13.33 & & 115.5 & 436.9 \\
\hline 79 & 25.57 & 3000 & 1520 & & 0.0027 & \begin{tabular}{l|l|}
91.42 \\
\end{tabular} & 82.04 & 5125 & 0.1 & 1950 & 7500 & 937500 & 0.1244 & \#\#\#\#曲 & 3.031 & $1.56 \mathrm{E}-02$ & 20.24 & & 0 & & $\begin{array}{l}44.6 \\
\end{array}$ & 607.5 \\
\hline 80 & 21.39 & 3000 & 1490 & 8.287934 & 0.2936 & $\begin{array}{l}106.3 \\
\end{array}$ & 70.57 & 5125 & 0.1 & 1950 & 7500 & 1175000 & $\begin{array}{l}0.1487 \\
\end{array}$ & \#\#\#\#曲 & 3.048 & $5.09 \mathrm{E}-03$ & 93.26 & 39.67 & 0 & & 136.1 & 730.2 \\
\hline 81 & 54.2 & 3000 & 1130 & & 0.4486 & 123 & 60.99 & 5125 & 0.1 & 1950 & 7500 & 1462500 & 0.0587 & \#\#\#\#+ & 4.014 & $9.35 \mathrm{E}-03$ & 52.36 & 54.31 & 0 & & 74.2 & 379.6 \\
\hline 82 & 43.28 & 3000 & 410 & 454.042118 & 16.873 & 111.5 & 67.27 & 5125 & 0.1 & 1950 & 7500 & 1262500 & 0.0735 & \#\#\#\#+ & 1.843 & $4.71 \mathrm{E}-03$ & 36.87 & $\begin{array}{l}98.9 \\
\end{array}$ & 88.99 & & 147.1 & 218.2 \\
\hline 83 & 23.12 & 3000 & 1310 & \begin{tabular}{|r|}
10.192102 \\
\end{tabular} & 0.5993 & $\begin{array}{l}176.4 \\
\end{array}$ & $\begin{array}{ll}42.52 \\
5.27 \\
\end{array}$ & 5125 & 0.1 & 1950 & 7500 & 2512500 & 0.1376 & \#\#\#\# & 2.521 & $6.18 \mathrm{E}-03$ & 19.94 & 50.94 & 0 & & 112.1 & 558.8 \\
\hline $\begin{array}{l}84 \\
85 \\
\end{array}$ & $\begin{array}{l}22.33 \\
32.7 \\
\end{array}$ & $\begin{array}{ll}3000 \\
3000\end{array}$ & 830 & \begin{tabular}{|l|l|}
4.073215 \\
22.777557
\end{tabular} & $\begin{array}{l}0.1873 \\
11189 \\
\end{array}$ & $\begin{array}{l}137.9 \\
1390\end{array}$ & $\begin{array}{l}54.37 \\
52.6\end{array}$ & $\begin{array}{l}5125 \\
525\end{array}$ & $\begin{array}{l}0.1 \\
0.1\end{array}$ & $\begin{array}{l}1950 \\
1950\end{array}$ & 7500 & $\begin{array}{l}1737500 \\
17700\end{array}$ & $\begin{array}{l}0.1425 \\
0.909\end{array}$ & 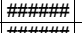 & \begin{tabular}{|l|}
2.01 \\
1902
\end{tabular} & $1.12 \mathrm{E}-02$ & $\begin{array}{l}31.85 \\
20.19\end{array}$ & \begin{tabular}{r|} 
\\
7807 \\
\end{tabular} & 0 & & \begin{tabular}{ll|}
61.8 \\
56.8
\end{tabular} & $\begin{array}{l}461.3 \\
3231\end{array}$ \\
\hline 85 & $\begin{array}{l}32.17 \\
21.77\end{array}$ & 3000 & $\begin{array}{l}5500 \\
890\end{array}$ & 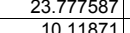 & \begin{tabular}{|c|c|}
1.1089 \\
0.4917
\end{tabular} & \begin{tabular}{|l|}
139.9 \\
145.8
\end{tabular} & \begin{tabular}{r|r}
53.6 & \\
51.45 &
\end{tabular} & $\begin{array}{l}5125 \\
5125\end{array}$ & $\begin{array}{l}0.1 \\
0.1 \\
\end{array}$ & $\begin{array}{l}1950 \\
1950\end{array}$ & $\begin{array}{l}7500 \\
7500\end{array}$ & \begin{tabular}{|l|}
1775000 \\
187500
\end{tabular} & 0.0989 & |\#\#\#\# & $\frac{1.902}{2.094}$ & $\begin{array}{r}1.22 \mathrm{E}-02 \\
788 \mathrm{E}-03\end{array}$ & \begin{tabular}{|c|c|}
29.19 \\
476
\end{tabular} & $\begin{array}{l}78.97 \\
50.59\end{array}$ & & & $\begin{array}{l}56.6 \\
87.9\end{array}$ & 303.1 \\
\hline 87 & 34.88 & 3000 & 600 & & 0.081 & \begin{tabular}{|l}
14.0 \\
170.5
\end{tabular} & 43.99 & 5125 & 0.1 & 1950 & 7500 & 2387500 & $\begin{array}{l}0.141 \\
0.0912 \\
\end{array}$ & 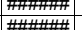 & $\frac{2.094}{2.601}$ & $\begin{array}{l}1.88 \mathrm{E}-03 \\
1.83 \mathrm{E}-02\end{array}$ & $\begin{array}{r}47.6 \\
99.18 \\
\end{array}$ & & & & 87.9 & 492.9 \\
\hline 88 & 21.72 & 3000 & 1390 & 0.079587 & 0.0031 & \begin{tabular}{|l|l|}
118.7 \\
\end{tabular} & \begin{tabular}{|l|l|}
63.17 \\
\end{tabular} & 5125 & 0.1 & 1950 & 7500 & 1387500 & 0.14 & 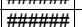 & 2.719 & & 26.10 & & & & 37.8 & 382.2 \\
\hline 89 & 35.11 & 3000 & 1210 & 3.826907 & 0.1989 & 155.9 & 48.11 & 5125 & 0.1 & 1950 & 7500 & 2087500 & 0.0906 & \#\#曲 & 3.332 & $\begin{array}{l}1.41 \mathrm{E}-0 \mathrm{~L} \\
.30 \mathrm{E}-03\end{array}$ & 50.87 & 0 & 0 & & 74.5 & $\frac{641.7}{486.3}$ \\
\hline 90 & 27.31 & 3000 & 620 & \begin{tabular}{|c|}
74.556706 \\
\end{tabular} & 2.1074 & 84.8 & 88.45 & 5125 & 0.1 & 1950 & 7500 & 837500 & 0.1165 & \#\#\#\#+ & 1.892 & $7.44 \mathrm{E}-03$ & 54.23 & 93.29 & 32.94 & & 93.2 & 355. \\
\hline 91 & 41.97 & 3000 & 1090 & $\begin{array}{l}1.512106 \\
\end{array}$ & 0.0695 & $\begin{array}{r}137.9 \\
\end{array}$ & $\begin{array}{l}54.43 \\
54.37 \\
\end{array}$ & 5125 & 0.1 & 1950 & 7500 & \begin{tabular}{|l|l|}
1737500 \\
\end{tabular} & 0.07 & \#\#巫曲 & 3.381 & $\begin{array}{l}1.445 \mathrm{E}-02 \\
\end{array}$ & 33.87 & $\begin{array}{r}90.25 \\
\end{array}$ & $\frac{32.54}{0}$ & & 47.9 & 412.8 \\
\hline 92 & 33.28 & 3000 & 1090 & 11.699945 & 0.3906 & 100.2 & 74.88 & 5125 & 0.1 & 1950 & 7500 & 1075000 & & \#\#\#\#+ & 2.961 & & 47.7 & 57.26 & 0 & & 82.5 & 45 \\
\hline & 58.37 & 3000 & 580 & 67.593219 & 2.6911 & \begin{tabular}{|l|}
119.4 \\
\end{tabular} & 62.79 & 5125 & 0.1 & 1950 & 7500 & 1400000 & 0.0 & \#\#\#\#+ & 2.644 & $1.09 \mathrm{E}-02$ & 31.4 & 92.6 & 26.03 & & 63.5 & 232. \\
\hline 94 & 22.02 & 3000 & 790 & 28.725261 & 1.1024 & 115.1 & 65.14 & 5125 & 0.1 & 1950 & 7500 & 1325000 & 0.1 & \#\#\#\#+ & 1.975 & & 59.75 & 82.59 & & & 104.6 & 459 \\
\hline 95 & 31.31 & 3000 & 440 & 26.134063 & 0.7893 & 90.61 & 82.78 & 5125 & 0.1 & 1950 & 7500 & 925000 & & \#\#\#\#+ & 1.721 & & 62.93 & 80.87 & & & 46.8 & 281. \\
\hline 96 & 42.82 & 3000 & 200 & 237.419 & 9.8423 & 124.4 & 60.31 & 5125 & 0.1 & 1950 & 7500 & 1487500 & & \#\#\#\#+ & 1.249 & & 75.81 & 97.89 & 78.94 & & 46.7 & 149. \\
\hline & 47.98 & 3000 & 2290 & & 0.0742 & 162.1 & 46.28 & 5125 & 0.1 & 1950 & 7500 & 2212500 & & & 5.307 & & 26.21 & 0 & & & 79.3 & 566. \\
\hline 98 & 38.89 & & 1350 & & 0.2795 & $\begin{array}{l}93.84 \\
\end{array}$ & 79. & 5125 & 0.1 & 1950 & 7500 & & & & & & 71.09 & 44.04 & & & 90.7 & 51 \\
\hline 99 & 62.75 & & 1450 & & 0.1974 & 104 & & 51 & 0.1 & 19 & 7500 & & & & & & & 12.21 & & & 66.1 & 410.7 \\
\hline 100 & 63. & & 1940 & & 06 & \begin{tabular}{|l|}
118.7 \\
\end{tabular} & & & 0.1 & & 750 & & & & & & & 0 & 0 & & & 476 \\
\hline 101 & & & 1720 & & & 10 & & & 0.1 & 19 & 750 & & & & & & & 51.53 & 0 & & 150.8 & 709.4 \\
\hline 102 & 25.95 & & 400 & & & 139 & & & 0.1 & & & & & & & & & 97.11 & 71.13 & & 104.2 & 280.9 \\
\hline 103 & 43 & & 1490 & & & \begin{tabular}{|l|}
129.9 \\
\end{tabular} & & & & & & & & & & & & 0 & 0 & & 74.9 & 530.1 \\
\hline 104 & 31.34 & & 2370 & & 0.1 & \begin{tabular}{|l|}
131.9 \\
\end{tabular} & 56. & 51. & & & & & & & & & 41. & & 0 & & 115.7 & 729.4 \\
\hline 105 & 23.6 & 3000 & 540 & & 1.6 & 154 & & 512 & 0.1 & & & & & & & & & 84.56 & 0 & & 67 & 344.1 \\
\hline 106 & 55.04 & 3000 & 560 & & & 118 & & 5125 & 0.1 & & & & & & & & & 90.21 & 2.1 & & 55.6 & 234.9 \\
\hline 107 & 29.62 & 3000 & 720 & & 0.09 & \begin{tabular}{l|l|}
187.3 \\
\end{tabular} & & 51 & & & & & & & & & & & & & 46.4 & 418.9 \\
\hline 108 & 26.42 & $\begin{array}{l}3000 \\
3000\end{array}$ & 1130 & & 0.6987 & $\begin{array}{l}182.8 \\
\end{array}$ & & 5125 & 0.1 & 19 & 7500 & & & & 22 & & & 56.4 & 0 & & 100 & 489.2 \\
\hline 109 & $\begin{array}{r}21.8 \\
\end{array}$ & 3000 & $\begin{array}{r}980 \\
1130\end{array}$ & & $\begin{array}{l}0.0401 \\
\end{array}$ & \begin{tabular}{|l|}
131.9 \\
1271
\end{tabular} & $\frac{56.8}{58 .}$ & $\begin{array}{l}5125 \\
5125\end{array}$ & 0.1 & $\begin{array}{l}1950 \\
1950\end{array}$ & 7500 & & 0.1. & & 2.3 & & 51. & 0 & $\begin{array}{l}0 \\
0 \\
\end{array}$ & & 55.7 & 549.7 \\
\hline $\begin{array}{l}110 \\
111\end{array}$ & $\begin{array}{l}47.41 \\
29.13\end{array}$ & 3000 & $\frac{1130}{1480}$ & & $\begin{array}{l}0.0526 \\
0.0043\end{array}$ & $\begin{array}{l}127.1 \\
07.02\end{array}$ & 58.99 & \begin{tabular}{|l|l|}
5125 \\
5125
\end{tabular} & 0.1 & $\frac{195}{195}$ & $\begin{array}{l}7500 \\
7500\end{array}$ & 1537500 & 0.0 & 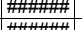 & $\begin{array}{l}4.398 \\
3516\end{array}$ & & $\begin{array}{ll}77 . \\
339\end{array}$ & 0 & $\begin{array}{l}0 \\
0 \\
\end{array}$ & & $\begin{array}{l}47.3 \\
15.3\end{array}$ & 475.4 \\
\hline \begin{tabular}{|c|}
111 \\
112
\end{tabular} & \begin{tabular}{r|r}
29.13 \\
28
\end{tabular} & $\begin{array}{l}3000 \\
3000\end{array}$ & $\frac{1480}{540}$ & $\begin{array}{r}0.1311689 \\
109.31499\end{array}$ & $\begin{array}{l}0.0043 \\
4.0085\end{array}$ & \begin{tabular}{r|}
97.02 \\
110
\end{tabular} & $\begin{array}{r}77.3 \\
68.18\end{array}$ & $\begin{array}{l}5125 \\
5125\end{array}$ & $\begin{array}{l}0.1 \\
0.1\end{array}$ & $\frac{195}{195}$ & $\begin{array}{l}7500 \\
77500\end{array}$ & $\frac{1025000}{11237500}$ & $\frac{0.1}{0.1}$ & 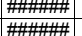 & $\begin{array}{l}3.516 \\
1.749\end{array}$ & & 39. & $\begin{array}{r}0 \\
95.43\end{array}$ & $\begin{array}{r}0 \\
54.26\end{array}$ & & $\begin{array}{r}45.3 \\
100.3\end{array}$ & $\frac{618.6}{320 .}$ \\
\hline 113 & 30.74 & 3000 & 1120 & & 0.0527 & \begin{tabular}{|c|}
132.6 \\
\end{tabular} & 56.5 & 5125 & 0.1 & 195 & $\begin{array}{l}7500 \\
\end{array}$ & & & \begin{tabular}{|l|l} 
\#\#\# \\
\end{tabular} & 3.0 & & 47. & & & & $\begin{array}{l}55 \\
\end{array}$ & 504.5 \\
\hline 114 & 35.47 & 300 & 900 & 65.70 & 3.30 & 150.9 & 49. & 5125 & 0.1 & 195 & 7500 & & & \#\#\# & 2.6 & & 47 & 92.33 & 23.9 & & $\begin{array}{l}163.4 \\
\end{array}$ & 381.8 \\
\hline 115 & 45.97 & & 610 & & 1.09 & 110 & & 512 & 0.1 & 195 & 7500 & & & \#\#\# & 2.352 & & 14. & 83.28 & & & 48 & 262.2 \\
\hline 116 & 47.77 & 3000 & 1420 & & 0.79 & \begin{tabular}{|l|}
131.9 \\
\end{tabular} & 56. & 512 & 0.1 & 195 & 750 & & & & 4.13 & & 50 & 72.26 & & & 121.4 & 443. \\
\hline 117 & 31.16 & 3000 & 740 & 146.3 & 4.61 & \begin{tabular}{|c|}
94.64 \\
\end{tabular} & 79. & 512 & 0.1 & 195 & 7500 & & & & 2.16 & & 38. & 96.58 & 65.83 & & 146.5 & 355.2 \\
\hline 118 & 26.8 & & 510 & & 1.5 & \begin{tabular}{|l|}
166.3 \\
\end{tabular} & 45 & 512 & 0.1 & 195 & 7500 & & & & 1.645 & & 21.2 & 81.92 & & & 60.3 & 314. \\
\hline 119 & 22.18 & & 1360 & & 0.88 & 157 & & 512 & 0.1 & 19 & 750 & & & & 2.6 & & & 70.21 & & & 157.4 & 604. \\
\hline 120 & 30.47 & 3000 & 870 & & & & & & 0.1 & 195 & 7500 & & & & 2.4 & & & 79.59 & & & 102.2 & 411. \\
\hline 121 & 43. & 3000 & 1210 & & 0.1 & 108 & & 512 & 0.1 & 195 & 7500 & & & & & & & 0 & 0 & & 58.1 & 436. \\
\hline 122 & & & 650 & & & & & & 0.1 & & 750 & & & & & & & 69.86 & & & 75 & 415.2 \\
\hline 123 & & & 970 & & & 15 & & & 0.1 & & & & & & & & & 68.44 & & & 69.5 & \\
\hline 124 & & & 1660 & & & & & & 0.1 & & & & & & & & & 0 & 0 & & 74.4 & 656.8 \\
\hline 125 & 60.37 & & 1100 & & 0.4211 & & & 5125 & 0.1 & 195 & 7500 & & & & 4.484 & & & 56.16 & 0 & & 72.3 & \\
\hline 126 & 23.88 & 3000 & 370 & 75.641408 & 2.0521 & 81.39 & 92.15 & 5125 & 0.1 & 1950 & 7500 & 787500 & 0.1332 & \#\#\#\#+1| & 1.295 & & 58.57 & 93.39 & 33.9 & & 57.5 & 277.9 \\
\hline
\end{tabular}




\begin{tabular}{|c|c|c|c|c|c|c|c|c|c|c|c|c|c|c|c|c|c|c|c|c|c|c|}
\hline Realization & $\begin{array}{c}\text { Velocity } \\
(\mathrm{m} / \mathrm{y})\end{array}$ & $\begin{array}{l}\text { Fixed Source } \\
\text { Concentration } \\
(\mathrm{mg} / \mathrm{L})\end{array}$ & $\begin{array}{c}\text { Time of } \\
\text { Maximum } \\
\text { at } \\
\text { Compliance } \\
\text { Boundary } \\
\text { (y) }\end{array}$ & $\mid \begin{array}{c}\text { Maximum } \\
\text { Concentration } \\
\text { Compliance } \\
\text { Boundary } \\
(\mu \mathrm{g} / \mathrm{L})\end{array}$ & $\begin{array}{c}\text { Maximum } \\
\text { Release Rate } \\
\text { at } \\
\text { Compliance } \\
\text { Boundary } \\
(\mathrm{Kg} / \mathrm{y})\end{array}$ & $\begin{array}{l} \\
\text { Sourc } \\
\text { e Rate } \\
\text { (Kg/y) }\end{array}$ & \begin{tabular}{|c|} 
Source \\
Duration \\
(y)
\end{tabular} & $\begin{array}{c}\text { 1-D } \\
\text { Streamtube } \\
\text { Length } \\
(\mathrm{m})\end{array}$ & $\mid \begin{array}{c}\text { Base } \\
\text { Porosity } \\
\left(\mathrm{m}^{3} / \mathrm{m}^{3}\right)\end{array}$ & $\begin{array}{c}\text { Bulk } \\
\text { Densty } \\
\left(\mathrm{Kg} / \mathrm{m}^{3}\right)\end{array}$ & \begin{tabular}{|c|} 
Remaining \\
Carbon \\
Tetrachloride \\
Source \\
$(\mathrm{Kg})$
\end{tabular} & $\begin{array}{c}\text { Volume at or } \\
\text { above Fixed } \\
\text { Source } \\
\begin{array}{c}\text { Concentratio } \\
n(m 3)\end{array}\end{array}$ & $\begin{array}{l}\text { Porosity } \\
\left(\mathrm{m}^{3} / \mathrm{m}^{3}\right)\end{array}$ & $\left(\begin{array}{c}\mathrm{Kd} \\
\left(\mathrm{m}^{3} / \mathrm{kg}\right)\end{array}\right.$ & Retardation & $\begin{array}{c}\text { First } \\
\text { Order } \\
\text { Rate } \\
\text { Constant } \\
(1 / y)\end{array}$ & $\begin{array}{l}\text { Longitudinal } \\
\text { Dispersivity } \\
(\mathrm{m})\end{array}$ & $\begin{array}{c}\text { Required } \\
\text { Source } \\
\text { Cleanup } \\
\text { Percentage } \\
(5 \mu \mathrm{g} / \mathrm{L} \\
\text { Compliance })\end{array}$ & $\begin{array}{c}\text { Required } \\
\text { Source } \\
\text { Cleanup } \\
\text { Percentage } \\
(50 \mu \mathrm{g} / \mathrm{L} \\
\text { Compliance })\end{array}$ & \begin{tabular}{|c} 
Required \\
Source Cleanup \\
Percentage \\
(500 $\mu \mathrm{g} / \mathrm{L}$ \\
Compliance)
\end{tabular} & \begin{tabular}{|c|} 
Abiotic \\
Reaction \\
Half Time \\
(y)
\end{tabular} & $\begin{array}{l}\text { Carbon } \\
\text { Tetrachloride } \\
\text { Travel Time } \\
\text { (y) }\end{array}$ \\
\hline & & & & \begin{tabular}{|l|}
9.233732 \\
\end{tabular} & $\begin{array}{r}0.3248 \\
\end{array}$ & 105.5 & \begin{tabular}{|l|}
71.08 \\
\end{tabular} & 5125 & & & & 1162500 & & \#\#\#\#+ & 3.104 & $1.65 \mathrm{E}-02$ & $\begin{array}{r}86.02 \\
\end{array}$ & 45.85 & & & & \\
\hline 128 & 62.62 & 3000 & 780 & \begin{tabular}{|l|}
147.688812 \\
\end{tabular} & 5.5965 & 1113.7 & 65.97 & 5125 & 0.1 & 1950 & 7500 & 1300000 & 0.0508 & 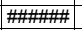 & 3.162 & $5.30 \mathrm{E}-03$ & 28.81 & 96.61 & 66.15 & & & 258.8 \\
\hline 129 & 28.84 & 3000 & 390 & 197.549082 & 5.5839 & \begin{tabular}{|l|}
84.8 \\
\end{tabular} & 88.45 & 5125 & 0.1 & 1950 & 7500 & 837500 & 0.1103 & \#\#\#\#+ & $\begin{array}{l}1.465 \\
\end{array}$ & $8.23 \mathrm{E}-03$ & 61.96 & 97.47 & 74.69 & & 84.2 & 260.4 \\
\hline 130 & 23.76 & 3000 & 340 & $\begin{array}{r}37.930642 \\
\end{array}$ & 1.7019 & $\begin{array}{l}134.6 \\
\end{array}$ & 55.72 & 5125 & 0.1 & 1950 & 7500 & 1675000 & 0.1339 & \#\#\#\#+1 & 1.258 & $1.39 \mathrm{E}-02$ & 41.03 & 86.82 & & & 49.7 & 271.3 \\
\hline 131 & 25.51 & 3000 & 520 & 9.368479 & 0.549 & $\begin{array}{ll}175.8 \\
\end{array}$ & 42.66 & 5125 & 0.1 & 1950 & 7500 & 2500000 & 0.1247 & \#\#\#\#+1 & 1.698 & $1.31 \mathrm{E}-02$ & 41.95 & 46.63 & & & 53 & 341 \\
\hline 132 & 47.27 & 3000 & 1630 & 0.287273 & 0.0118 & 123.7 & 60.65 & 5125 & 0.1 & 1950 & 7500 & 1475000 & 0.0673 & \#\#\#\#+ & 4.812 & $1.55 \mathrm{E}-02$ & 37.09 & & & & 44.9 & 521.7 \\
\hline 133 & 38.38 & 3000 & 1320 & 5.171792 & 0.2655 & 154 & 48.7 & 5125 & 0.1 & 1950 & 7500 & 2050000 & 0.0829 & \#\#\#\#+ & 3.842 & $\begin{array}{l}7.86 \mathrm{E}-03 \\
\end{array}$ & 70.23 & 3.32 & & & 88.2 & 513 \\
\hline 134 & 23.94 & 3000 & 2510 & $\begin{array}{r}0.395449 \\
\end{array}$ & 0.0224 & 169.9 & 44.15 & 5125 & 0.1 & 1950 & 7500 & 2375000 & 0.1329 & \#\#\#\# & 4.025 & $7.43 \mathrm{E}-03$ & 35.24 & & 0 & & 93.3 & 861.7 \\
\hline 135 & 34.5 & 3000 & 720 & \begin{tabular}{|l|l|}
126.236193 \\
2.2776
\end{tabular} & 5.4651 & 129.9 & 57.75 & 5125 & 0.1 & 1950 & 7500 & 1587500 & 0.0922 & \#\#\#\#年曲 & 2.352 & $3.30 \mathrm{E}-03$ & 74.31 & 96.04 & 60.39 & & 209.8 & 349.4 \\
\hline 136 & 27.14 & 3000 & 770 & 25.347663 & 0.8064 & $\begin{array}{l}95.44 \\
1279\end{array}$ & 78.58 & 5125 & 0.1 & 1950 & 7500 & 1000000 & 0.1172 & \#\#\#\# & 2.053 & $9.68 \mathrm{E}-03$ & 22.44 & 80.27 & 0 & & 71.6 & 387.7 \\
\hline 137 & $\begin{array}{r}61.3 \\
5.51\end{array}$ & 3000 & 910 & 88.681147 & \begin{tabular}{|l|l|}
3.7786 \\
1510
\end{tabular} & \begin{tabular}{l|l|}
127.8 \\
11.9
\end{tabular} & 58.67 & 5125 & 0.1 & 1950 & 7500 & 1550000 & 0.0519 & \#\#\#\# & 3.476 & $4.97 \mathrm{E}-03$ & 37.88 & $\begin{array}{l}94.36 \\
9830\end{array}$ & 43.62 & & \begin{tabular}{|l|l|}
139.5 \\
\end{tabular} & $\frac{290.6}{302}$ \\
\hline 138 & 45.51 & 3000 & 630 & 39.332416 & \begin{tabular}{|l|l|}
1.519 \\
\end{tabular} & 115.9 & \begin{tabular}{|l|l|}
64.73 \\
\end{tabular} & 5125 & 0.1 & 1950 & 7500 & 1337500 & & \begin{tabular}{|l|l|l|} 
\\
\end{tabular} & $\begin{array}{ll}2.682 \\
\end{array}$ & $9.09 \mathrm{E}-03$ & 84.52 & 87.29 & & & 76.3 & 302 \\
\hline 139 & 23.72 & & 970 & & $\begin{array}{l}1.187 \\
\end{array}$ & \begin{tabular}{|l|}
157.1 \\
\end{tabular} & & 5125 & 0.1 & 1950 & 7500 & & & \#\#\#\#+ & \begin{tabular}{|l|l|}
2.213 \\
\end{tabular} & $6.09 \mathrm{E}-03$ & 31.74 & 77.94 & & & 113.9 & 478 \\
\hline 140 & 22.28 & 3000 & 760 & 38.997347 & 1.8781 & $\begin{array}{l}144.5 \\
\end{array}$ & 51.91 & 5125 & 0.1 & 1950 & 7500 & 1862500 & & \#\#\#" & 1.869 & $6.37 \mathrm{E}-03$ & 33.22 & 87.18 & 0 & & 108.8 & 430 \\
\hline 141 & 25.64 & 3000 & 670 & 75.760894 & 3.2281 & \begin{tabular}{|l|}
127.8 \\
\end{tabular} & & 5125 & 0.1 & 1950 & 7500 & 1550000 & & \#\#\#\#+ & \begin{tabular}{|l|l|}
1.878 \\
\end{tabular} & $5.92 \mathrm{E}-03$ & 39.15 & & 34 & & 117.1 & 375.5 \\
\hline 142 & 29.93 & 3000 & 1230 & & \begin{tabular}{|c|}
0.3898 \\
\end{tabular} & \begin{tabular}{|l|}
160.8 \\
\end{tabular} & 46.63 & 5125 & 0.1 & 1950 & 7500 & 2187500 & & \#\#\#\#+ & 3.091 & $6.85 \mathrm{E}-03$ & 61.91 & \begin{tabular}{|l|}
31.23 \\
\end{tabular} & & & 101.2 & 529.2 \\
\hline $\begin{array}{l}143 \\
\end{array}$ & & 3000 & 1320 & 15.711529 & $\begin{array}{ll}0.6403 \\
\end{array}$ & $\begin{array}{l}122.3 \\
\end{array}$ & 61.34 & 5125 & 0.1 & & & 1450000 & 0.1442 & \#\#\#\#+ & $\begin{array}{l}2.646 \\
\end{array}$ & $4.84 \mathrm{E}-03$ & 58.49 & $\begin{array}{l}68.18 \\
\end{array}$ & & & 143.3 & \\
\hline \begin{tabular}{l|l|l|l|}
146 & \\
147
\end{tabular} & $\begin{array}{l}39.82 \\
345\end{array}$ & 3000 & \begin{tabular}{|l|l|l|l|}
1030 & \\
\end{tabular} & 2.260395 & \begin{tabular}{|c|}
0.0725 \\
\end{tabular} & $\begin{array}{l}96.23 \\
\end{array}$ & $\begin{array}{l}77.94 \\
\end{array}$ & 5125 & 0.1 & 1950 & 7500 & 1012500 & $\begin{array}{l}0.0799 \\
\end{array}$ & 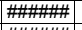 & 3.571 & $1.38 \mathrm{E}-02$ & 71.64 & & 0 & & 50.3 & 459.6 \\
\hline \begin{tabular}{|c|}
147 \\
148
\end{tabular} & $\begin{array}{l}33.45 \\
43.52\end{array}$ & 3000 & \begin{tabular}{r|r|r|}
1200 \\
760
\end{tabular} & $\begin{array}{r}0.623939 \\
240143\end{array}$ & \begin{tabular}{|c|c|} 
\\
1 \\
\end{tabular} & \begin{tabular}{|l|}
163.9 \\
144.5
\end{tabular} & \begin{tabular}{|l|l|}
55.77 \\
51.91
\end{tabular} & $\begin{array}{l}5125 \\
5525\end{array}$ & 0.1 & $\begin{array}{l}1950 \\
1950\end{array}$ & $7500 \mid$ & $\begin{array}{l}2250000 \\
18250\end{array}$ & $\begin{array}{r}0.0951 \\
0.0731\end{array}$ & |\#\#\#\#| & $\begin{array}{l}3.043 \\
2.808 \\
\end{array}$ & $\begin{array}{l}1.44 \mathrm{E}-02 \\
8\end{array}$ & $\begin{array}{l}22.51 \\
58.11\end{array}$ & \begin{tabular}{r|r}
0 \\
70.17
\end{tabular} & 0 & & $\begin{array}{ll}48.2 \\
78.3\end{array}$ & $\begin{array}{l}4666.2 \\
327\end{array}$ \\
\hline 149 & $\begin{array}{l}40.02 \\
21.89\end{array}$ & 3000 & 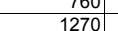 & $\begin{array}{r}24.00145 \\
30.064156\end{array}$ & $\begin{array}{l}1.1559 \\
0.9644\end{array}$ & $\begin{array}{r}144.5 \\
96.23\end{array}$ & $\begin{array}{l}51.91 \\
77.94\end{array}$ & $\begin{array}{l}5120 \\
5125\end{array}$ & $\begin{array}{l}0.1 \\
0.1\end{array}$ & $\frac{1950}{1950}$ & 7500 & $\begin{array}{l}1862500 \\
102500\end{array}$ & $\begin{array}{l}0.0731 \\
0.1453\end{array}$ & 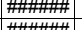 & 2.808 & $\begin{array}{l}8.86 \mathrm{E}-03 \\
307 \mathrm{~F}-03\end{array}$ & 58.11 & \begin{tabular}{|l|l|l|l|}
79.17 & 83.37 \\
\end{tabular} & & & $\begin{array}{r}78.3 \\
174.8\end{array}$ & $\begin{array}{l}330.7 \\
614\end{array}$ \\
\hline 150 & 36.78 & 3000 & 330 & 62.881297 & 2.4284 & 1115.9 & 64.73 & 5125 & 0.1 & 1950 & 7500 & 1337500 & $\begin{array}{l}.1405 \\
0.0865\end{array}$ & 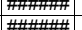 & $\mid \frac{2.625}{1607}$ & & $\begin{array}{r}9.41 \\
80.46 \\
\end{array}$ & $\frac{83.36}{92.05}$ & $\begin{array}{r}0 \\
20.49\end{array}$ & & $\begin{array}{l}1 / 4.8 \\
193\end{array}$ & $\begin{array}{r}614.5 \\
224\end{array}$ \\
\hline 151 & 39.57 & 3000 & $\begin{array}{l}640 \\
\end{array}$ & $\mid$\begin{tabular}{|c|}
4.297289 \\
\end{tabular} & \begin{tabular}{|c|}
0.1649 \\
\end{tabular} & 115.1 & $\begin{array}{l}65.14 \\
65\end{array}$ & 5125 & 0.1 & 1950 & 7500 & 1325000 & $0.0 \varepsilon$ & 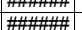 & $\frac{1.007}{2.588}$ & $\begin{array}{l}1.4 \mathrm{IE}-0 \mathrm{~L} \\
1.69 \mathrm{E}-0\end{array}$ & 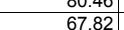 & & & & $\begin{array}{r}49.3 \\
41\end{array}$ & $\begin{array}{l}224 \\
335.2\end{array}$ \\
\hline 152 & 25.47 & 3000 & 1870 & 0.615958 & 0.0274 & 133.3 & 56.28 & 5125 & 0.1 & 1950 & 7500 & 1650000 & 0.1249 & \#\#曲 & 3.518 & $9.18 \mathrm{E}-03$ & 35.62 & & 0 & & 75.5 & 707.8 \\
\hline 153 & 24.47 & 3000 & 340 & 88.057958 & 2.8247 & \begin{tabular}{|l|}
96.23 \\
\end{tabular} & 77.94 & 5125 & 0.1 & 1950 & 7500 & 1012500 & 0.13 & 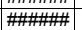 & 1.269 & $1.16 \mathrm{E}-02$ & 61.52 & 94.32 & 43.22 & & 59.7 & 265.7 \\
\hline 154 & 55.42 & 3000 & 1800 & 6.124974 & 0.1997 & 97.81 & 76.68 & 5125 & 0.1 & 1950 & 7500 & 1037500 & 0.0574 & \#\#\#\#+ & 4.996 & $8.70 \mathrm{E}-03$ & 30.88 & 18.37 & & & 79.6 & 462 \\
\hline 155 & 56.91 & 3000 & 630 & \begin{tabular}{|l|}
315.472485 \\
\end{tabular} & 12.031 & 114.4 & 65.55 & 5125 & 0.1 & 1950 & 7500 & 1312500 & 0.0559 & \#\#\#\#+ & 2.696 & & 37.69 & 98.42 & 84.15 & & 231.1 & 242.8 \\
\hline 156 & 21.57 & 3000 & 1360 & \begin{tabular}{|l|}
0.906889 \\
\end{tabular} & 0.0317 & 104.8 & 71.59 & 5125 & 0.1 & 1950 & 7500 & & & \#\#\#\#+ & 3.01 & & 73.1 & 0 & 0 & & 70.4 & 715. \\
\hline 157 & 28.05 & 3000 & 1410 & 7.117 & 0.2834 & 119.4 & 62.79 & 5125 & 0.1 & 1950 & 7500 & 1400 & & \#\#\#\#+ & 3.053 & & 38.86 & 29.75 & & & 98.1 & 557. \\
\hline 158 & 33.88 & 3000 & 410 & 91.41 & 4.7501 & 155.9 & 48.11 & 5125 & 0.1 & 1950 & 7500 & 2087 & & \#\#\#\#+ & 1.655 & & 27.32 & 94.53 & 45.3 & & 68.4 & 250 \\
\hline 159 & 25.09 & 3000 & 450 & 17.32 & 0.8492 & 147 & 51 & 5125 & 0.1 & 1950 & 7500 & 1912500 & & & 1.534 & & 41.12 & 71.14 & & & 52 & 313.3 \\
\hline 160 & 25.23 & 3000 & 1040 & 0.89 & 0.0395 & \begin{tabular}{|l|}
133.3 \\
\end{tabular} & 56.28 & 5125 & 0.1 & 1950 & 7500 & 1650 & & & 2.656 & & 54.92 & & & & 54.5 & 539.5 \\
\hline 161 & 39.92 & & 1030 & & 0.1159 & 169.3 & 44.3 & 5125 & 0.1 & 1950 & 7500 & & & & & & 33.49 & & 0 & & 51.9 & 405 \\
\hline 162 & 34.1 & & 440 & 331.30 & 10.18 & 92.23 & & 5125 & 0.1 & 19 & 7500 & & & & & & & 98.49 & 84.91 & & 105.6 & 250. \\
\hline 163 & 38.15 & & 890 & & & 95. & & & 0.1 & & 7500 & & & & & & & 93.41 & 34.14 & & 162.2 & \\
\hline 164 & 22.66 & & 2210 & & & 139 & & 51 & 0.1 & 19 & 75 & & & & & & & 0 & 0 & & 44.2 & 860.5 \\
\hline 165 & 28. & & 1280 & & 0.15 & 124 & 60.31 & & 0.1 & & 750 & & & & & & 34. & 0 & 0 & & 76.6 & 530.7 \\
\hline 166 & 26. & & 240 & 104.8 & & 133 & 56 & & 0.1 & 19 & 750 & & & & & & & 95.23 & 52.29 & & 53.6 & 220 \\
\hline 167 & 24.66 & & 380 & & & \begin{tabular}{ll|}
124.4 \\
\end{tabular} & 60. & 5125 & 0.1 & & & & & & & & 38 & 80.72 & 0 & & 47.5 & 284.2 \\
\hline 168 & 50.98 & 3000 & 750 & 109.0 & 3.1433 & $\begin{array}{l}86.48 \\
\end{array}$ & 86. & 5125 & 0.1 & & 75 & & & & 2.823 & & & 95.41 & 54.15 & & $\begin{array}{l}97.8 \\
\end{array}$ & 283.7 \\
\hline 169 & 24.49 & & $\begin{array}{l}950 \\
9\end{array}$ & & 0.3926 & 96.23 & & 51 & 0.1 & & & & & & $\begin{array}{l}2.38 \\
\end{array}$ & & & 59.15 & & & 86.4 & 498 \\
\hline 170 & 31.8 & & 1800 & & $\begin{array}{l}0.265 \\
\end{array}$ & \begin{tabular}{ll|}
100.2 \\
\end{tabular} & 74. & 51 & 0.1 & & & & & & & & & 37 & 0 & & 120.8 & 630.3 \\
\hline 171 & 60.25 & 30 & \begin{tabular}{|l|l|}
1960 \\
\end{tabular} & & 0.2461 & $\begin{array}{r}149 \\
\end{array}$ & 50.3 & 5125 & 0.1 & 19 & 7500 & & & & & & & 0 & 0 & & $\begin{array}{l}90.7 \\
\end{array}$ & 472.3 \\
\hline 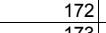 & $\begin{array}{l}24.74 \\
25.1\end{array}$ & 3000 & \begin{tabular}{|r|r|}
510 \\
1140
\end{tabular} & $\begin{array}{r}5.251369 \\
28.02510\end{array}$ & \begin{tabular}{|c|}
0.2391 \\
1212
\end{tabular} & \begin{tabular}{|l|l|}
136.6 \\
1521
\end{tabular} & 54 & $\begin{array}{l}5125 \\
5125\end{array}$ & 0.1 & $\begin{array}{l}1950 \\
1950\end{array}$ & 7500 & $\begin{array}{l}1712500 \\
201250\end{array}$ & $\begin{array}{l}0.12 \\
0.17\end{array}$ & & $\frac{1.6}{2.14}$ & & 26. & \begin{tabular}{|l|l|}
8.79 \\
82.16
\end{tabular} & 0 & & 41.5 & $\begin{array}{l}332.4 \\
5.25\end{array}$ \\
\hline $\begin{array}{l}173 \\
174 \\
\end{array}$ & $\frac{23.51}{21.64}$ & 3000 & \begin{tabular}{l|l}
1140 \\
1120
\end{tabular} & 28.025199 & \begin{tabular}{|l|l}
1.4212 \\
0.5609
\end{tabular} & $\begin{array}{r}152.1 \\
7524\end{array}$ & $\begin{array}{r}49.3 \\
99.68\end{array}$ & \begin{tabular}{|l|l|}
5125 \\
5125
\end{tabular} & 0.1 & $\frac{195}{195}$ & $\begin{array}{l}7500 \\
77500\end{array}$ & $\begin{array}{r}2012500 \\
770000\end{array}$ & 0.1353 & 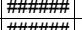 & $\begin{array}{l}2.409 \\
2.209\end{array}$ & & $\frac{38.4}{23 .}$ & \begin{tabular}{|l|l|l|}
82.16 & 77.64 \\
\end{tabular} & & & $\begin{array}{r}150 \\
989\end{array}$ & $\begin{array}{r}525 \\
5276\end{array}$ \\
\hline $\begin{array}{l}1 / 44 \\
175\end{array}$ & $\begin{array}{r}21.04 \\
42.25\end{array}$ & $\frac{3000}{3000}$ & $\begin{array}{l}11720 \\
1480\end{array}$ & & \begin{tabular}{|}
0.5609 \\
0.0999
\end{tabular} & $\begin{array}{r}r .24 \\
83.1\end{array}$ & $\begin{array}{l}99.68 \\
90.25 \\
\end{array}$ & \begin{tabular}{|l|}
5125 \\
5125
\end{tabular} & $\begin{array}{l}.1 \\
0.1\end{array}$ & $\frac{195}{195}$ & $\begin{array}{l}7500 \\
7500\end{array}$ & \begin{tabular}{l|}
100000 \\
812500
\end{tabular} & $\begin{array}{r}0.144 \\
0.0753\end{array}$ & 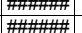 & $\begin{array}{r}2.228 \\
4.06\end{array}$ & & $\frac{23 .}{41.2}$ & & & & $\begin{array}{l}98.9 \\
65.9\end{array}$ & $\frac{527.6}{492.5}$ \\
\hline 176 & 25.43 & 300 & 1370 & & 0.12 & $\mid$\begin{tabular}{|c|}
116.6 \\
\end{tabular} & 64. & 512 & 0.1 & 195 & 7500 & 1350 & 0.1 & \#\# & \begin{tabular}{l|l}
2.832 \\
\end{tabular} & & 31. & 0 & & & 79.5 & 570.7 \\
\hline 177 & 24.0 & 300 & 690 & & 1.49 & $\begin{array}{l}146.4 \\
\end{array}$ & 51. & 512 & 0.1 & 195 & 7500 & & 0.1 & \#\#\# & 1.9 & & 86.3 & 83.68 & & & $\mid 107.6$ & 422.9 \\
\hline 178 & 29.9 & & 1180 & & 0.04 & 181 & 41. & 512 & 0.1 & 195 & 7500 & & & \#\#\# & 3.3 & & 67 & 0 & & & $\mid 57.8$ & 566.2 \\
\hline 179 & 23.12 & 3000 & 730 & $\begin{array}{r}1.1 \\
1.82 \\
\end{array}$ & 0.05 & $\begin{array}{r}92.23 \\
\end{array}$ & 81. & 512 & 0.1 & 195 & 7500 & & & & $\frac{0.5}{1.9}$ & & 43. & 0 & & & 45.2 & 439.2 \\
\hline 180 & 38.1 & 3000 & 520 & 44.30 & 1.8264 & 123.7 & & 512 & 0.1 & 19 & 7500 & & & & 2.0 & & 49. & 88.72 & & & \begin{tabular}{ll|l}
64.6 \\
\end{tabular} & 270 \\
\hline 181 & 59.58 & 3000 & 1750 & & 0.23 & 108 & 69. & 5125 & 0.1 & 195 & 7500 & & & & 6.026 & & 74. & 21.8 & & & 92.6 & 518.4 \\
\hline 182 & 22.79 & & 760 & 116.3 & & 92. & & 512 & 0.1 & 19 & & & & & 1.9 & & & 95.7 & 57.02 & & 173.2 & 433.4 \\
\hline 183 & & 30 & 1420 & & & & & & 0.1 & 195 & 7500 & & & & 4.41 & & & 36.68 & & & 72.3 & 425.5 \\
\hline 184 & 27 & 30 & 1380 & & 0.1 & 136 & 54 & 51 & 0.1 & 195 & 7500 & & & & & & & 0 & 0 & & 86 & 591.7 \\
\hline 185 & & & 1400 & & & & & & 0.1 & & & & & & & & & & & & 76.9 & 603.4 \\
\hline 186 & & & 76 & & & & & & 0.1 & & & & & & & & & & & & 4.7 & \\
\hline 18 & & & 60 & & & & & & 0.1 & & 750 & & & & & & & 87.41 & & & & \\
\hline 188 & 30.62 & 3000 & 3030 & & \begin{tabular}{|c|}
0.0009 \\
\end{tabular} & & 51.68 & 5125 & 0.1 & 1950 & 7500 & & & & & & & 0 & & & 56.8 & \\
\hline 189 & \begin{tabular}{|l|l|}
44.49 \\
\end{tabular} & 3000 & 790 & 6.456247 & 0.3368 & 156.5 & 47.92 & 5125 & 0.1 & 1950 & 7500 & 2100000 & 0.0715 & \#\#\#\#+1| & 2.894 & $1.33 \mathrm{E}-02$ & 39.81 & \begin{tabular}{|l|}
22.56 \\
\end{tabular} & & & 52.1 & 333.3 \\
\hline
\end{tabular}




\begin{tabular}{|c|c|c|c|c|c|c|c|c|c|c|c|c|c|c|c|c|c|c|c|c|c|c|}
\hline Realization & $\begin{array}{c}\text { Velocity } \\
(\mathrm{m} / \mathrm{y})\end{array}$ & $\begin{array}{l}\text { Fixed Source } \\
\text { Concentration } \\
(\mathrm{mg} / \mathrm{L})\end{array}$ & $\begin{array}{c}\text { Time of } \\
\text { Maximum } \\
\text { at } \\
\text { Compliance } \\
\text { Boundary } \\
\text { (y) }\end{array}$ & $\begin{array}{c}\text { Maximum } \\
\text { Concentration } \\
\text { Compliance } \\
\text { Boundary } \\
(\mu \mathrm{g} / \mathrm{L})\end{array}$ & $\begin{array}{c}\text { Maximum } \\
\text { Release Rate } \\
\text { at } \\
\text { Compliance } \\
\text { Boundary } \\
(\mathrm{Kg} / \mathrm{y})\end{array}$ & $\begin{array}{l} \\
\text { Sourc } \\
\text { e Rate } \\
\text { (Kg/y) }\end{array}$ & \begin{tabular}{|c|} 
Source \\
Duration \\
(y)
\end{tabular} & $\begin{array}{c}\text { 1-D } \\
\text { Streamtube } \\
\text { Length } \\
(\mathrm{m})\end{array}$ & $\begin{array}{c}\text { Base } \\
\text { Porosity } \\
\left(\mathrm{m}^{3} / \mathrm{m}^{3}\right)\end{array}$ & $\begin{array}{c}\text { Bulk } \\
\text { Densty } \\
\left(\mathrm{Kg} / \mathrm{m}^{3}\right)\end{array}$ & \begin{tabular}{|c|} 
Remaining \\
Carbon \\
Tetrachloride \\
Source \\
$(\mathrm{Kg})$
\end{tabular} & $\begin{array}{c}\text { Volume at or } \\
\text { above Fixed } \\
\text { Source } \\
\begin{array}{c}\text { Concentratio } \\
n(m 3)\end{array}\end{array}$ & $\begin{array}{l}\text { Porosity } \\
\left(\mathrm{m}^{3} / \mathrm{m}^{3}\right)\end{array}$ & $\left(\begin{array}{c}\mathrm{Kd} \\
\left(\mathrm{m}^{3} / \mathrm{kg}\right)\end{array}\right.$ & Retardation & $\begin{array}{c}\text { First } \\
\text { Order } \\
\text { Rate } \\
\text { Constant } \\
(1 / y)\end{array}$ & $\begin{array}{l}\text { Longitudinal } \\
\text { Dispersivity } \\
(\mathrm{m})\end{array}$ & $\begin{array}{c}\text { Required } \\
\text { Source } \\
\text { Cleanup } \\
\text { Percentage } \\
(5 \mu \mathrm{g} / \mathrm{L} \\
\text { Compliance })\end{array}$ & $\begin{array}{c}\text { Required } \\
\text { Source } \\
\text { Cleanup } \\
\text { Percentage } \\
(50 \mu \mathrm{g} / \mathrm{L} \\
\text { Compliance })\end{array}$ & \begin{tabular}{|c|} 
Required \\
Source Cleanup \\
Percentage \\
(500 $\mu \mathrm{g} / \mathrm{L}$ \\
Compliance)
\end{tabular} & \begin{tabular}{c|} 
Abiotic \\
Reaction \\
Half Time \\
(y)
\end{tabular} & $\begin{array}{l}\text { Carbon } \\
\text { Tetrachloride } \\
\text { Travel Time } \\
\text { (y) }\end{array}$ \\
\hline & & 3000 & 530 & \begin{tabular}{|l|}
338.263461 \\
\end{tabular} & 11.2051 & 99.38 & \begin{tabular}{|l|}
75.47 \\
\end{tabular} & 5125 & & 1950 & & 1062500 & \begin{tabular}{|r|}
0.0786 \\
\end{tabular} & \#\#\#\#+ & & $4.48 \mathrm{E}-03$ & \begin{tabular}{|r|}
36.19 \\
\end{tabular} & $\begin{array}{r}98.52 \\
\end{array}$ & & & & \\
\hline 191 & 21.76 & 3000 & 1240 & 3.952764 & 0.2594 & \begin{tabular}{|l|}
196.9 \\
\end{tabular} & 38.1 & 5125 & 0.1 & 1950 & 7500 & 2962500 & 0.1462 & 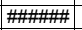 & 2.547 & $6.98 \mathrm{E}-03$ & 46.83 & & & & 99.3 & 600 \\
\hline 192 & 34.58 & 3000 & 2550 & 0.46064 & 0.0164 & 107 & 70.08 & 5125 & 0.1 & 1950 & 7500 & 1187500 & 0.092 & \#\#\#\#+1 & 4.794 & $9.50 \mathrm{E}-03$ & 25.42 & & 0 & & 73 & 710.5 \\
\hline 193 & 35.39 & 3000 & 330 & 83.298874 & 4.2241 & 152.1 & 49.3 & 5125 & 0.1 & 1950 & 7500 & 2012500 & 0.0899 & \#\#\#\#+1 & 1.534 & $1.23 \mathrm{E}-02$ & 42.8 & 94 & 39.98 & & 56.4 & 222.2 \\
\hline 194 & 21.63 & 3000 & 610 & $\begin{array}{r}2.29637 \\
\end{array}$ & 0.1184 & 154.6 & 48.5 & 5125 & 0.1 & 1950 & 7500 & 2062500 & 0.1471 & \#\#\#\#+1 & 1.796 & $1.43 \mathrm{E}-02$ & 65.09 & 0 & & & 48.6 & 425.6 \\
\hline 195 & 23.44 & 3000 & 350 & $\begin{array}{l}157.425285 \\
\end{array}$ & 6.1928 & 118 & 63.55 & 5125 & 0.1 & 1950 & 7500 & 1375000 & 0.1357 & \#\#\#\#+ & 1.266 & $8.20 \mathrm{E}-03$ & 50.11 & 96.82 & 68.24 & & 84.6 & 276.7 \\
\hline 196 & 29.19 & 3000 & 530 & 10.732077 & 0.5192 & 145.1 & 51.68 & 5125 & 0.1 & 1950 & 7500 & 1875000 & 0.109 & \#\#\#\#+ & 1.785 & $1.48 \mathrm{E}-02$ & 25.94 & 53.41 & & & 47 & 313.4 \\
\hline 197 & 28.23 & 3000 & 1050 & 2.579763 & 0.1129 & 131.2 & 57.15 & 5125 & 0.1 & 1950 & 7500 & 1612500 & 0.1127 & \#\#\#\# & 2.516 & $1.21 \mathrm{E}-02$ & 18.66 & & & & 57.1 & 456.8 \\
\hline 198 & 55.81 & 3000 & 1640 & $\begin{array}{c}0.453359 \\
\end{array}$ & 0.0154 & 101.7 & 73.75 & 5125 & 0.1 & 1950 & 7500 & 1100000 & 0.057 & \#\#\#\#年曲 & 5.052 & $1.62 \mathrm{E}-02$ & 29.39 & 0 & 0 & & 42.7 & 463.9 \\
\hline 199 & 33.81 & 3000 & 780 & 10.290012 & 0.5745 & 167.5 & 44.78 & 5125 & 0.1 & 1950 & 7500 & 2325000 & 0.0941 & \#\#\#\#+ & 2.448 & $1.04 \mathrm{E}-02$ & 40.07 & 51.41 & & & 66.8 & 371.2 \\
\hline 200 & 48.94 & 3000 & 510 & 54.095069 & 2.4991 & 138.6 & 54.11 & 5125 & 0.1 & 1950 & 7500 & 1750000 & & \#\#\#\#\#+ & 2.372 & $1.03 \mathrm{E}-02$ & 57.92 & & & & 67.5 & 248.3 \\
\hline 201 & 26.96 & 3000 & 490 & & & \begin{tabular}{ll|}
152.8 \\
\end{tabular} & 49.1 & 5125 & 0.1 & 1950 & 7500 & 2025000 & 0.118 & \#\#\#\#+ & 1.663 & $7.48 \mathrm{E}-03$ & 52.69 & $\begin{array}{l}92.97 \\
\end{array}$ & 29.67 & & 92.7 & 316.1 \\
\hline 202 & 44.37 & 3000 & 2210 & & \begin{tabular}{|c|}
0.0119 \\
\end{tabular} & \begin{tabular}{|l|l|}
131.9 \\
\end{tabular} & 56.85 & 5125 & 0.1 & 1950 & 7500 & & & \#\#\#\#+ & 5.351 & $1.22 \mathrm{E}-02$ & 31.54 & & & & 56.7 & 618.1 \\
\hline 203 & 24.05 & 3000 & 1320 & & \begin{tabular}{ll|}
0.3774 \\
\end{tabular} & $\begin{array}{l}137.3 \\
\end{array}$ & 54.64 & 5125 & 0.1 & 1950 & 7500 & 1725000 & & \#\#\#\#+ & 2.633 & $6.81 \mathrm{E}-03$ & 27.58 & 39.37 & & & 101.8 & 561.1 \\
\hline 204 & 39.08 & 3000 & 1040 & & \begin{tabular}{l|l|}
0.218 \\
\end{tabular} & $\begin{array}{l}116.6 \\
\end{array}$ & 64.33 & 5125 & 0.1 & 1950 & 7500 & 1350000 & & \#\#\#\#+ & 3.396 & $1.04 \mathrm{E}-02$ & \begin{tabular}{ll|}
68.97 \\
\end{tabular} & 10.85 & 0 & & 66.5 & 445.3 \\
\hline 205 & 56.31 & 3000 & 460 & 236.157888 & 9.8448 & 125.1 & 59.97 & 5125 & 0.1 & 1950 & 7500 & 1500000 & & \#\#\#\#曲 & 2.261 & $6.69 \mathrm{E}-03$ & 37.96 & $\begin{array}{ll}97.88 \\
\end{array}$ & 78.83 & & 103.6 & 205.8 \\
\hline 206 & 49.55 & 3000 & 1000 & 73.885899 & 3.2155 & $\begin{array}{l}130.6 \\
\end{array}$ & 57.44 & 5125 & 0.1 & 1950 & 7500 & & 0.0642 & \#\#\#\#曲 & 3.184 & $5.67 \mathrm{E}-03$ & 19.51 & $\begin{array}{l}93.23 \\
\end{array}$ & 32.33 & & 122.3 & 329.3 \\
\hline 207 & 24.14 & 3000 & 910 & & 0.0301 & $\begin{array}{l}137.3 \\
\end{array}$ & 54.64 & 5125 & 0.1 & 1950 & 7500 & 1725000 & 0.1318 & \#\#\#\#曲 & 2.365 & 1.45E-02 & 46.99 & & & & $\begin{array}{l}47.8 \\
\end{array}$ & 502.1 \\
\hline 208 & 32.01 & 3000 & 960 & $\begin{array}{r}1.560734 \\
\end{array}$ & $\begin{array}{l}0.0606 \\
\end{array}$ & $\begin{array}{l}116.6 \\
\end{array}$ & 64.33 & 5125 & 0.1 & 1950 & 7500 & & 0.0994 & \#\#\#\#曲 & 2.898 & $1.39 \mathrm{E}-02$ & 56.77 & & & & 50 & 464 \\
\hline 209 & 27.12 & 3000 & 400 & \begin{tabular}{|l|}
167.523932 \\
\end{tabular} & $\begin{array}{r}6.8275 \\
\end{array}$ & 122.3 & $\begin{array}{ll}61.34 \\
8270\end{array}$ & 5125 & 0.1 & 1950 & 7500 & 1450000 & 0.1173 & \#\#\#\# & \begin{tabular}{|l|l|}
1.47 \\
\end{tabular} & $7.13 \mathrm{E}-03$ & 47.97 & 97.02 & 70.15 & & 97.3 & 277.8 \\
\hline $\begin{array}{l}210 \\
211 \\
\end{array}$ & 52.24 & $\begin{array}{ll}3000 \\
3000\end{array}$ & $\begin{array}{r}360 \\
1180\end{array}$ & \begin{tabular}{|r|}
736.884312 \\
7430621
\end{tabular} & $\begin{array}{r}22.2554 \\
2519\end{array}$ & $\begin{array}{l}90.61 \\
1017\end{array}$ & $\begin{array}{l}82.78 \\
7375\end{array}$ & $\begin{array}{l}5125 \\
525\end{array}$ & $\begin{array}{l}0.1 \\
0.1\end{array}$ & $\begin{array}{l}1950 \\
1950\end{array}$ & 7500 & $\begin{array}{r}925000 \\
111000\end{array}$ & 0.0609 & 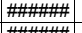 & \begin{tabular}{|c|c|}
1.807 \\
3.071 \\
\end{tabular} & 7.00E-03 & $\begin{array}{l}12.18 \\
4.0\end{array}$ & $\begin{array}{l}99.32 \\
9327 \\
\end{array}$ & 93.21 & 32.15 & $\begin{array}{r}99 \\
1706\end{array}$ & \\
\hline 211 & $\begin{array}{l}58.05 \\
36.48 \\
\end{array}$ & $\frac{3000}{3000}$ & $\frac{1180}{620}$ & $\begin{array}{r}74.306321 \\
36.13045 \\
\end{array}$ & $\begin{array}{r}2.519 \\
15724 \\
\end{array}$ & \begin{tabular}{|l|}
101.7 \\
1306
\end{tabular} & $\begin{array}{l}73.75 \\
57.44\end{array}$ & $\begin{array}{l}5125 \\
5125\end{array}$ & 0.1 & $\begin{array}{l}1950 \\
1950\end{array}$ & $\begin{array}{l}7500 \\
7500\end{array}$ & \begin{tabular}{|l|}
1100000 \\
1600000
\end{tabular} & $\begin{array}{ll}0.0588 \\
0.072\end{array}$ & 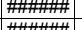 & 3.974 & 4.06E-03 & $\begin{array}{r}47.9 \\
68.28\end{array}$ & $\begin{array}{l}93.27 \\
86.16\end{array}$ & 32.71 & & $\begin{array}{c}170.6 \\
792\end{array}$ & $\begin{array}{ll}350.8 \\
322.5\end{array}$ \\
\hline 213 & 29.03 & 3000 & 1410 & 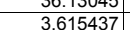 & 0.1654 & \begin{tabular}{|l}
130.0 \\
137.3
\end{tabular} & 54.64 & 5125 & 0.1 & 1950 & 7500 & 5000 & 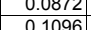 & 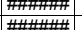 & $\frac{2.296}{3.241}$ & $\begin{array}{l}8.16 E-03 \\
7.98-03\end{array}$ & $\begin{array}{ll}68.28 \\
48.67\end{array}$ & 86.16 & & & $\frac{79.2}{86.8}$ & 322.5 \\
\hline 214 & 26.53 & 3000 & 820 & 0.903 & \begin{tabular}{|c|}
0.0449 \\
\end{tabular} & 149 & 50.35 & 5125 & 0.1 & 1950 & $\begin{array}{l}7500 \\
\end{array}$ & 1950000 & $\begin{array}{l}0.1190 \\
0.1199 \\
\end{array}$ & |m+m\#" & $\frac{0.241}{2.437}$ & & $\begin{array}{ll}48.67 \\
6320\end{array}$ & 0 & & & 86.8 & $\frac{572.2}{470.8}$ \\
\hline 215 & 39.18 & 3000 & 1250 & 16.508 & 0.6216 & 113 & $\mid 66.4$ & 5125 & 0.1 & 1950 & 7500 & 1287500 & 0.0812 & 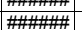 & $\frac{2.401}{3.195}$ & $\begin{array}{l}.00 \mathrm{E}-0 \mathrm{~L} \\
8.29 \mathrm{E}-03\end{array}$ & $\begin{array}{l}03.29 \\
15.83\end{array}$ & $\begin{array}{r}09.71 \\
\end{array}$ & & & $\begin{array}{l}8.0 \\
83.6\end{array}$ & 410.8 \\
\hline 216 & 27.5 & 3000 & 470 & $\begin{array}{l}79.63156 \\
\end{array}$ & 3.5195 & 132.6 & 56.56 & 5125 & 0.1 & 1950 & 7500 & 1637500 & 0.1157 & \#\#\#\#+ & 1.612 & $8.56 \mathrm{E}-03$ & 39.5 & 93.72 & 37.21 & & 81 & 300.5 \\
\hline 217 & 41.86 & 3000 & 350 & \begin{tabular}{|l|}
182.892384 \\
\end{tabular} & 11.0015 & 180.5 & 41.56 & 5125 & 0.1 & 1950 & 7500 & 2600000 & 0.076 & \#\#\#\#+ & 1.694 & $8.81 \mathrm{E}-03$ & 25.8 & 97.27 & 72.66 & & 78.7 & 207. \\
\hline 218 & 23.97 & 3000 & 480 & \begin{tabular}{|r|}
66.914879 \\
\end{tabular} & 2.8817 & $\begin{array}{l}129.2 \\
\end{array}$ & 58.05 & 5125 & 0.1 & 1950 & 7500 & & 0.1327 & \#\#\#\#+ & 1.507 & 8.944 & 31. & 92.53 & 25.28 & & 77.5 & 322 \\
\hline 219 & 32.73 & 3000 & 1400 & 0.453609 & 0.0167 & \begin{tabular}{|l|}
110.7 \\
\end{tabular} & 67.72 & 5125 & 0.1 & 1950 & 7500 & & 0.0972 & \#\#\#\#+ & 3.798 & & 56.08 & & 0 & & 51.8 & 594 \\
\hline 220 & 24.1 & 3000 & 730 & 1.53666 & 0.0525 & 102.5 & 73.19 & 5125 & 0.1 & 1950 & 7500 & 1112500 & 0. & \#\#\#\#+ & 2.28 & & 90.82 & & & & 46.6 & 484.8 \\
\hline 221 & 27.54 & 3000 & 1710 & & 0.0309 & 114.4 & 65.55 & 5125 & 0.1 & 1950 & 7500 & & & \#\#\#\#+ & 4.082 & & 77.28 & 0 & & & 74.4 & 759. \\
\hline 222 & 50.58 & 3000 & 1300 & & 0.45 & 155.3 & 48.3 & 5125 & 0.1 & 1950 & 7500 & 2075000 & & & 4.413 & & 72.24 & 42.5 & & & 93.9 & 447 \\
\hline 223 & 22.26 & 3000 & 1300 & & $\begin{array}{ll}0.1305 \\
\end{array}$ & \begin{tabular}{|l|l|}
114.4 \\
\end{tabular} & 65.55 & 5125 & 0.1 & 1950 & 7500 & & 0.1 & & 2.578 & & 33.03 & & & & 82.1 & 593. \\
\hline 224 & 42.42 & & 440 & \begin{tabular}{|l|l|}
245.02 \\
\end{tabular} & 8.3691 & 102.5 & 73.19 & 5125 & 0.1 & 1950 & 7500 & & & & 1.851 & & 11.89 & $\begin{array}{l}97.96 \\
\end{array}$ & 79.59 & & 72.6 & 223. \\
\hline 225 & 30.24 & & 1290 & & & $\begin{array}{l}143.2 \\
\end{array}$ & & 51 & 0.1 & & & & & & & & & & & & 56.1 & 56 \\
\hline 226 & 26. & & 2290 & & & 15. & & & 0.1 & & & & & & & & & 0 & 0 & & 94.8 & \\
\hline 227 & & & 780 & & & & & & 0.1 & & 75 & & & & & & & 40.88 & 0 & & 59.9 & 401.3 \\
\hline 228 & & & 950 & & & 150 & & & 0.1 & & & & & & & & & 0 & 0 & & 49.4 & 512.6 \\
\hline 229 & & & 930 & & & 134 & & & & & & & & & & & & \begin{tabular}{|l|}
61.39 \\
\end{tabular} & 0 & & & 409.4 \\
\hline 230 & & & 970 & 19.1 & 1.0039 & \begin{tabular}{|l|}
157.1 \\
\end{tabular} & & 51 & & & & & & & & & & 73.91 & 0 & & 87.4 & 366.2 \\
\hline 231 & 56.2 & 30 & 2670 & & & $\begin{array}{l}136.6 \\
\end{array}$ & 5 & 51 & 0.1 & & & & & & & & & & 0 & & $\begin{array}{l}81.8 \\
\end{array}$ & 613.7 \\
\hline 232 & & & 420 & & 2.6447 & 89. & & & & & & & & & & & & 94.34 & 43.42 & & 73.5 & 301.7 \\
\hline 233 & 46.9 & & 980 & & $\begin{array}{l}4.9333 \\
\end{array}$ & 116 & & & & & & & & & & & 34 & $\begin{array}{l}96.06 \\
\end{array}$ & 60.61 & & 203 & 339.8 \\
\hline 234 & 24.28 & 30 & 430 & & $\begin{array}{r}.939 \\
\end{array}$ & $\begin{array}{l}106.3 \\
\end{array}$ & & 51 & 0.1 & 19 & & & 0. & & & & 32. & $\begin{array}{l}3.97 \\
\end{array}$ & $\begin{array}{l}39.73 \\
\end{array}$ & & \begin{tabular}{l|l|}
69.2 \\
\end{tabular} & 298.3 \\
\hline 235 & $\begin{array}{l}28.43 \\
3769\end{array}$ & 3000 & $\frac{2190}{540}$ & & 0.0691 & $\begin{array}{l}124.4 \\
\end{array}$ & 60.3 & $\begin{array}{l}5125 \\
5125\end{array}$ & 0.1 & $\begin{array}{l}1950 \\
1950\end{array}$ & 7500 & & 0.11 & & & & & $\begin{array}{r}0 \\
\end{array}$ & 0 & & 103.1 & $\begin{array}{l}772.5 \\
32.57\end{array}$ \\
\hline $\begin{array}{l}236 \\
237 \\
\end{array}$ & $\begin{array}{l}37.69 \\
33.74 \\
\end{array}$ & 3000 & $\begin{array}{r}540 \\
1120\end{array}$ & $\begin{array}{r}14.882103 \\
0.79673\end{array}$ & \begin{tabular}{|c|}
0.4891 \\
0.026
\end{tabular} & $\begin{array}{r}98.6 \\
\end{array}$ & 76.4 & \begin{tabular}{|l|l|}
5125 \\
5125
\end{tabular} & 0.1 & $\frac{195}{199}$ & $\begin{array}{l}7500 \\
7500\end{array}$ & & 0.08 & 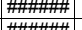 & $\begin{array}{l}2.307 \\
3256 \\
\end{array}$ & & 89. & 66.4 & & & 48.9 & 313.7 \\
\hline 237 & $\begin{array}{l}33.4 \\
29.65 \\
\end{array}$ & $\frac{3000}{3000}$ & $\frac{7120}{1450}$ & $\begin{array}{r}0.1896 \\
2.964\end{array}$ & $\mid$ & $\begin{array}{r}98.6 \\
100.2\end{array}$ & $\frac{76.6}{74.8}$ & \begin{tabular}{|l|}
5125 \\
5125
\end{tabular} & $\begin{array}{l}.1 \\
0.1\end{array}$ & $\frac{795}{195}$ & $\begin{array}{l}7500 \\
7500\end{array}$ & & $\frac{0.05}{0.16}$ & 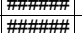 & $\frac{3.25}{3.1}$ & & $\frac{50 .}{27 .}$ & 0 & & & $\begin{array}{l}46.2 \\
72.4\end{array}$ & $\frac{494.6}{544.5}$ \\
\hline 239 & 30.2 & 300 & 1860 & 0.472 & 0.0282 & \begin{tabular}{|l|l|}
179.3 \\
\end{tabular} & 41. & 5125 & 0.1 & 195 & $\begin{array}{l}7500 \\
\end{array}$ & & 0.1 & \#\#\# & 4.3 & & 62. & 0 & & & 73.6 & 733. \\
\hline 240 & 35.7 & 300 & 1290 & 26.453 & 1.19 & \begin{tabular}{|l|}
135.3 \\
\end{tabular} & 55. & 512 & 0.1 & 195 & 750 & & 0. & 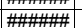 & 3.1 & & 26. & 81.1 & & & \begin{tabular}{|l|l|}
124.8 \\
\end{tabular} & 452.7 \\
\hline 241 & 46.3 & & 1510 & & 0.00 & \begin{tabular}{|l|}
116.6 \\
\end{tabular} & 64. & 512 & 0.1 & 195 & 7500 & & & \#\#車 & 5.24 & & 55. & & & & 36.2 & 580.8 \\
\hline 242 & 54.6 & 300 & 1420 & & 0.15 & 74.34 & 100. & 512 & 0.1 & 195 & 750 & & & & 5.1 & & 75 . & 21.02 & & & 69.2 & 483. \\
\hline 243 & 27.8 & 3000 & 1220 & 1.178 & 0.03 & 97.81 & 76. & 512 & 0.1 & 195 & 7500 & & & & & & 52.7 & & & & 58 & 562. \\
\hline 244 & 39. & 300 & 650 & 29.17 & 1.15 & 118 & 63. & 512 & 0.1 & 195 & 7500 & & & & & & 23 & 82.86 & & & 58.8 & 295. \\
\hline 245 & & & 660 & 169.83 & & 100 & & 512 & 0.1 & 19 & & & & & & & & 97.06 & 70.56 & & 179 & 261. \\
\hline 246 & & 3000 & 1710 & & 0.3 & & & & 0.1 & 195 & 7500 & & & & & & & 40.73 & 0 & & 110.5 & 526. \\
\hline 247 & & 3000 & 990 & & & & & & 0.1 & & 7500 & & & & & & & 0 & 0 & & 82.3 & 532. \\
\hline 248 & & & 620 & & & & & & 0.1 & & & & & & & & & 84.81 & & & 57 & 276. \\
\hline 24 & & & 93 & & & & & & 0.1 & & & & & & & & & 87 & 0 & & \begin{tabular}{|l|l|}
101.7 \\
\end{tabular} & \\
\hline 250 & & & 75 & & & & & & 0.1 & & 750 & & & & 97 & & & 0 & & & 39.9 & \\
\hline 251 & 30.13 & 3000 & 530 & 286.166122 & \begin{tabular}{|l|l|}
13.4088 \\
\end{tabular} & 140.6 & 53.35 & 5125 & 0.1 & 1950 & 7500 & & & & & & & 98.25 & 82.53 & & 205.4 & 301.6 \\
\hline 252 & \begin{tabular}{|l|}
29.4 \\
\end{tabular} & 3000 & 1840 & 0.323416 & 0.0177 & \begin{tabular}{|c|}
163.9 \\
\end{tabular} & 45.77 & 5125 & & 1950 & 7500 & 2250000 & 0.1082 & \#\#\#\#+1| & 3.949 & & 42.62 & & & & $\begin{array}{l}65.8 \\
\end{array}$ & 688.3 \\
\hline
\end{tabular}




\begin{tabular}{|c|c|c|c|c|c|c|c|c|c|c|c|c|c|c|c|c|c|c|c|c|c|c|}
\hline Realization & 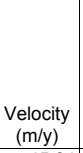 & $\begin{array}{c}\text { Fixed Source } \\
\text { Concentration } \\
(\mathrm{mg} / \mathrm{L})\end{array}$ & $\begin{array}{c}\text { Time of } \\
\text { Maximum } \\
\text { at } \\
\text { Compliance } \\
\text { Boundary } \\
\text { (y) }\end{array} \mid$ & $\begin{array}{c}\text { Maximum } \\
\text { Concentration } \\
\text { Compliance } \\
\text { Boundary } \\
(\mu \mathrm{g} / \mathrm{L})\end{array}$ & $\begin{array}{c}\text { Maximum } \\
\text { Release Rate } \\
\text { at } \\
\text { Compliance } \\
\text { Boundary } \\
\text { (Kg/y) }\end{array}$ & $\begin{array}{l} \\
\text { Sourc } \\
\text { e Rate } \\
\text { (Kg/y) } \\
\end{array}$ & \begin{tabular}{|c|} 
Source \\
Duration \\
(y)
\end{tabular} & $\begin{array}{c}\text { 1-D } \\
\text { Streamtube } \\
\text { Length } \\
(\mathrm{m})\end{array}$ & $\begin{array}{c}\text { Base } \\
\text { Porosity } \\
\left(\mathrm{m}^{3} / \mathrm{m}^{3}\right)\end{array}$ & $\begin{array}{c}\text { Bulk } \\
\text { Densty } \\
\left(\mathrm{Kg} / \mathrm{m}^{3}\right)\end{array}$ & \begin{tabular}{|c|}
$\begin{array}{c}\text { Remaining } \\
\text { Carbon } \\
\text { Tetrachloride } \\
\text { Source } \\
(\mathrm{Kg})\end{array}$ \\
\end{tabular} & $\begin{array}{c}\text { Volume at or } \\
\text { above Fixed } \\
\text { Source } \\
\text { Concentratio } \\
n(m 3)\end{array}$ & $\begin{array}{l}\text { Porosity } \\
\left(\mathrm{m}^{3} / \mathrm{m}^{3}\right) \\
\end{array}$ & $\left.\mid \begin{array}{c}\mathrm{Kd} \\
\left(\mathrm{m}^{3} / \mathrm{kg}\right)\end{array}\right)$ & Retardation & $\begin{array}{c}\text { First } \\
\text { Order } \\
\text { Rate } \\
\text { Constant } \\
(1 / y) \\
\end{array}$ & \begin{tabular}{|c|} 
Longitudinal \\
Dispersivity \\
$(\mathrm{m})$
\end{tabular} & $\begin{array}{c}\text { Required } \\
\text { Source } \\
\text { Cleanup } \\
\text { Percentage } \\
(5 \text { ug/L } \\
\text { Compliance) } \\
\end{array}$ & $\begin{array}{c}\text { Required } \\
\text { Source } \\
\text { Cleanup } \\
\text { Percentage } \\
\text { (50 ug/L } \\
\text { Compliance) } \\
\end{array}$ & \begin{tabular}{|c|} 
Required \\
Source Cleanup \\
Percentage \\
(500 $\mathrm{\mu g} / \mathrm{L}$ \\
Compliance) \\
\end{tabular} & $\begin{array}{c}\text { Abiotic } \\
\text { Reaction } \\
\text { Half Time } \\
(y) \\
\end{array}$ & $\begin{array}{c}\text { Carbon } \\
\text { Tetrachloride } \\
\text { Travel Time } \\
\text { (y) } \\
\end{array}$ \\
\hline & & 3000 & 1010 & & \begin{tabular}{|l|}
0.1743 \\
\end{tabular} & 82.25 & \begin{tabular}{|l|l|}
91.19 \\
\end{tabular} & 5125 & & 1950 & \begin{tabular}{|r|}
7500 \\
\end{tabular} & \begin{tabular}{|r|}
800000 \\
\end{tabular} & \begin{tabular}{|l|}
0.0694 \\
\end{tabular} & \#\#\#\#+ & \begin{tabular}{|r|}
3.515 \\
\end{tabular} & $1.27 \mathrm{E}-02$ & \begin{tabular}{|l|}
53.99 \\
\end{tabular} & & & & & \\
\hline 254 & 23.34 & 3000 & 490 & 43.241442 & \begin{tabular}{|l|l|}
1.499 \\
\end{tabular} & 104 & 72.12 & 5125 & 0.1 & 1950 & 7500 & 1137500 & 0.1363 & \#\#\#\#+ & 1.559 & $9.65 \mathrm{E}-03$ & 67.04 & \begin{tabular}{|l|l|}
88.44 \\
\end{tabular} & 0 & 0 & 71.8 & 342.4 \\
\hline 255 & 51.9 & 3000 & 900 & 59.554521 & 2.2277 & 112.2 & 66.83 & 5125 & 0.1 & 1950 & 7500 & 1275000 & 0.0613 & \#\#\#\#+ & 3.359 & $5.50 \mathrm{E}-03$ & 65.89 & $\begin{array}{l}91.6 \\
9\end{array}$ & 16.04 & 0 & 126.1 & 331.7 \\
\hline 256 & 51.06 & 3000 & 330 & \begin{tabular}{|r|}
599.642064 \\
\end{tabular} & 14.4974 & 72.53 & 103.4 & 5125 & 0.1 & 1950 & 7500 & 662500 & 0.0623 & \#\#\#\#+ & 1.735 & $6.89 \mathrm{E}-03$ & 63.56 & 99.17 & 91.66 & 16.62 & 100.6 & 174.2 \\
\hline 257 & 36.69 & 3000 & 310 & 461.845568 & 20.6196 & \begin{tabular}{|l|} 
\\
\end{tabular} & 56 & 5125 & 0.1 & 1950 & 7500 & 1662500 & 0.0867 & \#\#\#\#+1 & 1.465 & $5.08 \mathrm{E}-03$ & 55.95 & 98.92 & 89.17 & 0 & 136.4 & 204.6 \\
\hline 258 & 24.12 & 3000 & 460 & 56.775368 & \begin{tabular}{ll|}
1.8362 \\
\end{tabular} & 97.02 & 77.3 & 5125 & 0.1 & 1950 & 7500 & 1025000 & 0.1319 & \#\#\#\#+1 & 1.447 & $1.15 \mathrm{E}-02$ & 20.96 & 91.19 & 11.93 & 0 & 60.2 & 307.5 \\
\hline 259 & 54.76 & 3000 & 1220 & 0.943005 & \begin{tabular}{|l|l|}
0.0373 \\
\end{tabular} & 118.7 & 63.17 & 5125 & 0.1 & 1950 & 7500 & 1387500 & 0.0581 & \#\#\#\#+ & 4.564 & $1.64 \mathrm{E}-02$ & 50.34 & 0 & 0 & 0 & 42.3 & 427.2 \\
\hline 260 & 31.04 & 3000 & $\begin{array}{l}680 \\
\end{array}$ & 1.000353 & \begin{tabular}{|c|}
0.0649 \\
\end{tabular} & \begin{tabular}{|l|}
194.6 \\
\end{tabular} & 38.53 & 5125 & 0.1 & 1950 & 7500 & 2912500 & 0.1025 & \#\#\#\#+ & 2.307 & $1.75 \mathrm{E}-02$ & 49.61 & 0 & 0 & 0 & 39.5 & 381 \\
\hline 261 & 42.76 & 3000 & 430 & $\begin{array}{l}9.160549 \\
.9030\end{array}$ & 0.5314 & 174 & 43.09 & 5125 & 0.1 & 1950 & 7500 & 2462500 & 0.0744 & \#\#\#\# & 2.071 & 1.87E-02 & 53 & 45.42 & 0 & 0 & 37.1 & 248.2 \\
\hline 262 & 28.05 & 3000 & 330 & 67.203822 & 2.9551 & \begin{tabular}{|l|l|}
131.9 \\
\end{tabular} & 56.85 & 5125 & 0.1 & 1950 & 7500 & 1625000 & 0.1134 & \#\#\#\#曲 & 1.33 & $1.34 \mathrm{E}-02$ & 34.91 & 92.56 & 25.6 & & 51.7 & 242.9 \\
\hline 263 & 22.95 & 3000 & 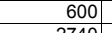 & $\begin{array}{l}42.034196 \\
\end{array}$ & 1.7131 & \begin{tabular}{|l|}
122.3 \\
\end{tabular} & 61.34 & 5125 & 0.1 & 1950 & 7500 & 1450000 & $\begin{array}{l}0.1386 \\
0.077\end{array}$ & 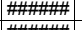 & 1.695 & 7.94E-03 & $\begin{array}{ll}47.16 \\
2 ? 05\end{array}$ & 88.1 & 0 & & 87.3 & 378.5 \\
\hline $\begin{array}{l}264 \\
265\end{array}$ & $\begin{array}{l}46.99 \\
37.04\end{array}$ & 3000 & $\begin{array}{rl}2740 & 490\end{array}$ & \begin{tabular}{|r|}
8.7222918 \\
83.270619
\end{tabular} & $\begin{array}{l}0.0308 \\
4.0281\end{array}$ & \begin{tabular}{|l|}
127.8 \\
145.1 \\
\end{tabular} & $\begin{array}{l}58.67 \\
51.68\end{array}$ & $\begin{array}{l}5125 \\
5125\end{array}$ & $\begin{array}{l}0.1 \\
0.1 \\
\end{array}$ & $\begin{array}{l}1950 \\
1950\end{array}$ & $\begin{array}{l}7500 \\
7500\end{array}$ & $\begin{array}{l}\frac{1550000}{1875000} \\
\end{array}$ & $\begin{array}{l}0.06877 \\
0.0859\end{array}$ & 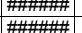 & $\begin{array}{l}5.818 \\
1.985 \\
\end{array}$ & $\begin{array}{r}9.24 \mathrm{E}-03 \\
7.57 \mathrm{E}-03 \\
\end{array}$ & $\begin{array}{l}23.95 \\
66.88 \\
\end{array}$ & \begin{tabular}{r|}
0 \\
94
\end{tabular} & $\begin{array}{r}0 \\
39.95\end{array}$ & & $\begin{array}{r}75 \\
916\end{array}$ & $\begin{array}{l}634.5 \\
274.7 \\
\end{array}$ \\
\hline 266 & 22.06 & 3000 & 1650 & 0.251907 & 0.0119 & \begin{tabular}{|l|}
140.9 \\
141.9
\end{tabular} & 52.86 & 5125 & 0.1 & 1950 & 7500 & 1812500 & 0.1442 & 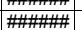 & 3.223 & $1.06 \mathrm{E}-02$ & 40.00 & $\frac{34}{0}$ & 0 & & 65.6 & $\begin{array}{l}274.1 \\
748.7\end{array}$ \\
\hline 267 & 23.15 & 3000 & 580 & 17.586672 & 0.7372 & 125.8 & 59.64 & 5125 & 0.1 & 1950 & 7500 & 1512500 & 0.1374 & \#\#\#\#| & $\begin{array}{l}1.647 \\
\end{array}$ & $1.14 \mathrm{E}-02$ & 27.37 & 71.57 & 0 & & 60.7 & 364.5 \\
\hline 268 & 455.32 & 3000 & \begin{tabular}{|l|l|}
1870 \\
\end{tabular} & 1.704438 & \begin{tabular}{|l|l|}
0.0427 \\
\end{tabular} & 75.24 & 99.68 & 5125 & 0.1 & 1950 & 7500 & 700000 & 0.0702 & \#\#\#\#| & \begin{tabular}{|l|l|}
4.857 \\
\end{tabular} & $1.10 \mathrm{E}-02$ & | 36.59 & 0 & 0 & & 63.3 & 549.3 \\
\hline 269 & 29.9 & 3000 & 1470 & $\begin{array}{ll}3.468628 \\
\end{array}$ & 0.1831 & \begin{tabular}{|l|}
158.4 \\
\end{tabular} & 47.36 & 5125 & 0.1 & 1950 & 7500 & 2137500 & 0.1064 & \#mm!n & 3.619 & $7.10 \mathrm{E}-03$ & 74.71 & & & & 97.6 & $\begin{array}{l}549.5 \\
620.4\end{array}$ \\
\hline & 55.33 & 3000 & 3700 & $\begin{array}{ll}0.214472 \\
\end{array}$ & $\begin{array}{l}0.0075 \\
\end{array}$ & 105.5 & 71.08 & 5125 & 0.1 & 1950 & 7500 & 1162500 & 0.0575 & \#\#mm & 8.05 & $1.03 \mathrm{E}-02$ & 26.45 & 0 & & & 67.4 & $\begin{array}{l}620.4 \\
745.7 \\
\end{array}$ \\
\hline & 23.55 & & 690 & 9.532449 & 0.4446 & | 139.9 & 53.6 & 5125 & 0.1 & 1950 & 7500 & 1775000 & 0.1351 & \#\#\#\#\# & 1.933 & $\begin{array}{l}1.02 \mathrm{E}-02 \\
\end{array}$ & 56.85 & 47.55 & 0 & & 679 & $\begin{array}{l}745.1 \\
420.7 \\
\end{array}$ \\
\hline & 48.13 & 3000 & 450 & \begin{tabular}{|l|}
179.914166 \\
\end{tabular} & \begin{tabular}{|l|}
6.73 \\
\end{tabular} & \begin{tabular}{l|l|}
312.2 \\
\end{tabular} & 66.83 & 5125 & 0.1 & 1950 & 7500 & 1275000 & & 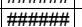 & 2.143 & $6.71 \mathrm{E}-03$ & 75.14 & 97.22 & 72.21 & & 1032 & $\begin{array}{l}428.1 \\
228.2 \\
\end{array}$ \\
\hline 273 & 27.03 & 3000 & 710 & & 0.3132 & 128.5 & 58.36 & 5125 & 0.1 & 1950 & 7500 & 1562500 & & \#\#\# & 2.173 & $1.13 \mathrm{E}-02$ & 65.03 & 31.61 & & & 61.4 & 248.4 \\
\hline 274 & 38.94 & 3000 & 2400 & & 0.011 & \begin{tabular}{|l|}
165.7 \\
\end{tabular} & \begin{tabular}{|l|l|}
45.26 \\
\end{tabular} & 5125 & & 1950 & 7500 & & 0.0817 & \#\#\#\#| & 5.106 & & \begin{tabular}{|l|l|}
28.19 \\
\end{tabular} & & 0 & & 62.7 & $\begin{array}{l}412.1 \\
672.1 \\
\end{array}$ \\
\hline 275 & 34.21 & 3000 & 760 & 51.2. & 1.8428 & \begin{tabular}{|l|l|}
3 & 107.8
\end{tabular} & \begin{tabular}{|c|}
699.59 \\
\end{tabular} & 5125 & 0.1 & 1950 & 7500 & 50000 & $\mid 0.093$ & 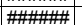 & 2.444 & & $\begin{array}{l}65.83 \\
65.83\end{array}$ & 90.25 & 2.52 & & 106.3 & 366.2 \\
\hline 276 & 29.9 & 3000 & 2350 & & 0.2617 & 118 & \begin{tabular}{l|l}
63.55 \\
\end{tabular} & 5125 & 0.1 & 1950 & 7500 & & 0.1064 & "\#\#\#\#" & $\begin{array}{l}4.083 \\
\end{array}$ & & \begin{tabular}{|l|}
30.7 \\
\end{tabular} & 24.84 & 0 & & 142.6 & $\begin{array}{ll}300.4 \\
699.8\end{array}$ \\
\hline 277 & 23.51 & 3000 & 970 & & 1.232 & 2115.9 & 64.73 & 5125 & 0.1 & 1950 & 7500 & & 0.13 & \#\#\#\#\# & 61 & & $|52.57|$ & 84,33 & 0 & & 127.9 & $\begin{array}{l}0959.0 \\
492.7 \\
\end{array}$ \\
\hline \begin{tabular}{|l|l|}
278 \\
\end{tabular} & 48.79 & 3000 & 760 & & 0.4232 & \begin{tabular}{ll|}
142.5 \\
\end{tabular} & 52.62 & 5125 & 0.1 & 1950 & 7500 & & 0.06 & \#\#\#\#\#| & 98 & & 32.64 & 43.87 & 0 & & 49.6 & $\begin{array}{l}432.91 \\
304.4 \\
\end{array}$ \\
\hline 279 & 50.74 & 3000 & $\begin{array}{ll}1190 \\
\end{array}$ & & \begin{tabular}{|l|l|l|}
0.3239 \\
\end{tabular} & 132.6 & 56.56 & 5125 & 0.1 & 1950 & 7500 & & 0.0627 & "\#\#\#\#" & \begin{tabular}{|c|}
3.908 \\
\end{tabular} & & 41,8 & 31.78 & 0 & & 69.7 & $\begin{array}{l}304.4 \\
394.7 \\
\end{array}$ \\
\hline 280 & 43.05 & 3000 & 430 & \begin{tabular}{|l|l|}
134.15 \\
\end{tabular} & 5.8081 & \begin{tabular}{|l|} 
\\
\end{tabular} & 57.75 & 5125 & 0.1 & 1950 & 7500 & & 0.07 & "\#\#"\#\#| & & & 58.6 & 96.27 & \begin{tabular}{|l|l|}
62.73 \\
\end{tabular} & & 86.9 & 234.9 \\
\hline 281 & 41.21 & 3000 & 1690 & & 0.0771 & \begin{tabular}{|c|} 
\\
\end{tabular} & 51.23 & 5125 & 0.1 & 1950 & 7500 & 0000 & 0.07 & "\#\#\#" & 3.924 & & \begin{tabular}{|l|l|}
14.68 \\
\end{tabular} & & 0 & & 62.9 & 488 \\
\hline 282 & 23.07 & 3000 & 450 & & 12.041 & \begin{tabular}{|l|l|}
143.2 \\
\end{tabular} & 52.38 & $\begin{array}{l}5125 \\
\end{array}$ & 0.1 & 1950 & 7500 & 7500 & 0.1379 & \#\#\#\#| & $\begin{array}{l}1.443 \\
\end{array}$ & & $\begin{array}{ll}62.94 \\
\end{array}$ & $\begin{array}{l}98.02 \\
\end{array}$ & 80.18 & & 193.1 & 320.5 \\
\hline 283 & 43.94 & 3000 & 880 & 3657 & 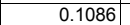 & \begin{tabular}{|l|}
139.9 \\
\end{tabular} & \begin{tabular}{|l|l|}
53.6 \\
\end{tabular} & 5125 & 0.1 & 1950 & 7500 & 1775000 & & 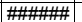 & 3.122 & & 42.05 & & & & 44.1 & 364.1 \\
\hline 284 & 25.97 & 3000 & 900 & & \begin{tabular}{|c|}
0.1099 \\
\end{tabular} & \begin{tabular}{|l|}
77.91 \\
\end{tabular} & $\overline{96.27}$ & 5125 & 0.1 & 1950 & 7500 & & & 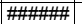 & \begin{tabular}{|l|l|}
2.39 \\
\end{tabular} & & 53.71 & 0 & 0 & & 57.5 & 471.7 \\
\hline 285 & \begin{tabular}{|l|l|l}
24.43 \\
\end{tabular} & 3000 & 960 & & 0.0293 & 123 & & 5125 & 0.1 & 1950 & 7500 & & 0.13 & |\#\#\#\#+ & 2.471 & & 49 & 0 & 0 & & \begin{tabular}{l|l|}
49.4 \\
\end{tabular} & 518.3 \\
\hline 286 & 39.28 & 3000 & 1580 & & 0.0933 & \begin{tabular}{|l|}
125.8 \\
\end{tabular} & & 5125 & 0.1 & 1950 & 7500 & & & 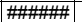 & 3.705 & & 16.77 & 0 & 0 & & 64.1 & 483.5 \\
\hline 287 & 23.71 & 3000 & 1790 & 2.362211 & \begin{tabular}{|c|}
0.0849 \\
\end{tabular} & \begin{tabular}{|l|}
107.8 \\
\end{tabular} & 69.59 & 5125 & 0.1 & 1950 & 7500 & & \begin{tabular}{l|l}
0.1342 \\
\end{tabular} & "\#\#\#\#| & 3.25 & & 38.38 & 0 & 0 & & 95.3 & 702.6 \\
\hline 288 & 32.43 & 3000 & 370 & 268.020565 & 12.1453 & \begin{tabular}{|l|}
135.9 \\
\end{tabular} & & 5125 & 0.1 & 1950 & 7500 & 1700000 & 0.0981 & & $\begin{array}{l}1.527 \\
\end{array}$ & & | 43.48 & \begin{tabular}{|c|}
98.13 \\
\end{tabular} & 81.34 & & 112 & 241. \\
\hline 289 & 34.06 & 3000 & 1740 & & & \begin{tabular}{|l|}
110.7 \\
\end{tabular} & & 5125 & 0.1 & 1950 & & & & & 3.872 & & 25.85 & 0 & 0 & & 53.9 & 582. \\
\hline 290 & 46.65 & 3000 & 360 & & 6.43 & \begin{tabular}{|l|}
103.2 \\
\end{tabular} & & 5125 & 0.1 & 1950 & 7500 & & & & & & & 97.32 & 73.25 & & 5 & 198.5 \\
\hline 291 & \begin{tabular}{|l|l|}
46.78 \\
\end{tabular} & 3000 & 260 & \begin{tabular}{|l|l}
1060. \\
\end{tabular} & & \begin{tabular}{|l|l|}
83.95 \\
\end{tabular} & & 5125 & 0.1 & 1950 & 756 & & & & & & & \begin{tabular}{|c|}
99.53 \\
\end{tabular} & \begin{tabular}{|l|l|}
95.29 \\
\end{tabular} & 52.87 & 140.3 & $157 .$. \\
\hline 292 & 31. & & $\begin{array}{ll}1490 \\
\end{array}$ & & & \begin{tabular}{|l|}
93.84 \\
\end{tabular} & & 5125 & 0.1 & & & & & & & & & 0 & 0 & 0 & 63 & 555.7 \\
\hline 293 & 26.71 & & 1030 & & & \begin{tabular}{|l|}
118.7 \\
\end{tabular} & & 5125 & 0.1 & & & & & & & & & 14.42 & 0 & & 3 & 507.1 \\
\hline 294 & 26.76 & & 1320 & & & \begin{tabular}{|l|} 
\\
\end{tabular} & & & 0.1 & & & & & & & & & 87.25 & 0 & & 161.3 & 521.6 \\
\hline 295 & 25.95 & & 550 & & & 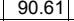 & & & 0.1 & & & & & & & & & & 23.69 & & 78.9 & 336. \\
\hline 296 & 34.02 & & 470 & & 5.1446 & 144.5 & & & 0.1 & & & & & & & & & & 53.2 & & 99.9 & 276. \\
\hline 297 & & & 460 & & 0.6 & \begin{tabular}{|l|l|} 
\\
\end{tabular} & & & 0.1 & & & & & & & & & 48.96 & & & 57 & 358.5 \\
\hline 298 & & & 960 & & $\begin{array}{ll}0.225 \\
\end{array}$ & \begin{tabular}{|l|}
155.9 \\
\end{tabular} & & & 0.1 & & & & & & & & & & & & 63.6 & 428.1 \\
\hline 299 & 22.23 & & 1180 & & 1.2426 & \begin{tabular}{|l|}
125.8 \\
\end{tabular} & 59. & & 0.1 & & & & & & & & & 83.13 & & & & 558.8 \\
\hline 300 & 39.37 & & 1220 & & 0.03 & \begin{tabular}{|l|l|}
129.9 \\
\end{tabular} & & & 0.1 & & & & & & & & & 0 & & & & 520.5 \\
\hline 301 & 23.29 & 3000 & $\begin{array}{r}380 \\
\end{array}$ & 11.29 & $\begin{array}{l}0.494 \\
\end{array}$ & \begin{tabular}{|l|}
131.2 \\
\end{tabular} & & 51 & 0.1 & & & & & & & & & 55.72 & & & 38.2 . & 285.9 \\
\hline 302 & 41.37 & & 1520 & 1.2 & & \begin{tabular}{|l|l|}
142.5 \\
$12 n$
\end{tabular} & & & 0. & & & & 0.0 & & & & 26.7 & 0 & & & 58.3 & 485.4 \\
\hline 303 & 52.76 & 3000 & $\begin{array}{r}740 \\
\end{array}$ & $\frac{20.79}{10}$ & 0.8525 & \begin{tabular}{|r|}
123 \\
\end{tabular} & 60.99 & & 0.1 & 1950 & & & 0.06 & & & & 55.2 & 75.96 & & & 62.8 & 300.4 \\
\hline 304 & 21.5 & 3000 & 1410 & $\begin{array}{r}1.93862 \\
\end{array}$ & 0.0983 & \begin{tabular}{|l|}
152.1 \\
\end{tabular} & 49.3 & 5125 & 0.1 & $\begin{array}{l}1950 \\
1050\end{array}$ & & & 0.1 & & 77 | & & 21.3 & $\begin{array}{r}0 \\
\end{array}$ & 0 & & 80 & 614.3 \\
\hline 305 & 49.02 & 3000 & 1520 & & 0.6779 & \begin{tabular}{|l|}
133.9 \\
93.9
\end{tabular} & $\begin{array}{r}56 \\
10271\end{array}$ & $\frac{5125}{5125}$ & 0.1 & $\frac{195}{195}$ & & & & & & & 17. & $\begin{array}{l}67.07 \\
08.49\end{array}$ & 0 & & $\frac{95}{70}$ & 418 \\
\hline 年 & 年 & 然 & $\frac{420}{1120}$ & & 6.1413 & \begin{tabular}{|l|l|} 
\\
\end{tabular} & $\frac{122.17}{28.19}$ & ST125 & 0.1 & & & & & & & & 6.8 & 90.49 & & & 18.5 & $\frac{186.6}{256}$ \\
\hline 年 & 50.42 & 3000 & 1130 & & 2.4816 & $\mid$ & 64.33 & $\frac{512}{512}$ & 0.1 & & & & & & & & & 92.11 & 27.11 & & $\frac{140}{50}$ & 356 \\
\hline 年 & $\frac{25.05}{34.39}$ & (300 & 1700 & & & $\mid$ & $\frac{50}{71}$ & $\frac{512}{512}$ & 0 & & & & & & & & & & t & & 23.4 & $\frac{486.6}{701}$ \\
\hline 310 & | & 300 & 240 & \begin{tabular}{|c|}
6371 \\
\end{tabular} & 20.2714 & $|705.04|$ & 78 & 515 & 0.1 & 195 & & & & & & & & 9922 & 9215 & 21.53 & 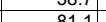 & 145 \\
\hline 311 & 247 & & 1590 & & 101 & 1502 & & 515 & 01 & 195 & & & & & & & 252 & 7528 & & & 1655 & $\frac{140.4}{601.5}$ \\
\hline 312 & 32.6 & & 960 & & 0.02 & \begin{tabular}{|l|}
126.4 \\
\end{tabular} & & 5125 & 0.1 & 195 & & & & & & & $\frac{2.0}{46.0}$ & 0 & 0 & & 42.4 & 455.2 \\
\hline 313 & 27.62 & 3000 & 1850 & & 0.00 & \begin{tabular}{|l|}
135.9 \\
\end{tabular} & 55.17 & 5125 & 0.1 & 1950 & 7500 & & & & 4.306 & & 45.57 & 0 & 0 & & 39.7 & 9. \\
\hline 314 & 21.82 & 3000 & 1480 & 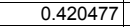 & \begin{tabular}{|l|}
0.022 \\
\end{tabular} & \begin{tabular}{|l|}
157.1 \\
\end{tabular} & 47.73 & 5125 & 0.1 & 1950 & 7500 & & 0.1458 & & 3.108 & $1.01 \mathrm{E}-02$ & 61.34 & 0 & 0 & & 68.9 & 730.1 \\
\hline 315 & 29.38 & 3000 & 1820 & 0.515903 & 0.0168 & \begin{tabular}{|l|}
97.81 \\
\end{tabular} & \begin{tabular}{|l|l|}
76.68 \\
\end{tabular} & 5125 & 0.1 & 1950 & 7500 & \begin{tabular}{|c|}
1037500 \\
\end{tabular} & 0.1083 & 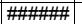 & 3.81 & $1.09 \mathrm{E}-02$ & 36.67 & & & & $\begin{array}{l}63.7 \\
\end{array}$ & 664.7 \\
\hline
\end{tabular}




\begin{tabular}{|c|c|c|c|c|c|c|c|c|c|c|c|c|c|c|c|c|c|c|c|c|c|c|}
\hline Realization & $\begin{array}{l}\text { Velocity } \\
(\mathrm{m} / \mathrm{y})\end{array}$ & $\begin{array}{l}\text { Fixed Source } \\
\text { Concentration } \\
(\mathrm{mg} / \mathrm{L})\end{array}$ & $\begin{array}{c}\text { Time of } \\
\text { Maximum } \\
\text { at } \\
\text { Compliance } \\
\text { Boundary } \\
\text { (y) }\end{array}$ & $\begin{array}{c}\text { Maximum } \\
\text { Concentration } \\
\text { Compliance } \\
\text { Boundary } \\
(\mu \mathrm{g} / \mathrm{L})\end{array}$ & $\begin{array}{c}\text { Maximum } \\
\text { Release Rate } \\
\text { at } \\
\text { Compliance } \\
\text { Boundary } \\
(\mathrm{Kg} / \mathrm{y})\end{array}$ & $\begin{array}{l} \\
\text { Sourc } \\
\text { e Rate } \\
\text { (Kg/y) }\end{array}$ & $\begin{array}{c}\text { Source } \\
\text { Duration } \\
\text { (y) }\end{array}$ & $\begin{array}{c}\text { 1-D } \\
\text { Streamtube } \\
\text { Length } \\
(\mathrm{m})\end{array}$ & $\mid \begin{array}{c}\text { Base } \\
\text { Porosity } \\
\left(\mathrm{m}^{3} / \mathrm{m}^{3}\right)\end{array}$ & $\begin{array}{c}\text { Bulk } \\
\text { Densty } \\
\left(\mathrm{Kg} / \mathrm{m}^{3}\right)\end{array}$ & \begin{tabular}{|c|} 
Remaining \\
Carbon \\
Tetrachloride \\
Source \\
$(\mathrm{Kg})$
\end{tabular} & $\begin{array}{c}\text { Volume at or } \\
\text { above Fixed } \\
\text { Source } \\
\begin{array}{c}\text { Concentratio } \\
n(m 3)\end{array}\end{array}$ & $\begin{array}{l}\text { Porosity } \\
\left(\mathrm{m}^{3} / \mathrm{m}^{3}\right)\end{array}$ & $\left(\begin{array}{c}\mathrm{Kd} \\
\left(\mathrm{m}^{3} / \mathrm{kg}\right)\end{array}\right.$ & Retardation & $\begin{array}{c}\text { First } \\
\text { Order } \\
\text { Rate } \\
\text { Constant } \\
(1 / y)\end{array}$ & $\begin{array}{l}\text { Longitudinal } \\
\text { Dispersivity } \\
(\mathrm{m})\end{array}$ & $\begin{array}{c}\text { Required } \\
\text { Source } \\
\text { Cleanup } \\
\text { Percentage } \\
(5 \mu \mathrm{g} / \mathrm{L} \\
\text { Compliance })\end{array}$ & $\begin{array}{c}\text { Required } \\
\text { Source } \\
\text { Cleanup } \\
\text { Percentage } \\
(50 \mu \mathrm{g} / \mathrm{L} \\
\text { Compliance })\end{array}$ & \begin{tabular}{|c} 
Required \\
Source Cleanup \\
Percentage \\
(500 $\mu \mathrm{g} / \mathrm{L}$ \\
Compliance)
\end{tabular} & \begin{tabular}{|c|} 
Abiotic \\
Reaction \\
Half Time \\
(y)
\end{tabular} & $\begin{array}{c}\text { Carbon } \\
\text { Tetrachloride } \\
\text { Travel Time } \\
\text { (y) }\end{array}$ \\
\hline & 24.82 & $\begin{array}{r}3000 \\
\end{array}$ & & \begin{tabular}{|l|}
12.320283 \\
\end{tabular} & $\begin{array}{r} \\
\end{array}$ & 141.9 & \begin{tabular}{|l|}
52.86 \\
\end{tabular} & 5125 & & & & 1812500 & \begin{tabular}{|r|}
0.1282 \\
\end{tabular} & \#\#\#\#+ & 3.804 & $3.08 \mathrm{E}-03$ & $\begin{array}{r}28.61 \\
\end{array}$ & 59.42 & & & & \\
\hline 317 & 45.77 & 3000 & 460 & 83.123283 & 2.4192 & 87.31 & 85.9 & 5125 & 0.1 & 1950 & 7500 & 875000 & 0.0695 & 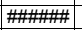 & 2.065 & $1.24 \mathrm{E}-02$ & 46.28 & 93.98 & 39.85 & & 55.9 & 231.2 \\
\hline 318 & 40.17 & 3000 & 590 & 72.932444 & 3.5593 & 146.4 & 51.23 & 5125 & 0.1 & 1950 & 7500 & 1900000 & 0.0792 & \#\#\#\#+ & 2.167 & $9.11 \mathrm{E}-03$ & 18.36 & 93.14 & 31.44 & & 76.1 & 276.5 \\
\hline 319 & 31.47 & 3000 & 510 & 177.626851 & 7.6091 & 128.5 & 58.36 & 5125 & 0.1 & 1950 & 7500 & 1562500 & 0.1011 & \#\#\#\#+ & 1.798 & $5.39 \mathrm{E}-03$ & 42.43 & 97.19 & 71.85 & & 128.6 & 292.8 \\
\hline 320 & 30.98 & 3000 & 590 & 32.691096 & 1.4227 & $\begin{array}{l}130.6 \\
\end{array}$ & 57.44 & 5125 & 0.1 & 1950 & 7500 & 1600000 & 0.1027 & 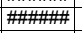 & 1.975 & $9.78 \mathrm{E}-03$ & 44.07 & 84.71 & & & 70.9 & 326.8 \\
\hline 321 & 38.01 & 3000 & 630 & 11.648458 & 0.6387 & \begin{tabular}{ll|}
164.5 \\
\end{tabular} & 45.6 & 5125 & 0.1 & 1950 & 7500 & 2262500 & 0.0837 & \#\#\#\#+1 & 2.264 & $1.34 \mathrm{E}-02$ & 29.69 & 57.08 & & & 51.7 & 305.3 \\
\hline 322 & 36.4 & 3000 & 1400 & 0.337714 & 0.013 & \begin{tabular}{ll|}
115.9 \\
\end{tabular} & 64.73 & 5125 & 0.1 & 1950 & 7500 & 1337500 & 0.0874 & \#\#\#\#+1 & 4.035 & $1.47 \mathrm{E}-02$ & 52.91 & & & & 47.1 & 568.1 \\
\hline 323 & 38.24 & 3000 & 1080 & 0.510576 & 0.0216 & 127.1 & 58.99 & 5125 & 0.1 & 1950 & 7500 & 1537500 & 0.0832 & 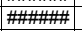 & 3.007 & $1.83 \mathrm{E}-02$ & 16 & & & & 37.9 & 403 \\
\hline 324 & 33.81 & 3000 & 800 & 70.961001 & 3.0235 & 127.8 & 58.67 & 5125 & 0.1 & 1950 & 7500 & 1550000 & 0.0941 & \#\#\#\#\#+ & 2.413 & $5.22 \mathrm{E}-03$ & 46.39 & 92.95 & 29.54 & & 132.9 & 365.8 \\
\hline 325 & 25.8 & 3000 & 700 & 2.373846 & 0.1263 & 159.6 & 46.99 & 5125 & 0.1 & 1950 & 7500 & 2162500 & 0.1233 & \#\#\#\#+ & 1.987 & $1.48 \mathrm{E}-02$ & 30.93 & & & & 46.9 & 394.8 \\
\hline 326 & 27.78 & 3000 & 670 & & 1.7132 & 159 & 47.17 & 5125 & 0.1 & 1950 & 7500 & 2150000 & 0.1145 & \#\#\#\#\#+ & 1.938 & $8.84 \mathrm{E}-03$ & 20.16 & 84.53 & & & 78.4 & 357.4 \\
\hline & 27.17 & 3000 & 1370 & 5.629183 & $\begin{array}{ll}0.2576 \\
\end{array}$ & \begin{tabular}{|l|}
137.3 \\
\end{tabular} & 54.64 & 5125 & 0.1 & 1950 & 7500 & 1725000 & & \#\#\#\#+ & 2.883 & $7.69 \mathrm{E}-03$ & 27.41 & 11.18 & & & 90.1 & 543.9 \\
\hline 328 & 27.4 & 3000 & 390 & & 2.8079 & $\begin{array}{l}130.6 \\
\end{array}$ & 57.44 & 5125 & 0.1 & 1950 & 7500 & 1600000 & & \#\#\#\#+ & 1.505 & $1.01 \mathrm{E}-02$ & 72.11 & 92.25 & 22.5 & & 68.9 & 281.4 \\
\hline 329 & 56.11 & 3000 & 2500 & 0.127118 & 0.0048 & $\begin{array}{l}113.7 \\
\end{array}$ & & 5125 & 0.1 & 1950 & 7500 & 1300000 & & \#\#\#\#+ & 6.568 & $1.46 \mathrm{E}-02$ & 27.25 & & 0 & & 47.4 & 599.9 \\
\hline 330 & 33.17 & 3000 & 430 & 135.003368 & $\begin{array}{l}7.4835 \\
\end{array}$ & \begin{tabular}{ll|}
166.3 \\
\end{tabular} & 45.1 & 5125 & 0.1 & 1950 & 7500 & 2300000 & & \#\#\#\#+ & 1.662 & $8.15 \mathrm{E}-03$ & 23.25 & 96.3 & \begin{tabular}{ll|}
62.96 \\
\end{tabular} & & 85.1 & 256.7 \\
\hline 331 & 38.84 & 3000 & 260 & 52.675688 & 2.8347 & \begin{tabular}{ll|}
161.4 \\
\end{tabular} & 46.46 & 5125 & 0.1 & 1950 & 7500 & 2200000 & & \#\#\#\#+ & 1.469 & $1.68 \mathrm{E}-02$ & 81.45 & 90.51 & 5.08 & & 41.2 & 193.8 \\
\hline 332 & 53.92 & 3000 & 1340 & & 0.6604 & \begin{tabular}{|l|l|}
110.7 \\
\end{tabular} & 67.72 & 5125 & 0.1 & 1950 & 7500 & & 0.059 & \#\#\#\#曲 & & $8.26 \mathrm{E}-03$ & 21.41 & 72.05 & & & 83.9 & 378.1 \\
\hline 333 & 22.61 & 3000 & 1400 & & $\begin{array}{l}0.4309 \\
\end{array}$ & $\begin{array}{l}134.6 \\
\end{array}$ & 55.72 & 5125 & 0.1 & 1950 & 7500 & 1675000 & 0.1407 & \#\#\#\#曲 & 2.576 & $6.45 \mathrm{E}-03$ & 18.19 & \begin{tabular}{ll|}
47.93 \\
\end{tabular} & 0 & & 107.5 & 583.8 \\
\hline 334 & 23.83 & 3000 & 570 & \begin{tabular}{|l|l|}
147.040241 \\
\end{tabular} & 5.5362 & 113 & $\begin{array}{r}66.4 \\
\end{array}$ & 5125 & 0.1 & 1950 & 7500 & 1287500 & 0.1335 & \#\#\#\# & 1.652 & $5.14 \mathrm{E}-03$ & 43.66 & $\begin{array}{l}96.6 \\
\end{array}$ & 66 & & 134.7 & 355.3 \\
\hline $\begin{array}{l}335 \\
336\end{array}$ & & & 2250 & \begin{tabular}{|r|}
2.582533 \\
\end{tabular} & $\begin{array}{ll}0.0552 \\
50202\end{array}$ & $\begin{array}{l}64.07 \\
\end{array}$ & 117.06 & 5125 & 0.1 & 1950 & 7500 & $\begin{array}{r}550000 \\
1500\end{array}$ & 0.091 & \#\#\#\# & $\begin{array}{l}5.06 \\
\end{array}$ & 7.60E-03 & 60.02 & 0 & 0 & & 91.2 & 741.7 \\
\hline $\begin{array}{l}336 \\
337\end{array}$ & 37.65 & $\begin{array}{ll}3000 \\
3000\end{array}$ & \begin{tabular}{|l|l}
570 \\
380
\end{tabular} & \begin{tabular}{|r|}
124.701975 \\
314.22325 \\
\end{tabular} & $\begin{array}{r}5.0823 \\
14.0243 \\
\end{array}$ & $\begin{array}{l}122.3 \\
1339 \\
\end{array}$ & $\begin{array}{ll}61.34 \\
\end{array}$ & $\begin{array}{l}5125 \\
525\end{array}$ & $\begin{array}{l}0.1 \\
0.1\end{array}$ & $\begin{array}{l}1950 \\
1950\end{array}$ & 7500 & 1450000 & \begin{tabular}{|l|l|l|l}
0.0845 \\
\end{tabular} & 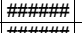 & $\begin{array}{l}2.144 \\
1.20 \\
\end{array}$ & 5.64E-03 & $\begin{array}{l}61.36 \\
2505 \\
\end{array}$ & $\begin{array}{l}95.99 \\
0.91\end{array}$ & 59.9 & & $\begin{array}{ll}122.8 \\
1103\end{array}$ & 291.9 \\
\hline 338 & $\begin{array}{r}28.92 \\
34.28\end{array}$ & 3000 & $\begin{array}{l}380 \\
320\end{array}$ & \begin{tabular}{|l|}
314.122325 \\
377.939625
\end{tabular} & $\mid \begin{array}{l}\frac{14.0243}{16.2761} \\
\end{array}$ & $\begin{array}{r}133.9 \\
129.2\end{array}$ & \begin{tabular}{r|}
56 \\
58.05 \\
\end{tabular} & $\begin{array}{l}5125 \\
5125\end{array}$ & 0.1 & $\begin{array}{l}1950 \\
1950\end{array}$ & $\begin{array}{l}7500 \\
7500\end{array}$ & \begin{tabular}{|r|}
1662500 \\
1575000
\end{tabular} & $\begin{array}{r}0.11 \\
0.098 \\
\end{array}$ & |\#\#\#\# & $\begin{array}{l}1.429 \\
1.429 \\
\mid\end{array}$ & $\begin{array}{c}6.29 \mathrm{E}-03 \\
6.34 \mathrm{E}-03 \\
\end{array}$ & $\begin{array}{l}25.05 \\
45.59\end{array}$ & $98.41 \mid$ & $\begin{array}{l}84.08 \\
86.77\end{array}$ & & $\begin{array}{ll}110.3 \\
109.3\end{array}$ & 253.2 \\
\hline 339 & 37.47 & 3000 & 620 & \begin{tabular}{|l|}
118.504109 \\
\end{tabular} & (6.1578 & \begin{tabular}{|l|}
155.9 \\
\end{tabular} & 48.11 & 5125 & 0.1 & 1950 & 7500 & 2087500 & $\begin{array}{l}.0920 \\
0.0849\end{array}$ & 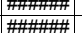 & $\frac{1.429}{2.335}$ & \begin{tabular}{|l|}
$6.34 \mathrm{E}-03$ \\
$3.62 \mathrm{E}-03$ \\
\end{tabular} & $\begin{array}{l}45.59 \\
69.24\end{array}$ & $\begin{array}{l}90.00 \\
95.78\end{array}$ & 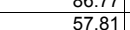 & & $\begin{array}{l}\frac{109.3}{191.4} \\
\end{array}$ & $\frac{213.7}{319.4}$ \\
\hline 340 & 23.64 & 3000 & 1510 & \begin{tabular}{|l|}
3.823289 \\
\end{tabular} & 0.1196 & 93.84 & 79.92 & 5125 & 0.1 & 1950 & 7500 & 975000 & 0.1346 & 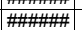 & 2.896 & $7.83 \mathrm{E}-03$ & 34.54 & & & & 88.5 & - \\
\hline 341 & 53.02 & 3000 & 2810 & 5.347731 & 0.126 & 70.69 & 106.09 & 5125 & 0.1 & 1950 & 7500 & 637500 & 0.06 & \#\#\#\#+ & 6.612 & $6.28 \mathrm{E}-03$ & 41.62 & 6.5 & & & 110.3 & 639. \\
\hline 342 & 38.47 & 3000 & 1090 & 30.706526 & 0.9605 & 93.84 & 79.92 & 5125 & 0.1 & 1950 & 7500 & 975000 & 0.0827 & \#\#\#\#+ & 3.098 & $6.74 \mathrm{E}-03$ & 42.48 & 83.72 & & & 102.8 & 412.8 \\
\hline 343 & 60.03 & 3000 & 2720 & $\begin{array}{l}.687185 \\
\end{array}$ & 0.0959 & 107 & 70.08 & 5125 & 0.1 & 1950 & 7500 & 1187500 & 0.053 & \#\#\#\#+ & 6.904 & $7.51 \mathrm{E}-03$ & 35.97 & 0 & & & 92.2 & 589. \\
\hline 344 & 35.95 & 3000 & 640 & 31.858473 & 1.3501 & 127.1 & 58.99 & 5125 & 0.1 & 1950 & 7500 & 1537500 & & \#\#\#\#+ & 2.363 & $8.67 \mathrm{E}-03$ & 76.5 & 84.31 & & & 80 & 336 \\
\hline 345 & 50.9 & 3000 & 1730 & 7.23602 & 0.3715 & 154 & $\begin{array}{l}48.7 \\
\end{array}$ & 5125 & 0.1 & 1950 & 7500 & 2050000 & 0.0625 & \#\#\#\#+ & 4.717 & & 37.46 & 30.9 & & & 101 & 474. \\
\hline 346 & 40.22 & 3000 & 260 & \begin{tabular}{|l|}
200.150682 \\
\end{tabular} & 5.9355 & 88.97 & 84.3 & 5125 & 0.1 & 1950 & 7500 & 900000 & & \#\#\#\#+ & 1.368 & & 54.12 & 97.5 & 75.02 & & 47.9 & 174. \\
\hline 347 & 51.64 & 3000 & 660 & 6.011 & 0.2738 & \begin{tabular}{|l|}
136.6 \\
\end{tabular} & 54.9 & 5125 & 0.1 & 1950 & 7500 & 1712500 & & \#\#\#\#+ & $\begin{array}{l}3.042 \\
\end{array}$ & & 65.7 & 16.83 & 0 & & 42.3 & 301. \\
\hline 348 & 38.56 & 3000 & 1590 & & 0.084 & 127.1 & 58.99 & 5125 & 0.1 & 1950 & 7500 & 1537500 & & & 4.194 & & 50.47 & 0 & & & 72.2 & 557. \\
\hline 349 & 50.66 & 3000 & 530 & & 1.4929 & 153.4 & 48.9 & 5125 & 0.1 & 1950 & 7500 & & & & 2.4 & & 44.75 & 82.88 & & & 54 & 247. \\
\hline 350 & & & 990 & & 0.1221 & 93.04 & 80.6 & 5125 & 0.1 & 1950 & 7500 & & & & & & & 0 & 0 & & 64.9 & 508. \\
\hline 351 & 56.71 & & 420 & 52.42 & & 109 & 68.6 & & 0.1 & & 7500 & & & & & & & 90.46 & 4.62 & & 44.6 & \\
\hline 352 & & & 760 & & & 12 & & & 0.1 & & 50 & & & & & & & 78.97 & 0 & & 98.4 & \\
\hline 353 & & & 1050 & & 0.725 & 159 & & & 0.1 & & 75 & & & & & & & 63.33 & 0 & & 87 & 386.8 \\
\hline 354 & 23. & & 1380 & & & & & & 0.1 & & 750 & & & & & & & 0 & 0 & & 70.3 & 1.3 .3 \\
\hline 355 & & & 2990 & & & 104 & & & & & 75 & & & & & & & & 0 & & & 7.2 \\
\hline 356 & 29.4 & & 370 & & & 93.84 & 79. & & & & & & & & & & & 95.83 & 58.33 & & & 45.5 \\
\hline 357 & 37.34 & & \begin{tabular}{ll|}
370 \\
\end{tabular} & & 5.6302 & $\begin{array}{l}102.5 \\
\end{array}$ & & 51 & 0.1 & & & & & & & & & 96 & \begin{tabular}{ll|}
69.67 \\
\end{tabular} & & & 226.5 \\
\hline 358 & 27.26 & & 730 & & & 142.5 & & 51 & 0.1 & & & & & & 2.0 & & & 15.02 & 0 & & 55.9 & 391.9 \\
\hline 359 & 23.37 & & 90 & & 3185 & $\begin{array}{r}84.8 \\
\end{array}$ & & 51 & 0.1 & & & & 0. & & & & & 55.62 & 0 & & 73.2 & 469.2 \\
\hline 360 & 21.7 & $\begin{array}{l}3000 \\
3000 \\
300\end{array}$ & 780 & & \begin{tabular}{|l|l|l|l|} 
\\
\end{tabular} & 178.7 & & 51 & 0.1 & 19 & 750 & & 0.1 & & 2.1 & & & 0 & 0 & & & 507.2 \\
\hline 361 & $\begin{array}{r}41.8 \\
37.12 \\
\end{array}$ & 3000 & $\begin{array}{l}450 \\
550\end{array}$ & \begin{tabular}{|l|}
142.655918 \\
32.20581
\end{tabular} & $\begin{array}{l}4.8726 \\
1.082\end{array}$ & $\begin{array}{l}102.5 \\
125.3\end{array}$ & $\begin{array}{l}73.19 \\
55.44 \\
\end{array}$ & $\begin{array}{l}5125 \\
5125\end{array}$ & 0.1 & $\begin{array}{l}1950 \\
1950\end{array}$ & 7500 & $\begin{array}{l}1112500 \\
167500\end{array}$ & 0.0 & & $\frac{1.9}{20}$ & & 43. & $\begin{array}{r}96.5 \\
89.95\end{array}$ & 64.95 & & 76.5 & 237.9 \\
\hline $\begin{array}{l}362 \\
363 \\
\end{array}$ & $\frac{37.12}{57.63}$ & 3000 & \begin{tabular}{r|r|r}
5500 & 4000
\end{tabular} & $\begin{array}{r}33.225841 \\
\end{array}$ & $\begin{array}{l}1.4982 \\
0.0069\end{array}$ & $\begin{array}{l}135.3 \\
8897\end{array}$ & 55.44 & $\begin{array}{l}5125 \\
5125\end{array}$ & 0.1 & $\frac{195}{195}$ & $\begin{array}{l}7500 \\
7500\end{array}$ & 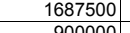 & 0.0 & 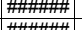 & 2.08 & & $\begin{array}{ll}36 . \\
18 .\end{array}$ & 84.95 & 0 & & 61.3 & 287.4 \\
\hline $\begin{array}{l}363 \\
364 \\
\end{array}$ & $\frac{57.63}{35.99}$ & $\frac{3000}{3000}$ & $\begin{array}{r}4000 \\
910\end{array}$ & \begin{tabular}{r|r|}
0.2311292 \\
31.031176
\end{tabular} & $|0.0069|$ & \begin{tabular}{|l|}
88.97 \\
127.8
\end{tabular} & \begin{tabular}{|r|r|r|}
58.67 & \\
\end{tabular} & $\begin{array}{l}5125 \\
5125\end{array}$ & $\begin{array}{l}0.1 \\
0.1\end{array}$ & $\frac{195}{195}$ & $\begin{array}{l}7500 \\
7500\end{array}$ & $\begin{array}{r}9900000 \\
1550000\end{array}$ & $\frac{0.0}{0.0}$ & "\#\#\#\#曲 & $\frac{8.06}{2.71}$ & & $\frac{18 .}{44 .}$ & \begin{tabular}{r|}
0 \\
83.89
\end{tabular} & & & $\begin{array}{r}67.1 \\
101.1\end{array}$ & $\begin{array}{c}717.2 \\
387\end{array}$ \\
\hline 365 & & 300 & 660 & & \begin{tabular}{|c|}
1.9228 \\
\end{tabular} & 125.8 & 59.6 & 512 & 0.1 & 195 & 7500 & 1512 & 0.1 & 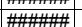 & 1.97 & & 38.2 & \begin{tabular}{|l|}
89.1 \\
\end{tabular} & & & 89.4 & 359.1 \\
\hline 366 & 25.43 & 300 & 900 & & 0.121 & 136.6 & 54 & 512 & 0.1 & 195 & 750 & 1712 & & \#\#\# & 2.2 & & 26. & 0 & 0 & & 55.8 & 450.2 \\
\hline 367 & 29.43 & 300 & 860 & & 0.1204 & \begin{tabular}{|l|}
133.3 \\
\end{tabular} & 56.28 & 512 & 0.1 & 195 & 7500 & & & \#\#車 & 2.3 & & 24. & 0 & 0 & & 50.1 & 409 \\
\hline 368 & 29.29 & 3000 & 580 & 126.3 & 6.19 & 147 & 51 & 512 & 0.1 & 195 & 7500 & & & & 1.8 & & 59. & 96.04 & 60.44 & & 155.6 & 331.2 \\
\hline 369 & 62.14 & 3000 & 1280 & & 0.50 & 150.2 & 49. & 512 & 0.1 & 195 & 7500 & & & & 4.6 & & 53. & 50.65 & 0 & & 83.3 & 383.4 \\
\hline 370 & 34.36 & 3000 & 2590 & & 0.00 & 175 & 42.6 & 512 & 0.1 & 195 & 7500 & & & & 4.9 & & 19. & & & & 46.2 & 733. \\
\hline 371 & & & 1290 & & 0.3 & & & 512 & 0.1 & 19 & 750 & & & & & & & 53.52 & & & 106.8 & 553. \\
\hline 372 & & 3000 & 1410 & & & & & & 0.1 & 195 & 7500 & & & & & & & & & & 70.1 & 486. \\
\hline 373 & & 3000 & 480 & & & 10 & & 51 & 0.1 & 19 & 7500 & & & & & & & 96.72 & 67.17 & & 76.6 & 6. \\
\hline 374 & & & 1550 & & & & & & 0.1 & & 750 & & & & & & & 58.06 & & & 87.8 & 429.8 \\
\hline 375 & & & 1690 & & & & & & 0.1 & & & & & & 3.4 & & & & 0 & & 130.4 & \\
\hline 376 & & & 840 & & 62 & 10 & & & 0.1 & & 7500 & & & & & & & & 0 & & 58 & \\
\hline 377 & 49.48 & 3000 & 310 & 135.349066 & 7.4482 & 165.1 & 45.43 & 5125 & 0.1 & 1950 & 7500 & & & & & & & 96.31 & 63.06 & & 68.6 & \\
\hline 378 & 25.55 & 3000 & 590 & 35.663503 & 1.1814 & $\begin{array}{l}99.38 \\
\end{array}$ & 75.47 & 5125 & & 1950 & 7500 & 1062500 & & \#\#\#\#+1| & 1.823 & & 66.37 & 85.98 & & & 76.4 & 365.7 \\
\hline
\end{tabular}




\begin{tabular}{|c|c|c|c|c|c|c|c|c|c|c|c|c|c|c|c|c|c|c|c|c|c|c|}
\hline Realization & $\begin{array}{c}\begin{array}{c}\text { Velocity } \\
(\mathrm{m} / \mathrm{y})\end{array} \\
\end{array}$ & $\begin{array}{c}\text { Fixed Source } \\
\text { Concentration } \\
(\mathrm{mg} / \mathrm{L})\end{array}$ & $\begin{array}{c}\text { Time of } \\
\text { Maximum } \\
\text { at } \\
\text { Compliance } \\
\text { Boundary } \\
\text { (y) } \\
\end{array}$ & $\begin{array}{c}\text { Maximum } \\
\text { Concentration } \\
\text { Compliance } \\
\text { Boundary } \\
(\mu \mathrm{g} / \mathrm{L})\end{array}$ & $\begin{array}{c}\text { Maximum } \\
\text { Release Rate } \\
\text { at } \\
\text { Compliance } \\
\text { Boundary } \\
\text { (Kg/y) }\end{array}$ & $\begin{array}{l} \\
\text { Sourc } \\
\text { e Rate } \\
(\mathrm{Kg} / \mathrm{y}) \\
\end{array}$ & \begin{tabular}{|c|} 
Source \\
Duration \\
(y)
\end{tabular} & $\begin{array}{c}1-\mathrm{D} \\
\text { Streamtube } \\
\text { Length } \\
(\mathrm{m})\end{array}$ & $\begin{array}{c}\text { Base } \\
\text { Porosity } \\
\left(\mathrm{m}^{3} / \mathrm{m}^{3}\right)\end{array}$ & $\begin{array}{c}\text { Bulk } \\
\text { Densty } \\
\left(\mathrm{Kg} / \mathrm{m}^{3}\right)\end{array}$ & \begin{tabular}{|c|}
$\begin{array}{c}\text { Remaining } \\
\text { Carbon } \\
\text { Tetrachloride } \\
\text { Source } \\
(\mathrm{Kg})\end{array}$ \\
\end{tabular} & $\begin{array}{c}\text { Volume at or } \\
\text { above Fixed } \\
\text { Source } \\
\text { Concentratio } \\
n(m 3)\end{array}$ & $\begin{array}{l}\text { Porosity } \\
\left(\mathrm{m}^{3} / \mathrm{m}^{3}\right) \\
\end{array}$ & $\left.\mid \begin{array}{c}\mathrm{Kd} \\
\left(\mathrm{m}^{3} / \mathrm{kg}\right)\end{array}\right)$ & Retardation & $\begin{array}{c}\text { First } \\
\text { Order } \\
\text { Rate } \\
\text { Constant } \\
(1 / y) \\
\end{array}$ & \begin{tabular}{|c} 
Longitudinal \\
Dispersivity \\
$(\mathrm{m})$
\end{tabular} & $\begin{array}{c}\text { Required } \\
\text { Source } \\
\text { Cleanup } \\
\text { Percentage } \\
(5 \text { ug/L } \\
\text { Compliance) } \\
\end{array}$ & $\begin{array}{c}\text { Required } \\
\text { Source } \\
\text { Cleanup } \\
\text { Percentage } \\
\text { (50 } \mathrm{\mu g} / \mathrm{L} \\
\text { Compliance) } \\
\end{array}$ & \begin{tabular}{|c|} 
Required \\
Source Cleanup \\
Percentage \\
(500 $\mathrm{\mu g} / \mathrm{L}$ \\
Compliance) \\
\end{tabular} & $\begin{array}{c}\text { Abiotic } \\
\text { Reaction } \\
\text { Half Time } \\
(y)\end{array}$ & $\begin{array}{c}\text { Carbon } \\
\text { Tetrachloride } \\
\text { Travel Time } \\
\text { (y) } \\
\end{array}$ \\
\hline & $\begin{array}{r}54.1 \\
28\end{array}$ & \begin{tabular}{|r|}
3000 \\
3000
\end{tabular} & \begin{tabular}{|r|}
640 \\
410
\end{tabular} & \begin{tabular}{|l|}
41.576013 \\
1950003
\end{tabular} & \begin{tabular}{|r|}
1.781 \\
10214
\end{tabular} & $\begin{array}{l}128.5 \\
1571\end{array}$ & $\begin{array}{r}58.36 \\
4733 \\
\end{array}$ & \begin{tabular}{|r|}
5125 \\
5125
\end{tabular} & $\begin{array}{l}0.1 \\
0.1\end{array}$ & $\begin{array}{l}1950 \\
1950\end{array}$ & $\begin{array}{r}7500 \\
\end{array}$ & $\begin{array}{l}1562500 \\
\end{array}$ & \begin{tabular}{|l|}
0.0588 \\
01136
\end{tabular} & 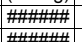 & \begin{tabular}{|r|}
2.958 \\
\end{tabular} & $8.92 \mathrm{E}-03$ & 80.6 & $\begin{array}{r}87.97 \\
\end{array}$ & 0 & & & \begin{tabular}{|r|}
280.2 \\
3031
\end{tabular} \\
\hline 380 & $\begin{array}{r}21.8 \\
21.8\end{array}$ & 3000 & \begin{tabular}{|l|}
4100 \\
430
\end{tabular} & \begin{tabular}{|r|}
19.500003 \\
212.499021
\end{tabular} & $\begin{array}{r}1.0214 \\
10.0496\end{array}$ & $\begin{array}{l}141.1 \\
141.9 \\
\end{array}$ & \begin{tabular}{|l|}
4.7 .3 \\
52.86 \\
\end{tabular} & \begin{tabular}{l|}
5125 \\
5125
\end{tabular} & $\begin{array}{l}0.1 \\
0.1\end{array}$ & $\begin{array}{l}1950 \\
1950\end{array}$ & $\begin{array}{l}7500 \\
7500\end{array}$ & \begin{tabular}{|l|}
2112500 \\
1812500
\end{tabular} & $\mid 0.1736$ | & "\#\#\#\#地 & $\begin{array}{r}1.656 \\
1.35\end{array}$ & \begin{tabular}{|l|l|}
$.28 \mathrm{E}-02$ \\
$5.33 \mathrm{E}-03$
\end{tabular} & $\begin{array}{l}94.67 \\
34.36\end{array}$ & $\begin{array}{l}74.36 \\
97.65\end{array}$ & $\begin{array}{r}0 \\
76.47\end{array}$ & $\begin{array}{l}\frac{0}{0} \\
\end{array}$ & $\begin{array}{r}54.3 \\
130\end{array}$ & $\begin{array}{l}303.1 \\
317.2 \\
\end{array}$ \\
\hline 382 & 24.59 & 3000 & 1330 & \begin{tabular}{|l|}
0.067534 \\
\end{tabular} & 0.0021 & 92.23 & 81.32 & 5125 & 0.1 & 1950 & 7500 & 950000 & 0.1294 & 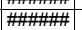 & 3.326 & $1.64 \mathrm{E}-02$ & 60.68 & & 0 & & 42.2 & 693.4 \\
\hline 383 & 30.15 & 3000 & 870 & 16.675201 & 0.7067 & 127.1 & 58.99 & 5125 & 0.1 & 1950 & 7500 & 1537500 & 0.1055 & 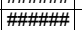 & 2.375 & $8.89 \mathrm{E}-03$ & 31.67 & 70.02 & 0 & & 78 & 403.6 \\
\hline 384 & 24.8 & 3000 & 800 & 0.327429 & 0.0102 & 93.84 & 79.92 & 5125 & 0.1 & 1950 & 7500 & 975000 & 0.1283 & \#\#\#\#+ & 2.528 & $1.85 \mathrm{E}-02$ & 83.92 & & 0 & & 37.4 & 522.4 \\
\hline 385 & 39.57 & 3000 & 330 & 96.603113 & 4.1822 & 129.9 & 57.75 & 5125 & 0.1 & 1950 & 7500 & 1587500 & 0.0804 & \#\#\#\#+ & 1.58 & $1.39 \mathrm{E}-02$ & 32.57 & 94.82 & 48.24 & & 49.8 & 204.6 \\
\hline 386 & 50.82 & 3000 & 2650 & 0.476106 & 0.0161 & 101.7 & 73.75 & 5125 & 0.1 & 1950 & 7500 & 1100000 & 0.0626 & \#\#\#\#+1 & 5.973 & $1.12 \mathrm{E}-02$ & 21.8 & & 0 & & 61.9 & 602.3 \\
\hline 387 & 56.41 & 3000 & 1050 & 1.579036 & 0.091 & 172.9 & 43.39 & 5125 & 0.1 & 1950 & 7500 & 2437500 & 0.0564 & 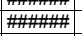 & 3.97 & $1.59 \mathrm{E}-02$ & 39.19 & & 0 & & 43.6 & 360.7 \\
\hline 388 & 25.41 & 3000 & 1790 & 0.141993 & 0.0051 & 108.5 & 69.11 & 5125 & 0.1 & 1950 & 7500 & 1212500 & 0.1252 & \#\#\#\#+ & 3.617 & $1.23 \mathrm{E}-02$ & 39.93 & & 0 & & 56.5 & 729.5 \\
\hline 389 & 36.32 & 3000 & 1940 & 4.320718 & 0.1781 & 123.7 & 60.65 & 5125 & 0.1 & 1950 & 7500 & 1475000 & 0.0876 & 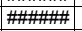 & 4.34 & $6.60 \mathrm{E}-03$ & 45.24 & 0 & 0 & & 105.1 & 612.5 \\
\hline 390 & 31.16 & 3000 & 950 & 6.354214 & 0.256 & 120.9 & 62.06 & 5125 & 0.1 & 1950 & 7500 & 1425000 & 0.1021 & 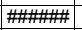 & 2.586 & $1.08 \mathrm{E}-02$ & 32.91 & 21.31 & 0 & & 64.1 & 425.4 \\
\hline 391 & 40.63 & 3000 & 1520 & 1.219917 & 0.0395 & 97.02 & 77.3 & 5125 & 0.1 & 1950 & 7500 & 1025000 & 0.0783 & 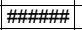 & 3.649 & $1.37 \mathrm{E}-02$ & 12.91 & 0 & 0 & & 50.6 & 460.2 \\
\hline 392 & 58.7 & 3000 & 700 & 6.468758 & 0.2741 & 127.1 & 58.99 & 5125 & 0.1 & 1950 & 7500 & 1537500 & 0.0542 & \#\#\#\#+ & 3.131 & $1.77 \mathrm{E}-02$ & 37.72 & 22.71 & 0 & & 39.1 & 273.4 \\
\hline 393 & 31.34 & 3000 & 540 & 149.52414 & 5.4089 & 108.5 & 69.11 & 5125 & 0.1 & 1950 & 7500 & 1212500 & 0.1015 & \#\#\#\#+ & 1.907 & $5.10 \mathrm{E}-03$ & 77.71 & 96.66 & 66.56 & & 135.8 & 311.8 \\
\hline 394 & 57.42 & 3000 & 1170 & 19.869732 & \begin{tabular}{|c|}
0.9739 \\
\end{tabular} & 147 & 51 & 5125 & 0.1 & 1950 & 7500 & 1912500 & & \#\#\#\#+ & 4.032 & $7.16 \mathrm{E}-03$ & 43.18 & 74.84 & 0 & & 96.8 & 359.9 \\
\hline 395 & 23.19 & 3000 & 1230 & 32.905486 & 1.5847 & 144.5 & 51.91 & 5125 & 0.1 & 1950 & 7500 & 1862500 & & & 2.499 & & 41.73 & 84.8 & & & 177 & 552.4 \\
\hline 396 & 21.34 & & 1240 & 0.219756 & 0.0061 & 83.1 & & 5125 & 0.1 & 1950 & 7500 & 812500 & & & 2.554 & $1.44 \mathrm{E}-02$ & 33.97 & 0 & & & 48.1 & 613.4 \\
\hline 397 & 24.3 & & 1410 & & & 81.39 & & 5125 & 0.1 & 1950 & 7500 & 787500 & & \#\#\#\#+ & 2.944 & $4.11 \mathrm{E}-03$ & 75.31 & 82.6 & & & 168.8 & 620.9 \\
\hline 398 & 22.48 & & 410 & & 0.2154 & 133.3 & & 5125 & 0.1 & 1950 & 7500 & 1650000 & & \#\#\#\#+ & 1.481 & $1.73 \mathrm{E}-02$ & 79.86 & 0 & 0 & & 40.2 & 337.7 \\
\hline 399 & 48.79 & 3000 & 520 & & 0.5133 & 132.6 & 56.56 & 5125 & 0.1 & 1950 & 7500 & 1637500 & & \#\#\#\# & 2.442 & $1.73 \mathrm{E}-02$ & 50.23 & 56.95 & & & 40 & 256.5 \\
\hline 400 & 44.74 & 3000 & 2120 & & $\begin{array}{l}0.0686 \\
\end{array}$ & 108.5 & $\begin{array}{ll}69.11 \\
\end{array}$ & 5125 & 0.1 & 1950 & 7500 & 1212500 & & \#\#\#曲 & 4.653 & 9.77E-03 & 16.18 & & 0 & $15=$ & 71 & 532.9 \\
\hline 401 & 40.42 & 3000 & 210 & \begin{tabular}{|r|}
589.906942 \\
\end{tabular} & 29.9146 & 152.1 & 49.3 & 5125 & 0.1 & 1950 & 7500 & 2012500 & 0.0787 & & 1.256 & $5.69 \mathrm{E}-03$ & \begin{tabular}{|l|}
92.87 \\
\end{tabular} & 99.15 & $\begin{array}{l}1.52 \\
\end{array}$ & 15.24 & \begin{tabular}{|l|l|}
121.8 \\
\end{tabular} & 159.2 \\
\hline $\begin{array}{ll}402 \\
403\end{array}$ & $\begin{array}{r}32.93 \\
40.89\end{array}$ & 3000 & \begin{tabular}{l|l|l|l|}
1270 \\
1240
\end{tabular} & $\begin{array}{l}87.740915 \\
\end{array}$ & $\begin{array}{r}.239 \\
\end{array}$ & 110.7 & $\begin{array}{l}67.72 \\
86.73\end{array}$ & $\begin{array}{l}5125 \\
5125\end{array}$ & $\begin{array}{l}0.1 \\
0.1\end{array}$ & 1950 & 7500 & 1250000 & $\begin{array}{l}0.0966 \\
\end{array}$ & \begin{tabular}{|l|l|} 
\#\#\#\# \\
.
\end{tabular} & 2.946 & $3.26 \mathrm{E}-03$ & 26.31 & $\begin{array}{l}94.3 \\
\end{array}$ & 43.01 & 0 & 212.6 & 458.4 \\
\hline \begin{tabular}{|l|l|}
403 \\
404
\end{tabular} & $\begin{array}{l}40.89 \\
26.67\end{array}$ & 3000 & \begin{tabular}{|c|c|}
1340 \\
910
\end{tabular} & $\begin{array}{r}4.889091 \\
16787914\end{array}$ & \begin{tabular}{l|l|l|l|}
0.1409 \\
0.9474
\end{tabular} & $\begin{array}{l}86.48 \\
110.3\end{array}$ & $\begin{array}{l}86.73 \\
4.3\end{array}$ & $\begin{array}{l}5125 \\
5125\end{array}$ & $\begin{array}{l}0.1 \\
0.1\end{array}$ & $\begin{array}{l}1950 \\
1950\end{array}$ & 7500 & $\begin{array}{r}862500 \\
232525\end{array}$ & \begin{tabular}{|c|}
0.0778 \\
0.1102
\end{tabular} & 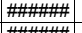 & $\begin{array}{l}3.845 \\
2302 \\
\end{array}$ & $1.01 \mathrm{E}-02$ & 49.14 & $\begin{array}{r}0 \\
\end{array}$ & 0 & & $\begin{array}{l}68.7 \\
07 .\end{array}$ & $\begin{array}{r}481.8 \\
42.7\end{array}$ \\
\hline 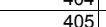 & 20.01 & 3000 & | & & \begin{tabular}{|l|l|l|}
0.9474 \\
0.3388
\end{tabular} & $\begin{array}{c}169.3 \\
1032\end{array}$ & $\begin{array}{r}44.3 \\
72.65\end{array}$ & \begin{tabular}{|l|l|l|l|}
5125 \\
5125
\end{tabular} & 0.1 & & $\begin{array}{l}7500 \\
77500\end{array}$ & 2362500 & 0.1193 & |\#\#\#\#| & 2.303 & 7.14E-03 & 36.38 & $\begin{array}{l}70.22 \\
19.22\end{array}$ & & & $\begin{array}{l}97.1 \\
05.7\end{array}$ & $\begin{array}{r}442.7 \\
558\end{array}$ \\
\hline \begin{tabular}{|l|}
406 \\
\end{tabular} & 52.85 & 300 & 1080 & & \begin{tabular}{|c|} 
\\
0.21980
\end{tabular} & $\begin{array}{l}0.03 .2 \\
167.5 \\
\end{array}$ & 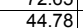 & 5125 & 0.1 & $\begin{array}{l}1950 \\
1950\end{array}$ & 7500 & $\frac{1125000}{2325000}$ & $\frac{0.1}{0.0}$ & 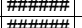 & & & $\frac{64.6}{46.6}$ & 49.22 & & & 95.7 & $\begin{array}{r}558 \\
3777 \\
\end{array}$ \\
\hline \begin{tabular}{|l|l|}
407 \\
\end{tabular} & 27.86 & & 1740 & & 0.588 & 87.31 & 85.9 & 5125 & 0.1 & & & 875000 & 0.1 & 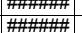 & $\begin{array}{l}3.8 \\
3.1\end{array}$ & & $\frac{46 .}{15 .}$ & $\begin{array}{r}0 \\
75.08\end{array}$ & $\frac{0}{0}$ & & $\begin{array}{r}5.6 \\
126.5\end{array}$ & $\begin{array}{l}377.7 \\
582.2\end{array}$ \\
\hline 408 & 41.21 & 00 & 1110 & & 1.4342 & 118.7 & 63.17 & 5125 & 0.1 & & & 7500 & & \#\#\#\#" & & & & 86.2 & & & 96.4 & 002.2 \\
\hline 409 & & 3000 & 1100 & & 0.2393 & 141.2 & 53.11 & 5125 & 0.1 & 1950 & 7500 & 00000 & & \#\#\#\#\#+ & & & 37.24 & $\begin{array}{l}0.4 \\
1.66\end{array}$ & & & 70.7 & 454.6 \\
\hline 410 & 50.1 & 3000 & 1690 & & 0.4654 & 136.6 & 54.9 & 5125 & 0.1 & 1950 & 756 & 1712500 & 0.0 & 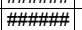 & 4.904 & & 61.03 & 51.08 & & & 120.2 & 501.7 \\
\hline 411 & 40.53 & 3000 & 1830 & & 1.0036 & 169.3 & 44.3 & 5125 & 0.1 & 1950 & & 2362500 & & \#\#\#\#+ & & & 35 & & & & 174.2 & 523.2 \\
\hline 412 & 38.75 & & 1380 & & 0.6909 & 75.24 & 99.68 & 5125 & 0.1 & 1950 & & 700000 & & \#\#\#\#+ & 3.609 & & 51.72 & 81.85 & 0 & & 118.6 & 477. \\
\hline 413 & 22.33 & 3000 & 380 & 62.122049 & 3.891 & 187.9 & 39.91 & 5125 & 0.1 & 1950 & 7500 & & & \#\#\#\#+ & 1.285 & & 26.23 & 91.95 & 19.51 & & 69.1 & 294. \\
\hline 414 & 36.65 & 3000 & 2080 & & 0.1308 & 105.5 & 71.08 & 5125 & 0.1 & 1950 & 7500 & & & \#\#\#\#+ & 5.304 & & 87.44 & & & & 112.7 & 741.6 \\
\hline 415 & 61.65 & 3000 & 1930 & & 0.0172 & 147 & 51 & 5125 & 0.1 & 1950 & & & & & 5.631 & & 22.67 & & & & 45.9 & 468 \\
\hline 416 & 26.85 & 3000 & 590 & & 0.0933 & 141.2 & 53.11 & 5125 & 0.1 & 1950 & 750 & & & & 67 & & 55.3 & & & & 40.7 & 375.5 \\
\hline 417 & 57.84 & 3000 & 1800 & & 0.1 & 151.5 & 49.5 & 5125 & 0.1 & 1950 & & & & & 39 & & & 0 & 0 & & 71.5 & 455.3 \\
\hline 418 & 26.49 & & $\begin{array}{l}490 \\
\end{array}$ & & 2.98 & 99.38 & 75. & 5125 & 0.1 & 19 & & & & & & & & \begin{tabular}{l|l|}
94.45 \\
\end{tabular} & \begin{tabular}{|l|l|}
44.47 \\
\end{tabular} & & 77 & 50 \\
\hline 419 & 24.64 & & 1130 & & 0.1 & 129.9 & & 5125 & 0.1 & 19 & & & & & & & & & 0 & & 72.7 & 514. \\
\hline 420 & 49.4 & & 800 & & \begin{tabular}{l|l|}
0.8827 \\
\end{tabular} & 91.42 & & 51 & 0.1 & & & & & & 2.841 & & & 82.74 & & & 57.7 & 294 \\
\hline 421 & 21.22 & & 770 & & & 146.4 & & 51 & 0.1 & & & & & & & & 36. & 65.32 & & & 83.7 & 45 \\
\hline 42 & 24.97 & & 1050 & & & 112.2 & & & 0.1 & & & & & & & & & & & & 51 & \\
\hline 3 & & & 770 & & & \begin{tabular}{|c|}
93.04 \\
\end{tabular} & & & 0.1 & & & 962500 & & & & & & 64.25 & & & 72 & \\
\hline & 22.12 & & 1120 & & & \begin{tabular}{ll|}
142.5 \\
\end{tabular} & & & 0.1 & & & & & & & & & & 0 & & 89.2 & 6.5 \\
\hline 5 & 38.47 & & $\begin{array}{l}770 \\
\end{array}$ & & & $\begin{array}{l}97.02 \\
\end{array}$ & 7 & & 0.1 & & & & & & 2.5 & & 42 & 78.89 & & & 67.2 & 342. \\
\hline 42 & 25.76 & & 720 & 13.34 & & 182.8 & 41. & 51 & 0.1 & & & & & & & & & 62.54 & 0 & & 86 & 423.8 \\
\hline 42 & 40.17 & & 2210 & 2. & & $\begin{array}{ll}94.64 \\
\end{array}$ & & & 0.1 & & & & & & 4.4 & & & & & & 77.4 & 562. \\
\hline 8 & 33.7 & & 400 & & 51 & 143.2 & & & 0.1 & & & 1837 & & & 1.7 & & 69 & 91.18 & 11.79 & & 64.4 & \\
\hline 429 & 42.08 & & 2200 & & & \begin{tabular}{|l|l|}
81.39 \\
7.21
\end{tabular} & 92. & 5125 & & & & & & & & & 23. & 0 & 0 & & 54.1 & 600.4 \\
\hline 430 & 29.48 & 3000 & $\begin{array}{r}400 \\
\end{array}$ & & 1.2415 & 74.34 & 100.88 & 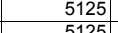 & & 195 & 750 & 6875 & & & 1.584 & & 80. & 90.02 & 0.2 & & 51.6 & 275.4 \\
\hline 431 & 21.93 & 3000 & 1000 & $\begin{array}{r}1.612535 \\
\end{array}$ & \begin{tabular}{|c|}
0.0913 \\
16352
\end{tabular} & 169.9 & 44.15 & 5125 & 0.1 & 1950 & & $\begin{array}{r}2375000 \\
77000\end{array}$ & 0.1 & & $\begin{array}{r}2.25 \\
1.077 \\
\end{array}$ & & 34. & 0 & 0 & & 63.4 & 525.9 \\
\hline 432 & $\begin{array}{ll}61.53 \\
22.39\end{array}$ & 300 & $\begin{array}{l}360 \\
770 \\
\end{array}$ & \begin{tabular}{|r|}
608.576044 \\
\end{tabular} & 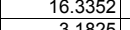 & 80.53 & 93.14 & 5125 & 0.1 & $\begin{array}{l}1950 \\
1050\end{array}$ & 750 & $\begin{array}{r}775000 \\
\end{array}$ & 0.0 & |\#\#\#+\#| & $1.977 \mid$ & & 26.8 & 99.18 & $\begin{array}{l}91.78 \\
52.12\end{array}$ & 17.84 & $\frac{90.3}{159 .}$ & 164.7 \\
\hline$\frac{435}{434}$ & $\frac{22.39}{3523}$ & & 170 & & 3.1825 & \begin{tabular}{|l|}
91.42 \\
1122
\end{tabular} & 82.04 & $\begin{array}{l}\frac{5125}{5125} \\
5125\end{array}$ & & & & & & & & & & & 52.12 & & $\frac{158.4}{1140}$ & $436 \mathrm{c}$ \\
\hline$\frac{45}{43}$ & $\frac{3.25}{2472}$ & & $\frac{1230}{580}$ & & $\frac{0.6214}{21024}$ & $\begin{array}{l}112.2 \\
023\end{array}$ & & $\frac{512}{514}$ & & & & & & & & & & & 260 & & & \\
\hline 436 & $\begin{array}{l}2.172 \\
55.91\end{array}$ & & 1050 & & 2.01024 & $\frac{5.4}{946}$ & & 512 & & & & & & & & & & & 0 & & & \\
\hline 437 & 2478 & & 86 & & & 1319 & & 512 & 0. & & & & & & & & & 7828 & 0 & & & \\
\hline | & 2357 & & 1180 & & & 149 & & 512 & & & & & & & & & & 0 & & & & \\
\hline | & $\frac{2.52}{5562}$ & 300 & 2570 & & 0 & 6683 & $108 \mathrm{c}$ & 5 & 0.1 & & & & & & & & & 0 & & & 534 & \\
\hline 440 & 36.19 & 3000 & 940 & & 0.1338 & 103.2 & 72.6 & 5125 & 0.1 & 195 & 7500 & 1125000 & 0.08 & & 2..4997 & & 55.8 & 0 & 0 & & $\begin{array}{l}50.4 \\
54.5 \\
\end{array}$ & 424. \\
\hline 441 & 34.65 & 3000 & 530 & 31.24439 & 1.5248 & 146.4 & 51.23 & 5125 & 0.1 & 1950 & 7500 & 1900000 & \begin{tabular}{|l|}
0.0918 \\
\end{tabular} & \#\#\#\#+ & 2.124 & $9.53 \mathrm{E}-03$ & 92.4 & 84 & & & 72.7 & 314 \\
\hline
\end{tabular}




\begin{tabular}{|c|c|c|c|c|c|c|c|c|c|c|c|c|c|c|c|c|c|c|c|c|c|c|}
\hline Realization & $\begin{array}{c}\begin{array}{c}\text { Velocity } \\
(\mathrm{m} / \mathrm{y})\end{array} \\
.\end{array}$ & $\begin{array}{c}\text { Fixed Source } \\
\text { Concentration } \\
(\mathrm{mg} / \mathrm{L})\end{array}$ & $\begin{array}{c}\text { Time of } \\
\text { Maximum } \\
\text { at } \\
\text { Compliance } \\
\text { Boundary } \\
\text { (y) }\end{array} \mid$ & $\begin{array}{c}\text { Maximum } \\
\text { Concentration } \\
\text { Compliance } \\
\text { Boundary } \\
(\mu \mathrm{g} / \mathrm{L})\end{array}$ & $\begin{array}{c}\text { Maximum } \\
\text { Release Rate } \\
\text { at } \\
\text { Compliance } \\
\text { Boundary } \\
\text { (Kg/y) }\end{array}$ & $\begin{array}{l} \\
\text { Sourc } \\
\text { e Rate } \\
(\mathrm{Kg} / \mathrm{y}) \\
\end{array}$ & $\begin{array}{c}\text { Source } \\
\text { Duration } \\
\text { (y) }\end{array}$ & $\begin{array}{c}\text { 1-D } \\
\text { Streamtube } \\
\text { Length } \\
(\mathrm{m})\end{array}$ & \begin{tabular}{|c} 
Base \\
Porosity \\
$\left(\mathrm{m}^{3} / \mathrm{m}^{3}\right)$
\end{tabular} & $\begin{array}{c}\text { Bulk } \\
\text { Densty } \\
\left(\mathrm{Kg} / \mathrm{m}^{3}\right)\end{array}$ & \begin{tabular}{|c|}
$\begin{array}{c}\text { Remaining } \\
\text { Carbon } \\
\text { Tetrachloride } \\
\text { Source } \\
(\mathrm{Kg})\end{array}$ \\
\end{tabular} & $\begin{array}{c}\text { Volume at or } \\
\text { above Fixed } \\
\text { Source } \\
\begin{array}{c}\text { Concentratio } \\
n(m 3)\end{array} \\
\end{array}$ & $\begin{array}{l}\text { Porosity } \\
\left(\mathrm{m}^{3} / \mathrm{m}^{3}\right) \\
\end{array}$ & $\left.\mid \begin{array}{c}\mathrm{Kd} \\
\left(\mathrm{m}^{3} / \mathrm{kg}\right)\end{array}\right)$ & Retardation & $\begin{array}{c}\text { First } \\
\text { Order } \\
\text { Rate } \\
\text { Constant } \\
(1 / y) \\
\end{array}$ & \begin{tabular}{|c|} 
Longitudinal \\
Dispersivity \\
$(\mathrm{m})$
\end{tabular} & $\begin{array}{c}\text { Required } \\
\text { Source } \\
\text { Cleanup } \\
\text { Percentage } \\
(5 \text { ug/L } \\
\text { Compliance) } \\
\end{array}$ & $\begin{array}{c}\text { Required } \\
\text { Source } \\
\text { Cleanup } \\
\text { Percentage } \\
\text { (50 } \mathrm{\mu g} / \mathrm{L} \\
\text { Compliance) } \\
\end{array}$ & \begin{tabular}{|c|} 
Required \\
Source Cleanup \\
Percentage \\
(500 $\mathrm{\mu g} / \mathrm{L}$ \\
Compliance) \\
\end{tabular} & $\begin{array}{c}\text { Abiotic } \\
\text { Reaction } \\
\text { Half Time } \\
(y)\end{array}$ & $\begin{array}{c}\text { Carbon } \\
\text { Tetrachloride } \\
\text { Travel Iime } \\
\text { (y) } \\
\end{array}$ \\
\hline $\begin{array}{r}442 \\
443 \\
\end{array}$ & $\begin{array}{r}23.31 \\
285 \\
\end{array}$ & \begin{tabular}{|r|}
3000 \\
\end{tabular} & $\begin{array}{r}640 \\
510\end{array}$ & \begin{tabular}{|r|}
2.297934 \\
\end{tabular} & \begin{tabular}{|l|}
0.0898 \\
36547
\end{tabular} & 117.3 & \begin{tabular}{|l|}
63.94 \\
7.5
\end{tabular} & $\begin{array}{r}5125 \\
5155\end{array}$ & 0.1 & $\begin{array}{l}1950 \\
1950\end{array}$ & $\begin{array}{r}7500 \\
7500\end{array}$ & \begin{tabular}{|r|}
1362500 \\
\end{tabular} & \begin{tabular}{|r|}
0.1365 \\
0.1126
\end{tabular} & 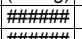 & \begin{tabular}{|r|}
1.865 \\
\end{tabular} & $1.54 \mathrm{E}-02$ & $\begin{array}{r}48.42 \\
\end{array}$ & & & & & $\begin{array}{r}410 \\
3173\end{array}$ \\
\hline $\begin{array}{l}443 \\
444 \\
\end{array}$ & \begin{tabular}{|l|l|}
28.25 \\
41.8
\end{tabular} & 3000 & \begin{tabular}{r|r|r|}
510 & \\
1930
\end{tabular} & \begin{tabular}{|l|}
110.330573 \\
0304053
\end{tabular} & $\begin{array}{l}3.6547 \\
0.165 \\
\end{array}$ & $\begin{array}{l}99.38 \\
113.3 \\
\end{array}$ & $\begin{array}{l}75.47 \\
4.94 \\
\end{array}$ & $\begin{array}{l}5125 \\
5125 \\
\end{array}$ & $\begin{array}{l}0.1 \\
0.1 \\
\end{array}$ & $\begin{array}{r}1950 \\
190 \\
\end{array}$ & 7500 & $\begin{array}{l}1062500 \\
223750 \\
\end{array}$ & $\begin{array}{l}0.1126 \\
0.0761\end{array}$ & 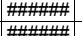 & \begin{tabular}{r|r}
1.749 \\
5.047
\end{tabular} & $\begin{array}{l}6.83 \mathrm{E}-03 \\
1.50\end{array}$ & 73.71 & $\begin{array}{l}95.47 \\
\end{array}$ & 54.68 & 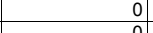 & $\begin{array}{l}101.6 \\
\end{array}$ & 317.3 \\
\hline 445 & \begin{tabular}{r|r}
41.0 \\
23.58
\end{tabular} & 3000 & 390 & \begin{tabular}{|l|}
0.304053 \\
50.95037
\end{tabular} & $\mid \begin{array}{l}0.0165 \\
1.2318\end{array}$ & $\begin{array}{l}163.3 \\
72.53 \\
\end{array}$ & $\begin{array}{l}45.94 \\
103.4\end{array}$ & 5125 & $\begin{array}{l}0.1 \\
0.1\end{array}$ & 1950 & $\begin{array}{l}7500 \\
7500\end{array}$ & $\begin{array}{r}22375000 \\
662500\end{array}$ & $\begin{array}{l}0.0761 \\
0.1349\end{array}$ & 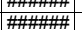 & $\begin{array}{l}5.04 t \\
1.291\end{array}$ & \begin{tabular}{|l|l|}
$1.21 \mathrm{E}-02$ \\
$1.41 \mathrm{E}-02$
\end{tabular} & $\begin{array}{l}43.14 \\
29.77\end{array}$ & $\begin{array}{r}0 \\
90.19\end{array}$ & $\begin{array}{r}0 \\
1.87\end{array}$ & $\frac{0}{0}$ & $\begin{array}{r}57.3 \\
49 \\
\end{array}$ & $\begin{array}{l}618.7 \\
280.5 \\
\end{array}$ \\
\hline 446 & 26.06 & 3000 & 720 & 8.528332 & 0.2645 & 93.04 & 80.61 & 5125 & 0.1 & 1950 & 7500 & 962500 & 0.1221 & 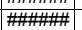 & 2.108 & $1.17 \mathrm{E}-02$ & 57.55 & 41.37 & 0 & 0 & 59.2 & 414.5 \\
\hline 447 & 33.07 & 3000 & 610 & \begin{tabular}{|l|l|}
82.997137 \\
\end{tabular} & 4.0682 & 147 & 51 & 5125 & 0.1 & 1950 & 7500 & 1912500 & 0.0962 & \#\#\#\#+ & 2.024 & $6.73 \mathrm{E}-03$ & 33.72 & 93.98 & 39.76 & & 103.1 & 313.7 \\
\hline 448 & 35.11 & 3000 & 1280 & 0.814678 & 0.0356 & 131.2 & 57.15 & 5125 & 0.1 & 1950 & 7500 & 1612500 & 0.0906 & 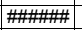 & 4.096 & $1.25 \mathrm{E}-02$ & 85.72 & 0 & 0 & & 55.3 & 597.8 \\
\hline 449 & 61.89 & 3000 & 690 & 121.163658 & 3.6594 & 90.61 & 82.78 & 5125 & 0.1 & 1950 & 7500 & 925000 & 0.0514 & \#\#\#\#+ & 2.962 & $8.10 \mathrm{E}-03$ & 28.93 & 95.87 & 58.73 & & 85.6 & 245.3 \\
\hline 450 & 26.21 & 3000 & $\begin{array}{l}410 \\
\end{array}$ & $\begin{array}{l}58.12295 \\
\end{array}$ & 3.3833 & $\begin{array}{l}174.6 \\
\end{array}$ & 42.95 & 5125 & 0.1 & 1950 & 7500 & 2475000 & 0.1214 & \#\#\#\#+ & 1.525 & $8.58 \mathrm{E}-03$ & \begin{tabular}{ll|}
67.37 \\
\end{tabular} & 91.4 & 13.98 & & 80.8 & 298.3 \\
\hline 451 & 22.56 & 3000 & 1580 & 1.086377 & 0.0443 & 122.3 & 61.34 & 5125 & 0.1 & 1950 & 7500 & 1450000 & 0.141 & \#\#\#\#曲 & 2.98 & $8.98 \mathrm{E}-03$ & 37.52 & 0 & 0 & & 77.2 & \\
\hline 452 & 23.34 & 3000 & 1650 & 0.231838 & $\begin{array}{l}0.0115 \\
07790\end{array}$ & 148.3 & & 5125 & 0.1 & 1950 & 7500 & 1937500 & 0.1363 & \#\#\#\#曲 & 3.521 & 1.07E-02 & 60.91 & 0 & 0 & & 64.6 & \\
\hline 453 & 26.8 & 3000 & 360 & 328.564695 & 17.8815 & $\begin{array}{l}163.3 \\
149\end{array}$ & 45.94 & 5125 & 0.1 & 1950 & 7500 & 2237500 & 0.1187 & 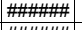 & 1.364 & $4.83 \mathrm{E}-03$ & 35.46 & 98.48 & 84.78 & & 143.6 & \\
\hline 454 & 38.61 & 3000 & $\begin{array}{l}600 \\
\end{array}$ & 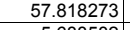 & 2.2468 & \begin{tabular}{ll|}
116.6 \\
1
\end{tabular} & 64.33 & 5125 & 0.1 & 1950 & 7500 & 1350000 & 0.0824 & \#\#\#\# & $\begin{array}{r}2.3 \\
\end{array}$ & $8.02 \mathrm{E}-03$ & $\begin{array}{l}66.48 \\
6.05\end{array}$ & \begin{tabular}{|c|}
91.35 \\
12.
\end{tabular} & 13.52 & & 86.5 & 305.3 \\
\hline 455 & 40.68 & 3000 & 1240 & $\begin{array}{r}.688582 \\
1.7902 \\
\end{array}$ & $\begin{array}{ll}0.2211 \\
1790\end{array}$ & \begin{tabular}{ll|}
116.6 \\
\end{tabular} & 64.33 & 5125 & 0.1 & 1950 & 7500 & 1350000 & 0.0782 & 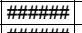 & $\begin{array}{l}3.851 \\
2.122\end{array}$ & 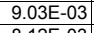 & 68.85 & 12.1 & 0 & & 76.7 & 485.2 \\
\hline 456 & 32.46 & 3000 & $\begin{array}{l}600 \\
60\end{array}$ & 41.702644 & 1.7288 & 124.4 & 60.31 & 5125 & 0.1 & 1950 & 7500 & 1487500 & 0.098 & 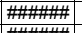 & 2.133 & $8.12 \mathrm{E}-03$ & $\begin{array}{l}78.92 \\
\end{array}$ & 88.01 & 0 & & 85.3 & 336.8 \\
\hline 457 & 44.81 & 3000 & $\begin{array}{ll}620 \\
\end{array}$ & 188.281815 & 5.5836 & 88.97 & $\begin{array}{l}84.3 \\
\end{array}$ & 5125 & 0.1 & 1950 & 7500 & 900000 & $\begin{array}{r}0.071 \\
\end{array}$ & 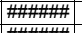 & \begin{tabular}{|l|l|}
2.317 \\
2302
\end{tabular} & $7.40 \mathrm{E}-03$ & 18 & 97.34 & 73.44 & & 93.6 & \\
\hline 458 & 21.73 & 3000 & \begin{tabular}{r|r|r}
930 \\
1710
\end{tabular} & $\begin{array}{r}0.799093 \\
\end{array}$ & $\begin{array}{l}0.0446 \\
\end{array}$ & $\begin{array}{l}167.5 \\
1000\end{array}$ & \begin{tabular}{|l|l|l|}
44.78 \\
7.31
\end{tabular} & $5125 \mid$ & 0.1 & 1950 & 7500 & 2325000 & 0.1464 & & 2.202 & & 40.25 & & & & 53.7 & \\
\hline 459 & $\begin{array}{r}22.99 \\
22.44\end{array}$ & $\begin{array}{l}3000 \\
3000\end{array}$ & & 4.957 & 0.1668 & $\begin{array}{c}100.9 \\
17\end{array}$ & 74.31 & 5125 & 0.1 & 1950 & 7500 & 1087500 & 0.1384 & 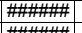 & \begin{tabular}{|l|l|}
2.98 \\
2111
\end{tabular} & $6.66 \mathrm{E}-03$ & 24.05 & & & & 104 & $\begin{array}{ll}664.4 \\
18.2\end{array}$ \\
\hline 460 & 22.44 & 3000 & 720 & & 0.1998 & 147 & 51 & 5125 & 0.1 & 1950 & 7500 & 1912500 & & \#册曲 & 2.111 & $1.09 \mathrm{E}-02$ & 93.45 & 0 & 0 & & 63.7. & 482.2 \\
\hline 461 & 50.74 & 3000 & 390 & 78.210484 & 2.9256 & 112.2 & 66.83 & 5125 & 0.1 & 1950 & 7500 & 1275000 & & \#\#\#\#\#! & 1.994 & $1.45 \mathrm{E}-02$ & 34.64 & 93.61 & 36.07 & & 47.7 & 201.4 \\
\hline 462 & 30.86 & 3000 & 520 & 14.679471 & $\begin{array}{l}0.6717 \\
\end{array}$ & 137.3 & 54.64 & 5125 & 0.1 & 1950 & 7500 & 1725000 & & \#\#\#\#+ & \begin{tabular}{|l|l|}
1.887 \\
\end{tabular} & $1.33 \mathrm{E}-02$ & 48.48 & 65.94 & 0 & & 52.1 & 313.3 \\
\hline $\begin{array}{l}463 \\
\end{array}$ & 25.17 & 3000 & \begin{tabular}{r|}
600 \\
1000
\end{tabular} & & $\begin{array}{l}0.8239 \\
\end{array}$ & $\begin{array}{l}130.6 \\
\end{array}$ & 57.44 & 5125 & $\begin{array}{l}0.1 \\
0.1\end{array}$ & $\begin{array}{l}1950 \\
1905\end{array}$ & 7500 & 1600000 & $\begin{array}{l}0.1264 \\
\end{array}$ & \#\#\#\# & & $1.08 \mathrm{E}-02$ & 35.56 & 73.59 & 0 & & 64.5 & 361.9 \\
\hline $\begin{array}{l}464 \\
\end{array}$ & $\begin{array}{ll}46.44 \\
\end{array}$ & 3000 & 1000 & 11.635861 & \begin{tabular}{|l|l|l|l|l|}
1.20 \\
\end{tabular} & \begin{tabular}{l|l|}
122.3 \\
807
\end{tabular} & $\begin{array}{l}61.34 \\
\end{array}$ & 5125 & $\begin{array}{l}0.1 \\
0.1\end{array}$ & 1950 & 7500 & 1450000 & $\begin{array}{l}0.0685 \\
\end{array}$ & 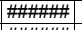 & \begin{tabular}{|l|l|}
3.167 \\
\end{tabular} & 1.11E-02 & 22.09 & 57.03 & 0 & & 62.2 & 349.5 \\
\hline $\begin{array}{l}465 \\
466 \\
\end{array}$ & $\begin{array}{l}25.45 \\
448\end{array}$ & 3000 & \begin{tabular}{|l|l|}
380 \\
520
\end{tabular} & \begin{tabular}{|c|c|}
48.689726 \\
1534929
\end{tabular} & \begin{tabular}{|l|l|}
1.4439 \\
42688
\end{tabular} & $\begin{array}{l}88.97 \\
813\end{array}$ & $\begin{array}{r}84.3 \\
0215\end{array}$ & 5125 & 0.1 & 1950 & 7500 & $\begin{array}{l}900000 \\
77507\end{array}$ & $\begin{array}{l}0.125 \\
\end{array}$ & 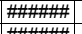 & 1.401 & $1.30 \mathrm{E}-02$ & 72.52 & 89.73 & $\begin{array}{r}0 \\
\end{array}$ & & 53.5 & 282 \\
\hline $\begin{array}{l}460 \\
467 \\
\end{array}$ & $\begin{array}{l}41.48 \\
21.37 \\
\end{array}$ & 3000 & \begin{tabular}{|l|l|}
520 \\
490
\end{tabular} & \begin{tabular}{|r|}
157.349229 \\
119.406133
\end{tabular} & $\begin{array}{l}4.2688 \\
7.4337\end{array}$ & $\begin{array}{l}81.39 \\
1868\end{array}$ & 92.15 & \begin{tabular}{|l|l|}
5125 \\
5125
\end{tabular} & 0.1 & $\begin{array}{c}1950 \\
11950 \\
\end{array}$ & $\begin{array}{l}7500 \\
7500 \\
\end{array}$ & 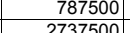 & & 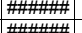 & $\begin{array}{l}96 \\
33\end{array}$ & 1.01E-02 & 17.62 & $\begin{array}{ll}96.82 \\
05.81\end{array}$ & & & $\begin{array}{r}68.8 \\
1144\end{array}$ & 246.6 \\
\hline 468 | & 50.26 & 3000 & $\begin{array}{l}780 \\
780\end{array}$ & 47.977512 & 2.6595 & 166.3 & \begin{tabular}{|c|}
4.10 \\
451
\end{tabular} & 5125 & 0.1 & 1950 & 7500 & 2300000 & $\begin{array}{l}0.1469 \\
0.0633\end{array}$ & 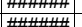 & 1.4 & & & $\begin{array}{ll}95.81 \\
89.58\end{array}$ & 58.13 & & 114.4 & $\begin{array}{l}343.7 \\
291.9\end{array}$ \\
\hline $\begin{array}{ll}469 \\
\end{array}$ & 53.92 & & 890 & 15.93 & 1.05 & 198 & & 5125 & 0.1 & 1950 & & 87500 & $\begin{array}{l}0.0059 \\
0.059\end{array}$ & |m+m|m| & & & & 89.58 & & & 89.6 & 291.8 \\
\hline |470| & 35.87 & & 800 & & & 117.3 & & 5125 & 0.1 & 50 & 7500 & 62500 & 0.0 & 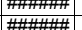 & & & & 68.62 & & & 67 & 304.2 \\
\hline 471 & 45.06 & & $\begin{array}{ll}1290 \\
\end{array}$ & & \begin{tabular}{|l|l|}
0.0996 \\
\end{tabular} & 152.8 & 49 & 5125 & 0.1 & 50 & 7500 & 25000 & 0.0 & 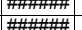 & $\frac{2.8}{43}$ & & $\begin{array}{ll}66.01 \\
71.44\end{array}$ & & & & $\begin{array}{ll}43.4 \\
6 ?\end{array}$ & $\begin{array}{l}406.8 \\
499\end{array}$ \\
\hline 472 & 41.53 & 00 & 950 & 3.161845 & \begin{tabular}{|l|l|}
0.1318 \\
\end{tabular} & 125.1 & 59.97 & 5125 & 0.1 & 1950 & 7500 & 1500000 & 0.0 & \#\#\#\#县 & $\begin{array}{r}4.395 \\
2.92 \\
\end{array}$ & & $\begin{array}{r}1.44 \\
181\end{array}$ & & & & $\frac{62}{458}$ & $\frac{499.9}{360.3}$ \\
\hline 473 & 48.13 & 3000 & 1790 & & 0.2501 & 140.6 & 53.35 & 5125 & 0.1 & 1950 & 7500 & 1787500 & & 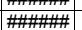 & $\begin{array}{l}2.92 \\
4.769\end{array}$ & $\begin{array}{l}1.07 \mathrm{E}-0 \mathrm{~L} \\
7.29 \mathrm{E}-03\end{array}$ & $\begin{array}{l}1.1 \\
41.6\end{array}$ & $\begin{array}{r}6.33 \\
\end{array}$ & 0 & & (40.0 & $\frac{360.3}{507.9}$ \\
\hline 474 & 34.32 & 3000 & 690 & 53.634482 & 06 & 88.97 & 84 & 5125 & 0.1 & 1950 & 7500 & 900000 & & \#\#\#\#+ & 2.321 & 7.80 & 64.81 & 90.68 & 6.78 & & 88.9 & 346.6 \\
\hline 475 & 21.54 & & 890 & & 0.4963 & $\begin{array}{l}155.3 \\
\end{array}$ & 48.3 & 5125 & 0.1 & 1950 & & & & \#\#\#\#+ & 2.046 & & 35.13 & 47.86 & & & 85.2 & 486.8 \\
\hline 476 & 26.01 & 3000 & 400 & 12.87 & 0.6118 & 142.5 & 52.62 & 5125 & 0.1 & 1950 & 7500 & & & \#\#\#\#" & 1.529 & & 68.6 & 61.17 & & & 45.6 & 301.2 \\
\hline 477 & 29.21 & 3000 & 1040 & & 0.0293 & 69.77 & 107.5 & 5125 & 0.1 & 1950 & 7500 & 000 & & \#\#\#\#+ & 3.153 & & 82.33 & & & & 49.1 & 553. \\
\hline 478 & 25.35 & & 2070 & & \begin{tabular}{|l|l|}
0.0044 \\
\end{tabular} & 134.6 & & 5125 & 0.1 & 1950 & 7500 & 000 & & 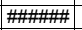 & 3.892 & & 36.76 & 0 & 0 & & 61 & 786.8 \\
\hline & 34.73 & & 500 & 15.93 & 0.59 & 112.2 & & 5125 & 0.1 & 1950 & 7500 & 1275000 & & & 1.927 & & 38 & 68.63 & & & 44.8 & 284. \\
\hline 480 & 47.41 & 3000 & 1610 & & 0.052 & 88.14 & & 5125 & 0.1 & 1950 & 75 & & & & 4.244 & & 21 & & & & 54.3 & 458. \\
\hline 481 & 23.24 & & 1050 & & $\begin{array}{ll}0.0194 \\
\end{array}$ & \begin{tabular}{ll|}
122.3 \\
\end{tabular} & 61.34 & 5125 & 0.1 & 195 & & & & & & & 30. & 0 & 0 & & 48.6 & 528.7 \\
\hline 482 & 23.04 & & 950 & & 0.4651 & 181.6 & 41 & 5125 & 0.1 & & & & & & & & & 34.92 & 0 & & 83.4 & 482.2 \\
\hline 483 & 21.88 & & 740 & & & 83.95 & 89. & 51 & 0.1 & & & & & & & & & 94.93 & 49.28 & & 134.2 & 427.5 \\
\hline 484 & 23.69 & & 650 & & 43 & \begin{tabular}{l|l|}
88.97 \\
\end{tabular} & 84 & 51 & 0.1 & & & & & & & & 17.2 & 0 & 0 & & 43.1 & 377. \\
\hline & & & 1460 & & & 13 & & & 0.1 & & & & & & & & & 0 & 0 & & 95.5 & $\begin{array}{l}594.4 \\
\end{array}$ \\
\hline 486 & & & 590 & & & 121 & & & 0.1 & & & & & & & & & 92.4 & 24.01 & & 75.3 & 259.4 \\
\hline & 54.95 & & 1270 & & & \begin{tabular}{ll|}
122.3 \\
\end{tabular} & & & 0.1 & & & & & & & & & 20.65 & 0 & & 69.6 & 414.8 \\
\hline 488 & 41.59 & & 750 & & 0.4 & $\begin{array}{l}145.1 \\
\end{array}$ & & & 0.1 & & & & & & & & 40. & 49.49 & 0 & & 56 & 330.8 \\
\hline 489 & 37.65 & & 1490 & & 21 & $\begin{array}{l}131.2 \\
\end{array}$ & 57. & 51 & 0.1 & & & & & & & & & 0 & 0 & & 53.8 & 648.2 \\
\hline 490 & 37.34 & & 880 & 2.19 & \begin{tabular}{|l|l|}
0.1024 \\
\end{tabular} & \begin{tabular}{|l|l|}
139.9 \\
\end{tabular} & 53 & & 0.1 & & & & & & & & & & 0 & & $\begin{array}{l}47.6 \\
\end{array}$ & 398.5 \\
\hline 491 & 38.1 & & $\begin{array}{r}660 \\
\end{array}$ & 80.4458 & \begin{tabular}{|l|l|}
3.1837 \\
\end{tabular} & $\begin{array}{l}118.7 \\
\end{array}$ & 63.17 & 51 & 0.1 & & & & 0.0 & & 77 & & 66. & 93.78 & 37.85 & & 115.3 & 319.8 \\
\hline 492 & 23.09 & 3000 & 1320 & $\begin{array}{l}0.83962 \\
\end{array}$ & \begin{tabular}{|c|}
0.042 \\
\end{tabular} & 150.2 & 49.92 & 5125 & & 195 & & & 0.1 & & 12 & & 34. & & 0 & & 65.7 & 602.1 \\
\hline 493 & 26.67 & 3000 & 830 & $\begin{array}{r}2.37994 \\
\end{array}$ & \begin{tabular}{|l|l|} 
\\
6 \\
6
\end{tabular} & \begin{tabular}{|c|}
166.3 \\
1261
\end{tabular} & $\begin{array}{r}45.1 \\
\end{array}$ & 5125 & & 195 & 7500 & & 0.1 & & \begin{tabular}{ll|}
98 \\
91
\end{tabular} & & 77.6 & $\begin{array}{r}0 \\
\end{array}$ & 0 & & 59.4 & 480.2 \\
\hline $\begin{array}{l}494 \\
495\end{array}$ & 25.39 & 3000 & 390 & 145.501524 & 6.1328 & $\begin{array}{l}126.4 \\
\end{array}$ & 59.31 & 5125 & 0.1 & 195 & 750 & 1525000 & 0.1 & 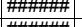 & 01 | & & $\frac{60.2}{3 ?}$ & 96.56 & 65.64 & & $\begin{array}{r}94.6 \\
106.7\end{array}$ & 282.7 \\
\hline 495 & $\frac{46.17}{24.97}$ & 3000 & 660 & & 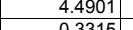 & $\begin{array}{l}150.2 \\
1107\end{array}$ & $\begin{array}{l}49.92 \\
67.72 \\
67\end{array}$ & 5125 & & $\begin{array}{l}1950 \\
1950 \\
\end{array}$ & & & 0.0 & 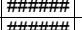 & 2.5 & & $\frac{32}{347}$ & $\begin{array}{l}94.42 \\
44.32\end{array}$ & 44.23 & & & 279.9 \\
\hline 年 & 24.97 & & 烈 & & 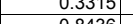 & $\frac{110.1}{8009}$ & & $\frac{512}{512}+2$ & & & & & & & & & & & 0 & & & 468.6 \\
\hline 年 & 30.5 & & 530 & & & 89.19 & & $\frac{512}{511}$ & & & & & & & & & & & t & & & \\
\hline 450 & $\frac{30.15}{635}$ & & 1610 & & 4 & 14c.5 & & $\frac{312}{512}+2$ & & & & & & & 4665 & & & & 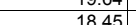 & & & \\
\hline 500 & 52.93 & 300 & 790 & & $\frac{2.45}{0.85}-3$ & 53.95 & 1390 & 515 & 01 & 19 & & & & & & & & 89.4 & 10.40 & & 64 & \\
\hline 501 & 36.86 & & 860 & & 0.8369 & 123 & 60. & 5125 & 0.1 & & & & & & 2.6 & & & 75 & 0 & & 78.9 & \\
\hline 502 & 34.21 & 3000 & 1000 & 16.882 & $\begin{array}{l}0.849 \\
\end{array}$ & 150.9 & 49.71 & 5125 & 0.1 & 195 & 7500 & & & & 2.721 & & 28.3 & 70.38 & 0 & & 88.1 & 407. \\
\hline 503 & 25.78 & 3000 & 1680 & 2.458891 & \begin{tabular}{|l|l|}
0.0756 \\
\end{tabular} & \begin{tabular}{|c|}
92.23 \\
\end{tabular} & 81.32 & 5125 & 0.1 & 1950 & 7500 & & 0.1234 & & \begin{tabular}{|l|l|}
3.706 \\
\end{tabular} & $7.55 \mathrm{E}-03$ & \begin{tabular}{|l|l|}
76.67 \\
\end{tabular} & 0 & 0 & & $\begin{array}{l}91.8 \\
\end{array}$ & 736. \\
\hline 504 & 22.48 & 3000 & 490 & 455.614771 & 2.1174 & 139.3 & \begin{tabular}{|l|}
53.86 \\
\end{tabular} & 5125 & 0.1 & 1950 & 7500 & 1762500 & 0.1415 & \#\#\#\#+ & 1.573 & $7.82 \mathrm{E}-03$ & 86.2 & 89.04 & & & \begin{tabular}{|l|}
88.6 \\
8
\end{tabular} & 358.6 \\
\hline
\end{tabular}




\begin{tabular}{|c|c|c|c|c|c|c|c|c|c|c|c|c|c|c|c|c|c|c|c|c|c|c|}
\hline $\begin{array}{r}\text { Realization } \\
505\end{array}$ & $\begin{array}{c}\text { Velocity } \\
(\mathrm{m} / \mathrm{y})\end{array}$ & $\begin{array}{c}\text { Fixed Source } \\
\text { Concentration } \\
\text { (mg/L } / \text { (n) }\end{array}$ & $\begin{array}{c}\text { Time of } \\
\text { Maximum } \\
\text { at } \\
\text { Compliance } \\
\text { Boundary } \\
\text { (y) }\end{array}$ & \begin{tabular}{|c|} 
Maximum \\
Concentration \\
Compliance \\
Boundary \\
$(\mu \mathrm{g} / \mathrm{L})$ \\
\end{tabular} & $\begin{array}{c}\text { Maximum } \\
\text { Release Rate } \\
\text { at } \\
\text { Compliance } \\
\text { Boundary } \\
(\mathrm{Kg} / \mathrm{y})\end{array}$ & \begin{tabular}{|l|} 
\\
\\
Sourc \\
e Rate \\
(Kg/y) \\
155. \\
\end{tabular} & \begin{tabular}{|c} 
Source \\
Duration \\
(y)
\end{tabular} & \begin{tabular}{|c|} 
1-D \\
Streamtube \\
Length \\
$(\mathrm{m})$
\end{tabular} & $\begin{array}{c}\text { Base } \\
\text { Porosity } \\
\left(\mathrm{m}^{3} / \mathrm{m}^{3}\right) \\
\end{array}$ & $\begin{array}{c}\text { Bulk } \\
\text { Densty } \\
\left(\mathrm{Kg} / \mathrm{m}^{3}\right) \\
(1050\end{array}$ & \begin{tabular}{|c|} 
Remaining \\
Carbon \\
Tetrachloride \\
Source \\
$(\mathrm{Kg})$
\end{tabular} & $\begin{array}{c}\text { Volume at or } \\
\text { above Fixed } \\
\text { Source } \\
\text { Concentratio } \\
n(m 3)\end{array}$ & $\begin{array}{l}\text { Porosity } \\
\left(\mathrm{m}^{3} / \mathrm{m}^{3}\right)\end{array}$ & $\begin{array}{c}\mathrm{Kd} \\
\left(\mathrm{m}^{3} / \mathrm{Kg}\right)\end{array}$ & Retardation & $\begin{array}{c}\text { First } \\
\text { Order } \\
\text { Rate } \\
\text { Constant } \\
(1 / y) \\
\end{array}$ & \begin{tabular}{|c|} 
Longitudinal \\
Dispersivity \\
$(\mathrm{m})$
\end{tabular} & \begin{tabular}{|c|} 
Required \\
Source \\
Cleanup \\
Percentage \\
$(5 \mu \mathrm{g} / \mathrm{L}$ \\
Compliance) \\
\end{tabular} & $\begin{array}{c}\text { Required } \\
\text { Source } \\
\text { Cleanup } \\
\text { Percentage } \\
(50 \mu \mathrm{g} / \mathrm{L} \\
\text { Compliance) } \\
\end{array}$ & \begin{tabular}{|c|} 
Required \\
Source Cleanup \\
Percentage \\
(500 $\mathrm{\mu g} / \mathrm{L}$ \\
Compliance) \\
\end{tabular} & \begin{tabular}{|c|}
$\begin{array}{c}\text { Abiotic } \\
\text { Reaction } \\
\text { Half TTime } \\
\text { (y) }\end{array}$ \\
\end{tabular} & $\begin{array}{c}\text { Carbon } \\
\text { Tetrachloride } \\
\text { Travel Time } \\
(y)\end{array}$ \\
\hline & 37.08 & & & & \begin{tabular}{|l|l} 
\\
\end{tabular} & 155.3 & & 5125 & & & 7500 & & & \#\#\#\#+ & & $6.30 \mathrm{E}-03$ & 58.17 & & & & \begin{tabular}{|l|}
110.1 \\
\end{tabular} & \\
\hline 506 & 24.19 & 3000 & 1190 & 33.468026 & 1.4106 & \begin{tabular}{|l|}
126.4 \\
\end{tabular} & 59.31 & 5125 & 0.1 & 1950 & 7500 & 1525000 & 0.1315 & \#\#\#\#曲 & 2.44 & $5.04 \mathrm{E}-03$ & 22.64 & 85.06 & & & 137.5 & 516.9 \\
\hline 507 & 24.43 & 3000 & 1440 & 0.241199 & 0.0093 & \begin{tabular}{|l|}
115.9 \\
\end{tabular} & 64.73 & 5125 & 0.1 & 1950 & 7500 & 1337500 & 0.1302 & \#\#\#\#\#+ & 3.256 & $1.26 \mathrm{E}-02$ & 53.51 & & 0 & c & 55.1 & \\
\hline 508 & 30.98 & 3000 & 400 & $\begin{array}{l}76.446087 \\
\end{array}$ & 3.9246 & 154 & 48.7 & 5125 & 0.1 & 1950 & 7500 & 2050000 & 0.1027 & \#\#\#曲 & 1.611 & $9.22 \mathrm{E}-03$ & 61.59 & 93.46 & & 0 & 75.2 & 266.6 \\
\hline 509 & 25.25 & 3000 & 630 & 68.102606 & 3.0405 & \begin{tabular}{|l|}
133.9 \\
\end{tabular} & 56 & 5125 & 0.1 & 1950 & 7500 & 1662500 & 0.126 & \#\#\#\#曲 & 1.83 & $6.13 \mathrm{E}-03$ & 47.11 & 92.66 & 26.58 & 0 & 113.1 & 371.5 \\
\hline 510 & 45.32 & 3000 & 440 & 109.809477 & 5.7968 & \begin{tabular}{|l|l|}
158.4 \\
\end{tabular} & 47.36 & 5125 & 0.1 & 1950 & 7500 & 2137500 & 0.0702 & \#\#\#曲 & 2.068 & $7.72 \mathrm{E}-03$ & 61.26 & 95.45 & 54.47 & 0 & 89.8 & 233.8 \\
\hline 511 & 43.82 & 3000 & 700 & 63.262245 & 2.8527 & \begin{tabular}{|l|l|}
135.3 \\
\end{tabular} & 55.44 & 5125 & 0.1 & 1950 & 7500 & 1687500 & 0.0726 & \#\#\#曲 & 2.662 & $6.20 \mathrm{E}-03$ & 65.42 & 92.1 & 20.96 & 0 & 111.8 & 311.4 \\
\hline 512 & 23.48 & 3000 & 530 & 3.595927 & 0.1778 & \begin{tabular}{|l|}
148.3 \\
\end{tabular} & 50.56 & 5125 & 0.1 & 1950 & 7500 & 1937500 & 0.1355 & \#\#\#\#正 & 1.73 & $1.52 \mathrm{E}-02$ & 65.29 & 0 & 0 & 0 & 45.7 & 377.5 \\
\hline 513 & 30.27 & 3000 & 560 & 36.191593 & 1.5503 & \begin{tabular}{|l|l|}
128.5 \\
\end{tabular} & 58.36 & 5125 & 0.1 & 1950 & 7500 & 1562500 & 0.1051 & \#\#\#\# & 1.957 & $9.13 \mathrm{E}-03$ & 64.31 & 86.18 & 0 & 0 & 75.9 & 331.3 \\
\hline 514 & 28.2 & 3000 & 510 & 82.055549 & 3.6818 & \begin{tabular}{|l|}
134.6 \\
\end{tabular} & 55.72 & 5125 & 0.1 & 1950 & 7500 & 1675000 & 0.1128 & \#\#曲 & 1.74 & $7.20 \mathrm{E}-03$ & 52.28 & 93.91 & 39.07 & & 96.2 & 316.1 \\
\hline 515 & 36.4 & 3000 & 1280 & $\begin{array}{l}0.67529 \\
\end{array}$ & 0.0241 & $\begin{array}{r}107 \\
1550\end{array}$ & $\begin{array}{l}70.08 \\
18.11 \\
\end{array}$ & 5125 & 0.1 & 1950 & 7500 & 1187500 & 0.0874 & \#\#\#\# & 3.441 & $1.47 \mathrm{E}-02$ & 32.68 & & 0 & & 47.1 & 484.5 \\
\hline $\begin{array}{l}5516 \\
517 \\
\end{array}$ & 22.27 & 3000 & $\begin{array}{r}910 \\
1340\end{array}$ & $\begin{array}{r}0.369462 \\
24.450345\end{array}$ & $\mid \begin{array}{l}0.0192 \\
1.135\end{array}$ & $\begin{array}{l}\frac{155.9}{139.3} \\
\end{array}$ & $\begin{array}{l}48.11 \\
53.86\end{array}$ & \begin{tabular}{|c|}
5125 \\
525 \\
\end{tabular} & $\begin{array}{l}0.1 \\
0.1 \\
\end{array}$ & $\begin{array}{l}1950 \\
1950\end{array}$ & $\begin{array}{l}7500 \\
7500\end{array}$ & \begin{tabular}{l|l|}
2087500 \\
172500
\end{tabular} & $\begin{array}{l}0.1496 \\
0.1411\end{array}$ & 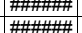 & $\begin{array}{l}2.456 \\
2.517 \\
\end{array}$ & \begin{tabular}{|l|}
$1.42 \mathrm{E}-02$ \\
$4.66 \mathrm{E}-03$ \\
\end{tabular} & $\begin{array}{r}74.9 \\
24.15\end{array}$ & $\begin{array}{r}0 \\
79.55 \\
\end{array}$ & $\frac{0}{0}$ & $\frac{0}{0}$ & \begin{tabular}{r|r|r|}
148.8 & \\
1487
\end{tabular} & $\frac{579.9}{572}$ \\
\hline 518 & 32.23 & 3000 & 1300 & 14.519981 & 0.5815 & \begin{tabular}{|l|}
120.1 \\
\end{tabular} & 62.42 & 5125 & 0.1 & 1950 & 7500 & 1412500 & 0.0987 & 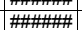 & 3.237 & \begin{tabular}{|l|}
$5.95 \mathrm{E}-03$ \\
\end{tabular} & 27.52 & 65.56 & & & $\begin{array}{l}140.1 \\
116.5\end{array}$ & $\begin{array}{l}5 / 2 \\
514.7 \\
\end{array}$ \\
\hline 519 & 22.92 & 3000 & 640 & 3.345679 & 0.2064 & \begin{tabular}{|l|}
185.1 \\
\end{tabular} & 40.53 & 5125 & 0.1 & 1950 & 7500 & 2700000 & 0.1388 & 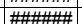 & 1.816 & $\frac{1.31 \mathrm{E}-02}{1.02}$ & 43.63 & & 0 & & 528 & \\
\hline 520 & 57.22 & 3000 & 1270 & 4.104604 & 0.1327 & \begin{tabular}{|l|}
97.02 \\
\end{tabular} & 77.3 & 5125 & 0.1 & 1950 & 7500 & 1025000 & 0.0556 & \#\#\#\#曲 & 5.019 & \begin{tabular}{|l|}
$1.17 \mathrm{E}-02$ \\
\end{tabular} & 74.79 & & 0 & & 59.1 & 449.5 \\
\hline 521 & 26.14 & 3000 & 630 & 103.175051 & 4.4668 & \begin{tabular}{|l|}
129.9 \\
\end{tabular} & 57.75 & 5125 & 0.1 & 1950 & 7500 & 1587500 & & \#\#\#\#曲 & 1.834 & $5.34 \mathrm{E}-03$ & 41.83 & 95.15 & 51.54 & 0 & 129.7 & 359.6 \\
\hline 522 & 23.02 & 3000 & 580 & & 0.5949 & \begin{tabular}{|l|}
100.2 \\
\end{tabular} & 74.88 & 5125 & 0.1 & 1950 & 7500 & 1075000 & & \#\#\#\#曲 & $\begin{array}{l}1.7 \\
\end{array}$ & 1.11E-02 & 53.74 & 71.94 & & 0 & 62.4 & 378.5 \\
\hline 523 & 32.17 & 3000 & 1360 & & & \begin{tabular}{|l|}
121.6 \\
\end{tabular} & 61.7 & & 0.1 & 1950 & 7500 & 1437500 & & 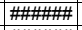 & 3.794 & $1.72 \mathrm{E}-02$ & 51.28 & & 0 & 0 & 40.3 & 604.4 \\
\hline 524 & 26.1 & 3000 & 620 & & $\begin{array}{l}0.078 \\
\end{array}$ & \begin{tabular}{|l|}
139.9 \\
\end{tabular} & 53.6 & & 0.1 & 1950 & 7500 & 1775000 & & \#\#\#\#| & 2.078 & $1.63 \mathrm{E}-02$ & 71.31 & 0 & 0 & 0 & 42.6 & 408.1 \\
\hline 525 & 34.39 & 3000 & 740 & & 0.5692 & \begin{tabular}{|l|l|}
188.5 \\
\end{tabular} & 39.79 & & 0.1 & 1950 & 7500 & 2775000 & & \#\#\#\#+ & 2.438 & & 48.24 & 44.81 & 0 & 0 & $\begin{array}{ll}64.8 \\
\end{array}$ & 363.4 \\
\hline 526 & 33.24 & 3000 & $\begin{array}{l}790 \\
1180\end{array}$ & \begin{tabular}{|l|}
106.412884 \\
105502
\end{tabular} & 4.6311 & \begin{tabular}{|l|l|}
130.6 \\
\end{tabular} & 57.44 & 5125 & 0.1 & 1950 & & 1600000 & 0.0957 & \#\#曲 & 2.274 & $5.52 \mathrm{E}-03$ & 17.92 & 95.3 & 53.01 & 0 & 125.6 & 350.6 \\
\hline 527 & 22.22 & 3000 & 1180 & $\begin{array}{l}1.055925 \\
\end{array}$ & $\begin{array}{ll}0.0292 \\
\end{array}$ & \begin{tabular}{|l|}
83.1 \\
\end{tabular} & 90.25 & 5125 & 0.1 & 1950 & 7500 & 812500 & 0.1432 & 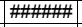 & 2.848 & $1.13 \mathrm{E}-02$ & \begin{tabular}{|l|l|}
78.8 \\
\end{tabular} & & 0 & 0 & $\begin{array}{l}61.3 \\
\end{array}$ & 657 \\
\hline $\begin{array}{l}528 \\
529 \\
\end{array}$ & $\begin{array}{l}24.87 \\
25.57 \\
\end{array}$ & 3000 & $\begin{array}{l}1230 \\
530\end{array}$ & 6.205094 & \begin{tabular}{l|l|}
0.2908 \\
13202
\end{tabular} & \begin{tabular}{|l|l|}
140.6 \\
81.39
\end{tabular} & $\begin{array}{l}53.35 \\
92.15\end{array}$ & $\begin{array}{l}5125 \\
5125\end{array}$ & $\begin{array}{l}0.1 \\
\end{array}$ & $\begin{array}{l}1950 \\
1950\end{array}$ & 7500 & \begin{tabular}{r|r|}
1787500 \\
7875
\end{tabular} & $\begin{array}{l}0.1279 \\
0.1244\end{array}$ & 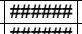 & $\begin{array}{l}2.669 \\
1642\end{array}$ & 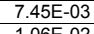 & 40.21 & $\begin{array}{l}19.42 \\
9077\end{array}$ & 0 & 0 & $\begin{array}{l}93.1 \\
\end{array}$ & 549.9 \\
\hline$\frac{529}{530}$ & $\begin{array}{l}25.57 \\
34.73\end{array}$ & 3000 & $\begin{array}{r}530 \\
1260\end{array}$ & & $\begin{array}{r}1.3262 \\
0.196\end{array}$ & \begin{tabular}{|l|}
81.39 \\
1237
\end{tabular} & $\begin{array}{l}92.15 \\
60.65 \\
\end{array}$ & $\frac{5125}{5125}$ & 0.1 & 1950 & $\begin{array}{l}7500 \\
77500\end{array}$ & $\begin{array}{r}787500 \\
147500\end{array}$ & $\begin{array}{l}0.1244 \\
0.916\end{array}$ & |\#\#\#曲 & $\begin{array}{r}1.643 \\
3.169\end{array}$ & & $\begin{array}{r}40.5 \\
32.81\end{array}$ & $\begin{array}{l}89.77 \\
5\end{array}$ & & & 65.5 & 329.3 \\
\hline 531 & 24.7 & 3000 & 1150 & & \begin{tabular}{|c|}
0.4196 \\
0.1248
\end{tabular} & \begin{tabular}{|r|}
$\mid 12.1$ \\
104
\end{tabular} & $\begin{array}{l}60.65 \\
72.12\end{array}$ & \begin{tabular}{|l|l|}
5125 \\
5125
\end{tabular} & $\begin{array}{l}0.1 \\
0.1\end{array}$ & $\begin{array}{l}190 \\
195\end{array}$ & $\begin{array}{l}5500 \\
7500\end{array}$ & $\begin{array}{l}1475000 \\
1137500\end{array}$ & $\begin{array}{l}0.0916 \\
0.1288\end{array}$ & 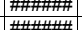 & & & & 50.88 & & & & $\begin{array}{l}467.6 \\
5132\end{array}$ \\
\hline 532 & 51.39 & 3000 & 1450 & 31.75 & 0.7869 & \begin{tabular}{|l|l|}
74.34 \\
\end{tabular} & 100.88 & 5125 & 0.1 & & 756 & & & 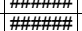 & $\begin{array}{l}2.474 \\
4.404\end{array}$ & \begin{tabular}{|l|}
$1.03 \mathrm{E}$ \\
$5.61 \mathrm{E}$ \\
\end{tabular} & & $\begin{array}{r}04.25 \\
8\end{array}$ & 0 & $\frac{0}{0}$ & $\begin{array}{r}\frac{67.2}{123.6} \\
\end{array}$ & $\begin{array}{l}513.3 \\
439.1\end{array}$ \\
\hline 533 & 39.42 & 3000 & 920 & & 0.4527 & \begin{tabular}{|l|}
93.04 \\
\end{tabular} & \begin{tabular}{|l|}
80.61 \\
\end{tabular} & 5125 & 0.1 & & 75 & & & "\#\#"\#! & 2.804 & & & 65.74 & & 0 & 63 & 364.5 \\
\hline 534 & 37.92 & 3000 & 1080 & & 1.054 & \begin{tabular}{|l|}
89.79 \\
\end{tabular} & 83.53 & 5125 & 0.1 & & 7500 & 912500 & & |\#\#\#\#| & 3.103 & & & 85.8 & & & 111.1 & 419.4 \\
\hline 535 & 31.25 & 3000 & 1150 & & 0.2676 & 110 & 68.18 & 5125 & 0.1 & & 7500 & 1237500 & & \#\#\#\#+ & 3.223 & $8.00 \mathrm{E}$ & & 31.49 & & 0 & 86.7 & 528.6 \\
\hline 536 & 31.59 & 3000 & 670 & & 2.62 & \begin{tabular}{|l|}
163.9 \\
\end{tabular} & 45.77 & 5125 & 0.1 & 1950 & 7500 & 2250000 & & \#\#\#\#" & 2.044 & & & 89.58 & & 0 & 81.5 & 33 \\
\hline 537 & 59.8 & 3000 & 980 & & 2.6463 & \begin{tabular}{|l|}
169.3 \\
\end{tabular} & 44.3 & & 0.1 & & 7500 & 2362500 & & \#\#\#\#+ & 3.7 & & & 89.34 & & 0 & 139.1 & 317 \\
\hline 538 & 53.56 & & 2160 & & 0.0531 & $\begin{array}{l}102.5 \\
\end{array}$ & & & 0.1 & & & & & \#\#\#\# & \begin{tabular}{|l|l|}
6.697 \\
\end{tabular} & 9.44 & & & & & 73.4 & 644 \\
\hline 539 & 39.72 & 3000 & 1850 & 3.14306 & $\begin{array}{l}0.0958 \\
\end{array}$ & \begin{tabular}{|l|l}
91.42 \\
\end{tabular} & 82.04 & 5125 & 0.1 & & 7500 & & & 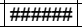 & 4.6 & \begin{tabular}{|l|l|}
8.37 \\
\end{tabular} & & 0 & 0 & 0 & 82.8 & 593. \\
\hline 540 & 39.72 & 3000 & 1120 & 17.448226 & 0.8214 & \begin{tabular}{|l|l|}
141.2 \\
\end{tabular} & & 5125 & 0.1 & & 7500 & & & \#\#\#\#+ & 3.062 & & & 71.34 & 0 & 0 & 84.7 & 395 \\
\hline 541 & & 3000 & 410 & & 2.5903 & 113 & 66 & & 0.1 & & 7500 & 128 & & \#\#\#\#+ & 1.416 & & & 92.73 & 27.32 & 0 & 74.3 & 309 \\
\hline 542 & 35.5 & & 390 & \begin{tabular}{|l|l|}
104.4 \\
\end{tabular} & 5.8097 & \begin{tabular}{|l|}
166.9 \\
\end{tabular} & & 512 & 0.1 & & 7500 & & & \#\#\#\#+ & 1.677 & & & 95.21 & 52.12 & 0 & 77.3 & 241 \\
\hline 543 & 24. & & 580 & & 2.2349 & \begin{tabular}{|l|}
171.1 \\
\end{tabular} & & 5125 & 0.1 & & 7500 & & & \#\#\#+\#| & 1.678 & & & 87.24 & 0 & 0 & 80.5 & 353 \\
\hline 544 & & & 1290 & & & \begin{tabular}{|l|}
113.7 \\
\end{tabular} & & & 0.1 & & 7500 & & & \#\#\# & 3.102 & & & 57 & 0 & 0 & 85 & 456 \\
\hline 545 & 26. & & 1270 & & & \begin{tabular}{l|l|}
147.7 \\
\end{tabular} & & & 0.1 & & & & & & 85 & & & 0 & & 0 & 54.8 & 541. \\
\hline 546 & 63. & & 2130 & & 0.04 & 156.5 & & & 0.1 & & & & & & & & & 0 & & 0 & 65.1 & 631.3 \\
\hline 547 & 45.13 & & 800 & & & $\begin{array}{l}115.1 \\
\end{array}$ & 65.14 & & 0.1 & & & & & & & & & 50.04 & & 0 & 60.2 & 365 \\
\hline 548 & & & 620 & & & & & & 0.1 & & & & & & & & & & 0 & 0 & 68.5 & \\
\hline 549 & & & 1650 & & $\begin{array}{l}0.3237 \\
\end{array}$ & 73. & 102 & & 0.1 & & & & & \#\#曲 & & & & .19 & 0 & 0 & 119.1 & \\
\hline 550 & & & 690 & & & \begin{tabular}{ll|}
106.3 \\
\end{tabular} & & & & & & & & & & & & & 0 & 0 & 53.1 & 392.2 \\
\hline 551 & 22.92 & 3000 & 450 & & & $\begin{array}{l}104.8 \\
\end{array}$ & & & 0.1 & & & & & & & & & \begin{tabular}{|l|l|}
93.13 \\
\end{tabular} & 31.25 & & & 317.1 \\
\hline 552 & 28.61 & 3000 & 2330 & & & 174 & & & 0.1 & & & & & & & & & & 0 & & $\begin{array}{l}94.7 \\
\end{array}$ & 768.2 \\
\hline 553 & 25.27 & 300 & 500 & & 0.3841 & $\begin{array}{l}157.1 \\
\end{array}$ & & & & & & & & & & & & 31.82 & 0 & & 45.1 & 7.5 .5 \\
\hline 554 & 58.59 & 300 & \begin{tabular}{ll|}
640 \\
740
\end{tabular} & 75 & 3.162 & \begin{tabular}{r|}
123 \\
\end{tabular} & & & & & & & & & & & & & 35.2 & & 93.5 & 57.2 \\
\hline 555 & 26.6 & & \begin{tabular}{r|}
710 \\
110
\end{tabular} & & 0.4 & $\begin{array}{l}79.66 \\
\end{array}$ & & & 0.1 & & & & 0.1 & & & & & 70.17 & 0 & t & $\begin{array}{r}67.9 \\
172 ?\end{array}$ & 412.7 \\
\hline 556 & $\begin{array}{l}22.42 \\
1.57\end{array}$ & 3000 & 1120 & & 1.67 & $\begin{array}{l}130.6 \\
112 ?\end{array}$ & 57.44 & & 0.1 & & 7500 & & & 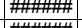 & 2.356 & \begin{tabular}{|l|}
$4.02 \mathrm{E}$ \\
1.52
\end{tabular} & & 87.04 & 0 & & 172.3 & 538.6 \\
\hline 557 & $\begin{array}{l}45.77 \\
25.15\end{array}$ & 3000 & $\begin{array}{r}530 \\
1190\end{array}$ & $\begin{array}{r}26.486574 \\
15\end{array}$ & 0.9908 & $\begin{array}{l}112.2 \\
100 .\end{array}$ & 66.83 & 512 & 0.1 & & 7500 & 1275000 & 0.0695 & $\mid$ |\#⿻ & 2.237 & $1.52 \mathrm{E}$ & & 81.12 & 0 & & 45.5 & 250.5 \\
\hline$\frac{558}{559}$ & 25.15 & 3000 & $\begin{array}{l}7190 \\
540\end{array}$ & & 0.0525 & $\begin{array}{l}100.9 \\
100 ?\end{array}$ & 74.31 & $\frac{512}{512}$ & 0.1 & & $7500 \mid$ & & 0.1265 & 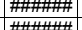 & $\begin{array}{l}2.556 \\
1787\end{array}$ & & & & & & $\begin{array}{r}57.9 \\
50.7\end{array}$ & 520.8 \\
\hline 5 & 20.00 & & S40 & & 0.3949 & 100.2 & & & & & & & & & & & & & & & & 347.2 \\
\hline 501 & & 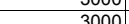 & 500 & & & 102.2 & & & 11 & & & & & & & & & 77.016 & & & & \\
\hline 562 & & 300 & 1800 & & & 11 & $\frac{3.5}{66}$ & & 0.1 & & & & & & & & & 10.40 & 0 & & & \\
\hline 563 & 278 & 3000 & 2100 & & & 1839 & & & 0.1 & & & & & & & & & & & & & \\
\hline 564 & 27.86 & & 640 & & 0.28 & 88.14 & & & 0.1 & & & & & & 1.9 & & & 48.71 & 0 & & & \\
\hline 565 & 32.53 & 3000 & 800 & 107.733976 & 3.624 & 100.9 & 74.31 & 5125 & 0.1 & & 75 & & & & 2.377 & 4.34 & 57. & $\begin{array}{l}95.36 \\
\end{array}$ & 53.59 & & 159.8 & 374.5 \\
\hline 566 & 27.1 & 3000 & 1380 & 29.818437 & \begin{tabular}{|l|l|}
1.0337 \\
\end{tabular} & 104 & 72.12 & 5125 & 0.1 & 1950 & 7500 & 1137500 & 0.1174 & & 2.808 & $5.07 \mathrm{E}-03$ & 24.23 & 83.23 & & j & 136.8 & 531 \\
\hline 567 & 22.45 & 3000 & 610 & \begin{tabular}{|l}
.926428 \\
\end{tabular} & $\begin{array}{ll}0.1664 \\
\end{array}$ & $\begin{array}{l}127.1 \\
\end{array}$ & 58.99 & 5125 & 0.1 & 1950 & 7500 & 1537500 & 0.1417 & \#\#\#\#+ & 1.792 & $1.38 \mathrm{E}-02$ & 56.93 & & & & \begin{tabular}{|l|}
50.3 \\
\end{tabular} & 409.2 \\
\hline
\end{tabular}




\begin{tabular}{|c|c|c|c|c|c|c|c|c|c|c|c|c|c|c|c|c|c|c|c|c|c|c|}
\hline & $\begin{array}{c}\text { Velocity } \\
(\mathrm{m} / \mathrm{y})\end{array}$ & $\begin{array}{l}\text { Fixed Source } \\
\text { Concentration } \\
(\mathrm{mg} / \mathrm{L})\end{array}$ & $\begin{array}{c}\text { Time of } \\
\text { Maximum } \\
\text { at } \\
\text { Compliance } \\
\text { Boundary } \\
\text { (y) }\end{array}$ & $\mid \begin{array}{c}\text { Maximum } \\
\text { Concentration } \\
\text { Compliance } \\
\text { Boundary } \\
(\mu \mathrm{g} / \mathrm{L})\end{array}$ & $\begin{array}{c}\text { Maximum } \\
\text { Release Rate } \\
\text { at } \\
\text { Compliance } \\
\text { Boundary } \\
(\mathrm{Kg} / \mathrm{y})\end{array}$ & $\begin{array}{l} \\
\text { Sourc } \\
\text { e Rate } \\
\text { (Kg/y) }\end{array}$ & \begin{tabular}{|c|} 
Source \\
Duration \\
(y)
\end{tabular} & $\begin{array}{c}\text { 1-D } \\
\text { Streamtube } \\
\text { Length } \\
(\mathrm{m})\end{array}$ & $\begin{array}{c}\text { Base } \\
\text { Porosity } \\
\left(\mathrm{m}^{3} / \mathrm{m}^{3}\right)\end{array}$ & $\begin{array}{c}\text { Bulk } \\
\text { Densty } \\
\left(\mathrm{Kg} / \mathrm{m}^{3}\right)\end{array}$ & \begin{tabular}{|c|} 
Remaining \\
Carbon \\
Tetrachloride \\
Source \\
$(\mathrm{Kg})$
\end{tabular} & $\begin{array}{c}\text { Volume at or } \\
\text { above Fixed } \\
\text { Source } \\
\text { Concentratio } \\
n(m 3)\end{array}$ & $\begin{array}{l}\text { Porosity } \\
\left(\mathrm{m}^{3} / \mathrm{m}^{3}\right)\end{array}$ & $\left.\mid \begin{array}{c}\mathrm{Kd} \\
\left(\mathrm{m}^{3} / \mathrm{Kg}\right.\end{array}\right)$ & Retardation & $\begin{array}{c}\text { First } \\
\text { Order } \\
\text { Rate } \\
\text { Constant } \\
(1 / y)\end{array}$ & $\begin{array}{l}\text { Longitudinal } \\
\text { Dispersivity } \\
(\mathrm{m})\end{array}$ & $\begin{array}{c}\text { Required } \\
\text { Source } \\
\text { Cleanup } \\
\text { Percentage } \\
(5 \mu \mathrm{g} / \mathrm{L} \\
\text { Compliance })\end{array}$ & $\begin{array}{c}\text { Required } \\
\text { Source } \\
\text { Cleanup } \\
\text { Percentage } \\
(50 \mu \mathrm{g} / \mathrm{L} \\
\text { Compliance })\end{array}$ & \begin{tabular}{|c|} 
Required \\
Source Cleanup \\
Percentage \\
(500 ug/L \\
Compliance)
\end{tabular} & \begin{tabular}{|c|} 
Abiotic \\
Reaction \\
Half Time \\
(y)
\end{tabular} & $\begin{array}{c}\text { Carbon } \\
\text { Tetrachloride } \\
\text { Travel Time } \\
\text { (y) }\end{array}$ \\
\hline & & & & \begin{tabular}{|l|}
8.769975 \\
\end{tabular} & $\begin{array}{l}0.3323 \\
\end{array}$ & 113.7 & & 5125 & & & & 1300000 & 0.1389 & \#\#\#立 & 2.455 & $7.34 \mathrm{E}-03$ & & 42.99 & & & & \\
\hline 569 & 48.72 & 3000 & 350 & 110.837868 & 4.7226 & 127.8 & 58.67 & 5125 & 0.1 & 1950 & 7500 & 1550000 & 0.0653 & 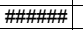 & 1.829 & $1.33 \mathrm{E}-02$ & 35.74 & 95.49 & 54.89 & 0 & 52 & 192.4 \\
\hline 570 & 23.15 & 3000 & 1250 & 10.756774 & 0.3507 & 97.81 & 76.68 & 5125 & 0.1 & 1950 & 7500 & 1037500 & 0.1374 & 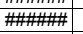 & 2.734 & $\begin{array}{ll}6.20 \mathrm{E}-03 \\
\end{array}$ & 70.98 & 53.52 & & 0 & 111.7 & 605.2 \\
\hline 571 & 37.78 & 3000 & 330 & 634.706556 & 27.7661 & $\begin{array}{l}131.2 \\
\end{array}$ & 57.15 & 5125 & 0.1 & 1950 & 7500 & 1612500 & 0.0842 & 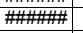 & 1.512 & $5.19 \mathrm{E}-03$ & 20.78 & 99.21 & 92.12 & 21.22 & 133.5 & 205.1 \\
\hline 572 & 49.32 & 3000 & 1090 & \begin{tabular}{|l|}
28.365043 \\
\end{tabular} & 1.1627 & 123 & 60.99 & 5125 & 0.1 & 1950 & 7500 & 1462500 & 0.0645 & 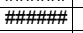 & 3.413 & $7.63 \mathrm{E}-03$ & 26.69 & 82.37 & & & 90.8 & 354.6 \\
\hline 573 & 23.27 & 3000 & 620 & 56.989632 & 1.6744 & \begin{tabular}{|l|l|}
88.14 \\
\end{tabular} & 85.09 & 5125 & 0.1 & 1950 & 7500 & 887500 & 0.1367 & 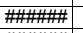 & 1.757 & $7.49 \mathrm{E}-03$ & 58.25 & 91.23 & 12.26 & & 92.5 & 387 \\
\hline 574 & 35.31 & 3000 & 2020 & $\begin{array}{r}4.217384 \\
\end{array}$ & 0.193 & $\begin{array}{l}137.3 \\
\end{array}$ & 54.64 & 5125 & 0.1 & 1950 & 7500 & 1725000 & 0.0901 & 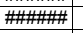 & 4.302 & $6.23 \mathrm{E}-03$ & 40.9 & & & 0 & 111.3 & 624.4 \\
\hline 575 & 47.84 & 3000 & 200 & 539.022423 & 25.7257 & \begin{tabular}{l|l}
143.2 \\
\end{tabular} & 52.38 & 5125 & 0.1 & 1950 & 7500 & 1837500 & 0.0665 & 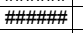 & 1.344 & $7.82 \mathrm{E}-03$ & 79.28 & 99.07 & 90.72 & 7.24 & 88.6 & 143.9 \\
\hline 576 & 35.31 & 3000 & 590 & 76.352504 & 2.0714 & 81.39 & 92.15 & 5125 & 0.1 & 1950 & 7500 & 787500 & 0.0901 & \#\#\#\#+ & 2.005 & $1.05 \mathrm{E}-02$ & 21.22 & 93.45 & 34.51 & 0 & 66.2 & \\
\hline 577 & 32.07 & 3000 & 450 & 86.712265 & 3.534 & $\begin{array}{l}122.3 \\
\end{array}$ & 61.34 & 5125 & 0.1 & 1950 & 7500 & 1450000 & 0.0992 & \#\#\#\#+ & 1.673 & $1.05 \mathrm{E}-02$ & 23.31 & 94.23 & 42.34 & & 66 & 267. \\
\hline 578 & 22.81 & 3000 & 1220 & 1.39886 & 0.054 & 115.9 & 64.73 & 5125 & 0.1 & 1950 & 7500 & 1337500 & 0.1395 & \#\#\#\#\#+ & 2.64 & $1.04 \mathrm{E}-02$ & 44.71 & & & & 66.6 & 593.2 \\
\hline 579 & 46.31 & 3000 & 860 & & 0.8671 & \begin{tabular}{|l|}
88.14 \\
\end{tabular} & & 5125 & 0.1 & 1950 & 7500 & & & \#\#\#\#+\#+ & 3.026 & $9.51 \mathrm{E}-03$ & 45.15 & 83.06 & & & 72.9 & 334.9 \\
\hline 580 & 35.95 & 3000 & 2170 & 0.968447 & 0.0428 & $\begin{array}{l}132.6 \\
\end{array}$ & 56.56 & 5125 & 0.1 & 1950 & 7500 & 1637500 & 0.0885 & 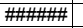 & 4.719 & $8.65 \mathrm{E}-03$ & 40.35 & & & 0 & 80.1 & 672.7 \\
\hline 581 & 42.08 & 3000 & 1970 & & 0.2428 & $\begin{array}{l}133.3 \\
\end{array}$ & 56.28 & 5125 & 0.1 & 1950 & 7500 & 1650000 & & 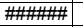 & 5.148 & $5.72 \mathrm{E}-03$ & 72.46 & 8.51 & & & 121.1 & 627 \\
\hline 582 & 21.22 & 3000 & 1170 & & $\begin{array}{l}0.1335 \\
\end{array}$ & $\begin{array}{l}156.5 \\
\end{array}$ & & 5125 & 0.1 & 1950 & 7500 & 2100000 & & \#\#\#\#曲 & 2.261 & $9.84 \mathrm{E}-03$ & 13.52 & & & 0 & 70.5 & 546 \\
\hline 583 & 56.81 & 3000 & 2180 & & 0.1831 & $\begin{array}{l}140.6 \\
\end{array}$ & & 5125 & 0.1 & 1950 & 7500 & 1787500 & 0.056 & \#\#\#\#曲 & & $7.38 \mathrm{E}-03$ & 35.61 & & 0 & 0 & 93.9 & 520.7 \\
\hline 584 & 49.25 & 3000 & 690 & & 2.8138 & $\begin{array}{l}154.6 \\
\end{array}$ & 48.5 & 5125 & 0.1 & 1950 & 7500 & & & \#\#\#\#曲 & 2.802 & $6.74 \mathrm{E}-03$ & 62.11 & \begin{tabular}{ll|}
90.84 \\
\end{tabular} & 8.4 & & 102.8 & 291.6 \\
\hline 585 & 37.74 & 3000 & 370 & 15.565157 & $\begin{array}{ll}0.7462 \\
\end{array}$ & $\begin{array}{l}143.8 \\
\end{array}$ & 52.15 & 5125 & 0.1 & 1950 & 7500 & 1850000 & 0.0843 & \#\#\#\#\# & 1.744 & $1.88 \mathrm{E}-02$ & 54.7 & \begin{tabular}{ll|}
67.88 \\
\end{tabular} & & 0 & 36.8 & 236.8 \\
\hline 586 & $\begin{array}{ll}39.62 \\
27.19\end{array}$ & 3000 & 1130 & $\begin{array}{r}4.865719 \\
\end{array}$ & 0.1784 & 110 & 68.18 & 5125 & 0.1 & 1950 & 7500 & 1237500 & $\begin{array}{ll}0.0803 \\
\end{array}$ & \#\#\#\# & 3.478 & $1.06 \mathrm{E}-02$ & 56.24 & & 0 & 0 & 65.2 & 449.9 \\
\hline \begin{tabular}{|c|}
587 \\
588
\end{tabular} & 27.74 & 3000 & \begin{tabular}{|l|l|l|l|}
680 \\
270
\end{tabular} & \begin{tabular}{|l|}
101.315743 \\
\end{tabular} & & $\begin{array}{l}134.6 \\
\end{array}$ & 55.72 & 5125 & 0.1 & 1950 & 7500 & & 0.1147 & \#\#\#\#+ & 2.039 & 3.95E-03 & 76.8 & $\begin{array}{ll}95.06 \\
\end{array}$ & 50.65 & 0 & 175.6 & 376.8 \\
\hline 588 & $\begin{array}{l}38.66 \\
3286 \\
\end{array}$ & $\begin{array}{ll}3000 \\
3000\end{array}$ & $\begin{array}{ll}370 \\
90\end{array}$ & \begin{tabular}{|r|}
241.291395 \\
68.901964
\end{tabular} & 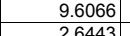 & $\begin{array}{l}119.4 \\
115 .\end{array}$ & $\begin{array}{l}62.79 \\
65.14 t\end{array}$ & 5125 & 0.1 & $\begin{array}{l}1950 \\
1050\end{array}$ & 7500 & $\begin{array}{l}1400000 \\
1225000\end{array}$ & 0.0823 & 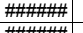 & \begin{tabular}{|c|}
1.681 \\
\end{tabular} & 7.03E-03 & 59.99 & 97.93 & $\begin{array}{ll}79.28 \\
27.23\end{array}$ & 0 & $\begin{array}{r}98.6 \\
170.7\end{array}$ & 222.9 \\
\hline 589 & $\begin{array}{ll}32.86 \\
48.28\end{array}$ & $\frac{3000}{3000}$ & $\begin{array}{l}950 \\
420\end{array}$ & $\begin{array}{r}68.901964 \\
76.94426\end{array}$ & $\begin{array}{l}2.6443 \\
2.4804 \\
\end{array}$ & \begin{tabular}{l|}
115.1 \\
97.02 \\
\end{tabular} & $\begin{array}{l}65.14 \\
773\end{array}$ & $\begin{array}{l}5125 \\
5125\end{array}$ & 0.1 & $\begin{array}{l}1950 \\
1950\end{array}$ & $\begin{array}{l}7500 \\
7500\end{array}$ & \begin{tabular}{|r|}
1325000 \\
125000
\end{tabular} & $\begin{array}{l}0.0968 \\
0.0659 \\
\end{array}$ & 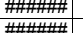 & $\begin{array}{r}2.69 \\
2.004 \\
\end{array}$ & $\begin{array}{r}3.88 \mathrm{E}-03 \\
1.3 \mathrm{~F}-02\end{array}$ & 69.65 & $\begin{array}{l}92.74 \\
93.48\end{array}$ & 27.43 & & & $\begin{array}{l}419.6 \\
2127\end{array}$ \\
\hline 591 & 31.97 & 3000 & $\begin{array}{ll}660 \\
660\end{array}$ & 40.171342 & 1.6653 & 124.4 & 60.31 & 5125 & 0.1 & 1950 & 7500 & \begin{tabular}{|l|l|l}
1487500 \\
148750
\end{tabular} & $\begin{array}{l}0.06599 \\
0.0955 \\
\end{array}$ & 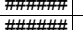 & 2.004 & & 33.89 & $\begin{array}{l}93.48 \\
87.55 \\
\end{array}$ & & & $\begin{array}{ll}48.5 \\
877 \\
\end{array}$ & $\frac{212.7}{348.7}$ \\
\hline 592 & 34.47 & 3000 & 390 & \begin{tabular}{|l|l|}
449.274652 \\
\end{tabular} & $\mid 12.1886$ & 81.39 & 92.15 & 5125 & 0.1 & 1950 & 7500 & 787500 & 0.0923 & 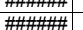 & $\frac{2.1 / 10}{1.581}$ & $\begin{array}{l}1.90 \mathrm{E}-0 \mathrm{~s} \\
5.67 \mathrm{E}-03\end{array}$ & 51.95 & $\begin{array}{l}01.05 \\
98.89 \\
\end{array}$ & 88.87 & & $\begin{array}{c}0.1 \\
122.2\end{array}$ & $\begin{array}{l}348.7 \\
235.1 \\
\end{array}$ \\
\hline 593 & 26.98 & 3000 & 870 & \begin{tabular}{|l|}
5.48381 \\
\end{tabular} & 0.2758 & 150.9 & 49.71 & 5125 & 0.1 & 1950 & 7500 & 1987500 & 0.1179 & \#\#\#\#立 & 2.307 & $1.05 \mathrm{E}-02$ & 37.35 & 8.82 & & & 65.9 & 438. \\
\hline 594 & 25.05 & 3000 & 1170 & 0.361937 & 0.0133 & 110 & 68.18 & 5125 & 0.1 & 1950 & 7500 & 1237500 & 0.127 & \#\#\#\#\# & 3.07 & $1.37 \mathrm{E}-02$ & 70.07 & 0 & 0 & 0 & 50.4 & 628. \\
\hline 595 & 23.9 & 3000 & 570 & $\begin{array}{l}366.140665 \\
\end{array}$ & 10.1424 & 83.1 & 90.25 & 5125 & 0.1 & 1950 & 7500 & 812500 & 0.13 & 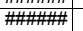 & 1.597 & $4.21 \mathrm{E}-03$ & 25.76 & 98.63 & 86.34 & & 164.8 & 342 . \\
\hline 596 & 50.42 & 3000 & 1650 & $\begin{array}{r}46.924116 \\
\end{array}$ & 1.7323 & \begin{tabular}{|c|}
110.7 \\
\end{tabular} & 67.72 & 5125 & 0.1 & 1950 & 7500 & 1250000 & & \#\#\#\#+ & 4.357 & & 37.39 & 89.34 & & & 200.3 & 442 \\
\hline 597 & 52.15 & 3000 & 520 & 228.592243 & 10.2057 & 133.9 & 56 & 5125 & 0.1 & 1950 & 7500 & & & \#\#\#\#+ & 2.372 & & 53.26 & 97.81 & 78.13 & & 160.5 & 233 \\
\hline 598 & 27.01 & 3000 & 1100 & 0.226426 & 0.0098 & 129.2 & 58.05 & 5125 & 0.1 & 1950 & 7500 & & 0.1 & \#\#\#\#+ & 3.103 & 1.56 & 67.99 & 0 & & & 44.3 & 588. \\
\hline 599 & 40.02 & 3000 & 430 & 126.34068 & 4.3476 & \begin{tabular}{|c|}
103.2 \\
\end{tabular} & 72.65 & 5125 & 0.1 & 1950 & 7500 & & & \#\#\#\#+ & 1.947 & & 85.12 & 96.04 & 60.42 & & 84 & 249. \\
\hline 600 & 42.3 & 3000 & 1570 & 24.227411 & 0.8088 & 100.2 & 74.88 & 5125 & 0.1 & 1950 & 7500 & 1075000 & & & 3.863 & & & 79.36 & & & 124.4 & 46 \\
\hline 601 & 34. & 00 & 2090 & & 0.0989 & 120.1 & 62.42 & 5125 & 0.1 & 1950 & 7500 & & & & 4.506 & & 46.4 & & & & 97.4 & 665. \\
\hline 602 & 44.43 & & 1240 & & $\begin{array}{ll}0.6215 \\
\end{array}$ & 125.1 & & 5125 & 0.1 & 1950 & 7500 & & & & & & & 66.46 & & & 92.2 & 415. \\
\hline 603 & & & 2090 & & & 77.02 & & 51 & 0.1 & & 7500 & & & & & & & & & & 112.4 & 597. \\
\hline 604 & & & $\begin{array}{l}410 \\
\end{array}$ & & 1.4 & $\begin{array}{l}107 \\
\end{array}$ & & & 0.1 & & & & & & & & & & 0 & & 47.6 & 251.8 \\
\hline 605 & 48. & & 320 & 432. & & 150.9 & & & 0.1 & & & & & & & & & & 88.44 & 0 & 135.4 & 104 \\
\hline 606 & & & 440 & & & \begin{tabular}{l|l|}
129.9 \\
\end{tabular} & & & & & & & & & & & & & 0 & 0 & 43.7 & 243.4 \\
\hline 607 & & & 200 & 1125. & 58.71 & $\begin{array}{l}156.5 \\
\end{array}$ & & & & & & & & & & & & & $\begin{array}{l}95.56 \\
\end{array}$ & 55.57 & 183 & 136.8 \\
\hline 608 & 40. & & 780 & & 71 & $\begin{array}{l}116.6 \\
\end{array}$ & & & & & & & & & & & & & 0 & 0 & 57.3 & 367 \\
\hline 609 & 24.53 & & 350 & & & $\begin{array}{l}86.48 \\
\end{array}$ & & 51 & 0.1 & & & & & & & & 54.57 & & 68.11 & & 71.2 & 264.2 \\
\hline 610 & & & 55 & & & $\begin{array}{l}113.7 \\
\end{array}$ & & & & & & & & & & & & & & & 54.1 & 326. \\
\hline 611 & 56.1 & & 550 & & & $\begin{array}{l}115.1 \\
150 .\end{array}$ & & & & & & & & & & & & & 63.13 & & & 245.2. \\
\hline 612 & 36.48 & 3000 & 1500 & & & $\begin{array}{l}169.3 \\
\end{array}$ & 44 & & & & & & & & & & & 73.19 & & $\frac{0}{n}$ & 144.6 & 492.2 \\
\hline 613 & $\begin{array}{l}36.28 \\
327 \\
\end{array}$ & 3000 & 2260 & & \begin{tabular}{|c|}
0.0048 \\
0.92 \\
\end{tabular} & $\begin{array}{l}133.3 \\
1571 \\
\end{array}$ & 56.28 & 5125 & 0.1 & $\begin{array}{l}1950 \\
1950 \\
\end{array}$ & & & & & 4.8 & & 29. & 0 & 0 & 0 & 54.6 & 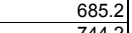 \\
\hline $\begin{array}{l}614 \\
615 \\
6\end{array}$ & \begin{tabular}{|l|}
32.7 \\
54.1 \\
\end{tabular} & $\frac{3000}{3000}$ & $\begin{aligned} 2420 \\
240\end{aligned}$ & \begin{tabular}{|r|}
0.941723 \\
1007.235592
\end{tabular} & $\begin{array}{r}0.0493 \\
42.9168\end{array}$ & \begin{tabular}{|l|}
157.1 \\
127.8
\end{tabular} & \begin{tabular}{|l|l|l|l|}
58.67 & \\
\end{tabular} & $\begin{array}{l}5125 \\
5125\end{array}$ & $\begin{array}{l}0.1 \\
0.1\end{array}$ & $\frac{195}{195}$ & $\begin{array}{l}7500 \\
7500\end{array}$ & $\frac{2112500}{1550000}$ & $\frac{0.05}{0.05}$ & 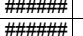 & $\frac{4.74}{1.52}$ & & $\begin{array}{l}41.51 \\
45.52\end{array}$ & \begin{tabular}{r|r}
0 \\
99.5
\end{tabular} & \begin{tabular}{r|r|}
0 & \\
95.04
\end{tabular} & 50.36 & \begin{tabular}{rl|}
94.2 & \\
167 &
\end{tabular} & $\frac{744.2}{144.2}$ \\
\hline $\begin{array}{l}010 \\
616 \\
\end{array}$ & 28.74 & 300 & 400 & \begin{tabular}{|l|l|l|l|l}
11.5882 \\
\end{tabular} & $\begin{array}{r}4.9100 \\
4.363 \\
\end{array}$ & $\begin{array}{l}12.0 \\
117.3 \\
\end{array}$ & 63. & 5125 & 0.1 & 195 & 7500 & & & \#冊曲! & $\frac{1.52}{1.53}$ & & $\begin{array}{l}45.02 \\
63.67 \\
\end{array}$ & $\begin{array}{r}95.52 \\
95.52\end{array}$ & 55.19 & & 81 & $\frac{144.5}{274}$ \\
\hline 617 & & & 400 & 129.99 & \begin{tabular}{|c|}
4.5723 \\
\end{tabular} & 105.5 & 71. & 512 & 0.1 & 195 & $\begin{array}{ll}7500 \\
\end{array}$ & & & \#\#車 & 1.3 & & 23. & 96.15 & 61.54 & & 69.7 & 271.8 \\
\hline 618 & 58.0 & & 320 & 269.81 & 10.1587 & 113 & 66 & 512 & 0.1 & 195 & 750 & & & \#\#! & 1.9 & & 45 & 98.15 & 81.47 & & 68.3 & 168.1 \\
\hline 619 & 27.4 & 30 & 1830 & & 0.0343 & 72.53 & 103 & 512 & 0.1 & 195 & 7500 & & & \#\#五 & & & 32. & 0 & 0 & & 73.6 & 661.7 \\
\hline 620 & 25.91 & 300 & 1040 & & 0.1233 & 163.9 & 45. & 512 & 0.1 & 195 & 750 & & & & 2.5 & & 34. & 0 & & & 64.3 & 495 . \\
\hline 621 & 28. & 3000 & 620 & & 1.62 & 109.3 & & 512 & 0.1 & 195 & 7500 & & & & 1.8 & & 31. & 88.82 & & & 74.6 & 338. \\
\hline 622 & 59.5 & 3000 & \begin{tabular}{|l|}
1740 \\
\end{tabular} & & 0.0507 & $\begin{array}{l}102.5 \\
\end{array}$ & 73. & 512 & 0.1 & 195 & 7500 & & & & & & 38. & & & 0 & 55.2 & 474. \\
\hline 623 & 51.31 & & 210 & & 52.3652 & \begin{tabular}{|l|}
163.9 \\
\end{tabular} & & 512 & 0.1 & 19 & & & & & & & & 99.48 & \begin{tabular}{|l|}
94.78 \\
\end{tabular} & 47.84 & 166.7 & 138. \\
\hline 624 & & 30 & 440 & & 7.8 & 179 & & & 0.1 & 195 & 7500 & & & & 1.4 & & 54. & 96.16 & 61.56 & & 129.3 & 318. \\
\hline 625 & 28.4 & 3000 & 730 & & 2.2307 & 17 & & & 0.1 & & 7500 & & & & & & 54 & 87 & 0 & & 114.7 & 385.8 \\
\hline 626 & & & 390 & & & 107 & & & 0.1 & & & & & & & & & & 84.71 & & 117.3 & 274. \\
\hline 62 & & & 80 & & & 130 & & & 0.1 & & & & & & & & & & 0 & & 87.4 & \\
\hline 62 & & & 81 & & & 1 & & & 0.1 & & & & & & & & & & 0 & & 78.1 & 306.3 \\
\hline 62 & & & 420 & & 13.5789 & 217.9 & & 5125 & 0.1 & 195 & 7500 & & & & & & & 33 & 73.25 & & 16.2 & \\
\hline 630 & 46.51 & 3000 & 2170 & $\mid 0.355913$ & 0.0184 & 155.3 & 48.3 & 5125 & 0.1 & 1950 & 7500 & 2075000 & & \#\#\#\#\#+ & 5.317 & & 29.55 & & & & \begin{tabular}{|l|}
58.7 \\
\end{tabular} & 585. \\
\hline
\end{tabular}




\begin{tabular}{|c|c|c|c|c|c|c|c|c|c|c|c|c|c|c|c|c|c|c|c|c|c|c|}
\hline Realization & $\begin{array}{c}\text { Velocity } \\
(\mathrm{m} / \mathrm{y})\end{array}$ & $\begin{array}{l}\text { Fixed Source } \\
\text { Concentration } \\
(\mathrm{mg} / \mathrm{L})\end{array}$ & $\begin{array}{c}\text { Time of } \\
\text { Maximum } \\
\text { at } \\
\text { Compliance } \\
\text { Boundary } \\
\text { (y) }\end{array}$ & $\mid \begin{array}{c}\text { Maximum } \\
\text { Concentration } \\
\text { Compliance } \\
\text { Boundary } \\
(\mu \mathrm{g} / \mathrm{L})\end{array}$ & $\begin{array}{c}\text { Maximum } \\
\text { Release Rate } \\
\text { at } \\
\text { Compliance } \\
\text { Boundary } \\
(\mathrm{Kg} / \mathrm{y})\end{array}$ & $\begin{array}{l} \\
\text { Sourc } \\
\text { e Rate } \\
\text { (Kg/y) }\end{array}$ & \begin{tabular}{|c|} 
Source \\
Duration \\
(y)
\end{tabular} & $\begin{array}{c}\text { 1-D } \\
\text { Streamtube } \\
\text { Length } \\
(\mathrm{m})\end{array}$ & $\mid \begin{array}{c}\text { Base } \\
\text { Porosity } \\
\left(\mathrm{m}^{3} / \mathrm{m}^{3}\right)\end{array}$ & $\begin{array}{c}\text { Bulk } \\
\text { Densty } \\
\left(\mathrm{Kg} / \mathrm{m}^{3}\right)\end{array}$ & \begin{tabular}{|c|} 
Remaining \\
Carbon \\
Tetrachloride \\
Source \\
$(\mathrm{Kg})$
\end{tabular} & $\begin{array}{c}\text { Volume at or } \\
\text { above Fixed } \\
\text { Source } \\
\begin{array}{c}\text { Concentratio } \\
n(m 3)\end{array}\end{array}$ & $\begin{array}{l}\text { Porosity } \\
\left(\mathrm{m}^{3} / \mathrm{m}^{3}\right)\end{array}$ & $\left(\begin{array}{c}\mathrm{Kd} \\
\left(\mathrm{m}^{3} / \mathrm{kg}\right)\end{array}\right.$ & Retardation & $\begin{array}{c}\text { First } \\
\text { Order } \\
\text { Rate } \\
\text { Constant } \\
(1 / y)\end{array}$ & $\begin{array}{l}\text { Longitudinal } \\
\text { Dispersivity } \\
(\mathrm{m})\end{array}$ & $\begin{array}{c}\text { Required } \\
\text { Source } \\
\text { Cleanup } \\
\text { Percentage } \\
(5 \mu \mathrm{g} / \mathrm{L} \\
\text { Compliance })\end{array}$ & $\begin{array}{c}\text { Required } \\
\text { Source } \\
\text { Cleanup } \\
\text { Percentage } \\
(50 \mu \mathrm{g} / \mathrm{L} \\
\text { Compliance })\end{array}$ & \begin{tabular}{|c} 
Required \\
Source Cleanup \\
Percentage \\
(500 $\mu \mathrm{g} / \mathrm{L}$ \\
Compliance)
\end{tabular} & \begin{tabular}{|c|} 
Abiotic \\
Reaction \\
Half Time \\
(y)
\end{tabular} & $\begin{array}{l}\text { Carbon } \\
\text { Tetrachloride } \\
\text { Travel Time } \\
\text { (y) }\end{array}$ \\
\hline & & & & \begin{tabular}{|l|}
5.588832 \\
\end{tabular} & $\begin{array}{l}0.3511 \\
\end{array}$ & 188.5 & \begin{tabular}{|l|l|}
197.79 \\
\end{tabular} & 5125 & & & & \begin{tabular}{|l|}
2775000 \\
\end{tabular} & 0.0982 & \#\#\#+ & 2.451 & $1.16 \mathrm{E}-02$ & $\begin{array}{r}73.32 \\
\end{array}$ & 10.54 & & & & \\
\hline 632 & 42.76 & 3000 & 1150 & 0.365691 & 0.0155 & 127.1 & 58.99 & 5125 & 0.1 & 1950 & 7500 & 1537500 & 0.0744 & 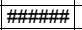 & 3.655 & $1.82 \mathrm{E}-02$ & 36.45 & & & & 38.2 & 438.1 \\
\hline 633 & 34.92 & 3000 & 790 & 17.714838 & 1.0894 & 184.5 & 40.65 & 5125 & 0.1 & 1950 & 7500 & 2687500 & 0.0911 & \#\#\#\#+ & 2.379 & $9.89 \mathrm{E}-03$ & 16.55 & 71.78 & & & 70.1 & 349.2 \\
\hline 634 & 29.16 & 3000 & 520 & 66.194651 & 2.6667 & 120.9 & 62.06 & 5125 & 0.1 & 1950 & 7500 & 1425000 & 0.1091 & \#\#\#\#+1 & 1.707 & $1.02 \mathrm{E}-02$ & 17.51 & 92.45 & 24.47 & & 67.7 & 299.9 \\
\hline $\begin{array}{l}635 \\
635 \\
\end{array}$ & 25.37 & 3000 & 1900 & $\begin{array}{r}.806792 \\
\end{array}$ & 0.0843 & $\begin{array}{l}139.9 \\
\end{array}$ & 53.6 & 5125 & 0.1 & 1950 & 7500 & 1775000 & 0.1254 & 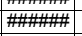 & 3.968 & $6.52 \mathrm{E}-03$ & 78.43 & 0 & & & 106.3 & 801.5 \\
\hline 636 & 34.58 & 3000 & 1800 & 5.555916 & 0.3615 & $\begin{array}{l}195.2 \\
\end{array}$ & 38.42 & 5125 & 0.1 & 1950 & 7500 & 2925000 & 0.092 & \#\#\#\#+1 & 3.754 & $\begin{array}{l}6.16 \mathrm{E}-03 \\
\end{array}$ & $\begin{array}{r}4.8 \\
24.8 \\
\end{array}$ & 10.01 & & & 112.5 & 556.3 \\
\hline 637 & 53.11 & 3000 & 820 & 5.558821 & 0.389 & \begin{tabular}{|l|}
209.9 \\
\end{tabular} & 35.72 & 5125 & 0.1 & 1950 & 7500 & 3262500 & 0.0599 & 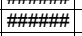 & 3.604 & $1.20 \mathrm{E}-02$ & 79.54 & 10.05 & & & 57.7 & 347.8 \\
\hline 638 & 60.03 & 3000 & 620 & 49.398015 & 2.505 & 152.1 & 49.3 & 5125 & 0.1 & 1950 & 7500 & 2012500 & 0.053 & \#\#\#\# & 2.91 & $9.20 \mathrm{E}-03$ & 52.04 & 89.88 & & & 75.4 & 248.5 \\
\hline 639 & 29.76 & 3000 & 1440 & 1.660286 & 0.0842 & 152.1 & 49.3 & 5125 & 0.1 & 1950 & 7500 & 2012500 & 0.1069 & \#\#\#\#\#+ & 3.618 & $8.91 \mathrm{E}-03$ & 68.67 & & 0 & & 77.8 & 623 \\
\hline 640 & 43.17 & 3000 & 730 & 102.563078 & 4.8505 & \begin{tabular}{|l|l|}
141.9 \\
\end{tabular} & 52.86 & 5125 & 0.1 & 1950 & 7500 & 1812500 & 0.0737 & & 2.7 & $3.67 \mathrm{E}-03$ & 78.3 & 95.12 & 51.25 & & 189.1 & 320.6 \\
\hline 641 & 42.03 & 3000 & 1230 & & \begin{tabular}{|c|}
0.0829 \\
\end{tabular} & 113 & 66.4 & 5125 & 0.1 & 1950 & 7500 & 1287500 & & \#\#\#\#\#+ & 3.679 & $1.26 \mathrm{E}-02$ & 41.14 & & & & 55 & 448.6 \\
\hline 642 & 21.39 & 3000 & 350 & & $\begin{array}{l}1.245 \\
\end{array}$ & \begin{tabular}{|l|}
135.3 \\
\end{tabular} & 55.44 & 5125 & 0.1 & 1950 & 7500 & 1687500 & 0.1487 & \#\#\#\#+ & 1.255 & $1.32 \mathrm{E}-02$ & \begin{tabular}{ll|}
62.44 \\
\end{tabular} & 81.89 & & & 52.6 & 300.6 \\
\hline 643 & 32.8 & & 390 & & \begin{tabular}{|l|l|}
7.3195 \\
\end{tabular} & $\begin{array}{l}127.1 \\
\end{array}$ & 58.99 & 5125 & 0.1 & 1950 & 7500 & 1537500 & & \#\#\#\#+ & 1.564 & & 27.16 & 97.11 & 71.05 & & 78.6 & 244.4 \\
\hline 644 & 26.21 & 3000 & 460 & & $\begin{array}{l}0.4366 \\
\end{array}$ & $\begin{array}{l}91.42 \\
\end{array}$ & 82.04 & 5125 & 0.1 & 1950 & 7500 & 937500 & & \#\#\#\#+ & $\begin{array}{l}1.58 \\
1.58\end{array}$ & $1.58 \mathrm{E}-02$ & 47.51 & 65.1 & & & 44 & 309 \\
\hline 645 & 31.07 & 3000 & 350 & & 11.0662 & 136.6 & 54.9 & 5125 & 0.1 & 1950 & 7500 & 1712500 & & \#\#\#\#+ & 1.452 & $6.57 \mathrm{E}-03$ & 55.88 & 97.94 & & & 105.5 & 239.5 \\
\hline 646 & 43.05 & 3000 & 660 & & 2.244 & \begin{tabular}{ll|}
114.4 \\
\end{tabular} & 65.55 & 5125 & 0.1 & 1950 & 7500 & 1312500 & & \#\#\#\#曲 & 2.484 & $8.25 \mathrm{E}-03$ & 48.75 & $\begin{array}{l}91.5 \\
9\end{array}$ & & & 84 & 295.8 \\
\hline 647 & 35.51 & 3000 & 1710 & $\begin{array}{l}6.632708 \\
\end{array}$ & 0.2734 & $\begin{array}{l}123.7 \\
\end{array}$ & 60.65 & 5125 & 0.1 & 1950 & 7500 & 1475000 & 0.0896 & \#\#\#\#+ & $\begin{array}{l}4.17 \\
\end{array}$ & $6.02 \mathrm{E}-03$ & 64.89 & 24.62 & & & 115.2 & 602 \\
\hline 648 & 22.95 & 3000 & 1700 & $\begin{array}{l}0.120717 \\
\end{array}$ & \begin{tabular}{ll|l}
0.0044 \\
\end{tabular} & 110 & 68.18 & 5125 & 0.1 & 1950 & 7500 & 1237500 & $\begin{array}{l}0.1386 \\
\end{array}$ & \#\#\#\#+ & 3.043 & $1.29 \mathrm{E}-02$ & 21.77 & 0 & & & 54 & 679.4 \\
\hline 649 & 21.25 & 3000 & 780 & 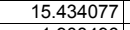 & 0.7333 & 142.5 & 52.62 & 5125 & 0.1 & 1950 & 7500 & 1825000 & 0.1497 & \#\#\#\#曲 & 2.023 & $7.04 \mathrm{E}-03$ & 81.91 & $\begin{array}{l}67.6 \\
\end{array}$ & & & \begin{tabular}{ll|}
98.4 \\
\end{tabular} & 487.8 \\
\hline $\begin{array}{l}650 \\
651 \\
651\end{array}$ & 21.58 & & \begin{tabular}{ll|}
1090 \\
550
\end{tabular} & $\begin{array}{r}.908496 \\
7\end{array}$ & & $\begin{array}{l}91.42 \\
111.5\end{array}$ & 82.04 & 5125 & 0.1 & & 7500 & $\begin{array}{r}937500 \\
12500\end{array}$ & 0.1474 & \#\#\#\# & $\begin{array}{l}2.38 \\
\end{array}$ & $1.10 \mathrm{E}-02$ & $\begin{array}{ll}43.06 \\
\end{array}$ & 0 & & & $\begin{array}{l}63.1 \\
\end{array}$ & 565.1 \\
\hline 651 & 28.18 & $\begin{array}{ll}3000 \\
3000\end{array}$ & $\begin{array}{l}550 \\
560\end{array}$ & \begin{tabular}{|r|}
73.866244 \\
\end{tabular} & \begin{tabular}{r|r|}
2.745 \\
107158
\end{tabular} & $\begin{array}{l}111.5 \\
1359\end{array}$ & \begin{tabular}{|c|c|}
67.27 \\
55.17
\end{tabular} & 5125 & 0.1 & $\begin{array}{l}1950 \\
1050\end{array}$ & 7500 & $\begin{array}{l}1262500 \\
17000\end{array}$ & $\begin{array}{l}0.1129 \\
0.059\end{array}$ & 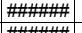 & 1.781 & $8.00 \mathrm{E}-03$ & \begin{tabular}{|l|l|}
44.1 \\
\end{tabular} & $\begin{array}{l}93.23 \\
0789\end{array}$ & 32.31 & 0 & $\begin{array}{r}86.6 \\
\end{array}$ & 323.9 \\
\hline 652 & $\begin{array}{l}54.6 \\
36.91\end{array}$ & $\frac{3000}{3000}$ & $\begin{array}{l}5600 \\
280\end{array}$ & \begin{tabular}{|l|}
236.474257 \\
524204169
\end{tabular} & \begin{tabular}{l|l|}
10.7158 \\
177707
\end{tabular} & \begin{tabular}{|l|}
135.9 \\
1017
\end{tabular} & $\begin{array}{l}55.17 \\
73.75 \\
\end{array}$ & $\begin{array}{l}5125 \\
5125\end{array}$ & 0.1 & $\begin{array}{l}1950 \\
1950\end{array}$ & $\begin{array}{l}7500 \\
7500\end{array}$ & \begin{tabular}{|l|}
1700000 \\
1110000
\end{tabular} & 0.0581 & 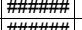 & $2.467 \mid$ & $\begin{array}{r}5.05 \mathrm{E}-03 \\
5.46 \mathrm{E}-0 .\end{array}$ & \begin{tabular}{rl|}
28.28 & \\
95 &
\end{tabular} & \begin{tabular}{|l|l|}
97.89 & 99.05 \\
9
\end{tabular} & & & 137.4 & 230.9 \\
\hline 654 & 52.07 & 3000 & 330 & \begin{tabular}{|l|}
482.738966 \\
\end{tabular} & 19.7872 & 123 & $\frac{70.15}{60.99}$ & $\begin{array}{l}3120 \\
5125\end{array}$ & 0.1 & 1950 & 7500 & \begin{tabular}{|l}
17100000 \\
1462500
\end{tabular} & $\begin{array}{l}.0062 \\
0.0611 \\
\end{array}$ & 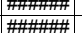 & $\begin{array}{r}1.41 \\
1.798\end{array}$ & \begin{tabular}{|c|}
$5.46 \mathrm{E}-03$ \\
$5.74 \mathrm{E}-03$
\end{tabular} & $\begin{array}{r}95 \\
45.68\end{array}$ & $\begin{array}{l}95.05 \\
98.96 \\
\end{array}$ & $\begin{array}{l}90.46 \\
89.64\end{array}$ & 4.02 & $\begin{array}{l}120.9 \\
120.7\end{array}$ & 195.8 \\
\hline 655 & 42.59 & 3000 & 620 & \begin{tabular}{|l|}
52.894324 \\
\end{tabular} & 2.4669 & \begin{tabular}{|l|}
139.9 \\
\end{tabular} & 53.6 & 5125 & 0.1 & 1950 & 7500 & 1775000 & 0.07 & 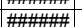 & 2.548 & $7.00 \mathrm{E}-03$ & 89.97 & 90.55 & 5.47 & & 99.1 & 306.6 \\
\hline 656 & 29.11 & 3000 & 860 & 4.689396 & 0.1911 & 122.3 & 61.34 & 5125 & 0.1 & 1950 & 7500 & 1450000 & 0.1093 & \#\#\#\#+ & 2.33 & $1.25 \mathrm{E}-02$ & 27.51 & 0 & 0 & & 55.6 & 410.3 \\
\hline 657 & 38.8 & 3000 & 310 & \begin{tabular}{|l}
325.646065 \\
\end{tabular} & 14.3193 & 131.9 & 56.85 & 5125 & 0.1 & 1950 & 7500 & 1625000 & 0.082 & \#\#\#\#+ & 1.509 & $8.23 \mathrm{E}-03$ & 29.84 & 98.46 & 84.65 & & 84.2 & 199.3 \\
\hline 658 & 22.72 & 3000 & 1510 & \begin{tabular}{|l|}
0.028068 \\
\end{tabular} & 0.0014 & 144.5 & 51.91 & 5125 & 0.1 & 1950 & 7500 & 1862500 & 0.14 & \#\#\#\#+ & 3.459 & $1.53 \mathrm{E}-02$ & 58.64 & & 0 & & 45.4 & 780.1 \\
\hline 659 & 34.02 & 3000 & 790 & 7.635347 & 0.2912 & 114.4 & 65.55 & 5125 & 0.1 & 1950 & 7500 & 1312500 & 0.0935 & \#\#\#\#+ & 2.795 & $1.10 \mathrm{E}-02$ & 91.71 & 34.52 & 0 & & 62.8 & 42 \\
\hline 660 & 25.66 & 3000 & 1540 & 3.402401 & 0.0812 & 71.62 & 104.73 & 5125 & 0.1 & 1950 & 7500 & & 0.124 & \#\#\#\#+ & 3.264 & & 54.63 & 0 & & & 83.4 & 00 \\
\hline 661 & 22.76 & 3000 & 1140 & 17.320094 & 0.596 & 103.2 & 72.65 & 5125 & 0.1 & 1950 & 7500 & 1125000 & & \#\#\#\#+ & 2.468 & & 55.03 & 71.13 & & & 117.4 & 555.7 \\
\hline 662 & 46.85 & 3000 & 1640 & 0.221283 & 0.0082 & \begin{tabular}{|l|}
110.7 \\
\end{tabular} & 67.72 & 5125 & 0.1 & 1950 & 7500 & 1250000 & & \#\#\#\#+ & 4.534 & & 26.49 & & 0 & & 41.6 & 496 \\
\hline 663 & 30.44 & 3000 & 700 & 72.80 & 2.139 & 88.14 & 85.09 & 5125 & 0.1 & 1950 & 7500 & 500 & & & 2.048 & & 24.5 & 93.13 & 31.32 & & 85.9 & 344. \\
\hline 664 & 41.1 & 00 & 630 & & 0.7921 & 129.9 & 57. & 5125 & 0.1 & 1950 & 7500 & & & & 2.319 & & 22.94 & 72.67 & & & 51 & 289.2 \\
\hline 665 & 30.5 & & 660 & & & 125.8 & & 5125 & 0.1 & 1950 & 7500 & & 0.1 & & & & 49.83 & 0 & & & 46.1 & 366.4 \\
\hline 666 & 22.72 & & 1290 & & 84 & \begin{tabular}{ll|}
155.9 \\
\end{tabular} & & 51 & 0.1 & & & & & & & & 31.9 & 25.43 & & & 102.5 & 57 \\
\hline 667 & 24.34 & & 2290 & & & \begin{tabular}{l|l|}
91.42 \\
\end{tabular} & & & 0.1 & & 750 & & & & & & & 0 & 0 & & 61.6 & \\
\hline 668 & & & 500 & & & $\begin{array}{l}157.1 \\
\end{array}$ & & & 0.1 & & 75 & & & & & & & 97.42 & 74.23 & & 118 & 2 \\
\hline 669 & & & 1890 & & & $\begin{array}{l}193.5 \\
\end{array}$ & & & & & & & & & & & & 0 & 0 & & 114.4 & 600.8 \\
\hline 670 & 48. & & 2200 & & & & & & & & & & & & & & & 10.29 & 0 & & 116.7 & 545.7 \\
\hline 671 & 22.37 & & 900 & & 64 & $\begin{array}{l}149.6 \\
\end{array}$ & 50.13 & 51. & & & & & & & & & 38. & 56.74 & 0 & & 89.3 & 482.3 \\
\hline 672 & 30.89 & 30 & 1370 & & 0.2062 & \begin{tabular}{ll|}
131.9 \\
\end{tabular} & 56.85 & 512 & 0.1 & & & & & & & & & & 0 & & $\begin{array}{l}77.6 \\
\end{array}$ & 504.1 \\
\hline 673 & 39.28 & & 260 & 127.6 & & 147 & & 51 & 0.1 & & & & & & & & & 96.08 & 60.83 & & 50.9 & 183.5 \\
\hline 674 & 21.45 & & 45 & & & \begin{tabular}{ll|}
135.9 \\
\end{tabular} & & & & & & & 0.1 & & & & & & $\begin{array}{l}41.16 \\
\end{array}$ & & 79 & 324.2 \\
\hline 675 & 22.05 & 300 & 500 & & $\begin{array}{l}0.4705 \\
\end{array}$ & $\begin{array}{l}143.8 \\
14.0\end{array}$ & 52. & & & 19 & & & 0.1 & & & & & 49.05 & 0 & & 52.5 & 356 \\
\hline $676 \mid$ & 53.47 & 3000 & \begin{tabular}{|r|r|}
400 & \\
1390
\end{tabular} & & 2.8727 & $\begin{array}{l}141.9 \\
05.9\end{array}$ & & $\begin{array}{l}5125 \\
5125\end{array}$ & 0.1 & 19 & 7500 & & 0.05 & & 2.2 & & 89.41 & $91.77 \mid$ & 17.68 & & 58.9 & 2611 \\
\hline \begin{tabular}{ll|}
677 \\
678
\end{tabular} & $\begin{array}{l}23.37 \\
44.25 \\
\end{array}$ & 3000 & \begin{tabular}{r|r|r}
1390 & \\
570 &
\end{tabular} & \begin{tabular}{|r|}
0.061884 \\
256.925016
\end{tabular} & & $\begin{array}{r}95.44 \\
81.39\end{array}$ & $\begin{array}{l}78.58 \\
92.15\end{array}$ & $\begin{array}{l}5125 \\
5125\end{array}$ & 0.1 & $\frac{195}{195}$ & $\begin{array}{l}7500 \\
7500\end{array}$ & 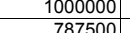 & 0.13 & 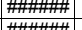 & $\frac{2.9}{2.1}$ & & $\begin{array}{l}31.52 \\
272 \\
\end{array}$ & \begin{tabular}{r|r}
0 \\
9805 \\
\end{tabular} & $\begin{array}{r}0 \\
80.54\end{array}$ & & $\begin{array}{r}43.3 \\
1057\end{array}$ & 636.8 \\
\hline 年 & $\begin{array}{l}44.25 \\
39.13 \\
\end{array}$ & $\frac{300}{300}$ & $\begin{array}{r}5 / 0 \\
1730\end{array}$ & & $\frac{6.9703}{0.0151}$ & $\begin{array}{r}81.39 \\
83.1\end{array}$ & $\begin{array}{l}92.15 \\
90.25\end{array}$ & $\begin{array}{l}\mid 5125 \\
5125\end{array}$ & $\begin{array}{l}0.1 \\
0.1\end{array}$ & $\frac{195}{195}$ & $\begin{array}{l}7500 \\
7500\end{array}$ & & & 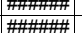 & $\frac{2.1}{4.3}$ & & $\begin{array}{l}2.2 \\
37.1\end{array}$ & \begin{tabular}{rl|}
98.05 \\
\end{tabular} & & & $\frac{105.1}{52.7}$ & $\frac{254.7}{574.4}$ \\
\hline 680 & & 300 & 890 & & 0.08 & \begin{tabular}{|l|}
133.9 \\
\end{tabular} & 56 & 512 & 0.1 & 195 & 7500 & 1662 & & \#\#\# & & & 39 & 0 & & & 39.2 & 341.4 \\
\hline 681 & 25.6 & 300 & 860 & 49.773 & 1.39 & 83.95 & 89.3 & 512 & 0.1 & 195 & 7500 & 825 & & \#\#\# & 2.7 & & 85. & 89.95 & & & 126.9 & 459.2 \\
\hline 682 & 28.2 & 30 & 720 & & & 107 & 70. & 512 & 0.1 & 195 & 7500 & 1187 & 0.1 & \#\#曲 & & & 37. & 0 & & & $\mid 43.9$ & 390.8 \\
\hline 683 & 26.91 & 3000 & 1040 & & 0.02 & 118 & 63. & 512 & 0.1 & 195 & 750 & & & & 2.5 & & 27. & & 0 & & 45.9 & 484. \\
\hline 684 & 51. & 3000 & 1300 & 10.65 & 0.4 & \begin{tabular}{|l|}
121.6 \\
\end{tabular} & & 512 & 0.1 & 19 & 7500 & & & & & & 45. & 53.09 & & & 83 & 410.3 \\
\hline 685 & 57.6 & & 350 & 324.2 & & 82.25 & 91. & 512 & 0.1 & 195 & 7500 & & & & & & & 98.46 & 84.58 & & 75.7 & 175. \\
\hline 686 & & & 3000 & & & 101 & & 512 & 0.1 & 19 & & & & & 4.1 & & 14. & & & & 108 & 824. \\
\hline 687 & & 3000 & 170 & & & 127 & & & 0.1 & 195 & 7500 & & & & & & & 98. & \begin{tabular}{|l|}
87.07 \\
\end{tabular} & & 43.7 & 120.9 \\
\hline 688 & & 30 & 880 & & & 178 & & & 0.1 & & 7500 & & & & & & 72 . & & 0 & & 85 & 38 \\
\hline $6 \varepsilon$ & & & 51 & & & 125 & & & 0.1 & & & & & & & & & & 0 & & 55.3 & 5. \\
\hline & & & 1240 & & & & & & 0.1 & & & & & & & & & & & & 95 & \\
\hline $6 s$ & & & 39 & & & & & & 0.1 & & & & & & & & & & 75.25 & & 77 & 87.5 \\
\hline 65 & & & 390 & & 5.5744 & 95.44 & 78.5 & 5125 & 0.1 & 195 & 7500 & & & & & & & 97.15 & 71.46 & & 86.2 & \\
\hline 693 & 27.08 & 3000 & 540 & 61.674841 & 1.4911 & 72.53 & 103.4 & 5125 & 0.1 & 1950 & 7500 & 662500 & 0.1175 & \#\#\#\#+1| & 1.683 & & 33.18 & 91.89 & 18.93 & & 65.2 & 318.5 \\
\hline
\end{tabular}




\begin{tabular}{|c|c|c|c|c|c|c|c|c|c|c|c|c|c|c|c|c|c|c|c|c|c|c|}
\hline & $\begin{array}{c}\text { Velocity } \\
(\mathrm{m} / \mathrm{y})\end{array}$ & $\begin{array}{l}\text { Fixed Source } \\
\text { Concentration } \\
(\mathrm{mg} / \mathrm{L})\end{array}$ & $\begin{array}{c}\text { Time of } \\
\text { Maximum } \\
\text { at } \\
\text { Compliance } \\
\text { Boundary } \\
\text { (y) }\end{array}$ & $\begin{array}{c}\text { Maximum } \\
\text { Concentration } \\
\text { Compliance } \\
\text { Boundary } \\
(\mu \mathrm{g} / \mathrm{L})\end{array}$ & $\begin{array}{c}\text { Maximum } \\
\text { Release Rate } \\
\text { at } \\
\text { Compliance } \\
\text { Boundary } \\
(\mathrm{Kg} / \mathrm{y})\end{array}$ & $\begin{array}{l} \\
\text { Sourc } \\
\text { e Rate } \\
\text { (Kg/y) }\end{array}$ & \begin{tabular}{|c|} 
Source \\
Duration \\
(y)
\end{tabular} & $\begin{array}{c}\text { 1-D } \\
\text { Streamtube } \\
\text { Length } \\
(\mathrm{m})\end{array}$ & $\mid \begin{array}{c}\text { Base } \\
\text { Porosity } \\
\left(\mathrm{m}^{3} / \mathrm{m}^{3}\right)\end{array}$ & $\begin{array}{c}\text { Bulk } \\
\text { Densty } \\
\left(\mathrm{Kg} / \mathrm{m}^{3}\right)\end{array}$ & \begin{tabular}{|c|} 
Remaining \\
Carbon \\
Tetrachloride \\
Source \\
$(\mathrm{Kg})$
\end{tabular} & $\begin{array}{c}\text { Volume at or } \\
\text { above Fixed } \\
\text { Source } \\
\begin{array}{c}\text { Concentratio } \\
n(m 3)\end{array}\end{array}$ & $\begin{array}{l}\text { Porosity } \\
\left(\mathrm{m}^{3} / \mathrm{m}^{3}\right)\end{array}$ & $\left.\mid \begin{array}{c}\mathrm{Kd} \\
\left(\mathrm{m}^{3} / \mathrm{Kg}\right.\end{array}\right)$ & Retardation & $\begin{array}{c}\text { First } \\
\text { Order } \\
\text { Rate } \\
\text { Constant } \\
(1 / y)\end{array}$ & $\begin{array}{l}\text { Longitudinal } \\
\text { Dispersivity } \\
(\mathrm{m})\end{array}$ & $\begin{array}{c}\text { Required } \\
\text { Source } \\
\text { Cleanup } \\
\text { Percentage } \\
(5 \mu \mathrm{g} / \mathrm{L} \\
\text { Compliance })\end{array}$ & $\begin{array}{c}\text { Required } \\
\text { Source } \\
\text { Cleanup } \\
\text { Percentage } \\
(50 \mu g / L \\
\text { Compliance })\end{array}$ & \begin{tabular}{|c|} 
Required \\
Source Cleanup \\
Percentage \\
(500 $\mu \mathrm{g} / \mathrm{L}$ \\
Compliance)
\end{tabular} & \begin{tabular}{|c|} 
Abiotic \\
Reaction \\
Half Time \\
(y)
\end{tabular} & $\begin{array}{c}\text { Carbon } \\
\text { Tetrachloride } \\
\text { Travel Time } \\
\text { (y) }\end{array}$ \\
\hline & & $\begin{array}{r}3000 \\
\end{array}$ & & & $\begin{array}{l} \\
0.1051 \\
\end{array}$ & 128.5 & \begin{tabular}{|l|l|}
58.36 \\
\end{tabular} & 5125 & & & & & & \#\#\#+ & 1.984 & $1.76 \mathrm{E}-02$ & & & & & & \\
\hline 695 & 48.42 & 3000 & 2620 & 20.180731 & 0.604 & 89.79 & 83.53 & 5125 & 0.1 & 1950 & 7500 & 912500 & 0.0657 & 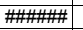 & 5.355 & $4.12 \mathrm{E}-03$ & 22.27 & 75.22 & & & 168.5 & 566.7 \\
\hline \begin{tabular}{l|l}
696 \\
\end{tabular} & 36.74 & 3000 & 1060 & 11.519378 & 0.4337 & 113 & 66.4 & 5125 & 0.1 & 1950 & 7500 & 1287500 & 0.0866 & \#\#\#\#\#+ & 3.08 & $8.68 \mathrm{E}-03$ & 48.33 & 56.59 & & & 79.9 & 429.7 \\
\hline 697 & 22.69 & 3000 & 450 & 28.778059 & 1.6468 & \begin{tabular}{|l|l|}
171.7 \\
\end{tabular} & 43.69 & 5125 & 0.1 & 1950 & 7500 & 2412500 & 0.1402 & 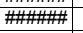 & 1.498 & $9.72 \mathrm{E}-03$ & 63.81 & 82.63 & & & 71.3 & 338.3 \\
\hline 698 & 33.24 & 3000 & 2320 & 10.861565 & 0.5137 & \begin{tabular}{|l|l|}
141.9 \\
\end{tabular} & 52.86 & 5125 & 0.1 & 1950 & 7500 & 1812500 & 0.0957 & 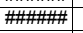 & 4.448 & $3.54 \mathrm{E}-03$ & 49.91 & 53.97 & & & 195.8 & 685.7 \\
\hline 699 & 28.58 & 3000 & 730 & 57.38879 & 2.6006 & \begin{tabular}{ll|}
135.9 \\
\end{tabular} & 55.17 & 5125 & 0.1 & 1950 & 7500 & 1700000 & 0.1113 & 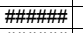 & 2.185 & $5.18 \mathrm{E}-03$ & 71.8 & 91.29 & & & 133.9 & 391.7 \\
\hline 700 & 21.31 & 3000 & 930 & \begin{tabular}{r|}
1.427703 \\
\end{tabular} & 0.0548 & \begin{tabular}{|l|}
115.1 \\
\end{tabular} & 65.14 & 5125 & 0.1 & 1950 & 7500 & 1325000 & 0.1493 & 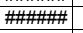 & 2.294 & $1.19 \mathrm{E}-02$ & 63.16 & & & & 58.2 & 551.7 \\
\hline 701 & 41.75 & 3000 & 340 & \begin{tabular}{|l|}
636.762256 \\
\end{tabular} & 14.0076 & $\begin{array}{ll}65.99 \\
\end{array}$ & 113.65 & 5125 & 0.1 & 1950 & 7500 & 575000 & 0.0762 & 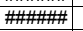 & 1.545 & $7.57 \mathrm{E}-03$ & 29.07 & 99.21 & & 21.48 & 91.6 & 189.7 \\
\hline 702 & 30.83 & 3000 & 410 & \begin{tabular}{|l|}
41.889779 \\
\end{tabular} & 1.5874 & 1113.7 & 65.97 & 5125 & 0.1 & 1950 & 7500 & 1300000 & 0.1032 & 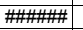 & 1.663 & $1.23 \mathrm{E}-02$ & 77.47 & 88.06 & & & 56.5 & 276.4 \\
\hline 703 & 28.53 & 3000 & 1650 & 7.877616 & 0.2443 & 93.04 & 80.61 & 5125 & 0.1 & 1950 & 7500 & 962500 & 0.1115 & \#\#\#\#+ & 3.423 & $6.31 \mathrm{E}-03$ & 46.11 & 36.53 & & & 109.8 & 614.8 \\
\hline 704 & 24.42 & 3000 & 630 & & 2.1898 & 186.8 & 40.16 & 5125 & 0.1 & 1950 & 7500 & 2737500 & 0.1303 & \#\#\#\#\#+ & 1.789 & $7.43 \mathrm{E}-03$ & 32.47 & 85.79 & & & 93.3 & 375.5 \\
\hline 705 & 34.77 & 3000 & 1520 & & $\begin{array}{l}0.016 \\
\end{array}$ & $\begin{array}{l}120.9 \\
\end{array}$ & 62.06 & 5125 & 0.1 & 1950 & 7500 & 1425000 & & \#\#\#\#+\#+ & 4.382 & $1.29 \mathrm{E}-02$ & 67.75 & & & & 53.6 & 645.9 \\
\hline 706 & 28.61 & & 680 & & 0.081 & \begin{tabular}{|l|l|}
127.8 \\
\end{tabular} & 58.67 & 5125 & 0.1 & 1950 & 7500 & & & 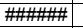 & 2.236 & $1.61 \mathrm{E}-02$ & 59.92 & & 0 & & 43.1 & 400.6 \\
\hline 707 & 41.69 & 3000 & 460 & & 2.422 & 90.61 & 82.78 & 5125 & 0.1 & 1950 & 7500 & 925000 & & 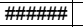 & 2.103 & $1.02 \mathrm{E}-02$ & $\begin{array}{l}96.19 \\
\end{array}$ & 93.77 & & & 68.2 & 258.5 \\
\hline 708 & 22.85 & 3000 & 940 & & 1.3371 & $\begin{array}{l}110.7 \\
\end{array}$ & & 5125 & 0.1 & 1950 & 7500 & 1250000 & 0.1392 & \#\#\#\#曲 & 2.133 & $5.82 \mathrm{E}-03$ & 36.31 & 86.2 & & & 119.2 & 478.4 \\
\hline 709 & 54.29 & 3000 & 1340 & \begin{tabular}{|l|l|l}
17.41399 \\
\end{tabular} & 0.5116 & $\begin{array}{l}88.14 \\
\end{array}$ & 85.09 & 5125 & 0.1 & 1950 & 7500 & 887500 & & \#\#\#\#曲 & & $6.88 \mathrm{E}-03$ & 80.79 & 71.29 & 0 & & 100.8 & 446.6 \\
\hline 710 & 33.52 & 3000 & 420 & & 0.6621 & \begin{tabular}{ll|}
135.3 \\
\end{tabular} & 55.44 & 5125 & 0.1 & 1950 & 7500 & & 0.0949 & \#\#\#\#曲 & 1.754 & $1.66 \mathrm{E}-02$ & 50.99 & 65.95 & 0 & & $\begin{array}{l}41.7 \\
\end{array}$ & 268.1 \\
\hline 711 & 25.03 & 3000 & 1130 & 15.523417 & 0.6861 & $\begin{array}{l}132.6 \\
\end{array}$ & 56.56 & 5125 & 0.1 & 1950 & 7500 & 1637500 & & \#\#\#\#\# & 2.583 & $5.89 \mathrm{E}-03$ & 52.88 & \begin{tabular}{|c|}
67.79 \\
\end{tabular} & 0 & & 117.7 & 528.8 \\
\hline 712 & 28.79 & 3000 & $\begin{array}{l}840 \\
820\end{array}$ & & 0.0911 & 123 & 60.99 & 5125 & 0.1 & 1950 & 7500 & 1462500 & 0.1105 & \#\#\#\#\# & 2.33 & $1.44 \mathrm{E}-02$ & 29.12 & & 0 & & 48.1 & 414.8 \\
\hline 713 & 25.68 & 3000 & $\begin{array}{l}2210 \\
400\end{array}$ & \begin{tabular}{|c|}
0.072718 \\
\end{tabular} & \begin{tabular}{|c|}
0.003 \\
28016
\end{tabular} & 125.1 & $\begin{array}{l}59.97 \\
57.15\end{array}$ & 5125 & 0.1 & 1950 & 7500 & 1500000 & 0.1239 & \#\#\#\#\# & $\begin{array}{l}4.198 \\
\end{array}$ & $1.15 \mathrm{E}-02$ & 40.79 & 0 & & & \begin{tabular}{ll|}
60.4 \\
\end{tabular} & 837.8 \\
\hline $\begin{array}{l}714 \\
715 \\
\end{array}$ & 27.93 & 3000 & $\begin{array}{l}400 \\
310\end{array}$ & \begin{tabular}{|r|}
64.1 \\
218.20 \\
\end{tabular} & $\begin{array}{r}2.8046 \\
13352\end{array}$ & $\begin{array}{r}131.2 \\
183.3\end{array}$ & $\begin{array}{l}57.15 \\
40.91\end{array}$ & $\begin{array}{l}5125 \\
5125\end{array}$ & 0.1 & $\begin{array}{l}1950 \\
1950\end{array}$ & $\begin{array}{l}7500 \\
7500\end{array}$ & $\begin{array}{l}1612500 \\
262500\end{array}$ & 0.1139 & \#\#\#\#" & $\begin{array}{l}1.479 \\
1204\end{array}$ & $\begin{array}{r}1.13 \mathrm{E}-02 \\
771 \mathrm{E}-3\end{array}$ & $\begin{array}{ll}36.26 \\
26.56\end{array}$ & $\begin{array}{r}92.2 \\
07.71\end{array}$ & & & 61.4 & 271.3 \\
\hline 716 & 22.53 & 3000 & 400 & \begin{tabular}{|r|}
21.2051026 \\
\end{tabular} & $\begin{array}{r}\frac{13.3552}{1.7543} \\
\end{array}$ & $\begin{array}{r}18.3 \\
181\end{array}$ & $\begin{array}{l}40.91 \\
41.43\end{array}$ & $\begin{array}{l}5120 \\
5125 \\
\end{array}$ & $\begin{array}{l}0.1 \\
0.1 \\
\end{array}$ & $\begin{array}{l}1950 \\
1950\end{array}$ & $\begin{array}{l}7500 \\
7500\end{array}$ & \begin{tabular}{|l|}
2662500 \\
2612500
\end{tabular} & $\begin{array}{l}.126 \\
0.1412 \\
\end{array}$ & \#\#\#\#曲 & $\begin{array}{l}\frac{1.204}{1.325} \\
\end{array}$ & $\begin{array}{r}.17 \mathrm{E}-03 \\
1.25 \mathrm{E}-02 \\
\end{array}$ & $\begin{array}{l}26.56 \\
23.92\end{array}$ & $\begin{array}{r}91.71 \\
82.8 \\
\end{array}$ & & & \begin{tabular}{r|}
90 \\
55.5
\end{tabular} & $\frac{247.5}{301.4}$ \\
\hline 717 & 62.38 & 3000 & 240 & \begin{tabular}{|r|}
589.749404 \\
\end{tabular} & 24.3111 & \begin{tabular}{|l|}
123.7 \\
\end{tabular} & 60.65 & 5125 & 0.1 & 1950 & 7500 & 1475000 & 0.051 & \#mmm & 1.665 & & 75.19 & 99.15 & 91.52 & 15.22 & 99.5 & 136.8 \\
\hline 718 & 32.46 & 3000 & 1260 & \begin{tabular}{|l|}
0.264099 \\
\end{tabular} & 0.0131 & 149 & 50.35 & 5125 & 0.1 & 1950 & 7500 & 1950000 & 0.098 & \#mmm & 2.947 & $1.68 \mathrm{E}-02$ & 10.71 & & & & 41.4 & 465.3 \\
\hline 719 & 30.04 & 3000 & 790 & 16.022217 & 0.6827 & \begin{tabular}{|c|}
127.8 \\
\end{tabular} & 58.67 & 5125 & 0.1 & 1950 & 7500 & 1550000 & 0.1059 & 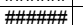 & $\begin{array}{l}2.507 \\
\end{array}$ & $8.10 \mathrm{E}-03$ & 90.4 & 68.79 & & & 85.5 & 427.8 \\
\hline 720 & 32.76 & 3000 & 920 & 7.57729 & 0.3315 & 131.2 & 57.15 & 5125 & 0.1 & 1950 & 7500 & 1612500 & 0.0971 & \#\#\#\#\# & 2.678 & $1.02 \mathrm{E}-02$ & 46.01 & 34.01 & & & 68 & 418.8 \\
\hline 721 & 22.87 & 3000 & 1060 & 8.607479 & 0.4754 & 165.7 & 45.26 & 5125 & 0.1 & 1950 & 7500 & 2287500 & & 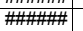 & 2.396 & $6.80 \mathrm{E}-03$ & 52.63 & 41.91 & 0 & & 102 & 536.9 \\
\hline 722 & 24.23 & 3000 & 490 & \begin{tabular}{|l|}
366.934333 \\
\end{tabular} & 14.2589 & \begin{tabular}{|c|}
116.6 \\
\end{tabular} & 64.33 & 5125 & 0.1 & 1950 & 7500 & & & \#\#\#\#+ & 1.534 & & 56.12 & 98.64 & 86.37 & & 249.8 & 324.6 \\
\hline 723 & 42.59 & 3000 & 890 & \begin{tabular}{|l|}
5.798348 \\
\end{tabular} & 0.2363 & 122.3 & 61.34 & 5125 & 0.1 & 1950 & 7500 & & 0.0 & \#\#\#\#+ & 2.996 & & 38.17 & 13.77 & 0 & & 52.8 & 360. \\
\hline 724 & 42.7 & 3000 & 640 & 26.052602 & 1.0372 & 119.4 & 62.79 & 5125 & 0.1 & 1950 & 7500 & & 0.0 & \#\#\#\#+ & 2.339 & & 15.2 & 80.81 & & & 51.6 & 280.7 \\
\hline 725 & 47.27 & 3000 & 1020 & 5.428 & 0.2084 & 115.1 & 65.14 & 5125 & 0.1 & 1950 & 7500 & & & \#\#\#\#+ & 3.321 & & 28.01 & 7.9 & & & 52.6 & 360 \\
\hline 726 & 22.64 & 3000 & 2010 & & 0.2318 & 102.5 & 73. & 5125 & 0.1 & 1950 & 7500 & 500 & & & 3.291 & & 37.59 & 26.32 & & & 138 & 744.9 \\
\hline 727 & 24.93 & 3000 & 1630 & & 0.0369 & $\begin{array}{l}73.44 \\
\end{array}$ & 102.12 & 5125 & 0.1 & 1950 & 7500 & & & & 3.08 & & 26.95 & & 0 & & 70.2 & 633.2 \\
\hline 728 & 27.96 & & 310 & \begin{tabular}{|l|l|}
445.97 \\
\end{tabular} & 13.3476 & \begin{tabular}{|l|}
89.79 \\
\end{tabular} & 83.53 & 5125 & 0.1 & 1950 & 7500 & & & & $\begin{array}{l}1.23 \\
\end{array}$ & & & 98.88 & 88.79 & & 91.3 & 225.4 \\
\hline 729 & 21.85 & & 750 & & 1.12 & \begin{tabular}{|l|}
136.6 \\
\end{tabular} & 54 & & 0.1 & & & & & & 1.846 & & & 79.74 & 0 & & 89.8 & 433 \\
\hline 730 & & & 770 & & & 125.1 & 59. & & 0.1 & & & & & & & & & 97.09 & 70.94 & & \begin{tabular}{|l|}
194.3 \\
\end{tabular} & \\
\hline 731 & 35.8 & & 1480 & & 0.5 & 58.1 & & & 0.1 & & & & & & & & & 82.1 & 0 & & 115.9 & \\
\hline 732 & & & 1760 & & & \begin{tabular}{|l|}
114.4 \\
\end{tabular} & & & 0.1 & & & & & & & & & & 0 & & 62.5 & 702.2 \\
\hline 733 & & & 550 & & & \begin{tabular}{l|l|}
162.1 \\
\end{tabular} & & & & & & 22125 & & & & & & 29.42 & & & 43 & 304 \\
\hline 734 & 25.86 & 3000 & 1590 & & & $\begin{array}{l}97.02 \\
\end{array}$ & & 51 & & & & & & & 3.3 & & & & 0 & & 59 & 664.8 \\
\hline 735 & $\begin{array}{l}27.76 \\
\end{array}$ & 3000 & $\begin{array}{l}710 \\
7\end{array}$ & & & 150.2 & 49. & 51 & 0.1 & & & & & & 2. & & & 87.92 & & & 104.5 & 380.3 \\
\hline 736 & $\begin{array}{l}21.67 \\
197\end{array}$ & & 790 & & & \begin{tabular}{|c|}
96.23 \\
\end{tabular} & & 51 & 0.1 & & & & 0.1 & & & & 64 & $\begin{array}{l}4.17 \\
\end{array}$ & & & 63.5 & 486 \\
\hline 737 & 27.43 & & 1040 & & & \begin{tabular}{|r|}
83.1 \\
\end{tabular} & & 51 & & & & & & & & & & 0 & 0 & & 59.3 & 557.1 \\
\hline 738 & 33.84 & $\begin{array}{l}3000 \\
3000\end{array}$ & 1220 & & 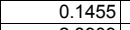 & $\begin{array}{l}172.9 \\
120 .\end{array}$ & & 5125 & 0.1 & 19 & 750 & & & & & & & 0 & 0 & & 75.8 & 557.8 \\
\hline 739 & \begin{tabular}{|l|l|}
42.53 \\
6.51
\end{tabular} & 3000 & $\begin{array}{l}1130 \\
1560\end{array}$ & \begin{tabular}{|c|c|}
67.128441 \\
5.89751
\end{tabular} & \begin{tabular}{|l|l|}
2.8909 \\
\end{tabular} & $\begin{array}{l}129.2 \\
\end{array}$ & 58.05 & $\begin{array}{l}5125 \\
5125\end{array}$ & 0.1 & $\begin{array}{l}1950 \\
1950\end{array}$ & 7500 & & \begin{tabular}{|c|c|}
0.0748 \\
\end{tabular} & & 3.2 & & & $\frac{92.55}{8.88}$ & 25.52 & & 184.8 & 391.9 \\
\hline $\begin{array}{l}740 \\
741\end{array}$ & $\begin{aligned} 26.51 \\
30.8\end{aligned}$ & 3000 & \begin{tabular}{r|r|r|}
1560 & \\
980
\end{tabular} & $\begin{array}{r}5.487531 \\
15.020984\end{array}$ & \begin{tabular}{l|l}
0.1612 \\
0.8506
\end{tabular} & $\begin{array}{r}88.14 \\
1699\end{array}$ & \begin{tabular}{|l|l|}
85.09 \\
415
\end{tabular} & \begin{tabular}{|l|l|}
5125 \\
5125
\end{tabular} & 0.1 & $\frac{195}{195}$ & $\begin{array}{l}7500 \\
77500\end{array}$ & $\begin{array}{r}887500 \\
23500\end{array}$ & $\begin{array}{r}0.12 \\
\end{array}$ & |m+\#\#+m| & $\frac{3.25}{261}$ & & 49.96 & $\begin{array}{r}8.88 \\
66.71\end{array}$ & & & $\begin{array}{l}97.5 \\
96.9\end{array}$ & 629.4 \\
\hline $\begin{array}{l}741 \\
742\end{array}$ & $\begin{array}{r}30.0 \\
24.99 \\
\end{array}$ & $\frac{300}{300}$ & \begin{tabular}{r|r|}
1620 \\
1620
\end{tabular} & & \begin{tabular}{|l}
$\mid$ \\
0.8506 \\
0.0128
\end{tabular} & \begin{tabular}{|l|}
169.9 \\
118.7
\end{tabular} & $\begin{array}{l}44.15 \\
63.17 \\
\end{array}$ & \begin{tabular}{|l|}
5125 \\
5125
\end{tabular} & $\begin{array}{l}.1 \\
0.1\end{array}$ & $\frac{195}{195}$ & $\begin{array}{l}7500 \\
7500\end{array}$ & $\begin{array}{l}2335000 \\
1387500\end{array}$ & & 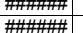 & $\frac{2.61}{3.2}$ & & $\begin{array}{r}40.67 \\
33.1\end{array}$ & & & & $\begin{array}{r}96.9 \\
60\end{array}$ & $\frac{434 . .}{660.3}$ \\
\hline 743 & 29.5 & 300 & 800 & & 0.2414 & 96.23 & 77. & 512 & 0.1 & 195 & 7500 & 1012 & & \#m! & 2.3 & & 56 & 33.55 & & & 59.5 & 415.2 \\
\hline 744 & 22.6 & 300 & 780 & & 0.0832 & $\begin{array}{l}170.5 \\
\end{array}$ & 43.5 & 512 & 0.1 & 195 & 750 & & 0.1 & \#\#! & 2.0 & & 35. & 0 & 0 & & 51.2 & 455 \\
\hline 745 & 47.6 & & 440 & & 3.05 & \begin{tabular}{|l|}
110.7 \\
\end{tabular} & \begin{tabular}{|c|c|}
67.72 \\
\end{tabular} & 512 & 0.1 & 195 & 7500 & & & \#\#! & 2.0 & & 34. & 93.95 & 39.49 & & 54.9 & 219.4 \\
\hline 746 & 29.8 & 3000 & 1670 & & 0.00 & 147 & & 512 & 0.1 & 195 & 750 & & & & & & 32. & & 0 & & 44.7 & 643.9 \\
\hline 747 & 37.0 & 3000 & 270 & 471.3 & 26.22 & 166.9 & 44. & 512 & 0.1 & 195 & 7500 & & & & & & 34. & 98.94 & 89.39 & & 111.5 & 188. \\
\hline 748 & 29.7 & 300 & 1830 & & & \begin{tabular}{|l|}
154.6 \\
\end{tabular} & 48 & 512 & 0.1 & 195 & 7500 & & & & 3.6 & & & & & & 49.5 & 625.7 \\
\hline 749 & & & 850 & & & 119 & & 512 & 0.1 & 19 & & & & & 1.9 & & 28. & 71.49 & & & 86.1 & 462.2 \\
\hline 750 & & 30 & 3630 & & & & 57. & & 0.1 & 195 & 7500 & & & & & & & & 0 & & 60.9 & 703.1 \\
\hline 751 & 29. & 3000 & 105 & & 0.5 & 176 & & 51 & 0.1 & 195 & 7500 & & & & 2.6 & & & 42.5 & 0 & & 88.2 & 463 \\
\hline 752 & & & 53 & & 4.24 & 103 & & & 0.1 & & 750 & & & & & & & 95.94 & 59.44 & & 97.9 & 312.2 \\
\hline & & & 450 & & \begin{tabular}{|l|}
3.3 \\
\end{tabular} & $1 C$ & & & 0.1 & & & & & & & & & 94.75 & 47.47 & & 59.6 & 212. \\
\hline 754 & & & 2290 & & 0.116 & 109 & & & 0.1 & & 750 & & & & & & & 0 & 0 & & 99.2 & \\
\hline 755 & & 3000 & 320 & & \begin{tabular}{|l|l|}
15.3106 \\
\end{tabular} & $\begin{array}{r}107 \\
\end{array}$ & & 5125 & 0.1 & 1950 & 7500 & & & & 1.4 & & & 98.83 & 88.35 & & 113.2 & \\
\hline 756 & 27.01 & 3000 & 600 & 6.343231 & 0.215 & \begin{tabular}{|l|l|}
101.7 \\
\end{tabular} & 73.75 & 5125 & & 1950 & 7500 & 0000 & 0.1178 & \#\#\#\#\#+ & 1.903 & $1.48 \mathrm{E}-02$ & 44.96 & 21.18 & & & 46.8 & 361. \\
\hline
\end{tabular}




\begin{tabular}{|c|c|c|c|c|c|c|c|c|c|c|c|c|c|c|c|c|c|c|c|c|c|c|}
\hline Realization & 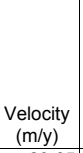 & $\begin{array}{c}\text { Fixed Source } \\
\text { Concentration } \\
(\mathrm{mg} / \mathrm{L})\end{array}$ & $\begin{array}{c}\text { Time of } \\
\text { Maximum } \\
\text { at } \\
\text { Compliance } \\
\text { Boundary } \\
\text { (y) }\end{array}$ & $\begin{array}{c}\text { Maximum } \\
\text { Concentration } \\
\text { Compliance } \\
\text { Boundary } \\
(\mu \mathrm{g} / \mathrm{L})\end{array}$ & $\begin{array}{c}\text { Maximum } \\
\text { Release Rate } \\
\text { at } \\
\text { Compliance } \\
\text { Boundary } \\
\text { (Kg/y) }\end{array}$ & $\begin{array}{l} \\
\text { Sourc } \\
\text { e Rate } \\
(\mathrm{Kg} / \mathrm{y})\end{array}$ & \begin{tabular}{|c|} 
Source \\
Duration \\
(y)
\end{tabular} & $\begin{array}{c}\text { 1-D } \\
\text { Streamtube } \\
\text { Length } \\
(\mathrm{m})\end{array}$ & \begin{tabular}{|c} 
Base \\
Porosity \\
$\left(\mathrm{m}^{3} / \mathrm{m}^{3}\right)$
\end{tabular} & $\begin{array}{c}\text { Bulk } \\
\text { Densty } \\
\left(\mathrm{Kg} / \mathrm{m}^{3}\right)\end{array}$ & \begin{tabular}{|c|}
$\begin{array}{c}\text { Remaining } \\
\text { Carbon } \\
\text { Tetrachloride } \\
\text { Source } \\
(\mathrm{Kg})\end{array}$ \\
\end{tabular} & $\begin{array}{c}\text { Volume at or } \\
\text { above Fixed } \\
\text { Source } \\
\text { Concentratio } \\
n(m 3)\end{array}$ & $\begin{array}{l}\text { Porosity } \\
\left(\mathrm{m}^{3} / \mathrm{m}^{3}\right)\end{array}$ & $\begin{array}{c}\mathrm{Kd} \\
\left(\mathrm{m}^{3} / \mathrm{kg}\right)\end{array}$ & Retardation & $\begin{array}{c}\text { First } \\
\text { Order } \\
\text { Rate } \\
\text { Constant } \\
(1 / y) \\
\end{array}$ & \begin{tabular}{|c|} 
Longitudinal \\
Dispersivity \\
$(\mathrm{m})$
\end{tabular} & $\begin{array}{c}\text { Required } \\
\text { Source } \\
\text { Cleanup } \\
\text { Percentage } \\
(5 \text { ug/L } \\
\text { Compliance) } \\
\end{array}$ & $\begin{array}{c}\text { Required } \\
\text { Source } \\
\text { Cleanup } \\
\text { Percentage } \\
\text { (50 ug/L } \\
\text { Compliance) } \\
\end{array}$ & \begin{tabular}{|c|} 
Required \\
Source Cleanup \\
Percentage \\
(500 $\mu \mathrm{g} / \mathrm{L}$ \\
Compliance) \\
\end{tabular} & $\begin{array}{c}\text { Abiotic } \\
\text { Reaction } \\
\text { Half Time } \\
(y)\end{array}$ & $\begin{array}{c}\text { Carbon } \\
\text { Tetrachloride } \\
\text { Travel Time } \\
\text { (y) } \\
\end{array}$ \\
\hline & 29.65 & 3000 & 630 & & & 95.44 & \begin{tabular}{|l|l|}
78.58 \\
\end{tabular} & $\begin{array}{r}125 \\
\end{array}$ & & 1950 & \begin{tabular}{|r|}
7500 \\
\end{tabular} & \begin{tabular}{|l|}
1000000 \\
\end{tabular} & 0.1073 & \#\#\#\#+ & & $4.56 \mathrm{E}-03$ & \begin{tabular}{|l|}
71.24 \\
\end{tabular} & 96.72 & & & & \\
\hline 758 & 31.69 & 3000 & 400 & \begin{tabular}{|r|}
612.035043 \\
\end{tabular} & \begin{tabular}{|l|l|}
18.8163 \\
\end{tabular} & 92.23 & 81.32 & 5125 & 0.1 & 1950 & 7500 & 950000 & 0.1004 & \#\#\#\#+ & 1.557 & $3.12 \mathrm{E}-03$ & 60.14 & $\begin{array}{l}99.18 \\
\end{array}$ & 91.83 & 18.31 & 222.5 & 251.9 \\
\hline 759 & 27.33 & 3000 & 370 & 27.617845 & 1.4918 & 162.1 & 46.28 & 5125 & 0.1 & 1950 & 7500 & 2212500 & 0.1164 & \#\#\#\#+ & 1.502 & $1.28 \mathrm{E}-02$ & 81.61 & 81.9 & 0 & 0 & 54.4 & 281.6 \\
\hline 760 & 59.8 & 3000 & 1900 & 35.37668 & 1.4168 & 120.1 & 62.42 & 5125 & 0.1 & 1950 & 7500 & 1412500 & 0.0532 & \#\#\#\#+ & 4.881 & $4.80 \mathrm{E}-03$ & 13.62 & 85.87 & 0 & 0 & 144.3 & 418.3 \\
\hline 761 & 21.37 & 3000 & 1460 & \begin{tabular}{|l|l|l|l}
0.037013 \\
\end{tabular} & \begin{tabular}{|l|l}
0.0013 \\
\end{tabular} & $\begin{array}{l}106.3 \\
\end{array}$ & 70.57 & \begin{tabular}{l|l}
5125 \\
\end{tabular} & 0.1 & 1950 & 7500 & 1175000 & 0.1489 & \#\#\#\#+1 & 2.949 & $1.54 \mathrm{E}-02$ & 37.62 & 0 & 0 & 0 & 45 & 707.5 \\
\hline 762 & 28.76 & 3000 & 790 & 44.187524 & 1.6203 & 110 & 68.18 & 5125 & 0.1 & 1950 & 7500 & 1237500 & 0.1106 & \#\#\#\#+ & 2.34 & $5.80 \mathrm{E}-03$ & 82.81 & 88.68 & 0 & 0 & 119.5 & 416.9 \\
\hline 763 & 47.55 & 3000 & 490 & 83.104639 & \begin{tabular}{|c|}
3.8758 \\
\end{tabular} & \begin{tabular}{ll|}
139.9 \\
\end{tabular} & 53.6 & 5125 & 0.1 & 1950 & 7500 & 1775000 & 0.0669 & \#\#\#\#+ & 2.295 & $8.21 \mathrm{E}-03$ & 70.16 & 93.98 & 39.83 & 0 & \begin{tabular}{ll|}
84.4 \\
\end{tabular} & 247.3 \\
\hline 764 & 22.68 & 3000 & 520 & 25.430226 & \begin{tabular}{|l|l|}
1.1297 \\
\end{tabular} & $\begin{array}{l}133.3 \\
\end{array}$ & 56.28 & 5125 & 0.1 & 1950 & 7500 & 1650000 & 0.1403 & \#\#\#\#+ & 1.541 & $1.09 \mathrm{E}-02$ & 33.13 & 80.34 & 0 & 0 & 63.9 & 348.4 \\
\hline 765 & 30.59 & 3000 & 1280 & 1.747191 & 0.0671 & $\begin{array}{l}115.1 \\
\end{array}$ & 65.14 & 5125 & 0.1 & 1950 & 7500 & 1325000 & 0.104 & \#\#\#\# & 2.944 & $1.19 \mathrm{E}-02$ & 20.49 & 0 & 0 & 0 & 58.4 & 493.3 \\
\hline 766 & 27.62 & 3000 & 1280 & 7.590055 & 0.2802 & $\begin{array}{l}110.7 \\
\end{array}$ & 67.72 & 5125 & 0.1 & 1950 & 7500 & 1250000 & 0.1152 & \#\#\#曲 & 3.056 & 7.06E-03 & 64.34 & 34.12 & 0 & & 98.2 & 567.2 \\
\hline 767 & 32.3 & 3000 & $\frac{1110}{640}$ & $\begin{array}{l}0.937993 \\
7089090\end{array}$ & 0.0391 & $\begin{array}{r}125.1 \\
110\end{array}$ & $59.97 \mid$ & 5125 & 0.1 & 1950 & 7500 & 1500000 & 0.0985 & 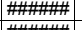 & \begin{tabular}{|c|}
3.407 \\
1.06
\end{tabular} & 1.33E-02 & 74.55 & 0 & 0 & & 52.2 & 540.6 \\
\hline $\begin{array}{l}768 \\
769\end{array}$ & $\begin{array}{l}24.01 \\
26.31\end{array}$ & 3000 & $\begin{array}{l}640 \\
760\end{array}$ & $\begin{array}{r}r .0889 / 8 \\
1.44924\end{array}$ & $\begin{array}{l}0.2789 \\
0.0786\end{array}$ & $\begin{array}{r}118 \\
162.7\end{array}$ & $\begin{array}{l}63.55 \\
46.11\end{array}$ & \begin{tabular}{|l|}
5125 \\
5125
\end{tabular} & $\begin{array}{l}0.1 \\
0.1\end{array}$ & $\begin{array}{l}1950 \\
1950\end{array}$ & $\begin{array}{l}7500 \\
7500\end{array}$ & \begin{tabular}{|l|}
2225000 \\
22500
\end{tabular} & $\begin{array}{l}\mid 0.125 \\
0.1209 \\
\end{array}$ & 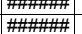 & $\frac{1.806}{2.259}$ & $\begin{array}{l}1.31 \mathrm{E}-02 \\
1.44 \mathrm{E}-02 \\
\end{array}$ & $\begin{array}{l}34.88 \\
56.61\end{array}$ & $\frac{29.47}{0}$ & 0 & & $\begin{array}{ll}53.1 \\
48.3 \\
\end{array}$ & 385.4 \\
\hline 770 & 28.35 & 3000 & 400 & 82.085461 & 3.9708 & 145.1 & 51.68 & 5125 & 0.1 & 1950 & 7500 & 1875000 & 0.1122 & 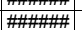 & 1.538 & $8.88 \mathrm{E}-03$ & 59.47 & 93.91 & 39.09 & & $\frac{40.0}{78}$ & $\frac{440.1}{277.9}$ \\
\hline 771 & 60.71 & 3000 & 490 & \begin{tabular}{|r|}
298.791723 \\
\end{tabular} & 9.6633 & 97.02 & 77.3 & 5125 & 0.1 & 1950 & 7500 & 1025000 & 0.0524 & \#\#\#\# & 2.392 & $6.96 \mathrm{E}-03$ & $\begin{array}{l}30.45 \\
30\end{array}$ & 98.33 & 83.27 & & 99.6 & $\begin{array}{l}271.9 \\
201.9\end{array}$ \\
\hline 772 & 21.45 & 3000 & 1210 & \begin{tabular}{|r|}
14.71938 \\
\end{tabular} & \begin{tabular}{|l|l|l|l|l|}
0.476 \\
\end{tabular} & 97.02 & 77.3 & 5125 & 0.1 & 1950 & 7500 & 1025000 & 0.1483 & \#\#\#\# & 2.502 & $5.78 \mathrm{E}-03$ & $\begin{array}{ll}61.17 \\
617\end{array}$ & 66.03 & 0 & & 119.9 & 597.7 \\
\hline 773 & 21.64 & & 770 & $\begin{array}{l}6.478698 \\
\end{array}$ & 0.2701 & $\begin{array}{l}125.1 \\
\end{array}$ & 59.97 & 5125 & 0.1 & 1950 & 7500 & 1500000 & 0.147 & 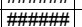 & 1.96 & $1.03 \mathrm{E}-02$ & 50.03 & 22.82 & & & 67.6 & $\frac{59.1}{464.2}$ \\
\hline & 30.33 & 3000 & 1740 & 15.328808 & \begin{tabular}{|l|l|}
0.463 \\
\end{tabular} & 90.61 & 82.78 & 5125 & 0.1 & 1950 & 7500 & 925000 & 0.1049 & \#\#冿 & 3.7 & $4.70 \mathrm{E}-03$ & 59.21 & 67.38 & & & 147.6 & $\frac{404.2}{6253}$ \\
\hline & 21.52 & & 1980 & 5.149544 & 0.1888 & 110 & 68.18 & 5125 & 0.1 & 1950 & 7500 & 1237500 & & \#\#\#\#\# & 3.241 & $5.16 \mathrm{E}-03$ & 43.6 & 2.9 & 0 & & 1344 & $\begin{array}{l}625.3 \\
771.8 \\
\end{array}$ \\
\hline 776 & 59.13 & 3000 & 600 & 40.423999 & 1.4623 & 108.5 & 69.11 & 5125 & 0.1 & 1950 & 7500 & 1212500 & & 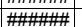 & 2729 & $1.35 \mathrm{E}-02$ & 28.09 & 87.63 & 0 & & 515 & $\begin{array}{l}231.8 \\
236.6 \\
\end{array}$ \\
\hline 777 & 62.38 & 3000 & 200 & 1240.6 & 42.6715 & 103.2 & 72.65 & 5125 & 0.1 & 1950 & 7500 & 1125000 & 0.051 & \#\#\#五 & $\begin{array}{ll}1.426 \\
\end{array}$ & | $5.62 \mathrm{E}-03$ & 2593 & 99.6 & 95.97 & 59.68 & 123.3 & $\begin{array}{l}230.0 \\
117.1 \\
\end{array}$ \\
\hline & 23.78 & 3000 & & & 0.2798 & 107 & \begin{tabular}{ll|l}
70.08 \\
\end{tabular} & 5125 & & 1950 & 7500 & & 0.1338 & 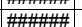 & 1.891 & $1.21 \mathrm{E}-02$ & 30.8 & 36.26 & & & 57.4 & 407.5 \\
\hline 779 & 31.44 & 3000 & 970 & & \begin{tabular}{|l|l|}
1.0049 \\
\end{tabular} & $\mid 149.6$ & 50.13 & 5125 & 0.1 & 1950 & 7500 & & 0.1012 & \#\#\#\#\#| & $\begin{array}{l}2.699 \\
\end{array}$ & & 59.84 & 75.19 & 0 & & 109.1 & 4440 \\
\hline 780 & 26.82 & 3000 & 1910 & & 0.1009 & \begin{tabular}{ll|l|}
146.4 \\
\end{tabular} & 51.23 & 5125 & 0.1 & 1950 & 7500 & & 0.1186 & \#\#\#\#\# & 3.62 & & & 0 & 0 & & 998 & $\begin{array}{r}491.6 \\
691\end{array}$ \\
\hline 781 & 49.63 & 3000 & 410 & \begin{tabular}{|l|}
182.508247 \\
\end{tabular} & 9.8957 & $\mid 162.7$ & 46.11 & 5125 & 0.1 & 1950 & 7500 & & 0.0641 & \#\#\#\#\# & 2.073 & & 68.4 & 97.26 & 72.6 & & 118.9 & 214 \\
\hline 782 & 25.53 & 3000 & 960 & 44.18 & 2.0022 & 135.9 & 55.17 & 5125 & 0.1 & 1950 & 7500 & 1700000 & 0.1246 & \#\#\#\#\#| & 2.288 & & & & 0 & & 141.3 & 459.3 \\
\hline 783 & 23.27 & 3000 & 2100 & & 0.512 & \begin{tabular}{|l|l|}
108.5 \\
\end{tabular} & 69.11 & 5125 & 0.1 & 1950 & 7500 & 1212500 & 0.1367 & 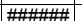 & 3.205 & & & 67 & 0 & & 158.1 & 705.7 \\
\hline 784 & 43.76 & 3000 & 2460 & & 0.2051 & 82.25 & 91.19 & 5125 & 0.1 & 1950 & 7500 & & & "\#\#\#\#\#| & \begin{tabular}{|l|l|}
4.958 \\
\end{tabular} & & & & 0 & & 108.5 & 580.7 \\
\hline 785 & 35.71 & 3000 & 660 & & 2.332 & \begin{tabular}{ll|l}
112.2 \\
\end{tabular} & 66.83 & \begin{tabular}{ll|l}
5125 \\
\end{tabular} & 0.1 & 1950 & 7500 & 1275000 & & 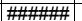 & 2.264 & & & 91.98 & 19.8 & & $\begin{array}{l}94.3 \\
\end{array}$ & 325 \\
\hline 786 & 59.35 & 3000 & 200 & 818.198834 & 36.346 & \begin{tabular}{|c|}
133.3 \\
\end{tabular} & 56.28 & $\begin{array}{l}5125 \\
\end{array}$ & 0.1 & 1950 & 7500 & & 0.0536 & \#\#\#\# & 1.455 & & & & \begin{tabular}{|c|c|}
93.89 \\
\end{tabular} & & 91 & 125.7 \\
\hline 787 & 39.82 & 3000 & 210 & 880.25 & 43.7094 & 149 & 50.35 & 5125 & 0.1 & 1950 & 7500 & 1950000 & 0.0799 & \#\#\#\#地 & 1.194 & & \begin{tabular}{ll|l}
25.69 &
\end{tabular} & 99.43 & 94.32 & 43.2 & 107.1 & 153.7 \\
\hline 788 & $\mid 46.04$ & 3000 & 940 & & 0.9923 & 144.5 & 51.91 & 5125 & 0.1 & 1950 & 7500 & 1862500 & 0.06 & \#\#\#\#年 & $\begin{array}{l}3.087 \\
\end{array}$ & & \begin{tabular}{|c|c|}
31.47 \\
\end{tabular} & 75 & 0 & & 78.7 & 343.7 \\
\hline 789 & 23.92 & 3000 & 450 & & \begin{tabular}{|l|l|}
7.5358 \\
\end{tabular} & 115.9 & 64.73 & 5125 & 0.1 & 1950 & 7500 & & & \#\#\#\#\#+ & & & & 97.44 & 74.38 & 0 & 131.6 & 314.3 \\
\hline 790 & 28.89 & 3000 & 310 & \begin{tabular}{|l|l|}
501.28 \\
\end{tabular} & 20.3128 & 121.6 & \begin{tabular}{|c|c|}
61.7 \\
\end{tabular} & 5125 & 0.1 & 1950 & 7500 & & & \#\#\#\#+ & 1.294 & & & $\begin{array}{lll}99 & \\
\end{array}$ & 90.03 & 0.26 & 131.2 & 229.5 \\
\hline 791 & 21.69 & 3000 & 1900 & & |0.0528 & 102.5 & 73.19 & 5125 & 0.1 & 1950 & 7500 & & 0.1. & "\#\#\#\#| & 3.565 & & 69.4 & 0 & 0 & 0 & 100.1 & 842.5 \\
\hline 792 & 32.5 & 3000 & 1110 & & 0.0171 & 115.9 & 64.73 & 5125 & 0.1 & 1950 & 7500 & & & & 3.668 & & $84.2 \mathrm{Y}$ & 0 & 0 & & 45.2 & 578.5 \\
\hline 793 & 22.36 & 3000 & 1090 & & 0.0108 & 124.4 & 60.31 & 5125 & 0.1 & 1950 & & & & & \begin{tabular}{ll|l}
2.642 \\
\end{tabular} & & & 0 & 0 & & | 48.7 & 605.8 \\
\hline 794 & 30.1 & 3000 & 1100 & & 0.1109 & 147 & 51 & 5125 & 0.1 & 1950 & 7500 & & & & 2.997 & & 58.78 & 0 & 0 & & 65 & 510.3 \\
\hline 795 & 441.05 & 30 & 1230 & & & 130.6 & 57.44 & 5125 & 0.1 & 1950 & & & & & 3.589 & & 67. & 81.89 & 0 & & 145 & 448 \\
\hline 796 & 26. & & 920 & & & 113.7 & & 5125 & 0.1 & 1950 & & & & & 2.3 & & & 0 & 0 & & $\begin{array}{ll}52.9 \\
\end{array}$ & 457.2 \\
\hline 797 & 24.89 & & 2110 & & & 78.78 & & 5125 & 0.1 & & & & & & & & & & 0 & & 81.3 & 811.3 \\
\hline 798 & 28.3 & & 700 & & & 89.79 & & & 0.1 & & & & & & & & & \begin{tabular}{|c|}
94.68 \\
\end{tabular} & \begin{tabular}{|l|l|}
46.76 \\
\end{tabular} & & 116.3 & 369.1 \\
\hline 799 & 29.82 & & 1100 & & & 159.6 & & & 0.1 & & & & & & & & & 0 & 0 & & 83.8 & 521.8 \\
\hline 800 & 22.82 & & 590 & & & \begin{tabular}{|l|l|}
96.23 \\
\end{tabular} & & & 0.1 & & & & & & & & & 87.93 & & & 84 & 387.9 \\
\hline 801 & 36.11 & & 600 & & 0.5151 & \begin{tabular}{|l|}
92.23 \\
\end{tabular} & & & 0.1 & & & & & & & & & & & & 54.7 & 5.1 \\
\hline 802 & & & 1460 & & $\begin{array}{l}0.636 \\
\end{array}$ & 181 & & & 0.1 & & & & & & & & & & & & 1 & 596.9 \\
\hline 803 & 46.58 & & 630 & & 0.9674 & $\begin{array}{l}150.9 \\
\end{array}$ & & & 0.1 & & & & & & 2.63 & & & & 0 & & 58 & 289.4 \\
\hline 804 & 29.21 & & 99 & & $\begin{array}{l}0.4369 \\
\end{array}$ & 92.23 & 81.3 & & 0.1 & & & & & & 22 & & & & 0 & & 77.9 & 442.5 \\
\hline 805 & 31.62 & & 880 & & 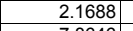 & $\begin{array}{l}99.38 \\
9.70\end{array}$ & & & 0.1 & & & & 0.1006 & & 11 & & & & 23.63 & & 138.9 & 407 \\
\hline 806 & 33.1 & & 420 & & $\begin{array}{l}7.8646 \\
1.040\end{array}$ & 107.8 & & & 0.1 & & & & & & & & & & 77.16 & & 1 & 256.9 \\
\hline 807 & 50.1 & 300 & 610 & 25.17 & 1.0495 & 125.1 & & & & & & & & & 2.551 & & & & 0 & & 51 & 261 \\
\hline 808 & 31.22 & 300 & 620 & 8.71 & 0.4046 & 139.3 & 53.86 & 512 & & & & 17625 & 0.1 & & 2.118 & & & & 0 & & & 347.7 \\
\hline 809 & 32.1 & 300 & 580 & & 2.1101 & 90.61 & 82.18 & 5125 & 0.1 & & & & & & $\begin{array}{l}15 \\
29\end{array}$ & & & 92.84 & & & & 321.7 \\
\hline 然 & 44.56 & & 1730 & & 0.2381 & $\begin{array}{r}133.9 \\
1230\end{array}$ & 56 & ST125 & 0.1 & & & & 0.0 & & & & & 6.24 & 0 & & 58 & 382.9 \\
\hline 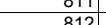 & 40.19 & & 2010 & & $\frac{0.1625}{41318}$ & 13.9 & $\begin{array}{r}56 \\
1417\end{array}$ & $\frac{512}{512}$ & 0.1 & & & & & & & & & 0 & & & $\mid$ & 649.2 \\
\hline$\frac{612}{813}$ & $\frac{2.45}{35.49}$ & (300 & 200 & & 年. & 1. & 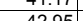 & $\frac{512}{512}$ & & & & & & & & & & 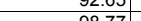 & 20.07 & & 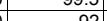 & 36.6 \\
\hline 814 & 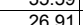 & 300 & 2170 & & 资. & $\begin{array}{l}14.0 \\
166.3\end{array}$ & $\frac{42.50}{451}$ & 515 & 0.1 & 195 & & & & 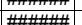 & & & & 10 & 20.14 & & 120. & 7658 \\
\hline 815 & 33.1 & & 540 & & 25731 & |100.04 & 80.61 & 515 & 0.1 & 195 & & & & & 1894 & & & 93.97 & 3974 & & 74.8 & 2928 \\
\hline 816 & 57.12 & 300 & 670 & & 1.50 & 154 & 48 & 512 & 0.1 & 195 & & & & & & & & 82.96 & 0 & & 67.3 & $\frac{292 .}{269.3}$ \\
\hline 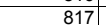 & 21.129 & 3000 & 620 & & $\mid 0.316$ & 9702 & 773 & 5125 & 0.1 & & 7500 & & & & & & & 48.83 & 0 & & 623 & 34.15 \\
\hline 818 & 55.62 & 3000 & 680 & 115.421012 & 3.3911 & 88.14 & 85.09 & 5125 & 0.1 & 1950 & 7500 & & 0.0572 & & 2.885 & $6.83 \mathrm{E}-0$ & 57.1 & 95.67 & 56.68 & & 101.4 & 265.8 \\
\hline 819 & 23.76 & 3000 & 490 & \begin{tabular}{|r|}
53.837608 \\
\end{tabular} & 2.7414 & \begin{tabular}{|l|}
152.8 \\
\end{tabular} & 49.1 & 5125 & $\begin{array}{l}0.1 \\
\end{array}$ & 1950 & 7500 & 2025000 & 0.1339 & \#\#\#\#+ & \begin{tabular}{l|l|}
1.582 \\
\end{tabular} & $7.66 \mathrm{E}-03$ & \begin{tabular}{|l|l|}
62.3 \\
\end{tabular} & 90.71 & 7.13 & & 90.4 & 341.3 \\
\hline
\end{tabular}




\begin{tabular}{|c|c|c|c|c|c|c|c|c|c|c|c|c|c|c|c|c|c|c|c|c|c|c|}
\hline & $\begin{array}{c}\begin{array}{c}\text { Velocity } \\
(\mathrm{m} / \mathrm{y})\end{array} \\
\text { (a) }\end{array}$ & $\begin{array}{l}\text { Fixed Source } \\
\text { Concentration } \\
(\mathrm{mg} / \mathrm{L})\end{array}$ & $\begin{array}{c}\text { Time of } \\
\text { Maximum } \\
\text { at } \\
\text { Compliance } \\
\text { Boundary } \\
\text { (y) }\end{array}$ & $\mid \begin{array}{c}\text { Maximum } \\
\text { Concentration } \\
\text { Compliance } \\
\text { Boundary } \\
(\mu \mathrm{g} / \mathrm{L})\end{array}$ & $\begin{array}{c}\text { Maximum } \\
\text { Release Rate } \\
\text { at } \\
\text { Compliance } \\
\text { Boundary } \\
(\mathrm{Kg} / \mathrm{y})\end{array}$ & $\begin{array}{l} \\
\text { Sourc } \\
\text { e Rate } \\
\text { (Kg/y) }\end{array}$ & \begin{tabular}{|c|} 
Source \\
Duration \\
(y)
\end{tabular} & $\begin{array}{c}\text { 1-D } \\
\text { Streamtube } \\
\text { Length } \\
(\mathrm{m})\end{array}$ & $\begin{array}{c}\text { Base } \\
\text { Porosity } \\
\left(\mathrm{m}^{3} / \mathrm{m}^{3}\right)\end{array}$ & $\begin{array}{c}\text { Bulk } \\
\text { Densty } \\
\left(\mathrm{Kg} / \mathrm{m}^{3}\right)\end{array}$ & \begin{tabular}{|c|} 
Remaining \\
Carbon \\
Tetrachloride \\
Source \\
$(\mathrm{Kg})$
\end{tabular} & $\begin{array}{c}\text { Volume at or } \\
\text { above Fixed } \\
\text { Source } \\
\text { Concentratio } \\
n(m 3)\end{array}$ & $\begin{array}{l}\text { Porosity } \\
\left(\mathrm{m}^{3} / \mathrm{m}^{3}\right)\end{array}$ & $\left|\begin{array}{c}\mathrm{Kd} \\
\left(\mathrm{m}^{3} / \mathrm{Kg}\right)\end{array}\right| \mathrm{F}$ & Retardation & $\begin{array}{c}\text { First } \\
\text { Order } \\
\text { Rate } \\
\text { Constant } \\
(1 / y)\end{array}$ & $\begin{array}{l}\text { Longitudinal } \\
\text { Dispersivity } \\
(\mathrm{m})\end{array}$ & $\begin{array}{c}\text { Required } \\
\text { Source } \\
\text { Cleanup } \\
\text { Percentage } \\
(5 \mu \mathrm{g} / \mathrm{L} \\
\text { Compliance })\end{array}$ & $\begin{array}{c}\text { Required } \\
\text { Source } \\
\text { Cleanup } \\
\text { Percentage } \\
(50 \mu \mathrm{g} / \mathrm{L} \\
\text { Compliance })\end{array}$ & \begin{tabular}{|c} 
Required \\
Source Cleanup \\
Percentage \\
(500 $\mu \mathrm{g} / \mathrm{L}$ \\
Compliance)
\end{tabular} & \begin{tabular}{|c|} 
Abiotic \\
Reaction \\
Half Time \\
(y)
\end{tabular} & $\begin{array}{c}\text { Carbon } \\
\text { Tetrachloride } \\
\text { Travel Time } \\
\text { (y) }\end{array}$ \\
\hline & 32.33 & 3000 & & 10.045975 & \begin{tabular}{|l|}
0.3855 \\
\end{tabular} & 115.1 & \begin{tabular}{|l|l|}
65.14 \\
\end{tabular} & 5125 & 0.1 & 1950 & & 1325000 & 0.0984 & \#\#\#立 & 3.385 & $6.51 \mathrm{E}-03$ & \begin{tabular}{|r|}
32.88 \\
\end{tabular} & 50.23 & & & & \\
\hline 821 & & 3000 & 520 & 181.849304 & 6.8024 & 112.2 & 66.83 & 5125 & 0.1 & 1950 & 7500 & 1275000 & 0.0909 & 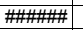 & 1.99 & $4.51 \mathrm{E}-03$ & 83.6 & 97.25 & 72.5 & & 153.7 & 291.5 \\
\hline 822 & 33.74 & 3000 & 490 & 40.196889 & 1.8393 & 137.3 & 54.64 & 5125 & 0.1 & 1950 & 7500 & 1725000 & 0.0943 & 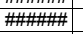 & 1.839 & $1.16 \mathrm{E}-02$ & 33.64 & 87.56 & & & 59.8 & 279.4 \\
\hline 823 & 25.31 & 3000 & 420 & 18.876951 & 0.7153 & 113.7 & 65.97 & 5125 & 0.1 & 1950 & 7500 & 1300000 & 0.1257 & \#\#\#+m & 1.487 & $1.47 \mathrm{E}-02$ & 47.59 & 73.51 & 0 & & 47.3 & 301.1 \\
\hline 824 & 37.6 & 3000 & 310 & 267.662032 & 13.9085 & 155.9 & 48.11 & 5125 & 0.1 & 1950 & 7500 & 2087500 & 0.0846 & \#\#\#+m & 1.537 & $6.11 \mathrm{E}-03$ & 76.18 & 98.13 & 81.32 & & \begin{tabular}{|c|}
113.5 \\
\end{tabular} & 209.5 \\
\hline 825 & 52.24 & 3000 & 2510 & $\begin{array}{r}0.312652 \\
\end{array}$ & 0.0155 & $\begin{array}{r}149 \\
\end{array}$ & 50.35 & 5125 & 0.1 & 1950 & 7500 & 1950000 & 0.0609 & \#\#\#\#+ & 6.029 & $1.17 \mathrm{E}-02$ & 24.9 & & & & 59.2 & 591.5 \\
\hline 826 & 47.7 & 3000 & 1360 & 0.602016 & 0.0301 & 150.2 & 49.92 & 5125 & 0.1 & 1950 & 7500 & 1975000 & 0.0667 & \#\#\#\#王 & 5.302 & $1.43 \mathrm{E}-02$ & 86.65 & & & & 48.3 & 569.7 \\
\hline 827 & 63.5 & 3000 & 1740 & \begin{tabular}{|l|l|}
1.740779 \\
\end{tabular} & 0.0726 & 125.1 & 59.97 & 5125 & 0.1 & 1950 & 7500 & 1500000 & 0.0501 & \#\#\#\#+ & 5.995 & $1.15 \mathrm{E}-02$ & 49.45 & 0 & 0 & & 60.1 & 483.9 \\
\hline 828 & 52.67 & 3000 & 430 & $\begin{array}{l}57.83032 \\
\end{array}$ & 3.4114 & 177 & 42.38 & 5125 & 0.1 & 1950 & 7500 & 2525000 & 0.0604 & \#\#\#\#\# & 2.236 & $1.12 \mathrm{E}-02$ & 55.31 & 91.35 & 13.54 & & 62.1 & 217.6 \\
\hline 829 & 32.66 & 3000 & 930 & 21.207828 & 0.5995 & 84.8 & 88.45 & 5125 & 0.1 & 1950 & 7500 & 837500 & 0.0974 & \#\#\#\#+ & 2.555 & $9.19 \mathrm{E}-03$ & 30.36 & 76.42 & & & 75.4 & 400.9 \\
\hline 830 & 21.66 & 3000 & 1140 & 43.856043 & 2.2055 & 150.9 & 49.71 & 5125 & 0.1 & 1950 & 7500 & 1987500 & 0.1469 & \#\#\#\#+ & 2.382 & $2.97 \mathrm{E}-03$ & & 88.6 & & & 233.7 & 563.7 \\
\hline 831 & 25.91 & 3000 & 1700 & 4.854915 & 0.199 & 123 & 60.99 & 5125 & 0.1 & 1950 & 7500 & 1462500 & & \#\#\#\#+ & 3.266 & $6.38 \mathrm{E}-03$ & 38.99 & & & & 108.7 & 646.1 \\
\hline 832 & 43.7 & 3000 & 260 & & 28.7513 & \begin{tabular}{|l|l|}
145.8 \\
\end{tabular} & 51.45 & 5125 & 0.1 & 1950 & 7500 & 1887500 & & 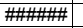 & 1.44 & $7.18 \mathrm{E}-03$ & 23.65 & 99.16 & 91.55 & & 96.5 & 168.9 \\
\hline 833 & 31.1 & 3000 & 710 & & 0.6491 & $\begin{array}{l}120.1 \\
\end{array}$ & 62.42 & 5125 & 0.1 & 1950 & 7500 & 1412500 & & 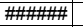 & 2.147 & $1.13 \mathrm{E}-02$ & 25.16 & 69.15 & & 0 & 61.2 & 353.9 \\
\hline 834 & 60.71 & 3000 & 860 & & 1.7956 & $\begin{array}{l}107.8 \\
\end{array}$ & & 5125 & 0.1 & 1950 & 7500 & 1200000 & & \#\#\#\#曲 & 3.68 & $6.59 \mathrm{E}-03$ & 78.25 & 90 & & & 105.2 & 310.7 \\
\hline 835 & 31.78 & 3000 & 750 & & 3.0288 & \begin{tabular}{ll|}
151.5 \\
\end{tabular} & 49.5 & 5125 & 0.1 & 1950 & 7500 & 2000000 & & \#\#\#\#曲 & 2.16 & $7.50 \mathrm{E}-03$ & 12.55 & 91.66 & 16.63 & & 92.4 & 348.3 \\
\hline 836 & 23.19 & 3000 & 430 & & 1.4465 & $\begin{array}{l}135.9 \\
\end{array}$ & 55.17 & 5125 & 0.1 & 1950 & 7500 & 1700000 & & \#\#\#\#\# & 1.475 & 1.07E-02 & 70.92 & 84.34 & & & 64.9 & 326 \\
\hline 837 & 25.72 & 3000 & 360 & 24.980669 & 1.3341 & 160.2 & 46.81 & 5125 & 0.1 & 1950 & 7500 & 2175000 & $\begin{array}{l}0.1237 \\
\end{array}$ & \#\#\#\#\# & 1.399 & $1.39 \mathrm{E}-02$ & 52.49 & 79.98 & & & 50 & 278.8 \\
\hline 839 & 38.99 & 3000 & 720 & 58.896203 & 1.7948 & 91.42 & 82.04 & 5125 & 0.1 & 1950 & 7500 & $\begin{array}{r}937500 \\
\end{array}$ & 0.0816 & \#\#\#\#\# & 2.523 & $7.56 \mathrm{E}-03$ & 60.33 & 91.51 & 15.1 & & 91.7 & 331.7 \\
\hline 840 & $\begin{aligned} 29.7 \\
\end{aligned}$ & $\begin{array}{l}3000 \\
3000 \\
300\end{array}$ & \begin{tabular}{|c|}
960 \\
1080
\end{tabular} & \begin{tabular}{|c|}
54.305475 \\
508077
\end{tabular} & $\begin{array}{l}2.4729 \\
0223 \\
\end{array}$ & $\begin{array}{l}136.6 \\
131\end{array}$ & $\begin{array}{r}54.9 \\
57.15\end{array}$ & 5125 & 0.1 & $\begin{array}{l}1950 \\
1050\end{array}$ & 7500 & $\begin{array}{l}1712500 \\
1612500\end{array}$ & $\begin{array}{l}0.1071 \\
0.1114\end{array}$ & 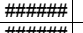 & 2.489 & 4.47E-03 & $\begin{array}{l}45.73 \\
32.77 \\
\end{array}$ & \begin{tabular}{|c|}
90.79 \\
159
\end{tabular} & 7.93 & & $\begin{array}{ll}155.2 \\
719\end{array}$ & $\begin{array}{r}429.4 \\
477\end{array}$ \\
\hline$\frac{841}{842}$ & $\begin{array}{l}28.56 \\
30.68\end{array}$ & 3000 & \begin{tabular}{r|r|r}
1080 & \\
880 &
\end{tabular} & \begin{tabular}{|l|}
5.080747 \\
8.97503
\end{tabular} & \begin{tabular}{ll|l}
0.2223 & \\
0.3595 &
\end{tabular} & $\begin{array}{l}131.2 \\
1201\end{array}$ & $\begin{array}{l}57.15 \\
62.42\end{array}$ & $\begin{array}{l}5125 \\
5125\end{array}$ & 0.1 & $\begin{array}{l}1950 \\
1950\end{array}$ & $\begin{array}{l}7500 \\
7500\end{array}$ & \begin{tabular}{|r|}
1612500 \\
142500
\end{tabular} & $\begin{array}{l}0.1114 \\
0.137\end{array}$ & \#\#\#\#+ & $\frac{2.658}{2411}$ & $9.64 \mathrm{E}$ & $33.77 \mid$ & $\begin{array}{r}1.59 \\
44.29\end{array}$ & & & 71.9 & $\begin{array}{r}477 \\
4028\end{array}$ \\
\hline 843 & 47.48 & 3000 & 340 & \begin{tabular}{|r|}
241.459886 \\
\end{tabular} & 12.092 & 150.2 & 49.92 & 5125 & 0.1 & 1950 & 7500 & $\begin{array}{l}1412000 \\
1975000\end{array}$ & $\begin{array}{l}0.1033 \\
0.067\end{array}$ & 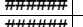 & 2.411 & & $\frac{21.12}{28.34}$ & $\begin{array}{l}44.29 \\
97.93\end{array}$ & $\begin{array}{r}0 \\
7929\end{array}$ & & 63.9 & 402.8 \\
\hline 844 & 25.07 & 3000 & 450 & \begin{tabular}{|r|}
90.110514 \\
\end{tabular} & 3.8395 & $\mid 127.8$ & $\begin{array}{l}58.67 \\
\end{array}$ & 5125 & 0.1 & 1950 & 7500 & 1550000 & $\begin{array}{l}0.001 \\
0.1269 \\
\end{array}$ & 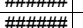 & $\frac{1.144}{1.454}$ & & $\frac{20.04}{16.63}$ & $\begin{array}{l}91.90 \\
94.45 \\
\end{array}$ & $\begin{array}{r}9.29 \\
451\end{array}$ & & 75.6 & $\frac{188.2}{2972}$ \\
\hline 845 & 29.98 & 3000 & $\begin{array}{l}1270 \\
\end{array}$ & 1.685221 & 0.0643 & 1114.4 & 65.55 & 5125 & 0.1 & 1950 & 7500 & 1312500 & 0.1061 & 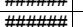 & $\begin{array}{l}3.454 \\
3.558\end{array}$ & & 80.00 & $\begin{aligned} 54.40 \\
0\end{aligned}$ & & & $\begin{array}{ll}0.6 \\
66.9\end{array}$ & 297.2 \\
\hline 846 & 21.94 & 3000 & 560 & \begin{tabular}{|r|}
146.726478 \\
\end{tabular} & 8.1922 & 167.5 & 44.78 & 5125 & 0.1 & 1950 & 7500 & 2325000 & 0.145 & 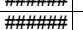 & $\begin{array}{r}0.500 \\
1.55 \\
\end{array}$ & $4.04 \mathrm{E}-0 \mathrm{Z}$ & 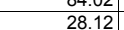 & 96.59 & 65.92 & & 150.2 & $\frac{608.2}{362.1}$ \\
\hline 847 & 24.03 & 3000 & 1840 & \begin{tabular}{|l|}
1.344737 \\
\end{tabular} & 0.0466 & 104 & 72.12 & 5125 & 0.1 & 1950 & 7500 & 1137500 & 0.13 & \#\#\#\#\#+1 & 3.263 & $8.36 \mathrm{E}-03$ & 29.98 & & & & 82.9 & 696 \\
\hline 848 & 31.85 & 3000 & 1420 & 10.00725 & 0.52 & 155.9 & 48.11 & 5125 & 0.1 & 1950 & 7500 & 2087500 & & \#\#\#\#+ & 3.422 & & 60.5 & 50.04 & & & 125 & 550.7 \\
\hline 849 & 23.97 & 3000 & 2100 & 1.004302 & 0.0402 & 120.1 & 62.42 & 5125 & 0.1 & 1950 & 7500 & 1412500 & & \#\#\#\#+ & 3.522 & & 30.03 & 0 & & & 89.5 & 753 \\
\hline 850 & 26.16 & 3000 & 640 & 3.79935 & 0.1879 & \begin{tabular}{|l|l|}
148.3 \\
\end{tabular} & 50.56 & 5125 & 0.1 & 1950 & 7500 & 1937500 & & 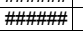 & 2.043 & & 64.16 & 0 & & & 51.1 & 400. \\
\hline 851 & 32.97 & 3000 & 1790 & 11.35 & 0.4109 & 108.5 & 69.11 & 5125 & 0.1 & 1950 & 7500 & 1212500 & & 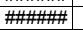 & 3.832 & & 46.31 & 55.98 & & & 133 & 595. \\
\hline 852 & 26.06 & 3000 & 1480 & & 0.0055 & $\begin{array}{l}104.8 \\
\end{array}$ & 71.59 & 5125 & 0.1 & 1950 & 7500 & 1150 & & & 3.4 & & 53. & & & & 50.3 & 684.8 \\
\hline 853 & 24.62 & 3000 & 1790 & & 0.0358 & 88.14 & 85.09 & 5125 & 0.1 & 1950 & 7500 & & & & & & 45. & & 0 & & 79.5 & 720. \\
\hline 854 & 35.35 & & 320 & \begin{tabular}{|l|}
273.12 \\
\end{tabular} & 11.9482 & 131.2 & 57. & 5125 & 0.1 & 1950 & 7500 & & & & & & 57. & 98.17 & 81.69 & & 96.1 & 215. \\
\hline 855 & 43.88 & & 1250 & & 0.3 & 104 & & 51 & 0.1 & & & & & & & & & 51 & 0 & & 71.7 & \\
\hline 856 & 30.41 & & 710 & & & & & & 0.1 & & 7500 & & & & & & & & 60.64 & & 152.7 & \\
\hline 857 & 44.43 & & 410 & & 3.7 & 14 & & & 0.1 & & & & & & & & 45. & & 34.75 & & 61.1 & \\
\hline 858 & 36.95 & & 410 & & 3.3691 & 81. & & & 0.1 & & 750 & & & & & & & & 59.74 & & 53.1 & 229.5 \\
\hline 859 & & & 870 & & & & & & & & 750 & 2337500 & & & & & & & 0 & & 74 & 324.7 \\
\hline 860 & 46.51 & & $\begin{array}{l}420 \\
\end{array}$ & & & 95. & & & & & & & & & & & & & 82.17 & & 103.5 & 217.2 \\
\hline 861 & 26.64 & 3000 & 1080 & & & 119 & & 512 & 0.1 & & & & & & & & 62.3 & & & & 180.3 & 492.9 \\
\hline 862 & 23.53 & & 390 & & & $\begin{array}{l}115.1 \\
\end{array}$ & & & & & & & & & & & 41.41 & & & & 56.2 & 3.5 \\
\hline 863 & 33.38 & & 820 & & & \begin{tabular}{|l|}
141.9 \\
\end{tabular} & & 51 & & & & & & & & & 29.3 & & 0 & & 60.5 & 4.9 \\
\hline 864 & 21.77 & 3000 & $\begin{array}{l}740 \\
\end{array}$ & 8.0 & 0.3387 & $\begin{array}{l}125.8 \\
71.97\end{array}$ & & 51 & & 19 & & & 0.1 & & & & & & 0 & & 71.4 & 463.9 \\
\hline 865 & 25.25 & 3000 & \begin{tabular}{|l|l|}
510 \\
1240
\end{tabular} & 152.329749 & $\begin{array}{l}3.6364 \\
1084\end{array}$ & 71.62 & 104.7 & $\begin{array}{l}5125 \\
5125\end{array}$ & 0.1 & $\frac{19}{19}$ & 7500 & & 0. & & $\begin{array}{l}1.544 \\
3235\end{array}$ & & & $\begin{array}{l}96.72 \\
\end{array}$ & 67.18 & & $\begin{array}{r}83.2 \\
152 .\end{array}$ & 313.5 \\
\hline \begin{tabular}{|l|l|}
866 & \\
867
\end{tabular} & $\begin{array}{l}40.02 \\
28.13\end{array}$ & 3000 & $\begin{array}{l}1340 \\
1790\end{array}$ & $\begin{array}{r}44.136975 \\
262037\end{array}$ & $\begin{array}{r}1.9804 \\
0.1399\end{array}$ & $\begin{array}{l}134.6 \\
1559\end{array}$ & 55.72 & $\begin{array}{l}5125 \\
5125\end{array}$ & 0.1 & $\frac{195}{195}$ & $\begin{array}{l}7500 \\
7500\end{array}$ & 1675000 & 0.0 & 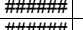 & $\begin{array}{l}3.365 \\
3.47 \\
3\end{array}$ & & 23.4 & $88.67 \mid$ & & & 153.1 & $\begin{array}{r}431 \\
6262\end{array}$ \\
\hline \begin{tabular}{|l|l|}
867 & \\
868
\end{tabular} & $\begin{array}{l}28.13 \\
32.66\end{array}$ & $\begin{array}{l}3000 \\
3000\end{array}$ & \begin{tabular}{|l|l|}
1790 \\
1380
\end{tabular} & $\begin{array}{r}2.6920037 \\
11.546101\end{array}$ & \begin{tabular}{|c|}
0.1399 \\
0.5976
\end{tabular} & \begin{tabular}{|l|}
155.9 \\
15.3
\end{tabular} & $\begin{array}{r}48.11 \\
48.3\end{array}$ & \begin{tabular}{|l|l|l|}
5125 \\
5125
\end{tabular} & $\begin{array}{l}0.1 \\
0.1\end{array}$ & $\frac{195}{195}$ & \begin{tabular}{|l|l|}
7500 & 7500 \\
\end{tabular} & & $\frac{0.1}{0.0}$ & 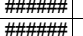 & $\begin{array}{l}3.437 \\
3.123 \\
\end{array}$ & & $\frac{28.7}{22.8}$ & $\begin{array}{r}0 \\
56.7\end{array}$ & & & $\begin{array}{r}96 \\
104.1\end{array}$ & $\frac{626.2}{490}$ \\
\hline 869 & 31.59 & 3000 & 600 & 101.150 & 4.6731 & \begin{tabular}{|c|}
138.6 \\
\end{tabular} & 54.1 & 5125 & 0.1 & 195 & $\begin{array}{ll}7500 \\
\end{array}$ & & & \#\#車 & 2.0 & & 79.9 & $\begin{array}{l}95.06 \\
\end{array}$ & 50.57 & & 145.4 & 332.2 \\
\hline 870 & 22.44 & 3000 & 600 & & 0.52 & 138.6 & 54. & 512 & 0.1 & 195 & 750 & & & \#\#! & 1.676 & & 29.9 & 55.93 & & & 59.6 & 382.8 \\
\hline 871 & 24.83 & 300 & 1570 & & 0.0 & 123 & 60.5 & 512 & 0.1 & 195 & 7500 & 1462 & & \#\#! & 3.473 & & 53.1 & 0 & & & 56.3 & 716.8 \\
\hline 872 & 27.1 & 3000 & 840 & & 0.34 & \begin{tabular}{|r|}
139.9 \\
\end{tabular} & & 512 & 0.1 & 195 & 750 & & & & 2.1 & & 22 & 33.12 & & & 62.9 & 412.9 \\
\hline 873 & 27.96 & 3000 & 780 & 14.36 & & 94.64 & 79.2 & 512 & 0.1 & 195 & 7500 & & & & 2.2 & & 47.7 & 65.19 & & & 68.6 & 408.1 \\
\hline 874 & 27.5 & & 850 & & 0.0 & 102.5 & 73. & 512 & 0.1 & 195 & 7500 & & & & 2.398 & & 42. & 0 & & & 43.5 & 447 \\
\hline 875 & 26.44 & & 1110 & & & & 42. & 512 & 0.1 & 19 & & & & & 3. & & & & & & 40.8 & 604.7 \\
\hline 876 & 30.8 & 3000 & 560 & & & 136 & & & 0.1 & 195 & 7500 & & & & 1.8 & & & & 60.52 & & 110.9 & 308.5 \\
\hline 877 & 23.85 & 3000 & 1060 & & & 124 & & 512 & 0.1 & 195 & 7500 & & & & & & 27. & & 0 & & 109.7 & 496.9 \\
\hline 878 & 30.21 & & 350 & & & & & & 0.1 & & & & & & & & & & 0 & & 40.5 & \\
\hline 87 & & & $\begin{array}{l}470 \\
\end{array}$ & & & 15 & & & 0.1 & & & & & & & & & 90.11 & 1.09 & & 81.4 & \\
\hline 880 & 57. & & 2050 & & & & & & 0.1 & & 750 & & & & & & & 0 & 0 & & 71 & \\
\hline 881 & 35.23 & 3000 & 670 & & & 143.8 & 52. & 5125 & 0.1 & 1950 & 7500 & & & & & & & 83.83 & & & 73.7 & \\
\hline 882 & 23.2 & 3000 & 1210 & 1.641804 & 0.0661 & 120.9 & 62.06 & 5125 & 0.1 & 1950 & 7500 & 1425000 & 0.1371 & \#\#\#\#\#+ & 2.945 & & 83.46 & & & & 72.9 & 650.4 \\
\hline
\end{tabular}




\begin{tabular}{|c|c|c|c|c|c|c|c|c|c|c|c|c|c|c|c|c|c|c|c|c|c|c|}
\hline Realization & $\begin{array}{c}\begin{array}{c}\text { Velocity } \\
(\mathrm{m} / \mathrm{y})\end{array} \\
56\end{array}$ & $\begin{array}{c}\text { Fixed Source } \\
\text { Concentration } \\
(\mathrm{mg} / \mathrm{L})\end{array}$ & $\begin{array}{c}\text { Time of } \\
\text { Maximum } \\
\text { at } \\
\text { Compliance } \\
\text { Boundary } \\
\text { (y) }\end{array}$ & \begin{tabular}{|c|} 
Maximum \\
Concentration \\
Compliance \\
Boundary \\
$(\mu \mathrm{g} / \mathrm{L})$ \\
\end{tabular} & \begin{tabular}{|c|} 
Maximum \\
Release Rate \\
at \\
Compliance \\
Boundary \\
(Kg/y)
\end{tabular} & $\begin{array}{l}\text { Sourc } \\
\text { e Rate } \\
(\mathrm{Kg} / \mathrm{y}) \\
\end{array}$ & $\begin{array}{c}\text { Source } \\
\text { Duration } \\
\text { (y) } \\
\end{array}$ & $\begin{array}{c}\text { 1-D } \\
\text { Streamtube } \\
\text { Length } \\
(\mathrm{m})\end{array}$ & \begin{tabular}{|c|} 
Base \\
Porosity \\
$\left(\mathrm{m}^{3} / \mathrm{m}^{3}\right)$
\end{tabular} & $\begin{array}{c}\text { Bulk } \\
\text { Densty } \\
\left(\mathrm{Kg} / \mathrm{m}^{3}\right)\end{array}$ & \begin{tabular}{|c|} 
Remaining \\
Carbon \\
Tetrachloride \\
Source \\
$(\mathrm{Kg})$
\end{tabular} & \begin{tabular}{|c|} 
Volume at or \\
above Fixed \\
Source \\
$\begin{array}{c}\text { Concentratio } \\
n(m 3)\end{array}$ \\
\end{tabular} & $\begin{array}{l}\text { Porosity } \\
\left(\mathrm{m}^{3} / \mathrm{m}^{3}\right) \\
\end{array}$ & $\begin{array}{c}\mathrm{Kd} \\
\left(\mathrm{m}^{3} / \mathrm{kg}\right)\end{array}$ & Retardation & $\begin{array}{c}\text { First } \\
\text { Order } \\
\text { Rate } \\
\text { Constant } \\
(1 / y) \\
1305-2\end{array}$ & \begin{tabular}{|c|} 
Longitudinal \\
Dispersivity \\
$(\mathrm{m})$
\end{tabular} & $\begin{array}{c}\text { Required } \\
\text { Source } \\
\text { Cleanup } \\
\text { Percentage } \\
\left(\begin{array}{c}\mathrm{g} g / \mathrm{L} \\
\text { Compliance) }\end{array}\right. \\
\end{array}$ & $\begin{array}{c}\text { Required } \\
\text { Source } \\
\text { Cleanup } \\
\text { Percentage } \\
\text { (50 } \mathrm{\mu g} / \mathrm{L} \\
\text { Compliance) } \\
\end{array}$ & \begin{tabular}{|c|} 
Required \\
Source Cleanup \\
Percentage \\
$(500$ ug/L \\
Compliance) \\
\end{tabular} & $\begin{array}{c}\begin{array}{c}\text { Abiotic } \\
\text { Reaction } \\
\text { Half Time } \\
(y)\end{array} \\
\end{array}$ & $\begin{array}{c}\text { Carbon } \\
\text { Tetrachloride } \\
\text { Travel Time } \\
(y)\end{array}$ \\
\hline & & \begin{tabular}{|r|}
3000 \\
3000
\end{tabular} & \begin{tabular}{|r|}
640 \\
1910
\end{tabular} & \begin{tabular}{|r|}
18.728183 \\
0064088
\end{tabular} & \begin{tabular}{|r|}
0.918 \\
0.029
\end{tabular} & $\begin{array}{r}147 \\
1379 \\
\end{array}$ & $\begin{array}{r}51 \\
5437 \\
\end{array}$ & \begin{tabular}{|r|}
5125 \\
5125
\end{tabular} & $\begin{array}{l}0.1 \\
0.1\end{array}$ & $\begin{array}{l}1950 \\
1950\end{array}$ & \begin{tabular}{|r|}
7500 \\
\end{tabular} & \begin{tabular}{|r|}
1912500 \\
1737500
\end{tabular} & \begin{tabular}{|l|}
0.0563 \\
0.072 \\
\end{tabular} & 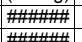 & $\begin{array}{r}2.924 \\
556\end{array}$ & $1.30 \mathrm{E}-02$ & \begin{tabular}{|r|}
48.97 \\
\end{tabular} & & & & 53.3 & \begin{tabular}{|r|}
265.2 \\
6421
\end{tabular} \\
\hline $\begin{array}{r}884 \\
885\end{array}$ & $\begin{array}{l}44.19 \\
24.59 \\
\end{array}$ & $\begin{array}{l}3000 \\
3000\end{array}$ & \begin{tabular}{r|r|r|r|}
1910 & \\
910 &
\end{tabular} & $\begin{array}{l}0.064488 \\
7.418041\end{array}$ & $\begin{array}{r}\mid .0029 \\
0.197\end{array}$ & $\begin{array}{l}173.9 \\
79.66\end{array}$ & $\begin{array}{l}54.34 \\
94.15 \\
\end{array}$ & \begin{tabular}{|l}
5125 \\
5125
\end{tabular} & 0.1 & $\begin{array}{l}1990 \\
1950\end{array}$ & $\begin{array}{l}7500 \\
7500\end{array}$ & $\begin{array}{r}1 / 3 / 500 \\
762500\end{array}$ & $\mid 0.1294:$ & "\#\#\#\#地 & $\begin{array}{l}5.536 \\
2.164 \\
\end{array}$ & $\begin{array}{l}\frac{1.65 \mathrm{E}-02}{1.12 \mathrm{E}-02} \\
\end{array}$ & \begin{tabular}{r|r|r|r|}
24.85 \\
\end{tabular} & \begin{tabular}{r|}
0 \\
32.6
\end{tabular} & $\begin{array}{ll}0 \\
0\end{array}$ & $\frac{0}{0}$ & $\begin{array}{l}42.1 \\
61.8 \\
\end{array}$ & $\begin{array}{l}642.1 \\
451.1 \\
\end{array}$ \\
\hline 886 & 51.64 & 3000 & 1550 & 7.375915 & 0.2989 & 121.6 & 61.7 & 5125 & 0.1 & 1950 & 7500 & 1437500 & 0.0616 & 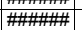 & 4.648 & $7.91 \mathrm{E}-03$ & 47.41 & 32.21 & & & 87.6 & 461.2 \\
\hline 887 & 21.48 & 3000 & 1060 & 16.457707 & 0.7531 & 137.3 & 54.64 & 5125 & 0.1 & 1950 & 7500 & 1725000 & 0.1481 & 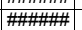 & 2.33 & $5.50 \mathrm{E}-03$ & 63.79 & 69.62 & 0 & & 126 & 555.8 \\
\hline 888 & 44 & 3000 & 680 & \begin{tabular}{|l|}
107.460492 \\
\end{tabular} & 3.9934 & 111.5 & 67.27 & 5125 & 0.1 & 1950 & 7500 & 1262500 & 0.0723 & \#\#\#\#+ & 2.584 & $5.36 \mathrm{E}-03$ & 63.25 & 95.35 & 53.47 & & 129.4 & 301 \\
\hline 889 & 21.85 & 3000 & 880 & 1.104002 & 0.046 & 125.1 & 59.97 & 5125 & 0.1 & 1950 & 7500 & 1500000 & 0.1456 & \#\#\#\#+ & 2.13 & $1.35 \mathrm{E}-02$ & 39.53 & & 0 & & 51.4 & 499.6 \\
\hline 890 & 23.48 & 3000 & 1920 & 3.686715 & 0.1415 & 115.1 & 65.14 & 5125 & 0.1 & 1950 & 7500 & 1325000 & 0.1355 & \#\#\#\#+1 & 3.45 & $5.79 \mathrm{E}-03$ & 51.59 & 0 & 0 & & 119.7 & 753.1 \\
\hline 891 & 45.51 & 3000 & 570 & 24.597393 & $\begin{array}{l}1.2832 \\
\end{array}$ & 156.5 & 47.92 & 5125 & 0.1 & 1950 & 7500 & 2100000 & 0.0699 & 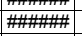 & 2.341 & $\begin{array}{l}1.30 \mathrm{E}-02 \\
\end{array}$ & 30.14 & 79.67 & 0 & & 53.5 & 263.6 \\
\hline 892 & 31.78 & 3000 & 240 & 131.55694 & 5.4842 & 125.1 & 59.97 & 5125 & 0.1 & 1950 & 7500 & 1500000 & 0.1001 & \#\#\#\#+ & 1.187 & 1.49E-02 & 40.64 & 96.2 & 61.99 & & 46.5 & 191.5 \\
\hline 893 & 54.57 & 3000 & 390 & 403.298556 & 13.3594 & 99.38 & 75.47 & 5125 & 0.1 & 1950 & 7500 & 1062500 & 0.0583 & 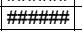 & 2.031 & $5.69 \mathrm{E}-03$ & 59.02 & 98.76 & 87.6 & & 121.7 & 190.7 \\
\hline 894 & 41.64 & 3000 & 370 & \begin{tabular}{|l|}
82.071445 \\
\end{tabular} & 4.9684 & 181.6 & 41.3 & 5125 & 0.1 & 1950 & 7500 & 2625000 & 0.0764 & \#\#\#\#\#+ & 1.745 & $1.19 \mathrm{E}-02$ & 30.05 & 93.91 & 39.08 & & 58.4 & 214.7 \\
\hline 895 & 35.15 & 3000 & 620 & 21.423458 & 0.8066 & 113 & 66.4 & 5125 & 0.1 & 1950 & 7500 & 1287500 & 0.0905 & \#\#\#\#\#+ & 2.222 & $1.14 \mathrm{E}-02$ & 51.34 & 76.66 & 0 & & 60.6 & 323.9 \\
\hline 896 & 33.56 & 3000 & 1020 & & \begin{tabular}{|l|}
0.0368 \\
\end{tabular} & 106.3 & 70.57 & 5125 & 0.1 & 1950 & 7500 & 1175000 & 0.0948 & \#\#\#\#+ & 2.991 & $1.52 \mathrm{E}-02$ & 43.35 & & 0 & & 45.7 & 456.8 \\
\hline 897 & 28.63 & 3000 & 2080 & 0.420056 & \begin{tabular}{|l|}
0.0228 \\
\end{tabular} & 162.7 & 46.11 & 5125 & 0.1 & 1950 & 7500 & 2225000 & 0.1111 & \#\#\#\#+ & 3.911 & $9.38 \mathrm{E}-03$ & 29.32 & & 0 & & 73.9 & 699.9 \\
\hline 898 & 23.43 & 3000 & 690 & 1.445684 & \begin{tabular}{|l|}
0.0709 \\
\end{tabular} & 147 & 51 & 5125 & 0.1 & 1950 & 7500 & 1912500 & \begin{tabular}{|c|}
0.1358 \\
\end{tabular} & \#\#\#\#+ & 2.009 & $1.49 \mathrm{E}-02$ & 57.93 & & & & 46.4 & 439.6 \\
\hline 899 & 33.31 & 3000 & 1500 & 1.185431 & \begin{tabular}{l|l|} 
\\
\end{tabular} & 140.6 & 53.35 & 5125 & 0.1 & 1950 & 7500 & & & & 3.601 & $1.06 \mathrm{E}-02$ & 37.42 & & & & 65.3 & 553.9 \\
\hline 900 & 58.59 & & 2570 & 1.741052 & $\begin{array}{l}0.0734 \\
\end{array}$ & 126.4 & 59.31 & 5125 & 0.1 & 1950 & 7500 & & & & 7.973 & & 70.51 & 0 & & & 90 & 697.4 \\
\hline 901 & 35.67 & & 1220 & & 1.1277 & 98.6 & & 5125 & 0.1 & 1950 & 7500 & 1050000 & & & 2.993 & $6.54 \mathrm{E}-03$ & $\begin{array}{l}18.9 \\
\end{array}$ & 85.43 & 0 & & 105.9 & 430.2 \\
\hline 902 & 22.39 & & 550 & 95.306747 & \begin{tabular}{|l|}
2.475 \\
\end{tabular} & 77.91 & & 5125 & 0.1 & 1950 & 7500 & 737500 & & \#\#\#\#+ & 1.555 & $7.81 \mathrm{E}-03$ & 40.44 & \begin{tabular}{|l|}
94.75 \\
\end{tabular} & 47.54 & & 88.8 & 356 \\
\hline 903 & 40.32 & 3000 & 2030 & 17.803394 & \begin{tabular}{ll|}
0.5032 \\
\end{tabular} & $\begin{array}{l}84.8 \\
\end{array}$ & & 5125 & 0.1 & 1950 & 7500 & 837500 & & \#\#\#\#+ & 4.732 & $4.26 \mathrm{E}-03$ & 60.55 & 71.92 & & & 162.9 & $\begin{array}{l}601.4 \\
\end{array}$ \\
\hline $\begin{array}{l}904 \\
905\end{array}$ & $\begin{array}{l}30.33 \\
5.37\end{array}$ & 3000 & \begin{tabular}{|l|l|}
630 \\
730
\end{tabular} & $\begin{array}{r}3.427425 \\
28201866\end{array}$ & \begin{tabular}{|c|c|}
0.183 \\
98481
\end{tabular} & $\begin{array}{l}160.2 \\
109 .\end{array}$ & $\begin{array}{l}46.81 \\
7159\end{array}$ & 5125 & $\begin{array}{l}0.1 \\
0.1\end{array}$ & $\begin{array}{l}1950 \\
1905\end{array}$ & 7500 & 2175000 & $\begin{array}{l}0.1049 \\
\end{array}$ & 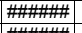 & \begin{tabular}{|l|l|}
2.157 \\
2073
\end{tabular} & $1.50 \mathrm{E}-02$ & & & 0 & & 46.1 & 364.5 \\
\hline 905 & 58.37 & 3000 & \begin{tabular}{r|}
730 \\
\end{tabular} & 282.01866 & 9.8481 & $\begin{array}{l}104.8 \\
\end{array}$ & 71.59 & 5125 & 0.1 & 1950 & 7500 & 1150000 & | 0.0545 & 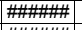 & 2.973 & $2.52 \mathrm{E}-03$ & 43.74 & 98.23 & \begin{tabular}{|l|l|}
82.27 \\
\end{tabular} & & 274.9 & 261 \\
\hline \begin{tabular}{|l|l|}
906 & \\
907
\end{tabular} & $\begin{array}{l}38.28 \\
3371 \\
3\end{array}$ & 3000 & \begin{tabular}{r|r|}
1220 \\
340
\end{tabular} & $\begin{array}{l}29.654142 \\
127.7023\end{array}$ & \begin{tabular}{|l|l|l|}
1.0874 \\
5.9823
\end{tabular} & $\begin{array}{r}110 \\
132 \\
\end{array}$ & $\begin{array}{l}68.18 \\
57.15\end{array}$ & $\begin{array}{l}5125 \\
5125\end{array}$ & 0.1 & 1950 & 7500 & 1237500 & $\begin{array}{l}0.0831 \\
\end{array}$ & 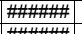 & \begin{tabular}{|l|l|l|}
3.137 \\
1.24
\end{tabular} & $6.58 \mathrm{E}-03$ & $\begin{array}{l}22.18 \\
\end{array}$ & $\begin{array}{l}83.14 \\
96.24\end{array}$ & 0 & & 105.3 & 419.9 \\
\hline $\begin{array}{l}901 \\
908\end{array}$ & $\begin{array}{l}30.71 \\
26.25 \\
\end{array}$ & $\begin{array}{l}3000 \\
33000 \\
\end{array}$ & \begin{tabular}{r|r}
340 \\
1020
\end{tabular} & $\frac{136.74}{23.12}$ & $5.9823 \mid$ & $\begin{array}{l}131.2 \\
1137\end{array}$ & \begin{tabular}{|c|}
57.15 \\
65.97
\end{tabular} & \begin{tabular}{|l|l|}
5125 \\
5125
\end{tabular} & $\begin{array}{l}0.1 \\
0.1\end{array}$ & $\begin{array}{l}1950 \\
11950\end{array}$ & $\begin{array}{l}7500 \\
77500\end{array}$ & & $\begin{array}{l}0.1036 \\
0\end{array}$ & |\#\#\#\#| & 1.424 & $\begin{array}{r}9.93 \mathrm{E}-03 \\
7\end{array}$ & & $\begin{array}{l}96.34 \\
78.38\end{array}$ & 63.44 & & 69.8 & 237.7 \\
\hline 909 & 36.23 & 3000 & 1890 & 0.06 & $\begin{array}{l}0.0023 \\
0.0023\end{array}$ & $\begin{array}{l}13.1 \\
110.7\end{array}$ & $\frac{65.97}{67.72}$ & \begin{tabular}{|l|l}
5125 \\
5125
\end{tabular} & $\begin{array}{l}0.1 \\
0.1\end{array}$ & $\begin{array}{l}950 \\
1950\end{array}$ & $\begin{array}{l}7500 \\
7500\end{array}$ & $\frac{1300000}{1250000}$ & \begin{tabular}{|c|}
0.121212 \\
0.0878
\end{tabular} & 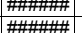 & $\begin{array}{l}2.369 \\
4.455 \\
\end{array}$ & $\begin{array}{c}.03 \mathrm{E}-03 \\
1.58 \mathrm{E}-02\end{array}$ & $\frac{25 .}{30}$ & $\begin{array}{r}78.38 \\
7\end{array}$ & \begin{tabular}{l|l}
0 \\
0
\end{tabular} & & $\begin{array}{l}98.6 \\
43.8 \\
\end{array}$ & $\begin{array}{l}462.6 \\
630.1\end{array}$ \\
\hline 910 & 37.96 & & 1150 & 81.37 & 2.2308 & 82.25 & & 5125 & 0.1 & 1950 & & & & \#m+m!m & 3 & & & 93.86 & 38.55 & & $\begin{array}{l}451.5 \\
151.5\end{array}$ & 4 411.1. \\
\hline 911 & 31.44 & & 1660 & & 0.0235 & 69.77 & & 5125 & 0.1 & & & 625000 & & 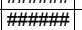 & & & & & 0 & & $\begin{array}{l} \\
64.2 \\
\end{array}$ & 720.7 \\
\hline 912 & 38.56 & & 2050 & 4.755 & 0.1487 & 93.84 & & 5125 & 0.1 & & 75 & $\begin{array}{l}975000 \\
\end{array}$ & & \#\#\#\#\#" & & & 18.44 & 0 & & & $\begin{array}{r}89 \\
89\end{array}$ & 558. \\
\hline 913 & 29.48 & 3000 & 660 & 5.211032 & 0.1859 & 107 & & 5125 & 0.1 & 1950 & 7500 & 1187500 & 0.1 & \#\#\#\#\# & 2.267 & & 77.21 & 4.05 & & & 50.1 & 394 \\
\hline 914 & 24.12 & 3000 & 950 & 42.351085 & 1.6151 & 114.4 & & 5125 & 0.1 & 1950 & 756 & 1312500 & & \#\#\#\#\#+ & 2.189 & & 32.75 & 88.19 & & & 124.1 & 465. \\
\hline 915 & 36.52 & 3000 & 380 & \begin{tabular}{|l|}
134.154471 \\
\end{tabular} & 6.4895 & 145.1 & & 5125 & 0.1 & 1950 & 756 & 1875000 & & \#\#\#\#+ & 1.653 & & 42.6 & 96.27 & 62.73 & & 76 & 231. \\
\hline 916 & 27.69 & & 800 & 46.19591 & 1.9043 & 123.7 & 60. & 5125 & 0.1 & 1950 & & & & \#\#\#\#+\# & 2.14 & & 30.5 & 89.18 & & & 103.9 & 396 \\
\hline 917 & 22.55 & 3000 & 1190 & 73.758561 & 2.5569 & 104 & 72. & 5125 & 0.1 & 1950 & 7500 & 1137500 & 0.1 & \#\#\#\#+ & 2.374 & & 38.3 & 93.22 & 32.21 & & 213.7 & 539. \\
\hline 918 & 48.5 & 3000 & 1510 & 2.55 & 0.0692 & 81.39 & 92. & 5125 & 0.1 & 1950 & 7500 & & 0.0 & 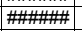 & 4.929 & & 63. & 0 & & & 60.8 & 520 \\
\hline 919 & 40.79 & & 290 & \begin{tabular}{|l|}
579.177 \\
\end{tabular} & 23.0589 & 119.4 & 62.7 & 5125 & 0.1 & 1950 & 750 & & & & 1.467 & & & 99.14 & 91.37 & 13.67 & 109.3 & 184 \\
\hline 920 & & & 730 & & 0.1113 & 82.25 & & 5125 & 0.1 & 1950 & 750 & & & & 2.134 & & & 0 & & & 52.3 & 451. \\
\hline 921 & 28.74 & 3000 & 580 & & 2.2397 & 100.9 & & 5125 & 0.1 & 1950 & & & & & 1.852 & & & 92.49 & 24.89 & & 82.6 & 330. \\
\hline 922 & 30.36 & & 860 & & 0.30 & 110.7 & & 5125 & 0.1 & 19 & & & & & & & & 39.89 & 0 & & 69.9 & 447 \\
\hline 923 & 22.29 & & 1400 & & 0.05 & 128.5 & & 5125 & 0.1 & 19 & & & & & & & & & & & 72 & 632. \\
\hline 924 & 33 & & 270 & 456.99 & 16.0738 & 105.5 & & 5125 & 0.1 & & & & & & & & 30. & 98.91 & 89.06 & & 82.4 & \\
\hline 925 & 23.64 & & 440 & & & 185.6 & & 5125 & 0.1 & & & & & & & & & 94.73 & 47.31 & & 108.4 & \\
\hline 926 & 43.46 & & 960 & & & 155.3 & & & 0.1 & & & & & & & & & 81.83 & & & 102 & \\
\hline 927 & & 3000 & 410 & & & 141 & & & 0.1 & & & & & & & & & 84.39 & & & 50.5 & 254.3 \\
\hline 928 & & & 2610 & & & \begin{tabular}{ll|}
158.4 \\
\end{tabular} & & & & & & & & & & & & 0 & & & 95.9 & 1.5 \\
\hline 929 & 21.28 & 300 & 770 & & 0.1715 & $\begin{array}{l}124.4 \\
\end{array}$ & & & 0.1 & & & & & & & & & 0 & & & 57 & 447.1 \\
\hline 930 & & & 480 & & $\begin{array}{l}4.6588 \\
\end{array}$ & 83.1 & & 51 & 0.1 & & & & & & & & & 97.03 & 70.27 & & 55 & 192.6 \\
\hline 931 & 32.23 & & 380 & & $\begin{array}{l}9.687 \\
\end{array}$ & 133.3 & 56. & & 0.1 & & & & & & & & & 97.71 & \begin{tabular}{ll|}
77.07 \\
\end{tabular} & & 96 & 44. \\
\hline 932 & 31.41 & & 660 & & $\begin{array}{l}0.542 \\
\end{array}$ & 152.8 & & & 0.1 & & & & & & 2. & & & 53.03 & 0 & & 6 & 0. \\
\hline 933 & 28.95 & $\begin{array}{ll}3000 \\
3000 \\
300\end{array}$ & $\begin{array}{l}830 \\
380 \\
38\end{array}$ & 0.961 & 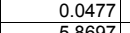 & $\begin{array}{r}149 \\
1104\end{array}$ & & & & & & & & & 2.3 & & & 0 & 0 & & 42 & 14.8 \\
\hline 934 & 24.34 & 3000 & 380 & 147.43023 & \begin{tabular}{|l|l|}
5.8697 & \\
13432
\end{tabular} & $\begin{array}{l}119.4 \\
\end{array}$ & & 5125 & & 195 & 750 & & 0.1 & & \begin{tabular}{l|l}
96 \\
27
\end{tabular} & & 14. & 96.61 & 66.09 & & $\frac{9}{3}$ & 2.5 \\
\hline 935 & 21.61 & 3000 & \begin{tabular}{r|r|r}
420 & 1500 \\
\end{tabular} & 41.19626 & 1.3432 & $\begin{array}{r}97.81 \\
126 f\end{array}$ & 76.68 & 5125 & 0.1 & 1950 & 750 & & 0.1. & 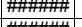 & 37 & & 16. & 87.86 & 0 & & 52.3 & 305.2. \\
\hline $936 \mid$ & $\begin{array}{l}22.59 \\
37.25\end{array}$ & $\frac{300}{300}$ & $\begin{array}{l}1500 \\
1270\end{array}$ & 3.71365 & 0.1691 & $\begin{array}{l}136.6 \\
172 ?\end{array}$ & $\begin{array}{r}54.9 \\
\end{array}$ & 5125 & 0.1 & 1950 & 750 & $\begin{array}{l}1712500 \\
\end{array}$ & 0.1 & |\#\#\#+\#| & 2.8. & & 41.5 & 0 & 0 & & 100.4 & $\frac{648.9}{517 .}$ \\
\hline $\begin{array}{c}931 \\
938 \\
\end{array}$ & $\frac{37.25}{2519}$ & 3000 & $\begin{array}{l}1270 \\
620\end{array}$ & $\frac{0.38}{14970}$ & $\begin{array}{l}0.0223 \\
56365 \\
\end{array}$ & $\begin{array}{r}172.3 \\
113\end{array}$ & $\begin{array}{l}43.54 \\
66.4\end{array}$ & $\begin{array}{l}\frac{5125}{5125} \\
5125\end{array}$ & & & 756 & & 0.0 & 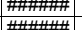 & 3.9 & & $\frac{62.6}{26 .}$ & 0 & 6 & & \begin{tabular}{r|r|r|}
128.4 \\
128.8
\end{tabular} & 547. \\
\hline | & & & 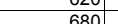 & & & $\frac{113}{113}$ & 60 & $\frac{512}{514}$ & & & & & & & & & & & & & & \\
\hline 940 & 21.3 & & 460 & & & 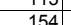 & & $\frac{112}{512}$ & & & & & & & & & & 2 & & & & \\
\hline 941 & 44.1 & & 520 & & 326 & 986 & & 512 & 0. & & & & & & 22 & & & & 966 & & & \\
\hline 942 & 610 & & 1720 & & 047 & So.0 & & 512 & & & & & & & & & & 7079 & & & $\frac{10}{118}$ & \\
\hline 943 & 281 & 300 & 1110 & & & 119 & & 5125 & 0.1 & 195 & & & & & & & & & & & 445 & \\
\hline 944 & 42.93 & 3000 & 290 & \begin{tabular}{|r|}
364.025124 \\
\end{tabular} & 13.2586 & 109.3 & 68.64 & 5125 & 0.1 & 195 & 7500 & & 0.07 & & 1.507 & $9.00 \mathrm{E}$ & 47.82 & 98.63 & 86.26 & & 77.1 & 179 \\
\hline \begin{tabular}{|c|}
945 \\
\end{tabular} & 34.13 & 3000 & 1090 & $\begin{array}{l}0.487091 \\
\end{array}$ & 0.0144 & 88.97 & 84.3 & 5125 & 0.1 & 1950 & 7500 & 900000 & 0.0932 & \#\#\#\#+1 & 3.357 & $1.68 \mathrm{E}-02$ & 56.3 & & & & 41.3 & 504.1 \\
\hline
\end{tabular}




\begin{tabular}{|c|c|c|c|c|c|c|c|c|c|c|c|c|c|c|c|c|c|c|c|c|c|c|}
\hline Realization & $\begin{array}{c}\text { Velocity } \\
(\mathrm{m} / \mathrm{y})\end{array}$ & $\begin{array}{c}\text { Fixed Source } \\
\begin{array}{c}\text { Concentration } \\
(\mathrm{mg} / \mathrm{L})\end{array} \\
\end{array}$ & $\begin{array}{c}\text { Time of } \\
\text { Maximum } \\
\text { at } \\
\text { Compliance } \\
\text { Boundary } \\
\text { (y) }\end{array}$ & \begin{tabular}{|c|} 
Maximum \\
Concentration \\
Compliance \\
Boundary \\
$(\mu \mathrm{g} / \mathrm{L})$ \\
\end{tabular} & $\begin{array}{c}\text { Maximum } \\
\text { Release Rate } \\
\text { at } \\
\text { Compliance } \\
\text { Boundary } \\
\text { (Kg/y) }\end{array}$ & $\begin{array}{l}\text { Sourc } \\
\text { e Rate } \\
\text { (Kg/y) } \\
\end{array}$ & $\begin{array}{c}\text { Source } \\
\text { Duration } \\
\text { (y) } \\
\end{array}$ & $\begin{array}{c}\text { 1-D } \\
\text { Streamtube } \\
\text { Length } \\
(\mathrm{m})\end{array}$ & $\begin{array}{c}\text { Base } \\
\text { Porosity } \\
\left(\mathrm{m}^{3} / \mathrm{m}^{3}\right)\end{array}$ & $\begin{array}{l}\text { Bulk } \\
\text { Densty } \\
\left(\mathrm{Kg} / \mathrm{m}^{3}\right)\end{array}$ & \begin{tabular}{|c|} 
Remaining \\
Carbon \\
Tetrachloride \\
Source \\
$(\mathrm{Kg})$
\end{tabular} & $\begin{array}{c}\text { Volume at or } \\
\text { above Fixed } \\
\text { Source } \\
\text { Concentratio } \\
n(m 3)\end{array}$ & $\begin{array}{l}\text { Porosity } \\
\left(\mathrm{m}^{3} / \mathrm{m}^{3}\right) \\
\end{array}$ & $\mid \begin{array}{c}\mathrm{Kd} \\
\left(\mathrm{m}^{3} / \mathrm{kg}\right)\end{array}$ & Retardation & $\begin{array}{c}\text { First } \\
\text { Order } \\
\text { Rate } \\
\text { Constant } \\
(1 / y) \\
\end{array}$ & $\begin{array}{c}\text { Longitudinal } \\
\text { Dispersivity } \\
(\mathrm{m})\end{array}$ & $\begin{array}{c}\text { Required } \\
\text { Source } \\
\text { Cleanup } \\
\text { Percentage } \\
(5 \mu \mathrm{g} / \mathrm{L} \\
\text { Compliance) } \\
\end{array}$ & $\begin{array}{c}\text { Required } \\
\text { Source } \\
\text { Cleanup } \\
\text { Percentage } \\
\text { (50 } \mathrm{\mu g} / \mathrm{L} \\
\text { Compliance) } \\
\end{array}$ & \begin{tabular}{|c|} 
Required \\
Source Cleanup \\
Percentage \\
$(500 \mu \mathrm{gg} / \mathrm{L}$ \\
Compliance) \\
\end{tabular} & $\begin{array}{c}\text { Abiotic } \\
\text { Reaction } \\
\text { Half Time } \\
(y)\end{array}$ & $\begin{array}{c}\text { Carbon } \\
\text { Tetrachloride } \\
\text { Travel Time } \\
\text { (y) } \\
\end{array}$ \\
\hline 946 & & 3000 & 690 & \begin{tabular}{|r|}
14.083155 \\
\end{tabular} & $\begin{array}{r}0.6537 \\
\end{array}$ & 139.3 & & 5125 & & 1950 & $\begin{array}{r}7500 \\
\end{array}$ & \begin{tabular}{|l|}
1762500 \\
\end{tabular} & & \#\#\#\#+ & \begin{tabular}{|r|}
1.748 \\
\end{tabular} & $1.04 \mathrm{E}-02$ & \begin{tabular}{|l|}
17.09 \\
\end{tabular} & & & & & \\
\hline 947 & 27.57 & 3000 & 880 & 8.665354 & 0.3632 & 125.8 & \begin{tabular}{|l|}
59.64 \\
\end{tabular} & 5125 & 0.1 & 1950 & 7500 & 1512500 & 0.1154 & \#\#\#\#+ & 2.595 & $8.63 \mathrm{E}-03$ & \begin{tabular}{l|l|}
89.95 \\
\end{tabular} & 42.3 & 0 & & 80.3 & 482.5 \\
\hline 948 & 43.46 & 3000 & 220 & 801.36864 & \begin{tabular}{|l|l}
32.4723 \\
\end{tabular} & 121.6 & 61.7 & 5125 & 0.1 & 1950 & 7500 & 1437500 & 0.0732 & \#\#\#\#+ & 1.287 & $7.37 \mathrm{E}-03$ & 31.32 & \begin{tabular}{|c|}
99.38 \\
\end{tabular} & 93.76 & 37.61 & 94.1 & \\
\hline 949 & 26.56 & 3000 & 1620 & 0.531165 & $\begin{array}{l}0.0149 \\
\end{array}$ & 83.95 & 89.34 & 5125 & 0.1 & 1950 & 7500 & 825000 & 0.1198 & \#\#\#\#+ & 3.586 & $1.12 \mathrm{E}-02$ & 51.89 & & 0 & & 61.7 & 692.1 \\
\hline 950 & 53.29 & 3000 & 1310 & 32.990012 & \begin{tabular}{|l|l|}
1.4207 \\
\end{tabular} & 129.2 & 58.05 & 5125 & 0.1 & 1950 & 7500 & 1575000 & 0.0597 & \#\#\#\#+ & 4.031 & $5.24 \mathrm{E}-03$ & 39.47 & 84.84 & 0 & & 132.3 & 387.7 \\
\hline 951 & 30.56 & 3000 & 720 & $\begin{array}{l}2.166966 \\
\end{array}$ & $\begin{array}{ll}0.0729 \\
\end{array}$ & 100.9 & 74.31 & 5125 & 0.1 & 1950 & 7500 & 1087500 & 0.1041 & \#\#\#\# & 2.352 & $1.65 \mathrm{E}-02$ & 55.64 & 0 & 0 & & 41.9 & 394.4 \\
\hline 952 & 29.29 & 3000 & 1410 & 1.874045 & 0.0442 & 70.69 & 106.09 & 5125 & 0.1 & 1950 & 7500 & 637500 & 0.1086 & \#\#曲! & 3.552 & $1.06 \mathrm{E}-02$ & 66.6 & 0 & 0 & & 65.7 & 621.5 \\
\hline 953 & 33.38 & 3000 & 1040 & 3.973698 & 0.2155 & 162.7 & 46.11 & 5125 & 0.1 & 1950 & 7500 & 2225000 & 0.0953 & \#\#\#\#! & 2.964 & $1.02 \mathrm{E}-02$ & 50.37 & 0 & 0 & & 68.2 & 455.1 \\
\hline 954 & 38.42 & 3000 & 750 & $\begin{array}{l}178.419992 \\
1502902\end{array}$ & 6.2304 & $\begin{array}{l}104.8 \\
125.2\end{array}$ & 71.59 & 5125 & 0.1 & 1950 & 7500 & 1150000 & 0.0828 & 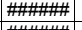 & $\begin{array}{l}2.445 \\
.166 \\
\end{array}$ & $3.70 \mathrm{E}-03$ & 44.81 & $\begin{array}{r}97.2 \\
06.96\end{array}$ & 71.98 & & 187.2 & \\
\hline 955 & 32.01 & 3000 & 420 & 159.38632 & 7.1871 & 135.3 & 55.44 & 5125 & 0.1 & 1950 & 7500 & 1687500 & 0.0994 & \#\#\#\#1 & 1.66 & 6.64E-03 & 54.49 & 96.86 & 68.63 & & 104.4 & \\
\hline 956 & 35.51 & 3000 & 1300 & 5.867964 & 0.3335 & 170.5 & 43.99 & 5125 & 0.1 & 1950 & 7500 & 2387500 & 0.0896 & 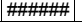 & 3.55 & $7.28 \mathrm{E}-03$ & 63.09 - & 14.79 & 0 & & $\begin{array}{ll}95.2 \\
\end{array}$ & 512.5 \\
\hline 957 . & 24.25 & 3000 & 490 & 15.289115 & 0.406 & 79.66 & 94.15 & 5125 & 0.1 & 1950 & 7500 & 762500 & 0.1312 & |\#\#\#\#\#| & 1.621 & $1.41 \mathrm{E}-02$ & 67.42 & 67.3 & 0 & & 49.2 & 342.6 \\
\hline 958 & 53.74 & 3000 & 540 & 70.63915 & 3.2635 & 138.6 & 54.11 & 5125 & 0.1 & 1950 & 7500 & 1750000 & 0.0592 & \#\#\#\#\#| & 2.624 & 8.03E-03 & 82.52 & 92.92 & 29.22 & & 86.3 & 250.3 \\
\hline 959 & 26.4 & 3000 & 500 & 137.397616 & 7.168 & 156.5 & 47.92 & 5125 & 0.1 & 1950 & 7500 & 2100000 & 0.1205 & \#\#\#\#\#" & 1.6 & $6.79 \mathrm{E}-03$ & 17.74 & 96.36 [ & 63.61 . & & 102.1 & 310.5 \\
\hline 960 & 40.27 & 3000 & 930 & \begin{tabular}{|l|}
109.667282 \\
\end{tabular} & 6.1011 & 166.9 & 44.94 & 5125 & 0.1 & 1950 & 7500 & 2312500 & | 0.079 & \#\#\#\#\# & 2.755 & $3.81 \mathrm{E}-03$ & 19.29 & 95.44 & 54.41 & & 181.9 & 350.6 \\
\hline 961 & 22.59 & 3000 & 1220 & 25.547588 & 1.1803 & 138.6 & 54.11 & 5125 & 0.1 & 1950 & 7500 & 1750000 & 0.1408 & \#\#\#\#\#| & 2.415 & 4.88E-03 & 28.44 & 80.43 & 0 & & 141.9 & 547.8 \\
\hline 962 & 28.35 & 3000 & 1540 & & $0.1968 \mid$ & 115.9 & 64.73 & 5125 & 0.1 & 1950 & 7500 & 1337500 & 0.1122 & \#冊自 & 3.659 & 6.43 & 82.77 . & 1.87 & 0 & & 107.8 & 661.3 \\
\hline 963 & 22.08 & 3000 & 380 & 156.174514 & 9.3341 & 179.3 & 41.83 & 5125 & 0.1 & 1950 & 7500 & 2575000 & 0.1441 & "\#\#\#\#\# & 1.285 & & 32.3 & 96.8 & 67.98 & & 106.9 & 298.4 \\
\hline 964 & 45 & 3000 & 390 & 250.751104 & 8.241 & 98.6 & 76.07 & 5125 & 0.1 & 1950 & 7500 & 1050000 & 0.0707 & |\#\#\#\#\#| & 1.809 & $9.39 \mathrm{E}-03$ & 29.22 & 98.01 & 80.06 & & 73.8 & 206 \\
\hline 965 & 22.09 & 3000 & 590 & & 2.1973 & \begin{tabular}{|l|l|}
141.9 \\
\end{tabular} & & 5125 & 0.1 & 1950 & 7500 & 1812500 & 0.144 & \#\#\#\#\# & 1.645 & $7.40 \mathrm{E}-03$ & 38.58 & 89.24 & 0 & & 93.7 & 381.6 \\
\hline 966 & 60.83 & 3000 & \begin{tabular}{r|r|}
230 \\
\end{tabular} & & \begin{tabular}{|c|}
9.731 \\
17305
\end{tabular} & $\begin{array}{ll}121.6 \\
1220\end{array}$ & $\begin{array}{r}61.7 \\
59.59\end{array}$ & 5125 & 0.1 & 1950 & 7500 & 1437500 & 0.0523 & 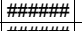 & 1.604 & 1.62E-02 & 42.56 & $\begin{array}{l}97.92 \\
9.72\end{array}$ & 79.18 & & $\begin{array}{r}42.9 \\
\end{array}$ & 135.1 \\
\hline $\begin{array}{l}967 \\
968\end{array}$ & $\begin{array}{l}36.69 \\
3122\end{array}$ & $\begin{array}{l}3000 \\
3000\end{array}$ & $\begin{array}{l}1140 \\
1410\end{array}$ & & $\begin{array}{r}1.7265 \\
0.029 \\
\end{array}$ & $\begin{array}{ll}132.6 \\
91.42\end{array}$ & $\begin{array}{l}56.56 \\
8204\end{array}$ & $\begin{array}{l}5125 \\
5125\end{array}$ & 0.1 & $\begin{array}{l}1950 \\
1950\end{array}$ & $\begin{array}{ll}7500 \\
7500\end{array}$ & \begin{tabular}{|l|l|l|l|l|l}
1637500 \\
927500
\end{tabular} & \begin{tabular}{|c|}
0.0867 \\
\end{tabular} & 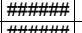 & $\begin{array}{l}3.006 \\
3641 \\
640\end{array}$ & $\begin{array}{l}5.25 \mathrm{E}-03 \\
1.8 \mathrm{E}-02\end{array}$ & $\begin{array}{l}30.78 \\
56.33 \\
\end{array}$ & 87.2 & 0 & & 132.1 & 419.9 \\
\hline 968 & 31.22 & 3000 & \begin{tabular}{|c|c}
1410 \\
550
\end{tabular} & \begin{tabular}{|r|}
0.951445 \\
\end{tabular} & $\begin{array}{r}0.029 \\
44261\end{array}$ & $\begin{array}{l}91.42 \\
121\end{array}$ & 82.04 & 5125 & 0.1 & $\begin{array}{l}1950 \\
1950 \\
1050\end{array}$ & 7500 & $\begin{array}{r}937500 \\
\end{array}$ & 0.1019 & 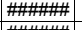 & 3.641 & $1.18 \mathrm{E}-02$ & 56.33 & 0 & 0 & & 58.6 & 597.8 \\
\hline $\begin{array}{l}969 \\
970\end{array}$ & $\begin{array}{l}53.47 \\
44.13\end{array}$ & $\begin{array}{l}3000 \\
3000\end{array}$ & $\begin{array}{r}550 \\
1900\end{array}$ & \begin{tabular}{|r|}
110.515613 \\
0.941793
\end{tabular} & \begin{tabular}{rl|}
4.4261 & \\
0.0366 &
\end{tabular} & $\begin{array}{l}120.1 \\
16.6\end{array}$ & & \begin{tabular}{|l|l|}
5125 & 5125
\end{tabular} & $\begin{array}{l}0.1 \\
0.1\end{array}$ & $\begin{array}{l}1950 \\
1950\end{array}$ & $\begin{array}{ll}7500 \\
7500\end{array}$ & $\begin{array}{l}1412500 \\
1350000\end{array}$ & & 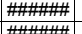 & $\begin{array}{l}2.382 \\
4847\end{array}$ & & $\begin{array}{l}14.64 \\
4352\end{array}$ & 95.48 & 54.76 & & 65.4 & 228.3 \\
\hline $\begin{array}{l}970 \\
971\end{array}$ & $\begin{array}{l}41.37 \\
51.56\end{array}$ & $\begin{array}{l}3000 \\
3000\end{array}$ & \begin{tabular}{r|r|r|r|r|r|}
450 &
\end{tabular} & \begin{tabular}{|r|}
0.941793 \\
425.373049 \\
\end{tabular} & \begin{tabular}{|l|l|l|l}
0.0366 \\
13.306
\end{tabular} & $\begin{array}{l}116.6 \\
9384\end{array}$ & 64.33 & \begin{tabular}{|l|l|}
5125 \\
5125
\end{tabular} & $\begin{array}{l}0.1 \\
0.1\end{array}$ & 1950 & $\begin{array}{l}7500 \\
7500\end{array}$ & \begin{tabular}{r|r|r|r|r|}
1350000 \\
975000
\end{tabular} & 0.0769 & 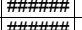 & $\begin{array}{r}4.847 \\
21\end{array}$ & 1.05E-02 & $\begin{array}{l}43.52 \\
36.81\end{array}$ & $\begin{array}{r}0 \\
9882\end{array}$ & \begin{tabular}{r|r}
0 \\
8825
\end{tabular} & & $\frac{66.2}{10.1}$ & $\frac{600.5}{208.9}$ \\
\hline 972 & 32.36 & 3000 & $\begin{array}{l}450 \\
690 \\
\end{array}$ & & $\begin{array}{l}13.306 \\
1.7694\end{array}$ & $\begin{array}{l}93.64 \\
1353\end{array}$ & & \begin{tabular}{|l|l|}
5125 \\
5125
\end{tabular} & $\frac{0.1}{0.1}$ & $\frac{1950}{1950}$ & $\begin{array}{l}7500 \\
7500\end{array}$ & & & 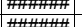 & & & & $\begin{array}{ll}98.82 \\
87.26\end{array}$ & 88.25 & & 128.1 & 208.8 \\
\hline $\begin{array}{l}973 \\
\end{array}$ & 35.87 & & 1000 & & & 1031 & & 5125 & & 1950 & & & & \#\#+ & & & & & & & & $\begin{array}{ll}339.8 \\
416.7\end{array}$ \\
\hline 974 & 49.17 & & 1490 & & & 1002 & & 55125 & & & & & & 世 & & & & & & & & 416.7 \\
\hline 975 & .22 & & 550 & & & 157.7 & & 55125 & & & & & & & & & & & 45 & & 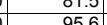 & \begin{tabular}{r|}
422 \\
2355 \\
\end{tabular} \\
\hline 976 & 33.92 & 3000 & 320 & \begin{tabular}{|l|}
292.898045 \\
\end{tabular} & 10.302 & 105.5 & & 5125 & 0. & & & & & & & & & & 82.93 & & $\begin{array}{c}90.0 \\
72 \\
\end{array}$ & $\frac{235.5}{210.5}$ \\
\hline 977 & 22.14 & 3000 & 790 & 35.261843 & \begin{tabular}{ll|l}
1.6753 \\
\end{tabular} & 142.5 & & 5125 & 0.1 & 1950 & & & & 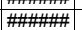 & 1.973 & & & 85.82 & 0 & & 123.9 & 210.5 \\
\hline 978 & & 300 & 1630 & & 0.08 & 124.4 & & \begin{tabular}{l|l}
5125 \\
\end{tabular} & & & & & & \#\#\#\#+ & & & & & 0 & & 87.1 & 669.3 \\
\hline 979 & 37.47 & & 490 & 318.027015 & & 98.6 & & & 0. & & & & & & 1.891 & & & 98.43 & 84.28 & & 142.4 & 258.7 \\
\hline 980 & 51.23 & 300 & 290 & 6293 & 13.5139 & 132.6 & & 5125 & 0.1 & & & & & & 1.721 & & 61. & \begin{tabular}{|c|c|}
98.36 \\
\end{tabular} & 83.65 & & 84.3 & 172.2 \\
\hline 981 & 22.11 & 300 & 2190 & & & 125.8 & & 5125 & 0.1 & & & & & & 3.359 & & & 11.69 & & & 148.3 & 778.7 \\
\hline 982 & 33.59 & & 1260 & & & 81.39 & & 5125 & 0.1 & & & & & & 43 & & 43. & & & & 54.8 & 51 \\
\hline 983 & & & 380 & & 5.8 & 13 & & & 0.1 & & & & & & 43 & & & 96.12 & \begin{tabular}{|c|}
61.18 \\
\end{tabular} & & 88.3 & 284.2 \\
\hline 984 & & & 400 & & & 13 & & & 0.1 & & & & & & 53 & & & & 70.53 & & 73.1 & 212.8 \\
\hline 985 & & & 530 & & & 128 & & & & & & & & & 44 & & & & 73.83 & & 113.7 & 225.2 \\
\hline 986 & & & 490 & & & 96.2 & & & & & & & & & & & & 89.36 & 0 & & 60 & \\
\hline 987 & 25.89 & & 840 & & & 135 & & & & & & & & & 2.2 & & & 90.3 & 2.99 & & 146.1 & 436.4 \\
\hline 988 & 24.82 & & 1020 & & & 89. & & & 0 & & & & & & & & & 0 & 0 & & 57.2 & 50 \\
\hline 989 & & & $\begin{array}{l}470 \\
\end{array}$ & & & 141 & & & 0.1 & & & & & & & & & 90.86 & 8.58 & & 4 & \\
\hline 990 & & 3000 & 2330 & & & 106 & & & 0.1 & & & & & & & & & & 0 & & \begin{tabular}{r|r}
80.4 \\
\end{tabular} & 779.8 \\
\hline 991 & 26.4 & & 440 & & $\begin{array}{ll}7.7384 \\
\end{array}$ & $\begin{array}{l}119.4 \\
\end{array}$ & & & 0.1 & & & & & & & & & 97.43 & 74.28 & 0 & 102.3 & 288.6 \\
\hline 992 & 30.62 & 0 & 430 & 589.15 & 27.8629 & 141.9 & & 51. & 0.1 & & & & & & & & 22. & 99.15 & 91.51 & 15.13 & 237.3 & 264 \\
\hline 993 & 47.98 & & 280 & 659.34 & 26.4066 & 120.1 & 62.42 & 5125 & 0.1 & & & & 0.0 & & 43 & & 24. & 99.24 & 92.42 & & 96.6 & 4.8 3.8 \\
\hline 994 & 57.95 & 3000 & 420 & 51.43 & 2.4323 & 141.9 & 52.86 & 5125 & 0.1 & 19 & & & 0.0 & & 17 | & & 30 & 90.28 & 2.78 & & 44.1 & 19 \\
\hline 995 & 38.84 & & 720 & & 0.1085 & 135.9 & & & 0.1 & & & & & & & & & & & & & \\
\hline 996 & 28.48 & & 520 & & \begin{tabular}{|l|l|}
2.658 \\
\end{tabular} & 135.9 & & 512 & & & & & & & & & & & 14.76] & & & \\
\hline 997 & 40.07 & 3000 & 640 & 66.03 & 2.1704 & 98.6 & 76.0 & 5125 & 0 & 195 & 7500 & & & \#\#\#五 & & & 28. & & & & .1 & \\
\hline 998 & 23.79 & 3000 & 520 & 108.397088 & \begin{tabular}{|l|l}
4.0548 \\
\end{tabular} & 112.2 & 66.8 & 5125 & 0.1 & 195 & 7500 & & 0.13 & \#\#\#! & 1.548 & & 30 & 95.39 & 87 & & 6 & \\
\hline $\begin{array}{l}999 \\
\end{array}$ & 44.81 & 3000 & 1260 & & 0.0602 & 161.4 & $46.4 .52>$ & 5125 & 01 & 195 & 7500 & & & & & & $39.8>>2$ & & 0 & & 51.3 & \\
\hline 1000 & 32.9 & 3000 & 320 & \begin{tabular}{|r|}
364.664162 \\
\end{tabular} & 19.3253 & 159 & \begin{tabular}{|l|l|}
47.17 \\
\end{tabular} & \begin{tabular}{|c|}
5125 \\
\end{tabular} & 0.1[ & 1950 & 7500 & 2150000 & \begin{tabular}{|l|}
0.0967 \\
\end{tabular} & \#\#\#\#曲 & 1.439 & $4.08 \mathrm{E}-03$ & 80.2 & 98.63 & 86.29 & & 169.9 & 224. \\
\hline
\end{tabular}




\begin{tabular}{|c|c|c|c|c|c|c|c|c|c|c|c|c|c|c|c|c|c|c|c|c|c|c|}
\hline Realization & $\begin{array}{c}\text { Velocity } \\
(\mathrm{m} / \mathrm{y})\end{array}$ & \begin{tabular}{|c|} 
Fixed Source \\
Concentratio \\
$\mathrm{n}(\mathrm{mg} / \mathrm{L})$
\end{tabular} & $\begin{array}{c}\text { Time of } \\
\text { Maximum at } \\
\text { Compliance } \\
\text { Boundary } \\
(y)\end{array}$ & \begin{tabular}{|c|} 
Maximum \\
Concentration \\
Compliance \\
Boundary \\
$(\mu \mathrm{g} / \mathrm{L})$
\end{tabular} & $\begin{array}{c}\text { Maximum } \\
\text { Release Rate } \\
\text { at Compliance } \\
\text { Boundary } \\
(\mathrm{Kg} / \mathrm{y})\end{array}$ & \begin{tabular}{|c|} 
\\
Source \\
Rate \\
$(\mathrm{Kg} / \mathrm{y})$ \\
\end{tabular} & $\begin{array}{c}\text { Source } \\
\text { Duration } \\
\text { (y) }\end{array}$ & $\begin{array}{c}\text { 1-D } \\
\text { Streamtube } \\
\text { Length } \\
\text { (m) }\end{array}$ & $\begin{array}{c}\text { Base } \\
\text { Porosity } \\
\left(\mathrm{m}^{3} / \mathrm{m}^{3}\right)\end{array}$ & $\begin{array}{c}\text { Bulk } \\
\text { Densty } \\
\left(\mathrm{Kg} / \mathrm{m}^{3}\right)\end{array}$ & $\begin{array}{c}\text { Remaining } \\
\text { Carbon } \\
\text { Tetrachloride } \\
\text { Source } \\
(\mathrm{Kg})\end{array}$ & $\begin{array}{c}\begin{array}{c}\text { Volume at or } \\
\text { above Fixed } \\
\text { Source } \\
\text { Concentration } \\
(\mathrm{m} 3)\end{array} \\
\end{array}$ & $\begin{array}{l}\text { Porosity } \\
\left(\mathrm{m}^{3} / \mathrm{m}^{3}\right)\end{array}$ & $\begin{array}{c}\mathrm{Kd} \\
\left(\mathrm{m}^{3} / \mathrm{Kg}\right)\end{array}$ & $\begin{array}{l}\text { Retardatio } \\
n\end{array}$ & \begin{tabular}{|c|} 
First \\
Order \\
Rate \\
Constant \\
$(1 / y)$ \\
\end{tabular} & $\begin{array}{l}\text { Longitudinal } \\
\text { Dispersivity } \\
\text { (m) }\end{array}$ & $\begin{array}{l}\text { Required } \\
\text { Source } \\
\text { Cleanup } \\
\text { Percentage } \\
\text { (5 ug/L } \\
\text { Compliance) }\end{array}$ & \begin{tabular}{|c} 
Required \\
Source \\
Cleanup \\
Percentage \\
$(50 \mu \mathrm{g} / \mathrm{L}$ \\
Compliance \\
)
\end{tabular} & \begin{tabular}{|c|} 
Required \\
Source \\
Cleanup \\
Percentage \\
$(500 \mu \mathrm{L} / \mathrm{L}$ \\
Compliance \\
) \\
\end{tabular} & \begin{tabular}{|c|} 
\\
Abiotic \\
Reaction \\
Half Time \\
$(y)$ \\
\end{tabular} & \begin{tabular}{|c|} 
Carbon \\
Tetrachloride \\
Travel Time \\
(y)
\end{tabular} \\
\hline & 58.91 & $\begin{array}{r}3000 \\
\end{array}$ & & \begin{tabular}{|l|}
12.561377 \\
\end{tabular} & $\begin{array}{r}0.5149 \\
\end{array}$ & \begin{tabular}{|l|}
122.968 \\
\end{tabular} & 609.91 & & & 1950 & 75000 & 1462500 & 0.054 & \#\#\#\# & \begin{tabular}{|l|} 
\\
\end{tabular} & $1.24 \mathrm{E}-02$ & 26.99 & 60.2 & 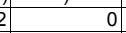 & & 55.8 & 453.1 \\
\hline 2 & 22.15 & 3000 & 1880 & 4.826098 & 0.0999 & \begin{tabular}{|l|}
62.1111 \\
\end{tabular} & 1207.51 & 5125 & 0.1 & 1950 & 75000 & 525000 & 0.1436 & \#\#\#\#\#曲 & 2.853 & $1.23 \mathrm{E}-02$ & 76.26 & 0 & 0 & 0 & 56.2 & 660 \\
\hline 3 & 30.44 & 3000 & 880 & 116.840515 & \begin{tabular}{|l|}
5.3722 \\
\end{tabular} & \begin{tabular}{|l|}
137.937 \\
\end{tabular} & 543.73 & 5125 & 0.1 & 1950 & 75000 & 1737500 & 0.1045 & \#\#\#\#\# & 1.975 & $1.04 \mathrm{E}-02$ & 55.4 & 95.72 & 57.21 & & 66.7 & 332.4 \\
\hline 4 & 22.99 & 3000 & 860 & 459.649464 & 26.0302 & \begin{tabular}{|l|}
169.892 \\
\end{tabular} & 441.46 & 5125 & 0.1 & 1950 & 75000 & 2375000 & 0.1384 & \#\#\#\#\#曲 & 1.704 & \begin{tabular}{|l|} 
\\
\end{tabular} & 35.33 & 98.91 & 89.12 & 0 & 138.6 & 379.8 \\
\hline & 45.25 & 3000 & 1660 & $\begin{array}{r}47.122104 \\
\end{array}$ & 1.4614 & \begin{tabular}{|l|}
93.0387 \\
\end{tabular} & 806.12 & 5125 & 0.1 & 1950 & 75000 & 962500 & \begin{tabular}{|l|}
0.0703 \\
\end{tabular} & \#\#\#\#曲 & 4.023 & \begin{tabular}{|l|}
$.02 \mathrm{E}-02$ \\
\end{tabular} & 63.48 & 89.39 & 0 & 0 & 67.8 & 455.6 \\
\hline 6 & 35.04 & 3000 & 1900 & 58.256399 & $\begin{array}{l}.40873 \\
\end{array}$ & \begin{tabular}{|l|}
158.985 \\
\end{tabular} & 471.74 & 5125 & 0.1 & 1950 & 75000 & 2150000 & \begin{tabular}{|l|}
0.0908 \\
\end{tabular} & 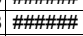 & 3.891 & \begin{tabular}{|l|}
$6.07 \mathrm{E}-03$ \\
\end{tabular} & 48.82 & 91.42 & 14.17 & 0 & 114.2 & 569.2 \\
\hline 7 & 59.13 & 3000 & 1190 & 258.618076 & 10.8409 & \begin{tabular}{|l|}
125.755 \\
\end{tabular} & 596.4 & 5125 & 0.1 & 1950 & 75000 & 1512500 & \begin{tabular}{|l|}
0.0538 \\
\end{tabular} & \#\#\#\#\# & 3.363 & $8.53 \mathrm{E}-03$ & 33 & 98.07 & 80.67 & 0 & 81.2 & 291.5 \\
\hline 9 & 26.53 & 3000 & 1330 & 29.084817 & 1.1232 & \begin{tabular}{|l|}
115.858 \\
\end{tabular} & 647.34 & 5125 & 0.1 & 1950 & 75000 & 1337500 & 0.1199 & \#\#\#\#\#曲 & 2.608 & $1.02 \mathrm{E}-02$ & 62.53 & 82.81 & 0 & & 68.1 & 503.7 \\
\hline 10 & 29.57 & 3000 & 2060 & 283.172977 & 10.173 & \begin{tabular}{|l|}
107.775 \\
\end{tabular} & 695.9 & 5125 & 0.1 & 1950 & 75000 & 1200000 & 0.1076 & \#\#\#\#曲 & 3.384 & 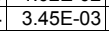 & 39.84 & 98.23 & 82.34 & & 201.2 & 586.6 \\
\hline 11 & 28.46 & 3000 & 1510 & 177.823326 & 8.8299 & \begin{tabular}{|l|}
148.966 \\
\end{tabular} & 503.47 & 5125 & 0.1 & 1950 & 75000 & 1950000 & \begin{tabular}{|l|}
0.1118 \\
\end{tabular} & 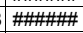 & 2.788 & $5.27 \mathrm{E}-03$ & 29.73 & 97.19 & 71.88 & 0 & 131.5 & 502.1 \\
\hline 12 & 21.41 & 3000 & 1150 & 185.517538 & 4.6529 & 75.2423 & 996.78 & 5125 & 0.1 & 1950 & 75000 & 700000 & \begin{tabular}{|l|}
0.1486 \\
\end{tabular} & \#\#\#\#\#曲 & 1.399 & $8.58 \mathrm{E}-03$ & 42.84 & 97.3 & 73.05 & 0 & 80.8 & 334.8 \\
\hline 13 & 62.5 & 3000 & 2560 & 2.354173 & 0.1134 & \begin{tabular}{|l|}
144.475 \\
\end{tabular} & 519.12 & 5125 & 0.1 & 1950 & 75000 & 1862500 & \begin{tabular}{|l|}
0.0509 \\
\end{tabular} & \#\#\#\#\#| & 7.691 & $\mid 1.36 \mathrm{E}-02$ & 49.38 & 0 & 0 & & 50.8 & 630.6 \\
\hline 14 & 28.87 & 3000 & 2510 & 11.22621 & 0.4144 & \begin{tabular}{|l|}
110.748 \\
\end{tabular} & 677.21 & 5125 & 0.1 & 1950 & 75000 & 1250000 & \begin{tabular}{|l|}
0.1102 \\
\end{tabular} & 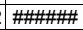 & 4.027 & $7.91 \mathrm{E}-03$ & 33.57 & 55.46 & 0 & 0 & 87.7 & 714.9 \\
\hline 15 & 41 & 3000 & 1860 & 17.515538 & 0.6932 & \begin{tabular}{|l|}
118.728 \\
\end{tabular} & 631.7 & 5125 & 0.1 & 1950 & 75000 & 1387500 & \begin{tabular}{|l|}
0.0776 \\
\end{tabular} & \#\#\#\#\# & 4.236 & $1.04 \mathrm{E}-02$ & 47.28 & 71.45 & 0 & 0 & 67 & 529.6 \\
\hline 16 & 37.83 & 3000 & 910 & 1282.963207 & \begin{tabular}{|c|}
49.8554 \\
\end{tabular} & \begin{tabular}{|l|}
116.579 \\
\end{tabular} & 643.34 & 5125 & 0.1 & 1950 & 75000 & 1350000 & 0.0841 & \#\#\#\#\#开 & 1.989 & $3.20 \mathrm{E}-03$ & 55.5 & 99.61 & 96.1 & 61.03 & 216.6 & 269.5 \\
\hline 17 & 59.24 & 3000 & 870 & 696.459267 & 33.6903 & \begin{tabular}{|l|}
145.121 \\
\end{tabular} & 516.81 & 5125 & 0.1 & 1950 & 75000 & 1875000 & \begin{tabular}{|l|}
0.0537 \\
\end{tabular} & \#\#\#\#\#曲 & 2.697 & $6.33 \mathrm{E}-03$ & 41.22 & 99.28 & 92.82 & 28.21 & 109.5 & 233.3 \\
\hline 18 & $\begin{array}{r}59.24 \\
52.5 \\
\end{array}$ & 3000 & 970 & \begin{tabular}{|l|}
095.452406 \\
200.241998
\end{tabular} & \begin{tabular}{|c|}
3.0903 \\
7.9248
\end{tabular} & \begin{tabular}{|l|}
148.12 \\
118.728 \\
\end{tabular} & $\begin{array}{l}10.01 \\
631.7 \\
\end{array}$ & $\begin{array}{l}5125 \\
5125\end{array}$ & $\begin{array}{l}0.1 \\
0.1\end{array}$ & $\frac{1950}{1950}$ & 75000 & $\frac{105000}{1387500}$ & \begin{tabular}{|l|}
0.0531 \\
0.0606 \\
\end{tabular} & $\mid$ & $\begin{array}{l}2.091 \\
2.617\end{array}$ & \begin{tabular}{|l|}
$.33 E-0 S$ \\
$1.12 E-02$ \\
\end{tabular} & $\begin{array}{l}41.22 \\
39.63 \\
\end{array}$ & $\begin{array}{r}99.20 \\
97.5\end{array}$ & $\begin{array}{ll}75.02 \\
75.03\end{array}$ & $\frac{28.21}{0}$ & $\begin{array}{r}\quad 09.5 \\
62.2 \\
\end{array}$ & 255.5 \\
\hline 19 & 29.59 & 3000 & 620 & 436.709687 & 19.8863 & 136.61 & 549.01 & 5125 & 0.1 & 1950 & 75000 & 1712500 & 0.1075 & \#\#\#\#曲 & 1.153 & $9.82 \mathrm{E}-03$ & 41.91 & 98.86 & 88.55 & 0 & 70.6 & 199.7 \\
\hline 20 & $\begin{array}{r}21.6 \\
20.0\end{array}$ & 3000 & 940 & $\begin{array}{r}13.342534 \\
\end{array}$ & $\begin{array}{l}0.8306 \\
\end{array}$ & \begin{tabular}{|l|}
186.767 \\
\end{tabular} & 401.57 & 5125 & 0.1 & 1950 & 75000 & 2737500 & 0.1473 & 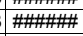 & 1.901 & \begin{tabular}{|l|l|}
$1.28 \mathrm{E}-02$ \\
\end{tabular} & 42.14 & 62.53 & 0 & & 54.2 & 451 \\
\hline 21 & 31.69 & 3000 & 2200 & 22.835159 & 1.2288 & \begin{tabular}{|l|}
161.44 \\
\end{tabular} & 464.57 & 5125 & 0.1 & 1950 & 75000 & 2200000 & 0.1004 & \#\#\#\# & 3.993 & $6.88 \mathrm{E}-03$ & 37.29 & 78.1 & 0 & 0 & 100.7 & 645.8 \\
\hline 22 & 26.1 & 3000 & 1350 & 17.45675 & 0.6096 & \begin{tabular}{|l|}
104.76 \\
\end{tabular} & 715.92 & 5125 & 0.1 & 1950 & 75000 & 1150000 & \begin{tabular}{|l|}
0.1219 \\
\end{tabular} & \#\#\#\#\#五 & 2.352 & $1.18 \mathrm{E}-02$ & 27.73 & 71.36 & 0 & 0 & 58.5 & 461.8 \\
\hline 23 & 22.18 & 3000 & 860 & 88.335134 & 4.6084 & 156.51 & 479.2 & 5125 & 0.1 & 1950 & 75000 & 2100000 & 0.1434 & \#\#\#\#\# & 1.682 & $9.48 \mathrm{E}-03$ & 42.19 & 94.34 & 43.4 & & 73.1 & 388.6 \\
\hline 24 & 28.87 & 3000 & 1830 & 133.172694 & 5.365 & \begin{tabular}{|l|}
120.857 \\
\end{tabular} & 620.57 & 5125 & 0.1 & 1950 & 75000 & 1425000 & 0.1102 & 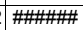 & 3.258 & $4.93 \mathrm{E}-03$ & 49.66 & 96.25 & 62.45 & 0 & 140.7 & 578.4 \\
\hline 25 & 29 & 3000 & 2100 & 179.125168 & 7.3422 & \begin{tabular}{|l|}
122.968 \\
\end{tabular} & 609.91 & 5125 & 0.1 & 1950 & 75000 & 1462500 & 0.1097 & \#\#\#\#" & 3.519 & $3.73 \mathrm{E}-03$ & 46.41 & 97.21 & 72.09 & 0 & 185.8 & 622 \\
\hline 26 & 22.76 & 3000 & 2970 & 156.246783 & 5.2163 & \begin{tabular}{|l|}
100.154 \\
\end{tabular} & 748.85 & 5125 & 0.1 & 1950 & 75000 & 1075000 & 0.1398 & \#\#\#\#\#五 & 3.771 & 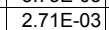 & 45.07 & 96.8 & 68 & 0 & 256.3 & 849.3 \\
\hline 27 & 43.28 & 3000 & 1700 & 68.260158 & 2.1352 & \begin{tabular}{|l|}
93.8425 \\
\end{tabular} & 799.21 & 5125 & 0.1 & 1950 & 75000 & 975000 & 0.0735 & \#\#\#\#曲 & 3.53 & $9.45 \mathrm{E}-03$ & 26.64 & 92.68 & 26.75 & & 73.3 & 417.9 \\
\hline 28 & 21.94 & 3000 & 650 & 95.219471 & 5.7827 & \begin{tabular}{|l|}
182.19 \\
\end{tabular} & 411.66 & 5125 & 0.1 & 1950 & 75000 & 2637500 & 0.145 & 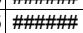 & 1.361 & \begin{tabular}{|l|}
$1.12 \mathrm{E}-02$ \\
\end{tabular} & 34.57 & 94.75 & 47.49 & & 61.9 & 317.9 \\
\hline 29 & 45.38 & 3000 & 730 & 838.048761 & 36.0909 & \begin{tabular}{|l|}
129.196 \\
\end{tabular} & 580.51 & 5125 & 0.1 & 1950 & 75000 & 1575000 & 0.0701 & \#\#\#\#\#曲 & 1.794 & \begin{tabular}{|l|}
$6.46 \mathrm{E}-03$ \\
\end{tabular} & & 99.4 & 94.03 & 40.34 & 107.3 & 202.6 \\
\hline 30 & 34.81 & 3000 & 920 & 39.514785 & 1.3998 & \begin{tabular}{|l|}
106.273 \\
\end{tabular} & 705.73 & 5125 & 0.1 & 1950 & 75000 & 1175000 & 0.0914 & \#\#\#\# & 2.034 & \begin{tabular}{|l|}
$1.66 \mathrm{E}-02$ \\
\end{tabular} & 85.44 & 87.35 & 0 & 0 & 41.8 & 299.4 \\
\hline 31 & 25.11 & 3000 & 1420 & 134.416681 & 4.2047 & \begin{tabular}{|l|}
93.8425 \\
\end{tabular} & 799.21 & 5125 & 0.1 & 1950 & 75000 & 975000 & 0.1267 & 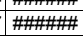 & 2.433 & \begin{tabular}{|l|}
$1.68 \mathrm{E}-03$ \\
\end{tabular} & 67.25 & 96.28 & 62.8 & 0 & 103.8 & 496.7 \\
\hline 32 & 21.44 & 3000 & 1180 & 5.403244 & 0.24 & \begin{tabular}{|l|}
133.266 \\
\end{tabular} & 562.78 & 5125 & 0.1 & 1950 & 75000 & 1650000 & 0.1484 & \#\#\#\#" & 2.126 & \begin{tabular}{|l|}
$1.37 \mathrm{E}-02$ \\
\end{tabular} & 42.06 & 7.46 & 0 & & 50.7 & 508.2 \\
\hline 33 & 49.71 & 3000 & 780 & 458.418892 & 19.4274 & \begin{tabular}{|l|}
127.137 \\
\end{tabular} & 589.91 & 5125 & 0.1 & 1950 & 75000 & 1537500 & 0.064 & \#\#\#\#\#曲 & 2.015 & \begin{tabular}{|l|} 
\\
\end{tabular} & 34.79 & 98.91 & 89.09 & & 74.7 & 207.7 \\
\hline 34 & 31.72 & 3000 & 1080 & 131.982806 & 6.5816 & \begin{tabular}{|l|}
149.602 \\
\end{tabular} & 501.33 & 5125 & 0.1 & 1950 & 75000 & 1962500 & 0.1003 & \#\#\#\#\# & 2.374 & \begin{tabular}{|l|} 
\\
\end{tabular} & 35.37 & 96.21 & 62.12 & & 83.1 & 383.5 \\
\hline 35 & 32.56 & 3000 & 1900 & 20.146403 & 0.8995 & \begin{tabular}{|l|}
133.938 \\
\end{tabular} & 559.96 & 5125 & 0.1 & 1950 & 75000 & 1662500 & 0.0977 & \#\#\#\#曲 & 3.649 & \begin{tabular}{|l|}
$8.74 \mathrm{E}-03$ \\
\end{tabular} & 36.93 & 75.18 & 0 & 0 & 79.4 & 574.3 \\
\hline 36 & 46.92 & 3000 & 2370 & 86.89421 & $\begin{array}{l}.0309 \\
.8209\end{array}$ & \begin{tabular}{|l|}
131.916 \\
\end{tabular} & 568.54 & 5125 & 0.1 & 1950 & 75000 & 1625000 & 0.0678 & \#\#\#\#曲 & 5.714 & 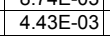 & 83.15 & 94.25 & 42.46 & 0 & 156.6 & 624 \\
\hline 37 & 55.81 & 3000 & 620 & 863.706 & 33.5632 & \begin{tabular}{|c|}
116.579 \\
\end{tabular} & 643.34 & & & & 75000 & 1350000 & 0.057 & \#\#\#\#曲 & 1.584 & \begin{tabular}{|l|}
$8.64 \mathrm{E}-03$ \\
\end{tabular} & 26.14 & 99.42 & 94.21 & 42.11 & 80.2 & 145.5 \\
\hline 38 & 29.79 & 3000 & 1050 & 532. & $\mid 17.3673$ & \begin{tabular}{|l|}
97.8112 \\
\end{tabular} & 766.78 & 512 & 0 & 195 & & & 0.1068 & \#\#\#\#\#五 & 1.858 & $5.53 \mathrm{E}-03$ & & 99.06 & 90.61 & 6.13 & 125.3 & 319.7 \\
\hline 39 & 24.51 & 3000 & 1290 & & 0.2209 & \begin{tabular}{|l|}
112.22 \\
\end{tabular} & 668.33 & 5125 & 0.1 & 1950 & 75000 & 1275000 & 0.1298 & & 2.543 & & 67.6 & 15.35 & 0 & 0 & 49.9 & 531.7 \\
\hline 40 & 23.9 & 3000 & 1150 & 135.043 & & \begin{tabular}{|l|}
88.1405 \\
\end{tabular} & 850.91 & & & 195 & & & 0.1 & \#\#\# & 1.7 & 8.60 & & 96.3 & 62.97 & & 80.6 & 38 \\
\hline 41 & 31.91 & 3000 & 1210 & $104.4 \varepsilon$ & 3.1842 & \begin{tabular}{|l|}
91.4206 \\
\end{tabular} & 820.38 & 5125 & 0.1 & 1950 & 75000 & 937500 & 0.0997 & \#\#\#\#\#曲 & 2.424 & 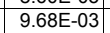 & 83.34 & 95.21 & 52.15 & 0 & 71.6 & 389.4 \\
\hline 42 & 25.8 & 3000 & 1530 & $\begin{array}{l}.042457 \\
\end{array}$ & 7.7634 & \begin{tabular}{|l|}
138.597 \\
\end{tabular} & 541.14 & 5125 & 0.1 & 1950 & 75000 & 1750000 & 0.1233 & \#\#\#\#曲 & 2.607 & \begin{tabular}{|l|}
$5.43 \mathrm{E}-03$ \\
\end{tabular} & 23.41 & 97.02 & 70.25 & & 127.6 & 517.9 \\
\hline 43 & 31.53 & 3000 & 960 & 148.5148 & 5.6997 & \begin{tabular}{|l|}
115.135 \\
\end{tabular} & 651.41 & 5125 & 0.1 & 1950 & 75000 & 1325000 & 0.1009 & \#\#\#\#\#曲 & 1.915 & $1.00 \mathrm{E}-02$ & 31. & 96.63 & 66.33 & 0 & 69.3 & 311.2 \\
\hline 44 & 22.5 & 3000 & 830 & 62.9482 & 2.6095 & \begin{tabular}{|l|}
124.366 \\
\end{tabular} & 603.06 & 5125 & 0.1 & 1950 & 75000 & 1487500 & 0.1414 & \#\#\#\# & 1.569 & \begin{tabular}{|c|}
$1.19 \mathrm{E}-02$ \\
\end{tabular} & 87.64 & 92.06 & 20.57 & 0 & 58.1 & 357.5 \\
\hline 45 & 31.94 & 3000 & 690 & 314.2216 & 12.9531 & \begin{tabular}{|l|}
123.668 \\
\end{tabular} & 606.46 & 5125 & 0.1 & 1950 & 75000 & 1475000 & 0.0996 & \#\#\#\#曲 & 1.287 & \begin{tabular}{|l|}
$1.13 \mathrm{E}-02$ \\
\end{tabular} & 53.8 & 98.41 & 84.09 & 0 & 61.6 & 206.5 \\
\hline 46 & 21.97 & 3000 & 1480 & 142.034524 & 4.251 & \begin{tabular}{|r|}
89.788 \\
\end{tabular} & 835.3 & 5125 & 0.1 & 1950 & 75000 & 912500 & 0.1448 & \#\#\#\#\# & 2256 & $\mid 6.12 \mathrm{E}-03$ & 53.24 & 96.48 & 64.8 & & 113.3 & 526.3 \\
\hline 47 & 24.28 & 3000 & 1310 & 17.659424 & 0.6606 & \begin{tabular}{|l|}
112.22 \\
\end{tabular} & 668.33 & 5125 & 0.1 & 1950 & 75000 & 1275000 & \begin{tabular}{|c|}
.1440 \\
0.131
\end{tabular} & 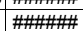 & 2.315 & \begin{tabular}{|l|}
$1.14 \mathrm{E}-02$ \\
\end{tabular} & 39.66 & 71.69 & 0 & 0 & 60.9 & 488.5 \\
\hline 48 & 26.73 & 3000 & 1670 & 63.666734 & 2.4434 & \begin{tabular}{|l|}
115.135 \\
\end{tabular} & 651.41 & 5125 & 0.1 & 1950 & 75000 & 1325000 & 0.119 & \#\#\#\#\# & 2.88 & 7.07E-03 & 37.04 & 92.15 & 21.47 & 0 & 98 & 552.1 \\
\hline 49 & 42.25 & & 2130 & & & \begin{tabular}{|l|}
131.916 \\
\end{tabular} & 568 & & & & & & 0.0753 & & 4.325 & & & 94.88 & 8.77 & & 122.6 & 246 \\
\hline 50 & 40.89 & 3000 & 11 & 143. & 7.2578 & \begin{tabular}{|l|}
151.501 \\
\end{tabular} & 495 & 512 & 0.1 & 19 & 75 & & & & 2.8 & & & 96.52 & 65.21 & 0 & 81 & 361.3 \\
\hline 51 & 33.99 & 3000 & 1620 & 40.572 & 2.5641 & \begin{tabular}{|l|}
189.599 \\
\end{tabular} & 395 & 512 & 0.1 & & 750 & 2800000 & 0.05 & & 3.784 & \begin{tabular}{|l|} 
\\
\end{tabular} & 74. & 87.68 & 0 & 0 & 101.3 & 570.6 \\
\hline 52 & 47.2 & 3000 & 1130 & 257.6390 & 11.2708 & \begin{tabular}{|l|}
131.239 \\
\end{tabular} & 571.48 & 5125 & 0.1 & 195 & 750 & 1612500 & 0.0674 & \#\#\#\# & 2.98 & \begin{tabular}{|c|} 
\\
\end{tabular} & 49.18 & 98.06 & 80.59 & 0 & 90.3 & 323.5 \\
\hline 53 & 55.14 & 3000 & 770 & 126.300305 & 4.9984 & \begin{tabular}{|l|}
118.728 \\
\end{tabular} & 631.7 & 5125 & 0.1 & 1950 & 75000 & 1387500 & 0.0577 & \#\#\#\#曲 & 2.141 & \begin{tabular}{|c|}
$1.75 \mathrm{E}-02$ \\
\end{tabular} & 73.54 & 96.04 & 60.41 & 0 & 39.7 & 199 \\
\hline 54 & 22.22 & 3000 & 1040 & 140.324853 & 5.9468 & \begin{tabular}{|l|}
127.137 \\
\end{tabular} & 589.91 & 5125 & 0.1 & 1950 & 75000 & 1537500 & 0.1432 & \#\#\#\#曲 & 1.797 & \begin{tabular}{|l|}
$7.61 \mathrm{E}-03$ \\
\end{tabular} & 28.5 & 96.44 & 64.37 & 0 & 91.1 & 414.5 \\
\hline 55 & 25.72 & 3000 & 1380 & $\begin{array}{r}6.989416 \\
\end{array}$ & 2.3376 & \begin{tabular}{|l|}
106.273 \\
\end{tabular} & 705.73 & 5125 & 0.1 & 1950 & 75000 & 1175000 & 0.1237 & 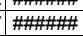 & 2.397 & \begin{tabular}{|l|}
$8.43 \mathrm{E}-03$ \\
\end{tabular} & 38.94 & 92.42 & 24.23 & 0 & 82.2 & 477.7 \\
\hline 56 & 48.57 & 3000 & 1640 & 22.949691 & 1.1638 & \begin{tabular}{|l|}
152.132 \\
\end{tabular} & 492.99 & 5125 & 0.1 & 1950 & 75000 & 2012500 & 0.0655 & \#\#\#\#\# & 4.175 & \begin{tabular}{|l|}
$1.11 \mathrm{E}-02$ \\
\end{tabular} & 35.94 & 78.21 & 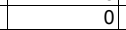 & & 62.5 & 440.5 \\
\hline 57 & 45.58 & 3000 & 790 & 624.483729 & 36.7 & \begin{tabular}{|l|}
176.387 \\
\end{tabular} & 425.2 & 5125 & 0 & 1950 & 75000 & 2512500 & 0.0698 & \#\#\#\#\# & 2.423 & & & 99.2 & 91.99 & .93 & 127.5 & 72.5 \\
\hline 58 & 30.04 & 3000 & 2310 & 52.81 & & & 806.12 & 5125 & & 1950 & & 2500 & & & 4.0 & & & 90.53 & 5.34 & & & 1 \\
\hline 59 & 23.24 & 3000 & 1100 & 137.324172 & 6.906 & \begin{tabular}{|l|}
150.869 \\
\end{tabular} & 497.12 & 5125 & 0.1 & 1950 & 75000 & 1987500 & 0.1369 & \#\#\#\#曲 & 2.144 & $1 \mathrm{E}-03$ & 68.12 & 96.36 & 63.59 & & 104.8 & 472.8 \\
\hline
\end{tabular}




\begin{tabular}{|c|c|c|c|c|c|c|c|c|c|c|c|c|c|c|c|c|c|c|c|c|c|c|}
\hline Realization & $\begin{array}{c}\text { Velocity } \\
(\mathrm{m} / \mathrm{y})\end{array}$ & $\begin{array}{c}\text { Fixed Source } \\
\text { Concentratio } \\
\mathrm{n}(\mathrm{mg} / \mathrm{L})\end{array}$ & $\begin{array}{c}\text { Time of } \\
\text { Maximum at } \\
\text { Compliance } \\
\text { Boundary } \\
(y)\end{array}$ & \begin{tabular}{|c|} 
Maximum \\
Concentration \\
Compliance \\
Boundary \\
$(\mu \mathrm{g} / \mathrm{L})$
\end{tabular} & $\begin{array}{c}\text { Maximum } \\
\text { Release Rate } \\
\text { at Compliance } \\
\text { Boundary } \\
(\mathrm{Kg} / \mathrm{y})\end{array}$ & \begin{tabular}{|c|} 
\\
Source \\
Rate \\
$(\mathrm{Kg} / \mathrm{y})$ \\
\end{tabular} & $\begin{array}{c}\text { Source } \\
\text { Duration } \\
\text { (y) }\end{array}$ & $\begin{array}{c}\text { 1-D } \\
\text { Streamtube } \\
\text { Length } \\
\text { (m) }\end{array}$ & $\begin{array}{c}\text { Base } \\
\text { Porosity } \\
\left(\mathrm{m}^{3} / \mathrm{m}^{3}\right)\end{array}$ & \begin{tabular}{|c|} 
Bulk \\
Densty \\
$\left(\mathrm{Kg} / \mathrm{m}^{3}\right)$ \\
\end{tabular} & $\begin{array}{c}\text { Remaining } \\
\text { Carbon } \\
\text { Tetrachloride } \\
\text { Source } \\
(\mathrm{Kg})\end{array}$ & $\begin{array}{c}\begin{array}{c}\text { Volume at or } \\
\text { above Fixed } \\
\text { Source } \\
\text { Concentration } \\
\text { (m3) }\end{array} \\
\end{array}$ & $\begin{array}{l}\text { Porosity } \\
\left(\mathrm{m}^{3} / \mathrm{m}^{3}\right)\end{array}$ & $\begin{array}{c}\mathrm{Kd} \\
\left(\mathrm{m}^{3} / \mathrm{Kg}\right)\end{array}$ & $\begin{array}{l}\text { Retardatio } \\
n\end{array}$ & \begin{tabular}{|c|} 
First \\
Order \\
Rate \\
Constant \\
$(1 / y)$ \\
\end{tabular} & $\begin{array}{l}\text { Longitudinal } \\
\text { Dispersivity } \\
\text { (m) }\end{array}$ & \begin{tabular}{|c|} 
Required \\
Source \\
Cleanup \\
Percentage \\
$(5 \mu \mathrm{g} / \mathrm{L}$ \\
Compliance)
\end{tabular} & \begin{tabular}{|c} 
Required \\
Source \\
Cleanup \\
Percentage \\
$(50 \mu \mathrm{g} / \mathrm{L}$ \\
Compliance \\
)
\end{tabular} & \begin{tabular}{|c|} 
Required \\
Source \\
Cleanup \\
Percentage \\
$(500 \mu \mathrm{L} / \mathrm{L}$ \\
Compliance \\
) \\
\end{tabular} & \begin{tabular}{|c|} 
\\
Abiotic \\
Reaction \\
Half Time \\
$(y)$ \\
\end{tabular} & \begin{tabular}{|c|} 
Carbon \\
Tetrachloride \\
Travel Time \\
(y)
\end{tabular} \\
\hline & 33.56 & 3000 & 770 & 111.661799 & 6.4997 & \begin{tabular}{|l|}
174.628 \\
\end{tabular} & \begin{tabular}{|l|} 
\\
\end{tabular} & & & & 75000 & 2475000 & \begin{tabular}{|l|}
0.0948 \\
\end{tabular} & 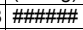 & \begin{tabular}{|l|l} 
& 2.125 \\
\end{tabular} & $1.10 \mathrm{E}-02$ & 88.57 & \begin{tabular}{l|l|}
7 & 95.52 \\
\end{tabular} & \begin{tabular}{|l|l|}
2 & 55.22 \\
\end{tabular} & 0 & \begin{tabular}{|r|} 
\\
\end{tabular} & 324.5 \\
\hline 61 & 27.69 & 3000 & 1370 & 4.578663 & 0.1176 & \begin{tabular}{|l|l|}
77.0232 \\
\end{tabular} & 973.73 & 5125 & 0.1 & 1950 & 75000 & 725000 & 0.1149 & \#\#\#\#\#+ & 2.441 & \begin{tabular}{|c|}
$1.72 \mathrm{E}-02$ \\
\end{tabular} & 65.36 & 0 & 0 & 0 & 40.2 & 451.8 \\
\hline 62 & 45.71 & 3000 & 3040 & 13.672589 & 0.5981 & \begin{tabular}{|l|}
131.239 \\
\end{tabular} & 571.48 & 5125 & 0.1 & 1950 & 75000 & 1612500 & \begin{tabular}{|l|}
0.0696 \\
\end{tabular} & \#\#\#\#\# & 5.954 & $7.46 \mathrm{E}-03$ & 33.26 & 63.43 & 0 & 0 & 92.9 & 667.6 \\
\hline 63 & 63 & 3000 & 830 & 550.369806 & 23.5763 & \begin{tabular}{|l|}
128.512 \\
\end{tabular} & 583.6 & 5125 & 0.1 & 1950 & 75000 & 1562500 & \begin{tabular}{|l|}
0.0505 \\
\end{tabular} & \#\#\#\#\#曲 & 2.457 & $8.69 \mathrm{E}-03$ & 30.09 & 99.09 & 90.92 & 9.15 & 79.8 & 199.9 \\
\hline 64 & 21.52 & 3000 & 740 & 29.57272 & 2.2354 & \begin{tabular}{|l|}
226.768 \\
\end{tabular} & 330.73 & 5125 & 0.1 & 1950 & 75000 & 3662500 & \begin{tabular}{|l|}
0.1478 \\
\end{tabular} & \#\#\#\#\# & 1.673 & $1.23 \mathrm{E}-02$ & 52.78 & 83.09 & 0 & 0 & 56.6 & 398.3 \\
\hline 65 & 24.66 & 3000 & 1490 & 107.662326 & $\begin{array}{l}.20745 \\
3.975\end{array}$ & \begin{tabular}{|l|}
110.748 \\
\end{tabular} & 677.21 & 5125 & 0.1 & 1950 & 75000 & 1250000 & $\begin{array}{l}0.1410 \\
0.129 \\
\end{array}$ & 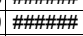 & 2.562 & $6.42 \mathrm{E}-03$ & 52.21 & 95.36 & 53.56 & 0 & 108 & 532.4 \\
\hline 66 & 46.17 & 3000 & 530 & 270.005197 & \begin{tabular}{|r|}
14.6398 \\
\end{tabular} & \begin{tabular}{|l|}
162.661 \\
\end{tabular} & 461.08 & 5125 & 0.1 & 1950 & 75000 & 2225000 & $\begin{array}{l}0.0689 \\
0.069\end{array}$ & \#\#曲 & $\begin{array}{l}2.002 \\
1.405 \\
\end{array}$ & \begin{tabular}{|l|l|}
$1.62 \mathrm{E}-02$ \\
\end{tabular} & 72.97 & 98.15 & 81.48 & 0 & $\begin{array}{r}42.8 \\
\end{array}$ & $\begin{array}{r}52.4 \\
156 \\
\end{array}$ \\
\hline 67 & 49.79 & 3000 & 1480 & 224.323656 & 7.5469 & \begin{tabular}{|l|}
100.929 \\
\end{tabular} & 743.1 & 5125 & 0.1 & 1950 & 75000 & 1087500 & 0.0639 & \#\#\#\#曲 & 3.368 & $7.65 \mathrm{E}-03$ & 21.89 & 97.77 & 77.71 & & 90.6 & 346.7 \\
\hline 68 & 26.34 & 3000 & 2160 & 5.149742 & 0.3548 & \begin{tabular}{|l|}
206.711 \\
\end{tabular} & 362.83 & 5125 & 0.1 & 1950 & 75000 & 3187500 & 0.1208 & \#\#\#\#\#五 & 3.918 & $8.11 \mathrm{E}-03$ & 50.27 & 2.91 & 0 & & 85.5 & 762.5 \\
\hline 69 & 41 & 3000 & 780 & 102.030167 & 4.6687 & \begin{tabular}{|l|}
137.274 \\
\end{tabular} & 546.35 & 5125 & 0.1 & 1950 & 75000 & 1725000 & 0.0776 & \#\#\#\#曲 & 2.075 & $1.42 \mathrm{E}-02$ & 64.47 & 95.1 & 50.99 & & 48.9 & 259.5 \\
\hline 70 & 56.81 & 3000 & 2010 & 1.878377 & 0.083 & \begin{tabular}{|l|}
132.592 \\
\end{tabular} & 565.65 & 5125 & 0.1 & 1950 & 75000 & 1637500 & 0.056 & \#\#\#\#曲 & 7.404 & $1.71 \mathrm{E}-02$ & 82.08 & 0 & 0 & 0 & 40.6 & 667.9 \\
\hline 71 & 25.31 & 3000 & 1480 & 0.592265 & 0.0263 & \begin{tabular}{|l|}
133.266 \\
\end{tabular} & 562.78 & 5125 & 0.1 & 1950 & 75000 & 1650000 & 0.1257 & \#\#\#\#\#曲 & 2.961 & \begin{tabular}{|l|}
$1.68 \mathrm{E}-02$ \\
\end{tabular} & 45.79 & 0 & 0 & 0 & 41.2 & 599.6 \\
\hline 72 & 27.21 & 3000 & 2390 & 7.888877 & 0.2814 & \begin{tabular}{|l|}
107.025 \\
\end{tabular} & 700.77 & 5125 & 0.1 & 1950 & 75000 & 1187500 & \begin{tabular}{|l|}
0.1169 \\
\end{tabular} & \#\#\#\#\#曲 & 3.975 & \begin{tabular}{|l|} 
\\
\end{tabular} & 44.95 & 36.62 & & 0 & 81.8 & 748.6 \\
\hline 73 & 29.32 & 3000 & 1620 & 34.754599 & 1.0018 & \begin{tabular}{|l|}
86.4774 \\
\end{tabular} & 867.28 & 5125 & 0.1 & 1950 & 75000 & 862500 & \begin{tabular}{|l|}
0.1085 \\
\end{tabular} & 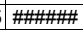 & 2.682 & $9.99 \mathrm{E}-03$ & 22.54 & 85.61 & 0 & 0 & 69.4 & 468.9 \\
\hline 74 & 46.04 & 3000 & 760 & 393.621665 & 19.2098 & \begin{tabular}{|l|}
146.408 \\
\end{tabular} & 512.27 & 5125 & 0.1 & 1950 & 75000 & 1900000 & 0.0691 & \#\#\#\#\# & 2.054 & \begin{tabular}{|l|} 
\\
\end{tabular} & 34.26 & 98.73 & 87.3 & 0 & 75.9 & 228.6 \\
\hline 75 & 33.49 & 3000 & 650 & 450.832377 & \begin{tabular}{|l|}
13.3696 \\
\end{tabular} & \begin{tabular}{|l|}
88.9661 \\
\end{tabular} & 843.02 & 5125 & 0.1 & 1950 & 75000 & 900000 & 0.095 & 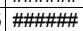 & 1.282 & $9.81 \mathrm{E}-03$ & 32.53 & 98.89 & 88.91 & 0 & 70.6 & 196.1 \\
\hline 76 & 23.85 & 3000 & 1520 & 79.224067 & 3.6775 & \begin{tabular}{|l|}
139.257 \\
\end{tabular} & 538.57 & 5125 & 0.1 & 1950 & 75000 & 1762500 & 0.1334 & \#\#\#\#州 & 2.644 & \begin{tabular}{|l|l|}
$6.26 \mathrm{E}-03$ \\
\end{tabular} & 46.73 & 93.69 & 36.89 & 0 & 110.8 & 568.2 \\
\hline 77 & 43.94 & 3000 & 950 & 279.418791 & 13.5765 & \begin{tabular}{|l|}
145.765 \\
\end{tabular} & 514.53 & 5125 & 0.1 & 1950 & 75000 & 1887500 & \begin{tabular}{|l|}
0.0724 \\
\end{tabular} & 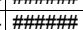 & 2.493 & \begin{tabular}{|l|l|} 
& $0.38 \mathrm{E}-03$ \\
\end{tabular} & $\begin{array}{ll}40.15 \\
32.24 \\
\end{array}$ & 98.21 & 82.11 & 0 & 82.7 & $\begin{array}{l}290.7 \\
290\end{array}$ \\
\hline 78 & 22.28 & 3000 & 1200 & 235.740438 & 8.1723 & \begin{tabular}{|l|}
103.999 \\
\end{tabular} & 721.16 & 5125 & 0.1 & 1950 & 75000 & 1137500 & \begin{tabular}{|l|}
0.1428 \\
\end{tabular} & \#\#\#\#\#五 & 1.899 & $6.00 \mathrm{E}-03$ & 33.34 & 97.88 & 78.79 & & 115.5 & 436.9 \\
\hline 79 & 25.57 & 3000 & 1950 & 0.53829 & 0.0164 & \begin{tabular}{|l|}
91.4206 \\
\end{tabular} & 820.38 & 5125 & 0.1 & 1950 & 75000 & 937500 & 0.1244 & 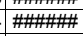 & 3.031 & \begin{tabular}{|l|}
$1.56 \mathrm{E}-02$ \\
\end{tabular} & 20.24 & 0 & 0 & & 44.6 & 607.5 \\
\hline 80 & 21.39 & 3000 & 1850 & 74.090958 & 2.6246 & \begin{tabular}{|l|}
106.273 \\
\end{tabular} & 705.73 & 5125 & 0.1 & 1950 & 75000 & 1175000 & 0.1487 & 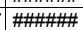 & 3.048 & $5.09 \mathrm{E}-03$ & 93.26 & 93.25 & 32.52 & 0 & 136.1 & 730.2 \\
\hline 81 & 54.2 & 3000 & 1450 & 91.506915 & 3.7508 & \begin{tabular}{|l|}
122.968 \\
\end{tabular} & 609.91 & 5125 & 0.1 & 1950 & 75000 & 1462500 & 0.0587 & \#\#\#\#曲 & 4.014 & \begin{tabular}{|c|} 
\\
\end{tabular} & 52.36 & 94.54 & 45.36 & 0 & 74.2 & 379.6 \\
\hline 82 & 43.28 & 3000 & 840 & 1087.902042 & 40.4283 & \begin{tabular}{|l|}
111.485 \\
\end{tabular} & 672.73 & 5125 & 0.1 & 1950 & 75000 & 1262500 & 0.0735 & \#\#\#\#\#曲 & 1.843 & $4.71 \mathrm{E}-03$ & 36.87 & 99.54 & 95.4 & 54.04 & 147.1 & 218.2 \\
\hline 83 & 23.12 & 3000 & 1520 & 81.705425 & 4.8039 & \begin{tabular}{|l|}
176.387 \\
\end{tabular} & 425.2 & 5125 & 0.1 & 1950 & 75000 & 2512500 & 0.1376 & \#\#\#\#曲 & 2.521 & \begin{tabular}{|c|}
$6.18 \mathrm{E}-03$ \\
\end{tabular} & 19.94 & 93.88 & 38.8 & 0 & 112.1 & 558.8 \\
\hline 84 & 22.33 & 3000 & 1120 & 22.194809 & 1.0205 & \begin{tabular}{|l|}
137.937 \\
\end{tabular} & 543.73 & 5125 & 0.1 & 1950 & 75000 & 1737500 & 0.1425 & \#\#\#\#曲 & 2.01 & \begin{tabular}{|l|}
$1.12 \mathrm{E}-02$ \\
\end{tabular} & 31.85 & 77.47 & 0 & 0 & 61.8 & 461.3 \\
\hline 85 & 32.17 & 3000 & 850 & 84.370441 & 3.9349 & \begin{tabular}{|l|}
139.914 \\
\end{tabular} & 536.04 & 5125 & 0.1 & 1950 & 75000 & 1775000 & 0.0989 & \#\#\#\#\#五 & 1.902 & $1.22 \mathrm{E}-02$ & 29.19 & 94.07 & 40.74 & 0 & 56.6 & 303.1 \\
\hline 86 & 21.77 & 3000 & 1150 & 70.557686 & & \begin{tabular}{|l|}
145.765 \\
\end{tabular} & 514.53 & 5125 & 0.1 & 1950 & 75000 & 1887500 & 0.1461 & 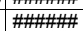 & 2.094 & \begin{tabular}{|l|} 
\\
$7.88 \mathrm{E}-03$ \\
\end{tabular} & 47.6 & 92.91 & 29.14 & & 87.9 & 492.9 \\
\hline 87 & 34.88 & 3000 & 840 & 10.453623 & 0.5941 & 170.487 & 439.92 & 5125 & 0.1 & 1950 & 75000 & 2387500 & 0.0912 & 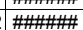 & 2.601 & \begin{tabular}{|l|}
$1.802-00$ \\
\end{tabular} & 99.18 & 52.17 & 0 & & 37.8 & 382.2 \\
\hline 88 & 21.72 & 3000 & 1710 & & 0.0226 & \begin{tabular}{|l|}
118.728 \\
\end{tabular} & 631.7 & 5125 & 0.1 & 1950 & 75000 & 1387500 & 0.1465 & \#\#\#\#\# & 2.719 & \begin{tabular}{|l|}
$1.47 \mathrm{E}-02$ \\
\end{tabular} & 26.72 & 0 & 0 & & 47.2 & 641.7 \\
\hline 89 & 35.11 & 3000 & 1450 & 33.843122 & 1.7586 & \begin{tabular}{|l|}
155.889 \\
\end{tabular} & 481.11 & 5125 & 0.1 & 1950 & 75000 & 2087500 & 0.0906 & \#\#\#\#曲 & 3.332 & \begin{tabular}{|l|} 
\\
\end{tabular} & 50.87 & 85.23 & 0 & & 74.5 & 486.3 \\
\hline 90 & 27.31 & 3000 & 1170 & 242.673242 & 6.8594 & \begin{tabular}{|l|}
84.7981 \\
\end{tabular} & 884.45 & 5125 & 0.1 & 1950 & 75000 & 837500 & 0.1165 & 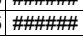 & 1.892 & \begin{tabular}{|l|}
$7.44 \mathrm{E}-03$ \\
\end{tabular} & 54.23 & 97.94 & 79.4 & 0 & 93.2 & 355.1 \\
\hline 91 & 41.97 & 3000 & 1370 & 11.647844 & 0.5356 & \begin{tabular}{|l|l|}
137.937 \\
\end{tabular} & 543.73 & 5125 & 0.1 & 1950 & 75000 & 1737500 & 0.0758 & \#\#\#\#\# & 3.381 & \begin{tabular}{|c|}
$1.45 \mathrm{E}-02$ \\
\end{tabular} & \begin{tabular}{ll|}
33.87 \\
\end{tabular} & 57.07 & 0 & 0 & 47.9 & 412.8 \\
\hline 92 & 33.28 & 3000 & 1500 & 80.848962 & 2.6991 & \begin{tabular}{|l|}
100.154 \\
\end{tabular} & 748.85 & 5125 & 0.1 & 1950 & 75000 & 1075000 & 0.0956 & \#\#\#\#\#曲 & 2.961 & \begin{tabular}{|l|}
$8.41 \mathrm{E}-03$ \\
\end{tabular} & 47.7 & 93.82 & 38.16 & & 82.5 & 456 \\
\hline 93 & 58.37 & 3000 & 940 & 261.738537 & 10.4207 & \begin{tabular}{|l|}
119.44 \\
\end{tabular} & 627.93 & 5125 & 0.1 & 1950 & 75000 & 1400000 & 0.0545 & \#\#\#\#\# & 2.644 & \begin{tabular}{|c|}
$.09 \mathrm{E}-02$ \\
\end{tabular} & 31.4 & 98.09 & 80.9 & & 63.5 & 232.1 \\
\hline 94 & 22.02 & 3000 & 1150 & 165.452318 & 6.3498 & \begin{tabular}{|l|}
115.135 \\
\end{tabular} & 651.41 & 5125 & 0.1 & 1950 & 75000 & 1325000 & 0.1445 & \#\#\#\#正 & 1.975 & \begin{tabular}{|l|}
$6.62 \mathrm{E}-03$ \\
\end{tabular} & 59.75 & 96.98 & 69.78 & 0 & 104.6 & 459.8 \\
\hline 95 & 31.31 & 3000 & 990 & 63.408567 & 1.9151 & 90.6061 & 827.76 & 5125 & 0.1 & 1950 & 75000 & 925000 & 0.1016 & \#\#\#\#\# & 1.721 & \begin{tabular}{|c|}
$1.48 \mathrm{E}-02$ \\
\end{tabular} & 62.93 & 92.11 & 21.15 & 0 & 46.8 & 281.6 \\
\hline 96 & 42.82 & 3000 & 640 & 355.247009 & 14.7269 & \begin{tabular}{|l|}
124.366 \\
\end{tabular} & 603 & & & 1950 & 75000 & 1487500 & 0.0743 & 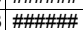 & 1.249 & \begin{tabular}{|l|}
$1.48 \mathrm{E}-02$ \\
\end{tabular} & 75.81 & 98.59 & 85.93 & & 46.7 & 149.5 \\
\hline 97 & 47.98 & 3000 & 2510 & & 0.7144 & 162.051 & 462.82 & 5125 & & 1950 & 75000 & 2212500 & 0.0663 & 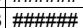 & 5.307 & \begin{tabular}{|l|}
$8.74 \mathrm{E}-03$ \\
\end{tabular} & 26.21 & 62.19 & 0 & & 79.3 & 566.8 \\
\hline 98 & 38.89 & 3000 & 1770 & & & \begin{tabular}{|l|}
93.8425 \\
\end{tabular} & 799.21 & 5125 & 0.1 & 1950 & 75000 & 975000 & 0.0818 & & 3.908 & & 71.09 & 93.32 & 33.24 & & 90.7 & 514.9 \\
\hline 99 & 62.75 & 3000 & 1820 & 48.944494 & 1.6967 & \begin{tabular}{|l|}
103.999 \\
\end{tabular} & 721.16 & & & 195 & & & & \#\#\# & 5.028 & 1.05 & 4 & 89.78 & 0 & & 66.1 & 410.7 \\
\hline 100 & 63.25 & 3000 & 2250 & .279683 & 0.8422 & \begin{tabular}{|l|}
118.728 \\
\end{tabular} & 631.7 & 5125 & 0.1 & 1950 & 75000 & 1387500 & 0.0503 & \#\#\#\#\#曲 & 5.874 & \begin{tabular}{|l|}
$1.04 \mathrm{E}-02$ \\
\end{tabular} & 35.04 & 76.5 & 0 & 0 & 66.6 & 476 \\
\hline 101 & 24.16 & 3000 & 2080 & 93.484923 & 3.2645 & \begin{tabular}{|l|}
104.76 \\
\end{tabular} & 715.92 & 5125 & 0.1 & 1950 & 75000 & 1150000 & 0.1317 & \#\#\#\#曲 & 3.344 & \begin{tabular}{|l|}
$4.60 \mathrm{E}-03$ \\
\end{tabular} & 70.34 & 94.65 & 46.52 & & 150.8 & 709.4 \\
\hline 102 & 25.95 & 3000 & 730 & 483.0548 & 22.4229 & \begin{tabular}{|l|}
139.257 \\
\end{tabular} & 538. & 5125 & 0.1 & 1950 & 75000 & 1762500 & 0.1226 & \#\#\#\#\#五 & 1.422 & 6.65E-03 & 45. & 98.96 & 89.65 & 0 & 104.2 & 280.9 \\
\hline 103 & 43.4 & 3000 & 1780 & 27.80239 & 1.2036 & \begin{tabular}{|l|}
129.879 \\
\end{tabular} & 577.46 & 5125 & 0.1 & 1950 & 7500 & 1587500 & 0.0733 & \#\#\#\#\# & 4.489 & \begin{tabular}{|l|}
$9.26 \mathrm{E}-03$ \\
\end{tabular} & 64. & 82.02 & 0 & 0 & 74.9 & 530.1 \\
\hline 104 & 31.34 & 3000 & 2640 & 24.665207 & 1.0846 & \begin{tabular}{|l|}
131.916 \\
\end{tabular} & 568.54 & 5125 & 0.1 & 1950 & 75000 & 1625000 & 0.1015 & \#\#\#\#曲 & 4.461 & \begin{tabular}{|c|}
$5.99 \mathrm{E}-03$ \\
\end{tabular} & 41.38 & 79.73 & 0 & 0 & 115.7 & 729.4 \\
\hline 105 & 23.6 & 3000 & 810 & 94.717951 & 4.8627 & \begin{tabular}{|l|}
154.016 \\
\end{tabular} & 486.96 & 5125 & 0.1 & 1950 & 75000 & 2050000 & 0.1348 & 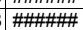 & 1.585 & \begin{tabular}{|l|}
$1.03 \mathrm{E}-02$ \\
\end{tabular} & 19.01 & 94.72 & 47.21 & & 67.7 & 344.1 \\
\hline 106 & 55.04 & 3000 & 930 & 179.734454 & 7.0704 & \begin{tabular}{|l|}
118.014 \\
\end{tabular} & 635.52 & 5125 & 0.1 & 1950 & 75000 & 1375000 & 0.0578 & 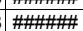 & 2.523 & \begin{tabular}{|l|}
$1.25 \mathrm{E}-02$ \\
\end{tabular} & 29.67 & 97.22 & 72.18 & 0 & 55.6 & 234.9 \\
\hline 107 & 29.62 & 3000 & 930 & 11.592288 & 0.7239 & \begin{tabular}{|l|}
187.335 \\
\end{tabular} & 400.35 & 5125 & 0.1 & 1950 & 75000 & 2750000 & \begin{tabular}{|l|}
0.1074 \\
\end{tabular} & \#\#\#\#曲 & 2.421 & \begin{tabular}{|c|}
$1.49 \mathrm{E}-02$ \\
\end{tabular} & 69.95 & 56.87 & 0 & 0 & 46.4 & 418.9 \\
\hline 108 & 26.42 & & 1340 & & & \begin{tabular}{|l|}
182.765 \\
\end{tabular} & & & & 19 & & & 0.1204 & & & & & 94.46 & 44.62 & & & \\
\hline 109 & 21.8 & 30 & 1280 & $\begin{array}{r}.495255 \\
\end{array}$ & 0.2856 & \begin{tabular}{|l|}
131.916 \\
\end{tabular} & 568.54 & 5125 & 0.1 & 19 & 75 & 000 & & \#\#\# & 2.339 & & & 23.02 & 0 & 0 & & 549 \\
\hline 110 & 47.41 & 3000 & 1440 & 10.7845 & 0.457 & \begin{tabular}{|l|}
127.137 \\
\end{tabular} & 589 & 512 & 0.1 & & 750 & & 0.0 & \#\#\# & 4.3 & & & 53.64 & 0 & 0 & 47.3 & 475.4 \\
\hline 111 & 29.13 & 30 & 1880 & 1.0037 & $\begin{array}{l}0.0325 \\
\end{array}$ & \begin{tabular}{|l|}
97.024 \\
\end{tabular} & 773 & 5125 & 0.1 & 1950 & 750 & 1025000 & 0.1092 & \#\#\#\#曲 & 3.516 & \begin{tabular}{|l|}
$1.53 \mathrm{E}-02$ \\
\end{tabular} & 39.76 & 0 & 0 & 0 & 45.3 & 618.6 \\
\hline 112 & 28 & 3000 & 950 & 351.551259 & \begin{tabular}{|l|l|}
12.8912 \\
\end{tabular} & \begin{tabular}{|l|}
110.009 \\
\end{tabular} & 681.77 & 5125 & 0.1 & 1950 & 75000 & 1237500 & 0.1136 & \#\#\#\#曲 & 1.749 & \begin{tabular}{|l|}
$6.91 \mathrm{E}-03$ \\
\end{tabular} & 43.41 & 98.58 & 85.78 & 0 & 100.3 & 320.1 \\
\hline 113 & 30.74 & 3000 & 1410 & $\begin{array}{l}9.477787 \\
\end{array}$ & \begin{tabular}{|l|l|}
0.4189 \\
\end{tabular} & \begin{tabular}{|l|}
132.592 \\
\end{tabular} & 565.65 & 5125 & 0.1 & 1950 & 75000 & 1637500 & 0.1035 & \#\#\#\#曲 & 3.026 & \begin{tabular}{|l|}
$1.26 \mathrm{E}-02$ \\
\end{tabular} & 47.36 & 47.25 & 0 & 0 & 55 & 504.5 \\
\hline 114 & 35.47 & 3000 & 1160 & 507.621585 & $\begin{array}{r}.45282 \\
\end{array}$ & \begin{tabular}{|l|}
150.869 \\
\end{tabular} & 497.12 & 5125 & 0.1 & 1950 & 75000 & 1987500 & 0.0897 & \#\#\#\#州 & 2.642 & 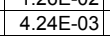 & 47.3 & 99.02 & 90.15 & 1.5 & 163.4 & 381.8 \\
\hline 115 & 45.97 & 3000 & 1010 & 74.356764 & 2.7266 & \begin{tabular}{|l|}
110.009 \\
\end{tabular} & 681.77 & 5125 & 0.1 & 1950 & 75000 & 1237500 & 0.0692 & 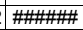 & 2.352 & $1.44 \mathrm{E}-02$ & 14.12 & 93.28 & 32.76 & 0 & 48 & 262.2 \\
\hline 116 & 47.77 & 3000 & 1700 & 163.638331 & 7.1955 & \begin{tabular}{|l|}
131.916 \\
\end{tabular} & 568.54 & 512 & 0.1 & 1950 & 75000 & 1625000 & 0.0666 & \#\#\#\#\# & 4.13 & $5.71 \mathrm{E}-03$ & 5 & 96.94 & 69.44 & 0 & 121.4 & 443.1 \\
\hline 117 & 31.16 & 3000 & 1200 & & 18.3713 & \begin{tabular}{|l|}
94.6429 \\
\end{tabular} & 792.45 & 5125 & 0.1 & 1950 & 750 & 7500 & & & 2.16 & & & 99.14 & & .14 & 146.5 & \\
\hline 118 & 26.8 & 3000 & 760 & 87.835622 & 4.8689 & \begin{tabular}{|l|}
166.296 \\
\end{tabular} & 451 & 5125 & 0.1 & 1950 & 75000 & 2300000 & 0.1187 & 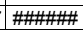 & 1.645 & $1.15 \mathrm{E}-02$ & 21.28 & 94.31 & 43.08 & & 60.3 & 314.6 \\
\hline
\end{tabular}




\begin{tabular}{|c|c|c|c|c|c|c|c|c|c|c|c|c|c|c|c|c|c|c|c|c|c|c|}
\hline Realization & $\begin{array}{c}\text { Velocity } \\
(\mathrm{m} / \mathrm{y})\end{array}$ & \begin{tabular}{|c|} 
Fixed Source \\
Concentratio \\
$\mathrm{n}(\mathrm{mg} / \mathrm{L})$
\end{tabular} & $\begin{array}{c}\text { Time of } \\
\text { Maximum at } \\
\text { Compliance } \\
\text { Boundary } \\
(y)\end{array}$ & \begin{tabular}{|c|} 
Maximum \\
Concentration \\
Compliance \\
Boundary \\
$(\mu \mathrm{g} / \mathrm{L})$
\end{tabular} & $\begin{array}{c}\text { Maximum } \\
\text { Release Rate } \\
\text { at Compliance } \\
\text { Boundary } \\
(\mathrm{Kg} / \mathrm{y})\end{array}$ & \begin{tabular}{|c|} 
\\
Source \\
Rate \\
$(\mathrm{Kg} / \mathrm{y})$ \\
\end{tabular} & $\begin{array}{c}\text { Source } \\
\text { Duration } \\
\text { (y) }\end{array}$ & $\begin{array}{c}\text { 1-D } \\
\text { Streamtube } \\
\text { Length } \\
\text { (m) }\end{array}$ & $\begin{array}{c}\text { Base } \\
\text { Porosity } \\
\left(\mathrm{m}^{3} / \mathrm{m}^{3}\right)\end{array}$ & \begin{tabular}{|c|} 
Bulk \\
Densty \\
$\left(\mathrm{Kg} / \mathrm{m}^{3}\right)$ \\
\end{tabular} & $\begin{array}{c}\text { Remaining } \\
\text { Carbon } \\
\text { Tetrachloride } \\
\text { Source } \\
(\mathrm{Kg})\end{array}$ & $\begin{array}{c}\begin{array}{c}\text { Volume at or } \\
\text { above Fixed } \\
\text { Source } \\
\text { Concentration } \\
(\mathrm{m} 3)\end{array} \\
\end{array}$ & $\begin{array}{l}\text { Porosity } \\
\left(\mathrm{m}^{3} / \mathrm{m}^{3}\right)\end{array}$ & $\begin{array}{c}\mathrm{Kd} \\
\left(\mathrm{m}^{3} / \mathrm{Kg}\right)\end{array}$ & $\begin{array}{l}\text { Retardatio } \\
n\end{array}$ & \begin{tabular}{|c|} 
First \\
Order \\
Rate \\
Constant \\
$(1 / y)$ \\
\end{tabular} & $\begin{array}{l}\text { Longitudinal } \\
\text { Dispersivity } \\
\text { (m) }\end{array}$ & \begin{tabular}{|c|} 
Required \\
Source \\
Cleanup \\
Percentage \\
$(5 \mu \mathrm{g} / \mathrm{L}$ \\
Compliance $)$ \\
\end{tabular} & \begin{tabular}{|c|} 
Required \\
Source \\
Cleanup \\
Percentage \\
$(50 \mu \mathrm{g} / \mathrm{L}$ \\
Compliance \\
) \\
\end{tabular} & \begin{tabular}{|c|} 
Required \\
Source \\
Cleanup \\
Percentage \\
$(500 \mu \mathrm{L} / \mathrm{L}$ \\
Compliance \\
) \\
\end{tabular} & \begin{tabular}{|c|} 
\\
Abiotic \\
Reaction \\
Half Time \\
$(y)$ \\
\end{tabular} & \begin{tabular}{|c|} 
Carbon \\
Tetrachloride \\
Travel Time \\
(y)
\end{tabular} \\
\hline 119 & 22.18 & 3000 & 1600 & 148.5437 & 7.8109 & 157.75 & \begin{tabular}{|l|} 
\\
\end{tabular} & & & & 75000 & 2125000 & \begin{tabular}{|l|}
0.1434 \\
\end{tabular} & \#\#\#\#曲 & 2.616 & 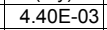 & 44.21 & \begin{tabular}{|c|}
96.63 \\
\end{tabular} & 66.34 & 0 & \begin{tabular}{|l|}
157.4 \\
\end{tabular} & 604.3 \\
\hline 120 & 30.47 & 3000 & 1130 & 182.138215 & 9.1212 & \begin{tabular}{|l|}
150.236 \\
\end{tabular} & 499.21 & 5125 & 0.1 & 1950 & 75000 & 1975000 & 0.1044 & \#\#\#\#\#曲 & 2.447 & \begin{tabular}{|c|}
$6.78 \mathrm{E}-03$ \\
\end{tabular} & 49.77 & 97.25 & 72.55 & 0 & 102.2 & 411.6 \\
\hline 121 & 43.58 & 3000 & 1570 & 25.102214 & 0.908 & \begin{tabular}{|l|}
108.522 \\
\end{tabular} & 691.1 & 5125 & 0.1 & 1950 & 75000 & 1212500 & 0.073 & \#\#\#\#\# & 3.711 & \begin{tabular}{|c|}
$1.19 \mathrm{E}-02$ \\
\end{tabular} & 42.27 & 80.08 & 0 & 0 & 58.1 & 436.4 \\
\hline 122 & 22.89 & 3000 & 1000 & 86.019785 & 3.5058 & \begin{tabular}{|l|}
122.267 \\
\end{tabular} & 613.41 & 5125 & 0.1 & 1950 & 75000 & 1450000 & 0.139 & \#\#\#\#\#曲 & 1.854 & \begin{tabular}{|l|}
$9.25 \mathrm{E}-03$ \\
\end{tabular} & 68.23 & 94.19 & 41.87 & & 75 & 415.2 \\
\hline 123 & 49.86 & 3000 & 1230 & \begin{tabular}{|l|l|}
111.462296 \\
\end{tabular} & 5.5819 & \begin{tabular}{|l|}
150.236 \\
\end{tabular} & 499.21 & 5125 & 0.1 & 1950 & 75000 & 1975000 & \begin{tabular}{|l|}
0.0638 \\
\end{tabular} & \#\#\#\#曲 & 3.239 & \begin{tabular}{|l|}
$9.97 \mathrm{E}-03$ \\
\end{tabular} & 23.78 & 95.51 & 55.14 & 0 & 69.5 & 332.9 \\
\hline 124 & 26.78 & 3000 & 1920 & 8.335562 & 0.3814 & \begin{tabular}{|l|}
137.274 \\
\end{tabular} & 546.35 & 5125 & 0.1 & 1950 & 75000 & 1725000 & \begin{tabular}{|l|}
0.1188 \\
\end{tabular} & 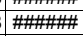 & 3.432 & \begin{tabular}{|l|l|} 
& $9.32 \mathrm{E}-03$ \\
\end{tabular} & 41.73 & 40.02 & 0 & 0 & 74.4 & 656.8 \\
\hline 125 & 60.37 & 3000 & 1460 & 96.640019 & 3.5676 & \begin{tabular}{|l|}
110.748 \\
\end{tabular} & 677.21 & 5125 & 0.1 & 1950 & 75000 & 1250000 & 0.0527 & \#\#\#\#曲 & 4.484 & \begin{tabular}{|l|}
$9.58 \mathrm{E}-03$ \\
\end{tabular} & 73.56 & 94.83 & 48.26 & 0 & 72.3 & 380.7 \\
\hline 126 & 23.88 & 3000 & 1020 & 122.606895 & 3.3263 & \begin{tabular}{|l|}
81.3886 \\
\end{tabular} & 921.51 & 5125 & 0.1 & 1950 & 75000 & 787500 & 0.1332 & \#\#\#\#曲 & 1.295 & \begin{tabular}{|l|}
$.21 \mathrm{E}-02$ \\
\end{tabular} & 58.57 & 95.92 & 59.22 & & 57.5 & 277.9 \\
\hline 127 & 53.74 & 3000 & 1040 & 51.151637 & 1.7991 & \begin{tabular}{|l|}
105.518 \\
\end{tabular} & 710.78 & 5125 & 0.1 & 1950 & 75000 & 1162500 & 0.0592 & \#\#\#\#\#五 & 3.104 & \begin{tabular}{|l|}
$1.65 \mathrm{E}-02$ \\
\end{tabular} & 86.02 & 90.23 & 2.25 & 0 & 42 & 296.1 \\
\hline 128 & 62.62 & 3000 & 1140 & 769.25838 & 29.1503 & \begin{tabular}{|l|}
113.682 \\
\end{tabular} & 659.73 & 5125 & 0.1 & 1950 & 75000 & 1300000 & 0.0508 & \#\#\#\#\#五 & 3.162 & \begin{tabular}{|l|}
$5.30 \mathrm{E}-03$ \\
\end{tabular} & 28.81 & 99.35 & 93.5 & 35 & 130.7 & 258.8 \\
\hline 129 & 28.84 & 3000 & 1000 & 379.551173 & 10.7284 & \begin{tabular}{|l|}
84.7981 \\
\end{tabular} & 884.45 & 5125 & 0.1 & 1950 & 75000 & 837500 & 0.1103 & \#\#\#\#曲 & 1.465 & 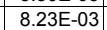 & 61.96 & 98.68 & 86.83 & 0 & 84.2 & 260.4 \\
\hline 130 & 23.76 & 3000 & 690 & 78.099195 & 3.5043 & \begin{tabular}{|l|}
134.609 \\
\end{tabular} & 557.17 & 5125 & 0.1 & 1950 & 75000 & 1675000 & 0.1339 & \#\#\#\#\#曲 & 1.258 & \begin{tabular}{|l|l|}
$1.39 \mathrm{E}-02$ \\
\end{tabular} & 41.03 & 93.6 & 35.98 & 0 & 49.7 & 271.3 \\
\hline 131 & 25.51 & 3000 & 760 & 43.898205 & 2.5725 & \begin{tabular}{|l|}
175.802 \\
\end{tabular} & 426.62 & 5125 & 0.1 & 1950 & 75000 & 2500000 & \begin{tabular}{|l|}
0.1247 \\
\end{tabular} & \#\#\#\#\# & 1.698 & $1.31 \mathrm{E}-02$ & 41.95 & 88.61 & 0 & & 53 & 341 \\
\hline 132 & 47.27 & 3000 & 1940 & 2.555846 & 0.1054 & \begin{tabular}{|l|}
123.668 \\
\end{tabular} & 606.46 & 5125 & 0.1 & 1950 & 75000 & 1475000 & \begin{tabular}{|l|}
0.0673 \\
\end{tabular} & \#\#\#\#\#曲 & 4.812 & \begin{tabular}{|l|}
$1.55 \mathrm{E}-02$ \\
\end{tabular} & 37.09 & 0 & 0 & 0 & 44.9 & 521.7 \\
\hline 133 & 38.38 & 3000 & 1560 & 48.054178 & 2.467 & \begin{tabular}{|l|}
154.016 \\
\end{tabular} & 486.96 & 5125 & 0.1 & 1950 & 75000 & 2050000 & \begin{tabular}{|l|}
0.0829 \\
\end{tabular} & \#\#\#\#\# & 3.842 & \begin{tabular}{|l|} 
\\
\end{tabular} & 70.23 & 89.6 & 0 & 0 & 88.2 & 513 \\
\hline 134 & 23.94 & 3000 & 2720 & 3.83672 & 0.2173 & \begin{tabular}{|l|}
169.892 \\
\end{tabular} & 441.46 & 5125 & 0.1 & 1950 & 75000 & 2375000 & 0.1329 & 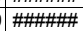 & 4.025 & \begin{tabular}{|l|}
$7.43 \mathrm{E}-03$ \\
\end{tabular} & 35.24 & 0 & 0 & 0 & 93.3 & 861.7 \\
\hline 135 & $\begin{array}{r}20.54 \\
34.5 \\
\end{array}$ & 3000 & 1040 & \begin{tabular}{|r|}
5.158198 \\
873.1588
\end{tabular} & $\begin{array}{r}.2710 \\
37.8016\end{array}$ & \begin{tabular}{|l|}
129.879 \\
\end{tabular} & $\begin{array}{l}577.46 \\
577.46\end{array}$ & 5125 & 0.1 & 1950 & 75000 & 1587500 & 0.0922 & \#\#\#\#曲 & 2.352 & \begin{tabular}{|l|}
$1.430 \mathrm{~L}-00$ \\
$3.30 \mathrm{E}$ \\
\end{tabular} & $\begin{array}{l}03.24 \\
74.31\end{array}$ & 99.43 & 94.27 & 42.74 & 209.8 & 349.4 \\
\hline 135 & \begin{tabular}{r|r|}
274.14 \\
27
\end{tabular} & 3000 & $\frac{1040}{1220}$ & \begin{tabular}{|}
0.178198 \\
79.241231
\end{tabular} & $\begin{array}{r}3.00100 \\
2.5209\end{array}$ & \begin{tabular}{|l|}
125.8399 \\
95.439 \\
\end{tabular} & $\begin{array}{l}011.46 \\
785.84\end{array}$ & $\begin{array}{l}5125 \\
5125\end{array}$ & $\begin{array}{l}0.1 \\
0.1\end{array}$ & $\frac{1950}{1950}$ & 75000 & $\frac{1500000}{10000}$ & $\begin{array}{l}0.0922 \\
0.1172\end{array}$ & 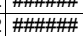 & $\frac{2.532}{2.053}$ & \begin{tabular}{|l|}
$3.608 \mathrm{E}-03$ \\
9.683 \\
\end{tabular} & $\begin{array}{l}24.34 \\
22.44\end{array}$ & \begin{tabular}{|l|}
93.45 \\
93.69
\end{tabular} & $\begin{array}{r}44.27 \\
36.9 \\
\end{array}$ & $\begin{array}{r}42 . .4 \\
0\end{array}$ & $\begin{array}{r}209.8 \\
71.6 \\
\end{array}$ & $\begin{array}{l}349.4 \\
387.7 \\
\end{array}$ \\
\hline 137 & 61.3 & 3000 & 1220 & 640.447461 & 27.2885 & \begin{tabular}{|l|}
127.826 \\
\end{tabular} & 586.74 & 5125 & 0.1 & 1950 & 75000 & 1550000 & 0.0519 & \#\#\#\#曲 & 3.476 & \begin{tabular}{|l|}
$4.97 E-03$ \\
\end{tabular} & 37.88 & 99.22 & 92.19 & 21.93 & 139.5 & 290.6 \\
\hline 138 & 45.51 & 3000 & 1000 & \begin{tabular}{|l|}
238.196175 \\
\end{tabular} & $\begin{array}{r}9.199 \\
\end{array}$ & \begin{tabular}{|l|}
115.858 \\
\end{tabular} & 647.34 & 5125 & 0.1 & 1950 & 75000 & 1337500 & 0.0699 & 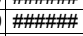 & 2.682 & \begin{tabular}{|l|} 
\\
\end{tabular} & 84.52 & 97.9 & 79.01 & 0 & 76.3 & 302 \\
\hline 139 & 23.72 & 3000 & 1210 & 160.130072 & 8.3871 & \begin{tabular}{|l|}
157.131 \\
\end{tabular} & 477.31 & 5125 & 0.1 & 1950 & 75000 & 2112500 & 0.1341 & \#\#\#\#曲 & 2.213 & \begin{tabular}{|l|l|}
$6.09 \mathrm{E}-03$ \\
\end{tabular} & 31.74 & 96.88 & 68.78 & 0 & 113.9 & 478 \\
\hline 140 & 22.28 & 3000 & 1030 & 206.652919 & 9.9521 & \begin{tabular}{|l|}
144.475 \\
\end{tabular} & 519.12 & 5125 & 0.1 & 1950 & 75000 & 1862500 & \begin{tabular}{|l|}
0.1428 \\
\end{tabular} & \#\#\#\#\# & 1.869 & \begin{tabular}{|l|}
$6.37 \mathrm{E}-03$ \\
\end{tabular} & 33.22 & 97.58 & 75.8 & 0 & 108.8 & 430 \\
\hline 141 & 25.64 & 3000 & 1000 & 344.906238 & 14.6959 & \begin{tabular}{|l|}
127.826 \\
\end{tabular} & 586.74 & 5125 & 0.1 & 1950 & 75000 & 1550000 & \begin{tabular}{|l|}
0.1241 \\
\end{tabular} & \#\#\#\#\#曲 & 1.878 & 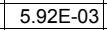 & 39.15 & 98.55 & 85.5 & & 117.1 & 375.5 \\
\hline 142 & 29.93 & 3000 & 1460 & 65.893323 & 3.5325 & \begin{tabular}{|l|}
160.828 \\
\end{tabular} & 466.34 & 5125 & 0.1 & 1950 & 75000 & 2187500 & 0.1063 & \#\#\#\#\# & 3.091 & \begin{tabular}{|l|}
$6.85 \mathrm{E}-03$ \\
\end{tabular} & 61.91 & 92.41 & 24.12 & 0 & 101.2 & 529.2 \\
\hline 143 & 22.06 & 3000 & 1630 & 133.041147 & 5.4222 & \begin{tabular}{|l|}
122.267 \\
\end{tabular} & 613.41 & 5125 & 0.1 & 1950 & 75000 & 1450000 & 0.1442 & \#\#\#\#曲 & 2.646 & 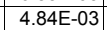 & 58.49 & 96.24 & 62.42 & 0 & 143.3 & 614.6 \\
\hline 144 & 42.19 & 3000 & 2030 & 0.396569 & 0.0193 & \begin{tabular}{|l|}
145.765 \\
\end{tabular} & 514.53 & 5125 & 0.1 & 1950 & 75000 & 1887500 & 0.0754 & 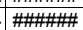 & 4.771 & \begin{tabular}{|l|}
$1.78 \mathrm{E}-02$ \\
\end{tabular} & 31.3 & 0 & 0 & 0 & 39 & 579.5 \\
\hline 145 & 44.31 & 3000 & 2170 & 1.54559 & 0.0516 & \begin{tabular}{|l|}
100.154 \\
\end{tabular} & 748.85 & 5125 & 0.1 & 1950 & 75000 & 1075000 & 0.0718 & 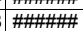 & 5.047 & \begin{tabular}{|l|}
$1.56 \mathrm{E}-02$ \\
\end{tabular} & 39.43 & 0 & 0 & 0 & 44.4 & 583.8 \\
\hline 146 & 39.82 & 3000 & 1450 & 16.309995 & 0.5232 & 96.2335 & 779.35 & 5125 & 0.1 & 1950 & 75000 & 1012500 & 0.0799 & 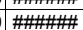 & 3.571 & \begin{tabular}{|l|}
$1.38 \mathrm{E}-02$ \\
\end{tabular} & $\begin{array}{ll}71.64 \\
71.64\end{array}$ & 69.34 & 0 & 0 & $\begin{array}{l}45.4 \\
50.3\end{array}$ & 459.6 \\
\hline 147 & 33.45 & 3000 & 1430 & 4.894969 & 0.2674 & \begin{tabular}{|l|}
163.877 \\
\end{tabular} & 457.66 & 5125 & 0.1 & 1950 & 75000 & 2250000 & 0.0951 & \#\#\#\#\#曲 & 3.043 & \begin{tabular}{|l|}
$1.44 \mathrm{E}-02$ \\
\end{tabular} & 22.51 & 0 & 0 & & 48.2 & 466.2 \\
\hline 148 & $\begin{array}{l}0.4 .52 \\
43.52\end{array}$ & 3000 & 1040 & $\begin{array}{l}4.00450919 \\
173.569\end{array}$ & 8.3588 & \begin{tabular}{|l|}
144.475 \\
\end{tabular} & 519.12 & 5125 & 0.1 & 1950 & 75000 & 1862500 & 0.0731 & 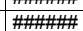 & 2.808 & \begin{tabular}{|l|} 
\\
\end{tabular} & 58.11 & 97.12 & 71.19 & & $\begin{array}{l}4.4 .3 \\
78.3\end{array}$ & 330.7 \\
\hline 149 & 21.89 & 3000 & 1680 & 242.319498 & 7.7731 & \begin{tabular}{|l|}
96.2335 \\
\end{tabular} & 779.35 & 5125 & 0.1 & 1950 & 75000 & 1012500 & 0.1453 & 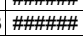 & 2.625 & \begin{tabular}{|l|}
$3.97 \mathrm{E}-03$ \\
\end{tabular} & 79.41 & 97.94 & 79.37 & 0 & 174.8 & 614.5 \\
\hline 150 & 36.78 & 3000 & 760 & 159.502043 & 6.1599 & \begin{tabular}{|l|}
115.858 \\
\end{tabular} & 647.34 & 5125 & 0.1 & 1950 & 75000 & 1337500 & 0.0865 & \#\#\#\#曲 & 1.607 & \begin{tabular}{|c|}
$1.41 \mathrm{E}-02$ \\
\end{tabular} & 80.46 & \begin{tabular}{|c|}
96.87 \\
\end{tabular} & 68.65 & & 49.3 & 224 \\
\hline 151 & 39.57 & 3000 & 1010 & 22.699888 & 0.8712 & \begin{tabular}{|l|}
115.135 \\
\end{tabular} & 651.41 & 5125 & 0.1 & 1950 & 75000 & 1325000 & 0.0804 & \#\#\#\#\# & 2.588 & $1.69 \mathrm{E}-02$ & 67.82 & 77.97 & 0 & 0 & 41 & 335.2 \\
\hline 152 & 25.47 & 3000 & 2140 & 5.591471 & 0.2484 & \begin{tabular}{|l|}
133.266 \\
\end{tabular} & 562.78 & 5125 & 0.1 & 1950 & 75000 & 1650000 & 0.1249 & \#\#\#\#\# & 3.518 & \begin{tabular}{|l|}
$9.18 \mathrm{E}-03$ \\
\end{tabular} & 35.62 & 10.58 & 0 & 0 & 75.5 & 707.8 \\
\hline 153 & 24.47 & 3000 & 880 & 156.700185 & 5.0266 & \begin{tabular}{|l|}
96.2335 \\
\end{tabular} & 779.35 & 5125 & 0.1 & 1950 & 75000 & 1012500 & 0.13 & \#\#\#\#曲 & 1.269 & \begin{tabular}{|l|}
$1.16 \mathrm{E}-02$ \\
\end{tabular} & 61.52 & 96.81 & 68.09 & 0 & 59.7 & 265.7 \\
\hline 154 & 55.42 & 3000 & 2180 & $\begin{array}{r}52.82223 \\
\end{array}$ & $\begin{array}{l}.0222 \\
1.722\end{array}$ & \begin{tabular}{|l|}
97.8112 \\
\end{tabular} & 766.78 & 5125 & 0.1 & 1950 & 75000 & 1037500 & 0.0574 & 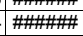 & 4.996 & \begin{tabular}{|l|l|} 
& $1.70 \mathrm{E}-03$ \\
\end{tabular} & 30.88 & 90.53 & 5.34 & 0 & $\begin{array}{l}39.6 \\
79.6\end{array}$ & 462 \\
\hline 155 & 56.91 & 3000 & 1010 & 1451.199984 & 55.3438 & \begin{tabular}{|l|}
114.41 \\
\end{tabular} & 655. & & & & 75000 & & 0.0559 & \#\#\#\#曲 & 2.696 & & 37.69 & 99.66 & 96.55 & 65.55 & 231.1 & 242.8 \\
\hline 156 & 21.57 & 3000 & 1740 & & 0.2621 & 104.76 & 715.92 & 5125 & 0 & 195 & 750 & & 0.1475 & \#\#\#\#\#五 & 3.01 & & 73.1 & 33.38 & 0 & & 70.4 & 715.3 \\
\hline 157 & 28.05 & 3000 & 1720 & & 2.3248 & \begin{tabular}{|l|}
119.44 \\
\end{tabular} & 627.93 & 512 & 0.1 & 1950 & 75000 & 1400000 & 0.1134 & & 3.053 & 7.0 & 38.86 & 91.44 & 14.37 & 0 & 98.1 & 557 \\
\hline 158 & 33.88 & 3000 & 700 & 250.74735 & 13.0295 & \begin{tabular}{|l|}
155.889 \\
\end{tabular} & 481.11 & 5125 & 0.1 & 1950 & 750 & & & \#\#\# & 1.655 & 1.01 & 27. & & 80.06 & & 68.4 & 250.4 \\
\hline 159 & 25.09 & 3000 & 750 & 55.872309 & \begin{tabular}{|l|}
2.7387 \\
\end{tabular} & \begin{tabular}{|l|}
147.05 \\
\end{tabular} & 510 & 5125 & 0.1 & 1950 & 75000 & 2500 & 0.1268 & 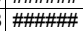 & 1.534 & \begin{tabular}{|l|}
$1.33 \mathrm{E}-02$ \\
\end{tabular} & 41.12 & 91.05 & 10.51 & 0 & 52 & 313.3 \\
\hline 160 & 25.23 & 3000 & 1330 & 6.83162 & 0.3035 & \begin{tabular}{|l|}
133.266 \\
\end{tabular} & 562. & 5125 & 0.1 & 1950 & 75000 & 1650000 & 0.1261 & \#\#\#\#曲 & 2.656 & \begin{tabular}{|l|}
$1.27 \mathrm{E}-02$ \\
\end{tabular} & 54.92 & 26.81 & 0 & 0 & 54.5 & 539.5 \\
\hline 161 & 39.92 & 3000 & 1250 & 16.645227 & 0.9393 & \begin{tabular}{|l|}
169.295 \\
\end{tabular} & 443. & 5125 & 0.1 & 1950 & 75000 & 2362500 & 0.0797 & \#\#\#\#\#曲 & 3.154 & \begin{tabular}{|c|}
$.34 \mathrm{E}-02$ \\
\end{tabular} & 33.49 & 69.96 & 0 & 0 & 51.9 & 405 \\
\hline 162 & 34.1 & 3000 & 970 & 591.9662 & \begin{tabular}{|l|l|}
18.1993 \\
\end{tabular} & \begin{tabular}{|l|}
92.2314 \\
\end{tabular} & 813.17 & 5125 & 0.1 & 1950 & 7500 & 950000 & 0.0933 & \#\#\#\#\# & 1.669 & \begin{tabular}{|l|}
$6.56 \mathrm{E}-03$ \\
\end{tabular} & 27.45 & 99.16 & 91.55 & 15.54 & 105.6 & 250.9 \\
\hline 163 & 38.15 & 3000 & 1340 & 546.9525 & 17.4004 & \begin{tabular}{|l|}
95.4399 \\
\end{tabular} & 785.84 & 5125 & 0.1 & 1950 & 7500 & 1000000 & 0.0834 & \#\#\#\# & 2.902 & 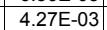 & 91.33 & 99.09 & 90.86 & 8.58 & 162.2 & 389.9 \\
\hline 164 & 22.66 & 3000 & 2470 & 0.025537 & 0.0012 & \begin{tabular}{|l|}
139.257 \\
\end{tabular} & 538.57 & 5125 & 0.1 & 1950 & 75000 & 1762500 & 0.1404 & \#\#\#\#曲 & 3.805 & $1.57 \mathrm{E}-02$ & 26.86 & 0 & & & 44.2 & 860.5 \\
\hline $\begin{array}{l}104 \\
165 \\
\end{array}$ & 28.13 & 3000 & 1590 & $\begin{array}{r}.05301 \\
28.972971 \\
\end{array}$ & $\begin{array}{l}0.2011 \\
1.2011 \\
\end{array}$ & \begin{tabular}{|l|}
13.24 .366 \\
124.36 \\
\end{tabular} & 603.06 & 5125 & $\begin{array}{l}0.1 \\
0.1 \\
\end{array}$ & 1950 & 75000 & 1487500 & \begin{tabular}{|l|}
0.1404 \\
0.1131 \\
\end{tabular} & 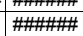 & $\begin{array}{l}0.005 \\
2.913 \\
\end{array}$ & \begin{tabular}{|l|}
$1.075 \mathrm{E}-02$ \\
$9.05 \mathrm{C}$ \\
\end{tabular} & $\begin{array}{l}30.000 \\
34.43\end{array}$ & 82.74 & 0 & 0 & $\begin{array}{l}4.4 \\
76.6\end{array}$ & 530.7 \\
\hline 166 & 26.01 & 3000 & 630 & 197.809533 & 8.8314 & \begin{tabular}{|l|}
133.938 \\
\end{tabular} & 559.96 & 5125 & 0.1 & 1950 & 75000 & 1662500 & \begin{tabular}{|l|}
0.1223 \\
\end{tabular} & \#\#\#\#\# & 1.117 & \begin{tabular}{|c|}
$1.29 \mathrm{E}-02$ \\
\end{tabular} & 78.7 & \begin{tabular}{|c|}
97.47 \\
\end{tabular} & 74.72 & 0 & 53.6 & 220 \\
\hline 167 & 24.66 & 3000 & 760 & & & \begin{tabular}{|l|}
124.366 \\
\end{tabular} & & & & 19 & & & 0.129 & & & & 38 & & & 0 & & 284.2 \\
\hline 168 & 50.98 & 3000 & 1270 & 430.225 & 12.4016 & \begin{tabular}{|l|}
86.4774 \\
\end{tabular} & 867 & 5125 & 0.1 & 19 & 750 & 2500 & 0.0624 & \#\# & 2.8 & & & & 88.38 & 0 & & 283 \\
\hline 169 & 24.49 & 3000 & 13 & 75.4 & & \begin{tabular}{|l|}
96.2335 \\
\end{tabular} & & 5125 & 0.1 & & 750 & 1012500 & 0.12 & \#\#\# & 2.38 & & 61. & 93. & 33.77 & 0 & 86.4 & 498 \\
\hline 170 & 31.85 & 3000 & 2180 & 71.3158 & 2.380 & \begin{tabular}{|l|}
100.154 \\
\end{tabular} & 748 & 5125 & 0.1 & 1950 & 750 & 1075000 & 0.09 & \#\#\#\#曲 & 3.917 & \begin{tabular}{|l|}
$5.74 \mathrm{E}-03$ \\
\end{tabular} & 55.14 & 92.99 & 29.89 & 0 & 120.8 & 630.3 \\
\hline 171 & 60.25 & 3000 & 2200 & 47.3361 & 2.3505 & \begin{tabular}{|l|}
148.966 \\
\end{tabular} & 503.47 & 5125 & 0.1 & 1950 & 75000 & 1950000 & 0.0528 & \#\#\#\#\# & 5.552 & \begin{tabular}{|l|} 
\\
\end{tabular} & 34.18 & 89.44 & 0 & 0 & 90.7 & 472.3 \\
\hline 172 & 24.74 & 3000 & 830 & 14.694078 & 0.6691 & \begin{tabular}{|l|}
136.61 \\
\end{tabular} & 549.01 & 5125 & 0.1 & 1950 & 75000 & 1712500 & 0.1286 & \#\#\#\#曲 & 1.604 & \begin{tabular}{|l|}
$.67 \mathrm{E}-02$ \\
\end{tabular} & 26.04 & 65.97 & 0 & 0 & 41.5 & 332.4 \\
\hline 173 & 23.51 & 3000 & 1380 & $\begin{array}{r}224.6222 \\
\end{array}$ & \begin{tabular}{|l|}
11.3907 \\
\end{tabular} & \begin{tabular}{|l|}
152.132 \\
\end{tabular} & 492.99 & 5125 & 0.1 & 1950 & 75000 & 2012500 & 0.1353 & 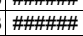 & 2.409 & \begin{tabular}{|l|l|}
$4.62 \mathrm{E}-03$ \\
\end{tabular} & 38.08 & 97.77 & 77.74 & 0 & 150 & 525 \\
\hline 174 & 21.64 & 3000 & 1680 & $\begin{array}{r}24.285596 \\
84.026\end{array}$ & 2.1139 & 75.2423 & $\begin{array}{l}492.35 \\
996.78 \\
\end{array}$ & 5125 & 0.1 & 1950 & 75000 & 700000 & \begin{tabular}{|c|}
0.147 \\
0.145
\end{tabular} & 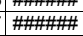 & 2.4228 & \begin{tabular}{|l|}
$4.02 L-00$ \\
$7.01 \mathrm{E}-03$ \\
\end{tabular} & 23.14 & 94.07 & 40.68 & & $\begin{array}{r}98.9 \\
\end{array}$ & 527.6 \\
\hline 175 & 42.25 & 3000 & 1950 & 27.222051 & 0.7541 & \begin{tabular}{|l|}
83.1021 \\
\end{tabular} & 902. & 512 & 0.1 & 1950 & 75000 & 812500 & 0.0753 & \#\#\#\#\# & 4.06 & \begin{tabular}{|l|}
$.05 \mathrm{E}-02$ \\
\end{tabular} & 41.28 & 81.63 & 0 & & 65.9 & 492.5 \\
\hline 176 & 25.43 & 3000 & 1690 & & & & 643.34 & 5125 & 0.1 & 1950 & & 1350000 & 0.1251 & & 2.832 & & & & 0 & & & \\
\hline 177 & 24.06 & 3000 & 970 & 214.014285 & 10.4445 & \begin{tabular}{|l|}
146.408 \\
\end{tabular} & 512.27 & 5125 & 0.1 & 1950 & 75000 & 1900000 & 0.1322 & \#\#\#\#曲 & 1.986 & $6.44 \mathrm{E}-03$ & 86.33 & 97.66 & 76.64 & & 107.6 & 422.9 \\
\hline
\end{tabular}




\begin{tabular}{|c|c|c|c|c|c|c|c|c|c|c|c|c|c|c|c|c|c|c|c|c|c|c|}
\hline Realization & $\begin{array}{c}\text { Velocity } \\
(\mathrm{m} / \mathrm{y})\end{array}$ & $\begin{array}{c}\text { Fixed Source } \\
\text { Concentratio } \\
\mathrm{n}(\mathrm{mg} / \mathrm{L})\end{array}$ & $\begin{array}{c}\text { Time of } \\
\text { Maximum at } \\
\text { Compliance } \\
\text { Boundary } \\
\text { (y) }\end{array}$ & $\begin{array}{c}\text { Maximum } \\
\text { Concentration } \\
\begin{array}{c}\text { Compliance } \\
\text { Boundary } \\
(\mu \mathrm{g} / \mathrm{L})\end{array} \\
\end{array}$ & $\begin{array}{c}\text { Maximum } \\
\text { Release Rate } \\
\text { at Compliance } \\
\text { Boundary } \\
(\mathrm{Kg} / \mathrm{y})\end{array}$ & \begin{tabular}{|c|} 
\\
Source \\
Rate \\
$(\mathrm{Kg} / \mathrm{y})$
\end{tabular} & $\begin{array}{c}\text { Source } \\
\text { Duration } \\
\text { (y) }\end{array}$ & $\begin{array}{c}\text { 1-D } \\
\text { Streamtube } \\
\text { Length } \\
\text { (m) }\end{array}$ & $\begin{array}{c}\text { Base } \\
\text { Porosity } \\
\left(\mathrm{m}^{3} / \mathrm{m}^{3}\right)\end{array}$ & $\begin{array}{c}\text { Bulk } \\
\text { Densty } \\
\left(\mathrm{Kg} / \mathrm{m}^{3}\right)\end{array}$ & $\begin{array}{c}\text { Remaining } \\
\text { Carbon } \\
\text { Tetrachloride } \\
\text { Source } \\
(\mathrm{Kg})\end{array}$ & $\begin{array}{c}\begin{array}{c}\text { Volume at or } \\
\text { above Fixed } \\
\text { Source } \\
\text { Concentration } \\
\text { (m3) }\end{array} \\
\end{array}$ & $\begin{array}{l}\text { Porosity } \\
\left(\mathrm{m}^{3} / \mathrm{m}^{3}\right)\end{array}$ & $\begin{array}{c}\mathrm{Kd} \\
\left(\mathrm{m}^{3} / \mathrm{Kg}\right)\end{array}$ & $\begin{array}{l}\text { Retardatio } \\
\text { n }\end{array}$ & \begin{tabular}{|c|} 
First \\
Order \\
Rate \\
Constant \\
$(1 / y)$ \\
\end{tabular} & $\begin{array}{l}\text { Longitudinal } \\
\text { Dispersivity } \\
\text { (m) }\end{array}$ & \begin{tabular}{|c|} 
Required \\
Source \\
Cleanup \\
Percentage \\
$(5 \mu \mathrm{g} / \mathrm{L}$ \\
Compliance $)$ \\
\end{tabular} & \begin{tabular}{|c|} 
Required \\
Source \\
Cleanup \\
Percentage \\
$(50 \mu \mathrm{g} / \mathrm{L}$ \\
Compliance \\
)
\end{tabular} & \begin{tabular}{|c|} 
Required \\
Source \\
Cleanup \\
Percentage \\
$(500 \mu \mathrm{L} / \mathrm{L}$ \\
Compliance \\
)
\end{tabular} & $\begin{array}{c} \\
\text { Abiotic } \\
\text { Reaction } \\
\text { Half Time } \\
\text { (y) }\end{array}$ & $\begin{array}{c}\text { Carbon } \\
\text { Tetrachloride } \\
\text { Travel Time } \\
\text { (y) }\end{array}$ \\
\hline 178 & 29.98 & 3000 & $\begin{array}{ll} & 1390 \\
\end{array}$ & \begin{tabular}{|l|}
6.584243 \\
\end{tabular} & \begin{tabular}{|l|l}
3 & 0.3973 \\
\end{tabular} & \begin{tabular}{|l|l|}
3 & 181.037 \\
\end{tabular} & \begin{tabular}{|l|} 
\\
\end{tabular} & \begin{tabular}{|l|r|}
3 & 5125 \\
\end{tabular} & 0.1 & 1950 & 75000 & 2612500 & 0.1061 & \#\#\#\#\# & 3.312 & \begin{tabular}{|l|l|}
$1.20 \mathrm{E}-02$ \\
\end{tabular} & 67.5 & \begin{tabular}{|l|l|}
5 & 24.06 \\
\end{tabular} & \begin{tabular}{|l|l|}
5 & 0 \\
\end{tabular} & 0 & \begin{tabular}{|l|} 
\\
\end{tabular} & \begin{tabular}{|l|l|} 
& 566.2
\end{tabular} \\
\hline 179 & 23.12 & 3000 & 1210 & 6.739583 & 0.2072 & 92.2314 & 813.17 & 5125 & 0.1 & 1950 & 75000 & 950000 & \begin{tabular}{|l|l}
0.1376 \\
\end{tabular} & 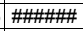 & 1.981 & \begin{tabular}{|l|}
$1.53 \mathrm{E}-02$ \\
\end{tabular} & 43.98 & 25.81 & 0 & 0 & 45.2 & 439.2 \\
\hline 180 & 38.1 & 3000 & 880 & 175.96896 & 7.2539 & 123.668 & 606.46 & 5125 & 0.1 & 1950 & 75000 & 1475000 & 0.0835 & \#\#\#\#\# & 2.074 & \begin{tabular}{|l|}
$1.07 E-02$ \\
\end{tabular} & 49.24 & 97.16 & 71.59 & 0 & 64.6 & 279 \\
\hline 181 & 59.58 & 3000 & 2100 & 60.352558 & 2.1832 & 108.522 & 691.1 & 5125 & 0.1 & 1950 & 75000 & 1212500 & 0.0534 & \#\#\#\#\#曲 & 6.026 & \begin{tabular}{|l|l}
$7.49 \mathrm{E}-03$ \\
\end{tabular} & 74.15 & 91.72 & 17.15 & 0 & 92.6 & 518.4 \\
\hline 182 & 22.79 & 3000 & 1240 & 560.005834 & 17.2167 & 92.2314 & 813.17 & 5125 & 0.1 & 1950 & 75000 & 950000 & 0.1396 & \#\#\#\#州 & 1.927 & $4.00 \mathrm{E}-03$ & 65.21 & 99.11 & 91.07 & 10.72 & 173.2 & 433.4 \\
\hline 183 & 53.11 & 3000 & 1850 & \begin{tabular}{|c|}
62.553179 \\
\end{tabular} & 1.9062 & 91.4206 & 820.38 & 5125 & 0.1 & 1950 & 75000 & 937500 & 0.0599 & 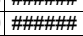 & 4.41 & \begin{tabular}{|l|l|}
$4.059-0$ \\
9.59
\end{tabular} & 41.02 & 92.01 & 20.07 & 0 & 72.3 & 425.5 \\
\hline 184 & 27.4 & 3000 & 1660 & 28.031246 & 1.2765 & 136.61 & 549.01 & 5125 & 0.1 & 1950 & 75000 & 1712500 & 0.1161 & \#\#\#\#\# & 3.164 & $8.06 \mathrm{E}-03$ & 53.97 & 82.16 & 0 & 0 & 86 & 591.7 \\
\hline 185 & 27.78 & 3000 & 1920 & 24.680797 & 0.6409 & 77.906 & 962.7 & 5125 & 0.1 & 1950 & 75000 & 737500 & 0.1145 & \#\#\#\#\#五 & 3.271 & $\begin{array}{l}9.02 \mathrm{E}-03 \\
\end{array}$ & 57.84 & 79.74 & 0 & 0 & 76.9 & 603.4 \\
\hline 186 & 27.57 & 3000 & 1030 & 50.988978 & 2.4665 & 145.121 & 516.81 & 5125 & 0.1 & 1950 & 75000 & 1875000 & 0.1154 & 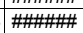 & 2.158 & \begin{tabular}{|l|l|}
3 & $1.07 \mathrm{E}-02$ \\
\end{tabular} & 39.34 & 90.19 & 1.94 & 0 & 64.7 & 401.2 \\
\hline 187 & 36.07 & 3000 & 850 & 239.166299 & 13.3534 & 167.499 & 447.76 & 5125 & 0.1 & 1950 & 75000 & 2325000 & 0.0882 & \#\#\#\#\# & 2.18 & $8.39 \mathrm{E}-03$ & 46.89 & 97.91 & 79.09 & 0 & 82.6 & 309.8 \\
\hline 188 & 30.62 & 3000 & 3270 & 0.183451 & 0.0089 & 145.121 & 516.81 & 5125 & 0.1 & 1950 & 75000 & 1875000 & $\begin{array}{l}0.1039 \\
\end{array}$ & \#\#\#\#\# & 4.985 & \begin{tabular}{|l|l|}
5 & $1.22 \mathrm{E}-02$ \\
\end{tabular} & 19.2 & 0 & 0 & 0 & 56.8 & 834.3 \\
\hline 189 & 44.49 & 3000 & 1050 & 45.478891 & 2.3726 & \begin{tabular}{|l|} 
\\
\end{tabular} & 479.2 & 5125 & 0.1 & 1950 & 75000 & 2100000 & 0.0715 & 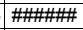 & 2.894 & \begin{tabular}{|l|}
$1.33 \mathrm{E}-02$ \\
\end{tabular} & 39.81 & 89.01 & 0 & 0 & 52.1 & 333.3 \\
\hline 190 & 40.47 & 3000 & 1000 & 960.551199 & 31.8186 & 99.3762 & 754.71 & 5125 & 0.1 & 1950 & 75000 & 1062500 & $\begin{array}{l}0.0786 \\
\end{array}$ & \#\#\#\#\# & 2.04 & $4.48 \mathrm{E}-03$ & 36.19 & 99.48 & 94.79 & 47.95 & 154.6 & 258.2 \\
\hline 191 & 21.76 & 3000 & 1430 & $\begin{array}{r}5.863273 \\
\end{array}$ & 2.3534 & 196.865 & 380.97 & 5125 & 0.1 & 1950 & 75000 & 2962500 & $\begin{array}{l}0.1462 \\
\end{array}$ & \#\#\#\#曲 & 2.547 & $6.98 \mathrm{E}-03$ & 46.83 & 86.06 & 0 & 0 & 99.3 & 600 \\
\hline 192 & 34.58 & 3000 & 2890 & $\begin{array}{r}4.238573 \\
\end{array}$ & 0.1512 & 107.025 & 700.77 & 5125 & 0.1 & 1950 & 75000 & 1187500 & 0.092 & \#\#\#\#\# & 4.794 & $9.50 \mathrm{E}-03$ & 25.42 & 0 & 0 & 0 & 73 & 710.5 \\
\hline 193 & 35.39 & 3000 & 640 & 213.496384 & 10.8265 & 152.132 & 492.99 & 5125 & 0.1 & 1950 & 75000 & 2012500 & $\begin{array}{l}0.0899 \\
0.08\end{array}$ & 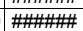 & 1.534 & $1.23 \mathrm{E}-02$ & 42.8 & 97.66 & 76.58 & 0 & 56.4 & 222.2 \\
\hline 194 & 21.63 & 3000 & 870 & 13.009316 & 0.6706 & 154.641 & 484.99 & 5125 & 0.1 & 1950 & 75000 & 2062500 & 0.1471 & \#\#m\#\# & 1.796 & \begin{tabular}{|l|l}
$1.43 \mathrm{E}-02$ \\
\end{tabular} & 65.09 & 61.57 & 0 & 0 & 48.6 & 425.6 \\
\hline 195 & 23.44 & 3000 & 770 & 330.02986 & 12.9827 & 118.014 & 635.52 & 5125 & 0.1 & 1950 & 75000 & 1375000 & 0.1357 & (m)m\#\# & 1.266 & $\begin{array}{l}.40 \mathrm{D}-0 \mathrm{C} \\
8.20 \mathrm{E}-03\end{array}$ & 50.11 & 98.48 & 84.85 & 0 & \begin{tabular}{|l|}
4.0 \\
84.6
\end{tabular} & $\begin{array}{l}40.0 \\
276.7\end{array}$ \\
\hline 196 & 29.19 & 3000 & 830 & 35.109443 & 1.6984 & \begin{tabular}{|l|}
45.121 \\
\end{tabular} & 516.81 & 5125 & 0.1 & 1950 & 75000 & 1875000 & 0.109 & \#\#\#\#曲 & 1.785 & \begin{tabular}{|l|l|}
5 & $1.48 \mathrm{E}-02$ \\
\end{tabular} & 25.94 & 85.76 & 0 & 0 & 47 & 313.4 \\
\hline 197 & 28.23 & 3000 & 1350 & 14.65399 & 0.6411 & 131.239 & 571.48 & 5125 & 0.1 & 1950 & 75000 & 1612500 & $\begin{array}{l}0.1127 \\
\end{array}$ & \#\#\#\#\#曲 & 2.516 & \begin{tabular}{|l|l|}
$1.21 \mathrm{E}-02$ \\
\end{tabular} & 18.66 & 65.88 & 0 & 0 & 57.1 & 456.8 \\
\hline 198 & 55.81 & 3000 & 2020 & 3.758324 & 0.1274 & \begin{tabular}{|l|} 
\\
\end{tabular} & 737.46 & 5125 & 0.1 & 1950 & 75000 & 1100000 & 0.057 & \#\#\#\#\# & 5.052 & $21.62 \mathrm{E}-02$ & 29.39 & 0 & 0 & 0 & 42.7 & 463.9 \\
\hline 199 & 33.81 & 3000 & 1020 & 72.09118 & 4.0251 & \begin{tabular}{|l|}
167.499 \\
\end{tabular} & 447.76 & 5125 & 0.1 & 1950 & 75000 & 2325000 & $\begin{array}{l}0.0941 \\
\end{array}$ & \#\#\#\#\# & 2.448 & \begin{tabular}{|l|l|}
$31.04 \mathrm{E}-02$ \\
\end{tabular} & 40.07 & 93.06 & 30.64 & 0 & 66.8 & 371.2 \\
\hline 200 & 48.94 & 3000 & 830 & 269.103884 & 12.4324 & 138.597 & 541.14 & 5125 & 0.1 & 1950 & 75000 & 1750000 & 0.065 & 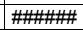 & 2.372 & $\begin{array}{l}2.03 \mathrm{E}-02 \\
\end{array}$ & 57.92 & 98.14 & 81.42 & 0 & 67.5 & 248.3 \\
\hline 201 & 26.96 & 3000 & 770 & 307.234718 & 15.6445 & 152.761 & 490.96 & 5125 & 0.1 & 1950 & 75000 & 2025000 & 0.118 & \#\#\#\#" & 1.663 & \begin{tabular}{|l|}
$7.48 \mathrm{E}-03$ \\
\end{tabular} & 52.69 & 98.37 & 83.73 & 0 & 92.7 & 316.1 \\
\hline 202 & 44.37 & 3000 & 2490 & 2.55516 & 0.1124 & 131.916 & 568.54 & 5125 & 0.1 & 1950 & 75000 & 1625000 & $\begin{array}{l}0.0717 \\
\end{array}$ & \#\#\#\#\#曲 & 5.351 & $1.22 \mathrm{E}-02$ & 31.54 & 0 & 0 & 0 & 56.7 & 618.1 \\
\hline 203 & 24.05 & 3000 & 1590 & 63.892205 & 2.9236 & 137.274 & 546.35 & 5125 & 0.1 & 1950 & 75000 & 1725000 & 0.1323 & 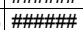 & 2.633 & \begin{tabular}{|l|l|}
3 & $6.81 \mathrm{E}-03$ \\
\end{tabular} & 27.58 & 92.17 & 21.74 & 0 & 101.8 & 561.1 \\
\hline 204 & 39.08 & 3000 & 1380 & 44.806489 & 1.7412 & 116.579 & 643.34 & 5125 & 0.1 & 1950 & 75000 & 1350000 & 0.0814 & 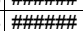 & 3.396 & $1.04 \mathrm{E}-02$ & 68.97 & 88.84 & 0 & 0 & 66.5 & 445.3 \\
\hline 205 & 56.31 & 3000 & 820 & 779.949386 & 32.5139 & 125.062 & 599.7 & 5125 & 0.1 & 1950 & 75000 & 1500000 & 0.0565 & 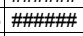 & 2.261 & $\mid 6.69 \mathrm{E}-03$ & 37.96 & 99.36 & 93.59 & 35.89 & 103.6 & 205.8 \\
\hline 206 & 49.55 & 3000 & 1300 & 456.154906 & \begin{tabular}{|l|}
19.8518 \\
\end{tabular} & \begin{tabular}{|l|}
130.56 \\
\end{tabular} & 574.45 & 5125 & 0.1 & 1950 & 75000 & 1600000 & 0.0642 & 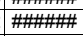 & 3.184 & $5.67 \mathrm{E}-03$ & 19.51 & 98.9 & 89.04 & 0 & 122.3 & 329.3 \\
\hline 207 & 24.14 & 3000 & 1200 & $\begin{array}{r}4.487036 \\
\end{array}$ & 0.2053 & 137.274 & 546.35 & 5125 & 0.1 & 1950 & 75000 & 1725000 & \begin{tabular}{|l|l|}
0.1318 \\
\end{tabular} & 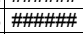 & 2.365 & $51.45 \mathrm{E}-02$ & 46.99 & 0 & 0 & 0 & 47.8 & 502.1 \\
\hline 208 & 32.01 & 3000 & 1300 & 11.005649 & 0.4277 & \begin{tabular}{|l|}
116.579 \\
\end{tabular} & 643.34 & 5125 & 0.1 & 1950 & 75000 & 1350000 & 0.0994 & \#\#\#\#\# & 2.898 & \begin{tabular}{|l|l|}
$31.39 \mathrm{E}-02$ \\
\end{tabular} & 56.77 & 54.57 & 0 & 0 & 50 & 464 \\
\hline 209 & 27.12 & 3000 & 790 & 436.188562 & 17.7771 & \begin{tabular}{|l|}
122.267 \\
\end{tabular} & 613.41 & 5125 & 0.1 & 1950 & 75000 & 1450000 & 0.1173 & \#\#\#\#\# & 1.47 & $7.13 \mathrm{E}-03$ & 47.97 & 98.85 & 88.54 & 0 & 97.3 & 277.8 \\
\hline 210 & 52.24 & 3000 & 670 & $\begin{array}{r}872.81002 \\
\end{array}$ & 26.3606 & 90.6061 & 827.76 & 5125 & 0.1 & 1950 & 75000 & 925000 & 0.0609 & \#\#\#\#" & 1.807 & $7.00 \mathrm{E}-03$ & 12.18 & 99.43 & 94.27 & 42.71 & 99 & 177.3 \\
\hline 211 & 58.05 & 3000 & 1570 & 592.419378 & 20.0832 & \begin{tabular}{|l|}
101.701 \\
\end{tabular} & 737.46 & 5125 & 0.1 & 1950 & 75000 & 1100000 & 0.0548 & \#\#\#\#" & 3.974 & $4.06 \mathrm{E}-03$ & 47.9 & 99.16 & 91.56 & 15.6 & 170.6 & 350.8 \\
\hline 212 & 36.48 & 3000 & 940 & 209.916358 & \begin{tabular}{|}
9.1356 \\
\end{tabular} & 130.56 & 574.45 & 5125 & 0.1 & 1950 & 75000 & 1600000 & 0.0872 & \#\#\#\#\#五 & 2.296 & $8.76 \mathrm{E}-03$ & 68.28 & 97.62 & 76.18 & 0 & 79.2 & 322.5 \\
\hline 213 & 29.03 & 3000 & 1680 & 31.929636 & 1.461 & \begin{tabular}{|l|}
137.274 \\
\end{tabular} & 546.35 & 5125 & 0.1 & 1950 & 75000 & 1725000 & 0.1096 & \#\#\#\#\# & 3.241 & $7.98 \mathrm{E}-03$ & 48.67 & 84.34 & 0 & 0 & 86.8 & 572.2 \\
\hline 214 & 26.53 & 3000 & 1080 & & 0.3256 & 148.966 & 503.47 & 5125 & 0.1 & 1950 & 75000 & 1950000 & 0.1199 & \#\#\#\#曲 & 2.437 & & 63.29 & 23.75 & 0 & 0 & 46.3 & 470.8 \\
\hline 215 & 39.18 & 3000 & 1590 & & 3.7499 & \begin{tabular}{|l|}
112.952 \\
\end{tabular} & 664 & 5125 & 0 & 195 & 75000 & 1287500 & 0.0812 & \#\#\#\#\#立 & 3.195 & 8.29 & 15.83 & 94.98 & 49.8 & 0 & 83.6 & 417.9 \\
\hline 216 & 27.5 & 3000 & 810 & 248.044 & 10.9629 & \begin{tabular}{|l|}
132.592 \\
\end{tabular} & 565.65 & 5125 & 0.1 & 1950 & & & 0.1157 & \#\# & 1.612 & 8.5 & 39. & 97.98 & 79.84 & 0 & 81 & \\
\hline 217 & 41.86 & 3000 & 600 & 496.266988 & 29.852 & \begin{tabular}{|l|}
180.459 \\
\end{tabular} & 415.61 & 5125 & 0.1 & 1950 & 75000 & 2600000 & 0.076 & \#\#\#\#\#曲 & 1.694 & \begin{tabular}{|l|}
$8.81 \mathrm{E}-03$ \\
\end{tabular} & 25.89 & 98.99 & 89.92 & 0 & 78.7 & 207.4 \\
\hline 218 & 23.97 & 3000 & 830 & 181.318495 & 7.8086 & \begin{tabular}{|l|}
129.196 \\
\end{tabular} & 580.51 & 5125 & 0.1 & 1950 & 75000 & 1575000 & $\begin{array}{l}0.1327 \\
\end{array}$ & \#\#\#\#" & 1.507 & \begin{tabular}{|l|}
8.94 \\
\end{tabular} & 31.91 & 97.24 & 72.42 & 0 & 77.5 & 322.2 \\
\hline 219 & 32.73 & 3000 & 1750 & \begin{tabular}{|l|}
3.82169 \\
\end{tabular} & 0.1411 & \begin{tabular}{|l|}
110.748 \\
\end{tabular} & 677.21 & 5125 & 0.1 & 1950 & 75000 & 1250000 & 0.0972 & \#\#\#\#\# & 3.798 & \begin{tabular}{|l|l|}
$31.34 \mathrm{E}-02$ \\
\end{tabular} & 56.08 & & 0 & 0 & 51.8 & 594.8 \\
\hline 220 & 24.1 & 3000 & 1150 & 8.497097 & 0.2902 & \begin{tabular}{|l|}
102.47 \\
\end{tabular} & 731.92 & 5125 & 0.1 & 1950 & 75000 & 1112500 & $\begin{array}{r}0.132 \\
\end{array}$ & \#\#\#\#\# & 2.28 & \begin{tabular}{|l|l|}
3 & $1.49 \mathrm{E}-02$ \\
\end{tabular} & 90.82 & 41.16 & 0 & 0 & 46.6 & 484.8 \\
\hline 221 & 27.54 & 3000 & 2040 & 7.462577 & 0.2846 & 114.41 & 655.54 & 5125 & 0.1 & 1950 & 75000 & 1312500 & \begin{tabular}{|l|}
0.1155 \\
\end{tabular} & \#\#\#\#\# & 4.082 & $9.32 \mathrm{E}-03$ & 77.28 & 33 & 0 & 0 & 74.4 & 759.4 \\
\hline 222 & 50.58 & 3000 & 1540 & 81.65827 & 4.2262 & 155.266 & 483.04 & 5125 & 0.1 & 1950 & 75000 & 2075000 & 0.0629 & \#\#\#\#\#五 & 4.413 & $37.38 \mathrm{E}-03$ & 72.24 & 93.88 & 38.77 & 0 & 93.9 & 447.1 \\
\hline 223 & 22.26 & 3000 & 1640 & 24.719327 & 0.9427 & $\mid 114.41$ & 655.54 & 5125 & 0.1 & 1950 & 75000 & 1312500 & 0.1429 & 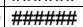 & 2.578 & $8.45 \mathrm{E}-03$ & 33.03 & 7777 & 0 & 0 & 821 & 5935 \\
\hline$\frac{223}{224}$ & $\frac{2.20}{42.42}$ & 3000 & $\frac{1040}{860}$ & 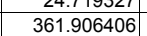 & \begin{tabular}{|}
.9427 \\
12.3615 \\
\end{tabular} & \begin{tabular}{|l|}
11.41 \\
102.47 \\
\end{tabular} & $\begin{array}{l}\quad 653.54 \\
731.92\end{array}$ & 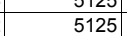 & $\begin{array}{l}0.1 \\
0.1\end{array}$ & $\begin{array}{l}1950 \\
1950\end{array}$ & 75000 & 1112500 & \begin{tabular}{|c|}
0.1429 \\
0.075
\end{tabular} & \#冊曲 & 1.851 & \begin{tabular}{|l|l|} 
& $0.45 \mathrm{E}-\mathrm{U}$ \\
\end{tabular} & $\begin{array}{l}35.05 \\
11.89\end{array}$ & 98.62 & 86.18 & 0 & $\begin{array}{ll}82.1 \\
72.6\end{array}$ & $\begin{array}{r}523.5 \\
223.6\end{array}$ \\
\hline 225 & 30.24 & 3000 & 1560 & & 0.2683 & \begin{tabular}{|l|}
143.18 \\
\end{tabular} & 523 & 512 & 0.1 & 1950 & 75000 & & 0.1052 & \#\#\#\#\#曲 & 3.312 & & 48. & 11.04 & 0 & 0 & 56.1 & 561.2 \\
\hline 226 & 26.16 & 30 & 2520 & 8.6 & 0.4065 & 152.132 & 492. & 512 & 0.1 & 19 & & & & & 3.9 & & & 37 & 0 & 0 & 94 & 7.4 \\
\hline 227 & 27.24 & 30 & 11 & 36.554 & 1.3 & 113.682 & 659 & 512 & 0.1 & 19 & 75 & 130 & 0.1 & \#\#\# & 2.133 & \begin{tabular}{|l|l|}
3 & 1.16 \\
\end{tabular} & 29 & 86.32 & 0 & 0 & 59.9 & 401.3 \\
\hline 228 & 21.97 & 30 & 1210 & 3.7010 & 0.1861 & 150.869 & 497. & 5125 & 0.1 & 1950 & 750 & 1987 & 0.1448 & \#\#\#\#\# & 2.198 & \begin{tabular}{|l|l|}
31.40 \\
\end{tabular} & 30. & & 0 & 0 & 49.4 & 512.6 \\
\hline 229 & 37.43 & 3000 & 1230 & 103.045196 & 4.6236 & \begin{tabular}{|l|}
134.609 \\
\end{tabular} & 557.17 & 5125 & 0.1 & 1950 & 75000 & 1675000 & 0.085 & \#\#\#\#\# & 2.99 & \begin{tabular}{|l|l|}
$9.49 \mathrm{E}-03$ \\
\end{tabular} & 64.65 & 95.15 & 51.48 & 0 & 81.6 & 409.4 \\
\hline 230 & 43.11 & 3000 & 1210 & 151.999215 & 7.9612 & $\mid 157.131$ & 477.31 & 5125 & 0.1 & 1950 & 75000 & 2112500 & $\begin{array}{l}0.0738 \\
\end{array}$ & \#\#\#\#" & 3.08 & \begin{tabular}{|l|l|}
$3.93 \mathrm{E}-03$ \\
\end{tabular} & 38.12 & 96.71 & 67.11 & 0 & 87.4 & 366.2 \\
\hline 231 & 56.21 & 3000 & 2940 & 12.115823 & 0.5517 & \begin{tabular}{|l|}
136.61 \\
\end{tabular} & 549.01 & 5125 & 0.1 & 1950 & 75000 & 1712500 & $\begin{array}{l}0.0566 \\
\end{array}$ & 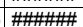 & 6.73 & $\begin{array}{ll}3 & 8.48 \mathrm{E}-03 \\
\end{array}$ & 36.22 & 58.73 & 0 & 0 & 81.8 & 613.7 \\
\hline 232 & 24.57 & 3000 & $\begin{array}{r}2540 \\
980\end{array}$ & \begin{tabular}{|r|}
201.610983 \\
\end{tabular} & $\begin{array}{l}0.0341 \\
\end{array}$ & \begin{tabular}{|l|}
159.788 \\
\end{tabular} & \begin{tabular}{|c|}
835.3 \\
835
\end{tabular} & 5125 & 0.1 & 1950 & 75000 & 912500 & 0.1295 & \#\#\#\#曲 & $\begin{array}{ll}0.476 \\
1.446\end{array}$ & \begin{tabular}{|l|l|}
$9.40-0$ \\
$9.43 \mathrm{E}-03$
\end{tabular} & $\begin{array}{l}0.22 \\
70.8 \\
\end{array}$ & 97.52 & 75.2 & 0 & 73.5 & 301.7 \\
\hline 233 & 46.99 & 3000 & 1330 & 866.791304 & 33.6831 & \begin{tabular}{|l|}
116.579 \\
\end{tabular} & 643.34 & 5125 & 0.1 & 1950 & 75000 & 1350000 & 0.0677 & \#\#\#\#\# & 3.116 & & 34.97 & 99.42 & 94.23 & 2.32 & 203 & 339.8 \\
\hline 234 & 24.28 & 3000 & 880 & 163.381206 & 5.7877 & \begin{tabular}{|l|}
106.273 \\
\end{tabular} & 705.73 & 5125 & 0.1 & 1950 & 75000 & 1175000 & 0.131 & \#\#\# & 1.414 & $1.00 \mathrm{E}-02$ & 32. & 96. & 69.4 & 0 & 69.2 & 298.3 \\
\hline 235 & 28.43 & 3000 & 2480 & & & & 603 & 5 & 0.1 & 19 & & & & & & & & & 0 & 0 & 103.1 & 772 \\
\hline 236 & 37.69 & 3000 & 1010 & 63.348786 & 2.082 & 98.5952 & 760.69 & 5125 & 0.1 & 1950 & 75000 & 1050000 & 0.0844 & \#\#\#\#\# & 2.307 & $1.42 \mathrm{E}-02$ & 89.31 & 92.11 & 21.07 & 0 & 48.9 & 313.7 \\
\hline
\end{tabular}




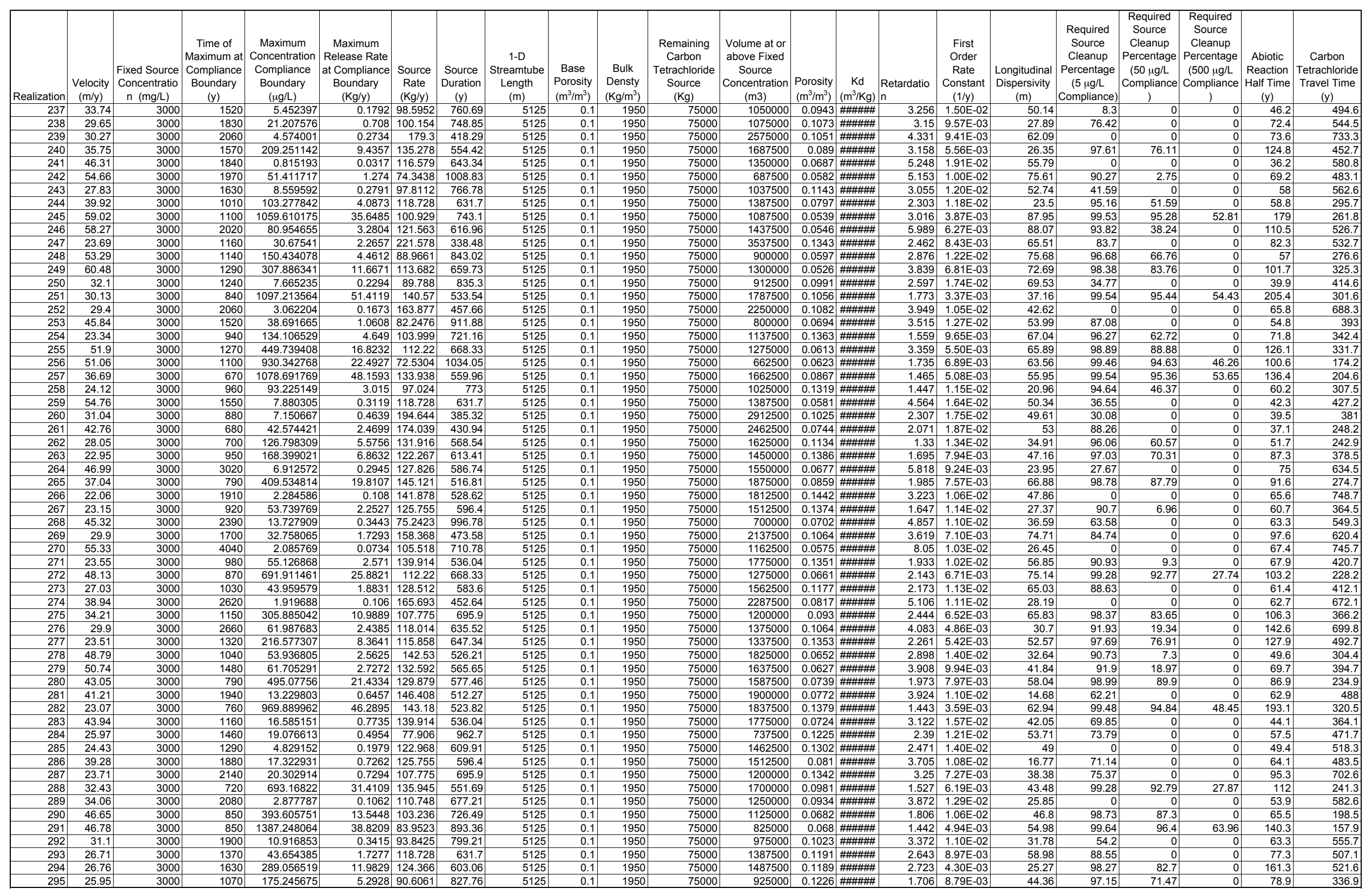




\begin{tabular}{|c|c|c|c|c|c|c|c|c|c|c|c|c|c|c|c|c|c|c|c|c|c|c|}
\hline Realization & $\begin{array}{c}\text { Velocity } \\
(\mathrm{m} / \mathrm{y})\end{array}$ & $\begin{array}{c}\text { Fixed Source } \\
\text { Concentratio } \\
\mathrm{n}(\mathrm{mg} / \mathrm{L})\end{array}$ & $\begin{array}{c}\text { Time of } \\
\text { Maximum at } \\
\text { Compliance } \\
\text { Boundary } \\
\text { (y) }\end{array}$ & $\begin{array}{c}\text { Maximum } \\
\text { Concentration } \\
\begin{array}{c}\text { Compliance } \\
\text { Boundary } \\
(\mu \mathrm{g} / \mathrm{L})\end{array} \\
\end{array}$ & $\begin{array}{c}\text { Maximum } \\
\text { Release Rate } \\
\text { at Compliance } \\
\text { Boundary } \\
(\mathrm{Kg} / \mathrm{y})\end{array}$ & \begin{tabular}{|c|} 
\\
Source \\
Rate \\
$(\mathrm{Kg} / \mathrm{y})$
\end{tabular} & $\begin{array}{c}\text { Source } \\
\text { Duration } \\
\text { (y) }\end{array}$ & $\begin{array}{c}\text { 1-D } \\
\text { Streamtube } \\
\text { Length } \\
\text { (m) }\end{array}$ & $\begin{array}{c}\text { Base } \\
\text { Porosity } \\
\left(\mathrm{m}^{3} / \mathrm{m}^{3}\right)\end{array}$ & $\begin{array}{c}\text { Bulk } \\
\text { Densty } \\
\left(\mathrm{Kg} / \mathrm{m}^{3}\right)\end{array}$ & $\begin{array}{c}\text { Remaining } \\
\text { Carbon } \\
\text { Tetrachloride } \\
\text { Source } \\
(\mathrm{Kg})\end{array}$ & $\begin{array}{c}\begin{array}{c}\text { Volume at or } \\
\text { above Fixed } \\
\text { Source } \\
\text { Concentration } \\
\text { (m3) }\end{array} \\
\end{array}$ & $\begin{array}{l}\text { Porosity } \\
\left(\mathrm{m}^{3} / \mathrm{m}^{3}\right)\end{array}$ & $\begin{array}{c}\mathrm{Kd} \\
\left(\mathrm{m}^{3} / \mathrm{Kg}\right)\end{array}$ & $\begin{array}{l}\text { Retardatio } \\
\text { n }\end{array}$ & \begin{tabular}{|c|} 
First \\
Order \\
Rate \\
Constant \\
$(1 / y)$ \\
\end{tabular} & $\begin{array}{c}\text { Longitudinal } \\
\text { Dispersivity } \\
\text { (m) }\end{array}$ & \begin{tabular}{|c|} 
Required \\
Source \\
Cleanup \\
Percentage \\
$(5 \mu \mathrm{\mu g} / \mathrm{L}$ \\
Compliance $)$
\end{tabular} & \begin{tabular}{|c|} 
Required \\
Source \\
Cleanup \\
Percentage \\
$(50 \mu \mathrm{g} / \mathrm{L}$ \\
Compliance \\
)
\end{tabular} & \begin{tabular}{|c|} 
Required \\
Source \\
Cleanup \\
Percentage \\
$(500 \mu \mathrm{L} / \mathrm{L}$ \\
Compliance \\
)
\end{tabular} & $\begin{array}{c} \\
\text { Abiotic } \\
\text { Reaction } \\
\text { Half Time } \\
\text { (y) }\end{array}$ & $\begin{array}{c}\text { Carbon } \\
\text { Tetrachloride } \\
\text { Travel Time } \\
\text { (y) }\end{array}$ \\
\hline 296 & 34.02 & 3000 & \begin{tabular}{l|l}
770 \\
\end{tabular} & 470.235288 & \begin{tabular}{|l|l}
8 & 22.6458 \\
\end{tabular} & 144.475 & \begin{tabular}{|r|}
519.12 \\
\end{tabular} & \begin{tabular}{|r|}
5125 \\
\end{tabular} & 0.1 & 1950 & 75000 & 1862500 & 0.0935 & \#\#\#\# & 1.838 & \begin{tabular}{|c|}
$6.94 \mathrm{E}-03$ \\
\end{tabular} & $\begin{array}{r}60.77 \\
\end{array}$ & \begin{tabular}{l|r}
7 & 98.94 \\
\end{tabular} & $\begin{array}{l}89.37 \\
\end{array}$ & 0 & $\begin{array}{l}99.9 \\
\end{array}$ & 276.9 \\
\hline 297 & 21.47 & 3000 & 680 & 51.295341 & 3.309 & \begin{tabular}{|l|}
193.528 \\
\end{tabular} & 387.54 & 5125 & 0.1 & 1950 & 75000 & 2887500 & 0.1482 & 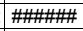 & 1.501 & $1.22 \mathrm{E}-02$ & 65.62 & 90.25 & 2.53 & 0 & 57 & 358.5 \\
\hline 298 & 35.71 & 3000 & 1210 & 35.583606 & 1.849 & 155.889 & 481.11 & 5125 & 0.1 & 1950 & 75000 & 2087500 & 0.0891 & \#\#\#\#\# & 2.982 & $1.09 \mathrm{E}-02$ & 54.43 & 85.95 & 0 & 0 & 63.6 & 428.1 \\
\hline 299 & 22.23 & 3000 & 1490 & 233.36276 & 9.7822 & \begin{tabular}{|l|}
125.755 \\
\end{tabular} & 596.4 & 5125 & 0.1 & 1950 & 75000 & 1512500 & $\begin{array}{l}0.1431 \\
\end{array}$ & 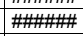 & 2.424 & \begin{tabular}{|l|}
$4.36 \mathrm{E}-03$ \\
\end{tabular} & 48.04 & 97.86 & 78.57 & 0 & 159 & 558.8 \\
\hline 300 & 39.37 & 3000 & 1510 & \begin{tabular}{|l|l|}
6.847629 \\
\end{tabular} & 0.2965 & \begin{tabular}{|l|l|}
129.879 \\
\end{tabular} & 577.46 & 5125 & 0.1 & 1950 & 75000 & 1587500 & $\begin{array}{l}0.0808 \\
\end{array}$ & \#\#\#\#州 & 3.998 & \begin{tabular}{|l|}
$1.40 \mathrm{E}-02$ \\
\end{tabular} & $\begin{array}{l}66.64 \\
\end{array}$ & 26.98 & 0 & 0 & \begin{tabular}{r|r|}
49.5 \\
\end{tabular} & 520.5 \\
\hline 301 & 23.29 & 3000 & 730 & \begin{tabular}{|c|}
0.047029 \\
19.163524 \\
\end{tabular} & 0.8383 & \begin{tabular}{|l|}
131.239 \\
\end{tabular} & 571.48 & 5125 & 0.1 & 1950 & 75000 & 1612500 & $\begin{array}{l}0.1366 \\
0\end{array}$ & \#\#\#\#曲 & 1.299 & \begin{tabular}{|l|}
$1.401 E-02$ \\
\end{tabular} & 20.41 & 73.91 & 0 & 0 & $\begin{array}{l}48.0 \\
38.2 \\
\end{array}$ & 285.9 \\
\hline 302 & 41.37 & 3000 & 1780 & 10.727389 & 0.5097 & \begin{tabular}{|l|}
142.53 \\
\end{tabular} & 526.21 & 5125 & 0.1 & 1950 & 75000 & 1825000 & $\begin{array}{l}0.0769 \\
\end{array}$ & \#\#\#\#" & 3.918 & \begin{tabular}{|l|}
$1.19 \mathrm{E}-02$ \\
\end{tabular} & 26.77 & 53.39 & 0 & 0 & 58.3 & 485.4 \\
\hline 304 & 21.5 & 3000 & 1660 & 15.245502 & 0.7731 & \begin{tabular}{|l|}
152.132 \\
\end{tabular} & 492.99 & 5125 & 0.1 & 1950 & 75000 & 2012500 & 0.148 & 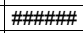 & 2.577 & $8.67 \mathrm{E}-03$ & 21.35 & 67.2 & 0 & 0 & 80 & 614.3 \\
\hline 305 & 49.02 & 3000 & 1800 & 122.858367 & 5.4851 & \begin{tabular}{|l|}
133.938 \\
\end{tabular} & 559.96 & 5125 & 0.1 & 1950 & 75000 & 1662500 & 0.0649 & \#\#\#\#\#曲 & 3.998 & $7.26 \mathrm{E}-03$ & 17.11 & 95.93 & 59.3 & 0 & 95.5 & 418 \\
\hline 306 & 61.77 & 3000 & 1320 & 621.846122 & 12.6693 & 61.1213 & \begin{tabular}{|l|}
1227.07 \\
\end{tabular} & 5125 & 0.1 & 1950 & 75000 & 512500 & 0.0515 & \#\#\#\#\#曲 & 2.249 & 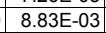 & 67.87 & 99.2 & 91.96 & 19.59 & 78.5 & 186.6 \\
\hline 307 & 50.42 & 3000 & 1460 & 469.711331 & 18.2528 & \begin{tabular}{|l|}
116.579 \\
\end{tabular} & $\begin{array}{l}643.34 \\
\end{array}$ & 5125 & 0.1 & 1950 & 75000 & 1350000 & 0.0631 & 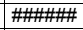 & 3.502 & $4.92 \mathrm{E}-03$ & 31.1 & 98.94 & 89.36 & 0 & 140.9 & 356 \\
\hline 308 & 25.03 & 3000 & 1590 & 14.059261 & 0.3692 & 78.7839 & 951.97 & 5125 & 0.1 & 1950 & 75000 & 750000 & 0.1271 & \#\#\#\#\# & 2.376 & $1.17 \mathrm{E}-02$ & 23.58 & 64.44 & 0 & 0 & 59.4 & 486.6 \\
\hline 309 & 34.39 & 3000 & 2060 & 0.2706 & 0.0095 & \begin{tabular}{|l|}
105.518 \\
\end{tabular} & 710.78 & 5125 & 0.1 & 1950 & 75000 & 1162500 & 0.0925 & \#\#\#\#\#曲 & 4.704 & \begin{tabular}{|l|}
$1.79 \mathrm{E}-02$ \\
\end{tabular} & 51.09 & 0 & 0 & 0 & 38.7 & 701 \\
\hline 310 & 52.58 & 3000 & 820 & 889.591382 & 28.3008 & 95.4399 & 785.84 & 5125 & 0.1 & 1950 & 75000 & 1000000 & 0.0605 & \#\#\#\#\#曲 & 1.491 & \begin{tabular}{|l|}
$8.54 \mathrm{E}-03$ \\
\end{tabular} & 59.4 & 99.44 & 94.38 & 43.79 & 81.1 & 145.3 \\
\hline 311 & 24.7 & 3000 & 1840 & 174.579251 & 8.7427 & \begin{tabular}{|l|l|}
150.236 \\
\end{tabular} & 499.21 & 5125 & 0.1 & 1950 & 75000 & 1975000 & $\begin{array}{l}0.1288 \\
\end{array}$ & 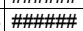 & 2.899 & \begin{tabular}{|l|}
$4.19 \mathrm{E}-03$ \\
\end{tabular} & 25.2 & 97.14 & 71.36 & 0 & 165.5 & 601.5 \\
\hline 312 & 32.6 & 3000 & 1280 & 4.503331 & 0.1898 & 126.447 & 593.13 & 5125 & 0.1 & 1950 & 75000 & 1525000 & 0.0976 & 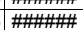 & 2.895 & \begin{tabular}{|l|l|} 
& $1.63 \mathrm{E}-02$ \\
\end{tabular} & 46.08 & 0 & 0 & 0 & 42.4 & 455.2 \\
\hline 313 & 27.62 & 3000 & 2120 & 0.066339 & 0.003 & \begin{tabular}{|l|}
135.945 \\
\end{tabular} & 551.69 & 5125 & 0.1 & 1950 & 75000 & 1700000 & 0.1152 & \#\#\#\#\# & 4.306 & \begin{tabular}{|l|l|}
$1.75 E-02$ \\
$1.75 E-02$
\end{tabular} & 40.00 & 0 & 0 & 0 & \begin{tabular}{|l|}
49.4 \\
39.7
\end{tabular} & $\begin{array}{l}790.2 \\
799.1\end{array}$ \\
\hline 314 & 21.82 & 3000 & 1710 & 3.868149 & 0.2026 & $\mid 157.131$ & 477.31 & 5125 & 0.1 & 1950 & 75000 & 2112500 & $\begin{array}{l}0.1458 \\
\end{array}$ & \#\#\#\# & 3.108 & \begin{tabular}{|l|}
$1.01 \mathrm{E}-02$ \\
\end{tabular} & 61.34 & 0 & 0 & 0 & 68.9 & 730.1 \\
\hline 315 & 29.38 & 3000 & 2210 & \begin{tabular}{|l}
4.339849 \\
\end{tabular} & 0.1415 & 97.8112 & 766.78 & 5125 & 0.1 & 1950 & 75000 & 1037500 & $\begin{array}{l}0.1083 \\
0.103\end{array}$ & 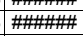 & 3.81 & \begin{tabular}{|l|}
$1.09 \mathrm{E}-02$ \\
\end{tabular} & 36.67 & 0 & 0 & 0 & 63.7 & 664.7 \\
\hline 316 & 24.82 & 3000 & 2850 & \begin{tabular}{|l|l|}
117.86224 \\
\end{tabular} & 5.574 & \begin{tabular}{|l|}
141.878 \\
\end{tabular} & 528.62 & 5125 & 0.1 & 1950 & 75000 & 1812500 & 0.1282 & \#\#\#\#\#曲 & 3.804 & $3.08 \mathrm{E}-03$ & 28.61 & 95.76 & 57.58 & 0 & 225.3 & 785.6 \\
\hline 317 & 45.77 & 3000 & 1030 & 196.34834 & 5.7144 & \begin{tabular}{|l|}
87.3109 \\
\end{tabular} & 859 & 5125 & 0.1 & 1950 & 75000 & 875000 & 0.0695 & \#\#\#\#\# & 2.065 & \begin{tabular}{|l|}
$1.24 \mathrm{E}-02$ \\
\end{tabular} & 46.28 & 97.45 & 74.54 & 0 & 55.9 & 231.2 \\
\hline 318 & 40.17 & 3000 & 880 & 253.325703 & 12.363 & \begin{tabular}{|l|}
146.408 \\
\end{tabular} & 512.27 & 5125 & 0.1 & 1950 & 75000 & 1900000 & $\begin{array}{l}0.0792 \\
\end{array}$ & \#\#\#\#\#曲 & 2.167 & $9.11 \mathrm{E}-03$ & 18.36 & 98.03 & 80.26 & 0 & 76.1 & 276.5 \\
\hline 319 & 31.47 & 3000 & 860 & 640.603401 & 27.4417 & 128.512 & 583.6 & 5125 & 0.1 & 1950 & 75000 & 1562500 & 0.1011 & \#\#\#\#\# & 1.798 & $5.39 \mathrm{E}-03$ & 42.43 & 99.22 & 92.19 & 21.95 & 128.6 & 292.8 \\
\hline 320 & 30.98 & 3000 & 920 & 142.821678 & 6.2156 & 130.56 & 574.45 & 5125 & 0.1 & 1950 & 75000 & 1600000 & 0.1027 & \#\#\#\#\#曲 & 1.975 & \begin{tabular}{|c|} 
\\
\end{tabular} & 44.07 & 96.5 & 64.99 & 0 & 70.9 & 326.8 \\
\hline 321 & 38.01 & 3000 & 880 & 59.874305 & 3.2828 & 164.483 & 455.97 & 5125 & 0.1 & 1950 & 75000 & 2262500 & 0.0837 & 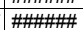 & 2.264 & \begin{tabular}{|l|} 
\\
\end{tabular} & 29.69 & 91.65 & 16.49 & 0 & 51.7 & 305.3 \\
\hline 322 & 36.4 & 3000 & 1730 & 2.879928 & 0.1112 & 115.858 & 647.34 & 5125 & 0.1 & 1950 & 75000 & 1337500 & o.0874 & 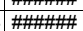 & $\begin{array}{ll}2.204 \\
4.035\end{array}$ & \begin{tabular}{|l|l|}
$1.47 \mathrm{E}-02$ \\
\end{tabular} & 52.91 & 0 & 0 & 0 & $\begin{array}{l}4.1 \\
47.1 \\
\end{array}$ & 568.1 \\
\hline 323 & 38.24 & 3000 & 1380 & 2.875395 & \begin{tabular}{|l|}
0.1219 \\
\end{tabular} & \begin{tabular}{|l|}
127.137 \\
\end{tabular} & 589.91 & 5125 & 0.1 & 1950 & 75000 & 1537500 & 0.0832 & \#\#\#\#\#曲 & 3.007 & \begin{tabular}{|c|} 
\\
\end{tabular} & 16 & 0 & 0 & 0 & 37.9 & 403 \\
\hline 324 & 33.81 & 3000 & 1120 & \begin{tabular}{|l|l|}
447.082906 \\
\end{tabular} & 19.0495 & \begin{tabular}{|l|}
27.826 \\
\end{tabular} & 586.74 & 5125 & 0.1 & 1950 & 75000 & 1550000 & 0.0941 & 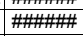 & 2.413 & \begin{tabular}{|l|}
$5.22 \mathrm{E}-03$ \\
\end{tabular} & 46.39 & 98.88 & 88.82 & 0 & 132.9 & 365.8 \\
\hline 325 & 25.8 & 3000 & 950 & $\begin{array}{r}12.379382 \\
\end{array}$ & 0.6586 & 159.6 & 469.92 & 5125 & 0.1 & 1950 & 75000 & 2162500 & \begin{tabular}{|l|}
0.1233 \\
\end{tabular} & \#\#\#\#曲 & 1.987 & \begin{tabular}{|l|} 
\\
\end{tabular} & 30.93 & 59.61 & 0 & 0 & 46.9 & \\
\hline 326 & 27.78 & 3000 & 920 & 136.51901 & 7.2348 & 158.985 & 471.74 & 5125 & 0.1 & 1950 & 75000 & 2150000 & $\begin{array}{l}0.1145 \\
\end{array}$ & \#\#\#\#\#曲 & 1.938 & 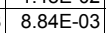 & 20.16 & 96.34 & 63.38 & 0 & 78.4 & 357.4 \\
\hline 327 & 27.17 & 3000 & 1640 & 45.003848 & 2.0593 & 137.274 & 546.35 & 5125 & 0.1 & 1950 & 75000 & 1725000 & 0.1171 & \#\#\#\#\# & 2.883 & $7.69 \mathrm{E}-03$ & 27.41 & 88.89 & 0 & 0 & 90.1 & 543.9 \\
\hline 328 & 27.4 & 3000 & 750 & 205.644641 & 8.9496 & 130.56 & 574.45 & 5125 & 0.1 & 1950 & 75000 & 1600000 & 0.1161 & \#\#\#\#\# & 1.505 & \begin{tabular}{|l|} 
\\
\end{tabular} & 72.11 & 97.57 & 75.69 & 0 & 68.9 & 281.4 \\
\hline 329 & 56.11 & 3000 & 2810 & 1.195224 & 0.0453 & 113.682 & 659.73 & 5125 & 0.1 & 1950 & 75000 & 1300000 & 0.0567 & \#\#\#\#" & 6.568 & \begin{tabular}{|l|}
$1.46 \mathrm{E}-02$ \\
\end{tabular} & 27.25 & 0 & 0 & 0 & 47.4 & 599.9 \\
\hline 330 & 33.17 & 3000 & 690 & 382.690071 & 21.2133 & \begin{tabular}{|l|}
166.296 \\
\end{tabular} & 451 & 5125 & 0.1 & 1950 & 75000 & 2300000 & 0.0959 & \#\#\#\#\#曲 & 1.662 & $8.15 \mathrm{E}-03$ & 23.25 & 98.69 & 86.93 & 0 & 85.1 & 256.7 \\
\hline 331 & 38.84 & 3000 & 560 & 142.350252 & 7.6603 & \begin{tabular}{|l|}
161.44 \\
\end{tabular} & 464.57 & 5125 & 0.1 & 1950 & 75000 & 2200000 & 0.0819 & 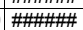 & 1.469 & 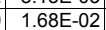 & 81.45 & 96.49 & 64.88 & 0 & 41.2 & 193.8 \\
\hline 332 & 53.92 & 3000 & 1690 & 130.809281 & 4.829 & 110.748 & 677.21 & 5125 & 0.1 & 1950 & 75000 & 1250000 & 0.059 & \#\#\#\#\#曲 & 3.978 & 8.26 & 21.41 & 96.18 & 61.78 & 0 & 83.9 & 378.1 \\
\hline 333 & 22.61 & 3000 & 1680 & & 3.0802 & 134.609 & 557.17 & 5125 & 0 & 19 & 75000 & 1675000 & 0.1407 & \#\#\#\#\#曲 & 2.576 & 6.45 & 18. & 92.72 & 27.17 & 0 & 107.5 & 583.8 \\
\hline 334 & 23.83 & 3000 & 960 & 504.323922 & \begin{tabular}{|c|}
18.9882 \\
18.98
\end{tabular} & \begin{tabular}{|l|l|}
112.952 \\
\end{tabular} & 664 & 5125 & 0.1 & 1950 & & 1287500 & 0.1335 & \#\# & 1.652 & & 43.66 & 99.01 & 90.09 & 0.86 & 134.7 & 355.3 \\
\hline 335 & 34.96 & 3000 & 2850 & 22.392351 & $\begin{array}{r}0.4782 \\
\end{array}$ & \begin{tabular}{|l|}
64.0676 \\
\end{tabular} & \begin{tabular}{|l|}
1170.64 \\
\end{tabular} & 5125 & 0.1 & 1950 & 75000 & 550000 & 0.091 & \#\#\#\#\#曲 & 5.06 & \begin{tabular}{|l|l|} 
& $7.60 \mathrm{E}-03$ \\
\end{tabular} & 60.02 & 77.67 & 0 & 0 & 91.2 & 741.7 \\
\hline 336 & 37.65 & 3000 & 930 & 607.853576 & 24.7734 & 122.267 & \begin{tabular}{|l|}
613.41 \\
\end{tabular} & 5125 & 0.1 & 1950 & 75000 & 1450000 & $\begin{array}{l}0.0845 \\
\end{array}$ & \#\#\#\#\# & 2.144 & $5.64 \mathrm{E}-03$ & 61.36 & \begin{tabular}{|c|c|}
99.18 \\
\end{tabular} & 91.77 & 17.74 & 122.8 & 291.9 \\
\hline 337 & 28.92 & 3000 & 730 & 621.487148 & 27.7469 & \begin{tabular}{|l|}
133.938 \\
\end{tabular} & 559.96 & 5125 & 0.1 & 1950 & 75000 & 1662500 & 0.11 & \#\#\#\#\#曲 & 1.429 & \begin{tabular}{|c|}
$6.29 \mathrm{E}-03$ \\
\end{tabular} & 25.05 & 99.2 & 91.95 & 19.55 & 110.3 & 253.2 \\
\hline 338 & 34.28 & 3000 & 700 & 791.379186 & 34.0811 & 129.196 & 580.51 & 5125 & 0.1 & 1950 & 75000 & 1575000 & 0.0928 & \#\#\#\#\# & 1.429 & \begin{tabular}{|l|}
$6.34 \mathrm{E}-03$ \\
\end{tabular} & 45.59 & 99.37 & 93.68 & 36.82 & 109.3 & 213.7 \\
\hline 339 & 37.47 & 3000 & 920 & 848.338854 & 44.0821 & \begin{tabular}{|l|}
155.889 \\
\end{tabular} & 481.11 & 5125 & 0.1 & 1950 & 75000 & 2087500 & 0.0849 & \#\#\#\#\#五 & 2.335 & $3.62 \mathrm{E}-03$ & 69.24 & 99.41 & 94.11 & 41.06 & 191.4 & 319.4 \\
\hline 340 & 23.64 & 3000 & 1920 & 27.933313 & 0.8738 & \begin{tabular}{|l|}
93.8425 \\
\end{tabular} & 799.21 & 5125 & 0.1 & 1950 & 75000 & 975000 & 0.1346 & 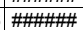 & 2.896 & $7.83 \mathrm{E}-03$ & 34.54 & 82.1 & 0 & 0 & 88.5 & 628 \\
\hline 341 & 53.02 & 3000 & 3340 & 49.589819 & 1.1686 & 70.6941 & 1060.91 & 5125 & 0.1 & 1950 & 75000 & 637500 & 0.06 & \#\#\#\#再 & 6.612 & \begin{tabular}{|l|}
$6.28 \mathrm{E}-03$ \\
\end{tabular} & 41.62 & 89.92 & 0 & 0 & 110.3 & 639.1 \\
\hline 342 & 38.47 & 3000 & 1530 & 202.064908 & 6.3208 & \begin{tabular}{|l|}
93.8425 \\
\end{tabular} & 799.21 & 5125 & 0.1 & 1950 & 75000 & 975000 & 0.0827 & \#\#\#\#曲 & 3.098 & 6.74 & 42.48 & 97.53 & 75.26 & 0 & 102.8 & 4128 \\
\hline 343 & 60.03 & 3000 & 3060 & & 0. & \begin{tabular}{|l|}
107.025 \\
\end{tabular} & 700. & 512 & 0. & 195 & 75000 & 1187500 & 0.053 & & 6.904 & & 35. & 80.65 & 0 & 0 & 92.2 & 589.5 \\
\hline 344 & 35.95 & 30 & 980 & 195. & $\begin{array}{l}8.2842 \\
\end{array}$ & \begin{tabular}{|l|l|}
127.137 \\
\end{tabular} & 589. & 5125 & 0.1 & 19 & & 1537500 & 0.0 & & 2.363 & & & 97 & 74.42 & 0 & 80 & \\
\hline 345 & 50.9 & 30 & 1970 & & 3.50 & 154.0 & 486 & & 0.1 & 19 & 75 & 2050000 & 0.06 & \#\#\# & 4. & & 37. & 92 & 26.83 & 0 & 101 & 474.9 \\
\hline 346 & 40.22 & 30 & 780 & 262.5957 & 7.78 & 88.9661 & 843 & 512 & 0.1 & 1950 & 750 & 900000 & \begin{tabular}{|c|}
0.0791 \\
\end{tabular} & \#\#\#\#\#| & 1.368 & $1.45 \mathrm{E}-02$ & 54.12 & \begin{tabular}{|l|}
98.1 \\
\end{tabular} & 80.96 & 0 & 47.9 & $\begin{array}{ll}174.4 \\
\end{array}$ \\
\hline 347 & 51.64 & 3000 & 960 & 39.413464 & 1.7948 & 136.61 & 549.01 & 5125 & 0.1 & 1950 & 75000 & 1712500 & 0.0616 & \#\#\#\#曲 & 3.042 & $1.64 \mathrm{E}-02$ & 65.7 & 87.31 & 0 & 0 & 42.3 & 301.8 \\
\hline 348 & 38.56 & 3000 & 1880 & 18.042585 & 0.7646 & 127.137 & 589.91 & 5125 & 0.1 & 1950 & 75000 & 1537500 & 0.0825 & \#\#\#\#\#曲 & 4.194 & $9.60 \mathrm{E}-03$ & 50.47 & 72.29 & 0 & 0 & 72.2 & 557.4 \\
\hline 349 & 50.66 & 3000 & 810 & 148.822073 & 7.6092 & 153.389 & 488.95 & 5125 & 0.1 & 1950 & 75000 & 2037500 & 0.0628 & \#\#\#\#" & 2.446 & \begin{tabular}{|c|} 
\\
\end{tabular} & 44.75 & 96.64 & 66.4 & 0 & 54 & 247.5 \\
\hline 350 & 23 & 3000 & 1430 & 19.970151 & 0.6193 & \begin{tabular}{|l|}
93.0387 \\
\end{tabular} & 806.12 & 5125 & 0.1 & 1950 & 75000 & 962500 & 0.1383 & \#\#\#\#\# & 2.282 & $1.07 E-02$ & 38.65 & 74.96 & 0 & 0 & $\mid 64.9$ & 508.3 \\
\hline 351 & 56.71 & 3000 & 860 & 154.53496 & 5.6285 & 109.267 & 686.4 & 5125 & 0.1 & 1950 & 75000 & 1225000 & 0.0561 & 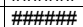 & 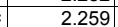 & \begin{tabular}{|c|}
$1.55 \mathrm{E}-02$ \\
\end{tabular} & 52.41 & 96.76 & 67.64 & 0 & 44.6 & 204.1 \\
\hline 352 & 21.25 & 3000 & 1080 & 138.716162 & 5.8468 & 126.447 & 593.13 & 5125 & 0.1 & 1950 & 75000 & 1525000 & 0.1497 & 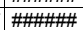 & 1.898 & $7.05 \mathrm{E}-03$ & 57.28 & 96.4 & 63.96 & 0 & 98.4 & 457.7 \\
\hline 353 & 45.06 & 3000 & 1290 & & & 159.6 & 469. & 5125 & 0.1 & 1950 & & & & & & & & 95 & 57.5 & 0 & 87 & 386.8 \\
\hline 354 & 23.65 & 3000 & 1900 & 14.048122 & 0.3607 & \begin{tabular}{|l|}
77.0232 \\
\end{tabular} & 973.73 & 5125 & 0.1 & 1950 & 75000 & 725000 & 0.1345 & \#\#\#\#\#曲 & 2.637 & $9.87 \mathrm{E}-03$ & 18.94 & 64.41 & 0 & 0 & 70.3 & 571.3 \\
\hline
\end{tabular}




\begin{tabular}{|c|c|c|c|c|c|c|c|c|c|c|c|c|c|c|c|c|c|c|c|c|c|c|}
\hline Realization & $\begin{array}{c}\text { Velocity } \\
(\mathrm{m} / \mathrm{y})\end{array}$ & $\begin{array}{c}\text { Fixed Source } \\
\text { Concentratio } \\
\mathrm{n}(\mathrm{mg} / \mathrm{L})\end{array}$ & $\begin{array}{c}\text { Time of } \\
\text { Maximum at } \\
\text { Compliance } \\
\text { Boundary } \\
\text { (y) }\end{array}$ & $\begin{array}{c}\text { Maximum } \\
\text { Concentration } \\
\begin{array}{c}\text { Compliance } \\
\text { Boundary } \\
(\mu \mathrm{g} / \mathrm{L})\end{array} \\
\end{array}$ & $\begin{array}{c}\text { Maximum } \\
\text { Release Rate } \\
\text { at Compliance } \\
\text { Boundary } \\
(\mathrm{Kg} / \mathrm{y})\end{array}$ & \begin{tabular}{|c|} 
\\
Source \\
Rate \\
$(\mathrm{Kg} / \mathrm{y})$
\end{tabular} & \begin{tabular}{|c|} 
Source \\
Duration \\
(y)
\end{tabular} & $\begin{array}{c}\text { 1-D } \\
\text { Streamtube } \\
\text { Length } \\
\text { (m) }\end{array}$ & $\begin{array}{c}\text { Base } \\
\text { Porosity } \\
\left(\mathrm{m}^{3} / \mathrm{m}^{3}\right)\end{array}$ & $\begin{array}{c}\text { Bulk } \\
\text { Densty } \\
\left(\mathrm{Kg} / \mathrm{m}^{3}\right)\end{array}$ & $\begin{array}{c}\text { Remaining } \\
\text { Carbon } \\
\text { Tetrachloride } \\
\text { Source } \\
(\mathrm{Kg})\end{array}$ & $\begin{array}{c}\begin{array}{c}\text { Volume at or } \\
\text { above Fixed } \\
\text { Source } \\
\text { Concentration } \\
\text { (m3) }\end{array} \\
\end{array}$ & $\begin{array}{l}\text { Porosity } \\
\left(\mathrm{m}^{3} / \mathrm{m}^{3}\right)\end{array}$ & $\begin{array}{c}\mathrm{Kd} \\
\left(\mathrm{m}^{3} / \mathrm{Kg}\right)\end{array}$ & $\begin{array}{l}\text { Retardatio } \\
\text { n }\end{array}$ & \begin{tabular}{|c|} 
First \\
Order \\
Rate \\
Constant \\
$(1 / y)$ \\
\end{tabular} & $\begin{array}{c}\text { Longitudinal } \\
\text { Dispersivity } \\
\text { (m) }\end{array}$ & \begin{tabular}{|c|} 
Required \\
Source \\
Cleanup \\
Percentage \\
$(5 \mu \mathrm{g} / \mathrm{L}$ \\
Compliance $)$ \\
\end{tabular} & \begin{tabular}{|c|} 
Required \\
Source \\
Cleanup \\
Percentage \\
$(50 \mu \mathrm{g} / \mathrm{L}$ \\
Compliance \\
)
\end{tabular} & \begin{tabular}{|c|} 
Required \\
Source \\
Cleanup \\
Percentage \\
$(500 \mu \mathrm{L} / \mathrm{L}$ \\
Compliance \\
)
\end{tabular} & \begin{tabular}{|c|} 
\\
Abiotic \\
Reaction \\
Half Time \\
$(y)$ \\
\end{tabular} & $\begin{array}{c}\text { Carbon } \\
\text { Tetrachloride } \\
\text { Travel Time } \\
\text { (y) }\end{array}$ \\
\hline 355 & 52.41 & 3000 & 3330 & $\begin{array}{l}19.775132 \\
\end{array}$ & \begin{tabular}{|l|l}
2 & 0.6905 \\
\end{tabular} & \begin{tabular}{|l|l|}
5 & 104.76 \\
\end{tabular} & 715.92 & 5125 & 0.1 & 1950 & 75000 & 1150000 & \begin{tabular}{|l|}
0.0607 \\
\end{tabular} & 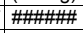 & 6.721 & \begin{tabular}{|l|}
$7.14 \mathrm{E}-03$ \\
\end{tabular} & 34.02 & 74.72 & $2 \quad 0$ & 0 & & 657.2 \\
\hline 356 & 29.4 & 3000 & 760 & 157.483074 & 4.9262 & 93.8425 & 799.21 & 5125 & 0.1 & 1950 & 75000 & 975000 & 0.1082 & 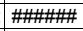 & 1.408 & $1.22 \mathrm{E}-02$ & 19.44 & 96.83 & 68.25 & 0 & 56.8 & 245.5 \\
\hline 357 & 37.34 & 3000 & 860 & 352.286947 & 12.0329 & 102.47 & 731.92 & 5125 & 0.1 & 1950 & 75000 & 1112500 & 0.0852 & \#\#\#\#\# & 1.65 & $9.78 \mathrm{E}-03$ & 49.31 & 98.58 & 85.81 & 0 & 70.9 & 226.5 \\
\hline 358 & 27.26 & 3000 & 1020 & $\begin{array}{r}30.005072 \\
\end{array}$ & 1.4255 & \begin{tabular}{|l|l|}
5 & 142.53 \\
\end{tabular} & 526.21 & 5125 & 0.1 & 1950 & 75000 & 1825000 & $\begin{array}{l}0.1167 \\
\end{array}$ & \#\#\#\#曲 & 2.084 & \begin{tabular}{|l|} 
\\
\end{tabular} & 31.36 & 83.34 & 0 & 0 & 55.9 & 391.9 \\
\hline 359 & 23.37 & 3000 & 1400 & 46.174808 & 1.3052 & 84.7981 & 884.45 & 5125 & 0.1 & 1950 & 75000 & 837500 & $\begin{array}{l}.1361 \\
\end{array}$ & 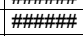 & 2.14 & \begin{tabular}{|l|}
$9.46 \mathrm{E}-03$ \\
\end{tabular} & 36.98 & 89.17 & 0 & 0 & 73.2 & 469.2 \\
\hline 360 & $\frac{20.01}{21.7}$ & 3000 & 990 & $\begin{array}{r}40.1780014 \\
\end{array}$ & 0.3206 & \begin{tabular}{|c|}
178.72 \\
\end{tabular} & $\begin{array}{l}419.65 \\
41.65\end{array}$ & 5125 & 0.1 & 1950 & 75000 & 2562500 & 0.1466 & \#\#\#\#曲 & 2.148 & \begin{tabular}{|l|}
$1.40 L-02$ \\
$1.42 E-02$ \\
\end{tabular} & 69.13 & $\begin{array}{l}7.111 \\
7.1 \\
\end{array}$ & 0 & 0 & 48.9 & 507.2 \\
\hline 361 & 41.8 & 3000 & 920 & 372.627864 & 12.7277 & 102.47 & 731.92 & 5125 & 0.1 & 1950 & 75000 & 1112500 & 0.0761 & \#\#\#\#\# & 1.941 & \begin{tabular}{|l|}
$9.07 \mathrm{E}-03$ \\
\end{tabular} & 43.28 & 98.66 & 86.58 & 0 & 76.5 & 237.9 \\
\hline 362 & 37.12 & 3000 & 870 & 133.896557 & 6.0377 & 135.278 & 554.42 & 5125 & 0.1 & 1950 & 75000 & 1687500 & 0.0857 & \#\#\#\#\#曲 & 2.082 & \begin{tabular}{|l|}
$1.13 \mathrm{E}-02$ \\
\end{tabular} & 36.73 & 96.27 & 62.66 & 0 & 61.3 & 287.4 \\
\hline 363 & 57.63 & 3000 & 4410 & 2.216366 & 0.0657 & 88.9661 & 843.02 & 5125 & 0.1 & 1950 & 75000 & 900000 & 0.0552 & 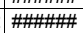 & 8.065 & $\begin{array}{l}1.03 \mathrm{E}-02 \\
\end{array}$ & 18.72 & 0 & 0 & 0 & 67.1 & 717.2 \\
\hline 364 & 35.99 & 3000 & 1230 & 216.425508 & 9.2216 & 127.826 & $\begin{array}{l}586.74 \\
\end{array}$ & 5125 & 0.1 & 1950 & 75000 & 1550000 & 0.0884 & \#\#\#\#\# & 2.718 & 6.86E-03 & 44.02 & 97.69 & 76.9 & 0 & 101.1 & 387 \\
\hline 365 & 28.2 & 3000 & 1000 & 204.799673 & 8.5849 & 125.755 & 596.4 & 5125 & 0.1 & 1950 & 75000 & 1512500 & \begin{tabular}{|l|}
0.1128 \\
\end{tabular} & \#\#\#\#州 & 1.976 & 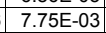 & 38.22 & 97.56 & 75.59 & 0 & 89.4 & 359.1 \\
\hline 366 & 25.43 & 3000 & 1190 & 14.880724 & 0.6776 & \begin{tabular}{|l|}
136.61 \\
\end{tabular} & 549.01 & 5125 & 0.1 & 1950 & 75000 & 1712500 & 0.1251 & \#\#\#\#\# & 2.234 & $1.24 \mathrm{E}-02$ & 26.39 & $\begin{array}{ll}66.4 \\
\end{array}$ & 0 & 0 & 55.8 & 450.2 \\
\hline 367 & 29.43 & 3000 & 1160 & 14.171978 & 0.6295 & 133.266 & 562.78 & 5125 & 0.1 & 1950 & 75000 & 1650000 & 0.1081 & \#\#\#\#\# & 2.348 & $1.38 \mathrm{E}-02$ & 24.36 & 64.72 & 0 & 0 & 50.1 & 409 \\
\hline 368 & 29.29 & 3000 & 870 & 695.927843 & 34.112 & 147.05 & 510.03 & 5125 & 0.1 & 1950 & 75000 & 1912500 & 0.1086 & \#\#\#\#曲 & 1.893 & \begin{tabular}{|l|l|}
$4.46 \mathrm{E}-03$ \\
\end{tabular} & 59.55 & 99.28 & 92.82 & 28.15 & 155.6 & 331.2 \\
\hline 369 & 62.14 & 3000 & 1530 & $\begin{array}{r}93.210793 \\
\end{array}$ & 4.6679 & 150.236 & 499.21 & 5125 & 0.1 & 1950 & 75000 & 1975000 & 0.0512 & \#\#\#\#\# & 4.648 & $8.32 \mathrm{E}-03$ & 53.33 & 94.64 & 46.36 & 0 & 83.3 & 383.4 \\
\hline 370 & 34.36 & 3000 & 2790 & 0.096311 & 0.0056 & 175.802 & 426.62 & 5125 & 0.1 & 1950 & 75000 & 2500000 & 0.0926 & 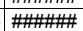 & 4.914 & \begin{tabular}{|l|}
$1.50 \mathrm{E}-02$ \\
\end{tabular} & 19.31 & 0 & 0 & 0 & 46.2 & 733.1 \\
\hline 371 & 26.89 & 3000 & 1630 & 86.499288 & 3.2145 & 111.485 & 672.73 & 5125 & 0.1 & 1950 & 75000 & 1262500 & 0.1183 & 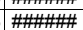 & 2.902 & \begin{tabular}{|l|l|} 
& $.049 \mathrm{E}-03$ \\
\end{tabular} & 51.73 & 94.22 & 42.2 & 0 & 106.8 & 553.1 \\
\hline 372 & 36.61 & 3000 & 1830 & 31.709354 & 0.9919 & 93.8425 & 799.21 & 5125 & 0.1 & 1950 & 75000 & 975000 & 0.0869 & \#冊曲 & 2.045 & \begin{tabular}{|l|l|}
$0.495-00$ \\
$9.88 E-03$ \\
\end{tabular} & 29.02 & $\begin{array}{l}34.24 \\
84.23 \\
\end{array}$ & $\begin{array}{ll}42.0 \\
0\end{array}$ & 0 & 70.1 & 486.5 \\
\hline 373 & 39.42 & 3000 & 920 & 335.700821 & 11.8919 & 106.273 & 705.73 & 5125 & 0.1 & 1950 & 75000 & 1175000 & 0.0807 & 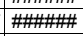 & 1.899 & \begin{tabular}{|c|}
$9.05 \mathrm{E}-03$ \\
\end{tabular} & 24.39 & 98.51 & 85.11 & 0 & 76.6 & 246.9 \\
\hline 374 & 54.38 & 3000 & 1950 & $\begin{array}{l}98.787016 \\
\end{array}$ & 3.1689 & 96.2335 & 779.35 & 5125 & 0.1 & 1950 & 75000 & 1012500 & $\begin{array}{l}0.0585 \\
0.058\end{array}$ & \#\#\#\#曲 & 4.56 & \begin{tabular}{|l|}
$7.89 \mathrm{E}-03$ \\
\end{tabular} & 35.86 & 94.94 & 49.39 & 0 & 87.8 & 429.8 \\
\hline 375 & 29.05 & 3000 & 2020 & 96.01123 & 3.5679 & 111.485 & \begin{tabular}{|c|c|}
672.73 \\
\end{tabular} & 5125 & 0.1 & 1950 & 75000 & 1262500 & $\begin{array}{l}0.1095 \\
\end{array}$ & 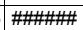 & 3.446 & $5.32 \mathrm{E}-03$ & 43.81 & 94.79 & 47.92 & 0 & 130.4 & 607.8 \\
\hline 376 & 42.42 & 3000 & 1220 & 65.75897 & 2.3624 & 107.775 & 695.9 & 5125 & 0.1 & 1950 & 75000 & 1200000 & 0.075 & \#\#\#\#\# & 2.811 & \begin{tabular}{|l|}
$1.20 \mathrm{E}-02$ \\
\end{tabular} & 31.96 & 92.4 & 23.96 & 0 & 58 & 339.6 \\
\hline 377 & 49.48 & 3000 & 590 & 492.99848 & 27.1295 & 165.089 & 454.3 & 5125 & 0.1 & 1950 & 75000 & 2275000 & 0.0643 & 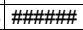 & 1.809 & \begin{tabular}{|l|}
$1.01 \mathrm{E}-02$ \\
\end{tabular} & 76.02 & 98.99 & 89.86 & 0 & 68.6 & 187.4 \\
\hline 378 & 25.55 & 3000 & 1040 & 136.04293 & 4.5065 & 99.3762 & 754.71 & 5125 & 0.1 & 1950 & 75000 & 1062500 & 0.1245 & \#\#\#\#\#曲 & 1.823 & \begin{tabular}{|l|}
$9.07 \mathrm{E}-03$ \\
\end{tabular} & 66.37 & 96.32 & 63.25 & 0 & 76.4 & 365.7 \\
\hline 379 & 54.1 & 3000 & 970 & 280.199514 & 12.003 & 128.512 & 583.6 & 5125 & 0.1 & 1950 & 75000 & 1562500 & 0.0588 & \#\#\#\#\# & 2.958 & $8.92 \mathrm{E}-03$ & 80.6 & 98.22 & 82.16 & 0 & 77.7 & 280.2 \\
\hline 380 & 28 & 3000 & 690 & 90.153732 & 4.722 & 157.131 & 477.31 & 5125 & 0.1 & 1950 & 75000 & 2112500 & 0.1136 & 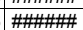 & 1.656 & \begin{tabular}{|l|l|} 
& $1.28 \mathrm{E}-02$ \\
\end{tabular} & 94.67 & 94.45 & 44.54 & 0 & 54.3 & 303.1 \\
\hline 381 & 21.8 & 3000 & 750 & \begin{tabular}{|c|}
566.615208 \\
\end{tabular} & 26.7967 & 141.878 & 528.62 & 5125 & 0.1 & 1950 & 75000 & 1812500 & 0.1459 & 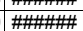 & 1.35 & \begin{tabular}{|l|l|} 
& $5.32 \mathrm{E}-03$ \\
\end{tabular} & 34.36 & $\begin{array}{l}49.4 \\
99.12 \\
\end{array}$ & $\begin{array}{l}4+.1 .18 \\
91.4\end{array}$ & 11.76 & $\begin{array}{l}340 \\
130\end{array}$ & 317.2 \\
\hline 382 & 24.59 & 3000 & 1760 & 0.499899 & 0.0154 & \begin{tabular}{|l|}
92.2314 \\
\end{tabular} & 813.17 & 5125 & 0.1 & 1950 & 75000 & 950000 & 0.1294 & \#\#\#\#\# & 3.326 & \begin{tabular}{|l|}
$1.64 \mathrm{E}-02$ \\
\end{tabular} & 60.68 & 0 & 0 & 0 & 42.2 & 693.4 \\
\hline 383 & 30.15 & 3000 & 1180 & 95.111464 & 4.0307 & \begin{tabular}{|l|}
127.137 \\
\end{tabular} & 589.91 & 5125 & 0.1 & 1950 & 75000 & 1537500 & 0.1055 & \#\#\#\#州 & 2.375 & \begin{tabular}{|c|}
$8.89 \mathrm{E}-03$ \\
\end{tabular} & 31.67 & 94.74 & 47.43 & 0 & 78 & 403.6 \\
\hline 384 & 24.8 & 3000 & 1260 & 1.764144 & 0.0552 & 93.8425 & 799.21 & 5125 & 0.1 & 1950 & 75000 & 975000 & 0.1283 & 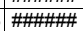 & 2.528 & 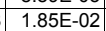 & 83.92 & 0 & 0 & 0 & 37.4 & 522.4 \\
\hline 385 & 39.57 & 3000 & 700 & 187.956624 & 8.1372 & 129.879 & 577.46 & 5125 & 0.1 & 1950 & 75000 & 1587500 & 0.0804 & \#\#\#\#\# & 1.58 & $1.39 \mathrm{E}-02$ & 32.57 & 97.34 & 73.4 & 0 & 49.8 & 204.6 \\
\hline 386 & 50.82 & 3000 & 3010 & 4.390803 & 0.1488 & \begin{tabular}{|l|}
101.701 \\
\end{tabular} & 737.46 & 5125 & 0.1 & 1950 & 75000 & 1100000 & $\begin{array}{l}0.0626 \\
\end{array}$ & \#\#\#\#\# & 5.973 & $1.12 \mathrm{E}-02$ & 21.8 & & 0 & 0 & 61.9 & 602.3 \\
\hline 387 & 56.41 & 3000 & 1270 & 13.61713 & 0.7846 & 172.859 & 433.88 & 5125 & 0.1 & 1950 & 75000 & 2437500 & 0.0564 & \#\#\#\#\#曲 & 3.97 & $1.59 \mathrm{E}-02$ & 39.19 & 63.28 & 0 & 0 & 43.6 & 360.7 \\
\hline 388 & 25.41 & 3000 & 2130 & 1.22186 & 0.0442 & 108.522 & 691.1 & 5125 & 0.1 & 1950 & 75000 & 1212500 & 0.1252 & \#\#\#\#" & 3.617 & \begin{tabular}{|l|}
$1.23 \mathrm{E}-02$ \\
\end{tabular} & 39.93 & 0 & 0 & 0 & 56.5 & 729.5 \\
\hline 389 & 36.32 & 3000 & 2240 & 40.397202 & 1.6653 & \begin{tabular}{|l|}
123.668 \\
\end{tabular} & 606.46 & 5125 & 0.1 & 1950 & 75000 & 1475000 & 0.0876 & \#\#\#\#\#曲 & 4.34 & \begin{tabular}{|l|}
$6.60 \mathrm{E}-03$ \\
\end{tabular} & 45.24 & 87.62 & 0 & 0 & 105.1 & 612.5 \\
\hline 390 & 31.16 & 3000 & 1280 & 38.833637 & 1.5644 & $\mid 120.857$ & 620.57 & 5125 & 0.1 & 1950 & 75000 & 1425000 & 0.1021 & 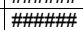 & 2.586 & $1.08 \mathrm{E}-02$ & 32.91 & 87.12 & 0 & 0 & 64.1 & 425.4 \\
\hline 391 & 40.63 & 3000 & 1910 & & 0.2352 & \begin{tabular}{|l|}
97.024 \\
\end{tabular} & 773 & 5125 & 0.1 & 1950 & 75000 & 1025000 & 0.0783 & \#\#\#\#\#曲 & 3.649 & \begin{tabular}{|l|}
$1.37 \mathrm{E}-02$ \\
\end{tabular} & 12.91 & 31.25 & 0 & 0 & 50.6 & 460.2 \\
\hline 392 & 58.7 & 3000 & 1030 & 35.8 & 1.5193 & \begin{tabular}{|l|}
127.137 \\
\end{tabular} & 589.91 & 5125 & 0.1 & 195 & 75000 & 1537500 & 0.0542 & \#\#\#\#\#立 & 3.131 & & 37.72 & 86.05 & 0 & 0 & 39.1 & 273.4 \\
\hline 393 & 31.34 & 3000 & 960 & 649.433507 & 23.4926 & 108.522 & $\begin{array}{l}691.1 \\
\end{array}$ & 5125 & 0.1 & 195 & & 1212 & 0.1015 & \#\#\#\#正 & 1.907 & \begin{tabular}{|l|}
5.1 \\
5.1
\end{tabular} & 77.71 & 99. & 92.3 & 3.01 & 135.8 & 311.8 \\
\hline 394 & 57.42 & 3000 & 1430 & $\begin{array}{l} \\
174.137066\end{array}$ & $\begin{array}{l}80.4520 \\
8.5356\end{array}$ & \begin{tabular}{|l|}
147.05 \\
\end{tabular} & 510.03 & 5125 & 0.1 & 1950 & 75000 & 1912500 & 0.0554 & \#\#\#\#曲 & 4.032 & \begin{tabular}{|l|l|}
$.16 \mathrm{E}-00$ \\
$7.6 \mathrm{E}$
\end{tabular} & 43.18 & 97.13 & 71.29 & 0 & 96.8 & 359.9 \\
\hline 395 & 23.19 & 3000 & 1490 & 272.867642 & 13.1409 & 144.475 & 519.12 & 5125 & 0.1 & 1950 & 75000 & 1862500 & 0.1372 & \#\#\#\#" & 2.499 & 3.92 & 41.73 & \begin{tabular}{|c|}
98.17 \\
\end{tabular} & 81.68 & 0 & 177 & 552.4 \\
\hline 396 & 21.34 & 3000 & 1730 & 1.182221 & 0.0327 & 83.1021 & 902.5 & 5125 & 0.1 & 1950 & 75000 & 812500 & 0.1491 & \#\#\#\#\#曲 & 2.554 & $1.44 \mathrm{E}-02$ & 33.97 & 0 & 0 & 0 & 48.1 & 613.4 \\
\hline 397 & 24.3 & 3000 & 1910 & 227.992292 & 6.1853 & 81.3886 & 921.51 & 5125 & 0.1 & 1950 & 75000 & 787500 & 0.1309 & \#\#\#\#\# & 2.944 & $4.11 \mathrm{E}-03$ & 75.31 & 97.81 & 78.07 & 0 & 168.8 & 620.9 \\
\hline 398 & 22.48 & 3000 & 760 & \begin{tabular}{|r|}
16.503828 \\
\end{tabular} & 0.7331 & 133.266 & 562.78 & 5125 & 0.1 & 1950 & 75000 & 1650000 & 0.1415 & 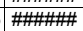 & 1.481 & \begin{tabular}{|c|}
$1.73 \mathrm{E}-02$ \\
\end{tabular} & 79.86 & 69.7 & 0 & 0 & 40.2 & 337.7 \\
\hline 399 & 48.79 & 3000 & 850 & 52.06285 & 2.301 & \begin{tabular}{|l|}
132.592 \\
\end{tabular} & 565.65 & 5125 & 0.1 & 1950 & 75000 & 1637500 & 0.0652 & \#\#\#\#\# & 2.442 & $1.73 \mathrm{E}-02$ & 50.23 & 90.4 & 3.96 & 0 & 40 & 256.5 \\
\hline 400 & 44.74 & 3000 & 2460 & 16.120043 & 0.5831 & \begin{tabular}{|l|}
108.522 \\
\end{tabular} & 691.1 & 5125 & 0.1 & 1950 & 75000 & 1212500 & 0.0711 & \#\#\#\#再 & 4.653 & \begin{tabular}{|l|l|}
$9.77 E-03$ \\
\end{tabular} & $\begin{array}{ll}16.18 \\
\end{array}$ & 68.98 & 0 & 0 & 71 & 532.9 \\
\hline 401 & 40.42 & 3000 & 550 & 1234.812961 & $\begin{array}{r}62.6182 \\
\end{array}$ & \begin{tabular}{|l|}
152.132 \\
\end{tabular} & 492.99 & 5125 & 0.1 & 1950 & 75000 & 2012500 & 0.0787 & \#\#\#\#曲 & 1.256 & \begin{tabular}{|l|l|}
5.69 \\
\end{tabular} & 92.87 & 99.6 & 95.95 & 59.51 & 121.8 & 159.2 \\
\hline 402 & 32.93 & 3000 & 1620 & 614.724 & $\begin{array}{l}22.6932 \\
\end{array}$ & \begin{tabular}{|l|}
10.748 \\
\end{tabular} & 677.21 & 512 & 0. & 195 & 75000 & 1250000 & 0.0966 & & 2.946 & & 26. & 99.19 & 91.87 & 18.66 & 212.6 & 458.4 \\
\hline 403 & 40.89 & 30 & 18 & & 1.0622 & 86.47 & 867 & 512 & 0 & 19 & & & & & & & 49 & & 0 & 0 & 68 & \\
\hline 404 & 26.67 & 30 & 11 & 124. & & 169.295 & 443 & 51 & 0.1 & 19 & 75 & 2362500 & 0.1 & \#\# & $\frac{2.3}{2.3}$ & 7.14 & 36 & 95 & 59.87 & 0 & 97.1 & 442.7 \\
\hline 405 & 22 & 30 & 1450 & 69.084 & 2.3773 & \begin{tabular}{|l|}
103.236 \\
\end{tabular} & 726. & 5125 & 0.1 & 1950 & 75 & 1125 & 0.1446 & \#\#\#\#曲 & 2.396 & 7.24 & 64. & 92. & 27.62 & 0 & 95.7 & 558 \\
\hline 406 & 52.85 & 3000 & 1300 & 34.666316 & 1.9355 & \begin{tabular}{|l|}
167.499 \\
\end{tabular} & 447.76 & 5125 & 0.1 & 1950 & 75000 & 2325000 & 0.0602 & \#\#\#\#\#曲 & 3.894 & $1.20 \mathrm{E}-02$ & 46.69 & 85.58 & 0 & 0 & 57.6 & 377.7 \\
\hline 407 & 27.86 & 3000 & 2180 & 126.826243 & 3.6911 & \begin{tabular}{|l|}
87.3109 \\
\end{tabular} & 859 & 5125 & 0.1 & 1950 & 75000 & 875000 & 0.1142 & \#\#\#\#" & 3.165 & 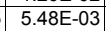 & 15.65 & 96.06 & 60.58 & 0 & 126.5 & 582.2 \\
\hline 408 & 41.21 & 3000 & 1440 & 202.172207 & 8.0011 & \begin{tabular}{|l|}
118.728 \\
\end{tabular} & $\begin{array}{l}631.7 \\
\end{array}$ & 5125 & 0.1 & 1950 & 75000 & 1387500 & 0.0772 & 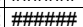 & 3.056 & \begin{tabular}{|l|} 
\\
\end{tabular} & 15.43 & 97.53 & 75.27 & 0 & 96.4 & 380 \\
\hline 409 & 33 & 3000 & 1370 & 39.703311 & 1.869 & \begin{tabular}{|l|}
141.225 \\
\end{tabular} & 531.07 & 5125 & 0.1 & 1950 & 75000 & 1800000 & 0.0964 & \#\#\#\# & 2.927 & \begin{tabular}{|l|l|}
$9.80 E-0$ \\
\end{tabular} & 37.24 & 87.41 & 0 & 0 & \begin{tabular}{|l|}
70.7 \\
\end{tabular} & 454.6 \\
\hline 410 & 50.1 & 3000 & 1960 & 97.295868 & 4.4305 & 136.61 & 549.01 & 5125 & 0.1 & 1950 & 75000 & 1712500 & 0.0635 & \#\#\#\#\# & 4.904 & & 61.03 & 94.86 & 48.61 & 0 & 120.2 & 501.7 \\
\hline 411 & 40.53 & 3000 & 2040 & 169.514568 & 9.566 & 169.295 & 443.01 & 5125 & 0.1 & 1950 & 75000 & 2362500 & \begin{tabular}{|l|l|}
0.0785 \\
\end{tabular} & & 4.137 & $3.98 \mathrm{E}-03$ & 35 & 97. & 70.5 & 0 & 174.2 & 523.2 \\
\hline 412 & 38.75 & 3000 & 1920 & & & & & 5125 & 0.1 & 19 & & & & & & & 51.72 & & & 0 & 118.6 & \\
\hline 413 & 22.33 & 3000 & 610 & 164.521332 & 10.3046 & \begin{tabular}{|l|}
187.902 \\
\end{tabular} & 399.14 & 5125 & 0.1 & 1950 & 75000 & 2762500 & 0.1425 & \#\#\#\#\# & 1.285 & $1.00 \mathrm{E}-02$ & 26.23 & 96.96 & 69.61 & 0 & 69.1 & 294.9 \\
\hline
\end{tabular}




\begin{tabular}{|c|c|c|c|c|c|c|c|c|c|c|c|c|c|c|c|c|c|c|c|c|c|c|}
\hline Realization & $\begin{array}{c}\text { Velocity } \\
(\mathrm{m} / \mathrm{y})\end{array}$ & $\begin{array}{c}\text { Fixed Source } \\
\text { Concentratio } \\
\mathrm{n}(\mathrm{mg} / \mathrm{L})\end{array}$ & $\begin{array}{c}\text { Time of } \\
\text { Maximum at } \\
\text { Compliance } \\
\text { Boundary } \\
\text { (y) }\end{array}$ & $\begin{array}{c}\text { Maximum } \\
\text { Concentration } \\
\begin{array}{c}\text { Compliance } \\
\text { Boundary } \\
(\mu \mathrm{g} / \mathrm{L})\end{array} \\
\end{array}$ & $\begin{array}{c}\text { Maximum } \\
\text { Release Rate } \\
\text { at Compliance } \\
\text { Boundary } \\
(\mathrm{Kg} / \mathrm{y})\end{array}$ & \begin{tabular}{|c|} 
\\
Source \\
Rate \\
$(\mathrm{Kg} / \mathrm{y})$
\end{tabular} & $\begin{array}{c}\text { Source } \\
\text { Duration } \\
\text { (y) }\end{array}$ & $\begin{array}{c}\text { 1-D } \\
\text { Streamtube } \\
\text { Length } \\
\text { (m) }\end{array}$ & $\begin{array}{c}\text { Base } \\
\text { Porosity } \\
\left(\mathrm{m}^{3} / \mathrm{m}^{3}\right)\end{array}$ & $\begin{array}{c}\text { Bulk } \\
\text { Densty } \\
\left(\mathrm{Kg} / \mathrm{m}^{3}\right)\end{array}$ & $\begin{array}{c}\text { Remaining } \\
\text { Carbon } \\
\text { Tetrachloride } \\
\text { Source } \\
(\mathrm{Kg})\end{array}$ & $\begin{array}{c}\begin{array}{c}\text { Volume at or } \\
\text { above Fixed } \\
\text { Source } \\
\text { Concentration } \\
\text { (m3) }\end{array} \\
\end{array}$ & $\begin{array}{l}\text { Porosity } \\
\left(\mathrm{m}^{3} / \mathrm{m}^{3}\right)\end{array}$ & $\begin{array}{c}\mathrm{Kd} \\
\left(\mathrm{m}^{3} / \mathrm{Kg}\right)\end{array}$ & $\begin{array}{l}\text { Retardatio } \\
\text { n }\end{array}$ & \begin{tabular}{|c|} 
First \\
Order \\
Rate \\
Constant \\
$(1 / y)$ \\
\end{tabular} & $\begin{array}{c}\text { Longitudinal } \\
\text { Dispersivity } \\
\text { (m) }\end{array}$ & \begin{tabular}{|c|} 
Required \\
Source \\
Cleanup \\
Percentage \\
$(5 \mu \mathrm{g} / \mathrm{L}$ \\
Compliance $)$ \\
\end{tabular} & \begin{tabular}{|c|} 
Required \\
Source \\
Cleanup \\
Percentage \\
$(50 \mu \mathrm{g} / \mathrm{L}$ \\
Compliance \\
)
\end{tabular} & \begin{tabular}{|c|} 
Required \\
Source \\
Cleanup \\
Percentage \\
$(500 \mu \mathrm{L} / \mathrm{L}$ \\
Compliance \\
)
\end{tabular} & \begin{tabular}{|c|} 
Abiotic \\
Reaction \\
Half Time \\
$(y)$ \\
\end{tabular} & $\begin{array}{c}\text { Carbon } \\
\text { Tetrachloride } \\
\text { Travel Time } \\
\text { (y) }\end{array}$ \\
\hline & 36.65 & \begin{tabular}{|l|}
5000 \\
\end{tabular} & $\begin{array}{l}2430 \\
\end{array}$ & \begin{tabular}{|l|}
35.568345 \\
\end{tabular} & \begin{tabular}{|l|l|}
5 & 1.251 \\
\end{tabular} & \begin{tabular}{|l|l|}
105.518 \\
\end{tabular} & \begin{tabular}{|l|} 
\\
\end{tabular} 10.78 & \begin{tabular}{|l|r|}
3 & 5125 \\
\end{tabular} & 0.1 & 1950 & 75000 & 1162500 & 0.0868 & 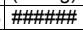 & 5.304 & \begin{tabular}{|c|}
$6.15 \mathrm{E}-03$ \\
\end{tabular} & $\begin{array}{r}87.44 \\
\end{array}$ & 85.94 & 0 & 0 & \begin{tabular}{|l|}
112.7 \\
\end{tabular} & 741.6 \\
\hline 415 & 61.65 & 3000 & 2180 & 3.250153 & 0.1593 & \begin{tabular}{|l|}
347.05 \\
\end{tabular} & 510.03 & 5125 & 0.1 & 1950 & 75000 & 1912500 & 0.0516 & 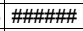 & 5.631 & \begin{tabular}{|l|}
$1.51 \mathrm{E}-02$ \\
\end{tabular} & 22.67 & 0 & 0 & 0 & 45.9 & 468 \\
\hline 416 & 26.85 & 3000 & 890 & 9.858124 & 0.4641 & \begin{tabular}{|l|}
141.225 \\
\end{tabular} & 531.07 & 5125 & 0.1 & 1950 & 75000 & 1800000 & 0.1185 & \#\#\#\#\# & 1.967 & $1.70 \mathrm{E}-02$ & 55.32 & 49.28 & 0 & 0 & 40.7 & 375.5 \\
\hline 417 & 57.84 & 3000 & 2040 & \begin{tabular}{|l|}
26.877967 \\
\end{tabular} & 1.3573 & \begin{tabular}{|l|l|}
3 & 151.501 \\
\end{tabular} & 495.05 & 5125 & 0.1 & 1950 & 75000 & 2000000 & 0.055 & \#\#\#\#曲 & 5.139 & \begin{tabular}{|l|}
$9.70 \mathrm{E}-03$ \\
\end{tabular} & 28.65 & 81.4 & 0 & 0 & 71.5 & 455.3 \\
\hline 418 & 26.49 & 3000 & 970 & 202.285018 & 6.7008 & \begin{tabular}{|l|l|}
39.3762 \\
\end{tabular} & 754.71 & 5125 & 0.1 & 1950 & 75000 & 1062500 & 0.1201 & 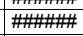 & 1.592 & 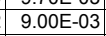 & 33.64 & 97.53 & 75.28 & 0 & 77 & 308 \\
\hline 419 & 24.64 & 3000 & 1430 & 26.593228 & 1.1513 & \begin{tabular}{|l|l|}
129.879 \\
\end{tabular} & 577.46 & 5125 & 0.1 & 1950 & 75000 & 1587500 & 0.1291 & \#曲曲 & 2.473 & \begin{tabular}{|l|} 
\\
$9.53 \mathrm{E}-03$ \\
\end{tabular} & 25.48 & \begin{tabular}{r|}
81.05 \\
81.2 \\
\end{tabular} & 0 & 0 & 72.7 & 514.3 \\
\hline 420 & 49.4 & 3000 & 1270 & 98.60094 & 3.0047 & 91.4206 & 820.38 & 5125 & 0.1 & 1950 & 75000 & 937500 & 0.0644 & \#\#\#\#" & 2.841 & \begin{tabular}{|l|}
$1.20 \mathrm{E}-02$ \\
\end{tabular} & 19.79 & 94.93 & 49.29 & 0 & 57.7 & 294.8 \\
\hline 421 & 21.22 & 3000 & 1050 & 80.888909 & 3.9476 & 146.408 & 512.27 & 5125 & 0.1 & 1950 & 75000 & 1900000 & 0.1499 & \#\#\#\#\#曲 & 1.875 & $8.28 \mathrm{E}-03$ & 36.15 & 93.82 & 38.19 & & 83.7 & 452.9 \\
\hline 422 & 24.97 & 3000 & 1400 & 4.374892 & 0.1636 & 112.22 & 668.33 & 5125 & 0.1 & 1950 & 75000 & 1275000 & 0.1274 & 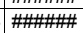 & 3.02 & $1.36 \mathrm{E}-02$ & $\begin{array}{l}91.3 \\
91\end{array}$ & 0 & 0 & 0 & 51 & 619.9 \\
\hline 423 & 24.03 & 3000 & 1240 & 60.854911 & 1.8873 & 93.0387 & 806.12 & 5125 & 0.1 & 1950 & 75000 & 962500 & 0.1324 & 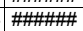 & 2.045 & $9.63 \mathrm{E}-03$ & 50.8 & 91.78 & 17.84 & 0 & 72 & 436.2 \\
\hline 424 & 22.12 & 3000 & 1380 & 33.272955 & 1.5808 & \begin{tabular}{|l|}
342.53 \\
\end{tabular} & 526.21 & 5125 & 0.1 & 1950 & 75000 & 1825000 & 0.1438 & \#\#\#\#州 & 2.661 & \begin{tabular}{|l|}
$7.77 \mathrm{E}-03$ \\
\end{tabular} & 86.8 & 84.97 & 0 & 0 & 89.2 & 616.5 \\
\hline 425 & 38.47 & 3000 & 1210 & 110.3439 & 3.5687 & 97.024 & 773 & 5125 & 0.1 & 1950 & 75000 & 1025000 & 0.0827 & 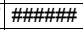 & 2.568 & $1.03 \mathrm{E}-02$ & 42.91 & 95.47 & 54.69 & & 67.2 & 342.1 \\
\hline 426 & 25.76 & 3000 & 930 & 104.887159 & 6.3899 & 182.765 & 410.36 & 5125 & 0.1 & 1950 & 75000 & 2650000 & 0.1235 & \#\#\#\#\# & 2.13 & $8.06 \mathrm{E}-03$ & 75.48 & 95.23 & 52.33 & 0 & 86 & 423.8 \\
\hline 427 & 40.17 & 3000 & 2600 & 20.086459 & 0.6337 & 94.6429 & 792.45 & 5125 & 0.1 & 1950 & 75000 & 987500 & 0.0792 & \#\#\#\#曲 & 4.406 & $8.95 \mathrm{E}-03$ & 13.19 & 75.11 & 0 & 0 & 77.4 & 562.1 \\
\hline 428 & 33.7 & 3000 & 720 & 213.312776 & 10.1807 & 143.18 & 523.82 & 5125 & 0.1 & 1950 & 75000 & $\begin{array}{r}1837500 \\
\end{array}$ & 0.0944 & \#\#\#\#\# & 1.714 & \begin{tabular}{|c|} 
\\
\end{tabular} & 69.34 & 97.66 & 76.56 & 0 & 64.4 & 260.6 \\
\hline 429 & 42.08 & 3000 & 2660 & 2.800323 & 0.076 & \begin{tabular}{|l|l|} 
& 17.3886 \\
\end{tabular} & 921.51 & 5125 & 0.1 & 1950 & 75000 & 787500 & 0.0756 & 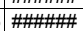 & 4.93 & \begin{tabular}{|l|l|}
$1.28 \mathrm{E}-02$ \\
\end{tabular} & 23.74 & 0 & 0 & 0 & 54.1 & 600.4 \\
\hline 430 & 29.48 & 3000 & 1120 & 98.835349 & 2.4493 & 74.3438 & 1008.83 & 5125 & 0.1 & 1950 & 75000 & 687500 & 0.1079 & \#\#\#\#\# & 1.584 & \begin{tabular}{|l|}
$1.35 \mathrm{E}-02$ \\
\end{tabular} & 80.36 & 94.94 & 49.41 & 0 & 51.6 & 275.4 \\
\hline 431 & 21.93 & 3000 & 1220 & 12.1909 & 0.6904 & 169.892 & 441.46 & 5125 & 0.1 & 1950 & 75000 & 2375000 & 0.1451 & \#\#\#\#\# & 2.25 & 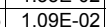 & 34.69 & 58.99 & 0 & 0 & 63.4 & 525.9 \\
\hline 432 & 61.53 & 3000 & 950 & 860.781927 & 23.1048 & \begin{tabular}{|l|l|}
3 & 80.525 \\
\end{tabular} & 931.39 & 5125 & 0.1 & 1950 & 75000 & 775000 & 0.0517 & \#\#\#\#曲 & 1.977 & $7.68 \mathrm{E}-03$ & 26.87 & 99.42 & 94.19 & 41.91 & 90.3 & 164.7 \\
\hline 433 & 22.39 & 3000 & 1250 & 473.237037 & 14.4212 & 91.4206 & 820.38 & 5125 & 0.1 & 1950 & 75000 & 937500 & 0.1421 & \#\#\#\#\#曲 & 1.909 & \begin{tabular}{|l|}
$4.38 \mathrm{E}-03$ \\
\end{tabular} & 57.67 & 98.94 & 89.43 & 0 & 158.4 & 436.9 \\
\hline 434 & 35.23 & 3000 & 1580 & 142.140495 & 5.317 & 112.22 & 668.33 & 5125 & 0.1 & 1950 & 75000 & 1275000 & 0.0903 & \#\#\#\#曲 & 3.401 & \begin{tabular}{|r|}
$6.03 \mathrm{E}-03$ \\
\end{tabular} & 72.85 & 96.48 & 64.82 & 0 & 114.9 & 494.8 \\
\hline 435 & 24.72 & 3000 & 1080 & 225.671131 & 6.938 & 92.2314 & 813.17 & 5125 & 0.1 & 1950 & 75000 & 950000 & 0.1287 & \#\#\#\#\#曲 & 1.743 & $7.53 \mathrm{E}-03$ & 58.72 & 97.78 & 77.84 & 0 & 92.1 & 361.4 \\
\hline 436 & 55.91 & 3000 & 1480 & 180.587293 & 5.6971 & \begin{tabular}{|l|}
4.6429 \\
\end{tabular} & 792.45 & 5125 & 0.1 & 1950 & 75000 & 987500 & 0.0569 & \#\#\#\#\# & 3.983 & \begin{tabular}{|l|}
$8.08 \mathrm{E}-03$ \\
\end{tabular} & 68.48 & 97.23 & 72.31 & 0 & 85.7 & 365.1 \\
\hline 437 & 24.78 & 3000 & 1160 & 125.144378 & 5.5029 & 131.916 & 568.54 & 5125 & 0.1 & 1950 & 75000 & 1625000 & 0.1284 & \#\#\#\#\#曲 & 2.116 & $7.47 \mathrm{E}-03$ & 29.05 & 96 & 60.05 & 0 & 92.8 & 437.8 \\
\hline 438 & 23.57 & 3000 & 1440 & 5.253565 & 0.2609 & 148.966 & 503.47 & 5125 & 0.1 & 1950 & 75000 & 1950000 & 0.135 & \#\#\#\#\#曲 & 2.95 & $1.15 \mathrm{E}-02$ & 73.84 & 4.83 & 0 & 0 & 60.5 & 641.6 \\
\hline 439 & 55.62 & 3000 & 3120 & 3.389522 & 0.0778 & 68.8336 & 1089.58 & 5125 & 0.1 & 1950 & 75000 & 612500 & 0.0572 & 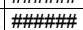 & 6.495 & \begin{tabular}{|l|l|}
$1.30 \mathrm{E}-02$ \\
\end{tabular} & 27.04 & & 0 & & 53.4 & 598.5 \\
\hline 440 & 36.19 & 3000 & 1340 & 25.443726 & 0.8756 & 103.236 & 726.49 & 5125 & 0.1 & 1950 & 75000 & 1125000 & 0.0879 & 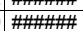 & $\begin{array}{l}2.4997 \\
2.997\end{array}$ & $\mid$\begin{tabular}{|l|l|}
$1.27 E-02$ \\
\end{tabular} & 55.88 & 80.35 & 0 & 0 & 54.5 & 424.4 \\
\hline 441 & 34.65 & 3000 & 820 & 188.32573 & 9.1908 & \begin{tabular}{|l|}
146.408 \\
\end{tabular} & 512.27 & 5125 & 0.1 & 1950 & 75000 & 1900000 & 0.0918 & \#\#\#\#" & 2.124 & \begin{tabular}{|l|} 
\\
\end{tabular} & 92.4 & 97.35 & 73.45 & 0 & 72.7 & 314 \\
\hline 442 & 23.31 & 3000 & 1010 & $\begin{array}{r}9.605885 \\
\end{array}$ & $\begin{array}{l}0.3756 \\
\end{array}$ & \begin{tabular}{|l|}
117.297 \\
\end{tabular} & 639.4 & 5125 & 0.1 & 1950 & 75000 & 1362500 & 0.1365 & 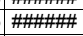 & 1.865 & \begin{tabular}{|l|}
$1.54 \mathrm{E}-02$ \\
\end{tabular} & 48.42 & 47.95 & 0 & 0 & 45 & 410 \\
\hline 443 & 28.25 & 3000 & 980 & 382.523501 & 12.6712 & 99.3762 & 754.71 & 5125 & 0.1 & 1950 & 75000 & 1062500 & 0.1126 & 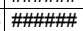 & 1.749 & \begin{tabular}{|l|}
$6.83 \mathrm{E}-03$ \\
\end{tabular} & 73.71 & 98.69 & 86.93 & 0 & 101.6 & 317.3 \\
\hline 444 & 41.8 & 3000 & 2150 & 2.915747 & 0.1587 & \begin{tabular}{|l|}
163.27 \\
\end{tabular} & 459.36 & 5125 & 0.1 & 1950 & 75000 & 2237500 & 0.0761 & \#\#\#\#\# & 5.047 & $1.21 \mathrm{E}-02$ & 43.14 & 0 & 0 & 0 & 57.3 & 618.7 \\
\hline 445 & 23.58 & 3000 & 870 & 63.580679 & 1.5372 & 72.5304 & 1034.05 & 5125 & 0.1 & 1950 & 75000 & 662500 & 0.1349 & \#\#\#\#\# & 1.291 & $1.41 \mathrm{E}-02$ & 29.77 & 92.14 & 21.36 & & 49 & 280.5 \\
\hline 446 & 26.06 & 3000 & 1200 & 36.697144 & 1.1381 & \begin{tabular}{|l|}
93.0387 \\
\end{tabular} & 806.12 & 5125 & 0.1 & 1950 & 75000 & 962500 & 0.1221 & 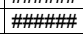 & 2.108 & \begin{tabular}{|l|}
$1.17 \mathrm{E}-02$ \\
\end{tabular} & 57.55 & 86.37 & 0 & 0 & 59.2 & 414.5 \\
\hline 447 & 33.07 & 3000 & 890 & 381.826165 & 18.7158 & 147.05 & 510.03 & 5125 & 0.1 & 1950 & 75000 & 1912500 & 0.0962 & \#\#\#\#" & 2.024 & $6.73 \mathrm{E}-03$ & 33.72 & 98.69 & 86.91 & 0 & 103.1 & 313.7 \\
\hline 448 & 35.11 & 3000 & 1570 & $\begin{array}{l}7.331142 \\
\end{array}$ & 0.3207 & \begin{tabular}{|l|}
131.239 \\
\end{tabular} & 571.48 & 5125 & 0.1 & 1950 & 75000 & 1612500 & 0.0906 & \#\#\#\#\#曲 & 4.096 & $1.25 \mathrm{E}-02$ & 85.72 & 31.8 & 0 & 0 & 55.3 & 597.8 \\
\hline 449 & 61.89 & 3000 & 1190 & 437.66608 & 13.2184 & 90.6061 & 827.76 & 5125 & 0.1 & 1950 & 75000 & 925000 & 0.0514 & \#\#\#\#\# & 2.962 & $8.10 \mathrm{E}-03$ & 28.93 & 98.86 & 88.58 & 0 & 85.6 & 245.3 \\
\hline 450 & 26.21 & 3000 & 660 & 260.085049 & 15.1394 & $\mid 174.628$ & 429.49 & 5125 & 0.1 & 1950 & 75000 & 2475000 & 0.1214 & \#\#\#\#\#曲 & 1.525 & $8.58 \mathrm{E}-03$ & 67.37 & 98.08 & 80.78 & & 80.8 & 298.3 \\
\hline 451 & 22.56 & 3000 & 1880 & 65964 & 0.3736 & 122.267 & 613.41 & 5125 & 0.1 & 195 & 75000 & 1450000 & 0.141 & \#\#\#\#西 & 2.98 & 8.98 & 37.52 & 45.45 & 0 & 0 & 77.2 & 677 \\
\hline 452 & 23.34 & 3000 & 1900 & & 0.1067 & \begin{tabular}{|l|}
148.328 \\
\end{tabular} & 505.63 & 5125 & 0.1 & 1950 & 75000 & 1937500 & 0.1363 & 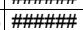 & 3.521 & & 60.91 & 0 & 0 & 0 & 64.6 & 773 \\
\hline 453 & 26.8 & 3000 & 640 & 864.54431 & $\begin{array}{r}0.10512 \\
\end{array}$ & \begin{tabular}{|r|}
4.0320 \\
163.27 \\
\end{tabular} & 459.36 & 5125 & 0.1 & 1950 & 75000 & 2237500 & 0.1187 & \#\#\#\#曲 & 1.364 & 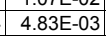 & 35.46 & 99.42 & 94.22 & 42.17 & 143.6 & 260.9 \\
\hline 454 & 38.61 & 3000 & 980 & 297.57734 & 11.5637 & $\mid 116.579$ & 643.34 & 5125 & 0.1 & 1950 & 75000 & 1350000 & 0.0824 & \#\#\#\#" & 2.3 & 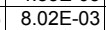 & 66.48 & 98.32 & 83.2 & 0 & 86.5 & 305.3 \\
\hline 455 & 40.68 & 3000 & 1570 & 49.183662 & 1.9113 & \begin{tabular}{|l|}
116.579 \\
\end{tabular} & 643.34 & 5125 & 0.1 & 1950 & 75000 & 1350000 & 0.0782 & \#\#\#\#曲 & 3.851 & \begin{tabular}{|c|}
$9.03 \mathrm{E}-03$ \\
\end{tabular} & 68.85 & 89.83 & 0 & 0 & 76.7 & 485.2 \\
\hline 456 & 32.46 & 3000 & 940 & 231.901849 & 9.6136 & \begin{tabular}{|l|}
124.366 \\
\end{tabular} & 603.06 & 5125 & 0.1 & 1950 & 75000 & 1487500 & 0.098 & \#\#\#\#\# & 2.133 & $8.12 \mathrm{E}-03$ & 78.92 & 97.84 & 78.44 & 0 & 85.3 & 336.8 \\
\hline 457 & 44.81 & 3000 & 1140 & 434.942011 & 12.8984 & 88.9661 & 843.02 & 5125 & 0.1 & 1950 & 75000 & 900000 & 0.071 & \#\#\#\#\#曲 & 2.317 & $7.40 \mathrm{E}-03$ & 18 & 98.85 & 88.5 & 0 & 93.6 & 265 \\
\hline 458 & 21.73 & 3000 & 1160 & 5.896834 & 0.3292 & \begin{tabular}{|l|}
167.499 \\
\end{tabular} & 447.76 & 5125 & 0.1 & 1950 & 75000 & 2325000 & 0.1464 & 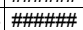 & 2.202 & $1.29 \mathrm{E}-02$ & 40.25 & 15.21 & 0 & 0 & 53.7 & 519.3 \\
\hline 459 & 22.99 & 3000 & 2120 & 37.721746 & 1.2691 & 100.929 & 743.1 & 5125 & 0.1 & 1950 & 75000 & 1087500 & 0.1384 & \#\#m\#\# & 2.98 & \begin{tabular}{|l|l|}
$6.66 \mathrm{E}-03$ \\
\end{tabular} & 24.05 & 86.75 & 0 & 0 & 104 & 664.4 \\
\hline 460 & 22.44 & 3000 & 1000 & 29.372595 & 1.4397 & \begin{tabular}{|l|}
147.05 \\
\end{tabular} & 510.03 & 5125 & 0.1 & 1950 & 75000 & 1912500 & 0.1418 & \#\#冊 & 2.111 & \begin{tabular}{|c|}
$0.00 \mathrm{E}-00$ \\
$1.09 \mathrm{E}$
\end{tabular} & $\begin{array}{l}4.40 \\
93.45\end{array}$ & $\begin{array}{l}0.1 \% \\
82.98\end{array}$ & 0 & 0 & $\begin{array}{ll}63.7 \\
63.7\end{array}$ & 484.4 \\
\hline 461 & 50.74 & 3000 & 830 & .77799 & 6.6875 & \begin{tabular}{|l|}
112.22 \\
\end{tabular} & 668.33 & 512 & 0.1 & 1950 & 75000 & 1275000 & 0.0627 & \#\#\#\#\#曲 & 1.994 & $\begin{array}{l}1.45 \mathrm{E}-02 \\
\end{array}$ & 34.64 & $\begin{array}{l}97.2 \\
9\end{array}$ & 72.03 & 0 & 47.7 & 201.4 \\
\hline 462 & 30.8 & 30 & 84 & & 2.7702 & 137.274 & 546 & 5125 & 0 & 19 & & & & & 1.8 & & 48 & 91 & 17.41 & 0 & 52 & \\
\hline 463 & 25. & 30 & 930 & 72.569 & 3.1582 & 130.56 & 574 & & 0.1 & 19 & & 1600000 & & \#\# & 1.7 & 1.08 & 35. & 93 & 31.1 & 0 & 64.5 & 361.9 \\
\hline 464 & 46.44 & 30 & 1320 & 70.073 & 2.8559 & \begin{tabular}{|l|}
122.267 \\
\end{tabular} & 613. & 5125 & 0.1 & 1950 & 750 & 1450000 & 0.06 & \#\#\#\#\# & 3.167 & $1.11 \mathrm{E}-02$ & 22.09 & 92. & 28.65 & 0 & 62.2 & 349.5 \\
\hline 465 & 25.45 & 3000 & 960 & 97.817719 & 2.9008 & 88.9661 & 843.02 & 5125 & 0.1 & 1950 & 75000 & 900000 & 0.125 & 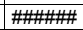 & 1.401 & \begin{tabular}{|c|}
$1.30 \mathrm{E}-02$ \\
\end{tabular} & 72.52 & 94.89 & 48.88 & 0 & 53.5 & 282 \\
\hline 466 & 41.48 & 3000 & 1120 & 260.751272 & 7.0741 & \begin{tabular}{|l|}
81.3886 \\
\end{tabular} & 921.51 & 5125 & 0.1 & 1950 & 75000 & 787500 & 0.0767 & \#\#\#\#" & 1.996 & \begin{tabular}{|l|}
$1.01 E-02$ \\
\end{tabular} & 17.62 & 98.08 & 80.82 & 0 & 68.8 & 246.6 \\
\hline 467 & 21.37 & 3000 & 720 & 382.942008 & 23.8403 & \begin{tabular}{|l|}
186.767 \\
\end{tabular} & 401.57 & 5125 & 0.1 & 1950 & 75000 & 2737500 & 0.1489 & \#\#\#\#曲 & 1.433 & \begin{tabular}{|l|l|}
$6.06 \mathrm{E}-03$ \\
\end{tabular} & 20.12 & 98.69 & 86.94 & 0 & 114.4 & 343.7 \\
\hline 468 & 50.26 & 3000 & 1010 & 310.884123 & 17.2329 & $|166.296|$ & 451 & 5125 & 0.1 & 1950 & 75000 & 2300000 & 0.0633 & \#\#\#\# & 2.862 & $7.74 \mathrm{E}-03$ & 25.75 & 98.39 & 83.92 & 0 & $\mid 89.6$ & 291.8 \\
\hline 469 & 53.92 & 3000 & 1080 & 120.77368 & 7.9699 & \begin{tabular}{|l|}
197.971 \\
\end{tabular} & 378.84 & 5125 & 0.1 & 1950 & 75000 & 2987500 & 0.059 & \#\#\#\#" & 3.201 & $1.03 \mathrm{E}-02$ & 21.47 & 95.86 & 58.6 & 5 & 67 & 304.2 \\
\hline 470 & 35.87 & 3000 & 1150 & 12.217544 & 0.4777 & 117.297 & 639.4 & 5125 & 0.1 & 1950 & 75000 & 1362500 & 0.0887 & \#\# & 2.847 & $1.60 \mathrm{E}-02$ & 66. & 59. & 0 & 0 & 43.4 & 406.8 \\
\hline 471 & 45.06 & 3000 & 1540 & & & & 490. & 51 & 0.1 & 195 & & & 0.07 & & & & 71 & & 0 & 0 & 62 & \\
\hline 472 & 41.53 & 3000 & 1270 & 16.622623 & 0.693 & 125.062 & 599.7 & 5125 & 0.1 & 1950 & 75000 & 1500000 & 0.0766 & \#\#\#\#" & 2.92 & $1.51 \mathrm{E}-02$ & 18.1 & 69.92 & 0 & 0 & 45.8 & 360.3 \\
\hline
\end{tabular}




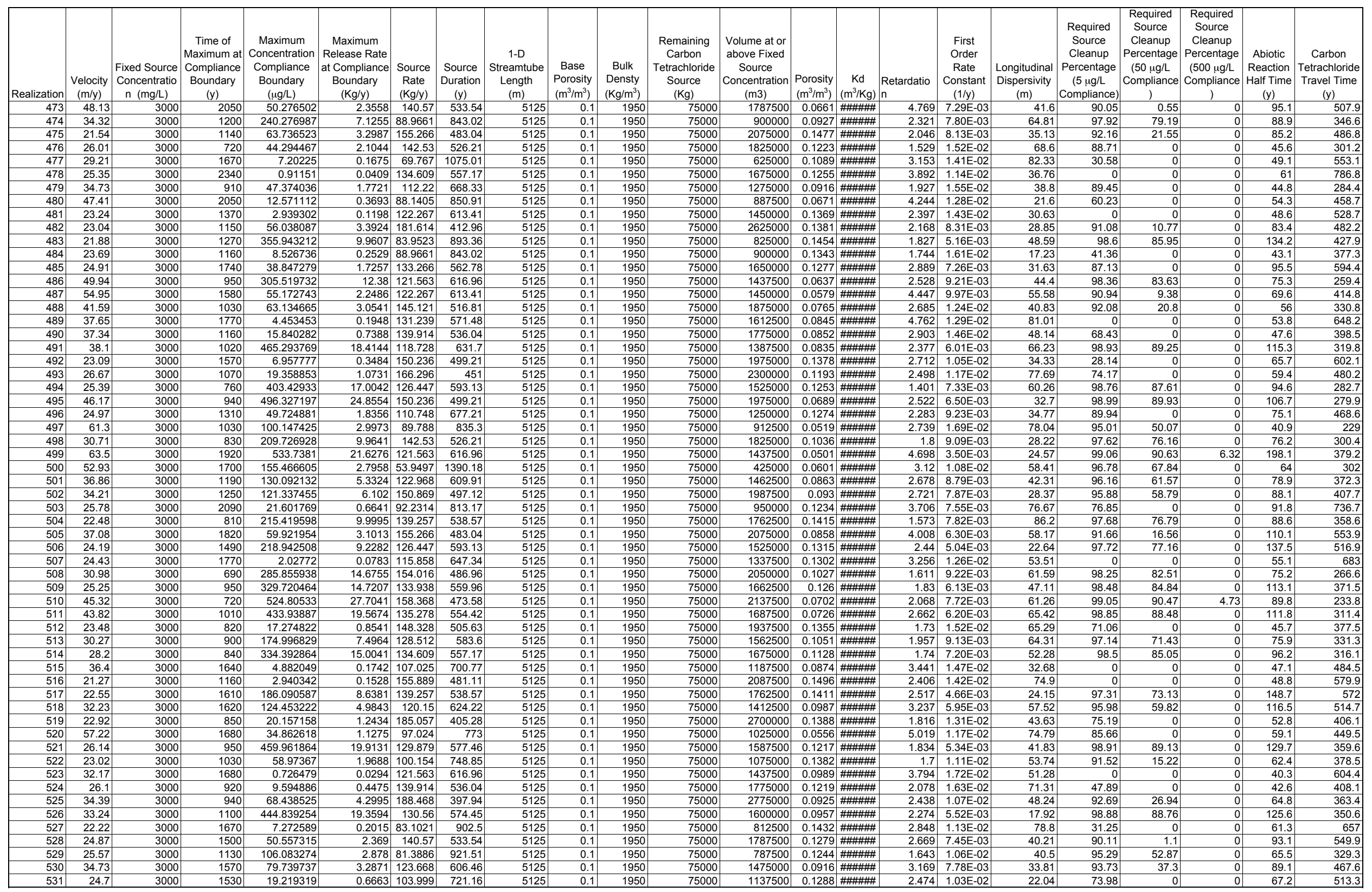




\begin{tabular}{|c|c|c|c|c|c|c|c|c|c|c|c|c|c|c|c|c|c|c|c|c|c|c|}
\hline Realization & $\begin{array}{c}\text { Velocity } \\
(\mathrm{m} / \mathrm{y})\end{array}$ & \begin{tabular}{|c|} 
Fixed Source \\
Concentratio \\
$\mathrm{n}(\mathrm{mg} / \mathrm{L})$
\end{tabular} & $\begin{array}{c}\text { Time of } \\
\text { Maximum at } \\
\text { Compliance } \\
\text { Boundary } \\
(y)\end{array}$ & \begin{tabular}{|c|} 
Maximum \\
Concentration \\
Compliance \\
Boundary \\
$(\mu \mathrm{g} / \mathrm{L})$
\end{tabular} & $\begin{array}{c}\text { Maximum } \\
\text { Release Rate } \\
\text { at Compliance } \\
\text { Boundary } \\
(\mathrm{Kg} / \mathrm{y})\end{array}$ & \begin{tabular}{|c|} 
\\
Source \\
Rate \\
$(\mathrm{Kg} / \mathrm{y})$ \\
\end{tabular} & $\begin{array}{c}\text { Source } \\
\text { Duration } \\
\text { (y) }\end{array}$ & $\begin{array}{c}\text { 1-D } \\
\text { Streamtube } \\
\text { Length } \\
\text { (m) }\end{array}$ & $\begin{array}{c}\text { Base } \\
\text { Porosity } \\
\left(\mathrm{m}^{3} / \mathrm{m}^{3}\right)\end{array}$ & $\begin{array}{c}\text { Bulk } \\
\text { Densty } \\
\left(\mathrm{Kg} / \mathrm{m}^{3}\right)\end{array}$ & $\begin{array}{c}\text { Remaining } \\
\text { Carbon } \\
\text { Tetrachloride } \\
\text { Source } \\
(\mathrm{Kg})\end{array}$ & $\begin{array}{c}\begin{array}{c}\text { Volume at or } \\
\text { above Fixed } \\
\text { Source } \\
\text { Concentration } \\
(\mathrm{m} 3)\end{array} \\
\end{array}$ & $\begin{array}{l}\text { Porosity } \\
\left(\mathrm{m}^{3} / \mathrm{m}^{3}\right)\end{array}$ & $\begin{array}{c}\mathrm{Kd} \\
\left(\mathrm{m}^{3} / \mathrm{Kg}\right)\end{array}$ & $\begin{array}{l}\text { Retardatio } \\
n\end{array}$ & \begin{tabular}{|c|} 
First \\
Order \\
Rate \\
Constant \\
$(1 / y)$ \\
\end{tabular} & $\begin{array}{l}\text { Longitudinal } \\
\text { Dispersivity } \\
\text { (m) }\end{array}$ & \begin{tabular}{|c|} 
Required \\
Source \\
Cleanup \\
Percentage \\
$(5 \mu \mathrm{g} / \mathrm{L}$ \\
Compliance $)$ \\
\end{tabular} & \begin{tabular}{|c|} 
Required \\
Source \\
Cleanup \\
Percentage \\
$(50 \mu \mathrm{g} / \mathrm{L}$ \\
Compliance \\
) \\
\end{tabular} & \begin{tabular}{|c|} 
Required \\
Source \\
Cleanup \\
Percentage \\
$(500 \mu \mathrm{L} / \mathrm{L}$ \\
Compliance \\
) \\
\end{tabular} & \begin{tabular}{|c|} 
\\
Abiotic \\
Reaction \\
Half Time \\
$(y)$ \\
\end{tabular} & \begin{tabular}{|c|} 
Carbon \\
Tetrachloride \\
Travel Time \\
(y)
\end{tabular} \\
\hline 532 & 51.39 & 3000 & & \begin{tabular}{|l|}
251.721525 \\
\end{tabular} & 6.238 & 74.3438 & 1008.83 & & & & 75000 & 687500 & \begin{tabular}{|l|}
0.0619 \\
\end{tabular} & \#\#\#\#曲 & 4.404 & 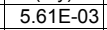 & 58.32 & 98.01 & 80.14 & 0 & \begin{tabular}{|l|}
123.6 \\
\end{tabular} & 439.1 \\
\hline 533 & 39.42 & 3000 & 1370 & 67.528755 & 2.0943 & \begin{tabular}{|l|}
93.0387 \\
\end{tabular} & \begin{tabular}{|l|}
806.12 \\
\end{tabular} & 5125 & 0.1 & 1950 & 75000 & 962500 & 0.0807 & 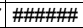 & 2.804 & \begin{tabular}{|c|}
$1.10 \mathrm{E}-02$ \\
\end{tabular} & 28.58 & 92.6 & 25.96 & 0 & 63 & 364.5 \\
\hline 534 & 37.92 & 3000 & 1540 & 238.218312 & $\begin{array}{ll}7.1297 \\
\end{array}$ & \begin{tabular}{|l|}
89.788 \\
\end{tabular} & 835.3 & 5125 & 0.1 & 1950 & 75000 & 912500 & 0.0839 & \#\#\#\#\# & 3.103 & $6.24 \mathrm{E}-03$ & 53.15 & 97.9 & 79.01 & 0 & \begin{tabular}{|c|}
111.1 \\
\end{tabular} & 419.4 \\
\hline 535 & 31.25 & 3000 & 1510 & 60.49608 & 2.2184 & \begin{tabular}{|l|}
110.009 \\
\end{tabular} & 681.77 & 5125 & 0.1 & 1950 & 75000 & 1237500 & \begin{tabular}{|l|}
0.1018 \\
\end{tabular} & \#\#\#\#\#曲 & 3.223 & \begin{tabular}{|l|}
$8.00 \mathrm{E}-03$ \\
\end{tabular} & 81.85 & 91.74 & 17.35 & 0 & 86.7 & 528.6 \\
\hline 536 & 31.59 & 3000 & 910 & 186.910685 & 10.2101 & \begin{tabular}{|l|}
163.877 \\
\end{tabular} & 457.66 & 5125 & 0.1 & 1950 & 75000 & 2250000 & \begin{tabular}{|l|}
0.1007 \\
\end{tabular} & \#\#\#\# & 2.044 & \begin{tabular}{|l|} 
\\
\end{tabular} & 15.12 & 97.32 & 73.25 & 0 & 81.5 & 331.6 \\
\hline 537 & 59.8 & 3000 & 1200 & 413.421637 & 23.3301 & \begin{tabular}{|l|}
169.295 \\
\end{tabular} & 443.01 & 5125 & 0.1 & 1950 & 75000 & 2362500 & \begin{tabular}{|l|}
0.0532 \\
\end{tabular} & 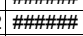 & 3.7 & \begin{tabular}{|l|l|}
$4.98 \mathrm{E}-03$ \\
\end{tabular} & 50.93 & 98.79 & 87.91 & 0 & 139.1 & 317.1 \\
\hline 538 & 53.56 & 3000 & 2530 & 14.799742 & 0.5055 & \begin{tabular}{|l|}
102.47 \\
\end{tabular} & 731.92 & 5125 & 0.1 & 1950 & 75000 & 1112500 & \begin{tabular}{|l|}
0.0594 \\
\end{tabular} & \#\#\#\#\# & 6.697 & \begin{tabular}{|c|}
$9.44 \mathrm{E}-03$ \\
\end{tabular} & 64.69 & 66.22 & 0 & & 73.4 & 640.8 \\
\hline 539 & 39.72 & 3000 & 2270 & 27.755119 & 0.8458 & \begin{tabular}{|l|}
91.4206 \\
\end{tabular} & 820.38 & 5125 & 0.1 & 1950 & 75000 & 937500 & 0.0801 & \#\#\#\#曲 & 4.6 & \begin{tabular}{|l|}
$8.37 \mathrm{E}-03$ \\
\end{tabular} & 48.92 & 81.99 & 0 & & 82.8 & 593.5 \\
\hline 540 & 39.72 & 3000 & 1390 & 118.865315 & 5.5956 & \begin{tabular}{|l|}
141.225 \\
\end{tabular} & 531.07 & 5125 & 0.1 & 1950 & 75000 & 1800000 & 0.0801 & \#\#\#\#\#五 & 3.062 & 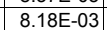 & 18.57 & 95.79 & 57.94 & & 84.7 & 395.1 \\
\hline 541 & 23.43 & 3000 & 830 & 193.612423 & 7.2896 & \begin{tabular}{|l|}
112.952 \\
\end{tabular} & 664 & 5125 & 0.1 & 1950 & 75000 & 1287500 & 0.1358 & \#\#\#\#曲 & 1.416 & \begin{tabular}{|l|} 
\\
\end{tabular} & 72.01 & 97.42 & 74.18 & & 74.3 & 309.7 \\
\hline 542 & 35.59 & 3000 & 650 & 367.461914 & 20.4429 & \begin{tabular}{|l|}
166.898 \\
\end{tabular} & 449.38 & 5125 & 0.1 & 1950 & 75000 & 2312500 & 0.0894 & \#\#\#\# & 1.677 & \begin{tabular}{|l|}
$8.96 \mathrm{E}-03$ \\
\end{tabular} & 45.27 & 98.64 & 86.39 & & 77.3 & 241.5 \\
\hline 543 & 24.36 & 3000 & 820 & 153.220184 & 8.7377 & \begin{tabular}{|l|}
171.082 \\
\end{tabular} & 438.39 & 5125 & 0.1 & 1950 & 75000 & 2400000 & 0.1306 & \#\#\#\#\#曲 & 1.678 & \begin{tabular}{|l|}
$8.61 \mathrm{E}-03$ \\
\end{tabular} & 23.16 & 96.74 & 67.37 & 0 & 80.5 & 353.1 \\
\hline 544 & 34.84 & 3000 & 1630 & 77.619837 & 2.9413 & \begin{tabular}{|l|}
113.682 \\
\end{tabular} & 659.73 & 5125 & 0.1 & 1950 & 75000 & 1300000 & \begin{tabular}{|l|}
0.0913 \\
\end{tabular} & \#\#\#\#\# & 3.102 & \begin{tabular}{|l|}
$8.16 \mathrm{E}-03$ \\
\end{tabular} & 20.61 & 93.56 & 35.58 & & 85 & 456.2 \\
\hline 545 & 26.34 & 3000 & 1520 & 4.44452 & 0.2188 & \begin{tabular}{|l|}
147.69 \\
\end{tabular} & 507.82 & 5125 & 0.1 & 1950 & 75000 & 1925000 & \begin{tabular}{|l|}
0.1208 \\
\end{tabular} & 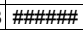 & 2.785 & \begin{tabular}{|l|}
$1.27 \mathrm{E}-02$ \\
\end{tabular} & 25.38 & 0 & 0 & 0 & 54.8 & 541.9 \\
\hline 546 & 63.25 & 3000 & 2360 & 8.535084 & 0.4453 & 156.51 & $\begin{array}{l}479.2 \\
\end{array}$ & 5125 & 0.1 & 1950 & 75000 & 2100000 & \begin{tabular}{|l|}
0.0503 \\
\end{tabular} & \#\#\#\#\# & 7.791 & \begin{tabular}{|c|}
$1.07 \mathrm{E}-02$ \\
\end{tabular} & 71.86 & 41.42 & 0 & 0 & 65.1 & 631.3 \\
\hline 547 & 45.13 & 3000 & 1160 & 72.993312 & 2.8014 & \begin{tabular}{|l|}
115.135 \\
\end{tabular} & 651.41 & 5125 & 0.1 & 1950 & 75000 & 1325000 & 0.0705 & 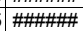 & 3.257 & \begin{tabular}{|l|}
$1.15 \mathrm{E}-02$ \\
\end{tabular} & 88.3 & 93.15 & 31.5 & 0 & 60.2 & 369.9 \\
\hline 548 & 22.77 & 3000 & 960 & 68.888615 & 2.9194 & \begin{tabular}{|l|}
127.137 \\
\end{tabular} & 589.91 & 5125 & 0.1 & 1950 & 75000 & 1537500 & \begin{tabular}{|l|}
0.01397 \\
\end{tabular} & 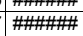 & 1.747 & \begin{tabular}{|l|}
$1.01 \mathrm{~L}-0 \mathrm{~L}$ \\
$1.01 \mathrm{E}$
\end{tabular} & 43.03 & 92.74 & 27.42 & 0 & $\begin{array}{l}0.4 \\
68.5 \\
\end{array}$ & 393.2 \\
\hline 549 & 24.53 & 3000 & 2190 & 87.872701 & 2.1511 & \begin{tabular}{|l|}
73.4399 \\
\end{tabular} & \begin{tabular}{|l|}
1021.24 \\
\end{tabular} & 5125 & 0.1 & 1950 & 75000 & 675000 & \begin{tabular}{|l|}
0.1297 \\
\end{tabular} & \#\#\#\#曲 & 3.012 & 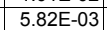 & 32.12 & 94.31 & 43.1 & & 119.1 & 629.2 \\
\hline 550 & 26.25 & 3000 & 1100 & 25.777712 & 0.9132 & \begin{tabular}{|l|}
106.273 \\
\end{tabular} & 705.73 & 5125 & 0.1 & 1950 & 75000 & 1175000 & 0.1212 & \#\#\#\#曲 & 2.009 & \begin{tabular}{|l|}
$1.30 \mathrm{E}-02$ \\
\end{tabular} & 40.72 & 80.6 & 0 & & 53.1 & 392.2 \\
\hline 551 & 22.92 & 3000 & 900 & \begin{tabular}{|l|l}
155.583982 \\
\end{tabular} & $\begin{array}{l}5.433 \\
\end{array}$ & \begin{tabular}{|l|}
104.76 \\
\end{tabular} & 715.92 & 5125 & 0.1 & 1950 & 75000 & 1150000 & 0.1388 & 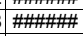 & 1.418 & 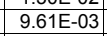 & 36.41 & 96.79 & 67.86 & 0 & 72.1 & 317.1 \\
\hline 552 & 28.61 & 3000 & 2530 & 7.518998 & 0.4362 & \begin{tabular}{|l|l|}
174.039 \\
\end{tabular} & 430.94 & 5125 & 0.1 & 1950 & 75000 & 2462500 & 0.1112 & \#\#\#\#\#五 & 4.288 & \begin{tabular}{|l|} 
\\
\end{tabular} & 40.12 & 33.5 & 0 & 0 & 94.7 & 768.2 \\
\hline 553 & 25.27 & 3000 & 770 & 24.087763 & \begin{tabular}{|l|}
1.2616 \\
\end{tabular} & \begin{tabular}{|l|}
157.131 \\
\end{tabular} & 477.31 & 5125 & 0.1 & 1950 & 75000 & 2112500 & \begin{tabular}{|l|}
0.1259 \\
\end{tabular} & \#\#\#\#\#曲 & 1.615 & $1.54 \mathrm{E}-02$ & 27.96 & 79.24 & 0 & 0 & 45.1 & 327.5 \\
\hline 554 & 58.59 & 3000 & 990 & 470.001449 & 19.2651 & \begin{tabular}{|l|}
122.968 \\
\end{tabular} & 609.91 & 5125 & 0.1 & 1950 & 75000 & 1462500 & 0.0543 & \#\#\#\#\# & 2.941 & 7.41E-03 & 61.01 & 98.94 & 89.36 & & 93.5 & 257.2 \\
\hline 555 & 26.6 & 3000 & 1290 & 68.594711 & 1.8213 & \begin{tabular}{|l|}
79.6568 \\
\end{tabular} & 941.54 & 5125 & 0.1 & 1950 & 75000 & 762500 & 0.1196 & 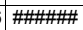 & 2.142 & \begin{tabular}{|l|}
$.02 \mathrm{E}-02$ \\
\end{tabular} & 73.92 & 92.71 & 27.11 & 0 & 67.9 & 412.7 \\
\hline 556 & 22.42 & 3000 & 1420 & 300.980962 & 13.0987 & \begin{tabular}{|l|}
130.56 \\
\end{tabular} & 574.45 & 5125 & 0.1 & 1950 & 75000 & 1600000 & 0.1419 & \#\#\#\#曲 & 2.356 & 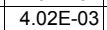 & 48.28 & 98.34 & 83.39 & 0 & 172.3 & 538.6 \\
\hline 557 & $\begin{array}{l}22.44 \\
45.77\end{array}$ & 3000 & 930 & \begin{tabular}{|c|}
78.501044 \\
78040
\end{tabular} & $\begin{array}{l}2.9365 \\
\end{array}$ & \begin{tabular}{|l|}
112.22 \\
\end{tabular} & 668.33 & 5125 & 0.1 & 1950 & 75000 & 1275000 & 0.0695 & 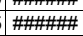 & 2.237 & \begin{tabular}{|l|}
$4.02 L-00$ \\
$1.52 \mathrm{E}-02$ \\
\end{tabular} & 29.49 & \begin{tabular}{|l|}
93.63 \\
\end{tabular} & 36.31 & 0 & 45.5 & 250.5 \\
\hline 558 & 25.15 & 3000 & 1590 & & 0.2763 & \begin{tabular}{|l|}
100.929 \\
\end{tabular} & 743.1 & 5125 & 0.1 & 1950 & 75000 & 1087500 & 0.1265 & \#\#\#\#曲 & 2.556 & \begin{tabular}{|l|}
$.20 \mathrm{E}-02$ \\
\end{tabular} & 20.67 & 39.11 & & & 57.9 & 520.8 \\
\hline 559 & 26.38 & 3000 & 1000 & 38.625609 & 1.2895 & \begin{tabular}{|l|}
100.154 \\
\end{tabular} & 748.85 & 5125 & 0.1 & 1950 & 75000 & 1075000 & 0.1206 & |\#m+m! & 1.787 & $1.37 \mathrm{E}-02$ & 60.23 & 87.06 & 0 & & 50.7 & 347.2 \\
\hline 560 & 30.92 & 3000 & 590 & .742391 & & \begin{tabular}{|l|}
182.19 \\
\end{tabular} & 411.66 & 5125 & 0.1 & 1950 & 75000 & 2637500 & 0.1029 & \#\#\#\#\#曲 & 1.58 & \begin{tabular}{|l|l|}
$1.80 \mathrm{E}-02$ \\
\end{tabular} & 73.24 & \begin{tabular}{|l|}
87.73 \\
\end{tabular} & 0 & & 38.5 & 261.9 \\
\hline 561 & 36.82 & 3000 & 720 & 77.391402 & 4.8473 & \begin{tabular}{|l|}
187.902 \\
\end{tabular} & 399.14 & 5125 & 0.1 & 1950 & 75000 & 2762500 & 0.0864 & \#\#\#\# & 1.936 & \begin{tabular}{|l|}
$1.40 \mathrm{E}-02$ \\
\end{tabular} & 21 & 93.54 & 35.39 & & 49.6 & 269.5 \\
\hline 562 & 38.05 & 3000 & 2130 & 25.48518 & 0.9595 & \begin{tabular}{|l|}
112.952 \\
\end{tabular} & 664 & 5125 & 0.1 & 1950 & 75000 & 1287500 & 0.0836 & 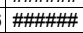 & 5.247 & \begin{tabular}{|l|}
$7.59 \mathrm{E}-03$ \\
\end{tabular} & 97.42 & 80.38 & 0 & 0 & 91.4 & 706.7 \\
\hline 563 & 27.88 & 3000 & 2290 & 0.422275 & 0.0259 & \begin{tabular}{|l|}
183.913 \\
\end{tabular} & 407.8 & 5125 & 0.1 & 1950 & 75000 & 2675000 & 0.1141 & \#\#\#\#曲 & 3.675 & $1.32 \mathrm{E}-02$ & 13.81 & 0 & 0 & 0 & 52.4 & 675.6 \\
\hline 564 & 27.86 & 3000 & 1160 & 29.608563 & 0.8699 & \begin{tabular}{|l|}
88.1405 \\
\end{tabular} & 850.91 & 5125 & 0.1 & 1950 & 75000 & 887500 & 0.1142 & \#\#\#\#\#曲 & 1.971 & \begin{tabular}{|c|}
$1.37 \mathrm{E}-02$ \\
\end{tabular} & 40.31 & 83.11 & 0 & 0 & 50.8 & 362.7 \\
\hline 565 & 32.53 & 3000 & 1220 & 607.777738 & 20.4475 & \begin{tabular}{|l|}
100.929 \\
\end{tabular} & 743.1 & 5125 & 0.1 & 1950 & 75000 & 1087500 & 0.0978 & \#\#\#\#\# & 2.377 & \begin{tabular}{|l|}
$4.34 \mathrm{E}-03$ \\
\end{tabular} & 57.03 & 99.18 & 91.77 & 17.73 & 159.8 & 374.5 \\
\hline 566 & 27.1 & 3000 & 1750 & 201.555879 & 6.9872 & \begin{tabular}{|l|}
103.999 \\
\end{tabular} & 721.16 & 5125 & 0.1 & 1950 & 75000 & 1137500 & 0.1174 & \#\#\#\# & 2.808 & \begin{tabular}{|l|}
$5.07 \mathrm{E}-03$ \\
\end{tabular} & 24.23 & 97.52 & 75.19 & 0 & 136.8 & 531 \\
\hline 567 & 22.45 & 3000 & 950 & \begin{tabular}{|l|}
17.915707 \\
\end{tabular} & 0.7593 & \begin{tabular}{|l|}
127.137 \\
\end{tabular} & 589.91 & 5125 & 0.1 & 1950 & 75000 & 1537500 & 0.1417 & \#\#\#\#曲 & 1.792 & \begin{tabular}{|l|l|} 
& $1.38 \mathrm{E}-02$ \\
\end{tabular} & 56.93 & 72.09 & 0 & 0 & 50.3 & 409.2 \\
\hline 568 & 22.9 & 3000 & 1460 & & 2.404 & \begin{tabular}{|l|}
113.682 \\
\end{tabular} & 659.73 & & & & 75000 & & 0.1389 & \#\#\#\#曲 & 2.455 & 7.34 & & 92.12 & 21.19 & & 94.4 & 549.3 \\
\hline 569 & 48.72 & 3000 & 730 & & 10.6536 & \begin{tabular}{|l|}
127.826 \\
\end{tabular} & 586.74 & 5125 & 0 & 195 & & 0000 & 0.0653 & 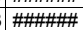 & 1.829 & & 35 & 98 & 80 & & 52 & 192.4 \\
\hline 570 & 23.15 & 3000 & 1650 & & 2.7486 & \begin{tabular}{|l|}
97.8112 \\
\end{tabular} & 766.78 & 5125 & 0.1 & 195 & 75000 & 1037500 & 0.13 & & 2.734 & & 70.98 & 94.07 & 40.69 & 0 & 111.7 & 605 \\
\hline 571 & 37.78 & 3000 & 700 & 1041.2663 & 45.5516 & \begin{tabular}{|l|}
131.239 \\
\end{tabular} & 571.48 & & 0.1 & 1950 & 750 & & 0.0842 & \#\#\# & 1.512 & 5.19 & & 99.52 & 95.2 & 51.98 & 133.5 & 205.1 \\
\hline 572 & 49.32 & 3000 & 1400 & 199.207756 & 8.1654 & \begin{tabular}{|l|}
122.968 \\
\end{tabular} & 609.91 & 5125 & 0.1 & 1950 & 75000 & 1462500 & 0.0645 & 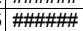 & 3.413 & \begin{tabular}{|l|l|} 
\\
\end{tabular} & 26.69 & \begin{tabular}{|l|}
97.49 \\
\end{tabular} & 74.9 & 0 & 90.8 & 354.6 \\
\hline 573 & 23.27 & 3000 & 1150 & 192.205165 & \begin{tabular}{|l|}
5.647 \\
\end{tabular} & \begin{tabular}{|l|}
88.1405 \\
\end{tabular} & 850. & 5125 & 0.1 & 1950 & 75000 & 887500 & 0.1367 & \#\#\#\#曲 & 1.757 & \begin{tabular}{|l|}
$7.49 E-03$ \\
\end{tabular} & 58.25 & 97.4 & 73.99 & 0 & 92.5 & 387 \\
\hline 574 & 35.31 & 3000 & 2290 & 39.9358 & 1.8274 & \begin{tabular}{|l|}
137.274 \\
\end{tabular} & 546. & 5125 & 0.1 & 1950 & 75000 & 1725000 & 0.0901 & \#\#\#\#\#曲 & 4.302 & $6.23 \mathrm{E}-03$ & 40.9 & 87.48 & 0 & 0 & 111.3 & 624.4 \\
\hline 575 & 47.84 & 3000 & 580 & \begin{tabular}{|c|}
998.058968 \\
\end{tabular} & 47.6339 & \begin{tabular}{|l|}
143.18 \\
\end{tabular} & 523.82 & 5125 & 0.1 & 1950 & 7500 & 1837500 & 0.0665 & 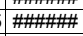 & 1.344 & \begin{tabular}{|l|} 
\\
\end{tabular} & 79.28 & 99.5 & 94.99 & 49.9 & 88.6 & 143.9 \\
\hline 576 & 35.31 & 3000 & 1170 & 153.618981 & \begin{tabular}{|r|}
4.1676 \\
\end{tabular} & \begin{tabular}{|l|}
81.3886 \\
\end{tabular} & 921.51 & 5125 & 0.1 & 1950 & 7500 & 787500 & 0.0901 & 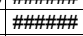 & 2.005 & \begin{tabular}{|l|}
$1.05 \mathrm{E}-02$ \\
\end{tabular} & 21.22 & 96.75 & 67.45 & 0 & 66.2 & 291 \\
\hline 577 & 32.07 & 3000 & 830 & 191.934987 & 7.8224 & \begin{tabular}{|l|}
122.267 \\
\end{tabular} & 613.41 & 5125 & 0.1 & 1950 & 75000 & 1450000 & 0.0992 & 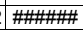 & 1.673 & \begin{tabular}{|l|}
$.05 \mathrm{E}-02$ \\
\end{tabular} & 23.31 & 97.39 & 73.95 & & 66 & 267.3 \\
\hline 578 & $\begin{array}{l}32.01 \\
22.81\end{array}$ & 3000 & $\begin{array}{l}0.00 \\
1550 \\
\end{array}$ & \begin{tabular}{|c|}
10.0462063 \\
10.462063
\end{tabular} & $\begin{array}{r}.0224 \\
0.404 \\
\end{array}$ & \begin{tabular}{|l|}
115.2058 \\
115.858 \\
\end{tabular} & $\begin{array}{l}647.34 \\
6474 \\
\end{array}$ & 5125 & $\begin{array}{l}0.1 \\
0.1 \\
\end{array}$ & 1950 & 75000 & 1337500 & \begin{tabular}{|l|}
0.0952 \\
0.1395 \\
\end{tabular} & (m) m\#\# & $\begin{array}{r}.015 \\
\end{array}$ & \begin{tabular}{|l|}
$1.050-02$ \\
$1.04 \mathrm{E}-02$ \\
\end{tabular} & 44.71 & 52.21 & $\begin{array}{r}r 5.90 \\
0\end{array}$ & & $\begin{array}{r}00 \\
66.6 \\
\end{array}$ & 593.2 \\
\hline 579 & 46.31 & 3000 & 1350 & 153.414616 & 4.5073 & \begin{tabular}{|l|}
88.1405 \\
\end{tabular} & 850.91 & 5125 & 0.1 & 1950 & 75000 & 887500 & \begin{tabular}{|l|}
0.0687 \\
\end{tabular} & \#\#\#\#" & 3.026 & \begin{tabular}{|l|} 
\\
\end{tabular} & 45.15 & 96.74 & 67.41 & 0 & 72.9 & 334.9 \\
\hline 580 & 35.95 & & 2440 & & 0.4064 & \begin{tabular}{|l|}
132.592 \\
\end{tabular} & & & & & & & & & & & & 45.63 & 0 & 0 & 80.1 & 672.7 \\
\hline 581 & 42.08 & 3000 & 2240 & 52.82 & 2.3467 & \begin{tabular}{|l|}
133.266 \\
\end{tabular} & 562 & 512 & 0.1 & 19 & 75 & & & & 5.148 & & 72.46 & 90. & 5.35 & 0 & 121.1 & 627 \\
\hline 582 & 21.22 & 3000 & 1410 & & 0.8 & 156.51 & & 512 & 0.1 & & 750 & 210 & 0.14 & & 2.2 & & 13. & \begin{tabular}{|c|}
67.88 \\
\end{tabular} & 0 & 0 & 70.5 & 546 \\
\hline 583 & 56.81 & 30 & 2430 & 37.5574 & \begin{tabular}{|c|}
1.7598 \\
\end{tabular} & 1440.57 & 533. & 5125 & $\begin{array}{l}0.1 \\
\end{array}$ & 195 & 750 & 1787500 & \begin{tabular}{|c|}
0.056 \\
\end{tabular} & |\#\#\#\#| & 5.772 & $7.38 \mathrm{E}-03$ & \begin{tabular}{|c|}
35.61 \\
\end{tabular} & 86.69 & 0 & 0 & \begin{tabular}{|l|}
93.9 \\
\end{tabular} & $\begin{array}{l}520.7 \\
\end{array}$ \\
\hline 584 & 49.25 & 3000 & 950 & 399.982653 & 20.618 & \begin{tabular}{|l|}
154.641 \\
\end{tabular} & 484.99 & 5125 & 0.1 & 1950 & 75000 & 2062500 & 0.0646 & \#\#\#\#曲 & 2.802 & \begin{tabular}{|l|} 
\\
\end{tabular} & 62.11 & 98.75 & 87.5 & 0 & 102.8 & 291.6 \\
\hline 585 & 37.74 & 3000 & 690 & 47.806725 & 2.292 & \begin{tabular}{|l|}
143.828 \\
\end{tabular} & 521.46 & 5125 & 0.1 & 1950 & 75000 & 1850000 & 0.0843 & \#\#\#\#曲 & 1.744 & \begin{tabular}{|l|}
$.88 \mathrm{E}-02$ \\
\end{tabular} & 54.7 & 89.54 & 0 & 0 & 36.8 & 236.8 \\
\hline 586 & 39.62 & 3000 & 1480 & \begin{tabular}{|l|l|}
38.058613 \\
\end{tabular} & 1.3956 & \begin{tabular}{|l|}
110.009 \\
\end{tabular} & 681.77 & 5125 & 0.1 & 1950 & 75000 & 1237500 & 0.0803 & 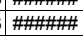 & 3.478 & \begin{tabular}{|l|}
$1.06 \mathrm{E}-02$ \\
\end{tabular} & 56.24 & $\begin{array}{l}86.06 \\
\end{array}$ & 0 & 0 & 65.2 & 449.9 \\
\hline 587 & 27.74 & 3000 & 990 & 660.855043 & 29.6523 & \begin{tabular}{|l|}
134.609 \\
\end{tabular} & 557.17 & 5125 & 0.1 & 1950 & 75000 & 1675000 & 0.1147 & 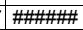 & 2.039 & $3.95 \mathrm{E}-03$ & 76.8 & 99.24 & 92.43 & 4.34 & 175.6 & 376.8 \\
\hline 588 & 38.66 & 3000 & 770 & 654.982618 & 26.077 & 119.44 & 627.93 & 5125 & 0.1 & 1950 & 75000 & 1400000 & 0.0823 & \#\#\#\#曲 & 1.681 & $7.03 \mathrm{E}-03$ & 59.99 & 99.24 & 92.37 & 23.66 & 98.6 & 222.9 \\
\hline 589 & 32.86 & 3000 & 1300 & & 20.1086 & \begin{tabular}{|l|}
115.135 \\
\end{tabular} & 651.41 & 5125 & 0. & 1950 & 75 & 5000 & 0.0968 & & 2.69 & & & 0005 & & 57 & 178 & \\
\hline 590 & 48.28 & 3000 & 930 & 160.685572 & 5.1968 & \begin{tabular}{|l|}
97.024 \\
\end{tabular} & 773 & 5125 & 0.1 & 1950 & 75000 & 1025000 & 0.0659 & 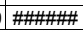 & 2.004 & $1.43 \mathrm{E}-02$ & 33.89 & 96.89 & 68.88 & 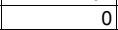 & 48.5 & 212.7 \\
\hline
\end{tabular}




\begin{tabular}{|c|c|c|c|c|c|c|c|c|c|c|c|c|c|c|c|c|c|c|c|c|c|c|}
\hline Realization & $\begin{array}{c}\text { Velocity } \\
(\mathrm{m} / \mathrm{y})\end{array}$ & \begin{tabular}{|c|} 
Fixed Source \\
Concentratio \\
$\mathrm{n}(\mathrm{mg} / \mathrm{L})$
\end{tabular} & $\begin{array}{c}\text { Time of } \\
\text { Maximum at } \\
\text { Compliance } \\
\text { Boundary } \\
(y)\end{array}$ & $\begin{array}{c}\text { Maximum } \\
\text { Concentration } \\
\begin{array}{c}\text { Compliance } \\
\text { Boundary } \\
(\mu \mathrm{g} / \mathrm{L})\end{array} \\
\end{array}$ & $\begin{array}{c}\text { Maximum } \\
\text { Release Rate } \\
\text { at Compliance } \\
\text { Boundary } \\
(\mathrm{Kg} / \mathrm{y})\end{array}$ & \begin{tabular}{c|} 
Source \\
Rate \\
$(\mathrm{Kg} / \mathrm{y})$ \\
\end{tabular} & $\begin{array}{c}\text { Source } \\
\text { Duration } \\
\text { (y) }\end{array}$ & $\begin{array}{c}\text { 1-D } \\
\text { Streamtube } \\
\text { Length } \\
(\mathrm{m})\end{array}$ & $\begin{array}{c}\text { Base } \\
\text { Porosity } \\
\left(\mathrm{m}^{3} / \mathrm{m}^{3}\right)\end{array}$ & $\begin{array}{c}\text { Bulk } \\
\text { Densty } \\
\left(\mathrm{Kg} / \mathrm{m}^{3}\right)\end{array}$ & $\begin{array}{c}\text { Remaining } \\
\text { Carbon } \\
\text { Tetrachloride } \\
\text { Source } \\
(\mathrm{Kg})\end{array}$ & \begin{tabular}{|c|}
$\begin{array}{c}\text { Volume at or } \\
\text { above Fixed } \\
\text { Source } \\
\text { Concentration } \\
(\mathrm{m} 3)\end{array}$ \\
\end{tabular} & $\begin{array}{l}\text { Porosity } \\
\left(\mathrm{m}^{3} / \mathrm{m}^{3}\right)\end{array}$ & $\begin{array}{c}\mathrm{Kd} \\
\left(\mathrm{m}^{3} / \mathrm{kg}\right)\end{array}$ & $\begin{array}{l}\text { Retardatio } \\
\mathrm{n}\end{array}$ & \begin{tabular}{|c|} 
First \\
Order \\
Rate \\
Constant \\
$(1 / y)$ \\
\end{tabular} & $\begin{array}{l}\text { Longitudinal } \\
\text { Dispersivity } \\
\text { (m) }\end{array}$ & \begin{tabular}{|c|} 
Required \\
Source \\
Cleanup \\
Percentage \\
$(5 \mu \mathrm{g} / \mathrm{L}$ \\
Compliance $)$ \\
\end{tabular} & \begin{tabular}{|c|} 
Required \\
Source \\
Cleanup \\
Percentage \\
$(50 \mu \mathrm{g} / \mathrm{L}$ \\
Compliance \\
) \\
\end{tabular} & \begin{tabular}{|c|} 
Required \\
Source \\
Cleanup \\
Percentage \\
$(500 \mu \mathrm{g} / \mathrm{L}$ \\
Compliance \\
)
\end{tabular} & $\begin{array}{c}\text { Abiotic } \\
\text { Reaction } \\
\text { Half Time } \\
\text { (y) }\end{array}$ & \begin{tabular}{|c|} 
Carbon \\
Tetrachloride \\
Travel Time \\
(y)
\end{tabular} \\
\hline & 31.97 & $\begin{array}{r}3000 \\
\end{array}$ & \begin{tabular}{r|}
1000 \\
\end{tabular} & \begin{tabular}{|l|}
217.964374 \\
\end{tabular} & $\begin{array}{r}9.0358 \\
\end{array}$ & 124.366 & 603.06 & $\begin{array}{r}5125 \\
\end{array}$ & $\begin{array}{l}0.1 \\
\end{array}$ & 1950 & 75000 & \begin{tabular}{|l|}
1487500 \\
\end{tabular} & \begin{tabular}{|l|}
0.0995 \\
\end{tabular} & \#\#\#\#+1 & \begin{tabular}{|r|}
2.176 \\
\end{tabular} & $7.90 \mathrm{E}-03$ & $\begin{array}{r}56.74 \\
\end{array}$ & $\begin{array}{r}97.71 \\
\end{array}$ & \begin{tabular}{|l|}
77.06 \\
\end{tabular} & 0 & 87.7 & 348.7 \\
\hline 592 & 34.47 & 3000 & 1030 & 812.57999 & 22.0449 & 81.3886 & 921.51 & 5125 & 0.1 & 1950 & 75000 & 787500 & $\begin{array}{ll}0.0923 \\
\end{array}$ & \#\#\#\#\#+ & 1.581 & $5.67 \mathrm{E}-03$ & 51.95 & 99.38 & 93.85 & 38.47 & 122.2 & 235.1 \\
\hline 593 & 26.98 & 3000 & 1130 & 36.903156 & 1.8559 & 150.869 & 497.12 & 5125 & 0.1 & 1950 & 75000 & 1987500 & 0.1179 & \#\#\#\#\#+ & 2.307 & \begin{tabular}{|c|}
$1.05 \mathrm{E}-02$ \\
\end{tabular} & 37.35 & 86.45 & 0 & 0 & 65.9 & 438.2 \\
\hline 594 & 25.05 & 3000 & 1530 & 2.833534 & $\begin{array}{l}.03039 \\
\end{array}$ & 110.009 & 681.77 & 5125 & 0.1 & 1950 & 75000 & 1237500 & 0.127 & \#\#\#\#+1 & 3.07 & \begin{tabular}{|l|}
$1.37 \mathrm{E}-02$ \\
\end{tabular} & 70.07 & 0 & 0 & 0 & 50.4 & 628.1 \\
\hline 595 & 23.9 & 3000 & 1140 & 722.53194 & 20.0146 & 83.1021 & 902.5 & 5125 & 0.1 & 1950 & 75000 & \begin{tabular}{|l|}
812500 \\
\end{tabular} & 0.1331 & \#\#\#\#\#+m & 1.597 & \begin{tabular}{|l|}
$4.21 \mathrm{E}-03$ \\
\end{tabular} & 25.76 & 99.31 & 93.08 & 30.8 & 164.8 & 342.4 \\
\hline 596 & 50.42 & 3000 & 1990 & \begin{tabular}{|l|}
417.12921 \\
\end{tabular} & $\begin{array}{l}15.3988 \\
\end{array}$ & $\begin{array}{l}0.110 .748 \\
04\end{array}$ & 677.21 & 5125 & 0.1 & 1950 & 75000 & 1250000 & \begin{tabular}{|l|}
0.10631 \\
\end{tabular} & 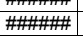 & 4.357 & \begin{tabular}{|l|}
$3.46 \mathrm{E}-03$ \\
\end{tabular} & 37.39 & \begin{tabular}{rl|}
958.8 \\
9.01
\end{tabular} & 88.01 & 0 & 200.3 & $\begin{array}{l}342.9 \\
442.9 \\
\end{array}$ \\
\hline 597 & 52.15 & 3000 & 850 & 1108.175282 & 49.4756 & 133.938 & 559.96 & 5125 & 0.1 & 1950 & 75000 & 1662500 & 0.061 & \#\#\#\#\# & 2.372 & \begin{tabular}{|l|}
$4.32 \mathrm{E}-03$ \\
\end{tabular} & 53.26 & 99.55 & 95.49 & 54.88 & 160.5 & 233.1 \\
\hline 598 & 27.01 & 3000 & 1400 & 1.830714 & 0.0788 & 129.196 & 580.51 & 5125 & 0.1 & 1950 & 75000 & 1575000 & 0.1178 & \#\#\#\#\# & 3.103 & $1.56 \mathrm{E}-02$ & 67.99 & & 0 & 0 & 44.3 & 588.8 \\
\hline 599 & 40.02 & 3000 & 900 & 432.977601 & 14.8996 & 103.236 & 726.49 & 5125 & 0.1 & 1950 & 75000 & 1125000 & 0.0795 & 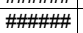 & 1.947 & $8.25 \mathrm{E}-03$ & 85.12 & 98.85 & 88.45 & 0 & 84 & 249.3 \\
\hline 600 & \begin{tabular}{|c|c|}
42.3 \\
42.3
\end{tabular} & 3000 & 1950 & 194.23897 & $\begin{array}{l} \\
6.4846 \\
\end{array}$ & 100.154 & 748.85 & 5125 & 0.1 & 1950 & 75000 & 1075000 & 0.0752 & \#\#m+m! & 3.863 & 5.57E-03 & 29.42 & 97.43 & $\begin{array}{l}30.40 \\
74.26 \\
\end{array}$ & 0 & 124.4 & r. \\
\hline 601 & $\begin{array}{ll}34.69 \\
\end{array}$ & 3000 & 2390 & 23.245128 & 0.931 & 120.15 & 624.22 & 5125 & 0.1 & 1950 & 75000 & 1412500 & \begin{tabular}{|l|}
0.0917 \\
\end{tabular} & 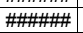 & 4.506 & \begin{tabular}{|l|}
$7.11 \mathrm{E}-03$ \\
\end{tabular} & 46.46 & 78.49 & 0 & 0 & 97.4 & 665.6 \\
\hline 602 & 44.43 & 3000 & 1550 & 122.141466 & 5.0917 & 125.062 & 599.7 & 5125 & 0.1 & 1950 & 75000 & 1500000 & \begin{tabular}{|l|}
0.0716 \\
\end{tabular} & \#\#\#\#\# & 3.602 & $7.52 \mathrm{E}-03$ & 38.04 & 95.91 & 59.06 & & 92.2 & 415.5 \\
\hline 603 & 38.24 & 3000 & 2590 & 74.022595 & 1.9005 & 77.0232 & 973.73 & 5125 & 0.1 & 1950 & 75000 & 725000 & \begin{tabular}{|l|}
0.0832 \\
\end{tabular} & 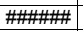 & 4.456 & \begin{tabular}{|l|}
$6.17 \mathrm{E}-03$ \\
\end{tabular} & 35.68 & 93.25 & 32.45 & 0 & 112.4 & 597.2 \\
\hline 604 & 34.5 & 3000 & 860 & 92.619974 & 3.3042 & 107.025 & 700.77 & 5125 & 0.1 & 1950 & 75000 & 1187500 & \begin{tabular}{|l|}
0.0922 \\
\end{tabular} & \#\#\#\#\# & 1.695 & \begin{tabular}{|l|}
$1.46 \mathrm{E}-02$ \\
\end{tabular} & 46.62 & 94.6 & 46.02 & 0 & 47.6 & 251.8 \\
\hline 605 & 48.35 & 3000 & 640 & 1185.78899 & 59.6331 & 150.869 & 497.12 & 5125 & 0.1 & 1950 & 75000 & 1987500 & \begin{tabular}{|l|}
0.0658 \\
\end{tabular} & \#\#\#\#\#" & 1.736 & \begin{tabular}{|l|}
$5.12 \mathrm{E}-03$ \\
\end{tabular} & 46.18 & 99.58 & 95.78 & 57.83 & 135.4 & 184 \\
\hline 606 & $\begin{array}{l}40.03 \\
39.03 \\
\end{array}$ & 3000 & 790 & \begin{tabular}{|l}
11.956672 \\
\end{tabular} & $\begin{array}{r}3.1152 \\
\end{array}$ & 129.879 & 577.46 & 5125 & 0.1 & 1950 & 75000 & 1587500 & \begin{tabular}{|l|}
0.0815 \\
0.0815
\end{tabular} & 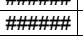 & 1.854 & \begin{tabular}{|l|}
$0.125 \mathrm{E}-02$ \\
\end{tabular} & 25.11 & 93.05 & 30.51 & 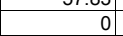 & $\begin{array}{l}43.4 \\
43.7 \\
\end{array}$ & 243.4 \\
\hline 607 & 51.15 & 3000 & 540 & 1791.854662 & 93.4812 & 156.51 & 479.2 & 5125 & 0.1 & 1950 & 75000 & 2100000 & \begin{tabular}{|l|}
0.0622 \\
\end{tabular} & \#\#\#\#\# & 1.365 & \begin{tabular}{|l|}
$3.79 \mathrm{E}-03$ \\
\end{tabular} & 40.58 & 99.72 & 97.21 & 72.1 & 183 & 136.8 \\
\hline 608 & 40.63 & 3000 & 1130 & 57.940202 & 2.2515 & 116.579 & 643.34 & 5125 & 0.1 & 1950 & 75000 & 1350000 & 0.0783 & \#\#\#\#\#" & 2.909 & \begin{tabular}{|l|}
$1.21 \mathrm{E}-02$ \\
\end{tabular} & 70.71 & 91.37 & 13.7 & 0 & 57.3 & 367 \\
\hline 609 & 24.53 & 3000 & 960 & 249.426548 & 7.1899 & 86.4774 & 867.28 & 5125 & 0.1 & 1950 & 75000 & 862500 & 0.1297 & 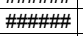 & 1.264 & $9.73 \mathrm{E}-03$ & 54.57 & 98 & 79.95 & 0 & 71.2 & 264.2 \\
\hline 610 & 27.64 & 3000 & 940 & 53.904345 & 2.0427 & 113.682 & 659.73 & 5125 & 0.1 & 1950 & 75000 & 1300000 & \begin{tabular}{|l|}
0.1151 \\
\end{tabular} & 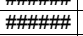 & 1.763 & \begin{tabular}{|l|}
$1.28 \mathrm{E}-02$ \\
\end{tabular} & 31 & 90.72 & 7.24 & 0 & 54.1 & 326.9 \\
\hline 611 & 56.11 & 3000 & 940 & 784.047638 & 30.0904 & 115.135 & 651.41 & 5125 & 0.1 & 1950 & 75000 & 1325000 & \begin{tabular}{|l|}
0.0567 \\
\end{tabular} & \#\#\#\#\# & 2.685 & \begin{tabular}{|l|}
$5.61 \mathrm{E}-03$ \\
\end{tabular} & 93.99 & 99.36 & 93.62 & 36.23 & 123.5 & 245.2 \\
\hline 612 & 36.48 & 3000 & 1720 & 170.588202 & 9.6266 & 169.295 & 443.01 & 5125 & 0.1 & 1950 & 75000 & 2362500 & \begin{tabular}{|l|}
0.0872 \\
\end{tabular} & \#\#\#\#\# & 3.504 & \begin{tabular}{|l|}
$4.79 \mathrm{E}-03$ \\
\end{tabular} & 32.02 & 97.07 & 70.69 & 0 & 144.6 & 492.2 \\
\hline 613 & 36.28 & 3000 & 2530 & 1.006926 & 0.0447 & 133.266 & 562.78 & 5125 & 0.1 & 1950 & 75000 & 1650000 & \begin{tabular}{|l|}
0.0877 \\
\end{tabular} & \#\#\#\#\# & 4.85 & \begin{tabular}{|l|}
$1.27 \mathrm{E}-02$ \\
\end{tabular} & 29.48 & 0 & 0 & 0 & 54.6 & 685.2 \\
\hline 614 & \begin{tabular}{|l|}
32.7 \\
\end{tabular} & 3000 & 2640 & 9.150862 & 0.4793 & 157.131 & 477.31 & 5125 & 0.1 & 1950 & 75000 & 2112500 & \begin{tabular}{|l|}
0.0973 \\
\end{tabular} & \#\#\#\#\# & 4.748 & \begin{tabular}{|l|}
$7.36 \mathrm{E}-03$ \\
\end{tabular} & 41.51 & 45.36 & 0 & 0 & 94.2 & 744.2 \\
\hline 615 & 54.1 & 3000 & 650 & 1657.122258 & \begin{tabular}{|l|}
.40 .6075 \\
\end{tabular} & 127.826 & 586.74 & 5125 & 0.1 & 1950 & 75000 & 1550000 & 0.0588 & 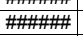 & 1.522 & 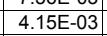 & 45.52 & 99.7 & 96.98 & 69.83 & 167 & 144.2 \\
\hline 616 & 28.74 & 3000 & 810 & 316.573515 & 12.3777 & 117.297 & 639.4 & 5125 & 0.1 & 1950 & 75000 & 1362500 & \begin{tabular}{|l|}
0.1107 \\
\end{tabular} & \#\#\#\#\# & 1.536 & \begin{tabular}{|l|}
$8.56 \mathrm{E}-03$ \\
\end{tabular} & \begin{tabular}{|c|}
63.67 \\
\end{tabular} & 98.42 & 84.21 & 0 & 81 & 274 \\
\hline 617 & 26.27 & 3000 & 860 & 210.239318 & 7.3947 & 105.518 & 710.78 & 5125 & 0.1 & 1950 & 75000 & 1162500 & \begin{tabular}{|l|}
0.1211 \\
\end{tabular} & \#\#\#\#\# & 1.393 & \begin{tabular}{|c|}
$9.95 \mathrm{E}-03$ \\
\end{tabular} & 23.87 & 97.62 & 76.22 & 0 & 69.7 & 271.8 \\
\hline 618 & 58.05 & 3000 & 770 & 570.611634 & 21.4839 & 112.952 & 664 & 5125 & 0.1 & 1950 & 75000 & 1287500 & 0.0548 & \#\#\#\#\#" & 1.904 & \begin{tabular}{|l|}
$1.02 \mathrm{E}-02$ \\
\end{tabular} & 45.35 & 99.12 & 91.24 & 12.37 & 68.3 & 168.1 \\
\hline 619 & 27.47 & 3000 & 2370 & & 0.2477 & 72.5304 & 1034.05 & 5125 & 0.1 & 1950 & 75000 & \begin{tabular}{|l|}
662500 \\
\end{tabular} & 0.1158 & 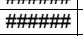 & 3.547 & $9.42 \mathrm{E}-03$ & 32.94 & 51.21 & 0 & 0 & 73.6 & 661.7 \\
\hline 620 & 25.91 & 3000 & 1270 & \begin{tabular}{|l|}
17.465607 \\
\end{tabular} & 0.9541 & 163.877 & 457.66 & 5125 & 0.1 & 1950 & 75000 & 2250000 & 0.1228 & 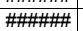 & 2.503 & $1.08 \mathrm{E}-02$ & 34.07 & 71.37 & 0 & 0 & 64.3 & 495.1 \\
\hline 621 & 28.69 & 3000 & 1020 & 143.965003 & 5.2435 & 109.267 & 686.4 & 5125 & 0.1 & 1950 & 75000 & 1225000 & \begin{tabular}{|l|}
0.1109 \\
\end{tabular} & \#\#\#\#\#升 & 1.894 & \begin{tabular}{|l|}
$9.29 \mathrm{E}-03$ \\
\end{tabular} & 31.13 & 96.53 & 65.27 & 0 & 74.6 & 338.3 \\
\hline 622 & 59.58 & 3000 & 2100 & 13.125242 & 0.4483 & \begin{tabular}{|l|}
102.47 \\
\end{tabular} & 731.92 & 5125 & 0.1 & 1950 & 75000 & 1112500 & \begin{tabular}{|l|}
0.0534 \\
\end{tabular} & \#\#\#\#\# & 5.511 & \begin{tabular}{|c|}
$1.26 \mathrm{E}-02$ \\
\end{tabular} & 38.46 & 61.91 & 0 & 0 & 55.2 & 474.1 \\
\hline 623 & 51.31 & 3000 & 520 & 1693.895738 & 92.5302 & 163.877 & 457.66 & 5125 & 0.1 & 1950 & 75000 & 2250000 & \begin{tabular}{|l|}
0.062 \\
\end{tabular} & \#\#\#\#\# & 1.386 & \begin{tabular}{|c|}
$4.16 \mathrm{E}-03$ \\
\end{tabular} & 46.56 & 99.7 & \begin{tabular}{|c|}
97.05 \\
\end{tabular} & 70.48 & 166.7 & 138.5 \\
\hline 624 & 23.07 & 3000 & 670 & 564.532093 & 33.8494 & 179.88 & 416.94 & 5125 & 0.1 & 1950 & 75000 & 2587500 & \begin{tabular}{|l|}
0.1379 \\
\end{tabular} & \#\#\#\#\# & 1.433 & \begin{tabular}{|l|}
$5.36 \mathrm{E}-03$ \\
\end{tabular} & 54.16 & 99.11 & 91.14 & 11.43 & 129.3 & 318.4 \\
\hline 625 & 28.4 & 3000 & 950 & 279.60254 & 16.2206 & 174.039 & 430.94 & 5125 & 0.1 & 1950 & 75000 & 2462500 & 0.112 & \#\#\#\#\#" & 2.138 & \begin{tabular}{|l|}
$6.04 \mathrm{E}-03$ \\
\end{tabular} & 54.3 & 98.21 & 82.12 & 0 & 114.7 & 385.8 \\
\hline 626 & 25.21 & 3000 & 840 & 605.150413 & 21.74 & 107.775 & 695.9 & 5125 & 0.1 & 1950 & 75000 & 1200000 & 0.1262 & \#\#\#\#\# & 1.352 & \begin{tabular}{|l|}
$5.91 \mathrm{E}-03$ \\
\end{tabular} & 34.29 & 99.17 & 91.74 & 17.38 & 117.3 & 274.9 \\
\hline 627 & 33.66 & 3000 & 1110 & & 7.9393 & 130.56 & 574.45 & 5125 & 0.1 & 1950 & 75000 & 1600000 & 0.0945 & \#\#\#\#\#" & 2.392 & \begin{tabular}{|c|}
$7.93 \mathrm{E}-03$ \\
\end{tabular} & 33.5 & 97.26 & 72.59 & 0 & 87.4 & 364.2 \\
\hline 628 & 47.13 & 3000 & 1160 & 214.86 & 8.4522 & 118.014 & 635.52 & 512 & 0.1 & 195 & 75000 & 1375000 & 0.0675 & \#\#\#\#\#吿 & 2.8 & 8.87E-03 & 24.98 & 97.67 & 76.73 & 0 & 78.1 & $\begin{array}{l}304.4 \\
306.3 \\
\end{array}$ \\
\hline 629 & 48.94 & 3000 & 610 & 861.7 & \begin{tabular}{|c|}
62.5913 \\
\end{tabular} & 217.909 & 344.18 & 5125 & 0.1 & 1950 & 75000 & & 0.065 & \#m & 2.016 & & 32.09 & 99.42 & 94.2 & 41.98 & 116.2 & 211.1 \\
\hline 630 & 46.51 & 3000 & 2400 & $\begin{array}{r}3.400415 \\
\end{array}$ & 0.176 & 155.266 & 483.04 & 5125 & 0.1 & 1950 & 75000 & 2075000 & 0.0684 & \#\#\#\#\#" & 5.317 & $1.18 \mathrm{E}-02$ & 29.55 & 0 & 0 & 0 & 58.7 & 585.9 \\
\hline 631 & \begin{tabular}{|l|}
32.4 \\
\end{tabular} & 3000 & 910 & 44.577768 & 2.8005 & 188.468 & 397.94 & 5125 & 0.1 & 1950 & 75000 & 2775000 & 0.0982 & \#\#\#\#\# & 2.451 & \begin{tabular}{|c|}
$1.16 \mathrm{E}-02$ \\
\end{tabular} & 73.32 & 88.78 & 0 & & 59.8 & 387.8 \\
\hline 632 & 42.76 & 3000 & 1450 & 2.812996 & 0.1192 & 127.137 & 589.91 & 5125 & 0.1 & 1950 & 75000 & 1537500 & 0.0744 & \#\#\#\#\# & 3.655 & \begin{tabular}{|l|}
$1.82 \mathrm{E}-02$ \\
\end{tabular} & 36.45 & 0 & 0 & & 38.2 & 438.1 \\
\hline 633 & 34.92 & 3000 & 1000 & 100.153021 & 6.1589 & 184.485 & 406.54 & 5125 & 0.1 & 1950 & 75000 & 2687500 & 0.0911 & 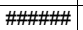 & 2.379 & \begin{tabular}{|c|}
$9.89 \mathrm{E}-03$ \\
\end{tabular} & 16.55 & 95.01 & 50.08 & 0 & 70.1 & 349.2 \\
\hline 634 & 29.16 & 3000 & 890 & 147.023313 & 5.9229 & 120.857 & 620.57 & 5125 & 0.1 & 1950 & 75000 & 1425000 & \begin{tabular}{|l|}
0.1091 \\
\end{tabular} & \#\#\#\#\# & 1.707 & \begin{tabular}{|l|}
$1.02 \mathrm{E}-02$ \\
\end{tabular} & 17.51 & 96.6 & 65.99 & & 67.7 & 299.9 \\
\hline 635 & 25.37 & 3000 & 2160 & 17.334493 & 0.8084 & 139.914 & 536.04 & 5125 & 0.1 & 1950 & 75000 & 1775000 & \begin{tabular}{|l|}
0.1254 \\
\end{tabular} & \#\#\#\#\#" & 3.968 & \begin{tabular}{|l|}
$6.52 \mathrm{E}-03$ \\
\end{tabular} & 78.43 & 71.16 & 0 & 0 & 106.3 & 801.5 \\
\hline 636 & 34.58 & 3000 & 1980 & 52.443073 & 3.4123 & 195.2 & 384.22 & 5125 & 0.1 & 1950 & 75000 & 2925000 & o. & \#\#mm地 & 3.754 & \begin{tabular}{|l|}
$0.16 \mathrm{E}-03$ \\
\end{tabular} & 24.8 & 90.47 & 4.66 & 0 & 1125 & 556.3 \\
\hline 637 & 53.11 & 3000 & 1000 & 50.389887 & 3.5263 & 209.941 & 357.24 & 5125 & 0.1 & 1950 & 75000 & 3262500 & \begin{tabular}{|l|}
0.0599 \\
0.0599
\end{tabular} & \#\#\#\#\#| & 3. 3.604 & \begin{tabular}{|l|}
$0.10 \mathrm{~L}-00$ \\
$1.20 \mathrm{E}$ \\
\end{tabular} & $\begin{array}{l}24.0 \\
79.54\end{array}$ & 90.08 & $\begin{array}{l}0.00 \\
0.77 \\
\end{array}$ & 0 & $\begin{array}{l}57.7 \\
57.7\end{array}$ & 347.8 \\
\hline 638 & 60.03 & 3000 & 890 & 321.342207 & 16.2955 & 152.132 & 492.99 & 512 & 0.1 & 1950 & 75000 & 2012500 & \begin{tabular}{|l|}
0.053 \\
\end{tabular} & & 2.91 & & 52.04 & 98.44 & 84.44 & 0 & 75.4 & 248.5 \\
\hline 639 & \begin{tabular}{|l|}
29.76 \\
\end{tabular} & 3000 & 1680 & 15.44 & 0.7832 & 152.132 & 492 & 512 & 0.1 & 1950 & 75000 & & 0.10 & & 3.618 & & & & $\begin{array}{r}04.44 \\
0\end{array}$ & 0 & 77 & $\begin{array}{r}-623 \\
6.03\end{array}$ \\
\hline 640 & 43.17 & 300 & 1020 & 786.3351 & & 141.878 & 528. & 512 & 0.1 & 1950 & 75000 & & 0.07 & \#\#\# & 2.7 & \begin{tabular}{|l|}
3.67 \\
\end{tabular} & 78.3 & 99.36 & 93.64 & 36.41 & 189.1 & 320.6 \\
\hline 641 & \begin{tabular}{|l|l|}
42.03 \\
\end{tabular} & & 1570 & 17.1900 & 0.6472 & 112.952 & 664 & 512 & 0.1 & 195 & 7500 & 12875 & 0.07 & \#\#\#\#\# & 3.679 & \begin{tabular}{|l|}
$1.26 \mathrm{E}-02$ \\
\end{tabular} & 41.14 & 70. & 0 & 0 & 55 & 448.6 \\
\hline 642 & 21.39 & 3000 & 700 & $71.0124 t$ & 3.2021 & 135.278 & 554.42 & 5125 & 0.1 & 1950 & 75000 & 1687500 & $\mid 0.1487$ & |\#\#\#\#" & 1.255 & \begin{tabular}{|l|}
$1.32 \mathrm{E}-02$ \\
\end{tabular} & 62.44 & \begin{tabular}{|c|}
92.96 \\
\end{tabular} & 29.59 & 0 & 52.6 & \begin{tabular}{|c|}
300.6 \\
\end{tabular} \\
\hline 643 & 32.8 & 3000 & 760 & 360.530532 & 15.279 & 127.137 & 589.91 & 5125 & 0.1 & 1950 & 75000 & 1537500 & 0.097 & \#\#\#\#\# & 1.564 & \begin{tabular}{|r|}
$8.82 \mathrm{E}-03$ \\
\end{tabular} & 27.16 & 98.61 & 86.13 & 0 & 78.6 & 244.4 \\
\hline 644 & 26.21 & 3000 & 990 & $\begin{array}{r}31.334955 \\
\end{array}$ & 0.9549 & 91.4206 & 820.38 & 5125 & 0.1 & 1950 & 75000 & 937500 & \begin{tabular}{|l|}
0.1214 \\
\end{tabular} & \#\#\#\#\# & 1.58 & \begin{tabular}{|l|}
$1.58 \mathrm{E}-02$ \\
\end{tabular} & 47.51 & 84.04 & 0 & 0 & 44 & 309 \\
\hline 645 & 31.07 & 3000 & 690 & 645.369603 & 29.388 & 136.61 & 549.01 & 5125 & 0.1 & 1950 & 75000 & \begin{tabular}{|l|l|}
1712500 \\
\end{tabular} & 0.1024 & \#\#\#\#\# & 1.452 & \begin{tabular}{|l|}
$6.57 \mathrm{E}-03$ \\
\end{tabular} & 55.88 & 99.23 & 92.25 & 22.53 & 105.5 & 239.5 \\
\hline 646 & 43.05 & 3000 & 1030 & 292.212957 & 11.144 & 114.41 & 655.54 & 5125 & 0.1 & 1950 & 75000 & 1312500 & 0.0739 & \#\#\#\#\# & 2.484 & $8.25 \mathrm{E}-03$ & 48.75 & 98.29 & 82.89 & 0 & 84 & 295.8 \\
\hline 647 & 35.51 & 3000 & 2010 & 62.08808 & 2.5594 & 123.668 & 606.46 & 5125 & 0.1 & 195 & 75000 & 1475000 & 0.0896 & & 4.17 & $6.02 \mathrm{E}-03$ & 64. & 91.95 & 9.47 & & 115.2 & \\
\hline 648 & 22.95 & 3000 & & & & & & 512 & & 195 & & & 0.13 & & 3.0 & & & & & & & \\
\hline 649 & 21.25 & 3000 & 1070 & 112.692354 & 5.354 & 142.53 & 526.21 & 5125 & 0.1 & 1950 & 75000 & 1825000 & 0.1497 & \#\#\#\#\# & 2.023 & $7.04 \mathrm{E}-03$ & 81.91 & 95.56 & 5.63 & 0 & 98.4 & 487.8 \\
\hline
\end{tabular}




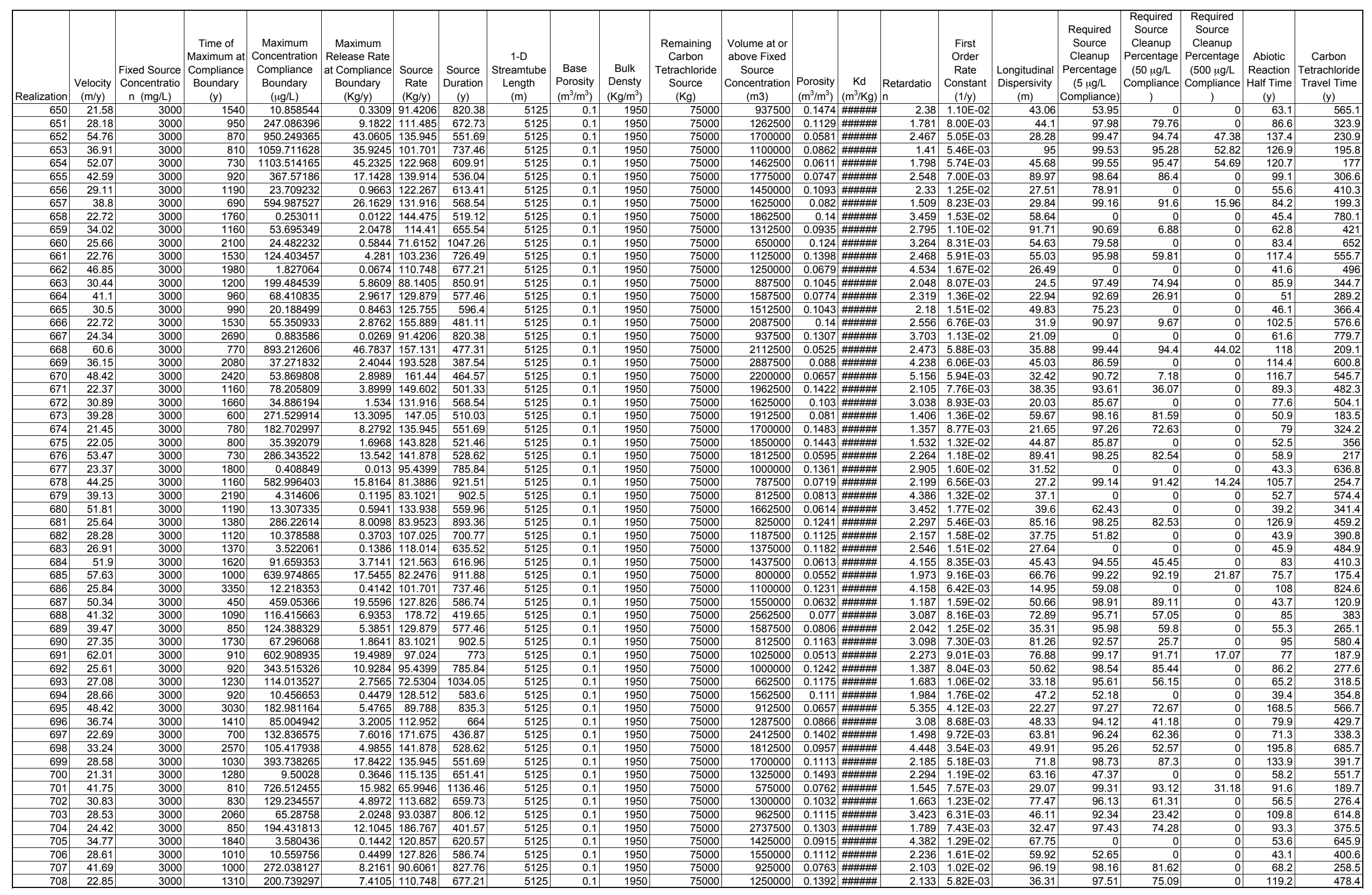




\begin{tabular}{|c|c|c|c|c|c|c|c|c|c|c|c|c|c|c|c|c|c|c|c|c|c|c|}
\hline Realization & $\begin{array}{c}\text { Velocity } \\
(\mathrm{m} / \mathrm{y})\end{array}$ & \begin{tabular}{|c|} 
Fixed Source \\
Concentratio \\
$\mathrm{n}(\mathrm{mg} / \mathrm{L})$
\end{tabular} & $\begin{array}{c}\text { Time of } \\
\text { Maximum at } \\
\text { Compliance } \\
\text { Boundary } \\
(y)\end{array}$ & $\begin{array}{c}\text { Maximum } \\
\text { Concentration } \\
\begin{array}{c}\text { Compliance } \\
\text { Boundary } \\
(\mu \mathrm{g} / \mathrm{L})\end{array} \\
\end{array}$ & $\begin{array}{c}\text { Maximum } \\
\text { Release Rate } \\
\text { at Compliance } \\
\text { Boundary } \\
(\mathrm{Kg} / \mathrm{y})\end{array}$ & \begin{tabular}{c|} 
\\
Source \\
Rate \\
$(\mathrm{Kg} / \mathrm{y})$ \\
\end{tabular} & $\begin{array}{c}\text { Source } \\
\text { Duration } \\
\text { (y) }\end{array}$ & $\begin{array}{c}\text { 1-D } \\
\text { Streamtube } \\
\text { Length } \\
(\mathrm{m})\end{array}$ & $\begin{array}{c}\text { Base } \\
\text { Porosity } \\
\left(\mathrm{m}^{3} / \mathrm{m}^{3}\right)\end{array}$ & $\begin{array}{c}\text { Bulk } \\
\text { Densty } \\
\left(\mathrm{Kg} / \mathrm{m}^{3}\right)\end{array}$ & $\begin{array}{c}\text { Remaining } \\
\text { Carbon } \\
\text { Tetrachloride } \\
\text { Source } \\
(\mathrm{Kg})\end{array}$ & \begin{tabular}{|c|}
$\begin{array}{c}\text { Volume at or } \\
\text { above Fixed } \\
\text { Source } \\
\text { Concentration } \\
(\mathrm{m} 3)\end{array}$ \\
\end{tabular} & $\begin{array}{l}\text { Porosity } \\
\left(\mathrm{m}^{3} / \mathrm{m}^{3}\right)\end{array}$ & $\begin{array}{c}\mathrm{Kd} \\
\left(\mathrm{m}^{3} / \mathrm{Kg}\right)\end{array}$ & $\begin{array}{l}\text { Retardatio } \\
n\end{array}$ & \begin{tabular}{|c|} 
First \\
Order \\
Rate \\
Constant \\
$(1 / y)$ \\
\end{tabular} & $\begin{array}{l}\text { Longitudinal } \\
\text { Dispersivity } \\
\text { (m) }\end{array}$ & \begin{tabular}{|c|} 
Required \\
Source \\
Cleanup \\
Percentage \\
$(5 \mu \mathrm{g} / \mathrm{L}$ \\
Compliance $)$ \\
\end{tabular} & \begin{tabular}{|c|} 
Required \\
Source \\
Cleanup \\
Percentage \\
$(50 \mu \mathrm{g} / \mathrm{L}$ \\
Compliance \\
) \\
\end{tabular} & \begin{tabular}{|c|} 
Required \\
Source \\
Cleanup \\
Percentage \\
$(500 \mu \mathrm{g} / \mathrm{L}$ \\
Compliance \\
)
\end{tabular} & $\begin{array}{c}\text { Abiotic } \\
\text { Reaction } \\
\text { Half Time } \\
\text { (y) }\end{array}$ & \begin{tabular}{|c|} 
Carbon \\
Tetrachloride \\
Travel Time \\
(y)
\end{tabular} \\
\hline & 54.29 & 3000 & $\begin{array}{r}1800 \\
\end{array}$ & 149.468205 & 4.3914 & 88.1405 & 850.91 & \begin{tabular}{|r|}
5125 \\
\end{tabular} & $\begin{array}{l}0.1 \\
\end{array}$ & 1950 & 75000 & $\begin{array}{r}887500 \\
\end{array}$ & 0.0586 & \#\#\# & \begin{tabular}{|l|}
4.731 \\
\end{tabular} & 6.88E-03 & \begin{tabular}{|r|}
80.79 \\
\end{tabular} & $\begin{array}{r}96.65 \\
\end{array}$ & $\begin{array}{r}66.55 \\
\end{array}$ & $\begin{array}{l} \\
\end{array}$ & 100.8 & $\begin{array}{r}446.6 \\
\end{array}$ \\
\hline 710 & 33.52 & 3000 & 760 & 47.24564 & 2.1304 & 135.278 & 554.42 & 5125 & 0.1 & 1950 & 75000 & 1687500 & 0.0949 & \#\#\#\#\#+ & 1.754 & $1.66 \mathrm{E}-02$ & 50.99 & 89.42 & 0 & 0 & 41.7 & 268.1 \\
\hline 711 & 25.03 & 3000 & 1420 & 125.622956 & 5.5522 & 132.592 & 565.65 & 5125 & 0.1 & 1950 & 75000 & 1637500 & 0.1271 & \#\#\#\#\#+ & 2.583 & \begin{tabular}{|l|}
$5.89 \mathrm{E}-03$ \\
\end{tabular} & 52.88 & 96.02 & 60.2 & 0 & \begin{tabular}{|l|l|}
117.7 \\
\end{tabular} & 528.8 \\
\hline 712 & 28.79 & 3000 & 1170 & 11.325693 & 0.4642 & 122.968 & 609.91 & 5125 & 0.1 & 1950 & 75000 & 1462500 & 0.1105 & 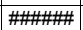 & 2.33 & \begin{tabular}{|l|}
$1.44 \mathrm{E}-02$ \\
\end{tabular} & 29.12 & \begin{tabular}{|l|}
55.85 \\
\end{tabular} & & 0 & 48.1 & 414.8 \\
\hline $\begin{array}{ll}713 \\
\end{array}$ & 25.68 & 3000 & 2500 & 0.678882 & 0.0283 & 125.062 & $\begin{array}{r}599.7 \\
\end{array}$ & 5125 & 0.1 & 1950 & 75000 & 1500000 & \begin{tabular}{|l|}
0.1239 \\
\end{tabular} & 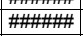 & 4.198 & \begin{tabular}{|l|}
$1.15 \mathrm{E}-02$ \\
\end{tabular} & 40.79 & 0 & 0 & 0 & 60.4 & 837.8 \\
\hline 714 & 27.93 & 3000 & $\begin{array}{r}750 \\
\end{array}$ & $\begin{array}{r}153.661122 \\
\end{array}$ & $\begin{array}{l}.0220 \\
6.7221 \\
\end{array}$ & 131.239 & 571.48 & 5125 & 0.1 & 1950 & 75000 & 1612500 & \begin{tabular}{|l|}
0.1139 \\
\end{tabular} & 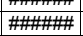 & $\begin{array}{l}4.150 \mid \\
1.479 \\
\end{array}$ & \begin{tabular}{|l|}
$1.13 \mathrm{E}-02$ \\
\end{tabular} & 36.26 & 96.75 & 67.46 & 0 & $\begin{array}{l}0.4 .4 \\
61.4\end{array}$ & 271.3 \\
\hline 715 & 24.93 & 3000 & 560 & 455.521213 & 27.8384 & 183.34 & 409.08 & 5125 & 0.1 & 1950 & 75000 & 2662500 & 0.1276 & \#\#\#\#覀 & 1.204 & \begin{tabular}{|l|}
$7.71 \mathrm{E}-03$ \\
\end{tabular} & 26.56 & 98.9 & 89.02 & 0 & 90 & 247.5 \\
\hline 716 & 22.53 & 3000 & 640 & 75.458988 & 4.5536 & 181.037 & \begin{tabular}{|l|l|}
414.28 \\
\end{tabular} & 5125 & 0.1 & 1950 & 75000 & 2612500 & 0.1412 & \#\#\#\#\#曲 & 1.325 & $1.25 \mathrm{E}-02$ & 23.92 & 93.37 & 33.74 & 0 & 55.5 & 301.4 \\
\hline 717 & 62.38 & 3000 & 670 & 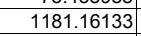 & 48.6907 & 123.668 & 606.46 & 5125 & 0.1 & 1950 & 75000 & 1475000 & 0.051 & 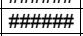 & 1.665 & 6.97E-03 & 75.19 & 99.58 & 95.77 & 57.67 & 99.5 & 136.8 \\
\hline 718 & 32.46 & 3000 & 1510 & 1.642608 & 0.0816 & 148.966 & 503.47 & 5125 & 0.1 & 1950 & 75000 & 1950000 & 0.098 & \#\#\#\#\#+ & 2.947 & $1.68 \mathrm{E}-02$ & 10.71 & 0 & 0 & 0 & 41.4 & 465.3 \\
\hline 719 & 30.04 & 3000 & \begin{tabular}{|c|}
1110 \\
\end{tabular} & 118.570536 & 5.0521 & 127.826 & 586.74 & 5125 & 0.1 & 1950 & 75000 & 1550000 & 0.1059 & \#\#\#\#正 & 2.507 & \begin{tabular}{|l|}
$8.10 \mathrm{E}-03$ \\
\end{tabular} & 90.4 & \begin{tabular}{|c|}
95.78 \\
\end{tabular} & 57.83 & 0 & 85.5 & 427.8 \\
\hline 720 & $\begin{array}{ll}32.76 \\
\end{array}$ & 3000 & 1220 & 53.310639 & 2.3321 & 131.239 & \begin{tabular}{|l|l|}
571.48 \\
\end{tabular} & 5125 & 0.1 & 1950 & 75000 & \begin{tabular}{|l|l|}
1612500 \\
\end{tabular} & 0.0971 & \#\#\#\#\#立 & 2.678 & \begin{tabular}{|l|}
$1.02 \mathrm{E}-02$ \\
\end{tabular} & 46.01 & 90.62 & 6.21 & & 68 & 418.8 \\
\hline 721 & 22.87 & 3000 & 1290 & $\begin{array}{l}2.456907 \\
\end{array}$ & 4.0019 & 165.693 & 452.64 & 5125 & 0.1 & 1950 & 75000 & 2287500 & \begin{tabular}{|l|}
0.1391 \\
\end{tabular} & 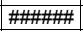 & 2.396 & \begin{tabular}{|l|}
$6.80 \mathrm{E}-03$ \\
\end{tabular} & 52.63 & 93.1 & 30.99 & 0 & 102 & 536.9 \\
\hline 722 & 24.23 & 3000 & 880 & 1234.886933 & 47.9871 & 116.579 & 643.34 & 5125 & 0.1 & 1950 & 75000 & 1350000 & \begin{tabular}{|l|}
0.1313 \\
\end{tabular} & \#\#\#\#\# & 1.534 & \begin{tabular}{|l|}
$2.78 \mathrm{E}-03$ \\
\end{tabular} & 56.12 & 99.6 & 95.95 & 59.51 & 249.8 & 324.6 \\
\hline 723 & 42.59 & 3000 & 1220 & 37.12663 & 1.5131 & 122.267 & 613.41 & 5125 & 0.1 & 1950 & 75000 & 1450000 & \begin{tabular}{|l|}
0.0747 \\
\end{tabular} & 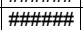 & 2.996 & \begin{tabular}{|l|}
$1.31 \mathrm{E}-02$ \\
\end{tabular} & 38.17 & 86.53 & 0 & 0 & 52.8 & 360.5 \\
\hline 724 & \begin{tabular}{|l|}
42.7 \\
42.7
\end{tabular} & 3000 & 1000 & $\begin{array}{l}76.109851 \\
76.1205\end{array}$ & 3.0302 & 119.44 & \begin{tabular}{|l|l|}
627.93 \\
627
\end{tabular} & 5125 & 0.1 & 1950 & 75000 & 1400000 & \begin{tabular}{|l|}
0.0745 \\
\end{tabular} & 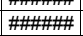 & 2.339 & \begin{tabular}{|l|}
$1.34 \mathrm{E}-02$ \\
\end{tabular} & $\begin{array}{l}30.2 \\
15.2 \\
\end{array}$ & 93.43 & 34.31 & 0 & 51.6 & 280.7 \\
\hline 725 & 47.27 & 3000 & 1360 & 34.488296 & 1.3236 & 115.135 & 651.41 & 5125 & 0.1 & 1950 & 75000 & 1325000 & \begin{tabular}{|l|}
0.0673 \\
\end{tabular} & \#\#\#\#再 & 3.321 & \begin{tabular}{|c|}
$1.32 \mathrm{E}-02$ \\
\end{tabular} & 28.01 & 85.5 & 0 & & 52.6 & 360 \\
\hline 726 & 22.64 & 3000 & 2370 & 59.640742 & 2.0371 & 102.47 & 731.92 & 5125 & 0.1 & 1950 & 75000 & 1112500 & 0.1405 & 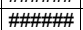 & 3.291 & $5.02 \mathrm{E}-03$ & 37.59 & 91.62 & 16.16 & 0 & 138 & 744.9 \\
\hline 727 & 24.93 & 3000 & 2170 & $\begin{array}{l}9.269881 \\
\end{array}$ & 0.2269 & 73.4399 & 1021.24 & 5125 & 0.1 & 1950 & 75000 & 675000 & 0.1276 & 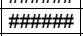 & 3.08 & \begin{tabular}{|l|}
$9.87 \mathrm{E}-03$ \\
\end{tabular} & 26.95 & 46.06 & 0 & 0 & 70.2 & 633.2 \\
\hline 728 & 27.96 & 3000 & 710 & 553.54963 & $\begin{array}{r}16.5674 \\
\end{array}$ & $\begin{array}{l}8.40 .788 \\
\end{array}$ & 835.3 & 5125 & 0.1 & 1950 & 75000 & $\begin{array}{l}912500 \\
\end{array}$ & \begin{tabular}{|l|}
0.1138 \\
\end{tabular} & 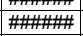 & 1.23 & \begin{tabular}{|l|}
$7.59 \mathrm{E}-03$ \\
\end{tabular} & 31.07 & 99.1 & 90.97 & 9.67 & 91.3 & 225.4 \\
\hline 729 & 21.85 & 3000 & 1050 & 117.809685 & 5.3647 & 136.61 & 549.01 & 5125 & 0.1 & 1950 & 75000 & \begin{tabular}{|l|l|}
1712500 \\
\end{tabular} & \begin{tabular}{|l|}
0.1456 \\
\end{tabular} & \#\#\#\#\# & 1.846 & 7.72E-03 & 30.42 & $\begin{array}{l}95.76 \\
\end{array}$ & 57.56 & 0 & 89.8 & 433 \\
\hline 730 & 37.3 & 3000 & 1100 & 913.998517 & 38.102 & 125.062 & \begin{tabular}{|l|}
599.7 \\
\end{tabular} & 5125 & 0.1 & 1950 & 75000 & 1500000 & \begin{tabular}{|l|}
0.0853 \\
\end{tabular} & \#\#\#\#\#立 & 2.42 & \begin{tabular}{|l|}
$3.57 \mathrm{E}-03$ \\
\end{tabular} & 32.14 & $\begin{array}{l}99.45 \\
\end{array}$ & 94.53 & 45.3 & 194.3 & 332.5 \\
\hline 731 & 35.83 & 3000 & 2200 & 172.224354 & 3.3355 & 58.1021 & \begin{tabular}{|l|}
1290.83 \\
\end{tabular} & 5125 & 0.1 & 1950 & 75000 & 475000 & \begin{tabular}{|l|}
0.0888 \\
\end{tabular} & \#\#\#\#覀 & 3.556 & \begin{tabular}{|l|}
$5.98 \mathrm{E}-03$ \\
\end{tabular} & 45.92 & 97.1 & 70.97 & 0 & 115.9 & 508.7 \\
\hline 732 & 30.13 & 3000 & 2090 & 3.664904 & 0.1398 & 114.41 & 655.54 & 5125 & 0.1 & 1950 & 75000 & 1312500 & \begin{tabular}{|l|}
0.1056 \\
\end{tabular} & \#\#\#\#再 & 4.128 & \begin{tabular}{|l|}
$1.11 \mathrm{E}-02$ \\
\end{tabular} & 54.76 & 0 & 0 & 0 & 62.5 & 702.2 \\
\hline 733 & 32.86 & 3000 & 810 & 27.374737 & 1.4787 & 162.051 & 462.82 & 5125 & 0.1 & 1950 & $\begin{array}{l}75000 \\
\end{array}$ & 2212500 & \begin{tabular}{|l|}
0.0968 \\
\end{tabular} & 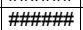 & 1.949 & \begin{tabular}{|c|}
$1.61 \mathrm{E}-02$ \\
\end{tabular} & 24.52 & 81.73 & 0 & 0 & 43 & 304 \\
\hline 734 & 25.86 & 3000 & 1980 & 3.111363 & 0.1006 & 97.024 & 773 & 5125 & 0.1 & 1950 & 75000 & 1025000 & \begin{tabular}{|l|}
0.123 \\
\end{tabular} & \#mmm & 3.355 & \begin{tabular}{|l|}
$1.17 \mathrm{E}-02$ \\
\end{tabular} & 41.31 & 0 & 0 & 0 & 59 & 664.8 \\
\hline $\begin{array}{l}734 \\
735\end{array}$ & 27.80 & 3000 & 980 & \begin{tabular}{|}
3.11305 \\
253.823525 \\
\end{tabular} & $\begin{array}{c}1.1000 \\
12.7112\end{array}$ & $\begin{array}{r}95.234 \\
150.236\end{array}$ & 499.21 & 5125 & $\begin{array}{l}0.1 \\
0.1\end{array}$ & 1950 & 75000 & $\frac{1025000}{1975000}$ & 0.1146 & 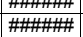 & \begin{tabular}{|l|}
3.3500 \\
2.06 \\
\end{tabular} & \begin{tabular}{|l|}
$1.17 \mathrm{E}-02$ \\
$6.63 \mathrm{E}-03$ \\
\end{tabular} & $\begin{array}{l}41.31 \\
47.04\end{array}$ & $\begin{array}{r}98.03 \\
\end{array}$ & 80.3 & 0 & $\begin{array}{r}594.5 \\
104\end{array}$ & $\begin{array}{l}604.0 \\
380.3 \\
\end{array}$ \\
\hline 736 & 21.67 & 3000 & 1240 & 26.053276 & 0.8357 & 96.2335 & 779.35 & 5125 & 0.1 & 1950 & 75000 & 1012500 & 0.1468 & 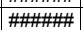 & 2.055 & $1.09 \mathrm{E}-02$ & 64.14 & 80.81 & 0 & 0 & 63.5 & 486 \\
\hline 737 & 27.43 & 3000 & 1550 & 15.22124 & 0.4216 & 83.1021 & 902.5 & 5125 & 0.1 & 1950 & 75000 & \begin{tabular}{|l|}
812500 \\
\end{tabular} & 0.116 & 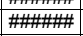 & 2.981 & \begin{tabular}{|c|}
$1.17 \mathrm{E}-02$ \\
\end{tabular} & 84.65 & 67.15 & 0 & 0 & 59.3 & 557.1 \\
\hline 738 & 33.84 & 3000 & 1430 & \begin{tabular}{|l|}
23.704919 \\
\end{tabular} & 1.3659 & 172.859 & 433.88 & 5125 & 0.1 & 1950 & 75000 & 2437500 & 0.094 & 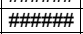 & 3.684 & $9.14 \mathrm{E}-03$ & 88.81 & 78.91 & 0 & 0 & 75.8 & 557.8 \\
\hline 739 & 42.53 & 3000 & 1430 & 545.283291 & 23.4829 & 129.196 & 580.51 & 5125 & 0.1 & 1950 & 75000 & 1575000 & \begin{tabular}{|l|}
0.0748 \\
\end{tabular} & \#\#\#\#\#立 & 3.252 & $3.75 \mathrm{E}-03$ & 42.49 & 99.08 & 90.83 & 8.3 & 184.8 & 391.9 \\
\hline 740 & 26.51 & 3000 & 2000 & 43.646896 & 1.2824 & 88.1405 & 850.91 & 5125 & 0.1 & 1950 & 75000 & 887500 & \begin{tabular}{|l|}
0.12 \\
\end{tabular} & \#\#\#\#\# & 3.256 & 7.11E-03 & 49.96 & 88.54 & 0 & 0 & 97.5 & 629.4 \\
\hline 741 & 30.8 & 3000 & 1200 & 121.295044 & 6.869 & 169.892 & 441.46 & 5125 & 0.1 & 1950 & 75000 & 2375000 & 0.1033 & 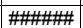 & 2.613 & $7.15 \mathrm{E}-03$ & 40.67 & 95.88 & 58.78 & & 96.9 & 434.9 \\
\hline 742 & 24.99 & 3000 & 1930 & 2.686482 & 0.1063 & 118.728 & 631.7 & 5125 & 0.1 & 1950 & 75000 & 1387500 & 0.1273 & \#\#\#\#覀 & 3.22 & $1.16 \mathrm{E}-02$ & 33.1 & 0 & 0 & 0 & 60 & 660.3 \\
\hline 743 & 29.51 & 3000 & 1240 & $\begin{array}{r}2.0006636 \\
38.26636 \\
\end{array}$ & $\begin{array}{l}0.2275 \\
1.2275 \\
\end{array}$ & 96.2335 & \begin{tabular}{l|l|l|}
779.35 \\
\end{tabular} & 5125 & 0.1 & 1950 & 75000 & 1012500 & \begin{tabular}{|l|}
0.1078 \\
0.1078
\end{tabular} & 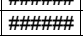 & $\begin{array}{l}2.324 \\
2.391\end{array}$ & 1.17E-02 & $\begin{array}{l}3.1 \\
56.4 \\
\end{array}$ & 86.93 & 0 & 0 & 59.5 & 415.2 \\
\hline 744 & 22.69 & 3000 & 1010 & $\begin{array}{r}9.356548 \\
\end{array}$ & 0.5317 & 170.487 & 439.92 & 5125 & 0.1 & 1950 & 75000 & 2387500 & \begin{tabular}{|l|}
0.1402 \\
\end{tabular} & \#\#\#\#\#立 & 2.015 & $1.35 \mathrm{E}-02$ & 35.77 & 46.56 & 0 & & 51.2 & 455 \\
\hline 745 & 47.62 & 3000 & 860 & 207.486227 & & 110.748 & 677.21 & 5125 & 0.1 & 1950 & 75000 & 1250000 & 0.0668 & 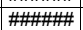 & 2.039 & 1.26E-02 & 34.46 & 97.59 & 75.9 & 0 & 54.9 & 219.4 \\
\hline 746 & 29.84 & 3000 & 1920 & 0.424822 & 0.0208 & 147.05 & & 512 & 0.1 & 195 & 75000 & 1912500 & 0.1066 & \#mmm & 3.749 & $1.55 \mathrm{E}-02$ & 32.23 & 0 & 0 & 0 & 44.7 & 643.9 \\
\hline 747 & 37.04 & 3000 & 560 & 940.637939 & 52.3302 & 166.898 & 449.38 & 5125 & 0.1 & 1950 & 75000 & 2312500 & 0.0859 & \#\#\# & 1.363 & & 34.72 & 99.47 & 94.68 & 46.84 & 111.5 & 188.6 \\
\hline 748 & 29.76 & 3000 & 2060 & 0.696108 & $\begin{array}{l}0.0359 \\
\end{array}$ & 154.641 & 484.99 & 5125 & 0.1 & 1950 & 75000 & 2062500 & 0.1069 & \#\#\#\#正 & 3.633 & $1.40 \mathrm{E}-02$ & 19.59 & 0 & 0 & 0 & 49.5 & 625.7 \\
\hline 749 & 21.88 & 3000 & 1190 & \begin{tabular}{|l|l|}
82.789197 \\
\end{tabular} & 3.2961 & 1119.44 & \begin{tabular}{|l|l|}
627.93 \\
\end{tabular} & 5125 & 0.1 & 1950 & 75000 & 1400000 & 0.1454 & \#\#\#\#覀 & 1.973 & $8.05 \mathrm{E}-03$ & 28.48 & 93.96 & 39.61 & 0 & 86.1 & 462.2 \\
\hline 750 & 61.18 & 3000 & 3900 & 1.3734 & 0.0598 & 130.56 & 574.45 & 5125 & 0.1 & 1950 & 75000 & 1600000 & 0.052 & 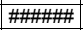 & 8.393 & $1.14 \mathrm{E}-02$ & 24.68 & 0 & 0 & 0 & 60.9 & 703.1 \\
\hline 751 & 29.24 & 3000 & 1260 & 72.0339 & 4.2353 & 176.387 & 425.2 & 5125 & 0.1 & 1950 & 75000 & 2512500 & 0.1088 & \#\#\#\#\#立 & 2.641 & $7.86 \mathrm{E}-03$ & 37.91 & $\begin{array}{l}93.06 \\
\end{array}$ & 30.59 & 0 & 88.2 & 463 \\
\hline 752 & 28.46 & 3000 & 980 & 350.4628 & 12.0601 & 103.236 & \begin{tabular}{|l|l|}
726.49 \\
\end{tabular} & 5125 & 0.1 & 1950 & 75000 & 1125000 & 0.1118 & 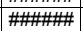 & 1.733 & $7.08 \mathrm{E}-03$ & 40.16 & 98.57 & 85.73 & 0 & 97.9 & 312.2 \\
\hline 753 & 54.95 & 3000 & 910 & 287.865929 & 9.9793 & 103.999 & 721.16 & 5125 & 0.1 & 1950 & 75000 & 1137500 & 0.0579 & 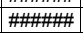 & 2.275 & $1.16 \mathrm{E}-02$ & 51.07 & 98.26 & 82.63 & 0 & 59.6 & 212.2 \\
\hline 754 & $\begin{array}{l}4.95 \\
42.93 \\
\end{array}$ & 3000 & 2620 & $\frac{201.003929}{30.092413}$ & \begin{tabular}{|c|}
1.096 \\
\end{tabular} & 109.267 & $\begin{array}{l}686.4 \\
686.4\end{array}$ & 5125 & $\begin{array}{l}0.1 \\
0.1\end{array}$ & 1950 & 75000 & $\frac{15000}{1225000}$ & $\begin{array}{l}0.0741 \\
0.0741\end{array}$ & "\#mm & 5.269 & $\begin{array}{l}1.10 \mathrm{E}-02 \\
6.99 \mathrm{E}-03 \\
\end{array}$ & 42.75 & \begin{tabular}{|l|}
0.20 \\
83.38 \\
\end{tabular} & $\begin{array}{l}02.00 \\
0\end{array}$ & 0 & $\begin{array}{l}59.2 \\
99.2\end{array}$ & $\begin{aligned}-12.29 \\
629 \\
\end{aligned}$ \\
\hline 755 & 37.17 & 3000 & 800 & 869.794892 & 31.03 & 107.025 & 700.77 & 5125 & 0.1 & 1950 & 75000 & 1187500 & 0.0856 & \#\#\#\#\#立 & 1.498 & $6.13 \mathrm{E}-03$ & 60. & 99.43 & 94.25 & 42.52 & 113.2 & 206.6 \\
\hline 756 & 27.01 & 3000 & 1040 & & 0.7244 & 101.701 & 737. & 512 & 0.1 & 195 & 75000 & 1100000 & 0.1178 & & 1.903 & & 44.96 & 76.6 & 0 & 0 & 46.8 & 361.1 \\
\hline 757 & 29.65 & 3000 & 1100 & 661.6 & $\begin{array}{r}21.0502 \\
\end{array}$ & 95.4399 & 785 & 512 & 0.1 & 1950 & 75000 & & & & 1.99 & & & 99.24 & 92.44 & 24.43 & & \\
\hline 758 & 31.69 & 3000 & 950 & 1384.0 & & 92.2314 & 813 & 512 & 0.1 & 195 & 75000 & & 0.1 & \#\#\# & 1.5 & $3.12 \mathrm{E}$ & 60.14 & 99.64 & 96.39 & 63.87 & 222.5 & 251.9 \\
\hline 759 & \begin{tabular}{|l|l|}
27.33 \\
\end{tabular} & 300 & 650 & 107.65234 & 5.8151 & 162.051 & 462. & 512 & 0.1 & 195 & 7500 & 2212500 & 0.11 & \#\#\#\#\#立 & 1.502 & $1.28 \mathrm{E}-02$ & 81.61 & 95.36 & 53.55 & 0 & 54.4 & 281.6 \\
\hline 760 & 59.8 & 3000 & 2210 & 297.9981 & 11.9348 & 120.15 & \begin{tabular}{|l|l|}
624.22 \\
\end{tabular} & 5125 & 0.1 & 1950 & 75000 & 1412500 & \begin{tabular}{|l|}
0.0532 \\
\end{tabular} & 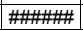 & 4.881 & $4.80 \mathrm{E}-03$ & 13.62 & 98.32 & 83.22 & 0 & 144.3 & 418.3 \\
\hline 761 & \begin{tabular}{|l|}
21.37 \\
\end{tabular} & 3000 & 1830 & 0.279152 & 0.0099 & 106.273 & 705.73 & 5125 & 0.1 & 1950 & 75000 & 1175000 & \begin{tabular}{|l|}
0.1489 \\
\end{tabular} & \#\#\#\#曲 & 2.949 & $1.54 \mathrm{E}-02$ & 37.62 & 0 & 0 & 0 & 45 & 707.5 \\
\hline 762 & 28.76 & 3000 & 1170 & 292.735676 & \begin{tabular}{|l|}
10.7345 \\
\end{tabular} & 110.009 & 681.77 & 5125 & 0.1 & 1950 & 75000 & 1237500 & \begin{tabular}{|l|}
0.1106 \\
\end{tabular} & \#\#\#\#立 & 2.34 & $5.80 \mathrm{E}-03$ & 82.81 & 98.29 & 82.92 & 0 & 119.5 & 416.9 \\
\hline 763 & 47.55 & 3000 & 810 & 432.353257 & 20.1641 & 139.914 & 536.04 & 5125 & 0.1 & 1950 & 75000 & 1775000 & 0.0669 & \#\#\#\#\#曲 & 2.295 & $8.21 \mathrm{E}-03$ & 70.16 & 98.84 & 88.44 & 0 & 84.4 & 247.3 \\
\hline 764 & 22.68 & 3000 & 840 & 78.096083 & 3.4692 & 133.266 & 562.78 & 5125 & 0.1 & 1950 & 75000 & & 0.1403 & \#\#\#\#覀 & 1.541 & $1.09 \mathrm{E}-02$ & & 93.6 & 35.98 & 0 & 63.9 & 348.4 \\
\hline 765 & 30.59 & 3000 & 1610 & 11.340333 & 0.4352 & 115.135 & 651.4 & 5125 & 0.1 & 195 & 75000 & 1325000 & 0.104 & & 2.944 & $1.19 \mathrm{E}-02$ & 20.49 & 55.91 & 0 & 0 & 58.4 & 3 \\
\hline 766 & 27.62 & 3000 & & & & & & 512 & & 195 & & & 0.1152 & & 3.0 & & & & 20.77 & 0 & & \\
\hline 767 & 32.3 & 3000 & 1420 & \begin{tabular}{|c|}
7.802703 \\
\end{tabular} & 0.3253 & 125.062 & 599.7 & 5125 & 0.1 & 1950 & 75000 & 1500000 & 0.0985 & \#\#\#\#! & 3.407 & $1.33 \mathrm{E}-02$ & 74.55 & 35.92 & 0 & 0 & 52.2 & 540.6 \\
\hline
\end{tabular}




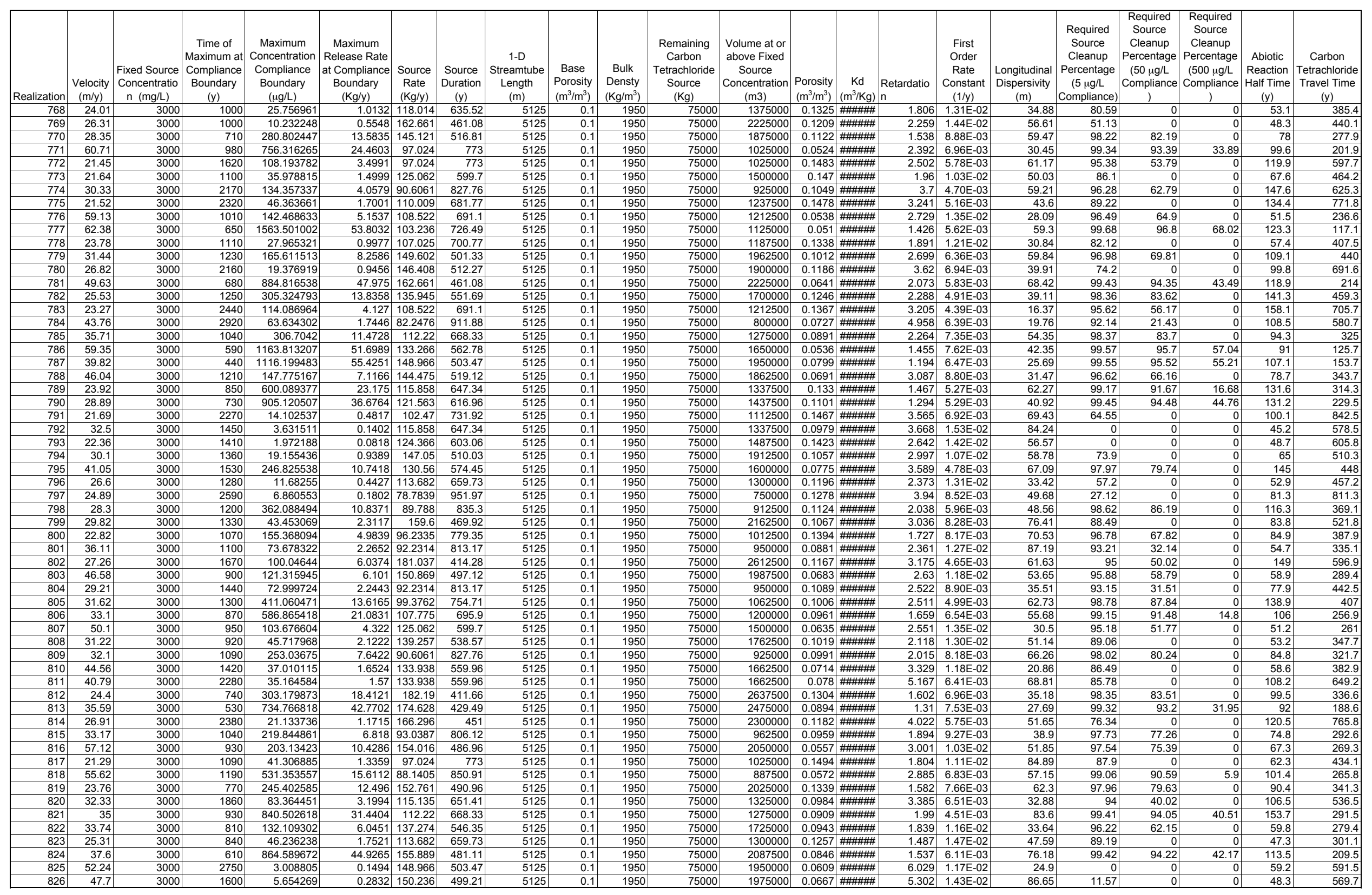




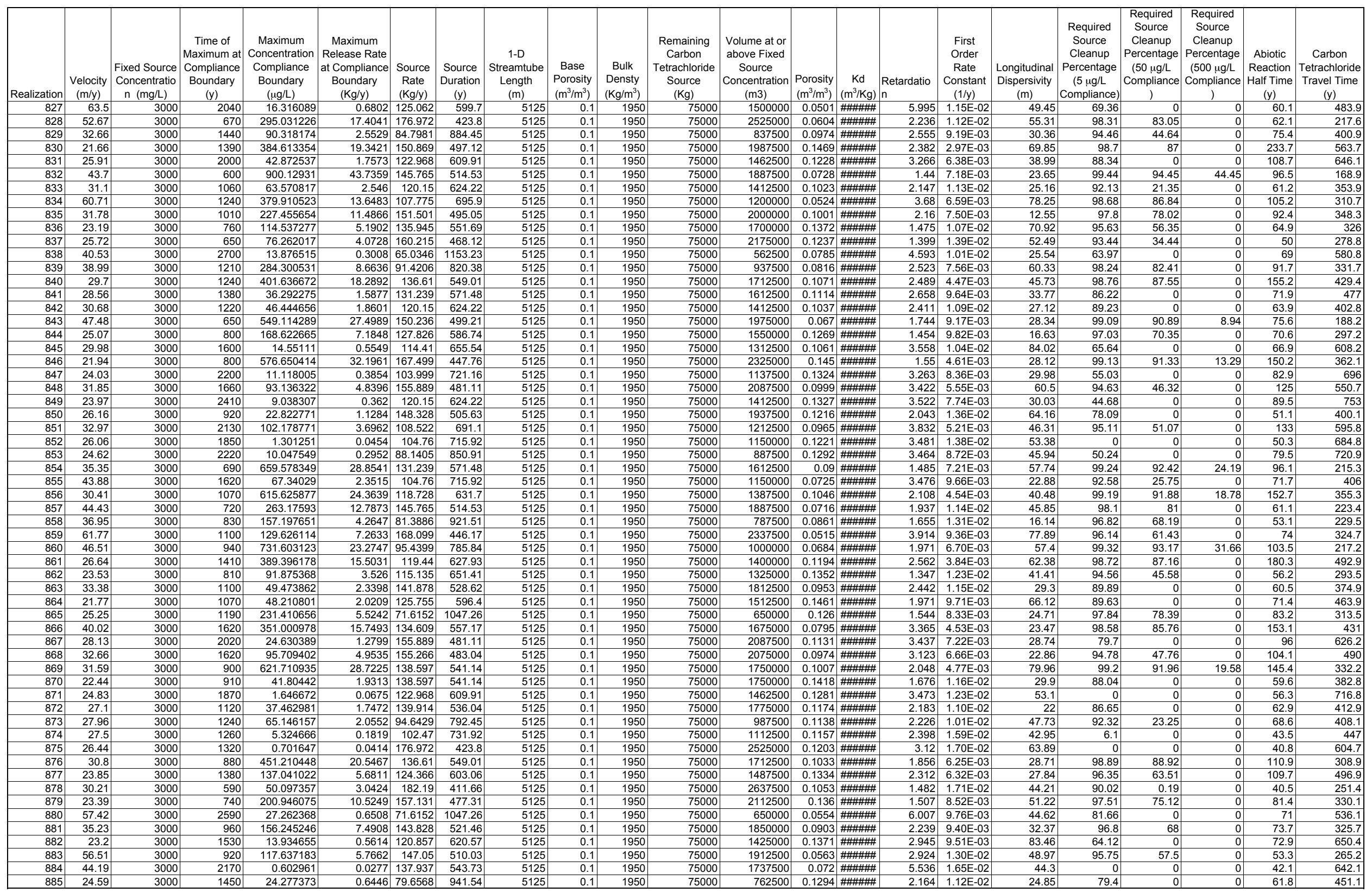




\begin{tabular}{|c|c|c|c|c|c|c|c|c|c|c|c|c|c|c|c|c|c|c|c|c|c|c|}
\hline Realization & $\begin{array}{c}\text { Velocity } \\
(\mathrm{m} / \mathrm{y})\end{array}$ & \begin{tabular}{|c|} 
Fixed Source \\
Concentratio \\
$\mathrm{n}(\mathrm{mg} / \mathrm{L})$
\end{tabular} & $\begin{array}{c}\text { Time of } \\
\text { Maximum at } \\
\text { Compliance } \\
\text { Boundary } \\
(y)\end{array}$ & \begin{tabular}{|c|} 
Maximum \\
Concentration \\
Compliance \\
Boundary \\
$(\mu \mathrm{g} / \mathrm{L})$
\end{tabular} & $\begin{array}{c}\text { Maximum } \\
\text { Release Rate } \\
\text { at Compliance } \\
\text { Boundary } \\
(\mathrm{Kg} / \mathrm{y})\end{array}$ & \begin{tabular}{|c|} 
\\
Source \\
Rate \\
$(\mathrm{Kg} / \mathrm{y})$ \\
\end{tabular} & $\begin{array}{c}\text { Source } \\
\text { Duration } \\
\text { (y) }\end{array}$ & $\begin{array}{c}\text { 1-D } \\
\text { Streamtube } \\
\text { Length } \\
\text { (m) }\end{array}$ & $\begin{array}{c}\text { Base } \\
\text { Porosity } \\
\left(\mathrm{m}^{3} / \mathrm{m}^{3}\right)\end{array}$ & $\begin{array}{c}\text { Bulk } \\
\text { Densty } \\
\left(\mathrm{Kg} / \mathrm{m}^{3}\right)\end{array}$ & $\begin{array}{c}\text { Remaining } \\
\text { Carbon } \\
\text { Tetrachloride } \\
\text { Source } \\
(\mathrm{Kg})\end{array}$ & $\begin{array}{c}\begin{array}{c}\text { Volume at or } \\
\text { above Fixed } \\
\text { Source } \\
\text { Concentration } \\
(\mathrm{m} 3)\end{array} \\
\end{array}$ & $\begin{array}{l}\text { Porosity } \\
\left(\mathrm{m}^{3} / \mathrm{m}^{3}\right)\end{array}$ & $\begin{array}{c}\mathrm{Kd} \\
\left(\mathrm{m}^{3} / \mathrm{Kg}\right)\end{array}$ & $\begin{array}{l}\text { Retardatio } \\
n\end{array}$ & \begin{tabular}{|c|} 
First \\
Order \\
Rate \\
Constant \\
$(1 / y)$ \\
\end{tabular} & $\begin{array}{l}\text { Longitudinal } \\
\text { Dispersivity } \\
\text { (m) }\end{array}$ & $\begin{array}{l}\text { Required } \\
\text { Source } \\
\text { Cleanup } \\
\text { Percentage } \\
\text { (5 ug/L } \\
\text { Compliance) }\end{array}$ & \begin{tabular}{|c} 
Required \\
Source \\
Cleanup \\
Percentage \\
$(50 \mu \mathrm{g} / \mathrm{L}$ \\
Compliance \\
)
\end{tabular} & \begin{tabular}{|c|} 
Required \\
Source \\
Cleanup \\
Percentage \\
$(500 \mu \mathrm{L} / \mathrm{L}$ \\
Compliance \\
) \\
\end{tabular} & \begin{tabular}{|c|} 
\\
Abiotic \\
Reaction \\
Half Time \\
$(y)$ \\
\end{tabular} & \begin{tabular}{|c|} 
Carbon \\
Tetrachloride \\
Travel Time \\
(y)
\end{tabular} \\
\hline 886 & 51.64 & $\begin{array}{r}3000 \\
\end{array}$ & & $\begin{array}{l}6.958603 \\
\end{array}$ & 2.7132 & \begin{tabular}{|l|}
121.563 \\
\end{tabular} & \begin{tabular}{|l|}
616.96 \\
\end{tabular} & & & 1950 & 75000 & 1437500 & \begin{tabular}{|l|}
0.0616 \\
\end{tabular} & 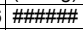 & 4.648 & $7.91 \mathrm{E}-03$ & 47.41 & 92.53 & \begin{tabular}{|l|l|}
3 & 25.33 \\
\end{tabular} & 0 & \begin{tabular}{|l|} 
\\
\end{tabular} & 461.2 \\
\hline 887 & 21.48 & 3000 & 1340 & 133.466885 & $\begin{array}{l}6.1072 \\
\end{array}$ & \begin{tabular}{|l|}
137.274 \\
\end{tabular} & 546.35 & 5125 & 0.1 & 1950 & 75000 & 1725000 & 0.1481 & \#\#\#\#\#曲 & 2.33 & $5.50 \mathrm{E}-03$ & 63.79 & 96.25 & 62.54 & 0 & 126 & 555.8 \\
\hline 888 & 44 & 3000 & 1070 & 618.507984 & 22.9848 & \begin{tabular}{|l|l|}
111.485 \\
\end{tabular} & 672.73 & 5125 & 0.1 & 1950 & 75000 & 1262500 & 0.0723 & \#\#\#\#\# & 2.584 & \begin{tabular}{|l|} 
\\
\end{tabular} & 63.25 & 99.19 & 91.92 & 19.16 & 129.4 & 301 \\
\hline 889 & 21.85 & 3000 & 1200 & 6.357583 & 0.265 & \begin{tabular}{|l|}
125.062 \\
\end{tabular} & 599.7 & 5125 & 0.1 & 1950 & 75000 & 1500000 & \begin{tabular}{|l|}
0.1456 \\
\end{tabular} & \#\#\#\#\#曲 & 2.13 & $1.35 \mathrm{E}-02$ & 39.53 & 21.35 & 0 & 0 & 51.4 & 499.6 \\
\hline 890 & 23.48 & 3000 & 2240 & \begin{tabular}{|l|}
33.823219 \\
\end{tabular} & 1.2981 & \begin{tabular}{|l|}
115.135 \\
\end{tabular} & 651.41 & 5125 & 0.1 & 1950 & 75000 & 1325000 & \begin{tabular}{|l|}
0.1355 \\
\end{tabular} & \#\#\#\#曲 & 3.45 & \begin{tabular}{|l|l|} 
& $5.79 \mathrm{E}-03$ \\
\end{tabular} & 51.59 & 85.22 & 0 & 0 & 119.7 & 753.1 \\
\hline 891 & 45.51 & 3000 & 840 & \begin{tabular}{|c|}
113.103463 \\
\end{tabular} & 5.9006 & \begin{tabular}{|l|}
156.51 \\
\end{tabular} & 479.2 & 5125 & 0.1 & 1950 & 75000 & 2100000 & \begin{tabular}{|l|}
0.0699 \\
\end{tabular} & \#\#\#\#曲 & 2.341 & \begin{tabular}{|l|}
$1.30 \mathrm{E}-02$ \\
\end{tabular} & 30.14 & 95.58 & 55.79 & 0 & 53.5 & 263.6 \\
\hline 892 & 31.78 & 3000 & 620 & 186.003086 & 7.7539 & \begin{tabular}{|l|}
125.062 \\
\end{tabular} & 599.7 & 5125 & 0.1 & 1950 & 75000 & 1500000 & 0.1001 & \#\#\#\#\# & 1.187 & $1.49 \mathrm{E}-02$ & 40.64 & 97.31 & 73.12 & 0 & 46.5 & 191.5 \\
\hline 893 & 54.57 & 3000 & 890 & 1039.42406 & 34.4313 & \begin{tabular}{|l|}
99.3762 \\
\end{tabular} & 754.71 & 5125 & 0.1 & 1950 & 75000 & 1062500 & 0.0583 & \#\#\#\#\# & 2.031 & \begin{tabular}{|l|}
$5.69 \mathrm{E}-03$ \\
\end{tabular} & 59.02 & 99.52 & 95.19 & 51.9 & 121.7 & 190.7 \\
\hline 894 & 41.64 & 3000 & 610 & 250.117782 & 15.1416 & \begin{tabular}{|l|}
181.614 \\
\end{tabular} & 412.96 & 5125 & 0.1 & 1950 & 75000 & 2625000 & 0.0764 & \#\#\#\#\# & 1.745 & $1.19 \mathrm{E}-02$ & 30.05 & 98 & 80.01 & & 58.4 & 214.7 \\
\hline 895 & 35.15 & 3000 & 1010 & 95.660444 & 3.6017 & \begin{tabular}{|l|}
112.952 \\
\end{tabular} & 664 & 5125 & 0.1 & 1950 & 75000 & 1287500 & 0.0905 & \#\#\#\#曲 & 2.222 & $1.14 \mathrm{E}-02$ & 51.34 & 94.77 & 47.73 & & 60.6 & 323.9 \\
\hline 896 & 33.56 & 3000 & 1400 & 6.753102 & 0.2392 & \begin{tabular}{|l|}
106.273 \\
\end{tabular} & 705.73 & 5125 & 0.1 & 1950 & 75000 & 1175000 & $\begin{array}{l}0.0948 \\
\end{array}$ & \#\#\#\#曲 & 2.991 & \begin{tabular}{|c|}
$1.52 \mathrm{E}-02$ \\
\end{tabular} & 43.35 & 25.96 & 0 & 0 & 45.7 & 456.8 \\
\hline 897 & 28.63 & 3000 & 2300 & 3.968586 & 0.2152 & \begin{tabular}{|l|}
162.661 \\
\end{tabular} & 461.08 & 5125 & 0.1 & 1950 & 75000 & 2225000 & \begin{tabular}{|l|}
0.1111 \\
\end{tabular} & \#\#\#\#\#曲 & 3.911 & \begin{tabular}{|l|}
$9.38 \mathrm{E}-03$ \\
\end{tabular} & 29.32 & 0 & 0 & 0 & 73.9 & 699.9 \\
\hline 898 & 23.43 & 3000 & 970 & 8.659516 & 0.4245 & \begin{tabular}{|l|}
147.05 \\
\end{tabular} & 510.03 & 5125 & 0.1 & 1950 & 75000 & 1912500 & \begin{tabular}{|l|}
0.1358 \\
\end{tabular} & \#\#\#\#\#立 & 2.009 & $1.49 \mathrm{E}-02$ & 57.93 & 42.26 & & & 46.4 & 439.6 \\
\hline 899 & 33.31 & 3000 & 1760 & 10.474642 & 0.4908 & 140.57 & 533.54 & 5125 & 0.1 & 1950 & 75000 & 1787500 & \begin{tabular}{|l|}
0.0955 \\
\end{tabular} & \#\#\#\#\#曲 & 3.601 & \begin{tabular}{|c|}
$.06 \mathrm{E}-02$ \\
\end{tabular} & 37.42 & 52.27 & 0 & 0 & 65.3 & 553.9 \\
\hline 900 & 58.59 & 3000 & 2850 & 17.08425 & 0.7201 & \begin{tabular}{|l|}
126.447 \\
\end{tabular} & 593.13 & 5125 & 0.1 & 1950 & 75000 & 1525000 & \begin{tabular}{|l|}
0.0543 \\
\end{tabular} & \#\#\#\#\# & 7.973 & $7.70 \mathrm{E}-03$ & 70.51 & 70.73 & 0 & 0 & 90 & 697.4 \\
\hline 901 & 35.67 & 3000 & 1620 & 189.710228 & 6.2348 & \begin{tabular}{|l|}
98.5952 \\
\end{tabular} & 760.69 & 5125 & 0.1 & 1950 & 75000 & 1050000 & 0.0892 & \#\#\#\#\#五 & 2.993 & $6.54 \mathrm{E}-03$ & 18.9 & 97.36 & 73.64 & 0 & 105.9 & 430.2 \\
\hline 902 & 22.39 & 3000 & 1180 & 203.413938 & 5.2824 & \begin{tabular}{|l|}
77.906 \\
\end{tabular} & 962.7 & 5125 & 0.1 & 1950 & 75000 & 737500 & 0.1421 & 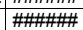 & 1.555 & $7.81 \mathrm{E}-03$ & 40.44 & 97.54 & 75.42 & 0 & 88.8 & 356 \\
\hline 903 & 40.32 & 3000 & 2480 & 162.973991 & 4.6066 & 84.7981 & 884.45 & 5125 & 0.1 & 1950 & 75000 & 837500 & 0.0789 & 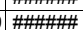 & 4.732 & 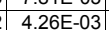 & $\begin{array}{l}40.44 \\
60.55\end{array}$ & 96.93 & $\begin{array}{l}0.4 .32 \\
69\end{array}$ & & 162.9 & $\begin{array}{r}601.4 \\
60\end{array}$ \\
\hline 904 & 30.33 & 3000 & 880 & 20.936062 & 1.1181 & \begin{tabular}{|l|}
160.215 \\
\end{tabular} & 468.12 & 5125 & 0.1 & 1950 & 75000 & 2175000 & 0.1049 & \#\#\#\#曲 & 2.157 & $1.50 \mathrm{E}-02$ & 56.52 & 76.12 & 0 & & 46.1 & 364.5 \\
\hline 905 & 58.37 & 3000 & 1140 & \begin{tabular}{|c|c|c|c|}
1526.587168 \\
\end{tabular} & 53.3084 & \begin{tabular}{|l|}
104.76 \\
\end{tabular} & 715.92 & 5125 & 0.1 & 1950 & 75000 & 1150000 & 0.0545 & \#\#\#\#\#曲 & 2.973 & $2.52 \mathrm{E}-03$ & 43.74 & 99.67 & 96.72 & 67.25 & 274.9 & 261 \\
\hline 906 & 38.28 & 3000 & 1570 & 192.463486 & $\begin{array}{l}7.0575 \\
\end{array}$ & \begin{tabular}{|l|}
110.009 \\
\end{tabular} & 681.77 & 5125 & 0.1 & 1950 & 75000 & 1237500 & \begin{tabular}{|l|}
0.0831 \\
\end{tabular} & \#\#\#\#曲 & 3.137 & $6.58 \mathrm{E}-03$ & 22.18 & 97.4 & 74.02 & 0 & 105.3 & 419.9 \\
\hline 907 & 30.71 & 3000 & 710 & 302.202729 & 13.2203 & \begin{tabular}{|l|}
131.239 \\
\end{tabular} & 571.48 & 5125 & 0.1 & 1950 & 75000 & 1612500 & \begin{tabular}{|l|}
0.1036 \\
\end{tabular} & \#\#\#\#\# & 1.424 & $9.93 \mathrm{E}-03$ & 43.85 & 98.35 & 83.45 & 0 & 69.8 & 237.7 \\
\hline 908 & 26.25 & 3000 & 1370 & 126.913252 & 4.8093 & \begin{tabular}{|l|}
113.682 \\
\end{tabular} & 659.73 & 5125 & 0.1 & 1950 & 75000 & 1300000 & 0.1212 & 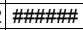 & 2.369 & $7.03 \mathrm{E}-03$ & 25.33 & 96.06 & 60.6 & & 98.6 & 462.6 \\
\hline 909 & 36.23 & 3000 & 2230 & 0.537053 & 0.0198 & \begin{tabular}{|l|}
110.748 \\
\end{tabular} & 677.21 & 5125 & 0.1 & 1950 & 75000 & 1250000 & 0.0878 & \#\#\#\#\# & 4.455 & $1.58 \mathrm{E}-02$ & 30.22 & 0 & 0 & 0 & 43.8 & 630.1 \\
\hline 910 & 37.96 & 3000 & 1650 & 469.582682 & 12.874 & \begin{tabular}{|l|}
82.2476 \\
\end{tabular} & 911.88 & 5125 & 0.1 & 1950 & 75000 & 800000 & 0.0838 & \#\#\#\#\# & 3.045 & $4.58 \mathrm{E}-03$ & 34.03 & 98.94 & 89.35 & 0 & 151.5 & 411.1 \\
\hline 911 & 31.44 & 3000 & 2230 & 8.137254 & 0.1892 & \begin{tabular}{|l|}
69.767 \\
\end{tabular} & 1075.01 & 5125 & 0.1 & 1950 & 75000 & 625000 & 0.1012 & \#\#\#\#\#五 & 4.421 & 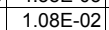 & 79.08 & 38.55 & 0 & 0 & 64.2 & 720.7 \\
\hline 912 & & 3000 & 2450 & & & \begin{tabular}{|l|}
93.8425 \\
\end{tabular} & $\begin{array}{l}599.21 \\
\end{array}$ & 5125 & 0.1 & 1950 & 75000 & 975000 & 0.0825 & m\#m & 4.205 & \begin{tabular}{|l|l|} 
& $7.790 \mathrm{E}-03$ \\
\end{tabular} & 18.44 & 86.91 & 0 & & 89 & 558.9 \\
\hline 913 & 29.48 & 3000 & 1070 & 26.766302 & 0.9549 & \begin{tabular}{|l|}
107.025 \\
\end{tabular} & 700.77 & 5125 & 0.1 & 1950 & 75000 & 1187500 & 0.1079 & 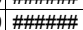 & 2.267 & \begin{tabular}{|l|}
$1.38 \mathrm{E}-02$ \\
\end{tabular} & 77.21 & 81.32 & 0 & & 50.1 & 394 \\
\hline 914 & 24.12 & 3000 & 1300 & 236.360527 & 9.014 & 114.41 & 655.54 & 5125 & 0.1 & 1950 & 75000 & 1312500 & 0.1319 & \#\#\#\#\#曲 & 2.189 & \begin{tabular}{|l|}
$5.59 \mathrm{E}-03$ \\
\end{tabular} & 32.75 & 97.88 & 78.85 & & 124.1 & 465.1 \\
\hline 915 & 36.52 & 3000 & 690 & 383.990965 & 18.5751 & 145.121 & 516.81 & 5125 & 0.1 & 1950 & 75000 & 1875000 & 0.0871 & \#\#\#\#曲 & 1.653 & \begin{tabular}{|l|}
$9.12 \mathrm{E}-03$ \\
\end{tabular} & 42.67 & 98.7 & 86.98 & & 76 & 231.9 \\
\hline 916 & 27.69 & 3000 & 1130 & \begin{tabular}{|l|}
229.342158 \\
\end{tabular} & $\begin{array}{l}.4541 \\
9.45\end{array}$ & \begin{tabular}{|l|}
123.668 \\
\end{tabular} & 606.46 & 5125 & 0.1 & 1950 & 75000 & 1475000 & 0.1149 & 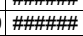 & 2.14 & \begin{tabular}{|l|} 
\\
$6.67 \mathrm{E}-03$ \\
\end{tabular} & 30.95 & 97.82 & 78.2 & 0 & 103.9 & 396.1 \\
\hline 917 & 22.55 & 3000 & 1570 & 496.707893 & 17.2191 & \begin{tabular}{|l|}
103.999 \\
\end{tabular} & 721.16 & 5125 & 0.1 & 1950 & 75000 & 1137500 & 0.1411 & \#\#\#\#曲 & 2.374 & \begin{tabular}{|l|}
$3.24 \mathrm{E}-03$ \\
\end{tabular} & 38.3 & 98.99 & 89.93 & 0 & 213.7 & 539.6 \\
\hline 918 & 48.5 & 3000 & 1990 & 21.06052 & 0.5714 & \begin{tabular}{|l|}
81.3886 \\
\end{tabular} & 921.51 & 5125 & 0.1 & 1950 & 75000 & 787500 & \begin{tabular}{|l|}
0.0656 \\
\end{tabular} & \#\#\#\#\# & 4.929 & \begin{tabular}{|c|}
$1.14 \mathrm{E}-02$ \\
\end{tabular} & 63.39 & 76.26 & 0 & 0 & 60.8 & 520.9 \\
\hline 919 & 40.79 & 3000 & 720 & 944.861707 & 37.618 & \begin{tabular}{|l|}
119.44 \\
\end{tabular} & 627.93 & 5125 & 0.1 & 1950 & 75000 & 1400000 & \begin{tabular}{|l|}
0.078 \\
\end{tabular} & \#\#\#\#\# & 1.467 & \begin{tabular}{|l|}
$6.34 \mathrm{E}-03$ \\
\end{tabular} & 35.12 & 99.47 & 94.71 & 47.08 & 109.3 & 184.3 \\
\hline 920 & 24.23 & 3000 & 1280 & 16.861273 & 0.4623 & \begin{tabular}{|l|}
82.2476 \\
\end{tabular} & 911.88 & 5125 & 0.1 & 1950 & 75000 & 800000 & 0.1313 & \#\#\#\#曲 & 2.134 & \begin{tabular}{|c|}
$.32 \mathrm{E}-02$ \\
\end{tabular} & 71.53 & 70.35 & 0 & & 52.3 & 451.3 \\
\hline 921 & 28.74 & 3000 & 1030 & 207.543021 & 6.9824 & \begin{tabular}{|l|}
100.929 \\
\end{tabular} & 743.1 & 5125 & 0.1 & 1950 & 75000 & 1087500 & 0.1107 & \#\#\#\#曲 & 1.852 & 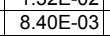 & 39.34 & 97.59 & 75.91 & 0 & 82.6 & 330.2 \\
\hline 922 & 30.36 & 3000 & 1230 & & & \begin{tabular}{|l|}
110.748 \\
\end{tabular} & 677.21 & & & & 75000 & 125 & & \#\#\#\#曲 & 2.651 & & 79.72 & 91.32 & 13.18 & & 69.9 & 447.6 \\
\hline 923 & 22.29 & 3000 & 1690 & & 0.4 & 128.512 & 583.6 & 5125 & 0 & 195 & 750 & & 0.1427 & 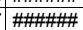 & 2.7 & & 3 & 48.17 & 0 & & 72.6 & 632.4 \\
\hline 924 & 33.1 & 3000 & 640 & 589.047399 & 20.7 & \begin{tabular}{|l|}
105.518 \\
\end{tabular} & 710.78 & 5125 & 0.1 & 1950 & 75000 & 1162500 & 0.0961 & & 1.265 & 8.42 & 30.34 & 99.15 & 91.51 & 15.12 & 82.4 & 195.8 \\
\hline 925 & 23.64 & 3000 & 670 & 418.902069 & 25 & \begin{tabular}{|l|}
185.628 \\
\end{tabular} & 404.03 & & & 19 & & & 0.1346 & \#\#\# & 1.457 & & & & 88.06 & 0 & 108.4 & 315. \\
\hline 926 & 43.46 & 3000 & 1200 & 071392 & 11.5451 & \begin{tabular}{|l|}
155.266 \\
\end{tabular} & 483.04 & 5125 & 0.1 & 1950 & 75000 & 2075000 & 0.0732 & \#\#\#\#\#曲 & 3.088 & & 44.61 & 97.76 & 77.59 & 0 & 102.7 & 364.1 \\
\hline 927 & 36.23 & 3000 & 730 & 113.3975 & 5.3629 & \begin{tabular}{|l|l|}
141.878 \\
\end{tabular} & 528.62 & 5125 & 0.1 & 1950 & 75000 & 1812500 & 0.0878 & \#\#\#\#\# & 1.798 & \begin{tabular}{|l|}
$1.37 \mathrm{E}-02$ \\
\end{tabular} & 57.08 & 95.59 & 55.91 & & 50.5 & 254.3 \\
\hline 928 & 44.87 & 3000 & 2840 & 12.0918 & 0.6383 & \begin{tabular}{|l|}
158.368 \\
\end{tabular} & 473. & 5125 & 0.1 & 1950 & 75000 & 2137500 & 0.070 & \#\#\#\#\#曲 & 6.229 & $7.23 \mathrm{E}-03$ & 51.49 & 58.65 & 0 & 0 & 95.9 & 711.5 \\
\hline 929 & 21.28 & 3000 & 1100 & 16.5591 & 0.6865 & \begin{tabular}{|l|}
124.366 \\
\end{tabular} & 603.06 & 5125 & 0.1 & 1950 & 7500 & 1487500 & 0.1495 & \#\#\#\#曲 & 1.856 & \begin{tabular}{|l|}
$.22 \mathrm{E}-02$ \\
\end{tabular} & 24.27 & 69.81 & 0 & 0 & 57 & 447.1 \\
\hline 930 & 63.12 & 3000 & 1070 & 282.165615 & $\begin{array}{l}7.8162 \\
\end{array}$ & \begin{tabular}{|l|}
83.1021 \\
\end{tabular} & $\begin{array}{l}902.5 \\
9\end{array}$ & 5125 & 0.1 & 1950 & 75000 & 812500 & 0.0504 & \#\#\#\# & 2.373 & \begin{tabular}{|l|}
$1.25 \mathrm{E}-02$ \\
\end{tabular} & 17.43 & 98.23 & 82.28 & 0 & 55.4 & 192.6 \\
\hline 931 & 32.23 & 3000 & 730 & 536.729357 & 23.8426 & \begin{tabular}{|l|}
133.266 \\
\end{tabular} & 562.78 & 5125 & 0.1 & 1950 & 75000 & 1650000 & 0.0987 & m m\#\#\# & 1.539 & \begin{tabular}{|l|}
$7.17 \mathrm{E}-03$ \\
\end{tabular} & 38.55 & 99.07 & 90.68 & 6.84 & 96.6 & 244.7 \\
\hline 932 & 31.41 & 3000 & 930 & $\begin{array}{r}73.229226 \\
\end{array}$ & 3.7289 & \begin{tabular}{|l|}
152.761 \\
\end{tabular} & 490.96 & 5125 & 0.1 & 1950 & 75000 & 2025000 & 0.1013 & 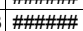 & 2.29 & \begin{tabular}{|l|}
$1.08 \mathrm{E}-02$ \\
\end{tabular} & 74.45 & 93.17 & 31.72 & 0.04 & $\begin{array}{ll}04.3 \\
6\end{array}$ & 373.7 \\
\hline 933 & 28.95 & 3000 & 1090 & 5.713062 & 0.2837 & \begin{tabular}{|l|}
148.966 \\
\end{tabular} & 503.47 & 5125 & 0.1 & 1950 & 75000 & 1950000 & \begin{tabular}{|l|}
0.1099 \\
\end{tabular} & \#\#\#\#\# & 2.343 & 1.63E-02 & 30.2 & 12.48 & 0 & 0 & 42.6 & 414.8 \\
\hline 934 & 24.34 & & 690 & & & \begin{tabular}{|l|}
119.44 \\
\end{tabular} & 627 & & & & & & 0.1 & & 1.2 & & & 97.57 & 75.69 & 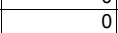 & & \\
\hline 935 & 21.61 & 3000 & 780 & 56 & 1.8316 & \begin{tabular}{|l|}
97.8112 \\
\end{tabular} & 766 & 512 & 0.1 & 19 & 75 & & & \#\#\# & 1.2 & & 16 & 91.1 & 10.99 & 0 & & 305.2 \\
\hline 936 & 22.59 & 3000 & 1770 & 32.343 & & \begin{tabular}{|l|}
136.61 \\
\end{tabular} & 549 & 512 & 0.1 & & 750 & & 0.14 & & 2.8 & & 41. & 84.54 & 0 & 0 & 100.4 & 648.9 \\
\hline 937 & 37.25 & 3000 & 1490 & 3.5892 & 0.2061 & \begin{tabular}{|l|}
172.268 \\
\end{tabular} & 435.37 & 5125 & 0.1 & 1950 & 750 & 2425000 & 0.0854 & \#\#\#\# & 3.977 & \begin{tabular}{|l|}
$1.43 \mathrm{E}-02$ \\
\end{tabular} & 62.66 & 0 & 0 & 0 & 48.4 & 547.1 \\
\hline 938 & 25.19 & 3000 & 1010 & 454.249482 & \begin{tabular}{|l|}
17.1028 \\
\end{tabular} & \begin{tabular}{|l|}
112.952 \\
\end{tabular} & 664 & 5125 & 0.1 & 1950 & 75000 & 1287500 & 0.1263 & \#\#\#\#\# & 1.753 & \begin{tabular}{|l|} 
\\
\end{tabular} & 26.07 & 98.9 & 88.99 & 0 & 128.8 & 356.6 \\
\hline 939 & 27.33 & 3000 & 1060 & 10.005136 & 0.3767 & \begin{tabular}{|l|}
112.952 \\
\end{tabular} & 664 & 5125 & 0.1 & 1950 & 75000 & 1287500 & 0.1164 & \#\#\#\#曲 & 2.168 & \begin{tabular}{|l|}
$.60 \mathrm{E}-02$ \\
\end{tabular} & 59.1 & 50.03 & 0 & 0 & 43.4 & 406.5 \\
\hline 940 & 21.34 & 3000 & 740 & $\begin{array}{l}73.233872 \\
\end{array}$ & $\begin{array}{l}.7597 \\
.750\end{array}$ & \begin{tabular}{|l|}
154.016 \\
\end{tabular} & 486.96 & 5125 & 0.1 & 1950 & 75000 & 2050000 & 0.1491 & \#\#\#\#曲 & 1.385 & \begin{tabular}{|l|}
$1.14 \mathrm{E}-02$ \\
\end{tabular} & 20.38 & 93.17 & 31.73 & 0 & $\begin{array}{l}6.4 \\
60.8 \\
\end{array}$ & 332.6 \\
\hline 941 & 44.12 & 3000 & 1000 & 346.110195 & 11.3749 & \begin{tabular}{|l|}
98.5952 \\
\end{tabular} & 760.69 & 5125 & 0.1 & 1950 & 75000 & 1050000 & 0.0721 & \#\#\#\#\# & 2.213 & \begin{tabular}{|l|}
$8.85 \mathrm{E}-03$ \\
\end{tabular} & 57.38 & 98.56 & 85.55 & 4 & 78.3 & 257.1 \\
\hline 942 & 61.06 & 3000 & 2180 & 154.9323 & 4.3356 & \begin{tabular}{|l|}
83.9523 \\
\end{tabular} & 893.36 & 5125 & 0.1 & 1950 & 75000 & 825000 & 0.0521 & \#\#\#\#曲 & 5.68 & 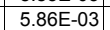 & 69.76 & 96.77 & 67.73 & & 118.3 & 476.7 \\
\hline 943 & 28.1 & 3000 & 1440 & & 0.0 & \begin{tabular}{|l|}
119.44 \\
\end{tabular} & 627.93 & 5125 & 0.1 & 1950 & & & & & 3.061 & & & & 0 & 0 & & 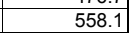 \\
\hline 944 & 42.93 & 3000 & 770 & 616.352643 & 22.4489 & \begin{tabular}{|l|}
109.267 \\
\end{tabular} & 686.4 & 5125 & 0.1 & 1950 & 75000 & 1225000 & 0.0741 & \#\#\#\#曲 & 1.507 & $9.00 \mathrm{E}-03$ & 47.82 & 99.19 & 91.89 & 18.88 & 77.1 & 179.8 \\
\hline
\end{tabular}




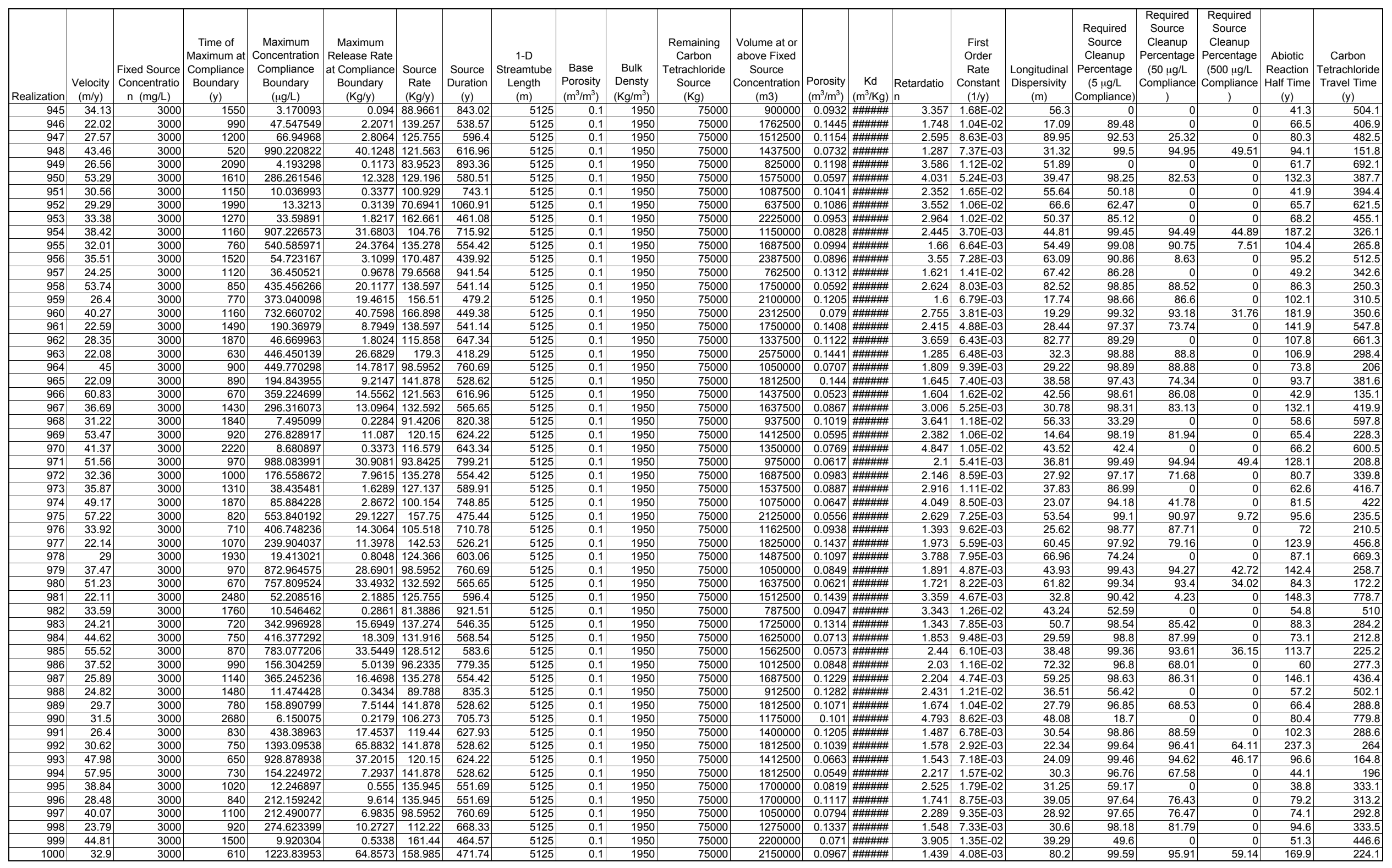




\begin{tabular}{|c|c|c|c|c|c|c|c|c|c|c|c|c|c|c|c|c|c|c|c|c|c|c|}
\hline $\begin{array}{c}\text { Realizatio } \\
n\end{array}$ & $\begin{array}{c}\text { Velocity } \\
(\mathrm{m} / \mathrm{y})\end{array}$ & $\begin{array}{c}\text { Fixed Source } \\
\text { Concentration } \\
\text { (mg/L) }\end{array}$ & $\begin{array}{c}\text { Time of } \\
\text { Maximum at } \\
\text { Compliance } \\
\text { Boundary } \\
(y)\end{array}$ & \begin{tabular}{|c|} 
Maximum \\
Concentration \\
Compliance \\
Boundary \\
$(\mu \mathrm{g} / \mathrm{L})$
\end{tabular} & \begin{tabular}{|c|} 
Maximum \\
Release Rate \\
at Compliance \\
Boundary \\
$(\mathrm{Kg} / \mathrm{y})$
\end{tabular} & \begin{tabular}{|c|} 
Source \\
Rate \\
$(\mathrm{Kg} / \mathrm{y})$ \\
\end{tabular} & \begin{tabular}{|c|} 
Source \\
Duration \\
(y)
\end{tabular} & \begin{tabular}{|c|} 
1-D \\
Streamtub \\
e Length \\
(m)
\end{tabular} & $\begin{array}{l}\text { Base } \\
\text { Porosity } \\
\left(\mathrm{m}^{3} / \mathrm{m}^{3}\right)\end{array}$ & $\begin{array}{c}\text { Bulk } \\
\text { Densty } \\
\left(\mathrm{Kg} / \mathrm{m}^{3}\right)\end{array}$ & $\begin{array}{c}\text { Remaining } \\
\text { Carbon } \\
\text { Tetrachloride } \\
\text { Source } \\
(\mathrm{Kg})\end{array}$ & \begin{tabular}{|c|} 
Volume at or \\
above Fixed \\
Source \\
$\begin{array}{c}\text { Concentration } \\
(\mathrm{m} 3)\end{array}$ \\
\end{tabular} & $\begin{array}{l}\text { Porosity } \\
\left(\mathrm{m}^{3} / \mathrm{m}^{3}\right)\end{array}$ & $\begin{array}{c}\mathrm{Kd} \\
\left(\mathrm{m}^{3} / \mathrm{Kg}\right)\end{array}$ & Retardation & $\left|\begin{array}{c}\text { First Order } \\
\text { Rate } \\
\text { Constant } \\
(1 / y)\end{array}\right|$ & $\begin{array}{c}\text { Longitudinal } \\
\text { Dispersivity } \\
(\mathrm{m})\end{array}$ & $\begin{array}{c}\text { Required } \\
\text { Source } \\
\text { Cleanup } \\
\text { Percentage } \\
(5 \mu g / L \\
\text { Compliance) } \\
\end{array}$ & \begin{tabular}{|c|} 
Required \\
Source \\
Cleanup \\
Percentage \\
(50 gig/L \\
Compliance)
\end{tabular} & \begin{tabular}{|c} 
Required \\
Source \\
Cleanup \\
Percentage \\
(500 ug/L \\
Compliance) \\
\end{tabular} & \begin{tabular}{|c|} 
Abiotic \\
Reaction \\
Half Time \\
(y)
\end{tabular} & $\begin{array}{c}\text { Carbon } \\
\text { Tetrachloride } \\
\text { Travel Time (y) }\end{array}$ \\
\hline & & & & \begin{tabular}{|r|}
20.884307 \\
\end{tabular} & & & \begin{tabular}{|l|}
1829.74 \\
\end{tabular} & & & & & 1462500 & 0.054 & $4.50 \mathrm{E}-04$ & & $1.24 \mathrm{E}-02$ & & 76.06 & & & & \\
\hline & 22.15 & 3000 & 3950 & 4.97586 & 0.103 & 62.1111 & \begin{tabular}{|r|}
3622.54 \\
\end{tabular} & 5125 & 0.1 & 1950 & 225000 & 525000 & 0.1436 & $5.26 \mathrm{E}-04$ & 2.853 & \begin{tabular}{|l|}
$1.23 \mathrm{E}-02$ \\
\end{tabular} & 76.26 & & & & 56.2 & 660 \\
\hline & 30.44 & 3000 & 1800 & \begin{tabular}{|l|l|}
118.468698 \\
\end{tabular} & $\begin{array}{l}.1003 \\
5.471 \\
\end{array}$ & \begin{tabular}{|l|}
137.9366 \\
\end{tabular} & \begin{tabular}{|l|l|}
1631.18 \\
1631
\end{tabular} & 5125 & 0.1 & 1950 & 225000 & 1737500 & $\begin{array}{l}0.1045 \\
0.1045\end{array}$ & $\begin{array}{l}.201-04 \\
2.01 E-04 \\
\end{array}$ & $\begin{array}{l}. .035 \\
1.975 \\
\end{array}$ & \begin{tabular}{|l|}
$\mid 1.05 \mathrm{E}-\mathrm{C}$ \\
$1.04 \mathrm{E}-02$ \\
\end{tabular} & 55.4 & 95.78 & 57.79 & 0 & $\begin{array}{ll}0.2 .7 \\
66.7\end{array}$ & 332.4 \\
\hline & 22.99 & 3000 & 1560 & 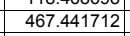 & 26.4715 & \begin{tabular}{|l|}
169.8917 \\
\end{tabular} & 1324.37 & 5125 & 0.1 & 1950 & 225000 & 2375000 & 0.1384 & $\begin{array}{l}2.03 \mathrm{E}-04 \\
\end{array}$ & 1.704 & \begin{tabular}{|l|}
$1.000 \mathrm{E}-03$ \\
\end{tabular} & 35.33 & 98.93 & 89.3 & & 138.6 & 379.8 \\
\hline & 45.25 & 3000 & 2910 & \begin{tabular}{|l|}
60.625998 \\
\end{tabular} & 1.8802 & \begin{tabular}{|r|}
93.0387 \\
\end{tabular} & \begin{tabular}{|l|}
2418.35 \\
\end{tabular} & 5125 & 0.1 & 1950 & 225000 & 962500 & 0.0703 & $4.20 \mathrm{E}-04$ & 4.023 & \begin{tabular}{|l|}
$1.02 \mathrm{E}-02$ \\
\end{tabular} & 63.48 & 91.75 & 17.53 & & 67.8 & 455.6 \\
\hline 6 & 35.04 & 3000 & 2500 & 122.485137 & 6.4911 & \begin{tabular}{|l|l|}
158.9847 \\
\end{tabular} & 1415.23 & 5125 & 0.1 & 1950 & 225000 & 2150000 & 0.0908 & $5.19 \mathrm{E}-04$ & 3.891 & \begin{tabular}{|c|}
$6.07 \mathrm{E}-03$ \\
\end{tabular} & 48.82 & 95.92 & 59.18 & & 114.2 & 569.2 \\
\hline & 59.13 & 3000 & 2120 & 281.623874 & 11.8052 & \begin{tabular}{|l|l|}
125.7554 \\
\end{tabular} & 1789.19 & 5125 & 0.1 & 1950 & 225000 & 1512500 & 0.0538 & $2.52 \mathrm{E}-04$ & 3.363 & $8.53 \mathrm{E}-03$ & 33 & 98.22 & 82.25 & & 81.2 & 291.5 \\
\hline & 49.32 & 3000 & 3320 & 26.762314 & 0.9814 & \begin{tabular}{|l|l|}
110.0086 \\
\end{tabular} & 2045.3 & 5125 & 0.1 & & 225000 & 1237500 & 0.0645 & $5.92 \mathrm{E}-04$ & 5.636 & \begin{tabular}{|l|} 
\\
\end{tabular} & & 81.32 & & & 71.2 & 585.6 \\
\hline & 26.53 & 3000 & 2330 & $\begin{array}{l}33.742146 \\
\end{array}$ & 1.3031 & \begin{tabular}{|l|l|}
115.8579 \\
\end{tabular} & 1942.03 & 5125 & 0.1 & 1950 & 225000 & 1337500 & 0.1199 & \begin{tabular}{|c|}
$3.81 \mathrm{E}-04$ \\
\end{tabular} & 2.608 & $1.02 \mathrm{E}-02$ & $\begin{array}{ll}62.53 \\
\end{array}$ & 85.18 & & & 68.1 & 503.7 \\
\hline 10 & 29.57 & 3000 & 3030 & 435.119264 & 15.6316 & \begin{tabular}{|l|l|}
107.7748 \\
\end{tabular} & \begin{tabular}{|l|}
2087.69 \\
\end{tabular} & 5125 & 0.1 & 1950 & 225000 & 1200000 & 0.1076 & $5.07 \mathrm{E}-04$ & 3.384 & \begin{tabular}{|l|}
$3.45 \mathrm{E}-03$ \\
\end{tabular} & 39.84 & 98.85 & 88.51 & & 201.2 & 586.6 \\
\hline$\frac{11}{12}$ & 28.46 & 3000 & 2210 & 235.920715 & 11.7147 & \begin{tabular}{|l|l|}
148.9657 \\
7
\end{tabular} & \begin{tabular}{|l|}
1510.42 \\
\end{tabular} & 5125 & 0.1 & $\begin{array}{l}1950 \\
1950\end{array}$ & 225000 & $\begin{array}{l}1950000 \\
\end{array}$ & 0.1118 & 3.95E-04 & 2.788 & \begin{tabular}{|l|}
$5.27 \mathrm{E}-03$ \\
\end{tabular} & 29.73 & 97.88 & & & 131.5 & 502.1 \\
\hline$\frac{12}{13}$ & 21.41 & 3000 & \begin{tabular}{ll|l}
1390 & \\
3300
\end{tabular} & \begin{tabular}{|r|}
185.517538 \\
582751
\end{tabular} & $\begin{array}{l}4.6529 \\
\end{array}$ & \begin{tabular}{|r|}
75.2423 \\
1441775 \\
\end{tabular} & \begin{tabular}{|l|}
2990.34 \\
15736
\end{tabular} & 5125 & 0.1 & $\begin{array}{l}1950 \\
1950\end{array}$ & 225000 & 700000 & 0.1486 & $\begin{array}{l}1.17 \mathrm{E}-04 \\
\end{array}$ & 1.399 & \begin{tabular}{|l|}
$8.58 \mathrm{E}-03$ \\
\end{tabular} & 42.84 & 97.3 & 73.05 & & $\begin{array}{l}80.8 \\
\end{array}$ & 334.8 \\
\hline$\frac{13}{14}$ & $\begin{array}{r}62.5 \\
28.87 \\
\end{array}$ & 3000 & \begin{tabular}{r|r|r|}
3200 \\
3390
\end{tabular} & $\begin{array}{r}5.822751 \\
1975743 \\
\end{array}$ & $\begin{array}{r}0.2804 \\
0.7334 \\
\end{array}$ & \begin{tabular}{|c|}
$\mid 144.4754$ \\
110781 \\
\end{tabular} & \begin{tabular}{|r|}
1557.36 \\
2031.64 \\
\end{tabular} & $\begin{array}{l}5125 \\
525 \\
\end{array}$ & 0.1 & $\begin{array}{r}1950 \\
1950 \\
1950\end{array}$ & $\begin{array}{r}225000 \\
225000\end{array}$ & \begin{tabular}{|l|}
1862500 \\
125000
\end{tabular} & 0.0509 & 6.74E-04 & 7.691 & \begin{tabular}{|l|}
$1.36 \mathrm{E}-02$ \\
$791 E-03$
\end{tabular} & \begin{tabular}{|c|c|}
49.38 \\
3.57
\end{tabular} & $\begin{array}{l}14.13 \\
7473\end{array}$ & & 0 & $\begin{array}{l}50.8 \\
877\end{array}$ & $\begin{array}{ll}630.6 \\
7149\end{array}$ \\
\hline 15 & 20.01 & 3000 & $\begin{array}{l}3390 \\
2740\end{array}$ & $\begin{array}{l}19.180543 \\
28.562124 \\
\end{array}$ & $\begin{array}{l}0.13344 \\
1.1304\end{array}$ & \begin{tabular}{|l|}
111.0 .481 \\
118.7276 \\
\end{tabular} & \begin{tabular}{|l|}
2031.64 \\
1895.09 \\
\end{tabular} & $\mid \begin{array}{l}5125 \\
5125\end{array}$ & $\begin{array}{l}0.1 \\
0.1\end{array}$ & $\begin{array}{l}1950 \\
1950\end{array}$ & $\begin{array}{l}225000 \\
225000\end{array}$ & $\begin{array}{l}1258000 \\
138500\end{array}$ & $\begin{array}{l}0.1102 \\
0.0776 \\
\end{array}$ & \begin{tabular}{|l|}
$.600-04$ \\
$4.97 \mathrm{E}-04$
\end{tabular} & $\frac{4.021}{4.236}$ & \begin{tabular}{|l|}
$1.91 \mathrm{E}-03$ \\
$1.04 \mathrm{E}-02$ \\
\end{tabular} & $\begin{array}{c}33.57 \\
47.28\end{array}$ & $\begin{array}{l}4.73 \\
82.49\end{array}$ & & & \begin{tabular}{rl|}
88.1 \\
67 \\
\end{tabular} & $\begin{array}{l}714.9 \\
529.6\end{array}$ \\
\hline 16 & 37.83 & 3000 & 2050 & \begin{tabular}{|l|}
1286.001247 \\
\end{tabular} & 49.9734 & \begin{tabular}{|l|}
116.5786 \\
\end{tabular} & \begin{tabular}{|l|}
1930.03 \\
\end{tabular} & 5125 & 0.1 & 1950 & 225000 & 1350000 & 0.0841 & $1.65 \mathrm{E}-04$ & 1.989 & \begin{tabular}{|l|}
$3.20 \mathrm{E}-03$ \\
\end{tabular} & 55.5 & 99.61 & 96.11 & 61.12 & 216.6 & 269.5 \\
\hline 17 & 59.24 & 3000 & 1730 & \begin{tabular}{|l|}
716.319339 \\
\end{tabular} & 34.651 & \begin{tabular}{|l|}
145.1211 \\
\end{tabular} & 1550.43 & 5125 & 0.1 & 1950 & 225000 & 1875000 & 0.0537 & $1.80 \mathrm{E}-04$ & 2.697 & \begin{tabular}{|l|}
$6.33 \mathrm{E}-03$ \\
\end{tabular} & 41.22 & 99.3 & 93.02 & 30.2 & 109.5 & 233.3 \\
\hline 18 & 52.5 & 3000 & 2060 & 201.416671 & 7.9712 & \begin{tabular}{|l|l|}
118.7276 \\
\end{tabular} & 1895.09 & 5125 & 0.1 & 1950 & 225000 & 1387500 & 0.0606 & $1.94 \mathrm{E}-04$ & 2.617 & \begin{tabular}{|l|}
$1.12 \mathrm{E}-02$ \\
\end{tabular} & 39.63 & 97.52 & 75.18 & & 62.2 & 255.5 \\
\hline 19 & 29.59 & 3000 & 650 & 436.709687 & 19.8863 & \begin{tabular}{|l|}
136.6103 \\
\end{tabular} & 1647.02 & 5125 & 0.1 & 1950 & 225000 & 1712500 & 0.1075 & $3.26 \mathrm{E}-05$ & 1.153 & $9.82 \mathrm{E}-03$ & 41.91 & 98.86 & 88.55 & & 70.6 & 199.7 \\
\hline 20 & 21.6 & 3000 & 1530 & 14.644185 & 0.9117 & \begin{tabular}{|l|l|}
186.7666 \\
\end{tabular} & 1204.71 & 5125 & 0.1 & 1950 & 225000 & 2737500 & 0.1473 & $2.62 \mathrm{E}-04$ & 1.901 & \begin{tabular}{|c|}
$1.28 \mathrm{E}-02$ \\
\end{tabular} & 42.14 & 65.86 & 0 & & 54.2 & 451 \\
\hline 21 & 31.69 & 3000 & 2770 & 49.577248 & 2.6679 & \begin{tabular}{|l|l|}
161.4401 \\
\end{tabular} & 1393.71 & 5125 & 0.1 & 1950 & 225000 & 2200000 & 0.1004 & 5.94E-04 & 3.993 & 6.88E-03 & 37.29 & 89.91 & & & 100.7 & 645.8 \\
\hline 22 & 26.1 & 3000 & 2500 & \begin{tabular}{|c|}
17.675777 \\
\end{tabular} & 0.6172 & \begin{tabular}{|l|l|}
104.7599 \\
\end{tabular} & 2147.77 & 5125 & 0.1 & 1950 & 225000 & 1150000 & & $3.26 \mathrm{E}-04$ & 2.352 & $1.18 \mathrm{E}-02$ & 27.73 & 71.71 & 0 & & 58.5 & 461.8 \\
\hline 23 & 22.18 & 3000 & 1640 & 89.296983 & 4.6586 & \begin{tabular}{|l|l|}
156.5102 \\
\end{tabular} & 1437.61 & 5125 & 0.1 & 1950 & 225000 & 2100000 & 0.1434 & 1.94E-04 & 1.682 & $9.48 \mathrm{E}-03$ & 42.19 & 94.4 & 44.01 & & 73.1 & 388.6 \\
\hline 24 & 28.87 & 3000 & 2690 & 213.361054 & 8.5954 & \begin{tabular}{|l|l|}
120.8573 \\
\end{tabular} & \begin{tabular}{|l|}
1861.7 \\
\end{tabular} & 5125 & 0.1 & 1950 & 225000 & 1425000 & 0.1102 & 4.92E-04 & 3.258 & $4.93 \mathrm{E}-03$ & $\begin{array}{l}49.66 \\
\end{array}$ & 97.66 & 76.57 & & 140.7 & 578.4 \\
\hline$\frac{25}{26}$ & 29 & 3000 & 2910 & \begin{tabular}{|l|}
329.500563 \\
32216256
\end{tabular} & $\begin{array}{l}13.5061 \\
107552\end{array}$ & \begin{tabular}{|l|l|}
122.9684 \\
\end{tabular} & \begin{tabular}{|l|}
1829.74 \\
\end{tabular} & 5125 & 0.1 & $\begin{array}{l}1950 \\
1050 \\
1050\end{array}$ & 225000 & 1462500 & 0.1097 & 5.47E-04 & 3.519 & \begin{tabular}{|c|}
$3.73 \mathrm{E}-03$ \\
\end{tabular} & 46.41 & 98.48 & 84.83 & & 185.8 & 622 \\
\hline 26 & 22.76 & 3000 & 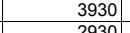 & $\begin{array}{r}322.163526 \\
7417946\end{array}$ & $\begin{array}{r}10.7553 \\
23203 \\
32\end{array}$ & \begin{tabular}{|l|l|}
100.1541 \\
\end{tabular} & \begin{tabular}{|l|}
2246.54 \\
\end{tabular} & 5125 & 0.1 & $\begin{array}{l}1950 \\
1950\end{array}$ & 225000 & 1075000 & 0.1398 & 7.66E-04 & 3.771 & \begin{tabular}{|l|}
$2.71 E-03$ \\
\end{tabular} & 45.07 & 98.45 & 84.48 & & 256.3 & 849.3 \\
\hline$\frac{27}{28}$ & $\begin{array}{l}\frac{43.28}{21.94} \\
-\end{array}$ & 3000 & $\begin{array}{l}2930 \\
1130\end{array}$ & $\begin{array}{r}74.174946 \\
953233404\end{array}$ & $\begin{array}{l}2.3203 \\
5.7835 \\
\end{array}$ & \begin{tabular}{|r|}
93.8425 \\
182.1901 \\
\end{tabular} & \begin{tabular}{|l|}
2397.63 \\
1234.97
\end{tabular} & $\begin{array}{l}5125 \\
5125 \\
\end{array}$ & 0.1 & $\begin{array}{l}1950 \\
1950 \\
\end{array}$ & $\begin{array}{l}225000 \\
225000\end{array}$ & $\begin{array}{r}975000 \\
263500\end{array}$ & $\begin{array}{l}0.0735 \\
\end{array}$ & $\begin{array}{l}3.68 \mathrm{E}-04 \\
104 \mathrm{~F}-04\end{array}$ & $\begin{array}{r}3.53 \\
1361\end{array}$ & \begin{tabular}{|l|}
$9.45 E-03$ \\
$112 E-02$
\end{tabular} & $\begin{array}{l}26.64 \\
34.57 \\
\end{array}$ & $\begin{array}{l}93.26 \\
9475\end{array}$ & $\begin{array}{r}32.59 \\
47.5 \\
\end{array}$ & $\frac{0}{0}$ & $\begin{array}{r}73.3 \\
619 \\
\end{array}$ & $\begin{array}{l}417.9 \\
3179\end{array}$ \\
\hline 29 & $\frac{41.94}{45.38}$ & 3000 & 1480 & 838.084464 & 36.0925 & \begin{tabular}{|c|}
18.12901 \\
129.1963 \\
\end{tabular} & \begin{tabular}{|l|}
1734.97 \\
1741.54 \\
\end{tabular} & 5125 & 0.1 & 1950 & 2250000 & $\begin{array}{l}2635000 \\
1575000\end{array}$ & $\begin{array}{l}0.145 \\
0.0701 \\
\end{array}$ & $\begin{array}{r}1.04 \mathrm{E}-04 \\
1.10 \mathrm{E}-04\end{array}$ & $\begin{array}{l}1.361 \\
1.794\end{array}$ & \begin{tabular}{|l|}
$1.12 \mathrm{E}-02$ \\
$6.46 \mathrm{E}-03$ \\
\end{tabular} & $\begin{array}{l}34.57 \\
58.83 \\
\end{array}$ & $\begin{array}{r}49.15 \\
99.4 \\
\end{array}$ & \begin{tabular}{r|r}
47.5 \\
94.03
\end{tabular} & $\begin{array}{r}0 \\
40.34\end{array}$ & $\begin{array}{r}61.9 \\
107.3 \\
\end{array}$ & $\begin{array}{l}3202.6 \\
202.6\end{array}$ \\
\hline 30 & 34.81 & 3000 & 2210 & 39.563262 & 1.4015 & \begin{tabular}{|l|}
106.2727 \\
\end{tabular} & \begin{tabular}{|l|}
2117.2 \\
\end{tabular} & 5125 & 0.1 & 1950 & 225000 & 1175000 & 0.0914 & $1.87 \mathrm{E}-04$ & 2.034 & \begin{tabular}{|l|} 
\\
$1.66 \mathrm{E}-02$ \\
\end{tabular} & 85.44 & 87.36 & & & 41.8 & 292.4 \\
\hline 31 & 25.11 & 3000 & 2730 & 145.565834 & 4.5534 & \begin{tabular}{|r|}
93.8425 \\
\end{tabular} & \begin{tabular}{|l|}
2397.63 \\
\end{tabular} & 5125 & 0.1 & 1950 & 225000 & 975000 & 0.1267 & $3.59 \mathrm{E}-04$ & 2.433 & \begin{tabular}{|l|} 
\\
\end{tabular} & 67.25 & 96.57 & 65.65 & & 103.8 & 496.7 \\
\hline 32 & 21.44 & 3000 & 2040 & 5.724448 & 0.2543 & \begin{tabular}{|l|}
133.2659 \\
\end{tabular} & \begin{tabular}{|l|}
1688.35 \\
\end{tabular} & 5125 & 0.1 & 1950 & 225000 & 1650000 & 0.1484 & $3.31 \mathrm{E}-04$ & 2.126 & $1.37 \mathrm{E}-02$ & 42.06 & 12.66 & & & 50.7 & 508.2 \\
\hline 33 & 49.71 & 3000 & 1360 & 458.422988 & 19.4276 & \begin{tabular}{|l|l|}
127.1374 \\
\end{tabular} & \begin{tabular}{|l|}
1769.74 \\
\end{tabular} & 5125 & 0.1 & 1950 & 225000 & 1537500 & 0.064 & $1.28 \mathrm{E}-04$ & 2.015 & $9.28 \mathrm{E}-03$ & 34.79 & 98.91 & 89.09 & & 74.7 & \\
\hline 34 & 31.72 & 3000 & 1850 & 142.402068 & 7.1012 & \begin{tabular}{|l|l|}
149.6016 \\
\end{tabular} & \begin{tabular}{|l|}
1503.99 \\
\end{tabular} & 5125 & 0.1 & 1950 & 225000 & 1962500 & 0.1003 & $2.73 \mathrm{E}-04$ & 2.374 & $8.34 \mathrm{E}-03$ & 35.37 & 96.49 & 64.89 & & 83.1 & 383.5 \\
\hline & 32.56 & 3000 & 2640 & 33.247014 & 1.4843 & \begin{tabular}{|l|l|}
133.9381 \\
\end{tabular} & \begin{tabular}{|l|}
1679.88 \\
\end{tabular} & 5125 & 0.1 & & 225000 & & 0.0977 & & 3.649 & & 36.93 & 84.96 & 0 & & 79.4 & 574.3 \\
\hline 36 & 46.92 & 3000 & 3100 & & 9.6325 & & 1705 & 5125 & 0.1 & & & & 0.0678 & 6.32E-04 & 5.714 & & 83.15 & & 77.18 & & 156.6 & 624 \\
\hline 37 & 55.81 & 3000 & $\begin{array}{ll}620 \\
\end{array}$ & \begin{tabular}{|l|}
863.706063 \\
\end{tabular} & 33.5632 & \begin{tabular}{|l|l|}
116.5786 \\
\end{tabular} & \begin{tabular}{|l|}
1930.03 \\
\end{tabular} & 5125 & 0.1 & 1950 & 225000 & 1350000 & 0.057 & 6.59E-05 & 1.584 & \begin{tabular}{|l|}
$8.64 \mathrm{E}-03$ \\
\end{tabular} & 26.14 & 99.42 & 94.21 & 42.11 & 80.2 & 145.5 \\
\hline 38 & 29.79 & 3000 & 1920 & 532.699315 & $\begin{array}{l}17.368 \\
10.718\end{array}$ & \begin{tabular}{|l|l|}
97.8112 \\
\end{tabular} & \begin{tabular}{|l|}
2300.35 \\
\end{tabular} & 5125 & 0.1 & 1950 & 225000 & 1037500 & & $1.81 \mathrm{E}-04$ & & \begin{tabular}{|l|}
$5.53 \mathrm{E}-03$ \\
\end{tabular} & 36.63 & & 90.61 & 6.14 & 125.3 & 319.7 \\
\hline 39 & $\frac{24.51}{230}$ & 3000 & $\begin{array}{l}2350 \\
2350 \\
2350\end{array}$ & $\begin{array}{r}6.542794 \\
13503235 \\
\end{array}$ & \begin{tabular}{|l|l|}
0.2447 \\
3968
\end{tabular} & \begin{tabular}{|c|}
112.2199 \\
88.145 \\
\end{tabular} & \begin{tabular}{|l|}
2004.99 \\
25274 \\
\end{tabular} & $\begin{array}{l}5125 \\
5125 \\
\end{array}$ & 0.1 & $\begin{array}{l}1950 \\
1950\end{array}$ & 225000 & 1275000 & 0.1298 & $3.96 \mathrm{E}-04$ & 2.543 & & 67.64 & 23.58 & 0 & & 49.9 & 531.7 \\
\hline$\frac{40}{41}$ & $\begin{array}{r}23.9 \\
31.91\end{array}$ & $\begin{array}{l}3000 \\
3000\end{array}$ & $\begin{array}{l}2350 \\
2640\end{array}$ & $\begin{array}{l}135.0633555 \\
107.058862\end{array}$ & $\begin{array}{l}3.9682 \\
3.2625 \\
\end{array}$ & \begin{tabular}{|l|}
88.1405 \\
91.206 \\
\end{tabular} & \begin{tabular}{|r|}
2552.74 \\
2461.15 \\
\end{tabular} & $\begin{array}{l}5125 \\
5125\end{array}$ & $\frac{0.1}{0.1}$ & $\begin{array}{l}\frac{1950}{1950} \\
\end{array}$ & $\frac{225000}{225000}$ & $\begin{array}{r}887500 \\
937500\end{array}$ & $\begin{array}{l}0.1331 \\
0.0997 \\
\end{array}$ & \begin{tabular}{|l|}
$2.04 E-04$ \\
$2.81-04$
\end{tabular} & $\frac{1.775}{2.424}$ & \begin{tabular}{|l|}
$8.60 \mathrm{E}-03$ \\
$9.68 \mathrm{E}-03$ \\
\end{tabular} & \begin{tabular}{|l|l|}
52.06 & 83.34 \\
\end{tabular} & \begin{tabular}{|r|}
96.3 \\
95.33
\end{tabular} & $\begin{aligned} 62.98 \\
53.3 \\
\end{aligned}$ & & \begin{tabular}{l|l|l}
80.6 & \\
71.6 &
\end{tabular} & $\begin{array}{l}380.6 \\
3894 \\
\end{array}$ \\
\hline 42 & 25.8 & & 2280 & $\begin{array}{l}196.751746 \\
\end{array}$ & $\begin{array}{l}.20898 \\
9.089\end{array}$ & \begin{tabular}{|r|}
138.5974 \\
\end{tabular} & \begin{tabular}{|l|}
1623.41 \\
\end{tabular} & 5125 & 0.1 & & 225000 & 1750000 & & $3.92 \mathrm{E}$ & 2.607 & \begin{tabular}{|l|}
$5.43 \mathrm{E}-03$ \\
\end{tabular} & 23.41 & 97.46 & 74.59 & & 127.6 & 309.4 \\
\hline 43 & 31.53 & 3000 & 1780 & 148.531 & 5.7004 & \begin{tabular}{|l|l|}
115.1349 \\
\end{tabular} & 1954 & 5125 & 0.1 & & 225000 & 1325000 & & $1.83 \mathrm{E}-04$ & 1.915 & $1.00 \mathrm{E}-02$ & 31.71 & 96.63 & 66.34 & & 69.3 & 311.2 \\
\hline 44 & 22.5 & 3000 & 1910 & 63.085456 & 2.6152 & \begin{tabular}{|l|}
124.3658 \\
\end{tabular} & \begin{tabular}{|l|}
1809.18 \\
\end{tabular} & 5125 & 0.1 & 1950 & 225000 & 1487500 & 0.1414 & $1.59 \mathrm{E}-04$ & 1.569 & \begin{tabular}{|l|}
$\mid 1.19 \mathrm{E}-02$ \\
\end{tabular} & 87.64 & 92.07 & 20.74 & & 58.1 & 357.5 \\
\hline 45 & 31.94 & & 870 & 314.2216 & 12.9531 & \begin{tabular}{|l|}
123.6681 \\
\end{tabular} & \begin{tabular}{|l|l|}
1819 \\
\end{tabular} & 5125 & 0.1 & 1950 & & 1475000 & 0.0996 & $5.65 \mathrm{E}-05$ & 1.287 & & 53.8 & 98.41 & 84.09 & & 61.6 & 206.5 \\
\hline 46 & 21.97 & 3000 & 2850 & 148.35 & 4.4402 & \begin{tabular}{|l|}
89.788 \\
\end{tabular} & & 5125 & 0.1 & 1950 & & 912500 & 0.1448 & 3.60 & 2.256 & & 53.24 & 96. & 66.3 & & 113.3 & 526.3 \\
\hline 47 & 24.28 & 3000 & 2360 & & 0.6888 & \begin{tabular}{|l|l|}
112.2199 \\
\end{tabular} & 2004 & 5125 & 0.1 & 1950 & 225000 & 1275000 & 0.131 & & 2.315 & & 39.6 & 72.85 & & & 60.9 & 488.5 \\
\hline 48 & 26.73 & 3000 & 2610 & 79.582736 & 3.0543 & \begin{tabular}{|l|l|}
115.1349 \\
\end{tabular} & 195 & 5125 & 0.1 & 1950 & 2250 & & 0.119 & & 2.88 & & 37.04 & 93.72 & 37.17 & & 98 & 552.1 \\
\hline 49 & & 3000 & 2870 & 183.1 & 8.0546 & & 170 & & 0.1 & & & & & & 4.3 & & & & 72.7 & & 122.6 & \\
\hline 50 & 40. & 3000 & 1850 & 180.1 & 9.09 & & & 5125 & 0 & 19 & 225 & & & 2.90 & 2.883 & & & 97.22 & 72.24 & & 81.9 & 361.3 \\
\hline 51 & 33.99 & 3000 & 2120 & \begin{tabular}{|c|}
92.601197 \\
\end{tabular} & 5.8524 & \begin{tabular}{|l|l|}
189.5986 \\
\end{tabular} & 1186. & 5125 & 0.1 & 1950 & 225000 & 2800000 & $\begin{array}{l}0.0936 \\
\end{array}$ & $5.16 \mathrm{E}$ & 3.784 & \begin{tabular}{|c|}
$6.84 \mathrm{E}-03$ \\
\end{tabular} & 74. & & 46.01 & & 101.3 & 570.6 \\
\hline 52 & $\begin{array}{r}47.2 \\
55.11 \\
\end{array}$ & & 2030 & & $\begin{array}{ll}12.8029 \\
\end{array}$ & & 1714. & 5125 & & & & & & $2.64 \mathrm{E}$ & & 7.67 & 49.1 & & & & 90.3 & 323.5 \\
\hline$\frac{53}{54}$ & $\frac{55.14}{2222}$ & $\begin{array}{l}3000 \\
3300 \\
3\end{array}$ & $\begin{array}{l}1850 \\
2010 \\
\end{array}$ & $\begin{array}{l}126.321225 \\
140635971\end{array}$ & \begin{tabular}{|l|}
4.9993 \\
5.9601 \\
\end{tabular} & \begin{tabular}{|l|l|}
118.7276 \\
1271271 \\
\end{tabular} & \begin{tabular}{|l|}
1895.09 \\
170.7 \\
\end{tabular} & $\frac{5125}{5125}$ & 0.1 & $\begin{array}{l}1950 \\
1950 \\
1950\end{array}$ & 225000 & 13875 & 0.05 & $1.30 \mathrm{E}-04$ & 2.141 & \begin{tabular}{|c|}
$1.75 E-02$ \\
\end{tabular} & 73.5 & 96.04 & 60.42 & & 39.7 & 199 \\
\hline $\begin{array}{r}54 \\
55\end{array}$ & 22.22 & 3000 & \begin{tabular}{|l|l|}
2010 \\
2490
\end{tabular} & \begin{tabular}{|c|c|}
140.636971 \\
691138354
\end{tabular} & $\begin{array}{l}5.9601 \\
2451 \\
\end{array}$ & \begin{tabular}{|l|l|}
127.1374 \\
1062077 \\
\end{tabular} & \begin{tabular}{|l|}
1769.74 \\
$217 ?$ \\
\end{tabular} & $\frac{5125}{5125}$ & 0.1 & $\frac{1950}{1950}$ & 225000 & $\frac{15375}{11750}$ & $\begin{array}{l}0.1432 \\
0.127\end{array}$ & $2.26 \mathrm{E}-04$ & 1.797 & $7.61 \mathrm{E}-03$ & 28.5 & 96.44 & $\begin{array}{l}64.45 \\
2771\end{array}$ & & 91.1 & 414.5 \\
\hline$\frac{55}{56}$ & $\frac{25.72}{48.57}$ & $\begin{array}{l}3000 \\
3000\end{array}$ & $2490 \mid$ & $\begin{array}{r}69.11638354 \\
38.49764\end{array}$ & $\begin{array}{l}2.4501 \\
1.9522 \\
\end{array}$ & \begin{tabular}{|l|}
106.2727 \\
152.1319 \\
\end{tabular} & \begin{tabular}{|l|}
$\mid 21117.2$ \\
1478.98 \\
\end{tabular} & $\begin{array}{l}55125 \\
5125\end{array}$ & $\frac{0.1}{0.1}$ & $\frac{1950}{1950}$ & $\frac{225000}{225000}$ & $\begin{array}{l}\frac{1175000}{2012500} \\
\end{array}$ & \begin{tabular}{|l|}
0.1237 \\
0.0655
\end{tabular} & $\frac{3.42 \mathrm{E}-04}{4.11-04}$ & $\begin{array}{l}2.397 \\
4.175\end{array}$ & $\mid$\begin{tabular}{|l}
$\mid 8.43 \mathrm{E}-03$ \\
$1.11 \mathrm{E}-02$
\end{tabular} & $\begin{array}{l}38.94 \\
35.94\end{array}$ & $\begin{array}{l}92.77 \\
8.01\end{array}$ & $\begin{aligned} 27.71 \\
0\end{aligned}$ & & $\frac{82.2}{62.5}$ & $\begin{array}{r}447.7 \\
440.5 \\
\end{array}$ \\
\hline 57 & 45. & 3000 & 1480 & 735.333512 & 43.2345 & \begin{tabular}{|l|}
176.3872 \\
\end{tabular} & \begin{tabular}{|l|}
1275.6 \\
\end{tabular} & 5125 & 0.1 & 1950 & 225000 & 2512500 & 0.0698 & $1.97 \mathrm{E}-04$ & 2.423 & $5.44 \mathrm{E}$ & 81.1 & 99.32 & 93.2 & 32 & 127.5 & 272.5 \\
\hline 58 & 30.04 & 3000 & 3440 & 91.95 & 2.85 & 93.0 & 2418 & 5125 & 0.1 & 1950 & 225 & & 0.1 & $6.38 \mathrm{E}-04$ & 4.045 & \begin{tabular}{|l|l|}
5.87 \\
\end{tabular} & 62.83 & 94.5 & 45.66 & & 118.1 & 690.1 \\
\hline 59 & 23.24 & & 1850 & 166.9 & 8.3. & \begin{tabular}{|l|l|}
150.8 \\
\end{tabular} & 1491 & 512 & 0.1 & & & & & 3.10 & 2.144 & & 68.11 & 97.01 & 70.06 & & 104.8 & 472.8 \\
\hline 60 & 33 & 3000 & 1470 & & & & 1288 & 5125 & 0.1 & 195 & & & & 2.11 & 2.1 & & & 95.97 & 59.67 & & 63.2 & \\
\hline 61 & 27.69 & 3000 & 3100 & 4.59 & & 77. & & 5125 & 0.1 & 1950 & 225000 & 725000 & & & 2.4 & & 65.36 & & 0 & & 40.2 & 451.8 \\
\hline 62 & 45.71 & & 3730 & & 1.44 & 131 & 1714 & & 0 & & & & & & & & & 84.84 & & & 92.9 & \\
\hline 63 & & 30 & $\begin{array}{l}1650 \\
1240\end{array}$ & \begin{tabular}{|r|}
550.490349 \\
324364
\end{tabular} & $\begin{array}{l}23.5815 \\
24547 \\
\end{array}$ & \begin{tabular}{|l|l|}
128.5118 \\
2267779
\end{tabular} & \begin{tabular}{|l|}
1750.81 \\
9922 \\
\end{tabular} & 5125 & 0.1 & & & $\begin{array}{l}1562500 \\
3662500\end{array}$ & & $\begin{array}{l}1.46 \mathrm{E}-04 \\
107 \mathrm{~F}-04\end{array}$ & 2.457 & \begin{tabular}{|l|}
$8.69 \mathrm{E}-03$ \\
$123 \mathrm{E}-02$
\end{tabular} & & & 90.92 & 9.17 & $\begin{array}{l}79.8 \\
56.6\end{array}$ & \\
\hline 64 & $\frac{21.52}{24.66}$ & 3000 & $\begin{array}{r}1240 \\
2520\end{array}$ & \begin{tabular}{|r|}
32.47364 \\
127.540191
\end{tabular} & \begin{tabular}{|l|}
2.4547 \\
47083 \\
\end{tabular} & \begin{tabular}{|l|}
226.7679 \\
1107481 \\
\end{tabular} & \begin{tabular}{|r|}
992.2 \\
2031.64
\end{tabular} & \begin{tabular}{|l|r|}
5125 \\
5125 \\
\end{tabular} & 0.1 & $\begin{array}{l}1950 \\
1950\end{array}$ & $\begin{array}{l}225000 \\
225000\end{array}$ & $\begin{array}{l}3662500 \\
1250000\end{array}$ & \begin{tabular}{|c|c|}
0.1478 \\
0.129
\end{tabular} & $\begin{array}{l}1.97 \mathrm{E}-04 \\
3.99-04\end{array}$ & $\begin{array}{l}1.673 \\
2562\end{array}$ & \begin{tabular}{|l|}
$1.23 \mathrm{E}-02$ \\
$6.42 \mathrm{E}-03$
\end{tabular} & $\begin{array}{l}52.78 \\
52.21\end{array}$ & \begin{tabular}{r|}
84.6 \\
96.08
\end{tabular} & \begin{tabular}{r|r}
0 \\
60.8 \\
\end{tabular} & & $\begin{array}{c}56.6 \\
108 \\
\end{array}$ & $\begin{array}{r}398.3 \\
52.4 \\
\end{array}$ \\
\hline
\end{tabular}




\begin{tabular}{|c|c|c|c|c|c|c|c|c|c|c|c|c|c|c|c|c|c|c|c|c|c|c|}
\hline $\begin{array}{c}\text { Realizatio } \\
n\end{array}$ & $\begin{array}{c}\text { Velocity } \\
(\mathrm{m} / \mathrm{y})\end{array}$ & $\begin{array}{c}\text { Fixed Source } \\
\text { Concentration } \\
\text { (mg/L) }\end{array}$ & $\begin{array}{c}\text { Time of } \\
\text { Maximum at } \\
\text { Compliance } \\
\text { Boundary } \\
(y)\end{array}$ & \begin{tabular}{|c|} 
Maximum \\
Concentration \\
Compliance \\
Boundary \\
$(\mu \mathrm{g} / \mathrm{L})$
\end{tabular} & \begin{tabular}{|c|} 
Maximum \\
Release Rate \\
at Compliance \\
Boundary \\
$(\mathrm{Kg} / \mathrm{y})$
\end{tabular} & \begin{tabular}{|c|} 
Source \\
Rate \\
$(\mathrm{Kg} / \mathrm{y})$ \\
\end{tabular} & \begin{tabular}{|c|} 
Source \\
Duration \\
(y)
\end{tabular} & \begin{tabular}{|c|} 
1-D \\
Streamtub \\
e Length \\
(m)
\end{tabular} & $\begin{array}{l}\text { Base } \\
\text { Porosity } \\
\left(\mathrm{m}^{3} / \mathrm{m}^{3}\right)\end{array}$ & $\begin{array}{c}\text { Bulk } \\
\text { Densty } \\
\left(\mathrm{Kg} / \mathrm{m}^{3}\right)\end{array}$ & $\begin{array}{c}\text { Remaining } \\
\text { Carbon } \\
\text { Tetrachloride } \\
\text { Source } \\
(\mathrm{Kg})\end{array}$ & $\begin{array}{c}\text { Volume at or } \\
\text { above Fixed } \\
\text { Source } \\
\begin{array}{c}\text { Concentration } \\
(\mathrm{m} 3)\end{array} \\
\end{array}$ & $\begin{array}{l}\text { Porosity } \\
\left(\mathrm{m}^{3} / \mathrm{m}^{3}\right)\end{array}$ & $\begin{array}{c}\mathrm{Kd} \\
\left(\mathrm{m}^{3} / \mathrm{Kg}\right)\end{array}$ & Retardation & $\begin{array}{c}\text { First Order } \\
\text { Rate } \\
\text { Constant } \\
(1 / y)\end{array}$ & $\begin{array}{c}\text { Longitudinal } \\
\text { Dispersivity } \\
(\mathrm{m})\end{array}$ & $\begin{array}{c}\text { Required } \\
\text { Source } \\
\text { Cleanup } \\
\text { Percentage } \\
(5 \mu g / L \\
\text { Compliance) } \\
\end{array}$ & \begin{tabular}{|c|} 
Required \\
Source \\
Cleanup \\
Percentage \\
(50 gig/L \\
Compliance)
\end{tabular} & \begin{tabular}{|c} 
Required \\
Source \\
Cleanup \\
Percentage \\
(500 ug/L \\
Compliance) \\
\end{tabular} & \begin{tabular}{|c|} 
Abiotic \\
Reaction \\
Half Time \\
(y)
\end{tabular} & $\begin{array}{c}\text { Carbon } \\
\text { Tetrachloride } \\
\text { Travel Time (y) }\end{array}$ \\
\hline & 46.17 & $\begin{array}{r}3000 \\
300\end{array}$ & & \begin{tabular}{|l|}
270.005206 \\
\end{tabular} & & \begin{tabular}{|l|}
162.6609 \\
\end{tabular} & 1383.25 & & & 1950 & & 2225000 & & & & $\mid 1.62 \mathrm{E}-02$ & \begin{tabular}{|l|}
72.97 \\
\end{tabular} & 98.15 & \begin{tabular}{|r|}
81.48 \\
\end{tabular} & & $\begin{array}{r}19.8 \\
42.8 \\
\end{array}$ & \\
\hline 67 & 49.79 & 3000 & 2650 & 232.270419 & 7.8143 & \begin{tabular}{|l|}
100.929 \\
\end{tabular} & 2229.29 & 5125 & 0.1 & 1950 & 225000 & 1087500 & 0.0639 & $2.99 \mathrm{E}-04$ & 3.368 & $7.65 \mathrm{E}-03$ & 21.89 & 97.85 & 78.47 & & 90.6 & 346.7 \\
\hline 68 & 26.34 & 3000 & 2590 & 12.855927 & 0.8858 & \begin{tabular}{|l|}
206.7108 \\
\end{tabular} & 1088.48 & 5125 & 0.1 & 1950 & 225000 & 3187500 & 0.1208 & $6.97 \mathrm{E}-04$ & 3.918 & $8.11 \mathrm{E}-03$ & 50.27 & 61.11 & 0 & & 85.5 & 762.5 \\
\hline 69 & 41 & 3000 & 1750 & 102.490738 & 4.6898 & \begin{tabular}{|l|l|}
137.2743 \\
\end{tabular} & 1639.05 & 5125 & 0.1 & 1950 & 225000 & 1725000 & 0.0776 & $1.65 \mathrm{E}-04$ & 2.075 & \begin{tabular}{|l|}
$1.42 \mathrm{E}-02$ \\
\end{tabular} & 64.47 & 95.12 & 51.22 & & 48.9 & 259.5 \\
\hline 70 & 56.81 & 3000 & 2750 & $\begin{array}{l}4.108232 \\
\end{array}$ & 0.1816 & \begin{tabular}{|r|}
132.592 \\
\end{tabular} & 1696.94 & 5125 & 0.1 & 1950 & 225000 & 1637500 & 0.056 & 7.09E-04 & 7.404 & $1.71 \mathrm{E}-02$ & 82.08 & & & & 40.6 & 667.9 \\
\hline 71 & 25.31 & 3000 & 2290 & 0.757937 & 0.0337 & \begin{tabular}{|l|}
133.2659 \\
\end{tabular} & 1688.35 & 5125 & 0.1 & 1950 & 225000 & 1650000 & 0.1257 & $4.88 \mathrm{E}-04$ & 2.961 & $\mid 1.68 \mathrm{E}-02$ & 45.79 & 0 & & 0 & 41.2 & 599.6 \\
\hline 72 & 27.21 & 3000 & 3320 & 13.93595 & 0.4972 & \begin{tabular}{|l|}
107.0251 \\
\end{tabular} & 2102.31 & 5125 & 0.1 & 1950 & 225000 & 1187500 & 0.1169 & $6.88 \mathrm{E}-04$ & 3.975 & \begin{tabular}{|l|}
$8.47 \mathrm{E}-03$ \\
\end{tabular} & 44.95 & 64.12 & & & 81.8 & 748.6 \\
\hline 73 & 29.32 & 3000 & 3010 & 35.062035 & 1.0107 & \begin{tabular}{|l|l|}
86.4774 \\
\end{tabular} & 2601.84 & 5125 & 0.1 & & 225000 & 862500 & 0.1085 & $3.61 \mathrm{E}-04$ & 2.682 & $9.99 \mathrm{E}-03$ & 22.54 & 85.74 & & & 69.4 & 468.9 \\
\hline 74 & 46.04 & 3000 & 1520 & $\begin{array}{l}393.79069 \\
\end{array}$ & 19.2181 & \begin{tabular}{|l|l|}
146.4082 \\
\end{tabular} & 1536.8 & 5125 & 0.1 & 1950 & 225000 & 1900000 & 0.0691 & $1.44 \mathrm{E}-04$ & 2.054 & \begin{tabular}{|c|}
$9.13 \mathrm{E}-03$ \\
\end{tabular} & 34.26 & 98.73 & 87.3 & & 75.9 & 228.6 \\
\hline 75 & 33.49 & 3000 & 650 & $\begin{array}{l}450.832377 \\
\end{array}$ & 13.3696 & \begin{tabular}{|l|}
88.9661 \\
\end{tabular} & 2529.05 & 5125 & 0.1 & 1950 & 225000 & 900000 & 0.095 & $5.29 \mathrm{E}-05$ & 1.282 & $9.81 \mathrm{E}-03$ & 32.53 & 98.89 & & & & 196.1 \\
\hline $\begin{array}{l}76 \\
777 \\
77\end{array}$ & 23.85 & 3000 & 2270 & \begin{tabular}{|l|l|}
111.162875 \\
28623825
\end{tabular} & $\begin{array}{r}5.1601 \\
130070\end{array}$ & \begin{tabular}{|l|l|}
139.2566 \\
\end{tabular} & 1615.72 & 5125 & 0.1 & $\begin{array}{l}1950 \\
1950\end{array}$ & 225000 & 1762500 & 0.1334 & 4.34E-04 & 2.644 & \begin{tabular}{|l|}
$6.26 \mathrm{E}-03$ \\
0.02
\end{tabular} & 46.73 & 95.5 & & & & 568.2 \\
\hline 77 & $\begin{array}{l}43.94 \\
22.0 \\
\end{array}$ & 3000 & \begin{tabular}{ll|l}
1780 & \\
2400 &
\end{tabular} & \begin{tabular}{|l|}
286.238735 \\
236.32511
\end{tabular} & \begin{tabular}{|l|l|}
13.9079 \\
81020
\end{tabular} & \begin{tabular}{|l|l|}
145.7654 \\
103009
\end{tabular} & \begin{tabular}{|l|}
1543.58 \\
213.27 \\
\end{tabular} & 5125 & 0.1 & $\begin{array}{l}1950 \\
1950\end{array}$ & 225000 & 1887500 & 0.0724 & $2.14 \mathrm{E}-04$ & 2.493 & \begin{tabular}{|l|}
$8.38 \mathrm{E}-03$ \\
\end{tabular} & 32.24 & 98.25 & 82.53 & & 82.7 & 290.7 \\
\hline $\begin{array}{r}78 \\
79 \\
\end{array}$ & $\frac{22.28}{22.57}$ & 3000 & $\begin{array}{l}2400 \\
3160\end{array}$ & $\begin{array}{r}236.326511 \\
0.568831\end{array}$ & \begin{tabular}{l|l}
8.19176 \\
0.0173
\end{tabular} & \begin{tabular}{|r|}
$\mid 103.9994$ \\
914206 \\
\end{tabular} & $\begin{array}{l}2163.47 \\
2461.15\end{array}$ & $\begin{array}{l}5125 \\
5125\end{array}$ & $\frac{0.1}{0.1}$ & $\frac{1950}{1950}$ & $\begin{array}{l}225000 \\
225000\end{array}$ & $\begin{array}{r}1137500 \\
937500\end{array}$ & 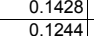 & $\begin{array}{r}2.54 \mathrm{E}-04 \\
5.00 \mathrm{E}-04\end{array}$ & $\begin{array}{l}1.899 \\
3.031\end{array}$ & \begin{tabular}{|l|}
$6.00 \mathrm{E}-03$ \\
$1.56 \mathrm{E}-02$ \\
\end{tabular} & $\begin{array}{l}33.34 \\
20.24\end{array}$ & $\begin{aligned} 97.88 \\
\end{aligned}$ & \begin{tabular}{rl|l}
788.84 & \\
\end{tabular} & & $\begin{array}{r}115.5 \\
44.6 \\
\end{array}$ & $\begin{array}{l}436.9 \\
607.5 \\
\end{array}$ \\
\hline 80 & 21.39 & 3000 & 2870 & $\begin{array}{r}124.269459 \\
\end{array}$ & 4.4021 & \begin{tabular}{|r|}
106.2727 \\
\end{tabular} & 2117.2 & 5125 & 0.1 & 1950 & 225000 & 1175000 & 0.1487 & $6.02 \mathrm{E}-04$ & 3.048 & \begin{tabular}{|l|}
$5.09 \mathrm{E}-03$ \\
\end{tabular} & 93.26 & 95.98 & 59.76 & & 136.1 & 730.2 \\
\hline 81 & 54.2 & 3000 & 2350 & \begin{tabular}{|l|}
129.145357 \\
\end{tabular} & 5.2936 & \begin{tabular}{|l|}
122.9684 \\
\end{tabular} & 1829.74 & 5125 & 0.1 & 1950 & 225000 & 1462500 & 0.0587 & $3.50 \mathrm{E}-04$ & 4.014 & 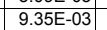 & 52.36 & 96.13 & 61.28 & 0 & 74.2 & 379.6 \\
\hline 82 & 43.28 & 3000 & 1330 & \begin{tabular}{|c|}
1087.902467 \\
\end{tabular} & 40.4284 & \begin{tabular}{|l|l|}
111.4852 \\
\end{tabular} & 2018.2 & 5125 & 0.1 & 1950 & 225000 & 1262500 & 0.0735 & $1.23 \mathrm{E}-04$ & 1.843 & 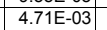 & 36.87 & 99.54 & 95.4 & 54.04 & 147.1 & 218.2 \\
\hline 83 & 23.12 & 3000 & 2070 & \begin{tabular}{|l|}
105.780098 \\
\end{tabular} & 6.2194 & \begin{tabular}{|l|}
176.3872 \\
\end{tabular} & 1275.6 & 5125 & 0.1 & 1950 & 225000 & 2512500 & 0.1376 & $4.14 \mathrm{E}-04$ & 2.521 & 6.18E-03 & 19.94 & 95.27 & 52.73 & & 112.1 & 558.8 \\
\hline 84 & 22.33 & 3000 & 1960 & 22.784665 & 1.0476 & \begin{tabular}{|l|}
137.9366 \\
\end{tabular} & 1631.18 & 5125 & 0.1 & 1950 & 225000 & 1737500 & 0.1425 & $2.85 \mathrm{E}-04$ & 2.01 & \begin{tabular}{|l|}
$1.12 \mathrm{E}-02$ \\
\end{tabular} & 31.85 & 78.06 & 0 & & 61.8 & 461.3 \\
\hline 85 & 32.17 & 3000 & 1630 & 84.409913 & 3.9367 & \begin{tabular}{|l|}
139.9143 \\
\end{tabular} & 1608.13 & 5125 & 0.1 & 1950 & 225000 & 1775000 & 0.0989 & $1.77 \mathrm{E}-04$ & 1.902 & $1.22 \mathrm{E}-02$ & 29.19 & 94.08 & 40.77 & & 56.6 & 303.1 \\
\hline 86 & 21.77 & 3000 & 1930 & $\begin{array}{l}79.601793 \\
\end{array}$ & 3.8677 & \begin{tabular}{|l|l|}
145.7654 \\
\end{tabular} & 1543.58 & 5125 & 0.1 & 1950 & 225000 & 1887500 & 0.1461 & $3.16 \mathrm{E}-04$ & 2.094 & \begin{tabular}{|l|}
$7.88 \mathrm{E}-03$ \\
\end{tabular} & 47.6 & 93.72 & 37.19 & & 87.9 & 492.9 \\
\hline 87 & 34.88 & 3000 & 1540 & 12.356346 & & \begin{tabular}{|l|l|}
170.4873 \\
\end{tabular} & 1319.75 & 5125 & 0.1 & 1950 & 225000 & 2387500 & & $2.89 \mathrm{E}-04$ & 2.601 & 1.83E-02 & 99.18 & 59.53 & 0 & & 37.8 & 382.2 \\
\hline 88 & 21.72 & 3000 & 2610 & $\begin{array}{r}0.65843 \\
519571 \\
\end{array}$ & 0.0261 & \begin{tabular}{|l|l|}
118.7276 \\
15.909
\end{tabular} & & 5125 & 0.1 & 1950 & 225000 & 1387500 & 0.1465 & $4.98 \mathrm{E}-04$ & 2.719 & \begin{tabular}{|l|}
$1.47 \mathrm{E}-02$ \\
\end{tabular} & 26.72 & 0 & 0 & & 47.2 & 641.7 \\
\hline 89 & 35.11 & 3000 & 2110 & 54.485715 & 2.8312 & \begin{tabular}{|l|l|}
155.8885 \\
\end{tabular} & 1443.34 & 5125 & 0.1 & 1950 & 225000 & 2087500 & 0.0906 & $4.18 \mathrm{E}-04$ & 3.332 & \begin{tabular}{|c|} 
\\
\end{tabular} & 50.87 & 90.82 & 8.23 & & 74.5 & 486.3 \\
\hline 90 & $\begin{array}{l}27.31 \\
41.97 \\
\end{array}$ & $\begin{array}{l}3000 \\
33000 \\
3\end{array}$ & 2530 & \begin{tabular}{|l|l|}
242.72278 \\
1436947
\end{tabular} & $\begin{array}{l}6.8608 \\
06607 \\
\end{array}$ & \begin{tabular}{|r|}
84.7981 \\
137.9366
\end{tabular} & \begin{tabular}{|l|}
2653.36 \\
1631.18 \\
\end{tabular} & $\frac{5125}{5125}$ & 0.1 & $\begin{array}{l}1950 \\
1950\end{array}$ & 225000 & \begin{tabular}{r|}
837500 \\
173700
\end{tabular} & 0.1165 & $2.06 \mathrm{E}-04$ & $\begin{array}{ll}1.892 \\
3281\end{array}$ & \begin{tabular}{|l|}
$7.44 \mathrm{E}-03$ \\
$1.5 \mathrm{E}-02$
\end{tabular} & $\begin{array}{l}54.23 \\
3207 \\
\end{array}$ & $\begin{array}{l}97.94 \\
65.2\end{array}$ & 79.4 & & 93.2 & 355.1 \\
\hline 91 & $\begin{array}{l}41.97 \\
3328 \\
\end{array}$ & 3000 & $2160 \mid$ & $\begin{array}{r}14.369474 \\
0.0101\end{array}$ & \begin{tabular}{|l|l|}
0.6607 \\
3035
\end{tabular} & \begin{tabular}{|l|l|}
137.9366 \\
100151
\end{tabular} & \begin{tabular}{|l|}
1631.18 \\
20654
\end{tabular} & 5125 & 0.1 & $\begin{array}{l}1950 \\
1950\end{array}$ & 225000 & 1737500 & 0.0758 & $3.57 \mathrm{E}-04$ & 3.381 & \begin{tabular}{|l|}
$1.45 \mathrm{E}-02$ \\
\end{tabular} & 33.87 & 65.2 & 0 & & 47.9 & 412.8 \\
\hline$\frac{92}{93}$ & $\begin{array}{l}38.28 \\
58.37\end{array}$ & $\begin{array}{l}3000 \\
3000\end{array}$ & 2670 & $\begin{array}{r}9.0101061 \\
262.139989\end{array}$ & $\begin{array}{r}3.035 \\
10.4366\end{array}$ & \begin{tabular}{|l|}
$\mid 100.1541$ \\
119.4396 \\
\end{tabular} & \begin{tabular}{rl|}
22446.54 & $\mid$ \\
1883.8
\end{tabular} & $\begin{array}{l}5125 \\
5125\end{array}$ & 0.1 & $\begin{array}{l}\frac{1950}{1950} \\
\end{array}$ & $\frac{2525000}{225000}$ & $\begin{array}{l}1075000 \\
1400000\end{array}$ & $\begin{array}{l}0.0956 \\
0.0545\end{array}$ & $\begin{array}{l}3.71 \mathrm{E}-04 \\
1.77 \mathrm{E}-04\end{array}$ & $\frac{2.9611}{2.644}$ & \begin{tabular}{|l|}
$8.41 \mathrm{E}-03$ \\
$1.09 \mathrm{E}-02$
\end{tabular} & $\begin{array}{r}47.7 \\
31.4 \\
\end{array}$ & $\begin{array}{r}94.5 \\
98.09 \\
\end{array}$ & $\begin{array}{r}45 \\
80.93\end{array}$ & & $\frac{82.5}{63.5}$ & $\begin{array}{r}456 \\
232.1 \\
\end{array}$ \\
\hline 94 & 22.02 & 3000 & 2220 & 172.258966 & 6.611 & \begin{tabular}{|l|}
115.1349 \\
\end{tabular} & \begin{tabular}{|l|}
1954.23 \\
\end{tabular} & 5125 & 0.1 & 1950 & 225000 & 1325000 & 0.1445 & $2.79 \mathrm{E}-04$ & 1.975 & \begin{tabular}{|l|l|}
$6.62 \mathrm{E}-03$ \\
\end{tabular} & 59.75 & 97.1 & 70.97 & & 104.6 & 459.8 \\
\hline 95 & 31.31 & 3000 & 1730 & 63.408631 & 1.9151 & \begin{tabular}{|l|}
90.6061 \\
\end{tabular} & \begin{tabular}{|l|}
2483.28 \\
\end{tabular} & 5125 & 0.1 & 1950 & 225000 & 925000 & 0.1016 & $1.45 \mathrm{E}-04$ & 1.721 & \begin{tabular}{|l|}
$1.48 \mathrm{E}-02$ \\
\end{tabular} & 62.93 & 92.11 & 21.15 & & 46.8 & 281.6 \\
\hline 96 & 42.82 & 3000 & 710 & 355.247009 & 14.7269 & \begin{tabular}{|l|l|}
124.3658 \\
\end{tabular} & \begin{tabular}{|l|}
1809.18 \\
\end{tabular} & 5125 & 0.1 & 1950 & 225000 & 1487500 & 0.0743 & $3.66 \mathrm{E}-05$ & 1.249 & \begin{tabular}{|l|}
$1.48 \mathrm{E}-02$ \\
\end{tabular} & 75.81 & 98.59 & 85.93 & & 46.7 & 149.5 \\
\hline 97 & 47.98 & 3000 & 3070 & 30.380417 & 1.6411 & \begin{tabular}{|l|}
162.0511 \\
\end{tabular} & \begin{tabular}{|l|}
1388.45 \\
\end{tabular} & 5125 & 0.1 & 1950 & 225000 & 2212500 & 0.0663 & $5.65 \mathrm{E}-04$ & 5.307 & \begin{tabular}{|l|}
$8.74 \mathrm{E}-03$ \\
\end{tabular} & 26.21 & 83.54 & 0 & & 79.3 & 566.8 \\
\hline 98 & 38.89 & 3000 & 2990 & 106.665358 & 3.3366 & \begin{tabular}{|l|}
93.8425 \\
\end{tabular} & \begin{tabular}{|l|}
2397.63 \\
\end{tabular} & 5125 & 0.1 & 1950 & 225000 & 975000 & 0.0818 & $4.70 \mathrm{E}-04$ & 3.908 & $7.64 \mathrm{E}-03$ & 71.09 & 95.31 & 53.12 & & \begin{tabular}{l|l}
90.7 \\
\end{tabular} & 514.9 \\
\hline 99 & 62.75 & 3000 & 2870 & 73.300271 & 2.5411 & \begin{tabular}{|l|l|}
103.9994 \\
\end{tabular} & \begin{tabular}{|l|}
2163.47 \\
\end{tabular} & 5125 & 0.1 & 1950 & 225000 & 1137500 & 0.0507 & $4.04 \mathrm{E}-04$ & 5.028 & $1.05 \mathrm{E}-02$ & 44.5 & 93.18 & 31.79 & & 66.1 & 410.7 \\
\hline 100 & 63.25 & 3000 & 3080 & $\begin{array}{l}41.061774 \\
\end{array}$ & 1.6251 & \begin{tabular}{|l|l|}
118.7276 \\
\end{tabular} & \begin{tabular}{|l|}
1895.09 \\
\end{tabular} & 5125 & 0.1 & 1950 & 225000 & 1387500 & 0.0503 & $4.85 \mathrm{E}-04$ & 5.874 & & 35.04 & 87.82 & & & 66.6 & 476 \\
\hline 101 & 24.16 & 3000 & 3080 & 163.736895 & 5.7177 & & & 5125 & 0.1 & & & & & $6.11 \mathrm{E}-04$ & 3.344 & \begin{tabular}{|l|}
$.60 \mathrm{E}-03$ \\
\end{tabular} & 70.34 & 96.95 & $\begin{array}{ll}69.46 \\
\end{array}$ & & 150.8 & 709.4 \\
\hline 102 & 25.95 & 3000 & 1250 & 483.060162 & 22.4231 & \begin{tabular}{|l|l|}
139.2566 \\
\end{tabular} & \begin{tabular}{|l|}
1615.72 \\
\end{tabular} & 5125 & 0.1 & 1950 & 225000 & 1762500 & $\begin{array}{l}0.1226 \\
\end{array}$ & $1.02 \mathrm{E}-04$ & 1.422 & $6.65 \mathrm{E}-03$ & 45.37 & 98.96 & 89.65 & & 104.2 & 280.9 \\
\hline 103 & 43.4 & & 2570 & 52.225715 & 2.261 & \begin{tabular}{|l|}
129.879 \\
\end{tabular} & \begin{tabular}{|l|}
1732.38 \\
\end{tabular} & 5125 & 0.1 & 1950 & 225000 & & & $5.06 \mathrm{E}-04$ & & \begin{tabular}{|c|}
$9.26 \mathrm{E}-03$ \\
\end{tabular} & $\begin{array}{l}64.56 \\
\end{array}$ & & 4.26 & & 74.9 & 530.1 \\
\hline$\frac{104}{105}$ & $\begin{array}{l}31.34 \\
23.6 \\
\end{array}$ & $\begin{array}{l}3000 \\
3000\end{array}$ & $\frac{3350}{1230}$ & 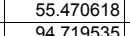 & $\begin{array}{l}2.4392 \\
48628 \\
\end{array}$ & \begin{tabular}{|l|}
131.9164 \\
1540159 \\
\end{tabular} & \begin{tabular}{|c|}
1705.63 \\
1460.89 \\
\end{tabular} & $\begin{array}{l}5125 \\
5125 \\
\end{array}$ & 0.1 & $\begin{array}{l}1950 \\
1950\end{array}$ & 225000 & 1625000 & 0.1015 & $6.95 \mathrm{E}-04$ & $\frac{4.4}{15}$ & \begin{tabular}{|l|}
$5.99 \mathrm{E}-03$ \\
$103 \mathrm{E}-02$ \\
\end{tabular} & $\begin{array}{l}41.38 \\
1001\end{array}$ & 90.99 & $\begin{array}{r}9.86 \\
9721\end{array}$ & & 115.7 & 729.4 \\
\hline$\frac{105}{106}$ & $\begin{array}{r}23.6 \\
55.04 \\
\end{array}$ & 3000 & $\begin{array}{l}1230 \\
1950\end{array}$ & $\begin{array}{r}94.7195355 \\
179.821317\end{array}$ & \begin{tabular}{|c|}
4.8628 \\
7.0738
\end{tabular} & \begin{tabular}{|l|}
154.0159 \\
118.0135 \\
\end{tabular} & \begin{tabular}{|l|}
1460.89 \\
1906.56 \\
\end{tabular} & $\begin{array}{l}5125 \\
5125\end{array}$ & $\frac{0.1}{0.1}$ & $\begin{array}{l}\frac{1950}{1950} \\
\end{array}$ & $\frac{225000}{225000}$ & $\begin{array}{l}20500000 \\
1375000\end{array}$ & $\begin{array}{l}0.1348 \\
0.0578 \\
\end{array}$ & $\begin{array}{r}\frac{1.56 E-04}{1.74 E-04} \\
\end{array}$ & $\frac{1.585}{2.523}$ & \begin{tabular}{|l|}
$1.03 \mathrm{E}-02$ \\
$1.25 \mathrm{E}-02$
\end{tabular} & $\begin{array}{l}19.01 \\
29.67 \\
\end{array}$ & $\begin{array}{l}94.72 \\
9.22 \\
\end{array}$ & $\begin{array}{l}47.21 \\
72.19\end{array}$ & & $\frac{67.7}{55.6}$ & 344.1 \\
\hline 107 & 29.62 & & 1520 & 14.617 & & \begin{tabular}{|l|}
187.3347 \\
\end{tabular} & \begin{tabular}{|l|}
1201.06 \\
\end{tabular} & 5125 & 0 & & 225000 & 2750000 & 0.1074 & $3.02 \mathrm{E}-04$ & 2.421 & \begin{tabular}{|l|}
$1.49 \mathrm{E}-02$ \\
\end{tabular} & 69.95 & 65.79 & & & 46.4 & $\begin{array}{l}2.34 .9 \\
418.9 \\
\end{array}$ \\
\hline 108 & 26.42 & 3000 & 1890 & 113.939 & 6.94 & \begin{tabular}{|l|l|}
182.7653 \\
\end{tabular} & 1231.09 & 5125 & 0.1 & 19 & 225000 & & 0.1204 & $3.63 \mathrm{E}-04$ & 2.5 & \begin{tabular}{|l|}
$6.93 \mathrm{E}-03$ \\
\end{tabular} & 22.75 & 95.61 & 56.12 & & 100 & 489.2 \\
\hline 109 & 21.8 & 3000 & 2130 & 7.440 & 0.3272 & \begin{tabular}{|l|l|}
131.9164 \\
\end{tabular} & 1705 & 5125 & 0.1 & 1950 & 225000 & 1625000 & 0.1459 & $3.86 \mathrm{E}-04$ & 2.339 & $1.25 \mathrm{E}-02$ & 51.43 & 32.8 & & & \begin{tabular}{|l|l|}
55.7 \\
\end{tabular} & 549.7 \\
\hline 110 & 47.41 & & 2310 & 16.648 & 0.7056 & \begin{tabular}{|l|}
127.1374 \\
\end{tabular} & \begin{tabular}{|l|}
1769.74 \\
\end{tabular} & 5125 & 0.1 & 1950 & & & & $4.51 \mathrm{E}-04$ & & & 77.02 & 69.97 & & & 47.3 & 475.4 \\
\hline 111 & 29.13 & 3000 & 3010 & & 0.0396 & \begin{tabular}{|l|}
97.024 \\
\end{tabular} & 2319 & 5125 & 0.1 & 1950 & & & 0.1092 & $5.44 \mathrm{E}-04$ & 3.5 & & 39.76 & 0 & & & 45.3 & 618.6 \\
\hline 112 & & 3000 & 1890 & 351.600966 & $\begin{array}{l}12.893 \\
\end{array}$ & \begin{tabular}{|l|}
110.0086 \\
\end{tabular} & & 5125 & 0.1 & 1950 & 225000 & 1237500 & 0.1136 & 1.68 & 1.749 & 6.9 & 43.41 & 98.58 & 85.78 & & 100.3 & 320.1 \\
\hline 113 & 30.74 & 3000 & 2240 & 12.189 & 0.5388 & \begin{tabular}{|l|}
132.592 \\
\end{tabular} & \begin{tabular}{|l|}
1696.94 \\
\end{tabular} & 5125 & 0.1 & & & & & 4.15 & & & 47.36 & 58.98 & & & 55 & 504.5 \\
\hline 114 & & 30 & 1900 & & 31.6864 & \begin{tabular}{|l|l|}
150.8694 \\
\end{tabular} & 1491 & & 0 & & & & & & 2.6 & & 47 & 99.21 & 92.06 & 20.64 & 163.4 & \\
\hline 115 & 45. & 30 & 1430 & & 2.7 & 110.0086 & 204 & 5125 & 0 & 19 & 225 & & & 1.85 & 2.3 & & 14. & 93 & 32.76 & 0 & 48 & 262.2 \\
\hline 116 & 47.77 & 3000 & 2490 & 288. & 12.6 & & 1705 & 5125 & 0.1 & 1950 & 225000 & & & & & & & & \begin{tabular}{|l|l|}
82.66 \\
\end{tabular} & 0 & & \\
\hline 117 & 31.16 & 3000 & 2570 & & & & & 5125 & & & & & & & & & & & & 14.32 & & \\
\hline 118 & 26.8 & 3000 & 1230 & & 4.86 & 166.2 & 1353 & 5125 & 0.1 & 19 & 2250 & & 0.1 & 1.52 & & & & & & & & \\
\hline 119 & 22.18 & 3000 & 2230 & 239.145214 & 12.575 & \begin{tabular}{|l|l|}
157.7499 \\
\end{tabular} & 1426.31 & 512 & 0.1 & 19 & 2250 & 21250 & 0.14 & $4.58 \mathrm{E}-04$ & 2.6 & 4.40 & 44. & & 79.09 & & & \\
\hline 120 & 30.47 & 300 & 1880 & 216.722162 & 10.85 & 150.2362 & $\mid 1497.64$ & 51 & & 19 & & & 0.10 & $2.99 \mathrm{E}$ & 2.4 & & 49 & 97 & $\begin{array}{ll}76.93 \\
\end{array}$ & & 10 & 411.6 \\
\hline 121 & $\begin{array}{l}43.58 \\
\end{array}$ & 300 & 2610 & 31.0802 & $\begin{array}{l}1.1243 \\
\end{array}$ & 108.52 & 2075 & 512 & & 19 & 225 & & 0.073 & $3.91 \mathrm{E}$ & 3.7 & 1.19 & & & & & 58.1 & 436.4 \\
\hline 122 & 22. & 3000 & 2030 & & 3.57 & 122.266 & & 512 & 0. & 19 & 22500 & 14500 & 0.1 & $2.35 \mathrm{t}$ & 1.8 & & 68. & 94.3 & \begin{tabular}{|l|l|}
43.06 \\
\end{tabular} & & 75 & 415.2 \\
\hline 123 & $\begin{array}{l}49.86 \\
\end{array}$ & 300 & 1960 & 126. & 6.32 & 150.2362 & & 51 & 0.1 & 19 & & & 0.0 & 2.83 & & & & 96 & 60.42 & & 69.5 & 332.9 \\
\hline 124 & 26. & & 2650 & & & & & & 0.1 & & & & & & & & & & & & 74.4 & 656.8 \\
\hline 125 & 60 & & 2500 & & & \begin{tabular}{|l|l|}
1110. \\
\end{tabular} & & & 0 & & & & & & & & & & & & 72.3 & \\
\hline 126 & & 3000 & 1150 & 122.60 & 3.3 & \begin{tabular}{|l|l}
81. \\
\end{tabular} & 276 & 5125 & 0.1 & 1950 & 225000 & & & & 1.2 & 1.2 & & 95.92 & 59.22 & & 57.5 & 277.9 \\
\hline 127 & & & 2290 & & 1.8 & & & & 0 & & & & & & & & & & 5.69 & & 42 & \\
\hline 128 & & 30 & 30 & & & \begin{tabular}{|l|}
113.6821 \\
8.701 \\
\end{tabular} & & & & & & 1300000 & $\begin{array}{l}0.0508 \\
0.112 \\
\end{array}$ & & & \begin{tabular}{|l|}
$5.30 \mathrm{E}-03$ \\
\end{tabular} & 28.81 & & 93.63 & 36.31 & \begin{tabular}{l|l|l|}
130.7 \\
8.2
\end{tabular} & \\
\hline$\frac{129}{130}$ & $\begin{array}{l}28.84 \\
2376\end{array}$ & 3000 & 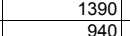 & \begin{tabular}{|l|}
379.551173 \\
78099195
\end{tabular} & \begin{tabular}{r|r}
10.7284 \\
35043
\end{tabular} & \begin{tabular}{|r|}
$\mid 84.7981$ \\
1346086 \\
\end{tabular} & \begin{tabular}{|l|}
$\mid 2653.36$ \\
167.51
\end{tabular} & $\begin{array}{l}5125 \\
5125\end{array}$ & 0.1 & $\begin{array}{l}1950 \\
1950\end{array}$ & $\begin{array}{l}225000 \\
225000\end{array}$ & $\begin{array}{r}837500 \\
1675000\end{array}$ & $\begin{array}{l}0.1103 \\
0.1339\end{array}$ & \begin{tabular}{l|l}
$1.02 E-04$ \\
$6.83 E-05$
\end{tabular} & $\begin{array}{l}1.465 \\
1.258\end{array}$ & \begin{tabular}{|l|}
$8.23 \mathrm{E}-03$ \\
$139 \mathrm{E}-02$
\end{tabular} & \begin{tabular}{ll|}
61.96 & 41.03 \\
4
\end{tabular} & $\begin{array}{c}98.68 \\
936\end{array}$ & \begin{tabular}{ll|l}
866.83 & \\
35.98
\end{tabular} & & \begin{tabular}{r|r|}
84.2 & 49.7 \\
\end{tabular} & $\begin{array}{l}260.4 \\
271.3 \\
\end{array}$ \\
\hline
\end{tabular}




\begin{tabular}{|c|c|c|c|c|c|c|c|c|c|c|c|c|c|c|c|c|c|c|c|c|c|c|}
\hline $\begin{array}{c}\text { Realizatio } \\
n\end{array}$ & $\begin{array}{c}\text { Velocity } \\
(\mathrm{m} / \mathrm{y})\end{array}$ & $\begin{array}{c}\text { Fixed Source } \\
\text { Concentration } \\
\text { (mg/L) }\end{array}$ & $\begin{array}{c}\text { Time of } \\
\text { Maximum at } \\
\text { Compliance } \\
\text { Boundary } \\
\text { (y) }\end{array}$ & \begin{tabular}{|c|} 
Maximum \\
Concentration \\
Compliance \\
Boundary \\
$(\mu \mathrm{g} / \mathrm{L})$
\end{tabular} & \begin{tabular}{|c|} 
Maximum \\
Release Rate \\
at Compliance \\
Boundary \\
$(\mathrm{Kg} / \mathrm{y})$
\end{tabular} & \begin{tabular}{|c|} 
Source \\
Rate \\
$(\mathrm{Kg} / \mathrm{y})$ \\
\end{tabular} & \begin{tabular}{|c|} 
Source \\
Duration \\
(y)
\end{tabular} & \begin{tabular}{|c|} 
1-D \\
Streamtub \\
e Length \\
(m)
\end{tabular} & $\begin{array}{l}\text { Base } \\
\text { Porosity } \\
\left(\mathrm{m}^{3} / \mathrm{m}^{3}\right)\end{array}$ & $\begin{array}{c}\text { Bulk } \\
\text { Densty } \\
\left(\mathrm{Kg} / \mathrm{m}^{3}\right)\end{array}$ & $\begin{array}{c}\text { Remaining } \\
\text { Carbon } \\
\text { Tetrachloride } \\
\text { Source } \\
(\mathrm{Kg})\end{array}$ & \begin{tabular}{|c|} 
Volume at or \\
above Fixed \\
Source \\
$\begin{array}{c}\text { Concentration } \\
(\mathrm{m} 3)\end{array}$ \\
\end{tabular} & $\begin{array}{l}\text { Porosity } \\
\left(\mathrm{m}^{3} / \mathrm{m}^{3}\right)\end{array}$ & $\begin{array}{c}\mathrm{Kd} \\
\left(\mathrm{m}^{3} / \mathrm{Kg}\right) \\
\end{array}$ & Retardation & $\begin{array}{c}\text { First Order } \\
\text { Rate } \\
\text { Constant } \\
(1 / y)\end{array}$ & $\begin{array}{c}\text { Longitudinal } \\
\text { Dispersivity } \\
(\mathrm{m})\end{array}$ & $\begin{array}{c}\text { Required } \\
\text { Source } \\
\text { Cleanup } \\
\text { Percentage } \\
(5 \mu g / L \\
\text { Compliance) } \\
\end{array}$ & \begin{tabular}{|c|} 
Required \\
Source \\
Cleanup \\
Percentage \\
(50 gig/L \\
Compliance)
\end{tabular} & 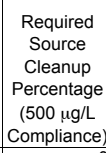 & \begin{tabular}{|c|} 
Abiotic \\
Reaction \\
Half Time \\
(y)
\end{tabular} & $\begin{array}{c}\text { Carbon } \\
\text { Tetrachloride } \\
\text { Travel Time (y) }\end{array}$ \\
\hline $\begin{array}{l}131 \\
132\end{array}$ & 25.51 & $\begin{array}{r}3000 \\
300\end{array}$ & 1450 & \begin{tabular}{|r|r|} 
& 44.283814 \\
\end{tabular} & $\begin{array}{r}2.5951 \\
\end{array}$ & 175.8017 & 1279.85 & 5125 & 0.1 & 1950 & 201000 & & 0.1247 & $1.72 \mathrm{E}-04$ & 1.698 & $1.31 \mathrm{E}-02$ & \begin{tabular}{|r|}
41.95 \\
\end{tabular} & 88.71 & & & 53 & 341 \\
\hline$\frac{132}{133}$ & \begin{tabular}{|l|}
47.27 \\
38.38 \\
\end{tabular} & $\begin{array}{l}3000 \\
3000 \\
\end{array}$ & $\begin{array}{l}2760 \\
2220 \\
\end{array}$ & 4.185304 & \begin{tabular}{|l|l|}
0.1725 \\
47678
\end{tabular} & \begin{tabular}{|l|}
123.6681 \\
1540150
\end{tabular} & \begin{tabular}{|r|}
$\mid 1819.39$ \\
140.89 \\
\end{tabular} & $\begin{array}{r}5125 \\
5125 \\
\end{array}$ & 0.1 & 1950 & 225000 & 1475000 & 0.0673 & \begin{tabular}{|l|}
$5.08 \mathrm{E}-04$ \\
\end{tabular} & 4.812 & $1.55 \mathrm{E}-02$ & 37.09 & & & & 44.9 & 521.7 \\
\hline 134 & $\begin{array}{l}38.38 \\
23.94 \\
\end{array}$ & 3000 & 3240 & \begin{tabular}{|c|c|}
92.869603 \\
9268195 \\
\end{tabular} & $\begin{array}{l}4.7678 \\
0.5249 \\
\end{array}$ & 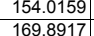 & $\begin{array}{l}\frac{1400.89}{1324.37} \\
\end{array}$ & 5125 & 0.1 & $\begin{array}{l}1950 \\
1950 \\
\end{array}$ & 225000 & 2050000 & 0.0829 & \begin{tabular}{|l|}
$4.66 \mathrm{E}-04$ \\
$7.95 \mathrm{E}-04$ \\
\end{tabular} & 3.842 & $7.86 \mathrm{E}-03$ & 70.23 & 94.62 & 46.16 & 0 & 88.2 & 513 \\
\hline 135 & 34.5 & 3000 & 1980 & 986.319876 & 42.7007 & \begin{tabular}{|l|}
129.879 \\
\end{tabular} & 1732.38 & 5125 & 0.1 & 1950 & 225000 & 1587500 & $\begin{array}{l}0.1329 \\
0.0922\end{array}$ & \begin{tabular}{|l|}
$1.90 \mathrm{E}-04$ \\
$2.47 \mathrm{E}-04$ \\
\end{tabular} & $\begin{array}{l}.025 \\
2.352 \\
\end{array}$ & \begin{tabular}{|l|l|}
$1.30 \mathrm{E}-\mathrm{O}$ \\
$3.30 \mathrm{E}-03$
\end{tabular} & $\begin{array}{l}35.24 \\
74.31\end{array}$ & 99.49 & 94.93 & 49.31 & \begin{tabular}{r|}
53.3 \\
209.8 \\
\end{tabular} & $\begin{array}{l}861.7 \\
349.4\end{array}$ \\
\hline 136 & 27.14 & 3000 & 2090 & 79.24651 & 2.5211 & \begin{tabular}{|l|}
95.4399 \\
\end{tabular} & 2357.51 & 5125 & 0.1 & 1950 & 225000 & 1000000 & 0.1172 & \begin{tabular}{|l|}
$2.44 \mathrm{E}-04$ \\
\end{tabular} & 2.053 & \begin{tabular}{|l|}
$9.68 \mathrm{E}-03$ \\
\end{tabular} & 22.44 & 93.69 & 36.91 & & 71.6 & $\begin{array}{l}349.4 \\
387.7 \\
\end{array}$ \\
\hline 137 & 61.3 & 3000 & 2130 & 743.94935 & 31.6986 & \begin{tabular}{|l|l|}
127.8255 \\
\end{tabular} & 1760.21 & 5125 & 0.1 & 1950 & 225000 & 1550000 & 0.0519 & \begin{tabular}{|l|}
$2.54 \mathrm{E}-04$ \\
\end{tabular} & 3.476 & $4.97 \mathrm{E}-03$ & $\begin{array}{ll}37.88 \\
\end{array}$ & 99.33 & 93.28 & 32.79 & 139.5 & $\frac{387.7}{290.6}$ \\
\hline & 45.51 & & & 252.978214 & & \begin{tabular}{|l|l|}
115.8579 \\
\end{tabular} & 1942.03 & & 0.1 & & 225000 & 1337500 & 0.0699 & \begin{tabular}{|l|}
$2.33 \mathrm{E}-04$ \\
\end{tabular} & 2.682 & $9.09 \mathrm{E}-03$ & 84.52 & 98.02 & & & 76.3 & $\frac{29.6}{302}$ \\
\hline 139 & 23.72 & 3000 & 1900 & 181.993882 & 9.5323 & \begin{tabular}{|l|l|}
157.1306 \\
\end{tabular} & 1431.93 & 5125 & 0.1 & 1950 & 225000 & 2112500 & 0.1341 & \begin{tabular}{|l|}
$3.22 \mathrm{E}-04$ \\
\end{tabular} & 2.213 & $6.09 \mathrm{E}-03$ & 31.74 & 97.25 & 72.53 & & 113.9 & 478 \\
\hline 140 & 22.28 & 3000 & 1850 & 211.192568 & 10.1707 & \begin{tabular}{|l|l|}
144.4754 \\
\end{tabular} & 1557.36 & 5125 & 0.1 & 1950 & 225000 & 1862500 & 0.1428 & \begin{tabular}{|l|}
$2.46 \mathrm{E}-04$ \\
\end{tabular} & 1.869 & \begin{tabular}{|l|l|}
$6.37 \mathrm{E}-03$ \\
\end{tabular} & 33.22 & 97.63 & & & & 430 \\
\hline $\begin{array}{l}141 \\
142\end{array}$ & $\begin{array}{l}25.64 \\
29.93 \\
29\end{array}$ & $\begin{array}{l}3000 \\
3300 \\
3000\end{array}$ & $\frac{1970}{2100}$ & \begin{tabular}{|l|l|}
347.320814 \\
115070143
\end{tabular} & \begin{tabular}{|l|l|}
14.7988 \\
6.688.
\end{tabular} & \begin{tabular}{|l|}
127.8255 \\
16.821 \\
\end{tabular} & \begin{tabular}{|l|}
1760.21 \\
130901
\end{tabular} & 5125 & 0.1 & $\begin{array}{l}1950 \\
1950\end{array}$ & 225000 & 1550000 & 0.1241 & \begin{tabular}{|l|l|}
$2.16 \mathrm{E}-04$ \\
\end{tabular} & $\begin{array}{ll}1.878 \\
3091\end{array}$ & \begin{tabular}{|l|l|}
$5.92 E-03$ \\
\end{tabular} & 39.15 & 98.56 & $\begin{array}{r}85.6 \\
\end{array}$ & & & 375.5 \\
\hline$\frac{142}{143}$ & $\begin{array}{l}29.93 \\
2206\end{array}$ & $\begin{array}{l}3000 \\
3300 \\
3300\end{array}$ & 2100 & \begin{tabular}{|c|}
115.070143 \\
\end{tabular} & $\begin{array}{l}6.1688 \\
78222 \\
\end{array}$ & \begin{tabular}{|l|}
160.8281 \\
1222668 \\
\end{tabular} & 1399.01 & 5125 & 0.1 & $\begin{array}{l}1950 \\
1950\end{array}$ & 225000 & 2187500 & 0.1063 & \begin{tabular}{|l|l|}
$4.40 \mathrm{E}-04$ \\
\end{tabular} & 3.091 & $6.85 \mathrm{E}-03$ & 61.91 & 95.65 & & & 101.2 & 529.2 \\
\hline$\frac{143}{144}$ & $\begin{array}{l}22.06 \\
42.19\end{array}$ & 3000 & $\frac{2510}{2690}$ & $\begin{array}{r}191.928734 \\
0720723\end{array}$ & $\begin{array}{r}7.8222 \\
0.035\end{array}$ & \begin{tabular}{|c|}
122.2668 \\
1457654 \\
\end{tabular} & $\begin{array}{l}1840.24 \\
15435\end{array}$ & $\begin{array}{l}5125 \\
525 \\
\end{array}$ & 0.1 & $\begin{array}{r}1950 \\
1950 \\
1950\end{array}$ & $\begin{array}{r}225000 \\
225000\end{array}$ & \begin{tabular}{|l|}
1450000 \\
1887500 \\
\end{tabular} & $\begin{array}{r}0.1442 \\
0.0754 \\
\end{array}$ & \begin{tabular}{|c|}
$4.69 \mathrm{E}-04$ \\
$562 \mathrm{E}-04$ \\
\end{tabular} & 2.646 & \begin{tabular}{|l|}
$4.84 \mathrm{E}-03$ \\
$17 \mathrm{E}-02$
\end{tabular} & $\begin{array}{r}58.49 \\
313\end{array}$ & $\begin{array}{r}97.39 \\
0\end{array}$ & 73.95 & & $\begin{array}{r}143.3 \\
39\end{array}$ & $\begin{array}{l}614.6 \\
579.5 \\
70.5\end{array}$ \\
\hline 145 & 44.31 & 3000 & 3220 & 2.370722 & 0.0791 & \begin{tabular}{|l|}
100.1541 \\
\end{tabular} & 2246.54 & 5125 & 0.1 & 1950 & 225000 & $\begin{array}{l}10075000 \\
1075000\end{array}$ & $\begin{array}{l}0.074 \\
0.0718 \\
\end{array}$ & \begin{tabular}{|l|}
$0.02 \mathrm{E}-04$ \\
$5.75 \mathrm{E}-04$ \\
\end{tabular} & 5.047 & \begin{tabular}{|l|}
$1.8 \mathrm{E}-02$ \\
$1.56 \mathrm{E}-02$
\end{tabular} & $\begin{array}{r}31.3 \\
39.43 \\
\end{array}$ & 0 & & & $\begin{array}{r}399 \\
44.4 \\
\end{array}$ & $\frac{579.5}{583.8}$ \\
\hline 146 & 39.82 & 3000 & 2710 & 19.023123 & 0.6102 & \begin{tabular}{|l|}
96.2335 \\
\end{tabular} & 2338.06 & 5125 & 0.1 & 1950 & 225000 & 1012500 & 0.0799 & \begin{tabular}{|l|}
$4.06 \mathrm{E}-04$ \\
\end{tabular} & 3.571 & $1.38 \mathrm{E}-02$ & 71.64 & 73.72 & & & 50.3 & 459.6 \\
\hline 147 & 33.45 & 3000 & 2050 & 6.156327 & 0.3363 & \begin{tabular}{|l|}
163.877 \\
\end{tabular} & 1372.98 & 5125 & 0.1 & 1950 & 225000 & 2250000 & 0.0951 & \begin{tabular}{|l|}
$3.84 \mathrm{E}-04$ \\
\end{tabular} & 3.043 & \begin{tabular}{|l|}
$1.44 \mathrm{E}-02$ \\
\end{tabular} & 22.51 & 18.78 & 0 & & 48.2 & 466.2 \\
\hline 148 & 43.52 & 3000 & 1850 & 202.210499 & 9.7381 & \begin{tabular}{|l|l|}
144.4754 \\
\end{tabular} & \begin{tabular}{|l|}
1557.36 \\
\end{tabular} & 5125 & 0.1 & 1950 & 225000 & 1862500 & 0.0731 & \begin{tabular}{|l|}
$2.61 \mathrm{E}-04$ \\
\end{tabular} & 2.808 & $8.86 \mathrm{E}-03$ & 58.11 & 97.53 & 75.27 & & 78.3 & 330.7 \\
\hline 149 & 21.89 & 3000 & 2880 & 320.945737 & 10.2952 & \begin{tabular}{|l|}
96.2335 \\
\end{tabular} & \begin{tabular}{|r|}
2338.06 \\
\end{tabular} & 5125 & 0.1 & 1950 & 225000 & 1012500 & 0.1453 & \begin{tabular}{|l|}
$4.67 \mathrm{E}-04$ \\
\end{tabular} & 2.625 & $3.97 \mathrm{E}-03$ & 79.41 & 98.44 & 84.42 & & 174.8 & 614.5 \\
\hline 150 & 36.78 & 3000 & 1490 & 159.502668 & 6.1599 & \begin{tabular}{|l|l|}
115.8579 \\
\end{tabular} & 1942.03 & 5125 & 0.1 & 1950 & 225000 & 1337500 & 0.0865 & $1.04 \mathrm{E}-04$ & 1.607 & $1.41 \mathrm{E}-02$ & 80.46 & 96.87 & 68.65 & & 49.3 & \\
\hline 151 & 39.57 & 3000 & 2130 & 23.25577 & 0.8925 & \begin{tabular}{|l|l|}
115.1349 \\
\end{tabular} & 1954.23 & 5125 & 0.1 & 1950 & 225000 & 1325000 & 0.0804 & \begin{tabular}{|l|}
$2.53 \mathrm{E}-04$ \\
\end{tabular} & 2.588 & $1.69 \mathrm{E}-02$ & 67.82 & 78.5 & & & 41 & 335.2 \\
\hline 152 & 25.47 & 3000 & 2870 & 9.776136 & & \begin{tabular}{|l|l|}
133.2659 \\
\end{tabular} & \begin{tabular}{|l|}
1688.35 \\
\end{tabular} & 5125 & 0.1 & 1950 & 225000 & & 0.1249 & \begin{tabular}{|l|l|}
$6.22 \mathrm{E}-04$ \\
\end{tabular} & 3.518 & $9.18 \mathrm{E}-03$ & 35.62 & 48.86 & & & 75.5 & 707.8 \\
\hline 153 & 24.47 & 3000 & 1130 & 156.700185 & 5.0266 & \begin{tabular}{|l|l|}
96.2335 \\
\end{tabular} & \begin{tabular}{|l|}
2338.06 \\
\end{tabular} & 5125 & 0.1 & 1950 & 225000 & 1012500 & 0.13 & \begin{tabular}{|l|}
$6.91 \mathrm{E}-05$ \\
\end{tabular} & 1.269 & \begin{tabular}{|l|l|}
$1.16 \mathrm{E}-02$ \\
\end{tabular} & 61.52 & 96.81 & 68.09 & 0 & $\begin{array}{l}59.7 \\
\end{array}$ & 265.7 \\
\hline 154 & $\begin{array}{l}55.42 \\
5.91\end{array}$ & 3000 & 3260 & \begin{tabular}{|r|}
79.423326 \\
\end{tabular} & 2.5895 & \begin{tabular}{|l|l|}
97.8112 \\
\end{tabular} & \begin{tabular}{|l|}
2300.35 \\
\end{tabular} & 5125 & 0.1 & 1950 & 225000 & 1037500 & 0.0574 & \begin{tabular}{|l|l|}
$4.54 \mathrm{E}-04$ \\
\end{tabular} & 4.996 & $8.70 \mathrm{E}-03$ & 30.88 & 93.7 & 37.05 & & 79.6 & 462 \\
\hline$\frac{155}{156}$ & 56.91 & 3000 & 2140 & \begin{tabular}{|l|}
1463.195101 \\
1
\end{tabular} & $\begin{array}{l}55.8012 \\
03619 \\
\end{array}$ & 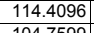 & & 5125 & 0.1 & $\begin{array}{l}1950 \\
1050 \\
1050\end{array}$ & 225000 & 1312500 & $\begin{array}{l}0.0559 \\
0.1475\end{array}$ & \begin{tabular}{|c|}
$1.88 \mathrm{E}-04$ \\
\end{tabular} & 2.696 & $3.00 \mathrm{E}-03$ & $\begin{array}{l}37.69 \\
\end{array}$ & 99.66 & 96.58 & 65.83 & 231.1 & 242.8 \\
\hline$\frac{156}{157}$ & 21.57 & 3000 & 2790 & $\begin{array}{r}10.359727 \\
7802613 \\
\end{array}$ & $\begin{array}{l}0.3618 \\
3.142\end{array}$ & \begin{tabular}{|l|l|}
104.7599 \\
119.436 \\
\end{tabular} & \begin{tabular}{|l|}
2147.77 \\
18838 \\
\end{tabular} & 5125 & 0.1 & $\begin{array}{l}1950 \\
1950\end{array}$ & 225000 & 1150000 & 0.1475 & \begin{tabular}{|l|}
$5.87 \mathrm{E}-04$ \\
\end{tabular} & 3.01 & \begin{tabular}{|l|}
$9.85 \mathrm{E}-03$ \\
70502
\end{tabular} & 73.1 & 51.74 & 0 & 0 & 70.4 & 715.3 \\
\hline$\frac{157}{158}$ & $\frac{28.05}{33.88}$ & $\begin{array}{l}3000 \\
3000\end{array}$ & $\begin{array}{l}2610 \\
1090\end{array}$ & $\begin{array}{r}78.922613 \\
250.748788\end{array}$ & \begin{tabular}{r|}
$\frac{3.1422}{13.0296}$ \\
\end{tabular} & \begin{tabular}{|l|}
119.4396 \\
155.8885 \\
\end{tabular} & \begin{tabular}{|r|}
1883.8 \\
1443.34
\end{tabular} & $\begin{array}{l}55125 \\
5125\end{array}$ & $\frac{0.1}{0.1}$ & $\begin{array}{l}1950 \\
1950\end{array}$ & $\frac{225000}{225000}$ & \begin{tabular}{|l|}
14000000 \\
2087500
\end{tabular} & $\begin{array}{l}0.1134 \\
0.0939 \\
\end{array}$ & \begin{tabular}{|l|}
$4.61 \mathrm{E}-04$ \\
$1.22 \mathrm{E}-04$ \\
\end{tabular} & $\begin{array}{l}3.053 \\
1.655 \\
\end{array}$ & \begin{tabular}{|l|l|}
$7.07 E-03$ \\
$1.01 E-02$ \\
\end{tabular} & $\begin{array}{l}38.86 \\
27.32\end{array}$ & $\begin{array}{l}93.66 \\
98.01 \\
\end{array}$ & $\begin{array}{l}36.65 \\
80.06\end{array}$ & & $\begin{array}{c}98.1 \\
68.4 \\
\end{array}$ & $\frac{557.7}{250.4}$ \\
\hline 159 & 25.09 & 3000 & 1380 & 55.879461 & 2.739 & \begin{tabular}{|l|}
147.0497 \\
\end{tabular} & 1530.1 & 5125 & 0.1 & 1950 & 225000 & 1912500 & 0.1268 & \begin{tabular}{|l|}
$1.34 \mathrm{E}-04$ \\
\end{tabular} & 1.534 & $1.33 \mathrm{E}-02$ & 41.12 & 91.05 & 10.52 & & 52 & 313.3 \\
\hline 160 & 25.23 & 3000 & 2160 & 8.405773 & 0.3734 & 133.2659 & \begin{tabular}{|l|}
1688.35 \\
\end{tabular} & 5125 & 0.1 & 1950 & 225000 & 1650000 & 0.1261 & \begin{tabular}{|l|}
$4.13 \mathrm{E}-04$ \\
\end{tabular} & 2.656 & \begin{tabular}{|l|}
$1.27 \mathrm{E}-02$ \\
\end{tabular} & 54.92 & 40.52 & & & 54.5 & 539.5 \\
\hline 161 & 39.92 & 3000 & 1880 & 22.022304 & 1.2428 & \begin{tabular}{|l|l|}
169.2951 \\
\end{tabular} & \begin{tabular}{|l|}
1329.04 \\
\end{tabular} & 5125 & 0.1 & 1950 & 225000 & 2362500 & 0.0797 & \begin{tabular}{|l|}
$3.40 \mathrm{E}-04$ \\
\end{tabular} & 3.154 & $1.34 \mathrm{E}-02$ & 33.49 & 77.3 & 0 & & 51.9 & 405 \\
\hline 162 & 34.1 & 3000 & 1160 & 591.966297 & 18.1993 & \begin{tabular}{|l|}
92.2314 \\
\end{tabular} & \begin{tabular}{|l|}
2439.52 \\
\end{tabular} & 5125 & 0.1 & 1950 & 225000 & 950000 & & \begin{tabular}{|c|}
$1.24 \mathrm{E}-04$ \\
\end{tabular} & 1.669 & $6.56 \mathrm{E}-03$ & 27.45 & 99.16 & 91.55 & 15.54 & 105.6 & 250.9 \\
\hline 163 & 38.15 & 3000 & 2650 & 640.983297 & 20.3918 & \begin{tabular}{|l|}
95.4399 \\
\end{tabular} & \begin{tabular}{|l|}
2357.51 \\
\end{tabular} & 5125 & 0.1 & 1950 & 225000 & 1000000 & 0.0834 & \begin{tabular}{|c|}
$3.14 \mathrm{E}-04$ \\
\end{tabular} & 2.902 & $4.27 \mathrm{E}-03$ & 91.33 & 99.22 & 92.2 & 21.99 & 162.2 & 389.9 \\
\hline 164 & 22.66 & 3000 & 3130 & 0.046152 & 0.0021 & \begin{tabular}{|l|l|}
139.2566 \\
\end{tabular} & 1615.72 & 5125 & 0.1 & 1950 & 225000 & 1762500 & 0.1404 & \begin{tabular}{|l|}
$7.79 \mathrm{E}-04$ \\
\end{tabular} & 3.805 & $1.57 \mathrm{E}-02$ & 26.86 & & & & 44.2 & 860.5 \\
\hline 165 & 28.13 & 3000 & 2450 & 36.060812 & & & \begin{tabular}{|l|}
1809.18 \\
\end{tabular} & 5125 & 0.1 & & 225000 & & 0.1131 & \begin{tabular}{|l|l|}
$4.28 \mathrm{E}-04$ \\
\end{tabular} & 2.913 & & 34.43 & 86.13 & 0 & & 76.6 & 530.7 \\
\hline 166 & 26.01 & 3000 & 880 & 197.809533 & 8.8314 & \begin{tabular}{|l|l|}
133.9381 \\
\end{tabular} & 1679.88 & 5125 & 0.1 & & & & & \begin{tabular}{|l|}
$2.83 \mathrm{E}-05$ \\
\end{tabular} & 1.117 & & 78.7 & 97.47 & 74.72 & & 53.6 & 220 \\
\hline 167 & 24.66 & 3000 & 1070 & $\begin{array}{l}55.756415 \\
\end{array}$ & $\begin{array}{r}2.3114 \\
\end{array}$ & \begin{tabular}{|l|l|}
124.3658 \\
8.177
\end{tabular} & \begin{tabular}{|l|}
1809.18 \\
\end{tabular} & 5125 & 0.1 & 1950 & 225000 & 1487500 & 0.129 & \begin{tabular}{|c|}
$9.38 \mathrm{E}-05$ \\
\end{tabular} & 1.367 & \begin{tabular}{|l|l|}
$1.46 \mathrm{E}-02$ \\
\end{tabular} & 38.69 & 91.03 & 10.32 & & 47.5 & 284.2 \\
\hline $\begin{array}{ll}168 \\
169\end{array}$ & 50.98 & $\begin{array}{ll}3000 \\
3000\end{array}$ & 2790 & \begin{tabular}{|l|l|}
431.08649 \\
80.31576
\end{tabular} & $\begin{array}{l}12.4264 \\
25769 \\
\end{array}$ & \begin{tabular}{|l|l|}
86.4774 \\
96.235 \\
\end{tabular} & \begin{tabular}{|l|}
2601.84 \\
23806
\end{tabular} & $\begin{array}{l}5125 \\
5125\end{array}$ & 0.1 & $\begin{array}{l}1950 \\
1950\end{array}$ & 225000 & \begin{tabular}{r|}
862500 \\
101500
\end{tabular} & $\begin{array}{ll}0.0624 \\
0.209\end{array}$ & \begin{tabular}{|l|}
$2.25 \mathrm{E}-04$ \\
$355 \mathrm{E}-1$
\end{tabular} & 2.823 & $7.09 \mathrm{E}-03$ & 34.15 & & 88.4 & & 97.8 & 283.7 \\
\hline$\frac{169}{170}$ & $\begin{array}{l}24.49 \\
31.85 \\
\end{array}$ & $\begin{array}{l}3000 \\
3000\end{array}$ & 2660 & 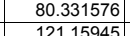 & \begin{tabular}{|l|}
2.5769 \\
4.049
\end{tabular} & \begin{tabular}{|r|}
96.2335 \\
100151
\end{tabular} & \begin{tabular}{|l|}
2338.06 \\
224654 \\
\end{tabular} & $\begin{array}{l}5125 \\
5125 \\
\end{array}$ & 0.1 & $\begin{array}{l}1950 \\
1950\end{array}$ & 225000 & $\begin{array}{l}1012500 \\
107500\end{array}$ & 0.1299 & \begin{tabular}{|l|}
$3.55 \mathrm{E}-04$ \\
$576 \mathrm{~F}-4$.
\end{tabular} & $\begin{array}{r}2.38 \\
3917 \\
\end{array}$ & \begin{tabular}{|l|l|}
$8.02 E-03$ \\
$574-03$
\end{tabular} & $\begin{array}{l}61.69 \\
55.14\end{array}$ & $\begin{array}{l}93.78 \\
95.87\end{array}$ & $\begin{array}{l}37.76 \\
5873\end{array}$ & & 86.4 & 498 \\
\hline 171 & $\begin{array}{l}3.00 \\
60.25 \\
\end{array}$ & 3000 & $\frac{3220}{2840}$ & $\begin{array}{r}121.4159445 \\
104.44148\end{array}$ & $\begin{array}{l}4.0489 \\
5.1861\end{array}$ & \begin{tabular}{|l|}
100.1541 \\
148.6657 \\
\end{tabular} & $\begin{array}{l}2246.54 \\
2510.42\end{array}$ & $\mid \begin{array}{l}5125 \\
5125\end{array}$ & $\begin{array}{l}0.1 \\
0.1\end{array}$ & $\frac{1950}{1950}$ & $\frac{225000}{225000}$ & & \begin{tabular}{|l|l}
0.09999 \\
0.0528
\end{tabular} & \begin{tabular}{|l|}
$5.76 \mathrm{E}-04$ \\
$4.76 \mathrm{E}-04$ \\
\end{tabular} & $\begin{array}{l}3.917 \\
5.552\end{array}$ & \begin{tabular}{|l|}
$5.74 \mathrm{E}-03$ \\
$7.64 \mathrm{E}-03$ \\
\end{tabular} & \begin{tabular}{|l|l|}
55.14 \\
34.18
\end{tabular} & \begin{tabular}{|l|l|}
95.87 \\
9.21
\end{tabular} & \begin{tabular}{|c|c|}
58.73 \\
52.13
\end{tabular} & & \begin{tabular}{rl|l}
$\frac{120.8}{90.7}$ & \\
9 &
\end{tabular} & $\frac{630.3}{472.3}$ \\
\hline 172 & 24.74 & & 1280 & 14.694 & 0.6691 & \begin{tabular}{|l|l|}
136.6103 \\
\end{tabular} & \begin{tabular}{|l|}
1647.02 \\
\end{tabular} & 5125 & 0 & & 225 & & & \begin{tabular}{|l|}
$1.54 \mathrm{E}-04$ \\
\end{tabular} & 1.604 & \begin{tabular}{|l|}
$1.67 E-02$ \\
\end{tabular} & & 65.97 & & & 41.5 & $\frac{472.5}{332.4}$ \\
\hline 173 & 23.51 & 3000 & 2080 & 291.878459 & \begin{tabular}{|l|l|}
14.8013 \\
\end{tabular} & \begin{tabular}{|l|l|}
152.1319 \\
\end{tabular} & \begin{tabular}{|l|}
1478.98 \\
\end{tabular} & 5125 & 0.1 & & 225000 & 2012500 & 0.1353 & \begin{tabular}{|l|}
$3.77 \mathrm{E}-04$ \\
\end{tabular} & 2.409 & \begin{tabular}{|l|l|}
$4.62 \mathrm{E}-03$ \\
\end{tabular} & 38.08 & 98.29 & 82.87 & & 150 & 525 \\
\hline 174 & 21. & 3000 & 3200 & 84.367189 & 2.116 & \begin{tabular}{|l|}
75.2423 \\
\end{tabular} & \begin{tabular}{|r|}
2990.34 \\
\end{tabular} & 5125 & 0.1 & 1950 & 225000 & 700000 & 0.147 & \begin{tabular}{|l|}
$3.57 \mathrm{E}-04$ \\
\end{tabular} & 2.228 & $7.01 \mathrm{E}-03$ & 23.14 & 94.07 & 40.74 & & 98.9 & 527.6 \\
\hline 175 & 42.25 & & 3330 & 32.956525 & 0.9129 & \begin{tabular}{|l|}
83.1021 \\
\end{tabular} & \begin{tabular}{|l|}
2707.51 \\
\end{tabular} & 5125 & 0.1 & 1950 & & & 0.0 & \begin{tabular}{|l|}
$4.56 \mathrm{E}-04$ \\
\end{tabular} & 4.06 & & 41.28 & 84.83 & & & 65.9 & 492.5 \\
\hline 176 & 25.43 & 3000 & 2610 & & 1.1616 & \begin{tabular}{|l|l|}
116.5786 \\
\end{tabular} & 1930 & 5125 & 0.1 & 1950 & & & & \begin{tabular}{|l|}
$4.53 \mathrm{E}-04$ \\
\end{tabular} & 2.832 & & 31.18 & 83.27 & & & 79.5 & 570.7 \\
\hline 177 & 24.06 & 3000 & 1790 & 243.016941 & 11.8599 & 146.4082 & & 5125 & 0.1 & 1950 & 225000 & 1900000 & & 2.58 & 1.986 & & 86.33 & 97.94 & 79.43 & & 107.6 & 422.9 \\
\hline 178 & 29.98 & 3000 & 1940 & & \begin{tabular}{|l|}
0.728 \\
\end{tabular} & & \begin{tabular}{|l|}
1242.84 \\
\end{tabular} & & 0.1 & & & & & 4.85 & 3.3 & & 67.5 & 58.55 & & & 57.8 & 566.2 \\
\hline 179 & $\begin{array}{l}23.12 \\
\end{array}$ & & 2570 & & 0.2074 & \begin{tabular}{|l|}
92.2 \\
\end{tabular} & & & 0 & & & & & 2.67 & & & & & & & 45.2 & 439.2 \\
\hline 180 & 38. & 30 & 1950 & & & 123.6 & 1816 & 51 & 0 & 19 & 225 & & & & & & & .16 & 71.65 & & $\begin{array}{l}64.6 \\
\end{array}$ & \\
\hline 181 & $\begin{array}{l}59.58 \\
2.70\end{array}$ & 3000 & 3060 & 126.074583 & $\begin{array}{l}4.5606 \\
17.0\end{array}$ & \begin{tabular}{|l|l|}
108219 \\
\end{tabular} & 2073.31 & 5125 & 0.1 & 1950 & 225000 & 1212500 & 0.0 & $5.31 \mathrm{E}$ & $\begin{array}{l}6.026 \\
1027\end{array}$ & & 74. & $\begin{array}{l}96.03 \\
0.12\end{array}$ & 60.34 & 0 & \begin{tabular}{|l|}
92.6 \\
\end{tabular} & 518.4 \\
\hline$\frac{182}{182}$ & \begin{tabular}{|l|l|}
22.79 \\
5311
\end{tabular} & & 2640 & 566.91 & $\begin{array}{l}17.429 \\
2.451\end{array}$ & \begin{tabular}{|l|l|}
92314 \\
\end{tabular} & 2439.52 & $\begin{array}{l}5125 \\
5125\end{array}$ & & & & & & $2.56 \mathrm{E}$ & 1.927 & & 65. & & $\begin{array}{l}91.18 \\
27.0\end{array}$ & 11.8 & $\begin{array}{l}173.2 \\
\end{array}$ & 433.4 \\
\hline$\frac{183}{184}$ & 53.11 & 3000 & $\begin{array}{l}3080 \\
2410\end{array}$ & $\begin{array}{c}80.449745 \\
4511409 \\
\end{array}$ & $\begin{array}{l}2.4516 \\
20533\end{array}$ & \begin{tabular}{|r|}
91.4206 \\
\end{tabular} & \begin{tabular}{|r|}
2461.15 \\
\end{tabular} & $\begin{array}{l}5125 \\
5125\end{array}$ & 0.1 & $\begin{array}{l}1950 \\
1950\end{array}$ & $\begin{array}{r}225000 \\
225000\end{array}$ & $\begin{array}{r}937500 \\
171250\end{array}$ & $\begin{array}{l}0.0599 \\
0.1161\end{array}$ & \begin{tabular}{|l|l|}
$4.04 \mathrm{E}-04$ \\
\end{tabular} & $\begin{array}{r}4.41 \\
2.16\end{array}$ & 9.59E-03 & $\begin{array}{ll}41.6 \\
5.5 .5\end{array}$ & 93.78 & 37.85 & & 72.3 & 425.5 \\
\hline$\frac{184}{185}$ & $\begin{array}{r}27.4 \\
2778 \\
\end{array}$ & 3000 & 2410 & $\begin{array}{r}45.114069 \\
28.819014\end{array}$ & \begin{tabular}{r|r|}
2.0543 \\
0.744
\end{tabular} & \begin{tabular}{|l|}
136.6103 \\
770
\end{tabular} & \begin{tabular}{|l|}
1647.02 \\
2880 \\
\end{tabular} & $\begin{array}{l}5125 \\
525 \\
\end{array}$ & 0.1 & $\frac{1950}{1950}$ & $\begin{aligned} 225000 \\
225000\end{aligned}$ & $\begin{array}{r}1712500 \\
73750\end{array}$ & $\begin{array}{l}0.1161 \\
\end{array}$ & \begin{tabular}{|l|}
$4.97 \mathrm{E}-04$ \\
$5.15-0$
\end{tabular} & $\begin{array}{l}3.164 \\
3.711\end{array}$ & $8.06 \mathrm{E}-03$ & 53.9 & 88.92 & 0 & & 86 & 591.7 \\
\hline$\frac{185}{186}$ & $\begin{array}{l}27.78 \\
27.57\end{array}$ & $\begin{array}{l}3000 \\
3000\end{array}$ & $\frac{3430}{1840}$ & $\begin{array}{l}28.8919014 \\
53.348711\end{array}$ & $\begin{array}{l}0.7484 \\
2.5807\end{array}$ & \begin{tabular}{|r|}
77.906 \\
145.1211 \\
\end{tabular} & \begin{tabular}{|r|}
2888.09 \\
1550.43
\end{tabular} & $\begin{array}{l}5125 \\
5125\end{array}$ & $\frac{0.1}{0.1}$ & $\frac{19}{19}$ & $\frac{2250}{2250}$ & $\begin{array}{l}7374 \\
1875\end{array}$ & $\begin{array}{l}0.1115 \\
0.1154\end{array}$ & \begin{tabular}{|c|}
$5.14 \mathrm{E}-04$ \\
$2.64 \mathrm{E}-04$ \\
\end{tabular} & $\begin{array}{l}3.2771 \\
2.158\end{array}$ & \begin{tabular}{|l|}
$9.02 \mathrm{E}$ \\
$1.07 \mathrm{E}$
\end{tabular} & $\begin{array}{l}57.84 \\
39.34\end{array}$ & \begin{tabular}{|l|l|}
92.65 \\
90.63
\end{tabular} & $\begin{array}{r}0 \\
6.28 \\
\end{array}$ & & $\begin{array}{l}76.9 \\
64.7 \\
\end{array}$ & $\frac{603.4}{401.2}$ \\
\hline 187 & 36. & 3000 & 1560 & 251.897 & 14.0642 & $167.49 \varepsilon$ & 1343. & 5125 & 0.1 & 1950 & 225000 & 2325 & 0.0 & \begin{tabular}{|l|}
$2.06 \mathrm{E}-04$ \\
\end{tabular} & 2.18 & 8.399 & 46.89 & 98.02 & 80.15 & & 82.6 & 309.8 \\
\hline 188 & 30. & 3000 & 3880 & 0.411094 & 0.0199 & \begin{tabular}{|l|l|}
145.1211 \\
\end{tabular} & \begin{tabular}{|l|}
1550.43 \\
\end{tabular} & 5125 & 0.1 & 1950 & 2250 & & 0.1 & $8.19 \mathrm{E}-04$ & 4.985 & 1.22 & 19.2 & & & & 56.8 & 834.3 \\
\hline 189 & 44. & & 1780 & & & 156.5 & 143 & 512 & 0.1 & & & & & 2.68 & 2.894 & & 39. & 90.33 & 3.29 & & 52.1 & 333.3 \\
\hline 190 & & & 1810 & 960.5 & 31.8192 & & 226 & 5125 & 0 & 195 & & & & 1.62 & & & 36. & 99.48 & 94.79 & 47.95 & 154.6 & 258.2 \\
\hline 191 & & 3000 & 1920 & & & \begin{tabular}{|l|l|}
196.8 \\
\end{tabular} & 1142 & 5125 & 0.1 & 1950 & 225000 & & 0.1 & 4.48 & 2.547 & & 46.83 & 92.02 & 20.15 & & \begin{tabular}{|l|l|}
99.3 \\
\end{tabular} & 600 \\
\hline 192 & & & 37 & & & & & & 0 & & & & & \begin{tabular}{|l|l|l|}
6.900 \\
\end{tabular} & & & & & & & 73 & 710.5 \\
\hline 193 & & & $\begin{array}{l}1050 \\
1660\end{array}$ & & & \begin{tabular}{|l|l|l|}
152.139 \\
15.
\end{tabular} & 1478 & & 0.1 & & & & & \begin{tabular}{|c|}
$9.50 \mathrm{E}-05$ \\
\end{tabular} & & & & $\begin{array}{l}97.66 \\
6291\end{array}$ & 76.58 & & 56.4 & \\
\hline$\frac{194}{195}$ & $\begin{array}{l}21.63 \\
2344\end{array}$ & 3000 & $\frac{1660}{1100}$ & \begin{tabular}{|r|}
13.481861 \\
30.029861 \\
\end{tabular} & \begin{tabular}{r|r|}
0.6955 \\
12.987
\end{tabular} & \begin{tabular}{|l|}
154.6414 \\
1180135 \\
\end{tabular} & \begin{tabular}{|c|}
$\mid 1454.98$ \\
1906.56 \\
\end{tabular} & $\begin{array}{l}5125 \\
5125\end{array}$ & 0.1 & $\begin{array}{l}1950 \\
1950\end{array}$ & 225000 & $\begin{array}{l}2062500 \\
1375000\end{array}$ & \begin{tabular}{|l|l|}
0.1471 \\
0.1375
\end{tabular} & \begin{tabular}{|l|}
$2.32 \mathrm{E}-04$ \\
$713 \mathrm{E}-05$ \\
\end{tabular} & \begin{tabular}{|l|l|}
1.796 \\
1226 \\
\end{tabular} & \begin{tabular}{|l|l|}
$1.43 \mathrm{E}-02$ \\
$820 \mathrm{E}-03$ \\
\end{tabular} & \begin{tabular}{|c|}
65.09 \\
50.11
\end{tabular} & $\begin{array}{ll}62.91 \\
988\end{array}$ & \begin{tabular}{r|}
0 \\
84.85
\end{tabular} & & $\begin{array}{ll}48.6 \\
8.6\end{array}$ & 425.6 \\
\hline
\end{tabular}




\begin{tabular}{|c|c|c|c|c|c|c|c|c|c|c|c|c|c|c|c|c|c|c|c|c|c|c|}
\hline $\begin{array}{c}\text { Realizatio } \\
n\end{array}$ & $\begin{array}{c}\text { Velocity } \\
(\mathrm{m} / \mathrm{y})\end{array}$ & $\begin{array}{c}\text { Fixed Source } \\
\text { Concentration } \\
\text { (mg/L) }\end{array}$ & $\begin{array}{c}\text { Time of } \\
\text { Maximum at } \\
\text { Compliance } \\
\text { Boundary } \\
\text { (y) }\end{array}$ & \begin{tabular}{|c|} 
Maximum \\
Concentration \\
Compliance \\
Boundary \\
$(\mu \mathrm{g} / \mathrm{L})$
\end{tabular} & \begin{tabular}{|c|} 
Maximum \\
Release Rate \\
at Compliance \\
Boundary \\
$(\mathrm{Kg} / \mathrm{y})$
\end{tabular} & \begin{tabular}{|c|} 
Source \\
Rate \\
$(\mathrm{Kg} / \mathrm{y})$ \\
\end{tabular} & \begin{tabular}{|c|} 
Source \\
Duration \\
(y)
\end{tabular} & \begin{tabular}{|c|} 
1-D \\
Streamtub \\
e Length \\
(m)
\end{tabular} & $\begin{array}{l}\text { Base } \\
\text { Porosity } \\
\left(\mathrm{m}^{3} / \mathrm{m}^{3}\right)\end{array}$ & $\begin{array}{c}\text { Bulk } \\
\text { Densty } \\
\left(\mathrm{Kg} / \mathrm{m}^{3}\right)\end{array}$ & $\begin{array}{c}\text { Remaining } \\
\text { Carbon } \\
\text { Tetrachloride } \\
\text { Source } \\
(\mathrm{Kg})\end{array}$ & \begin{tabular}{|c|} 
Volume at or \\
above Fixed \\
Source \\
$\begin{array}{c}\text { Concentration } \\
(\mathrm{m} 3)\end{array}$ \\
\end{tabular} & $\begin{array}{l}\text { Porosity } \\
\left(\mathrm{m}^{3} / \mathrm{m}^{3}\right)\end{array}$ & $\begin{array}{c}\mathrm{Kd} \\
\left(\mathrm{m}^{3} / \mathrm{Kg}\right)\end{array}$ & Retardation & $\left|\begin{array}{c}\text { First Order } \\
\text { Rate } \\
\text { Constant } \\
(1 / y)\end{array}\right|$ & $\begin{array}{c}\text { Longitudinal } \\
\text { Dispersivity } \\
(\mathrm{m})\end{array}$ & $\begin{array}{c}\text { Required } \\
\text { Source } \\
\text { Cleanup } \\
\text { Percentage } \\
(5 \mu g / L \\
\text { Compliance) } \\
\end{array}$ & \begin{tabular}{|c|} 
Required \\
Source \\
Cleanup \\
Percentage \\
(50 gig/L \\
Compliance)
\end{tabular} & \begin{tabular}{|c} 
Required \\
Source \\
Cleanup \\
Percentage \\
(500 ug/L \\
Compliance) \\
\end{tabular} & \begin{tabular}{|c|} 
Abiotic \\
Reaction \\
Half Time \\
(y)
\end{tabular} & $\begin{array}{c}\text { Carbon } \\
\text { Tetrachloride } \\
\text { Travel Time (y) }\end{array}$ \\
\hline & 29.19 & & & \begin{tabular}{|r|}
35.114395 \\
\end{tabular} & & 145.1211 & 1550.43 & & $\begin{array}{l}0.1 \\
\end{array}$ & 1950 & & 1875000 & 0.109 & $1.69 \mathrm{E}-04$ & & $\mid 1.48 \mathrm{E}-02$ & & 85.76 & & & & 313.4 \\
\hline 197 & 28.23 & 3000 & 2190 & 15.158705 & 0.6631 & \begin{tabular}{|l|}
131.239 \\
\end{tabular} & \begin{tabular}{|l|}
1714.43 \\
\end{tabular} & 5125 & 0.1 & 1950 & 225000 & 1612500 & 0.1127 & \begin{tabular}{|l|}
$3.38 \mathrm{E}-04$ \\
\end{tabular} & 2.516 & $1.21 \mathrm{E}-02$ & 18.66 & 67.02 & & & 57.1 & 456.8 \\
\hline 198 & 55.81 & 3000 & 3060 & 5.179683 & 0.1756 & \begin{tabular}{|l|l|}
101.7009 \\
\end{tabular} & \begin{tabular}{|l|}
2212.37 \\
\end{tabular} & 5125 & 0.1 & 1950 & 225000 & 1100000 & 0.057 & \begin{tabular}{|l|}
$4.57 \mathrm{E}-04$ \\
\end{tabular} & 5.052 & $1.62 \mathrm{E}-02$ & 29.39 & 3.47 & & 0 & 42.7 & 463.9 \\
\hline 199 & 33.81 & 3000 & 1690 & 81.597334 & 4.5558 & \begin{tabular}{|l|l|}
167.4988 \\
\end{tabular} & \begin{tabular}{|r|}
1343.29 \\
\end{tabular} & 5125 & 0.1 & 1950 & 225000 & 2325000 & 0.0941 & \begin{tabular}{|l|}
$2.70 \mathrm{E}-04$ \\
\end{tabular} & 2.448 & \begin{tabular}{|l|}
$1.04 \mathrm{E}-02$ \\
\end{tabular} & 40.07 & $\begin{array}{r}93.87 \\
\end{array}$ & & & 66.8 & 371.2 \\
\hline 200 & 48.94 & 3000 & 1760 & 273.362682 & 12.6291 & $\begin{array}{l}138.5974 \\
\end{array}$ & \begin{tabular}{|l|}
1623.41 \\
\end{tabular} & 5125 & 0.1 & 1950 & 225000 & 1750000 & 0.065 & $1.76 \mathrm{E}-04$ & 2.372 & \begin{tabular}{|l|l|}
$1.03 \mathrm{E}-02$ \\
\end{tabular} & 57.92 & 98.17 & & & 67.5 & 248.3 \\
\hline 201 & 26.96 & 3000 & 1610 & 308.652439 & 15.7167 & \begin{tabular}{|l|}
152.7612 \\
\end{tabular} & 1472.89 & 5125 & 0.1 & 1950 & 225000 & 2025000 & 0.118 & $1.55 \mathrm{E}-04$ & 1.663 & \begin{tabular}{|l|}
$7.48 \mathrm{E}-03$ \\
\end{tabular} & 52.69 & 98.38 & 83.8 & & 92.7 & 316.1 \\
\hline 202 & 44.37 & 3000 & 3200 & \begin{tabular}{|l|l|}
5.270166 \\
\end{tabular} & 0.2317 & 131.9164 & 1705.63 & 5125 & 0.1 & 1950 & 225000 & 1625000 & 0.0717 & \begin{tabular}{|l|}
$6.17 \mathrm{E}-04$ \\
\end{tabular} & 5.351 & \begin{tabular}{|c|}
$1.22 \mathrm{E}-02$ \\
\end{tabular} & 31.54 & 5.13 & 0 & & 56.7 & 618.1 \\
\hline & & & 2350 & 79.136375 & 3.6211 & \begin{tabular}{|l|l|}
137.2743 \\
\end{tabular} & 1639.05 & 5125 & 0.1 & & 225000 & 1725000 & 0.1323 & \begin{tabular}{|l|}
$4.27 \mathrm{E}-04$ \\
\end{tabular} & & $6.81 \mathrm{E}-03$ & 27.58 & 93.68 & & & & \\
\hline 204 & 39.08 & 3000 & 2370 & 58.516489 & 2.2739 & \begin{tabular}{|l|l|}
116.5786 \\
\end{tabular} & 1930.03 & 5125 & 0.1 & 1950 & 225000 & 1350000 & 0.0814 & \begin{tabular}{|l|}
$3.86 \mathrm{E}-04$ \\
\end{tabular} & 3.396 & $1.04 \mathrm{E}-02$ & $\begin{array}{ll}68.97 \\
\end{array}$ & $\begin{array}{l}91.46 \\
\end{array}$ & & & & 445.3 \\
\hline 205 & 56.31 & 3000 & 1710 & 780.12345 & 32.5212 & \begin{tabular}{|l|l|}
125.0616 \\
\end{tabular} & 1799.11 & 5125 & 0.1 & 1950 & 225000 & 1500000 & 0.0565 & \begin{tabular}{|l|l|}
$1.41 \mathrm{E}-04$ \\
\end{tabular} & 2.261 & \begin{tabular}{|l|}
$6.69 \mathrm{E}-03$ \\
\end{tabular} & $\begin{array}{l}37.96 \\
\end{array}$ & $\begin{array}{l}99.36 \\
\end{array}$ & & 35.91 & & 205.8 \\
\hline 206 & 49.55 & 3000 & 2160 & 483.522313 & \begin{tabular}{ll|l|}
21.0429 \\
\end{tabular} & \begin{tabular}{|l|l|}
130.5599 \\
\end{tabular} & $\begin{array}{l}1723.35 \\
19300\end{array}$ & 5125 & 0.1 & $\begin{array}{l}1950 \\
1950\end{array}$ & 225000 & 1600000 & 0.0642 & \begin{tabular}{|l|l|}
$2.77 \mathrm{E}-04$ \\
\end{tabular} & 3.184 & \begin{tabular}{|l|}
$5.67 \mathrm{E}-03$ \\
\end{tabular} & 19.51 & 98.97 & & 0 & & 329.3 \\
\hline 207 & 24.14 & $\begin{array}{l}3000 \\
3300 \\
3300\end{array}$ & 2030 & $\begin{array}{r}.99374 \\
1255223 \\
\end{array}$ & $\begin{array}{l}0.2285 \\
0.878 \\
\end{array}$ & \begin{tabular}{|l|l|}
137.2743 \\
116576 \\
\end{tabular} & $\begin{array}{r}1639.05 \\
193003\end{array}$ & 5125 & 0.1 & $\begin{array}{l}1950 \\
1950\end{array}$ & 225000 & 1725000 & 0.1318 & \begin{tabular}{|l|l|}
$3.56 \mathrm{E}-04$ \\
\end{tabular} & 2.365 & $1.45 \mathrm{E}-02$ & 46.99 & 0 & & 0 & 47.8 & 502.1 \\
\hline$\frac{208}{209}$ & $\frac{32.01}{27.12}$ & $\begin{array}{l}3000 \\
3000 \\
\end{array}$ & $\begin{array}{l}2310 \\
1330\end{array}$ & $\begin{array}{r}12.553323 \\
436.189728\end{array}$ & $\begin{array}{r}0.4878 \\
17.7772\end{array}$ & \begin{tabular}{|l|}
116.52866 \\
12.2668 \\
\end{tabular} & $\begin{array}{l}1930.03 \\
1840.24\end{array}$ & $\begin{array}{l}5125 \\
5125\end{array}$ & $\begin{array}{l}0.1 \\
0.1\end{array}$ & $\begin{array}{l}\frac{1950}{1950} \\
1950\end{array}$ & $\frac{225000}{225000}$ & $\begin{array}{l}1350000 \\
1450000\end{array}$ & \begin{tabular}{|l|l|}
0.0994 \\
0.173
\end{tabular} & \begin{tabular}{|l|}
$3.73 \mathrm{E}-04$ \\
$1.09 \mathrm{E}-04$ \\
\end{tabular} & \begin{tabular}{r|r}
2.898 \\
1.47
\end{tabular} & \begin{tabular}{|l|}
$1.39 \mathrm{E}-02$ \\
$7.31 \mathrm{E}-03$
\end{tabular} & \begin{tabular}{|l|l|}
56.77 & 47.97 \\
\end{tabular} & \begin{tabular}{|c|}
60.17 \\
98.85
\end{tabular} & $\begin{array}{r}0 \\
88.54 \\
\end{array}$ & & $\begin{array}{r}50 \\
97.3 \\
\end{array}$ & $\frac{464}{277.8}$ \\
\hline 210 & 52.24 & 3000 & 670 & 872.81002 & 26.3606 & \begin{tabular}{|l|}
90.6061 \\
\end{tabular} & \begin{tabular}{|l|}
104.24 \\
2483.28
\end{tabular} & 5125 & $\begin{array}{l}0.1 \\
0.1\end{array}$ & 1950 & 225000 & $\frac{1450000}{925000}$ & $\begin{array}{l}0.110 \\
0.0609 \\
\end{array}$ & \begin{tabular}{|l|}
$1.09 \mathrm{E}-04$ \\
$9.72 \mathrm{E}-05$ \\
\end{tabular} & $\begin{array}{r}1.84 \\
1.807 \\
\end{array}$ & \begin{tabular}{|l|}
$1.13 \mathrm{E}-03$ \\
$7.00 \mathrm{E}-03$ \\
\end{tabular} & $\begin{array}{l}41.97 \\
12.18\end{array}$ & $\begin{array}{l}90.60 \\
99.43\end{array}$ & $\begin{array}{l}80.54 \\
94.27\end{array}$ & 42.71 & $\begin{array}{r}71.5 \\
99 \\
\end{array}$ & $\frac{271.0}{177.3}$ \\
\hline 211 & 58.05 & 3000 & 2710 & 771.541327 & 26.1555 & \begin{tabular}{|r|}
101.7009 \\
\end{tabular} & \begin{tabular}{|r|}
2212.37 \\
\end{tabular} & 5125 & 0.1 & 1950 & 225000 & 1100000 & 0.0548 & \begin{tabular}{|l|}
$3.22 \mathrm{E}-04$ \\
\end{tabular} & 3.974 & 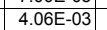 & 47.9 & 99.35 & 93.52 & 35.19 & 170.6 & 350.8 \\
\hline 212 & 36.48 & 3000 & 1910 & 219.468767 & 9.5513 & \begin{tabular}{|l|}
130.5599 \\
\end{tabular} & 1723.35 & 5125 & 0.1 & 1950 & 225000 & 1600000 & 0.0872 & \begin{tabular}{|l|}
$2.24 \mathrm{E}-04$ \\
\end{tabular} & 2.296 & $8.76 \mathrm{E}-03$ & 68.28 & 97.72 & 77.22 & 0 & & 322.5 \\
\hline 213 & 29.03 & 3000 & 2430 & 51.146443 & 2.3404 & \begin{tabular}{|l|}
137.2743 \\
\end{tabular} & 1639.05 & 5125 & 0.1 & 1950 & 225000 & 1725000 & 0.1096 & \begin{tabular}{|l|}
$4.86 \mathrm{E}-04$ \\
\end{tabular} & 3.241 & \begin{tabular}{|l|}
$7.98 \mathrm{E}-03$ \\
\end{tabular} & 48.67 & 90.22 & 2.24 & & 86.8 & 572.2 \\
\hline 214 & 26.53 & 3000 & 1850 & 7.63571 & 0.3792 & \begin{tabular}{|l|l|}
148.9657 \\
\end{tabular} & 1510.42 & 5125 & 0.1 & 1950 & 225000 & 1950000 & 0.1199 & $3.41 \mathrm{E}-04$ & 2.437 & $1.50 \mathrm{E}-02$ & 63.29 & 34.52 & 0 & & 46.3 & 470.8 \\
\hline 215 & 39.18 & 3000 & 2570 & 104.709233 & 3.9424 & \begin{tabular}{|l|}
112.9522 \\
\end{tabular} & 1991.99 & 5125 & 0.1 & 1950 & 225000 & 1287500 & 0.0812 & \begin{tabular}{|l|}
$3.53 \mathrm{E}-04$ \\
\end{tabular} & 3.195 & \begin{tabular}{|l|}
$.29 \mathrm{E}-03$ \\
\end{tabular} & 15.83 & 95.22 & 52.25 & & 83.6 & 417.9 \\
\hline 216 & 27.5 & 3000 & 1480 & 248.06424 & 10.9638 & \begin{tabular}{|l|}
132.592 \\
\end{tabular} & 1696.94 & 5125 & 0.1 & 1950 & 225000 & 1637500 & 0.1157 & \begin{tabular}{|l|l|}
$1.40 \mathrm{E}-04$ \\
\end{tabular} & 1.612 & $8.56 \mathrm{E}-03$ & 39.98 & 97.98 & 79.84 & & 81 & 300.5 \\
\hline 217 & 41.86 & 3000 & 930 & 496.268951 & 29.8521 & \begin{tabular}{|l|}
180.459 \\
\end{tabular} & 1246.82 & 5125 & 0.1 & 1950 & 225000 & 2600000 & 0.076 & \begin{tabular}{|l|l|}
$1.04 \mathrm{E}-04$ \\
\end{tabular} & 1.694 & $8.81 \mathrm{E}-03$ & 25.89 & 98.99 & 89.92 & & 78.7 & 207.4 \\
\hline 218 & 23.97 & 3000 & 1330 & 181.319361 & 7.8086 & \begin{tabular}{|l|l|}
129.1963 \\
\end{tabular} & \begin{tabular}{|l|l|}
1741.54 \\
\end{tabular} & 5125 & 0.1 & 1950 & 225000 & 1575000 & 0.1327 & \begin{tabular}{|l|l|}
$1.33 \mathrm{E}-04$ \\
\end{tabular} & 1.507 & $8.94 \mathrm{E}-03$ & 31.91 & $\begin{array}{l}97.24 \\
\end{array}$ & 72.42 & & 77.5 & 322.2 \\
\hline 219 & 32.73 & 3000 & 2720 & 5.458467 & 0.2015 & \begin{tabular}{|l|l|l|l|l|l|} 
\\
\end{tabular} & \begin{tabular}{|l|}
2031.64 \\
\end{tabular} & 5125 & 0.1 & 1950 & 225000 & 1250000 & 0.0972 & \begin{tabular}{|l|}
$5.38 \mathrm{E}-04$ \\
\end{tabular} & 3.798 & $1.34 \mathrm{E}-02$ & 56.08 & 8.4 & & & 51.8 & 594.8 \\
\hline 220 & $\begin{array}{r}24.1 \\
27.54 \\
\end{array}$ & $\begin{array}{l}3000 \\
33000 \\
3\end{array}$ & 2400 & \begin{tabular}{r|r|}
8.782058 \\
130918
\end{tabular} & $\begin{array}{r}0.3 \\
0.534 \\
\end{array}$ & \begin{tabular}{|l|l|}
102.4699 \\
1144096
\end{tabular} & \begin{tabular}{|l|}
2195.77 \\
1966.62 \\
\end{tabular} & 5125 & 0.1 & $\begin{array}{l}1950 \\
1050 \\
1050\end{array}$ & 225000 & 1112500 & $\begin{array}{l}0.132 \\
01155\end{array}$ & \begin{tabular}{|l|}
$3.34 \mathrm{E}-04$ \\
7050
\end{tabular} & \begin{tabular}{r|r}
2.28 \\
4008
\end{tabular} & \begin{tabular}{|l|}
$1.49 \mathrm{E}-02$ \\
0.22502
\end{tabular} & $\begin{array}{l}90.82 \\
7720\end{array}$ & 43.07 & 0 & & 46.6 & 484.8 \\
\hline 221 & $\begin{array}{l}27.54 \\
50.58 \\
\end{array}$ & 3000 & 2930 & $\begin{array}{r}13.909158 \\
16504975\end{array}$ & 0.5304 & 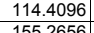 & \begin{tabular}{|l|}
1966.62 \\
14913 \\
\end{tabular} & $\begin{array}{l}5125 \\
51125 \\
\end{array}$ & 0.1 & $\begin{array}{l}1950 \\
1950\end{array}$ & 225000 & 1312500 & 0.1155 & 7.04E-04 & 4.082 & \begin{tabular}{|l|}
$9.32 \mathrm{E}-03$ \\
70502
\end{tabular} & 77.28 & 64.05 & 0 & & 74.4 & 759.4 \\
\hline$\frac{222}{223}$ & $\begin{array}{l}50.58 \\
22.26 \\
\end{array}$ & 3000 & $\frac{2200}{2590}$ & $\begin{array}{r}165.945975 \\
28.586878\end{array}$ & \begin{tabular}{|l|}
8.5886 \\
1.0902
\end{tabular} & \begin{tabular}{|c|}
155.2656 \\
114.4096 \\
\end{tabular} & \begin{tabular}{|l|}
1449.13 \\
1966.62
\end{tabular} & $\begin{array}{l}5125 \\
5125 \\
\end{array}$ & $\begin{array}{l}0.1 \\
0.1\end{array}$ & $\begin{array}{l}1950 \\
1950 \\
\end{array}$ & $\begin{array}{l}225000 \\
225000\end{array}$ & $\begin{array}{l}2075000 \\
1312500\end{array}$ & $\begin{array}{l}0.0629 \\
0.1429\end{array}$ & \begin{tabular}{|l|}
$4.25 \mathrm{E}-04$ \\
$4.46 \mathrm{E}-04$ \\
\end{tabular} & $\begin{array}{l}4.413 \\
258 \\
\end{array}$ & \begin{tabular}{|l|}
$7.38 \mathrm{E}-03$ \\
$8.45 \mathrm{E}-03$
\end{tabular} & $\begin{array}{l}72.24 \\
33.03\end{array}$ & $\frac{96.99}{82.51}$ & 69.87 & $t$ & $\begin{array}{l}93.9 \\
8.1\end{array}$ & $\begin{array}{l}447.1 \\
5935\end{array}$ \\
\hline 224 & $\begin{array}{l}22.20 \\
42.42\end{array}$ & 3000 & 860 & \begin{tabular}{|l}
20.000010 \\
361.906406
\end{tabular} & $\begin{array}{l}1.09615 \\
12.3615\end{array}$ & \begin{tabular}{|l|}
114.4090 \\
102.4699 \\
\end{tabular} & \begin{tabular}{|l|}
2195.77 \\
\end{tabular} & 5125 & $\begin{array}{l}0.1 \\
0.1 \\
\end{array}$ & 1950 & 2250000 & $\begin{array}{l}13112500 \\
112500\end{array}$ & $\begin{array}{r}0.1249 \\
0.075 \\
\end{array}$ & \begin{tabular}{|l|}
$4.46 \mathrm{E}-04$ \\
$1.26 \mathrm{E}-04$ \\
\end{tabular} & $\begin{array}{l}2.588 \\
1.851 \\
\end{array}$ & \begin{tabular}{|l|}
$8.45 \mathrm{E}-03$ \\
$9.54 \mathrm{E}-03$ \\
\end{tabular} & $\begin{array}{l}33.03 \\
11.89 \\
\end{array}$ & $\begin{array}{l}82.51 \\
98.62 \\
\end{array}$ & \begin{tabular}{r|}
0 \\
86.18
\end{tabular} & & $\begin{array}{l}82.1 \\
72.6\end{array}$ & $\frac{593.5}{223.6}$ \\
\hline 225 & 30.24 & 3000 & 2280 & 8.543132 & 0.4077 & \begin{tabular}{|l|}
143.1797 \\
\end{tabular} & \begin{tabular}{|l|}
1571.45 \\
\end{tabular} & 5125 & 0.1 & 1950 & 225000 & 1837500 & 0.1052 & \begin{tabular}{|l|}
$4.81 \mathrm{E}-04$ \\
\end{tabular} & 3.312 & \begin{tabular}{|l|} 
\\
$1.24 \mathrm{E}-02$ \\
\end{tabular} & 48.73 & 41.47 & & & 56.1 & 561.2 \\
\hline 226 & 26.16 & 3000 & 3120 & 17.828979 & 0.9041 & \begin{tabular}{|l|}
152.1319 \\
\end{tabular} & 1478.98 & 5125 & 0.1 & 1950 & 225000 & 2012500 & 0.1216 & \begin{tabular}{|l|}
$7.14 \mathrm{E}-04$ \\
\end{tabular} & 3.969 & \begin{tabular}{|l|}
$7.31 \mathrm{E}-03$ \\
\end{tabular} & 36.01 & 71.96 & & & 94.8 & 777.4 \\
\hline 227 & 27.24 & 3000 & 2230 & 36.710461 & 1.3911 & \begin{tabular}{|c|}
113.6821 \\
\end{tabular} & 1979.2 & 5125 & 0.1 & 1950 & 225000 & 1300000 & 0.1168 & \begin{tabular}{|l|}
$2.62 \mathrm{E}-04$ \\
\end{tabular} & 2.133 & & 29.89 & 86.38 & & & 59.9 & 401.3 \\
\hline 228 & 21.97 & 3000 & 1940 & 4.017248 & 0.202 & \begin{tabular}{|l|l|}
150.8694 \\
\end{tabular} & \begin{tabular}{|l|}
1491.36 \\
\end{tabular} & 5125 & 0.1 & 1950 & 225000 & 1987500 & 0.1448 & \begin{tabular}{|l|}
$3.43 \mathrm{E}-04$ \\
\end{tabular} & 2.198 & & 30.72 & 0 & 0 & & 49.4 & 512.6 \\
\hline 229 & 37.43 & 3000 & 2070 & 133.566513 & 5.9931 & \begin{tabular}{|l|l|}
134.6086 \\
\end{tabular} & 1671.51 & 5125 & 0.1 & 1950 & 225000 & 1675000 & 0.085 & \begin{tabular}{|c|}
$3.35 \mathrm{E}-04$ \\
\end{tabular} & 2.99 & $8.49 \mathrm{E}-03$ & 64.65 & 96.26 & 62.57 & & 81.6 & 409.4 \\
\hline 230 & 43.11 & 3000 & 1900 & & 10.2073 & \begin{tabular}{|l|l|}
157.1306 \\
\end{tabular} & \begin{tabular}{|l|}
1431.93 \\
\end{tabular} & 5125 & 0.1 & 1950 & 225000 & 2112500 & 0.0738 & & 3.08 & & 38.12 & 97.43 & 74.34 & & 87.4 & 366.2 \\
\hline 231 & & 3000 & 3600 & & & \begin{tabular}{|l|l|}
136.6103 \\
\end{tabular} & \begin{tabular}{|l|}
1647.02 \\
\end{tabular} & 5125 & 0.1 & & & & & \begin{tabular}{|l|l|}
$6.42 \mathrm{E}-04$ \\
\end{tabular} & 6.73 & & 36.22 & & 0 & & 81.8 & 613.7 \\
\hline 232 & 24. & 3000 & 1630 & \begin{tabular}{|l|}
201.611027 \\
\end{tabular} & 6.0341 & \begin{tabular}{|r|}
89.788 \\
\end{tabular} & \begin{tabular}{|r|}
2505.9 \\
\end{tabular} & 5125 & 0.1 & 1950 & 225000 & 912500 & 0.1295 & \begin{tabular}{|l|}
$1.14 \mathrm{E}-04$ \\
\end{tabular} & 1.446 & $9.43 \mathrm{E}-03$ & $\begin{array}{l}70.8 \\
\end{array}$ & 97.52 & 75.2 & & 73.5 & 301.7 \\
\hline 233 & 46.99 & 3000 & 2320 & 966.229814 & & & \begin{tabular}{|l|}
1930.03 \\
\end{tabular} & 5125 & 0.1 & & 225000 & 1350000 & 0.0677 & \begin{tabular}{|l|l|}
$2.83 \mathrm{E}-04$ \\
\end{tabular} & & & 34.97 & & 94.83 & 48.25 & 203 & 339.8 \\
\hline 234 & 24.28 & $\begin{array}{l}3000 \\
3000\end{array}$ & $\begin{array}{l}1130 \\
3250\end{array}$ & \begin{tabular}{|r|}
163.381206 \\
34501448
\end{tabular} & \begin{tabular}{|l|l|}
5.7877 \\
1.303
\end{tabular} & \begin{tabular}{|l|}
106.2727 \\
124359 \\
\end{tabular} & \begin{tabular}{|r|}
2117.2 \\
18018
\end{tabular} & $\begin{array}{l}5125 \\
5125 \\
\end{array}$ & 0.1 & $\begin{array}{l}1950 \\
1950\end{array}$ & 225000 & $\begin{array}{l}1175000 \\
148700\end{array}$ & $\begin{array}{l}0.131 \\
0119 \\
\end{array}$ & \begin{tabular}{|l|}
$1.07 \mathrm{E}-04$ \\
$727 \mathrm{~F}-04$ \\
\end{tabular} & 1.414 & & 32.42 & $\begin{array}{l}96.94 \\
85.51\end{array}$ & 69.4 & & 69.2 & 298.3 \\
\hline$\frac{235}{236}$ & $\begin{array}{l}28.43 \\
37.69 \\
\end{array}$ & 3000 & $\frac{3250}{2390}$ & $\frac{34.501}{66.681}$ & \begin{tabular}{|l|}
1.4303 \\
2.029 \\
\end{tabular} & \begin{tabular}{|r|}
124.3658 \\
9.8552 \\
\end{tabular} & \begin{tabular}{|l|}
1809.18 \\
2282.06 \\
\end{tabular} & $\begin{array}{l}5125 \\
5125\end{array}$ & $\begin{array}{l}0.1 \\
0.1\end{array}$ & $\begin{array}{l}\frac{1950}{1950} \\
\end{array}$ & $\frac{225000}{225000}$ & $\begin{array}{l}\frac{1487500}{1050000} \\
\end{array}$ & $\begin{array}{l}0.1119 \\
0.0844\end{array}$ & \begin{tabular}{|l|}
$7.27 \mathrm{E}-04$ \\
$2.18 \mathrm{E}-04$ \\
\end{tabular} & $\begin{array}{l}4.286 \\
2.307\end{array}$ & \begin{tabular}{|l|}
$\mid 6.72 \mathrm{E}-03$ \\
$1.42 \mathrm{E}-02$ \\
\end{tabular} & 53.87 & $\begin{array}{l}85.51 \\
92.15\end{array}$ & \begin{tabular}{r|r|}
0 & \\
21.48
\end{tabular} & & $\begin{array}{c}103.1 \\
48.9\end{array}$ & 772.5 \\
\hline 237 & 33.74 & & 2720 & 6.127 & 0.2014 & \begin{tabular}{|l|}
98.5952 \\
\end{tabular} & \begin{tabular}{|l|}
2282.06 \\
\end{tabular} & 5125 & 0.1 & & 225000 & 1050000 & 0.0943 & \begin{tabular}{|l|}
$4.21 \mathrm{E}-04$ \\
\end{tabular} & 3.256 & \begin{tabular}{|l|}
$1.425 \mathrm{E}-02$ \\
\end{tabular} & 50.14 & 18.41 & & & 46.2 & $\begin{array}{l}31.1 . \\
494.6\end{array}$ \\
\hline 238 & & 3000 & 2930 & 24.342 & 0.8127 & \begin{tabular}{|l|}
100.1541 \\
\end{tabular} & 2246 & 5125 & 0.1 & & 225000 & 1075000 & 0.1 & $4.56 \mathrm{E}-04$ & 3.15 & $9.57 \mathrm{E}$ & 27.8 & 79.46 & & & 72.4 & 544.5 \\
\hline 239 & 30.27 & 3000 & 2570 & 10.970017 & 0.6556 & \begin{tabular}{|l|}
179.3004 \\
\end{tabular} & 1254 & 5125 & 0.1 & 195 & 225000 & 2575000 & 0.1051 & \begin{tabular}{|l|}
$6.93 \mathrm{E}-04$ \\
\end{tabular} & 4.331 & \begin{tabular}{|c|}
$9.41 \mathrm{E}-03$ \\
\end{tabular} & 62.09 & 54.42 & & & 73.6 & 733.3 \\
\hline 240 & 35. & & 2350 & 266.563727 & 12.02 & \begin{tabular}{|l|}
135.2775 \\
\end{tabular} & 1663 & 5125 & 0.1 & 1950 & & & 0.089 & \begin{tabular}{|c|}
$3.80 \mathrm{E}-04$ \\
\end{tabular} & & & 26.35 & 98.12 & 81.24 & & 124.8 & 452.7 \\
\hline 241 & 46. & 3000 & 2740 & & & \begin{tabular}{|l|l|}
116.5786 \\
\end{tabular} & & 5125 & 0.1 & 1950 & & & & 5.77E-04 & 5.2 & & 55.79 & 0 & & & 36.2 & 580.8 \\
\hline 242 & 54.66 & 3000 & 3570 & 69.49997 & 1.7223 & \begin{tabular}{|l|l|}
74.3438 \\
\end{tabular} & \begin{tabular}{|l|}
3026.48 \\
\end{tabular} & 5125 & 0.1 & 1950 & 225000 & 687500 & & 4.78 & 5.153 & & 75.61 & 92.81 & 28.06 & & 69.2 & 483.1 \\
\hline 243 & 27.83 & 3000 & 2800 & $9.989 \mathrm{C}$ & & \begin{tabular}{|l|}
97.8112 \\
\end{tabular} & 2300 & 5125 & 0.1 & 1950 & & & 0.1 & & & & 52.74 & 49.95 & & & 58 & 562.6 \\
\hline 244 & & 3000 & 1880 & 103.3 & $\begin{array}{l}4.0892 \\
9230\end{array}$ & & & & 0.1 & & & & & & & & & 95.16 & 51.61 & & 58.8 & \\
\hline 245 & 59.02 & 3000 & 2400 & 1142.3 & 38.4305 & & 222 & 5125 & 0.1 & 19 & 225 & & & 2.15 & & & 87.95 & 99.56 & 95.62 & 56.23 & 179 & 261.8 \\
\hline 246 & 58.27 & 3000 & 2860 & \begin{tabular}{|l|}
184.417996 \\
\end{tabular} & 7.4728 & \begin{tabular}{|l|}
121.5631 \\
\end{tabular} & 1850 & 5125 & 0.1 & 1950 & 225000 & 1437500 & $\begin{array}{l}0.0546 \\
\end{array}$ & \begin{tabular}{|l|l|}
$5.39 \mathrm{E}-04$ \\
\end{tabular} & 5.989 & \begin{tabular}{|l|}
$6.27 \mathrm{E}-03$ \\
\end{tabular} & 88.07 & $\begin{array}{l}77.29 \\
\end{array}$ & 72.89 & & 110.5 & 526.7 \\
\hline 247 & & & 1610 & & & 221.578 & 1015 & 5125 & & & & & & 3.891 & & & & & $\begin{array}{l}7.56 \\
\end{array}$ & & $\begin{array}{l}82.3 \\
5\end{array}$ & \\
\hline$\frac{248}{249}$ & $\frac{53.29}{60.48}$ & $\begin{array}{l}3000 \\
3000\end{array}$ & $\frac{2660}{2340}$ & $\begin{array}{r}151.889343 \\
405.87538 \\
\end{array}$ & $\begin{array}{r}4.5043 \\
15.3802\end{array}$ & \begin{tabular}{|r|}
88.9661 \\
113621 \\
\end{tabular} & \begin{tabular}{|r|}
2529.05 \\
19792 \\
\end{tabular} & $\begin{array}{l}5125 \\
5125\end{array}$ & $\begin{array}{l}0.1 \\
0.1\end{array}$ & $\begin{array}{l}1950 \\
1950\end{array}$ & $\frac{225000}{225000}$ & \begin{tabular}{r|r|}
9000000 \\
1300000
\end{tabular} & \begin{tabular}{|c|}
0.0597 \\
0.0526
\end{tabular} & \begin{tabular}{|l|}
$2.22 \mathrm{E}-04$ \\
$2.95 \mathrm{E}-04$ \\
\end{tabular} & $\begin{array}{l}2.876 \\
3.389\end{array}$ & \begin{tabular}{|l|}
$1.22 \mathrm{E}-02$ \\
$6.81 \mathrm{E}-03$
\end{tabular} & $\begin{array}{ll}75.68 \\
72.69\end{array}$ & \begin{tabular}{|c|c|}
96.71 \\
98.77
\end{tabular} & $\begin{array}{l}67.08 \\
8.68\end{array}$ & & $\begin{array}{r}57 \\
101.7 \\
\end{array}$ & $\begin{array}{l}276.6 \\
325.3\end{array}$ \\
\hline 250 & 32.1 & 3000 & 2700 & $\begin{array}{l}7.759341 \\
\end{array}$ & 0.2322 & \begin{tabular}{|l|}
89.788 \\
\end{tabular} & 2505.9 & 5125 & 0.1 & 1950 & 225000 & 912500 & 0.0991 & \begin{tabular}{|l|}
$3.13 \mathrm{E}-04$ \\
\end{tabular} & 2.597 & \begin{tabular}{|l|l|} 
& $0.74 \mathrm{E}$ \\
\end{tabular} & 69.53 & 35.56 & & & 39.9 & (3.5.6. \\
\hline 251 & 30.13 & 3000 & 1750 & 1098.65332 & 51.4794 & 140.5704 & $\mid 1600.62$ & 5125 & 0.1 & 19 & 2250 & 1787500 & 0.1056 & $1.61 \mathrm{E}-04$ & 1.773 & \begin{tabular}{|l|l} 
\\
\end{tabular} & \begin{tabular}{|l|l|}
37.16 \\
\end{tabular} & 99.54 & 95.45 & 54.49 & 205.4 & 301.6 \\
\hline 252 & 29.4 & 3000 & 2630 & 6.439 & 0.3518 & 163.877 & 1372 & 5125 & 0.1 & 1950 & 225000 & 2250000 & $0.1 c$ & $6.31 \mathrm{E}-04$ & 3.949 & \begin{tabular}{|l|l|} 
& $1.05 E$ \\
\end{tabular} & 42.6 & 22.35 & 0 & & $\mid 65.8$ & 688.3 \\
\hline 253 & 45.84 & & 3050 & & 1.1251 & \begin{tabular}{|l|l|}
82.2 \\
\end{tabular} & 2735 & 5125 & 0.1 & 1950 & 2256 & 800 & 0.06 & \begin{tabular}{|l|}
$3.45 \mathrm{E}-04$ \\
\end{tabular} & 3.5 & 1.27 & 53.6 & 87.82 & & & 54.8 & 393 \\
\hline 254 & 23.34 & & 1960 & 134.12 & 4.6 & 103.9 & 216 & 512 & 0.1 & & & & & & & & 67. & 96.2 & 62.72 & & 71.8 & 342.4 \\
\hline 255 & 51.9 & 30 & 2340 & & 20.5 & 112.2 & & 5125 & 0.1 & 195 & & & & & & & 65. & 99.09 & 90.89 & 8.89 & 126.1 & 33 \\
\hline 256 & 51.06 & 3000 & 1270 & \begin{tabular}{|r|}
930.342768 \\
\end{tabular} & 22.4 & \begin{tabular}{|l|}
72.5304 \\
\end{tabular} & \begin{tabular}{|l|}
3102.15 \\
\end{tabular} & 5125 & 0.1 & 1950 & 225000 & 662500 & & & 1.7 & & 63.56 & $\begin{array}{l}99.46 \\
\end{array}$ & 94.63 & 46.26 & 100.6 & \\
\hline 257 & & & 11 & & 48.1 & 133.93 & & & 0.1 & & & & & & & & & & 95.36 & 53.65 & 136.4 & \\
\hline 258 & & 30 & $\frac{10}{24}$ & & & \begin{tabular}{|r|}
97.024 \\
1187776 \\
\end{tabular} & & & 0.1 & & & & & \begin{tabular}{|l|l|}
$1.17 \mathrm{E}-04$ \\
$410 \mathrm{E}-04$
\end{tabular} & \begin{tabular}{l|l|}
1.447 \\
454
\end{tabular} & & $\begin{array}{ll}20.96 \\
50.34\end{array}$ & $\begin{array}{l}94.64 \\
54.91 \\
\end{array}$ & $\begin{array}{ll}46.37 \\
\end{array}$ & & $\begin{array}{ll}60.2 \\
423\end{array}$ & \\
\hline$\frac{259}{260}$ & $\begin{array}{l}54.76 \\
31104\end{array}$ & 3000 & $\frac{2470}{1460}$ & 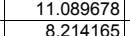 & $\begin{array}{l}0.4389 \\
0.5329\end{array}$ & \begin{tabular}{|l|l|}
118.7276 \\
1946437 \\
\end{tabular} & \begin{tabular}{|c|}
$\mid 1895.09$ \\
1155.96
\end{tabular} & $\begin{array}{l}5125 \\
5125\end{array}$ & $\begin{array}{l}0.1 \\
0.1\end{array}$ & $\begin{array}{l}1950 \\
1950\end{array}$ & $\begin{array}{l}225000 \\
225000\end{array}$ & $\begin{array}{l}1387500 \\
2912500\end{array}$ & $\begin{array}{l}0.0581 \\
0.125\end{array}$ & \begin{tabular}{|c|}
$4.10 \mathrm{E}-04$ \\
$265 \mathrm{E}-04$ \\
\end{tabular} & $\begin{array}{l}4.564 \\
2307\end{array}$ & \begin{tabular}{|l|}
$1.64 \mathrm{E}-02$ \\
$175 \mathrm{E}-02$
\end{tabular} & $\begin{array}{l}50.34 \\
49.61\end{array}$ & $\begin{array}{l}54.91 \\
39.13\end{array}$ & $\frac{0}{0}$ & & $\begin{array}{l}42.3 \\
3.5 \\
\end{array}$ & $\frac{427.2}{381}$ \\
\hline
\end{tabular}




\begin{tabular}{|c|c|c|c|c|c|c|c|c|c|c|c|c|c|c|c|c|c|c|c|c|c|c|}
\hline $\begin{array}{c}\text { Realizatio } \\
n\end{array}$ & $\begin{array}{c}\text { Velocity } \\
(\mathrm{m} / \mathrm{y})\end{array}$ & $\begin{array}{c}\text { Fixed Source } \\
\text { Concentration } \\
\text { (mg/L) }\end{array}$ & $\begin{array}{c}\text { Time of } \\
\text { Maximum at } \\
\text { Compliance } \\
\text { Boundary } \\
(y)\end{array}$ & \begin{tabular}{|c|} 
Maximum \\
Concentration \\
Compliance \\
Boundary \\
$(\mu \mathrm{g} / \mathrm{L})$
\end{tabular} & \begin{tabular}{|c|} 
Maximum \\
Release Rate \\
at Compliance \\
Boundary \\
$(\mathrm{Kg} / \mathrm{y})$
\end{tabular} & \begin{tabular}{|c|} 
Source \\
Rate \\
$(\mathrm{Kg} / \mathrm{y})$ \\
\end{tabular} & \begin{tabular}{|c|} 
Source \\
Duration \\
(y)
\end{tabular} & \begin{tabular}{|c|} 
1-D \\
Streamtub \\
e Length \\
(m)
\end{tabular} & $\begin{array}{l}\text { Base } \\
\text { Porosity } \\
\left(\mathrm{m}^{3} / \mathrm{m}^{3}\right)\end{array}$ & $\begin{array}{c}\text { Bulk } \\
\text { Densty } \\
\left(\mathrm{Kg} / \mathrm{m}^{3}\right)\end{array}$ & $\begin{array}{c}\text { Remaining } \\
\text { Carbon } \\
\text { Tetrachloride } \\
\text { Source } \\
(\mathrm{Kg})\end{array}$ & \begin{tabular}{|c|} 
Volume at or \\
above Fixed \\
Source \\
$\begin{array}{c}\text { Concentration } \\
(\mathrm{m} 3)\end{array}$ \\
\end{tabular} & $\begin{array}{l}\text { Porosity } \\
\left(\mathrm{m}^{3} / \mathrm{m}^{3}\right)\end{array}$ & $\begin{array}{c}\mathrm{Kd} \\
\left(\mathrm{m}^{3} / \mathrm{Kg}\right)\end{array}$ & Retardation & $\begin{array}{c}\text { First Order } \\
\text { Rate } \\
\text { Constant } \\
\text { (1/y) }\end{array}$ & $\begin{array}{c}\text { Longitudinal } \\
\text { Dispersivity } \\
(\mathrm{m})\end{array}$ & $\begin{array}{c}\text { Required } \\
\text { Source } \\
\text { Cleanup } \\
\text { Percentage } \\
(5 \mu g / L \\
\text { Compliance) } \\
\end{array}$ & \begin{tabular}{|c|} 
Required \\
Source \\
Cleanup \\
Percentage \\
(50 gig/L \\
Compliance)
\end{tabular} & \begin{tabular}{|c} 
Required \\
Source \\
Cleanup \\
Percentage \\
(500 ug/L \\
Compliance) \\
\end{tabular} & \begin{tabular}{|c|} 
Abiotic \\
Reaction \\
Half Time \\
(y)
\end{tabular} & $\begin{array}{c}\text { Carbon } \\
\text { Tetrachloride } \\
\text { Travel Time (y) }\end{array}$ \\
\hline & 42.76 & & & \begin{tabular}{|r|} 
\\
42.947434 \\
\end{tabular} & & \begin{tabular}{|l|l|}
174.0393 \\
\end{tabular} & \begin{tabular}{|l|}
1292.81 \\
\end{tabular} & & & 1950 & & & 0.0744 & & & \begin{tabular}{|l|}
$1.87 \mathrm{E}-02$ \\
\end{tabular} & & & & & & \\
\hline 262 & 28.05 & 3000 & 860 & \begin{tabular}{|c|}
126.798309 \\
\end{tabular} & 5.5756 & \begin{tabular}{|l|l|}
131.9164 \\
\end{tabular} & \begin{tabular}{|l|}
1705.63 \\
\end{tabular} & 5125 & 0.1 & 1950 & 225000 & 1625000 & 0.1134 & $7.40 \mathrm{E}-05$ & 1.33 & \begin{tabular}{|l|} 
\\
\end{tabular} & 34.91 & 96.06 & 60.57 & & 51.7 & 242.9 \\
\hline 263 & 22.95 & 3000 & 2000 & 168.758695 & $\begin{array}{l}\mid .0370 \\
6.8779 \\
\end{array}$ & \begin{tabular}{|l|}
122.2668 \\
\end{tabular} & \begin{tabular}{|l|}
1840.24 \\
\end{tabular} & 5125 & 0.1 & 1950 & 225000 & 1450000 & 0.1386 & \begin{tabular}{|l|l|}
$.401 \mathrm{E}-04$ \\
\end{tabular} & 1.695 & \begin{tabular}{|l|}
$1.04 \mathrm{E}-02$ \\
$7.94 \mathrm{E}-03$ \\
\end{tabular} & 年4..16 & 97.04 & 70.37 & & $\begin{array}{l}87.3 \\
87.3 \\
\end{array}$ & 378.5 \\
\hline 264 & 46.99 & 3000 & 3730 & 15.317338 & 0.6526 & \begin{tabular}{|l|}
127.8255 \\
\end{tabular} & 1760.21 & 5125 & 0.1 & 1950 & 225000 & 1550000 & 0.0677 & $6.45 \mathrm{E}-04$ & 5.818 & \begin{tabular}{|l|}
$1.24 \mathrm{E}-03$ \\
\end{tabular} & 23.95 & 67.36 & $\begin{array}{r}0 \\
\end{array}$ & & 75 & 634.5 \\
\hline 265 & 37.04 & 3000 & 1680 & 415.503657 & 20.0995 & \begin{tabular}{|l|}
145.1211 \\
\end{tabular} & 1550.43 & 5125 & 0.1 & 1950 & 225000 & 1875000 & 0.0859 & $1.67 \mathrm{E}-04$ & 1.985 & $7.57 \mathrm{E}-03$ & 66.88 & 98.8 & 87.97 & & 91.6 & 274.7 \\
\hline 266 & 22.06 & 3000 & 2600 & 3.985642 & 0.1885 & \begin{tabular}{|l|}
141.878 \\
\end{tabular} & 1585.87 & 5125 & 0.1 & 1950 & 225000 & 1812500 & 0.1442 & $6.34 \mathrm{E}-04$ & 3.223 & $\mid 1.06 \mathrm{E}-02$ & 47.86 & 0 & & & 65.6 & 748.7 \\
\hline 267 & 23.15 & 3000 & 1520 & 53.742142 & 2.2528 & \begin{tabular}{|l|l|}
125.7554 \\
\end{tabular} & 1789.19 & 5125 & 0.1 & 1950 & 225000 & 1512500 & 0.1374 & $1.76 \mathrm{E}-04$ & 1.647 & \begin{tabular}{|l|}
$1.14 \mathrm{E}-02$ \\
\end{tabular} & 27.37 & 90.7 & 6.96 & & 60.7 & 364.5 \\
\hline 268 & 45.32 & 3000 & 3860 & 18.063194 & 0.453 & \begin{tabular}{|l|}
75.2423 \\
\end{tabular} & 2990.34 & 5125 & 0.1 & & 225000 & 700000 & 0.0702 & $5.36 \mathrm{E}-04$ & 4.857 & \begin{tabular}{|l|}
$1.10 \mathrm{E}-02$ \\
\end{tabular} & 36.59 & 72.32 & & & 63.3 & 549.3 \\
\hline 269 & 29.9 & 3000 & 2320 & 68.241432 & 3.6024 & \begin{tabular}{|l|l|}
158.3679 \\
\end{tabular} & 1420.74 & 5125 & 0.1 & 1950 & 225000 & 2137500 & 0.1064 & 5.51E-04 & 3.619 & \begin{tabular}{|l|}
$7.10 \mathrm{E}-03$ \\
\end{tabular} & 74.71 & $\begin{array}{l}92.67 \\
\end{array}$ & 26.73 & & & 620.4 \\
\hline 270 & 55.33 & 3000 & 4890 & 5.118094 & 0.18 & \begin{tabular}{|l|l|}
105.5176 \\
\end{tabular} & 2132.35 & 5125 & 0.1 & 1950 & 225000 & 1162500 & 0.0575 & $8.02 \mathrm{E}-04$ & 8.05 & \begin{tabular}{|l|}
$1.03 \mathrm{E}-02$ \\
\end{tabular} & 26.45 & 2.31 & & 0 & & 745.7 \\
\hline 271 & 23.55 & 3000 & 1850 & 57.411454 & 2.6776 & \begin{tabular}{|l|l|}
139.9143 \\
\end{tabular} & \begin{tabular}{|l|}
1608.13 \\
\end{tabular} & 5125 & 0.1 & $\begin{array}{l}1950 \\
1950\end{array}$ & 225000 & 1775000 & 0.1351 & $2.49 \mathrm{E}-04$ & 1.933 & $1.02 \mathrm{E}-02$ & 56.85 & 91.29 & & & 67.9 & 420.7 \\
\hline$\frac{272}{273}$ & $\begin{array}{l}48.13 \\
27.03\end{array}$ & $\begin{array}{l}3000 \\
3300 \\
3300\end{array}$ & $\begin{array}{l}2090 \\
1990\end{array}$ & \begin{tabular}{r|r|}
693.308498 \\
\end{tabular} & $\begin{aligned} 25.9343 \\
1.9859 \\
\end{aligned}$ & \begin{tabular}{|l|l|}
112.2199 \\
128510 \\
\end{tabular} & \begin{tabular}{|l|}
2004.99 \\
17501 \\
\end{tabular} & 5125 & 0.1 & $\begin{array}{l}1950 \\
1950\end{array}$ & 225000 & 1275000 & 0.0661 & $\begin{array}{l}1.50 \mathrm{E}-04 \\
272-\end{array}$ & 2.143 & $6.71 \mathrm{E}-03$ & 75.14 & 99.28 & $\begin{array}{l}92.79 \\
9\end{array}$ & & 103.2 & 228.2 \\
\hline $\begin{array}{l}273 \\
274 \\
\end{array}$ & 27.03 & $\begin{array}{l}3000 \\
3000 \\
\end{array}$ & \begin{tabular}{|l|l|}
1990 & \\
3160
\end{tabular} & $\begin{array}{r}46.358588 \\
4490168 \\
\end{array}$ & $\begin{array}{r}1.9859 \\
0.28\end{array}$ & \begin{tabular}{|c|}
128.5118 \\
1656929 \\
\end{tabular} & \begin{tabular}{|l|}
1750.81 \\
137.93 \\
\end{tabular} & $\begin{array}{l}5125 \\
525 \\
\end{array}$ & 0.1 & $\begin{array}{c}1950 \\
1950 \\
-1950\end{array}$ & $\begin{array}{r}225000 \\
225000\end{array}$ & $\begin{array}{r}1562500 \\
2287500\end{array}$ & $\begin{array}{l}0.1177 \\
0.0817\end{array}$ & 2.73E-04 & 2.173 & \begin{tabular}{|l|}
$1.13 \mathrm{E}-02$ \\
$111 \mathrm{E}-2$
\end{tabular} & 65.03 & 89.21 & 0 & 0 & \begin{tabular}{ll|}
61.4 \\
627
\end{tabular} & $\begin{array}{l}412.1 \\
672 \\
\end{array}$ \\
\hline 275 & 34.21 & 3000 & $\begin{array}{l}3100 \\
2320\end{array}$ & $\begin{array}{r}4.400100 \\
322.260207\end{array}$ & $\begin{array}{r}0.248 \\
11.5772\end{array}$ & \begin{tabular}{|l|}
$\mid 166.692929$ \\
107.7748 \\
\end{tabular} & \begin{tabular}{|l|}
$1357 / .93$ \\
2087.69 \\
\end{tabular} & 5125 & $\begin{array}{l}0.1 \\
0.1\end{array}$ & $\begin{array}{l}\frac{1950}{1950} \\
1950\end{array}$ & $\begin{array}{l}225000 \\
225000\end{array}$ & $\begin{array}{l}2285000 \\
1200000\end{array}$ & $\begin{array}{l}0.08811 \\
0.093 \\
\end{array}$ & \begin{tabular}{|l|}
$.644-04$ \\
$2.66 \mathrm{E}-04$
\end{tabular} & $\begin{array}{l}5.106 \\
2.444\end{array}$ & \begin{tabular}{|l|}
$1.11 \mathrm{E}-02$ \\
$6.52 \mathrm{E}-03$ \\
\end{tabular} & 28.19 & $\begin{array}{r}0 \\
98.45 \\
\end{array}$ & \begin{tabular}{r|r}
0 & \\
84.48
\end{tabular} & & $\begin{array}{r}62 . .1 \\
106.3 \\
\end{array}$ & $\frac{672.1}{366.2}$ \\
\hline 276 & 29.9 & 3000 & 3470 & 120.592342 & 4.7438 & \begin{tabular}{|l|}
118.0135 \\
\end{tabular} & \begin{tabular}{|l|}
1906.56 \\
\end{tabular} & 5125 & 0.1 & 1950 & 225000 & 1375000 & 0.1064 & $6.49 \mathrm{E}-04$ & 4.083 & $4.86 \mathrm{E}-03$ & 30.7 & 95.85 & 58.54 & & 142.6 & 200.4. \\
\hline 277 & 23.51 & 3000 & 2330 & 240.68411 & 9.2951 & \begin{tabular}{|l|}
115.8579 \\
\end{tabular} & 1942.03 & 5125 & 0.1 & 1950 & 225000 & 1337500 & 0.1353 & $3.37 \mathrm{E}-04$ & 2.261 & \begin{tabular}{|l|}
$5.42 \mathrm{E}-03$ \\
\end{tabular} & 52.57 & 97.92 & 79.23 & & 127.9 & 492.7 \\
\hline 278 & 48.79 & 3000 & 1870 & 56.888718 & 2.7028 & \begin{tabular}{|l|}
142.5296 \\
\end{tabular} & 1578.62 & 5125 & 0.1 & 1950 & 225000 & 1825000 & 0.0652 & $2.45 \mathrm{E}-04$ & 2.898 & \begin{tabular}{|l|}
$1.40 \mathrm{E}-02$ \\
\end{tabular} & 32.64 & 91.21 & 12.11 & & 49.6 & 304.4 \\
\hline 279 & 50.74 & 3000 & 2290 & 87.918725 & 3.8858 & \begin{tabular}{|l|}
132.592 \\
\end{tabular} & 1696.94 & 5125 & 0.1 & 1950 & 225000 & 1637500 & 0.0627 & $3.61 \mathrm{E}-04$ & 3.908 & $9.94 \mathrm{E}-03$ & 41.84 & 94.31 & 43.13 & & 69.7 & 394.7 \\
\hline 280 & 43.05 & 3000 & 1820 & 495.608658 & 21.4564 & \begin{tabular}{|l|}
129.879 \\
\end{tabular} & 1732.38 & 5125 & 0.1 & 1950 & 225000 & 1587500 & 0.0739 & $1.42 \mathrm{E}-04$ & 1.973 & \begin{tabular}{|l|} 
\\
\end{tabular} & 58.04 & 98.99 & 89.91 & & 86.9 & 234.9 \\
\hline 281 & 41.21 & 3000 & 2610 & 18.456128 & $\begin{array}{l}0.9007 \\
\end{array}$ & \begin{tabular}{|l|l|}
146.4082 \\
\end{tabular} & 1536.8 & 5125 & 0.1 & 1950 & 225000 & 1900000 & 0.0772 & $4.46 \mathrm{E}-04$ & 3.924 & $1.10 \mathrm{E}-02$ & 14.68 & 72.91 & & 0 & 62.9 & 488 \\
\hline 282 & 23.07 & 3000 & 1680 & 971.346034 & 46.359 & \begin{tabular}{|l|l|}
143.1797 \\
\end{tabular} & 1571.45 & 5125 & 0.1 & 1950 & 225000 & 1837500 & & $1.21 \mathrm{E}-04$ & 1.443 & $3.59 \mathrm{E}-03$ & 62.94 & 99.49 & 94.85 & 48.53 & 193.1 & 320.5 \\
\hline 283 & 43.94 & 3000 & 1980 & \begin{tabular}{|l|}
19.020673 \\
\end{tabular} & 0.8871 & \begin{tabular}{|l|l|}
139.9143 \\
\end{tabular} & \begin{tabular}{|l|}
1608.13 \\
\end{tabular} & 5125 & 0.1 & 1950 & 225000 & 1775000 & 0.0724 & $3.04 \mathrm{E}-04$ & 3.122 & $1.57 \mathrm{E}-02$ & 42.05 & 73.71 & & 0 & 44.1 & 364.1 \\
\hline 284 & 25.97 & 3000 & 3120 & $\begin{array}{l}19.211832 \\
\end{array}$ & $\begin{array}{l}0.4989 \\
\end{array}$ & \begin{tabular}{|l|}
77.906 \\
\end{tabular} & \begin{tabular}{|l|}
2888.09 \\
\end{tabular} & 5125 & 0.1 & 1950 & 225000 & 737500 & 0.1225 & 3.37E-04 & 2.39 & \begin{tabular}{|l|}
$1.21 \mathrm{E}-02$ \\
\end{tabular} & 53.71 & 73.97 & & & 57.5 & 471.7 \\
\hline 285 & 24.43 & 3000 & 2230 & \begin{tabular}{|r|}
5.338943 \\
2159115 \\
\end{tabular} & $\begin{array}{l}0.2188 \\
\end{array}$ & \begin{tabular}{|l|l|}
122.9684 \\
\end{tabular} & \begin{tabular}{|l|l|}
1829.74 \\
\end{tabular} & 5125 & 0.1 & $\begin{array}{l}1950 \\
1050 \\
1050\end{array}$ & 225000 & 1462500 & 0.1302 & 3.79E-04 & 2.471 & \begin{tabular}{|l|}
$1.40 \mathrm{E}-02$ \\
\end{tabular} & $\begin{array}{r}49 \\
\end{array}$ & 6.35 & & & 49.4 & 518.3 \\
\hline 286 & $\begin{array}{l}39.28 \\
23.71 \\
\end{array}$ & 3000 & 2690 & $\begin{array}{l}21.558115 \\
30186115 \\
\end{array}$ & $\begin{array}{l}0.9037 \\
10844\end{array}$ & \begin{tabular}{|l|l|}
125.7554 \\
\end{tabular} & \begin{tabular}{|l|}
1789.19 \\
20769 \\
\end{tabular} & 5125 & 0.1 & $\begin{array}{l}1950 \\
1950\end{array}$ & 225000 & 1512500 & 0.081 & 4.34E-04 & 3.705 & \begin{tabular}{|l|}
$1.08 \mathrm{E}-02$ \\
\end{tabular} & 16.77 & 76.81 & & & 64.1 & 483.5 \\
\hline$\frac{287}{288}$ & $\begin{array}{l}23.71 \\
32.43 \\
\end{array}$ & 3000 & $\begin{array}{l}3090 \\
1200\end{array}$ & $\begin{array}{r}30.186115 \\
6931.69955\end{array}$ & $\begin{array}{r}\frac{1.0844}{31.4109} \\
\end{array}$ & \begin{tabular}{|l|l|}
107.7748 \\
135.947 \\
\end{tabular} & \begin{tabular}{|c|}
$\mid 2087.69$ \\
165508
\end{tabular} & $\begin{array}{l}5125 \\
5125 \\
\end{array}$ & $\begin{array}{l}0.1 \\
0.1\end{array}$ & $\begin{array}{l}1950 \\
1950 \\
\end{array}$ & $\begin{array}{l}225000 \\
225000\end{array}$ & $\begin{array}{l}1200000 \\
1700000\end{array}$ & $\begin{array}{l}0.1342 \\
0.091\end{array}$ & $\begin{array}{l}5.97 \mathrm{E}-04 \\
102 \mathrm{~F}-04\end{array}$ & $\begin{array}{r}3.25 \\
1527 \\
\end{array}$ & \begin{tabular}{|l|}
$7.27 E-03$ \\
$6.9 E-03$
\end{tabular} & $\begin{array}{l}38.38 \\
438\end{array}$ & $\begin{array}{l}83.44 \\
99.28\end{array}$ & \begin{tabular}{r|}
0 \\
9279
\end{tabular} & 0 & $\begin{array}{c}95.3 \\
112 \\
\end{array}$ & 702.6 \\
\hline $\begin{array}{l}280 \\
289 \\
\end{array}$ & $\begin{array}{l}34.40 \\
34.06\end{array}$ & 3000 & 3010 & 3.930889 & $\begin{array}{r}r 1.4149 \\
0.1451 \\
\end{array}$ & \begin{tabular}{|l|}
135.74441 \\
110.7481 \\
\end{tabular} & \begin{tabular}{|r|}
16553.08 \\
2031.64 \\
\end{tabular} & 5125 & $\begin{array}{l}0.1 \\
0.1\end{array}$ & $\begin{array}{l}1950 \\
1950\end{array}$ & 2250000 & $\begin{array}{l}1700000 \\
1250000\end{array}$ & $\begin{array}{l}0.0087 \\
0.0934 \\
\end{array}$ & \begin{tabular}{|l}
$1.02 \mathrm{E}-04$ \\
$5.31 \mathrm{E}-04$
\end{tabular} & $\begin{array}{l}1.521 \\
.872\end{array}$ & \begin{tabular}{|l|}
$6.19 \mathrm{E}-03$ \\
$1.29 \mathrm{E}-02$ \\
\end{tabular} & $\begin{array}{l}43.48 \\
25.85 \\
\end{array}$ & $\begin{array}{r}99.28 \\
0\end{array}$ & $\begin{aligned} 92.19 \\
0\end{aligned}$ & & $\begin{array}{r}172 \\
53.9\end{array}$ & $\frac{241.3}{582.6}$ \\
\hline 290 & 46.65 & 3000 & 1270 & \begin{tabular}{|l|}
393.605756 \\
\end{tabular} & 13.5448 & \begin{tabular}{|l|}
103.2361 \\
\end{tabular} & \begin{tabular}{|l|}
2179.47 \\
\end{tabular} & 5125 & 0.1 & 1950 & 225000 & 1125000 & 0.0682 & $1.09 \mathrm{E}-04$ & 1.806 & \begin{tabular}{|l|l|}
$1.06 \mathrm{E}-02$ \\
\end{tabular} & 46.8 & 98.73 & 87.3 & 0 & 65.5 & $\begin{array}{l}30<.0 \\
198.5\end{array}$ \\
\hline 291 & 46.78 & 3000 & 850 & $|1387.248064|$ & 38.8209 & \begin{tabular}{|l|}
83.9523 \\
\end{tabular} & \begin{tabular}{|l|}
2680.09 \\
\end{tabular} & 5125 & 0.1 & 1950 & 225000 & 825000 & 0.068 & $5.94 \mathrm{E}-05$ & 1.442 & \begin{tabular}{|l|}
$.94 \mathrm{E}-03$ \\
\end{tabular} & 54.98 & 99.64 & 96.4 & 63.96 & 140.3 & 157.9 \\
\hline 292 & 31.1 & 3000 & 3080 & \begin{tabular}{|l|}
12.754425 \\
\end{tabular} & 0.399 & \begin{tabular}{|l|}
93.8425 \\
\end{tabular} & \begin{tabular}{|l|}
2397.63 \\
\end{tabular} & 5125 & 0.1 & 1950 & 225000 & 975000 & 0.1023 & $4.80 \mathrm{E}-04$ & 3.372 & $1.10 \mathrm{E}-02$ & 31.78 & 60.8 & 0 & & 63.3 & 555.7 \\
\hline 293 & 26.71 & 3000 & 2330 & 52.313775 & 2.0704 & \begin{tabular}{|l|l|}
118.7276 \\
\end{tabular} & \begin{tabular}{|l|}
1895.09 \\
\end{tabular} & 5125 & 0.1 & 1950 & 225000 & 1387500 & 0.1191 & $3.87 \mathrm{E}-04$ & 2.643 & & 58.98 & 90.44 & 4.42 & & 77.3 & 507.1 \\
\hline 294 & 26.76 & 3000 & 2480 & 339.831223 & 14.0878 & \begin{tabular}{|l|l|}
124.3658 \\
\end{tabular} & \begin{tabular}{|l|}
1809.18 \\
\end{tabular} & 5125 & 0.1 & 1950 & 225000 & 1487500 & 0.1189 & $4.05 \mathrm{E}-04$ & 2.723 & 4.30 & 25.27 & 98.53 & 85.29 & & 161.3 & 521.6 \\
\hline 295 & 25.95 & 3000 & 1880 & 175.246611 & 5.2928 & \begin{tabular}{|l|}
90.6061 \\
\end{tabular} & \begin{tabular}{|l|}
2483.28 \\
\end{tabular} & 5125 & 0.1 & & & & 0.1226 & & 1.706 & & 44.36 & 97.15 & 71.47 & & 78.9 & 336.9 \\
\hline 296 & 34.02 & 3000 & 1680 & 473.00 & 22.7793 & \begin{tabular}{|l|l|}
144.4754 \\
\end{tabular} & & 5125 & 0.1 & & 225000 & 1862500 & & $1.55 \mathrm{E}-04$ & 1.838 & & & 98.94 & 89.43 & & 99.9 & 276.9 \\
\hline 297 & 21.47 & 3000 & 1310 & 52.380544 & $\begin{array}{r}3.379 \\
\end{array}$ & \begin{tabular}{|l|l|}
193.5283 \\
\end{tabular} & 1162 & 5125 & 0.1 & 1950 & 225000 & 2887500 & 0.1482 & 1.47E-04 & 1.501 & \begin{tabular}{|c|}
$1.22 \mathrm{E}-02$ \\
\end{tabular} & $\begin{array}{ll}65.62 \\
\end{array}$ & 90.45 & 4.54 & & 57 & 358.5 \\
\hline 298 & 35.71 & 3000 & 1910 & 48.389374 & 2.5144 & & 1443.34 & 5125 & 0.1 & & 225000 & 2087500 & & 3.49E-04 & 2.982 & & & & 0 & & 63.6 & 428.1 \\
\hline 299 & $\begin{array}{l}22.23 \\
39.37 \\
\end{array}$ & $\begin{array}{l}3000 \\
3000\end{array}$ & $\begin{array}{l}2360 \\
2340 \\
\end{array}$ & \begin{tabular}{r|r|}
296.282587 \\
1057972
\end{tabular} & $\begin{array}{l}12.4197 \\
0.48 \\
\end{array}$ & \begin{tabular}{|r|}
125.7554 \\
129879 \\
\end{tabular} & \begin{tabular}{|c|}
1789.19 \\
173.38
\end{tabular} & $\begin{array}{l}5125 \\
5125 \\
\end{array}$ & $\begin{array}{l}0.1 \\
0.1\end{array}$ & $\begin{array}{l}1950 \\
1950\end{array}$ & 225000 & $\begin{array}{l}1512500 \\
158700\end{array}$ & 0.1431 & $\begin{array}{l}4.03 E-04 \\
\end{array}$ & $\begin{array}{l}2.424 \\
2.098\end{array}$ & 4.36 & 48.04 & $\begin{array}{l}98.31 \\
5271\end{array}$ & 83.12 & & 159 & 558.8 \\
\hline $\begin{array}{l}300 \\
301\end{array}$ & $\frac{39.37}{23.29}$ & 3000 & $\begin{array}{r}2340 \\
790\end{array}$ & & $\begin{array}{r}0.4588 \\
0.8383\end{array}$ & \begin{tabular}{|l|}
129.879 \\
131.239 \\
\end{tabular} & $\begin{array}{l}\frac{1 / 32.38}{1714.43} \\
173\end{array}$ & $\begin{array}{l}\mid 5125 \\
5125\end{array}$ & $\begin{array}{l}0.1 \\
0.1\end{array}$ & $\begin{array}{l}\frac{1950}{1950} \\
\end{array}$ & $\frac{225000}{225000}$ & $\frac{1587500}{1612500}$ & $\frac{0.0}{0.1}$ & $\frac{3.79 E-04}{88.09-05}$ & $\frac{3.998}{1.299}$ & \begin{tabular}{|l|}
$\mid 1.40 \mathrm{E}-02$ \\
$1.81 \mathrm{E}-02$
\end{tabular} & $66.64 \mid$ & $\begin{array}{l}52.74 \\
7.91 \\
\end{array}$ & & & $\begin{array}{l}49.5 \\
38.2 \\
\end{array}$ & $\begin{array}{r}520.5 \\
285.9 \\
\end{array}$ \\
\hline 302 & 41.37 & & 2480 & 16.266 & 0.7728 & \begin{tabular}{|l|}
142.5296 \\
\end{tabular} & \begin{tabular}{|l|}
1578.62 \\
\end{tabular} & 5125 & 0.1 & & 2250 & 1825000 & 0.0 & 4.44E & 3.9 & \begin{tabular}{|l|}
$1.19 \mathrm{E}-02$ \\
\end{tabular} & 26.77 & 69.26 & & & 58.3 & 2805.9 \\
\hline 303 & 52.76 & 3000 & 2080 & \begin{tabular}{|l|}
147.155427 \\
\end{tabular} & & \begin{tabular}{|l|}
122.9684 \\
\end{tabular} & 1829 & 5125 & 0.1 & & 225000 & 1462500 & 0.0603 & $2.50 \mathrm{E}-04$ & & 1.11 & 55.24 & 96.6 & 66.02 & & 62.8 & 300.4 \\
\hline 304 & & 3000 & 2310 & 19.218 & 0.9746 & \begin{tabular}{|l|}
152.1319 \\
\end{tabular} & 1478 & 5125 & 0.1 & 195 & 225000 & 2012500 & 0.148 & $4.62 \mathrm{E}-04$ & 2.57 & & 21.35 & 73.98 & & & 80 & 614.3 \\
\hline & 49.02 & & 2560 & 161.578417 & 7.2138 & \begin{tabular}{|l|}
133.9381 \\
\end{tabular} & 1679 & 5125 & 0.1 & 1950 & & 1662500 & 0.0649 & $3.85 \mathrm{E}-04$ & 3.998 & & 17.11 & 96.91 & 69.06 & & 95.5 & 418 \\
\hline 306 & 61.77 & 3000 & 2040 & 621.846 & 12.6693 & \begin{tabular}{|l|}
61.1213 \\
\end{tabular} & 368 & 5125 & 0.1 & 1950 & & 512500 & & $1.27 \mathrm{E}-04$ & 2.249 & & 67.87 & 99.2 & 91.96 & 19.59 & 78.5 & 186.6 \\
\hline 307 & 50.42 & 3000 & 2430 & 553.26 & 21.4995 & \begin{tabular}{|l|l|}
116.5786 \\
\end{tabular} & 1930 & 5125 & 0.1 & 1950 & 225000 & 1350000 & & 3.12 & 3.502 & & 31.1 & 99.1 & 90.96 & 9.63 & 140.9 & 356 \\
\hline 308 & 25.03 & 3000 & 3050 & 14.07 & 0.3696 & \begin{tabular}{|l|l|}
78.7839 \\
\end{tabular} & 2855 & 5125 & 0.1 & 1950 & 2250 & & & 3.46 & 2.376 & & 23.58 & 64.47 & & & 59.4 & 486.6 \\
\hline 309 & 34.39 & 3000 & 3050 & & 0.0147 & \begin{tabular}{|l|l|}
105.5176 \\
\end{tabular} & 2132 & 5125 & 0.1 & & & 1162 & & & & & 51.09 & & & & 38.7 & \\
\hline 310 & 52.58 & 3000 & 820 & 889.5 & 28.3008 & \begin{tabular}{|l|l|}
95.4399 \\
\end{tabular} & 235 & 5125 & 0.1 & 19 & 225 & & & 5.87 & & & 59.4 & 99.44 & 94.38 & 43.79 & & 145.3 \\
\hline 311 & $\begin{array}{ll}24.7 \\
\end{array}$ & 3000 & 2490 & 261.4 & 13.0954 & \begin{tabular}{|l|}
150.2362 \\
\end{tabular} & 1497 & 5125 & 0.1 & 1950 & 225000 & 1975000 & 0.1288 & $4.84 \mathrm{E}$ & 2.899 & 4.19 & 25.2 & 98.09 & 80.88 & & 16 & 601.5 \\
\hline 312 & 32.6 & & 2180 & & & \begin{tabular}{|l|l|}
126.4473 \\
\end{tabular} & 177. & 5125 & & & & & & 3.66 & & 1.6 & 46. & 2.06 & & & 42.4 & 455.2 \\
\hline 313 & 27.62 & 3000 & $\begin{array}{ll}2830 \\
2340\end{array}$ & $\begin{array}{l}0.1217 \\
71366 \\
\end{array}$ & 0.005 & \begin{tabular}{|l|l|}
135.9447 \\
1571306
\end{tabular} & \begin{tabular}{|l|l|}
1655.0 \\
1210
\end{tabular} & $\frac{5125}{5125}$ & 0 & 195 & 22500 & 17000 & 0.1 & 7.53E-04 & 4.306 & & 45. & 0 & & & 39.7 & 9.1 \\
\hline $\begin{array}{r}314 \\
315 \\
\end{array}$ & $\begin{aligned} 21.82 \\
29.38 \\
\end{aligned}$ & 3000 & 2340 & $\begin{array}{l}7.136626 \\
6.154855\end{array}$ & $\begin{array}{l}0.3738 \\
02006\end{array}$ & \begin{tabular}{|l|l|}
157.1306 \\
07911 \\
\end{tabular} & \begin{tabular}{|l|}
1431.93 \\
\end{tabular} & $\begin{array}{l}5125 \\
525\end{array}$ & 0.1 & $\begin{array}{l}1950 \\
1050\end{array}$ & 225000 & 2112500 & 0.1458 & 6.08E-04 & 3.108 & \begin{tabular}{|l|}
$1.01 \mathrm{E}-02$ \\
\end{tabular} & 61.34 & 29.94 & 0 & & 68.9 & 730.1 \\
\hline $\begin{array}{l}315 \\
316 \\
\end{array}$ & $\begin{array}{r}29.38 \\
22.82 \\
\end{array}$ & $\begin{array}{l}3000 \\
3000 \\
\end{array}$ & $\begin{array}{l}3280 \\
3480\end{array}$ & $\begin{array}{r}6.154085 \\
2617205\end{array}$ & $\begin{array}{r}0.2006 \\
12.3776\end{array}$ & \begin{tabular}{|c|}
97.8112 \\
14.878 \\
\end{tabular} & \begin{tabular}{|l|}
2300.35 \\
15587 \\
\end{tabular} & $\begin{array}{l}5125 \\
5125 \\
\end{array}$ & 0.1 & $\frac{1950}{1950}$ & 225000 & \begin{tabular}{|l|l|}
1037500 \\
182500
\end{tabular} & $\begin{array}{l}0.1083 \\
0.1282\end{array}$ & $\begin{array}{ll}6.02 \mathrm{E}-04 \\
711-04\end{array}$ & $\begin{array}{r}3.81 \\
3.804 \\
\end{array}$ & \begin{tabular}{|l|}
$1.09 \mathrm{E}-02$ \\
$308 \mathrm{E}-03$
\end{tabular} & $\begin{array}{l}36.67 \\
2861\end{array}$ & $\begin{array}{r}18.75 \\
9809\end{array}$ & $\begin{array}{r}0 \\
809\end{array}$ & & \begin{tabular}{r|r|r}
63.7 \\
225.3
\end{tabular} & 664.7 \\
\hline 317 & $\begin{array}{l}4.02 \\
45.77 \\
\end{array}$ & 3000 & $\begin{array}{l}3400 \\
1720\end{array}$ & $\begin{array}{r}201.12405 \\
196.348433\end{array}$ & 年 & \begin{tabular}{|l|}
41.3109 \\
87.3109 \\
\end{tabular} & & 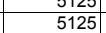 & $\begin{array}{l}0.1 \\
0.1\end{array}$ & 1950 & 225000 & \begin{tabular}{|c|}
1012500 \\
875000
\end{tabular} & $\begin{array}{l}0.1202 \\
0.0695\end{array}$ & $\begin{array}{l}1.111 \mathrm{E}-04 \\
1.46 \mathrm{E}-04\end{array}$ & $\begin{array}{l}0.044 \\
2.065\end{array}$ & \begin{tabular}{|l|}
$3.00 \mathrm{E}-03$ \\
$1.24 \mathrm{E}-02$ \\
\end{tabular} & $\begin{array}{l}\mid 8.01 \\
46.28\end{array}$ & $\begin{array}{l}0.09 \\
97.45 \\
\end{array}$ & $\begin{array}{l}00.9 \\
74.54\end{array}$ & & $\begin{array}{r}220.0 \\
55.9 \\
\end{array}$ & $\begin{array}{l}231.2 \\
231.2\end{array}$ \\
\hline 318 & 40. & & 1500 & 253.407662 & 12.367 & 146.4 & 1536.8 & 5125 & 0.1 & 195 & 225000 & & 0.0 & $1.83 \mathrm{E}-04$ & 2. & & 18.36 & 98.03 & 80.27 & & 76.1 & 276.5 \\
\hline 319 & 31. & & 1830 & 641.0 & 27.4 & & 1750 & 512 & 0.1 & & & & & & & & 42.43 & & 92.2 & & 128.6 & \\
\hline 320 & 30 & & 1890 & 143.544 & 6.2 & 130.5 & 172 & 5125 & 0.1 & 195 & & & & & & & & 96.52 & 65.17 & & 70.9 & \\
\hline 321 & 38. & 3000 & 1600 & & 3.3. & 164. & 136 & 5125 & 0.1 & 1950 & 225000 & 2262500 & & & 2.2 & 1.34 & 29.69 & 91.8 & 17.96 & & 51.7 & 305.3 \\
\hline & & & 2650 & & & & & & 0.1 & & & & & & & & 52.91 & & 0 & & 47.1 & \\
\hline 323 & & 30 & 2260 & & & \begin{tabular}{|l|l|}
127.1374 \\
127875
\end{tabular} & \begin{tabular}{|l|l|}
1769.74 \\
17921
\end{tabular} & & 0.1 & & & & & 3.30E-04 & & & $\begin{array}{r}16 \\
\end{array}$ & & 0 & & 37.9 & \\
\hline $\begin{array}{l}324 \\
325 \\
\end{array}$ & $\begin{array}{l}33.81 \\
25.8\end{array}$ & 3000 & $\begin{array}{l}2050 \\
1680\end{array}$ & \begin{tabular}{|l|}
478.975144 \\
12618722
\end{tabular} & $\begin{aligned} 20.4084 \\
0.6713\end{aligned}$ & \begin{tabular}{|c|}
127.8255 \\
1596003 \\
\end{tabular} & \begin{tabular}{|c|}
$\mid 1760.21$ \\
1409.77
\end{tabular} & \begin{tabular}{|l|r|}
5125 \\
5125 \\
\end{tabular} & $\begin{array}{l}0.1 \\
0.1\end{array}$ & $\begin{array}{l}1950 \\
1950\end{array}$ & $\begin{array}{l}225000 \\
225000\end{array}$ & $\begin{array}{l}1550000 \\
2162500\end{array}$ & $\begin{array}{l}0.0941 \\
0.1233\end{array}$ & $\begin{array}{l}2.63 \mathrm{E}-04 \\
2.41-04\end{array}$ & $\begin{array}{l}2.413 \\
1987 \\
\end{array}$ & \begin{tabular}{|l|}
$5.22 \mathrm{E}-03$ \\
$148 \mathrm{E}-02$
\end{tabular} & $\begin{array}{l}46.39 \\
30.93\end{array}$ & $\begin{array}{l}98.96 \\
60.38\end{array}$ & \begin{tabular}{r|r}
89.56 & \\
0 &
\end{tabular} & & $\begin{array}{ll}132.9 \\
4.9\end{array}$ & $\begin{array}{l}365.8 \\
394.8 \\
\end{array}$ \\
\hline
\end{tabular}




\begin{tabular}{|c|c|c|c|c|c|c|c|c|c|c|c|c|c|c|c|c|c|c|c|c|c|c|}
\hline $\begin{array}{c}\text { Realizatio } \\
n\end{array}$ & $\begin{array}{c}\text { Velocity } \\
(\mathrm{m} / \mathrm{y})\end{array}$ & $\begin{array}{c}\text { Fixed Source } \\
\text { Concentration } \\
\text { (mg/L) }\end{array}$ & $\begin{array}{c}\text { Time of } \\
\text { Maximum at } \\
\text { Compliance } \\
\text { Boundary } \\
(y)\end{array}$ & \begin{tabular}{|c|} 
Maximum \\
Concentration \\
Compliance \\
Boundary \\
$(\mu \mathrm{g} / \mathrm{L})$
\end{tabular} & \begin{tabular}{|c|} 
Maximum \\
Release Rate \\
at Compliance \\
Boundary \\
$(\mathrm{Kg} / \mathrm{y})$
\end{tabular} & \begin{tabular}{|c|} 
Source \\
Rate \\
$(\mathrm{Kg} / \mathrm{y})$ \\
\end{tabular} & \begin{tabular}{|c|} 
Source \\
Duration \\
(y)
\end{tabular} & \begin{tabular}{|c|} 
1-D \\
Streamtub \\
e Length \\
(m)
\end{tabular} & $\begin{array}{c}\text { Base } \\
\text { Porosity } \\
\left(\mathrm{m}^{3} / \mathrm{m}^{3}\right)\end{array}$ & $\begin{array}{c}\text { Bulk } \\
\text { Densty } \\
\left(\mathrm{Kg} / \mathrm{m}^{3}\right)\end{array}$ & $\begin{array}{c}\text { Remaining } \\
\text { Carbon } \\
\text { Tetrachloride } \\
\text { Source } \\
(\mathrm{Kg})\end{array}$ & \begin{tabular}{|c|} 
Volume at or \\
above Fixed \\
Source \\
$\begin{array}{c}\text { Concentration } \\
(\mathrm{m} 3)\end{array}$ \\
\end{tabular} & $\begin{array}{l}\text { Porosity } \\
\left(\mathrm{m}^{3} / \mathrm{m}^{3}\right)\end{array}$ & $\begin{array}{c}\mathrm{Kd} \\
\left(\mathrm{m}^{3} / \mathrm{Kg}\right) \\
\end{array}$ & Retardation & $\left|\begin{array}{c}\text { First Order } \\
\text { Rate } \\
\text { Constant } \\
(1 / y)\end{array}\right|$ & $\begin{array}{c}\text { Longitudinal } \\
\text { Dispersivity } \\
(\mathrm{m})\end{array}$ & $\begin{array}{c}\text { Required } \\
\text { Source } \\
\text { Cleanup } \\
\text { Percentage } \\
(5 \mu g / L \\
\text { Compliance) } \\
\end{array}$ & \begin{tabular}{|c|} 
Required \\
Source \\
Cleanup \\
Percentage \\
(50 gig/L \\
Compliance)
\end{tabular} & \begin{tabular}{|c} 
Required \\
Source \\
Cleanup \\
Percentage \\
(500 ug/L \\
Compliance) \\
\end{tabular} & \begin{tabular}{|c|} 
Abiotic \\
Reaction \\
Half Time \\
(y)
\end{tabular} & $\begin{array}{c}\text { Carbon } \\
\text { Tetrachloride } \\
\text { Travel Time (y) }\end{array}$ \\
\hline & 27.78 & & & & & \begin{tabular}{|c|}
158.9847 \\
\end{tabular} & \begin{tabular}{|l|}
1415.23 \\
\end{tabular} & & & 1950 & & & 0.1145 & $2.12 \mathrm{E}-04$ & & & & & & & & \\
\hline 327 & 27.17 & 3000 & 2390 & 58.171384 & 2.6618 & \begin{tabular}{|l|l|}
137.2743 \\
\end{tabular} & 1639.05 & 5125 & 0.1 & 1950 & 225000 & 1725000 & 0.1171 & \begin{tabular}{|l|}
$4.36 \mathrm{E}-04$ \\
\end{tabular} & 2.883 & \begin{tabular}{|l|}
$7.69 \mathrm{E}-03$ \\
\end{tabular} & 27.41 & 91.4 & 14.05 & 0 & 90.1 & 543.9 \\
\hline 328 & 27.4 & 3000 & 1620 & \begin{tabular}{|c|c|}
205.6774 \\
\end{tabular} & 8.9511 & \begin{tabular}{|l|}
130.5599 \\
\end{tabular} & $\begin{array}{l}10323.35 \\
1723\end{array}$ & 5125 & 0.1 & 1950 & 225000 & 1600000 & 0.1161 & \begin{tabular}{|l|}
$4.160 \mathrm{E}-\mathrm{-}-164$ \\
\end{tabular} & $\begin{array}{l}2.000 \\
1.505 \\
\end{array}$ & \begin{tabular}{|l|}
$1.091 \mathrm{E}-02$ \\
\end{tabular} & 72.11 & 97.57 & $\begin{array}{l}75.69 \\
75.6\end{array}$ & 0 & 68.9 & 281.4 \\
\hline 329 & 56.11 & 3000 & 3650 & 2.427501 & 0.092 & \begin{tabular}{|l|}
113.6821 \\
\end{tabular} & 1979.2 & 5125 & 0.1 & 1950 & 225000 & 1300000 & 0.0567 & \begin{tabular}{|c|}
$6.25 \mathrm{E}-04$ \\
\end{tabular} & 6.568 & $1.46 \mathrm{E}-02$ & 27.25 & 0 & & & 47.4 & 599.9 \\
\hline 330 & 33.17 & 3000 & 1090 & 382.693821 & 21.2135 & \begin{tabular}{|l|}
166.2959 \\
\end{tabular} & 1353.01 & 5125 & 0.1 & 1950 & 225000 & 2300000 & 0.0959 & \begin{tabular}{|l|}
$1.26 \mathrm{E}-04$ \\
\end{tabular} & 1.662 & \begin{tabular}{|l|}
$8.15 \mathrm{E}-03$ \\
\end{tabular} & 23.25 & 98.69 & 86.93 & & 85.1 & 256.7 \\
\hline 331 & 38.84 & 3000 & 1140 & 142.352003 & 7.6604 & \begin{tabular}{|l|}
161.4401 \\
\end{tabular} & 1393.71 & 5125 & 0.1 & 1950 & 225000 & 2200000 & 0.0819 & \begin{tabular}{|l|}
$7.60 \mathrm{E}-05$ \\
\end{tabular} & 1.469 & \begin{tabular}{|c|}
$1.68 \mathrm{E}-02$ \\
\end{tabular} & 81.45 & 96.49 & 64.88 & 3 & 41.2 & 193.8 \\
\hline 332 & 53.92 & 3000 & 2680 & 152.871911 & 5.6434 & \begin{tabular}{|l|}
110.7481 \\
\end{tabular} & 2031.64 & 5125 & 0.1 & 1950 & 225000 & 1250000 & 0.059 & \begin{tabular}{|l|}
$3.48 \mathrm{E}-04$ \\
\end{tabular} & 3.978 & \begin{tabular}{|l|} 
\\
\end{tabular} & 21.41 & 96.73 & 67.29 & 0 & 83.9 & 378.1 \\
\hline & 22.61 & & 2450 & 78.524025 & 3.5233 & \begin{tabular}{|l|l|}
134.6086 \\
\end{tabular} & 1671.51 & 5125 & 0.1 & & 225000 & 1675000 & 0.1407 & \begin{tabular}{|l|l|}
$4.39 \mathrm{E}-04$ \\
\end{tabular} & 2.576 & $6.45 \mathrm{E}-03$ & 18.19 & 93.63 & & & 107.5 & 583.8 \\
\hline 334 & 23.83 & 3000 & 1940 & $\begin{array}{l}504.498665 \\
\end{array}$ & 18.9947 & \begin{tabular}{|l|l|}
112.9522 \\
\end{tabular} & 1991.99 & 5125 & 0.1 & 1950 & 225000 & 1287500 & 0.1335 & \begin{tabular}{|l|}
$1.72 \mathrm{E}-04$ \\
\end{tabular} & 1.652 & $5.14 \mathrm{E}-03$ & 43.66 & 99.01 & 90.09 & 0.89 & 134.7 & 355.3 \\
\hline 335 & 34.96 & 3000 & 4580 & 34.393288 & 0.7345 & \begin{tabular}{|l|l|}
64.0676 \\
\end{tabular} & 3511.92 & 5125 & 0.1 & 1950 & 225000 & 550000 & 0.091 & \begin{tabular}{|l|l|}
$7.31 \mathrm{E}-04$ \\
\end{tabular} & 5.06 & \begin{tabular}{|l|}
$7.60 \mathrm{E}-03$ \\
\end{tabular} & 60.02 & 85.46 & & 0 & & 741.7 \\
\hline $\begin{array}{l}3366 \\
327 \\
\end{array}$ & 37.65 & 3000 & $\begin{array}{ll}1990 \\
900\end{array}$ & $\begin{array}{ll}616.189448 \\
\end{array}$ & 25.1132 & \begin{tabular}{|l|l|}
122.2668 \\
\end{tabular} & \begin{tabular}{|l|l|}
1840.24 \\
07.2
\end{tabular} & 5125 & 0.1 & $\begin{array}{l}1950 \\
1950\end{array}$ & 225000 & 1450000 & 0.0845 & \begin{tabular}{|l|}
$1.91 \mathrm{E}-04$ \\
\end{tabular} & 2.144 & \begin{tabular}{|l|}
$5.64 \mathrm{E}-03$ \\
\end{tabular} & $\begin{array}{l}61.36 \\
2.30\end{array}$ & $\begin{array}{l}99.19 \\
\end{array}$ & 91.89 & & 122.8 & 291.9 \\
\hline 337 & 28.92 & 3000 & \begin{tabular}{|l|l}
900 \\
980
\end{tabular} & \begin{tabular}{|l|}
621.487148 \\
70137901
\end{tabular} & 27.7469 & \begin{tabular}{|l|l|}
133.9381 \\
\end{tabular} & \begin{tabular}{|l|l|}
1679.88 \\
174154
\end{tabular} & 5125 & 0.1 & $\begin{array}{l}1950 \\
1950\end{array}$ & 225000 & 1662500 & $\begin{array}{r}0.11 \\
\end{array}$ & \begin{tabular}{|l|}
$9.33 \mathrm{E}-05$ \\
$79-0$
\end{tabular} & 1.429 & \begin{tabular}{|l|}
$6.29 \mathrm{E}-03$ \\
\end{tabular} & 25.05 & 99.2 & 91.95 & & 110.3 & 253.2 \\
\hline$\frac{338}{339}$ & $\begin{array}{l}33.28 \\
37.47\end{array}$ & $\begin{array}{l}3000 \\
3000 \\
\end{array}$ & $\begin{array}{r}980 \\
1690\end{array}$ & $\begin{array}{r}791.3919191 \\
981.42257\end{array}$ & $\begin{array}{l}34.0811 \\
50.9975\end{array}$ & \begin{tabular}{|l|}
129.1963 \\
155.8885 \\
\end{tabular} & $\begin{array}{l}17441.54 \\
1443.34\end{array}$ & $\begin{array}{l}5125 \\
5125\end{array}$ & $\begin{array}{l}0.1 \\
0.1\end{array}$ & $\begin{array}{l}\frac{1950}{1950} \\
1950\end{array}$ & $\begin{array}{l}225000 \\
225000\end{array}$ & $\begin{array}{l}1575000 \\
2087500\end{array}$ & $\begin{array}{l}0.0928 \\
0.0849\end{array}$ & \begin{tabular}{|l|}
$7.88 \mathrm{E}-05$ \\
$2.24 \mathrm{E}-04$ \\
\end{tabular} & $\begin{array}{l}\frac{1.429}{2.335} \\
\end{array}$ & \begin{tabular}{|l|}
$6.34 \mathrm{E}-03$ \\
$3.62 \mathrm{E}-03$
\end{tabular} & $\begin{array}{l}45.59 \\
69.24\end{array}$ & \begin{tabular}{|c|}
99.37 \\
99.49
\end{tabular} & \begin{tabular}{|c|}
93.68 \\
49.91
\end{tabular} & $\begin{array}{l}36.82 \\
44.05 \\
\end{array}$ & $\begin{array}{l}109.3 \\
191.4 \\
\end{array}$ & $\begin{array}{l}213.7 \\
319.4 \\
\end{array}$ \\
\hline 340 & 23.64 & 3000 & 3100 & 32.651591 & $\begin{array}{c}1.0214 \\
\end{array}$ & \begin{tabular}{|r|}
150.0000 \\
93.8425 \\
\end{tabular} & \begin{tabular}{|l}
144.54 \\
2397.63
\end{tabular} & 5125 & $\begin{array}{l}0.1 \\
0.1\end{array}$ & $\frac{1950}{1950}$ & 225000 & $\begin{array}{r}201500 \\
975000\end{array}$ & $\begin{array}{l}.04346 \\
0.1346\end{array}$ & \begin{tabular}{|l|}
$2.24 \mathrm{E}-04$ \\
$5.05 \mathrm{E}-04$ \\
\end{tabular} & $\begin{array}{l}2.3030 \\
2.896\end{array}$ & \begin{tabular}{|l|}
$3.02 \mathrm{E}-03$ \\
$7.83 \mathrm{E}-03$ \\
\end{tabular} & $\frac{69.24}{34.54}$ & $\begin{array}{l}99.49 \\
84.69\end{array}$ & & & $\begin{array}{r}91.4 \\
88.5 \\
\end{array}$ & $\begin{aligned} 519.4 \\
628 \\
\end{aligned}$ \\
\hline 341 & 53.02 & 3000 & 4780 & 95.071025 & 2.2403 & \begin{tabular}{|l|}
70.6941 \\
\end{tabular} & 3182.73 & 5125 & 0.1 & 1950 & 225000 & 637500 & 0.06 & \begin{tabular}{|l|}
$6.66 \mathrm{E}-04$ \\
\end{tabular} & 6.612 & $6.28 \mathrm{E}-03$ & 41.62 & 94.74 & 47.41 & 0 & 110.3 & 639.1 \\
\hline 342 & 38.47 & 3000 & 2800 & 220.913248 & 6.9103 & \begin{tabular}{|l|}
93.8425 \\
\end{tabular} & 2397.63 & 5125 & 0.1 & 1950 & 225000 & 975000 & 0.0827 & \begin{tabular}{|l|}
$3.43 \mathrm{E}-04$ \\
\end{tabular} & 3.098 & $6.74 \mathrm{E}-03$ & $\begin{array}{ll}42.48 \\
\end{array}$ & 97.74 & 77.37 & & 102.8 & 412.8 \\
\hline 343 & 60.03 & 3000 & 3940 & 59.310212 & 2.1159 & \begin{tabular}{|l|}
107.0251 \\
\end{tabular} & \begin{tabular}{|l|}
2102.31 \\
\end{tabular} & 5125 & 0.1 & 1950 & 225000 & 1187500 & 0.053 & \begin{tabular}{|c|}
$6.19 \mathrm{E}-04$ \\
\end{tabular} & 6.904 & $7.51 \mathrm{E}-03$ & 35.97 & 91.57 & 15.7 & & 92.2 & 589.5 \\
\hline 344 & 35.95 & 3000 & 1970 & 208.04895 & 8.8169 & \begin{tabular}{|l|l|}
127.1374 \\
\end{tabular} & \begin{tabular}{|l|}
1769.74 \\
\end{tabular} & 5125 & 0.1 & 1950 & 225000 & 1537500 & 0.0885 & \begin{tabular}{|l|}
$2.39 \mathrm{E}-04$ \\
\end{tabular} & 2.363 & $8.67 \mathrm{E}-03$ & 76.52 & 97.6 & 75.97 & & 80 & 336.8 \\
\hline 345 & 50.9 & 3000 & 2590 & 142.037514 & 7.292 & \begin{tabular}{|l|}
154.0159 \\
\end{tabular} & 1460.89 & 5125 & 0.1 & 1950 & 225000 & 2050000 & 0.0625 & \begin{tabular}{|l|}
$4.60 \mathrm{E}-04$ \\
\end{tabular} & 4.717 & \begin{tabular}{|l|}
$6.87 \mathrm{E}-03$ \\
\end{tabular} & 37.46 & 96.48 & 64.8 & & 101 & 474.9 \\
\hline 346 & 40.22 & 3000 & 780 & 262.595746 & 7.7874 & \begin{tabular}{|l|}
88.9661 \\
\end{tabular} & 2529.05 & 5125 & 0.1 & 1950 & 225000 & 900000 & 0.0791 & \begin{tabular}{|l|}
$5.76 \mathrm{E}-05$ \\
\end{tabular} & 1.368 & $1.45 \mathrm{E}-02$ & 54.12 & 98.1 & 80.96 & & 47.9 & 174.4 \\
\hline 347 & 51.64 & 3000 & 1870 & 43.144265 & 1.9647 & \begin{tabular}{|l|l|}
136.6103 \\
\end{tabular} & 1647.02 & 5125 & 0.1 & 1950 & 225000 & 1712500 & 0.0616 & \begin{tabular}{|l|}
$2.49 \mathrm{E}-04$ \\
\end{tabular} & 3.042 & & 65.7 & 88.41 & & & 42.3 & 301.8 \\
\hline 348 & 38.56 & 3000 & 2680 & \begin{tabular}{|l|l|}
31.973503 \\
\end{tabular} & 1.355 & \begin{tabular}{|l|l|}
127.1374 \\
\end{tabular} & & 5125 & 0.1 & 1950 & 225000 & 1537500 & 0.0825 & \begin{tabular}{|l|l|}
$5.21 \mathrm{E}-04$ \\
\end{tabular} & 4.194 & \begin{tabular}{|c|}
$9.60 \mathrm{E}-03$ \\
\end{tabular} & 50.47 & 84.36 & 0 & & 72.2 & 557.4 \\
\hline 349 & 50.66 & 3000 & 1630 & 151.488832 & 7.7456 & \begin{tabular}{|l|l|}
153.3892 \\
\end{tabular} & \begin{tabular}{|l|l|}
1466.86 \\
\end{tabular} & 5125 & 0.1 & 1950 & 225000 & 2037500 & 0.0628 & \begin{tabular}{|l|l|}
$1.80 \mathrm{E}-04$ \\
\end{tabular} & 2.446 & $1.28 \mathrm{E}-02$ & 44.75 & $\begin{array}{l}96.7 \\
\end{array}$ & $\begin{array}{ll}66.99 \\
\end{array}$ & & 54 & 247.5 \\
\hline 350 & $\begin{array}{r}23 \\
5671\end{array}$ & 3000 & $\begin{array}{l}2750 \\
1830\end{array}$ & $\begin{array}{r}20.300417 \\
\end{array}$ & $\begin{array}{l}0.6296 \\
5620 \\
\end{array}$ & \begin{tabular}{|r|}
93.0387 \\
100.655 \\
\end{tabular} & \begin{tabular}{|l|}
2418.35 \\
\end{tabular} & 5125 & 0.1 & $\begin{array}{l}1950 \\
1050 \\
1050\end{array}$ & 225000 & \begin{tabular}{|r|}
962500 \\
122500
\end{tabular} & 0.1383 & \begin{tabular}{|l|}
$3.51 \mathrm{E}-04$ \\
\end{tabular} & 2.282 & $1.07 \mathrm{E}-02$ & 38.65 & 75.37 & 0 & & 64.9 & 508.3 \\
\hline 351 & $\begin{array}{l}56.71 \\
21.25 \\
\end{array}$ & & $\begin{array}{l}1830 \\
2040 \\
\end{array}$ & $\begin{array}{r}154.543004 \\
144.83658\end{array}$ & $\begin{array}{l}5.6288 \\
6107\end{array}$ & \begin{tabular}{|l|l|}
109.2665 \\
126473 \\
\end{tabular} & \begin{tabular}{|r|}
2059.19 \\
17794 \\
\end{tabular} & 5125 & 0.1 & $\begin{array}{l}1950 \\
1950\end{array}$ & 225000 & 1225000 & 0.0561 & $1.40 \mathrm{E}-04$ & 2.259 & \begin{tabular}{|l|}
$1.55 \mathrm{E}-02$ \\
700502
\end{tabular} & 52.41 & $\begin{array}{l}96.76 \\
\end{array}$ & 67.65 & & 44.6 & 204.1 \\
\hline 355 & $\begin{array}{l}21.25 \\
45.06\end{array}$ & 3000 & $\begin{array}{l}2040 \\
1950\end{array}$ & $\begin{array}{l}144.836558 \\
177.398863\end{array}$ & \begin{tabular}{|l|l|}
6.1047 \\
9.476
\end{tabular} & \begin{tabular}{|l|}
126.4473 \\
159.6003 \\
\end{tabular} & \begin{tabular}{|r|}
1779.4 \\
109.77 \\
\end{tabular} & $\begin{array}{l}5125 \\
5125\end{array}$ & $\begin{array}{l}0.1 \\
0.1\end{array}$ & $\begin{array}{l}1950 \\
1950 \\
\end{array}$ & $\begin{array}{l}225000 \\
225000\end{array}$ & $\begin{array}{l}1525000 \\
2162500\end{array}$ & \begin{tabular}{|l|l|}
0.1497 \\
0.0706
\end{tabular} & \begin{tabular}{|l|}
$2.66 \mathrm{E}-04$ \\
$3.35 \mathrm{E}-04$ \\
\end{tabular} & $\begin{array}{l}1.898 \\
3.401 \\
\end{array}$ & \begin{tabular}{|l|}
$7.05 E-03$ \\
$7.97 E-03$
\end{tabular} & 57.28 & \begin{tabular}{|c|c|}
96.55 \\
97.18
\end{tabular} & $\begin{array}{l}65.48 \\
771.11\end{array}$ & 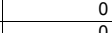 & \begin{tabular}{c|c}
98.4 \\
87
\end{tabular} & $\begin{array}{l}457.7 \\
388.8\end{array}$ \\
\hline 354 & 23.65 & 3000 & 3440 & 14.147407 & $\begin{array}{l}.45032 \\
0.3632\end{array}$ & \begin{tabular}{|l|}
77.00000 \\
77.0232 \\
\end{tabular} & 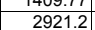 & 5125 & $\begin{array}{l}0.1 \\
0.1\end{array}$ & 1950 & 2250000 & $\begin{array}{r}21725000 \\
725000\end{array}$ & $\begin{array}{l}0.0706 \\
0.1345\end{array}$ & \begin{tabular}{|l|}
$3.35 E-04$ \\
$4.36 E-04$ \\
\end{tabular} & $\begin{array}{l}.4017 \\
2.637 \\
\end{array}$ & \begin{tabular}{|l|}
$1.97 \mathrm{E}-03$ \\
$9.87 \mathrm{E}-03$ \\
\end{tabular} & $\begin{array}{l}49.11 \\
18.94\end{array}$ & $\begin{array}{l}\mid 7 . .18 \\
64.66 \\
\end{array}$ & $\begin{array}{r}17.87 \\
0\end{array}$ & & $\begin{array}{r}81 \\
70.3 \\
\end{array}$ & $\begin{array}{l}356.8 \\
571.3 \\
\end{array}$ \\
\hline 355 & 52.41 & 3000 & 4220 & 46.074122 & 1.6089 & \begin{tabular}{|l|l|}
104.7599 \\
\end{tabular} & \begin{tabular}{|l|}
2147.77 \\
\end{tabular} & 5125 & 0.1 & 1950 & 225000 & 11150000 & 0.0607 & \begin{tabular}{|l|}
$6.87 \mathrm{E}-04$ \\
\end{tabular} & 6.721 & \begin{tabular}{|l|}
$7.14 \mathrm{E}-03$ \\
\end{tabular} & 34.02 & 89.15 & 0 & & 97.1 & 657.2 \\
\hline 356 & 29.4 & 3000 & 760 & 157.483074 & 4.9262 & \begin{tabular}{|r|}
93.8425 \\
\end{tabular} & \begin{tabular}{|l|}
2397.63 \\
\end{tabular} & 5125 & 0.1 & 1950 & 225000 & 975000 & 0.1082 & \begin{tabular}{|l|}
$8.74 \mathrm{E}-05$ \\
\end{tabular} & 1.408 & $1.22 \mathrm{E}-02$ & 19.44 & 96.83 & 68.25 & & 56.8 & 245.5 \\
\hline 357 & 37.34 & 3000 & 1310 & 352.286954 & 12.0329 & \begin{tabular}{|l|}
102.4699 \\
\end{tabular} & \begin{tabular}{|l|}
2195.77 \\
\end{tabular} & 5125 & 0.1 & 1950 & 225000 & 1112500 & 0.0852 & \begin{tabular}{|c|}
$1.10 \mathrm{E}-04$ \\
\end{tabular} & 1.65 & \begin{tabular}{|l|} 
\\
\end{tabular} & 49.31 & 98.58 & 85.81 & & 70.9 & 226.5 \\
\hline 358 & 27.26 & 3000 & 1850 & 30.502939 & 1.4492 & \begin{tabular}{|l|}
142.5296 \\
\end{tabular} & \begin{tabular}{|l|}
1578.62 \\
\end{tabular} & 5125 & 0.1 & 1950 & 225000 & 1825000 & 0.1167 & \begin{tabular}{|l|}
$2.50 \mathrm{E}-04$ \\
\end{tabular} & 2.084 & $1.24 \mathrm{E}-02$ & 31.36 & 83.61 & & & 55.9 & 391.9 \\
\hline 359 & 23.37 & 3000 & 2910 & 46.29893 & 1.3087 & \begin{tabular}{|l|}
84.7981 \\
\end{tabular} & 2653.36 & 5125 & 0.1 & 1950 & 225000 & 837500 & 0.1361 & \begin{tabular}{|l|}
$3.07 \mathrm{E}-04$ \\
\end{tabular} & 2.14 & \begin{tabular}{|c|}
$9.46 \mathrm{E}-03$ \\
\end{tabular} & 36.98 & 89.2 & & & 73.2 & 469.2 \\
\hline 360 & 21.7 & 3000 & 1620 & 6.644542 & 0.3958 & \begin{tabular}{|l|l|}
178.7197 \\
\end{tabular} & 1258.95 & 5125 & 0.1 & 1950 & 225000 & 2562500 & 0.1466 & \begin{tabular}{|l|}
$3.33 \mathrm{E}-04$ \\
\end{tabular} & 2.148 & & 69.13 & 24.75 & & & 48.9 & 507.2 \\
\hline 361 & 41.8 & 3000 & 1640 & 372.629298 & 12.7278 & & \begin{tabular}{|l|}
2195.77 \\
\end{tabular} & 5125 & 0.1 & & & 1112500 & & \begin{tabular}{|l|}
$1.42 \mathrm{E}-04$ \\
\end{tabular} & 1.941 & & 43.28 & $\begin{array}{l}98.66 \\
\end{array}$ & 86.58 & & 76.5 & 237.9 \\
\hline 362 & 37.12 & 3000 & 1820 & 134.196355 & 6.0512 & \begin{tabular}{|l|l|}
135.2775 \\
\end{tabular} & \begin{tabular}{|l|}
1663.25 \\
\end{tabular} & 5125 & 0.1 & 1950 & 225000 & 1687500 & 0.0857 & \begin{tabular}{|l|l|}
$1.83 \mathrm{E}-04$ \\
\end{tabular} & 2.082 & $1.13 \mathrm{E}-02$ & 36.73 & $\begin{array}{l}96.27 \\
\end{array}$ & 62.74 & & 61.3 & 287.4 \\
\hline 363 & 57.63 & 3000 & 5430 & 4.971072 & $\begin{array}{l}0.1474 \\
10.225\end{array}$ & \begin{tabular}{|r|}
88.9661 \\
1278255 \\
\end{tabular} & $\begin{array}{l}2529.05 \\
\end{array}$ & 5125 & 0.1 & & 225000 & 900000 & & 7.71E-04 & 8.065 & $1.03 \mathrm{E}-02$ & 18.72 & 0 & 0 & & 67.1 & 717.2 \\
\hline $\begin{array}{l}364 \\
365 \\
\end{array}$ & $\begin{array}{r}35.99 \\
28.2 \\
\end{array}$ & $\begin{array}{l}3000 \\
3000\end{array}$ & $\begin{array}{l}2130 \\
1990\end{array}$ & \begin{tabular}{|l|}
244.609972 \\
206.013193 \\
\end{tabular} & \begin{tabular}{|c|}
10.4225 \\
8.6358
\end{tabular} & \begin{tabular}{|l|}
127.8255 \\
1257554 \\
\end{tabular} & \begin{tabular}{|l|}
1760.21 \\
1789.19
\end{tabular} & $\begin{array}{l}5125 \\
5125 \\
\end{array}$ & $\begin{array}{l}0.1 \\
0.1\end{array}$ & $\begin{array}{l}1950 \\
1950\end{array}$ & 225000 & $\begin{array}{l}1550000 \\
1512500\end{array}$ & $\begin{array}{l}0.0884 \\
01128\end{array}$ & \begin{tabular}{|l|}
$3.00 \mathrm{E}-04$ \\
$218 \mathrm{E}-04$ \\
\end{tabular} & $\begin{array}{l}2.718 \\
1976 \\
\end{array}$ & \begin{tabular}{|l|}
$6.86 \mathrm{E}-03$ \\
$775 \mathrm{E}-3$ \\
\end{tabular} & $\begin{array}{l}44.02 \\
38.22\end{array}$ & $\begin{array}{l}97.96 \\
07.57\end{array}$ & $\begin{array}{ll}79.56 \\
77.73\end{array}$ & & \begin{tabular}{|l|l|}
101.1 \\
894
\end{tabular} & 387 \\
\hline 360 & \begin{tabular}{|l|}
28.2 \\
25.43
\end{tabular} & 3000 & $\begin{array}{l}1990 \\
2020\end{array}$ & $\begin{array}{r}206.01731 \\
153536\end{array}$ & $\begin{array}{l}8.6398 \\
0.6992 \\
\end{array}$ & \begin{tabular}{|l|}
125.7554 \\
136.6103 \\
\end{tabular} & \begin{tabular}{|l|l|}
1789.19 \\
1647.02
\end{tabular} & $\begin{array}{l}5125 \\
5125\end{array}$ & $\begin{array}{l}0.1 \\
0.1\end{array}$ & $\begin{array}{l}\frac{1950}{1950} \\
\end{array}$ & $\frac{225000}{225000}$ & $\begin{array}{l}\frac{1512500}{1712500} \\
\end{array}$ & $\begin{array}{l}0.1128 \\
0.1251\end{array}$ & \begin{tabular}{|l|}
$2.18 \mathrm{E}-04$ \\
$3.05 \mathrm{E}-04$ \\
\end{tabular} & $\begin{array}{c}1.976 \\
2.234\end{array}$ & \begin{tabular}{|l|}
$7.75 \mathrm{E}-03$ \\
$1.24 \mathrm{E}-02$ \\
\end{tabular} & $\begin{array}{l}38.22 \\
26.39\end{array}$ & $\begin{array}{c}97.57 \\
67.43\end{array}$ & \begin{tabular}{rl|}
75.73 & \\
0
\end{tabular} & & $\frac{89.4}{55.8}$ & 359.1 \\
\hline 367 & 29.43 & & 2030 & 14.4498 & 0.64 & \begin{tabular}{|l|}
133.2659 \\
\end{tabular} & \begin{tabular}{|l|}
1688.35 \\
\end{tabular} & 5125 & 0.1 & & 2250 & 1650000 & & \begin{tabular}{|l|l|}
$2.88 \mathrm{E}$ \\
\end{tabular} & 2.348 & \begin{tabular}{|l|l|} 
& $1.38 \mathrm{~L}-02$ \\
\end{tabular} & 24.36 & 65.4 & & & 50.1 & $\begin{array}{r}450.2 \\
409 \\
\end{array}$ \\
\hline 368 & 29.29 & 3000 & 1710 & 717.5077 & 35.1698 & \begin{tabular}{|l|l|}
147.0497 \\
\end{tabular} & 1530.1 & 5125 & 0.1 & & 225000 & 1912500 & & 1.92E-04 & $1.8 \mathrm{C}$ & 4.46 & 59.55 & 99.3 & 93.03 & 30.31 & 155.6 & 331.2 \\
\hline 369 & 62.14 & 3000 & 2210 & 172.8810 & 8.6577 & \begin{tabular}{|l|}
150.2362 \\
\end{tabular} & 1497.64 & 5125 & 0.1 & 1950 & 225000 & 1975000 & 0.0512 & \begin{tabular}{|l|}
$3.70 \mathrm{E}-04$ \\
\end{tabular} & 4.648 & $8.32 \mathrm{E}-03$ & 53.33 & 97.11 & 71.08 & & 83.3 & 383.4 \\
\hline 370 & 34.36 & 3000 & 3290 & 0.218063 & 0.0128 & \begin{tabular}{|c|}
175.8017 \\
\end{tabular} & 1279.85 & 5125 & 0.1 & 1950 & & & 0.0926 & \begin{tabular}{|l|l|}
$7.17 \mathrm{E}-04$ \\
\end{tabular} & 4.914 & & 19.31 & 0 & & & 46.2 & 733.1 \\
\hline 371 & 26.89 & 3000 & 2620 & 113.455437 & 4.2162 & 1111.4852 & 201 & 5125 & 0.1 & 1950 & & 1262500 & & $4.45 \mathrm{E}$ & 2.902 & & 51.73 & 95.59 & 55.93 & & 106.8 & 553.1 \\
\hline 372 & 36.61 & 3000 & 3020 & & $\begin{array}{l}1.1265 \\
\end{array}$ & \begin{tabular}{|r|}
93.8425 \\
\end{tabular} & \begin{tabular}{|l|}
2397 \\
\end{tabular} & 5125 & 0.1 & 1950 & 225000 & 975000 & 0.0 & & 3.475 & $9.8 \varepsilon$ & & 86.12 & & & 70.1 & 486.5 \\
\hline 373 & 39.42 & 3000 & 1280 & & 11.8919 & \begin{tabular}{|l|l|}
106.2727 \\
\end{tabular} & 211 & 5125 & 0.1 & & 2250 & & & 1.44 & 1.899 & & 24. & 98.51 & 85.11 & & 76.6 & 24 \\
\hline 374 & 54.3 & 3000 & 3090 & & & \begin{tabular}{|l|}
96.2335 \\
\end{tabular} & 2338 & & 0.1 & & & & 585 & & 4.56 & & & $\begin{array}{l}96.34 \\
\end{array}$ & $\begin{array}{l}63.37 \\
\end{array}$ & & 87.8 & \\
\hline 375 & 29.0 & 300 & 2950 & & & 111.4852 & 2018 & 512 & 0.1 & 19 & & & 0.1 & \begin{tabular}{|l|l|} 
& 5.30 \\
\end{tabular} & 3.4 & & & 96.71 & & & 130.4 & 607.8 \\
\hline 376 & $\begin{array}{l}42.42 \\
\end{array}$ & 3000 & 2360 & & 2.4044 & & 208 & 5125 & 0.1 & 1950 & 225000 & & & & & & & & & 0 & & \\
\hline 377 & $\begin{array}{l}49.48 \\
\end{array}$ & & 1420 & & 27.1583 & $165.08 \varepsilon$ & 136 & 5125 & 0.1 & & & & & & & & & & & & & \\
\hline 378 & 25.55 & 300 & 2390 & & $\begin{array}{l}.5133 \\
\end{array}$ & 9937 & 2264 & 51 & & & 2250 & 10625 & & 2003 & & & & 96. & \begin{tabular}{|c|c|}
63.3 \\
\end{tabular} & & 76.4 & \\
\hline 379 & \begin{tabular}{|l|}
54.1 \\
\end{tabular} & 3000 & 1950 & 3 & $\mid 13.3858$ & 128.51 & 1750.81 & 512 & & 19 & 2250 & 1562500 & $\mid 0.0588$ & 2 & 2.958 & \begin{tabular}{|l|l|} 
& 8.92 \\
\end{tabular} & 80 & $\mid$ & 84 & & 77.7 & 280.2 \\
\hline 380 & 28 & 30 & 1530 & 90.99 & \begin{tabular}{|l|l|}
4.7662 \\
\end{tabular} & 157.1306 & 1431.6 & 51 & & & & 2112 & 0.1136 & $\mid 1.47 \mathrm{E}-04$ & 1.656 & \begin{tabular}{|l|l|}
$.28 \mathrm{E}-02$ \\
\end{tabular} & \begin{tabular}{|l|l|}
94.67 \\
\end{tabular} & 94.51 & 45.05 & & 54.3 & 303.1 \\
\hline 381 & 21.8 & 3000 & \begin{tabular}{|l|l|}
1190 \\
\end{tabular} & $566.616 \mathrm{G}$ & 26.7968 & \begin{tabular}{|l|}
141.878 \\
\end{tabular} & \begin{tabular}{|l|l|}
1585.87 \\
\end{tabular} & 5125 & 0.1 & 19 & 2250 & 1812500 & 0.14 & \begin{tabular}{|l|l|}
$1.01 \mathrm{E}-04$ \\
\end{tabular} & 1.35 & \begin{tabular}{|l|l|} 
& $5.33 \mathrm{E}$ \\
\end{tabular} & 34.36 & 99.12 & 91.18 & 11.76 & 130 & 317.2 \\
\hline 382 & 24.59 & 3000 & 3000 & 0.593 & 0.0182 & 92.2314 & 2439 & 5125 & 0.1 & 1950 & 225000 & 950000 & 0.1294 & \begin{tabular}{|l|}
$5.95 \mathrm{E}-04$ \\
\end{tabular} & 3.326 & 1.64 & $\begin{array}{ll}60.68 \\
\end{array}$ & 0 & & & 42.2 & 693.4 \\
\hline 383 & 30.15 & 3000 & 2110 & 98.602 & 4.1787 & 127.1374 & \begin{tabular}{|l|}
1769.74 \\
\end{tabular} & 512 & 0.1 & 195 & 225000 & 1537 & 0.1 & \begin{tabular}{|l|}
$2.87 \mathrm{E}$ \\
\end{tabular} & 2.375 & 8.89 & 31.67 & 94.93 & 49.29 & & 78 & 403.6 \\
\hline 384 & 24.8 & & 2620 & & 0.0 & \begin{tabular}{|l|}
93.84 \\
\end{tabular} & & 512 & 0.1 & & & & & 3.88 & 2.528 & & 83.6 & & 0 & & 37.4 & 522.4 \\
\hline 385 & 39. & 30 & 890 & 187.9 & & & 173 & 5125 & 0.1 & 195 & & & & 9.22 & 1.58 & & 32.5 & 97.34 & 73.4 & & 49.8 & \\
\hline 386 & & 3000 & 3950 & & & \begin{tabular}{|l|l|}
101.7009 \\
\end{tabular} & & 5125 & 0.1 & 1950 & 225000 & 1100000 & & \begin{tabular}{|l|l|}
6.16 \\
\end{tabular} & 5.973 & & 21.8 & \begin{tabular}{|l|l|}
38.77 \\
\end{tabular} & 0 & & 61.9 & $\begin{array}{l}602.3 \\
\end{array}$ \\
\hline 87 & 56. & & 1870 & & 1.1 & & 130 & & 0.1 & & & & & & & & & 75.56 & 0 & & 43.6 & \\
\hline 388 & & 30 & 3080 & & & \begin{tabular}{|l|l|l|}
108.5219 \\
\end{tabular} & & & 0.1 & & & 1212500 & & \begin{tabular}{|c|c|}
$6.48 \mathrm{E}-04$ \\
\end{tabular} & & & & & & & $\begin{array}{r}56.5 \\
\end{array}$ & \\
\hline $\begin{array}{l}389 \\
390\end{array}$ & $\begin{array}{r}36.32 \\
31.16\end{array}$ & 3000 & $\begin{array}{l}3030 \\
2240\end{array}$ & $\begin{array}{r}80.0654 \\
41.060876\end{array}$ & $\begin{array}{l}3.3005 \\
16542\end{array}$ & \begin{tabular}{|c|}
123.6681 \\
1208573
\end{tabular} & \begin{tabular}{|r|}
1819.39 \\
18617
\end{tabular} & \begin{tabular}{|l|}
5125 \\
5125
\end{tabular} & $\begin{array}{l}0.1 \\
0.1\end{array}$ & $\begin{array}{l}1950 \\
1950\end{array}$ & $\begin{array}{l}225000 \\
225000\end{array}$ & $\begin{array}{l}1475000 \\
1425000\end{array}$ & $\begin{array}{l}0.0876 \\
0.121\end{array}$ & \begin{tabular}{|l|}
$5.79 \mathrm{E}-04$ \\
$320 \mathrm{E}-04$ \\
\end{tabular} & $\begin{array}{r}4.34 \\
258 \\
\end{array}$ & \begin{tabular}{|l|}
$6.60 \mathrm{E}-03$ \\
$108 \mathrm{E}-02$
\end{tabular} & $\begin{array}{l}45.24 \\
32.91\end{array}$ & \begin{tabular}{|c|c|}
93.76 \\
87.82
\end{tabular} & $\begin{array}{r}37.55 \\
0\end{array}$ & & $\begin{array}{r}105.1 \\
64.1 \\
\end{array}$ & $\begin{array}{r}612.5 \\
425.4 \\
\end{array}$ \\
\hline
\end{tabular}




\begin{tabular}{|c|c|c|c|c|c|c|c|c|c|c|c|c|c|c|c|c|c|c|c|c|c|c|}
\hline $\begin{array}{c}\text { Realizatio } \\
n\end{array}$ & $\begin{array}{c}\text { Velocity } \\
(\mathrm{m} / \mathrm{y})\end{array}$ & $\begin{array}{c}\text { Fixed Source } \\
\text { Concentration } \\
\text { (mg/L) }\end{array}$ & $\begin{array}{c}\text { Time of } \\
\text { Maximum at } \\
\text { Compliance } \\
\text { Boundary } \\
(y)\end{array}$ & \begin{tabular}{|c|} 
Maximum \\
Concentration \\
Compliance \\
Boundary \\
$(\mu \mathrm{g} / \mathrm{L})$
\end{tabular} & \begin{tabular}{|c|} 
Maximum \\
Release Rate \\
at Compliance \\
Boundary \\
$(\mathrm{Kg} / \mathrm{y})$
\end{tabular} & \begin{tabular}{|c|} 
Source \\
Rate \\
$(\mathrm{Kg} / \mathrm{y})$ \\
\end{tabular} & \begin{tabular}{|c|} 
Source \\
Duration \\
(y)
\end{tabular} & \begin{tabular}{|c|} 
1-D \\
Streamtub \\
e Length \\
(m)
\end{tabular} & $\begin{array}{l}\text { Base } \\
\text { Porosity } \\
\left(\mathrm{m}^{3} / \mathrm{m}^{3}\right)\end{array}$ & $\begin{array}{c}\text { Bulk } \\
\text { Densty } \\
\left(\mathrm{Kg} / \mathrm{m}^{3}\right)\end{array}$ & $\begin{array}{c}\text { Remaining } \\
\text { Carbon } \\
\text { Tetrachloride } \\
\text { Source } \\
(\mathrm{Kg})\end{array}$ & $\begin{array}{c}\text { Volume at or } \\
\text { above Fixed } \\
\text { Source } \\
\begin{array}{c}\text { Concentration } \\
(\mathrm{m} 3)\end{array} \\
\end{array}$ & $\begin{array}{l}\text { Porosity } \\
\left(\mathrm{m}^{3} / \mathrm{m}^{3}\right)\end{array}$ & $\begin{array}{c}\mathrm{Kd} \\
\left(\mathrm{m}^{3} / \mathrm{Kg}\right)\end{array}$ & Retardation & $\begin{array}{c}\text { First Order } \\
\text { Rate } \\
\text { Constant } \\
(1 / y)\end{array}$ & $\begin{array}{c}\text { Longitudinal } \\
\text { Dispersivity } \\
(\mathrm{m})\end{array}$ & $\begin{array}{c}\text { Required } \\
\text { Source } \\
\text { Cleanup } \\
\text { Percentage } \\
(5 \mu g / L \\
\text { Compliance) } \\
\end{array}$ & \begin{tabular}{|c|} 
Required \\
Source \\
Cleanup \\
Percentage \\
(50 gig/L \\
Compliance)
\end{tabular} & \begin{tabular}{|c} 
Required \\
Source \\
Cleanup \\
Percentage \\
(500 ug/L \\
Compliance) \\
\end{tabular} & \begin{tabular}{|c|} 
Abiotic \\
Reaction \\
Half Time \\
(y)
\end{tabular} & $\begin{array}{c}\text { Carbon } \\
\text { Tetrachloride } \\
\text { Travel Time (y) }\end{array}$ \\
\hline & 40.63 & & & & & \begin{tabular}{|l|}
97.024 \\
\end{tabular} & \begin{tabular}{|r|}
2319.01 \\
\end{tabular} & & & 1950 & & & & $4.10 \mathrm{E}-04$ & & \begin{tabular}{|l|}
$1.37 \mathrm{E}-02$ \\
\end{tabular} & & & & & & \\
\hline 392 & 58.7 & 3000 & 2000 & $\begin{array}{r}6.99138 \\
\end{array}$ & 1.5677 & \begin{tabular}{|l|l|}
127.1374 \\
\end{tabular} & \begin{tabular}{|l|}
1769.74 \\
\end{tabular} & 5125 & 0.1 & 1950 & 225000 & 1537500 & 0.0542 & $2.28 \mathrm{E}-04$ & 3.131 & \begin{tabular}{|l|l|}
$1.77 \mathrm{E}-02$ \\
\end{tabular} & 37.72 & 86.48 & & 0 & 39.1 & 273.4 \\
\hline 393 & $\begin{array}{l}30.34 \\
31.34 \\
\end{array}$ & 3000 & 2190 & \begin{tabular}{|r|}
653.192189 \\
\end{tabular} & 23.6286 & \begin{tabular}{|l|l|}
108.5219 \\
\end{tabular} & \begin{tabular}{|l|}
2073.31 \\
\end{tabular} & 5125 & 0.1 & 1950 & 225000 & 1212500 & $\begin{array}{l}0.0445 \\
0.1015 \\
\end{array}$ & $\begin{array}{l}2.202-04 \\
1.82 \mathrm{E}-04 \\
\end{array}$ & $\begin{array}{l}.1 .907 \\
\end{array}$ & \begin{tabular}{|l|}
$5.10 \mathrm{E}-03$ \\
\end{tabular} & 77.71 & $\begin{array}{l}90.40 \\
99.23 \\
\end{array}$ & 92.35 & 23.45 & 135.8 & 311.8 \\
\hline 394 & 57.42 & 3000 & 2150 & \begin{tabular}{|l|l|}
273.694332 \\
\end{tabular} & $\begin{array}{l}13.4156 \\
\end{array}$ & \begin{tabular}{|l|}
147.0497 \\
\end{tabular} & \begin{tabular}{|l|}
1530.1 \\
\end{tabular} & 5125 & 0.1 & 1950 & 225000 & 1912500 & 0.0554 & $3.32 \mathrm{E}-04$ & 4.032 & \begin{tabular}{|l|l|}
$7.16 \mathrm{E}-03$ \\
\end{tabular} & 43.18 & 98.17 & 81.73 & & 96.8 & 359.9 \\
\hline 395 & 23.19 & 3000 & 2220 & $\begin{array}{l}375.814232 \\
\end{array}$ & 18.0986 & \begin{tabular}{|l|}
144.4754 \\
\end{tabular} & 1557.36 & 5125 & 0.1 & 1950 & 225000 & 1862500 & $\begin{array}{l}0.0372 \\
\end{array}$ & $4.07 \mathrm{E}-04$ & 2.499 & \begin{tabular}{|l|}
$3.92 \mathrm{E}-03$ \\
\end{tabular} & 41.73 & 98.67 & 86.7 & & 177 & 552.4 \\
\hline 396 & 21.34 & 3000 & 3160 & 1.211582 & 0.0336 & \begin{tabular}{|l|}
83.1021 \\
\end{tabular} & 2707.51 & 5125 & 0.1 & 1950 & 225000 & 812500 & 0.1491 & $4.58 \mathrm{E}-04$ & 2.554 & $1.44 \mathrm{E}-02$ & 33.97 & 0 & 0 & & 48.1 & 613.4 \\
\hline 397 & 24.3 & 3000 & 3340 & 295.218018 & 8.0091 & \begin{tabular}{|l|}
81.3886 \\
\end{tabular} & 2764.52 & 5125 & 0.1 & 1950 & 225000 & 787500 & 0.1309 & $5.03 \mathrm{E}-04$ & 2.944 & \begin{tabular}{|l|} 
\\
\end{tabular} & 75.31 & 98.31 & 83.06 & & 168.8 & 620.9 \\
\hline & 22.48 & & & & 0.7334 & \begin{tabular}{|l|l|}
133.2659 \\
\end{tabular} & 1688.35 & 5125 & 0.1 & & 225000 & 1650000 & 0.1415 & $1.35 \mathrm{E}-04$ & 1.481 & \begin{tabular}{|c|}
$1.73 \mathrm{E}-02$ \\
\end{tabular} & 79.86 & 69.72 & & & & 337.7 \\
\hline 399 & 48.79 & 3000 & 1830 & 52.416225 & 2.3167 & \begin{tabular}{|l|}
132.592 \\
\end{tabular} & 1696.94 & 5125 & 0.1 & 1950 & 225000 & 1637500 & 0.0652 & \begin{tabular}{|c|}
$1.86 \mathrm{E}-04$ \\
\end{tabular} & 2.442 & $1.73 \mathrm{E}-02$ & 50.23 & 90.46 & 4.61 & & 40 & 256.5 \\
\hline 400 & 44.74 & 3000 & 3370 & 23.239648 & 0.8407 & \begin{tabular}{|l|l|}
108.5219 \\
\end{tabular} & 2073.31 & 5125 & 0.1 & 1950 & 225000 & 1212500 & 0.0711 & $5.14 \mathrm{E}-04$ & 4.653 & \begin{tabular}{|c|} 
\\
\end{tabular} & 16.18 & 78.49 & & 0 & 71 & 532.9 \\
\hline 401 & $\begin{array}{l}40.42 \\
2293\end{array}$ & 3000 & $\begin{array}{r}890 \\
8620\end{array}$ & 1234.813005 & $\begin{array}{ll}62.6182 \\
6652\end{array}$ & \begin{tabular}{|l|l|}
152.1319 \\
\end{tabular} & 1478.98 & 5125 & 0.1 & 1950 & 225000 & 2012500 & 0.0787 & $\begin{array}{l}3.98 \mathrm{E}-05 \\
\end{array}$ & 1.256 & \begin{tabular}{|l|}
$5.69 \mathrm{E}-03$ \\
\end{tabular} & $\begin{array}{l}92.87 \\
\end{array}$ & 99.6 & & & $\begin{array}{ll}121.8 \\
\end{array}$ & 159.2 \\
\hline \begin{tabular}{|l|l|}
402 \\
403
\end{tabular} & 32.93 & 3000 & 2620 & & $\begin{array}{l}25.6533 \\
12804 \\
\end{array}$ & \begin{tabular}{|l|l|}
110.7481 \\
\end{tabular} & 2031.64 & 5125 & 0.1 & $\begin{array}{l}1950 \\
1950\end{array}$ & 225000 & 1250000 & $\begin{array}{l}0.0966 \\
\end{array}$ & $\begin{array}{l}3.72 \mathrm{E}-04 \\
.20=\end{array}$ & 2.946 & \begin{tabular}{|l|}
$3.26 \mathrm{E}-03$ \\
\end{tabular} & 26.31 & 99.28 & 92.8 & & 212.6 & 458.4 \\
\hline \begin{tabular}{|l|l|}
403 \\
404
\end{tabular} & $\begin{array}{l}40.89 \\
26.67\end{array}$ & $\begin{array}{l}3000 \\
3000\end{array}$ & $\begin{array}{l}3150 \\
1780\end{array}$ & 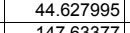 & $\begin{array}{l}1.2864 \\
83312 \\
\end{array}$ & \begin{tabular}{|r|}
86.4774 \\
1692915 \\
\end{tabular} & \begin{tabular}{|l|}
2601.84 \\
13904
\end{tabular} & $\begin{array}{l}5125 \\
5125 \\
\end{array}$ & 0.1 & $\begin{array}{l}1950 \\
1950\end{array}$ & 225000 & $\begin{array}{r}862500 \\
2362500\end{array}$ & $\begin{array}{l}0.0778 \\
0.1193 \\
\end{array}$ & $\begin{array}{l}4.38 \mathrm{E}-04 \\
308 E-04\end{array}$ & 3.845 & \begin{tabular}{|l|}
$1.01 \mathrm{E}-02$ \\
7
\end{tabular} & $\begin{array}{l}49.14 \\
36.38\end{array}$ & $\begin{array}{r}88.8 \\
96.61\end{array}$ & $\begin{array}{r}0 \\
\end{array}$ & 0 & 68.7 & 481.8 \\
\hline $\begin{array}{l}404 \\
405 \\
\end{array}$ & $\frac{26.67}{22}$ & 3000 & $\begin{array}{l}1780 \\
2590\end{array}$ & $\begin{array}{l}147.6334 / 1 \\
78.543412\end{array}$ & \begin{tabular}{|l|}
.33312 \\
2.7028 \\
\end{tabular} & \begin{tabular}{|l|}
169.2951 \\
103.2361 \\
\end{tabular} & $\begin{array}{l}1329.04 \\
2179.47\end{array}$ & \begin{tabular}{|l}
5125 \\
5125
\end{tabular} & $\frac{0.1}{0.1}$ & $\begin{array}{l}\frac{1950}{1950} \\
1950\end{array}$ & $\begin{array}{l}225000 \\
225000\end{array}$ & $\begin{array}{l}2362500 \\
1125000\end{array}$ & \begin{tabular}{|c|}
0.193 \\
0.1446
\end{tabular} & $\left|\frac{3.08 \mathrm{E}-04}{3.99 \mathrm{E}-04}\right|$ & $\frac{2.303}{2.396}$ & \begin{tabular}{|l|}
$7.14 \mathrm{E}-03$ \\
$7.24 \mathrm{E}-03$ \\
\end{tabular} & $\begin{array}{l}36.38 \\
64.02\end{array}$ & $\begin{array}{l}96.61 \\
93.63 \\
\end{array}$ & $\begin{array}{l}66.63 \\
6.34\end{array}$ & & $\begin{array}{l}99.1 \\
95.7 \\
\end{array}$ & $\begin{array}{r}442.7 \\
558 \\
\end{array}$ \\
\hline 406 & 52.85 & 3000 & 1930 & \begin{tabular}{|l|l|}
55.264928 \\
\end{tabular} & 3.0856 & \begin{tabular}{|l|}
167.4988 \\
\end{tabular} & \begin{tabular}{|l|}
1343.29 \\
\end{tabular} & 5125 & 0.1 & 1950 & 225000 & 2325000 & $\begin{array}{l}0.1400 \\
0.0602\end{array}$ & $\begin{array}{l}3.95 E-04 \\
3.45-04\end{array}$ & 3.894 & \begin{tabular}{|l|}
$1.24 \mathrm{E}-0$ \\
$1.20 \mathrm{E}-02$ \\
\end{tabular} & $\begin{array}{l}04.02 \\
46.69\end{array}$ & $\begin{array}{l}90.00 \\
90.95\end{array}$ & 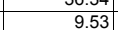 & & $\begin{array}{l}57.1 \\
57.6\end{array}$ & $\begin{array}{r}377.7 \\
377 \\
\end{array}$ \\
\hline 407 & 27.86 & 3000 & 3420 & 135.501152 & 3.9436 & \begin{tabular}{|r|}
87.3109 \\
\end{tabular} & $\begin{array}{r}2577 \\
\end{array}$ & 5125 & 0.1 & 1950 & 225000 & 875000 & 0.1142 & $\begin{array}{l}.489 \mathrm{E}-04 \\
\end{array}$ & 3.165 & \begin{tabular}{|l|}
$5.48 \mathrm{E}-03$ \\
\end{tabular} & 15.65 & 96.31 & $\begin{array}{l}6.3 .1 \\
63.1\end{array}$ & & 126.5 & 582.2 \\
\hline 408 & 41.21 & 3000 & 2380 & 208.352883 & 8.2457 & \begin{tabular}{|r|}
118.7276 \\
\end{tabular} & 1895.09 & 5125 & 0.1 & 1950 & 225000 & 1387500 & 0.0772 & $3.14 \mathrm{E}-04$ & 3.056 & \begin{tabular}{|l|} 
\\
\end{tabular} & 15.43 & 97.6 & 76 & & 96.4 & 380 \\
\hline 409 & 33 & 3000 & 2140 & 49.814191 & 2.345 & \begin{tabular}{|l|}
141.2249 \\
\end{tabular} & 1593.2 & 5125 & 0.1 & 1950 & 225000 & 1800000 & 0.0964 & $3.68 \mathrm{E}-04$ & 2.927 & $9.80 \mathrm{E}-03$ & 37.24 & 89.96 & 0 & & 70.7 & 454.6 \\
\hline 410 & 50.1 & 3000 & 2680 & 211.523627 & 9.6321 & \begin{tabular}{|l|}
136.6103 \\
\end{tabular} & 1647.02 & 5125 & 0.1 & 1950 & 225000 & 1712500 & 0.0635 & $4.90 \mathrm{E}-04$ & 4.904 & \begin{tabular}{|l|}
$5.77 \mathrm{E}-03$ \\
\end{tabular} & 61.03 & 97.64 & 76.36 & & 120.2 & 501.7 \\
\hline 411 & 40.53 & 3000 & 2590 & 369.444987 & 20.8484 & \begin{tabular}{|l|}
169.2951 \\
\end{tabular} & 1329.04 & 5125 & 0.1 & 1950 & 225000 & 2362500 & 0.0785 & $4.87 \mathrm{E}-04$ & 4.137 & \begin{tabular}{|c|}
$3.98 \mathrm{E}-03$ \\
\end{tabular} & 35 & 98.65 & 86.47 & & 174.2 & 523.2 \\
\hline 412 & 38.75 & 3000 & 3500 & 233.663071 & 5.8604 & \begin{tabular}{|l|}
75.2423 \\
\end{tabular} & 2990.34 & 5125 & 0.1 & 1950 & 225000 & 700000 & & 4.24E-04 & 3.609 & \begin{tabular}{|l|}
$5.84 \mathrm{E}-03$ \\
\end{tabular} & 51.72 & 97.86 & 78.6 & & 118.6 & 477.3 \\
\hline 413 & 22.33 & 3000 & 900 & 164.521655 & 10.3046 & \begin{tabular}{|l|l|}
187.9019 \\
\end{tabular} & \begin{tabular}{|l|}
1197.43 \\
\end{tabular} & 5125 & 0.1 & 1950 & 225000 & 2762500 & 0.1425 & $8.02 \mathrm{E}-05$ & 1.285 & $\mid 1.00 \mathrm{E}-02$ & 26.23 & 96.96 & 69.61 & & 69.1 & 294.9 \\
\hline 414 & 36.65 & 3000 & 3390 & 79.529949 & 2.7973 & \begin{tabular}{|l|l|}
105.5176 \\
\end{tabular} & 2132.35 & 5125 & 0.1 & 1950 & 225000 & 1162500 & 0.0868 & $7.39 \mathrm{E}-04$ & 5.304 & $6.15 \mathrm{E}-03$ & 87.44 & 93.71 & 37.13 & & 112.7 & 741.6 \\
\hline 415 & $\begin{array}{l}61.65 \\
26.5 \\
6\end{array}$ & 3000 & 2820 & \begin{tabular}{|r|r|}
6.201872 \\
1002179
\end{tabular} & 0.304 & \begin{tabular}{|l|l|}
147.0497 \\
\end{tabular} & 1530.1 & 5125 & 0.1 & $\begin{array}{l}1950 \\
1050 \\
1050\end{array}$ & 225000 & 1912500 & $\begin{array}{l}0.0516 \\
\end{array}$ & 4.73E-04 & 5.631 & \begin{tabular}{|c|}
$1.51 \mathrm{E}-02$ \\
\end{tabular} & 22.67 & 19.38 & & & 45.9 & 468 \\
\hline $\begin{array}{l}416 \\
417 \\
\end{array}$ & 26.85 & 3000 & 1780 & \begin{tabular}{|c|}
10.003179 \\
5323571 \\
\end{tabular} & \begin{tabular}{|l|l|}
0.4709 \\
26925 \\
\end{tabular} & 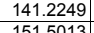 & $\begin{array}{l}1593.2 \\
190.2\end{array}$ & 5125 & 0.1 & $\begin{array}{l}1950 \\
1950\end{array}$ & 225000 & 1800000 & 0.1185 & $2.27 \mathrm{E}-04$ & 1.967 & \begin{tabular}{|l|}
$1.70 \mathrm{E}-02$ \\
\end{tabular} & 55.32 & 50.02 & 0 & & 40.7 & 375.5 \\
\hline$\frac{411}{418}$ & $\frac{57.84}{26.49}$ & $\begin{array}{l}3000 \\
3000\end{array}$ & $\begin{array}{l}2670 \\
1380\end{array}$ & $\begin{array}{r}53.335741 \\
202.285027\end{array}$ & $\begin{array}{l}2.6935 \\
6.7008\end{array}$ & \begin{tabular}{|r|}
$\mid 151.5013$ \\
99.3762 \\
\end{tabular} & \begin{tabular}{|l|}
1425.14 \\
2264.12 \\
\end{tabular} & $\begin{array}{l}55125 \\
5125\end{array}$ & $\frac{0.1}{0.1}$ & $\begin{array}{l}1950 \\
1950\end{array}$ & $\frac{225000}{225000}$ & $\begin{array}{l}20000000 \\
102500\end{array}$ & \begin{tabular}{|r|}
0.055 \\
0.1201
\end{tabular} & $\begin{array}{l}\frac{4.50 \mathrm{E}-04}{1.41-04} \\
\end{array}$ & 5.139 & \begin{tabular}{|l|}
$9.70 \mathrm{E}-03$ \\
$9.00 \mathrm{E}-03$
\end{tabular} & $\begin{array}{l}28.65 \\
33.64\end{array}$ & $\begin{array}{l}90.63 \\
9.53 \\
\end{array}$ & \begin{tabular}{r|r|r|}
75.25 \\
75.28
\end{tabular} & & $\begin{array}{r}71.5 \\
77 \\
\end{array}$ & $\begin{array}{r}455.3 \\
308 \\
\end{array}$ \\
\hline 419 & 24.64 & 3000 & 2270 & 29.019337 & 1.2563 & \begin{tabular}{|l|}
129.879 \\
\end{tabular} & \begin{tabular}{|l|}
1732.38 \\
\end{tabular} & 5125 & 0.1 & 1950 & 225000 & 1587500 & 0.1291 & $3.76 \mathrm{E}-04$ & 2.473 & \begin{tabular}{|c|} 
\\
\end{tabular} & 25.48 & 82.77 & & & 72.7 & $\begin{array}{r}514.3 \\
5\end{array}$ \\
\hline 420 & $\begin{array}{r}49.4 \\
\end{array}$ & 3000 & 2340 & 98.627376 & 3.0055 & \begin{tabular}{|l|}
91.4206 \\
\end{tabular} & \begin{tabular}{|l|}
2461.15 \\
\end{tabular} & 5125 & 0.1 & 1950 & 225000 & 937500 & 0.0644 & $2.35 \mathrm{E}-04$ & 2.841 & \begin{tabular}{|l|}
$1.20 \mathrm{E}-02$ \\
\end{tabular} & 19.79 & 94.93 & 49.3 & & 57.7 & 294.8 \\
\hline 421 & 21.22 & 3000 & 1840 & 83.521303 & 4.0761 & \begin{tabular}{|l|}
146.4082 \\
\end{tabular} & 1536.8 & 5125 & 0.1 & 1950 & 225000 & 1900000 & 0.1499 & $2.60 \mathrm{E}-04$ & 1.875 & \begin{tabular}{|l|}
$8.28 \mathrm{E}-03$ \\
\end{tabular} & 36.15 & 94.01 & 40.14 & & 83.7 & 452.9 \\
\hline 422 & 24.97 & 3000 & 2440 & 5.536844 & 0.2071 & \begin{tabular}{|l|}
112.2199 \\
\end{tabular} & \begin{tabular}{|l|}
2004.99 \\
\end{tabular} & 5125 & 0.1 & 1950 & 225000 & 1275000 & 0.1274 & $5.09 \mathrm{E}-04$ & 3.02 & $1.36 \mathrm{E}-02$ & 91.3 & 9.7 & & & 51 & 619.9 \\
\hline 423 & 24.03 & 3000 & 2630 & 61.161247 & 1.8968 & \begin{tabular}{|l|}
93.0387 \\
\end{tabular} & \begin{tabular}{|l|}
2418.35 \\
\end{tabular} & 5125 & 0.1 & 1950 & 225000 & 962500 & 0.1324 & $2.74 \mathrm{E}-04$ & 2.045 & $9.63 \mathrm{E}-03$ & 50.8 & 91.82 & 18.25 & & 72 & 436.2 \\
\hline 424 & 22.12 & 3000 & 2140 & 51.69871 & 2.4562 & \begin{tabular}{|l|l|}
142.5296 \\
\end{tabular} & 1578.62 & 5125 & 0.1 & 1950 & 225000 & 1825000 & 0.1438 & $4.73 \mathrm{E}-04$ & 2.661 & 7.77E-03 & 86.8 & 90.33 & 3.29 & & 89.2 & 616.5 \\
\hline 425 & 38.47 & 3000 & 2530 & 111.347343 & & 97.024 & \begin{tabular}{|l|}
2319.01 \\
\end{tabular} & 5125 & 0.1 & 1950 & 225000 & 1025000 & 0.0827 & $2.57 \mathrm{E}-04$ & 2.568 & & 42.91 & 95.51 & 55.1 & & 67.2 & 342.1 \\
\hline 426 & 25. & 3000 & 1550 & & & \begin{tabular}{|l|l|}
182.7653 \\
\end{tabular} & \begin{tabular}{|l|}
1231.09 \\
\end{tabular} & 5125 & 0.1 & & & 2650000 & & & 2.13 & & 75.48 & 96.25 & \begin{tabular}{|l|}
62.49 \\
\end{tabular} & & 86 & 423.8 \\
\hline $\begin{array}{ll}427 \\
\end{array}$ & $\begin{array}{l}40.17 \\
\end{array}$ & 3000 & 3660 & 11209 & $\begin{array}{l}0.7985 \\
\end{array}$ & \begin{tabular}{|l|}
94.6429 \\
\end{tabular} & \begin{tabular}{|l|l|}
2377.36 \\
\end{tabular} & 5125 & 0.1 & 1950 & 225000 & 987500 & 0.0792 & 5.34E-04 & 4.406 & $8.95 \mathrm{E}-03$ & \begin{tabular}{|l|l|}
13.19 \\
\end{tabular} & 80.25 & 0 & & \begin{tabular}{|l|l|}
77.4 \\
\end{tabular} & 562.1 \\
\hline 428 & 33.7 & 3000 & 1660 & & \begin{tabular}{|l|l|}
10.1941 \\
\end{tabular} & 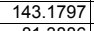 & \begin{tabular}{|l|l|}
1571.45 \\
\end{tabular} & 5125 & 0.1 & 1950 & 225000 & 1837500 & $\begin{array}{l}0.0944 \\
\end{array}$ & $1.33 \mathrm{E}-04$ & 1.714 & \begin{tabular}{|c|}
$1.08 \mathrm{E}-02$ \\
\end{tabular} & $\begin{array}{l}69.34 \\
\end{array}$ & 97.66 & 76.59 & & $\begin{array}{l}64.4 \\
\end{array}$ & 260.6 \\
\hline \begin{tabular}{|l|l|}
429 \\
430
\end{tabular} & $\begin{array}{l}42.08 \\
29.48\end{array}$ & $\begin{array}{l}3000 \\
3000\end{array}$ & \begin{tabular}{|l|l|}
3950 \\
1710
\end{tabular} & $\begin{array}{r}3.788064 \\
9883539\end{array}$ & \begin{tabular}{l|l|}
0.1028 \\
24493
\end{tabular} & \begin{tabular}{|l|}
81.3886 \\
74.3128 \\
\end{tabular} & \begin{tabular}{|r|}
2764.52 \\
\end{tabular} & $\begin{array}{l}5125 \\
5125\end{array}$ & 0.1 & $\begin{array}{l}1950 \\
1950\end{array}$ & $\begin{array}{l}225000 \\
225000\end{array}$ & $\begin{array}{l}787500 \\
6857500\end{array}$ & $\begin{array}{l}0.0756 \\
0.1079\end{array}$ & $5.88 \mathrm{E}-04$ & 4.93 & $1.28 \mathrm{E}-02$ & 23.74 & 0 & 0 & & 54.1 & 600.4 \\
\hline 4331 & $\begin{array}{l}29.48 \\
2.193\end{array}$ & $\begin{array}{l}3000 \\
3000 \\
\end{array}$ & \begin{tabular}{|l|}
1710 \\
1850
\end{tabular} & 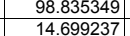 & \begin{tabular}{r|}
2.4493 \\
0.8324
\end{tabular} & \begin{tabular}{|r|}
74.3438 \\
169.917
\end{tabular} & \begin{tabular}{|l|}
3026.48 \\
124.37 \\
\end{tabular} & \begin{tabular}{|l|}
5125 \\
5125
\end{tabular} & $\frac{0.1}{0.1}$ & $\frac{\frac{195}{195}}{195}$ & $\begin{array}{l}225000 \\
225000\end{array}$ & $\begin{array}{r}637500 \\
2375000\end{array}$ & \begin{tabular}{|l|}
0.1079 \\
0.1451
\end{tabular} & $\frac{1.25 \mathrm{EE}-04}{3.59-04}$ & $\frac{1.584}{2.25}$ & \begin{tabular}{|l|}
$1.35 \mathrm{E}-02$ \\
$1.09 \mathrm{E}-02$ \\
\end{tabular} & \begin{tabular}{|l|l|l|}
80.36 & 34.69 \\
\end{tabular} & $\begin{array}{r}94.94 \\
6.58 \\
\end{array}$ & $\begin{array}{r}49.41 \\
0\end{array}$ & & \begin{tabular}{|l|l|l}
61.6 \\
63.4
\end{tabular} & $\begin{array}{l}275.4 \\
55.9 \\
\end{array}$ \\
\hline 432 & 61.53 & & 950 & 860.781927 & 23.1048 & \begin{tabular}{|r|}
80.525 \\
\end{tabular} & \begin{tabular}{|l|}
2794 \\
\end{tabular} & 5125 & 0 & & 225000 & 775000 & & $1.00 \mathrm{E}-04$ & 1.977 & \begin{tabular}{|l|l|}
$7.68 \mathrm{E}-03$ \\
\end{tabular} & 26.87 & 99.42 & 94.19 & 41.91 & $\begin{array}{l}0.3 \\
90.3\end{array}$ & $\begin{array}{l}025.9 \\
164.7 \\
\end{array}$ \\
\hline 433 & 22.39 & 3000 & 2660 & 476.7 & 14.528 & \begin{tabular}{|r|}
91.4206 \\
\end{tabular} & \begin{tabular}{|r|}
2461.15 \\
\end{tabular} & 5125 & 0.1 & 19 & 225000 & 937500 & 0.1 & $2.55 \mathrm{E}-04$ & 1.909 & $4.38 \mathrm{E}$ & 57.67 & 98.95 & 89.51 & & 158.4 & 436.9 \\
\hline 434 & 35.23 & 3000 & 2570 & 211.449421 & 7.9096 & \begin{tabular}{|l|}
112.2199 \\
\end{tabular} & \begin{tabular}{|l|}
2004.99 \\
\end{tabular} & 5125 & 0.1 & 1950 & 225000 & 1275000 & 0.0903 & $4.29 \mathrm{E}-04$ & 3.401 & $6.03 \mathrm{E}-03$ & 72.85 & 97.64 & 76.35 & & 114.9 & 494.8 \\
\hline 435 & 24.72 & & 2360 & 225.727588 & 6.9397 & 92.2314 & \begin{tabular}{|l}
2439.52 \\
\end{tabular} & 5125 & 0.1 & 1950 & & 950000 & 0.1287 & 1.89E-04 & & & 58.72 & 97.78 & 77.85 & & 92.1 & 361.4 \\
\hline 436 & 55.91 & 3000 & 2760 & 224.18 & & \begin{tabular}{|l|}
94.6429 \\
\end{tabular} & \begin{tabular}{|l|l|}
2377 \\
\end{tabular} & 5125 & 0.1 & 1950 & & & 0.0 & $3.36 \mathrm{E}-04$ & 3.983 & & 68.48 & 97.77 & 77.7 & & 85.7 & 365.1 \\
\hline 437 & 24.78 & 3000 & 2050 & 128.436 & & \begin{tabular}{|l|l|}
131.9164 \\
\end{tabular} & 1705 & 5125 & 0.1 & 1950 & 225000 & 1625000 & 0.1284 & 2.84 & 2.116 & & 29.6 & 96.11 & 61.07 & & 92.8 & 437.8 \\
\hline 438 & 23.57 & 3000 & 2140 & & 0.4 & \begin{tabular}{|l|l|}
148.9657 \\
\end{tabular} & 1510 & 5125 & 0.1 & 1950 & 2250 & & $\begin{array}{l}0.135 \\
\end{array}$ & 5.21 & 2.95 & & 73.84 & 39.85 & & & 60.5 & 64 \\
\hline 439 & & & 4650 & 5.057 & & & 3268 & & 0.1 & & & & 0.0572 & $6.22 \mathrm{E}$ & 6.4 & & & $\begin{array}{l}1.14 \\
\end{array}$ & & & \begin{tabular}{|l|l|}
53.4 \\
\end{tabular} & \\
\hline 440 & 36.19 & 3000 & 2510 & & & 103.23 & 2179 & 51 & 0 & 19 & 225 & & & $3.47 \mathrm{E}$ & & & 55. & 82 & 0 & & 54.5 & 424.4 \\
\hline 441 & 34.65 & 3000 & 1690 & 199.355736 & \begin{tabular}{|c|}
9.7291 \\
\end{tabular} & 146.4082 & \begin{tabular}{|r|}
1536.8 \\
\end{tabular} & 5125 & 0.1 & 1950 & 225000 & 1900000 & \begin{tabular}{|c|}
0.0918 \\
\end{tabular} & 2.04E-04 & 2.124 & \begin{tabular}{|c|} 
\\
\end{tabular} & $\begin{array}{l}92.4 \\
\end{array}$ & 97.49 & 74.92 & & 72.7 & 314 \\
\hline 442 & 23.31 & & 2100 & & $\begin{array}{l}0.3768 \\
\end{array}$ & \begin{tabular}{|l|l|}
117.2971 \\
\end{tabular} & 1918.2 & 5125 & & & & & & & & & & & & & 45 & 410 \\
\hline$\frac{443}{444}$ & $\begin{array}{r}28.25 \\
41.8\end{array}$ & 3000 & \begin{tabular}{r|r|}
2350 \\
2720
\end{tabular} & \begin{tabular}{|r|r|}
382.73656 \\
6.573998
\end{tabular} & $\begin{array}{r}r 2.6783 \\
0.3578 \\
\end{array}$ & \begin{tabular}{|r|}
99.3762 \\
1632695 \\
\end{tabular} & \begin{tabular}{|l|}
2264.12 \\
178.09
\end{tabular} & $\begin{array}{l}5125 \\
5125\end{array}$ & $\frac{0.1}{0.1}$ & $\begin{array}{l}1950 \\
1950 \\
\end{array}$ & $\frac{225000}{225000}$ & $\begin{array}{l}1062500 \\
2237500\end{array}$ & \begin{tabular}{|l|}
0.1126 \\
0.0761
\end{tabular} & \begin{tabular}{|l|l|}
$1.67 E-04$ \\
$609-04$
\end{tabular} & $\frac{1.749}{5.047}$ & \begin{tabular}{|l|}
$6.83 \mathrm{E}-03$ \\
$121 \mathrm{E}-02$
\end{tabular} & $\begin{array}{l}73.71 \\
43.14\end{array}$ & $\begin{array}{l}98.69 \\
23.94\end{array}$ & $\begin{array}{r}86.94 \\
0\end{array}$ & & $\begin{array}{r}101.6 \\
57.3\end{array}$ & $\begin{array}{l}317.3 \\
6618.7 \\
\end{array}$ \\
\hline 445 & 23.58 & 3000 & 870 & 63.580679 & 1.5372 & \begin{tabular}{|l|}
72.5304 \\
\end{tabular} & \begin{tabular}{|l|}
3102.15 \\
\end{tabular} & 5125 & 0.1 & 1950 & 225000 & 6625 & 0.1349 & $7.75 \mathrm{E}-05$ & 1.291 & \begin{tabular}{|l|}
$1.41 \mathrm{E}-02$ \\
\end{tabular} & 29.77 & 92.14 & 21.36 & & 49 & 280.5 \\
\hline 446 & 26.06 & 3000 & 2600 & 36.871007 & 1.1435 & \begin{tabular}{|l|}
93.0387 \\
\end{tabular} & \begin{tabular}{|l|}
2418.35 \\
\end{tabular} & 5125 & 0.1 & 19 & 2250 & 962500 & 0.1221 & $2.68 \mathrm{E}-04$ & 2.108 & \begin{tabular}{|l|}
1.177 \\
\end{tabular} & 57.55 & 86.44 & 0 & & 59.2 & 414.5 \\
\hline 447 & 33. & 3000 & 1730 & 384.671653 & 18.8553 & \begin{tabular}{||c|}
147.0497 \\
\end{tabular} & \begin{tabular}{|l|}
1530.1 \\
\end{tabular} & 5125 & 0.1 & 1950 & 225000 & 1912500 & 0.0 & $1.95 \mathrm{E}-04$ & 2.6 & 6.73 & 33.72 & 98.7 & 87 & & 103.1 & 313.7 \\
\hline 448 & 35.11 & & 2380 & 12.550381 & $\begin{array}{l}0.549 \\
\end{array}$ & 131.2 & 1714 & 5125 & 0.1 & 1950 & 225000 & 1612 & 0.0906 & $5.55 \mathrm{E}-04$ & 4.096 & 1.25 & 85.72 & 60.16 & 0 & & 55.3 & 597.8 \\
\hline 449 & 61.89 & & 2590 & 437.9 & 13.22 & & & 512 & 0.1 & & & & & & 2.9 & & & 98. & 88.58 & & 85.6 & 245.3 \\
\hline 450 & 26. & 30 & 1400 & & 15.2 & 174. & 128 & 5125 & 0.1 & 195 & & & 0.1 & 1.26 & 1.5 & & 67.3 & 98.09 & 80.9 & & 80.8 & 298.3 \\
\hline 451 & 22. & 3000 & 2720 & & & & 1840 & 5125 & 0.1 & 1950 & 225000 & 145 & 0. & & 2.98 & \begin{tabular}{|l|}
$8.98 \mathrm{E}-03$ \\
\end{tabular} & 37.52 & 61.71 & 0 & & 77.2 & 677 \\
\hline 452 & 23. & & 2550 & & & 148. & & & 0 & & & & & & & & & & & & 64.6 & 773 \\
\hline 453 & & 30 & 1020 & 864.5463 & 47.0514 & \begin{tabular}{|l|l|}
163.2695 \\
11.679
\end{tabular} & & & 0.1 & & & & & & 1.364 & & 35.46 & 99.42 & 94.22 & 42.17 & 143.6 & \\
\hline $\begin{array}{l}454 \\
455 \\
\end{array}$ & $\begin{array}{l}38.61 \\
40.68\end{array}$ & $\begin{array}{l}3000 \\
3000\end{array}$ & $\begin{array}{l}2090 \\
2520\end{array}$ & \begin{tabular}{|r|}
303.714619 \\
74.969757 \\
\end{tabular} & \begin{tabular}{r|}
$\frac{11.8022}{2.9133}$ \\
\end{tabular} & \begin{tabular}{|l|}
116.5786 \\
116.5786 \\
\end{tabular} & \begin{tabular}{|l|}
1930.03 \\
1930.03 \\
\end{tabular} & \begin{tabular}{|l|l|}
5125 \\
5125
\end{tabular} & $\frac{0.1}{0.1}$ & $\begin{array}{l}1950 \\
1950\end{array}$ & $\begin{array}{l}225000 \\
225000\end{array}$ & \begin{tabular}{|l|}
1350000 \\
1350000 \\
\end{tabular} & $\begin{array}{l}0.0824 \\
0.0782 \\
\end{array}$ & \begin{tabular}{|l|}
$2.12 \mathrm{E}-04$ \\
$441 \mathrm{E}-04$
\end{tabular} & $\begin{array}{r}2.3 \\
3.851\end{array}$ & \begin{tabular}{|l|}
$8.02 E-03$ \\
$9.03 E-03$
\end{tabular} & $\begin{array}{ll}66.48 \\
68.85\end{array}$ & $\begin{array}{l}98.35 \\
93.33\end{array}$ & $\begin{array}{l}83.54 \\
33.31\end{array}$ & & $\begin{array}{l}88.5 \\
7.7 \\
\end{array}$ & $\begin{array}{l}305.3 \\
485.2\end{array}$ \\
\hline
\end{tabular}




\begin{tabular}{|c|c|c|c|c|c|c|c|c|c|c|c|c|c|c|c|c|c|c|c|c|c|c|}
\hline $\begin{array}{c}\text { Realizatio } \\
n\end{array}$ & $\begin{array}{c}\text { Velocity } \\
(\mathrm{m} / \mathrm{y})\end{array}$ & $\begin{array}{c}\text { Fixed Source } \\
\text { Concentration } \\
\text { (mg/L) }\end{array}$ & $\begin{array}{c}\text { Time of } \\
\text { Maximum at } \\
\text { Compliance } \\
\text { Boundary } \\
(y)\end{array}$ & \begin{tabular}{|c|} 
Maximum \\
Concentration \\
Compliance \\
Boundary \\
$(\mu \mathrm{g} / \mathrm{L})$
\end{tabular} & \begin{tabular}{|c|} 
Maximum \\
Release Rate \\
at Compliance \\
Boundary \\
$(\mathrm{Kg} / \mathrm{y})$
\end{tabular} & $\begin{array}{c}\text { Source } \\
\text { Rate } \\
(\mathrm{Kg} / \mathrm{y})\end{array}$ & \begin{tabular}{|c|} 
Source \\
Duration \\
(y)
\end{tabular} & \begin{tabular}{|c|} 
1-D \\
Streamtub \\
e Length \\
(m)
\end{tabular} & $\begin{array}{c}\text { Base } \\
\text { Porosity } \\
\left(\mathrm{m}^{3} / \mathrm{m}^{3}\right)\end{array}$ & $\begin{array}{c}\text { Bulk } \\
\text { Densty } \\
\left(\mathrm{Kg} / \mathrm{m}^{3}\right)\end{array}$ & $\begin{array}{c}\text { Remaining } \\
\text { Carbon } \\
\text { Tetrachloride } \\
\text { Source } \\
(\mathrm{Kg})\end{array}$ & \begin{tabular}{|c|} 
Volume at or \\
above Fixed \\
Source \\
$\begin{array}{c}\text { Concentration } \\
(\mathrm{m} 3)\end{array}$ \\
\end{tabular} & $\begin{array}{l}\text { Porosity } \\
\left(\mathrm{m}^{3} / \mathrm{m}^{3}\right)\end{array}$ & $\begin{array}{c}\mathrm{Kd} \\
\left(\mathrm{m}^{3} / \mathrm{Kg}\right)\end{array}$ & Retardation & $\left|\begin{array}{c}\text { First Order } \\
\text { Rate } \\
\text { Constant } \\
(1 / y)\end{array}\right|$ & $\begin{array}{c}\text { Longitudinal } \\
\text { Dispersivity } \\
(\mathrm{m})\end{array}$ & $\begin{array}{c}\text { Required } \\
\text { Source } \\
\text { Cleanup } \\
\text { Percentage } \\
(5 \mu g / L \\
\text { Compliance) } \\
\end{array}$ & \begin{tabular}{|c|} 
Required \\
Source \\
Cleanup \\
Percentage \\
(50 gig/L \\
Compliance)
\end{tabular} & \begin{tabular}{|c} 
Required \\
Source \\
Cleanup \\
Percentage \\
(500 ug/L \\
Compliance) \\
\end{tabular} & \begin{tabular}{|c|} 
Abiotic \\
Reaction \\
Half Time \\
(y)
\end{tabular} & $\begin{array}{c}\text { Carbon } \\
\text { Tetrachloride } \\
\text { Travel Time (y) }\end{array}$ \\
\hline & & & & & & & & & & & & & & & & & & & & & & \\
\hline $\begin{array}{l}457 \\
457 \\
\end{array}$ & $\begin{array}{l}3.40 \\
44.81 \\
\end{array}$ & 3000 & $\begin{array}{l}1610 \\
1610 \\
\end{array}$ & \begin{tabular}{|l|}
400.034440 \\
434.942036 \\
\end{tabular} & 12.85984 & \begin{tabular}{|r|}
88.96061 \\
\end{tabular} & $\begin{array}{r}1009.10 \\
2529.05 \\
\end{array}$ & 5125 & $\begin{array}{l}0.1 \\
0.1 \\
\end{array}$ & 1950 & 225000 & 900000 & 0.071 & $\begin{array}{l}2.20=-04 \\
1.85 E-04 \\
\end{array}$ & $\frac{2.130}{2.317}$ & \begin{tabular}{|l|l|}
$0.12 \mathrm{E}-0$ \\
$7.40 \mathrm{E}-03$ \\
\end{tabular} & $\begin{array}{r}10.92 \\
\end{array}$ & $\begin{array}{l}98.85 \\
98.85 \\
\end{array}$ & 88.5 & & $\begin{array}{l}05.5 \\
93.6 \\
\end{array}$ & $\begin{array}{r}r 30.0 \\
265 \\
\end{array}$ \\
\hline 458 & 21.73 & 3000 & 1800 & $\begin{array}{r}\quad 53.4942020 \\
6.946274 \\
\end{array}$ & $\begin{array}{l}0.0584 \\
\end{array}$ & \begin{tabular}{|l|l|}
167.4988 \\
\end{tabular} & 1343.29 & 5125 & 0.1 & 1950 & 225000 & 2325000 & 0.1464 & $\begin{array}{l}1.035 \mathrm{E}-04 \\
.48-04\end{array}$ & 2.202 & \begin{tabular}{|l|}
$1.40 \mathrm{E}-00$ \\
$1.29 \mathrm{E}-02$ \\
\end{tabular} & $\begin{array}{r}40.25 \\
\end{array}$ & 28.02 & & & 53.7 & 519.3 \\
\hline 459 & 22.99 & 3000 & 3160 & 45.754485 & 1.5393 & $\begin{array}{l}100.929 \\
\end{array}$ & \begin{tabular}{|l|}
2229.29 \\
\end{tabular} & 5125 & 0.1 & 1950 & 225000 & 1087500 & 0.1384 & $5.42 \mathrm{E}-04$ & $\begin{array}{r}2.98 \\
\end{array}$ & \begin{tabular}{|l|}
$1.66 \mathrm{E}-03$ \\
\end{tabular} & 24.05 & 89.07 & & & $\begin{array}{l}104 \\
104 \\
\end{array}$ & 664.4 \\
\hline 460 & 22.44 & 3000 & 1800 & 34.149888 & 1.6739 & \begin{tabular}{|l|l|}
147.0497 \\
\end{tabular} & \begin{tabular}{|l|}
1530.1 \\
\end{tabular} & 5125 & 0.1 & 1950 & 225000 & $\begin{array}{l}1912500 \\
\end{array}$ & 0.1418 & $3.12 \mathrm{E}-04$ & 2.111 & \begin{tabular}{|l|}
$1.09 \mathrm{E}-02$ \\
\end{tabular} & 93.45 & 85.36 & 0 & & 63.7 & 482.2 \\
\hline 461 & 50.74 & 3000 & 1280 & 178.778008 & 6.6875 & 112.2199 & 2004.99 & 5125 & 0.1 & 1950 & 225000 & 1275000 & 0.0627 & $1.23 \mathrm{E}-04$ & 1.994 & \begin{tabular}{|c|}
$1.45 \mathrm{E}-02$ \\
\end{tabular} & 34.64 & 97.2 & 72.03 & & 47.7 & 201.4 \\
\hline 462 & 30.86 & 3000 & 1780 & 60.719498 & 2.7784 & 137.2743 & 1639.05 & 5125 & 0.1 & 1950 & 225000 & 1725000 & 0.1031 & $1.81 \mathrm{E}-04$ & 1.887 & \begin{tabular}{|c|}
$1.33 \mathrm{E}-02$ \\
\end{tabular} & 48.48 & 91.77 & 17.65 & & 52.1 & 313.3 \\
\hline & 25.17 & 3000 & 1850 & $\begin{array}{l}72.660948 \\
\end{array}$ & & 130.5599 & 1723.35 & 5125 & 0.1 & & 225000 & 1600000 & 0.1264 & $1.94 \mathrm{E}-04$ & 1.777 & \begin{tabular}{|c|}
$1.08 \mathrm{E}-02$ \\
\end{tabular} & 35.56 & 93.12 & & & & 361.9 \\
\hline 464 & 46.44 & 3000 & 2260 & 73.697015 & 3.0036 & $\begin{array}{l}122.2668 \\
\end{array}$ & 1840.24 & 5125 & 0.1 & 1950 & 225000 & 1450000 & 0.0685 & $2.94 \mathrm{E}-04$ & 3.167 & $\mid 1.11 \mathrm{E}-02$ & 22.09 & 93.22 & 32.15 & & & 349.5 \\
\hline 465 & 25.45 & 3000 & 1410 & 97.817719 & 2.9008 & 88.9661 & 2529.05 & 5125 & 0.1 & 1950 & 225000 & 900000 & 0.125 & $9.91 \mathrm{E}-05$ & 1.401 & \begin{tabular}{|c|}
$1.30 \mathrm{E}-02$ \\
\end{tabular} & & 94.89 & & 0 & & 282 \\
\hline 4666 & 41.48 & 3000 & 1180 & 260.751272 & $\begin{array}{l}7.0741 \\
\end{array}$ & \begin{tabular}{|l|l|}
81.3886 \\
\end{tabular} & 2764.52 & 5125 & 0.1 & $\begin{array}{l}1950 \\
1950\end{array}$ & 225000 & $\begin{array}{r}787500 \\
\end{array}$ & 0.0767 & $1.51 \mathrm{E}-04$ & 1.996 & $1.01 \mathrm{E}-02$ & $\begin{array}{l}17.62 \\
262\end{array}$ & 98.08 & 80.82 & & $\begin{array}{ll}68.8 \\
6\end{array}$ & 246.6 \\
\hline $\begin{array}{ll}467 \\
468\end{array}$ & $\begin{array}{l}21.37 \\
50.36\end{array}$ & $\begin{array}{l}3000 \\
3300 \\
3300\end{array}$ & \begin{tabular}{ll|l}
1120 & \\
1690 &
\end{tabular} & $\begin{array}{l}382.975605 \\
36238101\end{array}$ & $\begin{array}{l}23.8423 \\
186283 \\
\end{array}$ & $\begin{array}{l}186.7666 \\
1162059\end{array}$ & \begin{tabular}{|l|}
1204.71 \\
123201 \\
\end{tabular} & 5125 & 0.1 & $\begin{array}{l}1950 \\
1950\end{array}$ & 225000 & 2737500 & $\begin{array}{l}0.1489 \\
\end{array}$ & $1.28 \mathrm{E}-04$ & 1.433 & \begin{tabular}{|l|}
$6.06 \mathrm{E}-03$ \\
$77-0$ \\
\end{tabular} & $\begin{array}{l}20.12 \\
\end{array}$ & 98.69 & 86.94 & & 114.4 & 343.7 \\
\hline $\begin{array}{l}468 \\
469 \\
\end{array}$ & $\begin{array}{l}50.26 \\
53.92 \\
\end{array}$ & $\begin{array}{l}3000 \\
3000 \\
3000\end{array}$ & \begin{tabular}{l|l|l|}
1690 \\
1610
\end{tabular} & $\begin{array}{r}336.238101 \\
145.915675\end{array}$ & $\begin{array}{r}18.6383 \\
9629 \\
6\end{array}$ & \begin{tabular}{|l|l|}
166.2959 \\
197971
\end{tabular} & $\begin{array}{r}1353.01 \\
1136.52\end{array}$ & $\begin{array}{l}5125 \\
5125 \\
\end{array}$ & 0.1 & $\begin{array}{l}1950 \\
1950\end{array}$ & $\begin{array}{l}225000 \\
25000\end{array}$ & $\begin{array}{l}2300000 \\
308750\end{array}$ & $\begin{array}{l}0.0633 \\
\end{array}$ & $2.33 \mathrm{E}-04$ & 2.862 & \begin{tabular}{|l|}
$7.74 \mathrm{E}-03$ \\
$103 \mathrm{E}-02$
\end{tabular} & 25.75 & $\begin{array}{l}98.51 \\
96.57\end{array}$ & 85.13 & 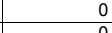 & 89.6 & 291.8 \\
\hline $\begin{array}{l}409 \\
470 \\
\end{array}$ & $\begin{array}{l}35.92 \\
35.87 \\
\end{array}$ & 3000 & $\begin{array}{l}610 \\
2190\end{array}$ & 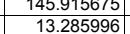 & $\begin{array}{r}9.6199 \\
0.5195 \\
\end{array}$ & \begin{tabular}{|l|}
199.99111 \\
117.2971
\end{tabular} & $\begin{array}{l}\frac{11366.53}{1918.21} \\
\end{array}$ & \begin{tabular}{|l}
5125 \\
5125
\end{tabular} & $\begin{array}{l}0.1 \\
0.1\end{array}$ & $\begin{array}{l}\frac{1950}{1950} \\
1950\end{array}$ & $\begin{array}{l}225000 \\
225000\end{array}$ & $\begin{array}{l}2987500 \\
1362500\end{array}$ & $\begin{array}{l}0.089 \\
0.087 \\
\end{array}$ & $\begin{array}{l}2.57 \mathrm{E}-04 \\
3.24 \mathrm{E}-04\end{array}$ & $\begin{array}{l}3.201 \\
2.847 \\
\end{array}$ & \begin{tabular}{|l|}
$1.03 \mathrm{E}-02$ \\
$1.60 \mathrm{E}-02$ \\
\end{tabular} & $21.47 \mid$ & $\frac{96.57}{62.37}$ & $\begin{array}{l}65.13 \\
0\end{array}$ & & $\begin{array}{r}67.4 \\
43.4 \\
\end{array}$ & $\begin{array}{l}304.2 \\
406.8 \\
\end{array}$ \\
\hline 471 & 45.06 & 3000 & 2210 & 34.797793 & 1.7719 & 152.7612 & 1472.89 & 5125 & 0.1 & 1950 & 225000 & 2025000 & 0.0706 & $4.74 \mathrm{E}-04$ & 4.395 & \begin{tabular}{|l|l|} 
& $1.12 \mathrm{E}-02$ \\
\end{tabular} & 71.44 & 85.63 & & & 62 & $\begin{array}{l}400.0 \\
499.9 \\
\end{array}$ \\
\hline 472 & 41.53 & 3000 & 2190 & 16.958704 & 0.707 & 125.0616 & 1799.11 & 5125 & 0.1 & 1950 & 225000 & 1500000 & 0.0766 & $2.91 \mathrm{E}-04$ & 2.92 & \begin{tabular}{|l|}
$1.51 \mathrm{E}-02$ \\
\end{tabular} & 18.1 & 70.52 & 0 & & 45.8 & 360.3 \\
\hline 473 & 48.13 & 3000 & 2740 & 103.185687 & 4.835 & 140.5704 & 1600.62 & 5125 & 0.1 & 1950 & 225000 & 1787500 & 0.0661 & $4.93 \mathrm{E}-04$ & 4.769 & \begin{tabular}{|l|l|}
$7.29 \mathrm{E}-03$ \\
\end{tabular} & 41.6 & 95.15 & 51.54 & & 95.1 & 507.9 \\
\hline 474 & 34.32 & 3000 & 2690 & 242.040042 & 7.1778 & 88.9661 & 2529.05 & 5125 & 0.1 & 1950 & 225000 & 900000 & 0.0927 & $2.42 \mathrm{E}-04$ & 2.321 & $7.80 \mathrm{E}-03$ & 64.81 & 97.93 & 79.34 & & 88.9 & 346.6 \\
\hline 475 & 21.54 & 3000 & 1860 & 69.761583 & 3.6105 & 155.2656 & 1449.13 & 5125 & 0.1 & 1950 & 225000 & 2075000 & 0.1477 & $3.06 \mathrm{E}-04$ & 2.046 & $8.13 \mathrm{E}-03$ & 35.13 & 92.83 & 28.33 & & 85.2 & 486.8 \\
\hline 476 & 26.01 & 3000 & 1610 & 44.312891 & 2.1053 & \begin{tabular}{ll|}
142.5296 \\
\end{tabular} & \begin{tabular}{|l|}
1578.62 \\
\end{tabular} & 5125 & 0.1 & 1950 & 225000 & 1825000 & 0.1223 & $1.28 \mathrm{E}-04$ & 1.529 & \begin{tabular}{|c|}
$1.52 \mathrm{E}-02$ \\
\end{tabular} & 68.6 & 88.72 & & & 45.6 & 301.2 \\
\hline 477 & 29.21 & 3000 & 3510 & 7.51063 & $\begin{array}{l}0.1747 \\
\end{array}$ & $\begin{array}{r}69.767 \\
\end{array}$ & \begin{tabular}{|l|}
3225.02 \\
\end{tabular} & 5125 & 0.1 & 1950 & 225000 & 625000 & & $4.64 \mathrm{E}-04$ & 3.153 & $1.41 \mathrm{E}-02$ & 82.33 & 33.43 & & & 49.1 & 553.1 \\
\hline 478 & 25.35 & 3000 & 3040 & 1.736884 & 0.0779 & $\begin{array}{l}134.6086 \\
\end{array}$ & \begin{tabular}{|l|}
1671.51 \\
\end{tabular} & 5125 & 0.1 & 1950 & 225000 & 1675000 & 0.1255 & $7.18 \mathrm{E}-04$ & 3.892 & $1.14 \mathrm{E}-02$ & 36.76 & 0 & & & 61 & 786.8 \\
\hline 479 & 34.73 & 3000 & 1670 & 47.375785 & 1.7722 & $\begin{array}{l}112.2199 \\
\end{array}$ & \begin{tabular}{|l|}
2004.99 \\
\end{tabular} & 5125 & 0.1 & 1950 & 225000 & 1275000 & $\begin{array}{l}0.0916 \\
\end{array}$ & $1.68 \mathrm{E}-04$ & 1.927 & $\mid 1.55 \mathrm{E}-02$ & 38.8 & 89.45 & & & 44.8 & 284.4 \\
\hline $\begin{array}{l}480 \\
481 \\
\end{array}$ & 47.41 & 3000 & 3300 & \begin{tabular}{|c|c|}
14.346053 \\
31179
\end{tabular} & $\begin{array}{l}0.4215 \\
\end{array}$ & $\begin{array}{r}88.1405 \\
122669\end{array}$ & \begin{tabular}{|l|}
2552.74 \\
1
\end{tabular} & $\begin{array}{l}5125 \\
5125 \\
\end{array}$ & 0.1 & $\begin{array}{l}1950 \\
1050 \\
1050\end{array}$ & 225000 & $\begin{array}{r}887500 \\
145000\end{array}$ & $\begin{array}{l}0.0671 \\
\end{array}$ & 4.31E-04 & \begin{tabular}{ll|l}
4.244 \\
\end{tabular} & $1.28 \mathrm{E}-02$ & 21.6 & 65.15 & 0 & & 54.3 & 458.7 \\
\hline $\begin{array}{l}481 \\
482\end{array}$ & 23.24 & 3000 & 2300 & $\begin{array}{r}3.11784 \\
65.252565 \\
\end{array}$ & $\begin{array}{l}0.1271 \\
39468\end{array}$ & \begin{tabular}{r|}
122.2668 \\
181.614
\end{tabular} & \begin{tabular}{|l|}
1840.24 \\
12389 \\
\end{tabular} & 5125 & 0.1 & $\begin{array}{l}1950 \\
1950\end{array}$ & 225000 & $\begin{array}{l}1450000 \\
262500\end{array}$ & $\begin{array}{l}0.1369 \\
0.1391\end{array}$ & $3.78 \mathrm{E}-04$ & 2.397 & \begin{tabular}{|l|}
$1.43 \mathrm{E}-02$ \\
\end{tabular} & 30.63 & 0 & 0 & & 48.6 & 528.7 \\
\hline \begin{tabular}{|c|}
482 \\
483
\end{tabular} & $\frac{23.04}{22.88}$ & $\begin{array}{l}3000 \\
3000\end{array}$ & $\begin{array}{r}1730 \\
2850\end{array}$ & $\begin{array}{r}65.225265 \\
356.201077\end{array}$ & $\begin{array}{r}3.9486 \\
9.968\end{array}$ & \begin{tabular}{|l|}
181.614 \\
83.9523
\end{tabular} & \begin{tabular}{|l|}
12388.89 \\
2680.09 \\
\end{tabular} & $\begin{array}{l}55125 \\
5125\end{array}$ & $\begin{array}{l}0.1 \\
0.1\end{array}$ & $\begin{array}{l}1950 \\
1950\end{array}$ & $\frac{225000}{225000}$ & $\frac{2625000}{825000}$ & $\begin{array}{l}0.1381 \\
0.1454\end{array}$ & $\begin{array}{l}3.19 \mathrm{E}-04 \\
2.38 \mathrm{E}-04\end{array}$ & $\begin{array}{l}2.168 \\
1.827 \\
\end{array}$ & \begin{tabular}{|l|}
$8.31 \mathrm{E}-03$ \\
$5.16 \mathrm{E}-03$ \\
\end{tabular} & $\begin{array}{l}28.85 \\
48.59\end{array}$ & $\frac{92.33}{98.6}$ & $\begin{array}{l}23.34 \\
85.96\end{array}$ & & $\frac{83.4}{134.2}$ & $\begin{array}{l}482.2 \\
427.9 \\
\end{array}$ \\
\hline 484 & 23.69 & 3000 & 1410 & 8.526736 & 0.2529 & 88.9661 & \begin{tabular}{|l|}
2529.05 \\
\end{tabular} & 5125 & 0.1 & 1950 & 225000 & 年 900000 & $\begin{array}{l}0.1344 \\
0.1343\end{array}$ & $\begin{array}{l}.00 \mathrm{E}-04 \\
1.98 \mathrm{E}-04 \\
\end{array}$ & $\begin{array}{l}1.027 \\
1.744\end{array}$ & \begin{tabular}{|l|}
$3.101 \mathrm{E}-02$ \\
1.61 \\
\end{tabular} & $\begin{array}{l}40.09 \\
17.23\end{array}$ & $\begin{array}{r}90.0 \\
41.36\end{array}$ & 0 & & $\frac{143.4}{43.1}$ & $\begin{array}{l}427.9 \\
377.3 \\
\end{array}$ \\
\hline 485 & 24.91 & 3000 & 2510 & 53.439918 & 2.3739 & 133.2659 & 1688.35 & 5125 & 0.1 & 1950 & 225000 & 1650000 & 0.1277 & $4.77 \mathrm{E}-04$ & 2.889 & \begin{tabular}{|l|}
$7.26 \mathrm{E}-03$ \\
\end{tabular} & 31.63 & 90.64 & 6.44 & & 95.5 & 594.4 \\
\hline 486 & 49.94 & 3000 & 2010 & 308.261068 & 12.4911 & 121.5631 & \begin{tabular}{|l|}
1850.89 \\
\end{tabular} & 5125 & 0.1 & 1950 & 225000 & 1437500 & 0.0637 & $1.93 \mathrm{E}-04$ & 2.528 & $9.21 \mathrm{E}-03$ & 44.4 & 98.38 & 83.78 & & 75.3 & 259.4 \\
\hline 487 & 54.95 & 3000 & 2470 & 86.792681 & 3.5373 & 122.2668 & \begin{tabular}{|l|}
1840.24 \\
\end{tabular} & 5125 & 0.1 & 1950 & 225000 & 1450000 & 0.0579 & $3.95 \mathrm{E}-04$ & 4.447 & \begin{tabular}{|c|}
$9.97 \mathrm{E}-03$ \\
\end{tabular} & 55.58 & 94.24 & 42.39 & & 69.6 & 414.8 \\
\hline 488 & 41.59 & 3000 & 1840 & 67.937271 & 3.2864 & 145.1211 & 1550.43 & 5125 & 0.1 & 1950 & 225000 & 1875000 & 0.0765 & $2.55 \mathrm{E}-04$ & 2.685 & $1.24 \mathrm{E}-02$ & 40.83 & 92.64 & 26.4 & & 56 & 330.8 \\
\hline 489 & 37.65 & 3000 & 2560 & 8.512287 & 0.3724 & \begin{tabular}{|l|}
131.239 \\
\end{tabular} & \begin{tabular}{|l|}
1714.43 \\
\end{tabular} & 5125 & 0.1 & 1950 & 225000 & 1612500 & 0.0845 & $6.29 \mathrm{E}-04$ & 4.762 & $1.29 \mathrm{E}-02$ & 81.01 & 41.26 & & & 53.8 & 648.2 \\
\hline 490 & 37.34 & 3000 & 1980 & 18.365777 & 0.8565 & 139.9143 & 1608.13 & 5125 & 0.1 & 1950 & 225000 & 1775000 & 0.0852 & $3.21 \mathrm{E}-04$ & 2.903 & & 48.14 & 72.78 & & & 47.6 & 398.5 \\
\hline 491 & 38.1 & 3000 & 2090 & 485.988942 & 19.2334 & & \begin{tabular}{|l|}
1895.09 \\
\end{tabular} & 5125 & 0.1 & & & 1387500 & & $2.28 \mathrm{E}-04$ & 2.377 & & 66.23 & & 89.71 & & 115.3 & 319.8 \\
\hline 492 & 23.09 & 3000 & 2240 & 9.55181 & 0.4783 & 150.2362 & \begin{tabular}{|l|}
1497.64 \\
\end{tabular} & 5125 & 0.1 & 1950 & 225000 & 1975000 & 0.1378 & 4.67E-04 & 2.712 & $1.05 \mathrm{E}-02$ & 34.33 & 47.65 & 0 & & 65.7 & 602.1 \\
\hline 493 & 26.67 & 3000 & 1740 & 25.903488 & $\begin{array}{r}1.4359 \\
17039 \\
\end{array}$ & & \begin{tabular}{|l|}
1353.01 \\
\end{tabular} & 5125 & 0.1 & 1950 & 225000 & & 0.1193 & 3.54E-04 & & \begin{tabular}{|l|}
$1.17 \mathrm{E}-02$ \\
\end{tabular} & 77.69 & & 0 & & \begin{tabular}{|l|l|}
59.4 \\
\end{tabular} & 480.2 \\
\hline $\begin{array}{l}494 \\
495\end{array}$ & 25.39 & 3000 & \begin{tabular}{ll|l|l|l|l|}
1400 \\
1730
\end{tabular} & \begin{tabular}{|l|l|l}
403.434284 \\
51154829
\end{tabular} & $\begin{array}{l}17.0044 \\
25617 \\
\end{array}$ & 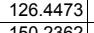 & $\begin{array}{r}1779.4 \\
1497.64\end{array}$ & $\begin{array}{l}5125 \\
5125 \\
\end{array}$ & 0.1 & $\begin{array}{l}1950 \\
1950\end{array}$ & 225000 & 1525000 & 0.1253 & $9.93 \mathrm{E}-05$ & 1.401 & & $\begin{array}{ll}60.26 \\
\end{array}$ & 98.76 & 87.61 & & $\begin{array}{l}94.6 \\
10.7\end{array}$ & 282.7 \\
\hline $\begin{array}{l}4495 \\
496\end{array}$ & $\frac{46.17}{22.97}$ & $\begin{array}{l}3000 \\
3000\end{array}$ & \begin{tabular}{|c|}
1730 \\
2380
\end{tabular} & $\begin{array}{r}511.548029 \\
51.227628\end{array}$ & $\begin{aligned} 25.6177 \\
1.8911\end{aligned}$ & \begin{tabular}{|l|}
$\frac{151.2362}{110.7481}$ \\
\end{tabular} & $\begin{array}{l}1497.64 \\
2031.64\end{array}$ & $\begin{array}{l}5125 \\
5125\end{array}$ & $\begin{array}{l}0.1 \\
0.1\end{array}$ & $\begin{array}{l}\frac{1950}{1950} \\
\end{array}$ & $\frac{225000}{225000}$ & $\begin{array}{l}1975000 \\
1250000\end{array}$ & $\begin{array}{l}0.0689 \\
0.1274\end{array}$ & $\begin{array}{l}2.07 \mathrm{E}-04 \\
3.23 \mathrm{E}-04\end{array}$ & $\frac{2.522}{2.283}$ & \begin{tabular}{|l|}
$\mid 6.50 \mathrm{E}-03$ \\
$9.23 \mathrm{E}-03$ \\
\end{tabular} & $\begin{array}{r}32.7 \\
34.77 \\
\end{array}$ & $\begin{array}{l}99.02 \\
9.24 \\
\end{array}$ & $\begin{array}{r}90.23 \\
2.4\end{array}$ & & $\begin{array}{c}106.7 \\
75.1\end{array}$ & 279.9 \\
\hline 497 & $\begin{array}{l}4.3 .3 \\
61.0\end{array}$ & & 2590 & 100.246418 & & 89.788 & 2505.9 & 5125 & 0.1 & & 225000 & 912500 & & $1.79 \mathrm{E}-04$ & 2.739 & \begin{tabular}{|l|}
$1.202-00$ \\
\end{tabular} & 78.04 & 95.01 & 50.12 & & 40.9 & $\begin{array}{r}400.0 \\
229 \\
\end{array}$ \\
\hline 498 & 30.71 & 3000 & & 209.77 & 9.96 & 142.5296 & 1578 & 5125 & 0.1 & 19 & 225000 & 1825000 & & $1.64 \mathrm{E}-04$ & 1.8 & \begin{tabular}{|l|l|} 
& $9.09 E$ \\
\end{tabular} & 28.22 & 97.62 & 76.16 & & 76.2 & 300.4 \\
\hline 499 & 63.5 & 3000 & 2760 & 820.221135 & 33.2362 & 121.5631 & 1850 & 5125 & 0.1 & 1950 & 225000 & 1437500 & 0.0 & $3.67 \mathrm{E}-04$ & 4.698 & $3.50 \mathrm{E}-03$ & 24.57 & 99.39 & 93.9 & 39.04 & 198.1 & 379.2 \\
\hline 500 & 52.93 & & 4290 & 155.520474 & 2.7968 & 53.9497 & 4170 & 5125 & 0.1 & 1950 & & & & $2.52 \mathrm{E}-04$ & 3.12 & & 58.41 & 96.78 & 67.85 & & 64 & 302 \\
\hline 501 & 36.86 & 3000 & 2150 & 139.969 & & 122.9684 & 1829 & 5125 & 0.1 & 1950 & & 146 & & $2.86 \mathrm{E}-04$ & 2.678 & & 42.31 & 96.43 & 64.28 & & 78.9 & 372.3 \\
\hline 502 & 34.21 & 3000 & 1980 & 139.76557 & 7.0 & 150.8694 & 1491 & 5125 & 0.1 & 1950 & 225000 & 1987500 & & & 2.72 & & 28.37 & 96.42 & 64.23 & & 88.1 & 407.7 \\
\hline 503 & 25.78 & 3000 & 3270 & 34.32 & 1.0553 & 92.2314 & 2439 & 5125 & 0.1 & 1950 & 2250 & 950000 & 0.1234 & 6.61 & 3.7 & & 76.67 & 85.43 & & & 91.8 & 736.7 \\
\hline 504 & 22.48 & 3000 & 1740 & & & $\begin{array}{l}139.2566 \\
\end{array}$ & & & 0.1 & & & 176 & & & & & 86.2 & & 77.04 & & 88.6 & 358.6 \\
\hline 505 & 37.08 & 3000 & 2450 & 126.5 & 6.55 & 155.2 & 1449 & 5125 & 0.1 & 19 & 225 & & & 5.10 & 4.008 & & 58.17 & 96.1 & 60.49 & & 11 & 553.9 \\
\hline 506 & 24.19 & 3000 & 2350 & 237.5 & 10.0 & $\begin{array}{l}126.4473 \\
\end{array}$ & 177 & 5125 & 0.1 & 1950 & 225000 & 1525000 & $\begin{array}{l}0.1315 \\
\end{array}$ & $3.75 \mathrm{E}$ & 2.44 & \begin{tabular}{|l|}
$5.04 \mathrm{E}-03$ \\
\end{tabular} & 22.64 & 97.9 & 78.95 & & 13 & 516.9 \\
\hline 507 & 24.43 & & 2690 & 2.87 & & & 1942 & 5125 & & & & & & 5.81 & & & 53.51 & & 0 & & 55.1 & 683 \\
\hline $\begin{array}{l}508 \\
509 \\
\end{array}$ & $\begin{array}{l}30.98 \\
2.25 \\
\end{array}$ & $\begin{array}{l}3000 \\
3000\end{array}$ & $\begin{array}{r}\frac{1550}{1880} \\
\end{array}$ & \begin{tabular}{|r|}
286.179106 \\
336.620871
\end{tabular} & \begin{tabular}{r|r|}
14.692 & 14.8949
\end{tabular} & \begin{tabular}{|l|}
$\mid 154.0159$ \\
133.9381
\end{tabular} & \begin{tabular}{|l|}
$\mid 1460.89$ \\
1679.88
\end{tabular} & $\begin{array}{l}5125 \\
5125\end{array}$ & $\begin{array}{l}0.1 \\
0.1\end{array}$ & $\begin{array}{l}1950 \\
1950\end{array}$ & $\frac{225000}{225000}$ & $\begin{array}{l}20500000 \\
162500\end{array}$ & \begin{tabular}{|c|}
0.1027 \\
0.126
\end{tabular} & $\frac{1.24 \mathrm{E}-04}{2.07 \mathrm{E}-04}$ & $\begin{array}{r}1.611 \\
1.83\end{array}$ & \begin{tabular}{|l|}
$9.22 \mathrm{E}-03$ \\
$6.13 \mathrm{E}-03$
\end{tabular} & $\begin{array}{l}61.59 \\
47.11\end{array}$ & $\begin{array}{r}98.25 \\
98.5 \\
\end{array}$ & 82.53 & $\frac{0}{0}$ & $\begin{array}{r}75.2 \\
113.1\end{array}$ & $\begin{array}{l}266.6 \\
371.5 \\
\end{array}$ \\
\hline 510 & 45.32 & 3000 & 1540 & \begin{tabular}{|l|}
530.981975 \\
\end{tabular} & 28.0302 & 158.3679 & \begin{tabular}{|l|}
1420.74 \\
\end{tabular} & 5125 & 0.1 & 1950 & 225000 & 2137500 & 0.0702 & $1.48 \mathrm{E}-04$ & 2.068 & \begin{tabular}{|l|} 
\\
\end{tabular} & 61.26 & 99.06 & 90.58 & 5.83 & 89.8 & 233.8 \\
\hline 511 & 43.82 & 3000 & 1910 & 487.2852 & 21.9729 & 135.2775 & $\mid 1663.25$ & 5125 & 0.1 & 19 & 2250 & 1687500 & 0.0726 & $2.39 E-04$ & 2.662 & $6.20 \mathrm{E}$ & 65.42 & 98.9 & 89.74 & & 111.8 & 311.4 \\
\hline 512 & 23.48 & 3000 & 1670 & 17.472 & 0.8639 & 148.3284 & 151 & 5125 & 0.1 & 1950 & 225000 & 1937500 & 0.1355 & $1.96 \mathrm{E}-04$ & 1.73 & 1.52 & 65.29 & 71.38 & 0 & & $\mid \begin{array}{l}\mid 45.7 \\
\end{array}$ & 377.5 \\
\hline 513 & 30.27 & 3000 & 1900 & \begin{tabular}{|l|l|}
177.190042 \\
\end{tabular} & 7.59 & 128.5 & 1750 & 5125 & 0.1 & 195 & 225000 & 1562500 & & $1.99 \mathrm{E}-04$ & 1.95 & 9.13 & 64.31 & 97.18 & 71.78 & & 75.9 & 331.3 \\
\hline 514 & & & 1800 & 335.28 & 15.0 & & & 512 & 0.1 & & & & & 1.65 & 1.74 & & 52.28 & 98.5 & 85.09 & & 96.2 & 316.1 \\
\hline 515 & 36.4 & 30 & 2680 & & & & & 5125 & 0.1 & 195 & & & & & 3.4 & & 32.68 & 11.62 & 0 & & 47.1 & \\
\hline 516 & 21.27 & 3000 & 1870 & & 0.1 & & 1443 & 5125 & 0.1 & 1950 & 225000 & & & & 2.40 & & 74.9 & 0 & 0 & & 48.8 & 579.9 \\
\hline 517 & & & 2350 & & 10.4 & & & & 0.1 & & & & & & & & .15 & 97.78 & 77.84 & & & \\
\hline 518 & 32.23 & 30 & 20 & 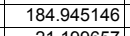 & & \begin{tabular}{|l|l|}
120.1495 \\
12507
\end{tabular} & & & 0.1 & & & 1412500 & & & & \begin{tabular}{|l|}
$5.95 \mathrm{E}-03$ \\
121503
\end{tabular} & & 97.3 & 72.96 & & 116.5 & 514.7 \\
\hline$\frac{519}{520}$ & $\begin{array}{l}22.92 \\
57.22 \\
\end{array}$ & 3000 & \begin{tabular}{r|r|r|}
1470 \\
2870
\end{tabular} & \begin{tabular}{|r|}
21.199657 \\
51.226332
\end{tabular} & \begin{tabular}{|l|l|}
1.3077 \\
1.6567
\end{tabular} & $\begin{array}{r}185.057 \\
97.024\end{array}$ & \begin{tabular}{|l|}
$\mid 1215.84$ \\
2319.01
\end{tabular} & \begin{tabular}{|l|r|}
5125 \\
5125 \\
\end{tabular} & $\begin{array}{l}0.1 \\
0.1\end{array}$ & $\begin{array}{l}1950 \\
1950\end{array}$ & $\begin{array}{l}225000 \\
225000\end{array}$ & $\begin{array}{l}2700000 \\
1025000\end{array}$ & $\begin{array}{l}0.1388 \\
0.056\end{array}$ & \begin{tabular}{l|l|}
$2.24 \mathrm{E}-04$ \\
$4.42 \mathrm{E}-04$
\end{tabular} & $\begin{array}{l}1.816 \\
5019\end{array}$ & \begin{tabular}{|l|}
$1.31 \mathrm{E}-02$ \\
$1.17 \mathrm{E}-02$
\end{tabular} & $\begin{array}{l}43.63 \\
74.79\end{array}$ & $\begin{array}{l}76.41 \\
9024\end{array}$ & \begin{tabular}{r|}
0 \\
239 \\
\end{tabular} & & $\begin{array}{l}52.8 \\
5.1 \\
\end{array}$ & $\begin{array}{r}406.1 \\
49.5 \\
\end{array}$ \\
\hline
\end{tabular}




\begin{tabular}{|c|c|c|c|c|c|c|c|c|c|c|c|c|c|c|c|c|c|c|c|c|c|c|}
\hline $\begin{array}{c}\text { Realizatio } \\
n\end{array}$ & \begin{tabular}{|c} 
Velocity \\
$(\mathrm{m} / \mathrm{y})$
\end{tabular} & $\begin{array}{c}\text { Fixed Source } \\
\text { Concentration } \\
\text { (mg/L) }\end{array}$ & $\begin{array}{c}\text { Time of } \\
\text { Maximum at } \\
\text { Compliance } \\
\text { Boundary } \\
(y)\end{array}$ & \begin{tabular}{|c|} 
Maximum \\
Concentration \\
Compliance \\
Boundary \\
$(\mu \mathrm{g} / \mathrm{L})$
\end{tabular} & \begin{tabular}{|c|} 
Maximum \\
Release Rate \\
at Compliance \\
Boundary \\
$(\mathrm{Kg} / \mathrm{y})$
\end{tabular} & \begin{tabular}{|c|} 
Source \\
Rate \\
$(\mathrm{Kg} / \mathrm{y})$ \\
\end{tabular} & \begin{tabular}{|c|} 
Source \\
Duration \\
(y)
\end{tabular} & \begin{tabular}{|c|} 
1-D \\
Streamtub \\
e Length \\
(m)
\end{tabular} & \begin{tabular}{|c|} 
Base \\
Porosity \\
$\left(\mathrm{m}^{3} / \mathrm{m}^{3}\right)$
\end{tabular} & $\begin{array}{c}\text { Bulk } \\
\text { Densty } \\
\left(\mathrm{Kg} / \mathrm{m}^{3}\right)\end{array}$ & \begin{tabular}{|c|}
$\begin{array}{c}\text { Remaining } \\
\text { Carbon } \\
\text { Tetrachloride } \\
\text { Source } \\
(\mathrm{Kg})\end{array}$ \\
\end{tabular} & \begin{tabular}{|c|} 
Volume at or \\
above Fixed \\
Source \\
$\begin{array}{c}\text { Concentration } \\
(\mathrm{m} 3)\end{array}$ \\
\end{tabular} & $\begin{array}{l}\text { Porosity } \\
\left(\mathrm{m}^{3} / \mathrm{m}^{3}\right)\end{array}$ & $\begin{array}{c}\mathrm{Kd} \\
\left(\mathrm{m}^{3} / \mathrm{Kg}\right) \\
\end{array}$ & Retardation & $\begin{array}{c}\text { First Order } \\
\text { Rate } \\
\text { Constant } \\
(1 / y)\end{array}$ & $\begin{array}{c}\text { Longitudinal } \\
\text { Dispersivity } \\
(\mathrm{m})\end{array}$ & $\begin{array}{c}\text { Required } \\
\text { Source } \\
\text { Cleanup } \\
\text { Percentage } \\
(5 \mu g / L \\
\text { Compliance) } \\
\end{array}$ & \begin{tabular}{|c|} 
Required \\
Source \\
Cleanup \\
Percentage \\
(50 gig/L \\
Compliance)
\end{tabular} & \begin{tabular}{|c} 
Required \\
Source \\
Cleanup \\
Percentage \\
(500 ug/L \\
Compliance) \\
\end{tabular} & \begin{tabular}{|c|} 
Abiotic \\
Reaction \\
Half Time \\
(y)
\end{tabular} & $\begin{array}{c}\text { Carbon } \\
\text { Tetrachloride } \\
\text { Travel Time (y) }\end{array}$ \\
\hline & & & & & & & & & & & & & & & & & & & & & & \\
\hline 522 & $\begin{array}{r}23.14 \\
23.02 \\
\end{array}$ & 3000 & 2140 & 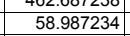 & $\begin{array}{r}2.0311 \\
.9693 \\
\end{array}$ & \begin{tabular}{|r|}
100.1541 \\
\end{tabular} & $\begin{array}{l}170.50 \\
2246.54 \\
\end{array}$ & 5125 & $\begin{array}{l}0.1 \\
0.1 \\
\end{array}$ & 1950 & 225000 & 1075000 & $\begin{array}{l}0.1211 \\
0.1382 \\
\end{array}$ & \begin{tabular}{|l|}
$2.01 \mathrm{E}^{-04}-$ \\
$1.91 \mathrm{E}$ \\
\end{tabular} & $\begin{array}{r}.054 \\
1.7 \\
\end{array}$ & \begin{tabular}{|l|}
$5.34 \mathrm{E}^{-}-0$ \\
$1.11 \mathrm{E}$
\end{tabular} & $\begin{array}{l}41.00 \\
53.74 \\
\end{array}$ & $\begin{array}{l}90.92 \\
91.52 \\
\end{array}$ & 15.24 & & $\frac{72.7}{62.4}$ & $\begin{array}{r}353.0 \\
378.5 \\
\end{array}$ \\
\hline 523 & 32.17 & 3000 & 2550 & \begin{tabular}{|r|}
1.03241 \\
\end{tabular} & $\begin{array}{l}0.0418 \\
0.048\end{array}$ & \begin{tabular}{|l|}
121.5631 \\
\end{tabular} & \begin{tabular}{|l|l|}
1850.89 \\
\end{tabular} & 5125 & 0.1 & 1950 & 225000 & 1437500 & $\begin{array}{l}0.1002 \\
0.0989 \\
\end{array}$ & \begin{tabular}{|l|}
$5.47 \mathrm{E}-04$ \\
\end{tabular} & $\begin{array}{r}1.1 .4 \\
.794\end{array}$ & \begin{tabular}{|l|}
$1.72 \mathrm{E}-02$ \\
\end{tabular} & 51.28 & 0 & & & 40.3 & 604.4 \\
\hline 524 & 26.1 & 3000 & 1810 & $\begin{array}{r}.984249 \\
\end{array}$ & 0.4656 & \begin{tabular}{|l|}
139.9143 \\
\end{tabular} & 1608.13 & 5125 & 0.1 & 1950 & 225000 & 1775000 & 0.1219 & \begin{tabular}{|l|}
$2.60 \mathrm{E}-04$ \\
\end{tabular} & 2.078 & \begin{tabular}{|l|}
$1.63 \mathrm{E}-02$ \\
\end{tabular} & 71.31 & 49.92 & & & 42.6 & 408.1 \\
\hline 525 & 34.39 & 3000 & 1530 & 82.712913 & 5.1963 & \begin{tabular}{|l|}
188.4683 \\
\end{tabular} & 1193.83 & 5125 & 0.1 & 1950 & 225000 & 2775000 & 0.0925 & \begin{tabular}{|c|}
$2.63 \mathrm{E}-04$ \\
\end{tabular} & 2.438 & \begin{tabular}{|c|}
$1.07 \mathrm{E}-02$ \\
\end{tabular} & 48.24 & 93.95 & 39.55 & & 64.8 & 363.4 \\
\hline 526 & 33.24 & 3000 & 2010 & 446.10414 & 19.4144 & \begin{tabular}{|l|}
130.5599 \\
\end{tabular} & 1723.35 & 5125 & 0.1 & 1950 & 225000 & 1600000 & 0.0957 & $2.41 \mathrm{E}-04$ & 2.274 & \begin{tabular}{|l|}
$5.52 \mathrm{E}-03$ \\
\end{tabular} & 17.92 & 98.88 & 88.79 & & 125.6 & 350.6 \\
\hline 527 & 22.22 & 3000 & 3130 & 8.182157 & 0.2267 & \begin{tabular}{|r|}
83.1021 \\
\end{tabular} & 2707.51 & 5125 & 0.1 & 1950 & 225000 & 812500 & 0.1432 & $5.23 \mathrm{E}-04$ & 2.848 & \begin{tabular}{|c|}
$1.13 \mathrm{E}-02$ \\
\end{tabular} & 78.8 & 38.89 & 0 & & 61.3 & 657 \\
\hline 528 & 24.87 & 3000 & 2250 & 67.477779 & 3.1618 & \begin{tabular}{|l|l|}
140.5704 \\
\end{tabular} & 1600.62 & 5125 & 0.1 & & 225000 & 1787500 & 0.1279 & \begin{tabular}{|l|l|}
$4.22 \mathrm{E}-04$ \\
\end{tabular} & 2.669 & $7.45 \mathrm{E}-03$ & 40.21 & 92.59 & & & 93.1 & 549.9 \\
\hline 529 & 25.57 & 3000 & 1630 & 106.083276 & 2.878 & \begin{tabular}{|l|l|}
81.3886 \\
\end{tabular} & 2764.52 & 5125 & 0.1 & 1950 & 225000 & 787500 & 0.1244 & \begin{tabular}{|l|}
$1.58 \mathrm{E}-04$ \\
\end{tabular} & 1.643 & $1.06 \mathrm{E}-02$ & 40.5 & 95.29 & & & 65.5 & 329.3 \\
\hline 530 & 34.73 & 3000 & 2440 & 100.463881 & 4.1414 & \begin{tabular}{|l|}
123.6681 \\
\end{tabular} & 1819.39 & 5125 & 0.1 & 1950 & 225000 & 1475000 & 0.0916 & \begin{tabular}{|l|}
$3.93 \mathrm{E}-04$ \\
\end{tabular} & 3.169 & \begin{tabular}{|l|}
$7.78 \mathrm{E}-03$ \\
\end{tabular} & 33.81 & 95.02 & 50.23 & & & 467.6 \\
\hline 5311 & $\begin{array}{r}24.7 \\
51.39 \\
\end{array}$ & 3000 & 2640 & \begin{tabular}{|l|l|}
19.65531 \\
\end{tabular} & 0.6814 & \begin{tabular}{|l|l|}
103.9994 \\
\end{tabular} & 2163.47 & 5125 & 0.1 & $\begin{array}{l}1950 \\
1950\end{array}$ & 225000 & 1137500 & 0.1288 & \begin{tabular}{|l|l|}
$3.76 \mathrm{E}-04$ \\
\end{tabular} & 2.474 & $1.03 \mathrm{E}-02$ & 22.04 & $\begin{array}{l}74.56 \\
\end{array}$ & & & 67.2 & 513.3 \\
\hline 532 & 51.39 & 3000 & $\begin{array}{l}3590 \\
2710 \\
\end{array}$ & $\begin{array}{l}326.58309 \\
\end{array}$ & 8.0931 & \begin{tabular}{|l|l|}
74.3438 \\
\end{tabular} & \begin{tabular}{|l|}
3026.48 \\
\end{tabular} & 5125 & 0.1 & $\begin{array}{l}1950 \\
1950\end{array}$ & 225000 & 687500 & $\begin{array}{l}0.0619 \\
\end{array}$ & \begin{tabular}{|l|l|}
$4.17 \mathrm{E}-04$ \\
\end{tabular} & 4.404 & $5.61 \mathrm{E}-03$ & 58.32 & 98.47 & & & & 439.1 \\
\hline$\frac{533}{534}$ & $\frac{39.42}{37.92}$ & $\begin{array}{l}3000 \\
3000 \\
3000\end{array}$ & \begin{tabular}{l|l|l|}
2710 \\
2890
\end{tabular} & $\begin{array}{r}68.07079 \\
264.97086\end{array}$ & $\begin{array}{l}2.1111 \\
79330 \\
\end{array}$ & \begin{tabular}{|r|}
93.0387 \\
80788 \\
\end{tabular} & \begin{tabular}{r|r|}
2418.35 \\
250.9 \\
\end{tabular} & $\begin{array}{l}5125 \\
5125 \\
\end{array}$ & $\begin{array}{l}0.1 \\
0.1 \\
\end{array}$ & $\begin{array}{l}1950 \\
1950\end{array}$ & 225000 & $\begin{array}{l}962500 \\
911500\end{array}$ & $\begin{array}{l}0.0807 \\
\end{array}$ & \begin{tabular}{|l|}
$2.88 \mathrm{E}-04$ \\
$3.40 \mathrm{E}-4$
\end{tabular} & 2.804 & \begin{tabular}{|l|}
$1.10 \mathrm{E}-02$ \\
\end{tabular} & $\begin{array}{l}28.58 \\
515 \\
\end{array}$ & $\begin{array}{l}92.65 \\
9.11\end{array}$ & 26.55 & 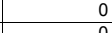 & $\begin{array}{r}63 \\
1111 \\
\end{array}$ & 364.5 \\
\hline $\begin{array}{l}534 \\
535 \\
\end{array}$ & $\begin{array}{l}31.92 \\
31.25\end{array}$ & 3000 & 2890 & $\begin{array}{r}264.9676086 \\
84.213632\end{array}$ & $\begin{array}{l}.93066 \\
3.0881 \\
\end{array}$ & \begin{tabular}{|l|}
89.788 \\
110.0086 \\
\end{tabular} & $\begin{array}{l}2045.9 \\
2045.3 \\
\end{array}$ & \begin{tabular}{|l}
5125 \\
5125
\end{tabular} & $\begin{array}{l}0.1 \\
0.1\end{array}$ & $\begin{array}{l}\frac{1950}{1950} \\
1950\end{array}$ & $\begin{array}{l}2250000 \\
225000\end{array}$ & $\begin{array}{r}912500 \\
1237500\end{array}$ & $\mid \begin{array}{l}0.0389 \\
0.1018\end{array}$ & \begin{tabular}{|l|}
$3.49 \mathrm{E}-04$ \\
$4.48 \mathrm{E}-04$ \\
\end{tabular} & $\begin{array}{l}3.103 \\
3.223 \\
\end{array}$ & \begin{tabular}{|l|}
$6.24 \mathrm{E}-03$ \\
$8.00 \mathrm{E}-03$ \\
\end{tabular} & $\begin{array}{l}53.15 \\
81.85\end{array}$ & $\begin{array}{c}98.11 \\
94.06\end{array}$ & \begin{tabular}{|c|c|}
41.13 \\
0.63
\end{tabular} & & $\begin{array}{r}\frac{111.1}{86.7} \\
86 .\end{array}$ & $\begin{array}{l}419.4 \\
528.6 \\
\end{array}$ \\
\hline 536 & 31.59 & 3000 & 1560 & $\begin{array}{l}187.151852 \\
\end{array}$ & 10.2233 & \begin{tabular}{|l|}
163.877 \\
\end{tabular} & 1372.98 & 5125 & 0.1 & 1950 & 225000 & 2250000 & 0.1007 & \begin{tabular}{|l|}
$2.08 \mathrm{E}-04$ \\
\end{tabular} & 2.044 & \begin{tabular}{|l|} 
\\
\end{tabular} & 15.12 & 97.33 & 73.28 & 0 & 81.5 & $\begin{array}{l}330.0 \\
331.6\end{array}$ \\
\hline 537 & 59.8 & 3000 & 1830 & 662.422096 & 37.3816 & \begin{tabular}{|l|}
169.2951 \\
\end{tabular} & \begin{tabular}{|l|}
1329.04 \\
\end{tabular} & 5125 & 0.1 & 1950 & 225000 & 2362500 & 0.0532 & \begin{tabular}{|l|}
$2.84 \mathrm{E}-04$ \\
\end{tabular} & 3.7 & \begin{tabular}{|l|}
$4.98 \mathrm{E}-03$ \\
\end{tabular} & 50.93 & 99.25 & 92.45 & 24.52 & 139.1 & 317.1 \\
\hline 538 & 53.56 & 3000 & 3500 & $\begin{array}{r}32.128229 \\
\end{array}$ & 1.0974 & \begin{tabular}{|l|}
102.4699 \\
\end{tabular} & \begin{tabular}{|l|}
2195.77 \\
\end{tabular} & 5125 & 0.1 & 1950 & 225000 & 1112500 & 0.0594 & \begin{tabular}{|l|}
$6.69 \mathrm{E}-04$ \\
\end{tabular} & 6.697 & \begin{tabular}{|l|}
$9.44 \mathrm{E}-03$ \\
\end{tabular} & 64.69 & 84.44 & & & 73.4 & 640.8 \\
\hline 539 & 39.72 & 3000 & 3430 & 44.631049 & 1.3601 & \begin{tabular}{|l|}
91.4206 \\
\end{tabular} & \begin{tabular}{|l|}
2461.15 \\
\end{tabular} & 5125 & 0.1 & 1950 & 225000 & 937500 & 0.0801 & \begin{tabular}{|l|}
$5.70 \mathrm{E}-04$ \\
\end{tabular} & 4.6 & \begin{tabular}{|l|} 
\\
\end{tabular} $.37 \mathrm{E}-03$ & 48.92 & 88.8 & 0 & & 82.8 & 593.5 \\
\hline 540 & 39.72 & 3000 & 2150 & 131.766275 & 6.2029 & \begin{tabular}{|l|}
141.2249 \\
\end{tabular} & 1593.2 & 5125 & 0.1 & 1950 & 225000 & 1800000 & 0.0801 & \begin{tabular}{|l|}
$3.27 \mathrm{E}-04$ \\
\end{tabular} & 3.062 & \begin{tabular}{|l|} 
\\
\end{tabular} & 18.57 & 96.21 & 62.05 & & 84.7 & 395.1 \\
\hline 541 & 23.43 & 3000 & 1630 & 193.616485 & 7.2898 & \begin{tabular}{|l|}
112.9522 \\
\end{tabular} & 1991.99 & 5125 & 0.1 & 1950 & 225000 & 1287500 & 0.1358 & \begin{tabular}{|c|}
$1.12 \mathrm{E}-04$ \\
\end{tabular} & 1.416 & $9.33 \mathrm{E}-03$ & 72.01 & 97.42 & 74.18 & & 74.3 & 309.7 \\
\hline 542 & 35.59 & 3000 & 1370 & 367.646802 & 20.4532 & \begin{tabular}{|l|l|}
166.8979 \\
\end{tabular} & \begin{tabular}{|l|l|}
1348.13 \\
\end{tabular} & 5125 & 0.1 & 1950 & 225000 & 2312500 & 0.0894 & \begin{tabular}{|l|}
$1.20 \mathrm{E}-04$ \\
\end{tabular} & 1.677 & $8.96 \mathrm{E}-03$ & 45.27 & 98.64 & 86.4 & & 77.3 & 241.5 \\
\hline 543 & 24.36 & 3000 & 1480 & 153.434897 & $\begin{array}{r}8.75 \\
\end{array}$ & \begin{tabular}{|l|l|}
171.0818 \\
\end{tabular} & 1315.16 & 5125 & 0.1 & 1950 & 225000 & 2400000 & 0.1306 & \begin{tabular}{|l|l|}
$1.75 \mathrm{E}-04$ \\
\end{tabular} & 1.678 & $8.61 \mathrm{E}-03$ & 23.16 & $\begin{array}{l}96.74 \\
\end{array}$ & 67.41 & & 80.5 & 353.1 \\
\hline 544 & 34.84 & 3000 & 2590 & & $\begin{array}{l}3.2252 \\
\end{array}$ & \begin{tabular}{|l|l|}
113.6821 \\
\end{tabular} & \begin{tabular}{|r|}
1979.2 \\
\end{tabular} & 5125 & 0.1 & 1950 & 225000 & 1300000 & \begin{tabular}{l|l|}
0.0913 \\
\end{tabular} & \begin{tabular}{|l|l|}
$3.80 \mathrm{E}-04$ \\
\end{tabular} & 3.102 & $8.16 \mathrm{E}-03$ & $\begin{array}{l}20.61 \\
0.6\end{array}$ & 94.13 & 41.25 & & 85 & 456.2 \\
\hline 545 & $\begin{array}{l}26.34 \\
6325\end{array}$ & 3000 & 2220 & 5.473705 & $\begin{array}{l}0.2695 \\
\end{array}$ & \begin{tabular}{|l|l|}
147.6897 \\
\end{tabular} & \begin{tabular}{|r|}
1523.46 \\
\end{tabular} & 5125 & 0.1 & $\begin{array}{l}1950 \\
1950\end{array}$ & 225000 & 1925000 & \begin{tabular}{|l|l|}
0.1208 \\
\end{tabular} & \begin{tabular}{|l|l|}
$4.27 \mathrm{E}-04$ \\
\end{tabular} & $\begin{array}{l}2.785 \\
770 \\
\end{array}$ & $1.27 \mathrm{E}-02$ & 25.38 & 8.65 & 0 & & 54.8 & 541.9 \\
\hline $\begin{array}{l}546 \\
547\end{array}$ & $\begin{array}{l}63.25 \\
45.13\end{array}$ & 3000 & 2950 & $\begin{array}{l}22.166963 \\
862201\end{array}$ & \begin{tabular}{|l|l|}
1.1565 \\
3301
\end{tabular} & \begin{tabular}{|l|}
156.5102 \\
115139
\end{tabular} & \begin{tabular}{|l|}
1437.61 \\
194523 \\
\end{tabular} & $\begin{array}{l}5125 \\
5125\end{array}$ & 0.1 & $\begin{array}{l}1950 \\
1950\end{array}$ & 225000 & 2100000 & \begin{tabular}{|c|}
0.0503 \\
\end{tabular} & \begin{tabular}{|l|l|}
$6.76 \mathrm{E}-04$ \\
\end{tabular} & 7.791 & \begin{tabular}{|l|}
$1.07 E-02$ \\
\end{tabular} & \begin{tabular}{ll|}
71.86 \\
\end{tabular} & $\begin{array}{l}77.44 \\
\end{array}$ & & & 65.1 & $\begin{array}{l}631.3 \\
\end{array}$ \\
\hline$\frac{547}{548}$ & $\begin{array}{l}\frac{45.13}{22.77} \\
\end{array}$ & $\begin{array}{l}3000 \\
3000 \\
\end{array}$ & $\begin{array}{l}2230 \\
1950\end{array}$ & $\begin{array}{l}86.223441 \\
66.9118074\end{array}$ & $\begin{array}{l}3.3091 \\
2.992\end{array}$ & \begin{tabular}{|l|}
115.1349 \\
127.1374
\end{tabular} & \begin{tabular}{|l|}
1954.23 \\
1769.74 \\
\end{tabular} & $\begin{array}{l}5125 \\
5125 \\
\end{array}$ & $\begin{array}{l}0.1 \\
0.1\end{array}$ & $\begin{array}{l}1950 \\
1950 \\
1950\end{array}$ & $\begin{array}{l}225000 \\
22500 \\
\end{array}$ & $\begin{array}{l}1325000 \\
1537500\end{array}$ & \begin{tabular}{l|l}
0.0705 \\
0.197
\end{tabular} & \begin{tabular}{|l|}
$3.15 \mathrm{E}-04$ \\
$206 \mathrm{E}-04$ \\
\end{tabular} & $\begin{array}{l}3.257 \\
1774\end{array}$ & \begin{tabular}{|l|}
$1.15 \mathrm{E}-02$ \\
$101 \mathrm{E}-02$
\end{tabular} & $\begin{array}{r}88.3 \\
43.03\end{array}$ & $\begin{array}{r}94.2 \\
99777\end{array}$ & $\begin{array}{l}42.01 \\
2766\end{array}$ & & \begin{tabular}{l|l}
60.2 \\
68.5
\end{tabular} & $\begin{array}{r}369.9 \\
3332\end{array}$ \\
\hline 549 & 24.53 & 3000 & 3750 & 96.329088 & $\begin{array}{l}2.59581 \\
2.3581\end{array}$ & \begin{tabular}{|l|}
73.13399 \\
\end{tabular} & \begin{tabular}{|l|}
1053.74 \\
\end{tabular} & 5125 & $\begin{array}{l}0.1 \\
0.1\end{array}$ & $\begin{array}{l}1950 \\
1950\end{array}$ & $\begin{array}{l}225000 \\
222000\end{array}$ & $\frac{1537500}{675000}$ & $\begin{array}{l}.1297 \\
0.1297\end{array}$ & \begin{tabular}{|l|}
$2.06 \mathrm{E}-04$ \\
$5.16 \mathrm{E}-04$ \\
\end{tabular} & $\begin{array}{l}.1 / 41 \\
3.012 \\
\end{array}$ & \begin{tabular}{|l|}
$1.01 \mathrm{E}-02$ \\
$5.82 \mathrm{E}-03$
\end{tabular} & \begin{tabular}{|l|}
33.03 \\
32.12
\end{tabular} & $\begin{array}{l}92.1 / \\
94.81\end{array}$ & $\begin{array}{l}27.66 \\
48.09\end{array}$ & & \begin{tabular}{|r|}
68.5 \\
119.1 \\
\end{tabular} & $\begin{array}{l}393.2 \\
629.2\end{array}$ \\
\hline 550 & 26.25 & 3000 & 2300 & 25.829295 & 0.915 & \begin{tabular}{|c|}
106.2727 \\
\end{tabular} & \begin{tabular}{|r|}
2117.2 \\
\end{tabular} & 5125 & 0.1 & 1950 & 225000 & $\begin{array}{r}1175000 \\
\end{array}$ & 0.1212 & \begin{tabular}{|l|}
$2.42 \mathrm{E}-04$ \\
\end{tabular} & 2.009 & \begin{tabular}{|l|l|} 
\\
\end{tabular} & 40.72 & 80.64 & & & 53.1 & $\begin{array}{l}29.42 \\
392.2\end{array}$ \\
\hline 551 & 22.92 & 3000 & 1260 & 155.583983 & 5.433 & \begin{tabular}{|c|}
104.7599 \\
\end{tabular} & \begin{tabular}{|r|}
2147.77 \\
\end{tabular} & 5125 & 0.1 & 1950 & 225000 & 1150000 & 0.1388 & \begin{tabular}{|c|}
$1.15 \mathrm{E}-04$ \\
\end{tabular} & 1.418 & 9.61E-03 & 36.41 & 96.79 & 67.86 & & 72.1 & 317.1 \\
\hline 552 & 28.61 & 3000 & 3040 & 18.411783 & 1.0681 & \begin{tabular}{|c|}
174.0393 \\
\end{tabular} & \begin{tabular}{|l|l}
1292.81 \\
\end{tabular} & 5125 & 0.1 & 1950 & 225000 & 2462500 & 0.1112 & \begin{tabular}{|l}
$7.23 \mathrm{E}-04$ \\
\end{tabular} & 4.288 & $7.32 \mathrm{E}-03$ & 40.12 & 72.84 & & & 94.7 & 768.2 \\
\hline 553 & 25.27 & 3000 & 1340 & 24.09125 & 1.2618 & \begin{tabular}{|l|l|}
157.1306 \\
\end{tabular} & 1431.93 & 5125 & 0.1 & 1950 & 225000 & 2112500 & 0.1259 & \begin{tabular}{|l|l}
$1.53 \mathrm{E}-04$ \\
\end{tabular} & 1.615 & & 27.96 & 79.25 & & & 45.1 & 327.5 \\
\hline 554 & 58.59 & 3000 & 2020 & 499.134278 & 20.4593 & 122.9684 & \begin{tabular}{|l|}
1829.74 \\
\end{tabular} & 5125 & 0.1 & 1950 & 225000 & 1462500 & 0.0543 & $2.09 \mathrm{E}-04$ & 2.941 & 7.41 & 61.01 & 99 & 89.98 & & 93.5 & 257.2 \\
\hline 555 & 26.6 & 3000 & 2980 & 68.823972 & $\begin{array}{l}1.8274 \\
1.970\end{array}$ & \begin{tabular}{|r|}
79.6568 \\
\end{tabular} & \begin{tabular}{|r|}
2824.62 \\
\end{tabular} & 5125 & 0.1 & 1950 & 225000 & 762500 & 0.1196 & \begin{tabular}{|l|l|}
$2.70 \mathrm{E}-04$ \\
\end{tabular} & 2.142 & & 73.92 & 92.74 & 27.35 & & 67.9 & 412.7 \\
\hline 556 & 22.42 & 3000 & 2260 & 377.728718 & 16.4387 & \begin{tabular}{|l|l|}
130.5599 \\
\end{tabular} & \begin{tabular}{|l|}
1723.35 \\
\end{tabular} & 5125 & 0.1 & & & 1600000 & & 3.81E-04 & 2.356 & & \begin{tabular}{|c|c|}
48.28 \\
\end{tabular} & \begin{tabular}{|c|}
98.68 \\
\end{tabular} & 86.76 & & 172.3 & 538.6 \\
\hline 557 & $\begin{array}{l}45.77 \\
\end{array}$ & 3000 & 1690 & 78.503651 & $\begin{array}{l}2.9366 \\
\end{array}$ & \begin{tabular}{|l|l|l|l|} 
\\
\end{tabular} & \begin{tabular}{|l|l|}
2004.99 \\
\end{tabular} & 5125 & 0.1 & 1950 & 225000 & 1275000 & 0.0695 & \begin{tabular}{|l|l|}
$1.70 \mathrm{E}-04$ \\
\end{tabular} & 2.237 & $\mid 1.52 \mathrm{E}-02$ & 29.49 & \begin{tabular}{|l|l}
93.63 \\
\end{tabular} & 36.31 & & 45.5 & 250.5 \\
\hline $\begin{array}{l}558 \\
559\end{array}$ & 25.15 & 3000 & 2720 & & & & 2229.29 & 5125 & 0.1 & & 225000 & & & \begin{tabular}{|l|l|}
$3.89 \mathrm{E}-04$ \\
\end{tabular} & & $1.20 \mathrm{E}-02$ & & & & & 57.9 & 520.8 \\
\hline 559 & 26.38 & $\begin{array}{l}3000 \\
3000\end{array}$ & \begin{tabular}{|l|l|}
2170 \\
1320
\end{tabular} & \begin{tabular}{|c|c|}
8.633688 \\
\end{tabular} & $\begin{array}{l}1.2898 \\
2481\end{array}$ & \begin{tabular}{|c|}
100.1541 \\
\end{tabular} & $\begin{array}{r}2246.54 \\
\end{array}$ & $\begin{array}{l}5125 \\
5125\end{array}$ & 0.1 & $\begin{array}{l}1950 \\
1950\end{array}$ & \begin{tabular}{ll|}
225000 \\
22500
\end{tabular} & & \begin{tabular}{l|}
0.1206 \\
\end{tabular} & \begin{tabular}{|c|}
$1.88 \mathrm{E}-04$ \\
$118 \mathrm{E}-0$
\end{tabular} & 1.787 & \begin{tabular}{|l|}
$1.37 \mathrm{E}-02$ \\
\end{tabular} & $\begin{array}{ll}60.23 \\
7220\end{array}$ & 87.06 & of & & 50.7 & 347.2 \\
\hline $\begin{array}{l}560 \\
561\end{array}$ & $\begin{array}{l}30.92 \\
36.82 \\
\end{array}$ & $\begin{array}{l}3000 \\
3000\end{array}$ & \begin{tabular}{|c|}
1320 \\
1310
\end{tabular} & $\begin{array}{l}40.838051 \\
77.468452\end{array}$ & $\begin{array}{l}2.4801 \\
4.8522\end{array}$ & \begin{tabular}{|l|}
182.1901 \\
187.9019 \\
\end{tabular} & \begin{tabular}{|l}
1234.97 \\
1197.43
\end{tabular} & $\begin{array}{l}5125 \\
5125\end{array}$ & $\begin{array}{l}0.1 \\
0.1\end{array}$ & $\begin{array}{l}\frac{1950}{1950} \\
\end{array}$ & 2250000 & $\frac{2637500}{2762500}$ & $\begin{array}{l}0.1029 \\
0.0864\end{array}$ & \begin{tabular}{|l|}
$1.18 \mathrm{E}-04$ \\
$1.60 \mathrm{E}-04$ \\
\end{tabular} & $\begin{array}{r}1.58 \\
1.936\end{array}$ & \begin{tabular}{|l|l|} 
& $1.80 \mathrm{E}$ \\
\end{tabular} & $\begin{aligned} 73.24 \\
21\end{aligned}$ & $\begin{array}{l}87.76 \\
93.55\end{array}$ & \begin{tabular}{r|}
0 \\
35.46
\end{tabular} & & $\begin{array}{l}38.5 \\
49.6\end{array}$ & $\begin{array}{ll}261.9 \\
269.5 \\
\end{array}$ \\
\hline 562 & 38.05 & 3000 & 3040 & 55.056 & 2.0729 & \begin{tabular}{|l|}
112.9522 \\
\end{tabular} & \begin{tabular}{|l|}
1991.99 \\
\end{tabular} & 5125 & 0.1 & & 225000 & 1287500 & 0.0836 & \begin{tabular}{|l|}
$7.02 \mathrm{E}-04$ \\
\end{tabular} & 5.247 & \begin{tabular}{|l|l|} 
& $7.59 \mathrm{E}$ \\
\end{tabular} & 97.42 & 90.92 & $\begin{array}{c}0.418 \\
9.18\end{array}$ & & 91.4 & $\begin{array}{l}209.0 \\
706.7\end{array}$ \\
\hline 563 & 27.88 & 3000 & 2780 & 0.755 & 0.0463 & \begin{tabular}{|l|}
183.9129 \\
\end{tabular} & \begin{tabular}{|l|}
1223.4 \\
\end{tabular} & 5125 & 0.1 & & 225000 & 2675000 & & \begin{tabular}{|l|}
$6.04 \mathrm{E}-04$ \\
\end{tabular} & 3.675 & $1.32 \mathrm{E}-02$ & 13.81 & 0 & & & 52.4 & 675.6 \\
\hline 564 & 27.86 & 3000 & 2190 & 29.610 & 0.87 & \begin{tabular}{|l|}
88.1405 \\
\end{tabular} & 2552.74 & 5125 & 0.1 & 195 & 225000 & 887500 & 0.1 & \begin{tabular}{|l|}
$2.19 \mathrm{E}-04$ \\
\end{tabular} & 1.971 & \begin{tabular}{|l|}
$1.37 \mathrm{E}-02$ \\
\end{tabular} & 40.31 & 83.11 & & & 50.8 & 362.7 \\
\hline 565 & 32.53 & & 2470 & 630.397146 & 21.2084 & 100.929 & \begin{tabular}{|l|l|}
2229 \\
\end{tabular} & 5125 & 0.1 & 1950 & & & 0.0 & \begin{tabular}{|l|}
$2.66 \mathrm{E}-04$ \\
\end{tabular} & 2.377 & & 57.03 & 99.21 & 92.07 & 20.68 & 159.8 & 374.5 \\
\hline 566 & 27.1 & 3000 & 2810 & 222.61151 & 7.7172 & \begin{tabular}{|l|l|}
103.9994 \\
\end{tabular} & 2163 & 5125 & 0.1 & 1950 & & 1137500 & 174 & \begin{tabular}{|l|}
$4.20 \mathrm{E}-04$ \\
\end{tabular} & 2.8 & & 24.23 & 97.75 & 77.54 & & 136.8 & 531 \\
\hline 567 & 22.45 & 3000 & 1950 & 18.049 & 0.7649 & \begin{tabular}{|l|l|}
127.1374 \\
\end{tabular} & \begin{tabular}{|l|}
1769.74 \\
\end{tabular} & 5125 & 0.1 & 1950 & 225000 & 1537500 & 0.1. & $2.22 \mathrm{E}$ & 1.792 & & 56.93 & 72.3 & & & 50.3 & 409.2 \\
\hline 568 & 22.9 & 3000 & 2460 & 73.6534 & 2.791 & \begin{tabular}{|l|}
113.6821 \\
\end{tabular} & & 5125 & 0.1 & 1950 & 2250 & & 0.1 & 4.00 & 2.455 & & & 93.21 & 32.11 & & 94.4 & \\
\hline 569 & 48.72 & & 1080 & & 10.6536 & 127.8 & 176 & & 0.1 & & & & & 1.07 & & & 35. & 98 & & & 52 & 92.4 \\
\hline 570 & 23.15 & 300 & 2830 & 106.9 & 3.4881 & \begin{tabular}{|l|l|}
97.8 \\
\end{tabular} & 2300 & 512 & 0.1 & 19 & & & & 4.71 & 2.734 & & & 95.33 & 53.26 & 0 & \begin{tabular}{|l|l|}
111.7 \\
\end{tabular} & 5.2 \\
\hline 571 & 37.78 & 3000 & 740 & 1041.2663 & 45.5516 & 131.2 & & 5125 & 0.1 & 1950 & 225000 & 1612500 & 0.0842 & 8.53 & 1.512 & 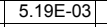 & 20. & 99.52 & 95.2 & 51.98 & 133.5 & 205.1 \\
\hline 572 & 49.32 & & 2320 & 225.767875 & 9.2541 & 122.96 & 1829.7 & 5125 & & & & & & 3.08 & & & & $\begin{array}{l}97.79 \\
\end{array}$ & & & 90.8 & 354.6 \\
\hline 573 & $\begin{array}{l}23.27 \\
3531 \\
3.5\end{array}$ & $\begin{array}{l}3000 \\
3300 \\
3\end{array}$ & $\begin{array}{l}2520 \\
2980 \\
298\end{array}$ & $\begin{array}{r}192.268059 \\
84110361\end{array}$ & \begin{tabular}{|l|}
5.6489 \\
3.8487
\end{tabular} & \begin{tabular}{|r|r|}
88.1405 \\
127
\end{tabular} & \begin{tabular}{|l|}
2552.74 \\
\end{tabular} & $\frac{5125}{5125}$ & 0.1 & $\begin{array}{l}1950 \\
1950 \\
1950\end{array}$ & 225000 & $\begin{array}{r}887500 \\
172000\end{array}$ & 0.1367 & \begin{tabular}{|l|}
$2.05 \mathrm{E}-04$ \\
58
\end{tabular} & & \begin{tabular}{|l|}
$7.49 \mathrm{E}-03$ \\
\end{tabular} & 58.25 & 97.4 & 73.99 & & $\begin{array}{r}92.5 \\
111 .\end{array}$ & \\
\hline 574 & 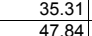 & 3000 & \begin{tabular}{rl|r|}
2980 \\
810
\end{tabular} & $\begin{array}{r}84.110361 \\
09805096\end{array}$ & 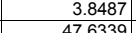 & \begin{tabular}{|l|}
137.2743 \\
1421707 \\
\end{tabular} & \begin{tabular}{|l|}
1639.05 \\
15145 \\
\end{tabular} & $\frac{5125}{5125}$ & 0.1 & $\begin{array}{l}1950 \\
1950 \\
1950\end{array}$ & 225000 & $\begin{array}{l}1725000 \\
183700\end{array}$ & $\begin{array}{l}0.0901 \\
\end{array}$ & \begin{tabular}{|l|}
$5.88 \mathrm{E}-04$ \\
\end{tabular} & $\begin{array}{l}4.302 \\
1344 \\
\end{array}$ & \begin{tabular}{|l|}
$6.23 \mathrm{E}-03$ \\
\end{tabular} & $\begin{array}{r}40.9 \\
70.02\end{array}$ & $\begin{array}{l}94.06 \\
995\end{array}$ & 40.55 & & 111.3 & 624.4 \\
\hline $\begin{array}{c}575 \\
576 \\
\end{array}$ & $\begin{array}{l}47.84 \\
35.31\end{array}$ & $\begin{array}{l}3000 \\
3000 \\
\end{array}$ & \begin{tabular}{r|r|r|}
1510 & \\
\end{tabular} & \begin{tabular}{|c|}
998.058968 \\
153619882
\end{tabular} & $\begin{array}{l}47.6339 \\
4.1676\end{array}$ & \begin{tabular}{|r|}
$\mid 143.1797$ \\
813886
\end{tabular} & \begin{tabular}{|l|}
1571.45 \\
276452 \\
\end{tabular} & $\begin{array}{l}5125 \\
5125 \\
\end{array}$ & 0.1 & $\frac{1950}{1950}$ & 225000 & $\begin{array}{r}1837500 \\
787500\end{array}$ & 0.0665 & \begin{tabular}{|l|}
$4.52 \mathrm{E}-05$ \\
$179 \mathrm{E}-04$ \\
\end{tabular} & $\frac{1.344}{2005}$ & \begin{tabular}{|l|}
$7.82 \mathrm{E}-03$ \\
$105 \mathrm{E}-02$
\end{tabular} & $\begin{array}{ll}79.28 \\
2122\end{array}$ & \begin{tabular}{r|r|r|}
99.5 & \\
9675 &
\end{tabular} & $\begin{array}{c}94.99 \\
67.45 \\
\end{array}$ & 49.9 & $\begin{array}{l}88.6 \\
66.2 \\
\end{array}$ & $\begin{array}{r}143.9 \\
291 \\
\end{array}$ \\
\hline 577 & $\begin{array}{l}30.07 \\
32.07\end{array}$ & 3000 & 15130 & $\begin{array}{r}191.93499 \\
\end{array}$ & $\begin{array}{l}\text { 4. } 1 \text { (1010 } \\
7.8224\end{array}$ & \begin{tabular}{|r|}
$\mid 01.32000$ \\
122.2668 \\
\end{tabular} & \begin{tabular}{|l|}
1840.52 \\
1840.24 \\
\end{tabular} & 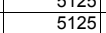 & $\begin{array}{l}0.1 \\
0.1\end{array}$ & 1950 & $\frac{2 \angle 0000}{225000}$ & $\begin{array}{l}1800000 \\
145000\end{array}$ & $\begin{array}{l}0.0991 \\
0.0992\end{array}$ & \begin{tabular}{|l|}
$1.19 \mathrm{E}-04$ \\
$1.32 \mathrm{E}-04$ \\
\end{tabular} & $\begin{array}{l}2.005 \\
1.673 \\
\end{array}$ & \begin{tabular}{|l|}
$1.05 \mathrm{E}-02$ \\
$1.05 \mathrm{E}-02$
\end{tabular} & $\frac{21.24}{23.31}$ & $\begin{array}{l}90.15 \\
97.39\end{array}$ & $\begin{array}{l}01.45 \\
73.95 \\
\end{array}$ & & $\begin{array}{c}0.0 .4 \\
66\end{array}$ & 267.3 \\
\hline 578 & 22.81 & 3000 & 2510 & 12.50 & 0.4829 & 115.85 & 1942 & 5125 & 0.1 & 1950 & 225000 & 1337500 & & \begin{tabular}{|l|}
$4.53 \mathrm{E}-04$ \\
\end{tabular} & 2.64 & 1.04 & 44.71 & 60.02 & & & 66.6 & 593.2 \\
\hline 579 & 46. & & 2800 & 156.7 & & 88.1 & & 512 & 0.1 & & & & & 2.75 & 3.0 & & & 96.81 & 68.09 & & 72.9 & 334.9 \\
\hline 580 & & 30 & 3160 & & & & & 5125 & 0.1 & 195 & & & & & & & 40.3 & 74.55 & & & 80.1 & 672.7 \\
\hline 581 & 42.08 & 3000 & 2970 & 125.1 & & 133.2 & 168 & 5125 & 0.1 & 1950 & 225000 & & & & 5.1 & & 72.46 & 96.01 & 60.06 & & 121.1 & 627 \\
\hline 582 & & & 2070 & & & & & & 0.1 & & & & & & & & & & & & 70.5 & 46 \\
\hline 583 & & 30 & 3100 & & 218 & \begin{tabular}{|l|l|}
140.5704 \\
\end{tabular} & & & 0.1 & & 225000 & 1787500 & & 5.29 & & & 35.61 & 94.17 & 41.75 & & 3.9 & \\
\hline 584 & 49.25 & 3000 & 1720 & 471.926085 & 24.3264 & \begin{tabular}{|l|l|}
154.6414 \\
\end{tabular} & 1454.98 & 5125 & 0.1 & 1950 & 225000 & 2062500 & 0.0646 & $2.30 \mathrm{E}-04$ & 2.802 & & 62.11 & 98.94 & 89.41 & & 102.8 & 291.6 \\
\hline & 37.74 & 3000 & & \begin{tabular}{|l}
47.810466 \\
\end{tabular} & 2.2922 & \begin{tabular}{|c|}
143.8283 \\
\end{tabular} & 1564.37 & 5125 & & 1950 & 225000 & 1850000 & 0.0843 & 1.24E-04 & & $1.88 \mathrm{E}-02$ & & 89.54 & & & & 236.8 \\
\hline
\end{tabular}




\begin{tabular}{|c|c|c|c|c|c|c|c|c|c|c|c|c|c|c|c|c|c|c|c|c|c|c|}
\hline $\begin{array}{c}\text { Realizatio } \\
n\end{array}$ & $\begin{array}{c}\text { Velocity } \\
(\mathrm{m} / \mathrm{y})\end{array}$ & $\begin{array}{c}\text { Fixed Source } \\
\text { Concentration } \\
\text { (mg/L) }\end{array}$ & $\begin{array}{c}\text { Time of } \\
\text { Maximum at } \\
\text { Compliance } \\
\text { Boundary } \\
(y)\end{array}$ & \begin{tabular}{|c|} 
Maximum \\
Concentration \\
Compliance \\
Boundary \\
$(\mu \mathrm{g} / \mathrm{L})$
\end{tabular} & \begin{tabular}{|c|} 
Maximum \\
Release Rate \\
at Compliance \\
Boundary \\
$(\mathrm{Kg} / \mathrm{y})$ \\
\end{tabular} & \begin{tabular}{|c|} 
Source \\
Rate \\
$(\mathrm{Kg} / \mathrm{y})$ \\
\end{tabular} & \begin{tabular}{|c|} 
Source \\
Duration \\
(y)
\end{tabular} & \begin{tabular}{|c|} 
1-D \\
Streamtub \\
e Length \\
(m)
\end{tabular} & $\begin{array}{c}\text { Base } \\
\text { Porosity } \\
\left(\mathrm{m}^{3} / \mathrm{m}^{3}\right)\end{array}$ & $\begin{array}{c}\text { Bulk } \\
\text { Densty } \\
\left(\mathrm{Kg} / \mathrm{m}^{3}\right)\end{array}$ & $\begin{array}{c}\text { Remaining } \\
\text { Carbon } \\
\text { Tetrachloride } \\
\text { Source } \\
(\mathrm{Kg})\end{array}$ & \begin{tabular}{|c|} 
Volume at or \\
above Fixed \\
Source \\
$\begin{array}{c}\text { Concentration } \\
(\mathrm{m} 3)\end{array}$ \\
\end{tabular} & $\begin{array}{l}\text { Porosity } \\
\left(\mathrm{m}^{3} / \mathrm{m}^{3}\right)\end{array}$ & $\begin{array}{c}\mathrm{Kd} \\
\left(\mathrm{m}^{3} / \mathrm{Kg}\right)\end{array}$ & Retardation & $\left|\begin{array}{c}\text { First Order } \\
\text { Rate } \\
\text { Constant } \\
(1 / y)\end{array}\right|$ & $\begin{array}{c}\text { Longitudinal } \\
\text { Dispersivity } \\
(\mathrm{m})\end{array}$ & $\begin{array}{c}\text { Required } \\
\text { Source } \\
\text { Cleanup } \\
\text { Percentage } \\
(5 \mu g / L \\
\text { Compliance) } \\
\end{array}$ & \begin{tabular}{|c|} 
Required \\
Source \\
Cleanup \\
Percentage \\
(50 gig/L \\
Compliance)
\end{tabular} & \begin{tabular}{|c} 
Required \\
Source \\
Cleanup \\
Percentage \\
(500 ug/L \\
Compliance) \\
\end{tabular} & \begin{tabular}{|c|} 
Abiotic \\
Reaction \\
Half Time \\
(y)
\end{tabular} & $\begin{array}{c}\text { Carbon } \\
\text { Tetrachloride } \\
\text { Travel Time (y) }\end{array}$ \\
\hline & & & & & & & & & & & & & & & & & & & & & & \\
\hline $\begin{array}{l}587 \\
587 \\
\end{array}$ & $\begin{array}{l}57.04 \\
27.74 \\
\end{array}$ & 3000 & 2500 & $\begin{array}{r}421.149542 \\
721.095322 \\
\end{array}$ & $\begin{array}{l}1.1050 \\
32.3552 \\
\end{array}$ & \begin{tabular}{|l|}
134.6086 \\
\end{tabular} & \begin{tabular}{|r|}
1671.51 \\
1671.51
\end{tabular} & 5125 & $\begin{array}{l}0.1 \\
0.1 \\
\end{array}$ & 1950 & 225000 & 1675000 & $\begin{array}{l}0.000 \\
0.1147 \\
\end{array}$ & $\begin{array}{l}.946=-04 \\
2.36 \mathrm{E}-04\end{array}$ & $\begin{array}{r}2.410 \\
2.039 \\
\end{array}$ & \begin{tabular}{|l|}
$1.00 \mathrm{E}-02$ \\
$3.95 \mathrm{E}-03$ \\
\end{tabular} & $\begin{array}{r}50.24 \\
76.8 \\
\end{array}$ & $\begin{array}{l}99.02 \\
99.31 \\
\end{array}$ & 93.07 & 30.66 & $\begin{array}{r}05.2 \\
175.6 \\
\end{array}$ & $\begin{array}{l}445.9 \\
376.8 \\
\end{array}$ \\
\hline 588 & 38.66 & 3000 & 1510 & 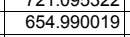 & 26.0773 & \begin{tabular}{|l|}
119.4396 \\
\end{tabular} & \begin{tabular}{|l|}
1883.8 \\
\end{tabular} & 5125 & 0.1 & 1950 & 225000 & 1400000 & 0.0823 & $\begin{array}{l}2.00 \mathrm{E}-04 \\
1.11 \mathrm{E}-04 \\
\end{array}$ & $\begin{array}{l}.0 .081 \\
1.681\end{array}$ & \begin{tabular}{|l|}
$3.03 E-03$ \\
\end{tabular} & $\begin{array}{r}59.99 \\
5\end{array}$ & 99.24 & 92.37 & 23.66 & $\begin{array}{l}17.0 \\
98.6 \\
\end{array}$ & 222.9 \\
\hline 589 & 32.86 & 3000 & 2330 & $\begin{array}{l}642.359468 \\
\end{array}$ & 24.6527 & \begin{tabular}{|l|l|}
115.1349 \\
\end{tabular} & \begin{tabular}{|l|}
1954.23 \\
\end{tabular} & 5125 & 0.1 & 1950 & 225000 & 1325000 & 0.0968 & $3.24 \mathrm{E}-04$ & 2.69 & \begin{tabular}{|l|}
$3.88 \mathrm{E}-03$ \\
\end{tabular} & 69.65 & 99.22 & 92.22 & 22.16 & 178.7 & 419.6 \\
\hline 590 & 48.28 & 3000 & 1340 & $\begin{array}{l}160.685573 \\
\end{array}$ & 5.1968 & \begin{tabular}{|r|}
97.024 \\
\end{tabular} & \begin{tabular}{|l|}
2319.01 \\
\end{tabular} & 5125 & 0.1 & 1950 & 225000 & 1025000 & 0.0659 & $1.31 \mathrm{E}-04$ & 2.004 & \begin{tabular}{|l|}
$1.43 \mathrm{E}-02$ \\
\end{tabular} & 33.89 & 96.89 & 68.88 & & 48.5 & 212.7 \\
\hline 591 & 31.97 & 3000 & 2010 & 224.115428 & 9.2908 & \begin{tabular}{|l|}
124.3658 \\
\end{tabular} & 1809.18 & 5125 & 0.1 & 1950 & 225000 & 1487500 & 0.0995 & $2.31 \mathrm{E}-04$ & 2.176 & \begin{tabular}{|l|}
$7.90 \mathrm{E}-03$ \\
\end{tabular} & 56.74 & 97.77 & 77.69 & & 87.7 & 348.7 \\
\hline 592 & 34.47 & 3000 & 1340 & 812.57999 & 22.0449 & \begin{tabular}{|l|}
81.3886 \\
\end{tabular} & 2764.52 & 5125 & 0.1 & 1950 & 225000 & 787500 & 0.0923 & $1.06 \mathrm{E}-04$ & 1.581 & \begin{tabular}{|l|}
$5.67 \mathrm{E}-03$ \\
\end{tabular} & 51.95 & 99.38 & 93.85 & 38.47 & 122.2 & 235.1 \\
\hline & & & 1880 & 40.693777 & & \begin{tabular}{|l|l|}
150.8694 \\
\end{tabular} & 1491.36 & 5125 & 0.1 & & 225000 & 1987500 & 0.1179 & $3.05 \mathrm{E}-04$ & 2.307 & $\mid 1.05 \mathrm{E}-02$ & 37.35 & 87.71 & & & 65.9 & 438.2 \\
\hline 594 & 25.05 & 3000 & 2560 & 3.584179 & 0.1314 & \begin{tabular}{|l|l|}
110.0086 \\
\end{tabular} & 2045.3 & 5125 & 0.1 & 1950 & 225000 & 1237500 & $\begin{array}{l}0.127 \\
\end{array}$ & $5.20 \mathrm{E}-04$ & 3.07 & \begin{tabular}{|c|}
$1.37 \mathrm{E}-02$ \\
\end{tabular} & 70.07 & & & & 50.4 & 628.1 \\
\hline 595 & 23.9 & 3000 & 1450 & 722.53194 & 20.0146 & \begin{tabular}{|l|}
83.1021 \\
\end{tabular} & 2707.51 & 5125 & 0.1 & 1950 & 225000 & 812500 & 0.1331 & 1.57E-04 & 1.597 & \begin{tabular}{|l|}
$4.21 \mathrm{E}-03$ \\
\end{tabular} & 25.76 & 99.31 & $\begin{array}{l}93.08 \\
\end{array}$ & & & 342.4 \\
\hline \begin{tabular}{|l|l|}
596 \\
507
\end{tabular} & 50.42 & 3000 & 2930 & \begin{tabular}{|r|}
683.20438 \\
\end{tabular} & 25.2212 & \begin{tabular}{|l|l|}
110.7481 \\
\end{tabular} & 2031.64 & 5125 & 0.1 & 1950 & 225000 & 1250000 & 0.0631 & 4.19E-04 & 4.357 & \begin{tabular}{|c|}
$3.46 \mathrm{E}-03$ \\
\end{tabular} & 37.39 & 99.27 & 92.68 & & & 442.9 \\
\hline 597 & 52.15 & 3000 & $\begin{array}{l}1820 \\
250\end{array}$ & 1122.769621 & 50.1272 & \begin{tabular}{|l|l|}
133.9381 \\
\end{tabular} & 1679.88 & 5125 & 0.1 & 1950 & 225000 & 1662500 & 0.061 & $\begin{array}{l}1.66 \mathrm{E}-04 \\
\end{array}$ & 2.372 & $4.32 \mathrm{E}-03$ & $\begin{array}{l}53.26 \\
\end{array}$ & 99.55 & 95.55 & & 160.5 & 233.1 \\
\hline $\begin{array}{l}598 \\
599 \\
\end{array}$ & $\frac{27.01}{40.02}$ & $\begin{array}{l}3000 \\
3000 \\
3000\end{array}$ & $\begin{array}{l}2250 \\
2250 \\
\end{array}$ & $\begin{array}{r}2.42399 \\
433.23343\end{array}$ & $\begin{array}{r}0.1044 \\
14.9084 \\
\end{array}$ & \begin{tabular}{|l|}
129.1963 \\
$10^{2} 2361$ \\
\end{tabular} & $\begin{array}{l}1741.54 \\
2179.77\end{array}$ & $\begin{array}{l}5125 \\
5125 \\
\end{array}$ & 0.1 & $\begin{array}{l}1950 \\
1950\end{array}$ & $\begin{array}{l}225000 \\
25000\end{array}$ & 1575000 & $\begin{array}{l}0.1178 \\
0075\end{array}$ & $\begin{array}{l}4.90 \mathrm{E}-04 \\
1.92-04\end{array}$ & $\begin{array}{l}3.103 \\
1947\end{array}$ & \begin{tabular}{|l|}
$1.56 \mathrm{E}-02$ \\
$825 \mathrm{E}-3$
\end{tabular} & $\begin{array}{l}67.99 \\
85.12\end{array}$ & 0 & 0 & 0 & $\begin{array}{l}44.3 \\
.84 \\
\end{array}$ & 588.8 \\
\hline 600 & $\begin{array}{r}40.02 \\
42.3 \\
\end{array}$ & 3000 & $\begin{array}{l}2250 \\
3030\end{array}$ & $\begin{array}{l}433.2333343 \\
253.032289\end{array}$ & $\begin{array}{r}14.9084 \\
8.4744\end{array}$ & \begin{tabular}{|l|}
103.2361 \\
10.1541 \\
\end{tabular} & 22174.474 & \begin{tabular}{|l}
5125 \\
5125
\end{tabular} & $\begin{array}{l}0.1 \\
0.1\end{array}$ & $\begin{array}{l}\frac{1950}{1950} \\
1950\end{array}$ & $\begin{array}{l}225000 \\
225000\end{array}$ & $\begin{array}{l}1125000 \\
1075000\end{array}$ & $\begin{array}{l}0.0795 \\
0.0752 \\
\end{array}$ & $\frac{1.496-04}{4.26 E-04} \mid$ & $\begin{array}{l}1.947 \\
3.863 \\
\end{array}$ & \begin{tabular}{|l|}
$8.25 \mathrm{E}-03$ \\
$5.57 \mathrm{E}-03$ \\
\end{tabular} & $\begin{array}{l}85.12 \\
29.42\end{array}$ & $\begin{array}{l}98.85 \\
98.02\end{array}$ & $\begin{array}{l}88.46 \\
80.24\end{array}$ & & $\begin{array}{r}84 \\
124.4 \\
\end{array}$ & $\begin{aligned} 249.3 \\
468\end{aligned}$ \\
\hline 601 & 34.69 & 3000 & 3200 & $\begin{array}{l}47.548389 \\
\end{array}$ & 1.9043 & \begin{tabular}{|l|}
120.1495 \\
\end{tabular} & 1872.67 & 5125 & 0.1 & 1950 & 225000 & 1412500 & 0.0917 & $6.36 \mathrm{E}-04$ & 4.506 & \begin{tabular}{|l|} 
\\
\end{tabular} & 46.46 & 89.48 & 0 & & $\begin{array}{l} \\
97.4 \\
\end{array}$ & $\begin{array}{r}465.6 \\
665.6\end{array}$ \\
\hline 602 & 44.43 & 3000 & 2410 & 165.049588 & 6.8805 & \begin{tabular}{|l|}
125.0616 \\
\end{tabular} & 1799.11 & 5125 & 0.1 & 1950 & 225000 & 1500000 & 0.0716 & $3.69 \mathrm{E}-04$ & 3.602 & $\mid 7.52 \mathrm{E}-03$ & 38.04 & 96.97 & 69.71 & & 92.2 & 415.5 \\
\hline 603 & 38.24 & 3000 & 3980 & 106.24095 & 2.7277 & \begin{tabular}{|l|}
77.0232 \\
\end{tabular} & 2921.2 & 5125 & 0.1 & 1950 & 225000 & 725000 & 0.0832 & $5.69 \mathrm{E}-04$ & 4.456 & \begin{tabular}{|l|} 
\\
\end{tabular} & 35.68 & 95.29 & 52.94 & & 112.4 & 597.2 \\
\hline 604 & 34.5 & 3000 & 1400 & 92.619999 & 3.3042 & \begin{tabular}{|l|}
107.0251 \\
\end{tabular} & 2102.31 & 5125 & 0.1 & 1950 & 225000 & 1187500 & 0.0922 & $1.27 \mathrm{E}-04$ & 1.695 & $1.46 \mathrm{E}-02$ & 46.62 & 94.6 & 46.02 & & 47.6 & 251.8 \\
\hline 605 & 48.35 & 3000 & 1190 & 1185.80152 & 59.6337 & \begin{tabular}{|l|l|}
150.8694 \\
\end{tabular} & 1491.36 & 5125 & 0.1 & 1950 & 225000 & 1987500 & 0.0658 & $9.58 \mathrm{E}-05$ & 1.736 & \begin{tabular}{|l|}
$5.12 \mathrm{E}-03$ \\
\end{tabular} & 46.18 & 99.58 & 95.78 & 57.83 & 135.4 & 184 \\
\hline 606 & 39.03 & 3000 & 1190 & \begin{tabular}{|r|}
71.956703 \\
\end{tabular} & 3.1152 & \begin{tabular}{|l|}
129.879 \\
\end{tabular} & 1732.38 & 5125 & 0.1 & 1950 & 225000 & 1587500 & 0.0815 & $1.38 \mathrm{E}-04$ & 1.854 & \begin{tabular}{|c|}
$1.59 \mathrm{E}-02$ \\
\end{tabular} & 25.11 & 93.05 & 30.51 & & 43.7 & 243.4 \\
\hline 607 & 51.15 & 3000 & 570 & \begin{tabular}{|l|}
1791.854662 \\
\end{tabular} & 93.4812 & \begin{tabular}{|l|l|}
156.5102 \\
\end{tabular} & 1437.61 & 5125 & 0.1 & 1950 & 225000 & 2100000 & & $4.50 \mathrm{E}-05$ & 1.365 & \begin{tabular}{|l|}
$3.79 \mathrm{E}-03$ \\
\end{tabular} & 40.58 & 99.72 & & 72.1 & 183 & 136.8 \\
\hline 608 & 40.63 & 3000 & 2190 & & $\begin{array}{l}2.4897 \\
\end{array}$ & \begin{tabular}{|l|l|}
116.5786 \\
\end{tabular} & \begin{tabular}{|l|}
1930.03 \\
\end{tabular} & 5125 & 0.1 & 1950 & 225000 & 1350000 & 0.0783 & $2.96 \mathrm{E}-04$ & 2.909 & \begin{tabular}{|c|}
$1.21 \mathrm{E}-02$ \\
\end{tabular} & 70.71 & 92.2 & 21.96 & & 57.3 & 367 \\
\hline 609 & 24.53 & 3000 & 1050 & 249.426548 & 7.1899 & \begin{tabular}{|l|l|}
86.4774 \\
\end{tabular} & \begin{tabular}{|l|}
2601.84 \\
\end{tabular} & 5125 & 0.1 & 1950 & 225000 & $\begin{array}{l}862500 \\
\end{array}$ & 0.1297 & 6.78E-05 & 1.264 & \begin{tabular}{|l|}
$9.73 \mathrm{E}-03$ \\
\end{tabular} & 54.57 & 98 & 79.95 & & 71.2 & 264.2 \\
\hline 610 & $\begin{array}{l}27.64 \\
5.11\end{array}$ & 3000 & 1570 & $\begin{array}{r}53.905155 \\
82202119\end{array}$ & \begin{tabular}{|l|}
2.0427 \\
215827
\end{tabular} & \begin{tabular}{|l|}
113.6821 \\
\end{tabular} & \begin{tabular}{|r|}
1979.2 \\
195.23 \\
\end{tabular} & 5125 & 0.1 & $\begin{array}{l}1950 \\
1050 \\
1050\end{array}$ & 225000 & 1300000 & 0.1151 & 1.74E-04 & 1.763 & $1.28 \mathrm{E}-02$ & 31 & 90.72 & 7.24 & & 54.1 & 326.9 \\
\hline \begin{tabular}{|c|c|}
611 \\
612
\end{tabular} & 56.11 & 3000 & \begin{tabular}{ll|l|l|l|}
2090 \\
2200
\end{tabular} & 822.931408 & \begin{tabular}{|c|}
11.5827 \\
1732
\end{tabular} & \begin{tabular}{|l|l|}
115.1349 \\
16.201
\end{tabular} & \begin{tabular}{|l|}
1954.23 \\
12020 \\
\end{tabular} & 5125 & 0.1 & $\begin{array}{l}1950 \\
1950\end{array}$ & 225000 & 1325000 & 0.0567 & 1.89E-04 & 2.685 & \begin{tabular}{|l|}
$5.61 \mathrm{E}-03$ \\
\end{tabular} & 93.99 & 99.39 & 93.92 & 39.24 & 123.5 & 245.2 \\
\hline$\frac{612}{613}$ & $\begin{array}{l}36.48 \\
36.28 \\
\end{array}$ & 3000 & \begin{tabular}{r|r|}
22290 \\
3230
\end{tabular} & $\begin{array}{r}306.920466 \\
2017212 \\
\end{array}$ & $\begin{array}{r}17.32 \\
0.0866\end{array}$ & \begin{tabular}{|c|}
169.2951 \\
13322599 \\
\end{tabular} & \begin{tabular}{|l|}
1329.04 \\
168.35 \\
\end{tabular} & $\begin{array}{l}5125 \\
5125 \\
\end{array}$ & $\begin{array}{l}0.1 \\
0.1\end{array}$ & $\begin{array}{l}1950 \\
1950 \\
\end{array}$ & $\begin{array}{l}225000 \\
225000\end{array}$ & $\begin{array}{l}2362500 \\
1650000\end{array}$ & $\begin{array}{l}0.0872 \\
0.087\end{array}$ & $\begin{array}{l}4.32 \mathrm{E}-04 \\
6.88 \mathrm{E}-04\end{array}$ & $\begin{array}{r}3.504 \\
4.55\end{array}$ & \begin{tabular}{|l|}
$4.79 E-03$ \\
$127 E-02$
\end{tabular} & $\begin{array}{l}32.02 \\
29.48 \\
\end{array}$ & 98.37 & 83.71 & 0 & $\begin{array}{c}144.6 \\
546 \\
\end{array}$ & 492.2 \\
\hline 614 & 32.7 & 3000 & $\frac{3<00}{3210}$ & 22.334078 & $\begin{array}{l}0.0869 \\
1.1698\end{array}$ & \begin{tabular}{|c|}
133.2659 \\
157.1306 \\
\end{tabular} & \begin{tabular}{|l|}
1400.00 \\
1431.93 \\
\end{tabular} & 5125 & $\begin{array}{l}0.1 \\
0.1\end{array}$ & $\begin{array}{l}1950 \\
1950\end{array}$ & 2250000 & $\begin{array}{l}1650000 \\
2112500\end{array}$ & $\begin{array}{l}.087 / \\
0.0973 \\
\end{array}$ & $\begin{array}{l}\mid .68 \mathrm{E}-04 \\
7.21 \mathrm{E}-04\end{array}$ & $\begin{array}{r}4.85 \\
4.748 \\
\end{array}$ & \begin{tabular}{|l|}
$1.27 \mathrm{E}-02$ \\
$7.36 \mathrm{E}-03$ \\
\end{tabular} & 29.48 & $\begin{array}{r}0 \\
77.61\end{array}$ & & & \begin{tabular}{l|}
54.6 \\
94.2 \\
\end{tabular} & $\frac{685.2}{744.2}$ \\
\hline 615 & 54.1 & 3000 & 750 & $\mid$\begin{tabular}{|l|}
1657.122258 \\
\end{tabular} & 70.6075 & \begin{tabular}{|l|}
127.8255 \\
\end{tabular} & \begin{tabular}{|l|}
1760.21 \\
\end{tabular} & 5125 & 0.1 & 1950 & 225000 & 1550000 & 0.0588 & $6.07 \mathrm{E}-05$ & 1.522 & \begin{tabular}{|l|l|} 
& $4.15 \mathrm{E}-03$ \\
\end{tabular} & 45.52 & 99.7 & 96.98 & 69.83 & 167 & $\begin{array}{l}1.44 .2 \\
144.2\end{array}$ \\
\hline 616 & 28.74 & 3000 & 1600 & \begin{tabular}{|r|}
316.580802 \\
\end{tabular} & 12.378 & \begin{tabular}{|l|}
117.2971 \\
\end{tabular} & \begin{tabular}{|l|}
1918.21 \\
\end{tabular} & 5125 & 0.1 & 1950 & 225000 & 1362500 & 0.1107 & $1.17 \mathrm{E}-04$ & 1.536 & $8.56 \mathrm{E}-03$ & 63.67 & 98.42 & 84.21 & & 81 & 274 \\
\hline 617 & 26.27 & 3000 & 900 & 210.239318 & 7.3947 & \begin{tabular}{|l|}
105.5176 \\
\end{tabular} & \begin{tabular}{|l|}
2132.35 \\
\end{tabular} & 5125 & 0.1 & 1950 & 225000 & 1162500 & 0.1211 & $9.42 \mathrm{E}-05$ & 1.393 & & 23.87 & 97.62 & 76.22 & & 69.7 & 271.8 \\
\hline 618 & 58.05 & 3000 & 1190 & 570.611644 & 21.4839 & \begin{tabular}{|l|}
112.9522 \\
\end{tabular} & \begin{tabular}{|l|}
1991.99 \\
\end{tabular} & 5125 & 0.1 & 1950 & 225000 & 1287500 & 0.0548 & $9.80 \mathrm{E}-05$ & 1.904 & & 45.35 & 99.12 & 91.24 & 12.37 & 68.3 & 168.1 \\
\hline 619 & 27.47 & 3000 & 3920 & 11.863108 & 0.2868 & \begin{tabular}{|l|}
72.5304 \\
\end{tabular} & \begin{tabular}{|l|}
3102.15 \\
\end{tabular} & 5125 & 0.1 & 1950 & 225000 & 662500 & 0.1158 & $5.84 \mathrm{E}-04$ & 3.547 & \begin{tabular}{|c|}
$9.42 \mathrm{E}-03$ \\
\end{tabular} & 32.94 & 57.85 & & & 73.6 & 661.7 \\
\hline 620 & 25.91 & 3000 & 1920 & 21.6 & & \begin{tabular}{|l|}
163.877 \\
\end{tabular} & \begin{tabular}{|l|}
1372.98 \\
\end{tabular} & 5125 & 0.1 & 1950 & 225000 & 2250000 & 0.1228 & 3.65E-04 & 2.503 & & 34.07 & 76.87 & & & 64.3 & 495.1 \\
\hline 621 & 28.69 & 3000 & 1890 & 143.9 & 5.2441 & & & 5125 & 0.1 & & & & & 1.96E-04 & 1.894 & & 31.13 & 96.53 & 65.27 & & 74.6 & 338.3 \\
\hline 622 & 59.58 & 3000 & 3130 & 21.1 & 0.7222 & \begin{tabular}{|l|l|}
102.4699 \\
\end{tabular} & \begin{tabular}{|l|l|}
2195.77 \\
\end{tabular} & 5125 & 0.1 & 1950 & 225000 & 1112500 & $\begin{array}{l}0.0534 \\
\end{array}$ & 4.77E-04 & 5.511 & \begin{tabular}{|c|}
$1.26 \mathrm{E}-02$ \\
\end{tabular} & $\begin{array}{l}38.46 \\
\end{array}$ & 76.35 & 0 & & 55.2 & 474.1 \\
\hline 623 & 51.31 & 3000 & 630 & \begin{tabular}{|l|l|}
1693.8 \\
\end{tabular} & & \begin{tabular}{|r|}
163.877 \\
\end{tabular} & & 5125 & 0.1 & & 225000 & 2250000 & 0.062 & 4.74E-05 & & & & & 97.05 & 70.48 & 166.7 & 138.5 \\
\hline 624 & 23.07 & $\begin{array}{l}3000 \\
3000\end{array}$ & \begin{tabular}{|l|l|l|l|l|l}
1380 \\
1610
\end{tabular} & 567.1 & 34.0092 & \begin{tabular}{|l|l|}
179.8802 \\
170202
\end{tabular} & \begin{tabular}{|l|}
1250.83 \\
\end{tabular} & 5125 & 0.1 & & 225000 & 2587500 & 0.1379 & $1.18 \mathrm{E}-04$ & 1.433 & & 54.16 & 99.12 & 91.18 & 11.85 & 129.3 & 318.4 \\
\hline 625 & $\begin{array}{r}28.4 \\
25.1 \\
\end{array}$ & 3000 & \begin{tabular}{|l|l|l|l|l|}
1610 & \\
1030 &
\end{tabular} & $\begin{array}{r}326.097793 \\
60515013\end{array}$ & $\begin{array}{l}18.9179 \\
2174\end{array}$ & \begin{tabular}{|l|l|l|}
174.0393 \\
1077780 \\
\end{tabular} & \begin{tabular}{|l|}
1292.81 \\
20769 \\
\end{tabular} & 5125 & & $\begin{array}{l}1950 \\
1950\end{array}$ & 225000 & 2462500 & $\begin{array}{l}0.112 \\
0.1262 \\
\end{array}$ & $2.52 \mathrm{E}-04$ & 2.138 & & 54.3 & 98.47 & & 0 & 114.7 & 385.8 \\
\hline $\begin{array}{l}626 \\
627 \\
\end{array}$ & $\begin{array}{l}25.21 \\
3.66 \\
\end{array}$ & $\begin{array}{l}3000 \\
3000 \\
\end{array}$ & $\begin{array}{r}1030 \\
2020\end{array}$ & 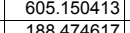 & $\begin{array}{r}21.74 \\
82024 \\
\end{array}$ & \begin{tabular}{|l|l|}
107.7748 \\
130.5599 \\
\end{tabular} & \begin{tabular}{|l|}
2087.69 \\
173.35 \\
\end{tabular} & \begin{tabular}{r|}
5125 \\
5125
\end{tabular} & 0.1 & $\begin{array}{r}1950 \\
1950 \\
\end{array}$ & 225000 & $\begin{array}{l}1200000 \\
160000\end{array}$ & $\begin{array}{l}0.1262 \\
0.995\end{array}$ & $\begin{array}{r}8.79 \mathrm{E}-05 \\
260 \mathrm{~F}-04\end{array}$ & $\begin{array}{l}1.352 \\
2392\end{array}$ & \begin{tabular}{|l|}
$5.91 E-03$ \\
$7.93 E-03$
\end{tabular} & $\begin{array}{l}34.29 \\
335 \\
\end{array}$ & 99.17 & $\begin{array}{l}91.74 \\
73.47\end{array}$ & $\begin{array}{r}17.38 \\
\end{array}$ & \begin{tabular}{|c|c|}
117.3 \\
87.4 \\
\end{tabular} & 274.9 \\
\hline $\begin{array}{l}27 \\
628 \\
\end{array}$ & & 3000 & $\begin{array}{l}2020 \\
2180\end{array}$ & \begin{tabular}{|l|}
8.48 .47617 \\
217.677052
\end{tabular} & $\begin{array}{l}8.2024 \\
8.5629 \\
\end{array}$ & \begin{tabular}{|c|}
118.05995 \\
118.0135 \\
\end{tabular} & \begin{tabular}{|l|}
123.35 \\
1906.56 \\
\end{tabular} & $\mid \begin{array}{l}5125 \\
5125\end{array}$ & & $\begin{array}{l}1950 \\
1950\end{array}$ & 225000 & $\begin{array}{l}1000000 \\
1375000\end{array}$ & $\begin{array}{l}0.0495 \\
0.0675 \\
\end{array}$ & \begin{tabular}{|l|}
$2.60 \mathrm{E} E-04$ \\
$2.43-04$
\end{tabular} & $\begin{array}{l}2.392 \\
2.817\end{array}$ & \begin{tabular}{|l|}
$1.93 \mathrm{E}-03$ \\
$8.87 \mathrm{E}-03$ \\
\end{tabular} & $\begin{array}{r}33.5 \\
24.98\end{array}$ & $\begin{array}{r}97.35 \\
97.7\end{array}$ & $\begin{array}{l}73.47 \\
77.03\end{array}$ & & $\begin{array}{l}87.4 \\
78.1\end{array}$ & $\begin{array}{l}364.2 \\
306.3 \\
\end{array}$ \\
\hline 629 & 48.94 & 3000 & 1170 & \begin{tabular}{|l|}
868.230408 \\
\end{tabular} & 63.0652 & \begin{tabular}{|l|}
217.9093 \\
\end{tabular} & 1032 & 5125 & 0.1 & 1950 & 225000 & 3450000 & 0.065 & $1.31 \mathrm{E}-04$ & 2.016 & $5.97 \mathrm{E}-03$ & 32.09 & 99.42 & 94.24 & 42.41 & 116.2 & 211.1 \\
\hline 630 & 46.51 & & 2990 & 7.500961 & & 155.2656 & 1449 & 5125 & 0.1 & 1950 & & & 0.0684 & $5.84 \mathrm{E}-04$ & 5.317 & & 29.55 & 33.34 & & & 58.7 & 585.9 \\
\hline 631 & 32.4 & 3000 & 1510 & 57.870092 & & \begin{tabular}{|l|}
188.4683 \\
\end{tabular} & 1193 & 5125 & 0.1 & 1950 & & & 0.0982 & $2.82 E$ & 2.4 & & 73.32 & 91.36 & 13.6 & & 59.8 & 387.8 \\
\hline 632 & 42.76 & 3000 & 2310 & & 0.1469 & & 1769 & 5125 & 0.1 & 1950 & 225000 & 1537500 & 0.0744 & 3.91 & 3.655 & & 36.45 & 0 & & & 38.2 & 438.1 \\
\hline 633 & & 3000 & 1590 & 103.457813 & & & & 5125 & 0.1 & 1950 & 2250 & & 0.0911 & 2.49 & 2.379 & & & 95.17 & 51.67 & & 70.1 & 349.2 \\
\hline 634 & & 30 & 1160 & & & & & & 0.1 & & & & & & & & 17.5 & 96.6 & 65.99 & & 67.7 & \\
\hline 635 & 25. & 30 & 2850 & & & & & 512 & 0.1 & & & & 0.1254 & 7.36 & 3.5 & & 78. & 87 & 0 & & 106.3 & 801.5 \\
\hline $\begin{array}{l}636 \\
327 \\
\end{array}$ & & 3000 & 2450 & & 7.0523 & \begin{tabular}{|l|l|}
195.2002 \\
\end{tabular} & 115 & 5125 & 0.1 & 1950 & 225000 & 2925000 & 0.092 & $5.01 \mathrm{E}$ & 3.754 & 6.1 & 24 & 95.39 & 53.87 & & 112.5 & 556.3 \\
\hline 637 & 53.11 & & & 88.486 & & & 1071 & 5125 & & & & & 0.0 & & 3.604 & & & & & & 57.7 & 347.8 \\
\hline $\begin{array}{l}638 \\
639 \\
\end{array}$ & $\begin{array}{l}60.03 \\
2976\end{array}$ & 3000 & & $\begin{array}{r}349.874684 \\
\end{array}$ & $\begin{array}{r}17.7424 \\
15102\end{array}$ & \begin{tabular}{|l|l|}
152.1319 \\
152121 \\
\end{tabular} & \begin{tabular}{|r|}
1478.98 \\
1178.00 \\
\end{tabular} & $\frac{5125}{5125}$ & 0.1 & $\begin{array}{l}1950 \\
1050\end{array}$ & 225000 & 2012500 & 0.053 & $2.00 \mathrm{E}-04$ & 2.91 & \begin{tabular}{|c|}
$9.20 \mathrm{E}-03$ \\
\end{tabular} & 52.04 & 98.57 & 85.71 & & 75.4 & 248.5 \\
\hline 639 & $\begin{array}{l}29.76 \\
4317\end{array}$ & 3000 & $\begin{array}{ll}2340 \\
1860\end{array}$ & \begin{tabular}{|r|}
29.960491 \\
\end{tabular} & $\begin{array}{r}1.5193 \\
46.1295 \\
\end{array}$ & \begin{tabular}{|l|l|}
152.1319 \\
11989 \\
\end{tabular} & \begin{tabular}{|l|}
1478.98 \\
159.97 \\
\end{tabular} & $\frac{5125}{5125}$ & 0.1 & $\begin{array}{l}1950 \\
1050\end{array}$ & 225000 & 2012500 & 0.1069 & 5.54E-04 & 3.618 & $8.91 \mathrm{E}-03$ & $\begin{array}{l}68.67 \\
793 \\
\end{array}$ & 83.31 & \begin{tabular}{r|r}
0 \\
\end{tabular} & & $\begin{array}{r}77.8 \\
1891\end{array}$ & 623 \\
\hline $\begin{array}{l}640 \\
641 \\
\end{array}$ & $\begin{array}{l}42.11 \\
42.03 \\
\end{array}$ & 3000 & $\begin{array}{l}2860 \\
2560\end{array}$ & \begin{tabular}{|r|}
915.40528 \\
\end{tabular} & $\begin{array}{r}46.1259 \\
0.8143\end{array}$ & \begin{tabular}{|r|}
111.988 \\
12.9522 \\
\end{tabular} & \begin{tabular}{|l|}
1985.81 .99 \\
\end{tabular} & $\begin{array}{l}5125 \\
5125\end{array}$ & $\begin{array}{l}0.1 \\
0.1\end{array}$ & $\frac{19}{19}$ & $\frac{2250}{2250}$ & $\begin{array}{l}1872500 \\
1287500\end{array}$ & $\begin{array}{l}0.0737 \\
0.0757\end{array}$ & $\begin{array}{l}2.48 \mathrm{E}-04 \\
4.01 \mathrm{E}-04\end{array}$ & $\begin{array}{r}2.1 \\
3.679\end{array}$ & \begin{tabular}{|l|}
$3.26 \mathrm{E}$ \\
$1.26 \mathrm{E}$
\end{tabular} & $\begin{array}{r}r 8.3 \\
41.14\end{array}$ & $\frac{99.49}{76.88}$ & $\begin{array}{l}94.87 \\
0\end{array}$ & & $\begin{array}{r}r 89.1 \\
55 \\
\end{array}$ & $\frac{320.6}{448.6}$ \\
\hline 642 & 21.39 & 3000 & 1220 & 71.01264 & 3.2021 & \begin{tabular}{|l|l|}
135.27 \\
\end{tabular} & 166 & 512 & 0.1 & 1950 & 225000 & 1687500 & 0.1 & $7.49 \mathrm{E}$ & 1.255 & 1.32 & $\mid 62.44$ & 92.96 & 29.59 & & 52.6 & 300.6 \\
\hline 643 & 32 & & 1010 & 360.530 & 15. & \begin{tabular}{|l|}
127.1374 \\
\end{tabular} & 1769 & 512 & 0.1 & 195 & 225000 & 1537 & 0. & $1.08 \mathrm{E}-04$ & 1.564 & & 27.16 & 98.61 & 86.13 & & 78.6 & 244.4 \\
\hline 644 & 26. & & 1470 & & & 91.4 & & 512 & 0.1 & & & & & & 1.58 & & & 84.04 & & & 44 & 309 \\
\hline 645 & & 30 & 1260 & 645.37 & 29.3 & 136. & & 5125 & 0.1 & 195 & & & & 9.15 & 1.4 & & 55. & 99 & 92.25 & 22.53 & 105.5 & \\
\hline 646 & 43.05 & 3000 & 2150 & & & 114. & 1966 & 5125 & 0.1 & 1950 & 225 & & & & 2.4 & & 48.7 & 98.31 & 83.15 & & 84 & 295.8 \\
\hline 647 & & & & & & & & & 0.1 & & & & & & & & & 95.97 & 59.71 & & 115.2 & \\
\hline 648 & & 30 & 2980 & & & \begin{tabular}{|l|l|}
110.0086 \\
112506 \\
\end{tabular} & & & 0.1 & & & & & $5.60 \mathrm{E}-04$ & 3.043 & & & & & & 54 & 679.4 \\
\hline 649 & $\begin{array}{l}21.25 \\
21.58\end{array}$ & 3000 & \begin{tabular}{r|r|r|r|}
2890 & \\
\end{tabular} & \begin{tabular}{|r|}
132.327306 \\
11260379
\end{tabular} & $\begin{array}{l}6.2869 \\
0.3431\end{array}$ & \begin{tabular}{|r|}
142.5296 \\
914206 \\
\end{tabular} & \begin{tabular}{|l|}
$\mid 1578.62$ \\
2461.15 \\
\end{tabular} & \begin{tabular}{|l|r|}
5125 \\
5125 \\
\end{tabular} & $\begin{array}{l}0.1 \\
0.1\end{array}$ & $\begin{array}{l}1950 \\
1950\end{array}$ & $\begin{array}{l}225000 \\
225000\end{array}$ & $\begin{array}{r}1825000 \\
937500\end{array}$ & \begin{tabular}{l|l|}
0.1497 & \\
0.1474
\end{tabular} & $\begin{array}{l}3.03 E-04 \\
4.02-04\end{array}$ & $\frac{2.023}{2.38}$ & \begin{tabular}{|l|}
$7.04 \mathrm{E}-03$ \\
$1.0 \mathrm{E}-02$
\end{tabular} & $\begin{array}{l}81.91 \\
43.06\end{array}$ & $\frac{96.22}{556}$ & \begin{tabular}{rl|r}
62.21 \\
0
\end{tabular} & & $\begin{array}{l}98.4 \\
663.1\end{array}$ & $\begin{array}{l}487.8 \\
565.1 \\
\end{array}$ \\
\hline
\end{tabular}




\begin{tabular}{|c|c|c|c|c|c|c|c|c|c|c|c|c|c|c|c|c|c|c|c|c|c|c|}
\hline $\begin{array}{c}\text { Realizatio } \\
\mathrm{n}\end{array}$ & \begin{tabular}{|c}
$\begin{array}{c}\text { Velocity } \\
(\mathrm{m} / \mathrm{y})\end{array}$ \\
28.0
\end{tabular} & $\begin{array}{c}\text { Fixed Source } \\
\text { Concentration } \\
\text { (mg/L) }\end{array}$ & $\begin{array}{c}\text { Time of } \\
\text { Maximum at } \\
\text { Compliance } \\
\text { Boundary } \\
\text { (y) }\end{array}$ & \begin{tabular}{|c|} 
Maximum \\
Concentration \\
Compliance \\
Boundary \\
$(\mu \mathrm{g} / \mathrm{L})$
\end{tabular} & \begin{tabular}{|c|} 
Maximum \\
Release Rate \\
at Compliance \\
Boundary \\
$(\mathrm{Kg} / \mathrm{y})$ \\
\end{tabular} & $\begin{array}{l}\text { Source } \\
\text { Rate } \\
(\mathrm{Kg} / \mathrm{y})\end{array}$ & $\begin{array}{c}\text { Source } \\
\text { Duration } \\
(y)\end{array}$ & \begin{tabular}{|c|}
$\begin{array}{c}\text { 1-D } \\
\text { Streatumb } \\
\text { e Length } \\
(\mathrm{m})\end{array}$ \\
5
\end{tabular} & $\begin{array}{c}\text { Base } \\
\text { Porosity } \\
\left(\mathrm{m}^{3} / \mathrm{m}^{3}\right)\end{array}$ & $\begin{array}{c}\text { Bulk } \\
\text { Densty } \\
\left(\mathrm{Kg} / \mathrm{m}^{3}\right) \\
1950\end{array}$ & $\begin{array}{c}\text { Remaining } \\
\text { Carbon } \\
\text { Tetrachloride } \\
\text { Source } \\
(\mathrm{Kg}) \\
\end{array}$ & $\begin{array}{c}\begin{array}{c}\text { Volume at or } \\
\text { above Fixed } \\
\text { Source } \\
\text { Concentration } \\
(\mathrm{m} 3)\end{array} \\
\end{array}$ & $\begin{array}{c}\text { Porosity } \\
\left(\mathrm{m}^{3} / \mathrm{m}^{3}\right)\end{array}$ & $\begin{array}{c}\mathrm{Kd} \\
\left(\mathrm{m}^{3} / \mathrm{Kg}\right)\end{array}$ & Retardation & $\begin{array}{c}\text { First Order } \\
\text { Rate } \\
\text { Constant } \\
(1 / y) \\
\end{array}$ & $\begin{array}{c}\text { Longitudinal } \\
\text { Dispersivity } \\
(\mathrm{m})\end{array}$ & $\begin{array}{c}\text { Required } \\
\text { Source } \\
\text { Cleanup } \\
\text { Percentage } \\
(5 \text { gg/L } \\
\text { Compliance }) \\
\end{array}$ & \begin{tabular}{|c|} 
Required \\
Source \\
Cleanup \\
Percentage \\
$(50 \mu \mathrm{\mu g} / \mathrm{L}$ \\
Compliance) \\
70
\end{tabular} & $\begin{array}{l}\text { Required } \\
\text { Source } \\
\text { Cleanup } \\
\text { Percentage } \\
\text { (500 ug/L } \\
\text { Compliance) } \\
\end{array}$ & $\begin{array}{c}\text { Abiotic } \\
\text { Reaction } \\
\text { Half Time } \\
\text { (y) }\end{array}$ & $\begin{array}{c}\text { Carbon } \\
\text { Tetrachloride } \\
\text { Travel Time }(y) \\
\end{array}$ \\
\hline $\begin{array}{l}651 \\
65 \\
65\end{array}$ & \begin{tabular}{|l|}
28.18 \\
54.76 \\
\end{tabular} & \begin{tabular}{|r|}
3000 \\
300
\end{tabular} & $\begin{array}{r}1930 \\
1810\end{array}$ & \begin{tabular}{|r|}
247.148542 \\
952.345109
\end{tabular} & \begin{tabular}{|r|}
9.1845 \\
43.1554
\end{tabular} & $\begin{array}{l}111.4852 \\
15.9447\end{array}$ & $\begin{array}{r}2018.2 \\
1655.08\end{array}$ & \begin{tabular}{|r|}
5125 \\
5525 \\
\end{tabular} & & $\begin{array}{r}1950 \\
1950\end{array}$ & \begin{tabular}{|r|}
225000 \\
22500
\end{tabular} & $\begin{array}{r}1262500 \\
1700000\end{array}$ & $\begin{array}{l}0.1129 \\
0.581\end{array}$ & \begin{tabular}{|l|l|}
$1.74 \mathrm{E}-04$ \\
$169 \mathrm{E}-04$
\end{tabular} & \begin{tabular}{|r|}
1.781 \\
2467
\end{tabular} & \begin{tabular}{|l|}
$8.00 \mathrm{E}-03$ \\
$505 \mathrm{E}-33$ \\
\end{tabular} & & $\begin{array}{r}97.98 \\
99.47\end{array}$ & \begin{tabular}{|r|}
79.77 \\
94.75
\end{tabular} & & $\begin{array}{r}86.6 \\
1374\end{array}$ & $\begin{array}{r}323.9 \\
329\end{array}$ \\
\hline 653 & 36.91 & 3000 & 1330 & \begin{tabular}{|c|}
1059.711651 \\
\end{tabular} & 35.9245 & $\begin{array}{l}150.944 t .7009 \\
01\end{array}$ & \begin{tabular}{|}
$\mid 103.00$ \\
2212.37 \\
\end{tabular} & $\begin{array}{l}5125 \\
5125\end{array}$ & $\begin{array}{l}0.1 \\
0.1 \\
\end{array}$ & $\begin{array}{l}\mid 950 \\
1950\end{array}$ & $\frac{2 \angle 3000}{225000}$ & 1100000 & $\begin{array}{l}.0081 \\
0.0862 \\
\end{array}$ & \begin{tabular}{|c|}
$1.695-04$ \\
$6.99 E-05$
\end{tabular} & $\begin{array}{l}2.46 r \\
1.41\end{array}$ & \begin{tabular}{|l|}
$5.05 \mathrm{E}-03$ \\
$5.46 \mathrm{E}-03$ \\
\end{tabular} & $\begin{array}{r}28.28 \\
95\end{array}$ & 99.47 & $\begin{array}{l}94.15 \\
95.28\end{array}$ & \begin{tabular}{r|}
47.5 \\
52.82
\end{tabular} & $\begin{array}{l}13.4 \\
126.9\end{array}$ & $\begin{array}{l}2305.9 \\
195.8\end{array}$ \\
\hline 654 & 52.07 & 3000 & 1170 & \begin{tabular}{|l|}
1103.514334 \\
\end{tabular} & 45.2325 & 122.9684 & 1829.74 & 5125 & 0.1 & 1950 & 225000 & 1462500 & 0.0611 & $9.64 \mathrm{E}-05$ & 1.798 & \begin{tabular}{|l|}
$5.74 \mathrm{E}-03$ \\
\end{tabular} & & 99.55 & & 54.69 & 120.7 & 177 \\
\hline 655 & 42.59 & 3000 & 1810 & \begin{tabular}{|l|}
417.825029 \\
\end{tabular} & 19.4866 & 139.9143 & 1608.13 & 5125 & 0.1 & 1950 & 225000 & 1775000 & 0.0747 & $2.29 \mathrm{E}-04$ & 2.548 & \begin{tabular}{|l|}
$7.00 \mathrm{E}-03$ \\
\end{tabular} & 89.97 & 98.8 & & 0 & 99.1 & 306.6 \\
\hline 656 & 29.11 & 3000 & 2160 & 24.077182 & 0.9813 & 122.2668 & 1840.24 & 5125 & 0.1 & 1950 & 225000 & 1450000 & 0.1093 & $2.88 \mathrm{E}-04$ & 2.33 & \begin{tabular}{|l|}
$1.25 \mathrm{E}-02$ \\
\end{tabular} & 27.51 & 79.23 & 0 & 0 & 55.6 & 410.3 \\
\hline 657 & 38.8 & 3000 & 830 & 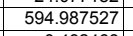 & 26.1629 & 131.9164 & 1705.63 & 5125 & 0.1 & 1950 & 225000 & 1625000 & 0.082 & $8.25 \mathrm{E}-05$ & 1.509 & \begin{tabular}{|l|}
$8.23 \mathrm{E}-03$ \\
\end{tabular} & 29.84 & 99.16 & \begin{tabular}{|l|l|}
91.6 \\
\end{tabular} & 15.96 & 84.2 & 199.3 \\
\hline 658 & 22.72 & 3000 & 2460 & 0.432468 & 0.0208 & 144.4754 & 1557.36 & 5125 & 0.1 & 1950 & 225000 & 1862500 & 0.14 & $\begin{array}{l}6.81 \mathrm{E}-04 \\
\end{array}$ & 3.459 & \begin{tabular}{|l|}
$1.53 \mathrm{E}-02$ \\
\end{tabular} & 58.64 & & & & 45.4 & 780.1 \\
\hline 659 & 34.02 & 3000 & 2230 & \begin{tabular}{ll|l|l}
61.480224 \\
\end{tabular} & 2.3446 & $\begin{array}{l}114.4096 \\
\end{array}$ & 1966.62 & \begin{tabular}{l|l}
5125 \\
\end{tabular} & 0.1 & 1950 & 225000 & 1312500 & 0.0935 & $3.32 \mathrm{E}-04$ & 2.795 & \begin{tabular}{|l|}
$1.10 \mathrm{E}-02$ \\
\end{tabular} & 91.71 & 91.87 & 18.67 & 0 & 62.8 & 421 \\
\hline 660 & 25.66 & 3000 & 3740 & \begin{tabular}{|l|l|l|}
28.389667 \\
\end{tabular} & 0.6777 & 71.6152 & 3141.79 & $\begin{array}{l}5125 \\
\end{array}$ & 0.1 & 1950 & 225000 & 650000 & 0.124 & $5.55 \mathrm{E}-04$ & 3.264 & $8.31 \mathrm{E}-03$ & 54.63 & 82.39 & 0 & 0 & 83.4 & 652 \\
\hline 661 & 22.76 & 3000 & 2650 & 143.822572 & 4.9492 & 103.2361 & 2179.47 & $\begin{array}{l}5125 \\
\end{array}$ & 0.1 & 1950 & 225000 & 1125000 & 0.1398 & $4.06 \mathrm{E}-04$ & 2.468 & \begin{tabular}{|l|}
$5.91 \mathrm{E}-03$ \\
\end{tabular} & 55.03 & 96.52 & $\begin{array}{l}65.23 \\
\end{array}$ & & 117.4 & 555.7 \\
\hline & & 3000 & & 2.49459 & & 110.7481 & 2031.64 & & 0.1 & 1950 & 225000 & 1250000 & $\begin{array}{l}0.0679 \\
\end{array}$ & 4.75E-04 & 4.534 & \begin{tabular}{|c|}
$1.67 \mathrm{E}-02$ \\
\end{tabular} & 26.49 & & & 0 & 41.6 & 496 \\
\hline 663 & 30.44 & 3000 & 1930 & 199.485722 & 5.8609 & 88.1405 & 2552.74 & 5125 & 0.1 & 1950 & 225000 & 887500 & 0.1045 & 2.17E-04 & 2.048 & \begin{tabular}{|l|} 
\\
\end{tabular} & 24.5 & 97.49 & 74.94 & 0 & 85.9 & 344.7 \\
\hline 664 & 41.1 & 3000 & 1820 & 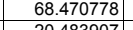 & $\begin{array}{l}2.9643 \\
\end{array}$ & $\begin{array}{r}129.879 \\
\end{array}$ & \begin{tabular}{|c|}
1732.38 \\
170.1
\end{tabular} & $\begin{array}{l}5125 \\
5125\end{array}$ & 0.1 & 1950 & 225000 & 1587500 & 0.0774 & 2.02E-04 & 2.319 & $1.36 \mathrm{E}-02$ & 22.94 & & 26.98 & 0 & 51 & 289.2 \\
\hline 665 & $\begin{array}{r}30.5 \\
2272\end{array}$ & 3000 & $\frac{1990}{2180}$ & $\begin{array}{r}20.483907 \\
754032\end{array}$ & $\mid \begin{array}{c}0.8587 \\
39181\end{array}$ & $\begin{array}{l}125.7554 \\
1155\end{array}$ & $\begin{array}{r}1789.19 \\
\end{array}$ & \begin{tabular}{|l|l}
5125 \\
5125
\end{tabular} & 0.1 & $\begin{array}{l}1950 \\
1950\end{array}$ & \begin{tabular}{|l|l|}
2255000 \\
22500
\end{tabular} & 1512500 & 0.1043 & 2.44E-04 & $\begin{array}{r}2.18 \\
2556\end{array}$ & $\mid$\begin{tabular}{|c|}
$\mid 1.51 \mathrm{E}-02$ \\
\end{tabular} & $\begin{array}{c}49.83 \\
310\end{array}$ & $\begin{array}{l}75.59 \\
03.37\end{array}$ & o & 0 & $\begin{array}{r}46.1 \\
1025\end{array}$ & $\begin{array}{l}366.4 \\
576 .\end{array}$ \\
\hline $666 \mid$ & $\frac{22.72}{22.34}$ & $\begin{array}{l}3000 \\
3000\end{array}$ & 2180 & $\begin{array}{r}75.40236 \\
1178212\end{array}$ & $\begin{array}{l}3.9181 \\
0.0359\end{array}$ & $\begin{array}{r}155.8885 \\
91.426\end{array}$ & $\begin{array}{r}1443.34 \\
2461.15\end{array}$ & \begin{tabular}{|l|l|}
5125 \\
5125
\end{tabular} & 0.1 & $\begin{array}{c}1950 \\
1950\end{array}$ & \begin{tabular}{r|}
2255000 \\
225000
\end{tabular} & $\begin{array}{r}2087500 \\
93750\end{array}$ & \begin{tabular}{r|r}
0.14 \\
0.1307
\end{tabular} & $\begin{array}{l}4.31 \mathrm{E}-04 \\
\end{array}$ & $\begin{array}{l}2.556 \\
3703\end{array}$ & $|6.76 \mathrm{E}-03|$ & $\begin{array}{r}31.9 \\
21.09\end{array}$ & 93.37 & 33.69 & 0 & 102.5 & $\begin{array}{l}576.6 \\
7797\end{array}$ \\
\hline $\begin{array}{l}668 \\
668 \\
\end{array}$ & $\begin{array}{r}24.34 \\
60.6\end{array}$ & 3000 & $\frac{3790}{1580}$ & $\begin{array}{r}9.1102012 \\
900.333045\end{array}$ & $\begin{array}{r}0.0359 \\
47.1566\end{array}$ & $\begin{array}{r}91.4206 \\
157.1306\end{array}$ & $\begin{array}{r}2461.15 \\
1431.93\end{array}$ & 5 & $\begin{array}{l}0.1 \\
0.1\end{array}$ & $\begin{array}{l}1950 \\
1950\end{array}$ & 22550000 & $\begin{array}{r}93 / 500 \\
2112500\end{array}$ & \begin{tabular}{|c|}
$0.130 \prime$ \\
0.0525
\end{tabular} & \begin{tabular}{|c|}
$.999-04$ \\
$1.53 E-04$
\end{tabular} & $\frac{3.03}{2.473}$ & \begin{tabular}{|l|}
$1.13 \mathrm{E}-02$ \\
$5.88 \mathrm{E}-03$ \\
\end{tabular} & $\begin{array}{l}21.09 \\
35.88\end{array}$ & $\begin{array}{r}99.44 \\
9\end{array}$ & $\begin{array}{r}94.45 \\
9\end{array}$ & $\begin{array}{r}0 \\
44.46\end{array}$ & $\begin{array}{r}61.6 \\
118 \\
\end{array}$ & $\begin{array}{l}7799.7 \\
209.1\end{array}$ \\
\hline 669 & 36.15 & 3000 & 2550 & 90.752619 & 5.8544 & 193.5283 & 1162.62 & 5125 & 0.1 & 1950 & 225000 & 2887500 & 0.088 & $5.64 \mathrm{E}-04$ & 4.238 & \begin{tabular}{|c|}
$6.06 \mathrm{E}-03$ \\
\end{tabular} & 45.03 & 94.49 & 44.91 & 0 & 114.4 & 600.8 \\
\hline 670 & 48.42 & 3000 & 2980 & 127.203761 & \begin{tabular}{|l|l|}
6.8453 \\
\end{tabular} & 161.4401 & \begin{tabular}{|c|}
1393.71 \\
\end{tabular} & 5125 & 0.1 & 1950 & 225000 & 2200000 & 0.0657 & $5.40 \mathrm{E}-04$ & 5.156 & $5.94 \mathrm{E}-03$ & 32.42 & 96.07 & 60.69 & & 116.7 & 545.7 \\
\hline 671 & 22.37 & 3000 & 1910 & 86.484121 & \begin{tabular}{|l|l|}
4.3127 \\
\end{tabular} & \begin{tabular}{|l|}
149.6016 \\
\end{tabular} & \begin{tabular}{|l|}
1503.99 \\
\end{tabular} & 5125 & 0.1 & 1950 & 225000 & 1962500 & 0.1422 & $3.11 \mathrm{E}-04$ & 2.105 & \begin{tabular}{|l|l|}
$7.76 \mathrm{E}-03$ \\
\end{tabular} & 38.35 & \begin{tabular}{l|l}
94.22 \\
\end{tabular} & 42.19 & 0 & \begin{tabular}{l|l}
89.3 \\
\end{tabular} & 482.3 \\
\hline 672 & 30.89 & 3000 & 2450 & 41.322742 & \begin{tabular}{|l|}
1.817 \\
\end{tabular} & \begin{tabular}{|l|l|}
131.9164 \\
\end{tabular} & 1705.63 & \begin{tabular}{l|l}
5125 \\
\end{tabular} & 0.1 & 1950 & 225000 & 1625000 & 0.103 & 4.15E-04 & 3.038 & $8.93 \mathrm{E}-03$ & 20.03 & 87.9 & 0 & & 77.6 & 504.1 \\
\hline 673 & 39.28 & 3000 & 890 & 271.529919 & 13.3095 & $\begin{array}{l}147.0497 \\
\end{array}$ & 1530.1 & 5125 & 0.1 & 1950 & 225000 & 1912500 & 0.081 & 6.51E-05 & 1.406 & \begin{tabular}{|c|}
$1.36 \mathrm{E}-02$ \\
\end{tabular} & 59.67 & 98.16 & 81.59 & & 50.9 & 183.5 \\
\hline 674 & 21.45 & 3000 & 1000 & \begin{tabular}{|l|l|}
182.702997 \\
\end{tabular} & 8.2792 & $\begin{array}{l}135.9447 \\
\end{array}$ & 1655.08 & 5125 & 0.1 & 1950 & 225000 & 1700000 & 0.1483 & 1.05E-04 & 1.357 & \begin{tabular}{|l|l|}
$8.77 \mathrm{E}-03$ \\
\end{tabular} & 21.65 & $\begin{array}{l}97.26 \\
\end{array}$ & 72.63 & & 79 & 324.2 \\
\hline 675 & 22.05 & 3000 & 1630 & $\begin{array}{r}35.413746 \\
\end{array}$ & \begin{tabular}{|c|}
1.6978 \\
1.700
\end{tabular} & $\begin{array}{l}143.8283 \\
\end{array}$ & \begin{tabular}{|l|l|l|}
1564.37 \\
159.37
\end{tabular} & 5125 & 0.1 & 1950 & 225000 & & 0.1443 & $\begin{array}{l}1.52 \mathrm{E}-04 \\
1.0-0\end{array}$ & 1.532 & & 44.87 & 85.88 & 0 & 0 & 52.5 & 356 \\
\hline 676 & 53.47 & 3000 & 1670 & 289.821997 & 13.7065 & \begin{tabular}{|l|l|l|}
141.878 \\
\end{tabular} & 1585.87 & 5125 & 0.1 & 1950 & 225000 & 1812500 & 0.0595 & 1.49E-04 & 2.264 & & 89.41 & 98.27 & 82.75 & 0 & 58.9 & 217 \\
\hline $677 \mid$ & 23.37 & 3000 & 2980 & $\begin{array}{r}0.446282 \\
\end{array}$ & $\begin{array}{r}0.0142 \\
158144\end{array}$ & \begin{tabular}{|l|l|}
95.4399 \\
\end{tabular} & \begin{tabular}{|l|}
2357.51 \\
\end{tabular} & $\begin{array}{l}5125 \\
5125\end{array}$ & 0.1 & 1950 & 225000 & 1000000 & 0.1361 & 5.13E-04 & 2.905 & \begin{tabular}{|l|}
$1.60 \mathrm{E}-02$ \\
\end{tabular} & 31.52 & 0 & 0 & 0 & 43.3 & 636.8 \\
\hline 678 & $\begin{array}{l}44.25 \\
39.13\end{array}$ & 3000 & 1740 & $\begin{array}{r}582.996442 \\
5525070\end{array}$ & 15.8164 & $\begin{array}{r}81.3886 \\
83.1021\end{array}$ & $\begin{array}{r}2764.52 \\
270751\end{array}$ & $\begin{array}{l}5125 \\
5125\end{array}$ & 0.1 & $\begin{array}{c}1950 \\
1050 \\
1050\end{array}$ & 225000 & $\begin{array}{r}787500 \\
\end{array}$ & 0.0719 & $\begin{array}{l}1.71 \mathrm{E}-04 \\
5.5 \mathrm{E}\end{array}$ & 2.199 & \begin{tabular}{|l|}
$6.56 \mathrm{E}-03$ \\
\end{tabular} & 27.2 & $\begin{array}{l}99.14 \\
0.52 \\
\end{array}$ & 91.42 & 14.24 & 105.7 & 254.7 \\
\hline 679 & $\begin{array}{l}33.13 \\
51.81\end{array}$ & $\begin{array}{l}3000 \\
3000\end{array}$ & $\frac{3520}{2050}$ & $\begin{array}{r}5.5252578 \\
15.165113\end{array}$ & $\begin{array}{l}0.1531 \\
0.6771\end{array}$ & $\begin{array}{r}83.1021 \\
133.9381\end{array}$ & \begin{tabular}{|l|}
2707.51 \\
167.88
\end{tabular} & $\begin{array}{l}5125 \\
5125\end{array}$ & 0.1 & $\begin{array}{l}\mid 1950 \\
1950\end{array}$ & 2250000 & $\begin{array}{r}812500 \\
162500\end{array}$ & $\begin{array}{l}0.0813 \\
0.0614\end{array}$ & \begin{tabular}{|l|}
$5.45 E-04$ \\
$2.98-04$
\end{tabular} & $\begin{array}{l}4.386 \\
3.452\end{array}$ & \begin{tabular}{|l|}
$\mid 1.32 \mathrm{E}-02$ \\
$177 \mathrm{E}-02$
\end{tabular} & \begin{tabular}{|c|}
37.1 \\
396
\end{tabular} & $\begin{array}{r}9.52 \\
67.03 \\
\end{array}$ & $\begin{array}{l}0 \\
0\end{array}$ & $\begin{array}{l}0 \\
0\end{array}$ & 52.7 & 574.4 \\
\hline 681 & 25.64 & 3000 & 2920 & $\begin{array}{r}15.1655113 \\
298.940605 \\
\end{array}$ & $\begin{array}{l}0.6371 \\
8.3656 \\
\end{array}$ & $\frac{1333.9381}{83.9523}$ & $\begin{array}{l}1698.88 \\
2680.09 \\
\end{array}$ & 5125 & 0.1 & $\frac{1950}{1950}$ & 2255000 & $\frac{16625000}{825000}$ & \begin{tabular}{|l|}
0.0614 \\
0.1241
\end{tabular} & $\begin{array}{l}2.98-04 \\
3.18 E-04\end{array}$ & $\begin{array}{l}3.452 \\
2.297\end{array}$ & \begin{tabular}{|l|}
$1.1 / 7 \mathrm{E}-02$ \\
$5.46 \mathrm{E}-03$ \\
\end{tabular} & $\begin{array}{r}39.6 \\
85.16\end{array}$ & $\begin{array}{l}67.03 \\
98.33\end{array}$ & $\begin{array}{r}83.27 \\
\end{array}$ & of & $\begin{array}{r}39.2 \\
126.9\end{array}$ & $\begin{array}{l}341.4 \\
459.2\end{array}$ \\
\hline 682 & 28.28 & 3000 & 2310 & 10.407833 & 0.3713 & 107.0251 & 2102.31 & 5125 & 0.1 & & 225000 & 1187500 & 0.1125 & $2.57 \mathrm{E}-04$ & 2.157 & \begin{tabular}{|c|}
$1.58 \mathrm{E}-02$ \\
\end{tabular} & 37.75 & 51.96 & & & 43.9 & 390.8 \\
\hline 683 & 26.91 & 3000 & 2340 & \begin{tabular}{|l|}
.678702 \\
\end{tabular} & \begin{tabular}{|l|l|l|l|l}
0.1447 & \\
\end{tabular} & \begin{tabular}{|l|l|}
118.0135 \\
\end{tabular} & \begin{tabular}{|l|}
1906.56 \\
\end{tabular} & 5125 & 0.1 & 1950 & 225000 & 1375000 & 0.1182 & $3.62 \mathrm{E}-04$ & 2.546 & \begin{tabular}{|l|}
$1.51 \mathrm{E}-02$ \\
\end{tabular} & 27.64 & & 0 & 0 & 45.9 & 484.9 \\
\hline 684 & 51.9 & 3000 & 2500 & 137.248655 & 5.5615 & 121.5631 & 1850.89 & 5125 & 0.1 & 1950 & 225000 & 1437500 & 0.0613 & $3.83 \mathrm{E}-04$ & 4.155 & $8.35 \mathrm{E}-03$ & 45.43 & 96.36 & 63.57 & 0 & 83 & 410.3 \\
\hline 685 & 57.63 & 3000 & 1600 & 639.974871 & 17.5455 & 82.2476 & $\begin{array}{l}2735.64 \\
\end{array}$ & 5125 & 0.1 & 1950 & 225000 & & 0.0552 & $\begin{array}{l}1.06 E-04 \\
\end{array}$ & 1.973 & & 66.76 & 99.22 & 92.19 & 21.87 & 75.7 & 175.4 \\
\hline 686 & 25.84 & 3000 & 4270 & 20.160754 & 0.6835 & $\begin{array}{l}101.7009 \\
\end{array}$ & $\begin{array}{l}2212.37 \\
\end{array}$ & 5125 & 0.1 & 1950 & 225000 & 1100000 & 0.1231 & 7.69E-04 & 4.158 & \begin{tabular}{|l|}
$6.42 \mathrm{E}-03$ \\
\end{tabular} & 14.95 & 75.2 & & 0 & 108 & 824.6 \\
\hline 687 & 50.34 & 3000 & 450 & $\begin{array}{l}459.05366 \\
\end{array}$ & 19.5596 & $\begin{array}{l}127.8255 \\
\end{array}$ & \begin{tabular}{|l|}
1760.21 \\
\end{tabular} & $\begin{array}{l}5125 \\
\end{array}$ & 0.1 & 1950 & 225000 & 1550000 & 0.0632 & $2.34 \mathrm{E}-05$ & 1.187 & 1.59 & 50.66 & 98.91 & 89.11 & 0 & 43.7 & 120.9 \\
\hline 688 & 41.32 & 3000 & 1700 & 183.292131 & 10.9193 & \begin{tabular}{|l|l|l|l|l|l|}
178.7197 \\
\end{tabular} & $\begin{array}{l}1258.95 \\
\end{array}$ & 5125 & 0.1 & & 225000 & 2562500 & 0.077 & $\begin{array}{l}3.18 \mathrm{E}-04 \\
\end{array}$ & 3.087 & & 72.89 & 97.27 & 72.72 & & 85 & 383 \\
\hline 689 & 39.47 & 3000 & 1690 & & 5.3871 & \begin{tabular}{|l|l|}
129.879 \\
\end{tabular} & \begin{tabular}{|l|l|}
1732.38 \\
\end{tabular} & 5125 & 0 & & 225000 & 1587500 & 0.0806 & 1.66E-04 & & & & 95. & 59.82 & 0 & 55.3 & 265.1 \\
\hline 690 & & 3000 & 3170 & $\begin{array}{r}81.952801 \\
\end{array}$ & & \begin{tabular}{|l|l|}
83.1021 \\
\end{tabular} & 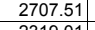 & $\begin{array}{l}5125 \\
5125\end{array}$ & 0 & 19 & 225000 & 812 & 0.1163 & & & & 81.26 & & 38.99 & 0 & 95 & 580.4 \\
\hline $691 \mid$ & 62.01 & $\begin{array}{ll}3000 \\
3000 \\
300\end{array}$ & 2250 & $\frac{602.97}{343.51}$ & $\begin{array}{r}19.501 \\
109284\end{array}$ & $\begin{array}{r}97.024 \\
95.4399\end{array}$ & $\begin{array}{r}2319.01 \\
2357.51\end{array}$ & \begin{tabular}{|l|l}
5125 \\
5125
\end{tabular} & 0.1 & $\frac{195}{195}$ & 225000 & 1025000 & 0.0513 & $\begin{array}{l}1.29 \mathrm{E}-04 \\
0.52 \mathrm{E}\end{array}$ & 2.273 & & 76.88 & 99.17 & $91.71 \mid$ & 17.08 & 77 & 187.9 \\
\hline \begin{tabular}{|c|c|}
692 & 693 \\
\end{tabular} & $25.61 \mid$ & $\begin{array}{l}3000 \\
3000 \\
300\end{array}$ & $\frac{1270}{1500}$ & $\frac{343.515}{114.01}$ & $\begin{aligned} 10.9284 \\
27655\end{aligned}$ & \begin{tabular}{|l}
95.4399 \\
72.5304
\end{tabular} & \begin{tabular}{|l|}
2357.51 \\
3102.15
\end{tabular} & $\begin{array}{l}5125 \\
5125\end{array}$ & & & 225000 & & & $\begin{array}{r}9.52 \mathrm{E}-05 \\
159 \mathrm{E}-04\end{array}$ & & & & & $85.44 \mid$ & 0 & 86.2 & 277.6 \\
\hline $\begin{array}{l}693 \\
694 \\
\end{array}$ & 27.08 & 30000 & $\frac{1500}{1920}$ & $\begin{array}{r}114.013527 \\
10.498442\end{array}$ & $\begin{array}{l}2.7565 \\
0.4497\end{array}$ & $\begin{array}{r}72.5304 \\
128.5118\end{array}$ & $\begin{array}{l}3102.15 \\
1750.81\end{array}$ & $\begin{array}{l}5125 \\
5125\end{array}$ & $\begin{array}{l}0.1 \\
0.1\end{array}$ & $\begin{array}{l}\frac{1950}{1950} \\
\end{array}$ & $\frac{225500}{225000}$ & $\begin{array}{r}\frac{662500}{1562500} \\
\end{array}$ & \begin{tabular}{|c|}
0.1175 \\
0.111
\end{tabular} & $\begin{array}{l}1.599 \mathrm{E}-04 \\
2.16 \mathrm{E}-04\end{array}$ & $\begin{array}{l}\frac{1.683}{1.984} \\
\end{array}$ & \begin{tabular}{|l|}
$1.06 \mathrm{E}-02$ \\
$1.76 \mathrm{E}-02$ \\
\end{tabular} & $\begin{array}{r}33.18 \\
47.2\end{array}$ & $\begin{array}{l}95.61 \\
52.37\end{array}$ & $\begin{array}{r}56.15 \\
0\end{array}$ & & $\begin{array}{l}65.2 \\
39.4 \\
\end{array}$ & $\begin{array}{l}3184.5 \\
354.8 \\
\end{array}$ \\
\hline 695 & 48.42 & 3000 & 4130 & & 9.5437 & 89.788 & 2505.9 & 5125 & & & & 912500 & 0.0657 & $5.66 \mathrm{E}-04$ & 5.355 & & 22.27 & 98.43 & 84.32 & & 168.5 & 566.7 \\
\hline 696 & 36.74 & 3000 & 2430 & 100.64 & 3.7892 & 112.9522 & \begin{tabular}{|l|l|}
1991.99 \\
\end{tabular} & 512. & 0.1 & & 225000 & 1287500 & 0.0866 & $3.56 \mathrm{E}-04$ & 3.08 & $8.68 \mathrm{E}$ & 48.33 & 95.03 & 50.32 & & 79.9 & 429.7 \\
\hline 697 & 22.69 & 3000 & 1440 & \begin{tabular}{|l|l|}
133.92072 \\
1
\end{tabular} & $\begin{array}{l}7.6636 \\
\end{array}$ & $\begin{array}{l}171.6754 \\
\end{array}$ & \begin{tabular}{|l|}
1310.61 \\
\end{tabular} & 5125 & 0.1 & 1950 & 225000 & 2412500 & 0.14 & $1.38 \mathrm{E}-04$ & 1.498 & & 63.81 & 96.27 & 62.66 & 0 & 71.3 & 338.3 \\
\hline 698 & 33.24 & 3000 & 3230 & 255.670472 & \begin{tabular}{|l|l|}
12.0913 \\
\end{tabular} & $\begin{array}{l}141.878 \\
\end{array}$ & 1585. & 5125 & 0.1 & 195 & & & 0.0957 & 6.53E-04 & 4.448 & & 49.91 & 98.04 & 80.44 & & 195.8 & 685.7 \\
\hline 699 & 28.58 & & 1920 & 441.6 & & 135.9 & & 5125 & 0.1 & & & & 0.11 & $2.61 \mathrm{E}-04$ & 2.18 & & 71.8 & 98.87 & 88.68 & & 133.9 & \\
\hline 700 & 21.3 & 30 & 2310 & & 0.4 & 115.1 & 195. & 512 & 0.1 & & 225 & & 0.1 & & 2.29 & & $\begin{array}{ll}63.16 \\
\end{array}$ & & & 0 & 58.2 & 551.7 \\
\hline 701 & 41.75 & 3000 & 810 & 726.5 & 15.6 & $\begin{array}{l}65.9946 \\
19.924\end{array}$ & \begin{tabular}{|l|l|}
3409.37 \\
\end{tabular} & 5125 & 0.1 & 195 & 225 & $\begin{array}{r}575000 \\
\end{array}$ & & $8.22 \mathrm{E}$ & 1.54 & & \begin{tabular}{|l|l|}
29.07 \\
77.77
\end{tabular} & 99.31 & 93.12 & 31.18 & \begin{tabular}{ll|}
91.6 \\
\end{tabular} & 189 \\
\hline 702 & 30.83 & & 1840 & & 4.89 & \begin{tabular}{|l|l|}
113.6821 \\
\end{tabular} & & 5125 & 0 & & & & & & & & & & 61.31 & 0 & 56.5 & \\
\hline 703 & 28. & 30 & 3230 & 90.15 & $\begin{array}{r}2.796 \\
\end{array}$ & & & 5125 & & & 2250 & & & & & & 46.11 & $\begin{array}{l}94.45 \\
07.5 \\
\end{array}$ & $\begin{array}{ll}44.54 \\
75.01\end{array}$ & 0 & 109.8 & \\
\hline 704 & 24.42 & 3000 & 1460 & 200.1 & $\begin{array}{l}12.458 \\
\end{array}$ & $\begin{array}{l}186.7666 \\
1\end{array}$ & 120 & 5125 & $\frac{0}{n}$ & & 225000 & 2737500 & 0.1303 & & & & 32.47 & $\begin{array}{r}97.5 \\
10.52\end{array}$ & 75.01 & 0 & 93.3 & 5.5 \\
\hline 705 & $34.77 \mid$ & & 2700 & $\begin{array}{r}6.213 \\
1090\end{array}$ & 0.2503 & 120.85 & $\frac{186}{1760}$ & \begin{tabular}{|l|l}
5125 \\
5125
\end{tabular} & & & & & & 6.12E-04 & 4.3 & & & $\begin{array}{l}19.53 \\
5414 \\
\end{array}$ & 0 & (2) & 53.6 & 645.9 \\
\hline $\begin{array}{l}706 \\
707\end{array}$ & 28.61 & 3000 & 1980 & $\begin{array}{r}10.902557 \\
27203719\end{array}$ & 0.4645 & $\begin{array}{r}127.8255 \\
\end{array}$ & \begin{tabular}{|r|r|}
1760.21 \\
248328
\end{tabular} & \begin{tabular}{|l|l}
5125 \\
5125
\end{tabular} & & & 2250 & & & $\begin{array}{r}2.72 \mathrm{E}-04 \\
167 \mathrm{E}-04\end{array}$ & 2.236 & & & 54.14 & 0 & & 43.1 & $\begin{array}{ll}400.6 \\
258.5\end{array}$ \\
\hline \begin{tabular}{|c|}
707 \\
708
\end{tabular} & |41.69| & $\begin{array}{l}3000 \\
3000\end{array}$ & 2550 & $\frac{272.203779}{206.879912}$ & $\begin{array}{l}8.2211 \\
7.6372\end{array}$ & $\begin{array}{r}90.6061 \\
10.7481\end{array}$ & \begin{tabular}{|l}
2483.28 \\
2031.64
\end{tabular} & $\frac{5125}{5125}$ & $\frac{0.1}{0.1}$ & $\begin{array}{l}\mid 1950 \\
1950\end{array}$ & $\begin{array}{l}225500 \\
225000\end{array}$ & $\begin{array}{r}9250000 \\
125000\end{array}$ & \begin{tabular}{|c|c|}
0.0763 \\
0.1392
\end{tabular} & \begin{tabular}{|l|}
$1.67 \mathrm{E}-04$ \\
$3.12 \mathrm{E}-04$
\end{tabular} & \begin{tabular}{|c|c|}
2.103 \\
2.133
\end{tabular} & \begin{tabular}{|l|}
$\mid 1.02 \mathrm{E}-02$ \\
$5.82 \mathrm{E}-03$ \\
\end{tabular} & $\frac{96.19}{36.31}$ & \begin{tabular}{|c|c|}
98.16 & 97.58 \\
9
\end{tabular} & $\begin{array}{l}81.63 \\
75.83\end{array}$ & 0 & \begin{tabular}{r|r|}
119.2 & \\
19.2
\end{tabular} & 258.5. \\
\hline 709 & 54.29 & 3000 & 3110 & 226.01515 & \begin{tabular}{|l|l|}
6.6404 \\
\end{tabular} & 88.1405 & 2552.74 & 5125 & 0.1 & 195 & 225000 & 887500 & 0.0586 & $4.33 \mathrm{E}-04$ & 4.731 & \begin{tabular}{|l|}
$6.88 \mathrm{E}-03$ \\
\end{tabular} & 80.79 & 97.79 & 77.88 & & 100.8 & 446.6 \\
\hline 710 & 33.52 & 3000 & 1530 & 47.25283 & 2.1307 & 135.2775 & $\begin{array}{l}1663.25 \\
\end{array}$ & 5125 & 0.1 & 1950 & 225000 & 1687500 & 0.0949 & $1.42 \mathrm{E}-04$ & 1.754 & $1.66 \mathrm{E}-$ & \begin{tabular}{|c|c|c|}
50.99 \\
\end{tabular} & 89.42 & 0 & 0 & 41.7 & 268.1 \\
\hline 711 & 25.03 & 3000 & 2250 & 166.308172 & 7.3504 & 132.592 & 1696.94 & 5125 & 0.1 & 1950 & 225000 & 1637500 & 0.1271 & $\begin{array}{l}3.98 \mathrm{E}-04 \\
\end{array}$ & 2.583 & $5.89 \mathrm{E}$ & 52.88 & 96.99 & 69.94 & & \begin{tabular}{|l|l|}
117.7 \\
\end{tabular} & 528.8 \\
\hline 712 & 28.79 & & 2140 & 11.5132 & 0.471 & 122.9684 & 1829. & 5125 & 0.1 & 195 & & & & $2.91 \mathrm{E}-04$ & 2.33 & 1.44 & 29.12 & 56.57 & 0 & & 48.1 & 414.8 \\
\hline 713 & 25.68 & 3000 & 3260 & $\begin{array}{r}1.33 \\
153.61\end{array}$ & & 125.06 & & $\begin{array}{l}5125 \\
5125\end{array}$ & 0.1 & 1950 & & & & & 4.1 & & 40.79 & & 0 & & 60.4 & \\
\hline $\begin{array}{ll}714 \\
7715\end{array}$ & 27.93 & $\begin{array}{l}3000 \\
3000 \\
3000\end{array}$ & $\frac{1140}{710}$ & \begin{tabular}{|r|}
153.661159 \\
4552114
\end{tabular} & $\begin{array}{l}6.7221 \\
278384 \\
27\end{array}$ & \begin{tabular}{|r|r|}
131.239 \\
18332396
\end{tabular} & \begin{tabular}{|l|l|l|}
1714.43 \\
122723
\end{tabular} & $\begin{array}{l}5125 \\
5125\end{array}$ & 0.1 & $\begin{array}{l}1950 \\
1950 \\
\end{array}$ & 225000 & $\begin{array}{l}1612500 \\
36250 \\
\end{array}$ & $\begin{array}{l}0.1139 \\
0.1766\end{array}$ & \begin{tabular}{|l|l|}
$1.08 E-04$ \\
$515 E-05$
\end{tabular} & \begin{tabular}{|l|l|}
1.479 \\
1204
\end{tabular} & \begin{tabular}{|l|l|}
1.131 \\
7711
\end{tabular} & $\begin{array}{l}36.26 \\
2656 \\
\end{array}$ & $\begin{array}{c}96.75 \\
989\end{array}$ & 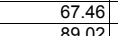 & & $\begin{array}{l}61.4 \\
9\end{array}$ & 271.3 \\
\hline 715 & 24.93] & 3000 & 710 & 455.521214 & $27.8384 \mid$ & 183.3396| & 1227.23 & $5125 \mid$ & & 1950 & 225000 & & 0.1276 & $5.15 \mathrm{E}-05$ & & |7.71E-03 & 26.56| & & 89.02 & & & 247.5 \\
\hline
\end{tabular}




\begin{tabular}{|c|c|c|c|c|c|c|c|c|c|c|c|c|c|c|c|c|c|c|c|c|c|c|}
\hline $\begin{array}{c}\text { Realizatio } \\
n\end{array}$ & $\begin{array}{c}\text { Velocity } \\
(\mathrm{m} / \mathrm{y})\end{array}$ & $\begin{array}{c}\text { Fixed Source } \\
\text { Concentration } \\
(\mathrm{mg} / \mathrm{L})\end{array}$ & $\begin{array}{c}\text { Time of } \\
\text { Maximum at } \\
\text { Compliance } \\
\text { Boundary } \\
(\mathrm{y})\end{array}$ & \begin{tabular}{|c|} 
Maximum \\
Concentration \\
Compliance \\
Boundary \\
$(\mu \mathrm{g} / \mathrm{L})$
\end{tabular} & \begin{tabular}{|c|} 
Maximum \\
Release Rate \\
at Compliance \\
Boundary \\
$(\mathrm{Kg} / \mathrm{y})$ \\
\end{tabular} & $\begin{array}{c}\text { Source } \\
\text { Rate } \\
(\mathrm{Kg} / \mathrm{y}) \\
\end{array}$ & \begin{tabular}{|c|} 
Source \\
Duration \\
(y)
\end{tabular} & $\begin{array}{c}\text { 1-D } \\
\text { Streamtub } \\
\text { e Length } \\
\text { (m) }\end{array}$ & $\begin{array}{l}\text { Base } \\
\text { Porosity } \\
\left(\mathrm{m}^{3} / \mathrm{m}^{3}\right)\end{array}$ & $\begin{array}{l}\text { Bulk } \\
\text { Densty } \\
\left(\mathrm{Kg} / \mathrm{m}^{3}\right)\end{array}$ & $\begin{array}{c}\text { Remaining } \\
\text { Carbon } \\
\text { Tetrachloride } \\
\begin{array}{c}\text { Source } \\
(\mathrm{Kg})\end{array} \\
\end{array}$ & \begin{tabular}{|c|}
$\begin{array}{c}\text { Volume at or } \\
\text { above Fixed } \\
\text { Source } \\
\text { Concentration } \\
(\mathrm{m} 3)\end{array}$ \\
\end{tabular} & $\begin{array}{l}\text { Porosity } \\
\left(\mathrm{m}^{3} / \mathrm{m}^{3}\right)\end{array}$ & $\begin{array}{c}\mathrm{Kd} \\
\left(\mathrm{m}^{3} / \mathrm{Kg}\right)\end{array}$ & Retardation & $\begin{array}{c}\text { First Order } \\
\text { Rate } \\
\text { Constant } \\
(1 / y)\end{array}$ & $\begin{array}{c}\text { Longitudinal } \\
\text { Dispersivity } \\
(\mathrm{m})\end{array}$ & $\begin{array}{c}\text { Required } \\
\text { Source } \\
\text { Cleanup } \\
\text { Percentage } \\
(5 \text { } \mathrm{g} / \mathrm{L} \\
\text { Compliance) } \\
\end{array}$ & \begin{tabular}{|c|} 
Required \\
Source \\
Cleanup \\
Percentage \\
(50 ug/L \\
Compliance)
\end{tabular} & \begin{tabular}{|c|} 
Required \\
Source \\
Cleanup \\
Percentage \\
$(500 \mu \mathrm{g} / \mathrm{L}$ \\
Compliance) \\
\end{tabular} & \begin{tabular}{|c|} 
Abiotic \\
Reaction \\
Half Time \\
(y)
\end{tabular} & $\begin{array}{c}\text { Carbon } \\
\text { Tetrachloride } \\
\text { Travel Time (y) }\end{array}$ \\
\hline & 22.53 & 3000 & $\begin{array}{r}920 \\
\end{array}$ & 75.45908 & 4.5536 & $\begin{array}{l}181.037 \\
\end{array}$ & \begin{tabular}{|l|}
1242.84 \\
\end{tabular} & 5125 & 0.1 & 1950 & 225000 & 2612500 & 0.1412 & $9.08 \mathrm{E}-05$ & 1.325 & $1.25 \mathrm{E}-02$ & $\begin{array}{r}23.92 \\
\end{array}$ & 93.37 & & & 55.5 & 301.4 \\
\hline 717 & 62.38 & 3000 & 1090 & \begin{tabular}{|l|}
1181.161344 \\
\end{tabular} & 48.6907 & 123.6681 & 1819.39 & 5125 & 0.1 & 1950 & 225000 & 1475000 & 0.051 & $6.71 \mathrm{E}-05$ & 1.665 & $6.97 \mathrm{E}-03$ & 75.19 & 99.58 & 95.77 & & 99.5 & 136.8 \\
\hline 718 & 32.46 & 3000 & 2200 & \begin{tabular}{|l|}
1.742395 \\
\end{tabular} & 0.0865 & 148.9657 & 1510.42 & 5125 & 0.1 & 1950 & 225000 & 1950000 & 0.098 & $3.77 \mathrm{E}-04$ & 2.947 & $1.68 \mathrm{E}-02$ & 10.71 & 0 & & 0 & 41.4 & 465.3 \\
\hline 719 & 30.04 & 3000 & 2050 & 141.539549 & 6.0308 & 127.8255 & 1760.21 & 5125 & 0.1 & 1950 & 225000 & 1550000 & 0.1059 & $3.16 \mathrm{E}-04$ & 2.507 & $8.10 \mathrm{E}-03$ & 90.4 & 96.47 & 64.67 & & 85.5 & 427.8 \\
\hline 720 & 32.76 & 3000 & 2100 & 60.597282 & 2.6509 & 131.239 & \begin{tabular}{|l|l|l|l|}
1714.43 \\
\end{tabular} & 5125 & 0.1 & 1950 & 225000 & 1612500 & 0.0971 & $3.22 \mathrm{E}-04$ & 2.678 & $1.02 \mathrm{E}-02$ & 46.01 & 91.75 & 17.49 & & 68 & 418.8 \\
\hline 721 & 22.87 & 3000 & 1920 & \begin{tabular}{|l|}
102.941934 \\
\end{tabular} & $\begin{array}{l}5.6856 \\
\end{array}$ & 165.6929 & 1357.93 & 5125 & 0.1 & 1950 & 225000 & 2287500 & 0.1391 & $3.84 \mathrm{E}-04$ & 2.396 & $6.80 \mathrm{E}-03$ & 52.63 & 95.14 & 51.43 & & 102 & 536.9 \\
\hline 722 & 24.23 & 3000 & 1870 & 1235.258928 & $\begin{array}{l}48.0016 \\
\end{array}$ & 116.5786 & 1930.03 & 5125 & 0.1 & 1950 & 225000 & 1350000 & 0.1313 & $1.39 \mathrm{E}-04$ & 1.534 & $2.78 \mathrm{E}-03$ & 56.12 & 99.6 & 95.95 & 59.52 & 249.8 & 324.6 \\
\hline 723 & 42.59 & 3000 & 2180 & \begin{tabular}{|l|}
40.018723 \\
\end{tabular} & 1.631 & \begin{tabular}{|l|l|}
122.2668 \\
\end{tabular} & 1840.24 & 5125 & 0.1 & 1950 & 225000 & 1450000 & 0.0747 & $2.95 \mathrm{E}-04$ & 2.996 & $1.31 \mathrm{E}-02$ & 38.17 & 87.51 & 0 & & 52.8 & 360.5 \\
\hline 724 & 42.7 & 3000 & 1560 & 76.111119 & 3.0302 & 119.4396 & \begin{tabular}{|l|l|}
1883.8 \\
\end{tabular} & 5125 & 0.1 & 1950 & 225000 & 1400000 & 0.0745 & 1.97E-04 & & $1.34 \mathrm{E}-02$ & 15.2 & 93.43 & 34.31 & & 51.6 & 280.7 \\
\hline 725 & 47.27 & 3000 & 2370 & \begin{tabular}{|l|l|}
37.008397 \\
\end{tabular} & \begin{tabular}{ll|}
1.4203 \\
\end{tabular} & \begin{tabular}{|l|l|}
115.1349 \\
\end{tabular} & \begin{tabular}{|l|l|l|l|}
1954.23 \\
\end{tabular} & 5125 & 0.1 & 1950 & 225000 & 1325000 & 0.0673 & 3.09E-04 & 3.321 & \begin{tabular}{|l|l|}
$1.32 E-02$ \\
\end{tabular} & 28.01 & 86.49 & & & 52.6 & 360 \\
\hline 726 & 22.64 & 3000 & 3360 & 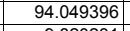 & 3.2124 & \begin{tabular}{|l|l|}
102.4699 \\
7
\end{tabular} & \begin{tabular}{|l|l|}
2195.77 \\
20.72
\end{tabular} & 5125 & 0.1 & 1950 & 225000 & 1112500 & 0.1405 & $\begin{array}{l}6.37 E-04 \\
\end{array}$ & 3.291 & $5.02 \mathrm{E}-03$ & 37.59 & 94.68 & $\begin{array}{ll}46.84 \\
\end{array}$ & & 138 & 744.9 \\
\hline 727 & 24.93 & $\begin{array}{l}3000 \\
3000 \\
3000\end{array}$ & 3740 & \begin{tabular}{|r|}
9.820231 \\
553540303
\end{tabular} & $\begin{array}{c}0.2404 \\
165674 \\
\end{array}$ & \begin{tabular}{|l|l|}
73.4399 \\
80709 \\
\end{tabular} & $\begin{array}{l}3063.73 \\
25050\end{array}$ & $\begin{array}{l}5125 \\
5125 \\
125\end{array}$ & 0.1 & $\begin{array}{l}1950 \\
1950\end{array}$ & 225000 & 675000 & $\begin{array}{l}0.1276 \\
0.1130\end{array}$ & $\begin{array}{l}5.25 \mathrm{E}-04 \\
5.77505\end{array}$ & $\begin{array}{l}3.08 \\
123 \\
\end{array}$ & $9.87 \mathrm{E}-03$ & 26.95 & $\begin{array}{l}49.08 \\
0.1\end{array}$ & 0 & & 70.2 & 633.2 \\
\hline $\begin{array}{l}728 \\
729\end{array}$ & 27.96 & $\begin{array}{l}3000 \\
3000 \\
\end{array}$ & $\begin{array}{r}710 \\
1920 \\
\end{array}$ & \begin{tabular}{|r|}
553.54463 \\
118.967872
\end{tabular} & $\begin{array}{r}16.5674 \\
5.4174\end{array}$ & \begin{tabular}{|r|}
89.788 \\
136.6103 \\
\end{tabular} & \begin{tabular}{r|}
2505.92 \\
1647.02
\end{tabular} & $\begin{array}{l}5125 \\
5125\end{array}$ & 0.1 & $\begin{array}{l}1950 \\
1950 \\
\end{array}$ & $\frac{225000}{225000}$ & \begin{tabular}{r|r|}
9112500 \\
1712500
\end{tabular} & $\mid 0.1148$ & $\begin{array}{l}\frac{5.17 E-05}{2.44-04} \\
\end{array}$ & $\begin{array}{r}1.23 \\
1.846\end{array}$ & $\begin{array}{l}7.592-03 \\
7.72 E-33 \\
\end{array}$ & $\begin{array}{l}31.07 \\
30.42\end{array}$ & $\begin{array}{l}99.1 \\
95.8 \\
\end{array}$ & $\begin{array}{l}90.97 \\
5.97 \\
\end{array}$ & \begin{tabular}{rl|l}
9.67 & \\
\end{tabular} & $\begin{array}{l}91.3 \\
89.8\end{array}$ & 225.4 \\
\hline 730 & 37.3 & 3000 & 2070 & \begin{tabular}{|l|}
934.989827 \\
\end{tabular} & 38.9771 & 125.0616 & 1799.11 & 5125 & 0.1 & 1950 & 225000 & 1500000 & 0.0853 & $2.40 \mathrm{E}-04$ & 2.42 & $3.57 \mathrm{E}-03$ & $\begin{array}{l}32.14 \\
32.14\end{array}$ & 99.47 & 94.65 & 46.52 & 194.3 & 332.5 \\
\hline 731 & 35.83 & 3000 & 4350 & \begin{tabular}{|l|}
183.447757 \\
\end{tabular} & 3.5529 & 58.1021 & 3872.49 & 5125 & 0.1 & 1950 & 225000 & 475000 & 0.0888 & $4.49 \mathrm{E}-04$ & 3.556 & \begin{tabular}{|l|l|} 
& $5.98 \mathrm{E}-03$ \\
\end{tabular} & 45.92 & 97.27 & 72.74 & & 115.9 & 508.7 \\
\hline 732 & 30.13 & 3000 & 2980 & 6.37756 & 0.2432 & \begin{tabular}{|r|}
114.4096 \\
\end{tabular} & $\begin{array}{l}1966.62 \\
1962\end{array}$ & 5125 & 0.1 & 1950 & 225000 & 1312500 & 0.1056 & $6.53 \mathrm{E}-04$ & 4.128 & $1.11 \mathrm{E}-02$ & 54.76 & 21.6 & 0 & & 62.5 & 702.2 \\
\hline 733 & 32.86 & 3000 & 1510 & 27.408425 & $\begin{array}{l}1.4805 \\
\end{array}$ & 162.0511 & 1388.45 & 5125 & 0.1 & 1950 & 225000 & 2212500 & 0.0968 & $1.82 \mathrm{E}-04$ & 1.949 & $1.61 \mathrm{E}-02$ & 24.52 & 81.76 & & & 43 & 304 \\
\hline 734 & 25.86 & 3000 & 3100 & 3.992773 & 0.1291 & 97.024 & 2319.01 & 5125 & 0.1 & 1950 & 225000 & 1025000 & 0.123 & $5.73 \mathrm{E}-04$ & 3.355 & $1.17 \mathrm{E}-02$ & 41.31 & & 0 & & 59 & 664.8 \\
\hline 735 & 27.76 & 3000 & 1760 & 268.950295 & \begin{tabular}{|l|l}
13.4687 \\
\end{tabular} & 150.2362 & 1497.64 & 5125 & 0.1 & 1950 & 225000 & 1975000 & 0.1146 & $2.40 \mathrm{E}-04$ & 2.06 & $6.63 \mathrm{E}-03$ & 47.04 & 98.14 & 81.41 & & 104.5 & 380.3 \\
\hline 736 & 21.67 & 3000 & 2570 & \begin{tabular}{|l|l|}
26.462797 \\
\end{tabular} & 0.8489 & \begin{tabular}{|l|}
96.2335 \\
\end{tabular} & $\begin{array}{l}2338.06 \\
30.61\end{array}$ & 5125 & 0.1 & 1950 & 225000 & 1012500 & 0.1468 & $3.06 \mathrm{E}-04$ & 2.055 & $1.09 \mathrm{E}-02$ & 64.14 & 81.11 & & & 63.5 & 486 \\
\hline 737 & 27.43 & 3000 & 3050 & 16.757434 & 0.4642 & 83.1021 & 2707.51 & 5125 & 0.1 & 1950 & 225000 & 812500 & 0.116 & $4.55 \mathrm{E}-04$ & 2.981 & \begin{tabular}{|l|l|}
$1.17 \mathrm{E}-02$ \\
\end{tabular} & 84.65 & 70.16 & 0 & & 59.3 & 557.1 \\
\hline 738 & 33.84 & 3000 & 2020 & 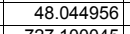 & \begin{tabular}{|r|}
2.7683 \\
21213
\end{tabular} & \begin{tabular}{|l|l|}
172.8593 \\
\end{tabular} & \begin{tabular}{|l|l|}
1301.64 \\
\end{tabular} & 5125 & 0.1 & 1950 & 225000 & 2437500 & 0.094 & 4.99E-04 & 3.684 & $9.14 \mathrm{E}-03$ & 88.81 & 89.59 & 0 & 0 & 75.8 & 557.8 \\
\hline 739 & 42.53 & 3000 & 2280 & 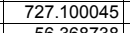 & \begin{tabular}{|l|l|}
31.3129 \\
\end{tabular} & \begin{tabular}{|l|l|}
129.1963 \\
\end{tabular} & \begin{tabular}{|l|l|}
1741.54 \\
907.7
\end{tabular} & 5125 & 0.1 & 1950 & 225000 & 1575000 & 0.0748 & 3.33E-04 & 3.252 & $3.75 \mathrm{E}-03$ & $\begin{array}{l}42.49 \\
\end{array}$ & 99.31 & 93.12 & 31.23 & 184.8 & 391.9 \\
\hline $\begin{array}{l}740 \\
774\end{array}$ & $\begin{array}{r}26.51 \\
30.8\end{array}$ & $\begin{array}{l}3000 \\
3000 \\
3000\end{array}$ & $\begin{array}{l}3270 \\
1830 \\
130\end{array}$ & \begin{tabular}{r|r|}
56.368738 \\
159683338
\end{tabular} & $\begin{array}{l}1.6561 \\
9043\end{array}$ & \begin{tabular}{|r|}
88.1405 \\
169
\end{tabular} & \begin{tabular}{|l|l|}
2552.74 \\
122.37
\end{tabular} & $\begin{array}{l}5125 \\
5125 \\
5\end{array}$ & 0.1 & $\begin{array}{l}1950 \\
1950 \\
\end{array}$ & 225000 & $\begin{array}{r}887500 \\
\end{array}$ & $\begin{array}{r}0.12 \\
0.103\end{array}$ & $5.36 \mathrm{E}-04$ & 3.256 & $7.11 \mathrm{E}-03$ & $\begin{array}{ll}49.96 \\
\end{array}$ & 91.13 & 11.3 & & 97.5 & 629.4 \\
\hline 741 & $\begin{array}{r}30.8 \\
2499\end{array}$ & 3000 & $\begin{array}{l}1830 \\
2800 \\
\end{array}$ & $\begin{array}{r}159.683338 \\
\end{array}$ & \begin{tabular}{|c|}
.043 \\
01408
\end{tabular} & 169.8917 & $\begin{array}{l}1324.37 \\
10.00\end{array}$ & 5125 & 0.1 & 1950 & 225000 & 2375000 & 0.1033 & 3.30E-04 & 2.613 & $7.15 \mathrm{E}-03$ & 40.67 & 96.87 & 68.69 & & 96.9 & 434.9 \\
\hline $\begin{array}{r}742 \\
743\end{array}$ & $\begin{array}{l}24.99 \\
29.51\end{array}$ & $\begin{array}{l}3000 \\
3000\end{array}$ & 2800 & $\begin{array}{r}3.710249 \\
38960569\end{array}$ & \begin{tabular}{|l|l|}
0.1468 \\
12498
\end{tabular} & \begin{tabular}{|r|}
118.7276 \\
96.2335 \\
\end{tabular} & $\begin{array}{l}1895.09 \\
2338.06 \\
\end{array}$ & $\begin{array}{l}5125 \\
5125 \\
\end{array}$ & 0.1 & $\begin{array}{l}1950 \\
1950\end{array}$ & $\begin{array}{l}225000 \\
25000\end{array}$ & $\begin{array}{r}1387500 \\
1012500\end{array}$ & $\begin{array}{l}0.1273 \\
0.178\end{array}$ & $\begin{array}{r}5.59 \mathrm{E}-04 \\
297 \mathrm{E}\end{array}$ & $\begin{array}{r}3.22 \\
2391 \\
\end{array}$ & $1.16 \mathrm{E}-02$ & $\begin{array}{r}33.1 \\
56.4\end{array}$ & $\frac{0}{8717}$ & 0 & & $\begin{array}{r}60 \\
595 \\
9\end{array}$ & $\begin{array}{l}660.3 \\
4152 \\
\end{array}$ \\
\hline 744 & 22.69 & 3000 & 1670 & $\mid$ & $\begin{array}{l}.2450 \\
0.5712\end{array}$ & \begin{tabular}{|r|}
170.4873 \\
\end{tabular} & $\begin{array}{l}2338.06 \\
1319.75 \\
\end{array}$ & $\begin{array}{l}5125 \\
5125 \\
\end{array}$ & $\begin{array}{l}0.1 \\
0.1\end{array}$ & $\begin{array}{l}1950 \\
1950 \\
\end{array}$ & $\frac{25000}{225000}$ & $\begin{array}{l}017500 \\
2387500\end{array}$ & $\begin{array}{l}0.100 \\
0.1402 \\
\end{array}$ & $\begin{array}{l}2.97 / \mathrm{E}-04 \\
2.81 \mathrm{E}-04\end{array}$ & $\begin{array}{l}2.391 \\
2.015\end{array}$ & \begin{tabular}{|l|l|}
$1.17 \mathrm{E}-02$ \\
$1.35 \mathrm{E}-02$
\end{tabular} & \begin{tabular}{|l}
56.4 \\
35.77 \\
\end{tabular} & $\frac{87.11}{50.25}$ & 0 & & $\begin{array}{l}59.5 \\
51.2\end{array}$ & $\begin{array}{r}415.2 \\
455 \\
\end{array}$ \\
\hline 745 & 47.62 & 3000 & 1410 & \begin{tabular}{|l|}
207.486483 \\
\end{tabular} & 7.6596 & 110.7481 & 2031.64 & 5125 & 0.1 & 1950 & 225000 & 1250000 & 0.0668 & $1.37 \mathrm{E}-04$ & 2.039 & $1.26 \mathrm{E}-02$ & 34.46 & 97.59 & 75.9 & & 54.9 & 219.4 \\
\hline 746 & 29.84 & 3000 & 2580 & \begin{tabular}{|l|}
0.706015 \\
\end{tabular} & 0.0346 & 147.0497 & 1530.1 & 5125 & 0.1 & 1950 & 225000 & 1912500 & 0.1066 & $5.80 \mathrm{E}-04$ & 3.749 & $1.55 \mathrm{E}-02$ & 32.23 & & 0 & 0 & 44.7 & 643.9 \\
\hline 747 & 37.04 & 3000 & 730 & 940.63794 & 52.3302 & 166.8979 & 1348.13 & 5125 & 0.1 & 1950 & 225000 & 2312500 & 0.0859 & $6.17 \mathrm{E}-05$ & 1.363 & $6.22 \mathrm{E}-03$ & 34.72 & 99.47 & 94.68 & 46.84 & 111.5 & 188.6 \\
\hline 748 & 29.76 & 3000 & 2670 & 1.108264 & 0.0571 & 154.6414 & 1454.98 & 5125 & 0.1 & 1950 & 225000 & 2062500 & 0.1069 & $5.57 \mathrm{E}-04$ & 3.633 & $1.40 \mathrm{E}-02$ & 19.59 & & 0 & & 49.5 & 625.7 \\
\hline 749 & 21.88 & 3000 & 2190 & 83.533177 & 3.3257 & 119.4396 & $\begin{array}{l}1883.8 \\
\end{array}$ & 5125 & 0.1 & 1950 & 225000 & 1400000 & 0.1454 & $2.80 \mathrm{E}-04$ & 1.973 & $8.05 \mathrm{E}-03$ & 28.48 & 94.01 & 40.14 & & 86.1 & 462.2 \\
\hline 750 & 61.18 & 3000 & 4560 & & $\begin{array}{l}0.1549 \\
\end{array}$ & 130.5599 & 1723.35 & 5125 & 0.1 & 1950 & 225000 & & & $7.60 \mathrm{E}-04$ & 8.393 & $1.14 \mathrm{E}-02$ & 24.68 & & & & 60.9 & 703.1 \\
\hline 751 & 29.24 & 3000 & 1850 & $98.8 \mathrm{C}$ & 5.8138 & 176.3872 & & & 0.1 & 1950 & 225000 & & 0.1088 & & 2.641 & & & 94.94 & 49.43 & & 88.2 & 463 \\
\hline 752 & 28.46 & 3000 & 1770 & \begin{tabular}{|l|}
350.468867 \\
\end{tabular} & \begin{tabular}{|l|l|}
12.0603 \\
\end{tabular} & 103.2361 & 2179.47 & 5125 & 0.1 & 1950 & 225000 & 1125000 & 0.1118 & $\begin{array}{l}1.62 \mathrm{E}-04 \\
\end{array}$ & 1.733 & $7.08 \mathrm{E}-03$ & 40.16 & 98.57 & 85.73 & 0 & 97.9 & 312.2 \\
\hline 753 & 54.95 & 3000 & $\begin{array}{l}1980 \\
2510\end{array}$ & & & 103.9994 & $\begin{array}{l}2163.47 \\
2050.19\end{array}$ & $\begin{array}{l}5125 \\
5125 \\
5\end{array}$ & 0.1 & $\begin{array}{l}1950 \\
1950\end{array}$ & 225000 & 1137500 & & $\begin{array}{l}1.46 \mathrm{E}-04 \\
.25-0\end{array}$ & 2.275 & $1.16 \mathrm{E}-02$ & 51.07 & & 82.63 & 0 & 59.6 & 212.2 \\
\hline $\begin{array}{l}754 \\
775 \\
\end{array}$ & $\begin{array}{l}42.93 \\
37.17 \\
\end{array}$ & $\begin{array}{l}3000 \\
3000\end{array}$ & $\begin{array}{l}3510 \\
1180\end{array}$ & \begin{tabular}{|c|}
62.804751 \\
86970989
\end{tabular} & $\begin{array}{l}2.2875 \\
3103 \\
\end{array}$ & \begin{tabular}{|l|l|}
109.2665 \\
1070251
\end{tabular} & $\begin{array}{l}2059.19 \\
210231\end{array}$ & $\begin{array}{l}5125 \\
5125 \\
125\end{array}$ & 0.1 & $\begin{array}{l}1950 \\
1950\end{array}$ & 225000 & $\begin{array}{l}1225000 \\
118700\end{array}$ & 0.0741 & $\begin{array}{l}6.26 \mathrm{E}-04 \\
\end{array}$ & 5.269 & $6.99 \mathrm{E}-03$ & 42.75 & 92.04 & 20.39 & 0 & 99.2 & 629 \\
\hline 756 & $\begin{array}{l}3.17 \\
27.01\end{array}$ & $\begin{array}{l}3000 \\
3000\end{array}$ & $\frac{1180}{2170}$ & $\begin{array}{r}869 . / 94849 \\
21.375578\end{array}$ & \begin{tabular}{|r|}
31.03 \\
0.7246
\end{tabular} & \begin{tabular}{|l|}
101.0251 \\
101.7009
\end{tabular} & $\begin{array}{l}2102.31 \\
2212.37\end{array}$ & $\begin{array}{l}5125 \\
5125 \\
\end{array}$ & $\frac{0.1}{0.1}$ & $\frac{1950}{1950}$ & $\frac{225000}{225000}$ & \begin{tabular}{|l|l|}
11800000 \\
1100000
\end{tabular} & $\begin{array}{l}0.0856 \\
0.1178 \\
\end{array}$ & \begin{tabular}{|l|}
$.433 E=-05$ \\
$2.10 E-04$
\end{tabular} & $\begin{array}{l}1.498 \\
1.903 \\
\end{array}$ & \begin{tabular}{|l|l}
$6.14 \mathrm{E}-03$ \\
$1.48 \mathrm{E}-02$
\end{tabular} & $\begin{array}{l}60.9 .9 \\
44.96\end{array}$ & $\begin{array}{l}99.43 \\
76.61\end{array}$ & \begin{tabular}{r|r|r|}
94.25 \\
\end{tabular} & re.52 & $\frac{113.2}{46.8}$ & $\begin{array}{l}206.6 \\
3611 \\
\end{array}$ \\
\hline 757 & 29.65 & 3000 & 2500 & \begin{tabular}{|l|}
665.399885 \\
\end{tabular} & 21.1686 & $\begin{array}{l}95.4399 \\
\end{array}$ & 2357 & 5125 & 0.1 & 1950 & 225000 & 1000000 & 0.1073 & $2.10 \mathrm{E}-04$ & 1.99 & $4.56 \mathrm{E}-03$ & 71.24 & 99.25 & 92.49 & 24.86 & 152 & 343.9 \\
\hline 758 & 31.69 & 3000 & 1580 & $\mid 1384.08131$ & 42.5519 & 92.2314 & 2439 & 5125 & 0.1 & 1950 & 225000 & 950000 & 0.1004 & $1.11 \mathrm{E}-04$ & 1.557 & $3.12 \mathrm{E}-03$ & 60.14 & 99.64 & 96.39 & 63.87 & 222.5 & 251.9 \\
\hline 759 & 27.33 & 3000 & 1470 & 107.85864 & \begin{tabular}{|l|}
5.8262 \\
\end{tabular} & 162.0511 & 1388 & 5125 & 0.1 & 1950 & 225000 & 2212500 & 0.1164 & $1.16 \mathrm{E}-04$ & 1.502 & $1.28 \mathrm{E}-02$ & 81.61 & 95.36 & 53.64 & & $\begin{array}{r}54.4 \\
\end{array}$ & 281.6 \\
\hline 760 & 59.8 & 3000 & 3030 & \begin{tabular}{|r|}
421.692967 \\
\end{tabular} & \begin{tabular}{|l|l|}
16.8887 \\
\end{tabular} & 120.1495 & 1872.67 & 5125 & 0.1 & 1950 & & 1412500 & 0.0532 & $4.08 \mathrm{E}-04$ & 4.881 & $4.80 \mathrm{E}-03$ & 13.62 & 98.81 & 88.14 & & 144.3 & 418.3 \\
\hline 761 & 21.37 & 3000 & 2840 & 0.336054 & & 106.2727 & & 5125 & 0.1 & 1950 & 225 & & 0.1489 & $5.74 \mathrm{E}-04$ & 2.949 & & & & 0 & & 45 & 707.5 \\
\hline 762 & 28.76 & 3000 & 2300 & 322.5 & & 110.0086 & 20 & 5125 & 0.1 & 1950 & 225000 & 1237500 & 0.1106 & $2.93 \mathrm{E}-04$ & 2.34 & 5.80 & 82 & 98.45 & 84.5 & & 119.5 & 416.9 \\
\hline 763 & 47.55 & 3000 & 1740 & 442. & & 139.9143 & 160 & & 0 & & & & 0.06 & & 2.295 & & & 98.87 & 88.69 & & 84.4 & 247.3 \\
\hline 764 & 22.68 & 3000 & 1470 & & & & & & 0 & 19 & & & 0.1 & & 1.541 & & & 93 & 35.98 & & 63.9 & 8.4 \\
\hline 765 & 30.59 & 3000 & 2560 & & & 115. & 1954 & 51 & 0.1 & 1950 & 225000 & $1325 \mathrm{C}$ & 0.104 & 4.00 & 2.944 & & & 59.22 & 0 & & 58.4 & 83.3 \\
\hline 766 & 27.62 & 3000 & 2630 & & & $\begin{array}{ll}110.7 \\
1250\end{array}$ & 203 & & 0 & 1950 & 225000 & & & $\begin{array}{l}4.69 \mathrm{E}-04 \\
\end{array}$ & 3.056 & & 64 & 94. & 43.15 & & 98.2 & 87.2 \\
\hline 767 & 32.3 & 3000 & 2310 & & & $\begin{array}{l}125.0616 \\
\end{array}$ & 1799.11 & 5125 & 0.1 & 1950 & & & & & & & & 54.1 & 0 & & 52.2 & 40.6 \\
\hline $\begin{array}{l}768 \\
769 \\
\end{array}$ & $\frac{24.01}{26.31}$ & $\begin{array}{l}3000 \\
3000 \\
\end{array}$ & $\frac{1950}{1700}$ & $\begin{array}{r}25.773565 \\
11656034\end{array}$ & \begin{tabular}{r|}
1.0139 \\
0.032
\end{tabular} & \begin{tabular}{|l|l|l|l|l}
18.0135 \\
162.6609
\end{tabular} & $\begin{array}{l}1906.56 \\
1383.5 \\
\end{array}$ & $\begin{array}{l}5125 \\
5125 \\
\end{array}$ & $\frac{0.1}{0.1}$ & $\frac{1950}{1950}$ & $\frac{222000}{225000}$ & $\begin{array}{l}1375000 \\
2225000\end{array}$ & $\begin{array}{l}0.1325 \\
0.1209\end{array}$ & \begin{tabular}{|l|}
$2.11 E-04$ \\
$301-04$
\end{tabular} & $\begin{array}{l}1.806 \\
2259\end{array}$ & \begin{tabular}{|l|l}
$1.31 \mathrm{E}-02$ \\
$1.4 \mathrm{E}-02$
\end{tabular} & \begin{tabular}{|c|}
34.88 \\
56.61
\end{tabular} & $\begin{array}{l}80.6 \\
57.1\end{array}$ & $\frac{0}{0}$ & $\frac{0}{0}$ & $\frac{53.1}{48.3}$ & $\frac{385.4}{40.1}$ \\
\hline 770 & 28.35 & 3000 & 1540 & \begin{tabular}{|l|}
280.910205 \\
\end{tabular} & \begin{tabular}{|l|}
13.5887 \\
\end{tabular} & 145.1211 & 1550.43 & 5125 & 0.1 & 1950 & 225 & 1875 & 0.1122 & $1.19 \mathrm{E}-04$ & 1.538 & $8.88 \mathrm{E}-03$ & 59.4 & 98.22 & 82.2 & & 78 & 277.9 \\
\hline 771 & 60.71 & 3000 & 1630 & \begin{tabular}{|l|}
756.317471 \\
\end{tabular} & 24.4603 & 97.024 & 2319.01 & 5125 & 0.1 & 1950 & 225000 & 102 & 0.0524 & $1.44 \mathrm{E}-04$ & 2.392 & $6.96 \mathrm{E}-03$ & 30.45 & 99.34 & 93.39 & 33.89 & 99.6 & 201.9 \\
\hline 772 & 21.45 & 3000 & 2810 & \begin{tabular}{|l|}
127.679791 \\
\end{tabular} & \begin{tabular}{|l|l|}
4.1293 \\
\end{tabular} & 97.024 & 2319.01 & 5125 & 0.1 & 1950 & 225000 & 1025000 & 0.1483 & $4.41 \mathrm{E}-04$ & 2.502 & $5.78 \mathrm{E}-03$ & 61.1 & 96. & 60.84 & 0 & 119.9 & 597.7 \\
\hline 773 & 21.6 & 3000 & 2070 & 37.1 & 1.5471 & 125.06 & 1799 & 5125 & 0.1 & 1950 & & 150 & & 2.79 & 1.96 & & 50 & 86 & 0 & & 67.6 & 464.2 \\
\hline 774 & 30.33 & 3000 & 3360 & 211. & 6.4 & & 248 & 5125 & 0 & 1950 & & & 0.1 & & 3.7 & & 59 & 97 & 76.4 & & 147.6 & 625.3 \\
\hline 775 & 21.52 & 3000 & 3230 & & 2.89 & 110. & & 5125 & 0.1 & 1950 & 225000 & 123 & 0.1 & 6.55 & 3.241 & & 43.6 & & 36.63 & 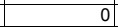 & 134.4 & 771.8 \\
\hline 776 & & & 2130 & 142 & & & 207 & & 0 & & & & & & 2.729 & & & & 64.92 & & 51.5 & 236.6 \\
\hline & 62.38 & & 650 & $1563.5 \mathrm{c}$ & & & & & 0 & 1950 & & & & 4.30 & 1.426 & 5.62 & 5 & & 96.8 & 68.02 & 123.3 & 117.1 \\
\hline 778 & \begin{tabular}{|l|l}
23.78 \\
31.44
\end{tabular} & 3000 & 2100 & \begin{tabular}{r|}
27.979387 \\
22553885
\end{tabular} & & $\begin{array}{l}107.0251 \\
149616\end{array}$ & \begin{tabular}{|l|l|l|l|l|}
2102.31 \\
1503.99
\end{tabular} & $\begin{array}{l}5125 \\
5125 \\
125\end{array}$ & 0.1 & $\begin{array}{l}1950 \\
1950\end{array}$ & 225000 & $\begin{array}{l}1187500 \\
1962500\end{array}$ & $\begin{array}{l}0.1338 \\
0.102\end{array}$ & \begin{tabular}{|l|l|}
$2.36 \mathrm{E}-04$ \\
$340 \mathrm{~F}-04$
\end{tabular} & 1.891 & $1.21 \mathrm{E}-02$ & $\begin{array}{l}30.84 \\
594 \\
\end{array}$ & 82.13 & $\begin{array}{r}0 \\
783 \\
\end{array}$ & & $\begin{aligned} 57.4 \\
1091\end{aligned}$ & 407.5 \\
\hline 778 & $\begin{array}{l}31.44 \\
26.82 \\
\end{array}$ & $\begin{array}{l}3000 \\
3000 \\
\end{array}$ & $\frac{1960}{2810}$ & \begin{tabular}{|r|}
225.5318855 \\
38656592
\end{tabular} & \begin{tabular}{r|}
11.2466 \\
1.8865
\end{tabular} & $\begin{array}{l}149.6016 \\
1446082\end{array}$ & $\begin{array}{r}1503.99 \\
1536.8\end{array}$ & $\frac{5125}{5125}$ & 0.1 & $\begin{array}{l}1950 \\
1950\end{array}$ & $\begin{array}{l}225000 \\
225000\end{array}$ & $\begin{array}{l}1962500 \\
1900000\end{array}$ & 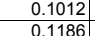 & \begin{tabular}{|l|}
$3.40 \mathrm{E}-04$ \\
$6.15 \mathrm{E}-04$
\end{tabular} & $\begin{array}{r}2.699 \\
362\end{array}$ & $\begin{array}{l}6.36 \mathrm{E}-03 \\
6.94-03 \\
\end{array}$ & $\begin{aligned} 59.84 \\
3391\end{aligned}$ & $\begin{array}{l}97.78 \\
87.07\end{array}$ & $\begin{aligned} 77.83 \\
0\end{aligned}$ & $\frac{0}{0}$ & $\frac{109.1}{998}$ & $\begin{array}{r}440 \\
6916 \\
\end{array}$ \\
\hline
\end{tabular}




\begin{tabular}{|c|c|c|c|c|c|c|c|c|c|c|c|c|c|c|c|c|c|c|c|c|c|c|}
\hline $\begin{array}{c}\text { Realizatio } \\
\text { n }\end{array}$ & $\begin{array}{c}\text { Velocity } \\
(\mathrm{m} / \mathrm{y})\end{array}$ & $\begin{array}{c}\text { Fixed Source } \\
\text { Concentration } \\
\text { (mg/L) }\end{array}$ & $\begin{array}{c}\text { Time of } \\
\text { Maximum at } \\
\text { Compliance } \\
\text { Boundary } \\
(y)\end{array}$ & $\begin{array}{c}\text { Maximum } \\
\text { Concentration } \\
\text { Compliance } \\
\text { Boundary } \\
(\mu \mathrm{g} / \mathrm{L})\end{array}$ & $\begin{array}{c}\text { Maximum } \\
\text { Release Rate } \\
\text { at Compliance } \\
\text { Boundary } \\
(\mathrm{Kg} / \mathrm{y})\end{array}$ & \begin{tabular}{|c|} 
Source \\
Rate \\
$(\mathrm{Kg} / \mathrm{y})$
\end{tabular} & \begin{tabular}{|c|} 
Source \\
Duration \\
(y)
\end{tabular} & \begin{tabular}{|c|} 
1-D \\
Streamtub \\
e Length \\
$(\mathrm{m})$
\end{tabular} & $\begin{array}{l}\text { Base } \\
\text { Porosity } \\
\left(\mathrm{m}^{3} / \mathrm{m}^{3}\right)\end{array}$ & $\begin{array}{c}\text { Bulk } \\
\text { Densty } \\
\left(\mathrm{Kg} / \mathrm{m}^{3}\right)\end{array}$ & $\begin{array}{c}\text { Remaining } \\
\text { Carbon } \\
\text { Tetrachloride } \\
\text { Source } \\
(\mathrm{Kg})\end{array}$ & $\begin{array}{c}\text { Volume at or } \\
\text { above Fixed } \\
\text { Source } \\
\begin{array}{c}\text { Concentration } \\
(\mathrm{m} 3)\end{array}\end{array}$ & $\begin{array}{l}\text { Porosity } \\
\left(\mathrm{m}^{3} / \mathrm{m}^{3}\right)\end{array}$ & $\begin{array}{c}\mathrm{Kd} \\
\left(\mathrm{m}^{3 /} / \mathrm{Kg}\right)\end{array}$ & Retardation & $\begin{array}{c}\text { First Order } \\
\text { Rate } \\
\text { Constant } \\
(1 / y)\end{array} \mid$ & $\begin{array}{l}\text { Longitudinal } \\
\text { Dispersivity } \\
\text { (m) }\end{array}$ & $\begin{array}{c}\text { Required } \\
\text { Source } \\
\text { Cleanup } \\
\text { Percentage } \\
(5 \mu \mathrm{\mu g} / \mathrm{L} \\
\text { Compliance })\end{array}$ & \begin{tabular}{|c|} 
Required \\
Source \\
Cleanup \\
Percentage \\
(50 $\mathrm{\mu g} / \mathrm{L}$ \\
Compliance)
\end{tabular} & \begin{tabular}{|c} 
Required \\
Source \\
Cleanup \\
Percentage \\
(500 uglL \\
Compliance)
\end{tabular} & \begin{tabular}{|c|} 
Abiotic \\
Reaction \\
Half Time \\
(y)
\end{tabular} & $\begin{array}{c}\text { Carbon } \\
\text { Tetrachloride } \\
\text { Travel Time (y) }\end{array}$ \\
\hline & & & & & & & & & & & & & & & & & & & & & & \\
\hline 782 & $\begin{array}{l}45.55 \\
25.53 \\
\end{array}$ & 3000 & 2080 & \begin{tabular}{|l|}
342.3029424 \\
\end{tabular} & $\begin{array}{l}40.0299 \\
15.5114 \\
\end{array}$ & \begin{tabular}{|l|}
120.20009 \\
135.9447 \\
\end{tabular} & \begin{tabular}{|r|}
1055.08 \\
165.08 \\
\end{tabular} & 5125 & $\begin{array}{l}0.1 \\
0.1 \\
\end{array}$ & 1950 & 225000 & 17700000 & $\begin{array}{l}0.0044 \\
0.1246 \\
\end{array}$ & $\begin{array}{l}.30-04 \\
3.18 E-04 \\
\end{array}$ & $\begin{array}{l}2.075 \\
2.288 \\
\end{array}$ & \begin{tabular}{|l|}
$5.035-0$ \\
$4.91 E-03$ \\
\end{tabular} & $\begin{array}{l}60.42 \\
39.11 \\
\end{array}$ & $\begin{array}{l}99.444 \\
\end{array}$ & $\begin{array}{l}84.424 \\
85.39 \\
\end{array}$ & & $\begin{array}{l}141.3 \\
141.3 \\
\end{array}$ & 459.3 \\
\hline 783 & $\begin{array}{l}23.27 \\
23.27\end{array}$ & 3000 & 3350 & 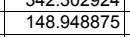 & 5.3881 & \begin{tabular}{|l|}
108.5219 \\
\end{tabular} & \begin{tabular}{|l|}
2073.31 \\
\end{tabular} & 5125 & 0.1 & 1950 & 225000 & 1212500 & $\begin{array}{l}0.1367 \\
0.1367\end{array}$ & $\begin{array}{l}.106-04 \\
5.96-04\end{array}$ & 3.205 & \begin{tabular}{|l|}
$4.39 \mathrm{E}-03$ \\
\end{tabular} & 16.37 & 96.64 & $\begin{array}{l}60.35 \\
66.43 \\
\end{array}$ & & 158.1 & 705.7 \\
\hline 784 & 43.76 & 3000 & 4150 & & 2.5295 & \begin{tabular}{|r|}
82.2476 \\
\end{tabular} & \begin{tabular}{|l|}
2735.64 \\
\end{tabular} & 5125 & 0.1 & 1950 & 225000 & 800000 & 0.0727 & 5.69E-04 & 4.958 & \begin{tabular}{|c|}
$6.39 \mathrm{E}-03$ \\
\end{tabular} & 19.76 & 94.58 & 45.81 & & 108.5 & 580.7 \\
\hline 785 & 35.71 & 3000 & 2190 & 311.08325 & 11.6366 & \begin{tabular}{|l|}
112.2199 \\
\end{tabular} & \begin{tabular}{|l|}
2004.99 \\
\end{tabular} & 5125 & 0.1 & 1950 & 225000 & 1275000 & 0.0891 & $2.23 \mathrm{E}-04$ & 2.264 & \begin{tabular}{|l|} 
\\
\end{tabular} & 54.35 & 98.39 & 83.93 & & 94.3 & 325 \\
\hline 786 & 59.35 & 3000 & 590 & \begin{tabular}{|c|}
1163.813207 \\
\end{tabular} & 51.6989 & \begin{tabular}{|l|}
133.2659 \\
\end{tabular} & 1688.35 & 5125 & 0.1 & 1950 & 225000 & 1650000 & 0.0536 & $4.83 \mathrm{E}-05$ & 1.455 & 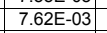 & 42.35 & 99.57 & 95.7 & 57.04 & 91 & 125.7 \\
\hline 787 & 39.82 & 3000 & 440 & \begin{tabular}{|l|}
1116.199483 \\
\end{tabular} & 55.4251 & \begin{tabular}{|l|l|}
148.9657 \\
\end{tabular} & 1510.42 & 5125 & 0.1 & 1950 & 225000 & 1950000 & 0.0799 & $3.06 \mathrm{E}-05$ & 1.194 & \begin{tabular}{|l|}
$6.47 \mathrm{E}-03$ \\
\end{tabular} & 25.69 & 99.55 & 95.52 & 55.21 & 107.1 & 153.7 \\
\hline 788 & 46.04 & & 1980 & 170.141566 & 8.1938 & \begin{tabular}{|l|l|}
144.4754 \\
\end{tabular} & 1557.36 & 5125 & 0.1 & & 225000 & 1862500 & 0.0691 & $2.85 \mathrm{E}-04$ & 3.087 & \begin{tabular}{|l|}
$8.80 \mathrm{E}-03$ \\
\end{tabular} & 31.47 & 97.06 & 70.61 & & 78.7 & 343.7 \\
\hline 789 & 23.92 & 3000 & 1750 & 600.139352 & 23.177 & \begin{tabular}{|l|l|}
115.8579 \\
\end{tabular} & 1942.03 & 5125 & 0.1 & 1950 & 225000 & 1337500 & 0.133 & \begin{tabular}{|c|}
$1.23 \mathrm{E}-04$ \\
\end{tabular} & 1.467 & \begin{tabular}{|l|}
$5.27 \mathrm{E}-03$ \\
\end{tabular} & $\begin{array}{ll}62.27 \\
\end{array}$ & $\begin{array}{l}99.17 \\
\end{array}$ & 91.67 & 16.69 & 131.6 & 314.3 \\
\hline 790 & 28.89 & 3000 & 870 & 905.120507 & 36.6764 & \begin{tabular}{|l|}
121.5631 \\
\end{tabular} & 1850.89 & 5125 & 0.1 & 1950 & 225000 & 1437500 & 0.1101 & $6.41 \mathrm{E}-05$ & 1.294 & \begin{tabular}{|l|}
$5.29 \mathrm{E}-03$ \\
\end{tabular} & 40.92 & 99.45 & 94.48 & & & 229.5 \\
\hline 791 & 21.69 & 3000 & 3270 & $\begin{array}{l}25.240247 \\
\end{array}$ & 0.8621 & \begin{tabular}{|l|l|}
102.4699 \\
\end{tabular} & \begin{tabular}{|l|}
2195.77 \\
\end{tabular} & 5125 & 0.1 & $\begin{array}{l}1950 \\
1950\end{array}$ & 225000 & 1112500 & 0.1467 & 7.44E-04 & 3.565 & $6.92 \mathrm{E}-03$ & $\begin{array}{l}69.43 \\
\end{array}$ & 80.19 & & 0 & & 842.5 \\
\hline 792 & 32.5 & 3000 & 2430 & $\begin{array}{l}4.956794 \\
2.289212\end{array}$ & 0.1914 & \begin{tabular}{|l|l|}
115.8579 \\
124369 \\
\end{tabular} & \begin{tabular}{|l|}
1942.03 \\
\end{tabular} & 5125 & 0.1 & $\begin{array}{l}1950 \\
1050 \\
1050\end{array}$ & 225000 & 1337500 & 0.0979 & 5.17E-04 & 3.668 & $1.53 \mathrm{E}-02$ & 84.24 & 0 & & & 45.2 & 578.5 \\
\hline 793 & $\begin{array}{r}22.36 \\
301 \\
\end{array}$ & $\begin{array}{l}3000 \\
3000 \\
3000\end{array}$ & 2300 & \begin{tabular}{|r|r|}
2.384212 \\
27618044 \\
\end{tabular} & $\begin{array}{l}0.0988 \\
1357 \\
\end{array}$ & \begin{tabular}{|l|l|}
124.3658 \\
1470497
\end{tabular} & \begin{tabular}{|l|l|}
1809.18 \\
15301
\end{tabular} & $\begin{array}{l}5125 \\
5125 \\
\end{array}$ & $\begin{array}{l}0.1 \\
0.1 \\
\end{array}$ & $\begin{array}{l}1950 \\
1950\end{array}$ & $\begin{array}{l}225000 \\
25000\end{array}$ & $\begin{array}{l}1487500 \\
191500\end{array}$ & $\begin{array}{l}0.1423 \\
0.107 \\
\end{array}$ & $\begin{array}{l}4.62 \mathrm{E}-04 \\
\end{array}$ & 2.642 & \begin{tabular}{|l|}
$1.42 \mathrm{E}-02$ \\
\end{tabular} & \begin{tabular}{|c|}
56.57 \\
5.78
\end{tabular} & $\begin{array}{r}0 \\
819\end{array}$ & 0 & & $\begin{array}{l}48.7 \\
65\end{array}$ & $\frac{605.8}{510.2}$ \\
\hline $\begin{array}{l}794 \\
795 \\
\end{array}$ & $\begin{array}{r}30.1 \\
41.05\end{array}$ & 3000 & $\begin{array}{l}2090 \\
2350\end{array}$ & $\begin{array}{r}2.6 .68044 \\
413.490917\end{array}$ & $\begin{array}{l}1.3534 \\
17.9951 \\
\end{array}$ & \begin{tabular}{|l|}
147.0497 \\
135.5599 \\
\end{tabular} & $\begin{array}{r}1750.1 \\
1723.35\end{array}$ & \begin{tabular}{|l}
5125 \\
5125
\end{tabular} & $\begin{array}{l}0.1 \\
0.1\end{array}$ & $\begin{array}{l}\frac{1950}{1950} \\
1950\end{array}$ & $\begin{array}{l}225000 \\
225000\end{array}$ & $\begin{array}{l}1912500 \\
1600000\end{array}$ & $\begin{array}{l}0.1057 \\
0.0775\end{array}$ & $\mid$ & 2.997 & \begin{tabular}{|l|}
$1.07 \mathrm{E}-02$ \\
$4.78 \mathrm{E}-03$ \\
\end{tabular} & \begin{tabular}{|c|c|}
68.78 \\
67.09
\end{tabular} & $\begin{array}{r}81.9 \\
98.79\end{array}$ & $\begin{array}{r}0 \\
87.91\end{array}$ & & $\begin{array}{r}65 \\
145 \\
\end{array}$ & 510.33 \\
\hline 796 & 26.6 & 3000 & 2320 & 12.000935 & 0.4548 & \begin{tabular}{|l|}
113.6821 \\
\end{tabular} & 1979.2 & 5125 & 0.1 & 1950 & 225000 & 1300000 & 0.1196 & $3.25 \mathrm{E}-04$ & 2.373 & \begin{tabular}{|l|l|} 
\\
\end{tabular} & 33.42 & 58.34 & & & 52.9 & 457.2 \\
\hline 797 & 24.89 & 3000 & 3940 & 10.176066 & 0.2672 & \begin{tabular}{|l|}
78.7839 \\
\end{tabular} & 2855.91 & 5125 & 0.1 & 1950 & 225000 & 750000 & 0.1278 & $7.43 \mathrm{E}-04$ & 3.94 & \begin{tabular}{|l|} 
\\
\end{tabular} & 49.68 & 50.87 & 0 & & 81.3 & 811.3 \\
\hline 798 & 28.3 & 3000 & 2670 & 362.660069 & 10.8542 & \begin{tabular}{|l|}
89.788 \\
\end{tabular} & 2505.9 & 5125 & 0.1 & 1950 & 225000 & 912500 & 0.1124 & $2.31 \mathrm{E}-04$ & 2.038 & 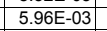 & 48.56 & 98.62 & 86.21 & & 116.3 & 369.1 \\
\hline 799 & 29.82 & 3000 & 1990 & 72.835074 & 3.8748 & \begin{tabular}{|l|l|}
159.6003 \\
\end{tabular} & 1409.77 & 5125 & 0.1 & 1950 & 225000 & 2162500 & 0.1067 & $4.30 \mathrm{E}-04$ & 3.036 & $8.28 \mathrm{E}-03$ & 76.41 & 93.14 & 31.35 & & 83.8 & 521.8 \\
\hline 800 & 22.82 & 3000 & 2460 & 155.566778 & 4.9902 & \begin{tabular}{|l|}
96.2335 \\
\end{tabular} & \begin{tabular}{|r|}
2338.06 \\
\end{tabular} & 5125 & 0.1 & 1950 & 225000 & 1012500 & 0.1394 & $2.01 \mathrm{E}-04$ & 1.727 & \begin{tabular}{|l|}
$8.17 \mathrm{E}-03$ \\
\end{tabular} & 70.53 & 96.79 & 67.86 & & 84.9 & 387.9 \\
\hline 801 & 36.11 & 3000 & 2570 & 74.184023 & 2.2807 & \begin{tabular}{|l|}
92.2314 \\
\end{tabular} & \begin{tabular}{|l|}
2439.52 \\
\end{tabular} & 5125 & 0.1 & 1950 & 225000 & 950000 & 0.0881 & $2.37 \mathrm{E}-04$ & 2.361 & \begin{tabular}{|c|}
$1.27 \mathrm{E}-02$ \\
\end{tabular} & 87.19 & 93.26 & 32.6 & & 54.7 & 335.1 \\
\hline 802 & 27.26 & 3000 & 2200 & 213.640821 & 12.8923 & \begin{tabular}{|l|l|}
181.037 \\
\end{tabular} & 1242.84 & 5125 & 0.1 & 1950 & 225000 & 2612500 & & $5.02 \mathrm{E}-04$ & 3.175 & & 61.63 & 97.66 & 76.6 & & 149 & 596.9 \\
\hline 803 & 46.58 & 3000 & 1710 & 130.189712 & 6.5472 & \begin{tabular}{|l|l|}
150.8694 \\
\end{tabular} & 1491.36 & 5125 & 0.1 & 1950 & 225000 & 1987500 & 0.0683 & $2.20 \mathrm{E}-04$ & 2.63 & $\mid 1.18 \mathrm{E}-02$ & 53.65 & $\begin{array}{l}96.16 \\
\end{array}$ & 61.59 & & 58.9 & 289.4 \\
\hline 804 & 29.21 & 3000 & 2770 & 74.335767 & 2.2854 & \begin{tabular}{|l|l|}
92.2314 \\
\end{tabular} & \begin{tabular}{|l|}
2439.52 \\
\end{tabular} & 5125 & 0.1 & 1950 & 225000 & 950000 & 0.1089 & $3.28 \mathrm{E}-04$ & 2.522 & $8.90 \mathrm{E}-03$ & 35.51 & 93.27 & 32.74 & & 77.9 & 442.5 \\
\hline $\begin{array}{l}805 \\
806 \\
\end{array}$ & $\begin{array}{l}31.62 \\
321\end{array}$ & 3000 & 2550 & \begin{tabular}{|l|l|}
440.937876 \\
596
\end{tabular} & $\begin{array}{l}14.6062 \\
210823 \\
\end{array}$ & \begin{tabular}{|l|l|}
99.3762 \\
\end{tabular} & \begin{tabular}{|l|}
2264.12 \\
\end{tabular} & 5125 & 0.1 & $\begin{array}{l}1950 \\
1050 \\
1050\end{array}$ & 225000 & 1062500 & $\begin{array}{l}0.1006 \\
\end{array}$ & 3.01E-04 & 2.511 & \begin{tabular}{|l|l|}
$4.99 \mathrm{E}-03$ \\
$.5-02$
\end{tabular} & $\begin{array}{l}62.73 \\
\end{array}$ & 98.87 & 88.66 & & 138.9 & 407 \\
\hline 806 & $\begin{array}{l}33.1 \\
501\end{array}$ & 3000 & $\begin{array}{l}1640 \\
1970\end{array}$ & \begin{tabular}{|l|}
586.870026 \\
$10^{3}$
\end{tabular} & $\begin{array}{l}21.0833 \\
43212\end{array}$ & \begin{tabular}{|l|l|}
107.7748 \\
1250616 \\
\end{tabular} & \begin{tabular}{|l|}
2087.69 \\
179911 \\
\end{tabular} & 5125 & 0.1 & $\begin{array}{l}1950 \\
1950\end{array}$ & 225000 & 1200000 & 0.0961 & $1.25 \mathrm{E}-04$ & 1.659 & \begin{tabular}{|c|}
$6.54 \mathrm{E}-03$ \\
\end{tabular} & 55.68 & 99.15 & 91.48 & 14.8 & 106 & 256.9 \\
\hline $\begin{array}{r}807 \\
808 \\
\end{array}$ & $\begin{array}{r}50.1 \\
31.22 \\
\end{array}$ & 3000 & \begin{tabular}{r|r|r|}
1970 \\
1810
\end{tabular} & $\begin{array}{r}103.969651 \\
46712642\end{array}$ & $\begin{array}{l}4.3342 \\
2.683 \\
\end{array}$ & \begin{tabular}{|c|}
125.0616 \\
1392566 \\
\end{tabular} & \begin{tabular}{|l|}
1799.11 \\
161572
\end{tabular} & $\begin{array}{l}5125 \\
5125 \\
\end{array}$ & $\begin{array}{l}0.1 \\
0.1\end{array}$ & $\begin{array}{l}1950 \\
1950 \\
\end{array}$ & $\begin{array}{l}225000 \\
225000\end{array}$ & $\begin{array}{l}1500000 \\
1762500\end{array}$ & $\begin{array}{l}0.0635 \\
01019\end{array}$ & $\begin{array}{l}1.95 \mathrm{E}-04 \\
25 \mathrm{~F}-04\end{array}$ & 2.551 & \begin{tabular}{|l|}
$1.35 \mathrm{E}-02$ \\
$130 \mathrm{E}-02$
\end{tabular} & $\begin{array}{r}30.5 \\
55114\end{array}$ & \begin{tabular}{|c|}
95.19 \\
893 \\
\end{tabular} & 51.91 & 0 & $\begin{array}{r}51.2 \\
5532 \\
\end{array}$ & 261 \\
\hline 809 & 32.1 & 3000 & 2600 & \begin{tabular}{|}
40.72042 \\
253.272917
\end{tabular} & $\begin{array}{l}2.1000 \\
7.6494 \\
\end{array}$ & \begin{tabular}{|r|}
159.20000 \\
90.6061 \\
\end{tabular} & \begin{tabular}{|l|}
10.12 \\
2483.28 \\
\end{tabular} & 5125 & $\begin{array}{l}0.1 \\
0.1\end{array}$ & 1950 & 2250000 & $\begin{array}{r}1 / 125000 \\
925000\end{array}$ & $\begin{array}{l}0.10991 \\
0.091\end{array}$ & $\begin{array}{l}2.25 \mathrm{E}-04 \\
1.99 \mathrm{E}-04\end{array}$ & $\begin{array}{l}2.118 \\
2.015\end{array}$ & \begin{tabular}{|l|l|}
$1.30 \mathrm{E}-02$ \\
$8.18 \mathrm{E}-03$ \\
\end{tabular} & $\begin{array}{l}51.14 \\
66.26\end{array}$ & $\begin{array}{r}89.3 \\
98.03 \\
\end{array}$ & $\begin{array}{r}0.26 \\
80 .\end{array}$ & & $\begin{array}{ll}53.2 \\
84.8 \\
\end{array}$ & 347.7 \\
\hline 810 & 44.56 & 3000 & 2230 & 41.532914 & 1.8543 & \begin{tabular}{|l|}
133.9381 \\
\end{tabular} & \begin{tabular}{|l|}
1679.88 \\
\end{tabular} & 5125 & 0.1 & 1950 & 225000 & 1662500 & 0.0714 & $3.29 \mathrm{E}-04$ & 3.329 & \begin{tabular}{|l|l|}
$1.18 \mathrm{E}-02$ \\
\end{tabular} & 20.86 & 87.96 & 0 & & 58.6 & 382.9 \\
\hline 811 & 40.79 & 3000 & 3000 & 83.020431 & 3.7065 & \begin{tabular}{|l|}
133.9381 \\
\end{tabular} & \begin{tabular}{|l|}
1679.88 \\
\end{tabular} & 5125 & 0.1 & 1950 & 225000 & 1662500 & 0.078 & $6.43 \mathrm{E}-04$ & 5.167 & \begin{tabular}{|l|}
$6.41 \mathrm{E}-03$ \\
\end{tabular} & 68.81 & 93.98 & 39.77 & & 108.2 & 649.2 \\
\hline 812 & 24.4 & 3000 & 1410 & 304.879955 & 18.5154 & \begin{tabular}{|l|}
182.1901 \\
\end{tabular} & \begin{tabular}{|l|}
1234.97 \\
\end{tabular} & 5125 & 0.1 & 1950 & 225000 & 2637500 & 0.1304 & $1.55 \mathrm{E}-04$ & 1.602 & $6.96 \mathrm{E}-03$ & 35.18 & 98.36 & 83.6 & & 99.5 & 336.6 \\
\hline 813 & 35.59 & 3000 & 620 & 734.766818 & 42.7702 & \begin{tabular}{|l|l|}
174.6277 \\
\end{tabular} & \begin{tabular}{|l|}
1288.46 \\
\end{tabular} & 5125 & 0.1 & 1950 & 225000 & 2475000 & 0.0894 & $5.48 \mathrm{E}-05$ & 1.31 & $7.53 \mathrm{E}-03$ & 27.69 & 99.32 & 93.2 & 31.95 & 92 & 188.6 \\
\hline 814 & 26.91 & 3000 & 2930 & 51.437735 & 2.8513 & \begin{tabular}{|l|l|}
166.2959 \\
\end{tabular} & 1353.01 & 5125 & 0.1 & 1950 & 225000 & 2300000 & 0.1182 & $7.07 \mathrm{E}-04$ & 4.022 & $5.75 \mathrm{E}-03$ & 51.65 & 90.28 & 2.8 & & 120.5 & 765.8 \\
\hline 815 & 33.17 & 3000 & 1790 & 219.845807 & & \begin{tabular}{|l|}
93.0387 \\
\end{tabular} & \begin{tabular}{|l|}
2418.35 \\
\end{tabular} & 5125 & 0.1 & 1950 & 225000 & & 0.0959 & 1.70E-04 & 1.894 & & 38.9 & 97.73 & 77.26 & & 74.8 & 292.6 \\
\hline 816 & 57.12 & 3000 & 1710 & 229.106184 & & \begin{tabular}{|l|l|}
154.0159 \\
\end{tabular} & \begin{tabular}{|l|}
1460.89 \\
\end{tabular} & 5125 & 0.1 & & & & & $2.21 \mathrm{E}-04$ & & & 51.85 & $\begin{array}{l}97.82 \\
\end{array}$ & 78.18 & & 67.3 & 269.3 \\
\hline 817 & 21.29 & 3000 & 2460 & 41.4 & 1.3418 & \begin{tabular}{|l|}
97.024 \\
\end{tabular} & 2319.01 & 5125 & 0.1 & 1950 & 225000 & 1025000 & 0.1494 & $2.38 \mathrm{E}-04$ & 1.804 & \begin{tabular}{|l|}
$1.11 \mathrm{E}-02$ \\
\end{tabular} & 84.89 & 87.95 & 0 & 0 & 62.3 & 434.1 \\
\hline 818 & 55.62 & 3000 & 2710 & 536.419945 & 15.7601 & \begin{tabular}{|r|}
88.1405 \\
\end{tabular} & \begin{tabular}{|l|}
2552.74 \\
\end{tabular} & 5125 & 0.1 & 1950 & 225000 & 887500 & & $2.13 \mathrm{E}-04$ & 2.885 & $6.83 \mathrm{E}-03$ & 57.15 & & 90.68 & 6.79 & 101.4 & 265.8 \\
\hline 819 & 23.76 & $\begin{array}{l}3000 \\
3000\end{array}$ & \begin{tabular}{|l|l|l|}
1610 \\
2770
\end{tabular} & & 12.5903 & \begin{tabular}{|l|l|}
152.7612 \\
115139 \\
\end{tabular} & \begin{tabular}{|l|l|}
1472.89 \\
1954.23 \\
\end{tabular} & $\begin{array}{l}5125 \\
5125 \\
\end{array}$ & 0.1 & & 225000 & $\begin{array}{l}2025000 \\
122000\end{array}$ & & $1.54 \mathrm{E}-04$ & 1.582 & & $\begin{array}{r}62.3 \\
\end{array}$ & 97.98 & 79.78 & & 90.4 & 341.3 \\
\hline 820 & $\begin{array}{r}32.33 \\
35\end{array}$ & 3000 & \begin{tabular}{ll|l|l|}
2770 & \\
2120 &
\end{tabular} & $\begin{array}{r}114.977251 \\
\end{array}$ & \begin{tabular}{r|}
4.4126 \\
317524
\end{tabular} & \begin{tabular}{|l|}
115.1349 \\
1122199 \\
\end{tabular} & \begin{tabular}{|l|}
1954.23 \\
\end{tabular} & 5125 & 0.1 & $\begin{array}{l}1950 \\
1950\end{array}$ & 225000 & 1325000 & & 4.64E-04 & 3.385 & 6.5 & 32.88 & 95.65 & 56.51 & 0 & 106.5 & 536.5 \\
\hline $\begin{array}{l}822 \\
822 \\
\end{array}$ & $\begin{array}{r}35 \\
33.74 \\
\end{array}$ & $\begin{array}{l}3000 \\
3000\end{array}$ & $\begin{array}{l}2120 \\
1510\end{array}$ & $\begin{array}{r}848.843276 \\
132.13181\end{array}$ & $\begin{aligned} 31.7524 \\
6.0461\end{aligned}$ & \begin{tabular}{|l|}
112.2199 \\
137.2743 \\
\end{tabular} & \begin{tabular}{|l|}
2004.99 \\
1639.05 \\
\end{tabular} & $\begin{array}{l}\mid 5125 \\
5125\end{array}$ & $\begin{array}{l}0.1 \\
0.1\end{array}$ & $\frac{19}{19}$ & $\frac{2250}{2250}$ & $\begin{array}{l}1275000 \\
1725000\end{array}$ & $\mid \begin{array}{l}0.0099 \\
0.0943\end{array}$ & $\begin{array}{l}\frac{1.78 E \mathrm{E}-04}{1.57 \mathrm{E}-04} \\
\end{array}$ & $\begin{array}{r}1.99 \\
1.839\end{array}$ & \begin{tabular}{|l|}
$4.51 \mathrm{E}-03$ \\
$1.16 \mathrm{E}-02$ \\
\end{tabular} & \begin{tabular}{r|r|r}
33.64 & \\
33
\end{tabular} & $\begin{array}{l}99.41 \\
96.22\end{array}$ & $\begin{array}{l}94.11 \\
62.16\end{array}$ & $\begin{aligned} 41.1 \\
0\end{aligned}$ & $\begin{array}{r}153.7 \\
59.8 \\
\end{array}$ & $\frac{291.5}{279.4}$ \\
\hline 823 & 25.31 & 3000 & 1350 & 46.236 & & \begin{tabular}{|l|}
113.6821 \\
\end{tabular} & & 5125 & 0.1 & & 225000 & & & $1.21 \mathrm{E}-04$ & 1.487 & \begin{tabular}{|l|}
1.47 \\
\end{tabular} & 47.59 & 89.19 & & & 47.3 & 301.1 \\
\hline 824 & 37.6 & 3000 & 1410 & 864.785924 & 44.9367 & \begin{tabular}{|l|}
155.8885 \\
\end{tabular} & 1443 & 5125 & 0.1 & 195 & 225000 & 2087500 & 0.0 & $8.99 \mathrm{E}-05$ & 1.537 & \begin{tabular}{|l|}
$6.11 \mathrm{E}-03$ \\
\end{tabular} & 76.18 & 99.42 & 94.22 & 42.18 & 113.5 & 209.5 \\
\hline 825 & 52.24 & & 3350 & & 0.3435 & \begin{tabular}{|l|}
148.9657 \\
\end{tabular} & 1510 & 5125 & 0.1 & 1950 & & & 0.0 & $6.06 \mathrm{E}-04$ & 6.029 & & 24.9 & 27.72 & & & 59.2 & 591.5 \\
\hline 826 & & 3000 & 2280 & 11.49 & & \begin{tabular}{|l|}
150.2362 \\
\end{tabular} & 1497 & 5125 & 0.1 & 1950 & & & & $5.68 \mathrm{E}$ & 5.3 & & 86.65 & 56.52 & & & 48.3 & 569.7 \\
\hline 827 & 63.5 & 3000 & 2840 & 32.79 & & \begin{tabular}{|l|l|}
125.0616 \\
\end{tabular} & 1799 & 5125 & 0.1 & 1950 & 225000 & 1500000 & & 4.95 & 5.995 & & 49.45 & 84.75 & & & 60.1 & 483.9 \\
\hline 828 & 52.67 & 3000 & 1390 & 300.5 & 17.7306 & 176.9 & 127 & 5125 & 0.1 & 1950 & 2250 & & & 1.48 & 2.236 & & 55.31 & 98.34 & 83.36 & & 62.1 & 217.6 \\
\hline 829 & 32.66 & 3000 & 2930 & 90.6 & $\begin{array}{l}2.5628 \\
2025\end{array}$ & & & & 0.1 & & & & & & & & & 94.49 & 44.85 & & 75.4 & 400.9 \\
\hline 830 & 21.66 & 3000 & 2090 & 605.6 & & & & 5125 & 0.1 & 19 & 225 & & & 4.02 & 2.3 & & 69. & 99.17 & 91.74 & 17.45 & 233.7 & 563.7 \\
\hline 831 & 25.91 & 3000 & 2820 & & $\begin{array}{l}2.8098 \\
\end{array}$ & 122.9 & 182 & 5125 & 0.1 & 1950 & 225000 & 1462500 & $\begin{array}{l}0.1228 \\
\end{array}$ & 5.50 & 3.266 & $6.38 \mathrm{E}-03$ & 38. & 92.71 & $\begin{array}{ll}27.06 \\
\end{array}$ & & 108.7 & 646.1 \\
\hline 832 & \begin{tabular}{|l|l|}
43.7 \\
31.1
\end{tabular} & & 600 & & 43.7359 & & 1543 & 5125 & & & & & & & & & & $\begin{array}{l}99.44 \\
\end{array}$ & 94.45 & 44.45 & 96.5 & 168.9 \\
\hline$\frac{833}{834}$ & $\begin{array}{r}31.1 \\
60.71 \\
\end{array}$ & $\begin{array}{l}3000 \\
3000\end{array}$ & $\begin{aligned} 2070 \\
2380\end{aligned}$ & \begin{tabular}{|r|}
63.668044 \\
469.285555 \\
\end{tabular} & \begin{tabular}{r|r|}
2.54999 \\
16.8592
\end{tabular} & \begin{tabular}{|c|}
120.1495 \\
10.7748 \\
\end{tabular} & \begin{tabular}{|l|}
$\mid 1872.67$ \\
2887.69
\end{tabular} & $\begin{array}{l}5125 \\
5125\end{array}$ & $\begin{array}{l}0.1 \\
0.1\end{array}$ & $\begin{array}{l}1950 \\
1950 \\
\end{array}$ & $\frac{225000}{225000}$ & $\begin{array}{l}1412500 \\
1200000\end{array}$ & $\begin{array}{l}0.1023 \\
0.0524 \\
\end{array}$ & $\begin{array}{l}2.32 \mathrm{E}-04 \\
278 \mathrm{E}-04\end{array}$ & $\frac{2.147}{368}$ & \begin{tabular}{|l|}
$1.13 \mathrm{E}-02$ \\
$6.59 \mathrm{E}-03$ \\
\end{tabular} & $\begin{array}{l}25.16 \\
78.25\end{array}$ & $\begin{array}{c}92.15 \\
98.93\end{array}$ & $\begin{array}{l}21.47 \\
89.35 \\
\end{array}$ & & $\begin{array}{r}61.2 \\
105.2\end{array}$ & 353.9 \\
\hline 835 & 31.78 & 3000 & 1630 & \begin{tabular}{|l|}
227.659983 \\
\end{tabular} & 11.4969 & \begin{tabular}{|l|}
151.5013 \\
\end{tabular} & \begin{tabular}{|l|}
1485.14 \\
\end{tabular} & 5125 & 0.1 & 1950 & 225000 & 2000 & 0.1001 & $2.30 \mathrm{E}-04$ & 2.16 & \begin{tabular}{|l|} 
\\
\end{tabular} & 12.55 & 97.8 & 78.04 & & $\begin{array}{r}92.4 \\
92\end{array}$ & 348.3 \\
\hline 836 & 23.19 & 3000 & \begin{tabular}{|l|l|}
1730 \\
\end{tabular} & 114.620644 & 5.194 & \begin{tabular}{|l|}
135.9447 \\
\end{tabular} & \begin{tabular}{|l|}
1655.08 \\
\end{tabular} & 5125 & 0.1 & 19 & 2250 & 1700 & 0.1372 & $1.29 \mathrm{E}-04$ & 1.475 & \begin{tabular}{|l|}
$1.07 \mathrm{E}-02$ \\
\end{tabular} & 70.92 & 95.64 & 56.38 & & 64.9 & 326 \\
\hline 837 & 25.72 & 3000 & 1230 & 76.26 & 4.073 & 160.21 & 1404 & 5125 & 0.1 & 1950 & 225000 & 2175000 & 0.1 & $9.77 \mathrm{E}$ & 1.399 & $1.39 !$ & 52.49 & 93.44 & 34.44 & & 50 & 278.8 \\
\hline 838 & 40.53 & & 4410 & 16.336 & 0.3542 & \begin{tabular}{|l|}
65.0346 \\
\end{tabular} & 3459 & 5125 & 0.1 & 1950 & 225000 & & 0.0 & $5.58 \mathrm{E}$ & 4.5 & 1.0 & 25.54 & 69.39 & 0 & & 69 & 580.8 \\
\hline 839 & 38.99 & & 2640 & & & 91.4 & & 512 & 0.1 & & & & & 2.46 & & & & 98.26 & 82.64 & & 91.7 & 331.7 \\
\hline 840 & & & 2070 & 475.5 & 21.6 & 136.6 & 164 & 5125 & 0.1 & 195 & & & & & 2.4 & & 45.73 & 98.95 & 89.49 & & 155.2 & 429.4 \\
\hline 841 & 28. & 3000 & 2220 & & & & 171 & 5125 & 0.1 & 1950 & 225000 & & & & 2.658 & & 33. & 87.99 & 0 & & 71.9 & 477 \\
\hline 842 & & & 2200 & & & & & & 0.1 & & & & & & & & & 89.43 & & & 63.9 & 22.8 \\
\hline 843 & & 30 & 920 & 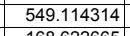 & & \begin{tabular}{|l|l|}
150.2362 \\
107025
\end{tabular} & \begin{tabular}{|l|}
1497.64 \\
17021
\end{tabular} & & 0.1 & & & & & $9.86 \mathrm{E}-05$ & 1.744 & & 28.34 & 99.09 & 90.89 & 8.94 & 75.6 & 188.2 \\
\hline$\frac{844}{845}$ & $\begin{array}{l}25.07 \\
29.98\end{array}$ & 3000 & $\begin{array}{r}910 \\
2570\end{array}$ & \begin{tabular}{|r|}
168.6226655 \\
22096173
\end{tabular} & $\begin{array}{l}7.1848 \\
0.847\end{array}$ & \begin{tabular}{|c|}
127.8255 \\
1144096 \\
\end{tabular} & \begin{tabular}{|l|}
$\mid 1760.21$ \\
1966.62
\end{tabular} & \begin{tabular}{|l|r|}
5125 \\
5125 \\
\end{tabular} & $\begin{array}{l}0.1 \\
0.1\end{array}$ & $\begin{array}{l}1950 \\
1950\end{array}$ & $\begin{array}{l}225000 \\
225000\end{array}$ & $\begin{array}{l}1550000 \\
1312500\end{array}$ & $\begin{array}{l}0.1269 \\
0.1061\end{array}$ & $\begin{array}{l}1.14 \mathrm{E}-04 \\
5.37 \mathrm{E}-04\end{array}$ & $\begin{array}{l}1.454 \\
3.558\end{array}$ & \begin{tabular}{|l|}
$9.82 \mathrm{E}-03$ \\
$104 \mathrm{E}-02$
\end{tabular} & $\begin{array}{l}\mid 16.63 \\
84.02\end{array}$ & \begin{tabular}{|c|c|}
97.03 & \\
77.37 &
\end{tabular} & $\begin{array}{r}70.35 \\
0\end{array}$ & & $\begin{array}{l}70.6 \\
66.9 \\
\end{array}$ & $\frac{297.2}{6082}$ \\
\hline
\end{tabular}




\begin{tabular}{|c|c|c|c|c|c|c|c|c|c|c|c|c|c|c|c|c|c|c|c|c|c|c|}
\hline $\begin{array}{c}\text { Realizatio } \\
n\end{array}$ & $\begin{array}{c}\text { Velocity } \\
(\mathrm{m} / \mathrm{y})\end{array}$ & $\begin{array}{c}\text { Fixed Source } \\
\text { Concentration } \\
(\mathrm{mg} / \mathrm{L})\end{array}$ & $\begin{array}{c}\text { Time of } \\
\text { Maximum at } \\
\text { Compliance } \\
\text { Boundary } \\
(y)\end{array}$ & $\begin{array}{c}\text { Maximum } \\
\begin{array}{c}\text { Concentration } \\
\text { Compliance } \\
\text { Boundary } \\
(\mu \mathrm{g} / \mathrm{L})\end{array} \\
\end{array}$ & $\begin{array}{c}\text { Maximum } \\
\text { Release Rate } \\
\text { at Compliance } \\
\text { Boundary } \\
(\mathrm{Kg} / \mathrm{y})\end{array}$ & \begin{tabular}{|c|} 
Source \\
Rate \\
(Kg/y) \\
\end{tabular} & \begin{tabular}{|c|} 
Source \\
Duration \\
$(y)$
\end{tabular} & $\begin{array}{c}\text { 1-D } \\
\text { Streamtub } \\
\text { e Length } \\
(\mathrm{m})\end{array}$ & $\begin{array}{c}\text { Base } \\
\text { Porosity } \\
\left(\mathrm{m}^{3} / \mathrm{m}^{3}\right) \\
\end{array}$ & $\begin{array}{c}\text { Bulk } \\
\text { Densty } \\
\left(\mathrm{Kg} / \mathrm{m}^{3}\right)\end{array}$ & \begin{tabular}{|c|} 
Remaining \\
Carbon \\
Tetrachloride \\
Source \\
$(\mathrm{Kg})$
\end{tabular} & $\begin{array}{c}\text { Volume at or } \\
\text { above Fixed } \\
\text { Source } \\
\begin{array}{c}\text { Concentration } \\
(\mathrm{m} 3)\end{array} \\
\end{array}$ & $\begin{array}{l}\text { Porosity } \\
\left(\mathrm{m}^{3} / \mathrm{m}^{3}\right)\end{array}$ & $\begin{array}{c}\mathrm{Kd} \\
\left(\mathrm{m}^{3} / \mathrm{Kg}\right)\end{array}$ & Retardation & $\begin{array}{c}\text { First Order } \\
\text { Rate } \\
\text { Constant } \\
(1 / y)\end{array}$ & $\begin{array}{c}\text { Longitudinal } \\
\text { Dispersivity } \\
(\mathrm{m})\end{array}$ & $\begin{array}{c}\text { Required } \\
\text { Source } \\
\text { Cleanup } \\
\text { Percentage } \\
(5 \mu \mathrm{g} / \mathrm{L} \\
\text { Compliance) } \\
\end{array}$ & \begin{tabular}{|c|} 
Required \\
Source \\
Cleanup \\
Percentage \\
(50 gg/L \\
Compliance)
\end{tabular} & \begin{tabular}{|c} 
Required \\
Source \\
Cleanup \\
Percentage \\
(500 ug/L \\
Compliance) \\
\end{tabular} & $\begin{array}{c}\text { Abiotic } \\
\text { Reaction } \\
\text { Half Time } \\
\text { (y) }\end{array}$ & $\begin{array}{c}\text { Carbon } \\
\text { Tetrachloride } \\
\text { Travel Time (y) }\end{array}$ \\
\hline $\begin{array}{l}846 \\
847 \\
\end{array}$ & \begin{tabular}{|l|}
21.94 \\
24.03 \\
\end{tabular} & \begin{tabular}{|r|}
3000 \\
3000 \\
\end{tabular} & \begin{tabular}{|r|}
1510 \\
3190 \\
\end{tabular} & \begin{tabular}{|r|r|}
577.504039 \\
\end{tabular} & \begin{tabular}{|r|}
32.2437 \\
\end{tabular} & \begin{tabular}{|l|}
167.4988 \\
103994 \\
\end{tabular} & \begin{tabular}{|r|}
1343.29 \\
2163.47
\end{tabular} & \begin{tabular}{|r|}
5125 \\
5125 \\
\end{tabular} & $\begin{array}{l}0.1 \\
0.1\end{array}$ & $\begin{array}{l}1950 \\
1950\end{array}$ & \begin{tabular}{|r|}
225000 \\
225000
\end{tabular} & \begin{tabular}{|l|}
2325000 \\
137500
\end{tabular} & $\begin{array}{r}0.145 \\
0.1324\end{array}$ & \begin{tabular}{|l|l|}
$1.58 \mathrm{E}-04$ \\
$5.93-04$
\end{tabular} & $\begin{array}{r}1.55 \\
3.263 \\
\end{array}$ & \begin{tabular}{|l|l|}
$4.61 \mathrm{E}-03$ \\
$836-03$
\end{tabular} & \begin{tabular}{|r|}
28.12 \\
29.98
\end{tabular} & $\begin{array}{r}99.13 \\
\end{array}$ & 91.34 & & \begin{tabular}{|l|}
150.2 \\
89
\end{tabular} & $\begin{array}{r}362.1 \\
696\end{array}$ \\
\hline $\begin{array}{l}841 \\
848 \\
\end{array}$ & $\begin{array}{l}24.00 \\
31.85 \\
\end{array}$ & 3000 & 2300 & $\begin{array}{c}10.202020 \\
180.985745 \\
\end{array}$ & $\begin{array}{l}0.527 \\
9.4045 \\
\end{array}$ & \begin{tabular}{|l|}
155.88845 \\
155.8
\end{tabular} & $\begin{array}{l}2100.434 \\
1443.34\end{array}$ & $\mid \begin{array}{l}5125 \\
5125\end{array}$ & $\begin{array}{l}0.1 \\
0.1\end{array}$ & 1950 & 2250000 & 2087500 & $\begin{array}{l}0.1524 \\
0.0999\end{array}$ & $\begin{array}{l}.953-04 \\
4.79 E-04\end{array}$ & $\begin{array}{l}3.203 \\
3.422 \\
\end{array}$ & \begin{tabular}{|l|l|}
$5.50 \mathrm{E}-\mathrm{U}-03$ \\
$5 . \mathrm{E}-3$
\end{tabular} & $\begin{array}{r}r 9.90 \\
60.5 \\
\end{array}$ & 97.24 & $\begin{array}{r}72.37 \\
\end{array}$ & & $\begin{array}{r}82.9 \\
125 \\
\end{array}$ & $\frac{696}{5507}$ \\
\hline 849 & 23.97 & 3000 & 3210 & 15.314406 & 0.6133 & \begin{tabular}{|l|}
120.1495 \\
\end{tabular} & 1872.67 & 5125 & 0.1 & 1950 & 225000 & 1412500 & 0.1327 & $\begin{array}{l}-6.15-04 \\
6.62 E-04\end{array}$ & $\begin{array}{l}0.424 \\
3.522\end{array}$ & $\begin{array}{l}.74 \mathrm{E}-03 \\
\end{array}$ & 30.03 & $\begin{array}{l}97.44 \\
67.35\end{array}$ & 0 & & 89.5 & 753 \\
\hline 850 & 26.16 & 3000 & 1740 & 24.042715 & 1.1887 & \begin{tabular}{|l|}
148.3284 \\
\end{tabular} & 1516.9 & 5125 & 0.1 & 1950 & 225000 & 1937500 & 0.1216 & $2.51 \mathrm{E}-04$ & $\frac{0.024}{2.043}$ & \begin{tabular}{|l|l|}
$1.36 \mathrm{E}-02$ \\
\end{tabular} & 64.16 & $\frac{0.5}{79.2}$ & 0 & & $\begin{array}{l}51.1 \\
51.1\end{array}$ & 400.1 \\
\hline 851 & 32.97 & 3000 & 3080 & 173.862751 & 6.2893 & \begin{tabular}{|l|}
108.5219 \\
\end{tabular} & 2073.31 & 5125 & 0.1 & 1950 & 225000 & 1212500 & 0.0965 & $5.41 \mathrm{E}-04$ & 3.832 & $5.21 \mathrm{E}-03$ & 46.31 & 97.12 & 71.24 & & 133 & 595.8 \\
\hline 852 & 26.06 & 3000 & 2880 & 1.768696 & 0.0618 & \begin{tabular}{|l|}
104.7599 \\
\end{tabular} & 2147.77 & 5125 & 0.1 & 1950 & 225000 & 1150000 & 0.1221 & $5.99 \mathrm{E}-04$ & 3.481 & \begin{tabular}{|l|l|} 
& $1.38 \mathrm{E}-02$ \\
\end{tabular} & 53.38 & & & & 50.3 & 684.8 \\
\hline 853 & 24.62 & 3000 & 3450 & 13.749981 & 0.404 & \begin{tabular}{|l|}
88.1405 \\
\end{tabular} & 2552.74 & 5125 & 0.1 & 1950 & 225000 & 887500 & 0.1292 & $6.30 \mathrm{E}-04$ & 3.464 & \begin{tabular}{|l|l|} 
& $.72 \mathrm{E}-03$ \\
\end{tabular} & 45.94 & 63.64 & 0 & 0 & 79.5 & 720.9 \\
\hline 854 & 35.35 & 3000 & 1180 & 659.578841 & 28.8542 & \begin{tabular}{|l|}
131.239 \\
\end{tabular} & 1714.43 & 5125 & 0.1 & 1950 & 225000 & 1612500 & $\begin{array}{r}0.09 \\
\end{array}$ & $8.63 \mathrm{E}-05$ & 1.485 & $7.21 \mathrm{E}-03$ & 57.74 & 99.24 & 92.42 & 24.19 & 96.1 & 215.3 \\
\hline 855 & 43.88 & 3000 & 2700 & 73.479648 & 2.5659 & \begin{tabular}{|l|l|}
104.7599 \\
\end{tabular} & \begin{tabular}{|l|}
2147.77 \\
\end{tabular} & 5125 & 0.1 & 1950 & 225000 & 1150000 & 0.0725 & $3.55 \mathrm{E}-04$ & 3.476 & $9.66 \mathrm{E}-03$ & 22.88 & 93.2 & 31.95 & & 71.7 & 406 \\
\hline 856 & 30.41 & 3000 & 2120 & 623.002757 & 24.6559 & \begin{tabular}{|l|l|}
118.7276 \\
\end{tabular} & \begin{tabular}{|l|}
1895.09 \\
\end{tabular} & 5125 & 0.1 & 1950 & 225000 & 1387500 & 0.1046 & $2.29 \mathrm{E}-04$ & 2.108 & $4.54 \mathrm{E}-03$ & 40.48 & 99.2 & 91.97 & 19.74 & 152.7 & 355.3 \\
\hline 857 & 44.43 & 3000 & 1530 & 263.277286 & 12.7922 & \begin{tabular}{|l|l|}
145.7654 \\
\end{tabular} & 1543.58 & 5125 & 0.1 & 1950 & 225000 & 1887500 & 0.0716 & $1.33 \mathrm{E}-04$ & 1.937 & $\mid 1.14 \mathrm{E}-02$ & 45.85 & 98.1 & 81.01 & & 61.1 & 223.4 \\
\hline 858 & 36.95 & 3000 & 830 & 157.197651 & 4.2647 & \begin{tabular}{|l|l|}
81.3886 \\
\end{tabular} & 2764.52 & 5125 & 0.1 & 1950 & 225000 & 787500 & 0.0861 & 1.12E-04 & 1.655 & $1.31 \mathrm{E}-02$ & 16.14 & 96.82 & 68.19 & & 53.1 & 229.5 \\
\hline 859 & 61.77 & 3000 & 1750 & 212.57173 & 11.911 & \begin{tabular}{|l|l|}
168.0986 \\
\end{tabular} & 1338.5 & 5125 & 0.1 & 1950 & 225000 & 2337500 & 0.0515 & 2.97E-04 & 3.914 & $9.36 \mathrm{E}-03$ & 77.89 & 97.65 & 76.48 & & 74 & 324.7 \\
\hline 860 & 46.51 & 3000 & 1840 & 731.607653 & 23.2748 & \begin{tabular}{|l|l|}
95.4399 \\
\end{tabular} & 2357.51 & 5125 & 0.1 & 1950 & 225000 & 1000000 & 0.0684 & 1.31E-04 & 1.971 & \begin{tabular}{|l}
$6.70 \mathrm{E}-03$ \\
\end{tabular} & 57.4 & 99.32 & 93.17 & 31.66 & 103.5 & 217.2 \\
\hline 861 & \begin{tabular}{ll|l}
26.64 \\
2352
\end{tabular} & 3000 & 2350 & \begin{tabular}{|l|l}
498.460466 \\
\end{tabular} & 19.8453 & 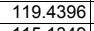 & 1883.8 & 5125 & 0.1 & 1950 & 225000 & 1400000 & 0.1194 & $\begin{array}{l}3.69 \mathrm{E}-04 \\
\end{array}$ & & $3.84 \mathrm{E}-03$ & 62.38 & 99 & 89.97 & & 180.3 & 492.9 \\
\hline $\begin{array}{l}862 \\
863 \\
\end{array}$ & $\begin{array}{l}23.53 \\
3.38 \\
\end{array}$ & $\begin{array}{l}3000 \\
3000\end{array}$ & $\begin{array}{l}1130 \\
1920\end{array}$ & \begin{tabular}{|l|l|}
91.875369 \\
51.51424 \\
\end{tabular} & $\begin{array}{r}3.526 \\
2.4356 \\
\end{array}$ & \begin{tabular}{|c|}
115.1349 \\
14.878 \\
\end{tabular} & \begin{tabular}{|c|}
1954.23 \\
1555.87
\end{tabular} & $\begin{array}{l}5125 \\
5125 \\
\end{array}$ & $\begin{array}{l}0.1 \\
0.1\end{array}$ & $\begin{array}{l}1950 \\
1950\end{array}$ & 225000 & $\begin{array}{l}1325000 \\
181500\end{array}$ & 0.1352 & $\begin{array}{l}9.29 \mathrm{E}-05 \\
272 \mathrm{E}\end{array}$ & 1.347 & $1.23 \mathrm{E}-02$ & $\begin{array}{l}41.41 \\
293 \\
\end{array}$ & $\begin{array}{l}94.56 \\
0.209\end{array}$ & $\begin{array}{ll}45.58 \\
292\end{array}$ & & 56.2 & 293.5 \\
\hline $\begin{array}{r}863 \\
864 \\
\end{array}$ & $\begin{array}{l}33.38 \\
2177 \\
\end{array}$ & $\begin{array}{l}3000 \\
3000 \\
3000\end{array}$ & $\begin{array}{l}1920 \\
2040 \\
\end{array}$ & \begin{tabular}{|l|l|}
51.501424 \\
50964416
\end{tabular} & \begin{tabular}{r|}
2.4356 \\
2125 \\
\end{tabular} & \begin{tabular}{|r|}
141.878 \\
125754 \\
\end{tabular} & \begin{tabular}{|l|}
1585.87 \\
178.19 \\
\end{tabular} & 5125 & 0.1 & $\begin{array}{l}1950 \\
1950\end{array}$ & $\begin{array}{ll}225000 \\
22500\end{array}$ & $\begin{array}{l}1812500 \\
151200\end{array}$ & 0.0953 & $\begin{array}{l}2.72 \mathrm{E}-04 \\
\end{array}$ & 2.442 & $1.15 \mathrm{E}-02$ & 29.3 & 90.29 & 2.92 & 0 & 60.5 & 374.9 \\
\hline 865 & 25.25 & $\begin{array}{l}3000 \\
3000\end{array}$ & $\begin{array}{l}2040 \\
1190\end{array}$ & $\begin{array}{r}50.649416 \\
231.410656\end{array}$ & $\begin{array}{r}2.125 \\
5.5242 \\
\end{array}$ & \begin{tabular}{|r|}
125.7554 \\
71.6152 \\
\end{tabular} & \begin{tabular}{|l|}
$\mid 1789.19$ \\
3141.79
\end{tabular} & $\begin{array}{l}5125 \\
5125\end{array}$ & $\frac{0.1}{0.1}$ & $\begin{array}{l}\frac{1950}{1950} \\
\end{array}$ & $\begin{array}{l}225000 \\
225000\end{array}$ & $\begin{array}{r}\frac{1512500}{650000} \\
6\end{array}$ & $\begin{array}{l}0.14161 \\
0.126\end{array}$ & $\begin{array}{l}2.8116-04 \\
1.36-04\end{array}$ & $\frac{1.971}{1.544}$ & \begin{tabular}{|l|}
$9.73 \mathrm{E}-03$ \\
$8.33 \mathrm{E}-03$ \\
\end{tabular} & $\frac{66.12}{24.71}$ & $\begin{array}{c}90.14 \\
97.84\end{array}$ & $\begin{array}{r}1.37 \\
78.39\end{array}$ & & $\begin{array}{r}71.4 \\
83.2\end{array}$ & $\frac{463.9}{313.5}$ \\
\hline 866 & 40.02 & 3000 & 2390 & 450.400686 & 20.2093 & \begin{tabular}{|l|}
134.6086 \\
\end{tabular} & \begin{tabular}{|l|}
1671.51 \\
\end{tabular} & 5125 & 0.1 & 1950 & 225000 & 1675000 & 0.0795 & $3.72 \mathrm{E}-04$ & 3.365 & $4.53 \mathrm{E}-03$ & 23.47 & 98.89 & 88.9 & & 153.1 & 431 \\
\hline 867 & 28.13 & 3000 & 2630 & $\begin{array}{r}4.31253 \\
\end{array}$ & 2.3026 & \begin{tabular}{|l|}
155.8885 \\
\end{tabular} & \begin{tabular}{|l|}
1443.34 \\
\end{tabular} & 5125 & 0.1 & 1950 & 225000 & 2087500 & 0.1131 & $5.45 \mathrm{E}-04$ & 3.437 & \begin{tabular}{|l|}
$7.22 \mathrm{E}-03$ \\
\end{tabular} & 28.74 & 88.72 & & & 96 & 626.2 \\
\hline 868 & 32.66 & 3000 & 2260 & 131.281795 & 6.7945 & \begin{tabular}{|c|}
155.2656 \\
\end{tabular} & 1449.13 & 5125 & 0.1 & 1950 & 225000 & 2075000 & 0.0974 & $4.09 \mathrm{E}-04$ & 3.123 & $6.66 \mathrm{E}-03$ & 22.86 & 96.19 & 61.91 & & 104.1 & \\
\hline 869 & 31.59 & 3000 & 1810 & 661.89038 & 30.5788 & \begin{tabular}{|l|l|}
138.5974 \\
\end{tabular} & 1623.41 & 5125 & 0.1 & 1950 & 225000 & 1750000 & 0.1007 & $2.09 \mathrm{E}-04$ & 2.048 & $4.77 \mathrm{E}-03$ & 79.96 & 99.24 & 92.45 & 24.46 & 145.4 & 332.2 \\
\hline 870 & 22.44 & 3000 & 1710 & 41.834579 & 1.9327 & \begin{tabular}{|l|l|}
138.5974 \\
\end{tabular} & \begin{tabular}{|l|}
1623.41 \\
\end{tabular} & 5125 & 0.1 & 1950 & 225000 & 1750000 & 0.1418 & $1.90 \mathrm{E}-04$ & 1.676 & $1.16 \mathrm{E}-02$ & 29.9 & 88.05 & & & 59.6 & 382.8 \\
\hline 871 & 24.83 & 3000 & 2710 & 2.609567 & 0.107 & \begin{tabular}{|l|l|}
122.9684 \\
\end{tabular} & \begin{tabular}{|l|}
1829.74 \\
\end{tabular} & 5125 & 0.1 & 1950 & 225000 & 1462500 & 0.1281 & 6.27E-04 & 3.473 & $1.23 \mathrm{E}-02$ & 53.1 & 0 & & & 56.3 & 716.8 \\
\hline 872 & $\begin{array}{ll}27.1 \\
\end{array}$ & 3000 & 1950 & 37.994084 & $\begin{array}{ll}1.772 \\
207\end{array}$ & \begin{tabular}{|l|l|}
139.9143 \\
\end{tabular} & \begin{tabular}{|l|}
1608.13 \\
\end{tabular} & 5125 & 0.1 & 1950 & 225000 & 1775000 & 0.1174 & $2.75 \mathrm{E}-04$ & 2.183 & $1.10 \mathrm{E}-02$ & 22 & 86.84 & & & 62.9 & 412.9 \\
\hline 873 & 27.96 & 3000 & 2590 & 65.6154 & 2.07 & \begin{tabular}{|l|}
94.6429 \\
\end{tabular} & \begin{tabular}{|l|}
2377.36 \\
\end{tabular} & 5125 & 0.1 & 1950 & 225000 & 987500 & 0.1138 & $2.76 \mathrm{E}-04$ & 2.226 & $1.01 \mathrm{E}-02$ & 47.73 & 92.38 & 23.8 & & 68.6 & 408.1 \\
\hline 874 & 27.5 & 3000 & 2470 & \begin{tabular}{|l|l|l|l}
5.40829 \\
\end{tabular} & 0.1847 & \begin{tabular}{|l|l|}
102.4699 \\
\end{tabular} & \begin{tabular}{|l|}
2195.77 \\
\end{tabular} & 5125 & 0.1 & 1950 & 225000 & 1112500 & 0.1157 & $3.20 \mathrm{E}-04$ & 2.398 & $1.59 \mathrm{E}-02$ & $\begin{array}{l}42.95 \\
\end{array}$ & 7.56 & 0 & & 43.5 & 447 \\
\hline $\begin{array}{l}875 \\
876 \\
\end{array}$ & \begin{tabular}{|l|}
26.44 \\
30.8 \\
\end{tabular} & $\begin{array}{l}3000 \\
3000\end{array}$ & $\begin{array}{l}1900 \\
1650\end{array}$ & $\begin{array}{r}1.129862 \\
451.42534 \\
\end{array}$ & \begin{tabular}{|c|c|}
0.0667 \\
20556
\end{tabular} & \begin{tabular}{|l|}
176.9718 \\
1366103 \\
\end{tabular} & \begin{tabular}{|c|}
1271.39 \\
164702
\end{tabular} & $\begin{array}{l}5125 \\
5125\end{array}$ & 0.1 & $\begin{array}{l}1950 \\
1950 \\
1950\end{array}$ & $\begin{array}{ll}225000 \\
22500\end{array}$ & \begin{tabular}{|l|l|}
2525000 \\
1712500
\end{tabular} & \begin{tabular}{|l|l|}
0.1203 \\
0.1033
\end{tabular} & $\begin{array}{l}5.05 \mathrm{E}-04 \\
175 \mathrm{E}\end{array}$ & $\begin{array}{r}3.12 \\
1956\end{array}$ & $1.70 \mathrm{E}-02$ & $\begin{array}{l}63.89 \\
2871\end{array}$ & & 0 & & 40.8 & 604.7 \\
\hline $\begin{array}{l}886 \\
877\end{array}$ & $\begin{array}{r}30.8 \\
23.85 \\
\end{array}$ & $\begin{array}{l}3000 \\
3000 \\
\end{array}$ & $\frac{1650}{2280}$ & $\begin{array}{l}4511.4325344 \\
145.879392\end{array}$ & \begin{tabular}{rl|}
20.5568 & \\
6.0475
\end{tabular} & \begin{tabular}{|l|}
$\mid 136.6103$ \\
124.3658 \\
\end{tabular} & \begin{tabular}{|l|}
$\mid 1647.02$ \\
1809.18
\end{tabular} & $\begin{array}{l}5125 \\
5125\end{array}$ & $\begin{array}{l}0.1 \\
0.1\end{array}$ & $\begin{array}{l}1950 \\
1950\end{array}$ & 225000 & $\begin{array}{l}1712500 \\
1487500\end{array}$ & \begin{tabular}{|c|}
0.1033 \\
0.1334
\end{tabular} & \begin{tabular}{|l|l|}
$.75 E-04$ \\
$3.46-04$
\end{tabular} & $\frac{1.856}{2.312}$ & \begin{tabular}{|l|l|}
$6.25 E-03$ \\
$6.32 E-03$ \\
\end{tabular} & $\begin{array}{l}28.71 \\
27.84\end{array}$ & $\begin{array}{l}98.89 \\
9.57\end{array}$ & \begin{tabular}{|c|c|}
88.92 \\
65.73
\end{tabular} & & \begin{tabular}{|l|l|l|}
110.9 \\
109.7
\end{tabular} & 308.9 \\
\hline 878 & 30.21 & 3000 & 1110 & $\begin{array}{l}50.101555 \\
\end{array}$ & 3.0427 & \begin{tabular}{|l|}
182.1901 \\
\end{tabular} & \begin{tabular}{|l|}
1234.97 \\
\end{tabular} & 5125 & 0.1 & 1950 & 225000 & 2637500 & $\begin{array}{l}0.1054 \\
0.1053\end{array}$ & $\begin{array}{l}.400-04 \\
1.00 \mathrm{E}-04\end{array}$ & $\begin{array}{l}.012 \\
1.482\end{array}$ & \begin{tabular}{|l|l|}
$.32 L-0$ \\
$.71 E-02$
\end{tabular} & $\begin{array}{l}\mid 74.24 \\
44.21\end{array}$ & $\begin{array}{l}0.0 .02 \\
90.02\end{array}$ & & & 40.5 & 251.4 \\
\hline 879 & 23.39 & 3000 & 1560 & 201.33633 & 10.5454 & \begin{tabular}{|l|}
157.1306 \\
\end{tabular} & \begin{tabular}{|l|}
1431.93 \\
\end{tabular} & 5125 & 0.1 & 1950 & 225000 & 2112500 & 0.136 & $1.36 \mathrm{E}-04$ & 1.507 & \begin{tabular}{|l|l|} 
& $8.52 \mathrm{E}-03$ \\
\end{tabular} & 51.22 & 97.52 & 75.17 & & 81.4 & 330.1 \\
\hline 880 & 57.42 & 3000 & 4130 & \begin{tabular}{|l|}
41.40948 \\
\end{tabular} & 0.9885 & \begin{tabular}{|l|}
71.6152 \\
\end{tabular} & \begin{tabular}{|l|}
3141.79 \\
\end{tabular} & 5125 & 0.1 & 1950 & 225000 & 650000 & 0.0554 & $5.49 \mathrm{E}-04$ & 6.007 & \begin{tabular}{|c|} 
\\
\end{tabular} & 44.62 & 87.93 & & & 71 & 536.1 \\
\hline 881 & 35.23 & 3000 & 1800 & 158.706034 & 7.6088 & \begin{tabular}{|l|}
143.8283 \\
\end{tabular} & \begin{tabular}{|l|}
1564.37 \\
\end{tabular} & 5125 & 0.1 & 1950 & 225000 & 1850000 & 0.0903 & $2.21 \mathrm{E}-04$ & 2.239 & $9.40 \mathrm{E}-03$ & 32.37 & 96.85 & 68.5 & & 73.7 & 325.7 \\
\hline 882 & 23.2 & & 2440 & 20.277693 & 0.8169 & \begin{tabular}{|l|}
120.8573 \\
\end{tabular} & \begin{tabular}{|l|}
1861.7 \\
\end{tabular} & 5125 & 0.1 & 1950 & 225000 & 1425000 & 0.1371 & $5.27 \mathrm{E}-04$ & 2.945 & $9.51 \mathrm{E}-03$ & 83.46 & 75.34 & & & 72.9 & 650.4 \\
\hline 883 & 56.51 & 3000 & 1750 & 126.033606 & 6.1777 & \begin{tabular}{|l|l|}
147.0497 \\
\end{tabular} & 1530.1 & 5125 & 0.1 & 1950 & 225000 & 1912500 & 0.0563 & $2.14 \mathrm{E}-04$ & 2.924 & & 48.97 & 96.03 & 60.33 & & 53.3 & 265.2 \\
\hline 884 & 44.19 & 3000 & 2870 & 1.230105 & 0.0566 & \begin{tabular}{|l|l|}
137.9366 \\
\end{tabular} & \begin{tabular}{|l|}
1631.18 \\
\end{tabular} & 5125 & 0.1 & 1950 & 225000 & 1737500 & 0.072 & 6.46E-04 & 5.536 & $1.65 \mathrm{E}-02$ & 44.3 & 0 & 0 & & 42.1 & 642.1 \\
\hline 885 & 24.59 & 3000 & 2580 & 24.280964 & 0.6447 & \begin{tabular}{|l|}
79.6568 \\
\end{tabular} & \begin{tabular}{|l|}
2824.62 \\
\end{tabular} & 5125 & 0.1 & & 225000 & & 0.1. & $2.98 \mathrm{E}-04$ & 2.164 & & 24.85 & 79.41 & 0 & & 61.8 & 451.1 \\
\hline 886 & 51.64 & 3000 & 2710 & 117.859 & & \begin{tabular}{|l|l|}
121.5 \\
1372
\end{tabular} & 1850 & 5125 & 0.1 & & & 1437500 & & & 4.648 & & 47.41 & 95.76 & 57.58 & & 87.6 & 461.2 \\
\hline 887 & 21.48 & 3000 & 2140 & 177.48 & 8.12 & 137.2 & 1639 & 5125 & 0.1 & 1950 & 225000 & 17250 & 0.1 & 3.90E-04 & 2.33 & 5.50 & 63.7 & & 71.83 & & 126 & 555.8 \\
\hline 888 & $\begin{array}{r}44 \\
2195\end{array}$ & & 2210 & 645.42 & & 1111.4852 & \begin{tabular}{|r|}
2018.2 \\
170.11
\end{tabular} & 5125 & 0.1 & 1950 & 225000 & & & $2.27 \mathrm{E}-04$ & 2.584 & $5.36 \mathrm{E}-03$ & $\begin{array}{ll}63.25 \\
2.52\end{array}$ & 99.23 & 92.25 & 22.53 & 129.4 & 301 \\
\hline $\begin{array}{l}889 \\
89 \\
\end{array}$ & 21.85 & $\begin{array}{l}3000 \\
3300\end{array}$ & 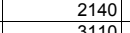 & 6.60673 & $\begin{array}{l}0.2754 \\
23713 \\
\end{array}$ & \begin{tabular}{|l|l|}
125.0616 \\
\end{tabular} & \begin{tabular}{|l|}
1799.11 \\
19453 \\
\end{tabular} & 5125 & 0.1 & & 225000 & 1500000 & 0.1456 & 3.25E-04 & 2.13 & & & 24.32 & 0 & & $\begin{array}{r}51.4 \\
110.7\end{array}$ & 499.6 \\
\hline $\begin{array}{r}899 \\
891\end{array}$ & $\begin{array}{l}23.48 \\
45.51\end{array}$ & $\begin{array}{l}3000 \\
3000\end{array}$ & $\begin{array}{l}3110 \\
1620\end{array}$ & $\begin{array}{r}61.78723 \\
11395437\end{array}$ & $\begin{array}{r}2.3713 \\
5.9453\end{array}$ & \begin{tabular}{|l|}
115.1349 \\
1565112 \\
\end{tabular} & \begin{tabular}{|l|}
1954.23 \\
14761
\end{tabular} & $\begin{array}{l}5125 \\
5125 \\
\end{array}$ & 0.1 & $\begin{array}{l}1950 \\
1950 \\
1950\end{array}$ & $\begin{array}{ll}225000 \\
22500\end{array}$ & 1325000 & 0.1355 & $\begin{array}{l}6.57 \mathrm{E}-04 \\
185 \mathrm{E}-04\end{array}$ & $\begin{array}{r}3.45 \\
2341\end{array}$ & $5.79 \mathrm{E}-03$ & \begin{tabular}{|c|c|}
51.59 \\
30.14
\end{tabular} & 91.91 & $\begin{array}{l}19.08 \\
5.12 \\
\end{array}$ & & \begin{tabular}{r|r|r|}
119.7 & \\
535 \\
\end{tabular} & 753.1 \\
\hline $\begin{array}{l}8911 \\
892 \\
\end{array}$ & $\begin{array}{l}45.51 \\
31.78 \\
\end{array}$ & 3000 & $\begin{array}{r}1620 \\
620\end{array}$ & \begin{tabular}{|l|}
136.959433 \\
\end{tabular} & $\begin{array}{l}5.94433 \\
7.7539 \\
\end{array}$ & \begin{tabular}{|l|}
156.5502 \\
125.0616 \\
\end{tabular} & \begin{tabular}{|l|}
173799.61 \\
1799.11 \\
\end{tabular} & $\begin{array}{l}5125 \\
5125\end{array}$ & $\begin{array}{l}0.1 \\
0.1\end{array}$ & $\begin{array}{l}\frac{1950}{1950} \\
\end{array}$ & 2250000 & $\begin{array}{l}21000000 \\
1500000\end{array}$ & \begin{tabular}{|l|}
0.06999 \\
0.1001
\end{tabular} & $\frac{1.85-5-04}{3.71 E-05}$ & $\frac{2.341}{1.187}$ & \begin{tabular}{|l|}
$1.30 \mathrm{E}-02$ \\
$1.49 \mathrm{E}-02$
\end{tabular} & $\begin{array}{l}30.14 \\
40.64\end{array}$ & $\begin{array}{l}99.61 \\
97.31 \\
\end{array}$ & $\begin{array}{l}56.12 \\
73.12\end{array}$ & & $\begin{array}{l}53.5 \\
46.5\end{array}$ & $\frac{263.6}{191.5}$ \\
\hline 893 & 54.57 & 30 & 1740 & \begin{tabular}{|l|}
1039.4295 \\
\end{tabular} & \begin{tabular}{|l|}
34.4315 \\
\end{tabular} & \begin{tabular}{|l|}
99.3762 \\
\end{tabular} & \begin{tabular}{|l|}
2264.12 \\
\end{tabular} & 5125 & 0.1 & 1950 & 225000 & 1062500 & 0.0583 & $1.19 \mathrm{E}-04$ & 2.031 & \begin{tabular}{|l|}
$5.69 \mathrm{E}-03$ \\
\end{tabular} & 59.02 & 99.52 & 95.19 & 51.9 & 121.7 & 190.7 \\
\hline 894 & 41.64 & 3000 & 1050 & \begin{tabular}{|r|}
250.129251 \\
\end{tabular} & 15.1423 & \begin{tabular}{|l|}
181.614 \\
\end{tabular} & \begin{tabular}{|l|}
1238.89 \\
\end{tabular} & 5125 & 0.1 & 1950 & 225000 & 2625000 & 0.0764 & $1.13 \mathrm{E}-04$ & 1.74 & $1.19 \mathrm{E}-02$ & 30.05 & 98 & 80.01 & & 58.4 & 214.7 \\
\hline 895 & 35.15 & & 2160 & \begin{tabular}{r|}
96.278985 \\
\end{tabular} & 3.625 & \begin{tabular}{|l|l|}
112.9522 \\
\end{tabular} & \begin{tabular}{|l|}
1991.99 \\
\end{tabular} & 5125 & 0.1 & & & & & $2.19 \mathrm{E}-04$ & 2.22 & $1.14 \mathrm{E}-02$ & 51.3 & 94.81 & 48.07 & & 60.6 & 323.9 \\
\hline 896 & 33.56 & & 2510 & & & 106.2 & & 512 & 0.1 & 1950 & & & 0.0948 & & 2.95 & & 43. & 31.7 & & & 45.7 & 456.8 \\
\hline 897 & 28.63 & 3000 & 2870 & 8.2 & & \begin{tabular}{|l|l|}
162.6609 \\
\end{tabular} & 1383 & 5125 & 0.1 & 1950 & 225000 & & & $6.40 \mathrm{t}$ & 3.9 & & 29.32 & 39.34 & & & 73.9 & 699.9 \\
\hline 898 & 23.43 & & 1780 & & & & & & 0.1 & 195 & & & & 2.71E-04 & 2.00 & & & 45.11 & & & 46.4 & 439.6 \\
\hline 899 & 33.31 & & 2480 & & & 140.5 & 1600 & & 0.1 & & & & & & & & 37. & & & & 65.3 & 553.9 \\
\hline 900 & 58.59 & 3000 & 3590 & $\begin{array}{r}4.564899 \\
\end{array}$ & 1.8784 & \begin{tabular}{|l|l|}
126.4473 \\
\end{tabular} & $\begin{array}{l}1779.4 \\
\end{array}$ & 5125 & 0.1 & 1950 & 225000 & 1525000 & 0.0543 & 7.49 & 7.973 & & 70. & 88.7 & 0 & & 90 & 697.4 \\
\hline 901 & 35.67 & & 2780 & $\begin{array}{l}195.232499 \\
\end{array}$ & $\begin{array}{l}6.4163 \\
\end{array}$ & \begin{tabular}{|l|l|}
98.5952 \\
770
\end{tabular} & \begin{tabular}{|l|}
2282.06 \\
\end{tabular} & 5125 & 0.1 & 1950 & & & 0.0892 & 3.52E-04 & 2.993 & & $\begin{array}{l}18.9 \\
\end{array}$ & 97.2. & 74.39 & & 105.9 & 430.2 \\
\hline 902 & 22.39 & 3000 & 1660 & $\begin{array}{r}203.41394 \\
\end{array}$ & 5.2824 & \begin{tabular}{|l|}
77.906 \\
91.701 \\
\end{tabular} & \begin{tabular}{|l|}
2888.09 \\
\end{tabular} & 5125 & 0.1 & & & 7375 & 0.1421 & $1.56 \mathrm{E}-04$ & 1.555 & & 40.44 & 97.54 & 75.42 & & 88.8 & 356 \\
\hline 903 & $\begin{array}{l}40.32 \\
3023\end{array}$ & 3000 & 3730 & 297.27764 & \begin{tabular}{|l|l|}
8.4029 \\
1130
\end{tabular} & \begin{tabular}{|r|r|}
84981 \\
\end{tabular} & \begin{tabular}{|l|}
2653.36 \\
\end{tabular} & 5125 & 0.1 & 1950 & 225000 & 837500 & 0.0789 & 5.82E-04 & 4.732 & $4.26 \mathrm{E}$ & $\begin{array}{l}60.55 \\
55.52 \\
\end{array}$ & 98.32 & 83.18 & & 162.9 & 601.4 \\
\hline $\begin{array}{r}904 \\
905 \\
\end{array}$ & $\begin{array}{l}30.33 \\
58.37 \\
\end{array}$ & $\begin{array}{l}3000 \\
3000\end{array}$ & $\begin{array}{l}1630 \\
2360\end{array}$ & \begin{tabular}{|r|}
22.162361 \\
1570.135516
\end{tabular} & $\begin{array}{r}1.1836 \\
54.8291\end{array}$ & \begin{tabular}{|l|}
160.2148 \\
104.7599 \\
\end{tabular} & \begin{tabular}{|l|}
1444.36 \\
2147.77 \\
\end{tabular} & $\begin{array}{l}5125 \\
5125\end{array}$ & $\begin{array}{l}0.1 \\
0.1\end{array}$ & $\begin{array}{l}1950 \\
1950\end{array}$ & $\frac{225000}{225000}$ & $\begin{array}{l}2175000 \\
1150000\end{array}$ & $\begin{array}{l}0.1049 \\
0.0545\end{array}$ & $\frac{2.4 \mathrm{E}-0-04}{2.13 \mathrm{E}-04}$ & $\frac{2.157}{2.973}$ & \begin{tabular}{|l|}
$.55 E-02$ \\
$2.52 E-03$ \\
\end{tabular} & $\begin{array}{l}56.52 \\
43.74\end{array}$ & $\begin{array}{ll}79.44 \\
99.68 \\
\end{array}$ & \begin{tabular}{r|}
0 \\
96.82
\end{tabular} & \begin{tabular}{r|}
0 \\
68.16
\end{tabular} & $\begin{array}{r}46.1 \\
274.9\end{array}$ & $\frac{364.5}{261}$ \\
\hline 906 & 38.28 & 300 & 2590 & \begin{tabular}{|r|}
208.219657 \\
\end{tabular} & \begin{tabular}{|l|}
7.6353 \\
\end{tabular} & \begin{tabular}{|l|}
110.0086 \\
\end{tabular} & 2045.3 & 5125 & 0.1 & 1950 & 2250 & 1237 & 0.0831 & $3.51 \mathrm{E}-04$ & 3.137 & \begin{tabular}{|l|l|}
$6.58 \mathrm{E}$ \\
\end{tabular} & 22.18 & $\begin{array}{l}97.6 \\
\end{array}$ & 75.99 & & 105.3 & 419.9 \\
\hline 907 & 30.71 & 3000 & 1050 & 302.202739 & 13.2203 & $\mid$\begin{tabular}{|l|}
131.239 \\
\end{tabular} & \begin{tabular}{|l|l|}
1714.43 \\
\end{tabular} & 5125 & 0.1 & 1950 & 225000 & 1612500 & 0.1036 & $8.69 \mathrm{E}$ & 1.424 & 9.93 & 43.85 & 98.35 & 83.45 & & 69.8 & 237.7 \\
\hline 908 & 26.25 & & 2390 & 130.443272 & 4.9 & 113.68 & 1979.2 & 5125 & 0.1 & 1950 & & & & $3.28 \mathrm{E}$ & 2.369 & 7.03 & 25.33 & $\begin{array}{l}96.17 \\
\end{array}$ & $|61.67|$ & & 98.6 & 462.6 \\
\hline 909 & 36.23 & 3000 & 3140 & \begin{tabular}{|l|}
0.820819 \\
\end{tabular} & $\begin{array}{l}0.0303 \\
\end{array}$ & 110.7481 & \begin{tabular}{|l|}
2031.64 \\
\end{tabular} & 5125 & 0.1 & 1950 & 225000 & 1250000 & 0.0878 & $6.00 \mathrm{E}-04$ & 4.455 & 1.58 & 30.22 & 0 & 0 & & 43.8 & 630.1 \\
\hline 910 & 37.96 & 3000 & 3130 & $\mid 488.930365$ & 13.4044 & 82.2476 & \begin{tabular}{|l|}
2735.64 \\
\end{tabular} & 5125 & 0.1 & 1950 & 225000 & 800000 & 0.0838 & 3.39E-04 & 3.045 & $4.58 \mathrm{E}-03$ & 34.03 & 98.98 & 89.77 & & 151.5 & 4111.1 \\
\hline
\end{tabular}




\begin{tabular}{|c|c|c|c|c|c|c|c|c|c|c|c|c|c|c|c|c|c|c|c|c|c|c|}
\hline $\begin{array}{c}\text { Realizatio } \\
n\end{array}$ & $\begin{array}{c}\text { Velocity } \\
(\mathrm{m} / \mathrm{y})\end{array}$ & $\begin{array}{c}\begin{array}{c}\text { Fixed Source } \\
\text { Concentration } \\
(\mathrm{mg} / \mathrm{L})\end{array}\end{array}$ & $\begin{array}{c}\text { Time of } \\
\text { Maximum at } \\
\text { Compliance } \\
\text { Boundary } \\
\text { (y) }\end{array}$ & $\begin{array}{c}\text { Maximum } \\
\text { Concentration } \\
\text { Compliance } \\
\text { Boundary } \\
(\mu \mathrm{g} / \mathrm{L})\end{array}$ & \begin{tabular}{|c|} 
Maximum \\
Release Rate \\
at Compliance \\
Boundary \\
(Kg/y)
\end{tabular} & $\begin{array}{c}\text { Source } \\
\text { Rate } \\
(\mathrm{Kg} / \mathrm{y})\end{array}$ & \begin{tabular}{|c|} 
Source \\
Duration \\
(y)
\end{tabular} & $\begin{array}{c}\text { 1-D } \\
\text { Streamtub } \\
\text { e Length } \\
\text { (m) }\end{array}$ & $\begin{array}{l}\text { Base } \\
\text { Porosity } \\
\left(\mathrm{m}^{3} / \mathrm{m}^{3}\right)\end{array}$ & $\begin{array}{l}\text { Bulk } \\
\text { Densty } \\
\left(\mathrm{Kg} / \mathrm{m}^{3}\right)\end{array}$ & $\begin{array}{c}\text { Remaining } \\
\text { Carbon } \\
\text { Tetrachloride } \\
\text { Source } \\
(\mathrm{Kg})\end{array}$ & $\begin{array}{c}\text { Volume at or } \\
\text { above Fixed } \\
\text { Source } \\
\begin{array}{c}\text { Concentration } \\
(\mathrm{m} 3)\end{array} \\
\end{array}$ & $\begin{array}{l}\text { Porosity } \\
\left(\mathrm{m}^{3} / \mathrm{m}^{3}\right)\end{array}$ & $\begin{array}{c}\mathrm{Kd} \\
\left(\mathrm{m}^{3} / \mathrm{Kg}\right)\end{array}$ & Retardation & $\begin{array}{c}\text { First Order } \\
\text { Rate } \\
\text { Constant } \\
\text { (1/y) }\end{array}$ & $\begin{array}{c}\text { Longitudinal } \\
\text { Dispersivity } \\
(\mathrm{m})\end{array}$ & $\begin{array}{c}\text { Required } \\
\text { Source } \\
\text { Cleanup } \\
\text { Percentage } \\
(5 \text { ug/L } \\
\text { Compliance) } \\
\end{array}$ & \begin{tabular}{|c|} 
Required \\
Source \\
Cleanup \\
Percentage \\
(50 g g/L/L \\
Compliance) \\
\end{tabular} & \begin{tabular}{|c|} 
Required \\
Source \\
Cleanup \\
Percentage \\
$(500 \mu \mathrm{gg} / \mathrm{L}$ \\
Compliance) \\
\end{tabular} & $\begin{array}{c}\text { Abiotic } \\
\text { Reaction } \\
\text { Half Time } \\
\text { (y) }\end{array}$ & $\begin{array}{c}\text { Carbon } \\
\text { Tetrachloride } \\
\text { Travel Time (y) }\end{array}$ \\
\hline & & 3000 & & & 0.2515 & $\begin{array}{l}69.767 \\
\end{array}$ & \begin{tabular}{|r|}
3225.02 \\
\end{tabular} & 5125 & 0.1 & & & $\begin{array}{r}625000 \\
\end{array}$ & 0.1012 & $6.85 \mathrm{E}-04$ & 4.421 & \begin{tabular}{|c|}
$1.08 \mathrm{E}-02$ \\
\end{tabular} & & 53.77 & & & & 720.7 \\
\hline 912 & 38.56 & 3000 & 3540 & 49.735507 & 1.5558 & 93.8425 & 2397.63 & 5125 & 0.1 & 1950 & 225000 & 975000 & 0.0825 & $5.23 \mathrm{E}-04$ & 4.205 & $7.79 \mathrm{E}-03$ & 18.44 & 89.95 & & & 89 & 558.9 \\
\hline 913 & 29.48 & 3000 & 2280 & 27.310058 & 0.9743 & 107.0251 & 2102.31 & 5125 & 0.1 & 1950 & 225000 & 1187500 & 0.1079 & $2.70 \mathrm{E}-04$ & 2.267 & $1.38 \mathrm{E}-02$ & 77.21 & 81.69 & 0 & & 50.1 & 394 \\
\hline 914 & 24.12 & 3000 & 2330 & 243.857533 & 9.2999 & 114.4096 & 1966.62 & 5125 & 0.1 & 1950 & 225000 & 1312500 & 0.1319 & $3.10 \mathrm{E}-04$ & 2.189 & $5.59 \mathrm{E}-03$ & 32.75 & 97.95 & 79.5 & & 124.1 & 465.1 \\
\hline 915 & 36.52 & 3000 & 1240 & 383.998231 & $\begin{array}{l}18.5754 \\
\end{array}$ & 145.1211 & 1550.43 & 5125 & 0.1 & 1950 & 225000 & 1875000 & 0.0871 & $1.12 \mathrm{E}-04$ & 1.653 & $9.12 \mathrm{E}-03$ & 42.67 & 98.7 & 86.98 & & 76 & 231.9 \\
\hline 916 & 27.69 & 3000 & 2100 & 232.481411 & 9.5835 & 123.6681 & 1819.39 & 5125 & 0.1 & 1950 & 225000 & 1475000 & 0.1149 & $2.59 \mathrm{E}-04$ & 2.14 & $6.67 \mathrm{E}-03$ & 30.95 & 97.85 & 78.49 & & 103.9 & 396.1 \\
\hline 917 & 22.55 & 3000 & 2660 & 548.415401 & 19.0116 & \begin{tabular}{|l|l|}
103.9994 \\
\end{tabular} & 2163.47 & 5125 & 0.1 & 1950 & 225000 & 1137500 & 0.1411 & $3.83 \mathrm{E}-04$ & 2.374 & $3.24 \mathrm{E}-03$ & 38.3 & 99.09 & 90.88 & 8.83 & 213.7 & 539.6 \\
\hline 918 & 48.5 & 3000 & 3410 & 29.111602 & 0.7898 & 81.3886 & 2764.52 & 5125 & 0.1 & 1950 & 225000 & 787500 & 0.0656 & $5.10 \mathrm{E}-04$ & 4.929 & $1.14 \mathrm{E}-02$ & 63.39 & 82.82 & & & 60.8 & 520.9 \\
\hline 919 & 40.79 & 3000 & 780 & 944.861707 & \begin{tabular}{ll|}
37.618 \\
\end{tabular} & \begin{tabular}{|l|l|}
119.4396 \\
\end{tabular} & \begin{tabular}{|l|l|}
1883.8 \\
\end{tabular} & 5125 & 0.1 & 1950 & 225000 & 1400000 & 0.078 & $7.20 \mathrm{E}-05$ & 1.467 & $6.34 \mathrm{E}-03$ & 35.12 & 99.47 & 94.71 & 47.08 & 109.3 & 184.3 \\
\hline 920 & 24.23 & 3000 & 2900 & $\begin{array}{l}16.923045 \\
20750271\end{array}$ & $\begin{array}{l}0.464 \\
\end{array}$ & \begin{tabular}{|l|}
82.2476 \\
\end{tabular} & 2735.64 & 5125 & 0.1 & 1950 & 225000 & 800000 & 0.1313 & $2.95 \mathrm{E}-04$ & 2.134 & $1.32 \mathrm{E}-02$ & 71.53 & 70.45 & & & 52.3 & 451.3 \\
\hline 921 & $\begin{array}{l}28.74 \\
3036\end{array}$ & $\begin{array}{l}3000 \\
3000 \\
3000\end{array}$ & $\begin{array}{l}1960 \\
2330 \\
230\end{array}$ & \begin{tabular}{|l|l|}
207.560371 \\
65088054
\end{tabular} & \begin{tabular}{|l|l|}
6.983 \\
2408 \\
\end{tabular} & \begin{tabular}{|r|}
100.929 \\
1107191 \\
\end{tabular} & $\begin{array}{l}2229.29 \\
20161\end{array}$ & $\frac{5125}{5125}$ & 0.1 & $\begin{array}{l}1950 \\
1950 \\
\end{array}$ & 225000 & 1087500 & 0.1107 & $\begin{array}{l}1.87 \mathrm{E}-04 \\
32504\end{array}$ & $\begin{array}{l}1.852 \\
2651\end{array}$ & $8.40 \mathrm{E}-03$ & $\begin{array}{l}39.34 \\
77077\end{array}$ & 97.59 & 75.91 & & 82.6 & 330.2 \\
\hline $\begin{array}{l}922 \\
923 \\
\end{array}$ & 30.36 & $\begin{array}{l}3000 \\
3000 \\
3000\end{array}$ & 2330 & \begin{tabular}{|l|l|}
65.088054 \\
1270161
\end{tabular} & $\begin{array}{l}2.4028 \\
05444 \\
\end{array}$ & \begin{tabular}{|l|}
110.7481 \\
128519 \\
\end{tabular} & \begin{tabular}{|l|l|}
2031.64 \\
1750.11
\end{tabular} & $\begin{array}{l}5125 \\
5125 \\
5\end{array}$ & 0.1 & $\begin{array}{l}1950 \\
1950\end{array}$ & 225000 & 1250000 & $\begin{array}{l}0.1048 \\
\end{array}$ & 3.42E-04 & 2.651 & \begin{tabular}{|c|}
$9.91 \mathrm{E}-03$ \\
\end{tabular} & 79.72 & 92.32 & 23.18 & & 69.9 & 447.6 \\
\hline $\begin{array}{l}923 \\
924 \\
\end{array}$ & $\begin{array}{r}22.29 \\
33.1\end{array}$ & $\begin{array}{l}3000 \\
3000 \\
\end{array}$ & $\frac{2500}{640}$ & \begin{tabular}{|l|l|l|}
12.709164 \\
589.047399
\end{tabular} & $\begin{array}{r}0.5444 \\
20.7183\end{array}$ & \begin{tabular}{|l}
$\mid 128.5118$ \\
105.5176 \\
\end{tabular} & $\begin{array}{l}\frac{17500.81}{2132.35} \\
\end{array}$ & $\begin{array}{l}5125 \\
5125\end{array}$ & 0.1 & $\begin{array}{l}1950 \\
1950 \\
\end{array}$ & $\frac{225000}{225000}$ & $\begin{array}{l}\frac{1562500}{1162500} \\
\end{array}$ & $|0.1427|$ & $\begin{array}{l}4.94 \mathrm{E}-04 \\
5.03-05\end{array}$ & $\frac{2.751}{1.265}$ & \begin{tabular}{|l|l|}
$9.552-03$ \\
$8.42 E-33$
\end{tabular} & $\begin{array}{r}36.1 \\
30.34 \\
\end{array}$ & $\begin{array}{l}60.66 \\
99.15 \\
\end{array}$ & \begin{tabular}{r|}
0 \\
91.51
\end{tabular} & $\begin{array}{r}0 \\
15.12\end{array}$ & $\begin{array}{r}72.6 \\
8.4\end{array}$ & $\frac{632.4}{195.8}$ \\
\hline 925 & 23.64 & 3000 & 1340 & \begin{tabular}{|l|l|} 
& 421.184601 \\
\end{tabular} & 26.0612 & \begin{tabular}{|l|}
185.6278 \\
\end{tabular} & 1212.1 & 5125 & 0.1 & 1950 & 225000 & 2712500 & 0.1346 & $1.22 \mathrm{E}-04$ & $\begin{array}{l}1.205 \\
1.457\end{array}$ & \begin{tabular}{|l|l}
$0.4 \mathrm{E}-\mathrm{U}$ \\
$6.40 \mathrm{E}-03$
\end{tabular} & 52.12 & 98.81 & 88.13 & & 108.4 & 315.9 \\
\hline 926 & 43.46 & 3000 & 1910 & 296.249028 & \begin{tabular}{|l|l|}
15.3324 \\
\end{tabular} & 155.2656 & 1449.13 & 5125 & 0.1 & 1950 & 225000 & 2075000 & 0.0732 & $3.02 \mathrm{E}-04$ & 3.088 & $6.75 \mathrm{E}-03$ & 44.61 & 98.31 & 83.12 & & 102.7 & 364.1 \\
\hline 927 & 36.23 & 3000 & 1630 & 113.465825 & 5.3661 & 141.878 & 1585.87 & 5125 & 0.1 & 1950 & 225000 & 1812500 & 0.0878 & $1.39 \mathrm{E}-04$ & 1.798 & $1.37 \mathrm{E}-02$ & 57.08 & 95.59 & 55.93 & & 50.5 & 254.3 \\
\hline 928 & 44.87 & 3000 & 3400 & 32.005511 & 1.6895 & 158.3679 & 1420.74 & 5125 & 0.1 & 1950 & 225000 & 2137500 & 0.0709 & $7.33 \mathrm{E}-04$ & 6.229 & $7.23 \mathrm{E}-03$ & 51.49 & 84.38 & & & 95.9 & 711.5 \\
\hline 929 & 21.28 & 3000 & 2040 & 16.589427 & 0.6877 & 124.3658 & 1809.18 & 5125 & 0.1 & 1950 & 225000 & 1487500 & 0.1495 & $2.53 \mathrm{E}-04$ & 1.856 & $1.22 \mathrm{E}-02$ & 24.27 & 69.86 & 0 & & 57 & 447.1 \\
\hline 930 & 63.12 & 3000 & 1190 & 282.165615 & $\begin{array}{l}7.8162 \\
\end{array}$ & 83.1021 & 2707.51 & 5125 & 0.1 & 1950 & 225000 & 812500 & 0.0504 & $1.37 \mathrm{E}-04$ & 2.373 & $1.25 \mathrm{E}-02$ & 17.43 & 98.23 & 82.28 & 0 & 55.4 & 192.6 \\
\hline 931 & 32.23 & 3000 & 1140 & \begin{tabular}{|l|}
536.729683 \\
\end{tabular} & $\begin{array}{l}23.8426 \\
\end{array}$ & \begin{tabular}{|l|l|}
133.2659 \\
\end{tabular} & $\begin{array}{l}1688.35 \\
147.3\end{array}$ & 5125 & 0.1 & 1950 & 225000 & 1650000 & 0.0987 & 1.05E-04 & 1.539 & $7.17 \mathrm{E}-03$ & 38.55 & 99.07 & 90.68 & 6.84 & 96.6 & 244.7 \\
\hline 932 & 31.41 & 3000 & 1710 & & $\begin{array}{r}4.1881 \\
\end{array}$ & 152.7612 & 1472.89 & 5125 & 0.1 & 1950 & 225000 & 2025000 & 0.1013 & $2.59 \mathrm{E}-04$ & 2.29 & $1.08 \mathrm{E}-02$ & 74.45 & 93.92 & 39.21 & & 64.3 & 373.7 \\
\hline 933 & 28.95 & 3000 & 1860 & \begin{tabular}{|l|l|}
5.982129 \\
256529 \\
\end{tabular} & & \begin{tabular}{|l|l|}
148.9657 \\
\end{tabular} & 1510.42 & 5125 & 0.1 & 1950 & 225000 & 1950000 & 0.1099 & $2.92 \mathrm{E}-04$ & 2.343 & $1.63 \mathrm{E}-02$ & 30.2 & 16.42 & 0 & & 42.6 & 414.8 \\
\hline 934 & 24.34 & 3000 & 6990 & 205.65435 & & \begin{tabular}{|l|l|}
119.4396 \\
\end{tabular} & 1883.8 & 5125 & 0.1 & 1950 & 225000 & 1400000 & 0.1307 & 7.66E-05 & 1.296 & $9.92 \mathrm{E}-03$ & $\begin{array}{ll}14.27 \\
\end{array}$ & 97.57 & 75.69 & & 69.9 & 272.9 \\
\hline 935 & $\begin{array}{l}21.61 \\
2259 \\
\end{array}$ & 3000 & $\begin{array}{r}780 \\
2510 \\
510\end{array}$ & $\begin{array}{l}56.176236 \\
49716906\end{array}$ & $\begin{array}{l}1.8316 \\
22369\end{array}$ & \begin{tabular}{|r|}
97.8112 \\
136613
\end{tabular} & $\begin{array}{l}2300.35 \\
191702 \\
\end{array}$ & $\begin{array}{l}5125 \\
5125 \\
5\end{array}$ & 0.1 & $\begin{array}{l}1950 \\
1950 \\
\end{array}$ & 225000 & $\begin{array}{l}1037500 \\
1712500\end{array}$ & 0.1472 & $\begin{array}{l}8.35 \mathrm{E}-05 \\
5.8501\end{array}$ & $\begin{array}{l}1.287 \\
2861 \\
\end{array}$ & $1.33 \mathrm{E}-02$ & $\begin{array}{ll}16.97 \\
156\end{array}$ & 91.1 & 10.99 & & 52.3 & 305.2 \\
\hline $\begin{array}{l}936 \\
937 \\
\end{array}$ & 22.59 & 3000 & 2510 & 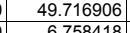 & 2.2639 & \begin{tabular}{|l|l|}
136.6103 \\
1722679
\end{tabular} & $\begin{array}{l}1647.02 \\
130611 \\
130\end{array}$ & $\begin{array}{l}5125 \\
5125 \\
5125\end{array}$ & 0.1 & $\begin{array}{l}1950 \\
1950 \\
1050\end{array}$ & 225000 & 1712500 & 0.1408 & 5.18E-04 & 2.861 & $6.91 \mathrm{E}-03$ & 41.56 & 89.94 & 0 & & 100.4 & 648.9 \\
\hline 938 & $\frac{37.25}{25.19}$ & $\begin{array}{l}3000 \\
3000\end{array}$ & $\frac{2070}{1680}$ & \begin{tabular}{|r|}
6.7584418 \\
454.267521
\end{tabular} & $\begin{array}{r}0.3881 \\
17.1035\end{array}$ & \begin{tabular}{|l}
$\mid 172.2679$ \\
112.9522 \\
\end{tabular} & $\begin{array}{l}\frac{1306.11}{1991.99} \\
\end{array}$ & $\begin{array}{l}5125 \\
5125\end{array}$ & $\frac{0.1}{0.1}$ & $\begin{array}{l}\frac{1950}{1950} \\
1950\end{array}$ & 225000 & $\begin{array}{l}2425000 \\
1287500\end{array}$ & $\begin{array}{l}0.0854 \\
0.1263\end{array}$ & \begin{tabular}{|l|}
$5.03 E E-04$ \\
$1.88-04$
\end{tabular} & $\begin{array}{l}3.977 \\
1.753 \\
\end{array}$ & $\begin{array}{l}1.43 \mathrm{E}-02 \\
5.38 \mathrm{E}-03 \\
\end{array}$ & $\begin{array}{l}62.66 \\
26.07 \\
\end{array}$ & $\frac{26.02}{98.9}$ & \begin{tabular}{r|}
0 \\
88.99
\end{tabular} & & $\frac{48.4}{128.8}$ & $\frac{547.1}{356.6}$ \\
\hline 939 & 27.33 & 3000 & 2190 & 10.137054 & 0.3817 & \begin{tabular}{|l|}
112.9522 \\
\end{tabular} & 1991.99 & 5125 & 0.1 & 1950 & 225000 & 1287500 & 0.1164 & $2.69 \mathrm{E}-04$ & 2.168 & \begin{tabular}{|l|l|}
$1.60 \mathrm{E}-02$ \\
\end{tabular} & 59.1 & 50.68 & & & 43.4 & 406.5 \\
\hline 940 & 21.34 & 3000 & 1010 & 73.233885 & 3.7597 & 154.0159 & 1460.89 & 5125 & 0.1 & 1950 & 225000 & 2050000 & 0.1491 & $1.13 \mathrm{E}-04$ & 1.385 & $1.14 \mathrm{E}-02$ & 20.38 & 93.17 & 31.73 & & 60.8 & 332.6 \\
\hline 941 & 44.12 & 3000 & 2370 & 346.315204 & 11.3817 & 98.5952 & 2282.06 & 5125 & 0.1 & 1950 & 225000 & 1050000 & 0.0721 & $1.73 \mathrm{E}-04$ & 2.213 & $8.85 \mathrm{E}-03$ & 57.38 & 98.56 & 85.56 & & 78.3 & 257.1 \\
\hline 942 & 61.06 & 3000 & 3500 & \begin{tabular}{|l|}
272.95458 \\
\end{tabular} & 7.6384 & 83.9523 & 2680.09 & 5125 & 0.1 & 1950 & 225000 & 825000 & 0.0521 & $4.82 \mathrm{E}-04$ & 5.68 & $5.86 \mathrm{E}-03$ & 69.76 & 98.17 & 81.68 & & 118.3 & 476.7 \\
\hline 943 & 28.1 & 3000 & 2380 & 2.687743 & $\begin{array}{l}0.107 \\
\end{array}$ & 119.4396 & 1883.8 & 5125 & 0.1 & 1950 & 225000 & 1400000 & 0.1132 & $4.61 \mathrm{E}-04$ & 3.061 & \begin{tabular}{|c|}
$1.56 \mathrm{E}-02$ \\
\end{tabular} & 57.22 & 0 & 0 & & 44.5 & 558.1 \\
\hline 944 & 42.93 & 3000 & 930 & 616.352643 & 22.4489 & 109.2665 & 2059.19 & 5125 & 0.1 & 1950 & 225000 & 1225000 & 0.0741 & $7.42 \mathrm{E}-05$ & 1.507 & $9.00 \mathrm{E}-03$ & 47.82 & 99.19 & 91.89 & 18.88 & 77.1 & 179.8 \\
\hline 945 & 34.13 & 3000 & 2910 & \begin{tabular}{|r|}
3.450361 \\
\end{tabular} & \begin{tabular}{|l|}
0.1023 \\
\end{tabular} & 88.9661 & 2529.05 & 5125 & 0.1 & 1950 & 225000 & 900000 & 0.0932 & 4.35E-04 & 3.357 & & 56.3 & & & & 41.3 & 504.1 \\
\hline 946 & 22.02 & 3000 & 1580 & 47.556406 & & 139.2566 & 1615.72 & 5125 & 0.1 & 1950 & 225000 & 1762500 & 0.1445 & $2.14 \mathrm{E}-04$ & 1.748 & \begin{tabular}{|l|l|l|l|} 
\\
\end{tabular} & $\begin{array}{l}17.09 \\
\end{array}$ & 89.49 & 0 & 0 & 66.5 & 406.9 \\
\hline 947 & 27.57 & 3000 & 2140 & $\begin{array}{r}83.66517 \\
\end{array}$ & $\begin{array}{r}3.5071 \\
\end{array}$ & 125.7554 & \begin{tabular}{|l|l|l|}
1789.19 \\
\end{tabular} & 5125 & 0.1 & 1950 & 225000 & 1512500 & 0.1154 & 3.64E-04 & 2.595 & $8.63 \mathrm{E}-03$ & 89.95 & 94.02 & 40.24 & 0 & 80.3 & 482.5 \\
\hline 948 & 43.46 & 3000 & 520 & 990.220822 & $\begin{array}{l}40.1248 \\
\end{array}$ & 121.5631 & $\begin{array}{l}1850.89 \\
30.0\end{array}$ & 5125 & 0.1 & 1950 & 225000 & 1437500 & 0.0732 & 4.16E-05 & 1.287 & $7.37 \mathrm{E}-03$ & & 99.5 & 94.95 & 49.51 & 94.1 & 151.8 \\
\hline$\frac{949}{950}$ & $\begin{array}{l}26.56 \\
53.29 \\
\end{array}$ & $\begin{array}{l}3000 \\
3000\end{array}$ & $\frac{3420}{2430}$ & $\begin{array}{r}5.357306 \\
437.673119\end{array}$ & $\begin{array}{r}0.1499 \\
188486\end{array}$ & 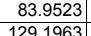 & $\begin{array}{l}2680.09 \\
171.54 \\
\end{array}$ & $\begin{array}{l}5125 \\
5125 \\
125\end{array}$ & 0.1 & $\begin{array}{l}1950 \\
1950\end{array}$ & 225000 & $\begin{array}{r}825000 \\
1575000\end{array}$ & 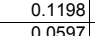 & $\begin{array}{l}6.13 \mathrm{E}-04 \\
358 \mathrm{E}-04\end{array}$ & 3.586 & \begin{tabular}{|l|l|l|l|l|} 
\\
$524 E-02$
\end{tabular} & $\begin{array}{l}51.89 \\
3947\end{array}$ & 6.67 & $\begin{array}{r}0 \\
0\end{array}$ & 0 & 61.7 & 692.1 \\
\hline 950 & $\frac{53.29}{30.56}$ & $\begin{array}{l}3000 \\
3000\end{array}$ & $\frac{2430}{2420}$ & $\begin{array}{r}437.6731199 \\
10.125498\end{array}$ & \begin{tabular}{r|}
18.8486 \\
0.3407
\end{tabular} & \begin{tabular}{|l|}
129.1963 \\
100.929
\end{tabular} & $\begin{array}{l}1741.54 \\
2229.29\end{array}$ & $\frac{5125}{5125}$ & $\frac{0.1}{0.1}$ & $\frac{1950}{1950}$ & 2250000 & \begin{tabular}{|l|}
1575000 \\
1087500
\end{tabular} & $|0.0597|$ & \begin{tabular}{|l|}
$3.58 \mathrm{E}-04$ \\
$2.78-04$
\end{tabular} & $\frac{4.031}{2.352}$ & \begin{tabular}{|l|l|}
$5.24 \mathrm{E}-03$ \\
$165-02$
\end{tabular} & $\begin{array}{l}39.47 \\
5.64\end{array}$ & $\frac{98.86}{50.62}$ & $\begin{array}{r}88.58 \\
0\end{array}$ & & $\frac{132.3}{41.9}$ & $\begin{array}{l}387.7 \\
394.4\end{array}$ \\
\hline 952 & 29.29 & 3000 & 3690 & 15.337874 & 0.3614 & $\begin{array}{l}70.6941 \\
7\end{array}$ & 3182.73 & 5125 & 0.1 & 1950 & 225000 & 637500 & 0.1086 & $5.48 \mathrm{E}-04$ & 3.552 & $1.06 \mathrm{E}-02$ & $\begin{array}{l}0.07 \\
66.6 \\
\end{array}$ & 67.4 & 0 & & 65.7 & 621.5 \\
\hline 953 & 33.38 & 3000 & 1920 & 48.231091 & 151 & 162.6609 & 1383.25 & 5125 & 0.1 & 1950 & 225000 & 2225000 & 0.0953 & $3.70 \mathrm{E}-04$ & 2.964 & \begin{tabular}{|l|l|}
$1.02 \mathrm{E}-02$ \\
\end{tabular} & 50.37 & 89.63 & 0 & & 68.2 & 455.1 \\
\hline 954 & 38.42 & 3000 & 2370 & 923.471683 & 32.2476 & 104.7599 & 2147.77 & 5125 & 0.1 & 1950 & 225000 & 1150000 & 0.0828 & $2.37 \mathrm{E}-04$ & 2.445 & $3.70 \mathrm{E}-03$ & 44.81 & 99.46 & 94.59 & $\mid 45.86$ & 187.2 & 326.1 \\
\hline & 32.01 & 3000 & 1640 & \begin{tabular}{|l|}
540.763597 \\
\end{tabular} & 24.3844 & 135.2775 & 1663.25 & 5125 & 0.1 & 1950 & 225000 & 1687500 & 0.0994 & $1.30 \mathrm{E}-04$ & 1.66 & $6.64 \mathrm{E}-03$ & 54.49 & 99. & 90.75 & 7.54 & 104.4 & 265.8 \\
\hline 956 & 35.51 & 3000 & 2100 & 107.341629 & & 170.4873 & 1319.75 & 5125 & 0.1 & 1950 & 225000 & 2387500 & 0.0896 & $4.52 \mathrm{E}-04$ & 3.55 & & 63.09 & 95.34 & 53.42 & & 95.2 & 512.5 \\
\hline 957 & 24.25 & 3000 & 1950 & 36.450545 & & 79.65 & 282 & 5125 & 0.1 & 1950 & 225000 & 762500 & 0.1312 & 1.61 & 1.621 & & 67.42 & 86.28 & 0 & & 49.2 & 342.6 \\
\hline 958 & 53.74 & 3000 & 1780 & 465.330463 & 21.4 & 138.5974 & 1623.41 & 5125 & 0.1 & 1950 & 225000 & 1750000 & & $1.90 \mathrm{E}$ & 2.624 & & & 98.93 & 89.25 & & 86.3 & 250.3 \\
\hline 959 & 26.4 & 3000 & 1120 & & & & 143 & & 0.1 & 1950 & & & & & 1.6 & & & 98.66 & 86.6 & & 102.1 & 310.5 \\
\hline 960 & 40.27 & 3000 & 1810 & & & 166.8 & 134 & & 0 & 195 & 225 & & & 2.74 & 2.755 & & & 99.38 & 93.77 & 37.75 & 181.9 & \\
\hline 961 & 22.59 & 3000 & 2260 & 226.094766 & 10.4 & 138.5 & 1623.41 & 5125 & 0.1 & 1950 & 225000 & & 0.1408 & & 2.415 & & 28.44 & & 77.89 & 0 & 141.9 & \\
\hline 962 & 28.35 & 3000 & 2770 & & & 115.8 & 1942 & 5125 & 0.1 & 1950 & & & & & 3.6 & & & & $\begin{array}{l}41.34 \\
\end{array}$ & & & \\
\hline 963 & 22.08 & 3000 & 990 & 446.455123 & 26.6832 & 179.36 & 1254 & 51 & 0 & 1950 & & & 0.1 & & 1.8 & & & & & & 106.9 & 298.4 \\
\hline 964 & 45 & 3000 & 1090 & 449.77 & 14.7817 & 98.5952 & 2282.06 & 512 & 0 & 1950 & 225000 & 1050 & 0.070 & $1.13 \mathrm{E}-04$ & 1.8 & \begin{tabular}{|c|}
$9.39 E-03$ \\
\end{tabular} & 29.4 & 98.89 & 88.88 & & 73.8 & 206 \\
\hline 965 & 22.09 & 3000 & 1770 & 195.459029 & 9.2438 & $\mid 141.878$ & 1585.87 & 51 & 0 & 1950 & 2250 & 18125 & \begin{tabular}{|c|c|}
0.144 \\
\end{tabular} & $1.84 \mathrm{E}-04$ & 1.645 & $\begin{array}{ll}77.40 E-03 \\
\end{array}$ & 38. & 97.44 & 74.42 & & 93.7 & 381.6 \\
\hline 966 & 60.83 & 3000 & 710 & 359.224699 & 14.5562 & 121.5631 & 1850.89 & 5125 & 0 & 1950 & 225000 & 1437500 & 0.0523 & $6.25 \mathrm{E}-05$ & 1.604 & $\mid 1.62 E-02$ & 42.56 & 98.61 & 86.08 & & 42.9 & 135.1 \\
\hline 967 & 36.69 & 3000 & 2250 & 359.099462 & 15.87 & 132. & 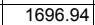 & 512 & 0.1 & 1950 & 225000 & 1637500 & 0.08 & $3.44 \mathrm{E}-04$ & 3.006 & $5.25 \mathrm{E}-03$ & 30.78 & 98.61 & 86.08 & & 132.1 & 419.9 \\
\hline 968 & 31.22 & 3000 & 3080 & 9.5624 & 0.25 & 91.42 & 2461 & 5125 & 0.1 & 1950 & 225000 & 93 & 0.1 & $5.32 \mathrm{E}-04$ & 3.641 & 1.18 & 56.3 & 47. & & & 58.6 & 597.8 \\
\hline 969 & 53.47 & & 1300 & 276.82 & & 120.1 & 187 & 5125 & 0.1 & 1950 & & 141 & & & 2.382 & & 14.64 & 98.19 & \begin{tabular}{l|l}
81.94 \\
\end{tabular} & & 65.4 & 228.3 \\
\hline 970 & 41.37 & 3000 & 3080 & & & 116. & 193 & 5125 & 0.1 & 1950 & 225 & & & 5.85 & 4.847 & & 43 & & 0 & 0 & 66.2 & 600.5 \\
\hline 971 & 51.56 & 3000 & 1550 & 988.08 & & 93.8 & 239 & 5125 & 0.1 & 1950 & 225000 & & & 1.34E-04 & 2.1 & 5.41 & 36.81 & 99. & 94.94 & 49.4 & 128.1 & 208.8 \\
\hline 972 & & & 1900 & & & 135. & & & 0 & & & & & & 2.146 & & & & 71.85 & & 80.7 & 339.8 \\
\hline 973 & 35 & 3000 & & & 3545 & & & & 0.1 & 1950 & 225000 & & & & 2.916 & $1.11 \mathrm{E}-02$ & \begin{tabular}{|l|}
37.83 \\
207
\end{tabular} & & $\begin{array}{r}0 \\
\end{array}$ & & $\begin{array}{l}62.6 \\
92 .\end{array}$ & 416.7 \\
\hline $\begin{array}{l}974 \\
975\end{array}$ & $\begin{array}{l}49.17 \\
57.22\end{array}$ & $\begin{array}{l}3000 \\
3000\end{array}$ & $\frac{2960}{1600}$ & \begin{tabular}{|l|}
102.239748 \\
585.750615
\end{tabular} & \begin{tabular}{|r|}
3.4132 \\
30.8007
\end{tabular} & \begin{tabular}{|l|}
100.1541 \\
157.7499 \\
\end{tabular} & \begin{tabular}{|l|}
2246.54 \\
1426.31 \\
\end{tabular} & $\frac{5125}{5125}$ & $\frac{0.1}{0.1}$ & $\begin{array}{l}\frac{1950}{1950} \\
1950\end{array}$ & $\begin{array}{l}225000 \\
225000\end{array}$ & $\begin{array}{l}1075000 \\
2125000 \\
\end{array}$ & \begin{tabular}{|l|l|}
0.0647 \\
0.0556
\end{tabular} & \begin{tabular}{|c|}
$3.90 \mathrm{E}-04$ \\
$179 \mathrm{E}-04$
\end{tabular} & $\begin{array}{l}4.049 \\
2.629 \\
\end{array}$ & \begin{tabular}{|l|l|}
$8.50 \mathrm{E}-03$ \\
$725 \mathrm{E}-03$
\end{tabular} & $\begin{array}{l}23.07 \\
5354 \\
\end{array}$ & $\begin{array}{l}95.11 \\
99.15\end{array}$ & $\begin{array}{r}51.1 \\
91.46\end{array}$ & \begin{tabular}{r|}
0 \\
14.64
\end{tabular} & $\begin{array}{l}81.5 \\
95.6\end{array}$ & $\begin{array}{r}422 \\
2355 \\
\end{array}$ \\
\hline
\end{tabular}




\begin{tabular}{|c|c|c|c|c|c|c|c|c|c|c|c|c|c|c|c|c|c|c|c|c|c|c|}
\hline $\begin{array}{c}\text { Realizatio } \\
\text { n }\end{array}$ & $\begin{array}{c}\text { Velocity } \\
(\mathrm{m} / \mathrm{y})\end{array}$ & $\begin{array}{c}\begin{array}{c}\text { Fixed Source } \\
\text { Concentration } \\
(\mathrm{mg} / \mathrm{L})\end{array} \\
\end{array}$ & $\begin{array}{c}\text { Time of } \\
\text { Maximum at } \\
\text { Compliance } \\
\text { Boundary } \\
\text { (y) }\end{array}$ & $\begin{array}{c}\text { Maximum } \\
\text { Concentration } \\
\text { Compliance } \\
\text { Boundary } \\
(\mu \mathrm{g} / \mathrm{L})\end{array}$ & $\begin{array}{c}\text { Maximum } \\
\text { Release Rate } \\
\text { at Compliance } \\
\text { Boundary } \\
(\mathrm{Kg} / \mathrm{y})\end{array}$ & $\begin{array}{c}\text { Source } \\
\text { Rate } \\
(\mathrm{Kg} / \mathrm{y}) \\
\end{array}$ & $\begin{array}{c}\text { Source } \\
\text { Duration } \\
\text { (y) }\end{array}$ & \begin{tabular}{|c|} 
1-D \\
Streamtub \\
e Length \\
$(\mathrm{m})$
\end{tabular} & $\begin{array}{c}\text { Base } \\
\text { Porosity } \\
\left(\mathrm{m}^{3} / \mathrm{m}^{3}\right)\end{array}$ & $\begin{array}{c}\text { Bulk } \\
\text { Densty } \\
\left(\mathrm{Kg} / \mathrm{m}^{3}\right)\end{array}$ & $\begin{array}{c}\text { Remaining } \\
\text { Carbon } \\
\text { Tetrachloride } \\
\text { Source } \\
(\mathrm{Kg})\end{array}$ & $\begin{array}{c}\text { Volume at or } \\
\text { above Fixed } \\
\text { Source } \\
\text { Concentration } \\
(\mathrm{m} 3)\end{array}$ & $\begin{array}{l}\text { Porosity } \\
\left(\mathrm{m}^{3} / \mathrm{m}^{3}\right)\end{array}$ & $\begin{array}{c}\mathrm{Kd} \\
\left(\mathrm{m}^{3} / \mathrm{Kg}\right)\end{array}$ & Retardation & $\begin{array}{c}\text { First Order } \\
\text { Rate } \\
\text { Constant } \\
(1 / y) \\
\end{array}$ & $\begin{array}{c}\text { Longitudinal } \\
\text { Dispersivity } \\
(\mathrm{m})\end{array}$ & 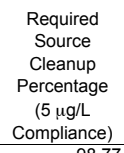 & \begin{tabular}{|c|} 
Required \\
Source \\
Cleanup \\
Percentage \\
(50 $\mu \mathrm{g} / \mathrm{L}$ \\
Compliance)
\end{tabular} & \begin{tabular}{|c|} 
Required \\
Source \\
Cleanup \\
Percentage \\
(500 $\mu g / L$ \\
Compliance) \\
\end{tabular} & \begin{tabular}{|c|}
$\begin{array}{c}\text { Abiotic } \\
\text { Reaction } \\
\text { Half fime } \\
(y)\end{array}$ \\
\end{tabular} & $\begin{array}{c}\text { Carbon } \\
\text { Tetrachloride } \\
\text { Travel Time (y) }\end{array}$ \\
\hline $\begin{array}{l}976 \\
997 \\
\end{array}$ & 33.92 & $\begin{array}{r}3000 \\
300\end{array}$ & $\begin{array}{r}710 \\
\end{array}$ & \begin{tabular}{|l|l|}
406.748236 \\
266909515 \\
\end{tabular} & $\begin{array}{r}14.3064 \\
12608\end{array}$ & $\begin{array}{l}105.5176 \\
12529\end{array}$ & \begin{tabular}{|l|}
2132.35 \\
15862
\end{tabular} & \begin{tabular}{|l|}
5125 \\
5125 \\
\end{tabular} & 0.1 & 1950 & $\begin{array}{r}225000 \\
225000\end{array}$ & $\begin{array}{r}1162500 \\
182500\end{array}$ & 0.0938 & \begin{tabular}{|l|l|}
$7.30 \mathrm{E}-05$ \\
$277-04$
\end{tabular} & $\begin{array}{r}1.393 \\
\end{array}$ & \begin{tabular}{|l|}
$9.62 E-03$ \\
$559 E-03$
\end{tabular} & $\begin{array}{r}25.62 \\
\end{array}$ & $\begin{array}{r}98.77 \\
\end{array}$ & \begin{tabular}{|r|}
87.71 \\
\end{tabular} & & \begin{tabular}{r|}
72 \\
\end{tabular} & \\
\hline 978 & 22.14 & & 27890 & & & $\mid \frac{\mid 142.5296}{124.3658}$ & & & 0.1 & & & & & $\begin{array}{r}2.7 / \mathrm{VE}-04 \\
6.05 \mathrm{E}-04\end{array}$ & $\begin{array}{l}1.973 \\
3.788 \\
\end{array}$ & $\begin{array}{c}5.59 \mathrm{E}-03 \\
7.95 \mathrm{E}-03\end{array}$ & & & & & $\begin{array}{r}\mid 23.9 \\
87.1\end{array}$ & \\
\hline 979 & 37.47 & & 1760 & & & \begin{tabular}{|c|}
24.0050 \\
98.5952
\end{tabular} & & & 0.1 & & & & & 1.0JE- -04 & & & & & 94.27 & & 142.4 & \\
\hline \begin{tabular}{|c|}
980 \\
980
\end{tabular} & $\frac{37.4 t}{51.23}$ & 3000 & $\frac{T r 60}{1220}$ & & & $\begin{array}{l}98.5952 \\
132.592 \\
\end{array}$ & $\begin{array}{l}228.206 \\
1696.94 \\
\end{array}$ & 5125 & $\frac{0.1}{0.1}$ & $\begin{array}{l}1950 \\
1950\end{array}$ & & & & 8.86 & & & & & $\begin{aligned} 94.27 \\
93.4\end{aligned}$ & $\frac{42.12}{34.02}$ & 售4.4.4 & \\
\hline 981 & 22.11 & 3000 & 3230 & & \begin{tabular}{|c|}
4.0678 \\
\end{tabular} & 125.7554 & 1789.19 & 5125 & 0.1 & & & & & 6.71 & & & & & 48.47 & 0 & 1483 & \\
\hline & 33.59 & & & & 0.31 & 81.3886 & 2764.52 & 5125 & 0.1 & & & & & $4.39 \mathrm{E}$ & 3.343 & & 43.24 & 56.25 & & & 54.8 & \\
\hline 983 & 24.21 & 3000 & 1240 & & \begin{tabular}{|c|}
15.695 \\
\end{tabular} & \begin{tabular}{|c|}
137.2743 \\
\end{tabular} & 1639 & 5125 & 0.1 & 1950 & & & & 8.911 & 1.343 & & 50.7 & 98.5 & 85.42 & & 88.3 & \\
\hline 984 & 44.62 & 3000 & 1140 & & 18.309 & 131.9164 & 1705. & 5125 & 0.1 & 1950 & & 162 & & $1.20 \mathrm{E}$ & 1.853 & 9.48 & & 98.8 & 87.99 & & 73.1 & \\
\hline & 55.52 & 3000 & 1880 & 785.3 & \begin{tabular}{|l|l|}
33.6418 \\
\end{tabular} & 128.5118 & 1750. & 5125 & 0.1 & 1950 & & & & $1.63 \mathrm{E}$ & 2.44 & & 38.48 & 99.36 & 93.63 & 36.33 & 113.7 & \\
\hline 986 & 37.52 & 3000 & 2410 & & \begin{tabular}{|l|l|}
5.0157 \\
\end{tabular} & 96.2335 & 2338 & 5125 & 0.1 & 195 & & & & 1.73 & & & 72.32 & 96.8 & 68.02 & & 60 & \\
\hline 987 & 25.89 & 3000 & 2000 & 417.8 & 18.843 & 135.2775 & 1663 & 5125 & 0.1 & 1950 & & & & & 2.204 & & 59.25 & 98.8 & 88.03 & & 146.1 & \\
\hline 988 & 24.82 & 30 & 2850 & & $\begin{array}{ll}0.3489 \\
\end{array}$ & \begin{tabular}{|l|}
89.788 \\
\end{tabular} & & 51 & 0.1 & 1950 & & & & & & & & & & & 57.2 & 502 \\
\hline & & & & & & 141.8 & & & & & & & & & & & & & 68.53 & & & 28 \\
\hline 990 & 31 & & 35 & & & & & & & & & & & & & & & & & & 80.4 & 779 \\
\hline 991 & & & 1150 & & & & & & & & & & & & & & & & 88. & & & 288 \\
\hline 992 & 30.62 & & & & & & & & & & & & & & & & & & & 64.11 & 237.3 & 26 \\
\hline 993 & 47.98 & & 650 & & 37.20 & & & & & & & & & & & & & & & 46.17 & 96.6 & 164 \\
\hline $\begin{array}{l}994 \\
995 \\
9\end{array}$ & $\begin{array}{l}57.95 \\
38.84\end{array}$ & $\begin{array}{ll}3000 \\
3000 \\
300\end{array}$ & 1360 & 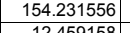 & $\begin{array}{r}7.294 \\
0.5646 \\
\end{array}$ & $\begin{array}{r}141.878 \\
1350447\end{array}$ & $\begin{array}{l}1585.87 \\
16550 .\end{array}$ & & & $\begin{array}{l}1950 \\
1950\end{array}$ & 00 & & & 1.32 & 17 & 1.57 & 30 & 96. & 67.58 & 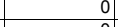 & 44.1 . & \\
\hline $\begin{array}{l}990 \\
996 \\
\end{array}$ & $\begin{array}{l}38.84 \\
28.48\end{array}$ & $\begin{array}{l}3000 \\
3000\end{array}$ & $\frac{1910}{1700}$ & $\begin{array}{r}12.4291198 \\
21296951\end{array}$ & $\begin{array}{l}0.5646 \\
96202 \\
\end{array}$ & $\mid \frac{135.944 !}{135.9447}$ & $\begin{array}{l}1655.08 \\
16508\end{array}$ & $\begin{array}{l}5^{1525} \\
5125 \\
\end{array}$ & 01 & $\frac{195}{199}$ & & & & $2.46 \mathrm{E}$ & $\frac{2.5}{17}$ & & & 59. & 76.45 & & 38.8 & \\
\hline 997 & 40.6 & 300 & 2060 & & 6.9 & 98.59 & 2288 & 512 & 0.1 & 19 & & & & $2.03 \mathrm{E}$ & 2.289 & 9.35 & 28.5 & 97. & 76.47 & & 74.1 & $\begin{array}{l}395 \\
292 \\
\end{array}$ \\
\hline 998 & 23.79 & & 1390 & & & \begin{tabular}{|l|}
112.2199 \\
\end{tabular} & & 5125 & 0.1 & 19 & & & & & 1.5 & & 30. & 98.1 & $\mid 81.79$ & & 94.6 & 333 \\
\hline 999 & 44.81 & 3000 & 2120 & \begin{tabular}{|l|}
16.086784 \\
\end{tabular} & $\begin{array}{l}0.8657 \\
\end{array}$ & 161.4401 & 1393.71 & 5125 & 0.1 & 19 & & & & $4.08 \mathrm{E}-04$ & 3.905 & \begin{tabular}{|l|}
$1.35 \mathrm{E}-02$ \\
\end{tabular} & 39.29 & 68.92 & 0 & & 51.3 & 446 \\
\hline 1000 & 32.9 & 3000 & 1470 & \begin{tabular}{|l|l}
1224.259845 \\
\end{tabular} & \begin{tabular}{|l|l|}
64.8795 \\
\end{tabular} & 158.9847 & 1415.23 & 5125 & 0.1 & 1950 & 225000 & 2150000 & 0.0967 & $8.39 \mathrm{E}-05$ & 1.439 & \begin{tabular}{|l|}
$4.08 \mathrm{E}-03$ \\
\end{tabular} & 80.2 & 99.59 & 95.92 & 59.16 & 169.9 & \\
\hline
\end{tabular}




\begin{tabular}{|c|c|c|c|c|c|c|c|c|c|c|c|c|c|c|c|c|c|c|c|c|c|c|}
\hline $\begin{array}{c}\text { Realizatio } \\
n\end{array}$ & $\begin{array}{c}\text { Velocity } \\
(\mathrm{m} / \mathrm{y})\end{array}$ & \begin{tabular}{|c} 
Fixed Source \\
Concentratio \\
n (mg/L)
\end{tabular} & $\begin{array}{c}\text { Time of } \\
\text { Maximum at } \\
\text { Compliance } \\
\text { Boundary } \\
\text { (y) }\end{array}$ & $\begin{array}{c}\text { Maximum } \\
\text { Concentratio } \\
\text { n Compliance } \\
\text { Boundary } \\
(\mu \mathrm{g} / \mathrm{L})\end{array}$ & \begin{tabular}{|c|} 
Maximum \\
Release \\
Rate at \\
Complianc \\
e Boundary \\
(Kg/y) \\
\end{tabular} & $\begin{array}{c}\text { Source } \\
\text { Rate } \\
(\mathrm{Kg} / \mathrm{y}) \\
\end{array}$ & $\begin{array}{c}\text { Source } \\
\text { Duration } \\
\text { (y) }\end{array}$ & \begin{tabular}{|c|} 
1-D \\
Streamtub \\
e Length \\
$(\mathrm{m})$
\end{tabular} & $\begin{array}{c}\text { Base } \\
\text { Porosity } \\
\left(\mathrm{m}^{3} / \mathrm{m}^{3}\right)\end{array}$ & $\begin{array}{c}\text { Bulk } \\
\text { Densty } \\
\left(\mathrm{Kg} / \mathrm{m}^{3}\right) \\
\end{array}$ & $\begin{array}{c}\text { Remaining } \\
\text { Carbon } \\
\text { Tetrachloride } \\
\text { Source } \\
(\mathrm{Kg}) \\
\end{array}$ & $\begin{array}{c}\text { Volume at or } \\
\text { above Fixed } \\
\text { Source } \\
\text { Concentratio } \\
n(m 3)\end{array}$ & $\begin{array}{l}\text { Porosity } \\
\left(\mathrm{m}^{3} / \mathrm{m}^{3}\right)\end{array}$ & $\begin{array}{c}\mathrm{Kd} \\
\left(\mathrm{m}^{3} / \mathrm{kg}\right)\end{array}$ & Retardation & $\begin{array}{c}\text { First } \\
\text { Order } \\
\text { Rate } \\
\text { Constant } \\
(1 / y) \\
105\end{array}$ & \begin{tabular}{|c} 
Longitudinal \\
Dispersivity \\
$(\mathrm{m})$
\end{tabular} & \begin{tabular}{|c|} 
Required \\
Source \\
Cleanup \\
Percentage \\
$(5 \mu \mathrm{\mu g} / \mathrm{L}$ \\
Compliance) \\
\end{tabular} & \begin{tabular}{|c|} 
Required \\
Source \\
Cleanup \\
Percentage \\
$(50 \mu \mathrm{g} / \mathrm{L}$ \\
Compliance) \\
\end{tabular} & $\begin{array}{c}\text { Required } \\
\text { Source } \\
\text { Cleanup } \\
\text { Percentage } \\
\text { (500 } \mathrm{\mu g} / \mathrm{L} \\
\text { Compliance) } \\
\end{array}$ & \begin{tabular}{|c} 
Abiotic \\
Reaction \\
Half Time \\
(y) \\
\end{tabular} & $\begin{array}{c}\text { Carbon } \\
\text { Tetrachloride } \\
\text { Travel Time } \\
\text { (y) } \\
\end{array}$ \\
\hline & & & 4630 & 21.221799 & & 122.9684 & 3964.43 & & & 1950 & 487500 & \begin{tabular}{|l|}
1462500 \\
\end{tabular} & 0.054 & $4.50 \mathrm{E}-04$ & \begin{tabular}{|l|}
5.208 \\
\end{tabular} & $1.24 \mathrm{E}-02$ & $\begin{array}{r}26.99 \\
\end{array}$ & $\begin{array}{r}76.44 \\
\end{array}$ & & & & 453.1 \\
\hline & \begin{tabular}{l|l}
22.15 \\
3044
\end{tabular} & $\begin{array}{l}3000 \\
3000 \\
3000\end{array}$ & 6530 & $\begin{array}{r}4.975861 \\
118468998\end{array}$ & \begin{tabular}{|r|}
0.103 \\
5471
\end{tabular} & \begin{tabular}{|r|}
62.1111 \\
1379366
\end{tabular} & \begin{tabular}{|l|l|}
7848.84 \\
352.23
\end{tabular} & 5125 & $\begin{array}{ll}0.1 \\
0.1\end{array}$ & \begin{tabular}{l|l|}
1950 \\
1900
\end{tabular} & 487500 & $\begin{array}{r}525000 \\
\end{array}$ & $\begin{array}{l}0.1436 \\
0.1015\end{array}$ & $5.26 \mathrm{E}-04$ & 2.853 & $1.23 \mathrm{E}-02$ & $\begin{array}{ll}76.26 \\
554\end{array}$ & \begin{tabular}{r|}
0 \\
0
\end{tabular} & 0 & 0 & 56.2 & 660 \\
\hline$\frac{3}{4}$ & $\begin{array}{l}30.44 \\
22.99 \\
\end{array}$ & 3000 & $\begin{array}{l}2440 \\
1940\end{array}$ & $\frac{1188.468698}{467441712}$ & $\begin{array}{r}5.4471 \\
26.4715\end{array}$ & $\begin{array}{l}137.9366 \\
1699817\end{array}$ & $\begin{array}{l}3534.23 \\
286947\end{array}$ & $\begin{array}{l}5125 \\
5125 \\
\end{array}$ & $\begin{array}{l}0.1 \\
0.1 \\
\end{array}$ & \begin{tabular}{|c|}
1950 \\
1950
\end{tabular} & $\begin{array}{l}487500 \\
48750\end{array}$ & $\begin{array}{l}1737500 \\
2375000\end{array}$ & \begin{tabular}{|l|l|}
0.1045 \\
0.1384
\end{tabular} & $\begin{aligned} 2.01 \mathrm{E}-04 \\
1.93 \mathrm{E}-04\end{aligned}$ & $\begin{array}{r}1.975 \\
1704\end{array}$ & \begin{tabular}{|l|}
$1.04 \mathrm{E}-02$ \\
$5.00 \mathrm{E}-03$ \\
\end{tabular} & $\begin{array}{r}55.4 \\
35.33\end{array}$ & $\begin{array}{l}95.78 \\
98.93\end{array}$ & \begin{tabular}{|c|c|}
57.79 \\
893
\end{tabular} & & $\frac{66.7}{138.6}$ & 332.4 \\
\hline 5 & 45.25 & 3000 & 5510 & 60.675825 & 1.8817 & 93.0387 & $\begin{array}{l}2009.41 \\
5239.76\end{array}$ & 5125 & 0.1 & 1950 & 487500 & | & $\begin{array}{l}0.104 \\
0.0703 \\
\end{array}$ & $4.20 \mathrm{E}-04$ & 4.023 & \begin{tabular}{|l|}
$0.00 \mathrm{E}-00$ \\
$1.02 \mathrm{E}-02$ \\
\end{tabular} & 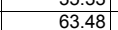 & 91.76 & $\begin{array}{l}0.0 \\
17.59\end{array}$ & & $\frac{10.0}{67.8}$ & 455.6 \\
\hline 6 & 35.04 & 3000 & 3790 & 135.138311 & 7.1616 & 158.9847 & 3066.33 & 5125 & 0.1 & 1950 & 487500 & 2150000 & 0.0908 & $5.19 \mathrm{E}-04$ & 3.891 & \begin{tabular}{|l|}
$1.02 \mathrm{~L}-02$ \\
$6.07 \mathrm{E}-03$ \\
\end{tabular} & $\begin{array}{l}0.480 \\
48.82\end{array}$ & 96.3 & 63 & & 114.2 & 569.2 \\
\hline 7 & 59.13 & 3000 & 3920 & 281.624411 & 11.8053 & 125.7554 & 3876.57 & 5125 & 0.1 & 1950 & 487500 & 1512500 & 0.0538 & $2.52 \mathrm{E}-04$ & 3.363 & \begin{tabular}{|l|}
$8.53 \mathrm{E}-03$ \\
\end{tabular} & $\frac{40.02}{33}$ & 98.22 & 82.25 & & 81.2 & 291.5 \\
\hline 8 & 49.32 & 3000 & 5250 & 28.738002 & 1.0538 & 110.0086 & 4431.47 & 5125 & 0.1 & 1950 & 487500 & 1237500 & 0.0645 & $5.92 \mathrm{E}-04$ & 5.636 & \begin{tabular}{|l|}
$9.74 \mathrm{E}-03$ \\
\end{tabular} & 44.48 & 82.6 & 0 & & 71.2 & 585.6 \\
\hline 9 & 26.53 & 3000 & 4420 & 33.743679 & 1.3032 & 115.8579 & 4207.74 & 5125 & 0.1 & 1950 & 487500 & 1337500 & 0.1199 & $3.81 \mathrm{E}-04$ & 2.608 & \begin{tabular}{|l|}
$1.02 \mathrm{E}-02$ \\
\end{tabular} & 62.53 & 85.18 & & & 68.1 & 503.7 \\
\hline 10 & 29.57 & 3000 & 5090 & 438.407689 & 15.7498 & 107.7748 & 4523.32 & 5125 & 0.1 & 1950 & 487500 & 1200000 & 0.1076 & $5.07 \mathrm{E}-04$ & 3.384 & \begin{tabular}{|l|l|}
$3.45 \mathrm{E}-03$ \\
\end{tabular} & 39.84 & 98.86 & 88.6 & & 201.2 & 586.6 \\
\hline 11 & 28.46 & 3000 & 3690 & 236.113198 & 11.7243 & 148.9657 & 3272.57 & 5125 & 0.1 & 1950 & 487500 & 1950000 & 0.1118 & $3.95 \mathrm{E}-04$ & 2.788 & \begin{tabular}{|l|}
$5.27 \mathrm{E}-03$ \\
\end{tabular} & 29.73 & 97.88 & & & 131.5 & 502.1 \\
\hline 12 & 21.41 & 3000 & 1390 & 185.517538 & 4.6529 & 75.2423 & 6479.07 & 5125 & 0.1 & 1950 & 487500 & 700000 & 0.1486 & $1.17 \mathrm{E}-04$ & 1.399 & \begin{tabular}{|l|}
$8.58 \mathrm{E}-03$ \\
\end{tabular} & 42.84 & 97.3 & 73.05 & & 80.8 & 334.8 \\
\hline 13 & 62.5 & 3000 & 4550 & 7.595494 & 0.3658 & 144.4754 & 3374.28 & 5125 & 0.1 & 1950 & 487500 & 1862500 & 0.0509 & $6.74 \mathrm{E}-04$ & 7.691 & \begin{tabular}{|l|}
$1.36 \mathrm{E}-02$ \\
\end{tabular} & 49.38 & 34.17 & & & 50.8 & 630.6 \\
\hline 14 & 28.87 & 3000 & 5280 & 20.314583 & 0.7499 & 110.7481 & 4401.88 & 5125 & 0.1 & 1950 & 487500 & 1250000 & 0.1102 & $6.60 \mathrm{E}-04$ & 4.027 & 7.91E-03 & 33.57 & 75.39 & & & 87.7 & 714.9 \\
\hline 15 & 41 & 3000 & 4620 & 28.976867 & 1.1468 & 118.7276 & 4106.04 & 5125 & 0.1 & 1950 & 487500 & 1387500 & 0.0776 & $4.97 \mathrm{E}-04$ & 4.236 & $1.04 \mathrm{E}-02$ & 47.28 & 82.74 & & & 67 & 529.6 \\
\hline 16 & 37.83 & 3000 & 2370 & 1286.001247 & 49.9734 & 116.5786 & 4181.73 & 5125 & 0.1 & 1950 & 487500 & 1350000 & 0.0841 & $1.65 \mathrm{E}-04$ & 1.989 & $3.20 \mathrm{E}-03$ & 55.5 & 99.61 & 96.11 & 61.12 & 216.6 & 269.5 \\
\hline 17 & 59.24 & 3000 & 2680 & \begin{tabular}{|l|}
716.319344 \\
\end{tabular} & 34.651 & 145.1211 & 3359.26 & 5125 & 0.1 & 1950 & 487500 & 1875000 & 0.0537 & $1.80 \mathrm{E}-04$ & 2.697 & 6.33E-03 & 41.22 & 99.3 & 93.02 & 30.2 & 109.5 & 233.3 \\
\hline 18 & 52.5 & 3000 & 2500 & 201.416671 & 7.9712 & 118.7276 & 4106.04 & 5125 & 0.1 & 1950 & 487500 & 1387500 & 0.0606 & $1.94 \mathrm{E}-04$ & 2.617 & $1.12 \mathrm{E}-02$ & 39.63 & 97.52 & 75.18 & 0 & 62.2 & 255.5 \\
\hline 19 & 29.59 & 3000 & 650 & 436.709687 & 19.8863 & 136.6103 & 3568.54 & 5125 & 0.1 & 1950 & 487500 & 1712500 & 0.1075 & $3.26 \mathrm{E}-05$ & 1.153 & $9.82 \mathrm{E}-03$ & 41.91 & $\begin{array}{l}98.86 \\
\end{array}$ & 88.55 & & 70.6 & 199.7 \\
\hline 20 & 21.6 & 3000 & 2550 & 14.64421 & 0.9117 & 186.7666 & 2610.21 & 5125 & 0.1 & 1950 & 487500 & 2737500 & 0.1473 & $2.62 \mathrm{E}-04$ & 1.901 & $1.28 \mathrm{E}-02$ & 42.14 & 65.86 & & & 54.2 & 451 \\
\hline 21 & 31.69 & 3000 & 3990 & 55.763392 & 3.0008 & 161.4401 & 3019.69 & 5125 & 0.1 & 1950 & 487500 & 2200000 & 0.1004 & $5.94 \mathrm{E}-04$ & 3.993 & $6.88 \mathrm{E}-03$ & 37.29 & 91.03 & 10.34 & & 100.7 & 645.8 \\
\hline 22 & 26.1 & 3000 & 3100 & \begin{tabular}{|l|l|l|}
17.675777 \\
90209
\end{tabular} & & $\begin{array}{l}104.7599 \\
\end{array}$ & 4653.5 & 5125 & 0.1 & 1950 & 487500 & 1150000 & 0.1219 & & & & 27.73 & 71.71 & & & 58.5 & 461.8 \\
\hline 23 & 22.18 & 3000 & 2030 & 89.296983 & 4.6586 & 156.5102 & 3114.81 & 5125 & 0.1 & 1950 & 487500 & 2100000 & 0.1434 & 1.94E-04 & 1.682 & & 42.19 & $\begin{array}{l}94.4 \\
\end{array}$ & 44.01 & & 73.1 & 388.6 \\
\hline 24 & 28.87 & 3000 & 4540 & 215.934961 & 8.6991 & 120.8573 & 4033.68 & 5125 & 0.1 & 1950 & 487500 & 1425000 & 0.1102 & $4.92 \mathrm{E}-04$ & 3.258 & $4.93 \mathrm{E}-03$ & 49.66 & $\begin{array}{l}97.68 \\
\end{array}$ & 76.84 & & 140.7 & 578.4 \\
\hline 25 & 29 & 3000 & 4650 & 342.553214 & 14.0411 & 122.9684 & 3964.43 & 5125 & 0.1 & 1950 & 487500 & 1462500 & 0.1097 & 5.47E-04 & 3.519 & $3.73 \mathrm{E}-03$ & 46.41 & $\begin{array}{l}98.54 \\
\end{array}$ & 85.4 & & 185.8 & 622 \\
\hline 26 & 22.76 & 3000 & 5990 & 351.206316 & 11.7249 & 100.1541 & 4867.5 & 5125 & 0.1 & 1950 & 487500 & 1075000 & 0.1398 & $7.66 \mathrm{E}-04$ & 3.771 & $2.71 \mathrm{E}-03$ & 45.07 & $\begin{array}{l}98.58 \\
\end{array}$ & 85.76 & & 256.3 & 849.3 \\
\hline 27 & 43.28 & 3000 & 5080 & $\begin{array}{l}74.175035 \\
0.22301\end{array}$ & $\begin{array}{l}2.3203 \\
57825\end{array}$ & 93.8425 & 5194.88 & 5125 & 0.1 & 1950 & 487500 & 975000 & 0.0735 & $3.68 \mathrm{E}-04$ & 3.53 & $9.45 \mathrm{E}-03$ & 26.64 & $\begin{array}{l}93.26 \\
\end{array}$ & 32.59 & 0 & 73.3 & 417.9 \\
\hline 28 & 21.94 & 3000 & 1130 & $\begin{array}{r}95.233404 \\
829\end{array}$ & $\begin{array}{r}5.7835 \\
360025\end{array}$ & 182.1901 & 2675.78 & 5125 & 0.1 & 1950 & 487500 & 2637500 & 0.145 & $1.04 \mathrm{E}-04$ & 1.361 & $1.12 \mathrm{E}-02$ & 34.57 & $\begin{array}{l}94.75 \\
\end{array}$ & 47.5 & 0 & 61.9 & 317.9 \\
\hline 29 & $\begin{array}{l}45.38 \\
24.19 \\
\end{array}$ & 3000 & 1480 & 838.084461 & 36.0925 & $\begin{array}{l}129.1963 \\
\end{array}$ & 3773.33 & 5125 & 0.1 & 1950 & 487500 & 1575000 & 0.0701 & $1.10 \mathrm{E}-04$ & 1.794 & $6.46 \mathrm{E}-03$ & 58.83 & $\begin{array}{l}99.4 \\
\end{array}$ & 94.03 & 40.34 & 107.3 & 202.6 \\
\hline 30 & $\begin{array}{l}34.81 \\
2511 \\
\end{array}$ & 3000 & 2440 & \begin{tabular}{|r|}
39.563262 \\
\end{tabular} & $\begin{array}{l}1.4015 \\
15521\end{array}$ & $\begin{array}{l}106.2727 \\
03.825 \\
\end{array}$ & $\begin{array}{l}4587.26 \\
5594.98\end{array}$ & 5125 & 0.1 & 1950 & 487500 & $\begin{array}{r}1175000 \\
07500\end{array}$ & 0.0914 & 1.87E-04 & 2.034 & $1.66 \mathrm{E}-02$ & 85.44 & \begin{tabular}{ll|l|l|l|}
87.36 \\
\end{tabular} & 0 & & 41.8 & 299.4 \\
\hline $\begin{array}{l}31 \\
32 \\
32\end{array}$ & $\frac{25.11}{22.14}$ & 3000 & 5370 & \begin{tabular}{|r|}
145.566186 \\
5.724449 \\
\end{tabular} & $\begin{array}{l}4.5534 \\
0.2543\end{array}$ & $\begin{array}{r}93.8425 \\
13.2659\end{array}$ & $\begin{aligned} 5194.88 \\
368.1 \\
\end{aligned}$ & $\begin{array}{l}5125 \\
5125\end{array}$ & $\begin{array}{l}0.1 \\
0.1\end{array}$ & $\begin{array}{l}1950 \\
1950\end{array}$ & $\begin{array}{l}487500 \\
487500\end{array}$ & $\begin{array}{r}975000 \\
1650000\end{array}$ & $\begin{array}{l}0.1267 \\
0.144\end{array}$ & \begin{tabular}{|l|}
$3.59 \mathrm{E}-04$ \\
$3.31 \mathrm{E}-04$
\end{tabular} & 2.433 & \begin{tabular}{|l|}
$6.68 \mathrm{E}-03$ \\
$1.37 \mathrm{E}-02$ \\
\end{tabular} & $\begin{array}{l}67.25 \\
42.06\end{array}$ & $\begin{array}{l}96.57 \\
12.66\end{array}$ & \begin{tabular}{rl|}
65.65 & \\
\end{tabular} & & $\frac{103.8}{50.7}$ & $\begin{array}{l}496.7 \\
508.2 \\
\end{array}$ \\
\hline 33 & 49.71 & 3000 & 1360 & $\begin{array}{r}.1244988 \\
458.422988\end{array}$ & 19.4276 & $\begin{array}{l}127.1374 \\
\end{array}$ & 3834.44 & 5125 & 0.1 & 1950 & & & $\begin{array}{r}0.1404 \\
0.064 \\
\end{array}$ & $1.28 \mathrm{E}-04$ & 2.015 & & 34.79 & & 89.09 & & 74.7 & $\begin{array}{l}207.7 \\
207.7\end{array}$ \\
\hline 34 & 31.72 & 3000 & 3120 & 142.40216 & 7.1012 & 149.6016 & 3258.66 & 5125 & 0.1 & 1950 & 4875 & & 0.1003 & $2.73 \mathrm{E}-04$ & 2.374 & 8.34 & 35.37 & 96.49 & 64.89 & & 83.1 & 383.5 \\
\hline 35 & 32.56 & 3000 & 4250 & 33.74652 & 1.5066 & 133.9381 & 3639.74 & 5125 & 0.1 & 1950 & 487500 & 1662500 & 0.0977 & $5.12 \mathrm{E}-04$ & 3.649 & \begin{tabular}{|l|}
$8.74 \mathrm{E}-03$ \\
\end{tabular} & 36.93 & 85.18 & & 0 & 79.4 & 574.3 \\
\hline 36 & 46.92 & 3000 & 4650 & 297.505594 & 13.082 & 131.9164 & 3695.52 & 5125 & 0.1 & 1950 & 487500 & 1625000 & 0.0678 & $6.32 \mathrm{E}-04$ & 5.714 & \begin{tabular}{|l|}
$4.43 \mathrm{E}-03$ \\
\end{tabular} & 83.15 & 98.32 & 83.19 & & 156.6 & 624 \\
\hline 37 & 55.81 & 3000 & 620 & 863.706063 & 33.5632 & 116.5786 & 4181.73 & 5125 & 0.1 & 1950 & 487500 & 1350000 & 0.057 & $6.59 \mathrm{E}-05$ & 1.584 & $8.64 \mathrm{E}-03$ & 26.14 & 99.42 & 94.21 & 42.11 & 80.2 & 145.5 \\
\hline 38 & 29.79 & 3000 & 1920 & 532.699315 & $\begin{array}{l}17.368 \\
\end{array}$ & 97.8112 & 4984.09 & 5125 & 0.1 & 1950 & 487500 & 1037500 & 0.1068 & $1.81 \mathrm{E}-04$ & 1.858 & $5.53 \mathrm{E}-03$ & 36.63 & 99.06 & 90.61 & 6.14 & 125.3 & 319.7 \\
\hline 39 & 24.51 & 3000 & 4530 & 6.542838 & 0.2447 & 112.2199 & 4344.15 & 5125 & 0.1 & 1950 & & 1275000 & 0.1298 & $3.96 \mathrm{E}-04$ & 2.543 & $1.39 \mathrm{E}-02$ & 67.64 & 23.58 & & 0 & 49.9 & 531.7 \\
\hline 40 & 23.9 & 3000 & 2350 & 135.063355 & 3.9682 & 88.1405 & 5530.94 & 5125 & 0.1 & 1950 & & 887500 & 0.1331 & $2.04 \mathrm{E}-04$ & 1.775 & $8.60 \mathrm{E}-03$ & 52.06 & 96.3 & 62.98 & & 80.6 & 380.6 \\
\hline 41 & 31.91 & 3000 & 4340 & 107.058863 & 3.2625 & 91.4206 & 5332.5 & 5125 & 0.1 & 1950 & & 937500 & 0.0997 & $2.81 \mathrm{E}-04$ & 2.424 & 9.68 & 83.34 & 95.33 & 53.3 & & 71.6 & 389.4 \\
\hline 42 & 25.8 & 3000 & 3910 & 196.757469 & 9.09 & 138.5974 & 3517.38 & 5125 & 0.1 & 1950 & & 1750000 & 0.1233 & & 2.607 & 5.43 & 23.41 & 97.46 & 74.59 & & 127.6 & 517.9 \\
\hline 43 & 31.53 & 3000 & 1780 & 148.5 & 5.7004 & 115.1349 & 4234.16 & 5125 & 0.1 & 1950 & & & 0.1009 & 1.83 & 1.915 & & 31.7 & 96.63 & 66.34 & & 69.3 & 311.2 \\
\hline 44 & 22.5 & 3000 & 2190 & & 2.6152 & 124.3658 & 3919.89 & 5125 & 0.1 & 1950 & 487 & 148 & 0.1414 & & 1.569 & & 87.64 & 92.07 & 20.74 & & 58.1 & 357.5 \\
\hline 45 & 31.94 & 3000 & 870 & 314.2 & 12.9531 & 123.6681 & 3942 & 5125 & 0.1 & 1950 & & 1475000 & 0.0996 & & 1.287 & & 53 & 98.41 & 84.09 & & 61.6 & 206.5 \\
\hline 46 & 21.97 & 3000 & 4720 & & & & 5429. & 5125 & 0.1 & & & & 0.14 & & 2.256 & & 53. & 96 & 66.3 & & 113.3 & 526.3 \\
\hline 47 & 24.28 & 3000 & 3600 & & & 112.21 & & 5125 & 0.1 & 195 & & & 0.131 & & 2.315 & & & 72.85 & 0 & & 60.9 & 88.5 \\
\hline 48 & 26.73 & 3000 & 4610 & & & 115.13 & & 5125 & 0.1 & 195 & & & 0.119 & & 2.88 & & & & 37.19 & & 98 & 62.1 \\
\hline 49 & 42.25 & 30 & 44 & & & 131.9 & & 5125 & 0.1 & 195 & & & 0.0753 & & 4.325 & & & & 73.88 & & 122.6 & 24.6 \\
\hline 50 & $\begin{array}{l}40.89 \\
\end{array}$ & & 3420 & & & 151.5 & & 5125 & 0.1 & 195 & & & 0.0778 & & 2.883 & & & & 2.25 & & 81.9 & 51.3 \\
\hline 51 & 33.99 & & 3200 & & 6.9 & 189. & 257 & 5125 & 0.1 & 1950 & & & 0.0936 & & 3.784 & & & & 54.34 & & 101.3 & 570.6 \\
\hline 52 & 47.2 & 3000 & 3880 & & 12.8 & 131.2 & 3714 & 5125 & 0.1 & 195 & & & 0.0674 & & 2.98 & & 49. & & 82.92 & 0 & 90.3 & 323.5 \\
\hline 53 & 55.14 & 3000 & 1850 & 126.3 & & 118.7 & 4106.04 & 5125 & 0.1 & 195 & & & 0.05 & 1.30 & 2.141 & 1.75 & 73.4 & \begin{tabular}{|l|}
96.04 \\
\end{tabular} & 60.42 & & 39.7 & 199 \\
\hline 54 & 22.22 & 3000 & 2070 & 140.6 & 5.9601 & 127.13 & 3834. & 5125 & 0.1 & 1950 & & & 0.14 & & 1.797 & & & \begin{tabular}{|l|}
96.44 \\
\end{tabular} & $\begin{array}{l}64.45 \\
\end{array}$ & & 91.1 & 414.5 \\
\hline 55 & 25.72 & 3000 & 3870 & 69.16 & 2.4501 & 106.2727 & 4587.26 & 5125 & 0.1 & 1950 & 4875 & 1175 & 0.1237 & $3.42 \mathrm{E}$ & 2.397 & 8.43 & 38.94 & 92.77 & 27.71 & & 82.2 & 477.7 \\
\hline 56 & 48.57 & 3000 & 3720 & 39.183717 & 1.987 & 152.1319 & 3204.46 & 5125 & 0.1 & 1950 & 487500 & 20125 & 0.0655 & 4.11E-04 & 4.175 & $1.11 \mathrm{E}-02$ & 35.94 & 87.24 & & & 62.5 & 440.5 \\
\hline 57 & 45.58 & 3000 & 2870 & 735.415021 & $\begin{array}{l}43.2393 \\
.0202\end{array}$ & 176.3872 & 2763.81 & 5125 & 0.1 & 1950 & 487500 & 2512500 & 0.0698 & 1.97E-04 & 2.423 & $5.44 \mathrm{E}-03$ & 81.1 & 99.32 & $\begin{array}{r}93.2 \\
97.0\end{array}$ & 32.01 & 127.5 & 272.5 \\
\hline $\begin{array}{l}58 \\
59 \\
\end{array}$ & $\begin{array}{l}30.04 \\
23.24\end{array}$ & 3000 & $\frac{5860}{3430}$ & $\begin{array}{r}94.4861685 \\
167.028584\end{array}$ & $\begin{array}{l}2.9302 \\
8.3998 \\
\end{array}$ & $\begin{array}{r}99.0387 \\
150.8694\end{array}$ & 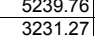 & $\begin{array}{l}5125 \\
5125\end{array}$ & $\begin{array}{l}0.1 \\
0.1 \\
\end{array}$ & $\begin{array}{l}1950 \\
1950\end{array}$ & $\begin{array}{l}487500 \\
487500\end{array}$ & $\begin{array}{r}962500 \\
1987500\end{array}$ & \begin{tabular}{|c|}
0.1559 \\
0.1369 \\
\end{tabular} & $\begin{array}{l}\frac{6.38 E-04}{3.10 E-04} \\
\end{array}$ & $\begin{array}{l}4.045 \\
2.144 \\
\end{array}$ & \begin{tabular}{|l|}
$5.87 \mathrm{E}-03$ \\
$6.61 \mathrm{E}-03$ \\
\end{tabular} & $\begin{array}{l}62.83 \\
68.12\end{array}$ & $94.71 \mid$ & $\begin{array}{l}47.08 \\
70.07\end{array}$ & 0 & $\frac{118.1}{104.8}$ & $\frac{690.1}{472.8}$ \\
\hline 60 & 33.56 & 3000 & 2890 & 123.975717 & $\begin{array}{l}0.090 \\
7.2165\end{array}$ & \begin{tabular}{|l|}
174.6277 \\
\end{tabular} & 2791.65 & 5125 & 0.1 & 1950 & 487500 & 2475000 & 0.0948 & $\begin{array}{l}2.10 E-04 \\
2.11 E-04\end{array}$ & $\begin{array}{l}2.144 \\
2.125\end{array}$ & \begin{tabular}{|l|}
$0.015-00$ \\
$1.10 \mathrm{E}-02$ \\
\end{tabular} & $\begin{array}{ll}00.12 \\
88.57\end{array}$ & $\begin{array}{l}91.01 \\
95.97\end{array}$ & 59.67 & 0 & $\frac{104.0}{63.2}$ & $\begin{array}{l}42.0 \\
324.5 \\
\end{array}$ \\
\hline 61 & 27.69 & 3000 & 3740 & 4.593 & $\mid 0.1179$ & 77.0232 & 6329.26 & 5125 & 0.1 & 1950 & 4875 & 725 & 0.1149 & $3.27 E-04$ & 2.441 & $1.72 \mathrm{E}-02$ & 65.3 & 0 & 0 & 0 & 40.2 & 451.8 \\
\hline 62 & 45.71 & 3000 & 5180 & 41.353101 & $\mid 1.809$ & 131.239 & 3714.6 & 5125 & 0.1 & 1950 & 487500 & 1612500 & \begin{tabular}{|c|}
0.0696 \\
\end{tabular} & $6.82 \mathrm{E}-04$ & 5.954 & $7.46 \mathrm{E}-03$ & 33.26 & 87.91 & 0 & 0 & 92.9 & 667.6 \\
\hline 63 & 63 & 3000 & 1650 & 550.490349 & 23.5815 & 128.5118 & 3793.42 & 5125 & 0.1 & 1950 & 487500 & 1562500 & 0.0505 & $1.46 \mathrm{E}-04$ & 2.457 & \begin{tabular}{|l|}
$8.69 \mathrm{E}-03$ \\
\end{tabular} & 30.09 & 99.09 & 90.92 & 9.17 & 79.8 & 199.9 \\
\hline
\end{tabular}




\begin{tabular}{|c|c|c|c|c|c|c|c|c|c|c|c|c|c|c|c|c|c|c|c|c|c|c|}
\hline $\begin{array}{c}\text { Realizatio } \\
n\end{array}$ & $\begin{array}{c}\text { Velocity } \\
(\mathrm{m} / \mathrm{y})\end{array}$ & $\begin{array}{c}\text { Fixed Source } \\
\text { Concentratio } \\
\mathrm{n}(\mathrm{mg} / \mathrm{L})\end{array}$ & $\begin{array}{c}\text { Time of } \\
\text { Maximum at } \\
\text { Compliance } \\
\text { Boundary } \\
\text { (y) }\end{array}$ & $\begin{array}{c}\text { Maximum } \\
\text { Concentratio } \\
\text { n Compliance } \\
\text { Boundary } \\
(\mu \mathrm{g} / \mathrm{L})\end{array}$ & \begin{tabular}{|c|} 
Maximum \\
Release \\
Rate at \\
Complianc \\
e Boundary \\
$(\mathrm{Kg} / \mathrm{y})$ \\
\end{tabular} & $\begin{array}{l} \\
\text { Source } \\
\text { Rate } \\
\text { (Kg/y) } \\
\end{array}$ & $\begin{array}{c}\text { Source } \\
\text { Duration } \\
\text { (y) } \\
\end{array}$ & \begin{tabular}{|c|} 
1-D \\
Streamtub \\
e Length \\
$(\mathrm{m})$
\end{tabular} & $\begin{array}{l}\text { Base } \\
\text { Porosity } \\
\left(\mathrm{m}^{3} / \mathrm{m}^{3}\right)\end{array}$ & $\begin{array}{c}\text { Bulk } \\
\text { Densty } \\
\left(\mathrm{Kg} / \mathrm{m}^{3}\right)\end{array}$ & $\begin{array}{c}\text { Remaining } \\
\text { Carbon } \\
\text { Tetrachloride } \\
\text { Source } \\
(\mathrm{Kg}) \\
\end{array}$ & $\begin{array}{c}\text { Volume at or } \\
\text { above Fixed } \\
\text { Source } \\
\begin{array}{c}\text { Concentratio } \\
n(m 3)\end{array} \\
\end{array}$ & $\begin{array}{l}\text { Porosity } \\
\left(\mathrm{m}^{3} / \mathrm{m}^{3}\right)\end{array}$ & $\begin{array}{c}\mathrm{Kd} \\
\left(\mathrm{m}^{3} / \mathrm{Kg}\right)\end{array}$ & Retardation & $\begin{array}{c}\text { First } \\
\text { Order } \\
\text { Rate } \\
\text { Constant } \\
(1 / y) \\
\end{array}$ & \begin{tabular}{|c|}
$\begin{array}{c}\text { Longitudinal } \\
\text { Dispersivity } \\
(\mathrm{m})\end{array}$ \\
\end{tabular} & $\begin{array}{c}\text { Required } \\
\text { Source } \\
\text { Cleanup } \\
\text { Percentage } \\
(5 \mu g / L \\
\text { Compliance }) \\
\end{array}$ & \begin{tabular}{|c|} 
Required \\
Source \\
Cleanup \\
Percentage \\
$(50 \mu \mathrm{g} / \mathrm{L}$ \\
Compliance)
\end{tabular} & $\begin{array}{c}\text { Required } \\
\text { Source } \\
\text { Cleanup } \\
\text { Percentage } \\
\text { (500 } \mathrm{\mu g} / \mathrm{L} \\
\text { Compliance) } \\
\end{array}$ & \begin{tabular}{|c|} 
Abiotic \\
Reaction \\
Half Time \\
(y) \\
\end{tabular} & \begin{tabular}{|c|} 
Carbon \\
Tetrachloride \\
Travel Time \\
(y) \\
\end{tabular} \\
\hline & & 3000 & 2130 & \begin{tabular}{|r|}
32.473706 \\
\end{tabular} & \begin{tabular}{|l|}
2.4547 \\
\end{tabular} & 226.7679 & 2149.78 & \begin{tabular}{|l|}
5125 \\
\end{tabular} & & 1950 & $\begin{array}{r}487500 \\
\end{array}$ & 3662500 & 0.1478 & 1.97E-04 & \begin{tabular}{|r|}
1.673 \\
\end{tabular} & $1.23 \mathrm{E}-02$ & \begin{tabular}{|r|}
52.78 \\
\end{tabular} & & & & 56.6 & 398.3 \\
\hline 65 & $\begin{array}{ll}24.66 \\
14.17\end{array}$ & 3000 & 4670 & \begin{tabular}{|l|}
127.550171 \\
\end{tabular} & $\begin{array}{r}4.7086 \\
146209\end{array}$ & 110.7481 & $\begin{array}{l}4401.88 \\
200702\end{array}$ & 5125 & 0.1 & 1950 & 487500 & 1250000 & 0.129 & 3.99E-04 & 2.562 & $6.42 \mathrm{E}-03$ & 52.21 & 96.08 & 60.8 & & 108 & 532.4 \\
\hline 66 & $\begin{array}{l}46.17 \\
1097\end{array}$ & 3000 & 830 & \begin{tabular}{|l|}
270.005206 \\
\end{tabular} & \begin{tabular}{|c|}
14.6398 \\
70103 \\
\end{tabular} & \begin{tabular}{|l|l|}
162.6609 \\
100020
\end{tabular} & 2997.03 & 5125 & 0.1 & 1950 & 487500 & 2225000 & $\begin{array}{ll}0.0689 \\
\end{array}$ & 5.52E-05 & 1.405 & $1.62 \mathrm{E}-02$ & 72.97 & 98.15 & $\begin{array}{l}81.48 \\
\end{array}$ & & 42.8 & 156 \\
\hline 67 & 49.79 & 3000 & 3770 & 232.270421 & 7.8143 & $\begin{array}{r}100.929 \\
\end{array}$ & 4830.13 & 5125 & 0.1 & 1950 & 487500 & 1087500 & 0.0639 & $2.99 \mathrm{E}-04$ & 3.368 & $7.65 \mathrm{E}-03$ & 21.89 & 97.85 & 78.47 & & 90.6 & 346.7 \\
\hline 68 & 26.34 & 3000 & 3480 & \begin{tabular}{|r|}
16.932378 \\
\end{tabular} & 1.1667 & 206.7108 & 2358.37 & 5125 & 0.1 & 1950 & 487500 & 3187500 & 0.1208 & 6.97E-04 & 3.918 & $8.11 \mathrm{E}-03$ & 50.27 & 70.47 & & & 85.5 & 762.5 \\
\hline 69 & & 3000 & 2150 & \begin{tabular}{|l|}
102.490738 \\
\end{tabular} & 4.6898 & 137.2743 & 3551.28 & 5125 & 0.1 & 1950 & 487500 & 1725000 & 0.0776 & 1.65E-04 & 2.075 & $1.42 \mathrm{E}-02$ & 64.47 & 95.12 & 51.22 & & 48.9 & 259.5 \\
\hline 70 & 56.81 & 3000 & 4360 & $\begin{array}{r}4.692344 \\
\end{array}$ & 0.2074 & 132.592 & 3676.69 & 5125 & 0.1 & 1950 & 487500 & 1637500 & 0.056 & 7.09E-04 & 7.404 & $1.71 \mathrm{E}-02$ & 82.08 & 0 & 0 & & 40.6 & 667.9 \\
\hline 71 & 25.31 & 3000 & 4010 & $\begin{array}{r}0.758277 \\
1.20707\end{array}$ & 0.0337 & 133.2659 & 3658.1 & 5125 & 0.1 & 1950 & 487500 & 1650000 & 0.1257 & 4.88E-04 & 2.961 & $1.68 \mathrm{E}-02$ & 45.79 & 0 & 0 & & 41.2 & 599.6 \\
\hline 72 & $\begin{array}{l}27.21 \\
2932\end{array}$ & $\begin{array}{l}3000 \\
3000 \\
3000\end{array}$ & 5330 & $\begin{array}{r}14.329763 \\
35062035\end{array}$ & $\begin{array}{l}0.5112 \\
1017 \\
\end{array}$ & \begin{tabular}{|l|l|}
107.0251 \\
86.4774
\end{tabular} & $\begin{array}{l}4555.01 \\
5637.31\end{array}$ & $\begin{array}{l}5125 \\
5125\end{array}$ & 0.1 & $\begin{array}{l}1950 \\
1900\end{array}$ & 487500 & $\begin{array}{l}1187500 \\
\end{array}$ & $\begin{array}{l}0.1169 \\
\end{array}$ & $6.88 \mathrm{E}-04$ & 3.975 & \begin{tabular}{|l|l|}
$8.47 \mathrm{E}-03$ \\
$0.095-03$
\end{tabular} & $\begin{array}{r}44.95 \\
2.54 \\
\end{array}$ & $\begin{array}{l}65.11 \\
857.1\end{array}$ & & & 81.8 & 748.6 \\
\hline $\begin{array}{l}73 \\
74\end{array}$ & $\begin{array}{l}29.32 \\
46.04\end{array}$ & $\begin{array}{l}3000 \\
3000\end{array}$ & $\frac{3500}{1520}$ & $\begin{array}{l}35.0620355 \\
393.79069\end{array}$ & $\begin{array}{r}\frac{1.0107}{19.2181} \\
\mid\end{array}$ & \begin{tabular}{|r|}
86.4774 \\
146.4082
\end{tabular} & 5637.31 & $\begin{array}{l}5125 \\
5125\end{array}$ & $\begin{array}{l}0.1 \\
0.1 \\
\end{array}$ & $\begin{array}{l}1950 \\
1950\end{array}$ & $\begin{array}{ll}487500 \\
487500\end{array}$ & 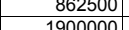 & $\begin{array}{l}0.1085 \\
0.0691 \\
\end{array}$ & $\begin{array}{r}3.61 \mathrm{E}-04 \\
1.44 \mathrm{E}-04 \\
\end{array}$ & $\begin{array}{l}2.682 \\
2054\end{array}$ & \begin{tabular}{|l|}
$9.99 \mathrm{E}-03$ \\
$913 \mathrm{E}-03$
\end{tabular} & $\begin{array}{l}22.54 \\
33.26\end{array}$ & 85.44 & 0 & & 69.4 & 468.9 \\
\hline 75 & 33.49 & 3000 & 650 & \begin{tabular}{|l}
450.832377 \\
\end{tabular} & 19.21896 & \begin{tabular}{|r|}
14.40682 \\
88.9661 \\
\end{tabular} & 5479.61 & 5125 & $\begin{array}{l}0.1 \\
0.1\end{array}$ & $\begin{array}{l}1950 \\
1950\end{array}$ & $\frac{487500}{487500}$ & $\frac{1900000}{900000}$ & \begin{tabular}{|c}
0.0691 \\
0.095
\end{tabular} & $\begin{array}{l}1.44 \mathrm{E}-04 \\
5.29 \mathrm{E}-05\end{array}$ & $\begin{array}{l}2.2054 \\
1.282\end{array}$ & \begin{tabular}{|l|}
$9.13 \mathrm{E}-03$ \\
$9.81 \mathrm{E}-03$ \\
\end{tabular} & $\begin{array}{l}34.26 \\
32.53\end{array}$ & $\begin{array}{l}98.13 \\
98.89 \\
\end{array}$ & $\begin{array}{r}88.31 \\
88.91\end{array}$ & $\frac{0}{0}$ & $\begin{array}{l}75.9 \\
70.6\end{array}$ & $\begin{array}{l}228.6 \\
196.1\end{array}$ \\
\hline 76 & 23.85 & 3000 & 3890 & 111.420926 & 5.172 & 139.2566 & 3500.73 & 5125 & 0.1 & 1950 & 487500 & 1762500 & 0.1334 & $4.34 \mathrm{E}-04$ & 2.644 & \begin{tabular}{|l|}
$6.26 \mathrm{E}-03$ \\
\end{tabular} & 46.73 & 95.51 & 55.13 & & 110.8 & 568.2 \\
\hline 77 & 43.94 & 3000 & 2490 & 286.238735 & 13.9079 & 145.7654 & 3344.42 & 5125 & 0.1 & 1950 & 487500 & 1887500 & 0.0724 & $2.14 \mathrm{E}-04$ & 2.493 & \begin{tabular}{|l|l|}
$8.38 \mathrm{E}-03$ \\
\end{tabular} & 32.24 & 98.25 & 82.53 & & 82.7 & 290.7 \\
\hline 78 & 22.28 & 3000 & 2510 & 236.326511 & 8.1926 & 103.9994 & 4687.53 & 5125 & 0.1 & 1950 & 487500 & 1137500 & 0.1428 & $2.54 \mathrm{E}-04$ & 1.899 & \begin{tabular}{|c|}
$6.00 \mathrm{E}-03$ \\
\end{tabular} & 33.34 & 97.88 & 78.84 & & 115.5 & 436.9 \\
\hline 79 & 25.57 & 3000 & 4620 & 0.568831 & 0.0173 & 91.4206 & 5332.5 & 5125 & 0.1 & 1950 & 487500 & 937500 & 0.1244 & $5.00 \mathrm{E}-04$ & 3.031 & $1.56 \mathrm{E}-02$ & 20.24 & 0 & & 0 & 44.6 & 607.5 \\
\hline 80 & 21.39 & 3000 & 5030 & \begin{tabular}{|l|l|}
126.875199 \\
\end{tabular} & 4.4945 & 106.2727 & 4587.26 & 5125 & 0.1 & 1950 & 487500 & 1175000 & 0.1487 & $6.02 \mathrm{E}-04$ & 3.048 & $5.09 \mathrm{E}-03$ & 93.26 & 96.06 & 60.59 & & 136.1 & 730.2 \\
\hline 81 & 54.2 & 3000 & 4260 & 129.550689 & 5.3102 & 122.9684 & 3964.43 & 5125 & 0.1 & 1950 & 487500 & 1462500 & 0.0587 & $3.50 \mathrm{E}-04$ & 4.014 & $9.35 \mathrm{E}-03$ & 52.36 & 96.14 & 61.41 & 0 & 74.2 & 379.6 \\
\hline 82 & 43.28 & 3000 & 1330 & \begin{tabular}{|l|}
1087.902467 \\
\end{tabular} & 40.4284 & 111.4852 & 4372.78 & 5125 & 0.1 & 1950 & 487500 & 1262500 & 0.0735 & $1.23 \mathrm{E}-04$ & 1.843 & $4.71 \mathrm{E}-03$ & 36.87 & 99.54 & 95.4 & 54.04 & 147.1 & 218.2 \\
\hline 83 & 23.12 & 3000 & 3270 & 105.824665 & 6.222 & 176.3872 & 2763.81 & 5125 & 0.1 & 1950 & 487500 & 2512500 & 0.1376 & $4.14 \mathrm{E}-04$ & 2.521 & $6.18 \mathrm{E}-03$ & 19.94 & 95.28 & 52.75 & & 112.1 & 558.8 \\
\hline 84 & 22.33 & 3000 & 2630 & 22.784665 & 1.0476 & 137.9366 & 3534.23 & 5125 & 0.1 & 1950 & 487500 & 1737500 & 0.1425 & $2.85 \mathrm{E}-04$ & 2.01 & $1.12 \mathrm{E}-02$ & 31.85 & 78.06 & 0 & & 61.8 & 461.3 \\
\hline 85 & 32.17 & 3000 & 1630 & 84.409913 & 3.9367 & 139.9143 & 3484.28 & 5125 & 0.1 & 1950 & 487500 & 1775000 & 0.0989 & 1.77E-04 & 1.902 & $1.22 \mathrm{E}-02$ & 29.19 & 94.08 & 40.77 & & 56.6 & 303.1 \\
\hline 86 & 21.77 & 3000 & 3560 & 79.60256 & 3.8678 & 145.7654 & 3344.42 & 5125 & 0.1 & 1950 & 487500 & 1887500 & 0.1461 & $3.16 \mathrm{E}-04$ & 2.094 & $7.88 \mathrm{E}-03$ & 47.6 & 93.72 & 37.19 & & 87.9 & 492.9 \\
\hline 87 & 34.88 & 3000 & 2980 & 12.357747 & 0.7023 & 170.4873 & 2859.45 & 5125 & 0.1 & 1950 & 487500 & 2387500 & 0.0912 & $2.89 \mathrm{E}-04$ & 2.601 & $1.83 \mathrm{E}-02$ & 99.18 & 59.54 & & & 37.8 & 382.2 \\
\hline 88 & 21.72 & 3000 & 4490 & 0.658439 & 0.0261 & 118.7276 & 4106.04 & 5125 & 0.1 & 1950 & 487500 & 1387500 & 0.1465 & $4.98 \mathrm{E}-04$ & 2.719 & $1.47 \mathrm{E}-02$ & 26.72 & 0 & 0 & & 47.2 & 641.7 \\
\hline 89 & 35.11 & 3000 & 3530 & 55.16802 & 2.8667 & 155.8885 & 3127.24 & 5125 & 0.1 & 1950 & 487500 & 2087500 & 0.0906 & $4.18 \mathrm{E}-04$ & 3.332 & $9.30 \mathrm{E}-03$ & 50.87 & 90.94 & 9.37 & & 74.5 & 486.3 \\
\hline 90 & 27.31 & 3000 & 2530 & 242.72278 & $\begin{array}{l}6.8608 \\
\end{array}$ & 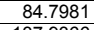 & 5748.95 & 5125 & 0.1 & 1950 & 487500 & $\begin{array}{l}837500 \\
\end{array}$ & 0.1165 & $2.06 \mathrm{E}-04$ & 1.892 & $7.44 \mathrm{E}-03$ & 54.23 & 97.94 & 79.4 & & 93.2 & 355.1 \\
\hline 91 & 41.97 & 3000 & 3840 & 14.372259 & 0.6608 & 137.9366 & 3534.23 & 5125 & 0.1 & 1950 & & 1737500 & 0.0758 & 3.57E-04 & 3.381 & & 33.87 & 65.21 & 0 & & 47.9 & 412.8 \\
\hline 92 & 33.28 & 3000 & 5090 & \begin{tabular}{|r|}
90.911757 \\
\end{tabular} & 3.0351 & 100.1541 & 4867.5 & 5125 & 0.1 & 1950 & & & & 3.71E-04 & 2.961 & & 47.7 & 94.5 & 45 & & 82.5 & 456 \\
\hline 93 & 58.37 & 3000 & 2110 & \begin{tabular}{|l|}
262.139989 \\
\end{tabular} & 10.4366 & 119.4396 & 4081.56 & 5125 & 0.1 & 1950 & 487500 & 1400000 & 0.0545 & 1.77E-04 & 2.644 & $1.09 \mathrm{E}-02$ & 31.4 & 98.09 & 80.93 & & 63.5 & 232.1 \\
\hline 94 & 22.02 & 3000 & 3620 & \begin{tabular}{|l|}
172.258973 \\
\end{tabular} & $\begin{array}{r}6.611 \\
\end{array}$ & 115.1349 & 4234.16 & 5125 & 0.1 & 1950 & 487500 & 1325000 & 0.1445 & $2.79 \mathrm{E}-04$ & 1.975 & 6.62E-03 & 59.75 & 97.1 & 70.97 & & 104.6 & 459.8 \\
\hline 95 & 31.31 & 3000 & 1730 & \begin{tabular}{|r|}
63.408631 \\
\end{tabular} & $\begin{array}{r}1.9151 \\
1.7002\end{array}$ & \begin{tabular}{|r|}
90.6061 \\
\end{tabular} & 5380.43 & 5125 & 0.1 & 1950 & 487500 & $\begin{array}{r}925000 \\
\end{array}$ & 0.1016 & 1.45E-04 & 1.721 & $1.48 \mathrm{E}-02$ & 62.93 & 92.11 & 21.15 & & 46.8 & 281.6 \\
\hline 96 & $\begin{array}{l}42.82 \\
4798\end{array}$ & 3000 & 710 & \begin{tabular}{|r|}
355.247009 \\
\end{tabular} & $\begin{array}{r}14.7269 \\
10271 \\
\end{array}$ & 124.3658 & $\begin{array}{l}3919.89 \\
2008.21\end{array}$ & 5125 & 0.1 & 1950 & 487500 & 1487500 & 0.0743 & $3.66 \mathrm{E}-05$ & \begin{tabular}{|c|c|c|}
1.249 \\
-5207
\end{tabular} & $1.48 \mathrm{E}-02$ & 75.81 & 98.59 & 85.93 & & 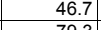 & 149.5 \\
\hline $\begin{array}{l}97 \\
98\end{array}$ & $\begin{array}{l}47.98 \\
3389\end{array}$ & $\begin{array}{l}3000 \\
3000 \\
\end{array}$ & $\begin{array}{l}4240 \\
5530\end{array}$ & \begin{tabular}{|r|}
35.867159 \\
10708972
\end{tabular} & $\begin{array}{l}1.9374 \\
33499 \\
\end{array}$ & \begin{tabular}{|r|}
162.0511 \\
938425
\end{tabular} & $\begin{array}{l}3008.31 \\
5194.88\end{array}$ & $\begin{array}{l}5125 \\
5525\end{array}$ & 0.1 & $\begin{array}{l}1950 \\
1950 \\
\end{array}$ & 487500 & $\begin{array}{r}2212500 \\
\end{array}$ & 0.0663 & 5.65E-04 & \begin{tabular}{|c|c|}
5.307 \\
3.009
\end{tabular} & \begin{tabular}{|l|l|}
$8.74 \mathrm{E}-03$ \\
$764 \mathrm{E}-03$
\end{tabular} & 26.21 & $\begin{array}{l}86.06 \\
0.53 \\
5.32\end{array}$ & \begin{tabular}{r|}
0 \\
531
\end{tabular} & & $\begin{array}{l}79.3 \\
007\end{array}$ & 566.8 \\
\hline $\begin{array}{l}98 \\
99 \\
\end{array}$ & $\begin{array}{l}38.89 \\
62.75 \\
\end{array}$ & 30000 & 55300 & $\begin{array}{r}107.089926 \\
73.773436 \\
\end{array}$ & $\begin{array}{l}3.3499 \\
2.5575 \\
\end{array}$ & \begin{tabular}{|r|}
93.83 .425 \\
103.9994 \\
\end{tabular} & $\begin{array}{l}5198.87 \\
4683.53\end{array}$ & $\frac{5125}{5125}$ & $\begin{array}{l}0.1 \\
0.1 \\
\end{array}$ & $\frac{1950}{1950}$ & $\frac{488}{487 !}$ & $\begin{array}{r}915000 \\
137500\end{array}$ & \begin{tabular}{|l}
0.0888 \\
0.0507
\end{tabular} & $\begin{array}{l}\mid 4.70 \mathrm{E}-04 \\
4.04 \mathrm{E}-04\end{array}$ & $\begin{array}{l}3.908 \\
5.028\end{array}$ & \begin{tabular}{|l|}
$1.64 \mathrm{E}-03$ \\
$1.05 \mathrm{E}-02$ \\
\end{tabular} & $\begin{array}{r}r 1.09 \\
44.5 \\
\end{array}$ & $\begin{array}{l}95.33 \\
93.22 \\
\end{array}$ & $\begin{array}{l}53.31 \\
32.22 \\
\end{array}$ & & $\begin{array}{l}90.7 \\
66.1\end{array}$ & $\begin{array}{l}514.9 \\
410.7 \\
\end{array}$ \\
\hline 100 & 63.25 & 3000 & 4870 & 43.406288 & & \begin{tabular}{|l|l|}
118.7276 \\
\end{tabular} & 4106.04 & 5125 & 0.1 & 1950 & 487 & & 0.0503 & $4.85 \mathrm{E}-04$ & 5.874 & \begin{tabular}{|c|}
$1.04 \mathrm{E}-02$ \\
\end{tabular} & 35.04 & 88.48 & & & 66.6 & $\begin{array}{r}40.1 \\
476\end{array}$ \\
\hline 101 & 24.16 & 3000 & 5220 & 168.440464 & & 104.7599 & 4653.5 & 5125 & 0.1 & 1950 & & 1150000 & 0.1317 & $6.11 \mathrm{E}-04$ & 3.344 & \begin{tabular}{|l|}
$4.60 \mathrm{E}-03$ \\
\end{tabular} & 70.34 & 97.03 & 70.32 & & 150.8 & 709.4 \\
\hline 102 & 25.95 & 3000 & 1250 & 483.060162 & 22.4231 & 139.2566 & 3500.73 & 5125 & 0.1 & 1950 & & 1762500 & 0.1226 & $1.02 \mathrm{E}$ & 1.422 & & & 98.96 & 89.65 & & 104.2 & 280.9 \\
\hline 103 & 43.4 & 3000 & 4270 & \begin{tabular}{|r|}
54.817261 \\
\end{tabular} & & \begin{tabular}{|l|}
129.879 \\
\end{tabular} & 3753.49 & 5125 & 0.1 & 1950 & & & & & 4.489 & & & 90.88 & 8.79 & & 74.9 & 530.1 \\
\hline 104 & 31.34 & 3000 & 4840 & 64.345794 & & \begin{tabular}{|l|l|}
131.9164 \\
\end{tabular} & 3695.52 & 5125 & 0.1 & 1950 & & 1625000 & & & 4.461 & & & & 22.29 & & 115.7 & 729.4 \\
\hline 105 & 23.6 & 3000 & 1230 & 94.719535 & 4.86 & 154.0159 & 3165.26 & 5125 & 0.1 & 1950 & 487 & 2050000 & 0.1348 & $1.56 \mathrm{E}-04$ & 1.585 & 1.03 & 19. & 94.72 & 47.21 & & 67.7 & 344.1 \\
\hline 106 & 55.04 & 3000 & 1950 & $\mid 179.821317$ & & 118.01 & 4130.88 & 5125 & 0.1 & 1950 & 487 & 1375000 & 0.0578 & $1.74 \mathrm{E}-04$ & 2.523 & $1.25 \mathrm{E}-02$ & 29. & 97.22 & 72.19 & & 55.6 & 234.9 \\
\hline 107 & 29.62 & 3000 & 2780 & 14.623199 & 0.91 & 187.3347 & 2602.29 & 5125 & 0.1 & 1950 & 487 & 2750000 & 0.1074 & $3.02 \mathrm{E}-04$ & 2.421 & $1.49 \mathrm{E}-02$ & 69.5 & 65.81 & 0 & & 46.4 & 418.9 \\
\hline 108 & 26.42 & 3000 & 3070 & 113.968 & 6.94 & 182.7653 & 2667.36 & 5125 & 0.1 & 1950 & & 2650000 & 0.1204 & $3.63 \mathrm{E}-04$ & 2.522 & 6.93 & 22. & 95.61 & 56.13 & & 100 & 489.2 \\
\hline 109 & 21.8 & & 3930 & & 0.3272 & 131.9164 & 3695.52 & 5125 & 0.1 & 1950 & & & 0.1459 & 3.86 & 2.339 & 1.25 & 51.43 & 32.8 & & & 55.7 & 549.7 \\
\hline 110 & 47.41 & & 4140 & 16.80 & & \begin{tabular}{|l|l|}
127.1374 \\
\end{tabular} & 383 & 5125 & 0.1 & 1950 & & & 0.0671 & & 4.398 & & 77.02 & 70.25 & & & 47.3 & 475.4 \\
\hline 111 & 29.13 & 3000 & 5420 & & 0.03 & & & 5125 & 0.1 & 1950 & & & & & 3.516 & & 39. & 0 & & & 45.3 & 618.6 \\
\hline 112 & & 30 & 1890 & 351.6 & 12.8 & 110.0086 & 443 & 5125 & 0.1 & 195 & & & 0.11 & & 1.7 & & 43. & 98.58 & 85.78 & & 100.3 & 320.1 \\
\hline 113 & 30.74 & 30 & 3990 & & 0.5 & & & 5125 & 0.1 & 195 & & & & & 3.6 & & & 59 & & & 55 & 504.5 \\
\hline 114 & & 30 & 34 & & 31.6 & 150.8 & & 5125 & 0.1 & 195 & & & & & & & & 99.21 & \begin{tabular}{ll|}
92.07 \\
\end{tabular} & 20.67 & 163.4 & 381.8 \\
\hline 115 & & 3000 & 1430 & & & & & 5125 & 0.1 & 195 & & & & & 2.3. & & 14. & 93.28 & 32.76 & & 48 & 262.2 \\
\hline 116 & 47.7 & 3000 & 4170 & & & & & 5125 & 0.1 & 195 & & & & & 4. & & 5 & 32 & 83.16 & & 121.4 & 44 \\
\hline 117 & 31.16 & & & & & & & 512 & 0.1 & 19 & & & & & 2 & & & 99.14 & 91.43 & 14.32 & 146.5 & \\
\hline 118 & 26.8 & 30 & 12 & & 4.8. & \begin{tabular}{|l|l|}
166.2959 \\
\end{tabular} & & 5125 & 0.1 & 19 & & & & & 1.645 & & & & 43.08 & & & \\
\hline 119 & 22.18 & 3000 & & & & & & 125 & 0.1 & 19 & & & 0.14 & & & & & & 79.33 & & & 04.3 \\
\hline 120 & 30.47 & 3000 & 3460 & 216.7 & 10.8541 & 150.2362 & & 5125 & 0.1 & 1950 & & 5000 & 0.1044 & & 2.447 & & & 97 & 76.93 & & 102 & 411.6 \\
\hline 121 & $\begin{array}{l}43.58 \\
2\end{array}$ & 3000 & 4790 & & $\begin{array}{l}1.1246 \\
25795\end{array}$ & \begin{tabular}{|l|l|}
108.5219 \\
122.0
\end{tabular} & 4492.18 & 5125 & 0.1 & $\frac{195}{105}$ & & & & & & & & 83.92 & & & 58.1 & 436.4 \\
\hline 122 & 22.89 & 3000 & 3020 & \begin{tabular}{|r|}
87.804172 \\
\end{tabular} & 3.5785 & \begin{tabular}{|l|}
122.2668 \\
150.2302
\end{tabular} & 3987.18 & 5125 & 0.1 & 1950 & 487500 & & 0.139 & 2.35E-04 & 1.854 & $9.25 \mathrm{E}-03$ & 68.2 & 94.31 & 43.06 & & 75 & 415.2 \\
\hline 123 & $\begin{array}{ll}49.86 \\
20.78\end{array}$ & 3000 & 3460 & \begin{tabular}{|r|}
126.325043 \\
14573091
\end{tabular} & 6.3262 & \begin{tabular}{|l|}
150.2362 \\
1372713
\end{tabular} & $\begin{array}{l}3244.89 \\
3251\end{array}$ & $\begin{array}{l}5125 \\
5125\end{array}$ & 0.1 & $\begin{array}{l}1950 \\
1900 \\
\end{array}$ & 487500 & 1975000 & 0.0638 & 2.83E-04 & 3.239 & 9.97E-03 & 23.7 & 96.04 & 60.42 & & 69.5 & 32.9 \\
\hline 124 & 26.78 & 3000 & 4190 & $\begin{array}{l}14.573091 \\
1.10919\end{array}$ & $\begin{array}{l}0.6668 \\
5241 \\
\end{array}$ & \begin{tabular}{|l|}
137.2743 \\
1107481
\end{tabular} & 3551.28 & $\begin{array}{l}5125 \\
5125\end{array}$ & 0.1 & $\begin{array}{c}1950 \\
1050 \\
\end{array}$ & 487500 & 1725000 & 0.1188 & 5.71E-04 & 3.432 & 9.32E-03 & 41.73 & 65.69 & 0 & & 74.4 & 56.8 \\
\hline$\frac{125}{126}$ & $\frac{60.37}{2388}$ & 3000 & 4670 & \begin{tabular}{|r|}
142.10919 \\
\end{tabular} & $\begin{array}{l}5.2461 \\
33203 \\
3\end{array}$ & 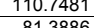 & $\begin{array}{l}4401.88 \\
5989.78\end{array}$ & $\begin{array}{l}5125 \\
5125\end{array}$ & 0.1 & $\begin{array}{l}1950 \\
1900 \\
\end{array}$ & & $\begin{array}{r}1250000 \\
78700 \\
\end{array}$ & 0.0527 & $\begin{array}{l}3.63 \mathrm{E}-04 \\
778 E-05 \\
\end{array}$ & $\begin{array}{l}4.484 \\
1295\end{array}$ & & $\begin{array}{l}73.56 \\
5.57 \\
\end{array}$ & $\begin{array}{l}96.48 \\
0909\end{array}$ & $\frac{64.82}{59.22}$ & & $\frac{12.3}{57.5}$ & $380.7 \mid$ \\
\hline 126 & 23.88 & 3000 & 1150 & \begin{tabular}{|l|}
122.606895 \\
\end{tabular} & 3.3263 & 81.3886 & 5989.78 & 5125 & & 1950 & 487500 & 787500 & 0.1332 & $7.78 \mathrm{E}-05$ & 1.295 & $1.21 \mathrm{E}-02$ & 58.57] & & 59.22 & & $57.5 \mid$ & 277.9 \\
\hline
\end{tabular}




\begin{tabular}{|c|c|c|c|c|c|c|c|c|c|c|c|c|c|c|c|c|c|c|c|c|c|c|}
\hline $\begin{array}{c}\text { Realizatio } \\
n\end{array}$ & $\begin{array}{c}\text { Velocity } \\
(\mathrm{m} / \mathrm{y})\end{array}$ & $\begin{array}{c}\text { Fixed Source } \\
\text { Concentratio } \\
\mathrm{n}(\mathrm{mg} / \mathrm{L})\end{array}$ & $\begin{array}{c}\text { Time of } \\
\text { Maximum at } \\
\text { Compliance } \\
\text { Boundary } \\
\text { (y) }\end{array}$ & $\begin{array}{c}\text { Maximum } \\
\text { Concentratio } \\
\text { n Compliance } \\
\text { Boundary } \\
(\mu \mathrm{g} / \mathrm{L})\end{array}$ & \begin{tabular}{|c|} 
Maximum \\
Release \\
Rate at \\
Complianc \\
e Boundary \\
$(\mathrm{Kg} / \mathrm{y})$ \\
\end{tabular} & $\begin{array}{c}\text { Source } \\
\text { Rate } \\
(\mathrm{Kg} / \mathrm{y}) \\
\end{array}$ & $\begin{array}{c}\text { Source } \\
\text { Duration } \\
\text { (y) }\end{array}$ & \begin{tabular}{|c|} 
1-D \\
Streamtub \\
e Length \\
$(\mathrm{m})$
\end{tabular} & $\begin{array}{l}\text { Base } \\
\text { Porosity } \\
\left(\mathrm{m}^{3} / \mathrm{m}^{3}\right)\end{array}$ & $\begin{array}{c}\text { Bulk } \\
\text { Densty } \\
\left(\mathrm{Kg} / \mathrm{m}^{3}\right)\end{array}$ & $\begin{array}{c}\text { Remaining } \\
\text { Carbon } \\
\text { Tetrachloride } \\
\text { Source } \\
(\mathrm{Kg}) \\
\end{array}$ & $\begin{array}{c}\text { Volume at or } \\
\text { above Fixed } \\
\text { Source } \\
\begin{array}{c}\text { Concentratio } \\
n(m 3)\end{array} \\
\end{array}$ & $\begin{array}{l}\text { Porosity } \\
\left(\mathrm{m}^{3} / \mathrm{m}^{3}\right)\end{array}$ & $\begin{array}{c}\mathrm{Kd} \\
\left(\mathrm{m}^{3} / \mathrm{Kg}\right)\end{array}$ & Retardation & $\begin{array}{c}\text { First } \\
\text { Order } \\
\text { Rate } \\
\text { Constant } \\
(1 / y) \\
\end{array}$ & \begin{tabular}{|c} 
Longitudinal \\
Dispersivity \\
$(\mathrm{m})$
\end{tabular} & \begin{tabular}{|c|} 
Required \\
Source \\
Cleanup \\
Percentage \\
$(5 \mu \mathrm{g} / \mathrm{L}$ \\
Compliance $)$ \\
\end{tabular} & \begin{tabular}{|c|} 
Required \\
Source \\
Cleanup \\
Percentage \\
$(50 \mu \mathrm{g} / \mathrm{L}$ \\
Compliance)
\end{tabular} & $\begin{array}{c}\text { Required } \\
\text { Source } \\
\text { Cleanup } \\
\text { Percentage } \\
\text { (500 } \mathrm{\mu g} / \mathrm{L} \\
\text { Compliance) } \\
\end{array}$ & \begin{tabular}{|c|}
$\begin{array}{c}\text { Abiotic } \\
\text { Reaction } \\
\text { Half Time } \\
(y)\end{array}$ \\
\end{tabular} & \begin{tabular}{|c|} 
Carbon \\
Tetrachloride \\
Travel Time \\
(y) \\
\end{tabular} \\
\hline & 53.74 & 3000 & 4220 & $\begin{array}{r}53.014972 \\
\end{array}$ & \begin{tabular}{|l|}
1.8647 \\
\end{tabular} & 105.5176 & \begin{tabular}{|l|l|}
4620.08 \\
\end{tabular} & 5125 & & 1950 & $\begin{array}{r}487500 \\
\end{array}$ & 1162500 & 0.0592 & $2.46 \mathrm{E}-04$ & 3.104 & $1.65 \mathrm{E}-02$ & \begin{tabular}{|r|}
86.02 \\
\end{tabular} & $\begin{array}{r}90.57 \\
\end{array}$ & & & & 296.1 \\
\hline 128 & 62.62 & 3000 & 3100 & $\begin{array}{r}785.012755 \\
370551173\end{array}$ & \begin{tabular}{|l|l|}
29.7473 \\
107284
\end{tabular} & \begin{tabular}{|l|l|}
113.6821 \\
917091
\end{tabular} & $\begin{array}{ll}4288.27 \\
5718.05\end{array}$ & 5125 & 0.1 & 1950 & 487500 & 1300000 & 0.0508 & 2.17E-04 & 3.162 & $5.30 \mathrm{E}-03$ & 28.81 & 99.36 & 93.63 & 36.31 & 130.7 & 258.8 \\
\hline 129 & 28.84 & 3000 & 1390 & 379.551173 & 10.7284 & \begin{tabular}{|l}
84.7981 \\
\end{tabular} & 5748.95 & 5125 & 0.1 & 1950 & 487500 & 837500 & 0.1103 & $1.02 \mathrm{E}-04$ & 1.465 & $8.23 \mathrm{E}-03$ & \begin{tabular}{ll|l|}
61.96 \\
\end{tabular} & $\begin{array}{l}98.68 \\
\end{array}$ & 86.83 & 0 & 84.2 & 260.4 \\
\hline 130 & 23.76 & 3000 & 940 & $\begin{array}{l}78.099195 \\
\end{array}$ & 3.5043 & 134.6086 & 3621.61 & 5125 & 0.1 & 1950 & 487500 & 1675000 & 0.1339 & $6.83 \mathrm{E}-05$ & 1.258 & $1.39 \mathrm{E}-02$ & 41.03 & $\begin{array}{l}93.6 \\
\end{array}$ & 35.98 & 0 & 49.7 & 271.3 \\
\hline 131 & 25.51 & 3000 & 1750 & 44.283814 & 2.5951 & \begin{tabular}{|l|l|}
175.8017 \\
\end{tabular} & 2773.01 & 5125 & 0.1 & 1950 & 487500 & 2500000 & 0.1247 & $1.72 \mathrm{E}-04$ & 1.698 & 1.31E-02 & 41.95 & 88.71 & & 0 & 53 & $\begin{array}{r}341 \\
\end{array}$ \\
\hline 132 & 47.27 & 3000 & 4520 & $\begin{array}{r}4.2453999 \\
\end{array}$ & 0.175 & 123.6681 & 3942 & 5125 & 0.1 & 1950 & 487500 & 1475000 & 0.0673 & $5.08 \mathrm{E}-04$ & 4.812 & $1.55 \mathrm{E}-02$ & 37.09 & & 0 & 0 & 44.9 & 521.7 \\
\hline 133 & 38.38 & 3000 & 3640 & 98.499568 & 5.0568 & 154.0159 & 3165.26 & 5125 & 0.1 & 1950 & 487500 & 2050000 & 0.0829 & 4.66E-04 & 3.842 & $7.86 \mathrm{E}-03$ & 70.23 & 94.92 & 49.24 & 0 & 88.2 & $\begin{array}{r}513 \\
\end{array}$ \\
\hline 134 & 23.94 & 3000 & 4320 & 11.601842 & 0.657 & \begin{tabular}{|l|l|}
169.8917 \\
\end{tabular} & 2869.47 & 5125 & 0.1 & 1950 & 487500 & 2375000 & 0.1329 & 7.95E-04 & 4.025 & 7.43E-03 & 35.24 & 56.9 & & 0 & 93.3 & 861.7 \\
\hline$\frac{135}{136}$ & $\begin{array}{r}34.5 \\
27.14\end{array}$ & $\begin{array}{l}3000 \\
3000 \\
3000\end{array}$ & 3890 & $\begin{array}{r}986.344518 \\
77924651 \\
\end{array}$ & $\begin{aligned} 42.7018 \\
25211 \\
52\end{aligned}$ & \begin{tabular}{|r|}
129.879 \\
05.399
\end{tabular} & $\begin{array}{l}3753.49 \\
5107.93\end{array}$ & $\begin{array}{l}5125 \\
5125\end{array}$ & 0.1 & $\begin{array}{l}1950 \\
1900\end{array}$ & 487500 & $\begin{array}{l}1587500 \\
\end{array}$ & 0.0922 & $2.47 \mathrm{E}-04$ & 2.352 & \begin{tabular}{|l|l|}
$3.30 \mathrm{E}-03$ \\
$0.68 \mathrm{E}-03$
\end{tabular} & & $\begin{array}{l}99.49 \\
93.69\end{array}$ & $\begin{array}{l}94.93 \\
36.01 \\
\end{array}$ & & $\begin{aligned} 209.8 \\
716\end{aligned}$ & $\begin{array}{l}349.4 \\
2077\end{array}$ \\
\hline $\begin{array}{l}136 \\
137\end{array}$ & $\begin{array}{r}27.14 \\
61.3 \\
\end{array}$ & $\begin{array}{l}3000 \\
3000\end{array}$ & 2090 & $\begin{array}{r}79.24651 \\
743938164\end{array}$ & $\begin{array}{r}2.5211 \\
317 \\
\end{array}$ & $\begin{array}{r}95.4399 \\
127.8255\end{array}$ & $\begin{array}{l}5107.93 \\
381379\end{array}$ & $\begin{array}{l}5125 \\
5125\end{array}$ & $\begin{array}{l}0.1 \\
0.1 \\
\end{array}$ & $\begin{array}{c}1950 \\
11950 \\
\end{array}$ & $\begin{array}{l}487500 \\
487500\end{array}$ & $\begin{array}{l}10000000 \\
150000\end{array}$ & 0.1172 & $\begin{array}{l}2.44 \mathrm{E}-04 \\
254 \mathrm{E}-04 \\
\end{array}$ & $\begin{array}{l}2.053 \\
3.47\end{array}$ & \begin{tabular}{|l|}
$9.68 \mathrm{E}-03$ \\
\end{tabular} & 22.44 & $\begin{array}{l}93.69 \\
0903\end{array}$ & 36.91 & & \begin{tabular}{rr|}
71.6 \\
130.5
\end{tabular} & 387.7 \\
\hline 138 & 45.51 & 3000 & 4280 & $\begin{array}{l}743.983164 \\
252.978554\end{array}$ & $\begin{array}{r}31.7 \\
9.7699\end{array}$ & \begin{tabular}{|l|}
12.85255 \\
115.8579 \\
\end{tabular} & $\begin{array}{l}3813.19 \\
4207.74\end{array}$ & $\begin{array}{l}3120 \\
5125\end{array}$ & $\begin{array}{l}0.1 \\
0.1\end{array}$ & $\begin{array}{l}1950 \\
1950\end{array}$ & $\frac{487500}{487500}$ & $\begin{array}{l}1550000 \\
137500\end{array}$ & \begin{tabular}{|c|}
0.0519 \\
0.0699 \\
\end{tabular} & $\begin{array}{l}2.54 \mathrm{E}-04 \\
2.33 \mathrm{E}-04\end{array}$ & $\begin{array}{l}3.468 \\
2.682 \\
\end{array}$ & \begin{tabular}{|l|}
$4.97 \mathrm{E}-03$ \\
$9.09 \mathrm{E}-03$ \\
\end{tabular} & $\begin{array}{l}37.88 \\
84.52\end{array}$ & $\begin{array}{l}99.33 \\
98.02\end{array}$ & $\begin{array}{l}93.28 \\
80.24\end{array}$ & $\begin{array}{rl}32.79 & 0\end{array}$ & $\begin{array}{r}\frac{139.5}{76.3} \\
\end{array}$ & $\begin{array}{r}290.6 \\
302\end{array}$ \\
\hline 139 & 23.72 & 3000 & 3360 & 181.995676 & 9.5324 & 157.1306 & 3102.51 & 5125 & 0.1 & 1950 & 487500 & 2112500 & 0.1341 & $3.22 \mathrm{E}-04$ & 2.213 & \begin{tabular}{|l|}
$6.09 \mathrm{E}-03$ \\
\end{tabular} & 31.74 & 97.25 & 72.53 & 0 & 113.9 & 478 \\
\hline 140 & 22.28 & 3000 & 2400 & 211.192568 & 10.1707 & 144.4754 & 3374.28 & 5125 & 0.1 & 1950 & 487500 & 1862500 & 0.1428 & $2.46 \mathrm{E}-04$ & 1.869 & $6.37 \mathrm{E}-03$ & 33.22 & \begin{tabular}{|l|l|}
97.63 \\
\end{tabular} & 76.32 & & 108.8 & 430 \\
\hline 141 & 25.64 & 3000 & 2350 & 347.320814 & 14.7988 & 127.8255 & 3813.79 & 5125 & 0.1 & 1950 & 487500 & 1550000 & 0.1241 & $2.16 \mathrm{E}-04$ & 1.878 & $5.92 \mathrm{E}-03$ & 39.15 & $\begin{array}{l}98.56 \\
\end{array}$ & 85.6 & & 117.1 & 375.5 \\
\hline 142 & 29.93 & 3000 & 3460 & 118.143484 & 6.3336 & 160.8281 & 3031.19 & 5125 & 0.1 & 1950 & 487500 & 2187500 & 0.1063 & $4.40 \mathrm{E}-04$ & 3.091 & \begin{tabular}{|c|}
$6.85 \mathrm{E}-03$ \\
\end{tabular} & 61.91 & 95.77 & 57.68 & 0 & 101.2 & 529.2 \\
\hline 143 & 22.06 & 3000 & 4380 & 192.640273 & 7.8512 & 122.2668 & 3987.18 & 5125 & 0.1 & 1950 & 487500 & 1450000 & 0.1442 & $4.69 \mathrm{E}-04$ & 2.646 & $4.84 \mathrm{E}-03$ & 58.49 & 97.4 & 74.04 & & 143.3 & 614.6 \\
\hline 144 & 42.19 & 3000 & 4110 & 0.74523 & 0.0362 & 145.7654 & 3344.42 & 5125 & 0.1 & 1950 & 487500 & 1887500 & 0.0754 & $5.62 \mathrm{E}-04$ & 4.771 & $1.78 \mathrm{E}-02$ & 31.3 & 0 & & & 39 & 579.5 \\
\hline 145 & 44.31 & 3000 & 5450 & 2.388547 & 0.0797 & 100.1541 & 4867.5 & 5125 & 0.1 & 1950 & 487500 & 1075000 & 0.0718 & $5.75 \mathrm{E}-04$ & 5.047 & $1.56 \mathrm{E}-02$ & 39.43 & 0 & & & 44.4 & 583.8 \\
\hline 146 & 39.82 & 3000 & 5260 & 19.024625 & 0.6103 & 96.2335 & 5065.8 & 5125 & 0.1 & 1950 & 487500 & 1012500 & 0.0799 & $4.06 \mathrm{E}-04$ & 3.571 & $1.38 \mathrm{E}-02$ & 71.64 & 73.72 & & & 50.3 & 459.6 \\
\hline 147 & 33.45 & 3000 & 3390 & $\begin{array}{r}6.157794 \\
\end{array}$ & 0.3364 & \begin{tabular}{|l|l|}
163.877 \\
\end{tabular} & 2974.79 & 5125 & 0.1 & 1950 & 487500 & 2250000 & 0.0951 & $3.84 \mathrm{E}-04$ & 3.043 & $1.44 \mathrm{E}-02$ & 22.51 & 18.8 & 0 & & 48.2 & 466.2 \\
\hline 148 & 43.52 & 3000 & 3530 & 202.222265 & 9.7387 & 144.4754 & 3374.28 & 5125 & 0.1 & 1950 & 487500 & 1862500 & 0.0731 & $2.61 \mathrm{E}-04$ & 2.808 & $8.86 \mathrm{E}-03$ & 58.11 & 97.53 & 75.27 & & 78.3 & 330.7 \\
\hline 149 & 21.89 & 3000 & 5360 & 321.362059 & 10.3086 & 96.2335 & 5065.8 & 5125 & 0.1 & 1950 & 487500 & 1012500 & 0.1453 & 4.67E-04 & 2.625 & 3.97E-03 & 79.41 & 98.44 & 84.44 & & 174.8 & 614.5 \\
\hline 150 & 36.78 & 3000 & 1490 & 159.502668 & 6.1599 & 115.8579 & 4207.74 & 5125 & 0.1 & 1950 & 487500 & 1337500 & 0.0865 & $1.04 \mathrm{E}-04$ & 1.607 & $1.41 \mathrm{E}-02$ & 80.46 & $\begin{array}{l}96.87 \\
\end{array}$ & 68.65 & & 49.3 & 224 \\
\hline 151 & 39.57 & 3000 & 3370 & 23.25577 & 0.8925 & 115.1349 & 4234.16 & 5125 & 0.1 & 1950 & 487500 & 1325000 & 0.0804 & $2.53 \mathrm{E}-04$ & 2.588 & $1.69 \mathrm{E}-02$ & 67.82 & 78.5 & & & 41 & 335.2 \\
\hline 152 & 25.47 & 3000 & 4430 & 10.016973 & 0.445 & 133.2659 & 3658.1 & 5125 & 0.1 & 1950 & 487500 & 1650000 & $\begin{array}{ll}0.1249 \\
\end{array}$ & $6.22 \mathrm{E}-04$ & 3.518 & $9.18 \mathrm{E}-03$ & 35.62 & 50.08 & & & 75.5 & 707.8 \\
\hline 153 & 24.47 & 3000 & 1130 & 156.700185 & $\begin{array}{l}5.0266 \\
\end{array}$ & 96.2335 & 5065.8 & 5125 & 0.1 & 1950 & 487500 & 1012500 & 0.13 & 6.91E-05 & 1.269 & $1.16 \mathrm{E}-02$ & 61.52 & 96.81 & $\begin{array}{l}68.09 \\
\end{array}$ & & 59.7 & 265.7 \\
\hline 154 & 55.42 & 3000 & 5560 & 79.899551 & 2.605 & \begin{tabular}{|l|l|l|}
97.8112 \\
\end{tabular} & 4984.09 & 5125 & 0.1 & 1950 & & 1037500 & 0.0574 & 4.54E-04 & 4.996 & & 30.88 & 93.74 & 37.42 & & 79.6 & 462 \\
\hline 155 & 56.91 & 3000 & 2730 & 1463.195101 & 55.8012 & 114.4096 & 4261 & 5125 & 0.1 & 1950 & & 1312500 & 0.0559 & $1.88 \mathrm{E}-04$ & 2.696 & & 37.69 & \begin{tabular}{ll|l|l|}
99.66 \\
\end{tabular} & 96.58 & 65.83 & 231.1 & 242.8 \\
\hline 156 & 21.57 & 3000 & 5020 & \begin{tabular}{|l|}
10.382068 \\
\end{tabular} & $\begin{array}{l}0.3625 \\
2120 \\
\end{array}$ & 104.7599 & 4653.5 & 5125 & 0.1 & 1950 & 487500 & 1150000 & 0.1475 & & 3.01 & & 73.1 & 51.84 & 0 & 0 & 70.4 & 715.3 \\
\hline 157 & 28.05 & 3000 & 4510 & & 3.1462 & 119.4396 & 4081.56 & 5125 & 0.1 & 1950 & 487500 & 1400000 & 0.1134 & & 3.053 & & 38.86 & & 36.73 & & 98.1 & 557.7 \\
\hline 158 & 33.88 & 3000 & 1090 & 250.748788 & 13.0296 & 155.8885 & 3127.24 & 5125 & 0.1 & 1950 & 487500 & 2087500 & 0.0939 & & 1.655 & 1.01E-02 & 27.32 & 98.01 & 80.06 & & 68.4 & 250.4 \\
\hline$\frac{159}{1160}$ & 25.09 & 3000 & 1380 & 55.8794 & $\begin{array}{r}2.739 \\
\end{array}$ & 147.0497 & $\begin{array}{l}3315.21 \\
3659.1\end{array}$ & 5125 & 0.1 & 1950 & & $\begin{array}{l}1912500 \\
\end{array}$ & 0.1268 & 1.34E-04 & 1.534 & $1.33 \mathrm{E}-02$ & $\begin{array}{l}41.12 \\
5.00\end{array}$ & $\begin{array}{l}91.05 \\
10.52 \\
\end{array}$ & 10.52 & & $\begin{array}{r}52 \\
515\end{array}$ & 313.3 \\
\hline$\frac{160}{161}$ & $\begin{array}{l}25.23 \\
39.92\end{array}$ & $\begin{array}{l}3000 \\
3000\end{array}$ & 3920 & $\begin{array}{r}\mid 8.4075414 \\
22.040466\end{array}$ & $\begin{array}{l}0.3735 \\
12438 \\
\end{array}$ & \begin{tabular}{|l|}
133.2659 \\
1692591 \\
\end{tabular} & $\begin{array}{r}3658.1 \\
2879.59\end{array}$ & \begin{tabular}{|l|l|}
5125 & 5125 \\
\end{tabular} & $\begin{array}{l}0.1 \\
0.1\end{array}$ & $\begin{array}{l}1950 \\
1950\end{array}$ & & & $\begin{array}{l}0.1261 \\
0.077\end{array}$ & & & & $\begin{array}{l}54.92 \\
3.49\end{array}$ & $\begin{array}{l}40.53 \\
77.31\end{array}$ & 0 & $\frac{0}{0}$ & $\begin{array}{l}54.5 \\
51.0\end{array}$ & 539.5 \\
\hline 162 & $\begin{array}{r}399.92 \\
34.1 \\
\end{array}$ & 30000 & $\frac{3200}{1160}$ & \begin{tabular}{|r|}
22.040496 \\
591.966297 \\
\end{tabular} & $\begin{array}{r}1.2438 \\
18.1993 \\
\end{array}$ & \begin{tabular}{|r|}
669.2251 \\
92.2314 \\
\end{tabular} & $\begin{array}{l}289.559 \\
5285.62\end{array}$ & $\begin{array}{l}5125 \\
5125 \\
\end{array}$ & $\begin{array}{l}0.1 \\
0.1 \\
\end{array}$ & $\frac{1950}{1950}$ & $\frac{487500}{487500}$ & $\begin{array}{r}2362500 \\
950000\end{array}$ & 0.0797 & $\begin{array}{l}3.40 \mathrm{E}-04 \\
1.24 \mathrm{E}-04\end{array}$ & $\begin{array}{l}3.154 \\
1.669\end{array}$ & \begin{tabular}{|l|}
$1.34 \mathrm{E}-02$ \\
$6.56 \mathrm{E}-03$ \\
\end{tabular} & $\begin{array}{l}33.49 \\
27.45\end{array}$ & $\begin{array}{l}77.31 \\
99.16\end{array}$ & \begin{tabular}{r|}
0 \\
91.55
\end{tabular} & & $\begin{array}{r}51.9 \\
105.6\end{array}$ & $\begin{array}{r}405 \\
250.9\end{array}$ \\
\hline 163 & 38.15 & 3000 & 5260 & \begin{tabular}{|l|}
641.073203 \\
\end{tabular} & 20.39 & \begin{tabular}{|l}
95.4399 \\
\end{tabular} & 5107.93 & 5125 & 0.1 & 1950 & & 1000000 & 0.0834 & $3.14 \mathrm{E}-04$ & 2.902 & \begin{tabular}{|l|}
$4.27 \mathrm{E}-03$ \\
\end{tabular} & 91.33 & $\begin{array}{l}99.22 \\
9\end{array}$ & 92.2 & & 162.2 & 389.9 \\
\hline 164 & 22.66 & 3000 & 4550 & \begin{tabular}{|l|}
0.047589 \\
\end{tabular} & 0.00 & 139.2566 & 3500.73 & 5125 & 0.1 & 195 & & 1762500 & 0.1404 & $7.79 \mathrm{E}-04$ & 3.805 & $1.57 \mathrm{E}-02$ & 26.86 & & & & 44.2 & 860.5 \\
\hline 165 & 28.13 & 3000 & 4290 & 36.069061 & & 124.3658 & 3919.89 & 5125 & 0.1 & 1950 & & 1487500 & 0.1131 & $4.28 \mathrm{E}-04$ & 2.913 & 9.05 & 34.43 & 86.14 & 0 & & 76.6 & 530.7 \\
\hline 166 & 26.01 & 3000 & 880 & 197.809533 & & 133.9381 & 3639.74 & 5125 & 0. & 1950 & & & 0.1223 & 2.83 & 1.117 & & & \begin{tabular}{|c|c|}
97.47 \\
\end{tabular} & 74.72 & & 53.6 & 220 \\
\hline 167 & 24.66 & 3000 & 1070 & 55.756415 & & 124.3658 & 3919.89 & 5125 & 0.1 & 1950 & & 14875 & 0.129 & & 1.367 & & 38.6 & & 10.32 & & 47.5 & 284.2 \\
\hline 168 & 50.98 & 3000 & 3030 & 431.08649 & 12.4264 & 86.4774 & 5637.31 & 5125 & 0.1 & 1950 & 487 & 862500 & 0.0624 & $2.25 \mathrm{E}-04$ & 2.823 & 7.09 & 34. & 98.84 & 88.4 & & 97.8 & 283.7 \\
\hline 169 & 24.49 & 3000 & 4760 & 80.33 & 2.5769 & 96.23 & 5065 & 5125 & 0.1 & 1950 & 487 & 1012500 & \begin{tabular}{|c|}
0.1299 \\
\end{tabular} & $3.55 \mathrm{E}-04$ & 2.38 & & 61. & \begin{tabular}{|c|}
93.78 \\
\end{tabular} & \begin{tabular}{|l|l|}
37.76 \\
\end{tabular} & & 86.4 & 498 \\
\hline 170 & 31.85 & 3000 & 5460 & \begin{tabular}{|l}
23.801 \\
\end{tabular} & & 100.1541 & 4867.5 & 5125 & 0.1 & 1950 & 487 & 1075000 & 0.0999 & $5.76 \mathrm{E}-04$ & 3.917 & $5.74 \mathrm{E}-03$ & 55.14 & 95.96 & 59.61 & & 120.8 & 630.3 \\
\hline 171 & $\begin{array}{ll}60.25 \\
\end{array}$ & 3000 & 4180 & 119.266874 & & 148.9657 & 3272.57 & 5125 & 0.1 & 1950 & & 1950000 & 0.0528 & $4.76 \mathrm{E}-04$ & 5.552 & $7.64 \mathrm{E}$ & 34. & 95.81 & 58.08 & & 90.7 & 472.3 \\
\hline 172 & 24.74 & 3000 & 1280 & & 0.6691 & 136.6103 & 3568.54 & 5125 & 0.1 & 1950 & & 1712500 & 0.1286 & $1.54 \mathrm{E}-04$ & 1.604 & 1.67 & 26. & 65.97 & & & 41.5 & 332.4 \\
\hline 173 & 23.51 & & 3560 & 292.04 & 14.8099 & 152.1319 & 3204.46 & 5125 & 0.1 & 1950 & & 2012500 & 0.1353 & 3.77 & 2.409 & & 38. & 98.2 & 82.88 & & 150 & 525 \\
\hline 174 & 21.64 & 30 & 3200 & & 2.1 & 75.24 & & 5125 & 0.1 & 195 & & & 0.1 & & 2.228 & & 23. & & 40.74 & & 98.9 & 527.6 \\
\hline 175 & & & 6210 & & & 83.1 & 5866 & 5125 & 0.1 & 195 & & 812500 & 0.07 & & 4.06 & & 41. & 84.8 & 0 & & 65.9 & 492.5 \\
\hline 176 & 25.43 & & 4580 & & & 116.5 & & 5125 & 0.1 & 195 & & & 0.1251 & & 2.832 & & 31. & 83.27 & & & 79.5 & 570.7 \\
\hline 177 & & & 3460 & & & 146.4 & & 5125 & 0.1 & 195 & & & & & 1.986 & & 86. & 97.94 & 79.43 & & 107.6 & 422.9 \\
\hline 178 & 29.98 & 3000 & 3130 & & & 181 & 2692.82 & 5125 & 0.1 & 195 & & 2612500 & 0.1 & & 3.312 & & 67 & 60.1 & 0 & & 57.8 & 566.2 \\
\hline 179 & 23.12 & 3000 & 2570 & & & 92.2 & 5285.62 & 5125 & 0.1 & 195 & & & & & & & 43. & & & & 45.2 & 439.2 \\
\hline 180 & 38 & & & & & & 35 & 512 & 0.1 & 19 & & & & & & & & & 71.65 & & 64.6 & \\
\hline 181 & 59.58 & & & 140.0 & & 108. & 4492.18 & 5125 & 0.1 & 19 & & & 0.0534 & & 6.026 & & & 96.43 & $\begin{array}{ll}64.29 \\
\end{array}$ & 0 & $\begin{array}{r}92.6 \\
\end{array}$ & 518.4 \\
\hline 182 & 22.79 & 30 & 36 & & & & & 5125 & 0.1 & 19 & & & 1396 & & 1.927 & & & & 91.18 & 11.8 & 173.2 & 433.4 \\
\hline 183 & 53.11 & 3000 & 5680 & & 2. & 91.4 & $\begin{array}{r}5332.5 \\
2568.54\end{array}$ & 5125 & 0.1 & 1950 & & $\begin{array}{r}937500 \\
171500\end{array}$ & 0.05 & & 4 & & & 3.79 & 37.89 & & 72.3 & 425.5 \\
\hline 184 & $\begin{array}{r}27.4 \\
\end{array}$ & 3000 & 4030 & & & \begin{tabular}{|l|l|}
136.6103 \\
7703
\end{tabular} & 3568.54 & 5125 & 0.1 & $\frac{195}{105}$ & & & 0.11 & & $\begin{array}{l}3.164 \\
2.271 \\
\end{array}$ & & & & 0 & & 86 & 591.7 \\
\hline$\frac{185}{186}$ & $\begin{array}{l}27.78 \\
27.57\end{array}$ & 3000 & 6550 & 28.820851 & $\begin{array}{l}0.7484 \\
2587\end{array}$ & \begin{tabular}{|r|}
77.906 \\
1451211
\end{tabular} & 6257.54 & 5125 & 0.1 & 195 & & $\begin{array}{r}737 \\
1975 \\
\end{array}$ & 0.1145 & 5.14E-04 & 3.271 & 9.02 & 57.84 & 82.65 & 0 & & 76.9 & 603.4 \\
\hline 186 & 27.57 & 3000 & 2780 & \begin{tabular}{|r|}
53.348713 \\
\end{tabular} & & 145.1211 & 3359.26 & $\begin{array}{l}5125 \\
5125\end{array}$ & 0.1 & $\begin{array}{c}1950 \\
1050 \\
\end{array}$ & 487500 & 1875 & 0.1154 & 2.64E-04 & 2.158 & 1.07E- & 39.34 & 90.63 & 6.28 & & 64.7 & 01.2 \\
\hline$\frac{188}{188}$ & $\begin{array}{l}36.07 \\
30.62\end{array}$ & 3000 & $\frac{2550}{5130}$ & \begin{tabular}{|r|}
251.897157 \\
0.472524 \\
\end{tabular} & $\begin{array}{r}\frac{14.0642}{0.0229} \\
0\end{array}$ & \begin{tabular}{|l|}
167.4888 \\
145.1211 \\
\end{tabular} & $\begin{array}{l}2910.47 \\
3359.26\end{array}$ & $\begin{array}{l}\mid 5125 \\
5125\end{array}$ & $\begin{array}{l}0.1 \\
0.1\end{array}$ & $\begin{array}{l}1950 \\
1950\end{array}$ & $\begin{array}{l}487500 \\
487500\end{array}$ & $\begin{array}{l}2325000 \\
1875000\end{array}$ & \begin{tabular}{|c|}
0.082 \\
0.1039 \\
\end{tabular} & $\begin{array}{l}2.06 \mathrm{E}-04 \\
8.19 \mathrm{E}-04\end{array}$ & \begin{tabular}{r|}
2.18 \\
4.985 \\
\end{tabular} & \begin{tabular}{|l|}
$1.39 \mathrm{E}-03$ \\
$1.22 \mathrm{E}-02$ \\
\end{tabular} & $\begin{array}{r}46.89 \\
19.2\end{array}$ & $\begin{array}{r}98.02 \\
0\end{array}$ & $\begin{array}{r}80.15 \\
0\end{array}$ & & $\begin{array}{l}82.6 \\
56.8\end{array}$ & $\begin{array}{l}309.8 \\
834.3 \\
\end{array}$ \\
\hline 189 & 44.49 & 3000 & 3300 & 51.702324 & 2.6973 & 156.5102 & 3114.81 & 5125 & 0.1 & 1950 & 487500 & 2100000 & 0.0715 & $2.68 \mathrm{E}-04$ & 2.894 & \begin{tabular}{|l|}
$1.33 \mathrm{E}-02$ \\
\end{tabular} & 39.81 & 90.33 & 3.29 & & 52.1 & 333.3 \\
\hline
\end{tabular}




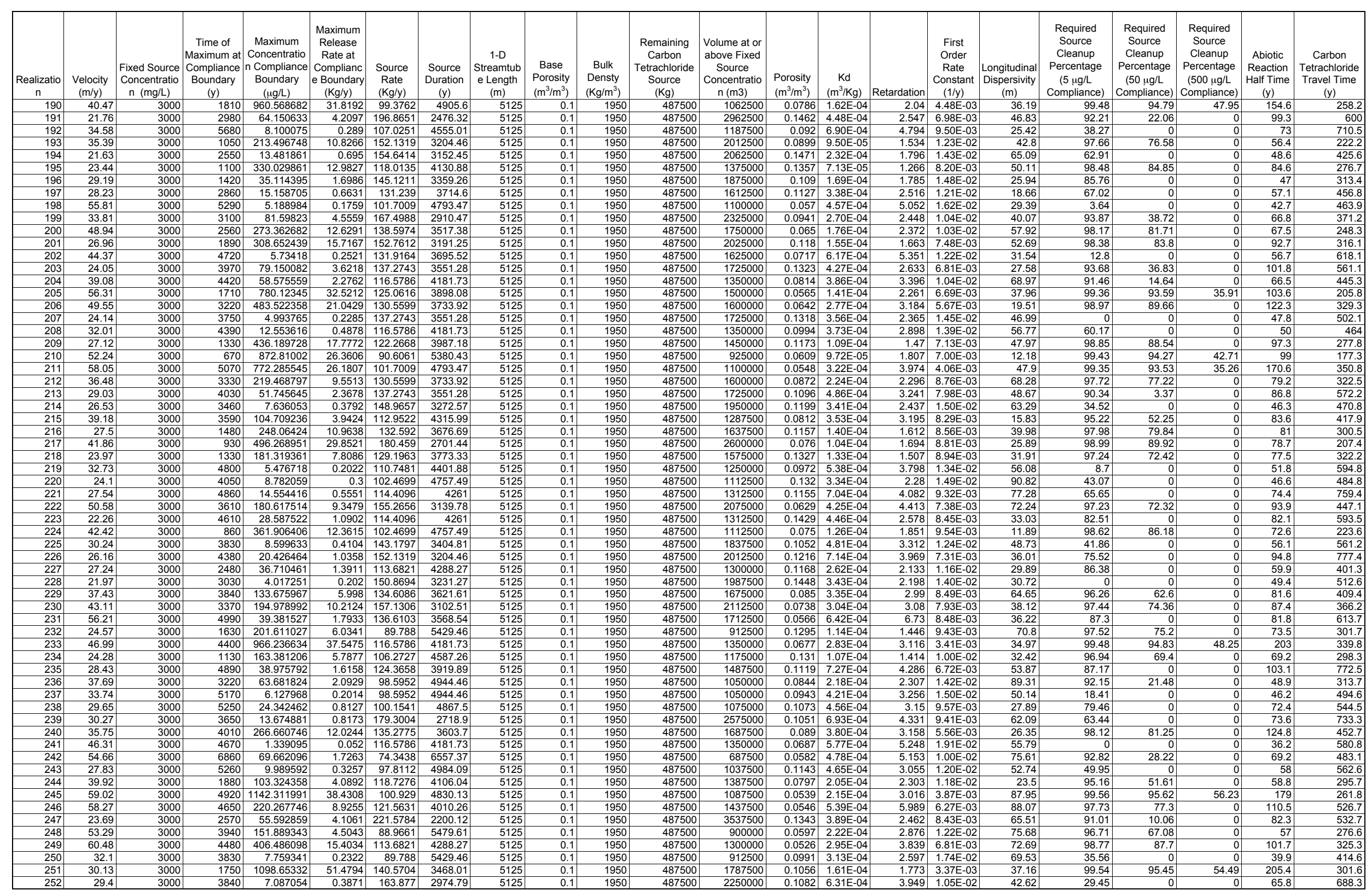




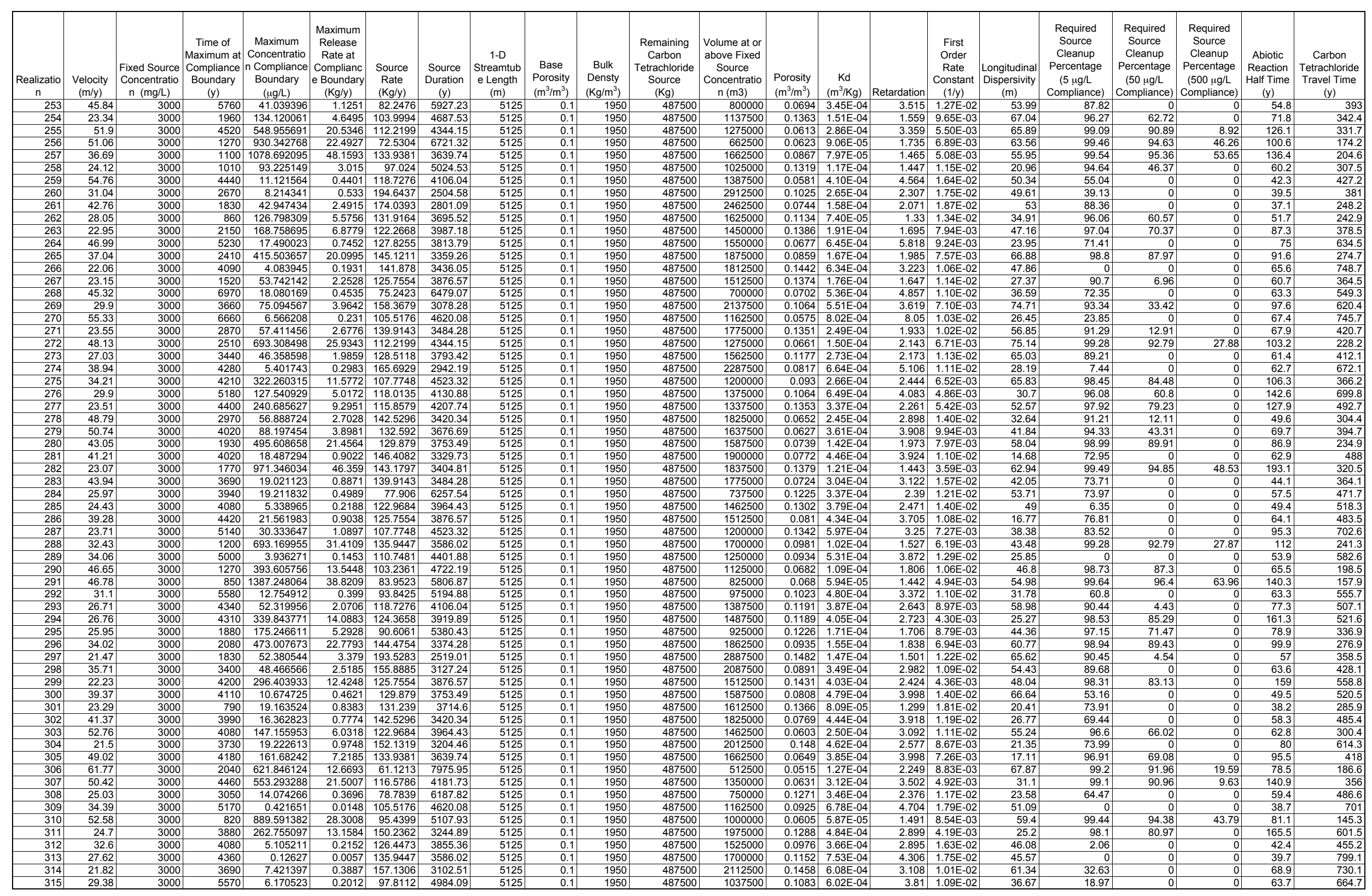




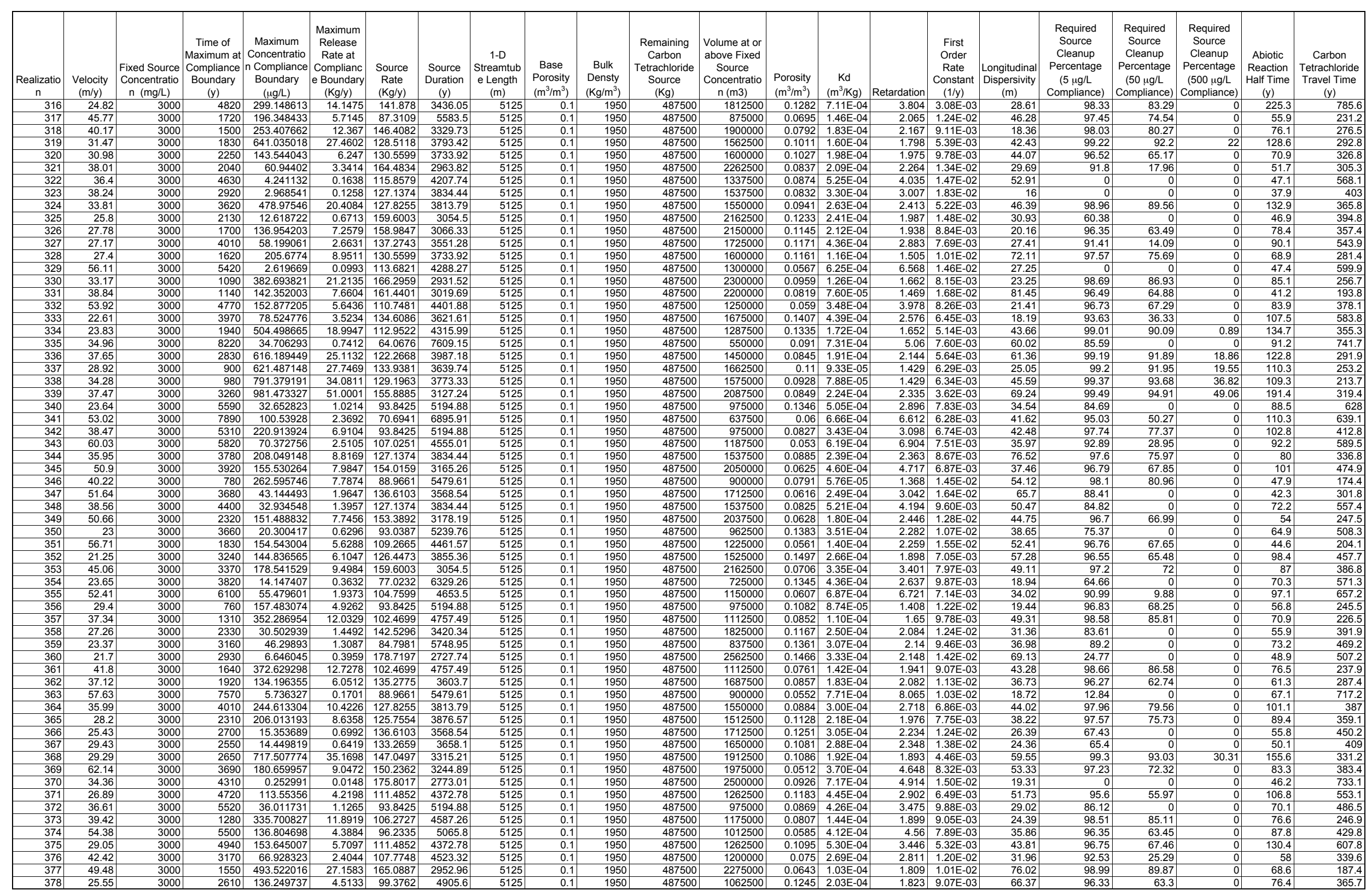




\begin{tabular}{|c|c|c|c|c|c|c|c|c|c|c|c|c|c|c|c|c|c|c|c|c|c|c|}
\hline $\begin{array}{c}\text { Realizatio } \\
n\end{array}$ & $\begin{array}{c}\text { Velocity } \\
(\mathrm{m} / \mathrm{y})\end{array}$ & $\begin{array}{c}\text { Fixed Source } \\
\text { Concentratio } \\
\mathrm{n}(\mathrm{mg} / \mathrm{L})\end{array}$ & $\begin{array}{c}\text { Time of } \\
\text { Maximum at } \\
\text { Compliance } \\
\text { Boundary } \\
\text { (y) }\end{array}$ & $\begin{array}{c}\text { Maximum } \\
\text { Concentratio } \\
\text { n Compliance } \\
\text { Boundary } \\
(\mu \mathrm{g} / \mathrm{L})\end{array}$ & \begin{tabular}{|c|} 
Maximum \\
Release \\
Rate at \\
Complianc \\
e Boundary \\
$(\mathrm{Kg} / \mathrm{y})$ \\
\end{tabular} & $\begin{array}{c}\text { Source } \\
\text { Rate } \\
(\mathrm{Kg} / \mathrm{y})\end{array}$ & $\begin{array}{c}\text { Source } \\
\text { Duration } \\
\text { (y) } \\
\end{array}$ & \begin{tabular}{|c|} 
1-D \\
Streamtub \\
e Length \\
$(\mathrm{m})$
\end{tabular} & $\begin{array}{c}\text { Base } \\
\text { Porosity } \\
\left(\mathrm{m}^{3} / \mathrm{m}^{3}\right)\end{array}$ & $\begin{array}{c}\text { Bulk } \\
\text { Densty } \\
\left(\mathrm{Kg} / \mathrm{m}^{3}\right)\end{array}$ & $\begin{array}{c}\text { Remaining } \\
\text { Carbon } \\
\text { Tetrachloride } \\
\text { Source } \\
(\mathrm{Kg}) \\
\end{array}$ & $\begin{array}{c}\text { Volume at or } \\
\text { above Fixed } \\
\text { Source } \\
\begin{array}{c}\text { Concentratio } \\
n(m 3)\end{array} \\
\end{array}$ & $\begin{array}{l}\text { Porosity } \\
\left(\mathrm{m}^{3} / \mathrm{m}^{3}\right)\end{array}$ & $\begin{array}{c}\mathrm{Kd} \\
\left(\mathrm{m}^{3} / \mathrm{kg}\right)\end{array}$ & Retardation & $\begin{array}{c}\text { First } \\
\text { Order } \\
\text { Rate } \\
\text { Constant } \\
(1 / y) \\
\end{array}$ & \begin{tabular}{|c} 
Longitudinal \\
Dispersivity \\
$(\mathrm{m})$
\end{tabular} & $\begin{array}{c}\text { Required } \\
\text { Source } \\
\text { Cleanup } \\
\text { Percentage } \\
(5 \mu g / L \\
\text { Compliance) } \\
\end{array}$ & \begin{tabular}{|c|} 
Required \\
Source \\
Cleanup \\
Percentage \\
$(50 \mu \mathrm{g} / \mathrm{L}$ \\
Compliance)
\end{tabular} & $\begin{array}{l}\text { Required } \\
\text { Source } \\
\text { Cleanup } \\
\text { Percentage } \\
(500 \mu g / L \\
\text { Compliance) } \\
\end{array}$ & \begin{tabular}{|c|} 
Abiotic \\
Reaction \\
Half Time \\
(y) \\
\end{tabular} & $\begin{array}{c}\text { Carbon } \\
\text { Tetrachloride } \\
\text { Travel Time } \\
\text { (y) } \\
\end{array}$ \\
\hline & & 3000 & 3900 & \begin{tabular}{|l|}
312.486233 \\
\end{tabular} & \begin{tabular}{|l|}
13.3861 \\
\end{tabular} & 128.5118 & \begin{tabular}{|l|l|}
3793.42 \\
210.51
\end{tabular} & 5125 & & 1950 & $\begin{array}{r}487500 \\
\end{array}$ & \begin{tabular}{r|}
1562500 \\
\end{tabular} & 0.0588 & $2.28 \mathrm{E}-04$ & 2.958 & $8.92 \mathrm{E}-03$ & $\begin{array}{r}80.6 \\
\end{array}$ & $\begin{array}{r}9.4 \\
\end{array}$ & 84 & & $\begin{array}{l}77.7 \\
\end{array}$ & 280.2 \\
\hline 380 & 28 & 3000 & 2110 & \begin{tabular}{|r|}
90.997611 \\
\end{tabular} & \begin{tabular}{|r|}
4.7662 \\
\end{tabular} & \begin{tabular}{|l|l|}
157.1306 \\
14.989
\end{tabular} & 3102.51 & $\begin{array}{l}5125 \\
5125 \\
\end{array}$ & 0.1 & 1950 & 487500 & 2112500 & 0.1136 & 1.47E-04 & 1.656 & $1.28 \mathrm{E}-02$ & $\begin{array}{l}94.67 \\
\end{array}$ & 94.51 & 45.05 & & 54.3 & 303.1 \\
\hline 381 & 21.8 & 3000 & 1190 & \begin{tabular}{|l|}
566.616948 \\
\end{tabular} & 26.7968 & $\begin{array}{l}141.878 \\
\end{array}$ & 3436.05 & 5125 & 0.1 & 1950 & 487500 & 1812500 & 0.1459 & 1.01E-04 & 1.35 & $5.33 \mathrm{E}-03$ & 34.36 & 99.12 & $\begin{array}{l}1.18 \\
\end{array}$ & 11.76 & 130 & 317.2 \\
\hline 382 & 24.59 & 3000 & 5590 & $\begin{array}{r}0.593358 \\
\end{array}$ & $\begin{array}{ll}0.0182 \\
\end{array}$ & 92.2314 & 5285.62 & 5125 & 0.1 & 1950 & 487500 & 950000 & 0.1294 & $5.95 \mathrm{E}-04$ & 3.326 & 1.64E-02 & 60.68 & & & 0 & 42.2 & 693.4 \\
\hline 383 & 30.15 & 3000 & 3020 & 98.602317 & $\begin{array}{l}4.1787 \\
\end{array}$ & 127.1374 & 3834.44 & 5125 & 0.1 & 1950 & 487500 & 1537500 & 0.1055 & $2.87 \mathrm{E}-04$ & 2.375 & $8.89 \mathrm{E}-03$ & 31.67 & 94.93 & 49.29 & & 78 & 403.6 \\
\hline 384 & 24.8 & 3000 & 4180 & 1.813441 & $\begin{array}{l}0.0567 \\
0.927 \\
\end{array}$ & 93.8425 & 5194.88 & 5125 & 0.1 & 1950 & 487500 & 975000 & 0.1283 & $3.88 \mathrm{E}-04$ & 2.528 & $1.85 \mathrm{E}-02$ & 83.92 & & 0 & & 37.4 & 522.4 \\
\hline 385 & 39.57 & 3000 & 890 & 187.956624 & 8.1372 & 129.879 & 3753.49 & 5125 & 0.1 & 1950 & 487500 & 1587500 & 0.0804 & $9.22 \mathrm{E}-05$ & 1.58 & $1.39 \mathrm{E}-02$ & 32.57 & 97.34 & 73.4 & & 49.8 & 204.6 \\
\hline 386 & 50.82 & 3000 & 5950 & $\begin{array}{r}8.498915 \\
\end{array}$ & 0.2881 & $\begin{array}{l}101.7009 \\
172950\end{array}$ & 4793.47 & 5125 & 0.1 & 1950 & 487500 & 1100000 & 0.0626 & $6.16 \mathrm{E}-04$ & 5.973 & 1.12E-02 & 21.8 & 41.17 & 0 & & 61.9 & 602.3 \\
\hline 387 & $\begin{array}{r}56.41 \\
22541\end{array}$ & 3000 & $\begin{array}{l}3160 \\
5110\end{array}$ & $\begin{array}{r}20.579086 \\
1831549\end{array}$ & $\begin{array}{l}1.1858 \\
0.063\end{array}$ & \begin{tabular}{|l|}
172.8593 \\
102519
\end{tabular} & $\begin{array}{l}2820.21 \\
4492.18\end{array}$ & $\begin{array}{l}5125 \\
5125\end{array}$ & 0.1 & $\begin{array}{l}1950 \\
1900\end{array}$ & 487500 & 2437500 & 0.0564 & $3.31 \mathrm{E}-04$ & $\begin{array}{r}3.97 \\
3617 \\
617\end{array}$ & \begin{tabular}{|l|}
$1.59 \mathrm{E}-02$ \\
$1.23 \mathrm{E}-02$
\end{tabular} & $\begin{array}{l}39.19 \\
30.03 \\
\end{array}$ & 75.7 & & & $\begin{array}{ll}43.6 \\
5.5 \\
\end{array}$ & $\begin{array}{l}360.7 \\
720.5\end{array}$ \\
\hline $\begin{array}{l}3388 \\
389\end{array}$ & $\begin{array}{l}25.41 \\
36.32\end{array}$ & 3000 & $\frac{5110}{4730}$ & $\begin{array}{r}1.831549 \\
85591659\end{array}$ & $\begin{array}{l}0.0663 \\
35823 \\
\end{array}$ & \begin{tabular}{|l|}
108.5219 \\
123681 \\
\end{tabular} & $\begin{array}{r}4492.18 \\
3942\end{array}$ & $\begin{array}{l}5125 \\
5125\end{array}$ & $\begin{array}{l}0.1 \\
0.1 \\
\end{array}$ & $\begin{array}{l}1950 \\
1950\end{array}$ & $\begin{array}{l}487500 \\
487500\end{array}$ & \begin{tabular}{|l|l|}
1212500 \\
145000
\end{tabular} & 0.1252 & $\begin{array}{l}6.48 \mathrm{E}-04 \\
579 \mathrm{E}-04 \\
\end{array}$ & $\begin{array}{l}3.617 \\
434\end{array}$ & \begin{tabular}{|l|}
$1.23 \mathrm{E}-02$ \\
$660 \mathrm{E}-03$
\end{tabular} & 39.93 & $\begin{array}{r}0 \\
9416\end{array}$ & $\begin{array}{r}0 \\
1158\end{array}$ & & $\begin{array}{r}56.5 \\
105.1\end{array}$ & $\begin{array}{l}729.5 \\
61.5\end{array}$ \\
\hline 390 & 31.16 & 3000 & $\frac{4730}{3540}$ & $\begin{array}{r}85.5916609 \\
41.06088\end{array}$ & $\begin{array}{l}3.5283 \\
1.6542\end{array}$ & $\begin{array}{l}12.36681 \\
120.8573\end{array}$ & $\begin{array}{r}3942 \\
4033.68 \\
\end{array}$ & $\begin{array}{l}3120 \\
5125\end{array}$ & $\begin{array}{l}0.1 \\
0.1\end{array}$ & $\begin{array}{l}1950 \\
1950\end{array}$ & $\frac{487500}{487500}$ & $\begin{array}{l}145000 \\
1425000\end{array}$ & \begin{tabular}{|l|}
0.0866 \\
0.1021
\end{tabular} & $\begin{array}{l}5.19 \mathrm{E}-04 \\
3.20 \mathrm{E}-04\end{array}$ & $\begin{array}{r}4.584 \\
2.586\end{array}$ & \begin{tabular}{|l|}
$6.60 \mathrm{E}-03$ \\
$1.08 \mathrm{E}-02$ \\
\end{tabular} & $\begin{array}{l}45.24 \\
32.91\end{array}$ & $\begin{array}{l}94.16 \\
87.82 \\
\end{array}$ & \begin{tabular}{r|r}
41.58 \\
0
\end{tabular} & $\frac{0}{0}$ & $\begin{array}{r}\frac{105.1}{64.1} \\
\end{array}$ & $\frac{612.5}{425.4}$ \\
\hline 391 & 40.63 & 3000 & 4110 & 7.615223 & 0.2463 & 97.024 & 5024.53 & 5125 & 0.1 & 1950 & 487500 & 1025000 & 0.0783 & $4.10 \mathrm{E}-04$ & 3.649 & $1.37 \mathrm{E}-02$ & 12.91 & 34.34 & & 0 & 50.6 & 460.2 \\
\hline 392 & 58.7 & 3000 & 3090 & 36.99138 & 1.5677 & 127.1374 & 3834.44 & 5125 & 0.1 & 1950 & 487500 & 1537500 & 0.0542 & $2.28 \mathrm{E}-04$ & 3.131 & $1.77 \mathrm{E}-02$ & 37.72 & 86.48 & & 0 & 39.1 & 273.4 \\
\hline 393 & 31.34 & 3000 & 2860 & \begin{tabular}{|r|}
653.192189 \\
\end{tabular} & 23.6286 & 108.5219 & 4492.18 & 5125 & 0.1 & 1950 & 487500 & 1212500 & 0.1015 & $1.82 \mathrm{E}-04$ & 1.907 & \begin{tabular}{|l|}
$5.10 \mathrm{E}-03$ \\
\end{tabular} & 77.71 & 99.23 & 92.35 & 23.45 & 135.8 & 311.8 \\
\hline 394 & 57.42 & 3000 & 3680 & 276.496765 & 13.5529 & $\begin{array}{l}147.0497 \\
\end{array}$ & 3315.21 & 5125 & 0.1 & 1950 & 487500 & 1912500 & 0.0554 & $3.32 \mathrm{E}-04$ & 4.032 & 7.16E-03 & 43.18 & 98.19 & 81.92 & 0 & 96.8 & 359.9 \\
\hline 395 & 23.19 & 3000 & 3770 & \begin{tabular}{|l|}
376.448771 \\
\end{tabular} & 18.1292 & 144.4754 & 3374.28 & 5125 & 0.1 & 1950 & 487500 & 1862500 & 0.1372 & $4.07 \mathrm{E}-04$ & 2.499 & $3.92 \mathrm{E}-03$ & 41.73 & 98.67 & 86.72 & & 177 & 552.4 \\
\hline 396 & 21.34 & 3000 & 4350 & 1.211582 & 0.0336 & 83.1021 & 5866.28 & 5125 & 0.1 & 1950 & 487500 & 812500 & 0.1491 & $4.58 \mathrm{E}-04$ & 2.554 & $1.44 \mathrm{E}-02$ & 33.97 & 0 & & 0 & 48.1 & 613.4 \\
\hline 397 & 24.3 & 3000 & 6310 & 295.490498 & 8.0165 & 81.3886 & 5989.78 & 5125 & 0.1 & 1950 & 487500 & 787500 & 0.1309 & $5.03 \mathrm{E}-04$ & 2.944 & $4.11 \mathrm{E}-03$ & 75.31 & 98.31 & 83.08 & & 168.8 & 620.9 \\
\hline 398 & 22.48 & 3000 & 1700 & 16.5102 & 0.7334 & 133.2659 & 3658.1 & 5125 & 0.1 & 1950 & 487500 & 1650000 & 0.1415 & $1.35 \mathrm{E}-04$ & 1.481 & $1.73 \mathrm{E}-02$ & 79.86 & 69.72 & & & 40.2 & 337.7 \\
\hline 399 & 48.79 & 3000 & 2310 & 52.416225 & 2.3167 & 132.592 & 3676.69 & 5125 & 0.1 & 1950 & 487500 & 1637500 & 0.0652 & $1.86 \mathrm{E}-04$ & 2.442 & $1.73 \mathrm{E}-02$ & 50.23 & 90.46 & 4.61 & & 40 & 256.5 \\
\hline 400 & 44.74 & 3000 & 5320 & \begin{tabular}{|r|}
23.307061 \\
\end{tabular} & 0.8431 & 108.5219 & 4492.18 & 5125 & 0.1 & 1950 & 487500 & 1212500 & 0.0711 & 5.14E-04 & 4.653 & $9.77 \mathrm{E}-03$ & 16.18 & 78.55 & 0 & & 71 & 532.9 \\
\hline 401 & 40.42 & 3000 & 890 & 1234.813005 & 62.6182 & \begin{tabular}{|l|l|}
152.1319 \\
\end{tabular} & 3204.46 & 5125 & 0.1 & 1950 & 487500 & 2012500 & 0.0787 & $3.98 \mathrm{E}-05$ & 1.256 & $5.69 \mathrm{E}-03$ & $\begin{array}{l}92.87 \\
\end{array}$ & 99.6 & $\begin{array}{l}95.95 \\
\end{array}$ & 59.51 & 121.8 & 159.2 \\
\hline 402 & 32.93 & 3000 & 4670 & \begin{tabular}{|l|}
694.914764 \\
\end{tabular} & 25.6535 & 110.7481 & 4401.88 & 5125 & 0.1 & 1950 & 487500 & 1250000 & 0.0966 & $3.72 \mathrm{E}-04$ & 2.946 & $3.26 \mathrm{E}-03$ & 26.31 & 99.28 & 92.8 & 28.05 & 212.6 & 458.4 \\
\hline 403 & 40.89 & 3000 & 5940 & 44.63608 & $\begin{array}{l}1.2867 \\
\end{array}$ & $\begin{array}{l}86.4774 \\
\end{array}$ & 5637.31 & 5125 & 0.1 & 1950 & 487500 & 862500 & 0.0778 & $4.38 \mathrm{E}-04$ & 3.845 & $1.01 \mathrm{E}-02$ & 49.14 & 88.8 & 0 & & 68.7 & 481.8 \\
\hline 404 & 26.67 & 3000 & 3140 & \begin{tabular}{|l|l}
147.641754 \\
\end{tabular} & 8.3317 & 169.2951 & 2879.59 & 5125 & 0.1 & 1950 & 487500 & 2362500 & 0.1193 & $3.08 \mathrm{E}-04$ & 2.303 & $7.14 \mathrm{E}-03$ & 36.38 & 96.61 & 66.13 & & 97.1 & 442.7 \\
\hline 405 & 22 & 3000 & 4940 & 78.545112 & 2.7029 & 103.2361 & 4722.19 & 5125 & 0.1 & 1950 & 487500 & 1125000 & 0.1446 & 3.99E-04 & 2.396 & $7.24 \mathrm{E}-03$ & $\begin{array}{ll}64.02 \\
\end{array}$ & 93.63 & 36.34 & & 95.7 & 558 \\
\hline 406 & 52.85 & 3000 & 3260 & 55.90417 & 3.1213 & $\begin{array}{l}167.4988 \\
\end{array}$ & 2910.47 & 5125 & 0.1 & 1950 & & 2325000 & 0.0602 & & 3.894 & & 46.69 & 91.06 & 10.56 & & 57.6 & 377.7 \\
\hline 407 & 27.86 & 3000 & 4940 & 135.501171 & 3.9436 & \begin{tabular}{|l|l|}
87.3109 \\
\end{tabular} & 5583.5 & 5125 & 0.1 & 1950 & & 875000 & 0.1142 & & 3.165 & $5.48 \mathrm{E}-03$ & 15.65 & 96.31 & \begin{tabular}{ll|}
63.1 \\
\end{tabular} & & 126.5 & 582.2 \\
\hline 408 & 41.21 & 3000 & 3090 & 208.352883 & & 118.7276 & 4106.04 & 5125 & 0.1 & 1950 & 487500 & 1387500 & & 3.14E-04 & 3.056 & 7.19E-03 & 15.43 & 97.6 & 76 & & 96.4 & 380 \\
\hline 409 & 33 & 3000 & 3760 & & 2.3457 & $\begin{array}{l}141.2249 \\
\end{array}$ & 3451.94 & 5125 & 0.1 & 1950 & 487500 & 1800000 & 0.0964 & & 2.927 & $9.80 \mathrm{E}-03$ & 37.24 & 89.97 & 0 & & 70.7 & 454.6 \\
\hline 410 & 50.1 & 3000 & 4240 & 240.30477 & 10.9427 & 136.6103 & 3568.54 & 5125 & 0.1 & 1950 & 487500 & 1712500 & 0.0635 & $4.90 \mathrm{E}-04$ & 4.904 & \begin{tabular}{|l|l|}
$5.77 \mathrm{E}-03$ \\
\end{tabular} & 61.03 & 97.92 & 79.19 & & 120.2 & 501.7 \\
\hline 411 & 40.53 & 3000 & 3760 & \begin{tabular}{|r|}
417.055662 \\
\end{tabular} & 23.5352 & $\begin{array}{l}169.2951 \\
7752123 \\
\end{array}$ & $\begin{array}{l}2879.59 \\
617007\end{array}$ & 5125 & 0.1 & 1950 & 487500 & 2362500 & 0.0785 & 4.87E-04 & 4.137 & $3.98 \mathrm{E}-03$ & 35 & 98.8 & 88.01 & & 174.2 & 523.2 \\
\hline $\begin{array}{r}412 \\
413 \\
13\end{array}$ & 38.75 & 3000 & 6750 & \begin{tabular}{|l|}
233.681189 \\
\end{tabular} & $\begin{array}{r}5.8609 \\
10304\end{array}$ & $\begin{array}{r}75.2423 \\
1870019 \\
\end{array}$ & $\begin{array}{l}6479.07 \\
259144\end{array}$ & $\begin{array}{l}5125 \\
525\end{array}$ & 0.1 & $\begin{array}{l}1950 \\
1900 \\
\end{array}$ & & $\begin{array}{r}700000 \\
2702500\end{array}$ & 0.0821 & 4.24E-04 & $\begin{array}{l}3.609 \\
1295\end{array}$ & \begin{tabular}{|l|}
$5.84 \mathrm{E}-03$ \\
$100 \mathrm{E}$
\end{tabular} & 51.72 & 97.86 & 78.6 & & \begin{tabular}{ll|l|l|l|}
118.6 \\
691
\end{tabular} & 477.3 \\
\hline$\frac{413}{444}$ & $\begin{array}{l}22.33 \\
36.65 \\
\end{array}$ & 3000 & $\begin{array}{r}900 \\
5430\end{array}$ & \begin{tabular}{|r|}
164.521655 \\
9288594
\end{tabular} & $\begin{aligned} 10.3046 \\
3267\end{aligned}$ & \begin{tabular}{|r|}
187.9019 \\
1055176
\end{tabular} & 2594.44 & $\begin{array}{l}5125 \\
5125\end{array}$ & 0.1 & $\begin{array}{c}1950 \\
11950 \\
\end{array}$ & 487500 & $\begin{array}{l}2762500 \\
1112500 \\
\end{array}$ & $\begin{array}{l}0.1425 \\
0.086\end{array}$ & $\begin{array}{l}8.02 \mathrm{E}-05 \\
730 \mathrm{E}-04\end{array}$ & $\begin{array}{l}1.285 \\
53204\end{array}$ & & 26.23 & 96.96 & 69.61 & & $\begin{array}{r}69.1 \\
112 .\end{array}$ & 294.9 \\
\hline \begin{tabular}{|c|}
414 \\
415 \\
\end{tabular} & $\begin{array}{ll}30.65 \\
61.65\end{array}$ & 3000 & $\frac{5430}{4190}$ & $\begin{array}{r}92.8859947 \\
6.507918\end{array}$ & $\begin{array}{l}3.2667 \\
0.319\end{array}$ & \begin{tabular}{|l}
105.5176 \\
147.0497
\end{tabular} & $\frac{4620.08}{3315.21}$ & $\begin{array}{l}5125 \\
5125\end{array}$ & $\begin{array}{l}0.1 \\
0.1\end{array}$ & $\begin{array}{l}1950 \\
1950\end{array}$ & $487 !$ & $\frac{11125000}{1912500}$ & $\mid$\begin{tabular}{|c|}
0.0688 \\
0.0516
\end{tabular} & \begin{tabular}{|l}
$7.39 \mathrm{E}-04$ \\
$4.73 \mathrm{E}-04$
\end{tabular} & $\begin{array}{l}5.304 \\
5.631 \\
\end{array}$ & \begin{tabular}{|l|}
$6.15 \mathrm{E}-03$ \\
$1.51 \mathrm{E}-02$ \\
\end{tabular} & $\begin{array}{l}87.44 \\
22.67\end{array}$ & $\begin{array}{l}94.62 \\
23.17\end{array}$ & $\begin{aligned} 46.17 \\
0\end{aligned}$ & & $\begin{array}{r}112.7 \\
45.9\end{array}$ & $\begin{array}{r}741.6 \\
468 \\
\end{array}$ \\
\hline 416 & 26.85 & & 2410 & & 0.47 & 141.2249 & 3451.94 & 5125 & 0.1 & 1950 & & & 0.1185 & $2.27 \mathrm{E}-04$ & 1.967 & & 55.32 & 50.02 & & & 40.7 & $\begin{array}{r}400 \\
375.5 \\
\end{array}$ \\
\hline 417 & 57.84 & 3000 & 4020 & 56.92219 & 2.87 & 151.5013 & 3217.79 & 5125 & 0.1 & 1950 & & & 0.055 & $4.50 \mathrm{E}-04$ & 5.139 & 9.70 & 28. & 91.22 & 12.16 & & 71.5 & 455.3 \\
\hline 418 & 26.49 & 3000 & 1380 & 202.285027 & & 99.3762 & 4905.6 & 5125 & 0. & 1950 & & & 0.1201 & & 1.592 & & 33. & 97.53 & 75.28 & & 77 & 308 \\
\hline 419 & 24.64 & 3000 & 3570 & \begin{tabular}{|r|}
29.019364 \\
\end{tabular} & 1.25 & 129.879 & 3753.49 & 5125 & 0.1 & 1950 & & 1587500 & & & 2.473 & & 25. & 82.77 & & & 72.7 & 514.3 \\
\hline 420 & 49.4 & 3000 & 2340 & 98.627376 & 3.00 & 91.4206 & 5332.5 & 5125 & 0.1 & 1950 & 487 & 937500 & 0.0644 & $2.35 \mathrm{E}-04$ & 2.841 & $1.20 \mathrm{E}$ & 19. & 94.93 & 49.3 & & 57.7 & 294.8 \\
\hline 421 & 21.22 & 3000 & 2580 & 83.5213 & 4.07 & 146.4082 & 3329.73 & 5125 & 0.1 & 1950 & 487 & 1900000 & 0.1499 & $2.60 \mathrm{E}-04$ & 1.875 & $8.28 \mathrm{E}-03$ & 36.15 & 94.01 & 40.14 & & 83.7 & 452.9 \\
\hline 422 & 24.97 & 3000 & 4580 & 5.5398 & 0.20 & 112.2199 & 4344.15 & 5125 & 0.1 & 1950 & 487 & 1275000 & 0.1274 & $5.09 \mathrm{E}-04$ & 3.02 & $1.36 \mathrm{E}-02$ & 91.3 & 9.75 & & & 51 & 619.9 \\
\hline 423 & 24.03 & 3000 & 3120 & 61.1612 & 1.8968 & 93.0387 & 5239.76 & 5125 & 0.1 & 1950 & & 962500 & 0.1324 & $2.74 \mathrm{E}-04$ & 2.045 & $9.63 \mathrm{E}-03$ & 50.8 & 91.82 & 18.25 & & 72 & 436.2 \\
\hline 424 & 22.12 & 3000 & 3750 & 52.191 & 2.4796 & 142.5296 & 3420.34 & 5125 & 0.1 & 1950 & & 1825000 & 0.1438 & $4.73 \mathrm{E}-04$ & 2.661 & & 86.8 & 90.42 & 4.2 & & 89.2 & 616.5 \\
\hline 425 & 38.47 & 3000 & 3220 & \begin{tabular}{|l|l|}
111.347 \\
\end{tabular} & 3.6 & 97.024 & 5024.53 & 5125 & 0.1 & 1950 & & & 0.08 & $2.57 \mathrm{E}-04$ & 2.568 & & 42. & 95.51 & 55.1 & & $\begin{array}{ll}67.2 \\
\end{array}$ & 342.1 \\
\hline 426 & 25.76 & 30 & 2850 & \begin{tabular}{|l|}
133.37 \\
\end{tabular} & & 182.7 & & 5125 & 0.1 & 1950 & & & 0.12 & & 2.13 & & 75. & 96.25 & 62.51 & & 86 & 423.8 \\
\hline 427 & 40.17 & & 5950 & & & 94.6 & & 5125 & 0.1 & 195 & & & & & 4.406 & & & 80.25 & & & 77.4 & 562.1 \\
\hline 428 & 33.7 & & 1790 & & & 143.1 & & 5125 & 0.1 & 195 & & & 0.09 & & 1.714 & & & 97.66 & 76.59 & & $\begin{array}{l}64.4 \\
\end{array}$ & 260.6 \\
\hline 429 & $\begin{array}{l}42.08 \\
\end{array}$ & & 6700 & & & & & 5125 & 0.1 & 195 & & & & & \begin{tabular}{|l|}
4.93 \\
\end{tabular} & & 23. & & 0 & & 54.1 & $\begin{array}{l}600.4 \\
\end{array}$ \\
\hline 430 & $\begin{array}{l}29.48 \\
\end{array}$ & 3000 & 1710 & & & & & 5125 & 0.1 & 195 & & & & & 1.584 & & 80. & 94.94 & \begin{tabular}{|l|}
49.41 \\
\end{tabular} & & 51.6 & 275.4 \\
\hline 431 & \begin{tabular}{|l|}
21.93 \\
\end{tabular} & 3000 & 3170 & & & 169.8 & & 5125 & 0.1 & 195 & & & & & 2.25 & & & 65.99 & & & 63.4 & 525.9 \\
\hline 432 & $\begin{array}{l}61.53 \\
202\end{array}$ & & 950 & & & & & 5124 & 0.1 & 19 & & & & & 1.977 & & & 99 & 94.19 & 41.91 & & 64.7 \\
\hline 433 & 22.39 & 30 & 3390 & & & 91.4206 & & 5 & 0.1 & 19 & & & & & 1.909 & & & & 89.51 & & 158.4 & 36.9 \\
\hline 434 & 35.23 & 30 & 4670 & & & 112.2 & 434 & 125 & 0.1 & 19 & & & & & 3.401 & & & & 76.51 & & 114.9 & 94.8 \\
\hline 435 & 24.72 & 3000 & 2360 & \begin{tabular}{|l|l|}
225.72 \\
\end{tabular} & \begin{tabular}{|l|l|}
6.9397 \\
\end{tabular} & & 5285.62 & 5125 & 0.1 & 1950 & & 950000 & 0.1287 & & 1.743 & & & $\begin{array}{l}97.78 \\
97.77\end{array}$ & $\begin{array}{l}77.85 \\
77.77\end{array}$ & & 92.1 & 61.4 \\
\hline 436 & 55.91 & & 5360 & 224.29 & $\begin{array}{r}7.076 \\
\end{array}$ & 94.6 & 5150.94 & 5125 & 0.1 & 195 & & & 0.0569 & & 3.983 & & & 97.77 & 77.71 & & & 365.1 \\
\hline 437 & 24.78 & 3000 & 2710 & 128.4361 & 5.6476 & 131.9164 & 3695.52 & 5125 & 0.1 & 195 & & 1625 & 0.1284 & 2.84E-04 & 2.116 & & 29. & 96.11 & 61.07 & & 92.8 & 437.8 \\
\hline 438 & 23.57 & 3000 & 3650 & $\begin{array}{r}8.403335 \\
\end{array}$ & 0.4173 & 148.9657 & $\begin{array}{l}3272.57 \\
70.02\end{array}$ & 5125 & 0.1 & 1950 & 487500 & 19500 & 0.135 & 5.21E-04 & 2.95 & 1.15E & 73.84 & 40.5 & 0 & & 60.5 & 641.6 \\
\hline 439 & 55.62 & 3000 & 7910 & $\begin{array}{r}5.084827 \\
\end{array}$ & 0.1167 & 68.8336 & $\begin{array}{r}7082.3 \\
\end{array}$ & 5125 & 0.1 & 1950 & 487500 & $\begin{array}{r}612500 \\
\end{array}$ & 0.0572 & $6.22 \mathrm{E}-04$ & 6.495 & $1.30 \mathrm{E}$ & 27.04 & $\begin{array}{c}1.67 \\
\end{array}$ & 0 & & 53.4 & \\
\hline 441 & 36.19 & 3000 & 4890 & $27 . .771756$ & 0.9557 & 103.2361 & 4722.19 & 5125 & 0.1 & 1950 & 487500 & 1125000 & 0.0879 & -04 & 2.997 & 1271 & 55.88 & 82 & 0 & & 54.5 & \\
\hline 4411 & 34.65 & 3000 & 3240 & \begin{tabular}{|c|}
199.355883 \\
\end{tabular} & 9.7291[ & 146.4082 & 3329.73 & 5125 & & 1950 & 487500 & 1900000 & 0.0918 & $2.04 \mathrm{E}-04$ & 2.124 & $9.53 \mathrm{E}-03$ & 92.4 & $97.49 \mid$ & 74.92 & & $72.7 \mid$ & 314 \\
\hline
\end{tabular}




\begin{tabular}{|c|c|c|c|c|c|c|c|c|c|c|c|c|c|c|c|c|c|c|c|c|c|c|}
\hline $\begin{array}{c}\text { Realizatio } \\
n\end{array}$ & $\begin{array}{c}\text { Velocity } \\
(\mathrm{m} / \mathrm{y})\end{array}$ & \begin{tabular}{|c} 
Fixed Source \\
Concentratio \\
n (mg/L)
\end{tabular} & \begin{tabular}{|c|} 
Time of \\
Maximum at \\
Compliance \\
Boundary \\
(y)
\end{tabular} & $\begin{array}{c}\text { Maximum } \\
\text { Concentratio } \\
\text { n Compliance } \\
\text { Boundary } \\
(\mu \mathrm{g} / \mathrm{L})\end{array}$ & \begin{tabular}{|c|} 
Maximum \\
Release \\
Rate at \\
Complianc \\
e Boundary \\
(Kg/y) \\
\end{tabular} & $\begin{array}{c}\text { Source } \\
\text { Rate } \\
(\mathrm{Kg} / \mathrm{y})\end{array}$ & $\begin{array}{c}\text { Source } \\
\text { Duration } \\
\text { (y) }\end{array}$ & \begin{tabular}{|c|} 
1-D \\
Streamtub \\
e Length \\
$(\mathrm{m})$
\end{tabular} & $\begin{array}{c}\text { Base } \\
\text { Porosity } \\
\left(\mathrm{m}^{3} / \mathrm{m}^{3}\right) \\
\end{array}$ & $\begin{array}{c}\text { Bulk } \\
\text { Densty } \\
\left(\mathrm{Kg} / \mathrm{m}^{3}\right) \\
\end{array}$ & $\begin{array}{c}\text { Remaining } \\
\text { Carbon } \\
\text { Tetrachloride } \\
\text { Source } \\
(\mathrm{Kg}) \\
\end{array}$ & $\begin{array}{c}\text { Volume at or } \\
\text { above Fixed } \\
\text { Source } \\
\text { Concentratio } \\
n(m 3)\end{array}$ & $\begin{array}{l}\text { Porosity } \\
\left(\mathrm{m}^{3} / \mathrm{m}^{3}\right)\end{array}$ & $\begin{array}{c}\mathrm{Kd} \\
\left(\mathrm{m}^{3} / \mathrm{Kg}\right)\end{array}$ & Retardation & $\begin{array}{c}\text { First } \\
\text { Order } \\
\text { Rate } \\
\text { Constant } \\
(1 / y) \\
15 / 50\end{array}$ & \begin{tabular}{|c|}
$\begin{array}{c}\text { Longitudinal } \\
\text { Dispersivity } \\
(\mathrm{m})\end{array}$ \\
\end{tabular} & \begin{tabular}{|c|} 
Required \\
Source \\
Cleanup \\
Percentage \\
$(5 \mu g / L$ \\
Compliance) \\
\end{tabular} & \begin{tabular}{|c|} 
Required \\
Source \\
Cleanup \\
Percentage \\
$(50 \mu \mathrm{g} / \mathrm{L}$ \\
Compliance) \\
\end{tabular} & $\begin{array}{c}\text { Required } \\
\text { Source } \\
\text { Cleanup } \\
\text { Percentage } \\
\text { (500 } \mathrm{\mu g} / \mathrm{L} \\
\text { Compliance) } \\
\end{array}$ & \begin{tabular}{|c|} 
Abiotic \\
Reaction \\
Half Time \\
$(y)$ \\
\end{tabular} & $\begin{array}{c}\text { Carbon } \\
\text { Tetrachloride } \\
\text { Travel Time } \\
(\mathrm{y}) \\
\end{array}$ \\
\hline & & 3000 & 2310 & 9.637674 & \begin{tabular}{|l|l|} 
& 0.3768 \\
\end{tabular} & 117.2971 & 4156.11 & & & 1950 & 487500 & 1362500 & 0.1365 & $2.33 \mathrm{E}-04$ & $\begin{array}{l}1.865 \\
\end{array}$ & $1.54 \mathrm{E}-02$ & $\begin{array}{r}48.42 \\
\end{array}$ & $\begin{array}{r}48.12 \\
\end{array}$ & & & & 410 \\
\hline $\begin{array}{r}443 \\
444 \\
\end{array}$ & $\begin{array}{r}28.25 \\
41.8\end{array}$ & 3000 & 2410 & $\begin{array}{r}382.73656 \\
7643617\end{array}$ & 12.6783 & \begin{tabular}{|r|}
99.3762 \\
1632695 \\
\end{tabular} & 4905.6 & 5125 & $\begin{array}{ll}0.1 \\
0.1\end{array}$ & $\begin{array}{l}1950 \\
1950\end{array}$ & 487500 & $\begin{array}{l}1062500 \\
223700\end{array}$ & 0.1126 & 1.67E-04 & 1.749 & \begin{tabular}{|l|l|}
$6.83 \mathrm{E}-03$ \\
121502
\end{tabular} & 73.71 & 98.69 & 86.94 & & 101.6 & 317.3 \\
\hline 445 & \begin{tabular}{r|r}
23.08 & \\
23.58
\end{tabular} & 3000 & $\begin{array}{r}3920 \\
870\end{array}$ & $\begin{array}{r}7.643617 \\
6350679\end{array}$ & \begin{tabular}{r|}
0.436 \\
15372 \\
\end{tabular} & $\begin{array}{r}r^{163.2695} \\
725304 \\
\end{array}$ & $\begin{array}{l}2985.86 \\
6721.32\end{array}$ & $\begin{array}{l}5125 \\
5125 \\
\end{array}$ & $\begin{array}{l}0.1 \\
0.1 \\
\end{array}$ & \begin{tabular}{|c|}
1950 \\
1950
\end{tabular} & $\begin{array}{l}487500 \\
48750\end{array}$ & $\begin{array}{r}2237500 \\
62500 \\
\end{array}$ & $\begin{array}{l}0.0761 \\
0.1349\end{array}$ & $\begin{array}{l}6.09 E-04 \\
775 E-05\end{array}$ & $\frac{5.047}{1291}$ & \begin{tabular}{|l|}
$1.21 \mathrm{E}-02$ \\
$141 \mathrm{E}-02$ \\
\end{tabular} & $\begin{array}{l}43.14 \\
29.77\end{array}$ & 34.59 & $\begin{array}{r}0 \\
21.36\end{array}$ & & $\begin{array}{l}57.3 \\
49 \\
\end{array}$ & \begin{tabular}{|c|c|}
618.7 \\
280.5 \\
\end{tabular} \\
\hline 446 & 26.06 & 3000 & 3180 & 36.871007 & 1.1435 & 93.0387 & 5239.76 & 5125 & 0.1 & 1950 & 487500 & 962500 & 0.1221 & $2.68 \mathrm{E}-04$ & 2.2.108 & \begin{tabular}{|l|}
$1.41 \mathrm{IE}-\mathrm{L}$ \\
$1.17 \mathrm{E}-02$ \\
\end{tabular} & 57.55 & 86.44 & 21.00 & & \begin{tabular}{c|}
59.2 \\
59
\end{tabular} & 400.5 \\
\hline 447 & 33.07 & 3000 & 2020 & 384.671653 & 18.8553 & $\begin{array}{r}147.0497 \\
\end{array}$ & 3315.21 & 5125 & 0.1 & 1950 & 487500 & 1912500 & 0.0962 & $1.95 \mathrm{E}-04$ & 2.024 & \begin{tabular}{|l|}
$6.73 \mathrm{E}-03$ \\
\end{tabular} & 33.72 & \begin{tabular}{|c|}
90.44 \\
98.7
\end{tabular} & 87 & & \begin{tabular}{r|r|}
103.1 \\
\end{tabular} & 313.7 \\
\hline 448 & 35.11 & 3000 & 4110 & 12.857262 & 0.5625 & 131.239 & 3714.6 & 5125 & 0.1 & 1950 & 487500 & 1612500 & 0.0906 & $5.55 \mathrm{E}-04$ & 4.096 & \begin{tabular}{|l|}
$1.25 \mathrm{E}-02$ \\
\end{tabular} & $\begin{array}{l}35.72 \\
85.72 \\
\end{array}$ & 61.11 & 0 & & 50.1 & 597.8 \\
\hline 449 & 61.89 & 3000 & 2590 & 437.978377 & 13.2278 & 90.6061 & 5380.43 & 5125 & 0.1 & 1950 & 487500 & 925000 & 0.0514 & $2.00 \mathrm{E}-04$ & 2.962 & $8.10 \mathrm{E}-03$ & $\begin{array}{l}28.12 \\
28.93 \\
\end{array}$ & 98.86 & 88.58 & & 85.6 & 245.3 \\
\hline 450 & 26.21 & 3000 & 1720 & 261.760809 & 15.2369 & 174.6277 & 2791.65 & 5125 & 0.1 & 1950 & 487500 & 2475000 & 0.1214 & $1.26 \mathrm{E}-04$ & 1.525 & $8.58 \mathrm{E}-03$ & 67.37 & 98.09 & 80.9 & & 80.8 & 298.3 \\
\hline 451 & 22.56 & 3000 & 4530 & 13.093697 & 0.5336 & 122.2668 & 3987.18 & 5125 & 0.1 & 1950 & 487500 & 1450000 & 0.141 & $5.52 \mathrm{E}-04$ & 2.98 & \begin{tabular}{|l|}
$8.98 \mathrm{E}-03$ \\
\end{tabular} & 37.52 & 61.81 & & & 77.2 & 677 \\
\hline 452 & 23.34 & 3000 & 3960 & 4.434821 & 0.2193 & 148.3284 & 3286.63 & 5125 & 0.1 & 1950 & 487500 & 1937500 & 0.1363 & $6.80 \mathrm{E}-04$ & 3.521 & \begin{tabular}{|l|}
$1.07 \mathrm{E}-02$ \\
\end{tabular} & 60.91 & 0 & 0 & 0 & 64.6 & 773 \\
\hline 453 & 26.8 & 3000 & 1020 & 864.546348 & 47.0514 & 163.2695 & 2985.86 & 5125 & 0.1 & 1950 & 487500 & 2237500 & 0.1187 & $8.55 \mathrm{E}-05$ & 1.364 & \begin{tabular}{|l}
$4.83 \mathrm{E}-03$ \\
\end{tabular} & 35.46 & 99.42 & 94.22 & & 143.6 & 260.9 \\
\hline 454 & 38.61 & 3000 & 3230 & 303.71462 & 11.8022 & 116.5786 & 4181.73 & 5125 & 0.1 & 1950 & 487500 & 1350000 & 0.0824 & $2.12 \mathrm{E}-04$ & 2.3 & \begin{tabular}{|l|l}
$8.02 \mathrm{E}-03$ \\
\end{tabular} & 66.48 & 98.35 & 83.54 & & 86.5 & 305.3 \\
\hline 455 & 40.68 & 3000 & 4520 & 75.590018 & 2.9374 & 116.5786 & 4181.73 & 5125 & 0.1 & 1950 & 487500 & 1350000 & 0.0782 & $4.41 \mathrm{E}-04$ & 3.851 & \begin{tabular}{|c|c|}
$9.03 \mathrm{E}-03$ \\
\end{tabular} & 68.85 & 93.39 & 33.85 & & 76.7 & 485.2 \\
\hline 456 & 32.46 & 3000 & 3400 & 240.034458 & 9.9507 & 124.3658 & 3919.89 & 5125 & 0.1 & 1950 & 487500 & 1487500 & 0.098 & $2.20 \mathrm{E}-04$ & 2.133 & \begin{tabular}{|l|l}
$8.12 \mathrm{E}-03$ \\
\end{tabular} & 78.92 & 97.92 & 79.17 & & 85.3 & 336.8 \\
\hline 457 & 44.81 & 3000 & 1610 & 434.942036 & 12.8984 & 88.9661 & 5479.61 & 5125 & 0.1 & 1950 & 487500 & 900000 & 0.071 & $1.85 \mathrm{E}-04$ & 2.317 & \begin{tabular}{|l}
$7.40 \mathrm{E}-03$ \\
\end{tabular} & 18 & 98.85 & 88.5 & & 93.6 & 265 \\
\hline 458 & 21.73 & 3000 & 3170 & 6.946572 & 0.3878 & 167.4988 & 2910.47 & 5125 & 0.1 & 1950 & 487500 & 2325000 & 0.1464 & $3.48 \mathrm{E}-04$ & 2.202 & $1.29 \mathrm{E}-02$ & 40.25 & 28.02 & 0 & & 53.7 & 519.3 \\
\hline 459 & 22.99 & 3000 & 5380 & 45.758934 & 1.5395 & 100.929 & 4830.13 & 5125 & 0.1 & 1950 & 487500 & 1087500 & 0.1384 & $5.42 \mathrm{E}-04$ & 2.98 & $6.66 \mathrm{E}-03$ & 24.05 & 89.07 & 0 & & 104 & 664.4 \\
\hline 460 & 22.44 & 3000 & 3460 & 34.151891 & 1.674 & 147.0497 & 3315.21 & 5125 & 0.1 & 1950 & 487500 & 1912500 & 0.1418 & $3.12 \mathrm{E}-04$ & 2.111 & $1.09 \mathrm{E}-02$ & 93.45 & 85.36 & 0 & & 63.7 & 482.2 \\
\hline 461 & 50.74 & 3000 & 1280 & 178.778008 & 6.6875 & 112.2199 & 4344.15 & 5125 & 0.1 & 1950 & 487500 & 1275000 & 0.0627 & $1.23 \mathrm{E}-04$ & 1.994 & $1.45 \mathrm{E}-02$ & 34.64 & 97.2 & 72.03 & & 47.7 & 201.4 \\
\hline 462 & 30.86 & 3000 & 1970 & 60.719498 & 2.7784 & 137.2743 & 3551.28 & 5125 & 0.1 & 1950 & 487500 & 1725000 & 0.1031 & $1.81 \mathrm{E}-04$ & 1.887 & $1.33 \mathrm{E}-02$ & 48.48 & \begin{tabular}{|l|l|}
91.77 \\
\end{tabular} & 17.65 & & 52.1 & 313.3 \\
\hline 463 & 25.17 & 3000 & 1910 & 72.660948 & 3.1622 & 130.5599 & 3733.92 & 5125 & 0.1 & 1950 & 487500 & 1600000 & 0.1264 & $1.94 \mathrm{E}-04$ & 1.777 & & 35.56 & 93.12 & 31.19 & & 64.5 & 361.9 \\
\hline 464 & 46.44 & 3000 & 3410 & 73.697018 & 3.0036 & 122.2668 & 3987.18 & 5125 & 0.1 & 1950 & & 1450000 & 0.0685 & $2.94 \mathrm{E}-04$ & 3.167 & $1.11 \mathrm{E}-02$ & 22.09 & 93.22 & 32.15 & & $\begin{array}{ll}62.2 \\
\end{array}$ & 349.5 \\
\hline 465 & 25.45 & 3000 & 1410 & 97.817719 & & 88.9661 & 5479.61 & 5125 & 0.1 & 1950 & 487500 & 900000 & 0.125 & & 1.401 & & 72.52 & \begin{tabular}{|l|}
94.89 \\
\end{tabular} & 48.88 & & 53.5 & 282 \\
\hline 466 & $\begin{array}{l}41.48 \\
\end{array}$ & 3000 & 1180 & 260.751272 & 7.0741 & 81.3886 & 5989.78 & 5125 & 0.1 & 1950 & 487500 & 787500 & 0.0767 & $1.51 \mathrm{E}-04$ & 1.996 & $1.01 \mathrm{E}-02$ & \begin{tabular}{|l|l|}
17.62 \\
\end{tabular} & \begin{tabular}{|l|l|}
98.08 \\
\end{tabular} & 80.82 & & \begin{tabular}{ll|}
68.8 \\
\end{tabular} & 246.6 \\
\hline 467 & 21.37 & 3000 & 1120 & 382.975605 & 23.8423 & 186.7666 & 2610.21 & 5125 & 0.1 & 1950 & 487500 & 2737500 & $\begin{array}{l}0.1489 \\
\end{array}$ & $1.28 \mathrm{E}-04$ & 1.433 & $6.06 \mathrm{E}-03$ & 20.12 & \begin{tabular}{|l|l|}
98.69 \\
\end{tabular} & 86.94 & & 114.4 & 343.7 \\
\hline 468 & $\begin{array}{l}50.26 \\
520\end{array}$ & 3000 & 2780 & $\begin{array}{l}336.238321 \\
1150202\end{array}$ & \begin{tabular}{|l|l|}
18.6384 \\
0.620
\end{tabular} & 166.2959 & 2931.52 & 5125 & 0.1 & 1950 & 487500 & 2300000 & $\begin{array}{l}0.0633 \\
\end{array}$ & $2.33 \mathrm{E}-04$ & $\begin{array}{l}2.862 \\
\end{array}$ & $7.74 \mathrm{E}-03$ & 25.75 & 98.51 & 85.13 & & $\begin{array}{l}89.6 \\
\end{array}$ & 291.8 \\
\hline 469 & $\begin{array}{l}53.92 \\
2.92\end{array}$ & 3000 & 2740 & 145.928274 & \begin{tabular}{|c|}
9.6299 \\
\end{tabular} & 197.9711 & 2462.48 & 5125 & 0.1 & 1950 & 487500 & 2987500 & $\begin{array}{l}0.059 \\
\end{array}$ & $2.57 \mathrm{E}-04$ & 3.201 & $1.03 \mathrm{E}-02$ & 21.47 & \begin{tabular}{|l|l|}
96.57 \\
9
\end{tabular} & 65.74 & & 67 & 304.2 \\
\hline 470 & 35.87 & 3000 & 4300 & $\begin{array}{l}13.286036 \\
368125\end{array}$ & $\begin{array}{l}0.5195 \\
1074\end{array}$ & $\begin{array}{l}117.2971 \\
15.2\end{array}$ & 4156.11 & 5125 & 0.1 & 1950 & 487500 & 1362500 & $\begin{array}{l}0.0887 \\
\end{array}$ & $3.24 \mathrm{E}-04$ & 2.847 & $1.60 \mathrm{E}-02$ & 66.01 & $\begin{array}{l}62.37 \\
\end{array}$ & & & 43.4 & 406.8 \\
\hline 471 & $\begin{array}{l}45.06 \\
115.5\end{array}$ & 3000 & 3650 & 36.811352 & $\begin{array}{l}1.8744 \\
\end{array}$ & 152.7612 & 3191.25 & 5125 & 0.1 & 1950 & 487500 & 2025000 & 0.0706 & 4.74E-04 & 4.395 & 1.12E-02 & 71.44 & 86.42 & 0 & & 62 & 499.9 \\
\hline 472 & \begin{tabular}{|l|l|}
41.53 \\
48.13
\end{tabular} & 3000 & 2730 & $\begin{array}{r}16.958704 \\
112020\end{array}$ & $\begin{array}{r}0.707 \\
5260 \\
\end{array}$ & 125.0616 & $\begin{array}{l}3898.08 \\
246801 \\
2408\end{array}$ & 5125 & 0.1 & $\begin{array}{l}1950 \\
1900\end{array}$ & 487500 & 1500000 & 0.0766 & 2.91E-04 & 2.92 & 1.51E-02 & 18.1 & 70.52 & 0 & & 45.8 & 360.3 \\
\hline $\begin{array}{l}443 \\
474 \\
\end{array}$ & $\begin{array}{l}48.13 \\
34.32\end{array}$ & 3000 & $\begin{array}{l}4220 \\
3620 \\
3\end{array}$ & $\frac{112.273445}{2420404}$ & $\begin{array}{l}5.2608 \\
71778 \\
\end{array}$ & $\begin{array}{r}140.5704 \\
88961\end{array}$ & $\begin{array}{l}3468.01 \\
547961\end{array}$ & $\begin{array}{l}5125 \\
5125 \\
\end{array}$ & 0.1 & $\begin{array}{l}1950 \\
1990\end{array}$ & $\begin{array}{r}487500 \\
487800\end{array}$ & 1787500 & 0.0661 & 4.93E-04 & $\begin{array}{l}4.769 \\
2321\end{array}$ & $7.29 \mathrm{E}-03$ & $\begin{array}{r}41.6 \\
\end{array}$ & 95.55 & 55.47 & & 95.1 & 507.9 \\
\hline $\begin{array}{l}444 \\
475 \\
\end{array}$ & $\begin{array}{l}34.32 \\
21.54\end{array}$ & 3000 & $\begin{array}{l}3620 \\
3030\end{array}$ & $\frac{242.040042}{69761677}$ & $\begin{array}{l}.1 .1 / 8 \\
3.6105\end{array}$ & $\begin{array}{r}88.9661 \\
155.6566\end{array}$ & $\frac{54 / 9.61}{313978}$ & $\begin{array}{l}5^{2125} \\
5125\end{array}$ & $\begin{array}{l}0.1 \\
0.1 \\
\end{array}$ & $\begin{array}{l}1950 \\
1950\end{array}$ & $\begin{array}{l}48 / 500 \\
487500\end{array}$ & $\begin{array}{r}900000 \\
2075000\end{array}$ & $\begin{array}{l}.09227 \\
0.1477 \\
\end{array}$ & $\begin{array}{l}2.42 \mathrm{E}-04 \\
3.06 \mathrm{E}-04\end{array}$ & $\frac{2.321}{2.046}$ & $\begin{array}{r}8.80 \mathrm{E}-03 \\
8.13 \mathrm{E}-03\end{array}$ & $\begin{array}{l}64.81 \\
35.13 \\
\end{array}$ & $\begin{array}{l}91.93 \\
92.83 \\
\end{array}$ & $\begin{array}{r}9.3 .34 \\
28.33 \\
\end{array}$ & & $\begin{array}{l}88.9 \\
85.2\end{array}$ & $\begin{array}{l}346.6 \\
486.8 \\
\end{array}$ \\
\hline 476 & 26.01 & 3000 & 1610 & 44.312891 & 2.1053 & 142.5296 & 3420.34 & 5125 & 0.1 & 1950 & 487500 & 1825000 & 0.1223 & $1.28 \mathrm{E}-04$ & 1.529 & $1.52 \mathrm{E}-02$ & $\begin{array}{l}68.6 \\
\end{array}$ & 88.72 & & & 45.6 & 301.2 \\
\hline 477 & 29.21 & 3000 & 6470 & 7.510632 & 0.1747 & 69.767 & 6987.55 & 5125 & 0.1 & 1950 & 487500 & 625000 & 0.1089 & $4.64 \mathrm{E}-04$ & 3.153 & \begin{tabular}{|l}
$1.41 \mathrm{E}-02$ \\
\end{tabular} & 82.33 & 33.43 & & & 49.1 & 553.1 \\
\hline 478 & 25.35 & 3000 & 4550 & 1.822081 & 0.0818 & 134.6086 & 3621.61 & 5125 & 0.1 & 1950 & 487500 & 1675000 & 0.1255 & $7.18 \mathrm{E}-04$ & 3.892 & \begin{tabular}{|c|}
$1.14 \mathrm{E}-02$ \\
\end{tabular} & 36.76 & 0 & & & 61 & 786.8 \\
\hline 479 & 34.73 & 3000 & 1670 & 47.375785 & 1.7722 & 112.2199 & 4344.15 & 5125 & 0.1 & 1950 & 487 & 1275000 & 0.0916 & $1.68 \mathrm{E}-04$ & 1.927 & \begin{tabular}{|c|}
$1.55 \mathrm{E}-02$ \\
\end{tabular} & 38.8 & 89.45 & & & 44.8 & 284.4 \\
\hline 480 & 47.41 & 3000 & 5900 & 14.346251 & 0.4215 & 88.1405 & 5530.94 & 5125 & 0.1 & 1950 & & 887500 & 0.0671 & $4.31 \mathrm{E}-04$ & 4.244 & 1.28 & 21.6 & 65.15 & & & 54.3 & 458.7 \\
\hline 481 & 23.24 & 3000 & 3460 & 3.11784 & 0.1271 & 122.2668 & 3987.18 & 5125 & 0.1 & 1950 & & 1450000 & 0.1369 & $3.78 \mathrm{E}-04$ & 2.397 & 1.43 & 30.63 & & & & 48.6 & 528.7 \\
\hline 482 & 23.04 & 3000 & 2970 & 65.226786 & 3.9487 & 181.614 & 2684.26 & 5125 & 0.1 & 1950 & & 2625000 & 0.1381 & $3.19 \mathrm{E}-04$ & 2.168 & & 28.85 & 92.33 & 23.34 & & 83.4 & 482.2 \\
\hline 483 & 21.88 & 3000 & 2850 & \begin{tabular}{|l|}
356.201077 \\
\end{tabular} & 9.968 & 83.9523 & 5806.87 & 5125 & 0.1 & 1950 & & & 0.14 & $2.38 \mathrm{E}-04$ & 1.827 & & 48.59 & 98.6 & 85.96 & & 134.2 & 427.9 \\
\hline 484 & 23.69 & 3000 & 1410 & & 0.2529 & 88.9 & 5479.61 & 5125 & 0.1 & 1950 & 487 & & 0.1343 & $1.98 \mathrm{E}-04$ & 1.744 & & 17.23 & 41.36 & & & 43.1 & 377.3 \\
\hline 485 & 24.91 & 3000 & 4160 & 53.522039 & 2.3776 & 133.2659 & & 5125 & 0.1 & 1950 & 487 & 000 & 0.12 & $4.77 \mathrm{E}$ & 2.889 & & 31.63 & 90.66 & 6.58 & & 95.5 & 594.4 \\
\hline 486 & 49.94 & 3000 & 2650 & 308.261068 & $12.4 \mathrm{~S}$ & 121.5631 & 4010.26 & 5125 & 0.1 & 1950 & 487 & 1437500 & 0.0637 & 1.93 & 2.528 & 9.21 & 44.4 & 98.38 & 83.78 & & 75.3 & 259.4 \\
\hline 487 & 54.95 & & 4350 & 87.761923 & & 122.2668 & & 5125 & 0.1 & & & & & & 4.447 & & & 94.3 & 43.03 & & 69.6 & 414.8 \\
\hline 488 & 41.59 & 3000 & 3190 & & & 145.1 & & 5125 & 0.1 & 19 & & & & & 2.6 & & 40 & & 26.4 & & 56 & \\
\hline 489 & 37.65 & 3000 & 4240 & & & 131. & & 5125 & 0.1 & 19 & & & & & 4.7 & & & & 0 & & 53.8 & \\
\hline 490 & 37.34 & & 3690 & & & & & 5125 & 0.1 & 195 & & & & & 2.903 & & 48. & & 0 & & 47.6 & 398.5 \\
\hline 491 & 38.1 & & 3720 & 485.9 & 19.23 & 118.7 & & 5125 & 0.1 & 195 & & & & & & & 66 & 97 & 89.71 & & 115.3 & \\
\hline 492 & 23.09 & & 3700 & & & 150.2 & 3244 & 5125 & 0.1 & 1950 & & & 0.13 & & & & & & 0 & & \begin{tabular}{ll|}
65.7 \\
\end{tabular} & \\
\hline 493 & 26.67 & 3000 & 3150 & 25.9 & 1.43 & 166.2 & 2931.52 & 5125 & 0.1 & 1950 & & & 0.1193 & 3.54 & 2.498 & & & 80.72 & & & 59.4 & 480.2 \\
\hline 494 & 25.39 & 3000 & 1400 & 403.43 & 17.0044 & 126.44 & 3855 & 5125 & 0.1 & 195 & & & 0.12 & 9.93 & 1.401 & 7.33 & 60.2 & $\begin{array}{l}98.76 \\
\end{array}$ & 87.61 & & $\begin{array}{l}94.6 \\
\end{array}$ & 282.7 \\
\hline 495 & 46.17 & 3000 & 2490 & 511.548031 & 25.6177 & 150.23 & 3244. & 5125 & 0.1 & 1950 & & & 0.0689 & 2.07 & 2.522 & 6.50 & 3 & 99.02 & 90.23 & 2.26 & 106.7 & 279.9 \\
\hline 496 & 24.97 & 3000 & 3340 & & 1.8911 & 110.7481 & 4401.88 & 5125 & 0.1 & 1950 & 4875 & 1250 & 0.1274 & 3.23 & 2.283 & $9.23 \mathrm{E}$ & 34. & 90.24 & 2.4 & & 75.1 & 468.6 \\
\hline 497 & 61.3 & 3000 & 2950 & 100.246418 & 3.00 & $\begin{array}{r}89.788 \\
\end{array}$ & 5429.4 & 5125 & 0.1 & 1950 & 487500 & 9125 & & & 2.739 & $1.69 \mathrm{E}$ & 78.04 & 95.01 & 50.12 & & 40.9 & \\
\hline $\begin{array}{l}4988 \\
499 \\
\end{array}$ & $\begin{aligned} 30.71 \\
63.5 \\
\end{aligned}$ & 3000 & \begin{tabular}{r|}
1490 \\
570
\end{tabular} & $\begin{array}{r}209.773662 \\
826.20882 \\
\end{array}$ & $\begin{array}{r}9.9663 \\
33.4788 \\
\end{array}$ & \begin{tabular}{|l|l|}
142.5296 \\
12.5631
\end{tabular} & $\begin{array}{l}3420.34 \\
4010.26\end{array}$ & $\begin{array}{l}5125 \\
5125\end{array}$ & $\begin{array}{l}0.1 \\
0.1\end{array}$ & $\begin{array}{l}1950 \\
1950\end{array}$ & $\begin{array}{l}487500 \\
487500\end{array}$ & $\begin{array}{l}1825000 \\
147500\end{array}$ & $\begin{array}{l}0.1036 \\
0.0501\end{array}$ & \begin{tabular}{|l|}
$1.64 E-04$ \\
$3.67 E-04$
\end{tabular} & \begin{tabular}{r|}
4.898 \\
4.698
\end{tabular} & \begin{tabular}{|l|}
$9.09 \mathrm{E}-03$ \\
$3.50 \mathrm{E}-03$ \\
\end{tabular} & $\begin{array}{l}28.22 \\
24.57 \\
\end{array}$ & $\begin{array}{l}97.62 \\
99.39\end{array}$ & $\begin{array}{l}76.16 \\
93.95 \\
\end{array}$ & 39.48 & $\begin{array}{r}76.2 \\
198.1\end{array}$ & $\begin{array}{r}300.4 \\
379.2 \\
\end{array}$ \\
\hline 500 & 52.93 & 3000 & 4400 & $\begin{array}{r}020.20002 \\
155.520474\end{array}$ & \begin{tabular}{|}
2.7968 \\
2.7968
\end{tabular} & 53.9497 & 9036.19 & 5125 & 0.1 & 1950 & 487500 & 425000 & 0.0601 & $2.52 \mathrm{E}-04$ & 3.12 & \begin{tabular}{|l|}
$0.00 \mathrm{E}-00$ \\
$1.08 \mathrm{E}-02$ \\
\end{tabular} & 58.41 & 96.78 & 67.85 & 0 & 64 & 302 \\
\hline 501 & 36.86 & 3000 & 3790 & 139.969514 & 5.7373 & 122.9684 & 3964.43 & 5125 & 0.1 & 1950 & 4875 & 1462500 & 0.0863 & $2.86 \mathrm{E}-04$ & 2.678 & $8.79 \mathrm{E}-03$ & 42.3 & 96.43 & 64.28 & & 78.9 & 372.3 \\
\hline 502 & 34.21 & 3000 & 3500 & 139.768169 & 7.028 & 150.8694 & 3231. & 5125 & 0.1 & 1950 & 4875 & $1987 \mathrm{G}$ & 0.093 & $3.17 E-04$ & 2.721 & $7.87 \mathrm{E}$ & 28.3 & 96.42 & 64.23 & & 88.1 & 407.7 \\
\hline 503 & 25.78 & 3000 & 5770 & 34.752305 & 1.0684 & 92.2314 & 5285.62 & 5125 & 0.1 & 1950 & 487500 & 950000 & 0.1234 & \begin{tabular}{|c|c|}
$6.61 \mathrm{E}-04$ \\
\end{tabular} & 3.706 & $7.55 \mathrm{E}-03$ & 76.67 & 85.61 & 0 & & 91.8 & 736.7 \\
\hline 504 & 22.48 & 3000 & 2370 & $\begin{array}{l}217.75682 \\
\end{array}$ & 10.108 & 139.2566 & 3500.73 & 5125 & 0.1 & 1950 & 487500 & 1762500 & 0.1415 & $1.60 \mathrm{E}-04$ & 1.573 & \begin{tabular}{|l|}
$7.82 \mathrm{E}-03$ \\
\end{tabular} & 86.2 & 97.7 & 77.04 & & 88.6 & 358.6 \\
\hline
\end{tabular}




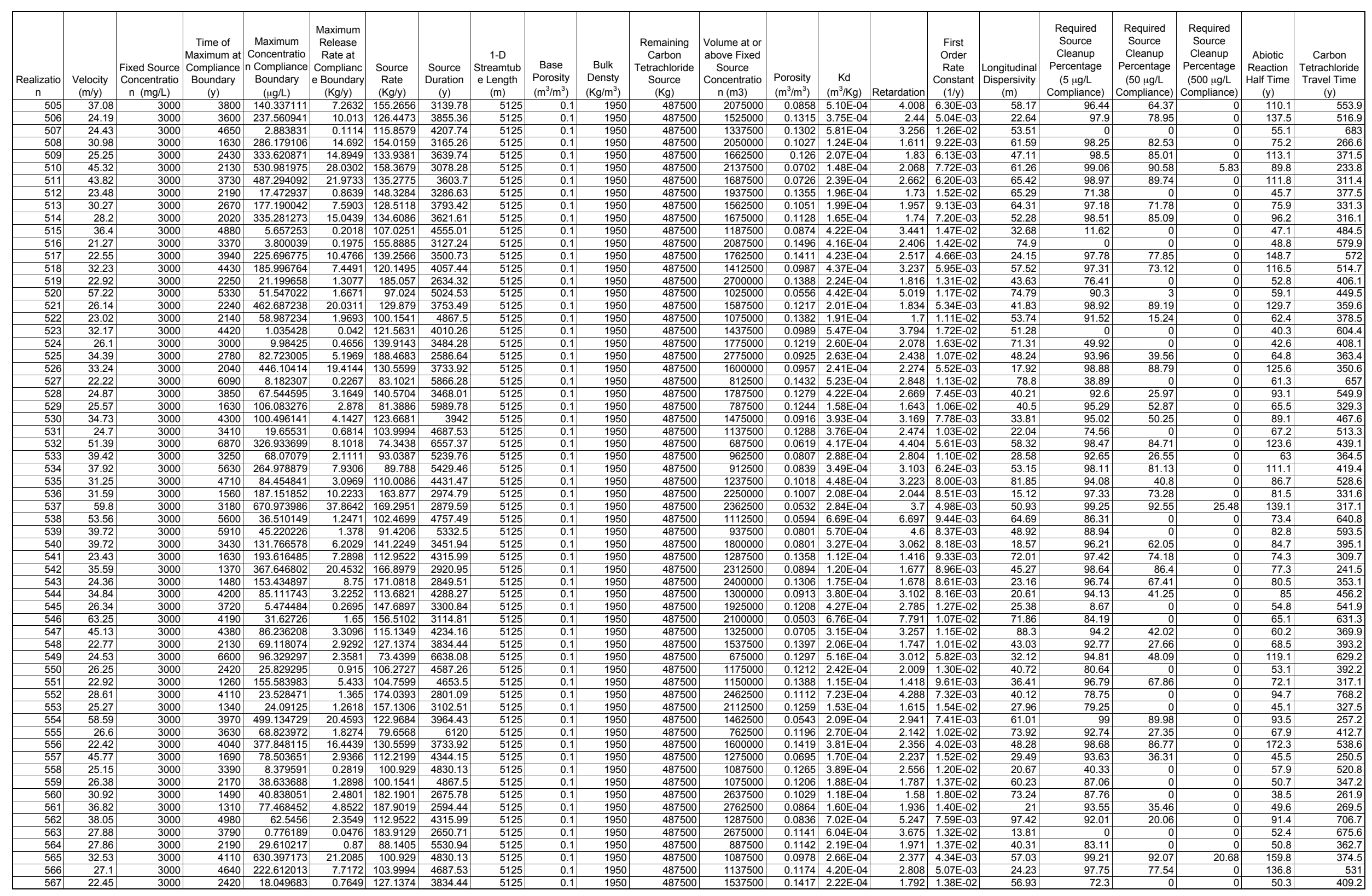




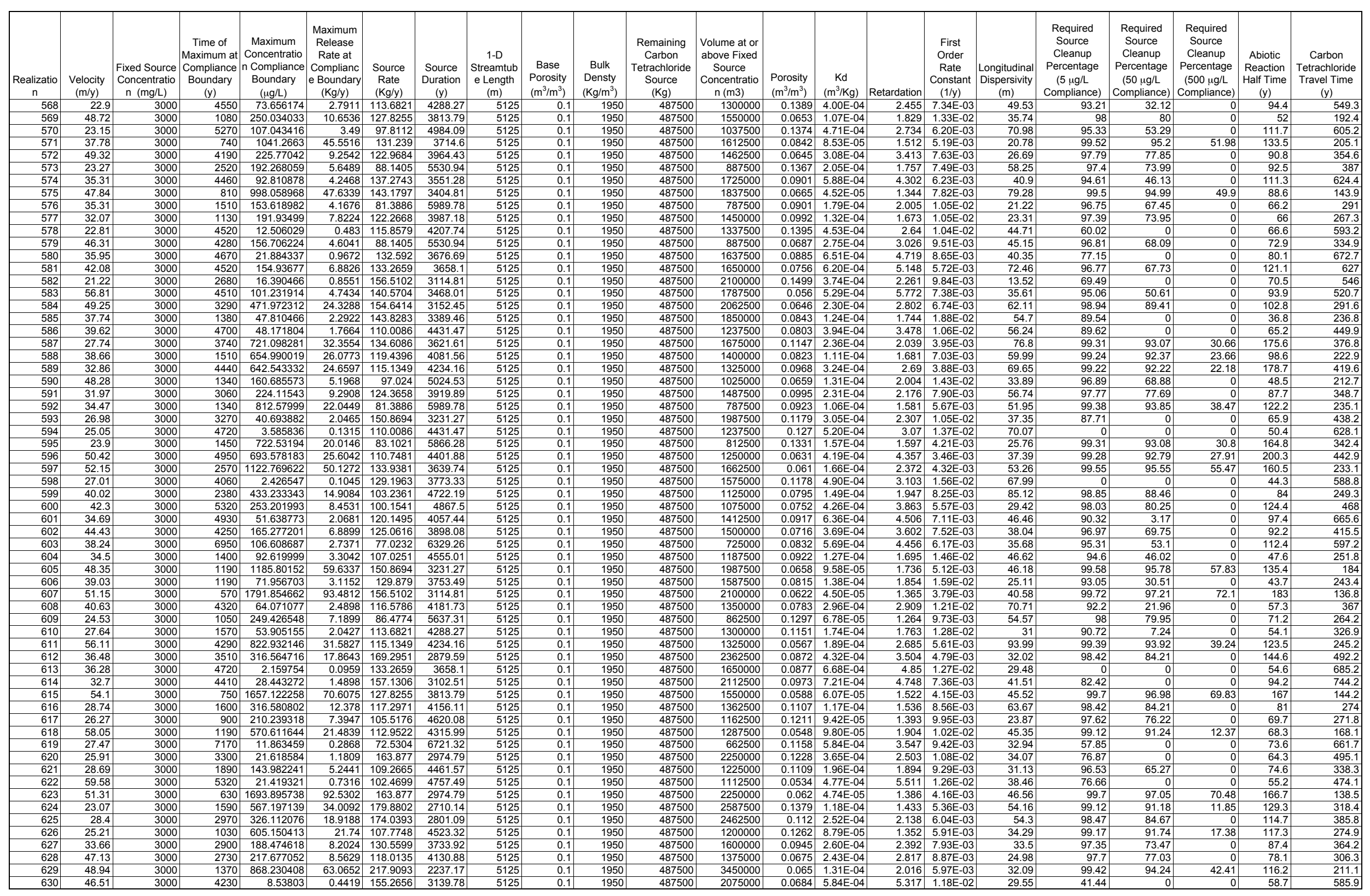




\begin{tabular}{|c|c|c|c|c|c|c|c|c|c|c|c|c|c|c|c|c|c|c|c|c|c|c|}
\hline $\begin{array}{c}\text { Realizatio } \\
n\end{array}$ & $\begin{array}{c}\text { Velocity } \\
(\mathrm{m} / \mathrm{y})\end{array}$ & $\begin{array}{c}\text { Fixed Source } \\
\text { Concentratio } \\
\mathrm{n}(\mathrm{mg} / \mathrm{L})\end{array}$ & $\begin{array}{c}\text { Time of } \\
\text { Maximum at } \\
\text { Compliance } \\
\text { Boundary } \\
\text { (y) }\end{array}$ & $\begin{array}{c}\text { Maximum } \\
\text { Concentratio } \\
\text { n Compliance } \\
\text { Boundary } \\
(\mu \mathrm{g} / \mathrm{L})\end{array}$ & \begin{tabular}{|c|} 
Maximum \\
Release \\
Rate at \\
Complianc \\
e Boundary \\
(Kg/y) \\
\end{tabular} & $\begin{array}{c}\text { Source } \\
\text { Rate } \\
(\mathrm{Kg} / \mathrm{y})\end{array}$ & $\begin{array}{c}\text { Source } \\
\text { Duration } \\
\text { (y) }\end{array}$ & \begin{tabular}{|c|} 
1-D \\
Streamtub \\
e Length \\
(m)
\end{tabular} & $\begin{array}{l}\text { Base } \\
\text { Porosity } \\
\left(\mathrm{m}^{3} / \mathrm{m}^{3}\right)\end{array}$ & $\begin{array}{c}\text { Bulk } \\
\text { Densty } \\
\left(\mathrm{Kg} / \mathrm{m}^{3}\right)\end{array}$ & \begin{tabular}{|c|}
$\begin{array}{c}\text { Remaining } \\
\text { Carbon } \\
\text { Tetrachloride } \\
\text { Source } \\
(\mathrm{Kg})\end{array}$ \\
\end{tabular} & $\begin{array}{c}\text { Volume at or } \\
\text { above Fixed } \\
\text { Source } \\
\begin{array}{c}\text { Concentratio } \\
n(m 3)\end{array} \\
\end{array}$ & $\begin{array}{l}\text { Porosity } \\
\left(\mathrm{m}^{3} / \mathrm{m}^{3}\right)\end{array}$ & $\begin{array}{c}\mathrm{Kd} \\
\left(\mathrm{m}^{3} / \mathrm{Kg}\right)\end{array}$ & Retardation & \begin{tabular}{|c|} 
First \\
Order \\
Rate \\
Constant \\
$(1 / y)$ \\
\end{tabular} & \begin{tabular}{|c|} 
Longitudinal \\
Dispersivity \\
$(\mathrm{m})$
\end{tabular} & \begin{tabular}{|c|} 
Required \\
Source \\
Cleanup \\
Percentage \\
(5 $\mu \mathrm{g} / \mathrm{L}$ \\
Compliance) \\
\end{tabular} & \begin{tabular}{|c|} 
Required \\
Source \\
Cleanup \\
Percentage \\
(50 ug/L \\
Compliance)
\end{tabular} & \begin{tabular}{|c} 
Required \\
Source \\
Cleanup \\
Percentage \\
$(500 \mu \mathrm{\mu g} / \mathrm{L}$ \\
Compliance) \\
\end{tabular} & \begin{tabular}{|c|} 
Abiotic \\
Reaction \\
Half Time \\
(y)
\end{tabular} & \begin{tabular}{|c} 
Carbon \\
Tetrachloride \\
Travel ITime \\
(y)
\end{tabular} \\
\hline 631 & & & $\begin{array}{r}2760 \\
\end{array}$ & \begin{tabular}{|r|}
57.914869 \\
\end{tabular} & & 188.4683 & \begin{tabular}{|l|}
2586.64 \\
\end{tabular} & 5125 & & 1950 & 487500 & 2775000 & 0.0982 & $2.82 \mathrm{E}-04$ & \begin{tabular}{|l|}
2.451 \\
\end{tabular} & \begin{tabular}{|l|}
$1.16 \mathrm{E}-02$ \\
\end{tabular} & 73.32 & \begin{tabular}{|l|}
91.37 \\
\end{tabular} & \begin{tabular}{|l|}
13.67 \\
\end{tabular} & & & \\
\hline 632 & 42.76 & 3000 & 4140 & $\mid$\begin{tabular}{|l|l|}
3.468053 \\
\end{tabular} & \begin{tabular}{|l|l|}
0.147 \\
\end{tabular} & \begin{tabular}{|l|}
127.1374 \\
\end{tabular} & 3834.44 & 5125 & 0.1 & 1950 & 487500 & 1537500 & 0.0744 & $3.91 \mathrm{E}-04$ & 3.655 & \begin{tabular}{|c|}
$1.82 \mathrm{E}-02$ \\
\end{tabular} & 36.45 & & & & 38.2 & 438.1 \\
\hline 633 & 34.92 & 3000 & 2030 & 103.457814 & 6.3622 & 184.4854 & 2642.48 & 5125 & 0.1 & 1950 & 487500 & 2687500 & 0.0911 & $2.49 \mathrm{E}-04$ & 2.379 & \begin{tabular}{|c|}
$9.89 \mathrm{E}-03$ \\
\end{tabular} & 16.55 & 95.17 & 51.67 & & 70.1 & 349.2 \\
\hline 634 & 29.16 & 3000 & 1160 & 147.023315 & 5.9229 & 120.8573 & 4033.68 & 5125 & 0.1 & 1950 & 487500 & 1425000 & 0.1091 & $1.53 \mathrm{E}-04$ & 1.707 & $1.02 \mathrm{E}-02$ & 17.51 & 96.6 & 65.99 & & & \\
\hline 635 & 25.37 & 3000 & 4320 & 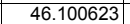 & 2.15 & 139.9143 & 3484.28 & 5125 & 0.1 & 1950 & 487500 & 1775000 & 0.1254 & $7.36 \mathrm{E}-04$ & 3.968 & \begin{tabular}{|l|l|}
$6.52 \mathrm{E}-03$ \\
\end{tabular} & 78.43 & 89.15 & 0 & & 106.3 & 801.5 \\
\hline 636 & 34.58 & 3000 & 3430 & 117.761708 & 7.6624 & 195.2002 & 2497.44 & 5125 & 0.1 & 1950 & 487500 & 2925000 & $\begin{array}{ll}0.092 \\
\end{array}$ & $5.01 \mathrm{E}-04$ & 3.754 & \begin{tabular}{|l|l|}
$6.16 \mathrm{E}-03$ \\
\end{tabular} & 24.8 & 95.75 & 57.54 & & 112.5 & 556.3 \\
\hline 637 & 53.11 & 3000 & 2580 & 91.16153 & 6.3795 & \begin{tabular}{|l|}
209.9408 \\
\end{tabular} & 2322.08 & 5125 & 0.1 & 1950 & 487500 & 3262500 & 0.0599 & $3.09 \mathrm{E}-04$ & 3.604 & $1.20 \mathrm{E}-02$ & 79.54 & $\begin{array}{ll}94.52 \\
\end{array}$ & 45.15 & & 57.7 & 347.8 \\
\hline 638 & 60.03 & 3000 & 3270 & 349.875801 & 17.7424 & \begin{tabular}{|l|l|}
152.1319 \\
\end{tabular} & 3204.46 & 5125 & 0.1 & 1950 & 487500 & 2012500 & $\begin{array}{l}0.053 \\
\end{array}$ & $2.00 \mathrm{E}-04$ & 2.91 & $9.20 \mathrm{E}-03$ & 52.04 & \begin{tabular}{l|l|l|}
98.57 \\
\end{tabular} & 85.71 & 0 & 75.4 & 248.5 \\
\hline 639 & 29.76 & 3000 & 3750 & 31.773059 & 1.6112 & \begin{tabular}{|l|l|}
152.1319 \\
\end{tabular} & 3204.46 & 5125 & 0.1 & 1950 & 487500 & 2012500 & 0.1069 & $5.54 \mathrm{E}-04$ & 3.618 & $8.91 \mathrm{E}-03$ & $\begin{array}{l}68.67 \\
\end{array}$ & \begin{tabular}{ll|l}
84.26 \\
\end{tabular} & 0 & 0 & \begin{tabular}{|c|c|}
77.8 \\
\end{tabular} & 623 \\
\hline 640 & 43.17 & 3000 & 3590 & 975.824271 & 46.1493 & \begin{tabular}{|l|l|l}
141.878 \\
\end{tabular} & 3436.05 & 5125 & 0.1 & 1950 & 487500 & 1812500 & 0.0737 & $2.48 \mathrm{E}-04$ & 2.7 & 3.67E-03 & 78.3 & 99.49 & 94.88 & 48.76 & 189.1 & 320.6 \\
\hline 641 & $\begin{array}{l}42.03 \\
\end{array}$ & 3000 & 4630 & 21.636503 & 0.8146 & 112.9522 & 4315.99 & 5125 & 0.1 & 1950 & 487500 & 1287500 & 0.0757 & 4.01E-04 & 3.679 & $1.26 \mathrm{E}-02$ & $\begin{array}{lll}41.14 \\
\end{array}$ & 76.89 & 0 & & 55 & 448.6 \\
\hline 642 & 21.39 & 3000 & 1220 & 71.01264 & 3.2021 & 135.2775 & 3603.7 & 5125 & 0.1 & 1950 & 487500 & 1687500 & 0.1487 & 7.49E-05 & 1.255 & $1.32 \mathrm{E}-02$ & 62.44 & $\begin{array}{l}92.96 \\
\end{array}$ & 29.59 & & 52.6 & 300.6 \\
\hline 643 & 32.8 & 3000 & 1010 & 360.530532 & 15.279 & \begin{tabular}{|l|l|}
127.1374 \\
\end{tabular} & 3834.44 & 5125 & 0.1 & 1950 & 487500 & 1537500 & 0.097 & $1.08 \mathrm{E}-04$ & 1.564 & 8.82E-03 & 27.16 & 98.61 & 86.13 & & 78.6 & 244.4 \\
\hline 644 & 26.21 & 3000 & 1470 & 31.334956 & 0.9549 & \begin{tabular}{|l|l|}
91.4206 \\
\end{tabular} & 5332.5 & 5125 & 0.1 & 1950 & 487500 & 937500 & 0.1214 & 1.39E-04 & 1.58 & $1.58 \mathrm{E}-02$ & 47.51 & 84.04 & 0 & & 44 & 309 \\
\hline 645 & 31.07 & 3000 & 1260 & 645.373055 & 29.3882 & \begin{tabular}{|c|}
136.6103 \\
\end{tabular} & 3568.54 & 5125 & 0.1 & 1950 & 487500 & 1712500 & 0.1024 & 9.15E-05 & 1.452 & $6.57 \mathrm{E}-03$ & 55.88 & 99.23 & $\begin{array}{l}92.25 \\
\end{array}$ & 22.53 & 105.5 & 239.5 \\
\hline 646 & 43.05 & 3000 & 3020 & 296.653735 & 11.3133 & 114.4096 & 4261 & 5125 & 0.1 & 1950 & 487500 & 1312500 & $0.073 \mathrm{C}^{-}$ & 2.17E-04 _ _ & 2.484 & .25E-03 & 48.75 & 98.31 & 83.15 & & 84 & 295.8 \\
\hline 647 & 35.51 & 3000 & 4580 & 133.621606 & 5.5082 & 123.6681 & 3942 & 5125 & 0.1 & 1950 & & & 0.089 & $5.62 \mathrm{E}-04$ & 4.17 & & & 96.26 & 62.58 & & 115.2 & 602 \\
\hline 648 & 22.95 & 3000 & 4990 & 1.092661 & 0.0401 & 110.0086 & 4431.47 & 5125 & 0.1 & 1950 & 487500 & 1237500 & 0.1386 & $5.60 \mathrm{E}-04$ & 3.043 & 1.29E- -02 & 21.77 & & & & 54 & 679.4 \\
\hline 649 & 21.25 & 3000 & 3590 & 132.337021 & 6.2873 & 142.5296 & 3420.34 & 5125 & 0.1 & 1950 & & 1825000 & 0.1497 & 3.03E-04 & 2.023 & $7.04 \mathrm{E}-03$ & 81.91 & 96.22 & 62.22 & & 98.4 & 487.8 \\
\hline 650 & 21.58 & 3000 & 4330 & & & 91.4206 & 5332.5 & 5125 & 0.1 & 1950 & & 937500 & 0.1474 & $4.02 \mathrm{E}-04$ & 2.38 & $1.10 \mathrm{E}-02$ & 43.06 & 55.6 & & 0 & 63.1 & 565.1 \\
\hline 651 & 28.18 & 3000 & 1930 & 247.148542 & $\begin{array}{r}9.1845 \\
\end{array}$ & 111.4852 & 4372.78 & 5125 & 0.1 & 1950 & & 1262500 & 0.1129 & $1.74 \mathrm{E}-04$ & 1.781 & $8.00 \mathrm{E}-03$ & 44.1 & 97.98 & 79.77 & 0 & $\begin{array}{l}86.6 \\
\end{array}$ & 323.9 \\
\hline 652 & 54.76 & 3000 & 1900 & & \begin{tabular}{|l|l|}
43.1554 \\
350215
\end{tabular} & 135.9447 & 3586.02 & & 0.1 & 1950 & & & 0.0581 & $1.69 \mathrm{E}-04$ & 2.467 & $5.05 \mathrm{E}-03$ & 28.28 & 99.47 & 94.75 & 47.5 & 137.4 & 230.9 \\
\hline 653 & $\begin{array}{l}36.91 \\
5207 \\
\end{array}$ & 3000 & 1330 & 1059.711651 & 35.9245 & 101.7009 & & 5125 & 0.1 & 1950 & & & 0.0862 & 6.99E-05 & 1.41 & $5.46 \mathrm{E}-03$ & 95 & 99.53 & 95.28 & 52.82 & 126.9 & 195.8 \\
\hline 654 & 52.07 & 3000 & 1170 & 1103.514334 & 45.2325 & 122.9684 & 3964.43 & 5125 & 0.1 & $\begin{array}{l}1950 \\
1950\end{array}$ & 487500 & 1462500 & $\begin{array}{ll}0.0611 \\
\end{array}$ & 9.64E-05 & 1.798 & $5.74 \mathrm{E}-03$ & 45.68 & 99.55 & 95.47 & 54.69 & 120.7 & 177 \\
\hline$\frac{655}{656}$ & $\begin{array}{l}42.59 \\
29.11 \\
\end{array}$ & $\begin{array}{l}3000 \\
33000\end{array}$ & $\begin{array}{l}3590 \\
2990\end{array}$ & \begin{tabular}{|r|}
417.842827 \\
24.077182
\end{tabular} & $\begin{array}{r}19.4874 \\
0.9813\end{array}$ & \begin{tabular}{|l|}
139.9143 \\
122.2668 \\
\end{tabular} & $\begin{array}{l}3484.28 \\
3987.18\end{array}$ & $\begin{array}{l}5125 \\
5125 \\
\end{array}$ & $\begin{array}{l}0.1 \\
0.1 \\
\end{array}$ & $\begin{array}{l}\frac{1950}{1} 1950 \\
\end{array}$ & $\begin{array}{l}487500 \\
487500\end{array}$ & $\begin{array}{l}1775000 \\
1450000\end{array}$ & $\begin{array}{l}0.0747 \\
0.1093 \\
\end{array}$ & $\begin{array}{l}2.29 \mathrm{E}-04 \\
288 \mathrm{E}-04\end{array}$ & $\begin{array}{r}2.548 \\
2.33 \\
\end{array}$ & \begin{tabular}{|l|}
$7.00 \mathrm{E}-03$ \\
$1.25 \mathrm{E}-02$ \\
\end{tabular} & $\frac{89 .}{27 .}$ & $\begin{array}{r}98.8 \\
79.23 \\
\end{array}$ & \begin{tabular}{rl|l}
88.03 & \\
\end{tabular} & $\frac{0}{0}$ & \begin{tabular}{|c|}
99.1 \\
55.6
\end{tabular} & $\begin{array}{r}306.6 \\
440.3 \\
\end{array}$ \\
\hline 657 & 38.8 & & 830 & 594. & 26.1629 & \begin{tabular}{|l|}
131.9164 \\
\end{tabular} & 3695.52 & 5125 & & & & & 0.082 & $8.25 \mathrm{E}-05$ & 1.509 & \begin{tabular}{|l|}
$8.23 \mathrm{E}-03$ \\
\end{tabular} & 29. & 99.16 & 91.6 & 15.96 & 84.2 & $\begin{array}{l}10.0 \\
199.3 \\
\end{array}$ \\
\hline 658 & 22.72 & & 3940 & & 0.0213 & 144.4754 & 3374.28 & 5125 & & & & & 0.14 & $6.81 \mathrm{E}-04$ & 3.459 & \begin{tabular}{|l|}
0.20 \\
1.53 \\
\end{tabular} & 58 & 0 & 0 & 0 & 45.4 & 780.1 \\
\hline 659 & 34.02 & & 4400 & 61.483301 & & 114.4096 & & 5125 & & & & & 0.0935 & $3.32 \mathrm{E}-04$ & 2.795 & & & 91.87 & 18.68 & & 62.8 & 421 \\
\hline 660 & 25.66 & & 7130 & & & 71.6152 & 6807.21 & 5125 & & & & & 0.124 & & 3.264 & & & & & & 83.4 & 652 \\
\hline 661 & 22.76 & 3000 & 4980 & 143.827801 & 4.9494 & 103.2361 & 4722.19 & 5125 & 0.1 & 19 & & 1125000 & 0.1398 & $4.06 \mathrm{E}-04$ & 2.468 & & 55.03 & 96.52 & 65.24 & & 117.4 & 555.7 \\
\hline 662 & 46.85 & 30 & 4930 & & 0.0922 & 110.7481 & 440 & 51 & 0.1 & & & 1250000 & 0.0679 & $4.75 \mathrm{E}-04$ & 4.534 & & 26.49 & 0 & 0 & 0 & 41.6 & 496 \\
\hline 663 & 30.44 & 3000 & 1930 & 199.4 & & 88.1405 & & & & & & & 0.1045 & $2.17 \mathrm{E}-04$ & 2.048 & & 24.5 & 97.49 & 74.94 & 0 & 85.9 & 344.7 \\
\hline 664 & & & 1820 & & 2.9643 & 129.879 & 375 & 5125 & & & & & 0.0774 & $2.02 \mathrm{E}-04$ & 2.319 & & 22.94 & 92.7 & 26.98 & & 51 & 289.2 \\
\hline 665 & 30.5 & 3000 & 2710 & & 0.8587 & 125.7554 & 3876 . & 5125 & 0.1 & & & & 0.1043 & $2.44 \mathrm{E}-04$ & 2.18 & & 49. & 75.59 & 0 & & 46.1 & 366.4 \\
\hline 666 & 22.72 & 3000 & 3580 & 75.498044 & 3.92 & 155.8885 & 3127.24 & 5125 & 0.1 & & & & 0.14 & & 2.556 & & 3 & 93.38 & 33.77 & & 102.5 & 576.6 \\
\hline 667 & 24.34 & 3000 & 6160 & & 0.0 & 91.4206 & & 5125 & 0.1 & & & & 0.1307 & & 3.703 & & & 0 & & 0 & 61.6 & 779.7 \\
\hline 668 & 60.6 & 3000 & 1930 & 900.3 & \begin{tabular}{|l|l|}
47.1566 \\
\end{tabular} & 157.1 & 310 & 5125 & 0.1 & 19 & & & 0.0525 & & 2.473 & & & 99.44 & 94.45 & 44.46 & 118 & 209.1 \\
\hline 669 & 36.15 & & 30 & & & 193.5 & & 5125 & 0.1 & & & & 0.0 & & 4.2 & & & & 56.62 & 0 & 114.4 & 600.8 \\
\hline 670 & 48.42 & & & & & & & & & & & & 0.06 & & & & & & 67.77 & & 116.7 & 545.7 \\
\hline 671 & 22.3 & & & & & & & & & & & & 0.14 & & & & & & 42.19 & & 89.3 & 482.3 \\
\hline 672 & 30.89 & & 4140 & & & & & & 0 & & & & 0.1 & & & & & 7.9 & & & 77.6 & 5 \\
\hline 673 & 39.28 & & 890 & & & & & & & & & & & & & & & & 81.59 & & 50.9 & 183.5 \\
\hline 674 & 21.45 & & 1000 & & & & & & & & & & & & & & & & 72.63 & & 79 & 324.2 \\
\hline 675 & & 30 & & & & & & & & & & & 0.14 & & & & & & & 0 & 52.5 & 356 \\
\hline 676 & 53.4 & & & & & & & & & & & 181 & & & 2.264 & & & \begin{tabular}{l|l|}
98.27 \\
\end{tabular} & 82.75 & 0 & 58.9 & \\
\hline 677 & 23.37 & & & 0. & & 95. & & & & & & & & & & & 31 & 0 & & 0 & 43.3 & 636.8 \\
\hline 678 & 44.25 & & & 582.9 & 15.8 & 81.3 & & & & & & & & & & & 2 & 99.14 & 91.42 & 14.24 & & 254.7 \\
\hline 679 & 39.13 & & 6330 & & & 83.1 & & & & & & & & & & & & $\begin{array}{l}9.56 \\
\end{array}$ & 0 & 0 & $\begin{array}{l}52.7 \\
\end{array}$ & 574.4 \\
\hline 680 & 51.81 & & 38 & & & 133. & & & & & & & & & & & & & 0 & 0 & 35 & 41.4 \\
\hline 681 & 25.64 & 3000 & 5390 & 298.9406 & & 83.9 & & & & & & & & & $2.297 \mid$ & & 85 & 98.33 & 83.27 & & 126.9 & 59.2 \\
\hline 682 & 28.28 & 3000 & 2520 & $\begin{array}{r}10.407833 \\
\end{array}$ & 0.37 & 107.02 & 4555.01 & 5125 & 0.1 & & & & 0.11 & 2.57 & 2.157 & & & 51.96 & 0 & & & 390.8 \\
\hline 683 & $\begin{array}{r}26.91 \\
519 \\
\end{array}$ & 3000 & $\begin{array}{l}33300 \\
4390\end{array}$ & \begin{tabular}{|r|}
$3.6 / 8 / 02$ \\
138097073
\end{tabular} & $\frac{0.1447}{55958}$ & \begin{tabular}{|l|l|}
18.01753 \\
121531
\end{tabular} & $\frac{4130.88}{401026}$ & $\begin{array}{l}125 \\
5125 \\
\end{array}$ & & & & 1345 & 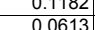 & $\frac{3.62}{3.83}$ & $\begin{array}{l}2.546 \\
4.555\end{array}$ & & & $\begin{array}{r}0 \\
9638\end{array}$ & $\begin{array}{r}0 \\
6379 \\
\end{array}$ & 0 & $\begin{array}{r}45.9 \\
83\end{array}$ & $\frac{484.9}{4403}$ \\
\hline 685 & 57.63 & 3000 & 1600 & \begin{tabular}{|l|}
639.974871 \\
\end{tabular} & 17.54 & $\mid 82.2476$ & 5927.23 & $\begin{array}{ll}5125 \\
\end{array}$ & 0.1 & & & & 0.05 & 1.06 & 1.973 & & 66 & 99.22 & 92.19 & 21.87 & 75.7 & 175.4 \\
\hline 686 & 25.84 & 3000 & 6210 & & 0.6 & 101.70 & 4793 & $\begin{array}{ll}5125 \\
\end{array}$ & & & & & 0.12 & 7.69 & 4.1 & & & 75.53 & 0 & 0 & 108 & 824.6 \\
\hline 687 & 50.34 & 30 & 450 & $459 . \mathrm{C}$ & 19.55 & 127.8 & & \begin{tabular}{|l|l|}
5125 \\
\end{tabular} & & & & & 0.0 & 2.34 & 1.18 & $1.5 \mathrm{~s}$ & 50 & 98.91 & 89.11 & 0 & 43.7 & 120.9 \\
\hline 688 & 41.32 & 3000 & 2980 & 185.3422 & 11.04 & 178.7 & & $\begin{array}{ll}5125 \\
\end{array}$ & 0.1 & 195 & & 256 & 0.6 & 3.18 & 3.0 & 8.16 & 72 & 97.3 & 73.02 & 0 & 85 & 383 \\
\hline 689 & 39.47 & 30 & 1690 & 124. & 5.3 & 129 & & 5125 & 0. & 19 & & 158 & 0.0806 & 1.66 & 2.0 & & 35. & $\mid 95.98$ & 59.82 & 0 & 55.3 & 265.1 \\
\hline 690 & 27.35 & 30 & 6110 & & 2.2 & 83. & & 5125 & 0.1 & & & & & 4.83 & 3.05 & & & 93.9 & 39.01 & 0 & 95 & 580.4 \\
\hline 691 & 62.01 & 3000 & 2250 & 602.974735 & 19. & 97. & 502 & 5125 & 0.1 & & & & 0.05 & & 2.273 & & & 99.17 & 91.7 & 17.08 & 77 & 187.9 \\
\hline 692 & 25.61 & 3000 & 1270 & 343.515327 & 10.9284 & \begin{tabular}{|l|l|}
95.4399 \\
\end{tabular} & 5107.93 & 5125 & 0.1 & 1950 & & & 0.1242 & $9.52 \mathrm{E}-05$ & 1.387 & $8.04 \mathrm{E}-03$ & 50.62 & $\begin{array}{l}98.54 \\
\end{array}$ & 85.44 & & 86.2 & 277.6 \\
\hline 693 & 27.08 & 3000 & 1500 & $\mid$\begin{tabular}{|l|l|l|}
114.013527 \\
\end{tabular} & 2.7565 & \begin{tabular}{|l|l|}
72.5304 \\
\end{tabular} & 6721.32 & 5125 & 0.1 & 1950 & 487500 & 662500 & 0.1175 & $1.59 \mathrm{E}-04$ & 1.683 & $1.06 \mathrm{E}-02$ & 33.18 & 95.61 & 56.15 & & 65.2 & 318.5 \\
\hline
\end{tabular}




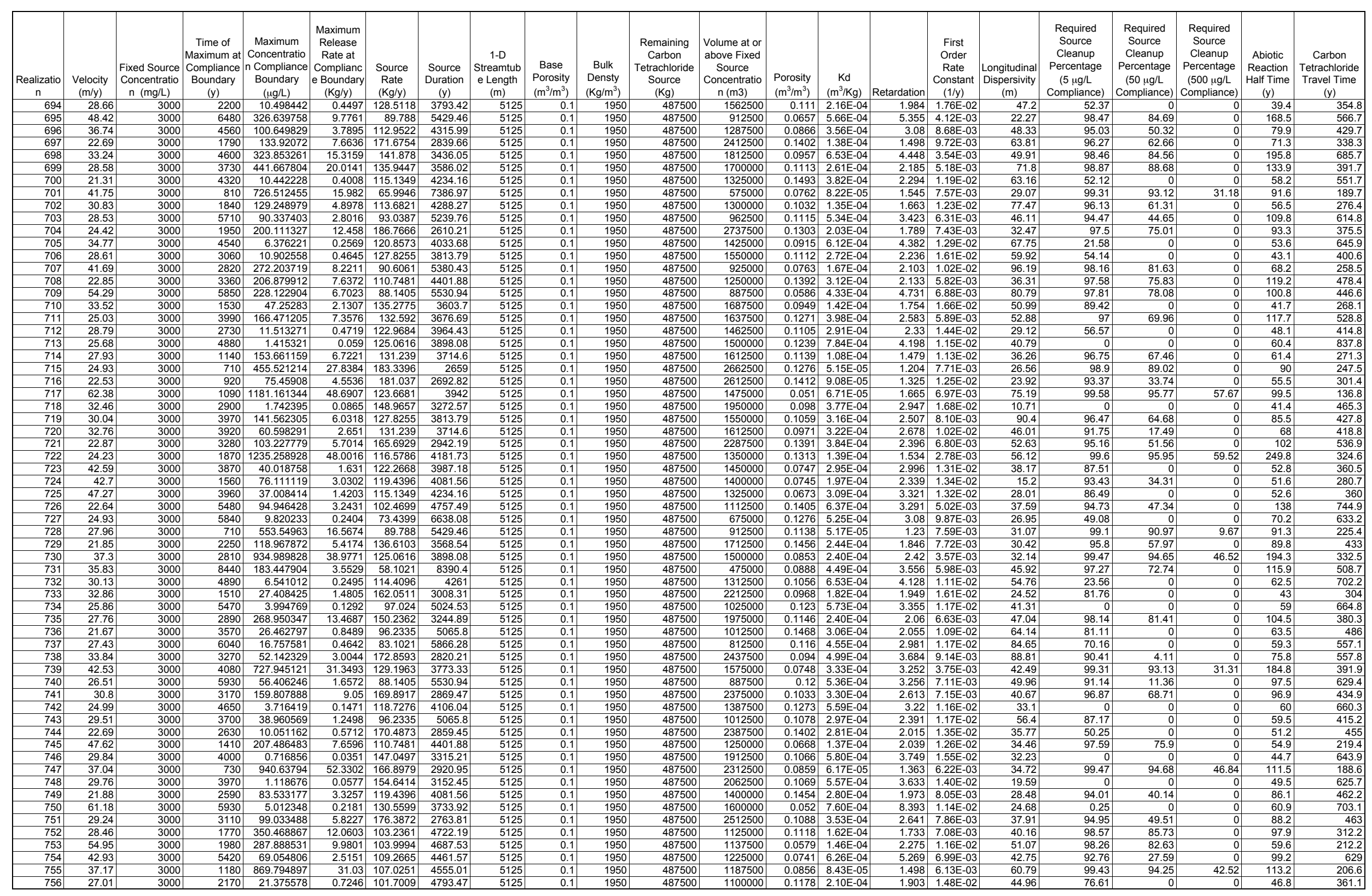




\begin{tabular}{|c|c|c|c|c|c|c|c|c|c|c|c|c|c|c|c|c|c|c|c|c|c|c|}
\hline $\begin{array}{c}\text { Realizatio } \\
n\end{array}$ & $\begin{array}{c}\text { Velocity } \\
(\mathrm{m} / \mathrm{y})\end{array}$ & $\begin{array}{c}\text { Fixed Source } \\
\text { Concentratio } \\
\mathrm{n}(\mathrm{mg} / \mathrm{L})\end{array}$ & $\begin{array}{c}\text { Time of } \\
\text { Maximum at } \\
\text { Compliance } \\
\text { Boundary } \\
\text { (y) }\end{array}$ & $\begin{array}{c}\text { Maximum } \\
\text { Concentratio } \\
\text { n Compliance } \\
\text { Boundary } \\
(\mu \mathrm{g} / \mathrm{L})\end{array}$ & \begin{tabular}{|c|} 
Maximum \\
Release \\
Rate at \\
Complianc \\
e Boundary \\
(Kg/y) \\
\end{tabular} & $\begin{array}{c}\text { Source } \\
\text { Rate } \\
(\mathrm{Kg} / \mathrm{y})\end{array}$ & $\begin{array}{c}\text { Source } \\
\text { Duration } \\
\text { (y) }\end{array}$ & \begin{tabular}{|c|} 
1-D \\
Streamtub \\
e Length \\
$(\mathrm{m})$
\end{tabular} & $\begin{array}{c}\text { Base } \\
\text { Porosity } \\
\left(\mathrm{m}^{3} / \mathrm{m}^{3}\right)\end{array}$ & $\begin{array}{c}\text { Bulk } \\
\text { Densty } \\
\left(\mathrm{Kg} / \mathrm{m}^{3}\right)\end{array}$ & $\begin{array}{c}\text { Remaining } \\
\text { Carbon } \\
\text { Tetrachloride } \\
\text { Source } \\
(\mathrm{Kg}) \\
\end{array}$ & $\begin{array}{c}\text { Volume at or } \\
\text { above Fixed } \\
\text { Source } \\
\begin{array}{c}\text { Concentratio } \\
n(m 3)\end{array} \\
\end{array}$ & $\begin{array}{l}\text { Porosity } \\
\left(\mathrm{m}^{3} / \mathrm{m}^{3}\right)\end{array}$ & $\begin{array}{c}\mathrm{Kd} \\
\left(\mathrm{m}^{3} / \mathrm{kg}\right)\end{array}$ & Retardation & $\begin{array}{c}\text { First } \\
\text { Order } \\
\text { Rate } \\
\text { Constant } \\
(1 / y) \\
\end{array}$ & \begin{tabular}{|c} 
Longitudinal \\
Dispersivity \\
$(\mathrm{m})$
\end{tabular} & $\begin{array}{c}\text { Required } \\
\text { Source } \\
\text { Cleanup } \\
\text { Percentage } \\
(5 \mu g / L \\
\text { Compliance) } \\
\end{array}$ & \begin{tabular}{|c|} 
Required \\
Source \\
Cleanup \\
Percentage \\
$(50 \mu \mathrm{g} / \mathrm{L}$ \\
Compliance)
\end{tabular} & $\begin{array}{c}\text { Required } \\
\text { Source } \\
\text { Cleanup } \\
\text { Percentage } \\
\text { (500 } \mathrm{\mu g} / \mathrm{L} \\
\text { Compliance) } \\
\end{array}$ & \begin{tabular}{|c|} 
Abiotic \\
Reaction \\
Half Time \\
(y) \\
\end{tabular} & $\begin{array}{c}\text { Carbon } \\
\text { Tetrachloride } \\
\text { Travel Time } \\
\text { (y) } \\
\end{array}$ \\
\hline & & 3000 & $\begin{array}{r}3310 \\
\end{array}$ & & & $\begin{array}{l}95.4399 \\
\end{array}$ & 5107.93 & 5125 & & 1950 & $\begin{array}{r}487500 \\
\end{array}$ & 1000000 & $\begin{array}{l}0.1073 \\
\end{array}$ & $2.10 \mathrm{E}-04$ & 1.99 & $4.56 \mathrm{E}-03$ & $\begin{array}{r}1.24 \\
\end{array}$ & 99.25 & $\begin{array}{r}92.49 \\
\end{array}$ & 24.86 & & 343.9 \\
\hline 758 & 31.69 & 3000 & 1580 & \begin{tabular}{|l|}
1384.08131 \\
\end{tabular} & \begin{tabular}{|l|l|}
42.5519 \\
5.202
\end{tabular} & $\begin{array}{r}92.2314 \\
162051\end{array}$ & \begin{tabular}{|l|l|}
5285.62 \\
\end{tabular} & $\begin{array}{l}5125 \\
5125 \\
\end{array}$ & 0.1 & 1950 & 487500 & 950000 & 0.1004 & $1.11 \mathrm{E}-04$ & 1.557 & $3.12 \mathrm{E}-03$ & 60.14 & 99.64 & 96.39 & $\begin{array}{ll}63.87 \\
\end{array}$ & 222.5 & 251.9 \\
\hline 759 & 27.33 & 3000 & 1640 & \begin{tabular}{|l|}
107.85864 \\
\end{tabular} & \begin{tabular}{r|}
5.8262 \\
160250
\end{tabular} & 162.0511 & 3008.31 & 5125 & 0.1 & 1950 & 487500 & 2212500 & 0.1164 & $1.16 \mathrm{E}-04$ & 1.502 & $1.28 \mathrm{E}-02$ & 81.61 & 95.36 & 53.64 & 0 & 54.4 & 281.6 \\
\hline 760 & $\begin{array}{l}59.8 \\
2.7\end{array}$ & 3000 & 4800 & \begin{tabular}{|l|l|l|l|}
422.62189 \\
\end{tabular} & 16.9259 & 120.1495 & 4057.44 & 5125 & 0.1 & 1950 & 487500 & 1412500 & 0.0532 & $4.08 \mathrm{E}-04$ & 4.881 & $4.80 \mathrm{E}-03$ & 13.62 & $\begin{array}{ll}98.82 \\
\end{array}$ & 88.17 & & $\begin{array}{l}144.3 \\
\end{array}$ & 418.3 \\
\hline 761 & 21.37 & 3000 & 5000 & $\begin{array}{r}0.336084 \\
\end{array}$ & \begin{tabular}{|c|c|}
0.0119 \\
14020
\end{tabular} & 106.2727 & 4587.26 & 5125 & 0.1 & 1950 & 487500 & 1175000 & 0.1489 & 5.74E-04 & 2.949 & 1.54E-02 & 37.62 & 0 & 0 & 0 & 45 & 707.5 \\
\hline 762 & 28.76 & 3000 & 4570 & \begin{tabular}{|r|}
322.515914 \\
\end{tabular} & 11.8265 & 110.0086 & 4431.47 & 5125 & 0.1 & 1950 & 487500 & 1237500 & 0.1106 & 2.93E-04 & 2.34 & $5.80 \mathrm{E}-03$ & 82.81 & 98.45 & 84.5 & 0 & 119.5 & 416.9 \\
\hline 763 & 47.55 & 3000 & 2750 & \begin{tabular}{|r|}
442.142336 \\
\end{tabular} & 20.6207 & 139.9143 & 3484.28 & 5125 & 0.1 & 1950 & 487500 & 1775000 & 0.0669 & 1.71E-04 & 2.295 & $8.21 \mathrm{E}-03$ & 70.16 & 98.87 & 88.69 & 0 & 84.4 & 247.3 \\
\hline 764 & 22.68 & 3000 & 1470 & \begin{tabular}{|l|}
78.100146 \\
\end{tabular} & 3.4694 & $\begin{array}{l}133.2659 \\
115120\end{array}$ & 3658.1 & 5125 & 0.1 & 1950 & 487500 & 1650000 & 0.1403 & $1.50 \mathrm{E}-04$ & 1.541 & 1.09E-02 & 33.13 & $\begin{array}{r}93.6 \\
50.20 \\
\end{array}$ & 35.98 & 0 & 63.9 & 348.4 \\
\hline 765 & $\begin{array}{l}30.59 \\
2760\end{array}$ & 3000 & 3910 & & $\begin{array}{l}0.4705 \\
3252 \\
3252\end{array}$ & \begin{tabular}{|l|}
115.1349 \\
1110781
\end{tabular} & 4234.16 & $\begin{array}{l}5125 \\
5125\end{array}$ & 0.1 & $\begin{array}{l}1950 \\
1900\end{array}$ & 487500 & $\begin{array}{l}1325000 \\
\end{array}$ & $\begin{array}{r}0.104 \\
\\
01152\end{array}$ & 4.00E-04 & 2.944 & \begin{tabular}{|l|l|}
$1.19 \mathrm{E}-02$ \\
7
\end{tabular} & 20.49 & 59.22 & & 0 & 58.4 & $\begin{array}{l}493.3 \\
57.2\end{array}$ \\
\hline $\begin{array}{l}766 \\
767 \\
\end{array}$ & $\begin{array}{r}27.62 \\
32.3 \\
\end{array}$ & $\begin{array}{l}3000 \\
3000\end{array}$ & $\begin{array}{l}4740 \\
4190\end{array}$ & $\begin{array}{r}88.1779099 \\
10.92199 \\
\end{array}$ & $\begin{array}{l}3.2552 \\
0.4553\end{array}$ & $\begin{array}{r}110.7481 \\
1250616\end{array}$ & $\begin{array}{l}4401.88 \\
389808\end{array}$ & $\begin{array}{l}5125 \\
5125\end{array}$ & $\begin{array}{l}0.1 \\
0.1 \\
\end{array}$ & $\begin{array}{l}1950 \\
1950\end{array}$ & $\begin{array}{l}487500 \\
487500\end{array}$ & \begin{tabular}{r|r|r|}
1250000 \\
1500000
\end{tabular} & $\begin{array}{l}0.1152 \\
00985\end{array}$ & $\begin{array}{r}4.69 \mathrm{E}-04 \\
469 \mathrm{E}-04 \\
\end{array}$ & $\begin{array}{l}3.056 \\
3.407\end{array}$ & \begin{tabular}{|l|}
$7.06 \mathrm{E}-03$ \\
$133 \mathrm{~F}-02$
\end{tabular} & 64.34 . & $\begin{array}{l}94.33 \\
5.21\end{array}$ & 43.3 & 0 & $\begin{array}{l}98.2 \\
52 ?\end{array}$ & 567.2 \\
\hline 768 & 24.01 & 3000 & $\begin{array}{l}4900 \\
1950 \\
\end{array}$ & $\begin{array}{l}\frac{10.9<199}{25.773565} \\
\end{array}$ & $\begin{array}{l}0.453 \\
1.0139 \\
\end{array}$ & 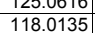 & 38980.08 & $\begin{array}{l}3120 \\
5125\end{array}$ & $\begin{array}{l}0.1 \\
0.1\end{array}$ & $\begin{array}{l}1950 \\
1950\end{array}$ & $\frac{487500}{487500}$ & $\frac{17500000}{1375000}$ & $\begin{array}{l}0.9085 \\
0.1325 \\
\end{array}$ & \begin{tabular}{|l}
$4.69 \mathrm{E}-04$ \\
$2.11 \mathrm{E}-04$
\end{tabular} & $\begin{array}{l}3.407 \\
.806\end{array}$ & \begin{tabular}{|l|}
$1.33 \mathrm{E}-02$ \\
$1.31 \mathrm{E}-02$ \\
\end{tabular} & \begin{tabular}{|c|}
74.55 \\
34.88
\end{tabular} & $\begin{array}{r}54.22 \\
80.6\end{array}$ & 0 & $\frac{0}{0}$ & $\begin{array}{l}52.2 \\
53.1\end{array}$ & $\begin{array}{l}530.6 \\
385.4 \\
\end{array}$ \\
\hline 769 & 26.31 & 3000 & 3170 & 11.656237 & 0.632 & 162.6609 & 2997.03 & 5125 & 0.1 & 1950 & 487500 & 2225000 & 0.1209 & $3.01 \mathrm{E}-04$ & 2.259 & $1.44 \mathrm{E}-02$ & 56.61 & 57.1 & 0 & 0 & 48.3 & 440.1 \\
\hline 770 & 28.35 & 3000 & 1540 & 280.910205 & 13.5887 & 145.1211 & 3359.26 & 5125 & 0.1 & 1950 & 487500 & 1875000 & 0.1122 & $1.19 \mathrm{E}-04$ & 1.538 & $8.88 \mathrm{E}-03$ & 59.47 & 98.22 & 82.2 & 舟 & 78 & 277.9 \\
\hline 771 & 60.71 & 3000 & 1630 & 756.317471 & 24.4603 & 97.024 & 5024.53 & 5125 & 0.1 & 1950 & 487500 & 1025000 & 0.0524 & $1.44 \mathrm{E}-04$ & 2.392 & $6.96 \mathrm{E}-03$ & 30.45 & 99.34 & $\begin{array}{lll}93.39 \\
\end{array}$ & 33.89 & 99.6 & 201.9 \\
\hline 772 & 21.45 & 3000 & 5290 & 127.689862 & 4.1297 & 97.024 & 5024.53 & 5125 & 0.1 & 1950 & 487500 & 1025000 & 0.1483 & $4.41 \mathrm{E}-04$ & 2.502 & \begin{tabular}{|l|l|}
$5.78 \mathrm{E}-03$ \\
\end{tabular} & 61.17 & 96.08 & 60.84 & & 119.9 & 597.7 \\
\hline 773 & 21.64 & 3000 & 3070 & 37.112745 & 1.5471 & 125.0616 & 3898.08 & 5125 & 0.1 & 1950 & 487500 & 1500000 & 0.147 & $2.79 \mathrm{E}-04$ & 1.96 & $1.03 \mathrm{E}-02$ & 50.03 & 86.53 & 0 & & 67.6 & 464.2 \\
\hline 774 & 30.33 & 3000 & 5890 & 214.300946 & 6.4723 & 90.6061 & 5380.43 & 5125 & 0.1 & 1950 & 487500 & 925000 & 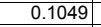 & $5.60 \mathrm{E}-04$ & 3.7 & $4.70 \mathrm{E}-03$ & 59.21 & 97.67 & 76.67 & & 147.6 & 625.3 \\
\hline 775 & 21.52 & 3000 & 5180 & 80.487494 & 2.9514 & 110.0086 & 4431.47 & 5125 & 0.1 & 1950 & 487500 & 1237500 & \begin{tabular}{|c|}
0.1478 \\
\end{tabular} & $6.55 \mathrm{E}-04$ & 3.241 & $5.16 \mathrm{E}-03$ & 43.6 & 93.79 & 37.88 & & 134.4 & 771.8 \\
\hline 776 & 59.13 & 3000 & 2130 & \begin{tabular}{|l|}
142.53827 \\
\end{tabular} & 5.1562 & 108.5219 & 4492.18 & 5125 & 0.1 & 1950 & 487500 & 1212500 & 0.0538 & $1.84 \mathrm{E}-04$ & 2.729 & $1.35 \mathrm{E}-02$ & 28.09 & 96.49 & 64.92 & 0 & 51.5 & 236.6 \\
\hline 777 & 62.38 & 3000 & 650 & \begin{tabular}{|l|}
1563.501002 \\
\end{tabular} & 53.8032 & 103.2361 & 4722.19 & 5125 & 0.1 & 1950 & 487500 & 1125000 & 0.051 & $4.30 \mathrm{E}-05$ & 1.426 & $5.62 \mathrm{E}-03$ & 59.3 & 99.68 & 96.8 & 68.02 & 123.3 & 117.1 \\
\hline 778 & 23.78 & 3000 & 2100 & \begin{tabular}{|l|}
27.979387 \\
\end{tabular} & 0.9982 & 107.0251 & 4555.01 & 5125 & 0.1 & 1950 & 487500 & 1187500 & 0.1338 & $2.36 \mathrm{E}-04$ & 1.891 & $1.21 \mathrm{E}-02$ & 30.84 & 82.13 & 0 & 0 & 57.4 & 407.5 \\
\hline 779 & 31.44 & 3000 & 3520 & 225.915543 & 11.2658 & 149.6016 & 3258.66 & 5125 & 0.1 & 1950 & 487500 & 1962500 & \begin{tabular}{ll|l}
0.1012 \\
\end{tabular} & $3.40 \mathrm{E}-04$ & 2.699 & $6.36 \mathrm{E}-03$ & 59.84 & 97.79 & 77.87 & & 109.1 & 440 \\
\hline 780 & 26.82 & 3000 & 4190 & \begin{tabular}{|r|}
41.347268 \\
\end{tabular} & $\begin{array}{l}2.0179 \\
\end{array}$ & 146.4082 & 3329.73 & 5125 & 0.1 & 1950 & 487500 & 1900000 & $\begin{array}{l}0.1186 \\
\end{array}$ & $6.15 \mathrm{E}-04$ & 3.62 & $6.94 \mathrm{E}-03$ & 39.91 & 87.91 & 0 & 0 & 99.8 & 691.6 \\
\hline 781 & 49.63 & 3000 & 2140 & \begin{tabular}{|l|}
896.765411 \\
\end{tabular} & 48.6229 & 162.6609 & 2997.03 & 5125 & 0.1 & 1950 & 487500 & 2225000 & 0.0641 & $1.36 \mathrm{E}-04$ & 2.073 & $5.83 \mathrm{E}-03$ & $\begin{array}{ll}68.42 \\
\end{array}$ & 99.44 & $\begin{array}{l}94.42 \\
\end{array}$ & 44.24 & 118.9 & 214 \\
\hline 782 & 25.53 & 3000 & 3770 & \begin{tabular}{|l|}
342.305278 \\
\end{tabular} & 15.5115 & 135.9447 & 3586.02 & 5125 & 0.1 & 1950 & 487500 & 1700000 & 0.1246 & $3.18 \mathrm{E}-04$ & 2.288 & $4.91 \mathrm{E}-03$ & 39.11 & 98.54 & 85.39 & 0 & 141.3 & 459.3 \\
\hline 783 & 23.27 & 3000 & 5310 & 149.024662 & 5.3908 & $\begin{array}{l}108.5219 \\
\end{array}$ & 4492.18 & 5125 & 0.1 & 1950 & 487500 & 1212500 & $\begin{array}{l}0.1367 \\
\end{array}$ & $5.96 \mathrm{E}-04$ & 3.205 & $4.39 \mathrm{E}-03$ & 16.37 & 96.64 & $\begin{array}{ll}66.45 \\
\end{array}$ & & 158.1 & 705.7 \\
\hline 784 & 43.76 & 3000 & 6800 & 92.577238 & 2.5381 & 82.2476 & 5927.23 & 5125 & 0.1 & 1950 & & 800000 & & 5.69E-04 & 4.958 & $6.39 \mathrm{E}-03$ & $\begin{array}{l}19.76 \\
\end{array}$ & 94.6 & 45.99 & & 108.5 & 580.7 \\
\hline 785 & 35.71 & 3000 & 3060 & 311.08325 & 11.6366 & $\begin{array}{l}112.2199 \\
12.20\end{array}$ & 4344.15 & 5125 & 0.1 & 1950 & & 1275000 & 0.0891 & $2.23 \mathrm{E}-04$ & 2.264 & $7.35 \mathrm{E}-03$ & 54.35 & 98.39 & 83.93 & 0 & 94.3 & \\
\hline 786 & 59.35 & 3000 & 590 & \begin{tabular}{|l|l|}
1163.813207 \\
\end{tabular} & 51.6989 & 133.2659 & 3658.1 & 5125 & 0.1 & 1950 & 487500 & 1650000 & 0.0536 & & 1.455 & $7.62 \mathrm{E}-03$ & 42.35 & & 95.7 & 57.04 & 91 & 125.7 \\
\hline 787 & 39.82 & 3000 & 440 & 1116.199483 & & 148.9657 & 3272.57 & 5125 & 0.1 & 1950 & 487500 & & 0.0799 & & 1.194 & & & 99.55 & 95.52 & 55.21 & 107.1 & 153.7 \\
\hline 788 & 46.04 & 3000 & 3610 & \begin{tabular}{|l|l|}
170.145379 \\
\end{tabular} & & 144.4754 & 3374.28 & 5125 & 0.1 & 1950 & 487500 & 1862500 & 0.0691 & $2.85 \mathrm{E}-04$ & 3.087 & $8.80 \mathrm{E}-03$ & & & 70.61 & 0 & 78.7 & 343.7 \\
\hline 789 & $\begin{array}{l}23.92 \\
28.99\end{array}$ & 3000 & 1750 & \begin{tabular}{|r|}
600.139352 \\
\end{tabular} & 23.177 & $\begin{array}{l}115.8579 \\
121561\end{array}$ & 4207.74 & 5125 & 0.1 & 1950 & & $\begin{array}{l}1337500 \\
127500\end{array}$ & $\begin{array}{r}0.133 \\
\end{array}$ & $1.23 \mathrm{E}-04$ & 1.467 & $5.27 \mathrm{E}-03$ & 62.27 & & 91.67 & 16.69 & 131.6 & 314.3 \\
\hline 7900 & $\begin{array}{l}28.89 \\
2169\end{array}$ & 3000 & $\begin{array}{r}870 \\
5420 \\
540\end{array}$ & \begin{tabular}{|r|}
905.120507 \\
26.07939
\end{tabular} & $\begin{array}{l}36.6764 \\
08908\end{array}$ & $\begin{array}{l}121.5631 \\
102469 \\
\end{array}$ & $\begin{array}{l}4010.26 \\
475749\end{array}$ & $\begin{array}{l}5125 \\
5525\end{array}$ & 0.1 & $\begin{array}{l}1950 \\
1950 \\
\end{array}$ & 487500 & $\begin{array}{l}1437500 \\
112500\end{array}$ & $\begin{array}{l}0.1101 \\
0.1467\end{array}$ & $6.41 \mathrm{E}-05$ & $\begin{array}{l}1.294 \\
3565\end{array}$ & \begin{tabular}{|l|}
$5.29 \mathrm{E}-03$ \\
$6.02 \mathrm{E}$
\end{tabular} & & 99.45 & 94.48 & 44.76 & $\begin{array}{ll}131.2 \\
100.1\end{array}$ & 229.5 \\
\hline $\begin{array}{l}791 \\
792 \\
\end{array}$ & $\begin{array}{r}21.69 \\
325 \\
\end{array}$ & $\begin{array}{l}3000 \\
3000\end{array}$ & $\begin{array}{r}5420 \\
448 \\
\end{array}$ & \begin{tabular}{|r|}
26.07939 \\
496687
\end{tabular} & $\begin{array}{l}0.8908 \\
0.1918 \\
\end{array}$ & \begin{tabular}{|c|}
102.4699 \\
115879
\end{tabular} & $\begin{array}{l}4757.49 \\
420774\end{array}$ & $\begin{array}{l}5125 \\
5125\end{array}$ & 0.1 & $\begin{array}{c}1950 \\
11950 \\
\end{array}$ & $\begin{array}{l}487500 \\
487500\end{array}$ & $\begin{array}{l}1112500 \\
137500\end{array}$ & $\begin{array}{l}0.1467 \\
00979\end{array}$ & $\begin{array}{l}7.44 \mathrm{E}-04 \\
5.17 \mathrm{E}-04\end{array}$ & $\begin{array}{l}3.565 \\
3669 \\
\end{array}$ & \begin{tabular}{|l|}
$6.92 \mathrm{E}-03$ \\
$153 \mathrm{E}-02$
\end{tabular} & 69.43 & 80.83 & 0 & 0 & $\begin{array}{l}100.1 \\
152\end{array}$ & 842.5 \\
\hline $\begin{array}{r}792 \\
793 \\
\end{array}$ & $\begin{array}{r}32.5 \\
22.36 \\
\end{array}$ & $\begin{array}{l}3000 \\
3000\end{array}$ & $\begin{array}{r}4480 \\
4200 \\
\end{array}$ & \begin{tabular}{|l|}
4.966877 \\
2.384517 \\
\end{tabular} & $\begin{array}{l}0.1918 \\
0.099\end{array}$ & \begin{tabular}{|l|l|l|l|l|}
15.8579 \\
124368
\end{tabular} & $\begin{array}{l}4207.74 \\
391989\end{array}$ & $\begin{array}{l}5125 \\
5125\end{array}$ & 0.1 & $\begin{array}{c}1950 \\
11950 \\
\end{array}$ & $\begin{array}{l}487500 \\
487500\end{array}$ & \begin{tabular}{|l|l|}
1337500 \\
1487500
\end{tabular} & 0.0979 & 5.17E-04 & $\begin{array}{l}3.668 \\
2642 \\
\end{array}$ & 1.53E-02 & $\begin{array}{l}84.24 \\
55.57 \\
\end{array}$ & 0 & 0 & $\frac{0}{0}$ & 45.2 & 578.5 \\
\hline $\begin{array}{l}795 \\
794\end{array}$ & $\begin{array}{r}\quad 2.36 \\
30.1\end{array}$ & 3000 & $\begin{array}{l}4200 \\
3640\end{array}$ & $\begin{array}{r}2.3845117 \\
27.720019\end{array}$ & \begin{tabular}{|c|}
0.0989 \\
1.3587
\end{tabular} & \begin{tabular}{|l|}
124.36568 \\
147.0497
\end{tabular} & $\begin{array}{l}3919.89 \\
3315.21\end{array}$ & $\begin{array}{l}\mid 5125 \\
5125\end{array}$ & $\begin{array}{l}0.1 \\
0.1\end{array}$ & $\begin{array}{l}1950 \\
1950\end{array}$ & $\frac{48 / 5}{4875}$ & $\frac{148 / 500}{1912500}$ & \begin{tabular}{|l|}
0.1423 \\
0.1057
\end{tabular} & $\begin{array}{l}\mid 4.62 \mathrm{E}-04 \\
4.18 \mathrm{E}-04\end{array}$ & $\begin{array}{l}2.642 \\
2.997\end{array}$ & \begin{tabular}{|l|l|}
$1.42 \mathrm{E}$ \\
$1.07 \mathrm{E}$
\end{tabular} & 58. & \begin{tabular}{r|r}
0 \\
81.96
\end{tabular} & $\frac{0}{0}$ & & $\begin{aligned} 48.7 \\
65 \\
\end{aligned}$ & $\frac{605.8}{510.3}$ \\
\hline 795 & 41.05 & 3000 & 4110 & 421.969242 & 18.36 & 130.5599 & 3733.92 & 5125 & 0.1 & 1950 & & 1600000 & 0.0775 & 3.97 & 3.589 & 4.78 & & 98.82 & 88.15 & 0 & 145 & 448 \\
\hline 796 & 26.6 & 3000 & 3190 & 12.0009 & & 113.6821 & 4288.27 & 5125 & 0. & 1950 & & 13000 & \begin{tabular}{|c|}
0.1196 \\
\end{tabular} & & 2.373 & & & 58.34 & & & 52.9 & 457.2 \\
\hline 797 & 24.89 & 3000 & 6830 & 10.2297 & & 78.7839 & 6187.82 & 5125 & 0.1 & 1950 & & 750000 & \begin{tabular}{|l|l|}
0.1278 \\
\end{tabular} & $7.43 \mathrm{E}-04$ & 3.94 & & & & & & 81.3 & 811.3 \\
\hline 798 & 28.3 & 3000 & 2850 & 362.660069 & 10.85 & 89.788 & 5429.46 & 5125 & 0.1 & 1950 & 487 & 912500 & 0.1124 & $2.31 \mathrm{E}-04$ & 2.038 & $5.96 \mathrm{E}$ & 48. & 98.62 & 86.21 & & 116.3 & 369.1 \\
\hline 799 & 29.82 & 3000 & 3400 & 74.2593 & 3.95 & 159.6003 & 305 & 5125 & 0.1 & 1950 & 487 & 2162500 & \begin{tabular}{|c|c|}
0.1067 \\
\end{tabular} & $4.30 \mathrm{E}-04$ & 3.036 & $8.28 \mathrm{E}-03$ & 76. & & \begin{tabular}{|l|l|}
32.67 \\
\end{tabular} & & 83.8 & 521.8 \\
\hline 800 & 22.82 & 3000 & 2640 & 155.5667 & 4.99 & 96.2335 & 5065.8 & 5125 & 0.1 & 1950 & 487 & 1012500 & 0.1394 & $2.01 \mathrm{E}-04$ & 1.727 & $8.17 \mathrm{E}-03$ & 70. & 96.79 & 67.86 & & 84.9 & 387.9 \\
\hline 801 & 36.11 & 3000 & 3580 & \begin{tabular}{|l|l|}
74.184023 \\
\end{tabular} & 2.28 & 92.2314 & 5285.62 & 5125 & 0.1 & 1950 & & 950000 & 0.0881 & $2.37 \mathrm{E}-04$ & 2.361 & $1.27 \mathrm{E}-02$ & 87. & 93.26 & 32.6 & & 54.7 & 335.1 \\
\hline 802 & 27.26 & 3000 & 3330 & 238.285583 & 14.3795 & 181.037 & 2692.82 & 5125 & 0.1 & 1950 & & 2612500 & 0.11 & $5.02 \mathrm{E}-04$ & 3.175 & & 61. & 97.9 & 79.02 & & 149 & 596.9 \\
\hline 803 & 46.58 & 3000 & 3160 & 130.189842 & 6.5 & 150.8694 & 3231.27 & 5125 & 0.1 & 1950 & & 198 & 0.0683 & 2.20 & 2.63 & & 53. & 96.16 & 61.59 & & 58.9 & 289.4 \\
\hline 804 & 29.21 & 30 & 3770 & & & 92.2314 & 528 & 5125 & 0.1 & 1950 & & & 0.10 & & 2.522 & & 35. & 93.27 & 32.74 & & 77.9 & 442.5 \\
\hline 805 & 31.62 & & 4900 & 440.9 & 14.6 & & & 5125 & 0.1 & 1950 & & & & & 2.511 & & 62. & & 88.66 & & 138.9 & 407 \\
\hline 806 & 33.1 & 30 & 1640 & & & 107.7 & 4523.32 & 5125 & 0.1 & 195 & & & 0.0 & & 1.659 & & 55. & 99. & $\begin{array}{l}91.48 \\
\end{array}$ & 14.8 & 106 & 256.9 \\
\hline 807 & 50.1 & 30 & 21 & & & & & 5125 & 0.1 & 195 & & & & & 2.551 & & & 95. & 51.91 & 0 & 51.2 & 261 \\
\hline 808 & 31.22 & 3000 & 2620 & & & & & 5125 & 0.1 & 195 & & 1762500 & & & 2.1 & & 51. & 89.3 & & 0 & 53.2 & 347.7 \\
\hline 809 & 32.1 & 3000 & & 253.2 & & & 538 & 5125 & 0.1 & 195 & & & 0.0 & & 2.0 & & & 98. & 80.26 & & 84.8 & 321.7 \\
\hline 810 & 44.56 & & & & & & & & 0.1 & 19 & & & 0.0714 & & & & & & & & 58.6 & \\
\hline 811 & 40.79 & & 4530 & 102.12 & & & & 5 & 0.1 & 19 & & & & & 5.167 & & & & 51.04 & & 108.2 & \\
\hline 812 & 24.4 & 30 & 1600 & 304.87 & 5154 & & & 5125 & 0.1 & 19 & & & 0.1304 & & 1.602 & & & 98.36 & 83.6 & 0 & 99.5 & \\
\hline 813 & 35.59 & 3000 & 620 & & & 174.6277 & & 5125 & 0.1 & 195 & & 2475000 & 0.0894 & & 1.31 & & & & 93.2 & 31.95 & 92 & 38.6 \\
\hline 814 & 26.91 & 3000 & 4070 & 65.32 & & & 2931 & 5125 & 0.1 & & & & & & 4.022 & & & 92. & 23.46 & 0 & 120.5 & 765.8 \\
\hline 815 & 33.17| & 3000 & 1790 & \begin{tabular}{|l|}
219.845807 \\
\end{tabular} & 6.8181 & $\begin{array}{r}93.0387 \\
\end{array}$ & 5239.76 & 5125 & 0.1 & 195 & & 9625 & 0.0959 & 1.70E-04 & 1.894 & $9.27 \mathrm{E}$ & 38 & 97.73 & 77.26 & 0 & 74.8 & 292.6 \\
\hline 816 & 57.12 & 3000 & 3300 & 229.110129 & 11.7622 & 154.0159 & 3165.26 & 5125 & 0.1 & 1950 & 487500 & 2050000 & 0.0557 & 2.21E-04 & 3.001 & 1.03E-02 & 51.85 & 97.82 & 78.18 & & 67.3 & 2693 \\
\hline 817 & 21.29 & 3000 & 3110 & $\begin{array}{r}41.48847 \\
50.001\end{array}$ & $\begin{array}{r}1.3418 \\
15.701 \\
\end{array}$ & 97.024 & 5024.53 & 5125 & 0.1 & 1950 & 487500 & 1025000 & 0.1494 & $2.38 \mathrm{E}-04$ & 1.804 & $1.11 \mathrm{E}-02$ & 84.89 & 87.95 & 0 & 0 & 62.3 & \\
\hline $\begin{array}{l}818 \\
819 \\
\end{array}$ & $\begin{array}{c}55.62 \\
23.76 \\
\end{array}$ & 3000 & 3880 & \begin{tabular}{|r|}
536.419945 \\
\end{tabular} & $\begin{array}{l}15.7601 \\
12503\end{array}$ & $\begin{array}{r}88.1405 \\
1527612 \\
\end{array}$ & 5530.94 & $\begin{array}{l}5125 \\
5125\end{array}$ & 0.1 & $\begin{array}{l}1950 \\
1050 \\
\end{array}$ & $\begin{array}{l}487500 \\
48750\end{array}$ & $\begin{array}{r}887500 \\
2825000\end{array}$ & 0.0572 & $2.13 \mathrm{E}-04$ & $\begin{array}{l}2.885 \\
158\end{array}$ & & $\begin{aligned} 57.15 \\
623 \\
\end{aligned}$ & 99.07 & $\begin{array}{l}90.68 \\
79.78\end{array}$ & 6.19 & 101.4 & 265.8 \\
\hline 819 & 23.76 & 3000 & 2000 & 247.254185 & 12.5903 & 152.7612 & 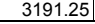 & 5125 & & 1950 & 487500 & 2025000 & & 1.54E-04 & & $7.66 \mathrm{E}-03$ & 62.3 & 97.98 & 79.78 & & 90.4 & 341.3 \\
\hline
\end{tabular}




\begin{tabular}{|c|c|c|c|c|c|c|c|c|c|c|c|c|c|c|c|c|c|c|c|c|c|c|}
\hline $\begin{array}{c}\text { Realizatio } \\
\mathrm{n}\end{array}$ & $\begin{array}{c}\text { Velocity } \\
(\mathrm{m} / \mathrm{y})\end{array}$ & \begin{tabular}{|c} 
Fixed Source \\
Concentratio \\
n (mg/L)
\end{tabular} & \begin{tabular}{|c|} 
Time of \\
Maximum at \\
Compliance \\
Boundary \\
(y)
\end{tabular} & $\begin{array}{c}\text { Maximum } \\
\text { Concentratio } \\
\text { n Compliance } \\
\text { Boundary } \\
(\mu \mathrm{g} / \mathrm{L})\end{array}$ & \begin{tabular}{|c|} 
Maximum \\
Release \\
Rate at \\
Complianc \\
e Boundary \\
(Kg/y) \\
\end{tabular} & $\begin{array}{c}\text { Source } \\
\text { Rate } \\
(\mathrm{Kg} / \mathrm{y}) \\
\end{array}$ & $\begin{array}{c}\text { Source } \\
\text { Duration } \\
\text { (y) }\end{array}$ & \begin{tabular}{|c|} 
1-D \\
Streamtub \\
e Length \\
$(\mathrm{m})$
\end{tabular} & $\begin{array}{c}\text { Base } \\
\text { Porosity } \\
\left(\mathrm{m}^{3} / \mathrm{m}^{3}\right) \\
\end{array}$ & $\begin{array}{c}\text { Bulk } \\
\text { Densty } \\
\left(\mathrm{Kg} / \mathrm{m}^{3}\right) \\
\end{array}$ & $\begin{array}{c}\text { Remaining } \\
\text { Carbon } \\
\text { Tetrachloride } \\
\text { Source } \\
(\mathrm{Kg}) \\
\end{array}$ & \begin{tabular}{|c|} 
Volume at or \\
above Fixed \\
Source \\
Concentratio \\
$n(m 3)$
\end{tabular} & $\begin{array}{l}\text { Porosity } \\
\left(\mathrm{m}^{3} / \mathrm{m}^{3}\right)\end{array}$ & $\begin{array}{c}\mathrm{Kd} \\
\left(\mathrm{m}^{3} / \mathrm{Kg}\right)\end{array}$ & Retardation & $\begin{array}{c}\text { First } \\
\text { Order } \\
\text { Rate } \\
\text { Constant } \\
(1 / y) \\
\end{array}$ & \begin{tabular}{|c} 
Longitudinal \\
Dispersivity \\
$(\mathrm{m})$
\end{tabular} & \begin{tabular}{|c|} 
Required \\
Source \\
Cleanup \\
Percentage \\
$(5 \mu g / L$ \\
Compliance) \\
\end{tabular} & \begin{tabular}{|c|} 
Required \\
Source \\
Cleanup \\
Percentage \\
$(50 \mu \mathrm{g} / \mathrm{L}$ \\
Compliance) \\
\end{tabular} & $\begin{array}{c}\text { Required } \\
\text { Source } \\
\text { Cleanup } \\
\text { Percentage } \\
\text { (500 } \mathrm{\mu g} / \mathrm{L} \\
\text { Compliance) } \\
\end{array}$ & \begin{tabular}{|c} 
Abiotic \\
Reaction \\
Half Time \\
(y) \\
\end{tabular} & \begin{tabular}{|c} 
Carbon \\
Tetrachloride \\
Travel Iime \\
(y)
\end{tabular} \\
\hline & & 3000 & \begin{tabular}{|r|}
4720 \\
\end{tabular} & \begin{tabular}{|l|l|}
115.176337 \\
\end{tabular} & \begin{tabular}{|l|}
4.4203 \\
\end{tabular} & 1115.1349 & 4234.16 & & & 1950 & 487500 & $\begin{array}{r}1325000 \\
\end{array}$ & 0.0984 & 4.64E- 04 & 3.385 & 6.51E-03 & $\begin{array}{r}32.88 \\
\end{array}$ & $\begin{array}{r}95.66 \\
\end{array}$ & $\begin{array}{r}56.59 \\
\end{array}$ & & 106.5 & 536.5 \\
\hline \begin{tabular}{r|r|}
821 \\
822
\end{tabular} & $\begin{array}{r}35 \\
3374 \\
\end{array}$ & $\begin{array}{l}3000 \\
3000 \\
3000\end{array}$ & 3050 & 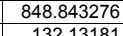 & \begin{tabular}{|l|}
31.7524 \\
60461
\end{tabular} & 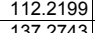 & $\begin{array}{r}4344.15 \\
2551.28\end{array}$ & 5125 & $\begin{array}{ll}0.1 \\
0.1\end{array}$ & $\begin{array}{l}1950 \\
1950\end{array}$ & 487500 & $\begin{array}{l}1275000 \\
175000\end{array}$ & 0.0909 & $1.78 \mathrm{E}-04$ & $\begin{array}{r}1.99 \\
1839\end{array}$ & 4.51E-03 & $\begin{array}{r}83.6 \\
32.61\end{array}$ & 99.41 & 94.11 & 41.1 & 153.7 & 291.5 \\
\hline$\frac{0.23}{823}$ & $\begin{array}{l}35.43 \\
25.31\end{array}$ & 3000 & $\begin{array}{l}1710 \\
1350 \\
\end{array}$ & $\begin{array}{r}132.13181 \\
46236267 \\
\end{array}$ & $\begin{array}{l}6.04611 \\
17521 \\
\end{array}$ & 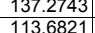 & $\begin{array}{l}3551.28 \\
428827\end{array}$ & $\begin{array}{l}5125 \\
5125 \\
\end{array}$ & $\begin{array}{l}0.1 \\
0.1 \\
\end{array}$ & \begin{tabular}{|c|}
1950 \\
1950
\end{tabular} & $\begin{array}{l}487500 \\
48750\end{array}$ & \begin{tabular}{r|r}
1725000 \\
1300000
\end{tabular} & $\begin{array}{l}0.0943 \\
0.1257\end{array}$ & \begin{tabular}{|l|l|}
$1.57 \mathrm{E}-04$ \\
$121 \mathrm{E}-04$
\end{tabular} & $\frac{1.839}{1.48}$ & \begin{tabular}{|l|}
$1.16 \mathrm{E}-02$ \\
$1.47 \mathrm{E}-02$ \\
\end{tabular} & $\begin{array}{l}33.64 \\
47.59\end{array}$ & $\begin{array}{l}96.22 \\
89.19\end{array}$ & \begin{tabular}{rl|l}
62.16 & \\
\end{tabular} & $\frac{0}{0}$ & 59.8 & $\frac{279.4}{301.1}$ \\
\hline 824 & 37.6 & 3000 & 1410 & 864.785924 & 44.9367 & 155.8885 & 3127.24 & 5125 & 0.1 & 1950 & 487500 & 2087500 & 0.0846 & $\begin{array}{l}1.2 \mathrm{TE}-04 \\
8.99 \mathrm{E}-05\end{array}$ & 1.537 & \begin{tabular}{|l|}
$1.41 \mathrm{~L}-\mathrm{L}-03$ \\
$6.11 \mathrm{E}-03$ \\
\end{tabular} & $\begin{array}{ll}47.09 \\
76.18\end{array}$ & $\begin{array}{ll}95.15 \\
99.42\end{array}$ & 94.22 & \begin{tabular}{r|r}
42.18 \\
\end{tabular} & $\frac{41.5}{113.5}$ & 209.5 \\
\hline 825 & 52.24 & 3000 & 4620 & 8.173413 & 0.4059 & 148.9657 & 3272.57 & 5125 & 0.1 & 1950 & 487500 & 1950000 & 0.0609 & $6.06 \mathrm{E}-04$ & 6.029 & \begin{tabular}{|l|}
$1.17 \mathrm{E}-02$ \\
\end{tabular} & $\begin{array}{r}24.9 \\
24.9\end{array}$ & 38.83 & 0 & $\frac{42.10}{0}$ & 59.2 & 591.5 \\
\hline 826 & 47.7 & 3000 & 3740 & 12.515404 & 0.6268 & 150.2362 & 3244.89 & 5125 & 0.1 & 1950 & 487500 & 1975000 & 0.0667 & $5.68 \mathrm{E}-04$ & 5.302 & \begin{tabular}{|l|}
$1.43 \mathrm{E}-02$ \\
\end{tabular} & 86.65 & 60.05 & 0 & $\frac{0}{0}$ & 48.3 & 569.7 \\
\hline 827 & 63.5 & 3000 & 4560 & 35.388302 & 1.4752 & 125.0616 & 3898.08 & 5125 & 0.1 & 1950 & 487500 & 1500000 & 0.0501 & $4.95 \mathrm{E}-04$ & 5.995 & \begin{tabular}{|l|}
$1.40 \mathrm{~L}-02$ \\
$1.15 \mathrm{E}-02$ \\
\end{tabular} & 49.45 & 85.87 & 0 & $\frac{0}{0}$ & 60.1 & 483.9 \\
\hline 828 & 52.67 & 3000 & 2020 & 300.566338 & 17.7306 & 176.9718 & 2754.68 & 5125 & 0.1 & 1950 & 487500 & 2525000 & 0.0604 & $1.48 \mathrm{E}-04$ & 2.236 & 1.12E-02 & 55.31 & 98.34 & & & 62.1 & 217.6 \\
\hline 829 & 32.66 & 3000 & 3290 & 90.667176 & 2.5628 & 84.7981 & 5748.95 & 5125 & 0.1 & 1950 & 487500 & | & 0.0974 & $3.00 \mathrm{E}-04$ & 2.555 & \begin{tabular}{|l|l|}
$9.19 \mathrm{E}-03$ \\
\end{tabular} & 30.36 & 94.49 & 44.85 & 0 & 75.4 & 400.9 \\
\hline 830 & 21.66 & 3000 & 3590 & 612.003743 & 30.7775 & 150.8694 & 3231.27 & 5125 & 0.1 & 1950 & 487500 & 1987500 & 0.1469 & $4.02 \mathrm{E}-04$ & 2.382 & \begin{tabular}{|c|c|}
$2.97 \mathrm{E}-03$ \\
\end{tabular} & 69.85 & 99.18 & 91.83 & 18.3 & 233.7 & 563.7 \\
\hline 831 & 25.91 & 3000 & 4580 & 69.303873 & 2.8407 & 122.9684 & 3964.43 & 5125 & 0.1 & 1950 & 487500 & 1462500 & 0.1228 & $5.50 \mathrm{E}-04$ & 3.266 & \begin{tabular}{|c|}
$6.38 \mathrm{E}-03$ \\
\end{tabular} & 38.99 & 92.79 & 27.85 & 0 & 108.7 & 646.1 \\
\hline 832 & 43.7 & 3000 & 600 & 900.12931 & 43.7359 & 145.7654 & 3344.42 & 5125 & 0.1 & 1950 & 487500 & 1887500 & 0.0728 & $6.34 \mathrm{E}-05$ & 1.44 & \begin{tabular}{|c|c|}
$7.18 \mathrm{E}-03$ \\
\end{tabular} & 23.65 & 99.44 & 94.45 & 44.45 & 96.5 & 168.9 \\
\hline 833 & 31.1 & 3000 & 2070 & 63.668044 & 2.5499 & 120.1495 & 4057.44 & 5125 & 0.1 & 1950 & 487500 & 1412500 & 0.1023 & $2.32 \mathrm{E}-04$ & 2.147 & \begin{tabular}{|c|}
$1.13 \mathrm{E}-02$ \\
\end{tabular} & 25.16 & 92.15 & 21.47 & & 61.2 & 353.9 \\
\hline 834 & 60.71 & 3000 & 4680 & 469.524499 & 16.8676 & 107.7748 & 4523.32 & 5125 & 0.1 & 1950 & 487500 & 1200000 & 0.0524 & $2.78 \mathrm{E}-04$ & 3.68 & \begin{tabular}{|c|c|}
$6.59 \mathrm{E}-03$ \\
\end{tabular} & 78.25 & 98.94 & 89.35 & & 105.2 & 310.7 \\
\hline 835 & 31.78 & 3000 & 1630 & 227.659983 & 11.4969 & 151.5013 & 3217.79 & 5125 & 0.1 & 1950 & 487500 & 2000000 & 0.1001 & $2.30 \mathrm{E}-04$ & 2.16 & \begin{tabular}{|l|l}
$7.50 \mathrm{E}-03$ \\
\end{tabular} & 12.55 & 97.8 & 78.04 & & 92.4 & 348.3 \\
\hline 836 & 23.19 & 3000 & 1730 & 114.620644 & 5.194 & 135.9447 & 3586.02 & 5125 & 0.1 & 1950 & 487500 & 1700000 & 0.1372 & $1.29 \mathrm{E}-04$ & 1.475 & 1.07E-02 & 70.92 & 95.64 & 56.38 & & 64.9 & 326 \\
\hline 837 & 25.72 & 3000 & 1230 & 76.2665 & 4.073 & 160.2148 & 3042.79 & 5125 & 0.1 & 1950 & 487500 & 2175000 & 0.1237 & $9.77 \mathrm{E}-05$ & 1.399 & $1.39 \mathrm{E}-02$ & 52.49 & 93.44 & 34.44 & & 50 & 278.8 \\
\hline 838 & 40.53 & 3000 & 8030 & 16.337566 & 0.3542 & 65.0346 & 7496 & 5125 & 0.1 & 1950 & 487500 & 562500 & 0.0785 & $5.58 \mathrm{E}-04$ & 4.593 & $1.01 \mathrm{E}-02$ & 25.54 & 69.4 & & & 69 & 580.8 \\
\hline 839 & 38.99 & 3000 & 3830 & 288.037927 & 8.7775 & 91.4206 & 5332.5 & 5125 & 0.1 & 1950 & 487500 & 937500 & 0.0816 & $2.46 \mathrm{E}-04$ & 2.523 & $7.56 \mathrm{E}-03$ & 60.33 & \begin{tabular}{|l|l|}
98.26 \\
\end{tabular} & 82.64 & & 91.7 & 331.7 \\
\hline 840 & 29.7 & 3000 & 3800 & 475.596703 & 21.6571 & 136.6103 & 3568.54 & 5125 & 0.1 & 1950 & 487500 & 1712500 & 0.1071 & $3.15 \mathrm{E}-04$ & 2.489 & $4.47 \mathrm{E}-03$ & 45.73 & $\begin{array}{l}98.95 \\
\end{array}$ & 89.49 & & 155.2 & 429.4 \\
\hline 841 & 28.56 & 3000 & 3990 & 41.619138 & & 131.239 & 3714.6 & 5125 & 0.1 & 1950 & 487500 & & 0.1114 & & 2.658 & $9.64 \mathrm{E}-03$ & 33.77 & 87.99 & & & 71.9 & 477 \\
\hline 842 & 30.68 & 3000 & 2810 & 47.306701 & 1.8946 & 120.1495 & 4057.44 & 5125 & 0.1 & 1950 & 487500 & 1412500 & $\begin{array}{l}0.1037 \\
\end{array}$ & $2.89 \mathrm{E}-04$ & 2.411 & $1.09 \mathrm{E}-02$ & 27.12 & \begin{tabular}{|l|}
89.43 \\
\end{tabular} & & 0 & 63.9 & 402.8 \\
\hline 843 & $\begin{array}{l}47.48 \\
\end{array}$ & 3000 & 920 & 549.114314 & 27.4989 & 150.2362 & 3244.89 & 5125 & 0.1 & 1950 & 487500 & 1975000 & 0.067 & $9.86 \mathrm{E}-05$ & 1.744 & 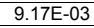 & 28.34 & \begin{tabular}{|l|}
99.09 \\
\end{tabular} & 90.89 & 8.94 & 75.6 & 188.2 \\
\hline 844 & \begin{tabular}{|l|}
25.07 \\
\end{tabular} & 3000 & 910 & 168.622665 & 7.1848 & 127.8255 & 3813.79 & 5125 & 0.1 & 1950 & 487500 & 1550000 & 0.1269 & 1.14E-04 & \begin{tabular}{|l|l|}
1.454 \\
\end{tabular} & $9.82 \mathrm{E}-03$ & \begin{tabular}{|l|}
16.63 \\
\end{tabular} & \begin{tabular}{|l|l|}
97.03 \\
\end{tabular} & 70.35 & 0 & 70.6 & 297.2 \\
\hline 845 & 29.98 & 3000 & 4610 & 22.271141 & \begin{tabular}{|c|c|} 
\\
\end{tabular} & 114.4096 & 4261 & 5125 & 0.1 & $\begin{array}{l}1950 \\
\end{array}$ & 487500 & 1312500 & 0.1061 & 5.37E-04 & 3.558 & $1.04 \mathrm{E}-02$ & 84.02 & 77.55 & 0 & 0 & 66.9 & 608.2 \\
\hline 846 & 21.94 & 3000 & 1510 & 577.504039 & 32.2437 & $\begin{array}{l}167.4988 \\
\end{array}$ & 2910.47 & 5125 & 0.1 & 1950 & 487500 & 2325000 & 0.145 & $1.58 \mathrm{E}-04$ & 1.55 & $4.61 \mathrm{E}-03$ & 28.12 & $\begin{array}{l}99.13 \\
\end{array}$ & 91.34 & 13.42 & 150.2 & 362.1 \\
\hline 847 & 24.03 & 3000 & 5310 & 15.223906 & 0.5278 & 103.9994 & 4687.53 & 5125 & 0.1 & 1950 & 487500 & 1137500 & 0.1324 & 5.93E-04 & 3.263 & $8.36 \mathrm{E}-03$ & 29.98 & $\begin{array}{l}67.16 \\
\end{array}$ & 0 & 0 & 82.9 & 696 \\
\hline 848 & $\begin{array}{l}31.85 \\
207 \\
207\end{array}$ & 3000 & 3670 & 192.037288 & 9.9788 & 155.8885 & 3127.24 & 5125 & 0.1 & 1950 & 487500 & 2087500 & $\begin{array}{l}0.0999 \\
\end{array}$ & 4.79E-04 & 3.422 & \begin{tabular}{|l|l|}
$5.55 \mathrm{E}-03$ \\
77502
\end{tabular} & 60.5 & 97.4 & 73.96 & & 125 & 550.7 \\
\hline $\begin{array}{l}849 \\
85 \\
85\end{array}$ & 23.97 & $\begin{array}{l}3000 \\
3000\end{array}$ & $\begin{array}{l}4940 \\
2030\end{array}$ & \begin{tabular}{|l|l|}
5.595512 \\
2402719
\end{tabular} & $\begin{array}{l}0.6246 \\
11897\end{array}$ & $\begin{array}{l}120.1495 \\
148.204\end{array}$ & 4057.44 & 5125 & 0.1 & 1950 & 487500 & 1412500 & 0.1327 & 6.62E-04 & 3.522 & 7.74E-03 & 30.03 & 67.94 & 0 & 0 & 89.5 & 753 \\
\hline $\begin{array}{l}850 \\
851\end{array}$ & $\begin{array}{l}26.16 \\
32.97 \\
\end{array}$ & 3000 & 2930 & $\begin{array}{r}24.042719 \\
177.562713\end{array}$ & $\begin{array}{r}1.1887 \\
6.432 \\
\end{array}$ & \begin{tabular}{|l|l|}
148.3284 \\
10.5219
\end{tabular} & 3286.63 & $\begin{array}{l}5125 \\
5125\end{array}$ & $\begin{array}{l}0.1 \\
0.1\end{array}$ & $\begin{array}{l}1950 \\
1950\end{array}$ & $\begin{array}{l}487500 \\
487500\end{array}$ & $\begin{array}{l}\frac{1937500}{1212500} \\
\end{array}$ & $\begin{array}{l}0.1216 \\
0.0655 \\
\end{array}$ & \begin{tabular}{|l|}
$2.51 \mathrm{E}-04$ \\
$5.41 \mathrm{E}-04$
\end{tabular} & $\begin{array}{l}2.043 \\
3.832 \\
\end{array}$ & $\frac{1.36 \mathrm{E}-02}{5.21 \mathrm{E}-03}$ & $\begin{array}{l}64.16 \\
6.31\end{array}$ & \begin{tabular}{r|r|r|}
99.2 & \\
97.18 &
\end{tabular} & $\begin{array}{r}0 \\
71.84\end{array}$ & & $\begin{array}{c}\frac{51.1}{133} \\
13\end{array}$ & $\frac{400.1}{559.8}$ \\
\hline 852 & 26.06 & 3000 & 5080 & 1.771399 & & $\mid \begin{array}{l}104.7599 \\
\end{array}$ & 4653.5 & 5125 & 0.1 & 1950 & & 1150000 & 0.1221 & & 3.481 & $1.38 \mathrm{E}-02$ & 53.38 & 0 & & & 50.3 & 684.8 \\
\hline 853 & 24.62 & 3000 & 6050 & 13.773199 & 0.4047 & 88.1405 & 5530.94 & 5125 & 0.1 & 1950 & 4875 & & 0.1292 & $6.30 \mathrm{E}-04$ & 3.464 & $8.72 \mathrm{E}-03$ & 45.94 & 63.7 & & & 79.5 & 720.9 \\
\hline 854 & 35.35 & 3000 & 1180 & 659.578841 & 28.8542 & 131.239 & 3714.6 & 5125 & 0.1 & 1950 & 487500 & 1612500 & 0.09 & $8.63 \mathrm{E}-05$ & 1.485 & $7.21 \mathrm{E}-03$ & 57.74 & 99.24 & 92.42 & 24.19 & 96.1 & 215.3 \\
\hline 855 & 43.88 & 3000 & 4540 & $\begin{array}{r}73.479739 \\
\end{array}$ & 2.5659 & 104.7599 & 4653.5 & 5125 & 0.1 & 1950 & 487500 & 1150000 & 0.0725 & $3.55 \mathrm{E}-04$ & 3.476 & $9.66 \mathrm{E}-03$ & 22.88 & 93.2 & 31.95 & & 71.7 & 406 \\
\hline 856 & 30.41 & 3000 & 2750 & 623.002757 & 24.6559 & 118.7276 & 4106.04 & 5125 & 0.1 & 1950 & 487500 & 1387500 & 0.1046 & $2.29 \mathrm{E}-04$ & 2.108 & $4.54 \mathrm{E}-03$ & 40.48 & 99.2 & 91.97 & 19.74 & 152.7 & 355.3 \\
\hline 857 & 44.43 & 3000 & 1530 & 263.277286 & 12.7922 & 145.7654 & 3344.42 & 5125 & 0.1 & 1950 & & 1887500 & 0.0716 & $1.33 \mathrm{E}-04$ & 1.937 & $1.14 \mathrm{E}-02$ & 45.85 & 98.1 & 81.01 & & 61.1 & 223.4 \\
\hline 858 & 36.95 & 3000 & 830 & 157.197651 & 4.2647 & 81.3886 & 5989.78 & 5125 & 0.1 & 1950 & & 787500 & 0.0861 & $1.12 \mathrm{E}-04$ & 1.655 & $1.31 \mathrm{E}-02$ & 16.14 & 96.82 & 68.19 & & 53.1 & 229.5 \\
\hline 859 & 61.77 & 3000 & 3140 & 216.402078 & 12.1256 & 168.0986 & 2900.08 & 5125 & 0.1 & 1950 & & 2337500 & 0.0515 & $2.97 \mathrm{E}-04$ & 3.914 & 9.36 & 77.89 & 97.69 & 76.89 & & 74 & 324.7 \\
\hline 860 & 46.51 & 3000 & 1840 & 731.607653 & 23.2748 & 95.4399 & 5107.93 & 5125 & 0.1 & 1950 & & & 0.0684 & $1.31 \mathrm{E}-04$ & 1.971 & & 57.4 & 99.32 & 93.17 & 31.66 & 103.5 & 217.2 \\
\hline 861 & 26.64 & 3000 & 4340 & 498.763195 & 19.8574 & 119.4396 & 408 & 5125 & 0.1 & 1950 & & & 0.1194 & $3.69 \mathrm{E}-04$ & 2.562 & & 62.38 & 99 & 89.98 & & 180.3 & 492.9 \\
\hline 862 & 23.53 & 3000 & 1130 & & & 115.1349 & 423 & 5125 & 0.1 & 1950 & & & & & 1.347 & & 41. & 94.56 & 45.58 & & 56.2 & 293.5 \\
\hline 863 & 33.38 & 3000 & 2750 & & 2.43 & 141. & 343 & 5125 & 0.1 & 1950 & & & 0.0 & 2.72 & 2.442 & & 29 & 90.29 & 2.92 & & 60.5 & 374.9 \\
\hline 864 & 21.77 & 3000 & 3400 & 50.6 & 2.125 & 125.7554 & & 5125 & 0.1 & 1950 & & 1512500 & 0.1. & & 1.971 & & 66. & 90.14 & 1.37 & & 71.4 & 463.9 \\
\hline 865 & 25.25 & 3000 & 1190 & 231.4 & 5.5242 & & & 5125 & 0.1 & & & & 0. & & 1.544 & & & 97.84 & 78.39 & & 83.2 & 313.5 \\
\hline 866 & 40.02 & 3000 & 4050 & & 20.21 & & & 5125 & 0.1 & 195 & & & & & 3.3 & & & & 88.9 & & 153.1 & 431 \\
\hline 867 & 28.13 & & 3930 & & & & & 5125 & 0.1 & 19 & & & & & & & & & 0 & 0 & 96 & 626.2 \\
\hline 868 & 32.66 & & 3650 & & & & & 5125 & 0.1 & 19 & & & & & & & & .2 & 61.97 & 0 & 104 & 490 \\
\hline 869 & 31.59 & & 3500 & & 30.57 & & & 5125 & 0.1 & 195 & & & 0.1007 & & 2.048 & & 79. & \begin{tabular}{ll|}
99.24 \\
\end{tabular} & 92.45 & 24.46 & 145 & \\
\hline 870 & 22.44 & & 1710 & & 1.93 & 138.5 & & 5125 & 0.1 & 195 & & & 0.14 & & 1.676 & & 29.9 & 88.05 & 0 & 0 & 59.6 & 382.8 \\
\hline 871 & 24.83 & 3000 & 4500 & & $\begin{array}{l}0.1081 \\
\end{array}$ & 122.9 & & 5125 & 0.1 & 195 & & & 0.1281 & & 3.473 & & 53.1 & & 0 & 0 & 56.3 & 6.8 \\
\hline 872 & 27.1 & 3000 & 2360 & 37.99 & 1.772 & 139.9 & 3484.28 & 5125 & 0.1 & 195 & & & 0.11 & & 2.183 & & & 86.84 & 0 & & 62.9 & 412.9 \\
\hline 873 & 27.96 & 500 & 3220 & 65.6154 & 2.4 & 94.6 & 5150. & 5125 & 0.1 & 195 & & & 0.1138 & & 2.226 & 1.0 & 47. & $\begin{array}{l}92.38 \\
\end{array}$ & 23.8 & & 68.6 & 408.1 \\
\hline 874 & 27.5 & 3000 & 3330 & 5.408829 & 0.18 & 102.46 & 4757. & 5125 & 0.1 & 1950 & 48 & 1112 & 0.1157 & & 2.398 & & 42. & 7.56 & 0 & & 43.5 & 447 \\
\hline 875 & 26.44 & 3000 & 3140 & 1.143714 & 0.06 & 176.9718 & 2754.68 & 5125 & 0.1 & 1950 & & 25250 & 0.1203 & $5.05 \mathrm{E}$ & 3.12 & 1.70 & 63. & 0 & & & 40.8 & 604.7 \\
\hline 876 & $\begin{array}{r}30.8 \\
\end{array}$ & 3000 & 1650 & $\begin{array}{r}451.432534 \\
\end{array}$ & 20.556 & 136.6103 & 3568.54 & 5125 & 0.1 & 1950 & 487500 & 17125 & 0.1033 & $1.75 \mathrm{E}$ & 1.856 & $6.25 \mathrm{E}$ & 28. & 98.89 & 88.92 & 0 & $\frac{110.9}{1007}$ & 308.9 \\
\hline $\begin{array}{l}877 \\
878 \\
\end{array}$ & $\begin{array}{l}23.85 \\
30.21\end{array}$ & 3000 & $\begin{array}{l}3510 \\
1110\end{array}$ & $\begin{array}{r}145.89 .19412 \\
50.101555\end{array}$ & $\begin{array}{l}6.0454 \\
3.0427 \\
\end{array}$ & 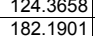 & $\begin{array}{l}3919.89 \\
2675.78\end{array}$ & $\begin{array}{l}5125 \\
5125\end{array}$ & $\begin{array}{l}0.1 \\
0.1 \\
\end{array}$ & $\begin{array}{l}1950 \\
1950\end{array}$ & $\begin{array}{l}487500 \\
487500\end{array}$ & $\begin{array}{l}\mid 1483500 \\
2637500\end{array}$ & \begin{tabular}{|c|}
0.1334 \\
0.1053
\end{tabular} & $\begin{array}{l}3.46 \mathrm{E}-04 \\
1.00 \mathrm{E}-04\end{array}$ & $\begin{array}{l}2.3122 \\
1.482\end{array}$ & \begin{tabular}{|l|}
$6.32 \mathrm{E}-03$ \\
$1.71 \mathrm{E}-02$ \\
\end{tabular} & 27.84 & 96.57 & $\begin{array}{r}65.73 \\
0.2 \\
\end{array}$ & & $\frac{1097}{40.5}$ & $\frac{496.9}{251.4}$ \\
\hline 879 & $\begin{array}{l}00.21 \\
23.39\end{array}$ & 3000 & 1630 & 201.33633 & 10.5454 & 157.1306 & 3102.51 & 5125 & 0.1 & 1950 & 487500 & 2112500 & 0.136 & $\begin{array}{l}1.00 \mathrm{E}-04 \\
1.36 \mathrm{E}-04\end{array}$ & $\begin{array}{l}1.507 \\
1.507 \\
\end{array}$ & \begin{tabular}{|l|}
$1.17 \mathrm{~L}-\mathrm{L}-03$ \\
$8.52 \mathrm{E}$
\end{tabular} & $\begin{array}{l}44.21 \\
51.22 \\
\end{array}$ & $\begin{array}{l}90.02 \\
97.52\end{array}$ & $\begin{array}{r}0.4 \\
75.17\end{array}$ & 0 & $\frac{80.0}{81.4}$ & 330.1 \\
\hline 880 & 57.42 & 3000 & 7380 & 41.7344 & \begin{tabular}{|l|l|}
0.9963 \\
\end{tabular} & 71.6152 & 6807. & 5125 & 0.1 & 1950 & 4875 & 650000 & 0.0554 & $5.49 E-04$ & 6.007 & \begin{tabular}{|l|}
$9.76 \mathrm{E}-03$ \\
\end{tabular} & 44.62 & 88.02 & 0 & 0 & 71 & 536.1 \\
\hline 881 & 35.23 & 3000 & 2330 & 158.706034 & 7.6088 & \begin{tabular}{|l|l|l}
143.8283 \\
\end{tabular} & 3389.46 & 5125 & 0.1 & 1950 & 487500 & 1850000 & 0.0903 & $2.21 \mathrm{E}-04$ & 2.239 & \begin{tabular}{|c|}
$9.40 \mathrm{E}-03$ \\
\end{tabular} & 32.37 & 96.85 & 68.5 & 0 & 73.7 & 325.7 \\
\hline 882 & 23.2 & 3000 & 4360 & 20.370895 & 0.8207 & 120.8573 & 4033.68 & 5125 & 0.1 & 1950 & 487500 & 1425000 & 0.1371 & $5.27 \mathrm{E}-04$ & 2.945 & \begin{tabular}{|c|}
$9.51 \mathrm{E}-03$ \\
\end{tabular} & 83.46 & 75.46 & 0 & 0 & 72.9 & 650.4 \\
\hline
\end{tabular}




\begin{tabular}{|c|c|c|c|c|c|c|c|c|c|c|c|c|c|c|c|c|c|c|c|c|c|c|}
\hline $\begin{array}{c}\text { Realizatio } \\
n\end{array}$ & $\begin{array}{c}\text { Velocity } \\
(\mathrm{m} / \mathrm{y})\end{array}$ & $\begin{array}{c}\text { Fixed Source } \\
\text { Concentratio } \\
\mathrm{n}(\mathrm{mg} / \mathrm{L})\end{array}$ & $\begin{array}{c}\text { Time of } \\
\text { Maximum at } \\
\text { Compliance } \\
\text { Boundary } \\
\text { (y) }\end{array}$ & $\begin{array}{c}\text { Maximum } \\
\text { Concentratio } \\
\text { n Compliance } \\
\text { Boundary } \\
(\mu \mathrm{g} / \mathrm{L})\end{array}$ & \begin{tabular}{|c|} 
Maximum \\
Release \\
Rate at \\
Complianc \\
e Boundary \\
(Kg/y) \\
\end{tabular} & $\begin{array}{c}\text { Source } \\
\text { Rate } \\
(\mathrm{Kg} / \mathrm{y})\end{array}$ & $\begin{array}{c}\text { Source } \\
\text { Duration } \\
\text { (y) }\end{array}$ & \begin{tabular}{|c|} 
1-D \\
Streamtub \\
e Length \\
$(\mathrm{m})$
\end{tabular} & $\begin{array}{c}\text { Base } \\
\text { Porosity } \\
\left(\mathrm{m}^{3} / \mathrm{m}^{3}\right)\end{array}$ & $\begin{array}{c}\text { Bulk } \\
\text { Densty } \\
\left(\mathrm{Kg} / \mathrm{m}^{3}\right)\end{array}$ & $\begin{array}{c}\text { Remaining } \\
\text { Carbon } \\
\text { Tetrachloride } \\
\text { Source } \\
(\mathrm{Kg}) \\
\end{array}$ & \begin{tabular}{|c|} 
Volume at or \\
above Fixed \\
Source \\
$\begin{array}{c}\text { Concentratio } \\
n(m 3)\end{array}$ \\
\end{tabular} & $\begin{array}{l}\text { Porosity } \\
\left(\mathrm{m}^{3} / \mathrm{m}^{3}\right)\end{array}$ & $\begin{array}{c}\mathrm{Kd} \\
\left(\mathrm{m}^{3} / \mathrm{kg}\right)\end{array}$ & Retardation & $\begin{array}{c}\text { First } \\
\text { Order } \\
\text { Rate } \\
\text { Constant } \\
(1 / y) \\
\end{array}$ & \begin{tabular}{|c|} 
Longitudinal \\
Dispersivity \\
$(\mathrm{m})$
\end{tabular} & $\begin{array}{c}\text { Required } \\
\text { Source } \\
\text { Cleanup } \\
\text { Percentage } \\
(5 \mu g / L \\
\text { Compliance) } \\
\end{array}$ & \begin{tabular}{|c|} 
Required \\
Source \\
Cleanup \\
Percentage \\
$(50 \mu \mathrm{g} / \mathrm{L}$ \\
Compliance)
\end{tabular} & $\begin{array}{c}\text { Required } \\
\text { Source } \\
\text { Cleanup } \\
\text { Percentage } \\
\text { (500 } \mathrm{\mu g} / \mathrm{L} \\
\text { Compliance) } \\
\end{array}$ & \begin{tabular}{|c|} 
Abiotic \\
Reaction \\
Half Time \\
(y) \\
\end{tabular} & $\begin{array}{c}\text { Carbon } \\
\text { Tetrachloride } \\
\text { Travel Time } \\
\text { (y) } \\
\end{array}$ \\
\hline & & 3000 & 3230 & & 6.1777 & $\begin{array}{l}147.0497 \\
127026\end{array}$ & $\begin{array}{r}3315.21 \\
352.22\end{array}$ & \begin{tabular}{|l|}
5125 \\
\end{tabular} & & 1950 & $\begin{array}{r}487500 \\
\end{array}$ & \begin{tabular}{|l|}
1912500 \\
\end{tabular} & 0.0563 & $2.14 \mathrm{E}-04$ & 2.924 & $1.30 \mathrm{E}-02$ & $\begin{array}{r}48.97 \\
\end{array}$ & 96.03 & 60.33 & & 53.3 & 265.2 \\
\hline 884 & $\begin{array}{l}44.19 \\
2.59\end{array}$ & 3000 & 4360 & 1.332493 & 0.0613 & $\begin{array}{l}137.9366 \\
70650\end{array}$ & 3534.23 & $\begin{array}{l}5125 \\
5125 \\
\end{array}$ & 0.1 & 1950 & 487500 & $\begin{array}{l}1737500 \\
\end{array}$ & 0.072 & $6.46 \mathrm{E}-04$ & 5.536 & $1.65 \mathrm{E}-02$ & 44.3 & & & & 42.1 & 642.1 \\
\hline 885 & 24.59 & 3000 & 2580 & \begin{tabular}{|r|}
24.280964 \\
\end{tabular} & $\begin{array}{l}0.6447 \\
10177\end{array}$ & \begin{tabular}{|l|l|}
79.6568 \\
121561
\end{tabular} & 6120 & 5125 & 0.1 & 1950 & 487500 & 762500 & 0.1294 & $2.98 \mathrm{E}-04$ & 2.164 & $1.12 \mathrm{E}-02$ & 24.85 & 79.41 & & 0 & 61.8 & 451.1 \\
\hline 886 & 51.64 & 3000 & 4540 & 121.311351 & 4.9157 & $\begin{array}{l}121.5631 \\
\end{array}$ & 4010.26 & 5125 & 0.1 & 1950 & 487500 & 1437500 & 0.0616 & 4.45E-04 & 4.648 & 7.91E-03 & 47.41 & 95.88 & 58.78 & 0 & 87.6 & 461.2 \\
\hline 887 & 21.48 & 3000 & 3840 & 177.681866 & 8.1304 & 137.2743 & 3551.28 & 5125 & 0.1 & 1950 & 487500 & 1725000 & 0.1481 & $3.90 \mathrm{E}-04$ & 2.33 & $5.50 \mathrm{E}-03$ & 63.79 & 97.19 & 71.86 & 0 & 126 & 555.8 \\
\hline 888 & & 3000 & 3940 & 645.420627 & 23.985 & 111.4852 & 4372.78 & 5125 & 0.1 & 1950 & 487500 & 1262500 & 0.0723 & $2.27 \mathrm{E}-04$ & 2.584 & $5.36 \mathrm{E}-03$ & 63.25 & 99.23 & 92.25 & 22.53 & 129.4 & 301 \\
\hline 889 & 21.85 & 3000 & 3110 & 6.606734 & 0.2754 & 125.0616 & 3898.08 & 5125 & 0.1 & 1950 & 487500 & 1500000 & 0.1456 & 3.25E-04 & 2.13 & $1.35 \mathrm{E}-02$ & 39.53 & 24.32 & & 0 & 51.4 & 499.6 \\
\hline 890 & $\begin{array}{l}23.48 \\
15.51\end{array}$ & 3000 & 4970 & \begin{tabular}{|r|}
64.09809 \\
\end{tabular} & $\begin{array}{r}2.46 \\
\end{array}$ & $\begin{array}{l}115.1349 \\
\end{array}$ & 4234.16 & 5125 & 0.1 & 1950 & 487500 & 1325000 & 0.1355 & 6.57E-04 & 3.45 & 5.79E-03 & 51.59 & 92.2 & 21.99 & 0 & 119.7 & 753.1 \\
\hline 8912 & $\begin{array}{l}45.51 \\
3178\end{array}$ & 3000 & $\frac{1900}{620}$ & \begin{tabular}{|l|}
113.959437 \\
18600308
\end{tabular} & $\begin{array}{l}5.9453 \\
77539 \\
\end{array}$ & $\begin{array}{l}156.5102 \\
125061\end{array}$ & 3114.81 & $\begin{array}{l}5125 \\
5125\end{array}$ & 0.1 & $\begin{array}{l}1950 \\
1900\end{array}$ & 487500 & 2100000 & 0.0699 & $\begin{array}{l}1.85 \mathrm{E}-04 \\
371 \mathrm{E}-05\end{array}$ & 2.341 & \begin{tabular}{|l|}
$1.30 \mathrm{E}-02$ \\
$1.0 \mathrm{E}$
\end{tabular} & 30.14 & $\begin{array}{l}95.61 \\
07.611\end{array}$ & $\begin{array}{l}56.12 \\
7212\end{array}$ & & 53.5 & $\begin{array}{l}263.6 \\
191.5\end{array}$ \\
\hline $\begin{array}{l}892 \\
893 \\
\end{array}$ & $\begin{array}{ll}31.78 \\
54.57\end{array}$ & 3000 & $\begin{array}{r}620 \\
1740\end{array}$ & \begin{tabular}{|r|r|}
186.0030386 \\
1039.429566
\end{tabular} & $\begin{array}{r}7 . .5339 \\
34.4315\end{array}$ & \begin{tabular}{|r|r|}
125.0616 \\
993762
\end{tabular} & $\begin{array}{l}3898.08 \\
49056\end{array}$ & $\begin{array}{l}5125 \\
5125\end{array}$ & $\begin{array}{l}0.1 \\
0.1 \\
\end{array}$ & $\begin{array}{l}1950 \\
1950\end{array}$ & $\begin{array}{l}487500 \\
487500\end{array}$ & \begin{tabular}{|l|l|}
15000000 \\
162500
\end{tabular} & $\begin{array}{l}0.1001 \\
0.0533 \\
\end{array}$ & $\begin{array}{l}3.71 \mathrm{E}-05 \\
1.9 \mathrm{E}-04\end{array}$ & $\frac{1.187}{2031}$ & \begin{tabular}{|l|}
$1.49 \mathrm{E}-02$ \\
$569 \mathrm{E}-03$
\end{tabular} & $\begin{array}{ll}40.64 \\
59.02\end{array}$ & 97.31 & $\frac{73.12}{05.19}$ & $\begin{array}{r}0 \\
519\end{array}$ & 46.5 & $\begin{array}{l}191.5 \\
1907\end{array}$ \\
\hline 894 & 41.64 & 3000 & 1050 & \begin{tabular}{|r|}
1039.429506 \\
250.129251
\end{tabular} & $\begin{array}{l}34.4315 \\
15.1423\end{array}$ & $\begin{array}{l}99.3162 \\
181.614 \\
\end{array}$ & $\begin{array}{r}4950.26 \\
2684.26\end{array}$ & $\begin{array}{l}3120 \\
5125\end{array}$ & $\begin{array}{l}0.1 \\
0.1\end{array}$ & $\begin{array}{l}1950 \\
1950\end{array}$ & $\frac{487500}{487500}$ & $\begin{array}{l}1025025000 \\
2625000\end{array}$ & \begin{tabular}{|c|}
0.0853 \\
0.0764 \\
\end{tabular} & $\begin{array}{l}1.19 \mathrm{E}-04 \\
1.13 \mathrm{E}-04\end{array}$ & $\begin{array}{l}2.031 \\
1.745\end{array}$ & \begin{tabular}{|l|}
$5.69 \mathrm{E}-03$ \\
$1.19 \mathrm{E}-02$ \\
\end{tabular} & $\begin{array}{l}59.02 \\
30.05 \\
\end{array}$ & \begin{tabular}{rl|}
99.52 & 98 \\
\end{tabular} & $\begin{array}{l}95.19 \\
80.01\end{array}$ & $\begin{aligned} 51.9 \\
0\end{aligned}$ & $\begin{aligned} \frac{121.7}{58.4} & \end{aligned}$ & $\begin{array}{l}\frac{190.7}{214.7} \\
\end{array}$ \\
\hline 895 & 35.15 & 3000 & 2680 & 96.278985 & 3.625 & 112.9522 & 4315.99 & 5125 & 0.1 & 1950 & 487500 & 1287500 & 0.0905 & $2.19 \mathrm{E}-04$ & 2.222 & $1.14 \mathrm{E}-02$ & 51.34 & 94.81 & 48.07 & 0 & 60.6 & 323.9 \\
\hline 896 & 33.56 & 3000 & 4520 & 7.33048 & 0.2597 & 106.2727 & 4587.26 & 5125 & 0.1 & 1950 & 487500 & 1175000 & 0.0948 & $3.73 \mathrm{E}-04$ & 2.991 & $1.52 \mathrm{E}-02$ & 43.35 & 31.79 & & & 45.7 & 456.8 \\
\hline 897 & 28.63 & 3000 & 4050 & 8.9857 & 0.4872 & 162.6609 & 2997.03 & 5125 & 0.1 & 1950 & 487500 & 2225000 & 0.1111 & $6.40 \mathrm{E}-04$ & 3.911 & $9.38 \mathrm{E}-03$ & 29.32 & 44.36 & & & 73.9 & 699.9 \\
\hline 898 & 23.43 & 3000 & 2920 & 9.109067 & 0.4465 & $\begin{array}{l}147.0497 \\
\end{array}$ & 3315.21 & 5125 & 0.1 & 1950 & 487500 & 1912500 & 0.1358 & $2.71 \mathrm{E}-04$ & 2.009 & $1.49 \mathrm{E}-02$ & 57.93 & 45.11 & & 0 & 46.4 & 439.6 \\
\hline 899 & 33.31 & 3000 & 4020 & 16.969379 & 0.7951 & 140.5704 & 3468.01 & 5125 & 0.1 & 1950 & 487500 & 1787500 & 0.0955 & $4.91 \mathrm{E}-04$ & 3.601 & $1.06 \mathrm{E}-02$ & 37.42 & 70.54 & & & 65.3 & 553.9 \\
\hline 900 & 58.59 & 3000 & 5140 & 64.233048 & 2.7074 & $\begin{array}{l}126.4473 \\
\end{array}$ & 3855.36 & 5125 & 0.1 & 1950 & 487500 & 1525000 & 0.0543 & $7.49 \mathrm{E}-04$ & 7.973 & $7.70 \mathrm{E}-03$ & 70.51 & 92.22 & 22.16 & & 90 & 697.4 \\
\hline 901 & 35.67 & 3000 & 3690 & 195.232499 & 6.4163 & 98.5952 & 4944.46 & 5125 & 0.1 & 1950 & 487500 & 1050000 & 0.0892 & $3.52 \mathrm{E}-04$ & 2.993 & $6.54 \mathrm{E}-03$ & 18.9 & 97.44 & 74.39 & & 105.9 & 430.2 \\
\hline 902 & 22.39 & 3000 & 1660 & 203.41394 & 5.2824 & 77.906 & 6257.54 & 5125 & 0.1 & 1950 & 487500 & 737500 & 0.1421 & $1.56 \mathrm{E}-04$ & 1.555 & $7.81 \mathrm{E}-03$ & 40.44 & 97.54 & 75.42 & & 88.8 & 356 \\
\hline 903 & 40.32 & 3000 & 6400 & 309.637574 & 8.7522 & 84.7981 & 5748.95 & 5125 & 0.1 & 1950 & 487500 & 837500 & 0.0789 & $5.82 \mathrm{E}-04$ & 4.732 & $4.26 \mathrm{E}-03$ & 60.55 & 98.39 & 83.85 & 0 & 162.9 & 601.4 \\
\hline 904 & 30.33 & 3000 & 2780 & 22.162366 & 1.1836 & 160.2148 & 3042.79 & 5125 & 0.1 & 1950 & 487500 & 2175000 & 0.1049 & $2.40 \mathrm{E}-04$ & 2.157 & $1.50 \mathrm{E}-02$ & 56.52 & 77.44 & 0 & 0 & 46.1 & 364.5 \\
\hline 905 & 58.37 & 3000 & 3730 & 1570.135536 & 54.8291 & $\begin{array}{l}104.7599 \\
\end{array}$ & 4653.5 & 5125 & 0.1 & 1950 & 487500 & 1150000 & 0.0545 & $2.13 \mathrm{E}-04$ & 2.973 & $2.52 \mathrm{E}-03$ & 43.74 & 99.68 & 96.82 & 68.16 & 274.9 & 261 \\
\hline 906 & 38.28 & 3000 & 4130 & 208.219783 & 7.6353 & 110.0086 & 4431.47 & 5125 & 0.1 & 1950 & 487500 & 1237500 & 0.0831 & $3.51 \mathrm{E}-04$ & 3.137 & $6.58 \mathrm{E}-03$ & 22.18 & 97.6 & 75.99 & 0 & 105.3 & 419.9 \\
\hline 907 & 30.71 & 3000 & 1050 & 302.202739 & 13.2203 & $\begin{array}{l}131.239 \\
\end{array}$ & 3714.6 & 5125 & 0.1 & 1950 & 487500 & 1612500 & 0.1036 & $8.69 \mathrm{E}-05$ & 1.424 & 9.93E-03 & 43.85 & 98.35 & 83.45 & & 69.8 & 237.7 \\
\hline 908 & 26.25 & 3000 & 3180 & 130.443272 & 4.943 & 113.6821 & 4288.27 & 5125 & 0.1 & 1950 & 487500 & 1300000 & 0.1212 & $3.28 \mathrm{E}-04$ & 2.369 & $7.03 \mathrm{E}-03$ & 25.33 & 96.17 & 61.67 & & 98.6 & 462.6 \\
\hline 909 & 36.23 & 3000 & 5090 & 0.826232 & 0.0305 & 110.7481 & 4401.88 & 5125 & 0.1 & 1950 & 487500 & 1250000 & 0.0878 & $6.00 \mathrm{E}-04$ & 4.455 & $1.58 \mathrm{E}-02$ & 30.22 & 0 & & & 43.8 & 630.1 \\
\hline 910 & 37.96 & 3000 & 4940 & 488.930382 & 13.4044 & \begin{tabular}{|l|}
82.2476 \\
\end{tabular} & 5927.23 & 5125 & 0.1 & 1950 & & 800000 & & 3.39E-04 & 3.045 & $4.58 \mathrm{E}-03$ & 34.03 & 98.98 & 89.77 & & 151.5 & 411.1 \\
\hline 911 & 31.44 & 3000 & 7360 & 10.831304 & 0.2519 & $\begin{array}{l}69.767 \\
60.925\end{array}$ & 6987.55 & 5125 & 0.1 & 1950 & & 625000 & & 6.85E-04 & 4.421 & $1.08 \mathrm{E}-02$ & 79.08 & 53.84 & & & $\begin{array}{l}64.2 \\
\end{array}$ & 720.7 \\
\hline 912 & 38.56 & 3000 & 5890 & 49.762547 & & 93.8425 & 5194.88 & 5125 & 0.1 & 1950 & 487500 & 975000 & 0.0825 & $5.23 \mathrm{E}-04$ & 4.205 & & 18.44 & 89.95 & & 0 & 89 & 558.9 \\
\hline 913 & 29.48 & 3000 & 3470 & $\begin{array}{r}27.310058 \\
\end{array}$ & 0.9743 & 107.0251 & 4555.01 & 5125 & 0.1 & 1950 & 487500 & 1187500 & 0.1079 & $2.70 \mathrm{E}-04$ & 2.267 & $1.38 \mathrm{E}-02$ & & 81.69 & 0 & 0 & 50.1 & 394 \\
\hline 914 & 24.12 & 3000 & 3260 & \begin{tabular}{|l|}
243.857534 \\
\end{tabular} & $\begin{array}{r}9.2999 \\
18.5751\end{array}$ & 114.4096 & 4261 & 5125 & 0.1 & 1950 & 487500 & 1312500 & 0.1319 & $3.10 \mathrm{E}-04$ & 2.189 & $5.59 \mathrm{E}-03$ & & 97.95 & 79.5 & 0 & 124.1 & 465.1 \\
\hline 915 & 36.52 & 3000 & 1240 & \begin{tabular}{|r|}
383.998231 \\
\end{tabular} & 18.5754 & $\begin{array}{l}145.1211 \\
123691 \\
\end{array}$ & $\begin{array}{r}3359.26 \\
3012 \\
\end{array}$ & 5125 & 0.1 & 1950 & & 1875000 & 0.0871 & $1.12 \mathrm{E}-04$ & 1.653 & \begin{tabular}{|c|}
$9.12 \mathrm{E}-03$ \\
\end{tabular} & 42.67 & $\begin{array}{r}98.7 \\
07.5 .5\end{array}$ & 86.98 & 0 & 76 & 231.9 \\
\hline $\begin{array}{l}916 \\
917 \\
\end{array}$ & $\begin{array}{l}27.69 \\
255 \\
\end{array}$ & $\begin{array}{l}3000 \\
3000\end{array}$ & 2610 & \begin{tabular}{|l|}
232.4814111 \\
548417195
\end{tabular} & \begin{tabular}{r|}
9.5835 \\
190117
\end{tabular} & $\begin{array}{l}123.6681 \\
103994 \\
\end{array}$ & $\begin{array}{r}3942 \\
468753 \\
\end{array}$ & $\begin{array}{l}5125 \\
5525\end{array}$ & 0.1 & $\begin{array}{l}1950 \\
1950 \\
\end{array}$ & & & 0.1149 & $2.59 \mathrm{E}-04$ & $\begin{array}{r}2.14 \\
2.371\end{array}$ & & $\begin{array}{l}30.95 \\
38.3 \\
\end{array}$ & & 78.49 & 0 & $\begin{array}{ll}103.9 \\
2137\end{array}$ & 396.1 \\
\hline \begin{tabular}{|l|l|}
918 \\
918
\end{tabular} & $\begin{array}{r}22.55 \\
48.5 \\
\end{array}$ & 3000 & $\begin{array}{l}4760 \\
6350\end{array}$ & \begin{tabular}{|r|}
548.417195 \\
29189257
\end{tabular} & $\begin{array}{c}19.0117 \\
07919\end{array}$ & $\begin{array}{r}103.9994 \\
813866\end{array}$ & $\begin{array}{l}4687.53 \\
598978\end{array}$ & $\begin{array}{l}5125 \\
5125\end{array}$ & 0.1 & $\begin{array}{c}1950 \\
11950 \\
\end{array}$ & $\begin{array}{l}487500 \\
487500\end{array}$ & $\begin{array}{r}1137500 \\
787500 \\
\end{array}$ & 0.1411 & $\begin{array}{r}3.83 \mathrm{E}-04 \\
5.0 \mathrm{E}-04 \\
\end{array}$ & 2.374 & & $\begin{array}{r}38.3 \\
63.39\end{array}$ & 99.09 & 90.88 & 8.83 & $213.7 \mid$ & 539.6 \\
\hline 919 & $\begin{array}{r}48.5 \\
40.79 \\
\end{array}$ & 3000 & $\begin{array}{r}6350 \\
780\end{array}$ & \begin{tabular}{|l|}
29.189 \\
944.861
\end{tabular} & $\begin{array}{l}0.19919 \\
37.618 \\
\end{array}$ & \begin{tabular}{|r}
81.13886 \\
119.4396
\end{tabular} & $\begin{array}{l}5989.18 \\
4081.56\end{array}$ & $\begin{array}{l}\mid 5125 \\
5125\end{array}$ & $\begin{array}{l}0.1 \\
0.1\end{array}$ & $\begin{array}{l}1950 \\
1950\end{array}$ & $487 !$ & $\begin{array}{r}887500 \\
1400000\end{array}$ & \begin{tabular}{|l}
0.0666 \\
0.078
\end{tabular} & $\begin{array}{l}5.10 \mathrm{E}-04 \\
7.20 \mathrm{E}-05\end{array}$ & \begin{tabular}{|l|l}
4.499 \\
1.467
\end{tabular} & \begin{tabular}{|l|}
$1.14 \mathrm{E}-02$ \\
$6.34 \mathrm{E}-03$ \\
\end{tabular} & $\begin{array}{l}63.39 \\
35.12\end{array}$ & $\begin{array}{l}82.88 \\
99.47\end{array}$ & \begin{tabular}{r|}
0 \\
94.71
\end{tabular} & $\begin{array}{r}0 \\
47.08\end{array}$ & $\begin{array}{r}60.8 \\
109.3\end{array}$ & $\begin{array}{l}\frac{520.9}{184.3} \\
\end{array}$ \\
\hline 920 & 24.23 & 3000 & 3510 & 16.923045 & & 82.2476 & 5927.23 & 5125 & 0.1 & 1950 & & & 0.1313 & $2.95 \mathrm{E}-04$ & 2.134 & \begin{tabular}{|l|l|}
$1.32 \mathrm{E}-02$ \\
\end{tabular} & 71.53 & 70.45 & & & 52.3 & 451.3 \\
\hline 921 & 28.74 & 3000 & 1960 & 207.56 & & 100.929 & 4830.13 & 5125 & 0.1 & 1950 & & 1087500 & 0.1107 & 1.87E-04 & 1.852 & $8.40 \mathrm{E}$ & 39. & 97.5 & 75.91 & 0 & 82.6 & 330.2 \\
\hline 922 & 30.36 & & 4560 & 65.089675 & 2.4029 & 110.7481 & 4401.88 & 5125 & 0. & 1950 & & & & 3.42 & 2.651 & & 79.7 & & 23.18 & & 69.9 & 447.6 \\
\hline 923 & 22.29 & 3000 & 4240 & 12.718571 & 0.5448 & $\mid 128.5118$ & 3793.42 & 5125 & 0.1 & 1950 & & 1562500 & & $4.94 \mathrm{E}-04$ & 2.751 & & 36.1 & & & 0 & 72.6 & 632.4 \\
\hline 924 & 33.1 & 3000 & 640 & 589.047399 & 20.7183 & 105.5176 & 4620.08 & 5125 & 0.1 & 1950 & 487 & 1162500 & 0.0961 & $5.03 \mathrm{E}-05$ & 1.265 & $8.42 \mathrm{E}$ & 30.34 & 99.15 & 91.51 & 15.12 & 82.4 & 195.8 \\
\hline 925 & 23.64 & 3000 & 1550 & 421.184601 & 26.06 & \begin{tabular}{|l|l|}
185.6278 \\
\end{tabular} & 2626.22 & 5125 & 0.1 & 1950 & 487 & 2712500 & 0.1346 & $1.22 \mathrm{E}-04$ & 1.457 & \begin{tabular}{|c|}
$6.40 \mathrm{E}-03$ \\
\end{tabular} & 52.12 & 98.81 & 88.13 & & 108.4 & 315.9 \\
\hline 926 & 43.46 & 3000 & 3400 & 296.561256 & 15.3486 & 155.2656 & 3139.78 & 5125 & 0.1 & 1950 & 487 & 2075000 & 0.0732 & $3.02 \mathrm{E}-04$ & 3.088 & 6.75E-03 & 44.6 & 98.31 & 83.14 & 0 & 102.7 & 364.1 \\
\hline 927 & 36.23 & 3000 & 1630 & 113.465825 & 5.36 & 141.878 & 3436.05 & 5125 & 0.1 & 1950 & & 1812500 & 0.0878 & $1.39 \mathrm{E}-04$ & 1.798 & $1.37 \mathrm{E}-02$ & 57. & 95.59 & 55.93 & & 50.5 & 254.3 \\
\hline 928 & 44.87 & 3000 & 4570 & & 2.49 & 158.3679 & 3078.28 & 5125 & 0.1 & 1950 & & & 0.0709 & & 6.229 & 7.23 & 51.49 & 89.42 & & & 95.9 & 711.5 \\
\hline 929 & 21.28 & 30 & 2040 & 16.58 & & $\begin{array}{l}124.3658 \\
\end{array}$ & 3919.89 & 5125 & 0.1 & 1950 & & & 0.1495 & $2.53 \mathrm{E}$ & 1.856 & & 24.27 & 69.86 & & & 57 & 447.1 \\
\hline 930 & 63.12 & 3000 & 1190 & 282.16 & 7.8 & 83.1 & 5866 & 5125 & 0.1 & 195 & & & & & 2.373 & & 17. & 98.23 & 82.28 & 0 & 55.4 & 192.6 \\
\hline 931 & 32.23 & 3000 & 1140 & 536.7 & 23.8 & 133.2 & & 5125 & 0.1 & 195 & & & & & 1.539 & & 38. & 99.07 & 90.68 & 6.84 & 96.6 & 244.7 \\
\hline 932 & 31.41 & 30 & 3320 & & & 152.7 & 319 & 5125 & 0.1 & 195 & & 202 & & & 2.2 & & 74. & 93 & 39.21 & 0 & 64.3 & 373.7 \\
\hline 933 & & 30 & 2690 & & & 148.5 & & 5125 & 0.1 & 195 & & & & & 2.343 & & 30 & 16.42 & & & 42.6 & 414.8 \\
\hline 934 & 24.34 & 3000 & 690 & & & 119.4 & & 5125 & 0.1 & 195 & & & & & 1.296 & & & 97. & 75.69 & & 69.9 & 272.9 \\
\hline 935 & 21.61 & 3000 & 780 & & & & 498 & 5125 & 0.1 & 195 & & & & & & & & & 10.99 & & 52.3 & \\
\hline 936 & & & 4110 & & & & & & 0.1 & 19 & & & & & 2.861 & & & & 0.14 & & & \\
\hline 937 & 37.25 & & 3310 & & $\frac{0.40}{17.10}$ & 172.2 & & 5 & 0.1 & 19 & & & & & & & & 29.4 & & & & 7.1 \\
\hline 938 & 25.19 & 3000 & 1680 & 454. & & 112.6 & & 125 & 0.1 & 19 & & & & & 1.7 & & & & 88.99 & 0 & 128.8 & 56.6 \\
\hline 939 & 27.33 & 3000 & 3010 & & & 112.9 & 4315. & 5125 & 0.1 & 1950 & & 1287500 & & & 2.168 & & & 50.68 & & 0 & 43 & 406.5 \\
\hline 940 & 21.34 & 3000 & 1010 & 73.2338 & 3.755 & 154.01 & & 5125 & 0.1 & 195 & & & & & & & & 93.17 & 31.73 & 0 & 60.8 & 332.6 \\
\hline 941 & 44.12 & 3000 & 2470 & \begin{tabular}{|l}
346.315204 \\
\end{tabular} & 11.3817 & $\begin{array}{l}98.5952 \\
\end{array}$ & 4944.46 & 5125 & 0.1 & 195 & & & 0.0721 & $1.73 \mathrm{E}-04$ & 2.213 & $8.85 \mathrm{E}-$ & 57.38 & 98.56 & 85.56 & & 78.3 & 57.1 \\
\hline 942 & 61.06 & 3000 & 6290 & \begin{tabular}{|r|}
282.499091 \\
\end{tabular} & 7.9055 & 83.9523 & 5806.87 & 5125 & 0.1 & 1950 & 487500 & 825 & 0.0521 & 4.82E-04 & 5.68 & $5.86 \mathrm{E}-03$ & 69.76 & 98.23 & 82.3 & & 118.3 & $\begin{array}{l}76.7 \\
6.9 .1\end{array}$ \\
\hline 943 & 28.1 & 3000 & 4360 & $\begin{array}{r}2.688336 \\
\end{array}$ & 0.107 & 119.4396 & 4081.56 & 5125 & 0.1 & 1950 & 487500 & 1400000 & 0.1132 & 4.61E-04 & 3.061 & 1.56E-02 & 57.22 & 0 & 0 & 0 & $\begin{array}{l}44.5 \\
-77.1\end{array}$ & 558.1 \\
\hline $\begin{array}{c}944 \\
945 \\
\end{array}$ & $\begin{array}{l}42.93 \\
3213\end{array}$ & 3000 & $\begin{array}{r}930 \\
5610\end{array}$ & \begin{tabular}{|r|}
616.352643 \\
345037
\end{tabular} & 22.4489 & $\begin{array}{r}109.2665 \\
88961\end{array}$ & $\begin{array}{l}4461.57 \\
547961\end{array}$ & $\begin{array}{l}5125 \\
5125\end{array}$ & 0.1 & $\begin{array}{l}1950 \\
1900 \\
\end{array}$ & $\begin{array}{l}487500 \\
87500\end{array}$ & 1225000 & 0.0741 & $\begin{array}{r}7.42 \mathrm{E}-05 \\
4.35 \mathrm{E}-04 \\
\end{array}$ & $\begin{array}{l}1.507 \\
3.357 \\
\end{array}$ & $\begin{array}{l}9.00 E-03 \\
168 E-02\end{array}$ & \begin{tabular}{rl|}
47.82 \\
56.3
\end{tabular} & $\begin{aligned} 99.19 \\
0\end{aligned}$ & 91.89 & 18.88 & & $\begin{array}{l}179.8 \\
5041\end{array}$ \\
\hline 945 & 34.13 & 3000 & 5610 & 3.45037 & 0.1023 & 88.9661 & 5479.61 & 5125 & 0.1 & 1950 & 487500 & 900000 & & $4.35 \mathrm{E}-04$ & 3.357 & 1.68E-02 & 56.3 & & & & 41.3 & 504.1 \\
\hline
\end{tabular}




\begin{tabular}{|c|c|c|c|c|c|c|c|c|c|c|c|c|c|c|c|c|c|c|c|c|c|c|}
\hline Realization & $\begin{array}{c}\text { Velocity } \\
(\mathrm{m} / \mathrm{y})\end{array}$ & $\begin{array}{l}\text { Fixed Source } \\
\text { Concentration } \\
(\mathrm{mg} / \mathrm{L})\end{array}$ & $\begin{array}{c}\text { Time of } \\
\text { Maximum } \\
\text { at } \\
\text { Compliance } \\
\text { Boundary } \\
\text { (y) }\end{array}$ & $\begin{array}{c}\text { Maximum } \\
\text { Concentration } \\
\text { Compliance } \\
\text { Boundary } \\
(\mu \mathrm{g} / \mathrm{L})\end{array}$ & $\begin{array}{c}\text { Maximum } \\
\text { Release Rate } \\
\text { at } \\
\text { Compliance } \\
\text { Boundary } \\
\text { (Kg/y) }\end{array}$ & \begin{tabular}{|l|} 
\\
\\
Sourc \\
e Rate \\
(Kg/y)
\end{tabular} & $\begin{array}{c}\text { Source } \\
\text { Duration } \\
\text { (y) }\end{array}$ & $\begin{array}{c}\text { 1-D } \\
\text { Streamtube } \\
\text { Length } \\
(\mathrm{m})\end{array}$ & $\begin{array}{c}\text { Base } \\
\text { Porosity } \\
\left(\mathrm{m}^{3} / \mathrm{m}^{3}\right)\end{array}$ & $\begin{array}{c}\text { Bulk } \\
\text { Densty } \\
\left(\mathrm{Kg} / \mathrm{m}^{3}\right)\end{array}$ & \begin{tabular}{|c|} 
Remaining \\
Carbon \\
Tetrachloride \\
Source \\
$(\mathrm{Kg})$
\end{tabular} & $\begin{array}{c}\text { Volume at or } \\
\text { above Fixed } \\
\text { Source } \\
\text { Concentratio } \\
n(m 3)\end{array}$ & $\begin{array}{l}\text { Porosity } \\
\left(\mathrm{m}^{3} / \mathrm{m}^{3}\right)\end{array}$ & $\begin{array}{c}\mathrm{Kd} \\
\left(\mathrm{m}^{3} / \mathrm{kg}\right)\end{array}$ & Retardation & \begin{tabular}{|c|} 
First \\
Order \\
Rate \\
Constant \\
$(1 / y)$ \\
\end{tabular} & $\begin{array}{l}\text { Longitudinal } \\
\text { Dispersivity } \\
(\mathrm{m})\end{array}$ & $\begin{array}{c}\text { Required } \\
\text { Source } \\
\text { Cleanup } \\
\text { Percentage } \\
(5 \mu \mathrm{g} / \mathrm{L} \\
\text { Compliance) }\end{array}$ & $\begin{array}{c}\text { Required } \\
\text { Source } \\
\text { Cleanup } \\
\text { Percentage } \\
(50 \mu \mathrm{g} / \mathrm{L} \\
\text { Compliance) }\end{array}$ & \begin{tabular}{|c} 
Required \\
Source Cleanup \\
Percentage \\
$(500 \mu \mathrm{g} / \mathrm{L}$ \\
Compliance)
\end{tabular} & \begin{tabular}{|c|} 
Abiotic \\
Reaction \\
Half Time \\
(y)
\end{tabular} & $\begin{array}{c}\text { Carbon } \\
\text { Tetrachloride } \\
\text { Travel Time } \\
\text { (y) }\end{array}$ \\
\hline & & & & & & & \begin{tabular}{|l|l|}
11 \\
\end{tabular} & & & & & & & & 5.208 & \begin{tabular}{|c|}
$1.24 \mathrm{E}-02$ \\
\end{tabular} & & & & & & \\
\hline & 22.15 & 3000 & 1190 & 0.883402 & 0.0183 & \begin{tabular}{|l|}
62.11 \\
\end{tabular} & 120.75 & 5125 & 0.1 & 1950 & 7500 & 525000 & 0.1436 & 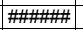 & 2.853 & $1.23 \mathrm{E}-02$ & 76.26 & 0 & & 0 & 56.2 & 660 \\
\hline 3 & 30.44 & 3000 & 570 & 23.721629 & 1.0907 & \begin{tabular}{|l|}
137.9 \\
\end{tabular} & \begin{tabular}{|l|}
54.37 \\
\end{tabular} & 5125 & 0.1 & 1950 & 7500 & 1737500 & 0.1045 & \#\#\#\#! & 1.975 & \begin{tabular}{|c|}
$1.04 \mathrm{E}-02$ \\
\end{tabular} & 55.4 & 78.92 & 0 & 0 & 66.7 & 332.4 \\
\hline 4 & 22.99 & 3000 & 620 & 89.847916 & 5.0881 & \begin{tabular}{|l|}
169.9 \\
\end{tabular} & 44.15 & 5125 & 0.1 & 1950 & 7500 & 2375000 & 0.1384 & \#\#\#+1 & 1.704 & \begin{tabular}{|l|l|}
$5.00 \mathrm{E}-03$ \\
\end{tabular} & 35.33 & 94.44 & 44.35040652 & & 138.6 & 379.8 \\
\hline & 45.25 & 3000 & 1230 & 5.966712 & 0.185 & \begin{tabular}{|l|}
93.04 \\
\end{tabular} & 80.61 & 5125 & 0.1 & 1950 & 7500 & 962500 & 0.0703 & 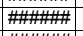 & 4.023 & \begin{tabular}{|c|}
$1.02 \mathrm{E}-02$ \\
\end{tabular} & 63.48 & 16.2 & & & 67.8 & 455.6 \\
\hline & 35.04 & 3000 & 1670 & 6.154983 & 0.3262 & 159 & 47.17 & 5125 & 0.1 & 1950 & 7500 & 2150000 & 0.0908 & \#\#\#\#+ & 3.891 & \begin{tabular}{|l|l|}
$6.07 \mathrm{E}-03$ \\
\end{tabular} & 48.82 & 18.77 & & & 114.2 & 569.2 \\
\hline & 59.13 & 3000 & 870 & 39.52725 & 1.6569 & 125.8 & 59.64 & 5125 & 0.1 & 1950 & 7500 & 1512500 & 0.0538 & \#\#\#\#+ & 3.363 & \begin{tabular}{|l|l|}
$8.53 \mathrm{E}-03$ \\
\end{tabular} & 33 & 87.35 & & & 81.2 & 291.5 \\
\hline 8 & 49.32 & 3000 & 2090 & 1.432062 & 0.0525 & 110 & 68.18 & 5125 & 0.1 & 1950 & 7500 & 1237500 & 0.0645 & 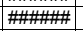 & 5.636 & \begin{tabular}{|c|}
$9.74 \mathrm{E}-03$ \\
\end{tabular} & 44.48 & & & 0 & 71.2 & 585.6 \\
\hline 9 & 26.53 & 3000 & 980 & 4.036136 & 0.1559 & \begin{tabular}{|l|}
115.9 \\
\end{tabular} & 64.73 & 5125 & 0.1 & 1950 & 7500 & 1337500 & 0.1199 & 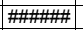 & 2.608 & $1.02 \mathrm{E}-02$ & 62.53 & & & 0 & 68.1 & 503.7 \\
\hline 10 & 29.57 & 3000 & 1710 & 32.54681 & 1.1692 & 107.8 & 69.59 & 5125 & 0.1 & 1950 & 7500 & 1200000 & 0.1076 & \#\#\#\#+ & 3.384 & $3.45 \mathrm{E}-03$ & 39.84 & 84.64 & & & 201.2 & 586.6 \\
\hline & 28.46 & 3000 & 1260 & & 1.0866 & 149 & 50.35 & 5125 & 0.1 & 1950 & 7500 & 1950000 & & \#\#\#\#\#+ & 2.788 & & 29.73 & 77.15 & & 0 & 131.5 & 502.1 \\
\hline & 21.41 & 3000 & 480 & 105.021888 & 2.634 & \begin{tabular}{|l|l|}
75.24 \\
\end{tabular} & 99.68 & 5125 & 0.1 & 1950 & 7500 & 700000 & 0.1486 & \#\#\#\#+ & 1.399 & $8.58 \mathrm{E}-03$ & 42.84 & $\begin{array}{l}95.24 \\
\end{array}$ & 52.39 & & 80.8 & 334.8 \\
\hline 13 & 62.5 & 3000 & 2320 & & 0.0116 & \begin{tabular}{|l|l|}
144.5 \\
\end{tabular} & 51.91 & 5125 & 0.1 & 1950 & 7500 & 1862500 & & \#\#\#\#+\#| & & & 49.38 & & & 0 & 50.8 & 630.6 \\
\hline & 28.87 & 3000 & 2170 & & $\begin{array}{l}0.0456 \\
\end{array}$ & \begin{tabular}{|l|l|}
110.7 \\
\end{tabular} & & 5125 & 0.1 & 1950 & 7500 & 1250000 & & \#\#\#\#曲 & 4.027 & $7.91 \mathrm{E}-03$ & 33.57 & & & 0 & 87.7 & 714.9 \\
\hline 15 & & 3000 & 1540 & & 0.0781 & \begin{tabular}{|l|l|}
118.7 \\
\end{tabular} & 63.17 & 5125 & 0.1 & 1950 & 7500 & 1387500 & & \#\#\#\#曲 & 4.236 & $1.04 \mathrm{E}-02$ & 47.28 & & & 0 & 67 & 529.6 \\
\hline & 37.83 & 3000 & 520 & \begin{tabular}{|l|l|}
22.384403 \\
\end{tabular} & 12.5277 & \begin{tabular}{|l|l|}
116.6 \\
\end{tabular} & 64.33 & 5125 & 0.1 & 1950 & 7500 & 1350000 & 0.0841 & \#\#\#\#+ & 1.989 & $3.20 \mathrm{E}-03$ & 55.5 & 98.45 & 84.49 & 0 & 216.6 & 269.5 \\
\hline & 59.24 & 3000 & 580 & 127.990848 & 6.1914 & \begin{tabular}{|l|l|}
145.1 \\
\end{tabular} & 51.68 & 5125 & 0.1 & 1950 & 7500 & & & \#\#\#\#+ & 2.697 & $6.33 \mathrm{E}-03$ & 41.22 & $\begin{array}{l}96.09 \\
\end{array}$ & 60.93 & & 109.5 & 233.3 \\
\hline & 52.5 & 3000 & 600 & 45.266497 & 1.7915 & \begin{tabular}{|l|l|}
118.7 \\
\end{tabular} & 63.17 & 5125 & 0.1 & 1950 & 7500 & 1387500 & 0.0606 & \#\#\#\#+ & 2.617 & $1.12 \mathrm{E}-02$ & 39.63 & 88.95 & & 0 & 62.2 & 255.5 \\
\hline 19 & 29.59 & 3000 & 250 & 280.884936 & $\begin{array}{l}12.7906 \\
\end{array}$ & \begin{tabular}{|l|l|}
136.6 \\
\end{tabular} & 54.9 & 5125 & 0.1 & 1950 & 7500 & 1712500 & 0.1075 & \#\#\#\#+ & 1.153 & \begin{tabular}{|c|}
$9.82 \mathrm{E}-03$ \\
\end{tabular} & 41.91 & 98.22 & 82.2 & & 70.6 & $\begin{array}{ll}199.7 \\
\end{array}$ \\
\hline 20 & 21.6 & 3000 & $\begin{array}{r}730 \\
1970 \\
\end{array}$ & $\begin{array}{r}1.998832 \\
\end{array}$ & $\begin{array}{l}0.1244 \\
\end{array}$ & \begin{tabular}{|l|}
186.8 \\
161.
\end{tabular} & 40.16 & 5125 & 0.1 & 1950 & 7500 & 2737500 & 0.1473 & \#\#\#+ & 1.901 & $1.28 \mathrm{E}-02$ & 42.14 & & & 0 & 54.2 & 451 \\
\hline $\begin{array}{ll}21 \\
22\end{array}$ & $\frac{31.69}{261}$ & 3000 & \begin{tabular}{r|r|}
1970 \\
970
\end{tabular} & $\begin{array}{r}2.397108 \\
355148 \\
\end{array}$ & $\begin{array}{r}0.129 \\
\end{array}$ & \begin{tabular}{|l|}
161.4 \\
104.8 \\
\end{tabular} & \begin{tabular}{ll|l}
46.46 \\
71.599
\end{tabular} & $\frac{5125}{5125}$ & 0.1 & $\begin{array}{c}1950 \\
11950 \\
\end{array}$ & 7500 & $\begin{array}{l}2200000 \\
1150000\end{array}$ & 0.1004 & 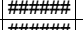 & $\begin{array}{l}3.993 \\
2.252 \\
\end{array}$ & \begin{tabular}{|l|}
$6.88 \mathrm{E}-03$ \\
$118 \mathrm{E}-02$
\end{tabular} & 37.29 & & & 0 & \begin{tabular}{r|r|}
100.7 \\
58.5
\end{tabular} & 645.8 \\
\hline 23 & $\frac{20.1}{22.18}$ & 3000 & 600 & $\begin{array}{r}3.5517448 \\
18.3426\end{array}$ & \begin{tabular}{|c|}
0.924 \\
0.9569
\end{tabular} & \begin{tabular}{|l|}
104.8 \\
156.5 \\
\end{tabular} & $\begin{array}{l}1.59 \\
7.92 \\
\end{array}$ & $\begin{array}{l}5125 \\
5125 \\
\end{array}$ & $\begin{array}{l}0.1 \\
0.1\end{array}$ & $\begin{array}{l}1950 \\
1950\end{array}$ & $\begin{array}{l}7500 \\
7500\end{array}$ & $\begin{array}{l}11150000 \\
2100000\end{array}$ & $\begin{array}{l}.1279 \\
0.1434 \\
\end{array}$ & 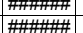 & $\begin{array}{l}2.352 \\
1.682 \\
\end{array}$ & \begin{tabular}{|l|}
$1.18 \mathrm{E}-02$ \\
$9.48 \mathrm{E}-03$ \\
\end{tabular} & $\frac{27.13}{42.19}$ & $\begin{array}{r}0 \\
72.74 \\
\end{array}$ & & $\frac{0}{0}$ & $\begin{array}{l}58.5 \\
73.1 \\
\end{array}$ & $\begin{array}{l}461.8 \\
388.6 \\
\end{array}$ \\
\hline 24 & 28.87 & 3000 & 1520 & 15.087007 & 0.6078 & \begin{tabular}{|l|}
120.9 \\
\end{tabular} & 62.06 & 5125 & 0.1 & 1950 & 7500 & 1425000 & 0.1 & "\#mmm & 3.258 & \begin{tabular}{|l|}
$4.93 \mathrm{E}-03$ \\
\end{tabular} & 49.66 & 66.86 & & & 140.7 & 578.4 \\
\hline 25 & 29 & 3000 & 1800 & 19.491104 & 0.7989 & 123 & 60.99 & 5125 & 0.1 & 1950 & 7500 & 1462500 & 0.1 & "\#mmm & 3.519 & \begin{tabular}{|l|}
$3.73 \mathrm{E}-03$ \\
\end{tabular} & 46.41 & 74.35 & & & 185.8 & 622 \\
\hline 26 & 22.76 & 3000 & 2610 & 16.571652 & 0.5532 & \begin{tabular}{|l|}
100.2 \\
\end{tabular} & 74.88 & 5125 & 0.1 & 1950 & 7500 & 1075000 & 0.1 & 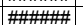 & 3.771 & 2.71 & 45.07 & 69.83 & & & 256.3 & 849.3 \\
\hline & 43.28 & 3000 & 1280 & 448816 & 0.3268 & \begin{tabular}{|l|}
93.84 \\
\end{tabular} & 79.92 & 5125 & 0.1 & 1950 & 7500 & 975000 & 0.0 & 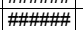 & 3.53 & \begin{tabular}{|l|}
$9.45 \mathrm{E}-03$ \\
\end{tabular} & 26. & 52.15 & & & 73.3 & 417.9 \\
\hline 28 & 21.94 & 3000 & 420 & 29.033846 & 1.7632 & \begin{tabular}{|l|}
182.2 \\
\end{tabular} & 41.17 & 5125 & 0.1 & 1950 & 7500 & 2637500 & & \#\#\#\#! & 1.361 & \begin{tabular}{|l|}
$1.12 \mathrm{E}-02$ \\
\end{tabular} & & 82.78 & & & 61.9 & 317.9 \\
\hline 29 & 45.38 & 3000 & 360 & \begin{tabular}{|l|}
286.734489 \\
\end{tabular} & 12.3483 & \begin{tabular}{|l|}
129.2 \\
\end{tabular} & 58.05 & 5125 & 0.1 & 1950 & 7500 & 1575000 & & \#\#\#\#+ & 1.794 & \begin{tabular}{|l|}
$6.46 \mathrm{E}-03$ \\
\end{tabular} & 58. & 98.26 & 82.56 & & 107.3 & 202 \\
\hline 30 & 34.81 & 3000 & 470 & \begin{tabular}{|r|}
10.735165 \\
\end{tabular} & 0.3803 & \begin{tabular}{|l|}
106.3 \\
\end{tabular} & 70.57 & 5125 & 0.1 & 1950 & 7500 & 1175000 & 0.0 & \#\#\#\#+ & 2.034 & \begin{tabular}{|l|}
$1.66 \mathrm{E}-02$ \\
\end{tabular} & 85.44 & 53.42 & & & 41.8 & 299.4 \\
\hline 31 & 25.11 & 3000 & 980 & 20.913635 & 0.6542 & \begin{tabular}{|l|}
93.84 \\
\end{tabular} & 79.92 & 5125 & 0.1 & 1950 & 7500 & 975000 & 0.1 & \#\#\#\#曲 & 2.433 & \begin{tabular}{|c|}
$6.68 \mathrm{E}-03$ \\
\end{tabular} & 67. & 76.09 & & & 103.8 & 496. \\
\hline & 21.44 & 3000 & 880 & 0.878 & 0.039 & \begin{tabular}{|l|}
133.3 \\
\end{tabular} & 56.28 & 5125 & 0.1 & 1950 & 7500 & 1650000 & 0.1 & \#\#\#\#曲 & 2.126 & & 42. & 0 & & 0 & 50.7 & 508. \\
\hline 33 & 49.71 & 3000 & 410 & \begin{tabular}{|l|}
166.471504 \\
\end{tabular} & 7.0549 & \begin{tabular}{|l|}
127.1 \\
\end{tabular} & 58.99 & 5125 & 0.1 & 1950 & 7500 & 1537500 & & \#\#\#\#曲 & 2.015 & & 34. & 97 & 69.96 & 0 & 74.7 & \\
\hline 34 & 31.72 & 3000 & 820 & 20.49509 & 1.022 & \begin{tabular}{|l|}
149.6 \\
\end{tabular} & 50.13 & 5125 & 0.1 & 1950 & 7500 & & & & 2.374 & & & 75.6 & & 0 & 83.1 & 383. \\
\hline & 32.56 & 3000 & 1620 & & 0.1 & \begin{tabular}{|l|}
133.9 \\
\end{tabular} & 56 & 512 & 0.1 & & & & & & & & & & & 0 & 79.4 & 574.3 \\
\hline 36 & & 30 & 2090 & & 0.3 & \begin{tabular}{|l|}
131.9 \\
\end{tabular} & 56.85 & & 0.1 & & & & & & & & & 43.83 & 0 & 0 & 156.6 & \\
\hline 37 & 55.81 & & 260 & 64 & & \begin{tabular}{|l|}
116.6 \\
\end{tabular} & & & 0.1 & & & & & & & & & 99.22 & 92.2 & 21.98 & .2 & \\
\hline 38 & 29.79 & & 590 & & & \begin{tabular}{|l|}
97.81 \\
\end{tabular} & & & 0.1 & & & & & & & & & 97.21 & 72.11 & 0 & 3 & \\
\hline 39 & 24.51 & & 930 & & & \begin{tabular}{|l|}
112.2 \\
\end{tabular} & & & 0.1 & & & & & & & & & & 0 & 0 & & 531.7 \\
\hline 40 & 23.9 & & 620 & & & \begin{tabular}{|l|}
88.14 \\
\end{tabular} & & & 0.1 & & & & & & & & & 88.17 & 0 & 0 & .6 & \\
\hline 41 & 31.91 & & 720 & & & \begin{tabular}{|l|}
91.42 \\
\end{tabular} & & & & & & & & & & & & 74.9 & 0 & 0 & & 389.4 \\
\hline 42 & 25.8 & 3000 & 1250 & & & \begin{tabular}{|l|l|}
138.6 \\
\end{tabular} & & & 0.1 & & & & & & & & & 78.15 & & 0 & 127.6 & 517.9 \\
\hline 43 & 31.53 & 3000 & 570 & & & \begin{tabular}{|l|}
115.1 \\
\end{tabular} & & & & & & & & & & & & & 0 & 0 & 69.3 & 311.2 \\
\hline 44 & 22.5 & 30 & 470 & & & \begin{tabular}{|l|l|}
124.4 \\
\end{tabular} & & & & & & & & & & & & & 0 & 0 & 58.1 & 57.5 \\
\hline 45 & 31.94 & 3000 & $\begin{array}{r}270 \\
1020\end{array}$ & 176.6 & & $\begin{array}{l}123.7 \\
\end{array}$ & & & 0.1 & & 7500 & 1475 & & & & & & $\begin{array}{l}97.17 \\
70.9\end{array}$ & 71.7 & 0 & $\begin{array}{l}61.6 \\
\end{array}$ & 206.5 \\
\hline 46 & 21.97 & 3000 & $\begin{array}{l}1020 \\
950\end{array}$ & & $\begin{array}{l}0.7291 \\
\end{array}$ & \begin{tabular}{|l|l|}
89.79 \\
112
\end{tabular} & 83. & $\begin{array}{l}5125 \\
5125\end{array}$ & 0.1 & & & & 0.1 & & 2.2 & & & 79.48 & 0 & ( & 113.3 & 526.3 \\
\hline $\begin{array}{l}47 \\
48 \\
8\end{array}$ & $\frac{24.28}{26.73}$ & $\frac{3000}{3000}$ & $\begin{array}{r}950 \\
1340 \\
\end{array}$ & $\begin{array}{l}3.029436 \\
8.17853\end{array}$ & \begin{tabular}{|c|}
0.1133 \\
0.3139
\end{tabular} & \begin{tabular}{|l|}
112.2 \\
115.
\end{tabular} & 66.83 & $\begin{array}{l}5125 \\
5125 \\
\end{array}$ & 0.1 & & $\begin{array}{l}7500 \\
7500\end{array}$ & 1275000 & $\begin{array}{l}0.131 \\
0.119\end{array}$ & 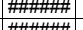 & $\begin{array}{r}2.315 \\
288 \\
\end{array}$ & & 39. & & & $\frac{0}{0}$ & $\begin{array}{l}60.9 \\
9.9 \\
\end{array}$ & $\begin{array}{l}488.5 \\
5521 .\end{array}$ \\
\hline $\begin{array}{l}48 \\
49\end{array}$ & $\frac{26.73}{42.25}$ & $\begin{array}{l}3000 \\
3000\end{array}$ & \begin{tabular}{|l|}
1340 \\
1850
\end{tabular} & $\begin{array}{r}8.17 \\
10.56\end{array}$ & $\frac{0.313}{0.464}$ & \begin{tabular}{|l|}
115.1 \\
131.9 \\
\end{tabular} & $\begin{array}{l}65.14 \\
56.85\end{array}$ & $\begin{array}{l}5125 \\
5125\end{array}$ & $\begin{array}{l}0.1 \\
0.1\end{array}$ & $\frac{19}{19}$ & $\begin{array}{l}7500 \\
77500\end{array}$ & $\frac{1325000}{1625000}$ & $\begin{array}{r}0.119 \\
0.0753 \\
\end{array}$ & 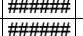 & \begin{tabular}{r|r}
2.38 \\
4.325 \\
\end{tabular} & \begin{tabular}{|l|}
7.07 \\
5.65 \\
\end{tabular} & & $\begin{array}{l}38.86 \\
52.67\end{array}$ & & $\frac{0}{0}$ & \begin{tabular}{r|}
98 \\
122.6
\end{tabular} & $\frac{552 .}{524 .}$ \\
\hline 50 & 40.89 & 300 & 850 & & 0.9 & \begin{tabular}{|l|l|}
151.5 \\
\end{tabular} & $4 !$ & 512 & 0.1 & 19 & 750 & & & "\#mmm & 2.883 & 8.46 & 54 & 72.95 & 0 & 0 & 81.9 & 361 . \\
\hline 51 & 33.99 & 3000 & 1430 & & 0.2 & \begin{tabular}{|l|}
189.6 \\
\end{tabular} & 39. & 512 & 0.1 & & 750 & & & \#\#m & 3.78 & & & & 0 & 0 & 101.3 & 570. \\
\hline 52 & 47.2 & 300 & \begin{tabular}{|l|}
420 \\
\end{tabular} & & 1.6 & \begin{tabular}{|l|}
131.2 \\
\end{tabular} & 57. & 51 & 0.1 & 19 & 750 & & & \#\#曲 & $\begin{array}{l}.104 \\
2.98 \\
\end{array}$ & \begin{tabular}{|l|}
0.04 \\
.67
\end{tabular} & 49 & 86.41 & 0 & 0 & $\begin{array}{l} \\
90.3 \\
\end{array}$ & 323. \\
\hline 53 & 55.14 & & 360 & & & \begin{tabular}{|l|}
118.7 \\
\end{tabular} & & 512 & 0.1 & & 75 & & & & 2.1 & & 73 & 87.6 & 0 & & 39.7 & 19 \\
\hline 54 & 22.22 & 300 & 710 & 34.52 & & \begin{tabular}{|l|}
127.1 \\
\end{tabular} & & 512 & 0.1 & & 75 & & & & & & $2 \varepsilon$ & 85.52 & & 0 & 91.1 & 414. \\
\hline 55 & 25.7 & & 1000 & & & \begin{tabular}{|l|}
106.3 \\
\end{tabular} & 70. & 512 & 0.1 & & 75 & & & & & & 38. & 55.05 & 0 & & 82.2 & 477. \\
\hline 56 & & & 1390 & & & \begin{tabular}{|l|}
152.1 \\
\end{tabular} & & 512 & 0.1 & & 7500 & & & & 4.1 & & 35. & & & & 62.5 & 440. \\
\hline 57 & & & 560 & & & \begin{tabular}{|l|}
176.4 \\
\end{tabular} & & & 0.1 & & 7500 & & & & & & & 94.16 & 41.63 & & 127.5 & 272. \\
\hline 58 & 30. & & 1900 & & & \begin{tabular}{|l|}
93.04 \\
\end{tabular} & & & 0.1 & & 75 & & & & & & & 14.37 & & & 118.1 & 66 \\
\hline 59 & 23.24 & & 830 & & & & & & 0.1 & & & & & & & & & 72.4 & & & 104.8 & \\
\hline 60 & & & 5 & & & \begin{tabular}{|l|}
174.6 \\
\end{tabular} & & & & & & & & & & & & 69.84 & & & 63.2 & \\
\hline 61 & & & 790 & & & & & & & & 50 & & & & & & & 0 & 0 & & 40.2 & \\
\hline 62 & 45.71 & & 2770 & & & \begin{tabular}{|l|}
131.2 \\
\end{tabular} & & 5125 & 0.1 & 1950 & 7500 & & & & 5.954 & & & 0 & 0 & & 92.9 & \\
\hline 63 & 63 & 3000 & 480 & 165.961253 & \begin{tabular}{|l|l|}
7.1093 \\
\end{tabular} & \begin{tabular}{|l|}
128.5 \\
\end{tabular} & 58.36 & 5125 & & 1950 & 7500 & 1562500 & 0.0505 & 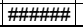 & 2.457 & $8.69 \mathrm{E}-03$ & 30.09 & 96.99 & 69.87 & & 79.8 & 199.9 \\
\hline
\end{tabular}




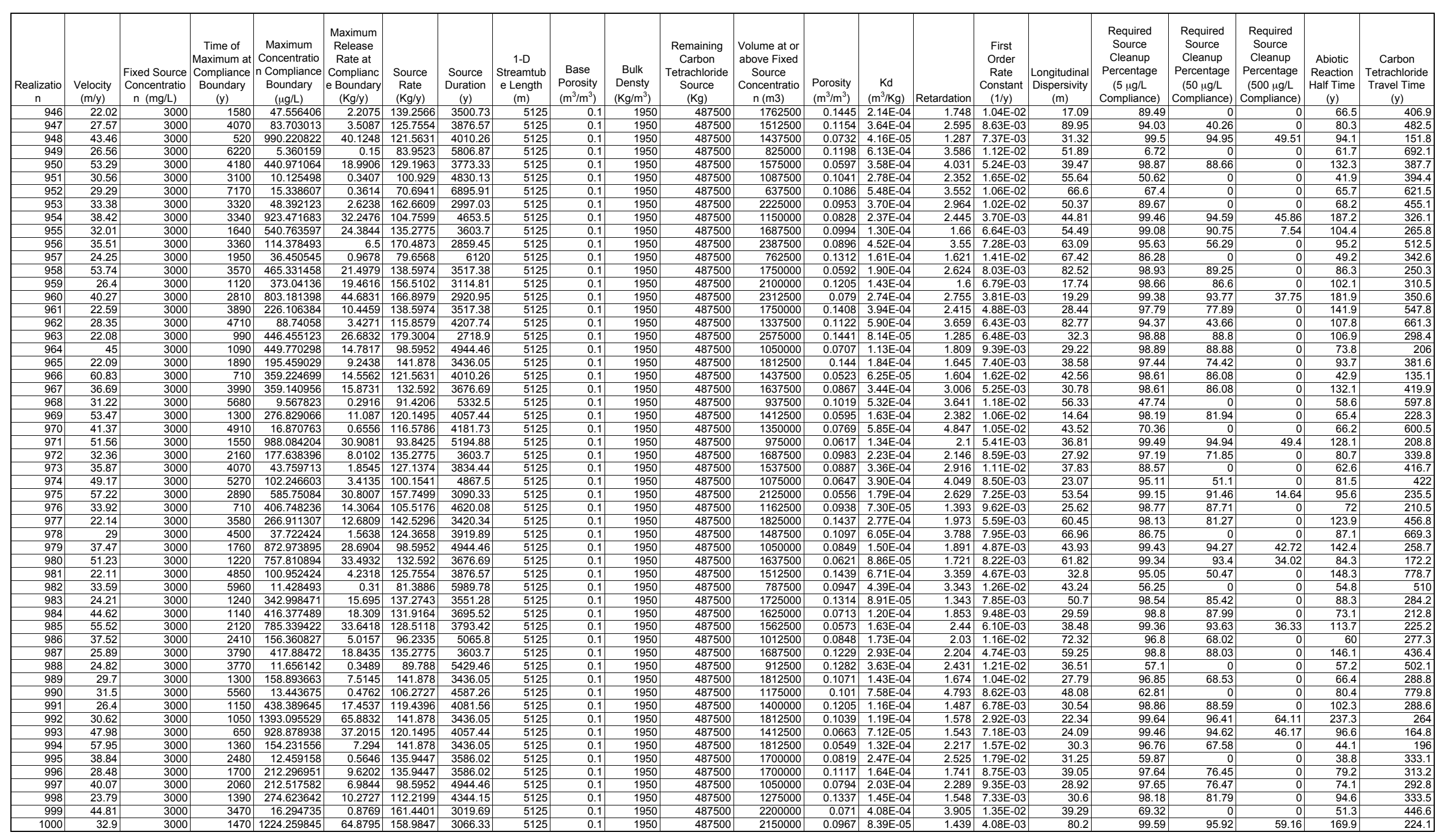




\section{Distribution}

No. of

Copies

OFFSITE

Sandia National Laboratories

P. O. Box 5800

Albuquerque, NM 87185

Attn: M. D. Siegel

Y. McClellan

ONSITE

1 DOE Richland Operations Office
A. C. Tortoso

H0-12

2 CH2M-HILL Hanford, Inc

C. C. Swanson

H9-02

V. J. Rohay

2 Bechtel Hanford Inc.

S. W. Petersen

H0-23

R. L. Jackson

H0-19

H0-19

1 U. S. Environmental Protection Agency

D. A. Faulk B5-01

Distr.1

No. of

Copies

59 Pacific Northwest National Laboratory
S. Q. Bennett
K7-90
R. J. Cameron
K6-96
K. J. Cantrell
K6-81
C. R. Cole
K9-36
C. D. Johnson
K2-10
M. D. Johnson
K6-96
C. J. Murray
K6-81
M. J. Truex
K6-96
Information Release (5)
K1-06 